Key Words:

high-level waste waste loading DWPF

EM

Retention:

Permanent

\title{
RESULTS OF THE FY09 ENHANCED DOE HIGH-LEVEL WASTE MELTER THROUGHPUT STUDIES AT SRNL
}

\author{
Fabienne C. Johnson \\ Thomas B. Edwards
}

JUNE 2010

Savannah River National Laboratory

Savannah River Nuclear Solutions

Aiken. SC 29808 


\section{DISCLAIMER}

This work was prepared under an agreement with and funded by the U.S. Government. Neither the U. S. Government or its employees, nor any of its contractors, subcontractors or their employees, makes any express or implied:

1. warranty or assumes any legal liability for the accuracy, completeness, or for the use or results of such use of any information, product, or process disclosed; or

2. representation that such use or results of such use would not infringe privately owned rights; or

3. endorsement or recommendation of any specifically identified commercial product, process, or service.

Any views and opinions of authors expressed in this work do not necessarily state or reflect those of the United States Government, or its contractors, or subcontractors.

Printed in the United States of America

Prepared for

U.S. Department of Energy 


\section{REVIEWS AND APPROVALS}

F.C. Johnson, Co-author, Process Technology Programs

Date

T.B. Edwards, Co-author, Applied Computational Engineering and Statistics

Date

D.K. Peeler, Peer Reviewer, Process Technology Programs

Date

C. C. Herman, Manager, Process Technology Programs

Date

S.L. Marra, Manager,

Date

Environmental \& Chemical Process Technology Research Programs

- ii - 


\section{TABLE OF CONTENTS}

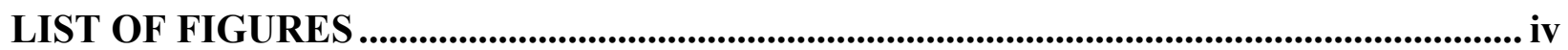

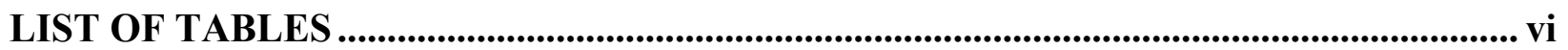

LIST OF ACRONYMS .................................................................................................................... vii

1.0 EXECUTIVE SUMMARY ..................................................................................... 1

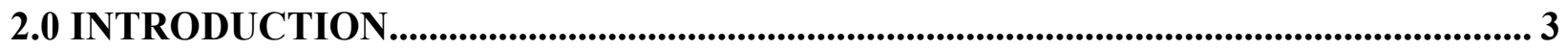

2.1 Glass Selection Strategy ……....................................................................................................... 4

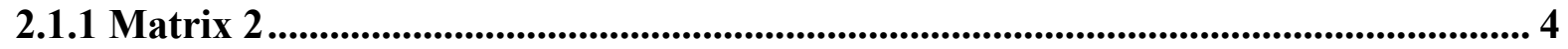

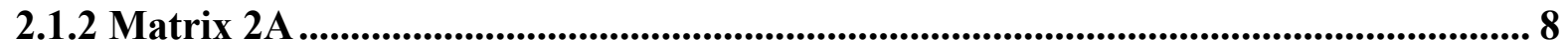

3.0 Objectives............................................................................................................................. 13

4.0 Experimental Procedures.................................................................................................. 13

4.1 Glass Fabrication ..................................................................................................................... 13

4.2 Chemical Composition Measurements ........................................................................................ 13

4.3 PCT ..................................................................................................................................... 14

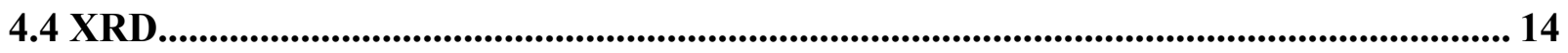

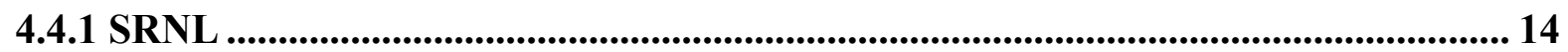

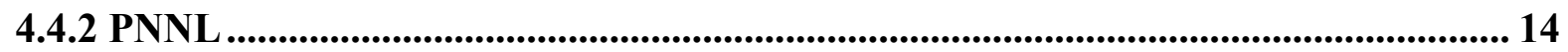

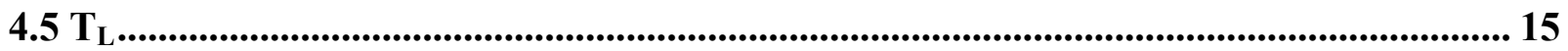

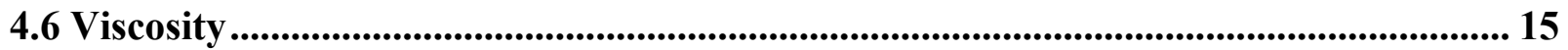

5.0 Results and Discussion................................................................................................................... 16

5.1 Test Matrix 2............................................................................................................................. 16

5.1.1 A Statistical Review of the Matrix 2 Chemical Composition Measurements of the

Non-Radioactive Glasses.......................................................................................................... 16

5.1.2 Matrix 2 Measurement Acceptability Region (MAR) Assessment ............................ 18

5.1.3 Matrix 2 XRD .................................................................................................................. 21

5.1.4 A Statistical Review of the PCT Results of the Matrix 2 Non-Radioactive Glasses

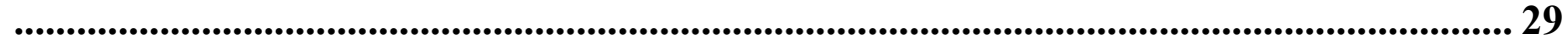

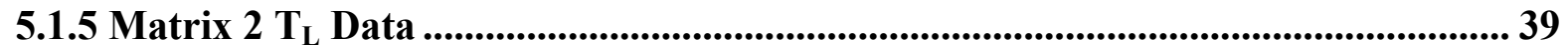

5.1.6 Matrix 2 Viscosity Data ........................................................................................................ 44

5.1.7 A Statistical Review of the Chemical Composition Measurements for the

Radioactive Glasses..................................................................................................................45

5.1.8 A Statistical Review of the PCT Results of the Matrix 2 Radioactive Glasses...... 46

5.2 Test Matrix 2A ....................................................................................................................... 47

5.2.1 A Statistical Review of the Matrix 2A Chemical Composition Measurements ..... 47

5.2.2 Matrix 2A MAR Assessment Results .................................................................. 49

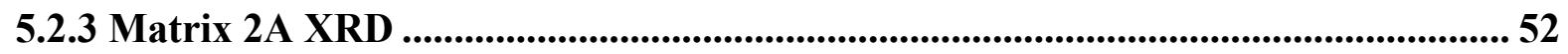

5.2.4 A Statistical Review of the Matrix 2A PCT Results..................................................... 69

5.2.5 Matrix 2A $T_{L}$ Data ........................................................................................................ 79

6.0 Comparison of Data to Model Predictions .................................................................................. 80

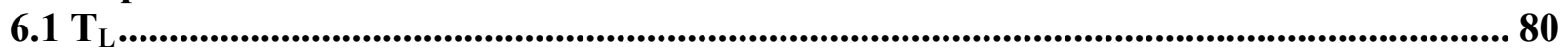

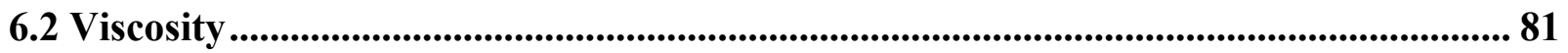

6.3 Chemical Durability ............................................................................................................ 82

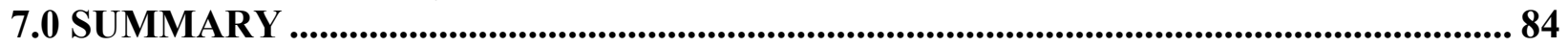

8.0 RECOMMENDATIONS/PATH FORWARD ........................................................................... 84

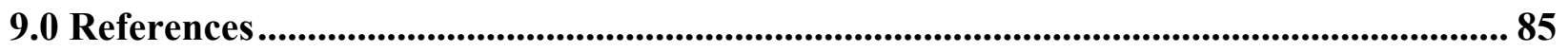




\section{LIST OF FIGURES}

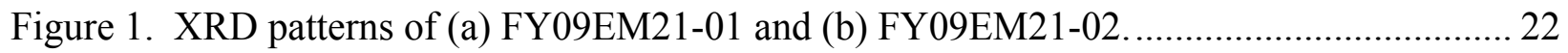

Figure 2. XRD patterns of (a) FY09EM21-03 and (b) FY09EM21-04 _.................................. 22

Figure 3. XRD patterns of (a) FY09EM21-05 and (b) FY09EM21-06..................................... 23

Figure 4. XRD patterns of (a) FY09EM21-07 and (b) FY09EM21-08 ...................................... 23

Figure 5. XRD patterns of (a) FY09EM21-09 and (b) FY09EM21-10 ....................................... 24

Figure 6. XRD patterns of (a) FY09EM21-11 and (b) FY09EM21-12 ...................................... 24

Figure 7. XRD patterns of (a) FY09EM21-13 and (b) FY09EM21-14 ....................................2 25

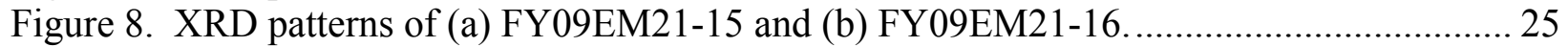

Figure 9. XRD patterns of (a) FY09EM21-17 and (b) FY09EM21-18 ...................................2 26

Figure 10. XRD patterns of (a) FY09EM21-19 and (b) FY09EM21-20 .................................... 26

Figure 11. XRD patterns of (a) FY09EM21-21 and (b) FY09EM21-22 _................................. 27

Figure 12. XRD patterns of (a) FY09EM21-23 and (b) FY09EM21-24 .................................. 27

Figure 13. XRD patterns of (a) FY09EM21-25 and (b) FY09EM21-26 .................................. 28

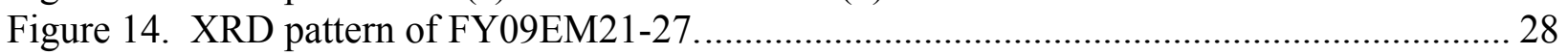

Figure 15. $\log$ NL $[\mathrm{B}(\mathrm{g} / \mathrm{L})]$ versus del Gp model with $95 \%$ Confidence Interval for Individual

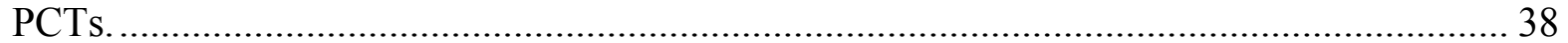

Figure 16. (a) Optical micrograph of FY09EM21-08 treated at $1000^{\circ} \mathrm{C}$ showing spinel crystals and (b) XRD pattern of FY09EM21-08 treated at $1000^{\circ} \mathrm{C}$ showing that the spinel is magnesium iron oxide.

Figure 17. (a) Optical micrograph of FY09EM $21-23$ treated at $1050^{\circ} \mathrm{C}$ showing iron titanate crystals and (b) XRD pattern of FY09EM21-23 treated at $1050^{\circ} \mathrm{C}$ showing that the crystals are iron titanium oxide.

Figure 18. (a) Optical micrograph of FY09EM21-18 treated at $816^{\circ} \mathrm{C}$. These clear geometric crystals take on a shape of cuboctahedrons (6 square and 8 triangular faces). (b) Optical micrograph of FY09EM21-19 treated at $740^{\circ} \mathrm{C}$. Structure and chemical identification of both of these crystals is unknown at this time as their concentrations were below the detection limit of the XRD...

Figure 19. (a) Optical micrograph of FY09EM21-06 treated at $849^{\circ} \mathrm{C}$ showing spinel and large rods and (b) XRD pattern showing evidence of iron oxide. Note that the terbium iron oxide

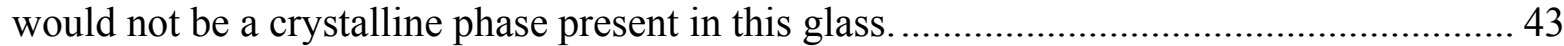

Figure 20. XRD patterns of (a) quenched and (b) ccc EM-01 ................................................ 54

Figure 21. XRD patterns of (a) quenched and (b) ccc EM-02 ………........................................ 54

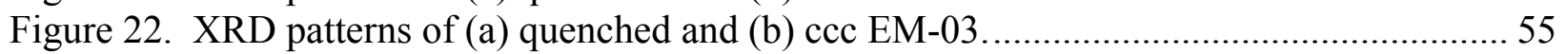

Figure 23. XRD patterns of (a) quenched and (b) ccc EM-04 ................................................. 55

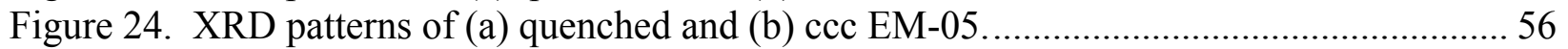

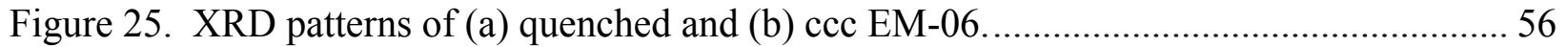

Figure 26. XRD patterns of (a) quenched and (b) ccc EM-07 .................................................... 57

Figure 27. XRD patterns of (a) quenched and (b) ccc EM-08 ……............................................ 57

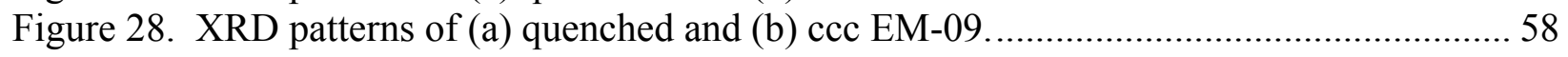

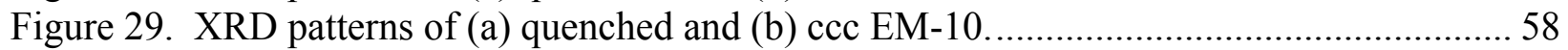

Figure 30. XRD patterns of (a) quenched and (b) ccc EM-11 ................................................... 59

Figure 31. XRD patterns of (a) quenched and (b) ccc EM-12 …............................................... 59

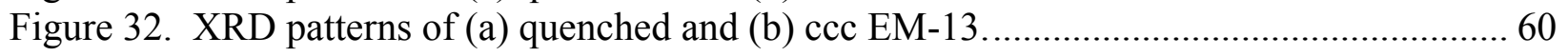




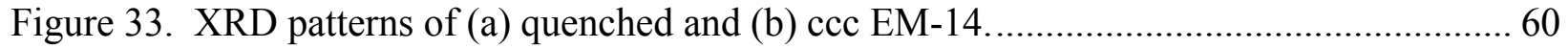

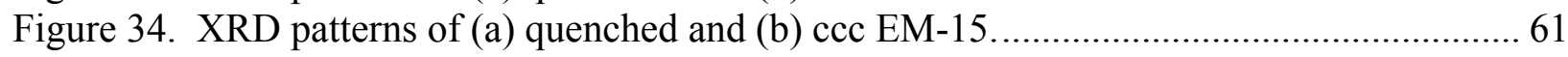

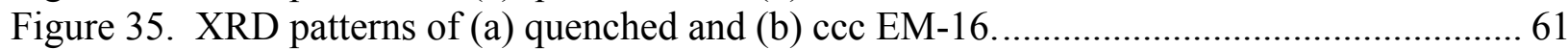

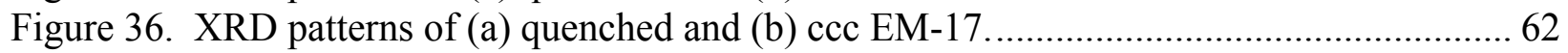

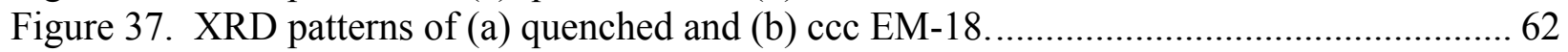

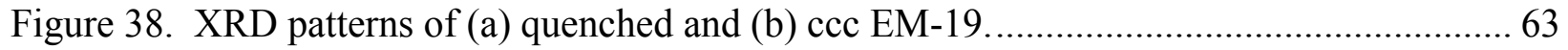

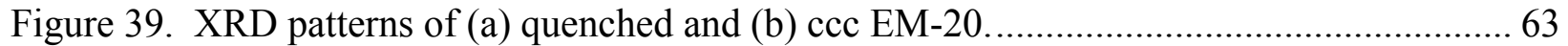

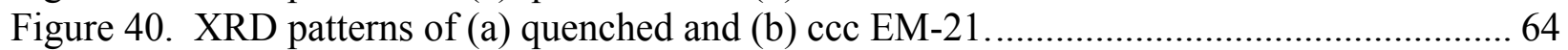

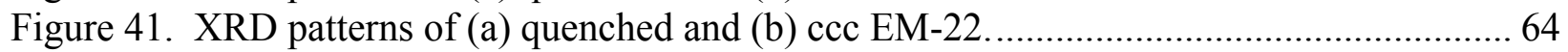

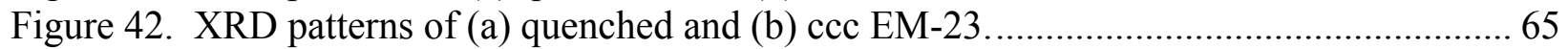

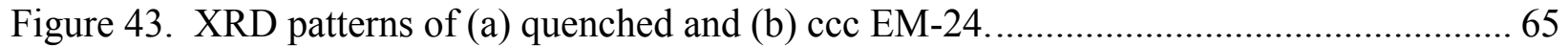

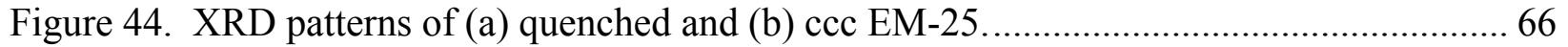

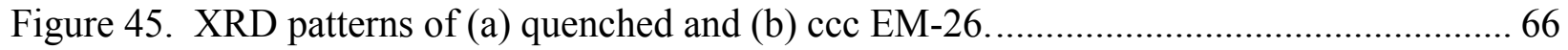

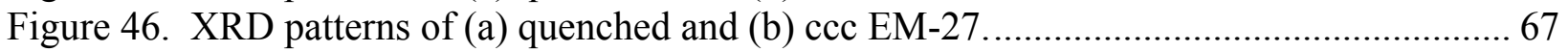

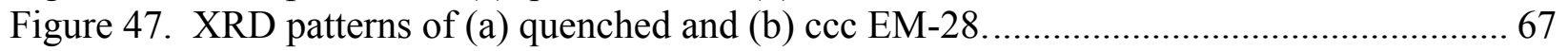

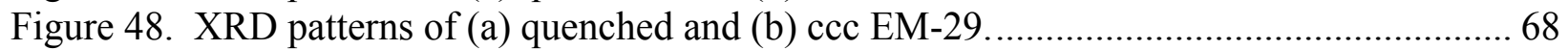

Figure 49. XRD patterns of (a) quenched and (b) ccc EM-30 .................................................. 68

Figure 50. $\log \mathrm{NL}[\mathrm{B}(\mathrm{g} / \mathrm{L})]$ versus del Gp model with $95 \%$ confidence interval for individual

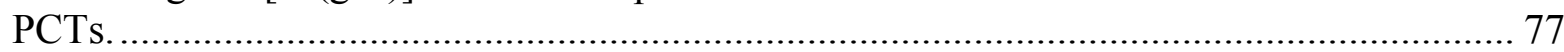

Figure 51. $\log$ NL [B (g/L)] versus del Gp model with 95\% confidence interval for individual

PCTs. Data points corresponding to glasses containing nepheline were removed. .............. 78

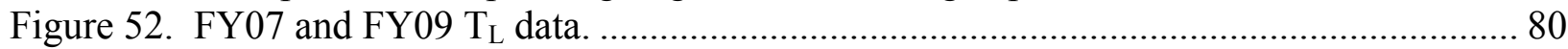

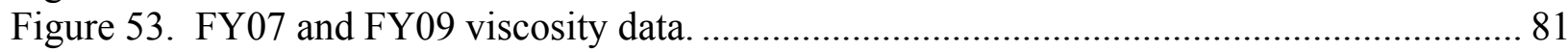

Figure 54. Comparison of durability data from the FY07 and FY09 tasks to the model predictions. Glasses containing nepheline have been removed from the plot. 82

Figure 55. Comparison of quenched durability data from the FY07 and FY09 tasks to the durability model. Questionable glasses from Matrix 2 have been removed from the plot (FY09EM21-02, -03, -07 and -21).

Figure 56. Figure 57. Comparison of cce durability data from the FY07 and FY09 tasks to the durability model. Questionable glasses from Matrix 2 have been removed from the plot (FY09EM21-02, -03, -07 and -21). 


\section{LIST OF TABLES}

Table 1. Projection Ranges Based on the HLW System Plan Revision ....................................... 5

Table 2. Oxide Interval Used to Develop the Glass Compositions …….................................... 5

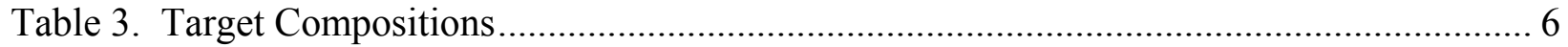

Table 4. Matrix 2 MAR Assessment Results..................................................................... 7

Table 5. Sludge Compositions Used to Develop the Matrix 2A Glasses ....................................... 9

Table 6. Target Glass Compositions of the Matrix 2A Glasses (wt\%) ........................................ 10

Table 7. Matrix 2A MAR Assessment Results................................................................... 12

Table 8. Predicted Properties and MAR Assessment Results of the Non-Radioactive Matrix 2

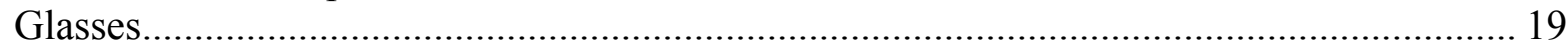

Table 9. XRD Results of the Matrix 2 Quenched Glasses........................................................... 21

Table 10. Multi-Element Solution Standard Results of the Non-Radioactive Matrix 2 Glasses. 30

Table 11. Normalized PCT Results of the Non-Radioactive Matrix 2 Glasses........................... 32

Table 12. Samples Exhibiting a Statistically Significant Difference Between the Quenched and

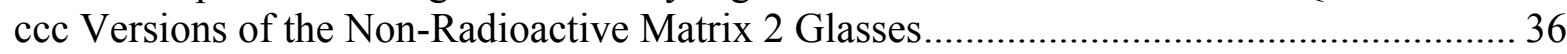

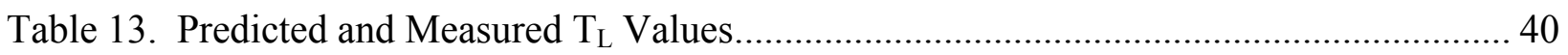

Table 14. Crystals Formed during $T_{L}$ Measurements ............................................................... 41

Table 15. Predicted and Measured Viscosity Data at $1150^{\circ} \mathrm{C}$....................................................... 44

Table 16. MAR Assessment Results of the Radioactive Matrix 2 Glasses ................................. 45

Table 17. Samples Exhibiting a Statistically Significant Difference Between the Quenched and ccc Versions of the Radioactive Matrix 2 Glasses............................................................... 46

Table 18. Normalized PCT Results of the Radioactive Matrix 2 Glasses ...................................... 47

Table 19. MAR Assessment Results of the Matrix 2A Glasses .................................................. 50

Table 20. XRD Results of the Matrix 2A Quenched and ccc Glasses........................................... 53

Table 21. Multi-Element Solution Standard Results of the Matrix 2A Glasses ............................ 70

Table 22. Normalized PCT Results of the Matrix 2A Glasses .................................................... 72

Table 23. Samples Exhibiting a Statistically Significant Difference Between the Quenched and

ccc Versions of the Matrix 2A Glasses ................................................................................. 76

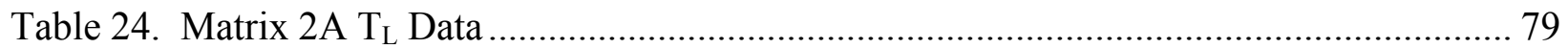




\section{LIST OF ACRONYMS}

$\begin{array}{ll}\text { AD } & \text { Analytical Development } \\ \text { ARM } & \text { Approved Reference Material } \\ \text { AR } & \text { As Received } \\ \text { ccc } & \text { Centerline Canister Cooling } \\ \text { DOE } & \text { Department of Energy } \\ \text { DWPF } & \text { Defense Waste Processing Facility } \\ \text { EA } & \text { Environmental Assessment } \\ \text { EM } & \text { Environmental Management } \\ \text { HLW } & \text { High-level Waste } \\ \text { ICP-AES } & \text { Inductively Coupled Plasma-Atomic Emission Spectroscopy } \\ \text { LM } & \text { Lithium Metaborate } \\ \text { MAR } & \text { Measurement Acceptability Region } \\ \text { PCCS } & \text { Product Composition Control System } \\ \text { PCT } & \text { Product Consistency Test } \\ \text { PF } & \text { Peroxide Fusion } \\ \text { PNNL } & \text { Pacific Northwest National Laboratory } \\ \text { PSAL } & \text { Process Science Analytical } \\ \text { SRNL } & \text { Savannah River National Laboratory } \\ \text { SRS } & \text { Savannah River Site } \\ \text { SWPF } & \text { Salt Waste Processing Facility } \\ \text { TCR } & \text { Task Change Request } \\ \text { T } & \text { Liquidus Temperature } \\ \text { WL } & \text { Waste Loading } \\ \text { WTP } & \text { Waste Treatment and Immobilization Plant } \\ \text { XRD } & \text { X-ray Diffraction } \\ \end{array}$




\subsection{EXECUTIVE SUMMARY}

High-level waste (HLW) throughput (i.e., the amount of waste processed per unit time) is a function of two critical parameters: waste loading (WL) and melt rate. For the Waste Treatment and Immobilization Plant (WTP) at the Hanford Site and the Defense Waste Processing Facility (DWPF) at the Savannah River Site (SRS), increasing HLW throughput would significantly reduce the overall mission life cycle costs for the Department of Energy (DOE).

The objective of this task is to develop data, assess property models, and refine or develop the necessary models to support increased WL of HLW at SRS. It is a continuation of the studies initiated in FY07, but is under the specific guidance of a Task Change Request (TCR)/Work Authorization received from DOE headquarters (Project Number RV071301). Using the data generated in FY07, FY08 and historical data, two test matrices (60 glasses total) were developed at the Savannah River National Laboratory (SRNL) in order to generate data in broader compositional regions. These glasses were fabricated and characterized using chemical composition analysis, $\mathrm{X}$-ray Diffraction (XRD), viscosity, liquidus temperature $\left(\mathrm{T}_{\mathrm{L}}\right)$ measurement and durability as defined by the Product Consistency Test (PCT).

The results of this study are summarized below:

- In general, the current durability model predicts the durabilities of higher waste loading glasses quite well. A few of the glasses exhibited poorer durability than predicted.

- Some of the glasses exhibited anomalous behavior with respect to durability (normalized leachate for boron (NL [B])). The quenched samples of FY09EM21-02, -07 and -21 contained no nepheline or other wasteform affecting crystals, but have unacceptable NL $[B]$ values $(>10 \mathrm{~g} / \mathrm{L})$. The ccc sample of FY09EM21-07 has a NL [B] value that is more than one half the value of the quenched sample. These glasses also have lower concentrations of $\mathrm{Al}_{2} \mathrm{O}_{3}$ and $\mathrm{SiO}_{2}$.

- Five of the ccc samples (EM-13, -14, -15, -29 and -30) completely crystallized with both magnetite and nepheline, and still had extremely low NL [B] values. These particular glasses have more $\mathrm{CaO}$ present than any of the other glasses in the matrix. It appears that while all of the glasses contain nepheline, the $\mathrm{NL}$ [B] values decrease as the $\mathrm{CaO}$ concentration increases from $2.3 \mathrm{wt} \%$ to $4.3 \mathrm{wt} \%$. A different form of nepheline may be created at higher concentrations of $\mathrm{CaO}$ that does not significantly reduce glass durability.

- The $\mathrm{T}_{\mathrm{L}}$ model appears to be under-predicting the measured values of higher waste loading glasses. Trends in $\mathrm{T}_{\mathrm{L}}$ with composition are not evident in the data from these studies.

- A small number of glasses in the FY09 matrix have measured viscosities that are much lower than the viscosity range over which the current model was developed. The decrease in viscosity is due to a higher concentration of non-bridging oxygens (NBO). A high iron concentration is the cause of the increase in NBO.

Durability, viscosity and $\mathrm{T}_{\mathrm{L}}$ data collected during FY07 and FY09 that specifically targeted higher waste loading glasses was compiled and assessed. It appears that additional data may be required to expand the coverage of the $\mathrm{T}_{\mathrm{L}}$ and viscosity models for higher waste loading glasses. 
In general, the compositional regions of the higher waste loading glasses are very different than those used to develop these models. On the other hand, the current durability model seems to be applicable to the new data. At this time, there is no evidence to modify this model; however additional experimental studies should be conducted to determine the cause of the anomalous durability data. 


\subsection{INTRODUCTION}

High-level waste (HLW) throughput (i.e., the amount of waste processed per unit time) is a function of two critical parameters: waste loading (WL) and melting rate. The melting rate is in turn a function of WL, feed chemistry and heat transfer characteristics within the melter. For the Waste Treatment and Immobilization Plant (WTP) at Hanford and the Defense Waste Processing Facility (DWPF) at the Savannah River Site (SRS), increasing HLW throughput would significantly reduce the overall mission life cycle costs for the Department of Energy (DOE).

Alternative melter technologies and strategic glass formulation could lead to significant improvements in both waste loading and melt rate, thus increasing waste throughput. Assuming that an alternative melter technology would eliminate the dependence of melt rate on waste loading, access to higher waste loadings would then be limited by specific process or product performance constraints, such as liquidus temperature $\left(\mathrm{T}_{\mathrm{L}}\right)$, viscosity, durability, or nepheline formation. Glass composition-property prediction models are currently employed by DWPF to demonstrate that these constraints are successfully and confidently being met for each process batch. ${ }^{1}$ A similar model-based approach is planned for WTP. The DWPF models also play a key role in frit development efforts for each new sludge batch, as well as providing important feedback on batching, washing and blending scenarios to describe the resulting sludge composition. While the reliable performance of these models to date is immediately apparent in the successful operation of DWPF, a push toward processing glass systems having higher WLs may result in a shift to glass compositional regions that have not been previously evaluated. Several questions are associated with reliable performance of the models in these broader compositional regions:

- Do the models help avoid glass compositional regions that are prone to processing or product quality issues?

- Do they allow entry into glass compositional regions that attain the target higher WLs while maintaining processability and product quality?

In order to answer these questions, data are needed in these broader compositional regions to ensure that the current models are applicable or that adequate data are available to refine the models or develop alternative models. Preemptive assessments of the process control models in the projected compositional regions of interest must be performed in order to ensure access to higher WL regions which may be required to meet contractual agreements. This assessment is currently being performed through DOE's Environmental Management (EM) program as both Savannah River National Laboratory (SRNL) and Pacific Northwest National Laboratory (PNNL) are focusing on the development of glass composition-property data in the anticipated regions covering higher waste loadings for DWPF.

A preliminary assessment of the current DWPF process control models for specific sludge compositions targeting higher WLs was completed in FY08. ${ }^{2}$ Glasses were fabricated and characterized in a joint SRNL-PNNL study to assess the applicability of the current process control models outside of the compositional region over which the current models were developed or validated. The results identified the inability of certain DWPF process control models to adequately predict physical properties related to processability, particular, the 
viscosity and liquidus temperature of select higher WL glasses that were outside of the compositional regions over which the models were developed or validated. In general, as the compositional overlap between the model validation ranges diverged from the target glass compositions, the difference between the measured and the predicted property values increased. This implies that there are individual oxides or oxide combinations that are outside of the model validation range, which include $\mathrm{B}_{2} \mathrm{O}_{3}, \mathrm{SiO}_{2}, \mathrm{MnO}$, and $\mathrm{TiO}_{2}$ or their combinations. Additional data are needed in these broader compositional regions to assess and/or refine current model predictions or to develop alternative models. Data generation, model development or refinement and model validation will be required prior to the implementation of updated alternative models into the DWPF process control system. The timing of these efforts must be coordinated with the DWPF schedule for higher waste loadings to ensure that there is no disconnect between contractual expectations and a control system that can support higher waste loadings during melter feed acceptability decisions.

This task is a continuation of the studies initiated in FY07, but is under the specific guidance of a Task Change Request (TCR)/Work Authorization received from DOE headquarters. ${ }^{3-6}$ Using the data generated in FY07, FY08 and historical data, two test matrices (Matrix 2 and Matrix 2A 60 glasses total) were developed in order to generate data in broader compositional regions. These glasses were fabricated and characterized using chemical composition analysis, X-ray Diffraction (XRD), viscosity, $\mathrm{T}_{\mathrm{L}}$ measurement and the Product Consistency Test (PCT). ${ }^{7}$

\subsection{GLASS SELECTION STRATEGY}

\subsubsection{Matrix 2}

The HLW System Plan (Revision 13) ${ }^{8}$ was used as the compositional basis for future flowsheets to be processed at the DWPF. The minimum and maximum ranges for each sludge oxide were determined from the "with and without Al-dissolution cases" and are shown in Table 1. The bounding glass compositional region was developed by (i) applying a 30\% WL to the minimum values of each sludge component, and (ii) applying a 50\% WL to the maximum values of each sludge component. The resulting oxide intervals are shown in Table 2. Minimum and maximum concentrations for the frit components $\left(\mathrm{B}_{2} \mathrm{O}_{3}, \mathrm{Li}_{2} \mathrm{O}, \mathrm{Na}_{2} \mathrm{O}\right.$ and $\left.\mathrm{SiO}_{2}\right)$ were selected by considering the Product Composition Control System (PCCS) constraints. The maximum $\mathrm{CaO}$ content was raised to gauge the affect of Ca-containing frit. In order to account for the Salt Waste Processing Facility (SWPF) flowsheet, the $\mathrm{TiO}_{2}$ content was raised from $0-2 \mathrm{wt} \%$ to $2-6$ $\mathrm{wt} \%$ and the upper $\mathrm{Na}_{2} \mathrm{O}$ concentration was increased to $18 \mathrm{wt}^{\%}$. JMP ${ }^{\mathrm{TM}}$ was used to Doptimally $^{\dagger}$ select 30 glasses based on the oxide ranges in Table 2; three of these glasses contained varying amounts of $\mathrm{U}_{3} \mathrm{O}_{8}(2,4$ and $6 \mathrm{wt} \%) .{ }^{9}$ Target compositions and a summary of the Measurement Acceptability Region (MAR) assessment results are provided in Table 3. Glasses were chosen to focus on specific technical issues that have been identified for future sludge batches to be processed at DWPF, which include glass systems with WLs restricted by $T_{L}$ predictions and $\mathrm{TiO}_{2}$ retention and model applicability of glass systems for coupled operations ${ }^{\sharp}$.

\footnotetext{
* "Others" in Table 2 includes $\mathrm{BaO}, \mathrm{Ce}_{2} \mathrm{O}_{3}, \mathrm{CuO}, \mathrm{La}_{2} \mathrm{O}_{3}, \mathrm{PbO}, \mathrm{SO}_{4}, \mathrm{ZnO}$ and $\mathrm{ZrO}_{2}$.

${ }^{\dagger} \mathrm{D}$-optimality is an experimental design method that minimizes the variance of the estimates of the coefficients of the proposed model. In this study, the proposed model was taken to be a linear function of the oxides in Table 2 .

* Addition of the Actinide Removal Process (ARP) stream to the sludge.
} 
Table 1. Projection Ranges Based on the HLW System Plan Revision

\begin{tabular}{|c|c|c|}
\hline \multirow{2}{*}{ Oxide } & Mininum & Maximum \\
\hline & \multicolumn{2}{|c|}{$\mathrm{wt} \%$} \\
\hline $\mathrm{Al}_{2} \mathrm{O}_{3}$ & 11.95 & 34.31 \\
\hline $\mathrm{BaO}$ & 0.07 & 0.28 \\
\hline $\mathrm{CaO}$ & 1.74 & 3.66 \\
\hline $\mathrm{Ce}_{2} \mathrm{O}_{3}$ & 0.08 & 0.96 \\
\hline $\mathrm{Cr}_{2} \mathrm{O}_{3}$ & 0.19 & 0.41 \\
\hline $\mathrm{CuO}$ & 0.04 & 0.14 \\
\hline $\mathrm{Fe}_{2} \mathrm{O}_{3}$ & 18.71 & 41.22 \\
\hline $\mathrm{K}_{2} \mathrm{O}$ & 0.07 & 0.41 \\
\hline $\mathrm{La}_{2} \mathrm{O}_{3}$ & 0.03 & 0.31 \\
\hline $\mathrm{MgO}$ & 0.27 & 2.65 \\
\hline $\mathrm{MnO}$ & 1.21 & 10.66 \\
\hline $\mathrm{Na}_{2} \mathrm{O}$ & 19.12 & 27.85 \\
\hline $\mathrm{NiO}$ & 0.17 & 4.51 \\
\hline $\mathrm{PbO}$ & 0.04 & 0.39 \\
\hline $\mathrm{SO}_{4}$ & 0.09 & 1.88 \\
\hline $\mathrm{SiO}_{2}$ & 1.79 & 8.27 \\
\hline $\mathrm{TiO}_{2}$ & 0.00 & 3.89 \\
\hline $\mathrm{U}_{3} \mathrm{O}_{8}$ & 0.54 & 18.80 \\
\hline $\mathrm{ZnO}$ & 0.05 & 0.24 \\
\hline $\mathrm{ZrO}_{2}$ & 0.10 & 0.67 \\
\hline
\end{tabular}

Table 2. Oxide Interval Used to Develop the Glass Compositions

\begin{tabular}{|c|c|c|}
\hline \multirow{2}{*}{ Oxide } & Mininum & Maximum \\
\hline & \multicolumn{2}{|c|}{$\mathrm{wt} \%$} \\
\hline $\mathrm{Al}_{2} \mathrm{O}_{3}$ & 3.25 & 18 \\
\hline $\mathrm{B}_{2} \mathrm{O}_{3}$ & 4.5 & 14 \\
\hline $\mathrm{CaO}$ & 0 & 4 \\
\hline $\mathrm{Cr}_{2} \mathrm{O}_{3}$ & 0 & 0.2 \\
\hline $\mathrm{Fe}_{2} \mathrm{O}_{3}$ & 5 & 21 \\
\hline $\mathrm{Li}_{2} \mathrm{O}$ & 4 & 7 \\
\hline $\mathrm{MgO}$ & 0 & 1.5 \\
\hline $\mathrm{MnO}$ & 0.3 & 5.5 \\
\hline $\mathrm{Na}_{2} \mathrm{O}$ & 10 & 18 \\
\hline $\mathrm{NiO}$ & 0 & 2.5 \\
\hline $\mathrm{SiO}_{2}$ & 30 & 55 \\
\hline $\mathrm{TiO}_{2}$ & 2 & 6 \\
\hline $\mathrm{U}_{3} \mathrm{O}_{8}$ & 0 & 9.5 \\
\hline Others & 0 & 2 \\
\hline
\end{tabular}


SRNL-STI-2009-00778, REVISION 0

Table 3. Target Compositions

\begin{tabular}{|c|c|c|c|c|c|c|c|c|c|c|c|c|c|c|c|c|c|c|c|c|c|c|}
\hline Glass ID & $\mathrm{Al}_{2} \mathrm{O}_{3}$ & $\mathrm{~B}_{2} \mathrm{O}_{3}$ & $\mathrm{BaO}$ & $\mathrm{CaO}$ & $\mathrm{CdO}$ & $\mathrm{Ce}_{2} \mathrm{O}_{3}$ & $\mathrm{Cr}_{2} \mathrm{O}_{3}$ & $\mathrm{CuO}$ & $\mathrm{Fe}_{2} \mathrm{O}_{3}$ & $\mathrm{La}_{2} \mathrm{O}_{3}$ & $\mathrm{Li}_{2} \mathrm{O}$ & $\mathrm{MgO}$ & $\mathrm{MnO}$ & $\mathrm{Na}_{2} \mathrm{O}$ & $\mathrm{NiO}$ & $\mathrm{PbO}$ & $\mathrm{SO}_{4}$ & $\mathrm{SiO}_{2}$ & $\mathrm{TiO}_{2}$ & $\mathrm{U}_{3} \mathrm{O}_{8}$ & $\mathrm{ZnO}$ & $\mathrm{ZrO}_{2}$ \\
\hline FY09EM21-01 & 9.97 & 4.50 & 0.00 & 0.00 & 0.00 & 0.00 & 0.20 & 0.00 & 18.35 & 0.00 & 4.00 & 0.00 & 5.50 & 11.39 & 2.50 & 0.00 & 0.00 & 37.99 & 5.60 & 0.00 & 0.00 & 0.00 \\
\hline FY09EM21-02 & 4.01 & 5.07 & 0.08 & 0.00 & 0.30 & 0.36 & 0.00 & 0.13 & 20.63 & 0.10 & 4.00 & 1.50 & 0.30 & 17.98 & 2.50 & 0.22 & 0.48 & 40.02 & 2.00 & 0.00 & 0.13 & 0.21 \\
\hline FY09EM21-03 & 6.97 & 4.50 & 0.00 & 0.00 & 0.00 & 0.00 & 0.00 & 0.00 & 12.52 & 0.00 & 7.00 & 1.50 & 5.50 & 14.13 & 2.50 & 0.00 & 0.00 & 40.65 & 4.73 & 0.00 & 0.00 & 0.00 \\
\hline FY09EM21-04 & 7.03 & 14.00 & 0.00 & 4.00 & 0.00 & 0.00 & 0.00 & 0.00 & 16.49 & 0.00 & 7.00 & 1.50 & 0.30 & 10.74 & 2.50 & 0.00 & 0.00 & 32.45 & 4.00 & 0.00 & 0.00 & 0.00 \\
\hline FY09EM21-05 & 3.60 & 4.50 & 0.00 & 4.00 & 0.00 & 0.00 & 0.00 & 0.00 & 13.77 & 0.00 & 4.00 & 0.00 & 0.30 & 10.00 & 2.50 & 0.00 & 0.00 & 51.50 & 5.83 & 0.00 & 0.00 & 0.00 \\
\hline FY09EM21-06 & 4.39 & 11.49 & 0.08 & 0.00 & 0.30 & 0.36 & 0.20 & 0.13 & 5.30 & 0.10 & 4.00 & 0.00 & 5.50 & 14.78 & 2.50 & 0.22 & 0.48 & 43.84 & 6.00 & 0.00 & 0.13 & 0.21 \\
\hline FY09EM21-07 & 4.87 & 13.92 & 0.00 & 3.92 & 0.00 & 0.00 & 0.00 & 0.00 & 17.98 & 0.00 & 4.00 & 1.50 & 5.50 & 12.71 & 0.00 & 0.00 & 0.00 & 33.60 & 2.00 & 0.00 & 0.00 & 0.00 \\
\hline FY09EM21-08 & 3.25 & 4.97 & 0.00 & 0.50 & 0.00 & 0.00 & 0.20 & 0.00 & 20.48 & 0.00 & 7.00 & 1.50 & 1.39 & 10.00 & 0.00 & 0.00 & 0.00 & 44.71 & 6.00 & 0.00 & 0.00 & 0.00 \\
\hline FY09EM21-09 & 12.97 & 9.48 & 0.00 & 3.03 & 0.00 & 0.00 & 0.20 & 0.00 & 10.96 & 0.00 & 7.00 & 0.00 & 2.01 & 10.00 & 0.68 & 0.00 & 0.00 & 41.19 & 2.47 & 0.00 & 0.00 & 0.00 \\
\hline FY09EM21-10 & 6.40 & 4.72 & 0.08 & 4.00 & 0.30 & 0.36 & 0.00 & 0.13 & 17.73 & 0.10 & 4.00 & 0.00 & 5.46 & 10.39 & 2.50 & 0.22 & 0.48 & 40.40 & 2.40 & 0.00 & 0.13 & 0.21 \\
\hline FY09EM21-11 & 3.25 & 4.58 & 0.08 & 4.00 & 0.30 & 0.36 & 0.00 & 0.13 & 6.04 & 0.10 & 5.63 & 1.50 & 5.50 & 12.21 & 0.00 & 0.22 & 0.48 & 49.30 & 6.00 & 0.00 & 0.13 & 0.21 \\
\hline FY09EM21-12 & 4.55 & 5.29 & 0.00 & 0.00 & 0.00 & 0.00 & 0.00 & 0.00 & 16.86 & 0.00 & 6.79 & 0.00 & 4.67 & 10.00 & 1.91 & 0.00 & 0.00 & 47.92 & 2.00 & 0.00 & 0.00 & 0.00 \\
\hline FY09EM21-13 & 10.38 & 5.88 & 0.08 & 4.00 & 0.30 & 0.36 & 0.20 & 0.13 & 14.08 & 0.10 & 7.00 & 0.00 & 0.30 & 10.52 & 2.50 & 0.22 & 0.48 & 37.14 & 6.00 & 0.00 & 0.13 & 0.21 \\
\hline FY09EM21-14 & 3.25 & 13.65 & 0.08 & 0.00 & 0.30 & 0.36 & 0.20 & 0.13 & 20.54 & 0.10 & 4.00 & 0.00 & 0.30 & 10.00 & 0.00 & 0.22 & 0.48 & 40.35 & 5.71 & 0.00 & 0.13 & 0.21 \\
\hline FY09EM21-15 & 5.32 & 4.50 & 0.08 & 3.19 & 0.30 & 0.36 & 0.20 & 0.13 & 8.29 & 0.10 & 6.00 & 1.50 & 5.36 & 10.00 & 2.50 & 0.22 & 0.48 & 49.14 & 2.00 & 0.00 & 0.13 & 0.21 \\
\hline FY09EM21-16 & 3.68 & 6.43 & 0.08 & 3.82 & 0.30 & 0.36 & 0.20 & 0.13 & 9.05 & 0.10 & 4.00 & 1.50 & 0.30 & 15.82 & 2.50 & 0.22 & 0.48 & 48.71 & 2.00 & 0.00 & 0.13 & 0.21 \\
\hline FY09EM21-17 & 7.19 & 4.50 & 0.08 & 0.10 & 0.30 & 0.36 & 0.00 & 0.13 & 13.91 & 0.10 & 6.74 & 0.00 & 5.50 & 14.85 & 0.00 & 0.22 & 0.48 & 39.20 & 6.00 & 0.00 & 0.13 & 0.21 \\
\hline FY09EM21-18 & 10.92 & 4.84 & 0.08 & 0.44 & 0.30 & 0.36 & 0.00 & 0.13 & 6.79 & 0.10 & 5.49 & 0.00 & 0.83 & 17.02 & 0.00 & 0.22 & 0.48 & 49.66 & 2.00 & 0.00 & 0.13 & 0.21 \\
\hline FY09EM21-19 & 5.72 & 4.50 & 0.00 & 4.00 & 0.00 & 0.00 & 0.00 & 0.00 & 5.00 & 0.00 & 4.00 & 0.00 & 0.30 & 18.00 & 0.98 & 0.00 & 0.00 & 51.50 & 6.00 & 0.00 & 0.00 & 0.00 \\
\hline FY09EM21-20 & 6.02 & 5.31 & 0.00 & 0.00 & 0.00 & 0.00 & 0.20 & 0.00 & 11.83 & 0.00 & 4.00 & 1.27 & 0.30 & 18.00 & 2.50 & 0.00 & 0.00 & 48.57 & 2.00 & 0.00 & 0.00 & 0.00 \\
\hline FY09EM21-21 & 4.86 & 9.01 & 0.08 & 0.00 & 0.30 & 0.36 & 0.20 & 0.13 & 19.95 & 0.10 & 7.00 & 0.00 & 1.00 & 14.67 & 0.00 & 0.22 & 0.48 & 39.31 & 2.01 & 0.00 & 0.13 & 0.21 \\
\hline FY09EM21-22 & 14.04 & 13.20 & 0.08 & 0.00 & 0.30 & 0.36 & 0.00 & 0.13 & 9.18 & 0.10 & 4.00 & 0.00 & 0.30 & 10.00 & 2.50 & 0.22 & 0.48 & 42.74 & 2.04 & 0.00 & 0.13 & 0.21 \\
\hline FY09EM21-23 & 13.96 & 5.78 & 0.08 & 0.00 & 0.30 & 0.36 & 0.00 & 0.13 & 12.11 & 0.10 & 4.00 & 1.50 & 0.30 & 10.00 & 0.00 & 0.22 & 0.48 & 44.35 & 6.00 & 0.00 & 0.13 & 0.21 \\
\hline FY09EM21-24 & 10.51 & 4.50 & 0.08 & 4.00 & 0.30 & 0.36 & 0.20 & 0.13 & 20.56 & 0.10 & 4.00 & 1.50 & 0.92 & 10.00 & 0.00 & 0.22 & 0.48 & 37.88 & 3.94 & 0.00 & 0.13 & 0.21 \\
\hline FY09EM21-25 & 6.24 & 4.73 & 0.00 & 4.00 & 0.00 & 0.00 & 0.20 & 0.00 & 7.83 & 0.00 & 4.00 & 0.00 & 5.50 & 15.46 & 0.00 & 0.00 & 0.00 & 50.03 & 2.00 & 0.00 & 0.00 & 0.00 \\
\hline FY09EM21-26 & 13.66 & 7.58 & 0.00 & 0.00 & 0.00 & 0.00 & 0.20 & 0.00 & 5.00 & 0.00 & 4.00 & 1.50 & 5.05 & 11.96 & 0.00 & 0.00 & 0.00 & 45.70 & 5.36 & 0.00 & 0.00 & 0.00 \\
\hline FY09EM21-27 & 7.19 & 6.98 & 0.04 & 1.81 & 0.16 & 0.19 & 0.10 & 0.07 & 13.12 & 0.05 & 5.10 & 0.68 & 2.62 & 12.72 & 1.29 & 0.12 & 0.26 & 43.38 & 3.93 & 0.00 & 0.07 & 0.11 \\
\hline FY09EM21-28 & 7.05 & 6.84 & 0.04 & 1.77 & 0.16 & 0.19 & 0.10 & 0.07 & 12.86 & 0.05 & 5.00 & 0.67 & 2.57 & 12.46 & 1.27 & 0.11 & 0.25 & 42.51 & 3.85 & 2.00 & 0.07 & 0.11 \\
\hline FY09EM21-29 & 6.90 & 6.70 & 0.04 & 1.74 & 0.15 & 0.19 & 0.10 & 0.07 & 12.60 & 0.05 & 4.90 & 0.66 & 2.52 & 12.21 & 1.24 & 0.11 & 0.25 & 41.64 & 3.77 & 4.00 & 0.07 & 0.11 \\
\hline FY09EM21-30 & 6.76 & 6.56 & 0.04 & 1.70 & 0.15 & 0.18 & 0.09 & 0.06 & 12.34 & 0.05 & 4.80 & 0.64 & 2.47 & 11.95 & 1.21 & 0.11 & 0.24 & 40.78 & 3.69 & 6.00 & 0.07 & 0.10 \\
\hline
\end{tabular}

${ }^{a}$ newlv/newhv = viscosity; Homg = homogeneity; $\mathrm{R}_{2} \mathrm{O}=$ alkali constraint; 1 frit $=$ associated with

homogeneity 
Table 4. Matrix 2 MAR Assessment Results

\begin{tabular}{|c|c|c|c|c|}
\hline Glass ID & $\mathrm{Al}_{2} \mathrm{O}_{3}(\mathrm{wt} \%)$ & $\mathrm{R}_{2} \mathrm{O}(\mathrm{wt} \%)$ & Nepheline Value & MAR Status \\
\hline FY09EM21-01 & 9.97 & 15.39 & 0.64 & $\mathrm{~T}_{\mathrm{L}}$ newlv $\mathrm{TiO}_{2}$ \\
\hline FY09EM21-02 & 4.01 & 21.98 & 0.65 & $\mathrm{~T}_{\mathrm{L}}$ newlv $\mathrm{TiO}_{2}$ IFrit \\
\hline FY09EM21-03 & 6.97 & 21.13 & 0.66 & newlv $\mathrm{TiO}_{2}$ \\
\hline FY09EM21-04 & 7.03 & 17.74 & 0.65 & $\mathrm{~T}_{\mathrm{L}}$ newlv $\mathrm{TiO}_{2}$ \\
\hline FY09EM21-05 & 3.60 & 14.00 & 0.79 & $\mathrm{~T}_{\mathrm{L}} \mathrm{TiO}_{2}$ lFrit \\
\hline FY09EM21-06 & 4.39 & 18.78 & 0.70 & newlv $\mathrm{TiO}_{2}$ Homg \\
\hline FY09EM21-07 & 4.87 & 16.71 & 0.66 & newlv $\mathrm{TiO}_{2}$ \\
\hline FY09EM21-08 & 3.25 & 17.00 & 0.77 & newlv $\mathrm{TiO}_{2} 1$ Frit \\
\hline FY09EM21-09 & 12.97 & 17.00 & 0.64 & $\mathrm{~T}_{\mathrm{L}}$ newly $\mathrm{TiO}_{2}$ \\
\hline FY09EM21-10 & 6.40 & 14.39 & 0.71 & $\mathrm{~T}_{\mathrm{L}} \mathrm{TiO}_{2}$ \\
\hline FY09EM21-11 & 3.25 & 17.83 & 0.76 & $\mathrm{TiO}_{2}$ Homg lFrit \\
\hline FY09EM21-12 & 4.55 & 16.79 & 0.77 & newlv $\mathrm{TiO}_{2} 1$ Frit \\
\hline FY09EM21-13 & 10.38 & 17.52 & 0.64 & $\mathrm{~T}_{\mathrm{L}}$ newly $\mathrm{TiO}_{2}$ \\
\hline FY09EM21-14 & 3.25 & 14.00 & 0.75 & newlv $\mathrm{TiO}_{2}$ 1Frit \\
\hline FY09EM21-15 & 5.32 & 16.00 & 0.76 & $\mathrm{~T}_{\mathrm{L}} \mathrm{TiO}_{2}$ Homg \\
\hline FY09EM21-16 & 3.68 & 19.82 & 0.71 & $\mathrm{TiO}_{2} \mathrm{R}_{2} \mathrm{O}$ \\
\hline FY09EM21-17 & 7.19 & 21.59 & 0.64 & newlv $\mathrm{TiO}_{2}$ \\
\hline FY09EM21-18 & 10.92 & 22.51 & 0.64 & $\mathrm{TiO}_{2}$ \\
\hline FY09EM21-19 & 5.72 & 22.00 & 0.68 & $\mathrm{TiO}_{2}$ Homg \\
\hline FY09EM21-20 & 6.02 & 22.00 & 0.67 & $\mathrm{~T}_{\mathrm{L}}$ newlv $\mathrm{TiO}_{2}$ \\
\hline FY09EM21-21 & 4.86 & 21.67 & 0.67 & newlv $\mathrm{TiO}_{2}$ \\
\hline FY09EM21-22 & 14.04 & 14.00 & 0.64 & $\mathrm{~T}_{\mathrm{L}} \mathrm{TiO}_{2}$ \\
\hline FY09EM21-23 & 13.96 & 14.00 & 0.65 & newhv $\mathrm{TiO}_{2}$ \\
\hline FY09EM21-24 & 10.51 & 14.00 & 0.65 & $\mathrm{~T}_{\mathrm{L}} \mathrm{TiO}_{2}$ \\
\hline FY09EM21-25 & 6.24 & 19.46 & 0.70 & $\mathrm{TiO}_{2}$ \\
\hline FY09EM21-26 & 13.66 & 15.96 & 0.64 & newhv $\mathrm{TiO}_{2}$ \\
\hline FY09EM21-27 & 7.19 & 17.82 & 0.69 & newlv $\mathrm{TiO}_{2}$ \\
\hline FY09EM21-28 & 7.05 & 17.46 & 0.69 & newlv $\mathrm{TiO}_{2}$ \\
\hline FY09EM21-29 & 6.90 & 17.11 & 0.69 & newlv $\mathrm{TiO}_{2}$ \\
\hline FY09EM21-30 & 6.76 & 16.75 & 0.69 & newlv $\mathrm{TiO}_{2}$ \\
\hline
\end{tabular}




\subsubsection{Matrix 2A}

$\mathrm{JMP}^{\mathrm{TM}}$ was used to develop a non-radioactive centroid sludge composition based on the sludge oxide intervals provided in Table $1 .^{\S}$ In order to assess the impact of several sludge components on glass properties, 14 sludge compositions were developed by systematically altering the centroid composition. These compositions are shown Table 5. A summary of the method is provided below. For each of the compositions, $\mathrm{Na}_{2} \mathrm{O}$ and $\mathrm{SiO}_{2}$ were adjusted in order to compensate for the changes listed below. It should be noted that the adjustments to the oxide components still fall within the sludge oxide bounds in Table 1.

- Sludge 1: centroid

- Sludge 2: $\mathrm{Al}_{2} \mathrm{O}_{3}$ was increased by $5 \mathrm{wt} \%$.

- Sludge 3: $\mathrm{Al}_{2} \mathrm{O}_{3}$ was decreased by $5 \mathrm{wt} \%$.

- Sludge 4: $\mathrm{Al}_{2} \mathrm{O}_{3}$ was decreased by $10 \mathrm{wt} \%$.

- Sludge 5: $\mathrm{Al}_{2} \mathrm{O}_{3}$ was decreased by $10 \mathrm{wt} \% . \mathrm{Fe}_{2} \mathrm{O}_{3}$ was increased by $5 \mathrm{wt} \%$.

- Sludge 6: $\mathrm{Al}_{2} \mathrm{O}_{3}$ was decreased by $10 \mathrm{wt} \%$. $\mathrm{Fe}_{2} \mathrm{O}_{3}$ was increased by $10 \mathrm{wt} \%$.

- Sludge 7: $\mathrm{CaO}$ was increased by $4 \mathrm{wt} \%$. $\mathrm{Al}_{2} \mathrm{O}_{3}$ decreased by $10 \mathrm{wt} \%$. $\mathrm{Fe}_{2} \mathrm{O}_{3}$ was increased by $10 \mathrm{wt} \%$.

- Sludge 8: $\mathrm{CaO}$ was increased by $6 \mathrm{wt} \% . \mathrm{Al}_{2} \mathrm{O}_{3}$ decreased by 10 wt $\%$. $\mathrm{Fe}_{2} \mathrm{O}_{3}$ was increased by $10 \mathrm{wt} \%$.

- Sludge 9: $\mathrm{Fe}_{2} \mathrm{O}_{3}$ was increased by $5 \mathrm{wt} \%$.

- Sludge 10: $\mathrm{Fe}_{2} \mathrm{O}_{3}$ was decreased by $5 \mathrm{wt} \%$.

- Sludge 11: $\mathrm{MnO}$ was increased by $2 \mathrm{wt} \%$.

- Sludge 12: $\mathrm{MnO}$ was decreased by $2 \mathrm{wt} \%$.

- Sludge 13: $\mathrm{CaO}$ was increased by $2 \mathrm{wt} \%$. $\mathrm{Al}_{2} \mathrm{O}_{3}$ was increased by $5 \mathrm{wt} \%$. $\mathrm{Fe}_{2} \mathrm{O}_{3}$ was decreased by $5 \mathrm{wt} \%$.

- Sludge 14: $\mathrm{CaO}$ was increased by $4 \mathrm{wt} \% . \quad \mathrm{Al}_{2} \mathrm{O}_{3}$ was increased by $5 \mathrm{wt} \%$. $\mathrm{Fe}_{2} \mathrm{O}_{3}$ was decreased by $5 \mathrm{wt} \%$.

- Sludge 15: $\mathrm{CaO}$ was increased by $6 \mathrm{wt} \% . \mathrm{Al}_{2} \mathrm{O}_{3}$ was increased by $5 \mathrm{wt} \%$. $\mathrm{Fe}_{2} \mathrm{O}_{3}$ was decreased by $5 \mathrm{wt} \%$.

These sludges were each combined with Frit 418 and Frit 510 at a 50\% WL in order to study the affects of $\mathrm{B}_{2} \mathrm{O}_{3}$ in the frit. ${ }^{* *}$ The target glass compositions are provided in Table 6 and MAR assessment results are shown in Table Table 4.

\footnotetext{
$\S$ The "Others" components $\left(\mathrm{BaO}, \mathrm{Ce}_{2} \mathrm{O}_{3}, \mathrm{Cr}_{2} \mathrm{O}_{3}, \mathrm{CuO}, \mathrm{K}_{2} \mathrm{O}, \mathrm{La}_{2} \mathrm{O}_{3}, \mathrm{PbO}, \mathrm{SO}_{4}, \mathrm{ZnO}\right.$ and $\left.\mathrm{ZrO}_{2}\right)$ were fixed at atal sum of $3 \mathrm{wt} \%$ of the sludge composition. The non-radioactive centroid was obtained by removing $\mathrm{U}_{3} \mathrm{O}_{8}$ from the sludge composition and renormalizing. In addition, the $\mathrm{TiO}_{2}$ content in the centroid sludge composition was raised to $4 \mathrm{wt} \%$ so that the $\mathrm{TiO}_{2}$ content of the glass would be $2 \mathrm{wt} \%$ at $50 \% \mathrm{WL}$. This renormalization caused the other oxide components to slightly decrease.

${ }^{* *}$ Frit $418=8 w t \% \mathrm{~B}_{2} \mathrm{O}_{3}-8 w t \% \mathrm{Li}_{2} \mathrm{O}-8 \mathrm{wt} \% \mathrm{Na}_{2} \mathrm{O}-70 \mathrm{wt} \% \mathrm{SiO}_{2}$ and Frit $510=14 \mathrm{wt} \% \mathrm{~B}_{2} \mathrm{O}_{3}-8 \mathrm{wt} \% \mathrm{Li}_{2} \mathrm{O}-$ $8 \mathrm{wt} \% \mathrm{Na}_{2} \mathrm{O}-70 \mathrm{wt} \% \mathrm{SiO}_{2}$.
} 
SRNL-STI-2009-00778, REVISION 0

Table 5. Sludge Compositions Used to Develop the Matrix 2A Glasses

\begin{tabular}{|c|c|c|c|c|c|c|c|c|c|c|c|c|c|c|c|}
\hline \multirow{2}{*}{ Oxide } & Sludge 1 & Sludge 2 & Sludge 3 & Sludge 4 & Sludge 5 & Sludge 6 & $\begin{array}{l}\text { Sludge } 7 \\
\end{array}$ & Sludge 8 & Sludge 9 & Sludge 10 & Sludge 11 & Sludge 12 & Sludge 13 & Sludge 14 & Sludge 15 \\
\hline & \multicolumn{15}{|c|}{$(\mathrm{wt} \%)$} \\
\hline $\mathrm{Al}_{2} \mathrm{O}_{3}$ & 23.18 & 28.18 & 18.18 & 13.18 & 13.18 & 13.18 & 13.18 & 13.18 & 23.18 & 23.18 & 23.18 & 23.18 & 28.18 & 28.18 & 28.18 \\
\hline $\mathrm{CaO}$ & 2.64 & 2.64 & 2.64 & 2.64 & 2.64 & 2.64 & 6.64 & 8.64 & 2.64 & 2.64 & 2.64 & 2.64 & 4.64 & 6.64 & 8.64 \\
\hline $\mathrm{Fe}_{2} \mathrm{O}_{3}$ & 29.89 & 29.89 & 29.89 & 29.89 & 34.89 & 39.89 & 39.89 & 39.89 & 34.89 & 24.89 & 29.89 & 29.89 & 25.89 & 25.89 & 25.89 \\
\hline $\mathrm{MgO}$ & 1.42 & 1.42 & 1.42 & 1.42 & 1.42 & 1.42 & 1.42 & 1.42 & 1.42 & 1.42 & 1.42 & 1.42 & 1.42 & 1.42 & 1.42 \\
\hline $\mathrm{MnO}$ & 5.85 & 5.85 & 5.85 & 5.85 & 5.85 & 5.85 & 5.85 & 5.85 & 5.85 & 5.85 & 7.85 & 3.85 & 5.85 & 5.85 & 5.85 \\
\hline $\mathrm{Na}_{2} \mathrm{O}$ & 22.96 & 19.20 & 27.01 & 27.85 & 24.54 & 19.54 & 19.20 & 19.20 & 19.20 & 27.01 & 19.20 & 27.01 & 21.11 & 20.11 & 19.11 \\
\hline $\mathrm{NiO}$ & 2.27 & 2.27 & 2.27 & 2.27 & 2.27 & 2.27 & 2.27 & 2.27 & 2.27 & 2.27 & 2.27 & 2.27 & 2.27 & 2.27 & 2.27 \\
\hline $\mathrm{SiO}_{2}$ & 4.85 & 3.61 & 5.80 & 9.96 & 8.27 & 8.27 & 4.61 & 2.61 & 3.61 & 5.80 & 6.61 & 2.80 & 3.70 & 2.70 & 1.70 \\
\hline $\mathrm{TiO}_{2}$ & 4.00 & 4.00 & 4.00 & 4.00 & 4.00 & 4.00 & 4.00 & 4.00 & 4.00 & 4.00 & 4.00 & 4.00 & 4.00 & 4.00 & 4.00 \\
\hline Others & 2.94 & 2.94 & 2.94 & 2.94 & 2.94 & 2.94 & 2.94 & 2.94 & 2.94 & 2.94 & 2.94 & 2.94 & 2.94 & 2.94 & 2.94 \\
\hline
\end{tabular}


SRNL-STI-2009-00778, REVISION 0

Table 6. Target Glass Compositions of the Matrix 2A Glasses (wt\%)

\begin{tabular}{|c|c|c|c|c|c|c|c|c|c|c|c|c|c|c|c|}
\hline Glass ID & EM-01 & EM-02 & EM-03 & EM-04 & EM-05 & EM-06 & EM-07 & EM-08 & EM-09 & EM-10 & EM-11 & EM-12 & EM-13 & EM-14 & EM-15 \\
\hline Frit & \multicolumn{15}{|c|}{418} \\
\hline WL & \multicolumn{15}{|c|}{50} \\
\hline Sludge & 1 & 2 & 3 & 4 & 5 & 6 & 7 & 8 & 9 & 10 & 11 & 12 & 13 & 14 & 15 \\
\hline $\mathrm{Al}_{2} \mathrm{O}_{3}$ & 11.59 & 14.09 & 9.09 & 6.59 & 6.59 & 6.59 & 6.59 & 6.59 & 11.59 & 11.59 & 11.59 & 11.59 & 14.09 & 14.09 & 14.09 \\
\hline $\mathrm{B}_{2} \mathrm{O}_{3}$ & 4.00 & 4.00 & 4.00 & 4.00 & 4.00 & 4.00 & 4.00 & 4.00 & 4.00 & 4.00 & 4.00 & 4.00 & 4.00 & 4.00 & 4.00 \\
\hline $\mathrm{BaO}$ & 0.08 & 0.08 & 0.08 & 0.08 & 0.08 & 0.08 & 0.08 & 0.08 & 0.08 & 0.08 & 0.08 & 0.08 & 0.08 & 0.08 & 0.08 \\
\hline $\mathrm{CaO}$ & 1.32 & 1.32 & 1.32 & 1.32 & 1.32 & 1.32 & 3.32 & 4.32 & 1.32 & 1.32 & 1.32 & 1.32 & 2.32 & 3.32 & 4.32 \\
\hline $\mathrm{Ce}_{2} \mathrm{O}_{3}$ & 0.24 & 0.24 & 0.24 & 0.24 & 0.24 & 0.24 & 0.24 & 0.24 & 0.24 & 0.24 & 0.24 & 0.24 & 0.24 & 0.24 & 0.24 \\
\hline $\mathrm{Cr}_{2} \mathrm{O}_{3}$ & 0.14 & 0.14 & 0.14 & 0.14 & 0.14 & 0.14 & 0.14 & 0.14 & 0.14 & 0.14 & 0.14 & 0.14 & 0.14 & 0.14 & 0.14 \\
\hline $\mathrm{CuO}$ & 0.04 & 0.04 & 0.04 & 0.04 & 0.04 & 0.04 & 0.04 & 0.04 & 0.04 & 0.04 & 0.04 & 0.04 & 0.04 & 0.04 & 0.04 \\
\hline $\mathrm{Fe}_{2} \mathrm{O}_{3}$ & 14.95 & 14.95 & 14.95 & 14.95 & 17.45 & 19.95 & 19.95 & 19.95 & 17.45 & 12.45 & 14.95 & 14.95 & 12.95 & 12.95 & 12.95 \\
\hline $\mathrm{K}_{2} \mathrm{O}$ & 0.11 & 0.11 & 0.11 & 0.11 & 0.11 & 0.11 & 0.11 & 0.11 & 0.11 & 0.11 & 0.11 & 0.11 & 0.11 & 0.11 & 0.11 \\
\hline $\mathrm{La}_{2} \mathrm{O}_{3}$ & 0.08 & 0.08 & 0.08 & 0.08 & 0.08 & 0.08 & 0.08 & 0.08 & 0.08 & 0.08 & 0.08 & 0.08 & 0.08 & 0.08 & 0.08 \\
\hline $\mathrm{Li}_{2} \mathrm{O}$ & 4.00 & 4.00 & 4.00 & 4.00 & 4.00 & 4.00 & 4.00 & 4.00 & 4.00 & 4.00 & 4.00 & 4.00 & 4.00 & 4.00 & 4.00 \\
\hline $\mathrm{MgO}$ & 0.71 & 0.71 & 0.71 & 0.71 & 0.71 & 0.71 & 0.71 & 0.71 & 0.71 & 0.71 & 0.71 & 0.71 & 0.71 & 0.71 & 0.71 \\
\hline $\mathrm{MnO}$ & 2.92 & 2.92 & 2.92 & 2.92 & 2.92 & 2.92 & 2.92 & 2.92 & 2.92 & 2.92 & 3.92 & 1.92 & 2.92 & 2.92 & 2.92 \\
\hline $\mathrm{Na}_{2} \mathrm{O}$ & 15.48 & 13.60 & 17.51 & 17.93 & 16.27 & 13.77 & 13.60 & 13.60 & 13.60 & 17.51 & 13.60 & 17.51 & 14.56 & 14.06 & 13.56 \\
\hline $\mathrm{NiO}$ & 1.14 & 1.14 & 1.14 & 1.14 & 1.14 & 1.14 & 1.14 & 1.14 & 1.14 & 1.14 & 1.14 & 1.14 & 1.14 & 1.14 & 1.14 \\
\hline $\mathrm{PbO}$ & 0.10 & 0.10 & 0.10 & 0.10 & 0.10 & 0.10 & 0.10 & 0.10 & 0.10 & 0.10 & 0.10 & 0.10 & 0.10 & 0.10 & 0.10 \\
\hline $\mathrm{SiO}_{2}$ & 40.42 & 39.81 & 40.90 & 42.98 & 42.14 & 42.14 & 40.31 & 39.31 & 39.81 & 40.90 & 41.31 & 39.40 & 39.85 & 39.35 & 38.85 \\
\hline $\mathrm{SO}_{4}$ & 0.45 & 0.45 & 0.45 & 0.45 & 0.45 & 0.45 & 0.45 & 0.45 & 0.45 & 0.45 & 0.45 & 0.45 & 0.45 & 0.45 & 0.45 \\
\hline $\mathrm{TiO}_{2}$ & 2.00 & 2.00 & 2.00 & 2.00 & 2.00 & 2.00 & 2.00 & 2.00 & 2.00 & 2.00 & 2.00 & 2.00 & 2.00 & 2.00 & 2.00 \\
\hline $\mathrm{ZnO}$ & 0.07 & 0.07 & 0.07 & 0.07 & 0.07 & 0.07 & 0.07 & 0.07 & 0.07 & 0.07 & 0.07 & 0.07 & 0.07 & 0.07 & 0.07 \\
\hline $\mathrm{ZrO}_{2}$ & 0.17 & 0.17 & 0.17 & 0.17 & 0.17 & 0.17 & 0.17 & 0.17 & 0.17 & 0.17 & 0.17 & 0.17 & 0.17 & 0.17 & 0.17 \\
\hline
\end{tabular}


SRNL-STI-2009-00778, REVISION 0

Table 6 cont. Target Glass Compositions of the Matrix 2 A Glasses (wt\%)

\begin{tabular}{|c|c|c|c|c|c|c|c|c|c|c|c|c|c|c|c|}
\hline Glass ID & EM-16 & EM-17 & EM-18 & EM-19 & EM-20 & EM-21 & EM-22 & EM-23 & EM-24 & EM-25 & EM-26 & EM-27 & EM-28 & EM-29 & EM-30 \\
\hline Frit & \multicolumn{15}{|c|}{510} \\
\hline $\mathrm{WL}$ & \multicolumn{15}{|c|}{50} \\
\hline Sludge & 1 & 2 & 3 & 4 & 5 & 6 & 7 & 8 & 9 & 10 & 11 & 12 & 13 & 14 & 15 \\
\hline $\mathrm{Al}_{2} \mathrm{O}_{3}$ & 11.59 & 14.09 & 9.09 & 6.59 & 6.59 & 6.59 & 6.59 & 6.59 & 11.59 & 11.59 & 11.59 & 11.59 & 14.09 & 14.09 & 14.09 \\
\hline $\mathrm{B}_{2} \mathrm{O}_{3}$ & 7.00 & 7.00 & 7.00 & 7.00 & 7.00 & 7.00 & 7.00 & 7.00 & 7.00 & 7.00 & 7.00 & 7.00 & 7.00 & 7.00 & 7.00 \\
\hline $\mathrm{BaO}$ & 0.08 & 0.08 & 0.08 & 0.08 & 0.08 & 0.08 & 0.08 & 0.08 & 0.08 & 0.08 & 0.08 & 0.08 & 0.08 & 0.08 & 0.08 \\
\hline $\mathrm{CaO}$ & 1.32 & 1.32 & 1.32 & 1.32 & 1.32 & 1.32 & 3.32 & 4.32 & 1.32 & 1.32 & 1.32 & 1.32 & 2.32 & 3.32 & 4.32 \\
\hline $\mathrm{Ce}_{2} \mathrm{O}_{3}$ & 0.24 & 0.24 & 0.24 & 0.24 & 0.24 & 0.24 & 0.24 & 0.24 & 0.24 & 0.24 & 0.24 & 0.24 & 0.24 & 0.24 & 0.24 \\
\hline $\mathrm{Cr}_{2} \mathrm{O}_{3}$ & 0.14 & 0.14 & 0.14 & 0.14 & 0.14 & 0.14 & 0.14 & 0.14 & 0.14 & 0.14 & 0.14 & 0.14 & 0.14 & 0.14 & 0.14 \\
\hline $\mathrm{CuO}$ & 0.04 & 0.04 & 0.04 & 0.04 & 0.04 & 0.04 & 0.04 & 0.04 & 0.04 & 0.04 & 0.04 & 0.04 & 0.04 & 0.04 & 0.04 \\
\hline $\mathrm{Fe}_{2} \mathrm{O}_{3}$ & 14.95 & 14.95 & 14.95 & 14.95 & 17.45 & 19.95 & 19.95 & 19.95 & 17.45 & 12.45 & 14.95 & 14.95 & 12.95 & 12.95 & 12.95 \\
\hline $\mathrm{K}_{2} \mathrm{O}$ & 0.11 & 0.11 & 0.11 & 0.11 & 0.11 & 0.11 & 0.11 & 0.11 & 0.11 & 0.11 & 0.11 & 0.11 & 0.11 & 0.11 & 0.11 \\
\hline $\mathrm{La}_{2} \mathrm{O}_{3}$ & 0.08 & 0.08 & 0.08 & 0.08 & 0.08 & 0.08 & 0.08 & 0.08 & 0.08 & 0.08 & 0.08 & 0.08 & 0.08 & 0.08 & 0.08 \\
\hline $\mathrm{Li}_{2} \mathrm{O}$ & 4.00 & 4.00 & 4.00 & 4.00 & 4.00 & 4.00 & 4.00 & 4.00 & 4.00 & 4.00 & 4.00 & 4.00 & 4.00 & 4.00 & 4.00 \\
\hline $\mathrm{MgO}$ & 0.71 & 0.71 & 0.71 & 0.71 & 0.71 & 0.71 & 0.71 & 0.71 & 0.71 & 0.71 & 0.71 & 0.71 & 0.71 & 0.71 & 0.71 \\
\hline $\mathrm{MnO}$ & 2.92 & 2.92 & 2.92 & 2.92 & 2.92 & 2.92 & 2.92 & 2.92 & 2.92 & 2.92 & 3.92 & 1.92 & 2.92 & 2.92 & 2.92 \\
\hline $\mathrm{Na}_{2} \mathrm{O}$ & 15.48 & 13.60 & 17.51 & 17.93 & 16.27 & 13.77 & 13.60 & 13.60 & 13.60 & 17.51 & 13.60 & 17.51 & 14.56 & 14.06 & 13.56 \\
\hline $\mathrm{NiO}$ & 1.14 & 1.14 & 1.14 & 1.14 & 1.14 & 1.14 & 1.14 & 1.14 & 1.14 & 1.14 & 1.14 & 1.14 & 1.14 & 1.14 & 1.14 \\
\hline $\mathrm{PbO}$ & 0.10 & 0.10 & 0.10 & 0.10 & 0.10 & 0.10 & 0.10 & 0.10 & 0.10 & 0.10 & 0.10 & 0.10 & 0.10 & 0.10 & 0.10 \\
\hline $\mathrm{SiO}_{2}$ & 37.42 & 36.81 & 37.90 & 39.98 & 39.14 & 39.14 & 37.31 & 36.31 & 36.81 & 37.90 & 38.31 & 36.40 & 36.85 & 36.35 & 35.85 \\
\hline $\mathrm{SO}_{4}$ & 0.45 & 0.45 & 0.45 & 0.45 & 0.45 & 0.45 & 0.45 & 0.45 & 0.45 & 0.45 & 0.45 & 0.45 & 0.45 & 0.45 & 0.45 \\
\hline $\mathrm{TiO}_{2}$ & 2.00 & 2.00 & 2.00 & 2.00 & 2.00 & 2.00 & 2.00 & 2.00 & 2.00 & 2.00 & 2.00 & 2.00 & 2.00 & 2.00 & 2.00 \\
\hline $\mathrm{ZnO}$ & 0.07 & 0.07 & 0.07 & 0.07 & 0.07 & 0.07 & 0.07 & 0.07 & 0.07 & 0.07 & 0.07 & 0.07 & 0.07 & 0.07 & 0.07 \\
\hline $\mathrm{ZrO}_{2}$ & 0.17 & 0.17 & 0.17 & 0.17 & 0.17 & 0.17 & 0.17 & 0.17 & 0.17 & 0.17 & 0.17 & 0.17 & 0.17 & 0.17 & 0.17 \\
\hline
\end{tabular}


Table 7. Matrix 2A MAR Assessment Results

\begin{tabular}{|c|c|c|c|c|}
\hline Glass ID & $\mathrm{Al}_{2} \mathrm{O}_{3}(\mathrm{wt} \%)$ & $\mathrm{R}_{2} \mathrm{O}(\mathrm{wt} \%)$ & Nepheline Value & MAR Status \\
\hline EM-01 & 11.59 & 19.59 & 0.60 & $\mathrm{~T}_{\mathrm{L}}$ newlv $\mathrm{TiO}_{2} \mathrm{Neph}$ \\
\hline EM-02 & 14.09 & 17.71 & 0.60 & $\mathrm{~T}_{\mathrm{L}} \mathrm{TiO}_{2} \mathrm{Neph}$ \\
\hline EM-03 & 9.09 & 21.62 & 0.61 & newlv $\mathrm{TiO}_{2} \mathrm{Neph}$ \\
\hline EM-04 & 6.59 & 22.04 & 0.64 & Del $\mathrm{G}_{\mathrm{p}}$ newlv $\mathrm{TiO}_{2}$ \\
\hline EM-05 & 6.59 & 20.38 & 0.65 & $\mathrm{~T}_{\mathrm{L}}$ newlv $\mathrm{TiO}_{2}$ \\
\hline EM-06 & 6.59 & 17.88 & 0.67 & $\mathrm{~T}_{\mathrm{L}}$ newlv $\mathrm{TiO}_{2}$ \\
\hline EM-07 & 6.59 & 17.71 & 0.67 & $\mathrm{~T}_{\mathrm{L}}$ newlv $\mathrm{TiO}_{2}$ \\
\hline EM-08 & 6.59 & 17.71 & 0.67 & $\mathrm{~T}_{\mathrm{L}}$ newlv $\mathrm{TiO}_{2}$ \\
\hline EM-09 & 11.59 & 17.71 & 0.61 & $\mathrm{~T}_{\mathrm{L}} \mathrm{TiO}_{2} \mathrm{Neph}$ \\
\hline EM-10 & 11.59 & 21.62 & 0.58 & newlv $\mathrm{TiO}_{2} \mathrm{Neph}$ \\
\hline EM-11 & 11.59 & 17.71 & 0.62 & $\mathrm{~T}_{\mathrm{L}} \mathrm{TiO}_{2} \mathrm{Neph}$ \\
\hline EM-12 & 11.59 & 21.62 & 0.58 & $\mathrm{~T}_{\mathrm{L}}$ newlv $\mathrm{TiO}_{2} \mathrm{Neph}$ \\
\hline EM-13 & 14.09 & 18.67 & 0.58 & $\mathrm{~T}_{\mathrm{L}} \mathrm{TiO}_{2} \mathrm{Neph}$ \\
\hline EM-14 & 14.09 & 18.17 & 0.58 & $\mathrm{~T}_{\mathrm{L}} \mathrm{TiO}_{2} \mathrm{Neph}$ \\
\hline EM-15 & 14.09 & 17.67 & 0.58 & $\mathrm{~T}_{\mathrm{L}} \mathrm{TiO}_{2} \mathrm{Neph}$ \\
\hline EM-16 & 11.59 & 19.59 & 0.58 & $\mathrm{~T}_{\mathrm{L}}$ newlv $\mathrm{TiO}_{2} \mathrm{Neph}$ \\
\hline EM-17 & 14.09 & 17.71 & 0.57 & $\mathrm{~T}_{\mathrm{L}}$ newlv $\mathrm{TiO}_{2} \mathrm{Neph}$ \\
\hline EM-18 & 9.09 & 21.62 & 0.59 & newlv $\mathrm{TiO}_{2} \mathrm{Neph}$ \\
\hline EM-19 & 6.59 & 22.04 & 0.62 & Del $\mathrm{G}_{\mathrm{p}}$ newlv $\mathrm{TiO}_{2} \mathrm{Neph}$ \\
\hline EM-20 & 6.59 & 20.38 & 0.63 & $\mathrm{~T}_{\mathrm{L}}$ newlv $\mathrm{TiO}_{2}$ \\
\hline EM-21 & 6.59 & 17.88 & 0.66 & $\mathrm{~T}_{\mathrm{L}}$ newlv $\mathrm{TiO}_{2}$ \\
\hline EM-22 & 6.59 & 17.71 & 0.65 & $\mathrm{~T}_{\mathrm{L}}$ newlv $\mathrm{TiO}_{2}$ \\
\hline EM-23 & 6.59 & 17.71 & 0.64 & $\mathrm{~T}_{\mathrm{L}}$ newlv $\mathrm{TiO}_{2}$ \\
\hline EM-24 & 11.59 & 17.71 & 0.59 & $\mathrm{~T}_{\mathrm{L}}$ newlv $\mathrm{TiO}_{2} \mathrm{Neph}$ \\
\hline EM-25 & 11.59 & 21.62 & 0.57 & newlv $\mathrm{TiO}_{2} \mathrm{Neph}$ \\
\hline EM-26 & 11.59 & 17.71 & 0.60 & $\mathrm{~T}_{\mathrm{L}}$ newlv $\mathrm{TiO}_{2} \mathrm{Neph}$ \\
\hline EM-27 & 11.59 & 21.62 & 0.56 & $\mathrm{~T}_{\mathrm{L}}$ newlv $\mathrm{TiO}_{2} \mathrm{Neph}$ \\
\hline EM-28 & 14.09 & 18.67 & 0.56 & $\mathrm{~T}_{\mathrm{L}}$ newlv $\mathrm{TiO}_{2} \mathrm{Neph}$ \\
\hline EM-29 & 14.09 & 18.17 & 0.56 & $\mathrm{~T}_{\mathrm{L}}$ newlv $\mathrm{TiO}_{2} \mathrm{Neph}$ \\
\hline EM-30 & 14.09 & 17.67 & 0.56 & $\mathrm{~T}_{\mathrm{L}}$ newlv $\mathrm{TiO}_{2} \mathrm{Neph}$ \\
\hline
\end{tabular}


SRNL-STI-2009-00778, REVISION 0

\subsection{OBJECTIVES}

The objective of this task is to develop data, assess property models, and determine if the current models need to be refined or re-developed to support increased WL of HLW at SRS.

\subsection{EXPERIMENTAL PROCEDURES}

\subsection{GLASS FABRICATION}

Each glass was prepared from the proper proportions of reagent-grade metal oxides, carbonates, $\mathrm{H}_{3} \mathrm{BO}_{3}$, and salts in (i) $250 \mathrm{~g}$ batches for FY09EM21-01 though 27, (ii) $200 \mathrm{~g}$ batches for EM-01 through 30, and (iii) $150 \mathrm{~g}$ for the radioactive glasses (FY09EM21-28 through 30). ${ }^{10}$ The raw materials were thoroughly mixed and placed into a platinum alloy crucible. Batched materials were placed into a high-temperature furnace at $1150^{\circ} \mathrm{C}$ for 1 hour. ${ }^{11}$ The molten glass was quenched by pouring the liquid onto a clean, stainless steel plate. It should be noted that a number of glasses ${ }^{6}$ appeared to be crystallized and were re-melted at $1150^{\circ} \mathrm{C}$ for 30 minutes. The molten glass of these samples was poured directly into an ice bath. The glass pour patties were used as a sampling stock for the various property measurements (i.e., chemical composition, durability testing and XRD).

Approximately $25 \mathrm{~g}$ of each glass was heat-treated to simulate cooling along the centerline of a DWPF-type canister to gauge the effects of thermal history on product performance. ${ }^{12}$ This cooling schedule is referred to as the canister centerline cooling (ccc) curve.

\subsection{CHEMICAL COMPOSITION MEASUREMENTS}

To confirm that the as-fabricated glasses met the target compositions, a representative sample from each non-radioactive glass was submitted to the SRNL Process Science Analytical Laboratory (PSAL) for chemical analysis under the auspices of two analytical plans: SRNLL5200-2009-00007 for glasses FY09EM21-01 though 27 and SRNL-L5200-2009-00025 for EM-01 through 30. ${ }^{13,14}$ Representative samples from each radioactive glass were submitted to Analytical Development (AD) under the auspices of a separate analytical plan: SRNL-L51002009-00003 for FY09EM21-28 through 30. ${ }^{15}$ Two dissolution techniques were utilized by PSAL: sodium peroxide fusion (PF) and lithium metaborate fusion (LM). Each glass was prepared in duplicate for each cation dissolution technique (PF and LM). All of the prepared samples were analyzed (twice for each element of interest) by Inductively Coupled PlasmaAtomic Emission Spectroscopy (ICP-AES) with the instrumentation being re-calibrated between the duplicate analyses. Each analytical plan was developed in such a way as to provide the opportunity to evaluate potential sources of bias and error. Glass standards were also intermittently measured to assess the performance of the ICP-AES instrument over the course of these analyses.

The elemental concentrations were converted to oxide concentrations by multiplying the values for each element by the gravimetric factor for the corresponding oxide. During this process, an

${ }^{6}$ FY09EM21-01, -04, -13, -22, -23 and -24. 
elemental concentration that was determined to be below the detection limit of the analytical procedures used by the PSAL/AD was reduced to half of that detection limit as the oxide concentration was determined. ${ }^{16}$

\subsection{PCT}

The PCT was performed in triplicate on each quenched and ccc glass to assess chemical durability using Method A of the PCT procedure. ${ }^{17}$ Also included in the experimental test matrix was the Environmental Assessment (EA) glass, the Approved Reference Material (ARM) glass, and blanks from the sample cleaning batch. ${ }^{18}$ Samples were ground, washed, and prepared according to the standard procedure. ${ }^{19}$ The resulting non-radioactive solutions were sampled (filtered and acidified) and analyzed by PSAL under the auspices of two analytical plans: SRNLL5200-2009-00008 and SRNL-L5200-2009-00024 for the non-radioactive samples. ${ }^{20,21}$ The solutions containing uranium were analyzed by AD under the auspices of one analytical plan (SRNL-L5200-2009-00006). ${ }^{22}$ Samples of a multi-element, standard solution were also included in the analytical plans (as a check on the accuracy of the ICP-AES instrument).

PCT leachate concentrations were normalized using the measured cation composition (expressed as a weight percent) in the glass to obtain a grams-per-liter $(\mathrm{g} / \mathrm{L})$ leachate concentration. For completeness, the target cation and the measured bias-corrected cation compositions were also used to conduct this normalization.

\subsection{XRD}

\subsubsection{SRNL}

Representative samples of the quenched and $\operatorname{ccc}$ glasses ${ }^{7}$ were submitted to AD for XRD analysis. The glasses were ground in an agate mortar and pestle to reduce the particle size and to homogenize the samples. Ground powder was then placed on a glass slide. A few drops of a $10 \%$ Amyl Acetate Collidion solution were added to the ground powder to affix the powder to the glass slide. Data were collected between 5 and $70^{\circ} 2 \theta$ with a step size of $0.02^{\circ} 2 \theta$ and a $1 \mathrm{~s}$ dwell time. Note that the detection limit of the instrument is approximately $0.5 \mathrm{vol} \%$.

\subsubsection{PNNL}

The amount and type of crystalline phases that formed during the ccc heat treatment on twenty two glass samples were analyzed by powdered XRD according to the PNNL procedures. ${ }^{23,24}$ Samples were prepared by mixing $5 \mathrm{wt} \% \mathrm{CaF}_{2}$ (internal standard) with approximately 1.5 to 2.5 $\mathrm{g}$ of glass. The glass $/ \mathrm{CaF}_{2}$ mixture was milled to a fine powder for 2 minutes in a $10 \mathrm{~cm}^{3}$ tungsten carbide disc mill. Samples were then loaded in round aluminum XRD sample holders and mounted in the automated 12 stage XRD sample platform. Each XRD scan was processed at a scan rate of $0.04^{\circ} 2 \theta$ between 10 and $70^{\circ} 2 \theta$, with a $4 \mathrm{~s}$ dwell time. Data were analyzed with Jade 6.0 Software (MDI, Inc.) for phase identification.

\footnotetext{
${ }^{7}$ Only the quenched glasses of the FY09EM21-xx series were measured, while both the quenched and ccc glasses of the EM-xx series were measured.
} 


\section{$4.5 T_{L}$}

The $T_{L}$ and equilibrium crystal fraction as a function of temperature were measured in Pt-alloy crucibles with tight-fitting lids (to avoid volatility) according to PNNL procedure GDL-LQT. ${ }^{25}$ The heat treatment times were roughly $24 \mathrm{~h}$ or longer to ensure equilibrium was achieved without excessive volatilization. Heat treated specimens were air quenched and analyzed to determine the type of crystalline phases according to PNNL procedure GDL-XRD. ${ }^{24}$ The temperature was varied so that the temperature at which the specimens were crystal-free could be determined to within $10^{\circ} \mathrm{C}$ for the $\mathrm{T}_{\mathrm{L}}$. For the equilibrium crystal fraction as a function of temperature, heat treatments were performed for the temperature range from the $\mathrm{T}_{\mathrm{L}}$ to $\sim 5 \mathrm{mass} \%$ crystallization. Notes were taken on the location of crystals within the crucible to distinguish between surface and bulk crystallization.

A National Bureau of Standards (NBS) liquidus temperature standard glass (SRM-773) was used to validate the $T_{L}$ measurement technique and profile the furnaces used in the experimentation. Based on the SRM-773 standard measurements (tested in all furnaces used to support the $\mathrm{T}_{\mathrm{L}}$ measurements), the $T_{L}$ values reported for the study glasses are estimated to be within \pm 5 to $10^{\circ} \mathrm{C}$ of the actual values. Calibrated thermocouples and temperature readouts were used for taking temperature measurements for all furnaces.

\subsection{VISCOSITY}

Viscosity was measured as a function of temperature using a Brookfield DV-II+ high temperature spindle viscometer for each as-fabricated glass. The measurements were obtained using standard procedures, which are compliant with ASTM C 965-81. ${ }^{26}$

In general, the glass was heated to $\sim 1200^{\circ} \mathrm{C}$ or $\sim 1250^{\circ} \mathrm{C}$ in a platinum alloy crucible and maintained until thermal equilibrium was achieved. An initial torque reading (at a constant spindle speed) was taken at this temperature with subsequent measurements at both higher and lower temperatures ranging from $\sim 1050^{\circ} \mathrm{C}$ to $1300^{\circ} \mathrm{C}$ using a hysteresis approach (to the extent possible). The hysteresis approach provides the opportunity to detect any issues associated with either volatilization and/or devitrification over the temperature range of interest. For example, assume the initial measurement was at $1200^{\circ} \mathrm{C}$ followed by multiple higher temperature measurements. Prior to going to temperatures less than $1200^{\circ} \mathrm{C}$, a second measurement was obtained at $1200^{\circ} \mathrm{C}$ to assess the potential impacts of volatility. If significant volatility occurred, then the second measurement at $1200^{\circ} \mathrm{C}$ would not be consistent with the initial measurement, leading to questionable data. A glass viscosity standard was used to determine the geometric constant of the spindle/cup based on torque and speed. This constant is corrected for temperature effects. 
SRNL-STI-2009-00778, REVISION 0

\subsection{RESULTS AND DISCUSSION}

\subsection{TEST MATRIX 2}

\subsubsection{A Statistical Review of the Matrix 2 Chemical Composition Measurements of the Non-Radioactive Glasses}

Table A1 (in two parts) in Appendix A provides the elemental concentration measurements from the study glasses that were prepared using LM, and Table A2 provides the measurements from the samples of these glasses prepared using PF. Measurements for samples of the standard Batch 1 glass that were included in the analytical plan along with the study glasses are also provided in these two tables.

\subsubsection{Measurements in Analytical Sequence}

Exhibit A1 in Appendix A provides plots in analytical sequence of the non-radioactive sample measurements generated by PSAL for each oxide over both preparation methods (i.e., LM and PF). These plots are in analytical sequence and include all of the measurement data from Tables A1 and A2. There do not appear to be any obvious patterns or trends due to the analytical sequence.

\subsubsection{Composition Measurements by Glass Identifier}

Exhibit A2 in Appendix A provide plots of the oxide concentration measurements by Glass ID/Lab ID for each analytical set (including Batch 1). These plots show the individual measurements across the duplicates of each preparation method and the two ICP-AES calibrations for each glass for each oxide. A review of the plots presented in these exhibits reveals the repeatability of the four individual values for each oxide of each glass. In general, there appears to be good repeatability among the measurements for each of the oxides for most of the glasses. However, there are some inconsistencies, which include: (i) in Set 1 the measurements for $\mathrm{B}_{2} \mathrm{O}_{3}$ and $\mathrm{Li}_{2} \mathrm{O}$ for glass FY09EM21-14 indicate a dissolution effect, (ii) the measurements for glasses FY09EM21-01, -02, and -03 indicate little or no NiO while the target concentration for this oxide for these glasses was $2.5 \mathrm{wt} \%^{8}$, (iii) in Set 2 the $\mathrm{MnO}$ measurements for FY09EM21-17 are well below the target value of $5.5 \mathrm{wt} \%$, and (iv) in the Set 2 glasses there appears to be a calibration effect for the $\mathrm{SiO}_{2}$ measurements.

\subsubsection{Results of the Batch 1 Standard}

Exhibit A3 in Appendix A provides statistical analyses of the Batch 1 results by analytical set and calibration block for each oxide of interest over both preparation methods. The results also include analysis of variance (ANOVA) investigations, which determine statistically significant differences among the means of these groups for each of the oxides for each of the standards.

\footnotetext{
${ }^{8} \mathrm{NiO}$ was not present in FY09EM21-01, -02 and -03 due to batching error. See page 111 of WSRC-NB-200300050 .

${ }^{9}$ There appears to be a batching error for the MnO in glass FY09EM21-17 based on the batching sheet or the measurement.
} 
The following components of the Batch 1 standard indicate a significant ICP-AES calibration effect on the block averages at the 5\% significance level:

$$
\text { - } \mathrm{BaO}, \mathrm{CaO}, \mathrm{Cr}_{2} \mathrm{O}_{3}, \mathrm{CuO}, \mathrm{Fe}_{2} \mathrm{O}_{3}, \mathrm{Li}_{2} \mathrm{O}, \mathrm{MgO}, \mathrm{MnO}, \mathrm{NiO}, \mathrm{SiO}_{2} \text {, and } \mathrm{ZrO}_{2}
$$

Results from these statistical analyses provide incentive for adjusting the measurements by the effects of the ICP-AES calibration. Thus, bias correction of these data was pursued in order to determine if the adjusted values impacted the conclusions of this study. ${ }^{10}$ Batch 1 results were used to bias correct all of the oxides as long as the reference value for the oxide concentration in the Batch 1 glass was greater than or equal to $0.1 \mathrm{wt} \%$. By applying this approach, the Batch 1 results were used to bias correct the $\mathrm{Al}_{2} \mathrm{O}_{3}, \mathrm{~B}_{2} \mathrm{O}_{3}, \mathrm{BaO}, \mathrm{CaO}, \mathrm{Cr}_{2} \mathrm{O}_{3}, \mathrm{CuO}, \mathrm{Fe}_{2} \mathrm{O}_{3}, \mathrm{~K}_{2} \mathrm{O}, \mathrm{Li}_{2} \mathrm{O}$, $\mathrm{MgO}, \mathrm{MnO}, \mathrm{Na}_{2} \mathrm{O}, \mathrm{NiO}, \mathrm{SiO}_{2}$ and $\mathrm{TiO}_{2}$ measurements. Bias correction was not conducted on $\mathrm{CdO}, \mathrm{Ce}_{2} \mathrm{O}_{3}, \mathrm{La}_{2} \mathrm{O}_{3}, \mathrm{PbO}, \mathrm{SO}_{4}, \mathrm{ThO}_{2}, \mathrm{ZnO}$, or $\mathrm{ZrO}_{2}$.

The bias correction was conducted as follows. For each oxide, let $\bar{a}_{i j}$ be the average measurement for the $i^{\text {th }}$ oxide at analytical block $\mathrm{j}$ for Batch 1 , and let $t_{i}$ be the reference value for the $i^{\text {th }}$ oxide for Batch 1. The averages and reference values are provided in Exhibit A3. Let $\bar{c}_{i j k}$ be the average measurement for the $\mathrm{i}^{\text {th }}$ oxide at analytical block $\mathrm{j}$ for the $\mathrm{k}^{\text {th }}$ glass. The bias adjustment was conducted as follows:

$$
\bar{c}_{i j k} \bullet\left(1-\frac{\bar{a}_{i j}-t_{i}}{\bar{a}_{i j}}\right)=\bar{c}_{i j k} \bullet \frac{t_{i}}{\bar{a}_{i j}}
$$

Bias-corrected measurements are indicated by a "bc" suffix in the remainder of the document. Both measured and measured "bc" values are included in the following discussion. For completeness, the original values of $\mathrm{CdO}, \mathrm{Ce}_{2} \mathrm{O}_{3}, \mathrm{La}_{2} \mathrm{O}_{3}, \mathrm{PbO}, \mathrm{SO}_{4}, \mathrm{ThO}_{2}, \mathrm{ZnO}$, and $\mathrm{ZrO}_{2}$ were included in the bias-corrected results in order to calculate a "bc" sum of oxides.

\subsubsection{Measured Versus Target Compositions}

With only minor problems in the repeatability of the measurements being revealed during the review process, all of the measurements for each oxide for each glass (i.e., all of the measurements in Tables A1 through A2) were averaged to determine a representative chemical composition for each glass. These determinations were conducted both for the measured and for the bias-corrected data. A sum of oxides was also computed for each glass based upon both the measured and bias-corrected values. Exhibit A4 in Appendix A provides plots showing results for each glass for each oxide to help highlight the comparisons among the measured, biascorrected, and target values. In general, there appear to have been few difficulties in hitting the target concentrations of the major oxides ${ }^{11}$ for the study glasses. The average of the $\mathrm{B}_{2} \mathrm{O}_{3}$ measurements for FY09EM21-14 was higher than the target concentration. A preparation effect

\footnotetext{
${ }^{10}$ It should be emphasized that bias correction is considered in order to demonstrate that the results and conclusions from this study are not affected by the compositional view (target, measured, or measured-bc). Demonstrating that the compositional view does not alter or change the conclusions shows a degree of robustness for the objective of the study.

${ }^{11}$ Greater than $0.5 \mathrm{wt} \%$.
} 
for this glass was previously identified and would account for the higher than expected value. The average $\mathrm{MnO}$ measurement for FY09EM21-17 was very low compared to the target value of $5.5 \mathrm{wt} \%$, which is probably due to a batching error since very little was detected. The average measurements for $\mathrm{NiO}$ for glasses FY09EM21-01, -02, -03 were much lower than their target values of $2.5 \mathrm{wt} \%$ due omission during batching (see Section 5.1.1.2).

Table A4 in Appendix A provides a summary of the average compositions as well as the target compositions and some associated differences and relative differences. Notice that the target sums of oxides for the standard glasses do not sum to $100 \%$ due to an incomplete coverage of the oxides in the Batch 1 glass. All of the sums of oxides (both measured and bias-corrected) for the Matrix 2 glasses fall within the interval of 95 to $105 \mathrm{wt} \%$. Entries in Table A4 show the relative differences between the measured or bias-corrected values and the target values. These differences are shaded when they are greater than or equal to $5 \%$. Overall, these comparisons between the measured and target compositions suggest only minor difficulties in meeting the target compositions for the study glasses.

\subsubsection{Matrix 2 Measurement Acceptability Region (MAR) Assessment}

MAR assessment results are provided in Table 8. The columns in the table give the percent waste loading (\%WL), the frit, the glass identifier with compositional view, the Del Gp value for boron (B Del Gp Value), the normalized leachate for boron in grams/Liter (NL[B (g/L)]), the liquidus temperature prediction in degrees Celsius $\left(\mathrm{T}_{\mathrm{L}}\right.$ Pred $\left.\left({ }^{\circ} \mathrm{C}\right)\right)$, the viscosity prediction in Poise (Visc Pred (P)), the sum of oxides (in wt\%), the nepheline value, and the overall MAR assessment. In general, the MAR assessments of the measured compositions are consistent with those of the target compositions despite the compositional variation observed in Exhibit A4. All of the study glasses fail the $\mathrm{TiO}_{2}$ constraint in order to study the effects of the addition of a secondary waste stream ${ }^{12}$ on glass properties. Other properties such as $\mathrm{T}_{\mathrm{L}}$ and viscosity were also challenged to determine the applicability of the current models.

\footnotetext{
${ }^{12}$ Future sludge batches to be processed at the DWPF may include as much as $6 \mathrm{wt} \% \mathrm{TiO}_{2}$ due to the waste stream coming from the Salt Waste Processing Facility (SWPF).
} 
SRNL-STI-2009-00778, REVISION 0

Table 8. Predicted Properties and MAR Assessment Results of the Non-Radioactive Matrix 2 Glasses

\begin{tabular}{|c|c|c|c|c|c|c|c|c|c|c|c|}
\hline Glass ID & \begin{tabular}{|c|}
$\begin{array}{c}\text { Compositional } \\
\text { View }\end{array}$ \\
\end{tabular} & B Del $G_{p}$ & \begin{tabular}{|c|}
$\mathbf{N L}$ \\
{$[\mathrm{B}(\mathrm{g} / \mathrm{L})]$} \\
\end{tabular} & $\mathbf{T}_{\mathrm{L}}\left({ }^{\circ} \mathbf{C}\right)$ & \begin{tabular}{|c|} 
Viscosity \\
$(\mathbf{P})$
\end{tabular} & $\begin{array}{l}\mathrm{Al}_{2} \mathrm{O}_{3} \\
(\mathrm{wt} \%) \\
\end{array}$ & $\begin{array}{c}\mathrm{TiO}_{2} \\
(\mathrm{wt} \%) \\
\end{array}$ & $\begin{array}{c}\mathrm{R}_{2} \mathrm{O} \\
(\mathrm{wt} \%)\end{array}$ & $\begin{array}{c}\text { Homogeneity } \\
(w t \%)\end{array}$ & \begin{tabular}{|c|}
$\begin{array}{c}\text { Nepheline } \\
\text { Value }\end{array}$ \\
\end{tabular} & MAR Status \\
\hline \multirow{3}{*}{ FY09EM21-01 } & Measured & -6.69 & 0.21 & 1076 & 26 & 10.25 & 5.62 & 15.66 & 252.9 & 0.64 & $\mathrm{~T}_{\mathrm{L}} \mathrm{TiO}_{2}$ \\
\hline & Measured bc & -6.54 & 0.19 & 1079 & 28 & 10.81 & 5.94 & 15.91 & 260.9 & 0.63 & $\mathrm{~T}_{\mathrm{L}} \mathrm{TiO}_{2}$ \\
\hline & Target & -6.46 & 0.19 & 1232 & 25 & 9.97 & 5.60 & 15.39 & 252.8 & 0.64 & $\mathrm{~T}_{\mathrm{L}}$ newlv $\mathrm{TiO}_{2}$ \\
\hline \multirow{3}{*}{ FY09EM21-02 } & Measured & -12.50 & 2.31 & 899 & 5 & 4.11 & 2.06 & 21.08 & 246.9 & 0.66 & newlv $\mathrm{TiO}_{2} 1$ Frit \\
\hline & Measured bc & -12.61 & 2.42 & 905 & 5 & 4.34 & 2.18 & 21.47 & 253.9 & 0.66 & newlv $\mathrm{TiO}_{2}$ IFrit \\
\hline & Target & -13.28 & 3.20 & 1019 & 4 & 4.01 & 2.00 & 21.98 & 249.3 & 0.65 & $\mathrm{~T}_{\mathrm{L}}$ newlv $\mathrm{TiO}_{2}$ IFrit \\
\hline \multirow{3}{*}{ FY09EM21-03 } & Measured & -13.29 & 3.21 & 863 & 6 & 6.93 & 4.98 & 21.32 & 219.9 & 0.65 & newlv $\mathrm{TiO}_{2}$ \\
\hline & Measured bc & -13.04 & 2.90 & 876 & 6 & 7.17 & 5.33 & 21.26 & 222.8 & 0.65 & newlv $\mathrm{TiO}_{2}$ \\
\hline & Target & -13.12 & 2.99 & 994 & 7 & 6.97 & 4.73 & 21.13 & 216.4 & 0.66 & newlv $\mathrm{TiO}_{2}$ \\
\hline \multirow{3}{*}{ FY09EM21-04 } & Measured & -11.16 & 1.32 & 1105 & 1 & 7.01 & 3.95 & 17.63 & 252.7 & 0.64 & $\mathrm{~T}_{\mathrm{L}}$ newlv $\mathrm{TiO}_{2}$ \\
\hline & Measured bc & -11.01 & 1.24 & 1130 & 1 & 7.25 & 4.23 & 17.61 & 256.8 & 0.64 & $\mathrm{~T}_{\mathrm{L}}$ newlv $\mathrm{TiO}_{2}$ \\
\hline & Target & -11.14 & 1.31 & 1126 & 1 & 7.03 & 4.00 & 17.74 & 258.3 & 0.65 & $\mathrm{~T}_{\mathrm{L}}$ newlv $\mathrm{TiO}_{2}$ \\
\hline \multirow{3}{*}{ FY09EM21-05 } & Measured & -6.34 & 0.18 & 1015 & 69 & 3.69 & 5.83 & 13.90 & 228.5 & 0.79 & $\mathrm{~T}_{\mathrm{L}} \mathrm{TiO}_{2}$ IFrit \\
\hline & Measured bc & -6.31 & 0.17 & 1031 & 72 & 3.89 & 6.15 & 14.12 & 235.2 & 0.78 & $\mathrm{~T}_{\mathrm{L}} \mathrm{TiO}_{2}$ IFrit \\
\hline & Target & -6.29 & 0.17 & 1020 & 73 & 3.60 & 5.83 & 14.00 & 233.0 & 0.79 & $\mathrm{~T}_{\mathrm{L}} \mathrm{TiO}_{2}$ IFrit \\
\hline \multirow{3}{*}{ FY09EM21-06 } & Measured & -13.21 & 3.11 & 892 & 19 & 4.44 & 5.90 & 18.66 & 174.2 & 0.69 & newlv $\mathrm{TiO}_{2}$ Homg IFrit \\
\hline & Measured bc & -12.95 & 2.78 & 921 & 20 & 4.59 & 6.30 & 18.56 & 175.6 & 0.69 & newlv $\mathrm{TiO}_{2}$ Homg lFrit \\
\hline & Target & -13.22 & 3.12 & 900 & 20 & 4.39 & 6.00 & 18.78 & 176.1 & 0.70 & newlv $\mathrm{TiO}_{2}$ Homg \\
\hline \multirow{3}{*}{ FY09EM21-07 } & Measured & -13.27 & 3.19 & 940 & 2 & 4.81 & 1.99 & 16.70 & 246.7 & 0.65 & newlv $\mathrm{TiO}_{2}$ \\
\hline & Measured bc & -13.08 & 2.95 & 955 & 2 & 4.98 & 2.13 & 16.60 & 250.2 & 0.65 & newlv $\mathrm{TiO}_{2}$ \\
\hline & Target & -13.18 & 3.07 & 954 & 2 & 4.87 & 2.00 & 16.71 & 254.2 & 0.66 & newlv $\mathrm{TiO}_{2}$ \\
\hline \multirow{3}{*}{ FY09EM21-08 } & Measured & -8.11 & 0.37 & 985 & 8 & 3.26 & 5.95 & 16.39 & 237.8 & 0.77 & newlv $\mathrm{TiO}_{2} 1$ Frit \\
\hline & Measured bc & -8.06 & 0.36 & 989 & 9 & 3.43 & 6.28 & 16.61 & 244.3 & 0.77 & newlv $\mathrm{TiO}_{2} 1$ Frit \\
\hline & Target & -8.47 & 0.43 & 987 & 9 & 3.25 & 6.00 & 17.00 & 243.8 & 0.77 & newlv $\mathrm{TiO}_{2} 1$ Frit \\
\hline \multirow{3}{*}{ FY09EM21-09 } & Measured & -7.98 & 0.35 & 998 & 23 & 12.97 & 2.49 & 16.87 & 256.9 & 0.64 & newlv $\mathrm{TiO}_{2}$ \\
\hline & Measured bc & -7.71 & 0.31 & 1007 & 24 & 13.41 & 2.67 & 16.81 & 261.3 & 0.63 & newlv $\mathrm{TiO}_{2}$ \\
\hline & Target & -7.97 & 0.35 & 1024 & 23 & 12.97 & 2.47 & 17.00 & 260.8 & 0.64 & $\mathrm{~T}_{\mathrm{L}}$ newlv $\mathrm{TiO}_{2}$ \\
\hline \multirow{3}{*}{ FY09EM21-10 } & Measured & -8.82 & 0.50 & 1129 & 27 & 6.39 & 2.44 & 14.56 & 249.8 & 0.70 & $\mathrm{~T}_{\mathrm{L}} \mathrm{TiO}_{2}$ \\
\hline & Measured bc & -8.62 & 0.46 & 1159 & 28 & 6.61 & 2.60 & 14.48 & 254.0 & 0.70 & $\mathrm{~T}_{\mathrm{L}} \mathrm{TiO}_{2}$ \\
\hline & Target & -8.52 & 0.44 & 1161 & 27 & 6.40 & 2.40 & 14.39 & 256.9 & 0.71 & $\mathrm{~T}_{\mathrm{L}} \mathrm{TiO}_{2}$ \\
\hline \multirow{3}{*}{ FY09EM21-11 } & Measured & -12.36 & 2.18 & 719 & 37 & 3.23 & 6.76 & 17.78 & 189.0 & 0.75 & $\mathrm{TiO}_{2}$ Homg IFrit \\
\hline & Measured bc & -12.20 & 2.04 & 733 & 38 & 3.34 & 7.23 & 17.73 & 191.5 & 0.76 & $\mathrm{TiO}_{2}$ Homg IFrit \\
\hline & Target & -12.53 & 2.34 & 708 & 40 & 3.25 & 6.00 & 17.84 & 192.6 & 0.76 & $\mathrm{TiO}_{2}$ Homg IFrit \\
\hline \multirow{3}{*}{ FY09EM21-12 } & Measured & -9.50 & 0.66 & 989 & 20 & 4.57 & 1.99 & 16.42 & 221.6 & 0.76 & newlv $\mathrm{TiO}_{2} 1$ Frit \\
\hline & Measured bc & -9.47 & 0.65 & 1010 & 21 & 4.82 & 2.11 & 16.65 & 227.8 & 0.76 & newlv $\mathrm{TiO}_{2}$ \\
\hline & Target & -9.57 & 0.68 & 1010 & 19 & 4.55 & 2.00 & 16.79 & 233.2 & 0.77 & newlv $\mathrm{TiO}_{2}$ IFrit \\
\hline \multirow{3}{*}{ FY09EM21-13 } & Measured & -7.78 & 0.32 & 1099 & 10 & 10.31 & 5.97 & 17.37 & 255.0 & 0.64 & $\mathrm{~T}_{\mathrm{L}}$ newlv $\mathrm{TiO}_{2}$ \\
\hline & Measured bc & -7.69 & 0.31 & 1109 & 11 & 10.88 & 6.30 & 17.66 & 263.4 & 0.64 & $\mathrm{~T}_{\mathrm{L}}$ newlv $\mathrm{TiO}_{2}$ \\
\hline & Target & -7.77 & 0.32 & 1124 & 10 & 10.38 & 6.00 & 17.52 & 260.4 & 0.64 & $\mathrm{~T}_{\mathrm{L}}$ newlv $\mathrm{TiO}_{2}$ \\
\hline
\end{tabular}


SRNL-STI-2009-00778, REVISION 0

Table 8 continued.

\begin{tabular}{|c|c|c|c|c|c|c|c|c|c|c|c|}
\hline Glass ID & $\begin{array}{c}\text { Compositional } \\
\text { View }\end{array}$ & B Del $G_{p}$ & $\left|\begin{array}{c}\text { NL } \\
{[\mathrm{B}(\mathrm{g} / \mathrm{L})]}\end{array}\right|$ & $\mathbf{T}_{\mathrm{L}}\left({ }^{\circ} \mathbf{C}\right)$ & $\begin{array}{c}\text { Viscosity } \\
\text { (P) }\end{array}$ & $\begin{array}{l}\mathrm{Al}_{2} \mathrm{O}_{3} \\
(\mathrm{wt} \%)\end{array}$ & $\begin{array}{c}\mathrm{TiO}_{2} \\
(\mathrm{wt} \%)\end{array}$ & $\begin{array}{c}\mathrm{R}_{2} \mathrm{O} \\
(\mathrm{wt} \%)\end{array}$ & $\begin{array}{c}\text { Homogeneity } \\
\text { (wt\%) }\end{array}$ & $\begin{array}{c}\text { Nepheline } \\
\text { Value }\end{array}$ & MAR Status \\
\hline \multirow{3}{*}{ FY09EM21-14 } & Measured & -7.47 & 0.28 & 959 & 6 & 3.22 & 5.60 & 14.12 & 241.5 & 0.75 & newlv $\mathrm{TiO}_{2}$ IFrit \\
\hline & Measured bc & -7.42 & 0.28 & 959 & 6 & 3.39 & 5.91 & 14.36 & 247.6 & 0.75 & newlv $\mathrm{TiO}_{2} 1$ Frit \\
\hline & Target & -6.99 & 0.23 & 975 & 8 & 3.25 & 5.71 & 14.00 & 246.0 & 0.75 & newlv $\mathrm{TiO}_{2}$ IFrit \\
\hline \multirow{3}{*}{ FY09EM21-15 } & Measured & -10.27 & 0.91 & 1003 & 60 & 5.37 & 1.86 & 15.72 & 206.8 & 0.76 & $\mathrm{~T}_{\mathrm{L}}$ Homg \\
\hline & Measured bc & -10.35 & 0.94 & 1033 & 58 & 5.51 & 1.97 & 15.83 & 209.0 & 0.76 & $\mathrm{~T}_{\mathrm{L}} \mathrm{TiO}_{2}$ Homg \\
\hline & Target & -10.51 & 1.01 & 1046 & 53 & 5.32 & 2.00 & 16.00 & 209.2 & 0.76 & $\mathrm{~T}_{\mathrm{L}} \mathrm{TiO}_{2}$ Homg \\
\hline \multirow{3}{*}{ FY09EM21-16 } & Measured & -12.75 & 2.56 & 962 & 30 & 3.74 & 1.97 & 19.25 & 213.2 & 0.72 & $\mathrm{TiO}_{2} \mathrm{R}_{2} \mathrm{O}$ \\
\hline & Measured bc & -12.69 & 2.50 & 992 & 29 & 3.81 & 2.10 & 19.21 & 215.0 & 0.71 & $\mathrm{~T}_{\mathrm{L}} \mathrm{TiO}_{2} \mathrm{R}_{2} \mathrm{O}$ \\
\hline & Target & -13.15 & 3.03 & 982 & 28 & 3.68 & 2.00 & 19.82 & 216.2 & 0.71 & $\mathrm{TiO}_{2} \mathrm{R}_{2} \mathrm{O}$ \\
\hline \multirow{3}{*}{ FY09EM21-17 } & Measured & -11.37 & 1.44 & 857 & 6 & 7.67 & 6.13 & 22.03 & 233.9 & 0.65 & newlv $\mathrm{TiO}_{2}$ \\
\hline & Measured bc & -11.14 & 1.31 & 866 & 6 & 7.83 & 6.53 & 21.94 & 236.5 & 0.64 & newlv $\mathrm{TiO}_{2}$ \\
\hline & Target & -12.99 & 2.83 & 852 & 5 & 7.19 & 6.00 & 21.60 & 227.1 & 0.64 & newlv $\mathrm{TiO}_{2}$ \\
\hline \multirow{3}{*}{ FY09EM21-18 } & Measured & -10.77 & 1.12 & 759 & 49 & 11.07 & 1.96 & 21.44 & 226.8 & 0.65 & $\mathrm{TiO}_{2}$ \\
\hline & Measured bc & -10.76 & 1.12 & 763 & 49 & 11.36 & 2.08 & 21.56 & 228.8 & 0.64 & $\mathrm{TiO}_{2}$ \\
\hline & Target & -11.74 & 1.69 & 743 & 40 & 10.92 & 2.00 & 22.51 & 228.6 & 0.64 & $\mathrm{TiO}_{2}$ \\
\hline \multirow{3}{*}{ FY09EM21-19 } & Measured & -12.03 & 1.90 & 748 & 55 & 5.84 & 5.85 & 20.68 & 207.2 & 0.70 & $\mathrm{TiO}_{2}$ Homg \\
\hline & Measured bc & -12.06 & 1.92 & 761 & 53 & 5.99 & 6.20 & 20.79 & 209.6 & 0.69 & $\mathrm{TiO}_{2}$ Homg \\
\hline & Target & -13.13 & 3.01 & 732 & 45 & 5.72 & 6.00 & 22.00 & 208.2 & 0.69 & $\mathrm{TiO}_{2}$ Homg \\
\hline \multirow{3}{*}{ FY09EM21-20 } & Measured & -11.89 & 1.79 & 994 & 26 & 6.16 & 1.98 & 20.97 & 218.3 & 0.67 & $\mathrm{~T}_{\mathrm{L}} \mathrm{TiO}_{2}$ \\
\hline & Measured bc & -11.84 & 1.76 & 1020 & 25 & 6.32 & 2.09 & 20.97 & 218.6 & 0.67 & $\mathrm{~T}_{\mathrm{L}} \mathrm{TiO}_{2}$ \\
\hline & Target & -12.73 & 2.54 & 1003 & 23 & 6.02 & 2.00 & 22.00 & 222.5 & 0.67 & $\mathrm{~T}_{\mathrm{L}}$ newlv $\mathrm{TiO}_{2}$ \\
\hline \multirow{3}{*}{ FY09EM21-21 } & Measured & -13.05 & 2.90 & 921 & 2 & 4.86 & 1.98 & 21.31 & 247.7 & 0.67 & newlv $\mathrm{TiO}_{2}$ \\
\hline & Measured bc & -13.01 & 2.86 & 928 & 2 & 4.96 & 2.11 & 21.40 & 251.9 & 0.67 & newlv $\mathrm{TiO}_{2}$ \\
\hline & Target & -13.23 & 3.14 & 930 & 2 & 4.86 & 2.01 & 21.67 & 254.9 & 0.67 & newlv $\mathrm{TiO}_{2}$ \\
\hline \multirow{3}{*}{ FY09EM21-22 } & Measured & -4.59 & 0.09 & 1031 & 83 & 14.26 & 2.00 & 14.11 & 245.6 & 0.64 & $\mathrm{~T}_{\mathrm{L}} \mathrm{TiO}_{2}$ \\
\hline & Measured bc & -4.39 & 0.08 & 1051 & 85 & 14.56 & 2.13 & 14.07 & 247.8 & 0.64 & $\mathrm{~T}_{\mathrm{L}} \mathrm{TiO}_{2}$ \\
\hline & Target & -4.56 & 0.08 & 1054 & 76 & 14.04 & 2.04 & 14.00 & 245.9 & 0.64 & $\mathrm{~T}_{\mathrm{L}} \mathrm{TiO}_{2}$ \\
\hline \multirow{3}{*}{ FY09EM21-23 } & Measured & -2.46 & 0.04 & 952 & 136 & 13.98 & 5.71 & 13.73 & 247.6 & 0.65 & newhv $\mathrm{TiO}_{2}$ \\
\hline & Measured bc & -2.31 & 0.03 & 957 & 133 & 14.27 & 6.08 & 13.78 & 251.4 & 0.65 & newhv $\mathrm{TiO}_{2}$ \\
\hline & Target & -2.56 & 0.04 & 956 & 123 & 13.96 & 6.00 & 14.00 & 252.7 & 0.65 & newhv $\mathrm{TiO}_{2}$ \\
\hline \multirow{3}{*}{ FY09EM21-24 } & Measured & -4.97 & 0.10 & 1103 & 35 & 10.53 & 3.54 & 13.89 & 283.7 & 0.65 & $\mathrm{~T}_{\mathrm{L}} \mathrm{TiO}_{2}$ \\
\hline & Measured bc & -4.97 & 0.10 & 1103 & 35 & 10.80 & 3.75 & 13.97 & 287.4 & 0.65 & $\mathrm{~T}_{\mathrm{L}} \mathrm{TiO}_{2}$ \\
\hline & Target & -4.91 & 0.10 & 1122 & 29 & 10.51 & 3.94 & 14.00 & 291.0 & 0.65 & $\mathrm{~T}_{\mathrm{L}} \mathrm{TiO}_{2}$ \\
\hline \multirow{3}{*}{ FY09EM21-25 } & Measured & -13.36 & 3.31 & 752 & 48 & 6.41 & 2.01 & 19.41 & 219.5 & 0.69 & $\mathrm{~T}_{\mathrm{L}} \mathrm{TiO}_{2}$ \\
\hline & Measured bc & -13.30 & 3.23 & 761 & 47 & 6.54 & 2.14 & 19.36 & 221.5 & 0.69 & $\mathrm{~T}_{\mathrm{L}} \mathrm{TiO}_{2}$ \\
\hline & Target & -13.24 & 3.14 & 764 & 51 & 6.24 & 2.00 & 19.46 & 221.1 & 0.70 & $\mathrm{~T}_{\mathrm{L}} \mathrm{TiO}_{2}$ \\
\hline \multirow{3}{*}{ FY09EM21-26 } & Measured & -7.09 & 0.24 & 928 & 128 & 13.73 & 4.86 & 16.02 & 215.3 & 0.64 & newhv $\mathrm{TiO}_{2}$ \\
\hline & Measured bc & -6.91 & 0.22 & 938 & 130 & 14.02 & 5.18 & 15.97 & 216.7 & 0.63 & newhv $\mathrm{TiO}_{2}$ \\
\hline & Target & -6.89 & 0.22 & 953 & 130 & 13.66 & 5.36 & 15.96 & 216.4 & 0.64 & newhv $\mathrm{TiO}_{2}$ \\
\hline \multirow{3}{*}{ FY09EM21-27 } & Measured & -9.77 & 0.74 & 1003 & 22 & 7.29 & 3.89 & 17.58 & 232.5 & 0.68 & newlv $\mathrm{TiO}_{2}$ \\
\hline & Measured bc & -9.77 & 0.74 & 1016 & 21 & 7.48 & 4.12 & 17.69 & 234.7 & 0.68 & newlv $\mathrm{TiO}_{2}$ \\
\hline & Target & -9.93 & 0.79 & 1016 & 20 & 7.19 & 3.93 & 17.82 & 235.7 & 0.69 & newlv $\mathrm{TiO}_{2}$ \\
\hline
\end{tabular}




\subsubsection{Matrix 2 XRD}

A summary of the types of crystals present in the quenched glasses is provided in Table 9 and XRD patterns of the quenched non-radioactive glasses are shown in Figure 1 through Figure 14. ${ }^{13}$ In general, most of the glasses were amorphous after quenching. Glasses that were not amorphous contained crystals that were either Fe-based spinels or Fe/Ni-based spinels. After the initial melting of the Matrix 2 glasses, FY09EM21-01, -04, -10, -13, -22, -23 and -24 all appeared to be crystallized throughout. These glasses were then re-melted at $1150^{\circ} \mathrm{C}$ for an additional 30 minutes and then poured directly into a cold water bath. The XRD results suggest that a faster quenching rate did not prevent crystallization in these glasses. It is also interesting to note the predicted $T_{L}$ values of the glasses that crystallized (Table 9). ${ }^{14}$ According to these values, all of the glasses except FY09EM21-10 should have been free of crystals when melted at $1150^{\circ} \mathrm{C}$.

Table 9. XRD Results of the Matrix 2 Quenched Glasses

\begin{tabular}{|c|c|c|c|}
\hline \multirow{2}{*}{ Glass ID } & \multirow{2}{*}{ Predicted $T_{L}\left({ }^{\circ} \mathrm{C}\right)$} & XRD Results & \multirow{2}{*}{ Water Quenched } \\
\hline & & quenched & \\
\hline FY09EM21-01 & 1079 & Magnetite $\left(\mathrm{Fe}^{+2} \mathrm{Fe}^{2+3} \mathrm{O}_{4}\right)$ & $\mathrm{x}$ \\
\hline FY09EM21-02 & 905 & Amorphous & \\
\hline FY09EM21-03 & 876 & Magnetite $\left(\mathrm{Fe}^{+2} \mathrm{Fe}^{2+3} \mathrm{O}_{4}\right)$ & \\
\hline FY09EM21-04 & 1130 & Trevorite $\left(\mathrm{NiFe}_{2} \mathrm{O}_{4}\right)$ & $\mathrm{x}$ \\
\hline FY09EM21-05 & 1031 & Amorphous & \\
\hline FY09EM21-06 & 921 & Amorphous & \\
\hline FY09EM21-07 & 955 & Amorphous & \\
\hline FY09EM21-08 & 989 & Amorphous & \\
\hline FY09EM21-09 & 1007 & Amorphous & \\
\hline FY09EM21-10 & 1159 & Magnetite $\left(\mathrm{Fe}^{+2} \mathrm{Fe}^{2+3} \mathrm{O}_{4}\right)$ & $\mathrm{x}$ \\
\hline FY09EM21-11 & 733 & Amorphous & \\
\hline FY09EM21-12 & 1010 & Amorphous & \\
\hline FY09EM21-13 & 1109 & Trevorite $\left(\mathrm{NiFe}_{2} \mathrm{O}_{4}\right)$ & $x$ \\
\hline FY09EM21-14 & 959 & Amorphous & \\
\hline FY09EM21-15 & 1033 & Amorphous & \\
\hline FY09EM21-16 & 992 & Amorphous & \\
\hline FY09EM21-17 & 866 & Amorphous & \\
\hline FY09EM21-18 & 763 & Amorphous & \\
\hline FY09EM21-19 & 761 & Amorphous & \\
\hline FY09EM21-20 & 1020 & Amorphous & \\
\hline FY09EM21-21 & 928 & Amorphous & \\
\hline FY09EM21-22 & 1051 & Trevorite $\left(\mathrm{NiFe}_{2} \mathrm{O}_{4}\right)$ & $\mathrm{x}$ \\
\hline FY09EM21-23 & 957 & Amorphous & $\mathrm{x}$ \\
\hline FY09EM21-24 & 1103 & Trevorite $\left(\mathrm{NiFe}_{2} \mathrm{O}_{4}\right)$, Hematite $\left(\mathrm{Fe}_{2} \mathrm{O}_{3}\right)$ & $\mathrm{x}$ \\
\hline FY09EM21-25 & 761 & Amorphous & \\
\hline FY09EM21-26 & 938 & Amorphous & \\
\hline FY09EM21-27 & 1016 & Amorphous & \\
\hline
\end{tabular}

\footnotetext{
${ }^{13}$ Samples of the ccc glasses were sent to PNNL for semi-quantitative XRD analysis; however, no measurements were made due to instrumental issues.

${ }^{14}$ The predicted $\mathrm{T}_{\mathrm{L}}$ is of the bias-corrected composition.
} 
SRNL-STI-2009-00778, REVISION 0

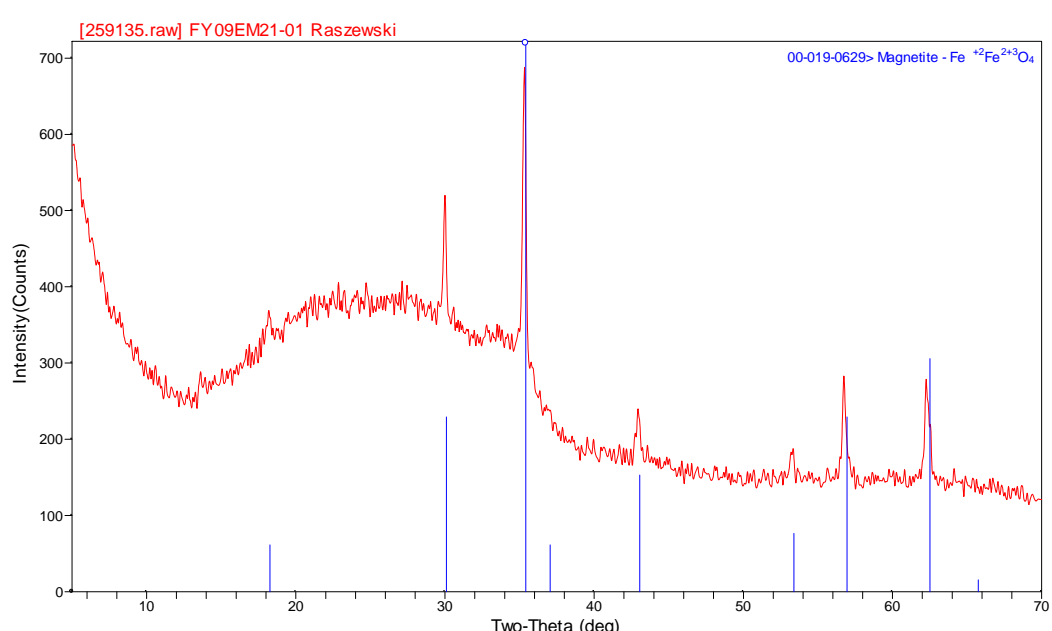

(a)

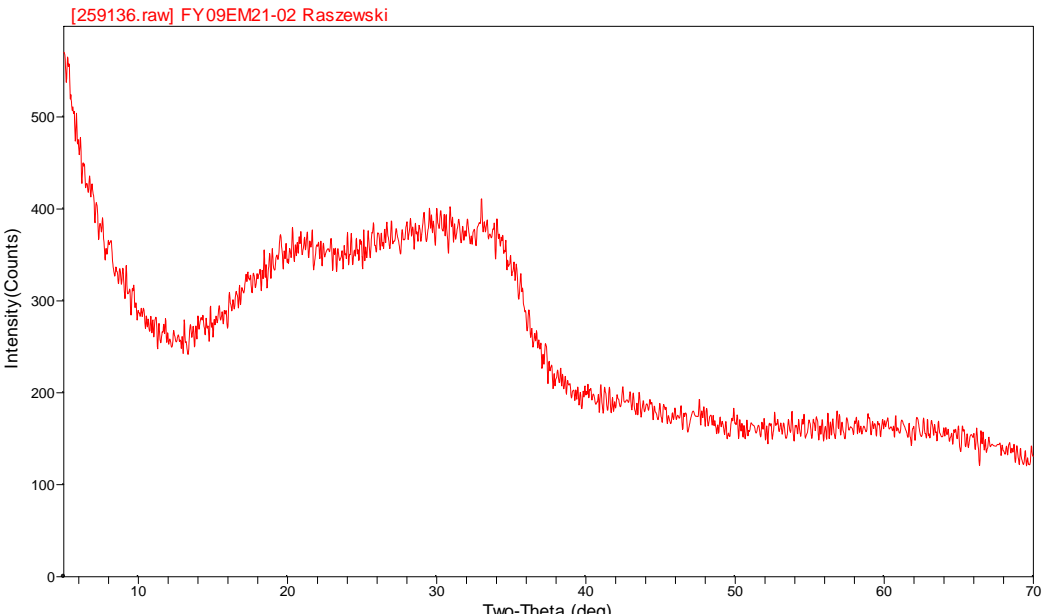

(b)

Figure 1. XRD patterns of (a) FY09EM21-01 and (b) FY09EM21-02.

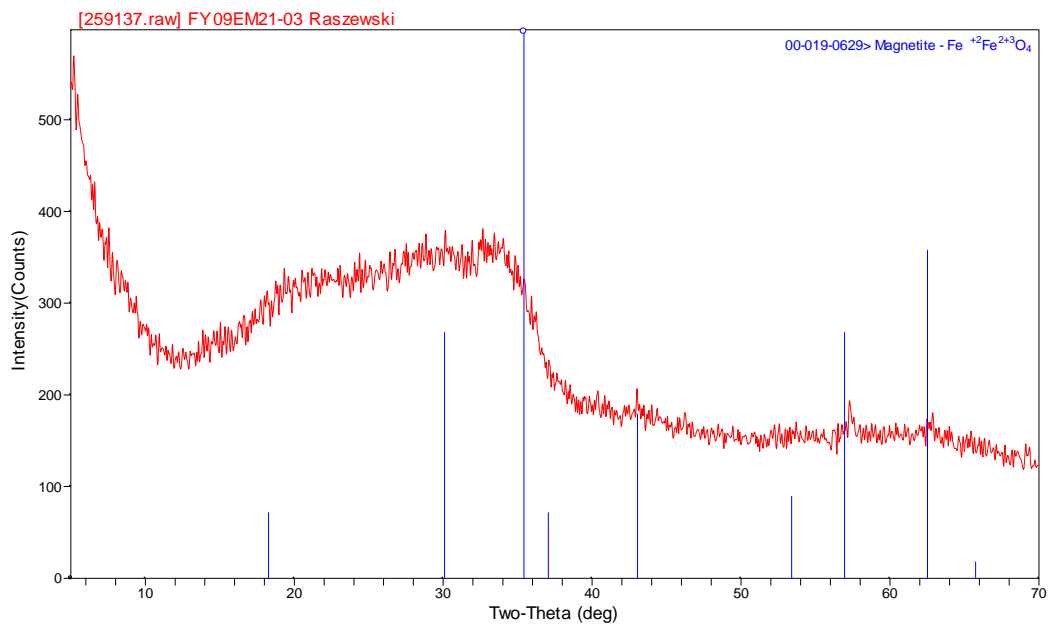

(a)

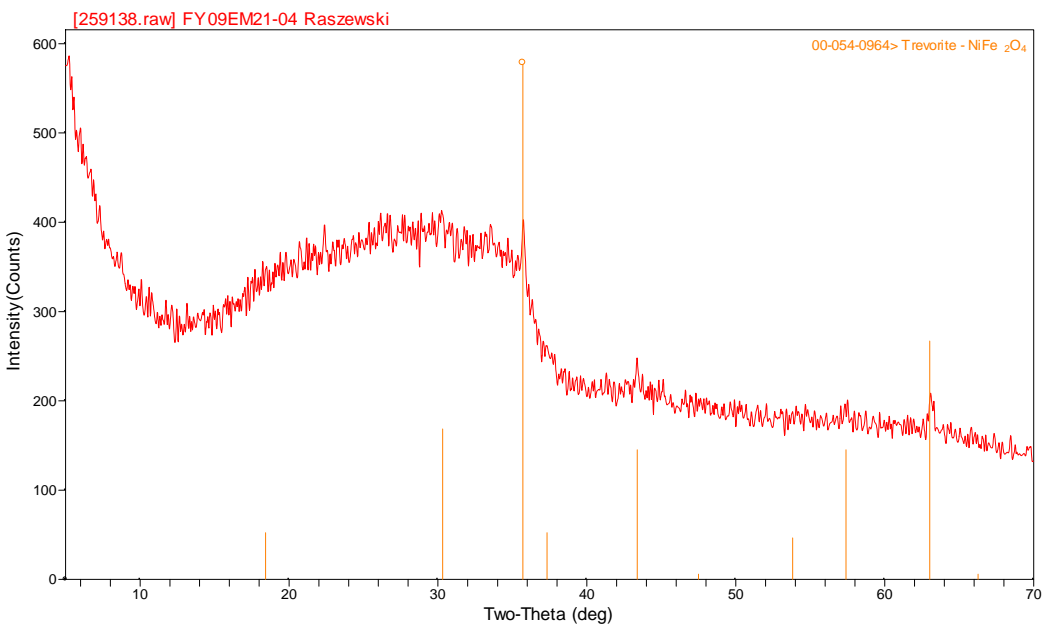

(b)

Figure 2. XRD patterns of (a) FY09EM21-03 and (b) FY09EM21-04. 
SRNL-STI-2009-00778, REVISION 0

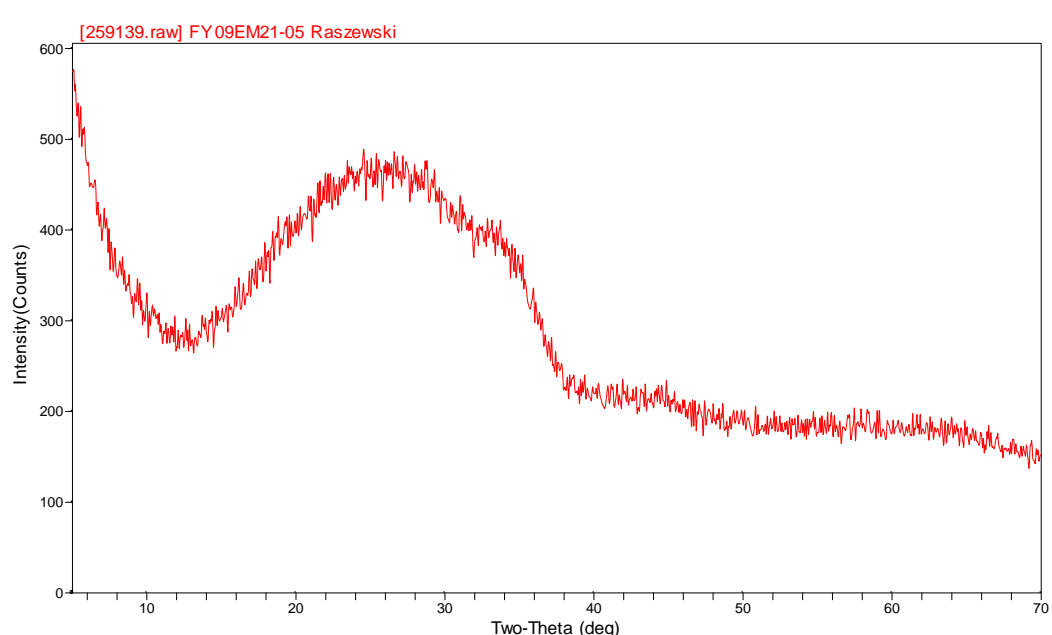

(a)

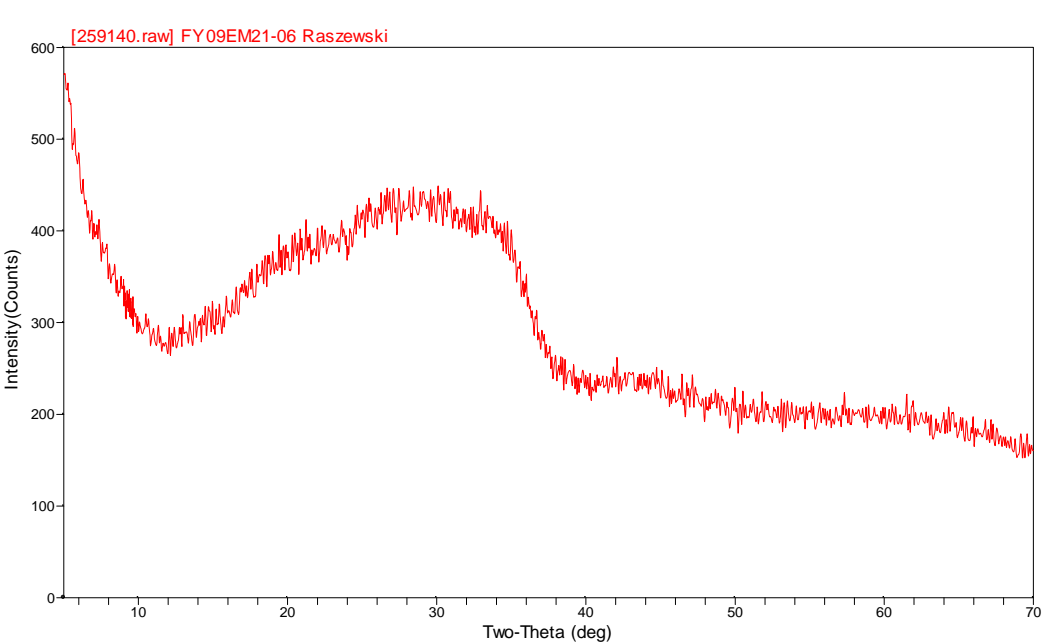

(b)

Figure 3. XRD patterns of (a) FY09EM21-05 and (b) FY09EM21-06.

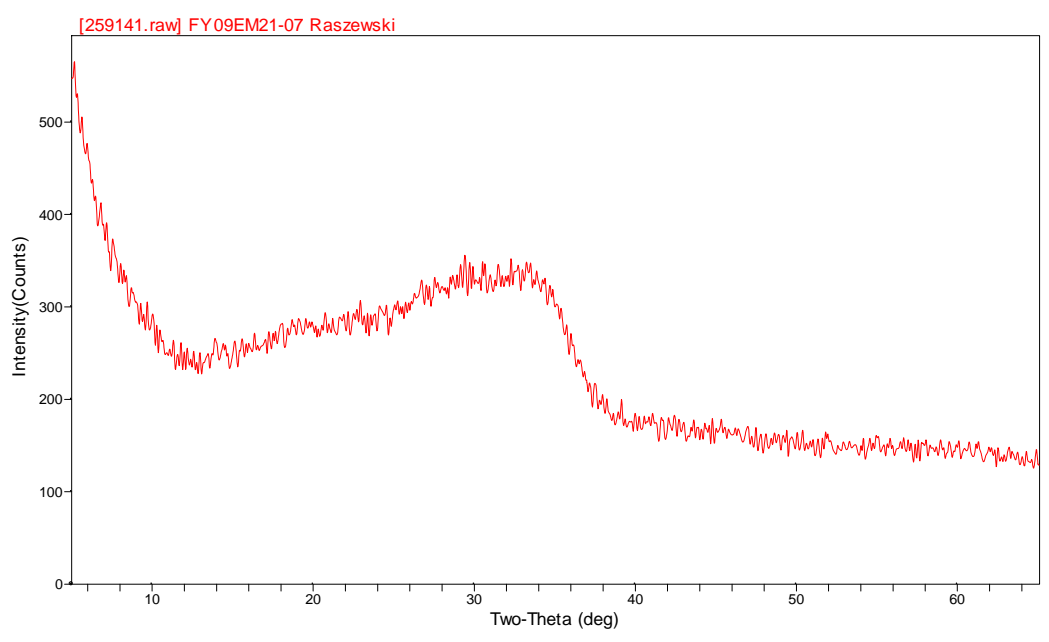

(a)

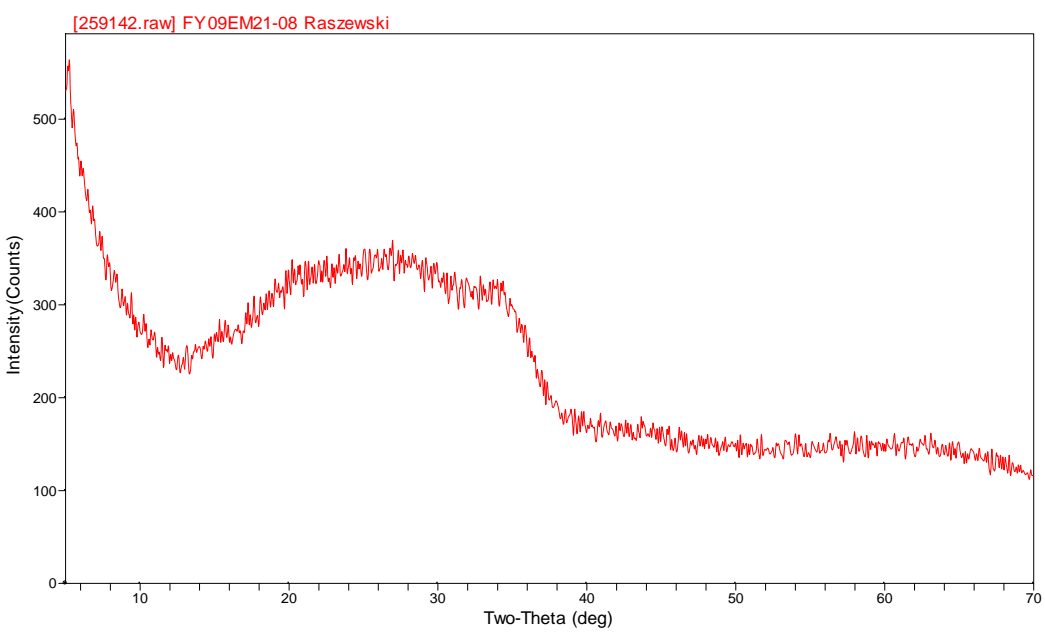

(b)

Figure 4. XRD patterns of (a) FY09EM21-07 and (b) FY09EM21-08. 
SRNL-STI-2009-00778, REVISION 0

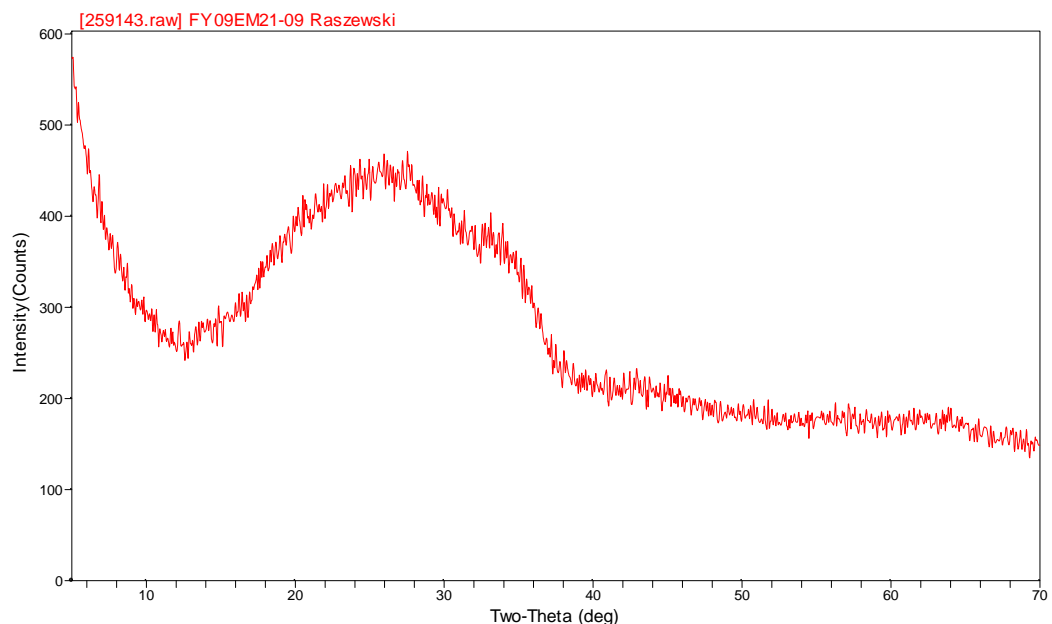

(a)

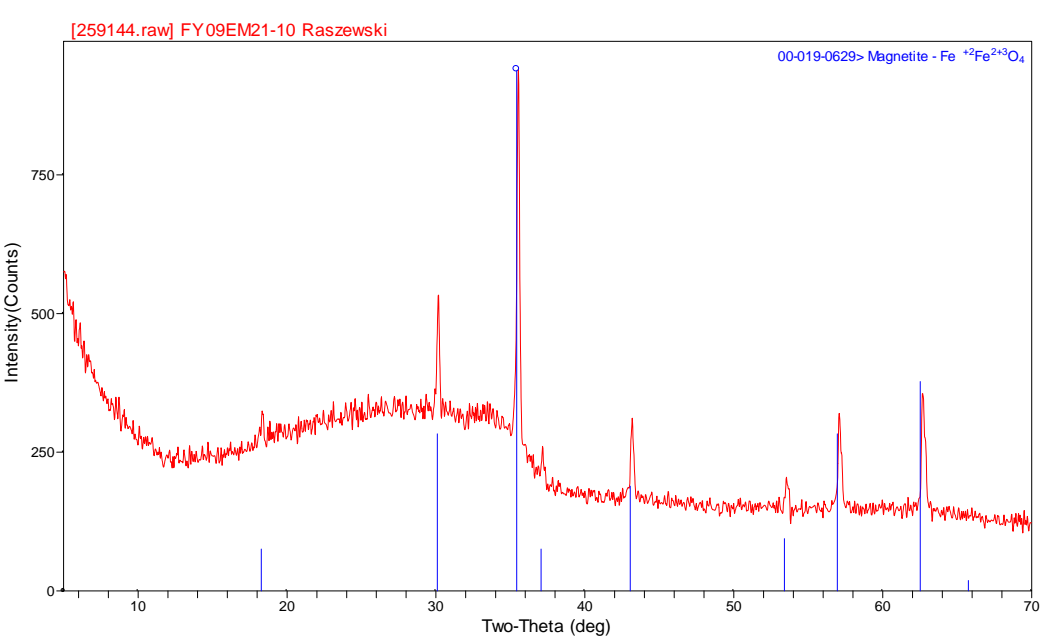

(b)

Figure 5. XRD patterns of (a) FY09EM21-09 and (b) FY09EM21-10.

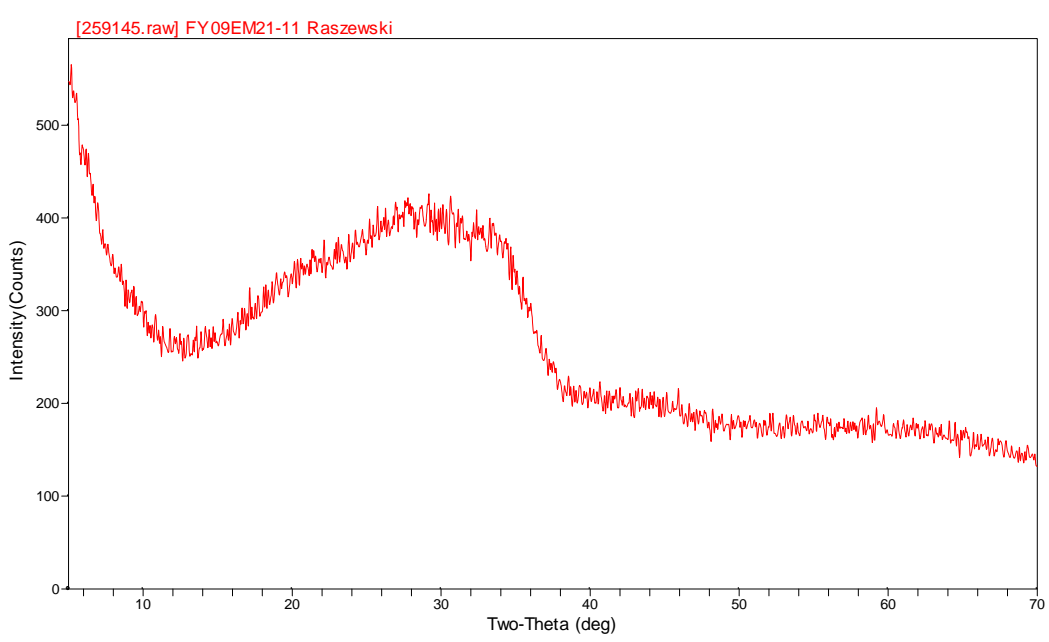

(a)

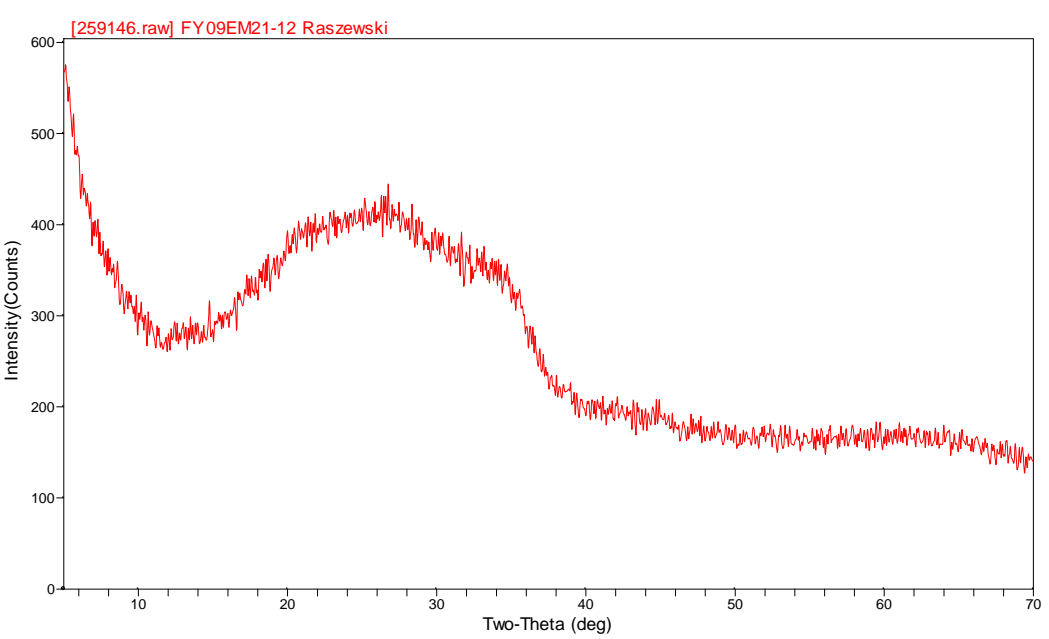

(b)

Figure 6. XRD patterns of (a) FY09EM21-11 and (b) FY09EM21-12. 
SRNL-STI-2009-00778, REVISION 0

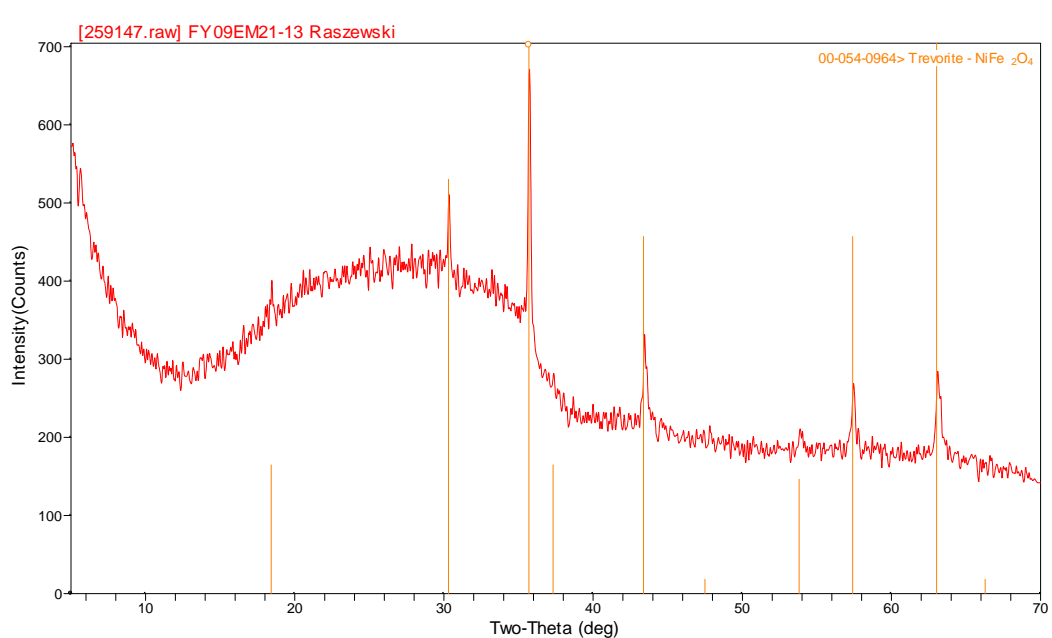

(a)

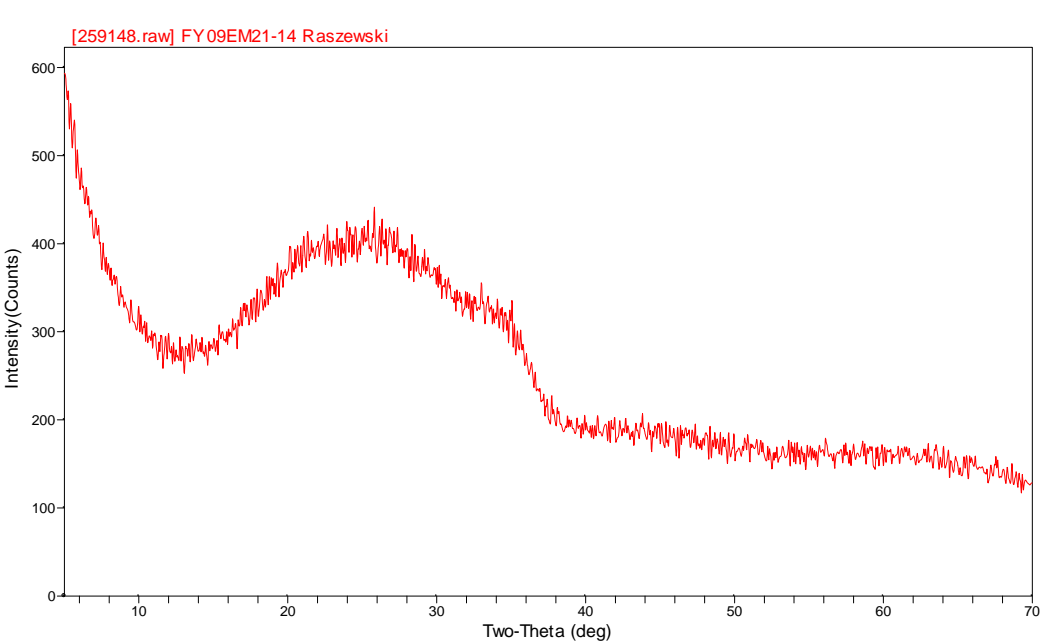

(b)

Figure 7. XRD patterns of (a) FY09EM21-13 and (b) FY09EM21-14.

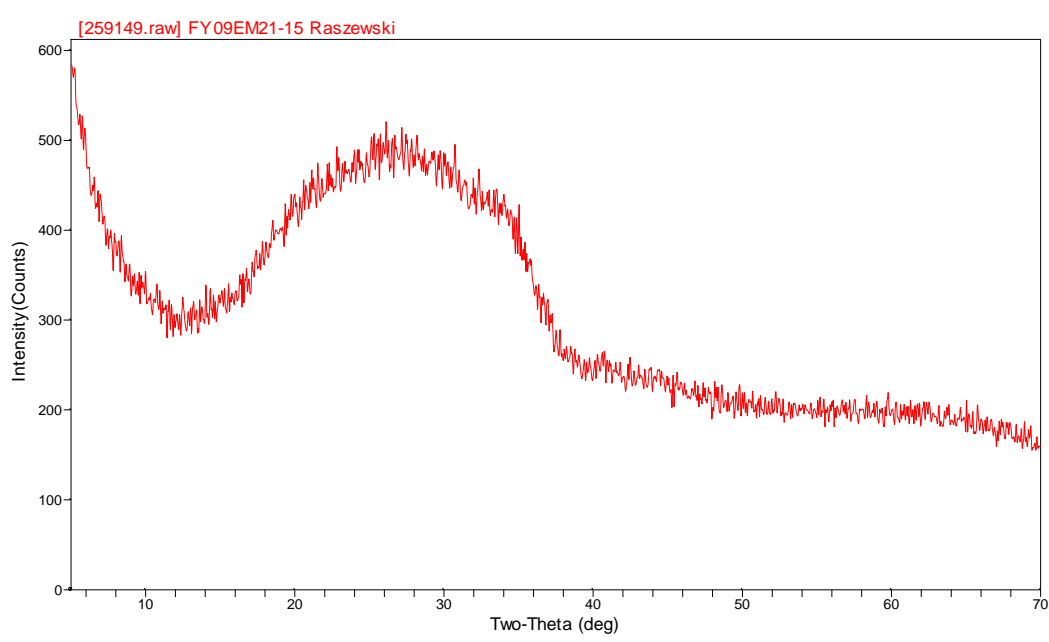

(a)

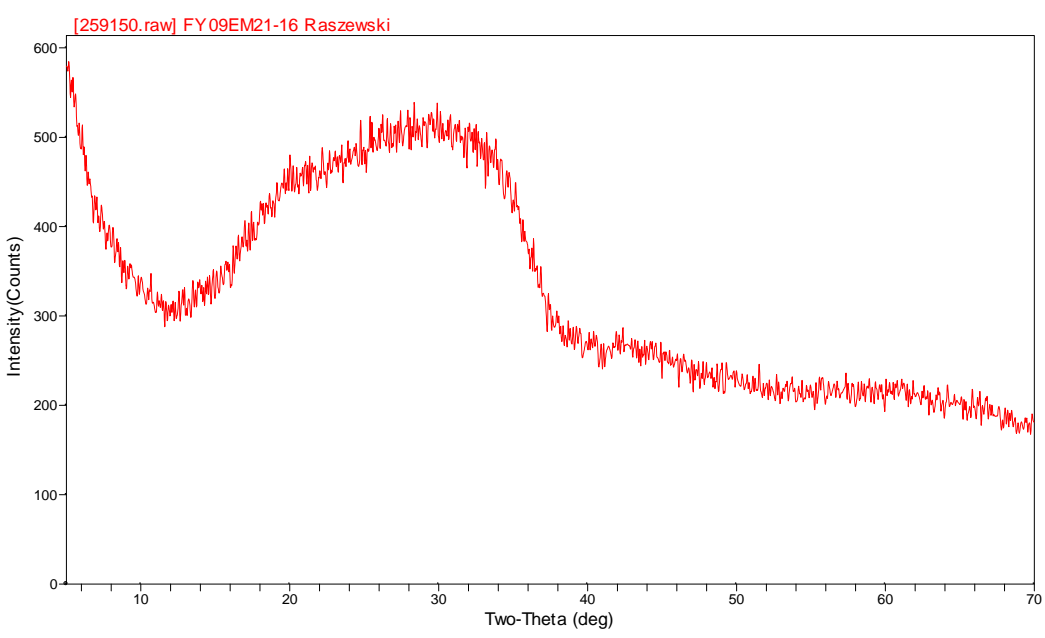

(b)

Figure 8. XRD patterns of (a) FY09EM21-15 and (b) FY09EM21-16. 
SRNL-STI-2009-00778, REVISION 0

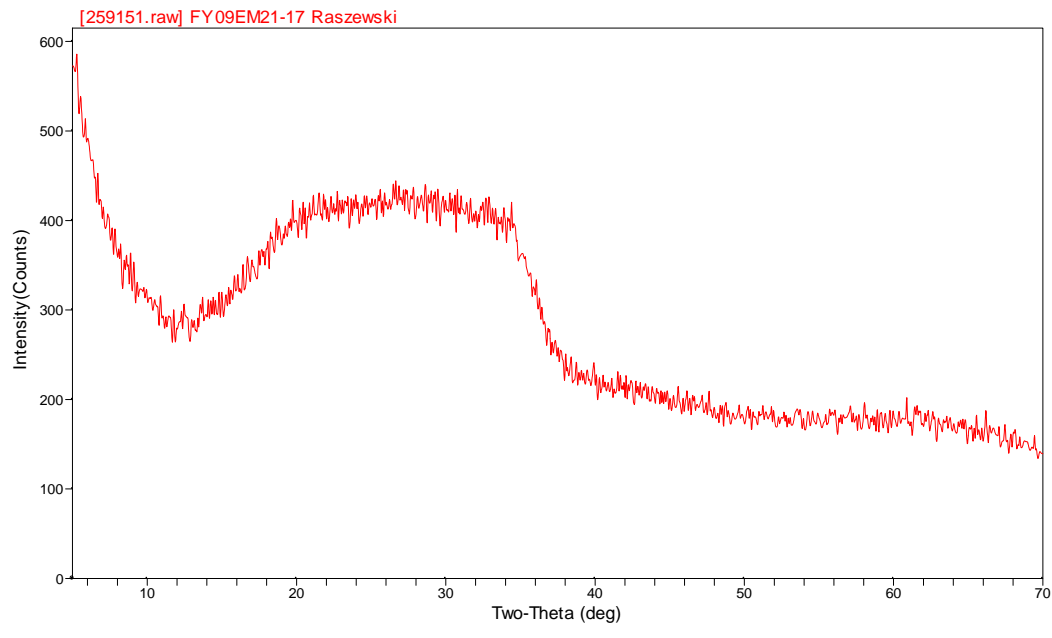

(a)

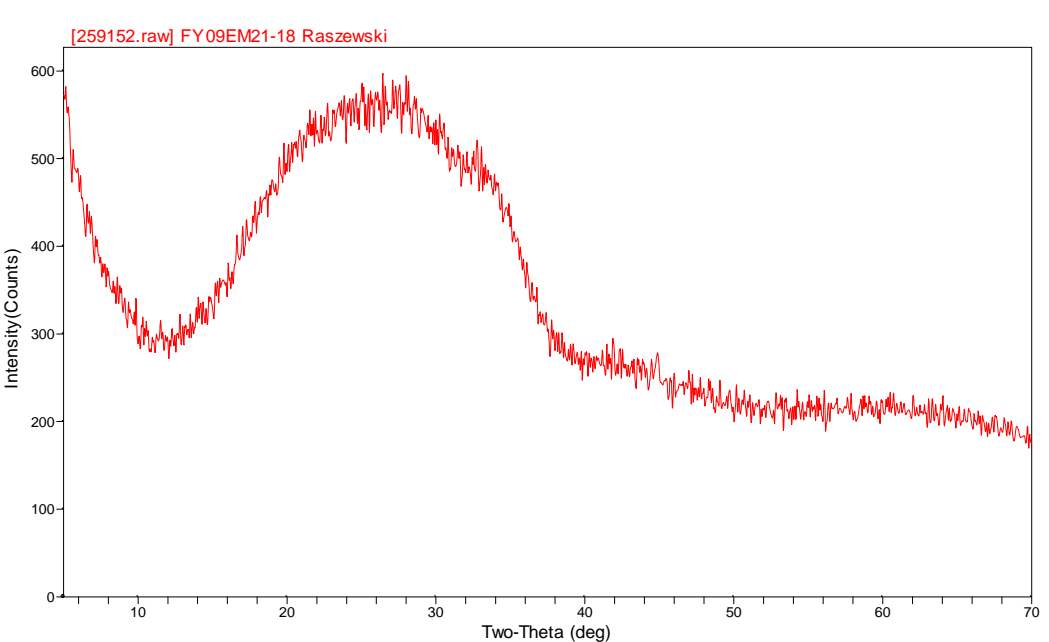

(b)

Figure 9. XRD patterns of (a) FY09EM21-17 and (b) FY09EM21-18.

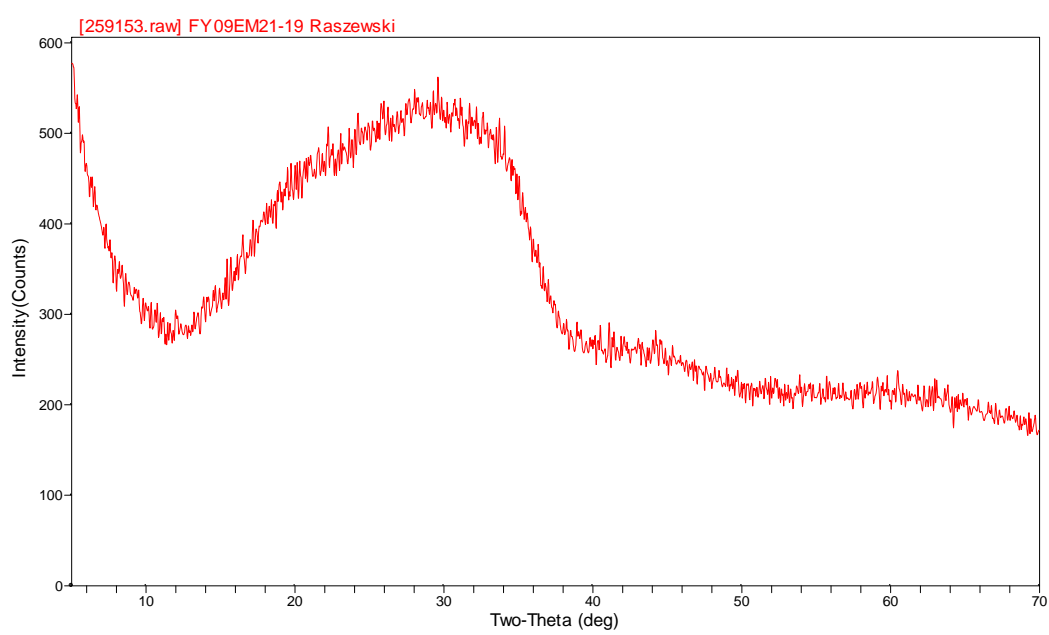

(a)

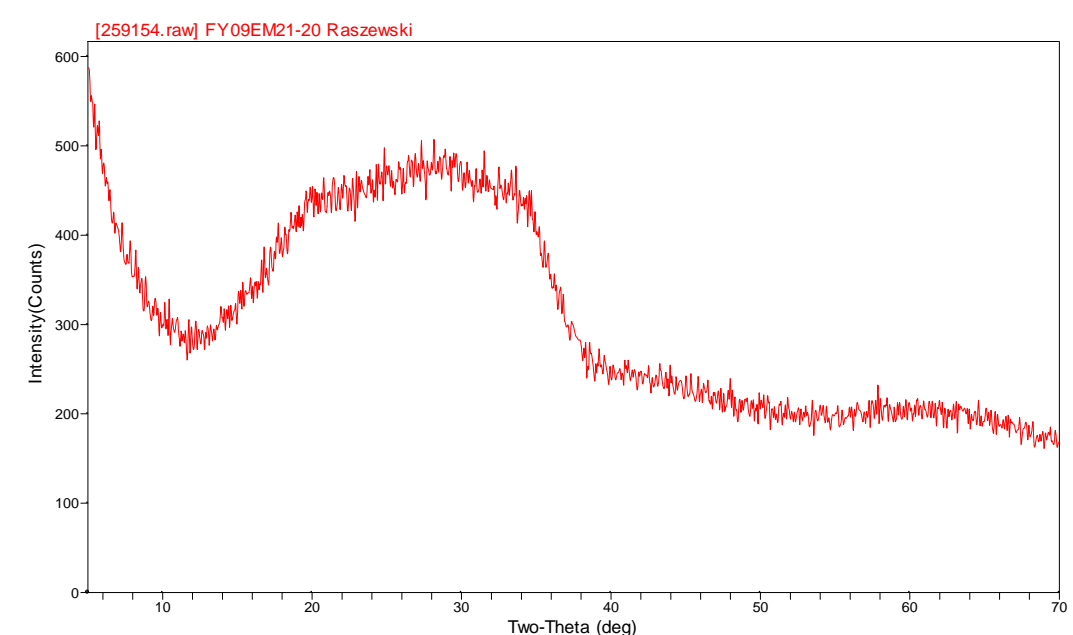

(b)

Figure 10. XRD patterns of (a) FY09EM21-19 and (b) FY09EM21-20. 
SRNL-STI-2009-00778, REVISION 0

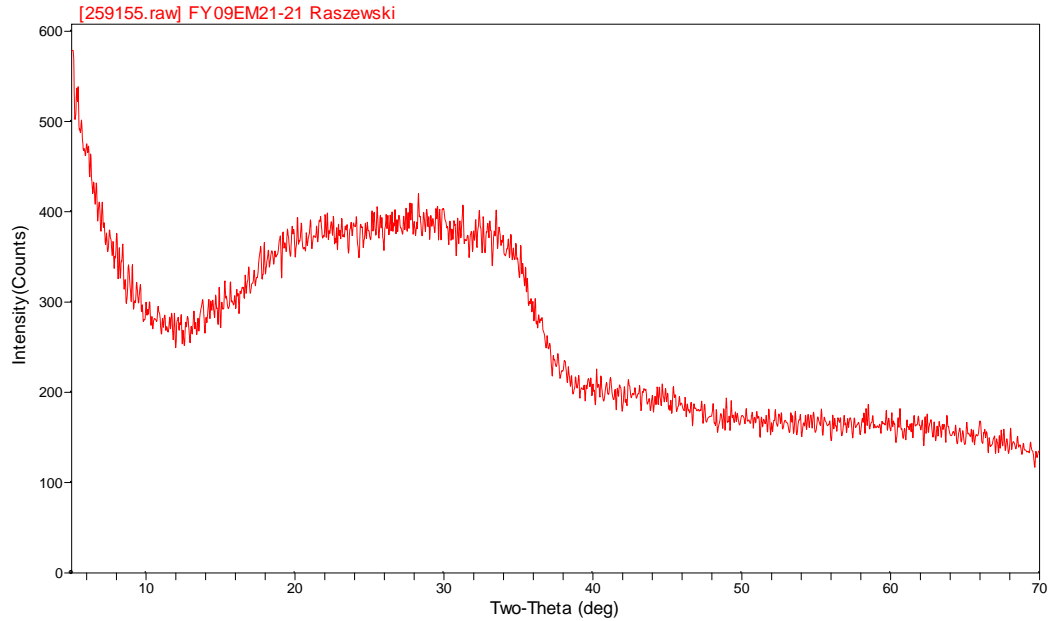

(a)

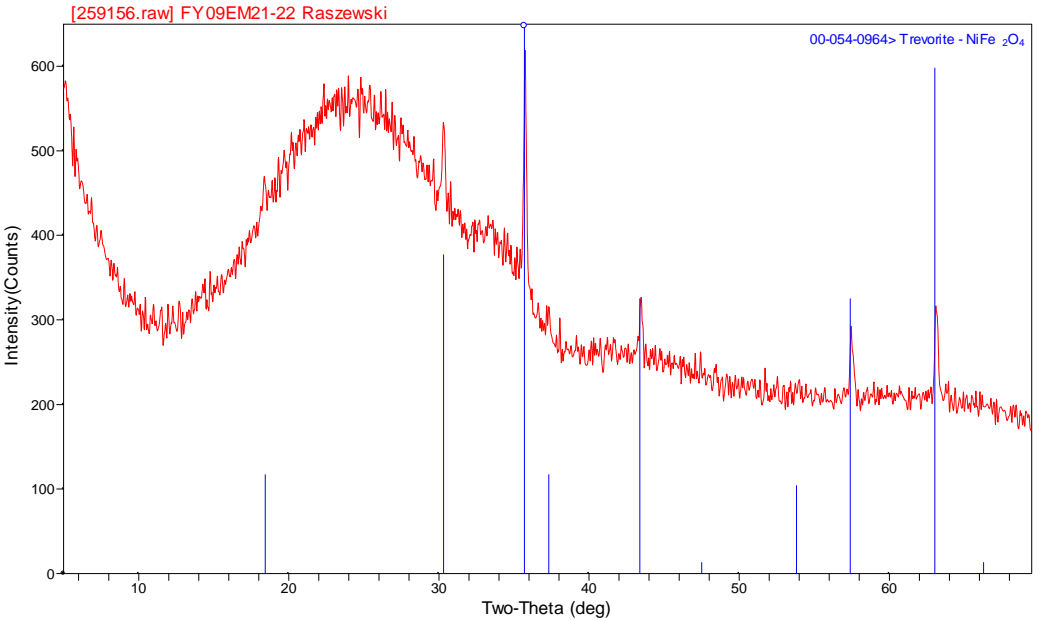

(b)

Figure 11. XRD patterns of (a) FY09EM21-21 and (b) FY09EM21-22.

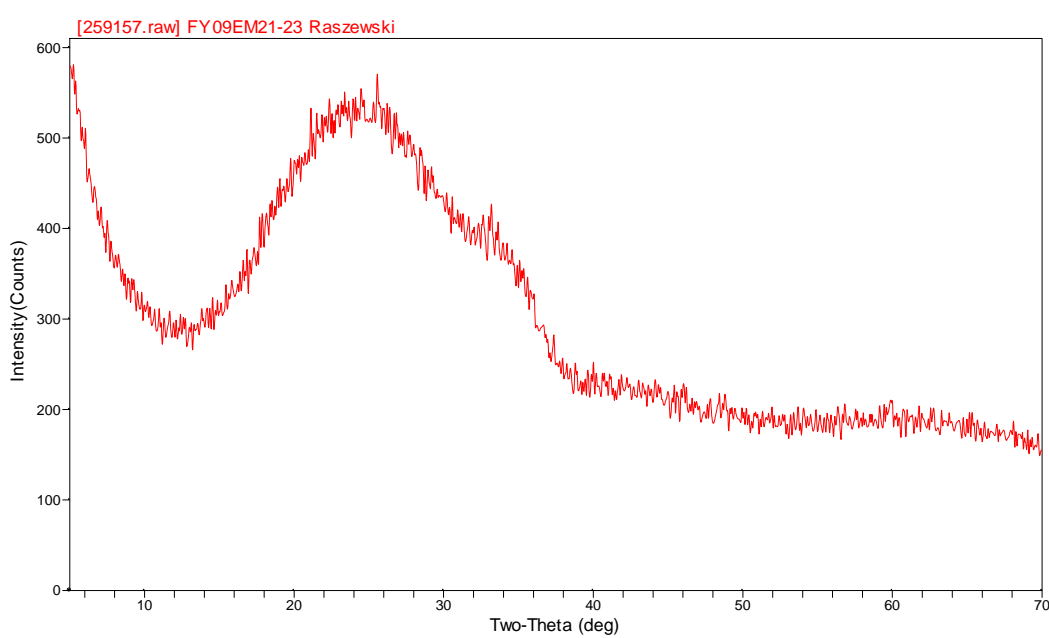

(a)

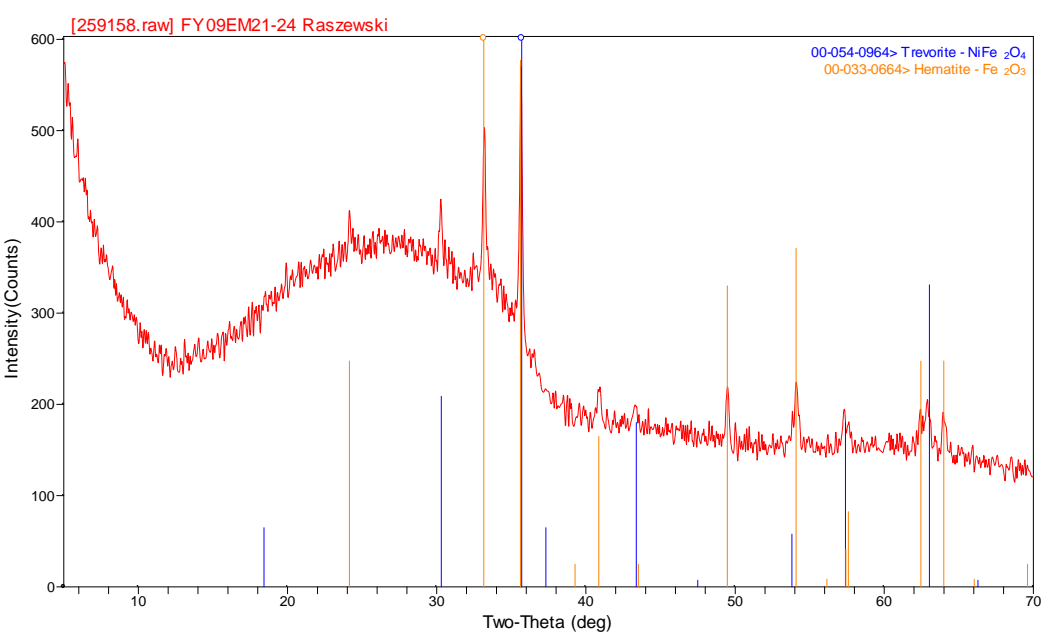

(b)

Figure 12. XRD patterns of (a) FY09EM21-23 and (b) FY09EM21-24. 
SRNL-STI-2009-00778, REVISION 0

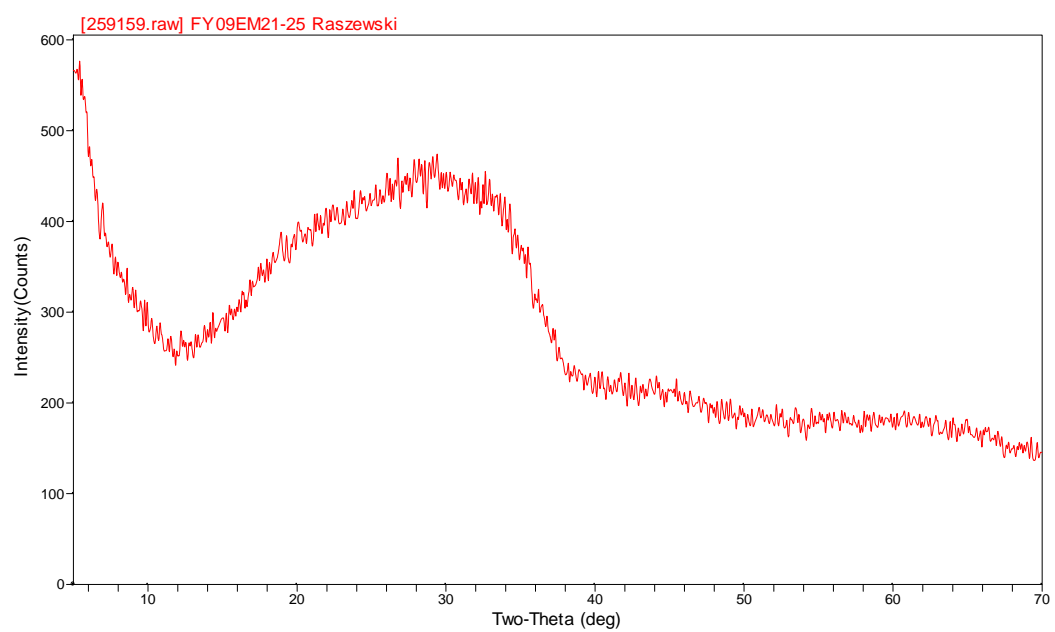

(a)

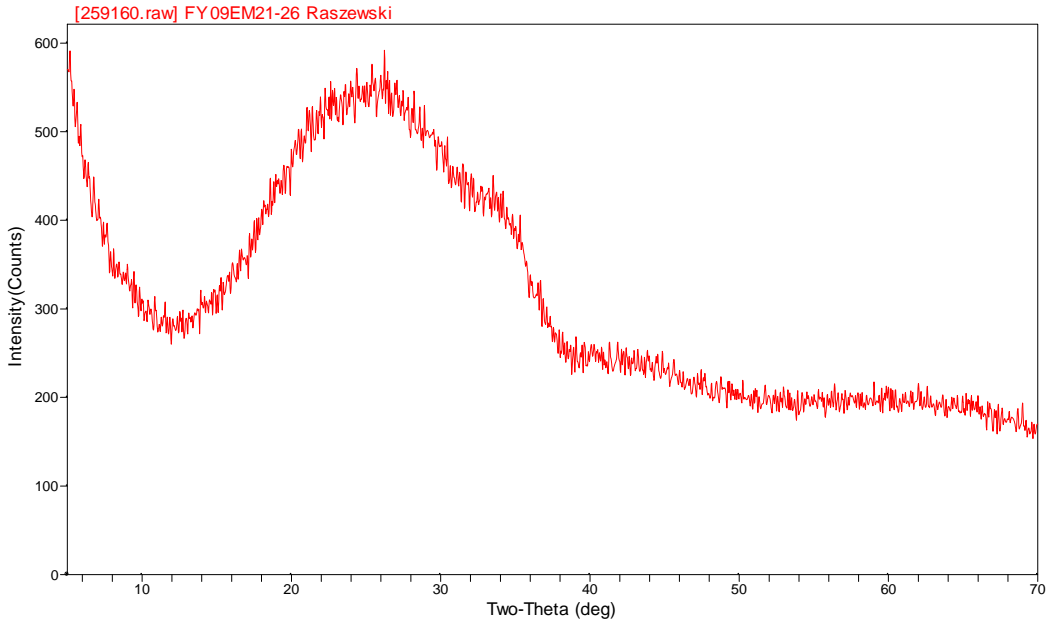

(b)

Figure 13. XRD patterns of (a) FY09EM21-25 and (b) FY09EM21-26.

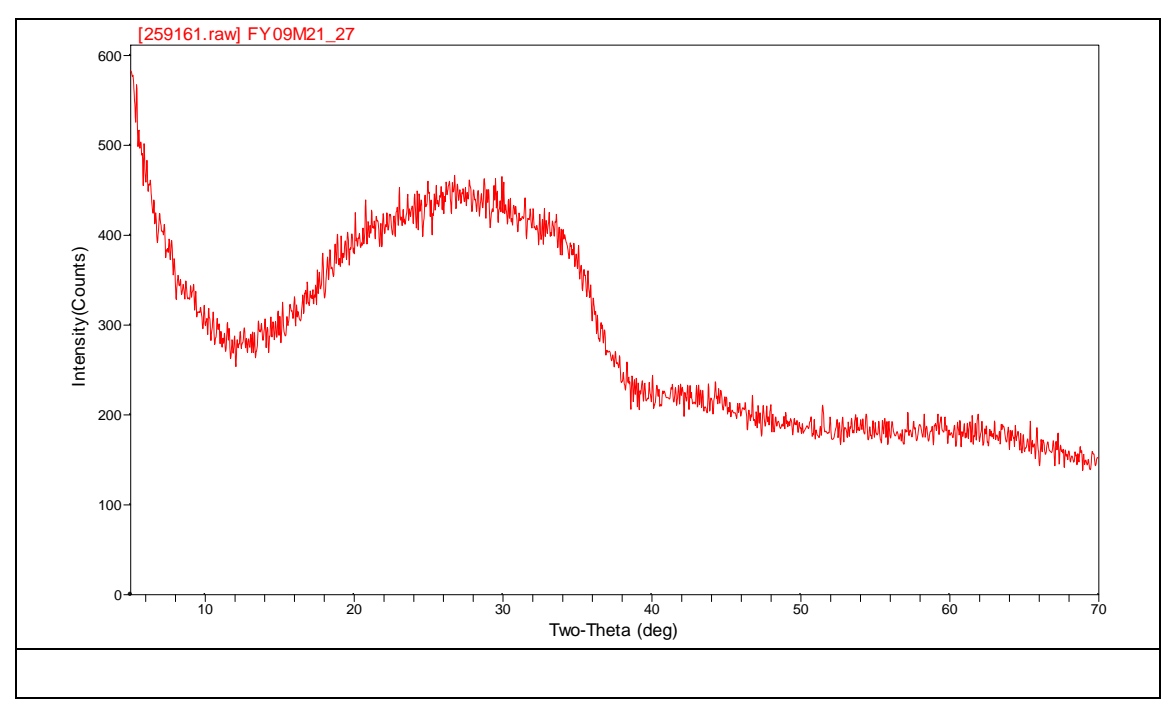

Figure 14. XRD pattern of FY09EM21-27. 


\subsubsection{A Statistical Review of the PCT Results of the Matrix 2 Non-Radioactive Glasses}

Table B1 in Appendix B provides the as-received (AR) elemental leachate concentration measurements for the solution samples generated by the PCTs. Any measurement below the detection limit of the analytical procedure (indicated by a "<") was replaced by one half of the detection limit in subsequent analyses. In addition to adjustments for detection limits, the values were adjusted for the acid dilution factors: study glasses, blanks and the ARM glass were multiplied by 1.6667 to determine the values in parts per million (ppm) and the values for EA were multiplied by 16.6667. The last four columns of Table B1 in Appendix B provide the resulting ppm measurements.

One of the quality control checkpoints for the PCT procedure is solution-weight loss over the course of the 7-day test. None of the PCTs from these glasses indicated a solution-weight loss problem.

\subsubsection{Measurements in Analytical Sequence}

Exhibit B1 in Appendix B provides plots of the leachate concentrations in analytical sequence as generated by the PSAL for all of the data from all three sets of PCTs. Some scatter in the measurement of the EA glass is observed (blue X). No other issues are observed in these plots.

\subsubsection{Results for the Samples of the Multi-Element Solution Standard}

Exhibit B2 in Appendix B provides analyses of measurements of the multi-element solution standard by analytical set/ICP-AES calibration block, and an ANOVA investigation for each element of interest. A statistically significant difference (at a 5\% level) among the averages of these measurements was indicated for $\mathrm{Li}$ and $\mathrm{Si}$. No attempt was made to bias correct for these effects since averaging the ppm values for each set of triplicates helps to minimize the impact of any potential effects of the instrument.

Table 10 summarizes the average measurements and the reference values for the 4 elements of interest. The results indicate consistent and accurate measurements from the PSAL processes used to conduct these analyses.

\subsubsection{Measurements by Glass Identifier}

Exhibit B3 in Appendix B provides plots of the leachate concentrations for both the quenched and ccc version of each glass, as well as the reference samples (EA, $\mathrm{ARM}^{\mathrm{O}}$, multi-element solution standard and blanks). ${ }^{27}$ Two units of measure are used in these plots: ppm and the common logarithms of the ppm values. The common logarithm plots allow for the assessment of the repeatability of the measurements.

For some of the glasses, scatter in the triplicate values of some analytes is observed. For example, there is some scatter in the Si values for the ccc version of FY07EM21-17.

\footnotetext{
${ }^{\mathrm{O}}$ The concentrations of each element of interest for ARM are within the control limits in THERMO ${ }^{\mathrm{TM}}$.
} 
Table 10. Multi-Element Solution Standard Results of the Non-Radioactive Matrix 2 Glasses

\begin{tabular}{|c|c|c|c|c|c|}
\hline Set & Block & B (ppm) & Li (ppm) & Na (ppm) & Si (ppm) \\
\hline 1 & 1 & 21.5 & 10.0 & 82.7 & 52.7 \\
\hline 1 & 1 & 20.5 & 10.0 & 81.9 & 51.5 \\
\hline 1 & 1 & 19.9 & 10.0 & 84.0 & 52.1 \\
\hline 1 & 2 & 20.4 & 9.9 & 84.4 & 52.5 \\
\hline 1 & 2 & 19.2 & 10.0 & 85.1 & 52.6 \\
\hline 1 & 2 & 18.2 & 9.6 & 79.9 & 51.3 \\
\hline 1 & 3 & 22.0 & 10.2 & 83.4 & 51.9 \\
\hline 1 & 3 & 19.9 & 9.9 & 77.7 & 50.6 \\
\hline 1 & 3 & 20.4 & 10.1 & 80.6 & 51.5 \\
\hline \multicolumn{2}{|c|}{ Average } & 20.2 & 10.0 & 82.2 & 51.9 \\
\hline 2 & 1 & 21.4 & 9.8 & 81.7 & 50.1 \\
\hline 2 & 1 & 20.5 & 9.9 & 81.4 & 50.5 \\
\hline 2 & 1 & 20.1 & 9.6 & 78.7 & 49.3 \\
\hline 2 & 2 & 21.6 & 10.0 & 82.4 & 50.4 \\
\hline 2 & 2 & 19.9 & 9.9 & 80.7 & 50.2 \\
\hline 2 & 2 & 20.0 & 9.9 & 81.3 & 50.6 \\
\hline 2 & 3 & 21.3 & 9.9 & 83.0 & 51.6 \\
\hline 2 & 3 & 20.3 & 9.9 & 82.5 & 51.7 \\
\hline 2 & 3 & 20.1 & 9.9 & 82.7 & 51.6 \\
\hline \multicolumn{2}{|c|}{ Average } & 20.6 & 9.9 & 81.6 & 50.7 \\
\hline 3 & 1 & 21.2 & 10.1 & 83.1 & 51.1 \\
\hline 3 & 1 & 20.2 & 9.9 & 82.7 & 49.5 \\
\hline 3 & 1 & 20.7 & 10.2 & 86.4 & 50.1 \\
\hline 3 & 2 & 21.0 & 10.2 & 81.9 & 51.3 \\
\hline 3 & 2 & 20.2 & 10.1 & 80.8 & 50.8 \\
\hline 3 & 2 & 20.4 & 10.2 & 81.6 & 51.6 \\
\hline 3 & 3 & 20.3 & 10.0 & 82.8 & 50.4 \\
\hline 3 & 3 & 20.2 & 10.1 & 83.8 & 50.6 \\
\hline 3 & 3 & 22.9 & 10.1 & 82.1 & 50.8 \\
\hline \multicolumn{2}{|c|}{ Average } & 20.8 & 10.1 & 82.8 & 50.7 \\
\hline \multicolumn{2}{|c|}{ Grand Average } & 20.5 & 10.0 & 82.2 & 51.1 \\
\hline \multicolumn{2}{|c|}{ Reference Value } & 20 & 10 & 81 & 50 \\
\hline \multicolumn{2}{|c|}{$\%$ difference } & $2.7 \%$ & $-0.2 \%$ & $1.5 \%$ & $2.1 \%$ \\
\hline
\end{tabular}




\subsubsection{Normalized PCT Results}

PCT leachate concentrations were normalized using the target, measured and bias-corrected cation compositions $(\mathrm{wt} \%)$ in the glass to obtain a grams-per-liter $(\mathrm{g} / \mathrm{L})$ leachate concentration.

As is the usual convention, the common logarithm of the normalized PCT (normalized leachate, $\mathrm{NL}$ ) for each element of interest was determined and used for comparison. To accomplish this computation, one must:

1. Determine the common logarithm of the elemental leachate concentration (ppm) for each of the triplicates and each of the elements of interest (these values are provided in Table B1 of Appendix B).

2. Average the common logarithms over the triplicates for each element of interest.

Normalizing Using Measured Composition

3. Subtract a quantity equal to 1 plus the common logarithm of the average cation measured concentration (expressed as a weight percent of the glass) from the average computed in step 2 .

Or Normalizing Using Target Composition

3. Subtract a quantity equal to 1 plus the common logarithm of the target cation concentration (expressed as a weight percent of the glass) from the average computed in step 2 .

Or Normalizing Using Measured Bias-Corrected Composition

3. Subtract a quantity equal to 1 plus the common logarithm of the measured biascorrected cation concentration (expressed as a weight percent of the glass) from the average computed in step 2.

Exhibit B4 in Appendix B provides scatter plots for these results and offers an opportunity to investigate the consistency in the leaching across the elements for the glasses of this study. All combinations of the normalizations of the PCTs (i.e., those generated using the target, measured, and bias-corrected compositional views) and both heat treatments are represented in the series of scatter plots. Consistency in the leaching across the elements is typically demonstrated by a high degree of linear correlation among the values for pairs of these elements. The smallest correlation in this plot is that for $\mathrm{Na}$ and $\mathrm{Si}$, with a value of $\sim 91.3 \%$.

Table 11 summarizes the normalized PCTs for the glasses of this study. ${ }^{\mathrm{P}}$ The PCTs are listed by heat treatment and compositional view for each glass.

\footnotetext{
${ }^{\mathrm{P}}$ The concentrations of each element of interest for ARM are within the control limits in THERMO ${ }^{\mathrm{TM}}$.
} 
SRNL-STI-2009-00778, REVISION 0

Table 11. Normalized PCT Results of the Non-Radioactive Matrix 2 Glasses

\begin{tabular}{|c|c|c|c|c|c|c|c|c|c|c|c|}
\hline Set & Glass ID & \begin{tabular}{c|c|} 
Heat \\
Treatment \\
\end{tabular} & $\begin{array}{l}\text { Comp } \\
\text { View }\end{array}$ & $\begin{array}{c}\log \mathrm{NL} \\
{[\mathrm{B}(\mathrm{g} / \mathrm{L})]} \\
\end{array}$ & $\begin{array}{c}\log \mathrm{NL} \\
{[\mathrm{Li}(\mathrm{g} / \mathrm{L})]}\end{array}$ & \begin{tabular}{|c|}
$\log \mathrm{NL}$ \\
{$[\mathrm{Na}(\mathrm{g} / \mathrm{L})]$} \\
\end{tabular} & $\begin{array}{c}\log \mathrm{NL} \\
{[\mathrm{Si}(\mathrm{g} / \mathrm{L})]} \\
\end{array}$ & $\begin{array}{c}\text { NL } \\
\text { B (g/L) }\end{array}$ & $\begin{array}{c}\text { NL } \\
\mathrm{Li}(\mathrm{g} / \mathrm{L}) \\
\end{array}$ & $\begin{array}{c}\mathrm{NL} \\
\mathrm{Na}(\mathrm{g} / \mathrm{L}) \\
\end{array}$ & $\begin{array}{c}\text { NL } \\
\text { Si }(g / L) \\
\end{array}$ \\
\hline 1 & ARM & ref & ref & -0.27 & -0.27 & -0.32 & -0.55 & 0.54 & 0.54 & 0.48 & 0.28 \\
\hline 2 & ARM & ref & ref & -0.34 & -0.27 & -0.35 & -0.58 & 0.46 & 0.53 & 0.45 & 0.27 \\
\hline 3 & ARM & ref & ref & -0.24 & -0.20 & -0.26 & -0.53 & 0.58 & 0.63 & 0.55 & 0.29 \\
\hline 1 & EA & ref & ref & 1.27 & 0.98 & 1.14 & 0.62 & 18.70 & 9.54 & 13.85 & 4.15 \\
\hline 2 & EA & ref & ref & 1.02 & 0.82 & 0.92 & 0.45 & 10.44 & 6.53 & 8.26 & 2.83 \\
\hline 3 & EA & ref & ref & 1.25 & 0.97 & 1.13 & 0.60 & 17.87 & 9.35 & 13.37 & 3.98 \\
\hline 1 & \multirow{6}{*}{ FY09EM21-01 } & \multirow{3}{*}{ quenched } & Measured & -0.09 & -0.11 & -0.14 & -0.29 & 0.82 & 0.78 & 0.72 & 0.51 \\
\hline 1 & & & Measured bc & -0.08 & -0.11 & -0.15 & -0.30 & 0.83 & 0.78 & 0.71 & 0.50 \\
\hline 1 & & & Target & -0.08 & -0.11 & -0.13 & -0.28 & 0.84 & 0.78 & 0.74 & 0.52 \\
\hline 1 & & \multirow{3}{*}{$\operatorname{ccc}$} & Measured & 0.05 & -0.09 & -0.22 & -0.21 & 1.13 & 0.81 & 0.61 & 0.61 \\
\hline 1 & & & Measured bc & 0.06 & -0.09 & -0.23 & -0.22 & 1.14 & 0.81 & 0.60 & 0.60 \\
\hline 1 & & & Target & 0.06 & -0.09 & -0.21 & -0.21 & 1.15 & 0.81 & 0.62 & 0.62 \\
\hline 1 & \multirow{6}{*}{ FY09EM21-02 } & \multirow{3}{*}{ quenched } & Measured & 1.07 & 0.76 & 1.01 & 0.47 & 11.72 & 5.79 & 10.31 & 2.92 \\
\hline 1 & & & Measured bc & 1.07 & 0.76 & 1.01 & 0.46 & 11.82 & 5.73 & 10.11 & 2.85 \\
\hline 1 & & & Target & 1.08 & 0.77 & 0.99 & 0.47 & 11.99 & 5.83 & 9.78 & 2.94 \\
\hline 1 & & \multirow{3}{*}{$\operatorname{ccc}$} & Measured & 1.08 & 0.78 & 1.01 & 0.47 & 11.93 & 5.97 & 10.16 & 2.97 \\
\hline 1 & & & Measured bc & 1.08 & 0.77 & 1.00 & 0.46 & 12.03 & 5.90 & 9.96 & 2.90 \\
\hline 1 & & & Target & 1.09 & 0.78 & 0.98 & 0.48 & 12.21 & 6.01 & 9.64 & 3.00 \\
\hline 1 & \multirow{6}{*}{ FY09EM21-03 } & \multirow{3}{*}{ quenched } & Measured & 0.42 & 0.36 & 0.34 & 0.10 & 2.65 & 2.29 & 2.16 & 1.26 \\
\hline 1 & & & Measured bc & 0.43 & 0.35 & 0.34 & 0.10 & 2.68 & 2.26 & 2.19 & 1.25 \\
\hline 1 & & & Target & 0.44 & 0.34 & 0.35 & 0.09 & 2.73 & 2.20 & 2.23 & 1.24 \\
\hline 1 & & \multirow{3}{*}{$\mathrm{ccc}$} & Measured & 1.07 & 0.77 & 0.86 & 0.45 & 11.83 & 5.88 & 7.15 & 2.84 \\
\hline 1 & & & Measured bc & 1.08 & 0.77 & 0.86 & 0.45 & 11.92 & 5.81 & 7.22 & 2.84 \\
\hline 1 & & & Target & 1.09 & 0.75 & 0.87 & 0.45 & 12.16 & 5.67 & 7.38 & 2.81 \\
\hline 1 & \multirow{6}{*}{ FY09EM21-04 } & \multirow{3}{*}{ quenched } & Measured & 0.79 & 0.71 & 0.70 & 0.07 & 6.15 & 5.18 & 5.02 & 1.16 \\
\hline 1 & & & Measured bc & 0.79 & 0.71 & 0.71 & 0.06 & 6.20 & 5.12 & 5.07 & 1.16 \\
\hline 1 & & & Target & 0.79 & 0.71 & 0.70 & 0.06 & 6.15 & 5.14 & 5.00 & 1.15 \\
\hline 1 & & \multirow{3}{*}{$\mathrm{ccc}$} & Measured & 0.31 & 0.29 & 0.28 & -0.07 & 2.05 & 1.97 & 1.89 & 0.86 \\
\hline 1 & & & \begin{tabular}{|l|} 
Measured bc \\
\end{tabular} & 0.32 & 0.29 & 0.28 & -0.07 & 2.06 & 1.95 & 1.91 & 0.85 \\
\hline 1 & & & Target & 0.31 & 0.29 & 0.28 & -0.07 & 2.05 & 1.95 & 1.88 & 0.85 \\
\hline 1 & \multirow{6}{*}{ FY09EM21-05 } & \multirow{3}{*}{ quenched } & Measured & 0.05 & -0.02 & -0.07 & -0.28 & 1.12 & 0.95 & 0.86 & 0.52 \\
\hline 1 & & & Measured bc & 0.06 & -0.02 & -0.08 & -0.29 & 1.14 & 0.95 & 0.84 & 0.51 \\
\hline 1 & & & Target & 0.03 & -0.03 & -0.07 & -0.30 & 1.08 & 0.94 & 0.85 & 0.50 \\
\hline 1 & & \multirow{3}{*}{$\mathrm{ccc}$} & Measured & -0.10 & -0.11 & -0.20 & -0.41 & 0.80 & 0.78 & 0.63 & 0.39 \\
\hline 1 & & & Measured bc & -0.09 & -0.11 & -0.21 & -0.42 & 0.81 & 0.78 & 0.62 & 0.38 \\
\hline 1 & & & Target & -0.12 & -0.11 & -0.20 & -0.42 & 0.77 & 0.77 & 0.63 & 0.38 \\
\hline 1 & \multirow{6}{*}{ FY09EM21-06 } & & Measured & 0.73 & 0.64 & 0.57 & 0.15 & 5.38 & 4.37 & 3.75 & 1.40 \\
\hline 1 & & quenched & Measured bc & 0.74 & 0.64 & 0.58 & 0.15 & 5.43 & 4.32 & 3.79 & 1.40 \\
\hline 1 & & & Target & 0.73 & 0.63 & 0.58 & 0.14 & 5.38 & 4.21 & 3.76 & 1.37 \\
\hline 1 & & & Measured & 0.81 & 0.73 & 0.66 & 0.24 & 6.50 & 5.34 & 4.57 & 1.72 \\
\hline 1 & & $\mathrm{ccc}$ & Measured bc & 0.82 & 0.72 & 0.66 & 0.24 & 6.55 & 5.28 & 4.61 & 1.72 \\
\hline 1 & & & Target & 0.81 & 0.71 & 0.66 & 0.23 & 6.49 & 5.14 & 4.57 & 1.69 \\
\hline 1 & & & Measured & 1.14 & 1.03 & 1.00 & 0.05 & 13.87 & 10.69 & 10.00 & 1.11 \\
\hline 1 & & quenched & Measured bc & 1.15 & 1.03 & 1.00 & 0.04 & 14.06 & 10.63 & 10.09 & 1.11 \\
\hline 1 & FV0OFM 107 & & Target & 1.14 & 1.01 & 1.00 & 0.04 & 13.64 & 10.34 & 10.09 & 1.10 \\
\hline 1 & FYO9E1M21-07 & & Measured & 0.78 & 0.71 & 0.67 & -0.01 & 6.02 & 5.14 & 4.70 & 0.98 \\
\hline 1 & & $\mathrm{ccc}$ & Measured bc & 0.79 & 0.71 & 0.68 & -0.01 & 6.11 & 5.11 & 4.75 & 0.97 \\
\hline 1 & & & Target & 0.77 & 0.70 & 0.68 & -0.02 & 5.93 & 4.97 & 4.75 & 0.96 \\
\hline 1 & & & Measured & 0.72 & 0.56 & 0.56 & 0.25 & 5.28 & 3.61 & 3.59 & 1.76 \\
\hline 1 & & quenched & Measured bc & 0.73 & 0.56 & 0.55 & 0.24 & 5.36 & 3.59 & 3.52 & 1.72 \\
\hline 1 & 00F & & Target & 0.71 & 0.54 & 0.54 & 0.23 & 5.13 & 3.47 & 3.47 & 1.68 \\
\hline 1 & F YOYEIVIL1-08 & & Measured & 0.63 & 0.66 & 0.53 & 0.40 & 4.27 & 4.55 & 3.37 & 2.53 \\
\hline 1 & & $\mathrm{ccc}$ & Measured bc & 0.64 & 0.66 & 0.52 & 0.39 & 4.34 & 4.52 & 3.30 & 2.47 \\
\hline 1 & & & Target & 0.62 & 0.64 & 0.51 & 0.38 & 4.15 & 4.37 & 3.25 & 2.42 \\
\hline
\end{tabular}


SRNL-STI-2009-00778, REVISION 0

Table 11 continued.

\begin{tabular}{|c|c|c|c|c|c|c|c|c|c|c|c|}
\hline Set & Glass ID & $\begin{array}{c}\text { Heat } \\
\text { Treatment }\end{array}$ & $\begin{array}{c}\text { Comp } \\
\text { View }\end{array}$ & $\begin{array}{c}\log N L \\
{[B(g / L)]}\end{array}$ & $\begin{array}{c}\log \mathrm{NL} \\
{[\mathrm{Li}(\mathrm{g} / \mathrm{L})]}\end{array}$ & \begin{tabular}{|c|}
$\log \mathrm{NL}$ \\
{$[\mathrm{Na}(\mathrm{g} / \mathrm{L})]$}
\end{tabular} & $\begin{array}{c}\log \mathrm{NL} \\
{[\mathrm{Si}(\mathrm{g} / \mathrm{L})]}\end{array}$ & $\begin{array}{c}\text { NL } \\
B(g / L)\end{array}$ & $\begin{array}{c}\mathrm{NL} \\
\mathrm{Li}(\mathrm{g} / \mathrm{L})\end{array}$ & \begin{tabular}{|c|}
$\mathrm{NL}$ \\
$\mathrm{Na}(\mathrm{g} / \mathrm{L})$
\end{tabular} & $\begin{array}{c}\mathrm{NL} \\
\mathrm{Si}(\mathrm{g} / \mathrm{L})\end{array}$ \\
\hline 1 & \multirow{6}{*}{ FY09EM21-09 } & \multirow{3}{*}{ quenched } & Measured & -0.07 & -0.05 & -0.14 & -0.34 & 0.85 & 0.89 & 0.72 & 0.46 \\
\hline 1 & & & Measured bc & -0.06 & -0.06 & $\begin{array}{c}-0.14 \\
\end{array}$ & -0.34 & 0.87 & 0.88 & 0.73 & 0.46 \\
\hline 1 & & & Target & -0.07 & -0.07 & -0.14 & -0.35 & 0.86 & 0.86 & 0.72 & 0.45 \\
\hline 1 & & \multirow{3}{*}{$\mathrm{ccc}$} & Measured & -0.32 & -0.23 & -0.31 & -0.45 & 0.48 & 0.59 & 0.49 & 0.36 \\
\hline 1 & & & Measured bc & -0.31 & -0.23 & -0.31 & -0.45 & 0.49 & 0.58 & 0.49 & 0.35 \\
\hline 1 & & & Target & $\begin{array}{c}-0.31 \\
\end{array}$ & -0.25 & $\begin{array}{c}-0.31 \\
\end{array}$ & -0.46 & 0.48 & 0.57 & 0.49 & 0.35 \\
\hline 2 & \multirow{6}{*}{ FY09EM21-10 } & \multirow{3}{*}{ quenched } & Measured & 0.10 & 0.10 & 0.09 & -0.24 & 1.27 & 1.26 & 1.23 & 0.57 \\
\hline 2 & & & \begin{tabular}{|l|} 
Measured bc \\
\end{tabular} & 0.11 & 0.10 & 0.09 & -0.25 & 1.29 & 1.25 & 1.24 & 0.57 \\
\hline 2 & & & Target & 0.10 & 0.09 & 0.10 & -0.25 & 1.26 & 1.22 & 1.26 & 0.56 \\
\hline 2 & & \multirow{3}{*}{$\mathrm{ccc}$} & Measured & -0.14 & 0.03 & -0.07 & -0.32 & 0.73 & 1.08 & 0.86 & 0.48 \\
\hline 2 & & & Measured bc & -0.13 & 0.03 & -0.06 & -0.32 & 0.74 & 1.07 & 0.87 & 0.48 \\
\hline 2 & & & Target & -0.15 & 0.02 & -0.05 & -0.33 & 0.72 & 1.04 & 0.88 & 0.47 \\
\hline 2 & \multirow{6}{*}{ FY09EM21-11 } & \multirow{3}{*}{ quenched } & Measured & 0.40 & 0.33 & 0.35 & 0.08 & 2.53 & 2.15 & 2.21 & 1.20 \\
\hline 2 & & & \begin{tabular}{|l|} 
Measured bc \\
\end{tabular} & 0.41 & 0.33 & 0.35 & 0.08 & 2.55 & 2.13 & 2.23 & 1.20 \\
\hline 2 & & & Target & 0.37 & 0.32 & 0.35 & 0.07 & 2.37 & 2.10 & 2.23 & 1.16 \\
\hline 2 & & \multirow{3}{*}{$\mathrm{ccc}$} & Measured & 0.38 & 0.32 & 0.30 & 0.07 & 2.39 & 2.10 & 1.99 & 1.17 \\
\hline 2 & & & Measured bc & 0.38 & 0.32 & 0.30 & 0.07 & 2.41 & 2.08 & 2.01 & 1.17 \\
\hline 2 & & & Target & 0.35 & 0.31 & 0.30 & 0.06 & 2.23 & 2.05 & 2.01 & 1.14 \\
\hline 2 & \multirow{6}{*}{ FY09EM21-12 } & \multirow{3}{*}{ quenched } & Measured & 0.15 & 0.12 & 0.08 & -0.08 & 1.42 & 1.33 & 1.21 & 0.84 \\
\hline 2 & & & Measured bc & 0.16 & 0.12 & 0.08 & -0.09 & 1.44 & 1.32 & 1.19 & 0.82 \\
\hline 2 & & & Target & 0.15 & 0.11 & 0.08 & -0.09 & 1.40 & 1.30 & 1.19 & 0.81 \\
\hline 2 & & \multirow{3}{*}{$\mathrm{ccc}$} & Measured & 0.25 & 0.50 & 0.20 & 0.11 & 1.76 & 3.18 & 1.58 & 1.29 \\
\hline 2 & & & Measured bc & 0.25 & 0.50 & 0.19 & 0.10 & 1.78 & 3.17 & 1.54 & 1.26 \\
\hline 2 & & & Target & 0.24 & 0.49 & 0.19 & 0.09 & 1.74 & 3.10 & 1.54 & 1.24 \\
\hline 2 & \multirow{6}{*}{ FY09EM21-13 } & \multirow{3}{*}{ quenched } & Measured & 0.07 & 0.13 & 0.13 & -0.20 & 1.19 & 1.35 & 1.33 & 0.64 \\
\hline 2 & & & Measured bc & 0.08 & 0.12 & 0.12 & -0.21 & 1.19 & 1.33 & 1.31 & 0.62 \\
\hline 2 & & & Target & 0.07 & 0.12 & 0.12 & -0.20 & 1.19 & 1.33 & 1.32 & 0.63 \\
\hline 2 & & \multirow{3}{*}{$\mathrm{ccc}$} & Measured & -0.09 & 0.09 & -0.04 & -0.33 & 0.81 & 1.23 & 0.91 & 0.46 \\
\hline 2 & & & Measured bc & -0.09 & 0.08 & -0.05 & -0.34 & 0.82 & 1.21 & 0.89 & 0.45 \\
\hline 2 & & & Target & -0.09 & 0.08 & -0.04 & -0.34 & 0.81 & 1.21 & 0.90 & 0.46 \\
\hline 2 & \multirow{6}{*}{ FY09EM21-14 } & & Measured & 0.01 & 0.01 & -0.12 & -0.33 & 1.02 & 1.02 & 0.76 & 0.46 \\
\hline 2 & & quenched & \begin{tabular}{|l|} 
Measured bc \\
\end{tabular} & 0.01 & 0.00 & -0.13 & -0.34 & 1.02 & 1.00 & 0.74 & 0.45 \\
\hline 2 & & & Target & 0.05 & 0.04 & -0.13 & -0.34 & 1.12 & 1.10 & 0.74 & 0.45 \\
\hline 2 & & & Measured & 0.59 & 0.52 & 0.43 & -0.01 & 3.87 & 3.33 & 2.70 & 0.99 \\
\hline 2 & & $\mathrm{ccc}$ & Measured bc & 0.59 & 0.52 & 0.42 & -0.02 & 3.90 & 3.29 & 2.65 & 0.96 \\
\hline 2 & & & Target & 0.63 & 0.56 & 0.42 & -0.02 & 4.25 & 3.62 & 2.64 & 0.96 \\
\hline 2 & & & Measured & 0.05 & 0.05 & 0.04 & -0.23 & 1.13 & 1.12 & 1.09 & 0.59 \\
\hline 2 & & quenched & Measured bc & 0.05 & 0.04 & 0.04 & -0.23 & 1.13 & 1.10 & 1.08 & 0.59 \\
\hline 2 & FY00FM21-15 & & Target & 0.06 & 0.03 & 0.03 & -0.23 & 1.15 & 1.08 & 1.08 & 0.59 \\
\hline 2 & FY09EM21-15 & & Measured & 0.22 & 0.37 & 0.16 & -0.05 & 1.65 & 2.35 & 1.46 & 0.89 \\
\hline 2 & & $\mathrm{ccc}$ & Measured bc & 0.22 & 0.37 & 0.16 & -0.05 & 1.65 & 2.32 & 1.45 & 0.90 \\
\hline 2 & & & Target & 0.23 & 0.36 & 0.16 & -0.05 & 1.69 & 2.28 & 1.44 & 0.90 \\
\hline 2 & & & Measured & 0.45 & 0.37 & 0.46 & 0.10 & 2.82 & 2.33 & 2.88 & 1.27 \\
\hline 2 & & quenched & \begin{tabular}{|l|} 
Measured bc \\
\end{tabular} & 0.46 & 0.37 & 0.46 & 0.11 & 2.88 & 2.36 & 2.88 & 1.28 \\
\hline 2 & FY00FM21-16 & & Target & 0.46 & 0.37 & 0.44 & 0.10 & 2.91 & 2.32 & 2.78 & 1.25 \\
\hline 2 & FYOSEIVIL-10 & & Measured & 0.23 & 0.19 & 0.28 & -0.06 & 1.71 & 1.55 & 1.90 & 0.87 \\
\hline 2 & & $\mathrm{ccc}$ & Measured bc & 0.24 & 0.20 & 0.28 & -0.06 & 1.74 & 1.57 & 1.90 & 0.88 \\
\hline 2 & & & Target & 0.25 & 0.19 & 0.26 & -0.07 & 1.76 & 1.54 & 1.84 & 0.86 \\
\hline 2 & & & Measured & 0.05 & 0.09 & 0.22 & -0.04 & 1.11 & 1.24 & 1.64 & 0.92 \\
\hline 2 & & quenched & Measured bc & 0.06 & 0.10 & 0.22 & -0.03 & 1.14 & 1.25 & 1.64 & 0.93 \\
\hline 2 & FY00F 17 & & Target & 0.08 & 0.11 & 0.22 & -0.02 & 1.19 & 1.28 & 1.66 & 0.96 \\
\hline 2 & FY09EV21-17 & & Measured & 0.44 & 0.55 & 0.36 & 0.33 & 2.76 & 3.54 & 2.29 & 2.13 \\
\hline 2 & & $\mathrm{ccc}$ & \begin{tabular}{|l|} 
Measured bc \\
\end{tabular} & 0.45 & 0.56 & 0.36 & 0.33 & 2.82 & 3.59 & 2.29 & 2.15 \\
\hline 2 & & & Target & 0.47 & 0.57 & 0.37 & 0.35 & 2.95 & 3.68 & 2.32 & 2.24 \\
\hline
\end{tabular}


SRNL-STI-2009-00778, REVISION 0

Table 11 continued.

\begin{tabular}{|c|c|c|c|c|c|c|c|c|c|c|c|}
\hline Set & Glass ID & $\begin{array}{c}\text { Heat } \\
\text { Treatment }\end{array}$ & $\begin{array}{l}\text { Comp } \\
\text { View }\end{array}$ & $\begin{array}{c}\log N \mathrm{~L} \\
{[\mathrm{~B}(\mathrm{~g} / \mathrm{L})]}\end{array}$ & \begin{tabular}{|c}
$\log \mathrm{NL}$ \\
{$[\mathrm{Li}(\mathrm{g} / \mathrm{L})]$}
\end{tabular} & \begin{tabular}{|c|}
$\log \mathrm{NL}$ \\
{$[\mathrm{Na}(\mathrm{g} / \mathrm{L})]$}
\end{tabular} & $\begin{array}{c}\log \mathrm{NL} \\
{[\mathrm{Si}(\mathrm{g} / \mathrm{L})]}\end{array}$ & $\begin{array}{c}\text { NL } \\
B(g / L)\end{array}$ & $\begin{array}{c}\mathrm{NL} \\
\mathrm{Li}(\mathrm{g} / \mathrm{L})\end{array}$ & \begin{tabular}{|c|}
$\mathrm{NL}$ \\
$\mathrm{Na}(\mathrm{g} / \mathrm{L})$
\end{tabular} & $\begin{array}{c}\mathrm{NL} \\
\mathrm{Si}(\mathrm{g} / \mathrm{L})\end{array}$ \\
\hline 2 & \multirow{6}{*}{ FY09EM21-18 } & \multirow{3}{*}{ quenched } & Measured & -0.21 & -0.12 & 0.07 & -0.24 & 0.62 & 0.76 & 1.18 & 0.57 \\
\hline 2 & & & \begin{tabular}{|l|} 
Measured bc \\
\end{tabular} & -0.21 & -0.13 & 0.07 & -0.24 & 0.62 & 0.75 & 1.17 & 0.57 \\
\hline 2 & & & Target & -0.20 & -0.13 & 0.05 & -0.24 & 0.62 & 0.74 & 1.12 & 0.57 \\
\hline 2 & & \multirow{3}{*}{$\mathrm{ccc}$} & Measured & -0.20 & -0.10 & 0.03 & -0.24 & 0.63 & 0.79 & 1.06 & 0.57 \\
\hline 2 & & & Measured bc & -0.20 & -0.11 & 0.03 & -0.24 & 0.63 & 0.78 & 1.06 & 0.57 \\
\hline 2 & & & Target & -0.20 & -0.11 & 0.00 & -0.24 & 0.63 & 0.77 & 1.01 & 0.57 \\
\hline 3 & \multirow{6}{*}{ FY09EM21-19 } & \multirow{3}{*}{ quenched } & Measured & -0.17 & 0.02 & 0.19 & -0.25 & 0.68 & 1.05 & 1.54 & 0.56 \\
\hline 3 & & & Measured bc & -0.17 & 0.02 & 0.19 & -0.25 & 0.68 & 1.04 & 1.53 & 0.56 \\
\hline 3 & & & Target & -0.16 & 0.02 & 0.16 & -0.25 & 0.70 & 1.04 & 1.43 & 0.56 \\
\hline 3 & & \multirow{3}{*}{$\mathrm{ccc}$} & Measured & -0.13 & 0.05 & 0.19 & -0.26 & 0.74 & 1.12 & 1.53 & 0.55 \\
\hline 3 & & & Measured bc & -0.13 & 0.04 & 0.18 & -0.26 & 0.74 & 1.10 & 1.53 & 0.55 \\
\hline 3 & & & Target & -0.12 & 0.05 & 0.15 & -0.26 & 0.75 & 1.11 & 1.42 & 0.55 \\
\hline 3 & \multirow{6}{*}{ FY09EM21-20 } & \multirow{3}{*}{ quenched } & Measured & 0.05 & -0.01 & 0.20 & -0.06 & 1.12 & 0.98 & 1.59 & 0.88 \\
\hline 3 & & & \begin{tabular}{|l|} 
Measured bc \\
\end{tabular} & 0.06 & 0.00 & 0.20 & -0.05 & 1.14 & 0.99 & 1.59 & 0.89 \\
\hline 3 & & & Target & 0.06 & -0.01 & 0.18 & -0.06 & 1.14 & 0.98 & 1.50 & 0.87 \\
\hline 3 & & \multirow{3}{*}{$\mathrm{ccc}$} & Measured & 0.04 & -0.01 & 0.18 & -0.08 & 1.11 & 0.98 & 1.53 & 0.84 \\
\hline 3 & & & Measured bc & 0.05 & 0.00 & 0.18 & -0.07 & 1.13 & 0.99 & 1.52 & 0.85 \\
\hline 3 & & & Target & 0.05 & -0.01 & 0.16 & -0.08 & 1.13 & 0.99 & 1.44 & 0.83 \\
\hline 3 & \multirow{6}{*}{ FY09EM21-21 } & \multirow{3}{*}{ quenched } & Measured & 1.48 & 1.16 & 1.34 & 0.58 & 30.16 & 14.33 & 21.91 & 3.79 \\
\hline 3 & & & \begin{tabular}{|l|} 
Measured bc \\
\end{tabular} & 1.48 & 1.15 & 1.34 & 0.58 & 30.15 & 14.14 & 21.90 & 3.80 \\
\hline 3 & & & Target & 1.48 & 1.15 & 1.33 & 0.58 & 29.96 & 14.10 & 21.55 & 3.78 \\
\hline 3 & & \multirow{3}{*}{$\mathrm{ccc}$} & Measured & 1.43 & 1.13 & 1.29 & 0.55 & 26.71 & 13.54 & 19.55 & 3.57 \\
\hline 3 & & & Measured bc & 1.43 & 1.13 & 1.29 & 0.55 & 26.71 & 13.36 & 19.54 & 3.58 \\
\hline 3 & & & Target & 1.42 & 1.12 & 1.28 & 0.55 & 26.54 & 13.32 & 19.22 & 3.56 \\
\hline 3 & \multirow{6}{*}{ FY09EM21-22 } & & Measured & -0.22 & -0.06 & -0.55 & -0.38 & 0.60 & 0.87 & 0.28 & 0.42 \\
\hline 3 & & quenched & Measured bc & -0.21 & -0.06 & -0.55 & -0.37 & 0.61 & 0.88 & 0.28 & 0.42 \\
\hline 3 & & & Target & -0.22 & -0.05 & -0.55 & -0.37 & 0.60 & 0.88 & 0.29 & 0.43 \\
\hline 3 & & & Measured & -0.28 & -0.15 & -0.50 & -0.40 & 0.52 & 0.71 & 0.31 & 0.40 \\
\hline 3 & & $\mathrm{ccc}$ & Measured bc & -0.27 & -0.14 & -0.50 & -0.39 & 0.53 & 0.72 & 0.31 & 0.41 \\
\hline 3 & & & Target & -0.28 & -0.14 & -0.50 & -0.39 & 0.53 & 0.72 & 0.32 & 0.41 \\
\hline 3 & & & Measured & -0.14 & -0.04 & -0.51 & -0.40 & 0.72 & 0.92 & 0.31 & 0.40 \\
\hline 3 & & quenched & \begin{tabular}{|l|} 
Measured bc \\
\end{tabular} & -0.14 & -0.04 & -0.51 & -0.40 & 0.72 & 0.91 & 0.31 & 0.40 \\
\hline 3 & FY09FM21-23 & & Target & -0.14 & -0.05 & -0.52 & -0.40 & 0.72 & 0.90 & 0.30 & 0.40 \\
\hline 3 & FY09EM21-23 & & Measured & -0.20 & -0.06 & -0.37 & -0.32 & 0.63 & 0.87 & 0.43 & 0.48 \\
\hline 3 & & $\mathrm{ccc}$ & Measured bc & -0.20 & -0.07 & -0.37 & -0.31 & 0.63 & 0.85 & 0.43 & 0.49 \\
\hline 3 & & & Target & -0.20 & -0.08 & -0.37 & -0.32 & 0.63 & 0.84 & 0.42 & 0.48 \\
\hline 3 & & & Measured & -0.03 & 0.00 & -0.03 & -0.40 & 0.94 & 1.01 & 0.95 & 0.40 \\
\hline 3 & & quenched & Measured bc & -0.03 & 0.00 & -0.03 & -0.40 & 0.94 & 1.00 & 0.94 & 0.40 \\
\hline 3 & FYoorMa1 2 & & Target & -0.02 & -0.01 & -0.03 & -0.39 & 0.96 & 0.99 & 0.94 & 0.40 \\
\hline 3 & 09EM21-24 & & Measured & -0.42 & -0.24 & -0.26 & -0.56 & 0.38 & 0.57 & 0.55 & 0.27 \\
\hline 3 & & $\mathrm{ccc}$ & Measured bc & -0.42 & -0.25 & -0.26 & -0.56 & 0.38 & 0.57 & 0.55 & 0.27 \\
\hline 3 & & & Target & -0.41 & -0.25 & -0.26 & -0.56 & 0.39 & 0.56 & 0.55 & 0.28 \\
\hline 3 & & & Measured & -0.01 & 0.08 & 0.11 & -0.21 & 0.99 & 1.21 & 1.30 & 0.62 \\
\hline 3 & & quenched & Measured bc & 0.00 & 0.09 & 0.11 & -0.20 & 1.01 & 1.23 & 1.30 & 0.63 \\
\hline 3 & FVOOFM21 25 & & Target & 0.02 & 0.08 & 0.11 & -0.22 & 1.04 & 1.21 & 1.29 & 0.60 \\
\hline 3 & FY09EM21-25 & & Measured & 0.01 & 0.10 & 0.11 & -0.21 & 1.03 & 1.26 & 1.28 & 0.62 \\
\hline 3 & & $\mathrm{ccc}$ & Measured bc & 0.02 & 0.11 & 0.11 & -0.20 & 1.05 & 1.27 & 1.28 & 0.63 \\
\hline 3 & & & Target & 0.04 & 0.10 & 0.11 & -0.22 & 1.08 & 1.26 & 1.28 & 0.61 \\
\hline 3 & & & Measured & -0.21 & -0.15 & -0.26 & -0.36 & 0.62 & 0.71 & 0.55 & 0.44 \\
\hline 3 & & quenched & Measured bc & -0.20 & -0.15 & -0.26 & -0.36 & 0.63 & 0.72 & 0.55 & 0.44 \\
\hline 3 & 120 & & Target & -0.21 & $\begin{array}{l}-0.14 \\
\end{array}$ & -0.26 & -0.36 & 0.62 & 0.72 & 0.55 & 0.43 \\
\hline 3 & FY09EM21-26 & & Measured & -0.17 & -0.18 & -0.28 & -0.38 & 0.67 & 0.66 & 0.53 & 0.42 \\
\hline 3 & & $\mathrm{ccc}$ & Measured bc & -0.16 & -0.17 & -0.28 & -0.37 & 0.69 & 0.67 & 0.53 & 0.43 \\
\hline 3 & & & Target & -0.18 & -0.17 & -0.28 & -0.38 & 0.67 & 0.68 & 0.53 & 0.42 \\
\hline 3 & & & Measured & 0.04 & 0.03 & 0.03 & -0.23 & 1.10 & 1.07 & 1.07 & 0.59 \\
\hline 3 & & quenched & Measured bc & 0.04 & 0.03 & 0.03 & -0.23 & 1.10 & 1.06 & 1.07 & 0.59 \\
\hline 3 & FVOOFM21 & & Target & 0.03 & 0.02 & 0.03 & -0.23 & 1.08 & 1.04 & 1.06 & 0.59 \\
\hline 3 & FY09EM21-27 & & Measured & 0.00 & 0.02 & 0.01 & -0.22 & 1.01 & 1.04 & 1.02 & 0.60 \\
\hline 3 & & $\mathrm{ccc}$ & Measured bc & 0.00 & 0.01 & 0.01 & -0.22 & 1.01 & 1.03 & 1.02 & 0.60 \\
\hline 3 & & & Target & 0.00 & 0.01 & 0.01 & -0.23 & 0.99 & 1.01 & 1.02 & 0.59 \\
\hline
\end{tabular}




\subsubsection{Effects of Heat Treatment}

Exhibit B5 in Appendix B provides a series of plots and statistical comparisons that demonstrate the effects of heat treatment on the common logarithm ppm-responses of interest of the triplicate PCTs for each element for each non-radioactive study glass. The quenched version of a given glass yielded measurements indicating a significantly (at the 5\% significance level) different mean $\log (\mathrm{ppm})$ response than the ccc version of the glass for a given element if the Prob $>|\mathbf{t}|$ value in the exhibit is 0.05 or smaller. Table 12 summaries the comparisons between the quenched and ccc versions of the study glasses for the four elements of the PCTs. The less durable heat treatment is noted.

Many of these glasses exhibit a statistically significant difference between the ccc versus the quenched versions for one or more of the PCT elements. Exhibit B6 in Appendix B provides plots of the normalized PCT responses between the two heat treatments grouped by compositional view. These plots provide a basis for judging the practical impact of differences in the PCT response due to the heat treatment of the glass.

The NL [B] values range from $0.61 \mathrm{~g} / \mathrm{L}$ (FY09EM21-22) to $30.15 \mathrm{~g} / \mathrm{L}$ (FY09EM21-21) for the quenched glasses and $0.38 \mathrm{~g} / \mathrm{L}$ (FY09EM21-24) to 26.71 $\mathrm{g} / \mathrm{L}$ (FY09EM21-21) for the ccc glasses. It is interesting to note that three of the quenched glasses are unacceptable ${ }^{\mathrm{Q}}$ (FY09EM21-02, -07 and -21) and nepheline was not detected in any of them. ${ }^{28}$ The poor durability could be due to phase separation; however, further characterization would be required to verify this hypothesis. Three of the slow cooled glasses are also unacceptable (FY09EM21$02,-03$ and -21$)$, but it is unknown at this time if these glasses contain nepheline as XRD measurements could not be completed.

\footnotetext{
${ }^{\mathrm{Q}}$ Historical glasses of interest to DWPF were found to be acceptable using a normalized boron release (NL [B]) of $10 \mathrm{~g} / \mathrm{L}$ as a benchmark. This value was chosen so that the boron releases of the study glasses were well below that of the Environmental Assessment (EA) glass when accounting for measurement uncertainty. It should be emphasized that this limit was selected only as a guide to develop the $\mathrm{Al}_{2} \mathrm{O}_{3}$ and/or sum of alkali criteria used in PCCS.
} 
Table 12. Samples Exhibiting a Statistically Significant Difference Between the Quenched and cce Versions of the Non-Radioactive Matrix 2 Glasses

\begin{tabular}{|l|c|c|c|c|}
\hline \multicolumn{1}{|c|}{ Glass ID } & $\mathbf{B}$ & $\mathbf{L i}$ & $\mathbf{N a}$ & $\mathbf{S i}$ \\
\hline FY09EM21-01 & $\mathrm{ccc}$ & & & $\mathrm{ccc}$ \\
\hline FY09EM21-02 & & & & \\
\hline FY09EM21-03 & $\mathrm{ccc}$ & $\mathrm{ccc}$ & $\mathrm{ccc}$ & $\mathrm{ccc}$ \\
\hline FY09EM21-04 & $\mathrm{Q}$ & $\mathrm{Q}$ & $\mathrm{Q}$ & $\mathrm{Q}$ \\
\hline FY09EM21-05 & & $\mathrm{Q}$ & $\mathrm{Q}$ & $\mathrm{Q}$ \\
\hline FY09EM21-06 & & $\mathrm{ccc}$ & $\mathrm{ccc}$ & $\mathrm{ccc}$ \\
\hline FY09EM21-07 & $\mathrm{Q}$ & $\mathrm{Q}$ & $\mathrm{Q}$ & $\mathrm{Q}$ \\
\hline FY09EM21-08 & $\mathrm{Q}$ & $\mathrm{ccc}$ & & $\mathrm{ccc}$ \\
\hline FY09EM21-09 & $\mathrm{Q}$ & $\mathrm{Q}$ & $\mathrm{Q}$ & $\mathrm{Q}$ \\
\hline FY09EM21-10 & $\mathrm{Q}$ & $\mathrm{Q}$ & $\mathrm{Q}$ & $\mathrm{Q}$ \\
\hline FY09EM21-11 & & & $\mathrm{Q}$ & \\
\hline FY09EM21-12 & $\mathrm{ccc}$ & $\mathrm{ccc}$ & $\mathrm{ccc}$ & $\mathrm{ccc}$ \\
\hline FY09EM21-13 & $\mathrm{Q}$ & $\mathrm{Q}$ & $\mathrm{Q}$ & $\mathrm{Q}$ \\
\hline FY09EM21-14 & $\mathrm{ccc}$ & $\mathrm{ccc}$ & $\mathrm{ccc}$ & $\mathrm{ccc}$ \\
\hline FY09EM21-15 & $\mathrm{ccc}$ & $\mathrm{ccc}$ & $\mathrm{ccc}$ & $\mathrm{ccc}$ \\
\hline FY09EM21-16 & $\mathrm{Q}$ & $\mathrm{Q}$ & $\mathrm{Q}$ & $\mathrm{Q}$ \\
\hline FY09EM21-17 & $\mathrm{ccc}$ & $\mathrm{ccc}$ & $\mathrm{ccc}$ & $\mathrm{ccc}$ \\
\hline FY09EM21-18 & & $\mathrm{ccc}$ & $\mathrm{Q}$ & \\
\hline FY09EM21-19 & & $\mathrm{ccc}$ & & \\
\hline FY09EM21-20 & & & & \\
\hline FY09EM21-21 & & & & \\
\hline FY09EM21-22 & & $\mathrm{Q}$ & $\mathrm{ccc}$ & \\
\hline FY09EM21-23 & & $\mathrm{Q}$ & $\mathrm{ccc}$ & $\mathrm{ccc}$ \\
\hline FY09EM21-24 & $\mathrm{Q}$ & $\mathrm{Q}$ & $\mathrm{Q}$ & $\mathrm{Q}$ \\
\hline FY09EM21-25 & & $\mathrm{ccc}$ & & \\
\hline FY09EM21-26 & & & & \\
\hline FY09EM21-27 & $\mathrm{Q}$ & & $\mathrm{Q}$ & \\
\hline
\end{tabular}




\subsubsection{Predicted versus Measured PCTs}

Exhibits B7 through B10 in Appendix B provides plots of the DWPF models ${ }^{\mathrm{R}}$ for B, Li, Na, and $\mathrm{Si}$, respectively, that relate the logarithm of the normalized PCT (for each element of interest) to a linear function of a free energy of hydration term $\left(\Delta \mathrm{G}_{\mathrm{p}}, \mathrm{kcal} / 100 \mathrm{~g}\right.$ glass $)$ derived for each of the compositional views and heat treatments for the Matrix 2 non-radioactive glasses. ${ }^{27}$ Prediction limits (at a 95\% confidence) for an individual PCT result are also plotted along with the linear fit. The EA and ARM results are also indicated on these plots.

There are a number of glasses that fall above the confidence limits for the non-radioactive study glasses. Exhibit B7 is repeated as Figure 15 below. As stated previously, three of the quenched glasses have unacceptable NL [B] values, one of which is greater than the EA glass. These glasses are of concern as no nepheline was detected by XRD. If these glasses are phase separated then the durability model would not be expected to predict their durability. It is highly recommended to use other characterization techniques in future studies to determine the cause of the poor durabilities of these glasses. These glasses do contain lower concentrations of $\mathrm{SiO}_{2}$ and $\mathrm{Al}_{2} \mathrm{O}_{3}$ compared to some of the other glasses. A few of the glasses with log NL [B] values in the range of 0.6 to 0.8 are also unpredictable, but still acceptable with respect to the $10 \mathrm{~g} / \mathrm{L}$ limit for boron release. Glasses at higher $\Delta \mathrm{G}_{\mathrm{p}}$ values (-8 to -6) are also unpredictable; however, none of these glasses have normalized boron releases greater than $1.5 \mathrm{~g} / \mathrm{L}$.

\footnotetext{
${ }^{\mathrm{R}}$ It should be noted that the DWPF models for durability only apply to amorphous glasses. These models would not be expected to predict the durabilities of phase separated glasses or those glasses containing nepheline.
} 
SRNL-STI-2009-00778, REVISION 0

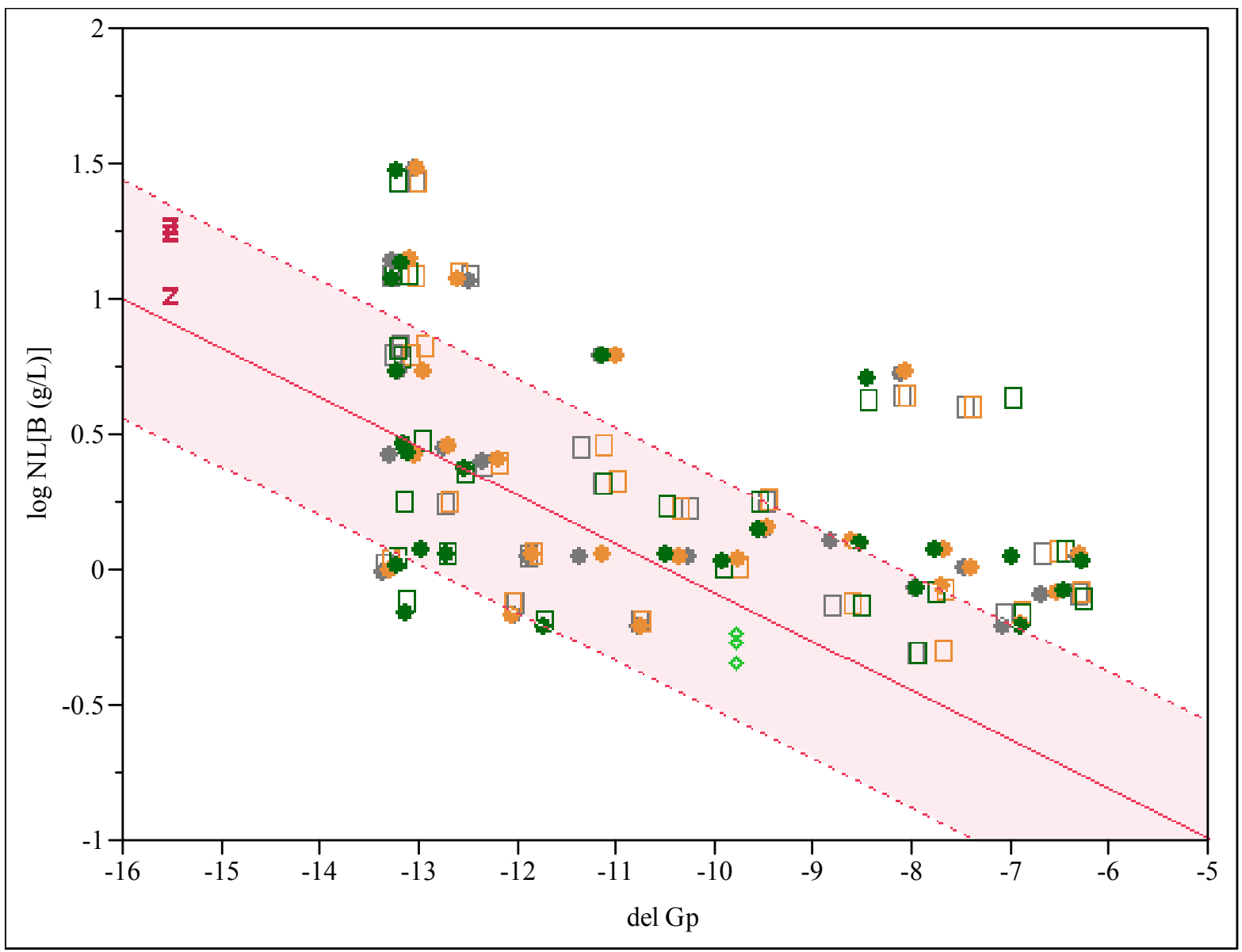

\begin{tabular}{|c|c|}
\hline Symbol & $\begin{array}{c}\text { Standard/ } \\
\text { Comp View-Heat Treatment }\end{array}$ \\
\hline $\mathrm{Z}$ & EA \\
\hline$\diamond$ & ARM \\
\hline$\square$ & Measured-ccc \\
\hline$\square$ & Measured bc -ccc \\
\hline$\square$ & Targeted-ccc \\
\hline$\bullet$ & Measured-quenched \\
\hline$\bullet$ & Measured bc - quenched \\
\hline$\bullet$ & Targeted- quenched \\
\hline
\end{tabular}

Figure 15. $\log$ NL [B (g/L)] versus del Gp model with $95 \%$ Confidence Interval for Individual PCTs. 


\subsubsection{Matrix 2 $\mathrm{T}_{\mathrm{L}}$ Data}

A comparison of the predicted and measured $T_{L}$ values is shown in Table 13. Oxides that are outside of the ranges used to develop the original model are also noted. In general, the current $\mathrm{T}_{\mathrm{L}}$ model predicts values to be lower than those actually measured. The data do not suggest any trends with composition; however, compositions vary greatly from one composition to another and it is therefore difficult to make any conclusions. It is probable that this glass property is extremely sensitive to even small compositional changes. Unlike the series of glasses from the FY07 series , most of these compositions of this series have many components that are outside of the model development ranges. As this property is sensitive to compositional variation, with each component having a different magnitude in its influence, then the current liquidus model would not be expected to accurately predict glasses in higher waste loading regions.

Primary crystalline phases formed during the measurements are listed in Table 14. In general, spinel formed at temperatures near the $\mathrm{T}_{\mathrm{L}}$; however, there are some exceptions (FY09EM21-05, $06,-11,-12,-14,-18,-19$ and -23$)$. Iron titanate crystals formed in some of the glasses with $\mathrm{TiO}_{2}$ concentrations of approximately $6 \mathrm{wt} \%$ (FY09EM-05, -14, and -23). Unidentifiable crystals were formed in FY09EM21-06, $-11,-12,-18$ and -19 . The current $\mathrm{T}_{\mathrm{L}}$ model is only applicable to glasses containing spinels, so it would not be expected to accurately predict glasses with unidentifiable crystals present. Optical micrographs and corresponding XRD patterns (if available) of all of the crystal types are shown in Figure 16 through Figure 19.

\footnotetext{
${ }^{\mathrm{S}}$ In FY07, it was mostly limited to variation in $\mathrm{MgO}$ and $\mathrm{TiO}_{2}$ (with some exceptions).
} 
SRNL-STI-2009-00778, REVISION 0

Table 13. Predicted and Measured $T_{L}$ Values

\begin{tabular}{|c|c|c|c|c|}
\hline Glass ID & $\mathbf{T}_{\mathbf{L}}$ Predicted $\left({ }^{\circ} \mathbf{C}\right)$ & $T_{L}$ Measured & Measured - Predicted $\left({ }^{\circ} \mathrm{C}\right)$ & $\begin{array}{c}\text { Compositional Assessment Relative to Model } \\
\text { Development Ranges }\end{array}$ \\
\hline FY09EM21-02 & 905 & 980 & 75 & $\begin{array}{c}\text { Lower: } \mathrm{CaO}, \mathrm{MnO}, \mathrm{NiO} \text { and } \mathrm{SiO}_{2} \\
\text { Higher: } \mathrm{Fe}_{2} \mathrm{O}_{3}, \mathrm{Na}_{2} \mathrm{O} \text { and } \mathrm{TiO}_{2}\end{array}$ \\
\hline FY09EM21-05 & 1031 & 1115 & 84 & $\begin{array}{c}\text { Lower: } \mathrm{B}_{2} \mathrm{O}_{3}, \mathrm{MgO} \text { and } \mathrm{MnO} \\
\text { Higher: } \mathrm{CaO} \text { and } \mathrm{TiO}_{2}\end{array}$ \\
\hline FY09EM21-06 & 921 & 995 & 74 & $\begin{array}{l}\text { Lower: } \mathrm{CaO} \text { and } \mathrm{MgO} \\
\text { Higher: } \mathrm{MnO} \text { and } \mathrm{TiO}_{2}\end{array}$ \\
\hline FY09EM21-07 & 955 & 1055 & 100 & $\begin{array}{c}\text { Lower: } \mathrm{SiO}_{2} \\
\text { Higher: } \mathrm{B}_{2} \mathrm{O}_{3}, \mathrm{CaO}, \mathrm{Fe}_{2} \mathrm{O}_{3}, \mathrm{MnO} \text { and } \mathrm{TiO}_{2}\end{array}$ \\
\hline FY09EM21-08 & 989 & 1096 & 107 & $\begin{array}{c}\text { Lower: } \mathrm{B}_{2} \mathrm{O}_{3} \text { and } \mathrm{NiO} \\
\text { Higher: } \mathrm{Fe}_{2} \mathrm{O}_{3}, \mathrm{Li}_{2} \mathrm{O} \text { and } \mathrm{TiO}_{2}\end{array}$ \\
\hline FY09EM21-09 & 1007 & 1075 & 68 & $\begin{array}{c}\text { Lower: } \mathrm{MgO} \text { and } \mathrm{SiO}_{2} \\
\text { Higher: } \mathrm{CaO}, \mathrm{Li}_{2} \mathrm{O} \text { and } \mathrm{TiO}_{2}\end{array}$ \\
\hline FY09EM21-11 & 733 & 858 & 125 & $\begin{array}{c}\text { Lower: } \mathrm{B}_{2} \mathrm{O}_{3} \text { and } \mathrm{NiO} \\
\text { Higher: } \mathrm{CaO}, \mathrm{MnO} \text { and } \mathrm{TiO}_{2}\end{array}$ \\
\hline FY09EM21-12 & 1010 & 1096 & 86 & $\begin{array}{c}\text { Lower: } \mathrm{CaO} \text { and } \mathrm{MgO} \\
\text { Higher: } \mathrm{Li}_{2} \mathrm{O}, \mathrm{MnO} \text { and } \mathrm{TiO}_{2}\end{array}$ \\
\hline FY09EM21-14 & 959 & 1078 & 119 & $\begin{array}{c}\text { Lower: } \mathrm{CaO}, \mathrm{MgO}, \mathrm{MnO}, \mathrm{NiO} \text { and } \mathrm{SiO}_{2} \\
\text { Higher: } \mathrm{B}_{2} \mathrm{O}_{3}, \mathrm{Fe}_{2} \mathrm{O}_{3} \text { and } \mathrm{TiO}_{2} \\
\end{array}$ \\
\hline FY09EM21-15 & 1033 & 1116 & 83 & $\begin{array}{c}\text { Lower: } \mathrm{B}_{2} \mathrm{O}_{3} \\
\text { Higher: } \mathrm{CaO}, \mathrm{MnO} \text { and } \mathrm{TiO}_{2}\end{array}$ \\
\hline FY09EM21-16 & 992 & 965 & -27 & $\begin{array}{c}\text { Lower: } \mathrm{MnO} \\
\text { Higher: } \mathrm{CaO}, \mathrm{Na}_{2} \mathrm{O} \text { and } \mathrm{TiO}_{2} \\
\end{array}$ \\
\hline FY09EM21-17 & 866 & 898 & 32 & $\begin{array}{c}\text { Lower: } \mathrm{B}_{2} \mathrm{O}_{3}, \mathrm{CaO}, \mathrm{MgO}, \mathrm{MnO}, \mathrm{NiO} \text { and } \mathrm{SiO}_{2} \\
\text { Higher: } \mathrm{Li}_{2} \mathrm{O}, \mathrm{Na}_{2} \mathrm{O} \text { and } \mathrm{TiO}_{2}\end{array}$ \\
\hline FY09EM21-18 & 763 & 829 & 66 & $\begin{array}{c}\text { Lower: } \mathrm{B}_{2} \mathrm{O}_{3}, \mathrm{MgO} \text { and } \mathrm{NiO} \\
\text { Higher: } \mathrm{Na}_{2} \mathrm{O} \text { and } \mathrm{TiO}_{2}\end{array}$ \\
\hline FY09EM21-19 & 761 & 779 & 18 & $\begin{array}{l}\text { Lower: } \mathrm{B}_{2} \mathrm{O}_{3}, \mathrm{MgO} \text { and } \mathrm{MnO} \\
\text { Higher: } \mathrm{CaO}, \mathrm{Na}_{2} \mathrm{O} \text { and } \mathrm{TiO}_{2}\end{array}$ \\
\hline FY09EM21-20 & 1020 & 1045 & 25 & $\begin{array}{l}\text { Lower: } \mathrm{CaO} \text { and } \mathrm{MnO} \\
\text { Higher: } \mathrm{Na}_{2} \mathrm{O} \text { and } \mathrm{TiO}_{2}\end{array}$ \\
\hline FY09EM21-21 & 928 & 933 & 5 & $\begin{array}{c}\text { Lower: } \mathrm{CaO}, \mathrm{MgO}, \mathrm{NiO} \text { and } \mathrm{SiO}_{2} \\
\text { Higher: } \mathrm{Fe}_{2} \mathrm{O}_{3}, \mathrm{Li}_{2} \mathrm{O} \text { and } \mathrm{TiO}_{2} \\
\end{array}$ \\
\hline FY09EM21-23 & 957 & 1145 & 188 & $\begin{array}{l}\text { Lower: } \mathrm{CaO}, \mathrm{MnO} \text { and } \mathrm{NiO} \\
\text { Higher: } \mathrm{Al}_{2} \mathrm{O}_{3} \text { and } \mathrm{TiO}_{2}\end{array}$ \\
\hline FY09EM21-25 & 761 & 858 & 97 & $\begin{array}{c}\text { Lower: } \mathrm{B}_{2} \mathrm{O}_{3}, \mathrm{MgO} \text { and } \mathrm{NiO} \\
\text { Higher: } \mathrm{CaO}, \mathrm{MnO}, \mathrm{Na}_{2} \mathrm{O} \text { and } \mathrm{TiO}_{2} \\
\end{array}$ \\
\hline FY09EM21-26 & 938 & 943 & 5 & $\begin{array}{l}\text { Lower: } \mathrm{CaO} \text { and } \mathrm{NiO} \\
\text { Higher: } \mathrm{MnO} \text { and } \mathrm{TiO}_{2}\end{array}$ \\
\hline FY09EM21-27 & 1016 & 1117 & 101 & Higher: $\mathrm{TiO}_{2}$ \\
\hline
\end{tabular}


SRNL-STI-2009-00778, REVISION 0

Table 14. Crystals Formed during $T_{L}$ Measurements

\begin{tabular}{|l|c|}
\hline Glass ID & Observations \\
\hline FY09EM21-02 & spinel \\
\hline FY09EM21-05 & iron titanate \\
\hline FY09EM21-06 & spinel and large rods \\
\hline FY09EM21-07 & spinel \\
\hline FY09EM21-08 & spinel \\
\hline FY09EM21-09 & spinel \\
\hline FY09EM21-11 & $\begin{array}{c}\text { clear cuboctahedrons at } 855^{\circ} \mathrm{C} \\
\text { spinel }<855^{\circ} \mathrm{C}\end{array}$ \\
\hline FY09EM21-12 & $\begin{array}{c}\text { clear cuboctahedrons at } 1075^{\circ} \mathrm{C} \\
\text { spinel at } 1050^{\circ} \mathrm{C}\end{array}$ \\
\hline FY09EM21-14 & iron titanate \\
\hline FY09EM21-15 & \\
\hline
\end{tabular}

\begin{tabular}{|c|c|}
\hline Glass ID & Observations \\
\hline FY09EM21-16 & spinel \\
\hline FY09EM21-17 & spinel \\
\hline FY09EM21-18 & clear cuboctahedrons \\
\hline FY09EM21-19 & $\begin{array}{c}\text { very few clear rods and clear panes } \\
\text { at } 776^{\circ} \text { C }\end{array}$ \\
\hline FY09EM21-20 & spinel \\
\hline FY09EM21-21 & spinel \\
\hline FY09EM21-23 & iron titanate \\
\hline FY09EM21-25 & spinel \\
\hline FY09EM21-26 & spinel \\
\hline FY09EM21-27 & spinel \\
\hline
\end{tabular}




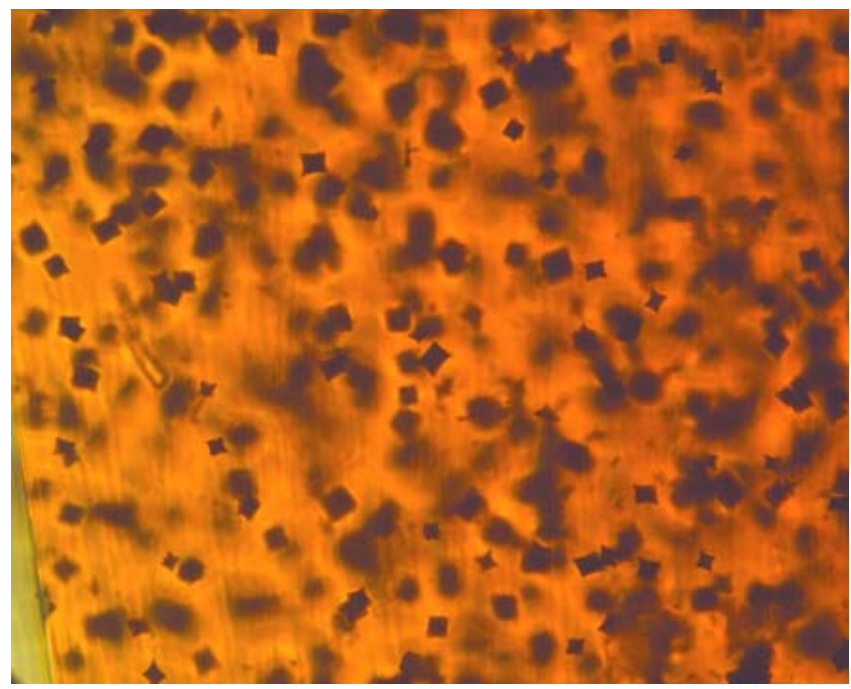

(a)

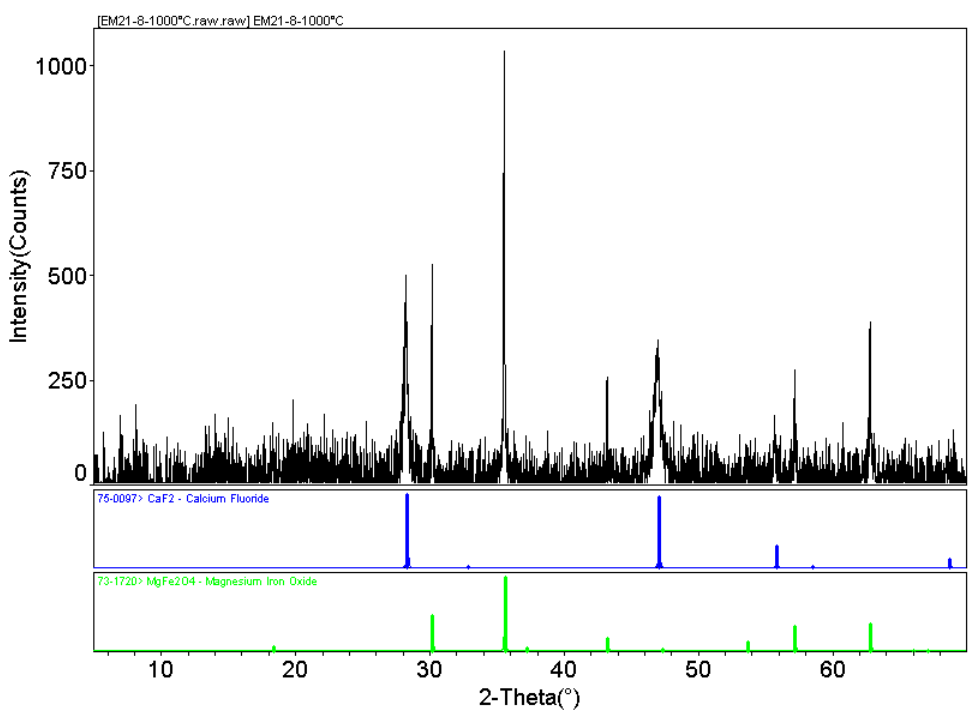

(b)

Figure 16. (a) Optical micrograph of FY09EM21-08 treated at $1000^{\circ} \mathrm{C}$ showing spinel crystals and (b) XRD pattern of FY09EM21-08 treated at $1000^{\circ} \mathrm{C}$ showing that the spinel is magnesium iron oxide.

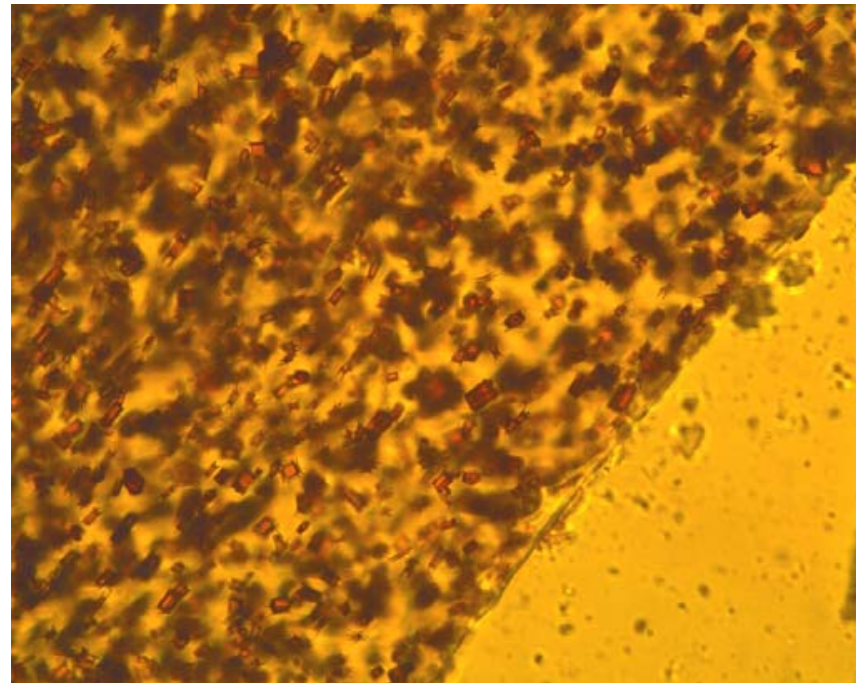

(a)

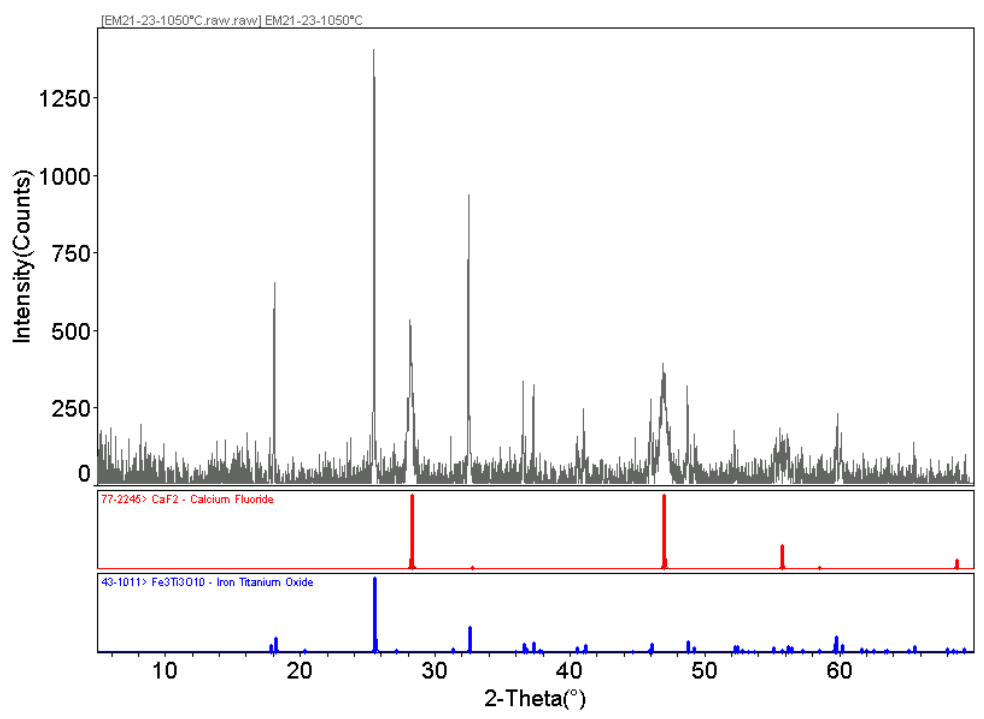

(b)

Figure 17. (a) Optical micrograph of FY09EM21-23 treated at $1050^{\circ} \mathrm{C}$ showing iron titanate crystals and (b) XRD pattern of FY09EM21-23 treated at $1050^{\circ} \mathrm{C}$ showing that the crystals are iron titanium oxide. 


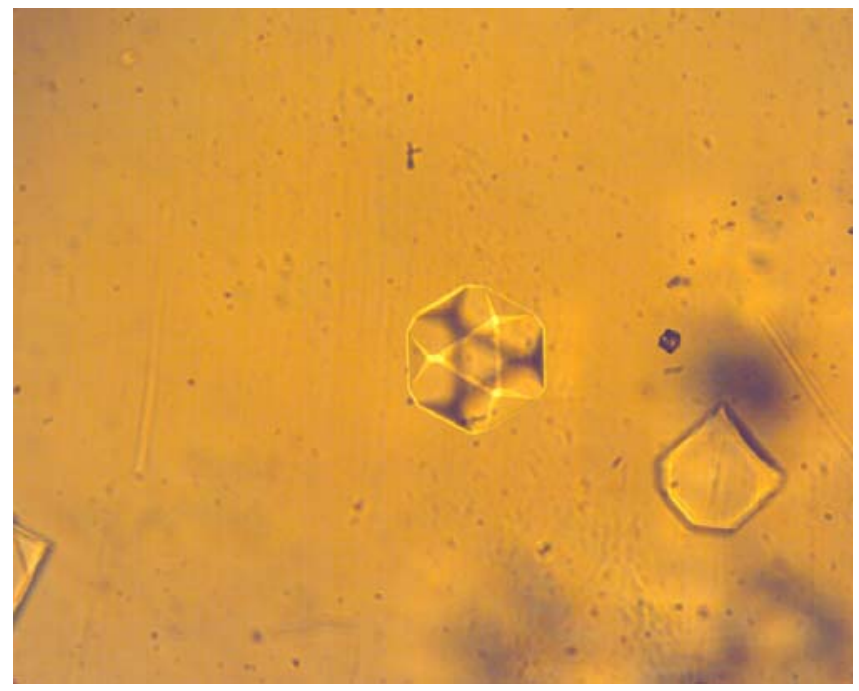

(a)

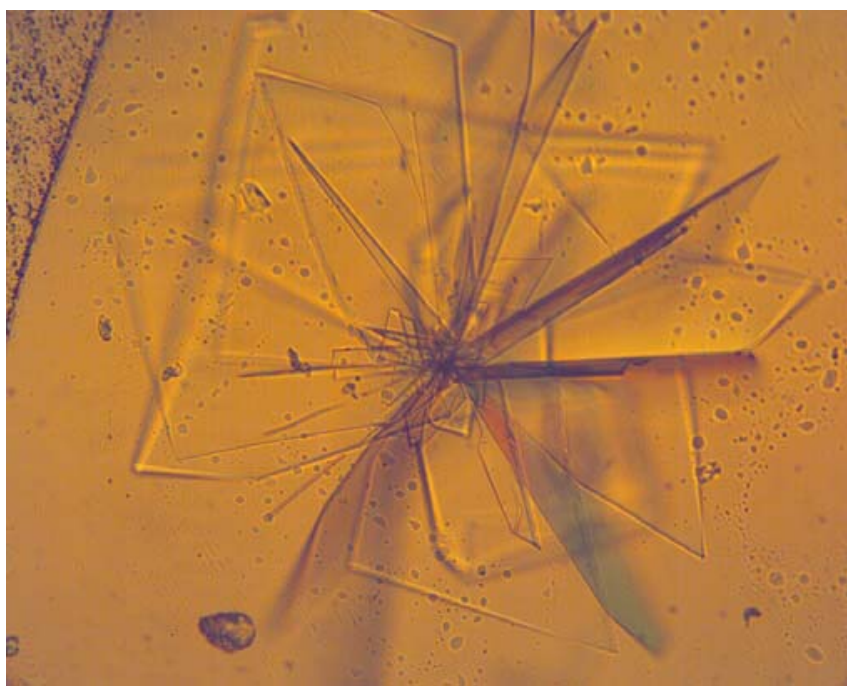

(b)

Figure 18. (a) Optical micrograph of FY09EM21-18 treated at $816^{\circ} \mathrm{C}$. These clear geometric crystals take on a shape of cuboctahedrons (6 square and 8 triangular faces). (b) Optical micrograph of FY09EM21-19 treated at $740^{\circ} \mathrm{C}$. Structure and chemical identification of both of these crystals is unknown at this time as their concentrations were below the detection limit of the XRD.

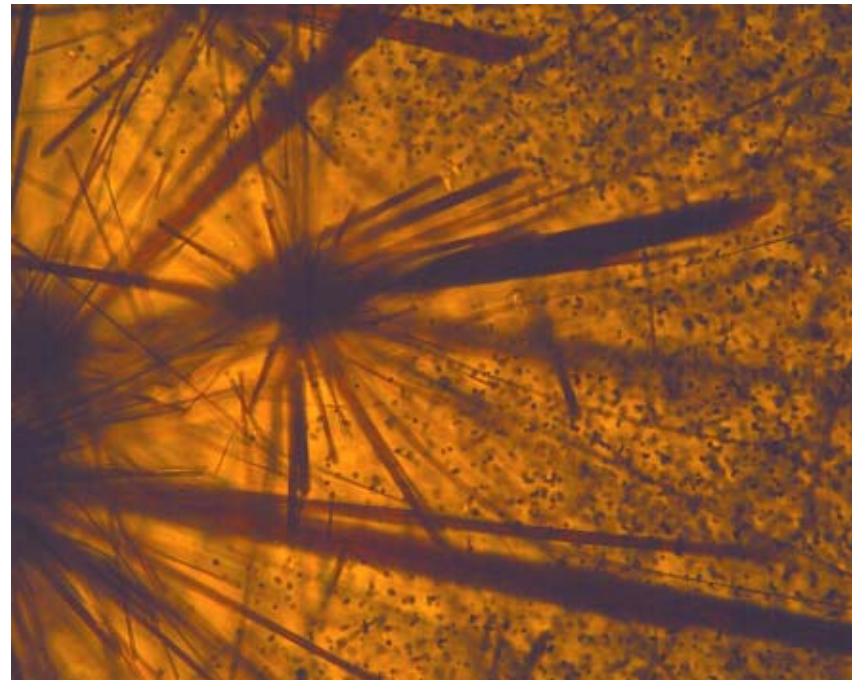

(a)

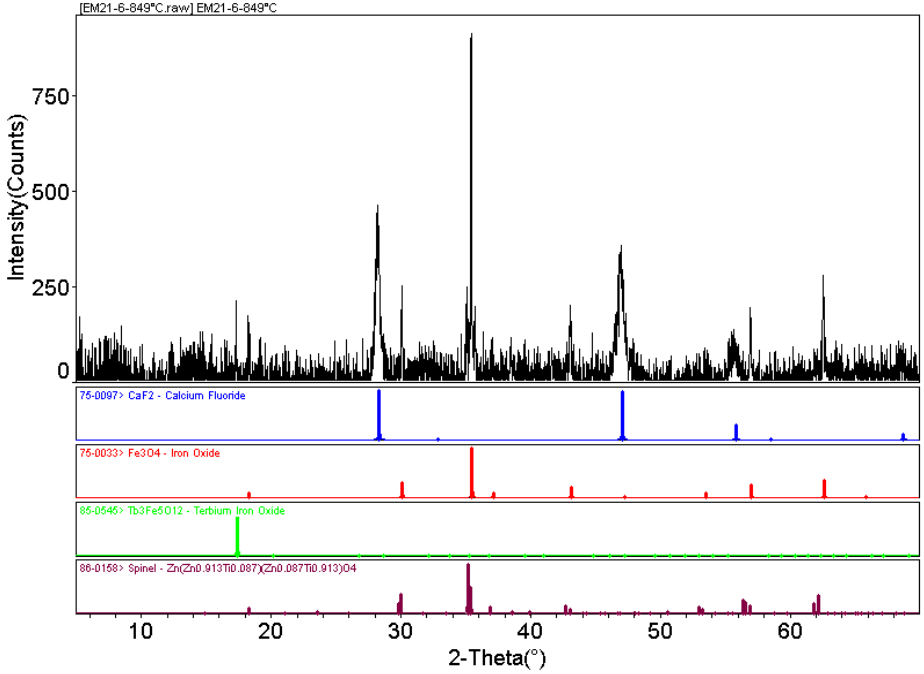

(b)

Figure 19. (a) Optical micrograph of FY09EM21-06 treated at $849^{\circ} \mathrm{C}$ showing spinel and large rods and (b) XRD pattern showing evidence of iron oxide. Note that the terbium iron oxide would not be a crystalline phase present in this glass. 


\subsubsection{Matrix 2 Viscosity Data}

Measured viscosity data were fit using the Vogel-Fulcher-Tammann (VFT) equation, given by:

$$
\ln \eta=A+\frac{B}{T-T_{0}}
$$

where $\mathrm{A}, \mathrm{B}$ and $\mathrm{T}_{0}$ are constants.

A summary of the measured viscosity data and the predicted viscosities at $1150^{\circ} \mathrm{C}$ are shown in Table 15. Note that there are not values for all of the samples, as some of the quenched glasses contained crystals and could not be measured. Plots of the fitted data are shown in Exhibits $\mathrm{C} 1$ through C18 in Appendix C. The fit of HWL-18 above $1200^{\circ} \mathrm{C}$ deviates slightly from linearity.

Table 15. Predicted and Measured Viscosity Data at $1150^{\circ} \mathrm{C}$

\begin{tabular}{|c|c|c|}
\hline Glass ID & $\begin{array}{c}\text { MAR Assessment } \\
\text { Viscosity Prediction (P) }\end{array}$ & $\begin{array}{c}\text { Measured } \\
\text { Viscosity }(\mathrm{P})\end{array}$ \\
\hline FY09EM21-02 & 5 & 12 \\
\hline FY09EM21-05 & 72 & 44 \\
\hline FY09EM21-06 & 20 & 11 \\
\hline FY09EM21-07 & 2 & 5 \\
\hline FY09EM21-08 & 9 & 10 \\
\hline FY09EM21-09 & 24 & 16 \\
\hline FY09EM21-11 & 38 & 18 \\
\hline FY09EM21-12 & 21 & 17 \\
\hline FY09EM21-14 & 6 & 15 \\
\hline FY09EM21-15 & 58 & 23 \\
\hline FY09EM21-16 & 29 & 22 \\
\hline FY09EM21-17 & 6 & 10 \\
\hline FY09EM21-18 & 49 & 53 \\
\hline FY09EM21-19 & 53 & 28 \\
\hline FY09EM21-20 & 25 & 28 \\
\hline FY09EM21-21 & 2 & 6 \\
\hline FY09EM21-25 & 47 & 28 \\
\hline FY09EM21-27 & 21 & 17 \\
\hline
\end{tabular}

Some of the measured viscosities are quite low, which include FY09EM21-02, -07, -08, -14, -17 and -21, and would be below DWPF's current lower viscosity limit. All of these glasses, except FY09EM21-08, are outside of the compositional region over which the model was developed. A calculation of the non-bridging oxygen (NBO) ratio indicates that these glasses have considerably more NBOs than the glasses used to develop the current viscosity model. This ratio is given by, 


$$
\mathrm{NBO}=\frac{2\left(\mathrm{Fe}_{2} \mathrm{O}_{3}(\text { molar })-\mathrm{Al}_{2} \mathrm{O}_{3}(\text { molar })+\mathrm{M}_{2} \mathrm{O}(\text { molar })\right)+\mathrm{B}_{2} \mathrm{O}_{3}(\text { molar })}{\mathrm{SiO}_{2}(\text { molar })}
$$

where $\mathrm{M}=$ alkali cations ( $\mathrm{Li}, \mathrm{Na}, \mathrm{K}$ and $\mathrm{Cs}$ ). A higher concentration of NBOs would account for the low viscosity as the presence of NBOs break up the linkages between network polyhedra. Higher concentrations of $\mathrm{Fe}_{2} \mathrm{O}_{3}$ are the cause of the increased number of NBOs in these glasses.

\subsubsection{A Statistical Review of the Chemical Composition Measurements for the Radioactive Glasses}

Table A5 in Appendix A provides a listing of the measurements, the measurements biascorrected using the standards included as part of the analytical plan, and the target concentrations for the samples of the Matrix 2 rad glasses. Exhibit A5 provides a plot of the measured values for these samples for each oxide. As seen in these plots, there is an indication of a difference in the measurements for the LM oxides between the two preparations for the FY09EM21-28 glass. However, all of the measurements were utilized in determining the chemical compositions for these radioactive glasses. These determinations were conducted both for the measured and for the bias-corrected data. A sum of oxides was also computed for each glass based upon both the measured and bias-corrected values. Exhibit A6 in Appendix A provides plots showing the results for each glass for each oxide to help highlight the comparisons among the measured, biascorrected, and target values.

Table A6 in Appendix A provides a summary of the average compositions as well as the target compositions and some associated differences and relative differences for these radioactive glasses. All of the sums of oxides (both measured and bias-corrected) fall within the interval of 95 to $105 \mathrm{wt} \%$. Entries in Table A6 show the relative differences between the measured or biascorrected values and the target values. These differences are shaded when they are greater than or equal to $5 \%$. Overall, the measured compositions are consistent with the target compositions and will serve the objectives of this study.

In Table 16, the MAR assessments of these radioactive glasses are presented in the same format as was used in Section 5.1.2 for the non-radioactive glasses. As in the non-radioactive glasses, these glasses also fail the $\mathrm{TiO}_{2}$ constraint in order to determine its influence on glass properties.

Table 16. MAR Assessment Results of the Radioactive Matrix 2 Glasses

\begin{tabular}{|c|c|c|c|c|c|c|c|c|c|c|c|}
\hline Glass ID & $\begin{array}{c}\text { Compositional } \\
\text { View }\end{array}$ & B Del $G_{p}$ & $\begin{array}{c}\text { NL } \\
{[B(g / L)]}\end{array}$ & $\mathbf{T}_{\mathrm{L}}\left({ }^{\circ} \mathrm{C}\right)$ & $\begin{array}{c}\text { Viscosity } \\
\text { (P) }\end{array}$ & $\begin{array}{l}\mathrm{Al}_{2} \mathrm{O}_{3} \\
(\mathrm{wt} \%)\end{array}$ & $\begin{array}{c}\mathrm{TiO}_{2} \\
(\mathrm{wt} \%)\end{array}$ & $\begin{array}{c}\mathbf{R}_{2} \mathrm{O} \\
(\mathbf{w t} \%)\end{array}$ & $\begin{array}{l}\text { Homogeneity } \\
\text { (wt\%) }\end{array}$ & $\begin{array}{c}\text { Nepheline } \\
\text { Value }\end{array}$ & MAR Status \\
\hline \multirow{3}{*}{ FY09EM21-28 } & Measured & -9.45 & 0.65 & 1015 & 19 & 7.17 & 3.40 & 16.92 & 224.5 & 0.68 & newlv $\mathrm{TiO}_{2}$ \\
\hline & Measured bc & -9.54 & 0.67 & 1016 & 18 & 7.42 & 3.60 & 17.17 & 229.9 & 0.68 & newlv $\mathrm{TiO}_{2}$ \\
\hline & Target & -9.79 & 0.75 & 1016 & 20 & 7.05 & 3.85 & 17.46 & 231.0 & 0.69 & newlv $\mathrm{TiO}_{2}$ \\
\hline \multirow{3}{*}{ FY09EM21-29 } & Measured & -9.68 & 0.71 & 1005 & 20 & 7.24 & 3.57 & 17.27 & 226.6 & 0.68 & newlv $\mathrm{TiO}_{2}$ \\
\hline & Measured bc & -9.75 & 0.73 & 1001 & 20 & 7.26 & 3.77 & 17.35 & 228.2 & 0.68 & newlv $\mathrm{TiO}_{2}$ \\
\hline & Target & -9.64 & 0.70 & 1016 & 20 & 6.91 & 3.77 & 17.11 & 226.2 & 0.69 & newlv $\mathrm{TiO}_{2}$ \\
\hline \multirow{3}{*}{ FY09EM21-30 } & Measured & -9.15 & 0.57 & 1017 & 18 & 6.93 & 3.61 & 16.34 & 219.2 & 0.68 & newlv $\mathrm{TiO}_{2}$ \\
\hline & Measured bc & -9.26 & 0.60 & 1018 & 18 & 7.18 & 3.72 & 16.63 & 225.3 & 0.68 & newlv $\mathrm{TiO}_{2}$ \\
\hline & Target & -9.50 & 0.66 & 1016 & 20 & 6.76 & 3.69 & 16.75 & 221.5 & 0.69 & newlv $\mathrm{TiO}_{2}$ \\
\hline
\end{tabular}




\subsubsection{A Statistical Review of the PCT Results of the Matrix 2 Radioactive Glasses}

Table B2 in Appendix B provides the measurements in ppm resulting from the PCTs. Exhibit B11 in Appendix B provides a series of plots and statistical comparisons that demonstrate the effects of heat treatment on the common logarithm ppm-responses of interest of the triplicate PCTs for each element for each radioactive glass. The quenched version of a given glass yielded measurements indicating a significantly different mean $\log (\mathrm{ppm})$ response (at the 5\% significance level) than the ccc version of the glass for a given element if the Prob $>|\mathbf{t}|$ value in the exhibit is 0.05 or smaller. Table 17 summarizes the comparisons between the quenched and ccc versions of the radioactive glasses for the four elements of the PCTs. The less durable heat treatment is noted.

Table 17. Samples Exhibiting a Statistically Significant Difference Between the Quenched and ccc Versions of the Radioactive Matrix 2 Glasses

\begin{tabular}{|c|c|c|c|c|}
\hline Glass ID & B & Li & Na & Si \\
\hline FY09EM21-28 & Q & Q & Q & Q \\
\hline FY09EM21-29 & Q & Q & Q & Q \\
\hline FY09EM21-30 & Q & Q & Q & Q \\
\hline
\end{tabular}

The PCT responses for the radioactive glasses are provided in Table 18. The PCT results were normalized following the approach outlined in Section 5.1.4.4. The resulting values are provided in Table 18. Differences in PCT responses due to heat treatment of the glass are shown in Exhibit B12 in Appendix B. The NL [B] values range from $1.03 \mathrm{~g} / \mathrm{L}$ (FY09EM21-28) to 1.17 $\mathrm{g} / \mathrm{L}$ (FY09EM21-29) for the quenched glasses and $0.75 \mathrm{~g} / \mathrm{L}$ (FY09EM21-28) to $0.83 \mathrm{~g} / \mathrm{L}$ (FY09EM21-29, -30) for the ccc glasses. While the NL [B] values of the quenched glasses are slightly higher than the ccc values, all of the release rates are well below the EA glass.

The predictability of the durabilities of the radioactive glasses is addressed in plots presented in Exhibit B13 of Appendix B. All of these glasses are acceptable and predictable with respect to the DWPF models for durability. 
Table 18. Normalized PCT Results of the Radioactive Matrix 2 Glasses

\begin{tabular}{|c|c|c|c|c|c|c|c|c|c|c|}
\hline Glass ID & $\begin{array}{c}\text { Heat } \\
\text { Treatment }\end{array}$ & $\begin{array}{l}\text { Comp } \\
\text { View }\end{array}$ & $\begin{array}{c}\log \mathrm{NL} \\
{[\mathrm{B}(\mathrm{g} / \mathrm{L})]}\end{array}$ & \begin{tabular}{|c|}
$\log \mathrm{NL}$ \\
{$[\mathrm{Li}(\mathrm{g} / \mathrm{L})]$}
\end{tabular} & \begin{tabular}{|c|}
$\log \mathrm{NL}$ \\
{$[\mathrm{Na}(\mathrm{g} / \mathrm{L})]$}
\end{tabular} & $\begin{array}{c}\log \mathrm{NL} \\
{[\mathrm{Si}(\mathrm{g} / \mathrm{L})]}\end{array}$ & $\begin{array}{c}\text { NL } \\
\text { B (g/L) }\end{array}$ & $\begin{array}{c}\mathrm{NL} \\
\mathrm{Li}(\mathrm{g} / \mathrm{L})\end{array}$ & $\begin{array}{c}\mathrm{NL} \\
\mathrm{Na}(\mathrm{g} / \mathrm{L})\end{array}$ & $\begin{array}{c}\mathrm{NL} \\
\mathrm{Si}(\mathrm{g} / \mathrm{L})\end{array}$ \\
\hline \multirow{6}{*}{ FY09EM21-28 } & \multirow{3}{*}{ quenched } & Measured & 0.04 & 0.05 & 0.03 & -0.20 & 1.09 & 1.12 & 1.08 & 0.63 \\
\hline & & Measured bc & 0.01 & 0.03 & 0.03 & -0.21 & 1.03 & 1.06 & 1.08 & 0.62 \\
\hline & & Target & 0.03 & 0.03 & 0.02 & -0.23 & 1.07 & 1.07 & 1.05 & 0.60 \\
\hline & \multirow{3}{*}{$\mathrm{ccc}$} & Measured & -0.10 & -0.02 & -0.04 & -0.23 & 0.80 & 0.96 & 0.91 & 0.59 \\
\hline & & Measured bc & -0.13 & -0.05 & -0.04 & -0.23 & 0.75 & 0.90 & 0.91 & 0.59 \\
\hline & & Target & -0.11 & -0.04 & -0.05 & -0.25 & 0.78 & 0.91 & 0.88 & 0.56 \\
\hline \multirow{6}{*}{ FY09EM21-29 } & \multirow{3}{*}{ quenched } & Measured & 0.07 & 0.06 & 0.04 & -0.19 & 1.17 & 1.15 & 1.09 & 0.65 \\
\hline & & Measured bc & 0.04 & 0.05 & 0.04 & -0.20 & 1.10 & 1.12 & 1.09 & 0.64 \\
\hline & & Target & 0.06 & 0.06 & 0.04 & -0.19 & 1.14 & 1.14 & 1.10 & 0.64 \\
\hline & \multirow{3}{*}{$\mathrm{ccc}$} & Measured & -0.08 & -0.04 & -0.05 & -0.23 & 0.83 & 0.91 & 0.89 & 0.59 \\
\hline & & Measured bc & -0.11 & -0.05 & -0.05 & -0.24 & 0.78 & 0.89 & 0.89 & 0.58 \\
\hline & & Target & -0.09 & -0.04 & -0.05 & -0.24 & 0.81 & 0.91 & 0.90 & 0.58 \\
\hline \multirow{6}{*}{ FY09EM21-30 } & \multirow{3}{*}{ quenched } & Measured & 0.05 & 0.06 & 0.03 & -0.19 & 1.12 & 1.15 & 1.08 & 0.64 \\
\hline & & Measured bc & 0.02 & 0.04 & 0.03 & -0.20 & 1.06 & 1.09 & 1.08 & 0.63 \\
\hline & & Target & 0.05 & 0.05 & 0.02 & -0.21 & 1.11 & 1.11 & 1.05 & 0.61 \\
\hline & \multirow{3}{*}{$\mathrm{ccc}$} & Measured & -0.08 & 0.00 & -0.04 & -0.20 & 0.83 & 1.00 & 0.91 & 0.62 \\
\hline & & Measured bc & -0.11 & -0.03 & -0.04 & -0.22 & 0.78 & 0.94 & 0.91 & 0.61 \\
\hline & & Target & -0.08 & -0.02 & -0.05 & -0.22 & 0.83 & 0.96 & 0.88 & 0.60 \\
\hline
\end{tabular}

\subsection{TEST MATRIX 2A}

\subsubsection{A Statistical Review of the Matrix 2A Chemical Composition Measurements}

Table D1 (in two parts) in Appendix D provides the elemental concentration measurements from the study glasses that were prepared using LM, and Table D2 provides the measurements from the samples of these glasses prepared using PF. Measurements for samples of the standard Batch 1 glass that were included in the analytical plan along with the study glasses are also provided in these two tables.

\subsubsection{Measurements in Analytical Sequence}

Exhibit D1 in Appendix D provides plots in analytical sequence of the sample measurements generated by PSAL for each oxide over both preparation methods (i.e., LM and PF). These plots include all of the measurement data from Tables D1 and D2. Some scatter is observed in the Batch 1 values for $\mathrm{Li}$ and $\mathrm{Si}$ and there is also quite a bit of scatter in the $\mathrm{Cr}$ measurements; however, its concentration is quite low. There do not appear to be any obvious patterns or trends due to the analytical sequence.

\subsubsection{Composition Measurements by Glass Identifier}

Exhibit D2 in Appendix D provides plots of the oxide concentration measurements by Glass ID (including the Batch 1 standard) by Lab ID. These plots demonstrate the individual measurements across the duplicates of each preparation method and the two ICP calibrations for each glass for each oxide. A review of the plots presented in these exhibits reveals the repeatability of the four individual values for each oxide for each glass. In general, there appears to be good repeatability among the measurements for each of the oxides for most of the glasses; 
however, there are some inconsistencies, which include: (i) in Set \#1 there appears to be preparation effects for EM-14 affecting the CaO measurements, and for EM-12 affecting the $\mathrm{Fe}_{2} \mathrm{O}_{3}$ and $\mathrm{NiO}$ measurements, and there appear to be calibration effects for several glasses affecting the $\mathrm{Li}_{2} \mathrm{O}$ and $\mathrm{SiO}_{2}$ measurements, (ii) in Set \#2 there appears to be a great deal of scatter in the $\mathrm{B}_{2} \mathrm{O}_{3}$ measurements ${ }^{\mathrm{T}}$ and there appears to be preparation effects for EM-19 affecting the $\mathrm{Li}_{2} \mathrm{O}$ and $\mathrm{B}_{2} \mathrm{O}_{3}$ measurements, and for EM-22 and EM-28 affecting the NiO measurements for these glasses. None of the observations discussed here suggest a significant issue in the batching of the study glasses or in the analytical process used to provide representative measurements of their compositions.

\subsubsection{Results for the Batch 1 Standard}

Exhibit D3 in Appendix D provides statistical analyses of the Batch 1 results by analytical set and calibration block for each oxide of interest over both preparation methods. The results also include ANOVA investigations. The following components of the Batch 1 standard indicate a significant ICP-AES calibration effect on the block averages at the 5\% significance level:

$$
\text { - } \mathrm{Al}_{2} \mathrm{O}_{3}, \mathrm{BaO}, \mathrm{CaO}, \mathrm{CuO}, \mathrm{Fe}_{2} \mathrm{O}_{3}, \mathrm{Li}_{2} \mathrm{O}, \mathrm{MgO}, \mathrm{MnO}, \mathrm{SiO}_{2}, \mathrm{TiO}_{2} \text {, and } \mathrm{ZrO}_{2}
$$

As a result, bias correction of the measured data was pursued to evaluate if the compositional view provided by the bias-corrected measurements has an impact of the conclusions of this study. The method for bias correction is discussed in Section 5.1.1.3.

\subsubsection{Measured versus Target Compositions}

With only minor problems in the repeatability of the measurements being revealed during the review process, all of the measurements for each oxide for each glass (i.e., all of the measurements in Tables D1 through D2) were averaged to determine a representative chemical composition for each glass. These determinations were conducted both for the measured and for the bias-corrected data. A sum of oxides was also computed for each glass based upon both the measured and bias-corrected values. Exhibit D4 in Appendix D provides plots showing results for each glass for each oxide to help highlight the comparisons among the measured, biascorrected, and target values. Some observations from the plots of Exhibit D4 for the major oxides (those oxides at concentrations greater than $0.5 \mathrm{wt} \%$ ) are offered: In general, there appear to have been few difficulties in hitting the target concentrations for the study glasses. The average of the $\mathrm{B}_{2} \mathrm{O}_{3}$ measurements for EM-19 was higher than the target concentration. A preparation issue was noted earlier for this glass. For all of the study glasses, the measured values for $\mathrm{Fe}_{2} \mathrm{O}_{3}, \mathrm{Cr}_{2} \mathrm{O}_{3}$ and for $\mathrm{NiO}$ were below the target concentrations.

Table D3 in Appendix D provides a summary of the average compositions as well as the target compositions and some associated differences and relative differences. Notice that the target sums of oxides for the standard glasses do not sum to $100 \%$ due to an incomplete coverage of the oxides in the Batch 1 glass. In fact, the only glass whose average measured value for sum of oxides fell below 95\% was the Batch 1 glass for both Set \#1 and for Set \#2. All of the sums of

\footnotetext{
${ }^{\mathrm{T}}$ Note the small scale.
} 
oxides (both measured and bias-corrected) for the Matrix 2A glasses fall within the interval of 95 to $105 \mathrm{wt} \%$. Entries in Table D3 show the relative differences between the measured or biascorrected values and the target values. These differences are shaded when they are greater than or equal to $5 \%$. Overall, these comparisons between the measured and target compositions suggest only minor difficulties in hitting the target compositions for the study glasses with the exceptions/potential issues already noted.

\subsubsection{Matrix 2A MAR Assessment Results}

MAR assessment results are provided in Table 19. In general, the results of the measured compositions are consistent with those of the target compositions. As in Matrix 2, the $\mathrm{TiO}_{2}$ constraint was intentionally failed for all of the glasses to determine model applicability. 
SRNL-STI-2009-00778, REVISION 0

Table 19. MAR Assessment Results of the Matrix 2A Glasses

\begin{tabular}{|c|c|c|c|c|c|c|c|c|c|c|c|}
\hline Glass ID & \begin{tabular}{|c|} 
Compositional \\
View
\end{tabular} & B Del $G_{p}$ & $\begin{array}{c}\text { NL } \\
{[\mathrm{B}(\mathrm{g} / \mathrm{L})]}\end{array}$ & $\mathrm{T}_{\mathrm{L}}\left({ }^{\circ} \mathrm{C}\right)$ & $\begin{array}{c}\text { Viscosity } \\
\text { (P) }\end{array}$ & $\begin{array}{l}\mathrm{Al}_{2} \mathrm{O}_{3} \\
(\mathrm{wt} \%)\end{array}$ & $\begin{array}{c}\mathrm{TiO}_{2} \\
(\mathrm{wt} \%)\end{array}$ & $\begin{array}{c}\mathbf{R}_{2} \mathrm{O} \\
(w t \%)\end{array}$ & $\begin{array}{c}\text { Homogeneity } \\
(w t \%)\end{array}$ & $\begin{array}{c}\text { Nepheline } \\
\text { Value }\end{array}$ & MAR Status \\
\hline \multirow{3}{*}{ EM-01 } & Measured & -9.80 & 0.75 & 1025 & 27 & 11.49 & 2.07 & 19.45 & 257.8 & 0.60 & $\mathrm{~T}_{\mathrm{L}} \mathrm{TiO}_{2} \mathrm{Neph}$ \\
\hline & Measured bc & -9.21 & 0.58 & 1042 & 38 & 11.81 & 2.16 & 19.12 & 264.1 & 0.62 & $\mathrm{~T}_{\mathrm{L}} \mathrm{TiO}_{2} \mathrm{Neph}$ \\
\hline & Target & -9.75 & 0.74 & 1057 & 24 & 11.59 & 2.00 & 19.48 & 261.6 & 0.60 & $\mathrm{~T}_{\mathrm{L}}$ newlv $\mathrm{TiO}_{2} \mathrm{Neph}$ \\
\hline \multirow{3}{*}{ EM-02 } & Measured & -7.28 & 0.26 & 1074 & 48 & 14.14 & 2.06 & 17.55 & 267.6 & 0.59 & $\mathrm{~T}_{\mathrm{L}} \mathrm{TiO}_{2} \mathrm{Neph}$ \\
\hline & Measured bc & -6.68 & 0.20 & 1089 & 65 & 14.54 & 2.15 & 17.26 & 274.3 & 0.60 & $\mathrm{~T}_{\mathrm{L}} \mathrm{TiO}_{2} \mathrm{Neph}$ \\
\hline & Target & -7.26 & 0.26 & 1101 & 43 & 14.09 & 2.00 & 17.60 & 271.7 & 0.59 & $\mathrm{~T}_{\mathrm{L}} \mathrm{TiO}_{2} \mathrm{Neph}$ \\
\hline \multirow{3}{*}{ EM-03 } & Measured & -12.24 & 2.07 & 976 & 14 & 9.09 & 2.00 & 21.17 & 246.0 & 0.61 & newlv $\mathrm{TiO}_{2} \mathrm{Neph}$ \\
\hline & Measured bc & -11.71 & 1.66 & 997 & 20 & 9.37 & 2.06 & 20.90 & 252.2 & 0.62 & newlv $\mathrm{TiO}_{2} \mathrm{Neph}$ \\
\hline & Target & -12.39 & 2.20 & 1000 & 13 & 9.09 & 2.00 & 21.51 & 251.5 & 0.61 & newlv $\mathrm{TiO}_{2} \mathrm{Neph}$ \\
\hline \multirow{3}{*}{ EM-04 } & Measured & -13.23 & 3.14 & 939 & 13 & 6.58 & 1.98 & 21.40 & 235.0 & 0.64 & newlv $\mathrm{TiO}_{2}$ \\
\hline & Measured bc & -12.77 & 2.59 & 959 & 18 & 6.78 & 2.04 & 21.21 & 242.1 & 0.65 & newlv $\mathrm{TiO}_{2}$ \\
\hline & Target & -13.53 & 3.55 & 956 & 12 & 6.59 & 2.00 & 21.93 & 241.4 & 0.64 & Del Gp newlv $\mathrm{TiO}_{2}$ \\
\hline \multirow{3}{*}{ EM-05 } & Measured & -11.80 & 1.72 & 991 & 14 & 6.59 & 1.99 & 19.91 & 243.8 & 0.65 & newlv $\mathrm{TiO}_{2}$ \\
\hline & Measured bc & -11.35 & 1.43 & 1012 & 19 & 6.79 & 2.05 & 19.73 & 251.1 & 0.66 & newlv $\mathrm{TiO}_{2}$ \\
\hline & Target & -11.94 & 1.83 & 1018 & 12 & 6.59 & 2.00 & 20.27 & 251.5 & 0.65 & $\mathrm{~T}_{\mathrm{L}}$ newlv $\mathrm{TiO}_{2}$ \\
\hline \multirow{3}{*}{ EM-06 } & Measured & -9.87 & 0.77 & 1053 & 17 & 6.71 & 2.03 & 17.93 & 251.9 & 0.67 & $\mathrm{~T}_{\mathrm{L}}$ newlv $\mathrm{TiO}_{2}$ \\
\hline & Measured bc & -9.38 & 0.63 & 1071 & 24 & 6.90 & 2.11 & 17.70 & 259.2 & 0.68 & $\mathrm{~T}_{\mathrm{L}}$ newly $\mathrm{TiO}_{2}$ \\
\hline & Target & -9.57 & 0.68 & 1094 & 16 & 6.59 & 2.00 & 17.77 & 261.6 & 0.67 & $\mathrm{~T}_{\mathrm{L}}$ newly $\mathrm{TiO}_{2}$ \\
\hline \multirow{3}{*}{ EM-07 } & Measured & -10.23 & 0.90 & 1049 & 16 & 6.67 & 1.91 & 17.56 & 258.9 & 0.67 & $\mathrm{~T}_{\mathrm{L}}$ newlv \\
\hline & Measured bc & -9.81 & 0.75 & 1068 & 22 & 6.87 & 1.97 & 17.41 & 266.5 & 0.68 & $\mathrm{~T}_{\mathrm{L}}$ newlv $\mathrm{TiO}_{2}$ \\
\hline & Target & -10.04 & 0.83 & 1100 & 13 & 6.59 & 2.00 & 17.60 & 269.7 & 0.67 & $\mathrm{~T}_{\mathrm{L}}$ newlv $\mathrm{TiO}_{2}$ \\
\hline \multirow{3}{*}{ EM-08 } & Measured & -10.64 & 1.07 & 1049 & 12 & 6.64 & 2.25 & 17.77 & 265.0 & 0.66 & $\mathrm{~T}_{\mathrm{L}}$ newlv $\mathrm{TiO}_{2}$ \\
\hline & Measured bc & -10.18 & 0.88 & 1066 & 18 & 6.83 & 2.35 & 17.54 & 272.3 & 0.67 & $\mathrm{~T}_{\mathrm{L}}$ newly $\mathrm{TiO}_{2}$ \\
\hline & Target & -10.35 & 0.94 & 1102 & 12 & 6.59 & 2.00 & 17.60 & 273.8 & 0.66 & $\mathrm{~T}_{\mathrm{L}}$ newly $\mathrm{TiO}_{2}$ \\
\hline \multirow{3}{*}{ EM-09 } & Measured & -8.22 & 0.39 & 1076 & 28 & 11.67 & 2.08 & 17.74 & 263.4 & 0.61 & $\mathrm{~T}_{\mathrm{L}} \mathrm{TiO}_{2} \mathrm{Neph}$ \\
\hline & Measured bc & -7.69 & 0.31 & 1090 & 39 & 12.00 & 2.17 & 17.52 & 271.0 & 0.62 & $\mathrm{~T}_{\mathrm{L}} \mathrm{TiO}_{2} \mathrm{Neph}$ \\
\hline & Target & -7.96 & 0.35 & 1119 & 27 & 11.59 & 2.00 & 17.60 & 271.7 & 0.61 & $\mathrm{~T}_{\mathrm{L}} \mathrm{TiO}_{2} \mathrm{Neph}$ \\
\hline \multirow{3}{*}{ EM-10 } & Measured & -11.55 & 1.55 & 967 & 20 & 11.51 & 2.07 & 21.16 & 245.5 & 0.58 & newlv $\mathrm{TiO}_{2} \mathrm{Neph}$ \\
\hline & Measured bc & -10.99 & 1.23 & 983 & 29 & 11.83 & 2.16 & 20.89 & 252.5 & 0.59 & $\mathrm{TiO}_{2} \mathrm{Neph}$ \\
\hline & Target & -11.69 & 1.65 & 991 & 21 & 11.59 & 2.00 & 21.51 & 251.5 & 0.58 & newlv $\mathrm{TiO}_{2} \mathrm{Neph}$ \\
\hline
\end{tabular}


SRNL-STI-2009-00778, REVISION 0

Table 19 continued.

\begin{tabular}{|c|c|c|c|c|c|c|c|c|c|c|c|}
\hline Glass ID & $\begin{array}{c}\text { Compositional } \\
\text { View }\end{array}$ & B Del $G_{p}$ & \begin{tabular}{|c|}
$\mathbf{N L}$ \\
{$[\mathrm{B}(\mathrm{g} / \mathrm{L})]$}
\end{tabular} & $\mathbf{T}_{\mathbf{L}}\left({ }^{\circ} \mathrm{C}\right)$ & $\begin{array}{c}\text { Viscosity } \\
(\mathrm{P})\end{array}$ & $\begin{array}{l}\mathrm{Al}_{2} \mathrm{O}_{3} \\
(\mathrm{wt} \%)\end{array}$ & $\begin{array}{c}\mathrm{TiO}_{2} \\
(\mathrm{wt} \%)\end{array}$ & $\begin{array}{c}\mathrm{R}_{2} \mathrm{O} \\
(\mathrm{wt} \%)\end{array}$ & $\begin{array}{c}\text { Homogeneity } \\
(w t \%)\end{array}$ & $\begin{array}{c}\text { Nepheline } \\
\text { Value }\end{array}$ & MAR Status \\
\hline \multirow{3}{*}{ EM-11 } & Measured & -8.39 & 0.42 & 1047 & 45 & 11.49 & 1.97 & 17.39 & 252.4 & 0.63 & $\mathrm{~T}_{\mathrm{L}} \mathrm{TiO}_{2} \mathrm{Neph}$ \\
\hline & Measured bc & -7.86 & 0.33 & 1066 & 60 & 11.84 & 2.03 & 17.16 & 259.2 & 0.64 & $\mathrm{~T}_{\mathrm{L}} \mathrm{TiO}_{2}$ \\
\hline & Target & -8.43 & 0.42 & 1091 & 37 & 11.59 & 2.00 & 17.60 & 260.0 & 0.62 & $\mathrm{~T}_{\mathrm{L}} \mathrm{TiO}_{2} \mathrm{Neph}$ \\
\hline \multirow{3}{*}{ EM-12 } & Measured & -11.34 & 1.42 & 984 & 15 & 11.49 & 2.02 & 21.49 & 257.0 & 0.58 & newlv $\mathrm{TiO}_{2} \mathrm{Neph}$ \\
\hline & Measured bc & -10.72 & 1.10 & 1000 & 22 & 11.81 & 2.10 & 21.13 & 263.1 & 0.59 & newlv $\mathrm{TiO}_{2} \mathrm{Neph}$ \\
\hline & Target & -11.22 & 1.35 & 1019 & 15 & 11.59 & 2.00 & 21.51 & 263.2 & 0.58 & $\mathrm{~T}_{\mathrm{L}}$ newlv $\mathrm{TiO}_{2} \mathrm{Neph}$ \\
\hline \multirow{3}{*}{ EM-13 } & Measured & -8.75 & 0.48 & 1014 & 46 & 14.30 & 1.93 & 18.73 & 262.6 & 0.58 & $\mathrm{~T}_{\mathrm{L}} \mathrm{TiO}_{2} \mathrm{Neph}$ \\
\hline & Measured bc & -8.19 & 0.38 & 1031 & 62 & 14.73 & 1.99 & 18.48 & 269.5 & 0.59 & $\mathrm{~T}_{\mathrm{L}} \mathrm{TiO}_{2} \mathrm{Neph}$ \\
\hline & Target & -8.51 & 0.44 & 1061 & 42 & 14.09 & 2.00 & 18.56 & 267.7 & 0.58 & $\mathrm{~T}_{\mathrm{L}} \mathrm{TiO}_{2} \mathrm{Neph}$ \\
\hline \multirow{3}{*}{ EM-14 } & Measured & -8.53 & 0.44 & 1026 & 47 & 14.25 & 2.01 & 18.06 & 268.5 & 0.58 & $\mathrm{~T}_{\mathrm{L}} \mathrm{TiO}_{2} \mathrm{Neph}$ \\
\hline & Measured bc & -7.99 & 0.35 & 1043 & 64 & 14.69 & 2.08 & 17.83 & 275.3 & 0.59 & $\mathrm{~T}_{\mathrm{L}} \mathrm{TiO}_{2} \mathrm{Neph}$ \\
\hline & Target & -8.36 & 0.41 & 1071 & 43 & 14.09 & 2.00 & 18.06 & 271.7 & 0.58 & $\mathrm{~T}_{\mathrm{L}} \mathrm{TiO}_{2} \mathrm{Neph}$ \\
\hline \multirow{3}{*}{ EM-15 } & Measured & -8.45 & 0.43 & 1025 & 55 & 14.31 & 1.89 & 17.75 & 271.8 & 0.59 & $\mathrm{~T}_{\mathrm{L}}$ Neph \\
\hline & Measured bc & -7.91 & 0.34 & 1042 & 73 & 14.74 & 1.94 & 17.51 & 278.8 & 0.60 & $\mathrm{~T}_{\mathrm{L}} \mathrm{TiO}_{2} \mathrm{Neph}$ \\
\hline & Target & -8.21 & 0.39 & 1080 & 45 & 14.09 & 2.00 & 17.56 & 275.8 & 0.58 & $\mathrm{~T}_{\mathrm{L}} \mathrm{TiO}_{2} \mathrm{Neph}$ \\
\hline \multirow{3}{*}{ EM-16 } & Measured & -10.17 & 0.87 & 1024 & 15 & 11.39 & 2.02 & 19.00 & 252.1 & 0.59 & $\mathrm{~T}_{\mathrm{L}}$ newlv $\mathrm{TiO}_{2} \mathrm{Neph}$ \\
\hline & Measured bc & -9.71 & 0.72 & 1042 & 22 & 12.17 & 2.18 & 19.05 & 265.1 & 0.59 & $\mathrm{~T}_{\mathrm{L}}$ newlv $\mathrm{TiO}_{2} \mathrm{Neph}$ \\
\hline & Target & -10.41 & 0.96 & 1061 & 13 & 11.59 & 2.00 & 19.48 & 261.6 & 0.58 & $\mathrm{~T}_{\mathrm{L}}$ newlv $\mathrm{TiO}_{2} \mathrm{Neph}$ \\
\hline \multirow{3}{*}{ EM-17 } & Measured & -7.73 & 0.32 & 1073 & 27 & 13.58 & 1.84 & 17.00 & 257.9 & 0.58 & $\mathrm{~T}_{\mathrm{L}}$ Neph \\
\hline & Measured bc & -7.21 & 0.25 & 1088 & 39 & 14.51 & 1.99 & 17.04 & 271.5 & 0.59 & $\mathrm{~T}_{\mathrm{L}} \mathrm{TiO}_{2} \mathrm{Neph}$ \\
\hline & Target & -7.91 & 0.34 & 1106 & 24 & 14.09 & 2.00 & 17.60 & 271.7 & 0.57 & $\mathrm{~T}_{\mathrm{L}}$ newlv $\mathrm{TiO}_{2} \mathrm{Neph}$ \\
\hline \multirow{3}{*}{ EM-18 } & Measured & -12.84 & 2.66 & 975 & 7 & 8.97 & 1.98 & 21.07 & 242.0 & 0.59 & newlv $\mathrm{TiO}_{2} \mathrm{Neph}$ \\
\hline & Measured bc & -12.05 & 1.92 & 1002 & 12 & 9.36 & 2.08 & 20.57 & 251.5 & 0.61 & newlv $\mathrm{TiO}_{2} \mathrm{Neph}$ \\
\hline & Target & -13.04 & 2.89 & 1004 & 7 & 9.09 & 2.00 & 21.51 & 251.5 & 0.59 & newlv $\mathrm{TiO}_{2} \mathrm{Neph}$ \\
\hline \multirow{3}{*}{ EM-19 } & Measured & -13.82 & 4.00 & 940 & 7 & 6.53 & 2.01 & 21.27 & 232.9 & 0.62 & Del Gp newlv $\mathrm{TiO}_{2} \mathrm{Neph}$ \\
\hline & Measured bc & -13.48 & 3.47 & 962 & 10 & 6.98 & 2.16 & 21.32 & 244.3 & 0.64 & Del Gp newlv $\mathrm{TiO}_{2}$ \\
\hline & Target & -14.18 & 4.66 & 959 & 6 & 6.59 & 2.00 & 21.93 & 241.4 & 0.62 & Del Gp newlv $\mathrm{TiO}_{2} \mathrm{Neph}$ \\
\hline \multirow{3}{*}{ EM-20 } & Measured & -12.39 & 2.20 & 991 & 7 & 6.59 & 1.98 & 19.82 & 240.1 & 0.63 & newlv $\mathrm{TiO}_{2} \mathrm{Neph}$ \\
\hline & Measured bc & -11.71 & 1.66 & 1016 & 11 & 6.87 & 2.08 & 19.45 & 250.4 & 0.65 & newlv $\mathrm{TiO}_{2}$ \\
\hline & Target & -12.59 & 2.40 & 1022 & 6 & 6.59 & 2.00 & 20.27 & 251.5 & 0.63 & $\mathrm{~T}_{\mathrm{L}}$ newlv $\mathrm{TiO}_{2}$ \\
\hline
\end{tabular}


SRNL-STI-2009-00778, REVISION 0

Table 19 continued.

\begin{tabular}{|c|c|c|c|c|c|c|c|c|c|c|c|}
\hline Glass ID & $\begin{array}{c}\text { Compositional } \\
\text { View }\end{array}$ & B Del $G_{p}$ & $\left|\begin{array}{c}\text { NL } \\
{[B(g / L)]}\end{array}\right|$ & $\mathbf{T}_{\mathrm{L}}\left({ }^{\circ} \mathrm{C}\right)$ & \begin{tabular}{|c|} 
Viscosity \\
(P)
\end{tabular} & $\begin{array}{l}\mathrm{Al}_{2} \mathrm{O}_{3} \\
(\mathrm{wt} \%)\end{array}$ & $\begin{array}{c}\mathrm{TiO}_{2} \\
(\mathrm{wt} \%)\end{array}$ & $\begin{array}{c}\mathrm{R}_{2} \mathrm{O} \\
(\mathrm{wt} \%)\end{array}$ & $\begin{array}{c}\text { Homogeneity } \\
\text { (wt\%) }\end{array}$ & \begin{tabular}{|c|}
$\begin{array}{c}\text { Nepheline } \\
\text { Value }\end{array}$ \\
\end{tabular} & MAR Status \\
\hline \multirow{3}{*}{ EM-21 } & Measured & -10.46 & 0.98 & 1068 & 9 & 6.96 & 1.90 & 17.95 & 256.0 & 0.65 & $\mathrm{~T}_{\mathrm{L}}$ newlv \\
\hline & Measured bc & -9.81 & 0.75 & 1092 & 14 & 7.26 & 2.00 & 17.64 & 267.3 & 0.67 & $\mathrm{~T}_{\mathrm{L}}$ newlv $\mathrm{TiO}_{2}$ \\
\hline & Target & -10.22 & 0.89 & 1099 & 9 & 6.59 & 2.00 & 17.77 & 261.6 & 0.66 & $\mathrm{~T}_{\mathrm{L}}$ newlv $\mathrm{TiO}_{2}$ \\
\hline \multirow{3}{*}{ EM-22 } & Measured & -11.01 & 1.24 & 1055 & 7 & 6.53 & 1.97 & 17.71 & 260.9 & 0.65 & $\mathrm{~T}_{\mathrm{L}}$ newlv $\mathrm{TiO}_{2}$ \\
\hline & Measured bc & -10.39 & 0.96 & 1078 & 11 & 6.81 & 2.07 & 17.41 & 272.0 & 0.66 & $\mathrm{~T}_{\mathrm{L}}$ newlv $\mathrm{TiO}_{2}$ \\
\hline & Target & -10.69 & 1.08 & 1105 & 7 & 6.59 & 2.00 & 17.60 & 269.7 & 0.65 & $\mathrm{~T}_{\mathrm{L}}$ newlv $\mathrm{TiO}_{2}$ \\
\hline \multirow{3}{*}{ EM-23 } & Measured & -10.76 & 1.12 & 1059 & 9 & 6.64 & 1.94 & 17.26 & 265.4 & 0.65 & $\mathrm{~T}_{\mathrm{L}}$ newlv $\mathrm{TiO}_{2}$ \\
\hline & Measured bc & -10.49 & 1.00 & 1076 & 13 & 7.10 & 2.09 & 17.40 & 279.4 & 0.67 & $\mathrm{~T}_{\mathrm{L}}$ newlv $\mathrm{TiO}_{2}$ \\
\hline & Target & -11.00 & 1.24 & 1106 & 6 & 6.59 & 2.00 & 17.60 & 273.8 & 0.64 & $\mathrm{~T}_{\mathrm{L}}$ newlv $\mathrm{TiO}_{2}$ \\
\hline \multirow{3}{*}{ EM-24 } & Measured & -8.71 & 0.48 & 1077 & 17 & 11.73 & 2.02 & 17.66 & 264.6 & 0.60 & $\mathrm{~T}_{\mathrm{L}}$ newlv $\mathrm{TiO}_{2} \mathrm{Neph}$ \\
\hline & Measured bc & -7.95 & 0.35 & 1099 & 26 & 12.23 & 2.12 & 17.25 & 275.4 & 0.61 & $\mathrm{~T}_{\mathrm{L}} \mathrm{TiO}_{2} \mathrm{Neph}$ \\
\hline & Target & -8.61 & 0.46 & 1124 & 14 & 11.59 & 2.00 & 17.60 & 271.7 & 0.59 & $\mathrm{~T}_{\mathrm{L}}$ newlv $\mathrm{TiO}_{2} \mathrm{Neph}$ \\
\hline \multirow{3}{*}{ EM-25 } & Measured & -12.10 & 1.95 & 973 & 12 & 11.49 & 1.99 & 21.04 & 244.7 & 0.56 & newlv $\mathrm{TiO}_{2} \mathrm{Neph}$ \\
\hline & Measured bc & -11.34 & 1.42 & 996 & 19 & 11.99 & 2.10 & 20.64 & 255.0 & 0.58 & newlv $\mathrm{TiO}_{2} \mathrm{Neph}$ \\
\hline & Target & -12.34 & 2.16 & 995 & 11 & 11.59 & 2.00 & 21.51 & 251.5 & 0.57 & newlv $\mathrm{TiO}_{2} \mathrm{Neph}$ \\
\hline \multirow{3}{*}{ EM-26 } & Measured & -8.90 & 0.52 & 1044 & 24 & 11.25 & 1.95 & 17.07 & 246.8 & 0.61 & $\mathrm{~T}_{\mathrm{L}}$ newlv $\mathrm{TiO}_{2} \mathrm{Neph}$ \\
\hline & Measured bc & -8.51 & 0.44 & 1059 & 34 & 12.02 & 2.10 & 17.21 & 260.4 & 0.62 & $\mathrm{~T}_{\mathrm{L}} \mathrm{TiO}_{2} \mathrm{Neph}$ \\
\hline & Target & -9.08 & 0.56 & 1096 & 21 & 11.59 & 2.00 & 17.60 & 260.0 & 0.60 & $\mathrm{~T}_{\mathrm{L}}$ newlv $\mathrm{TiO}_{2} \mathrm{Neph}$ \\
\hline \multirow{3}{*}{ EM-27 } & Measured & -11.54 & 1.55 & 995 & 8 & 11.13 & 2.02 & 20.86 & 252.6 & 0.56 & newlv $\mathrm{TiO}_{2} \mathrm{Neph}$ \\
\hline & Measured bc & -11.09 & 1.28 & 1012 & 13 & 11.90 & 2.18 & 20.91 & 265.4 & 0.57 & $\mathrm{~T}_{\mathrm{L}}$ newlv $\mathrm{TiO}_{2} \mathrm{Neph}$ \\
\hline & Target & -11.87 & 1.78 & 1023 & 7 & 11.59 & 2.00 & 21.51 & 263.2 & 0.56 & $\mathrm{~T}_{\mathrm{L}}$ newlv $\mathrm{TiO}_{2} \mathrm{Neph}$ \\
\hline \multirow{3}{*}{ EM-28 } & Measured & -9.31 & 0.61 & 1018 & 24 & 14.02 & 2.03 & 18.53 & 258.2 & 0.56 & $\mathrm{~T}_{\mathrm{L}}$ newlv $\mathrm{TiO}_{2} \mathrm{Neph}$ \\
\hline & Measured bc & -8.58 & 0.45 & 1037 & 38 & 14.62 & 2.14 & 18.19 & 269.1 & 0.58 & $\mathrm{~T}_{\mathrm{L}} \mathrm{TiO}_{2} \mathrm{Neph}$ \\
\hline & Target & -9.16 & 0.57 & 1067 & 23 & 14.09 & 2.00 & 18.56 & 267.7 & 0.56 & $\mathrm{~T}_{\mathrm{L}}$ newlv $\mathrm{TiO}_{2} \mathrm{Neph}$ \\
\hline \multirow{3}{*}{ EM-29 } & Measured & -8.84 & 0.50 & 1034 & 29 & 13.77 & 1.99 & 17.60 & 262.4 & 0.57 & $\mathrm{~T}_{\mathrm{L}} \mathrm{TiO}_{2} \mathrm{Neph}$ \\
\hline & Measured bc & -8.41 & 0.42 & 1047 & 41 & 14.72 & 2.15 & 17.74 & 277.0 & 0.58 & $\mathrm{~T}_{\mathrm{L}} \mathrm{TiO}_{2} \mathrm{Neph}$ \\
\hline & Target & -9.01 & 0.54 & 1076 & 24 & 14.09 & 2.00 & 18.06 & 271.7 & 0.56 & $\mathrm{~T}_{\mathrm{L}}$ newlv $\mathrm{TiO}_{2} \mathrm{Neph}$ \\
\hline \multirow{3}{*}{ EM-30 } & Measured & -8.98 & 0.53 & 1034 & 28 & 14.09 & 2.00 & 17.58 & 270.1 & 0.57 & $\mathrm{~T}_{\mathrm{L}} \mathrm{TiO}_{2} \mathrm{Neph}$ \\
\hline & Measured bc & -8.22 & 0.39 & 1054 & 43 & 14.69 & 2.11 & 17.18 & 280.6 & 0.58 & $\mathrm{~T}_{\mathrm{L}} \mathrm{TiO}_{2} \mathrm{Neph}$ \\
\hline & Target & -8.86 & 0.51 & 1085 & 25 & 14.09 & 2.00 & 17.56 & 275.8 & 0.57 & $\mathrm{~T}_{\mathrm{L}}$ newlv $\mathrm{TiO}_{2} \mathrm{Neph}$ \\
\hline
\end{tabular}

\subsubsection{Matrix 2A XRD}

A summary of the types of crystals present in the quenched and ccc glasses is provided in Table 20. An " $X$ " is used to indicate those glasses in which nepheline was predicted to form during slow cooling. XRD patterns of the quenched and ccc glasses are shown in Figure 20 through Figure 49. Most of the glasses crystallized upon quenching and contained Fe-based and/or $\mathrm{Fe} / \mathrm{Ni}$-based spinels. According to the $\mathrm{T}_{\mathrm{L}}$ predictions, all of these glasses should have melted at $1150^{\circ} \mathrm{C}$ without any crystals. After the ccc treatment, almost all of the glasses contained Febased and/or Fe/Ni-based spinels and some of the glasses contained nepheline. For the Matrix 2A glasses, the model predictions for nepheline (Table 20) may be somewhat conservative as nepheline was not detected in all of the glasses that were predicted to contain nepheline. From these data, it appears that nepheline tended to form in glasses with higher concentrations of $\mathrm{Al}_{2} \mathrm{O}_{3}$ (14.09 $\mathrm{wt} \%$ in glass). There also does not appear to be any dependence of the nepheline formation on the frit composition. ${ }^{\mathrm{U}}$ In previous studies, higher concentrations of $\mathrm{B}_{2} \mathrm{O}_{3}(\sim 18$ $\mathrm{wt} \%$ ) have been shown to suppress nepheline ${ }^{29}$, which is higher than the amounts used in this study. Glasses formulated with the same sludges contain nepheline with both frits.

\footnotetext{
${ }^{\mathrm{U}}$ Frit $418\left(8 \mathrm{~B}_{2} \mathrm{O}_{3}-8 \mathrm{Li}_{2} \mathrm{O}-8 \mathrm{Na}_{2} \mathrm{O}-76 \mathrm{SiO}_{2}\right)$ was used for EM-01 through EM-15 and Frit $510\left(14 \mathrm{~B}_{2} \mathrm{O}_{3}-8 \mathrm{Li} 2 \mathrm{O}-8 \mathrm{Na}_{2} \mathrm{O}-\right.$ $70 \mathrm{SiO}_{2}$ ) was used for EM-16 through EM-30.
} 
SRNL-STI-2009-00778, REVISION 0

Table 20. XRD Results of the Matrix 2A Quenched and ccc Glasses

\begin{tabular}{|c|c|c|c|c|}
\hline \multirow{2}{*}{ Glass ID } & \multirow{2}{*}{$\begin{array}{c}\text { Predicted } \mathrm{T}_{\mathrm{L}} \\
\left({ }^{\circ} \mathrm{C}\right)\end{array}$} & \multicolumn{2}{|r|}{ XRD Results } & \multirow{2}{*}{$\begin{array}{l}\text { Nepheline } \\
\text { Prediction }\end{array}$} \\
\hline & & quenched & cec & \\
\hline EM-01 & 1042 & Trevorite $\left(\mathrm{NiFe}_{2} \mathrm{O}_{4}\right)$, Magnetite $\left(\mathrm{Fe}^{+2} \mathrm{Fe}^{2+3} \mathrm{O}_{4}\right)$ & Trevorite $\left(\mathrm{NiFe}_{2} \mathrm{O}_{4}\right)$, Magnetite $\left(\mathrm{Fe}^{+2} \mathrm{Fe}^{2+3} \mathrm{O}_{4}\right)$ & $\mathrm{X}$ \\
\hline EM-02 & 1089 & Trevorite $\left(\mathrm{NiFe}_{2} \mathrm{O}_{4}\right)$, Magnetite $\left(\mathrm{Fe}^{+2} \mathrm{Fe}^{2+3} \mathrm{O}_{4}\right)$ & Trevorite $\left(\mathrm{NiFe}_{2} \mathrm{O}_{4}\right)$, Magnetite $\left(\mathrm{Fe}_{+2} \mathrm{Fe}_{2+3} \mathrm{O}_{4}\right)$, Nepheline $\left(\mathbf{N a A I S i O}{ }_{4}\right)$ & $\mathrm{X}$ \\
\hline EM-03 & 997 & Amorphous & Trevorite $\left(\mathrm{NiFe}_{2} \mathrm{O}_{4}\right)$, Magnetite $\left(\mathrm{Fe}^{+2} \mathrm{Fe}^{2+3} \mathrm{O}_{4}\right)$ & $\mathrm{X}$ \\
\hline EM-04 & 959 & Amorphous & Trevorite $\left(\mathrm{NiFe}_{2} \mathrm{O}_{4}\right)$, Magnetite $\left(\mathrm{Fe}^{+2} \mathrm{Fe}^{2+3} \mathrm{O}_{4}\right)$ & \\
\hline EM-05 & 1012 & Amorphous & Trevorite $\left(\mathrm{NiFe}_{2} \mathrm{O}_{4}\right)$, Magnetite $\left(\mathrm{Fe}^{+2} \mathrm{Fe}^{2+3} \mathrm{O}_{4}\right)$ & \\
\hline EM-06 & 1071 & Trevorite $\left(\mathrm{NiFe}_{2} \mathrm{O}_{4}\right)$, Magnetite $\left(\mathrm{Fe}^{+2} \mathrm{Fe}^{2+3} \mathrm{O}_{4}\right)$ & Trevorite $\left(\mathrm{NiFe}_{2} \mathrm{O}_{4}\right)$, Magnetite $\left(\mathrm{Fe}^{+2} \mathrm{Fe}^{2+3} \mathrm{O}_{4}\right)$ & \\
\hline EM-07 & 1068 & Trevorite $\left(\mathrm{NiFe}_{2} \mathrm{O}_{4}\right)$, Magnetite $\left(\mathrm{Fe}^{+2} \mathrm{Fe}^{2+3} \mathrm{O}_{4}\right)$ & Trevorite $\left(\mathrm{NiFe}_{2} \mathrm{O}_{4}\right)$, Magnetite $\left(\mathrm{Fe}^{+2} \mathrm{Fe}^{2+3} \mathrm{O}_{4}\right)$ & \\
\hline EM-08 & 1066 & Trevorite $\left(\mathrm{NiFe}_{2} \mathrm{O}_{4}\right)$, Magnetite $\left(\mathrm{Fe}^{+2} \mathrm{Fe}^{2+3} \mathrm{O}_{4}\right)$ & Trevorite $\left(\mathrm{NiFe}_{2} \mathrm{O}_{4}\right)$, Magnetite $\left(\mathrm{Fe}^{+2} \mathrm{Fe}^{2+3} \mathrm{O}_{4}\right)$ & \\
\hline EM-09 & 1090 & Trevorite $\left(\mathrm{NiFe}_{2} \mathrm{O}_{4}\right)$, Magnetite $\left(\mathrm{Fe}^{+2} \mathrm{Fe}^{2+3} \mathrm{O}_{4}\right)$ & Trevorite $\left(\mathrm{NiFe}_{2} \mathrm{O}_{4}\right)$, Magnetite $\left(\mathrm{Fe}^{+2} \mathrm{Fe}^{2+3} \mathrm{O}_{4}\right)$, Nepheline $\left(\mathbf{N a A l S i O}_{4}\right)$ & $\mathrm{X}$ \\
\hline EM-10 & 983 & Trevorite $\left(\mathrm{NiFe}_{2} \mathrm{O}_{4}\right)$, Bunsenite $(\mathrm{NiO})$ & Trevorite $\left(\mathrm{NiFe}_{2} \mathrm{O}_{4}\right)$, Magnetite $\left(\mathrm{Fe}^{+2} \mathrm{Fe}^{2+3} \mathrm{O}_{4}\right)$, Nepheline $\left(\mathrm{NaAlSiO}_{4}\right)$ & $\mathrm{X}$ \\
\hline EM-11 & 1066 & Trevorite $\left(\mathrm{NiFe}_{2} \mathrm{O}_{4}\right)$, Magnetite $\left(\mathrm{Fe}^{+2} \mathrm{Fe}^{2+3} \mathrm{O}_{4}\right)$ & Magnetite $\left(\mathrm{Fe}^{+2} \mathrm{Fe}^{2+3} \mathrm{O}_{4}\right)$ & $\mathrm{X}$ \\
\hline EM-12 & 1000 & Trevorite $\left(\mathrm{NiFe}_{2} \mathrm{O}_{4}\right)$, Bunsenite $(\mathrm{NiO})$ & Trevorite $\left(\mathrm{NiFe}_{2} \mathrm{O}_{4}\right)$, Magnetite $\left(\mathrm{Fe}^{+2} \mathrm{Fe}^{2+3} \mathrm{O}_{4}\right)$, Nepheline $\left(\mathrm{NaAlSiO}_{4}\right)$ & $\mathrm{X}$ \\
\hline EM-13 & 1031 & Trevorite $\left(\mathrm{NiFe}_{2} \mathrm{O}_{4}\right)$, Magnetite $\left(\mathrm{Fe}^{+2} \mathrm{Fe}^{2+3} \mathrm{O}_{4}\right)$ & Magnetite $\left(\mathrm{Fe}^{+2} \mathrm{Fe}^{2+3} \mathrm{O}_{4}\right)$, Nepheline $\left(\mathrm{NaAlSiO}_{4}\right)$ & $\mathrm{X}$ \\
\hline EM-14 & 1043 & Trevorite $\left(\mathrm{NiFe}_{2} \mathrm{O}_{4}\right)$, Magnetite $\left(\mathrm{Fe}^{+2} \mathrm{Fe}^{2+3} \mathrm{O}_{4}\right)$ & Magnetite $\left(\mathrm{Fe}^{+2} \mathrm{Fe}^{2+3} \mathrm{O}_{4}\right)$, Nepheline $\left(\mathrm{NaAlSiO}_{4}\right)$ & $\mathrm{X}$ \\
\hline EM-15 & 1042 & Trevorite $\left(\mathrm{NiFe}_{2} \mathrm{O}_{4}\right)$, Magnetite $\left(\mathrm{Fe}^{+2} \mathrm{Fe}^{2+3} \mathrm{O}_{4}\right)$ & Magnetite $\left(\mathrm{Fe}^{+2} \mathrm{Fe}^{2+3} \mathrm{O}_{4}\right)$, Nepheline $\left(\mathrm{NaAlSiO}_{4}\right)$ & $\mathrm{X}$ \\
\hline EM-16 & 1042 & Trevorite $\left(\mathrm{NiFe}_{2} \mathrm{O}_{4}\right)$, Magnetite $\left(\mathrm{Fe}^{+2} \mathrm{Fe}^{2+3} \mathrm{O}_{4}\right)$ & Magnetite $\left(\mathrm{Fe}^{+2} \mathrm{Fe}^{2+3} \mathrm{O}_{4}\right)$ & $\mathrm{X}$ \\
\hline EM-17 & 1088 & Trevorite $\left(\mathrm{NiFe}_{2} \mathrm{O}_{4}\right)$, Magnetite $\left(\mathrm{Fe}^{+2} \mathrm{Fe}^{2+3} \mathrm{O}_{4}\right)$ & Magnetite $\left(\mathrm{Fe}^{+2} \mathrm{Fe}^{2+3} \mathrm{O}_{4}\right)$ & $\mathrm{X}$ \\
\hline EM-18 & 1002 & Amorphous & Magnetite $\left(\mathrm{Fe}^{+2} \mathrm{Fe}^{2+3} \mathrm{O}_{4}\right)$ & $\mathrm{X}$ \\
\hline EM-19 & 962 & Amorphous & Magnetite $\left(\mathrm{Fe}^{+2} \mathrm{Fe}^{2+3} \mathrm{O}_{4}\right)$ & $\mathrm{X}$ \\
\hline EM-20 & 1016 & Hematite $\left(\mathrm{Fe}_{2} \mathrm{O}_{3}\right)$ & Magnetite $\left(\mathrm{Fe}^{+2} \mathrm{Fe}^{2+3} \mathrm{O}_{4}\right)$ & \\
\hline EM-21 & 1092 & Trevorite $\left(\mathrm{NiFe}_{2} \mathrm{O}_{4}\right)$, Magnetite $\left(\mathrm{Fe}^{+2} \mathrm{Fe}^{2+3} \mathrm{O}_{4}\right)$ & Magnetite $\left(\mathrm{Fe}^{+2} \mathrm{Fe}^{2+3} \mathrm{O}_{4}\right)$ & \\
\hline EM-22 & 1078 & Trevorite $\left(\mathrm{NiFe}_{2} \mathrm{O}_{4}\right)$, Magnetite $\left(\mathrm{Fe}^{+2} \mathrm{Fe}^{2+3} \mathrm{O}_{4}\right)$ & Magnetite $\left(\mathrm{Fe}^{+2} \mathrm{Fe}^{2+3} \mathrm{O}_{4}\right)$ & \\
\hline EM-23 & 1076 & Trevorite $\left(\mathrm{NiFe}_{2} \mathrm{O}_{4}\right)$, Magnetite $\left(\mathrm{Fe}^{+2} \mathrm{Fe}^{2+3} \mathrm{O}_{4}\right)$ & Magnetite $\left(\mathrm{Fe}^{+2} \mathrm{Fe}^{2+3} \mathrm{O}_{4}\right)$ & \\
\hline EM-24 & 1099 & Trevorite $\left(\mathrm{NiFe}_{2} \mathrm{O}_{4}\right)$, Magnetite $\left(\mathrm{Fe}^{+2} \mathrm{Fe}^{2+3} \mathrm{O}_{4}\right)$ & Magnetite $\left(\mathrm{Fe}^{+2} \mathrm{Fe}^{2+3} \mathrm{O}_{4}\right)$ & $\mathrm{X}$ \\
\hline EM-25 & 996 & Bunsenite $(\mathrm{NiO})$ & Magnetite $\left(\mathrm{Fe}^{+2} \mathrm{Fe}^{2+3} \mathrm{O}_{4}\right)$, Nepheline $\left(\mathrm{NaAlSiO}_{4}\right)$ & $\mathrm{X}$ \\
\hline EM-26 & 1059 & Trevorite $\left(\mathrm{NiFe}_{2} \mathrm{O}_{4}\right)$, Magnetite $\left(\mathrm{Fe}^{+2} \mathrm{Fe}^{2+3} \mathrm{O}_{4}\right)$ & Trevorite $\left(\mathrm{NiFe}_{2} \mathrm{O}_{4}\right)$, Magnetite $\left(\mathrm{Fe}^{+2} \mathrm{Fe}^{2+3} \mathrm{O}_{4}\right)$, Nepheline (NaAlSiO $\left.{ }_{4}\right)$ & $\mathrm{X}$ \\
\hline EM-27 & 1012 & Bunsenite $(\mathrm{NiO})$ & Magnetite $\left(\mathrm{Fe}^{+2} \mathrm{Fe}^{2+3} \mathrm{O}_{4}\right)$ & $\mathrm{X}$ \\
\hline EM-28 & 1037 & Trevorite $\left(\mathrm{NiFe}_{2} \mathrm{O}_{4}\right)$, Magnetite $\left(\mathrm{Fe}^{+2} \mathrm{Fe}^{2+3} \mathrm{O}_{4}\right)$ & Magnetite $\left(\mathrm{Fe}^{+2} \mathrm{Fe}^{2+3} \mathrm{O}_{4}\right)$, Nepheline $\left(\mathrm{NaAlSiO}_{4}\right)$ & $\mathrm{X}$ \\
\hline EM-29 & 1047 & Trevorite $\left(\mathrm{NiFe}_{2} \mathrm{O}_{4}\right)$, Magnetite $\left(\mathrm{Fe}^{+2} \mathrm{Fe}^{2+3} \mathrm{O}_{4}\right)$ & Magnetite $\left(\mathrm{Fe}^{+2} \mathrm{Fe}^{2+3} \mathrm{O}_{4}\right)$, Nepheline $\left(\mathrm{NaAlSiO}_{4}\right)$ & $\mathrm{X}$ \\
\hline EM-30 & 1054 & Trevorite $\left(\mathrm{NiFe}_{2} \mathrm{O}_{4}\right)$, Magnetite $\left(\mathrm{Fe}^{+2} \mathrm{Fe}^{2+3} \mathrm{O}_{4}\right)$ & Magnetite $\left(\mathrm{Fe}^{+2} \mathrm{Fe}^{2+3} \mathrm{O}_{4}\right)$, Nepheline $\left(\mathrm{NaAlSiO}_{4}\right)$ & $\mathrm{X}$ \\
\hline
\end{tabular}


SRNL-STI-2009-00778, REVISION 0

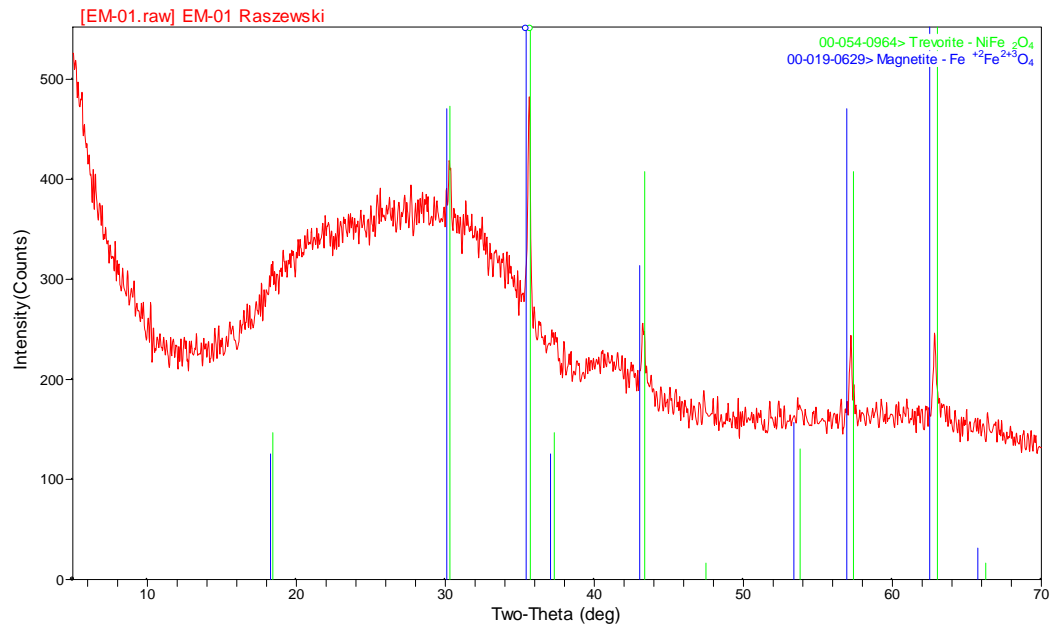

(a)

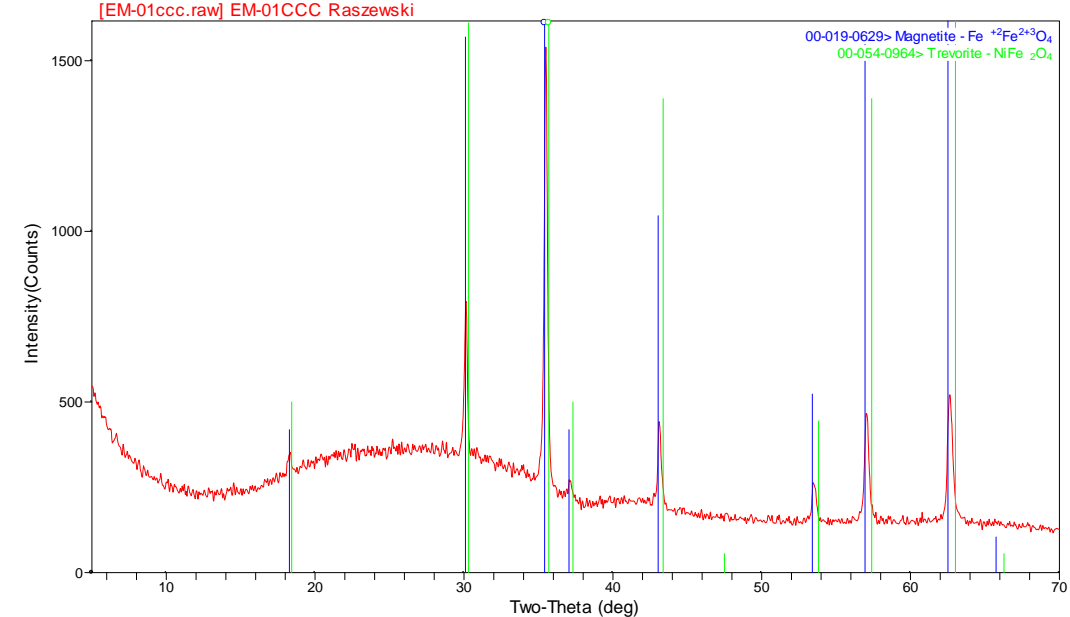

(b)

Figure 20. XRD patterns of (a) quenched and (b) cce EM-01.

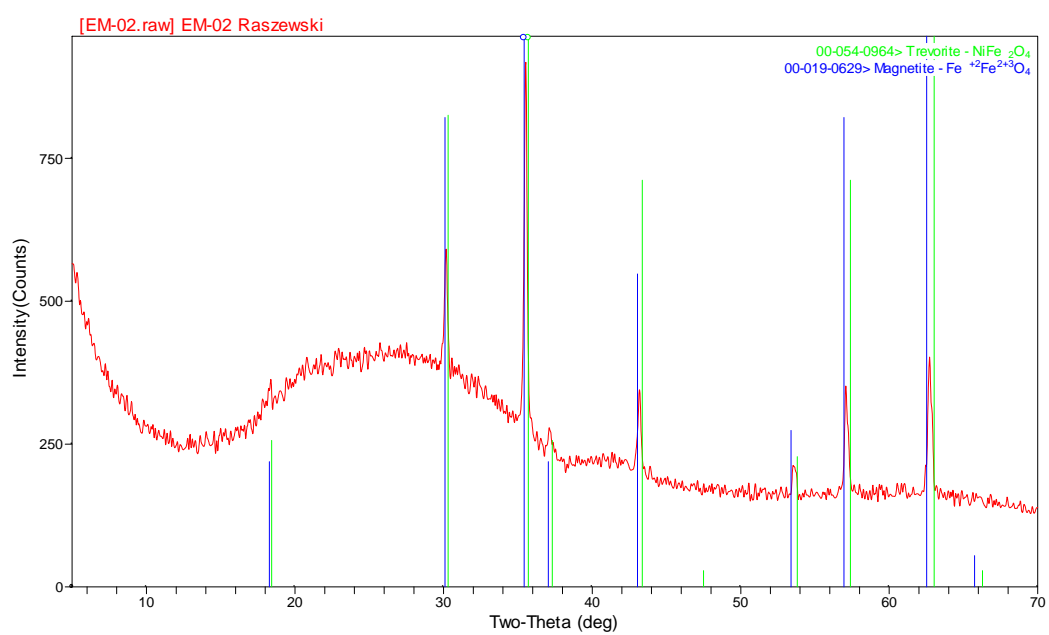

(a)

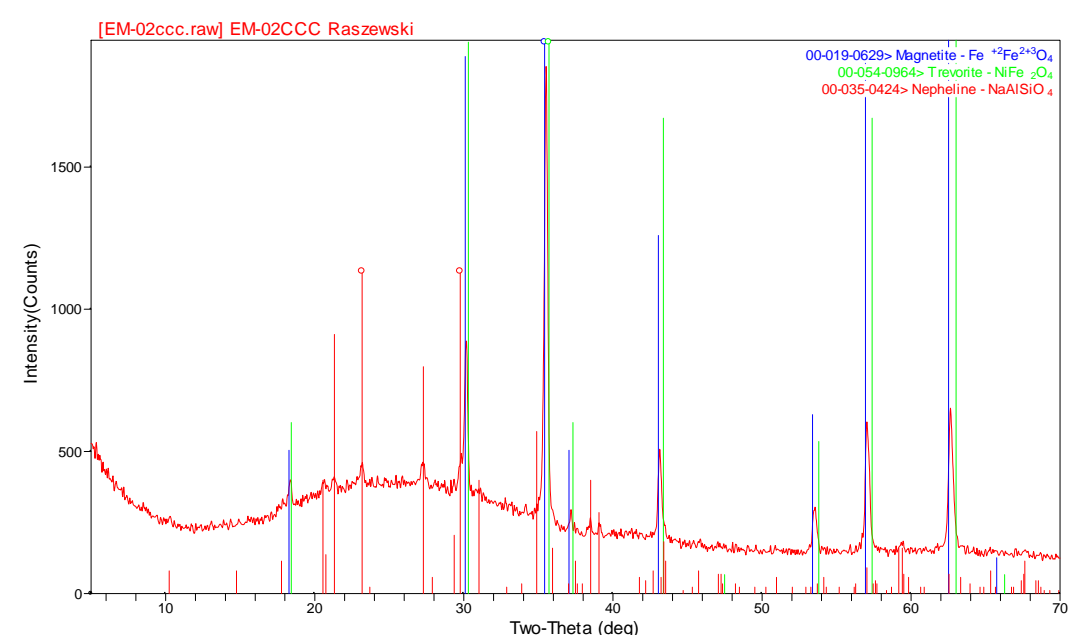

(b)

Figure 21. XRD patterns of (a) quenched and (b) cec EM-02. 
SRNL-STI-2009-00778, REVISION 0

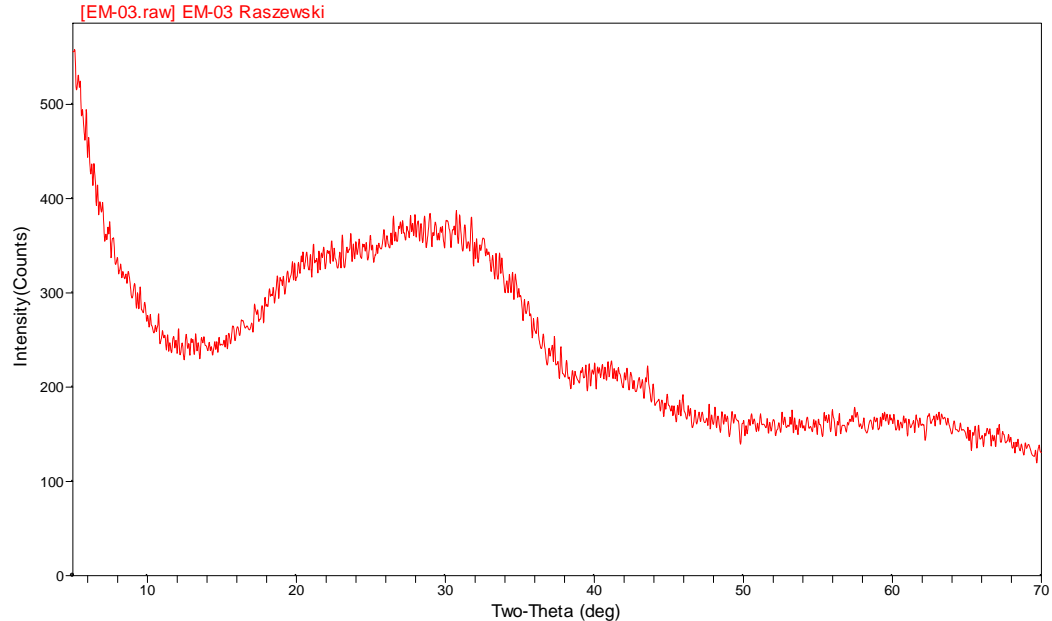

(a)

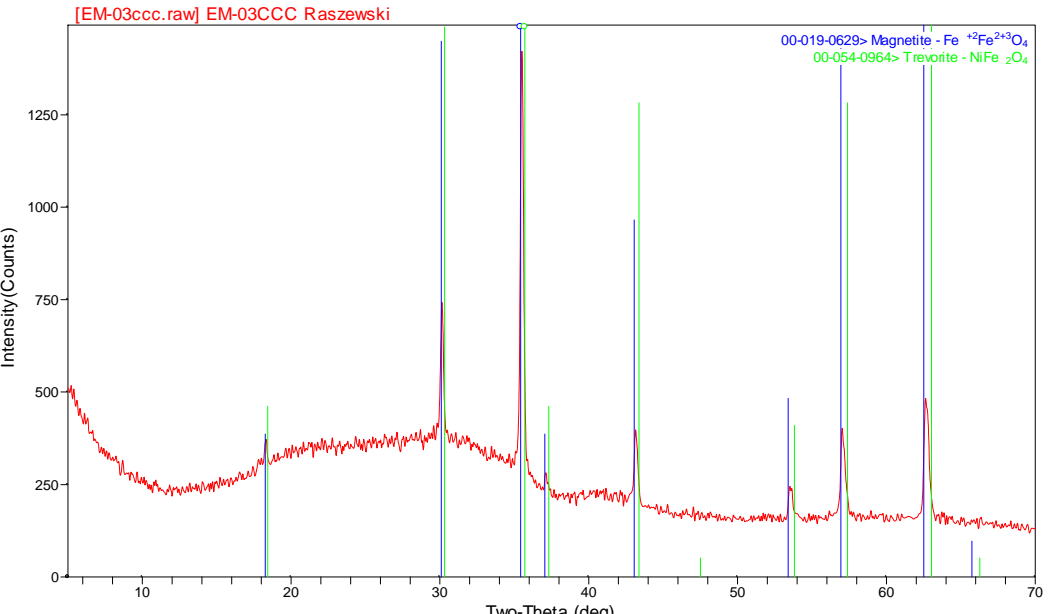

(b)

Figure 22. XRD patterns of (a) quenched and (b) cec EM-03.

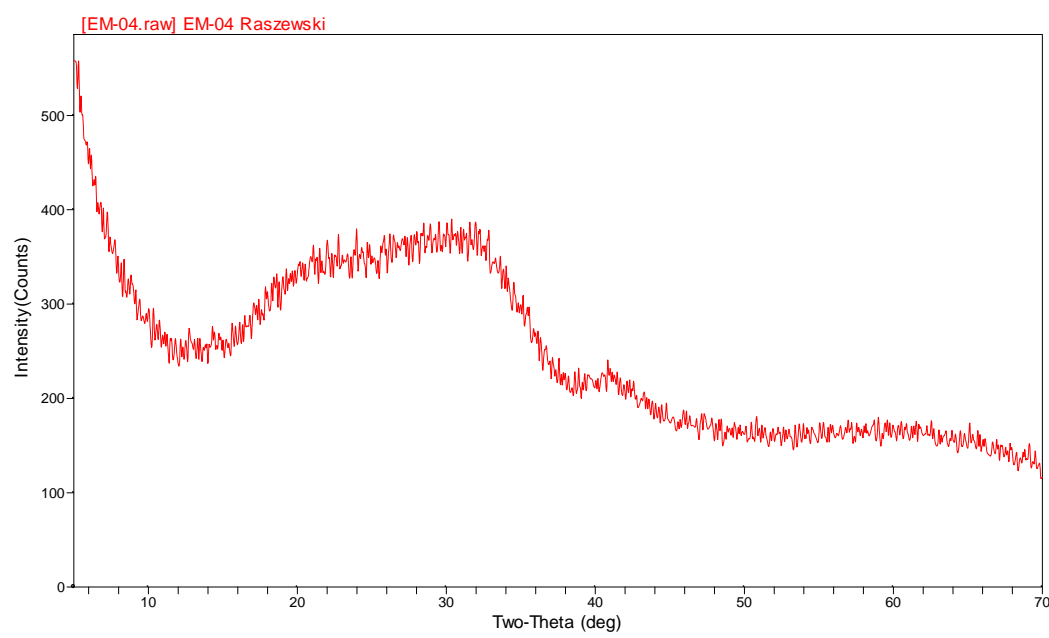

(a)

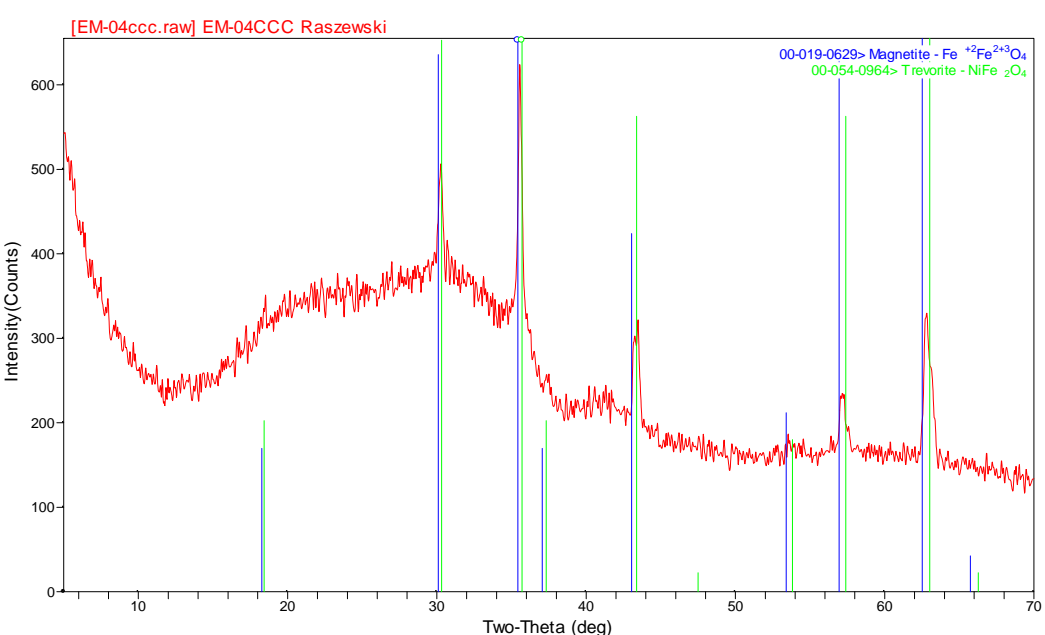

(b)

Figure 23. XRD patterns of (a) quenched and (b) cce EM-04. 
SRNL-STI-2009-00778, REVISION 0

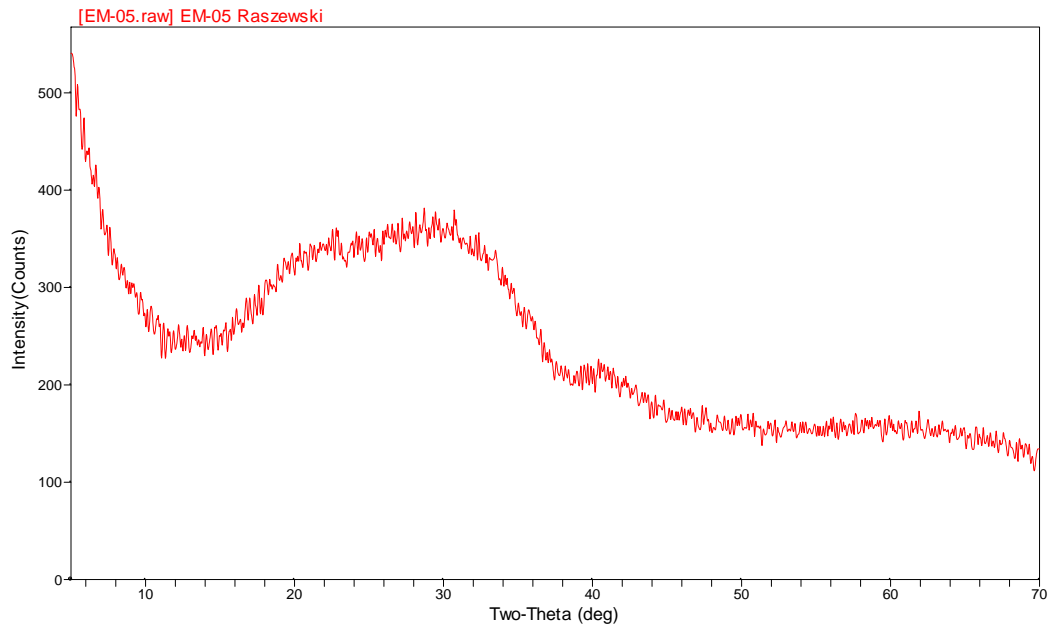

(a)

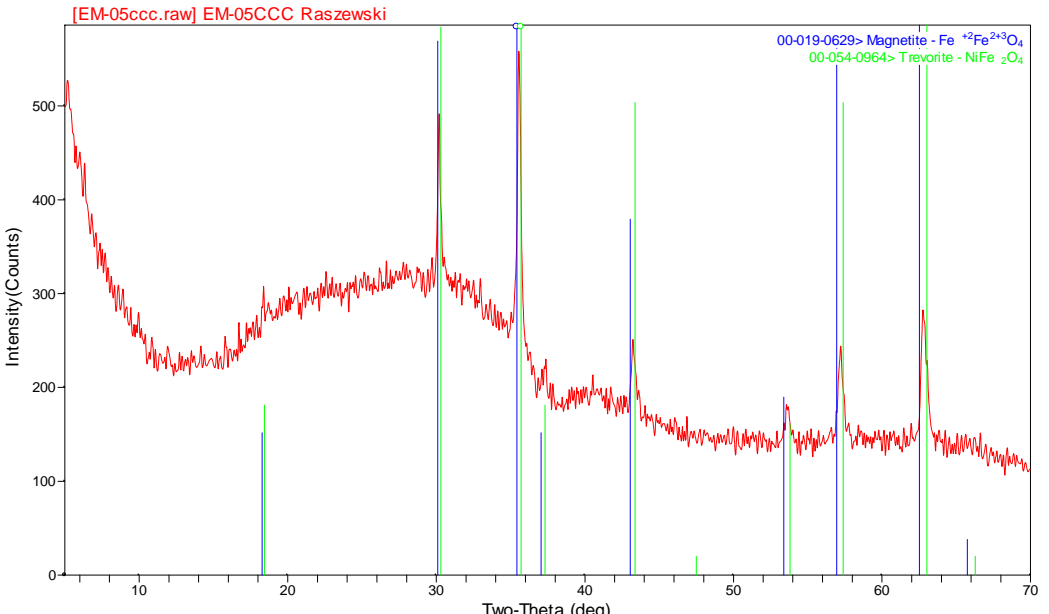

(b)

Figure 24. XRD patterns of (a) quenched and (b) cec EM-05.

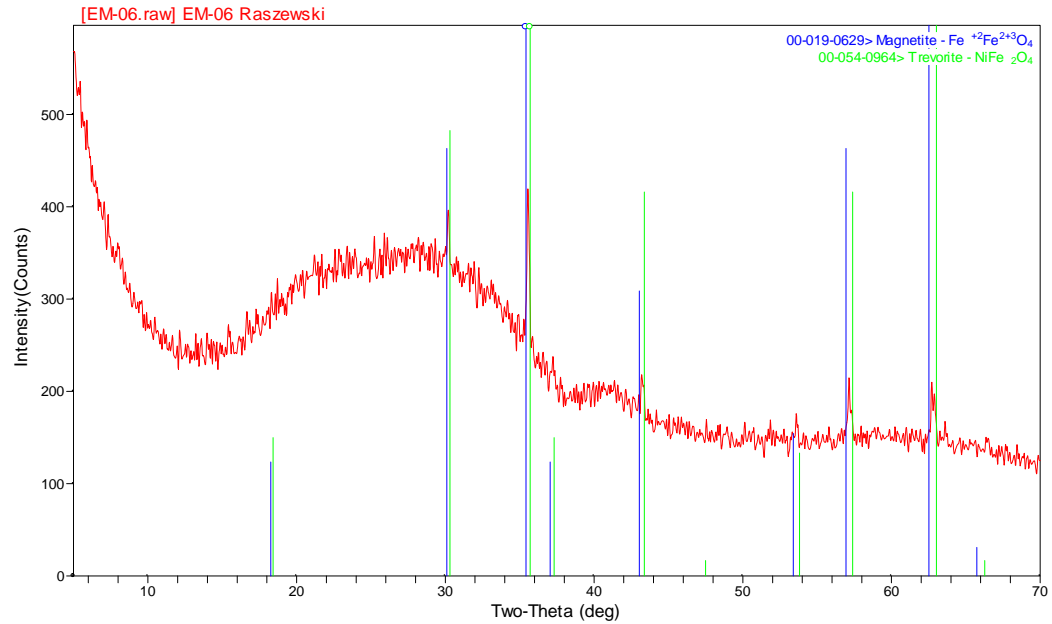

(a)

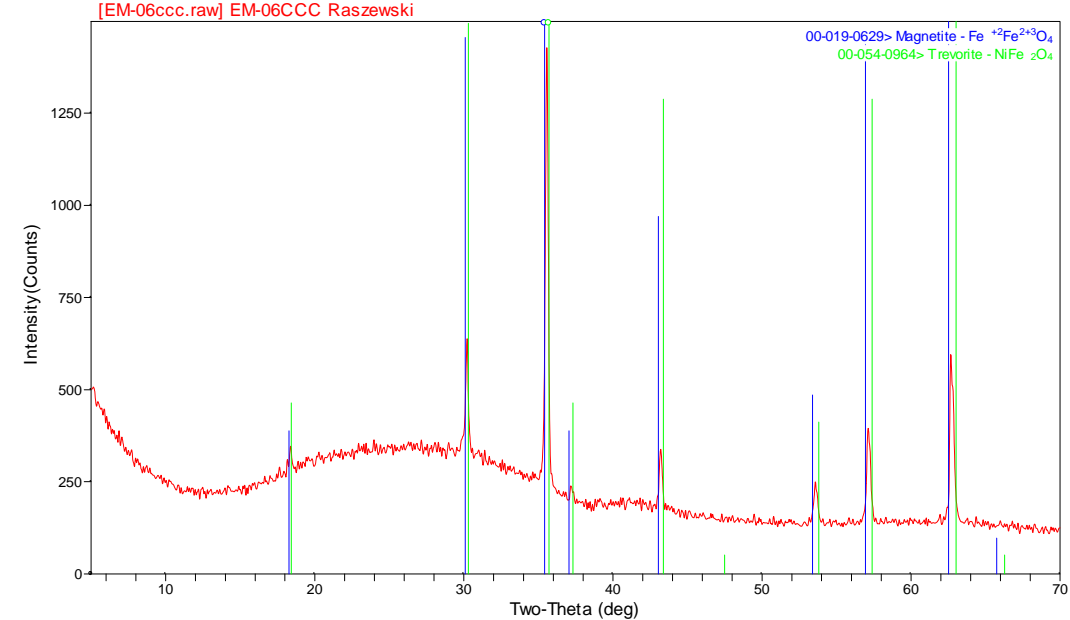

(b)

Figure 25. XRD patterns of (a) quenched and (b) ccc EM-06. 
SRNL-STI-2009-00778, REVISION 0

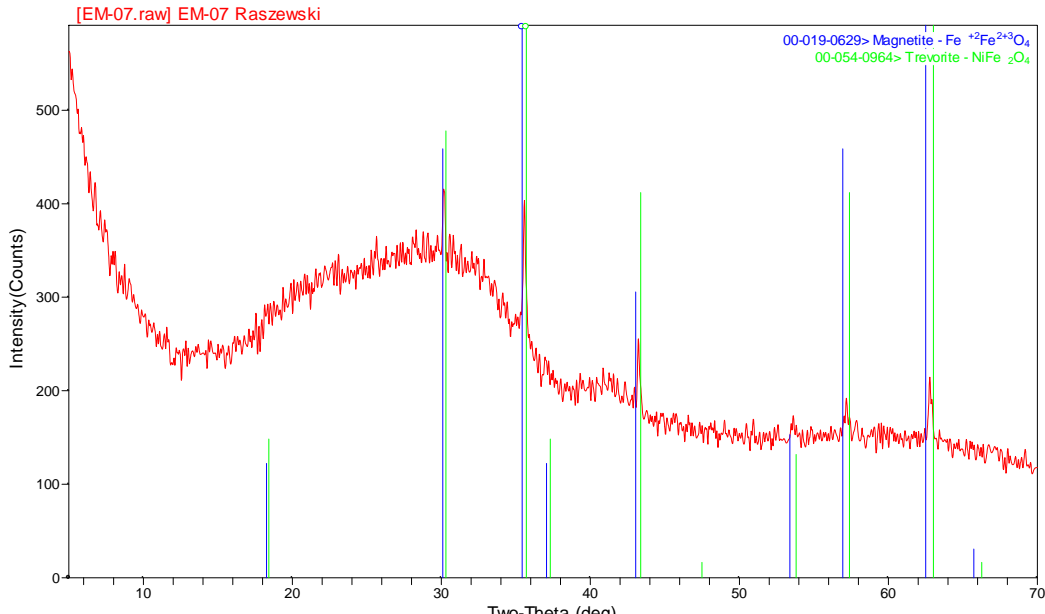

(a)

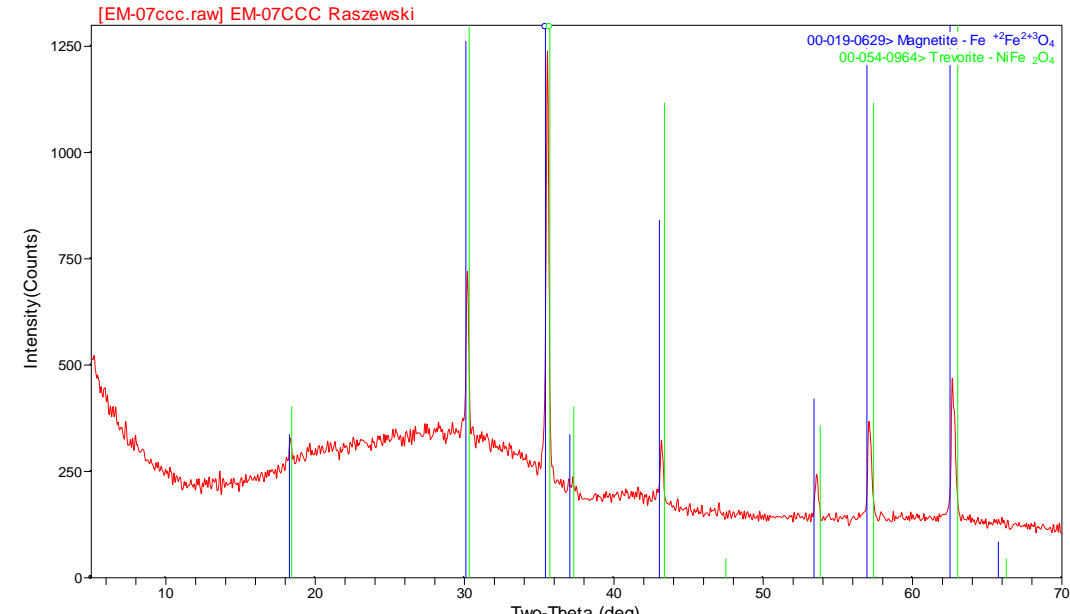

(b)

Figure 26. XRD patterns of (a) quenched and (b) cce EM-07.

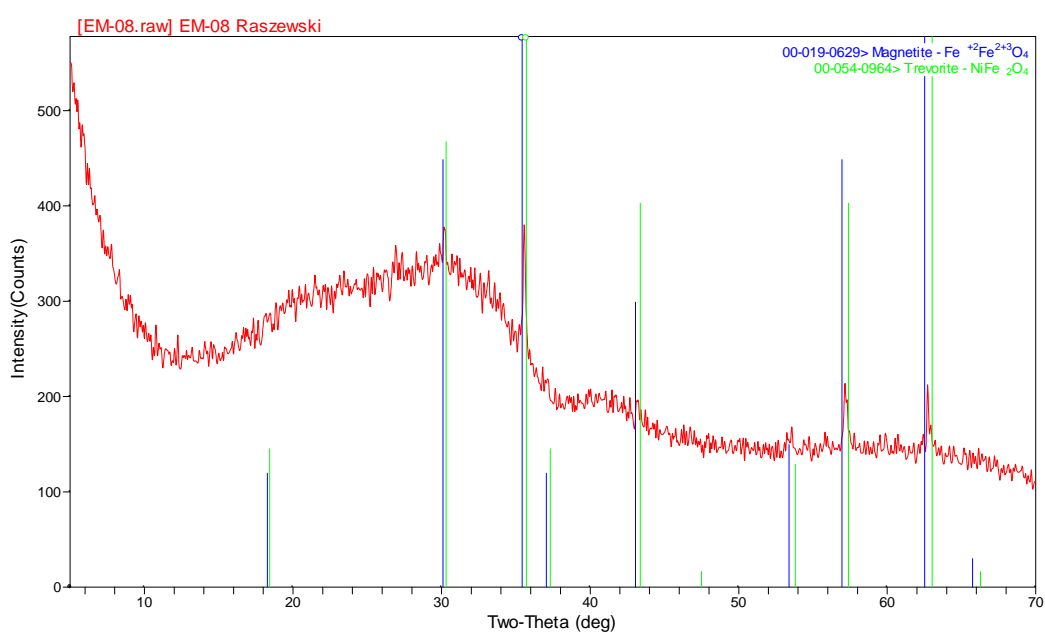

(a)

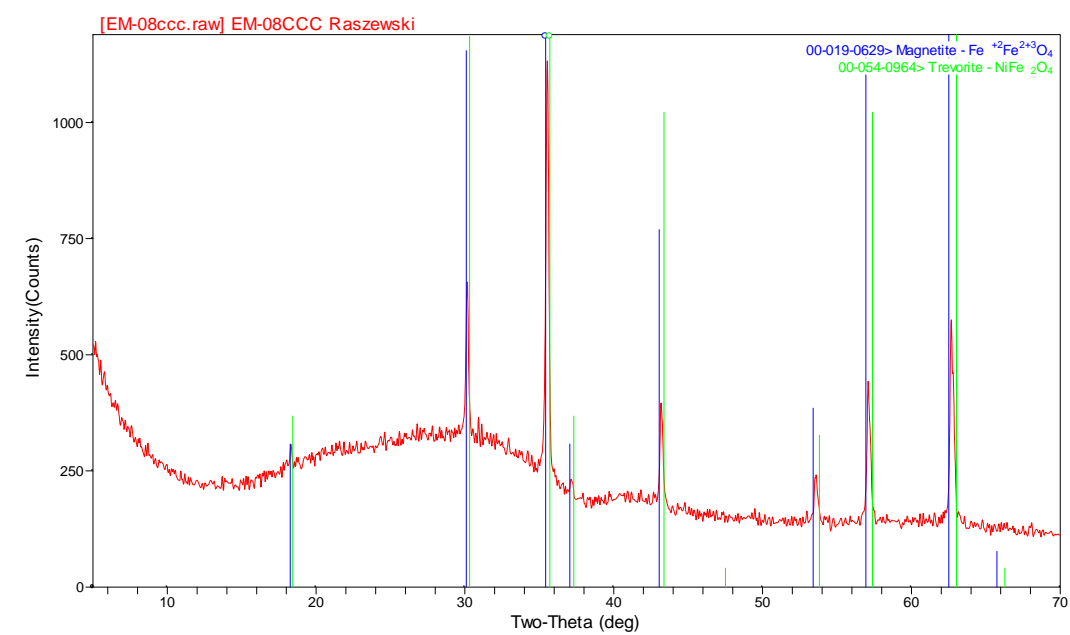

(b)

Figure 27. XRD patterns of (a) quenched and (b) cec EM-08. 
SRNL-STI-2009-00778, REVISION 0

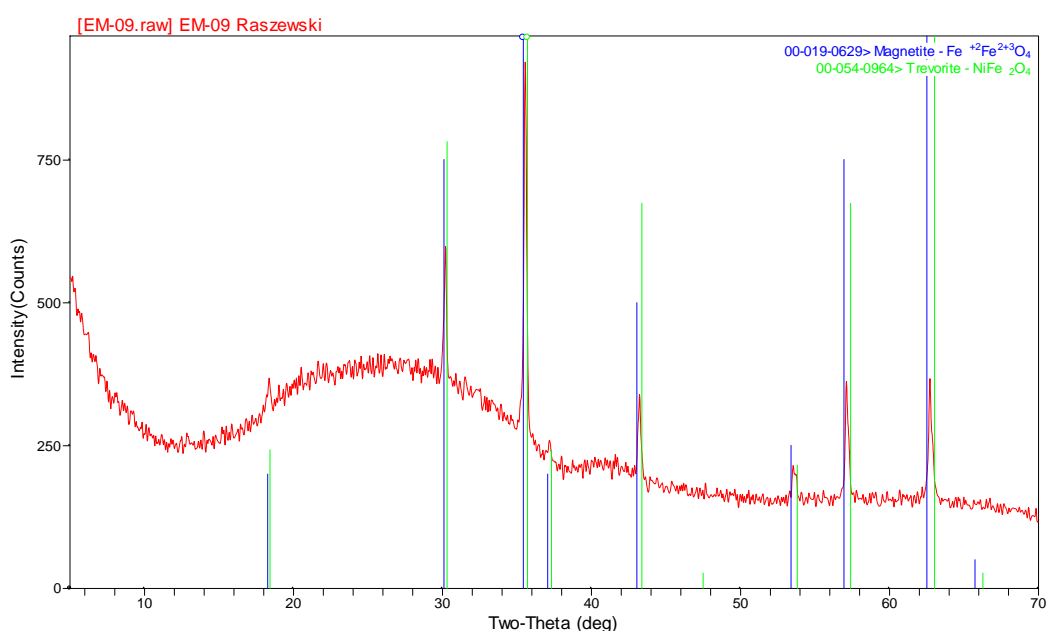

(a)

Figure 28. XRD patterns of (a) quenched and (b) ccc EM-09.

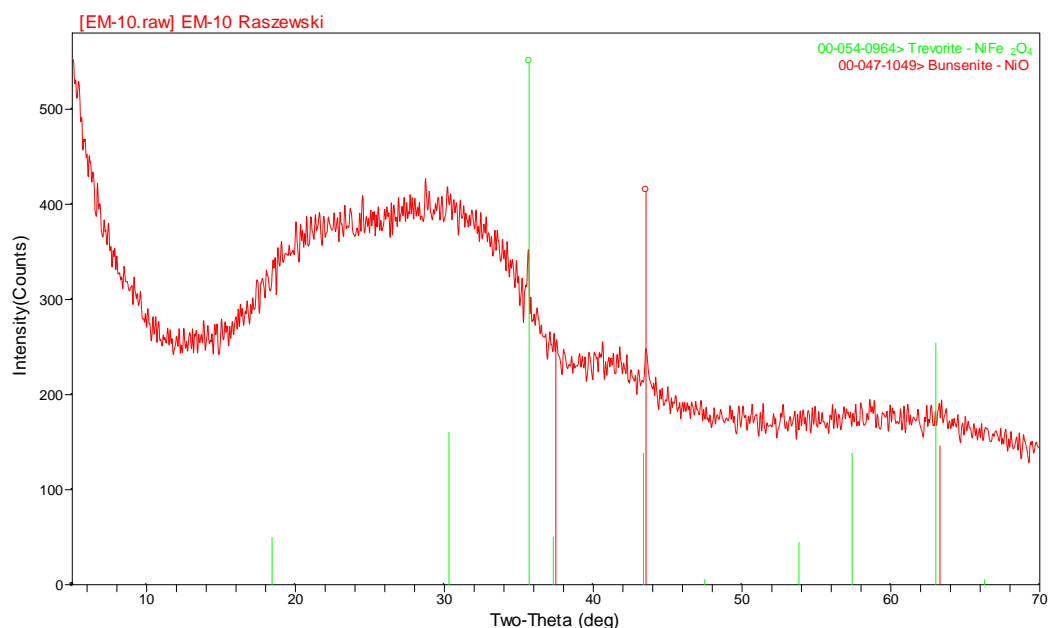

(a)

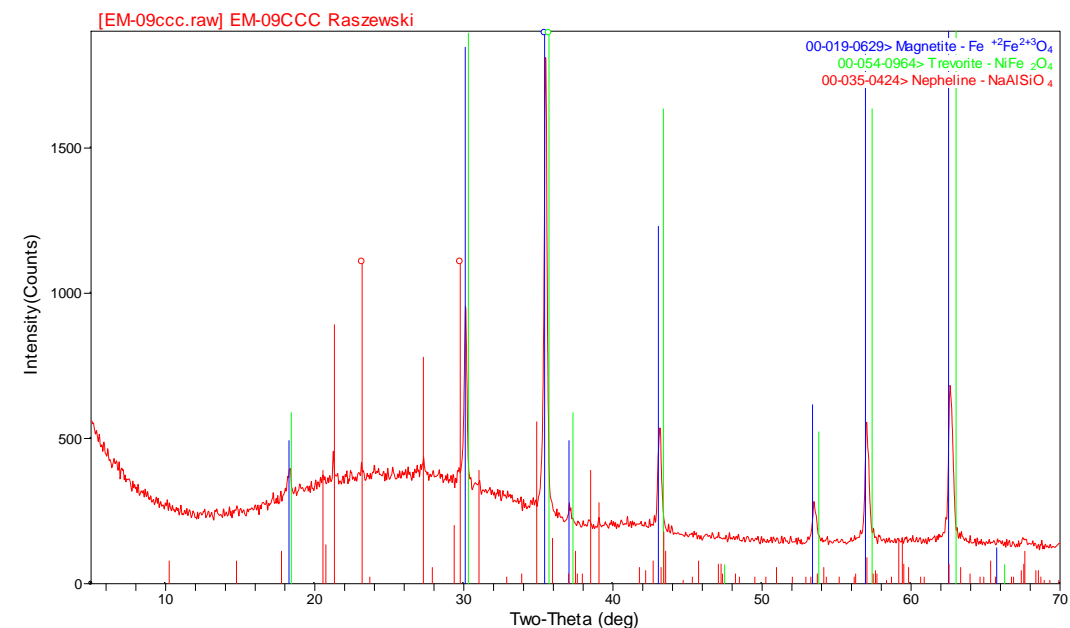

(b)

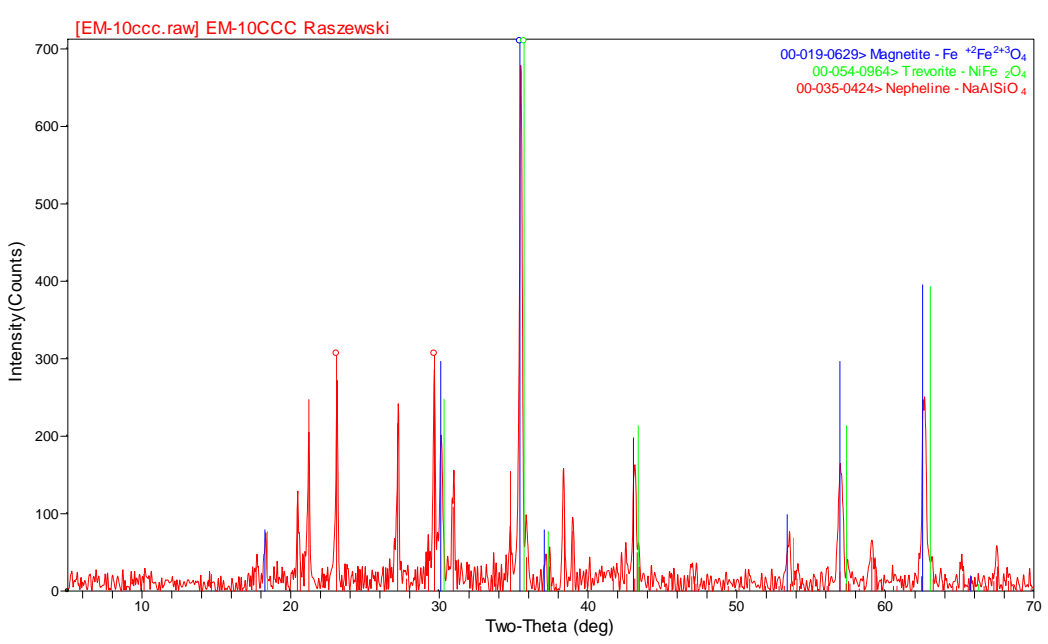

(b)

Figure 29. XRD patterns of (a) quenched and (b) cec EM-10. 
SRNL-STI-2009-00778, REVISION 0

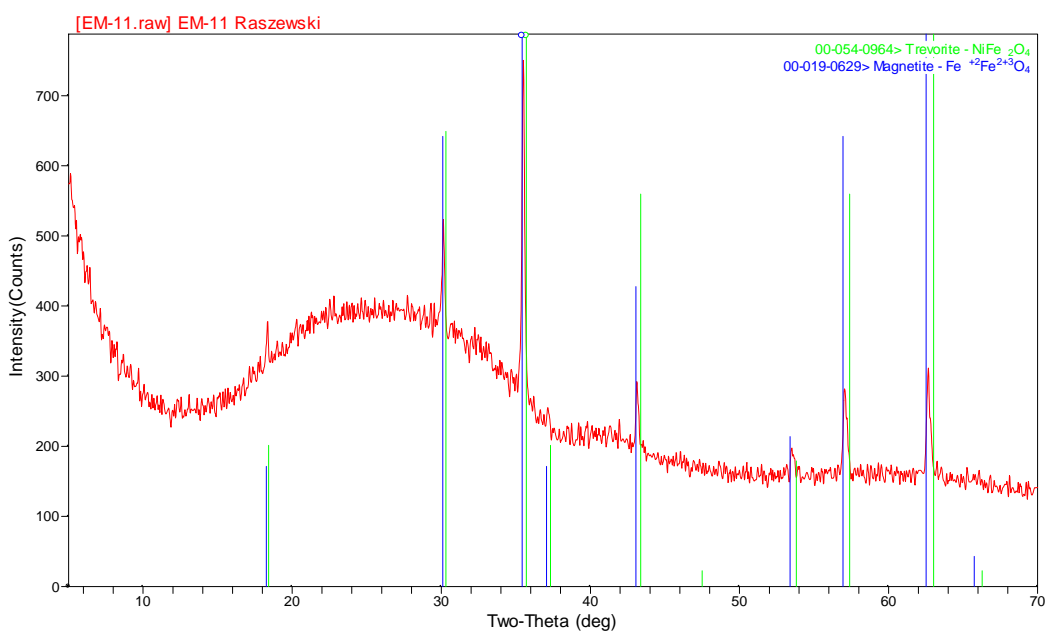

(a)

Figure 30. XRD patterns of (a) quenched and (b) ccc EM-11.

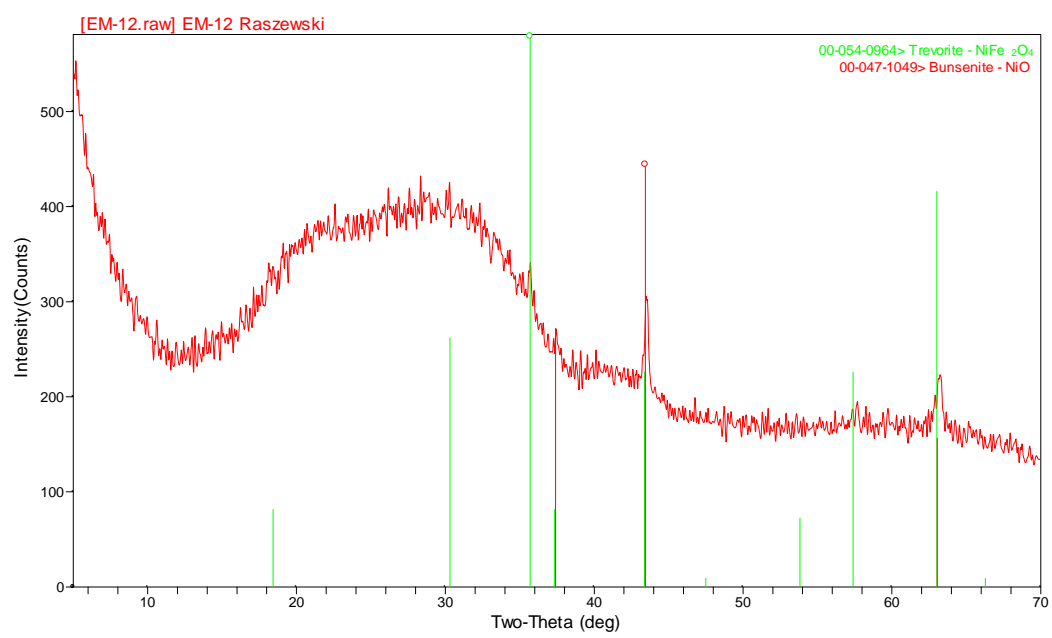

(a)

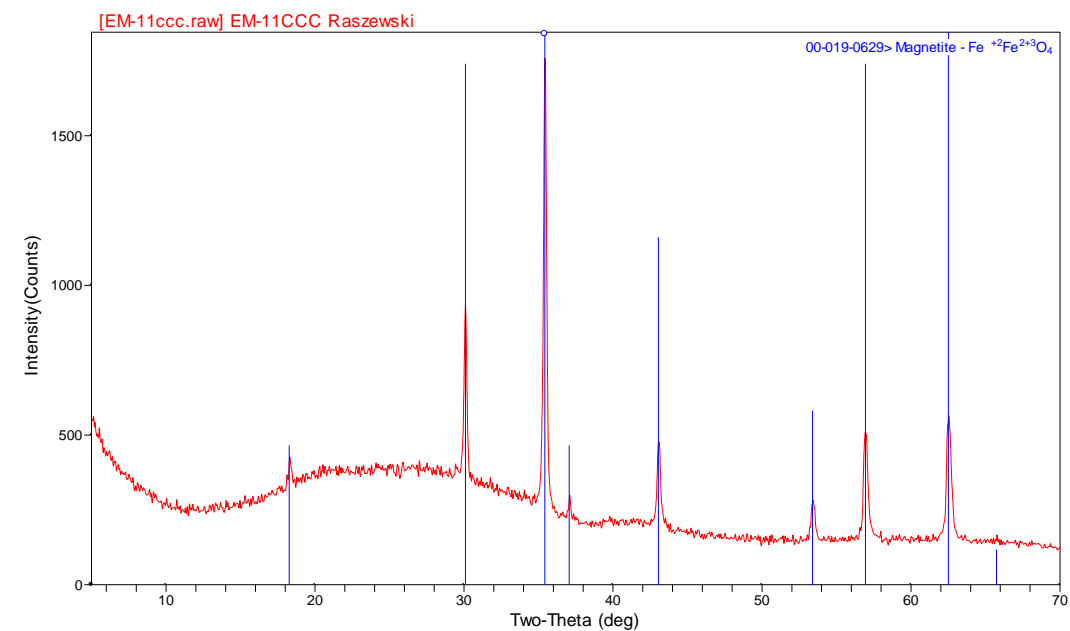

(b)

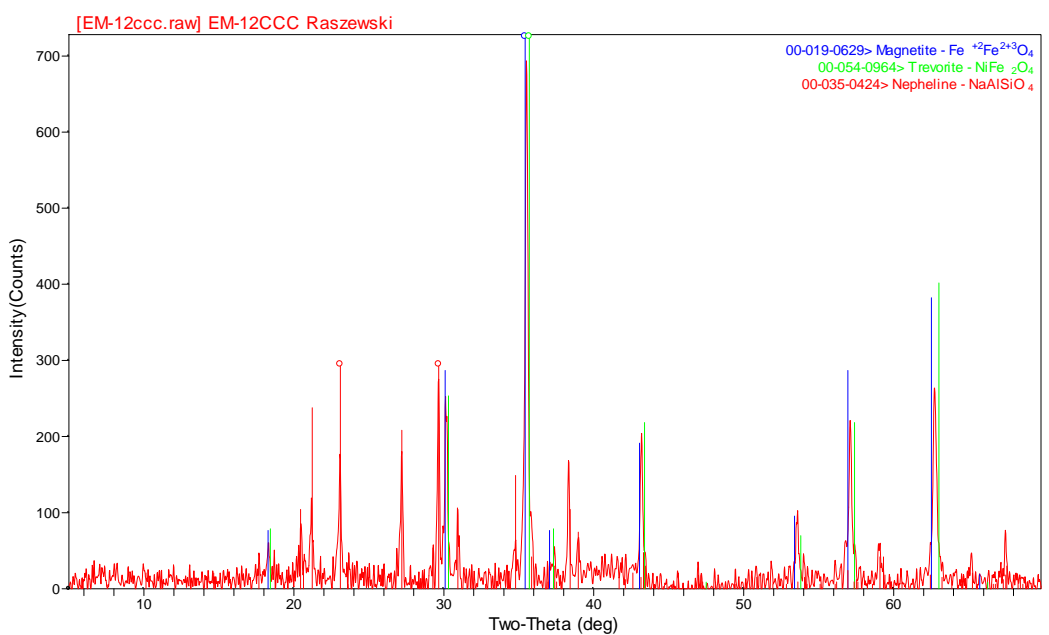

(b)

Figure 31. XRD patterns of (a) quenched and (b) cec EM-12. 
SRNL-STI-2009-00778, REVISION 0

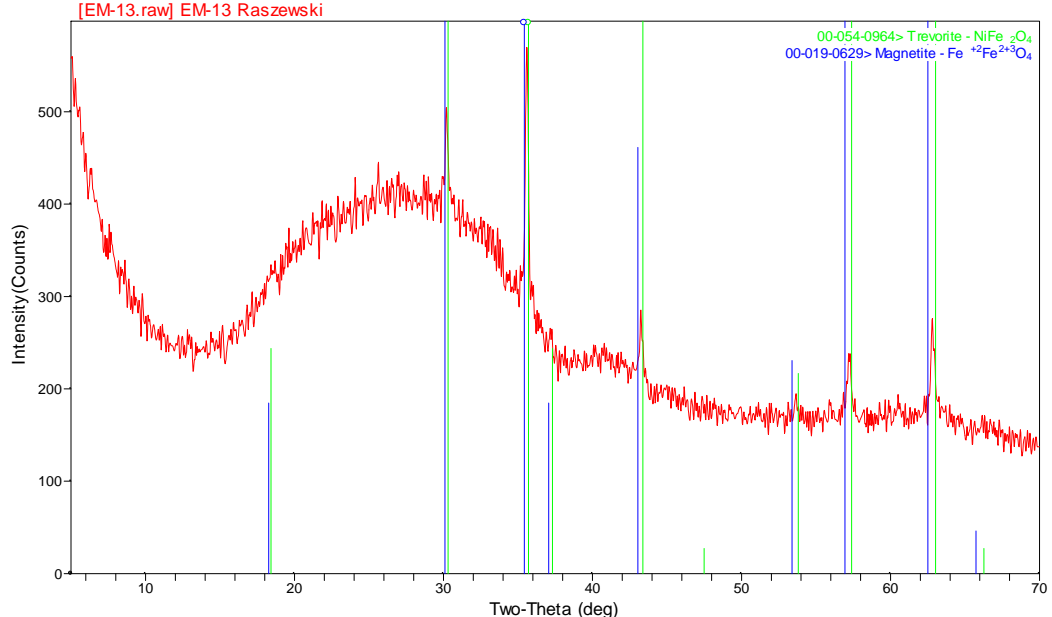

(a)

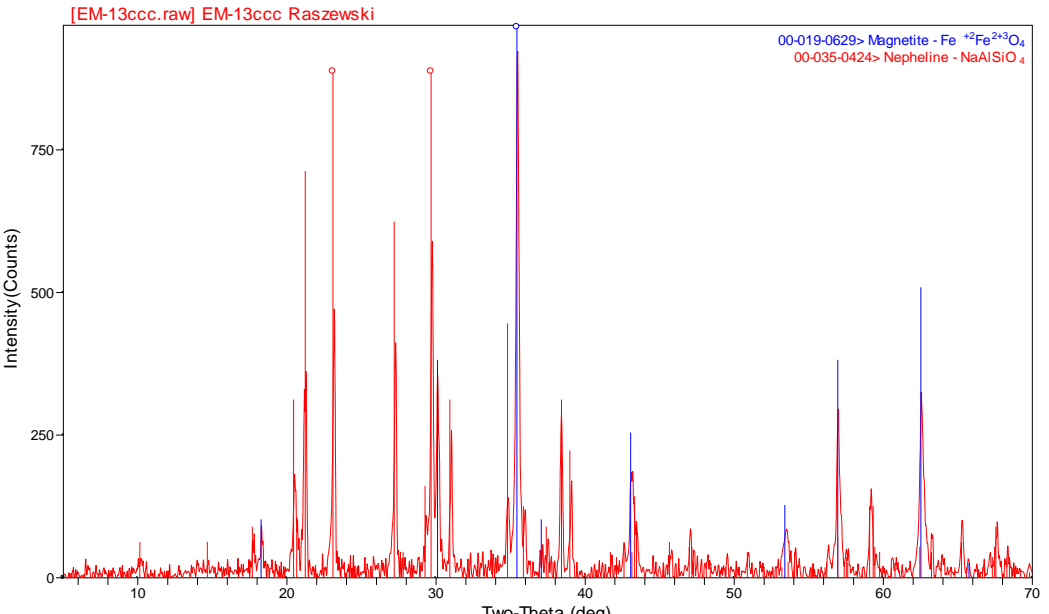

(b)

Figure 32. XRD patterns of (a) quenched and (b) cec EM-13.

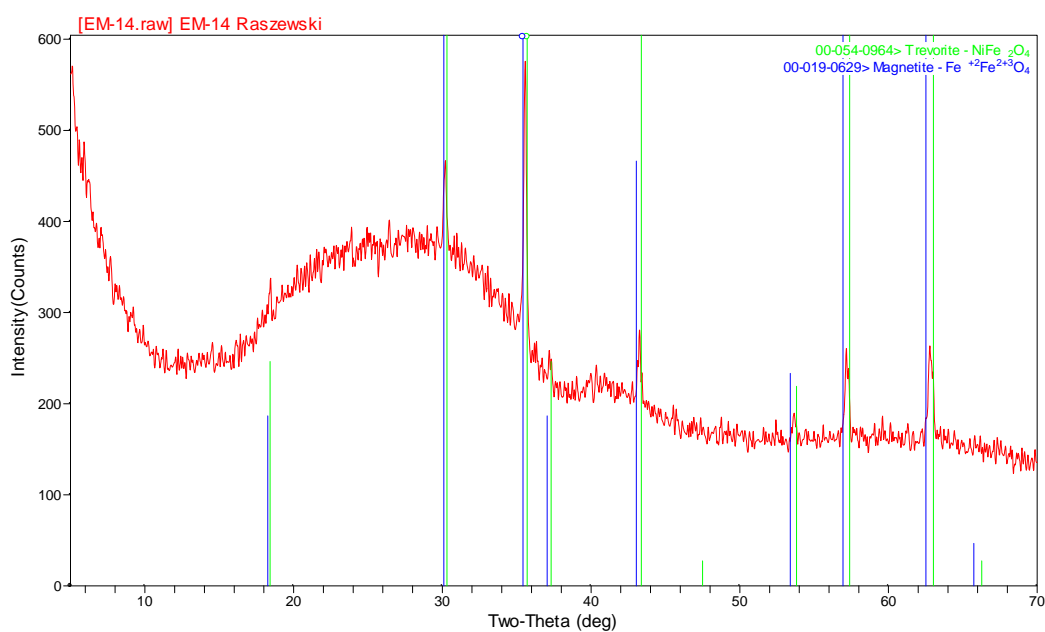

(a)

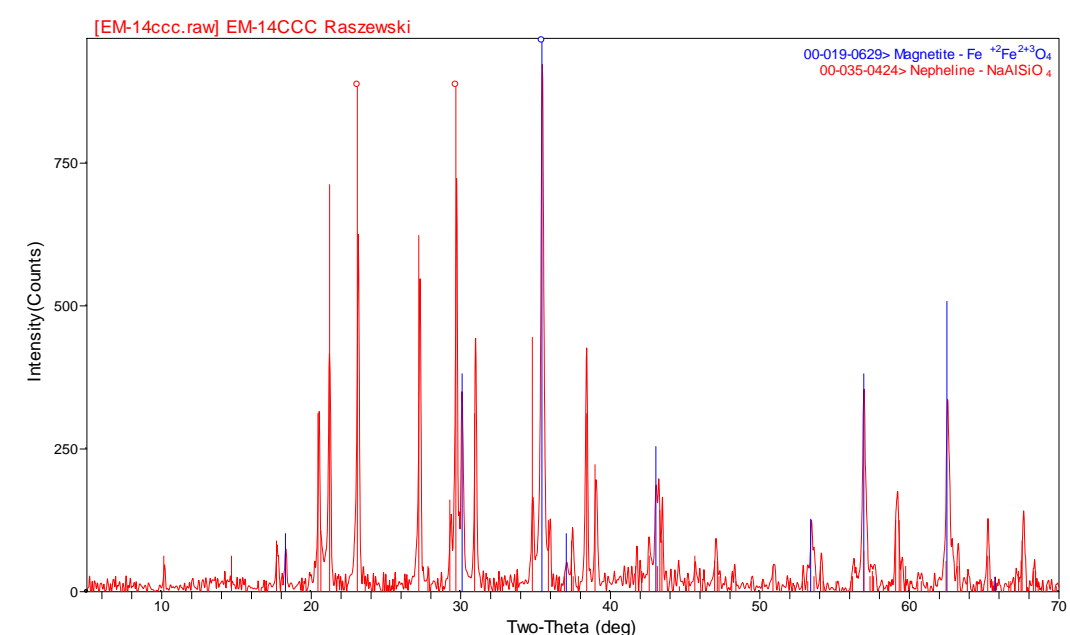

(b)

Figure 33. XRD patterns of (a) quenched and (b) cec EM-14. 
SRNL-STI-2009-00778, REVISION 0

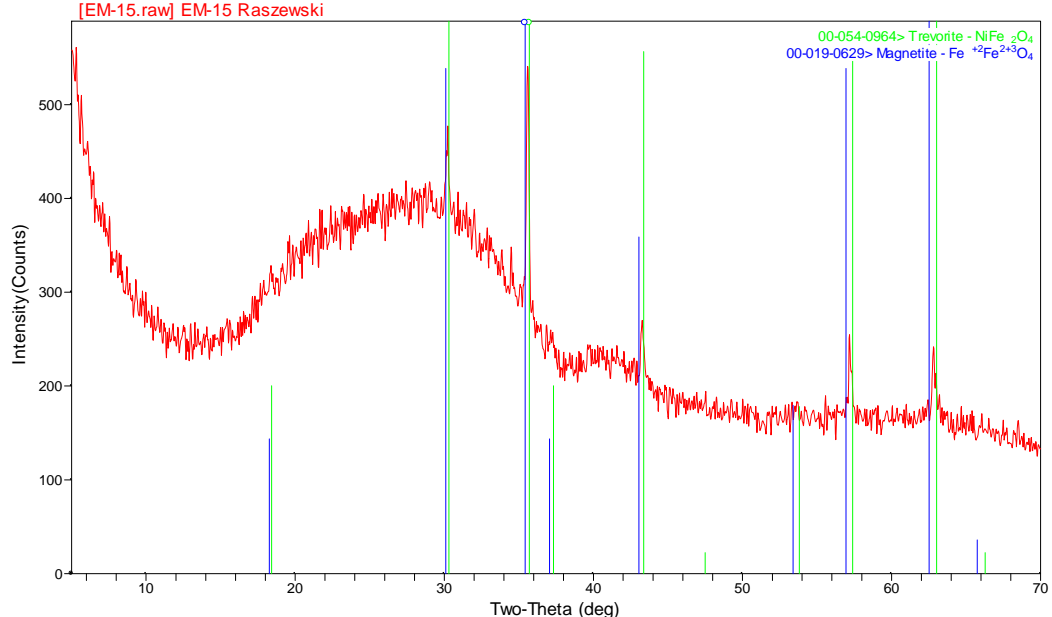

(a)

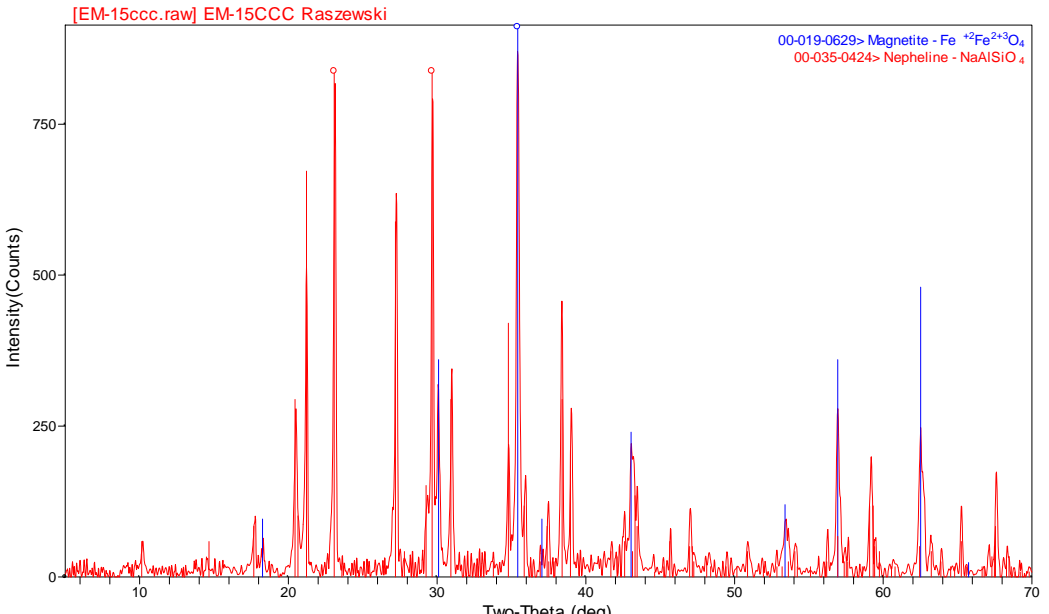

(b)

Figure 34. XRD patterns of (a) quenched and (b) cec EM-15.

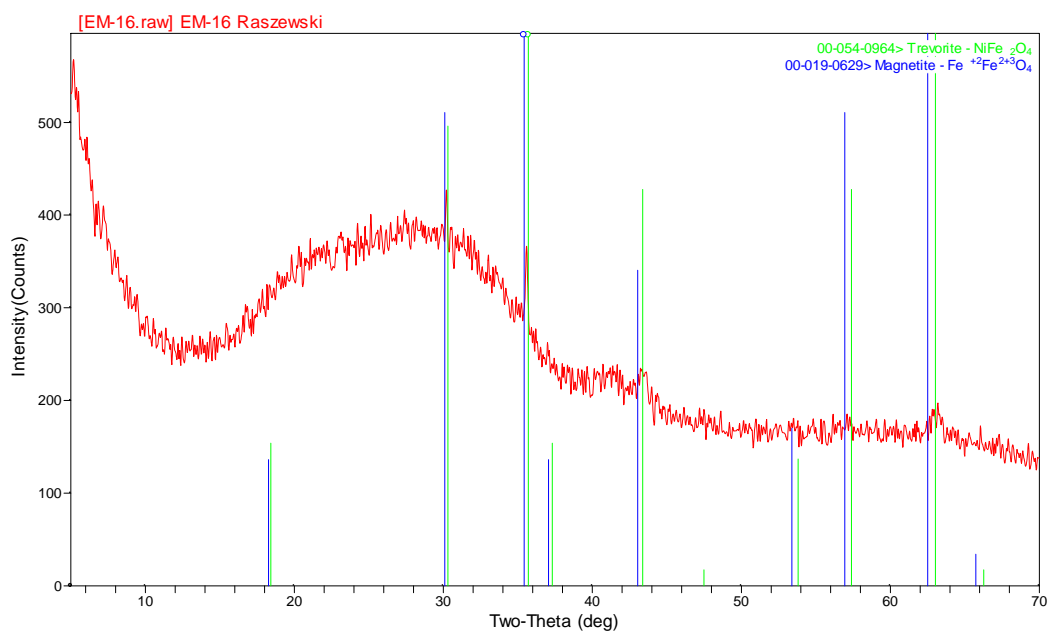

(a)

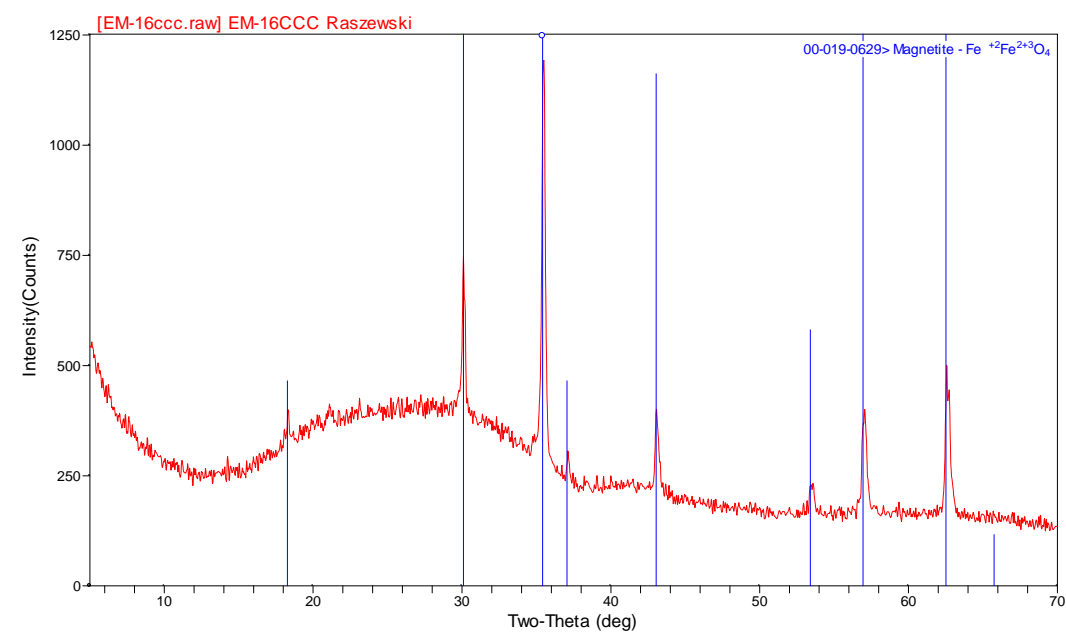

(b)

Figure 35. XRD patterns of (a) quenched and (b) cec EM-16. 
SRNL-STI-2009-00778, REVISION 0

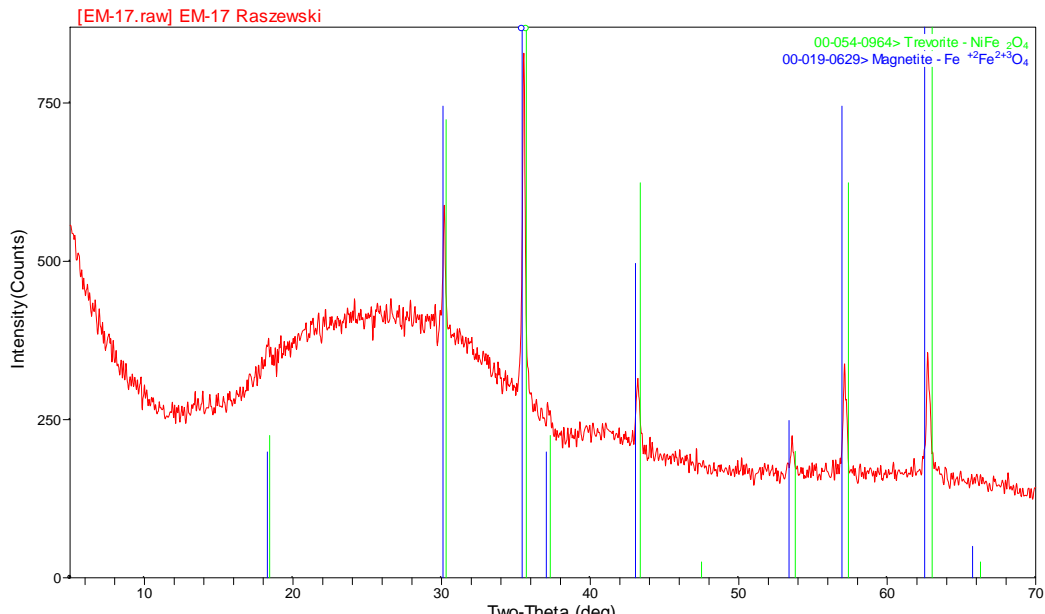

(a)

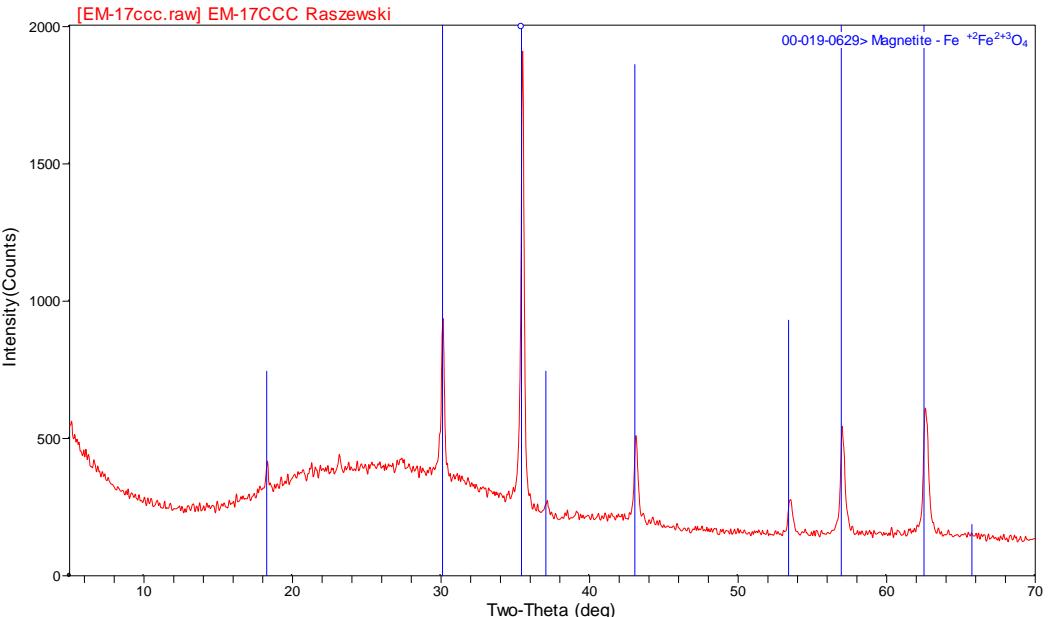

(b)

Figure 36. XRD patterns of (a) quenched and (b) cec EM-17.

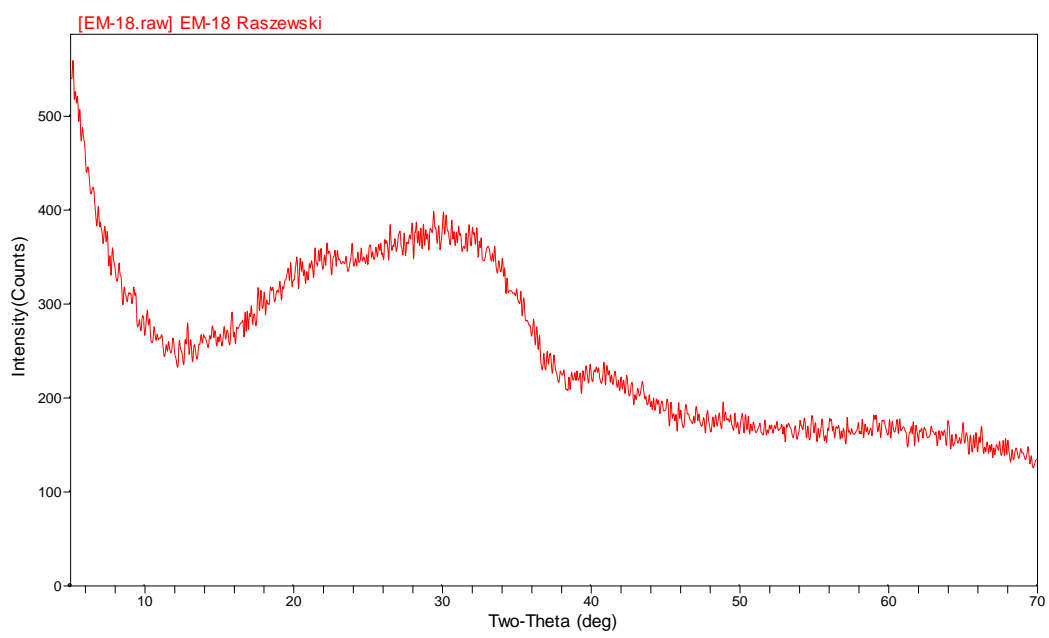

(a)

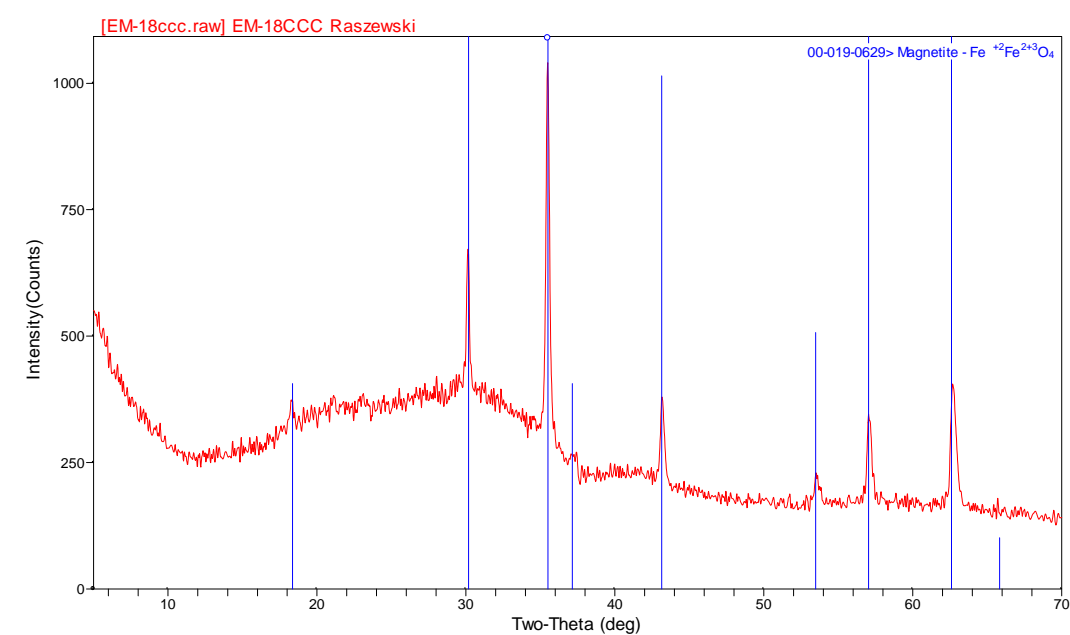

(b)

Figure 37. XRD patterns of (a) quenched and (b) cec EM-18. 
SRNL-STI-2009-00778, REVISION 0

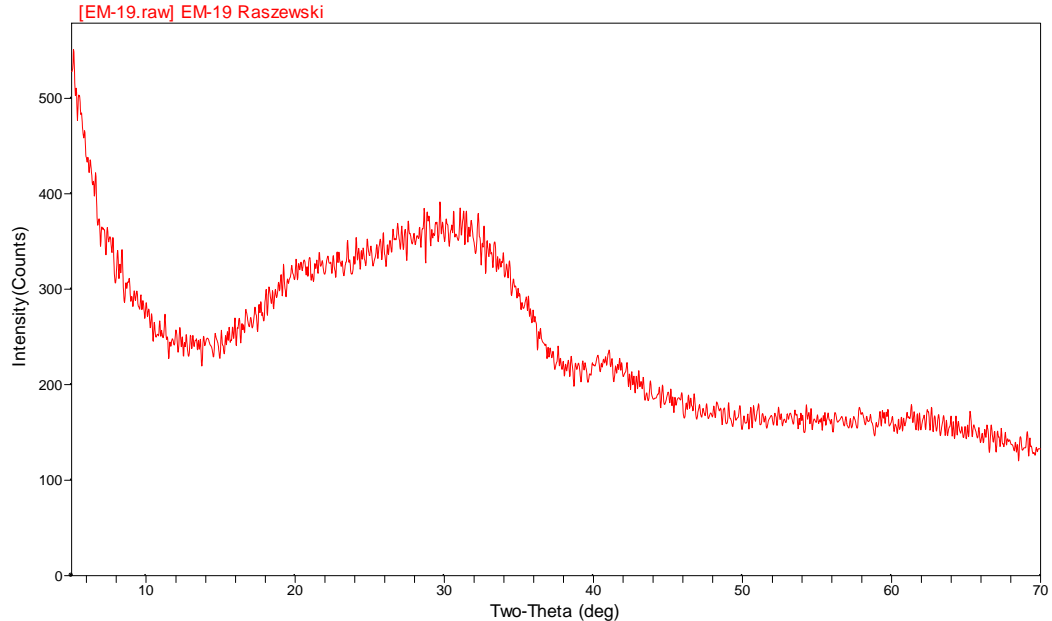

(a)

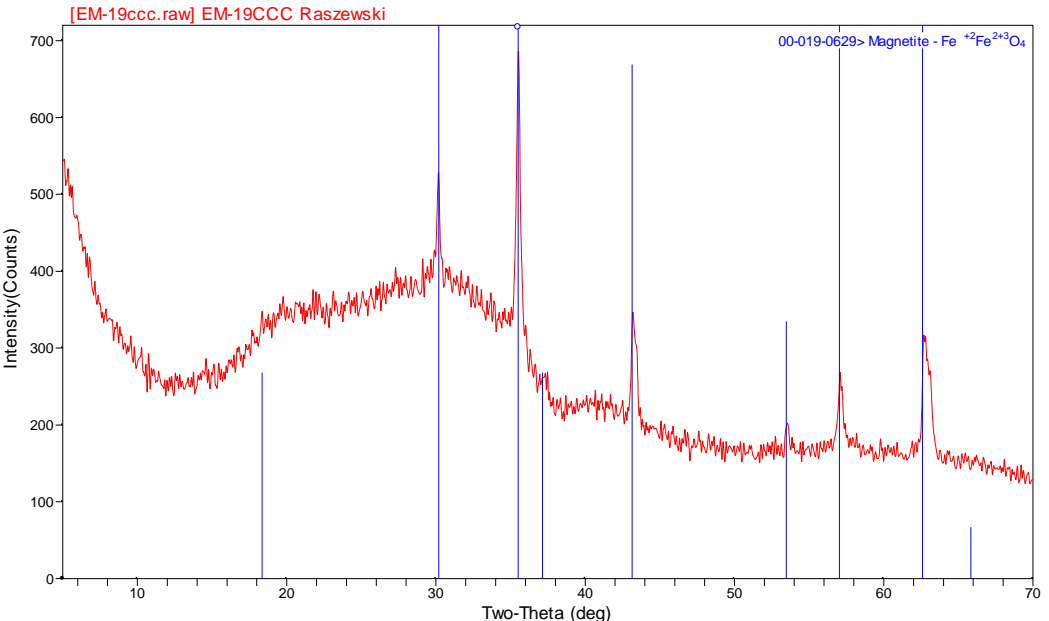

(b)

Figure 38. XRD patterns of (a) quenched and (b) cec EM-19.

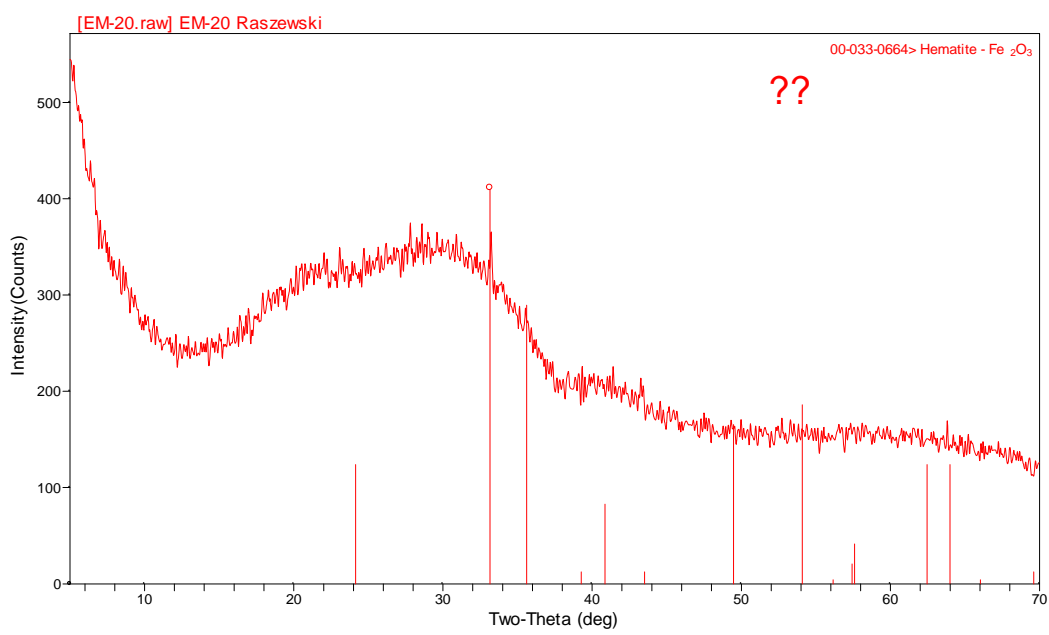

(a)

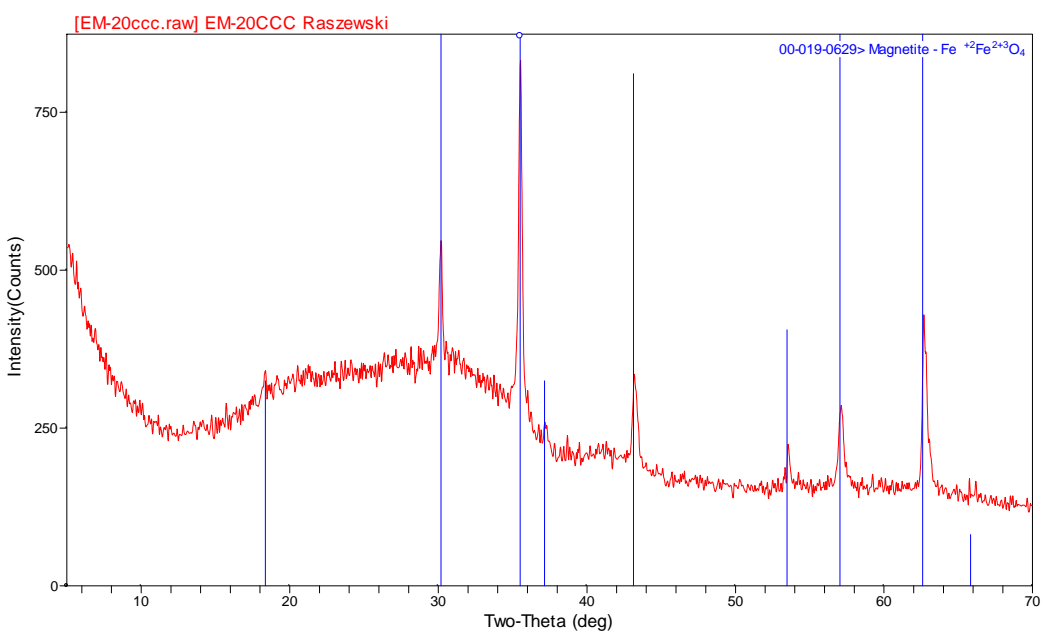

(b)

Figure 39. XRD patterns of (a) quenched and (b) cce EM-20. 
SRNL-STI-2009-00778, REVISION 0

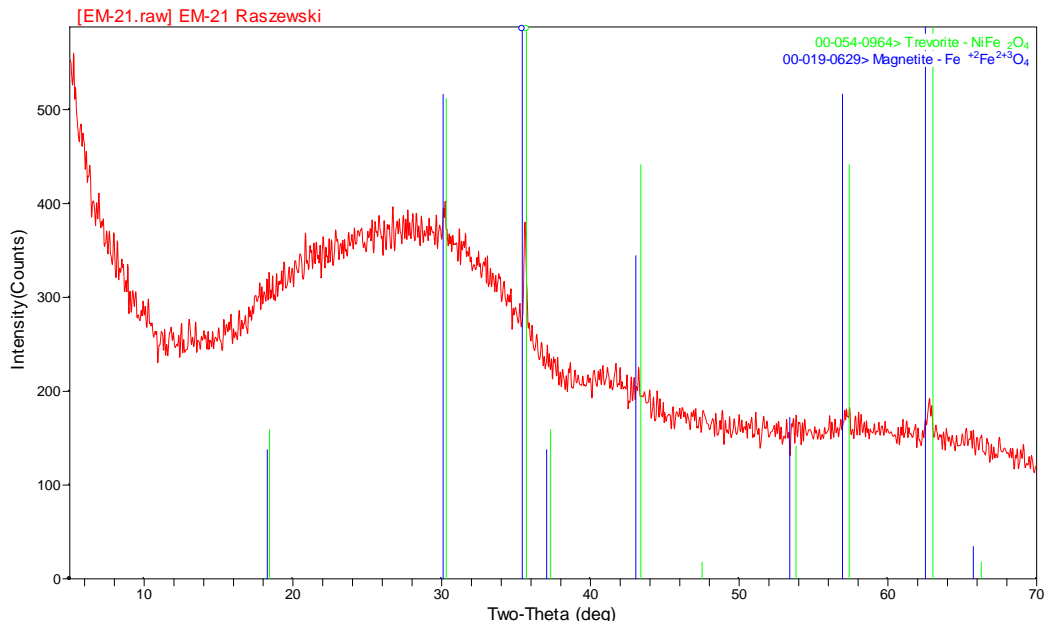

(a)

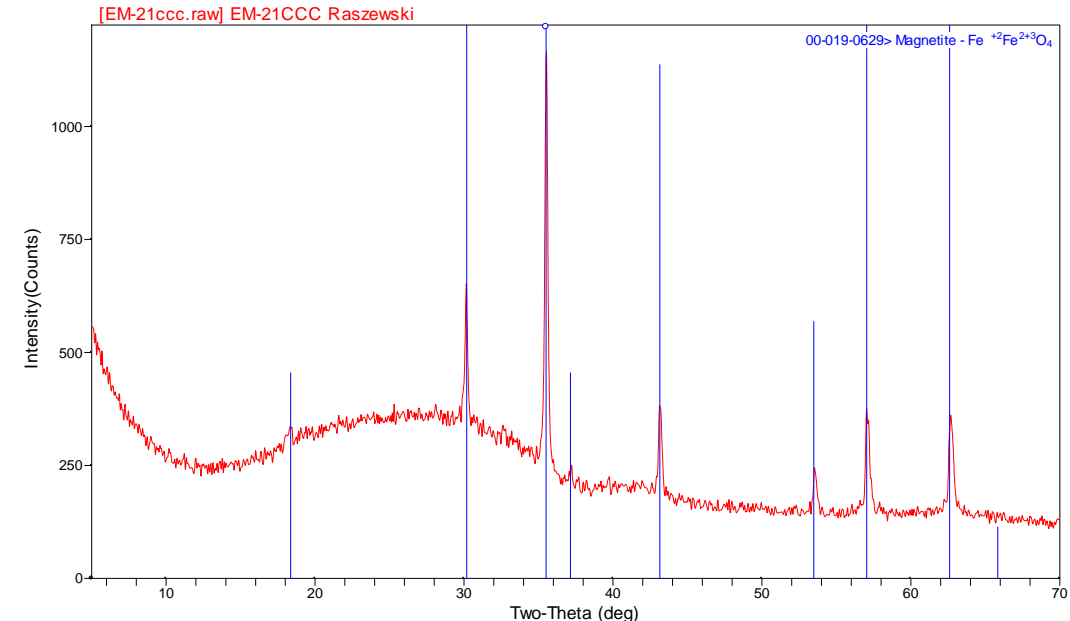

(b)

Figure 40. XRD patterns of (a) quenched and (b) cec EM-21.

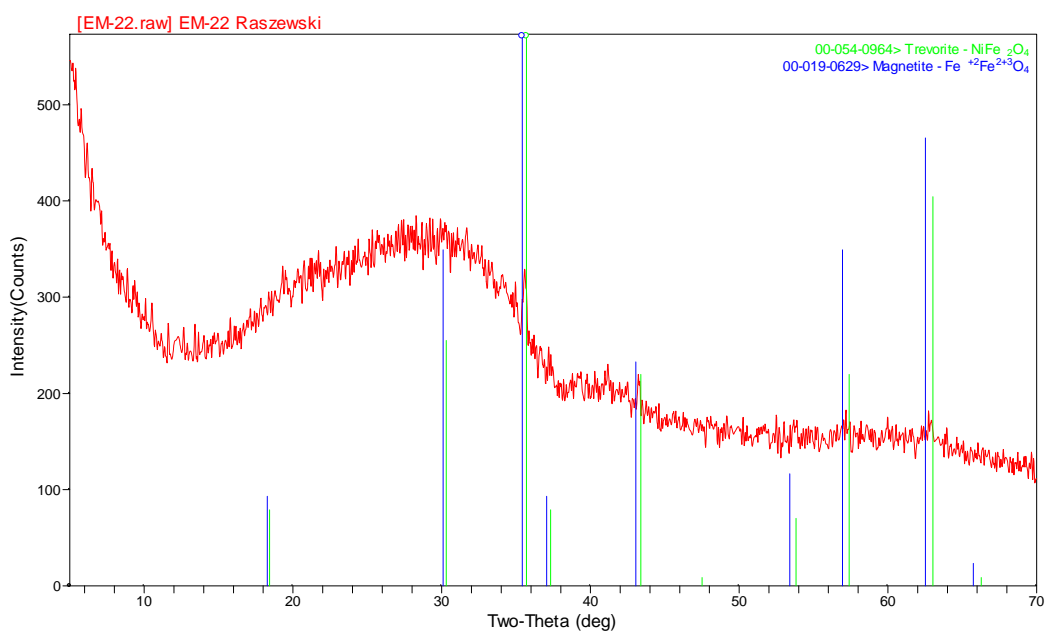

(a)

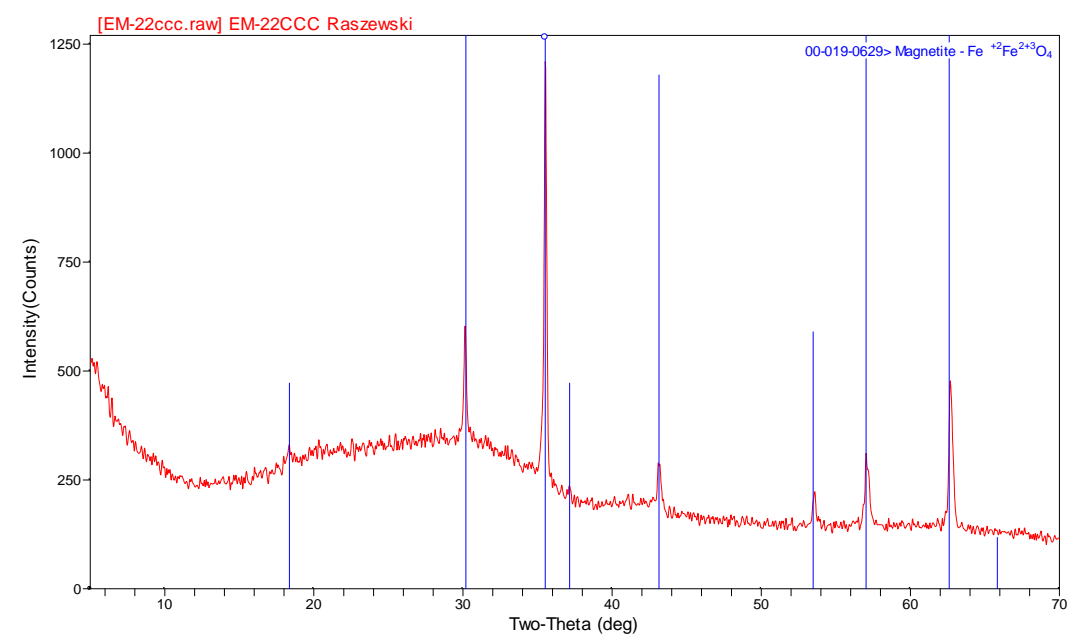

(b)

Figure 41. XRD patterns of (a) quenched and (b) cec EM-22. 
SRNL-STI-2009-00778, REVISION 0

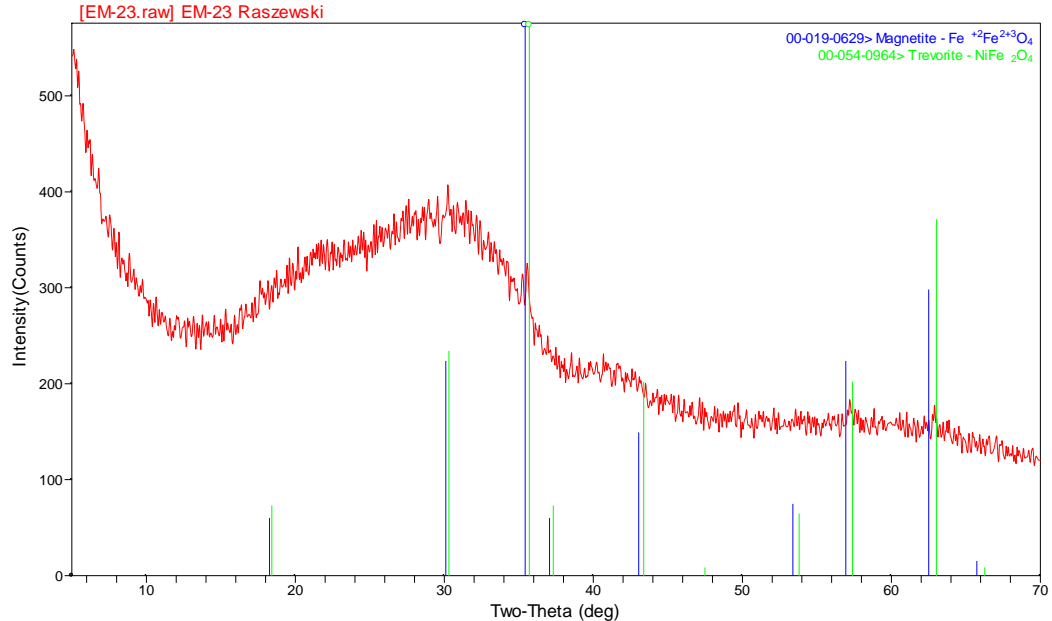

(a)

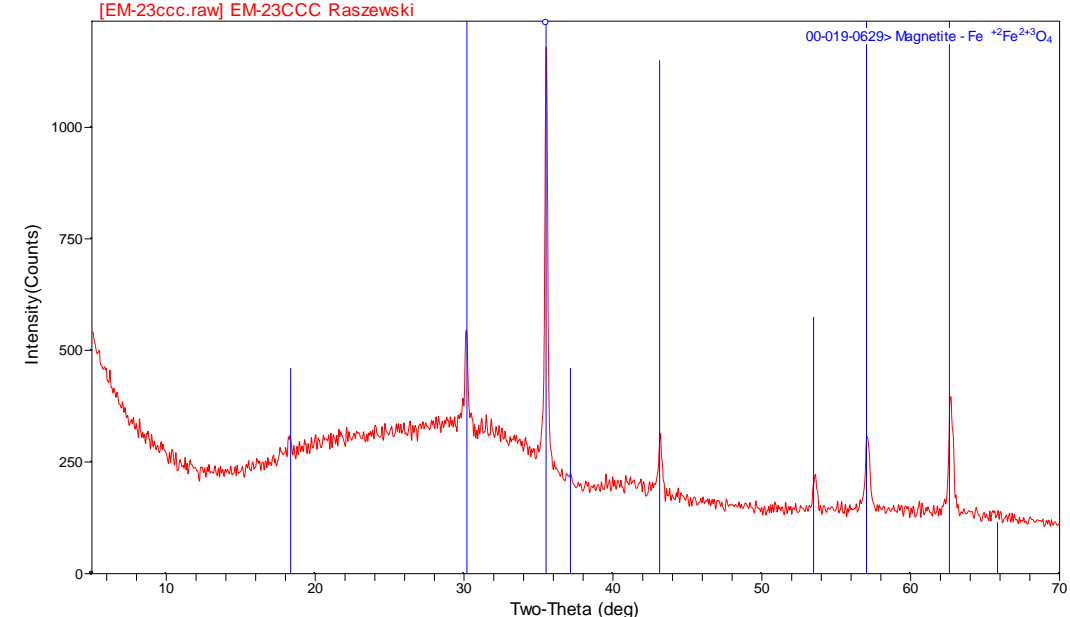

(b)

Figure 42. XRD patterns of (a) quenched and (b) cec EM-23.

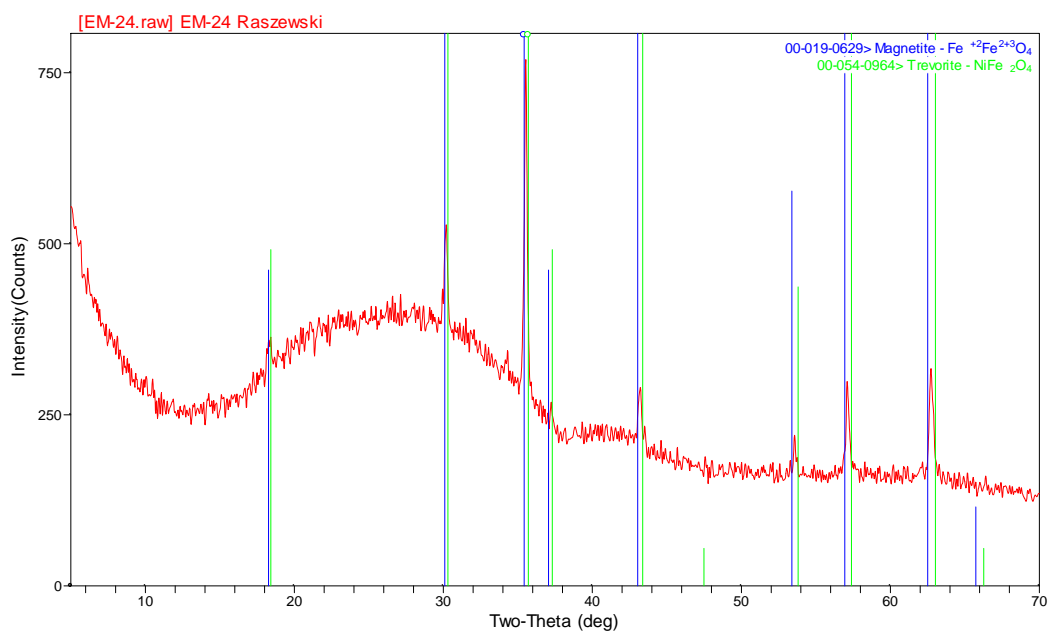

(a)

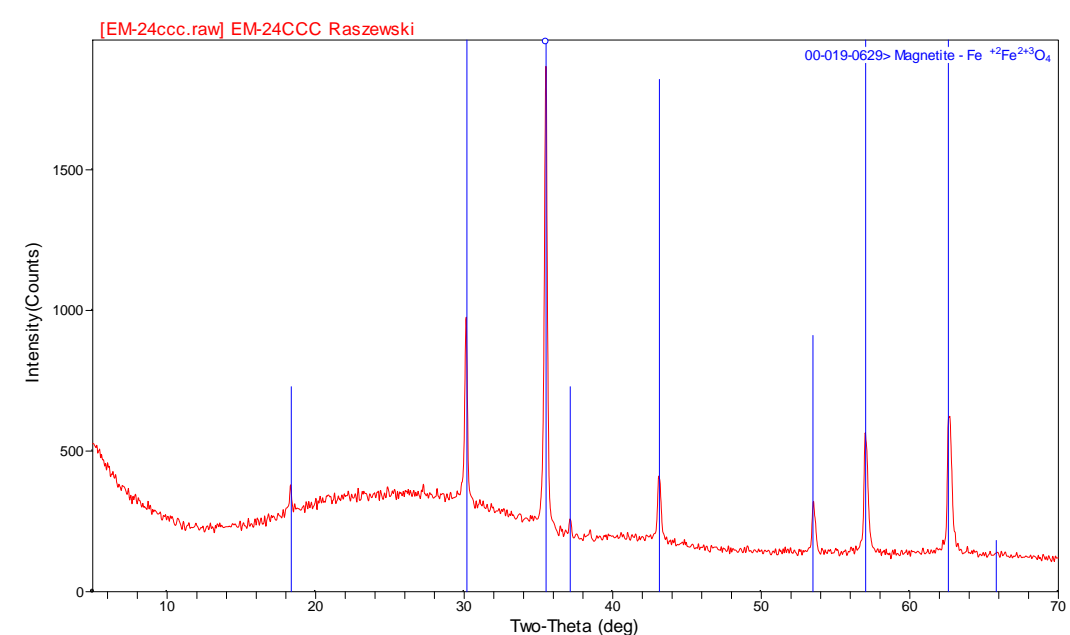

(b)

Figure 43. XRD patterns of (a) quenched and (b) cec EM-24. 
SRNL-STI-2009-00778, REVISION 0

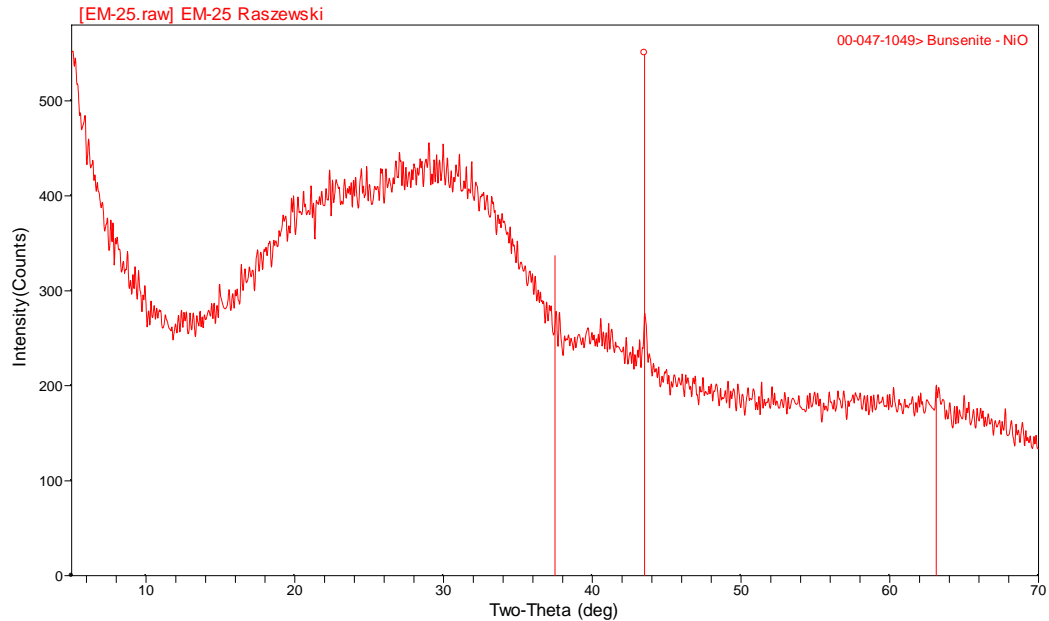

(a)

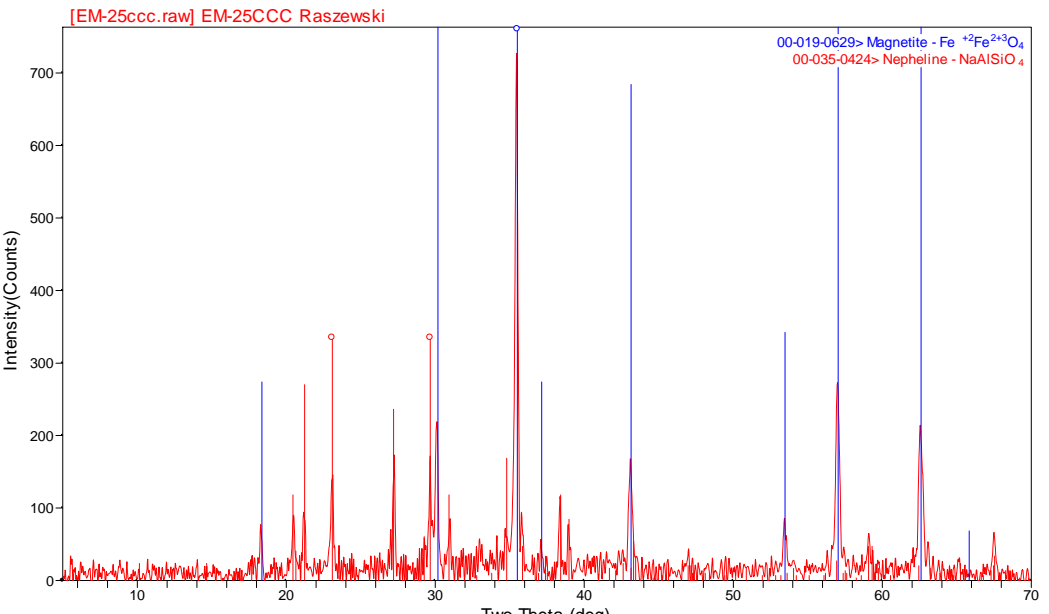

(b)

Figure 44. XRD patterns of (a) quenched and (b) cec EM-25.

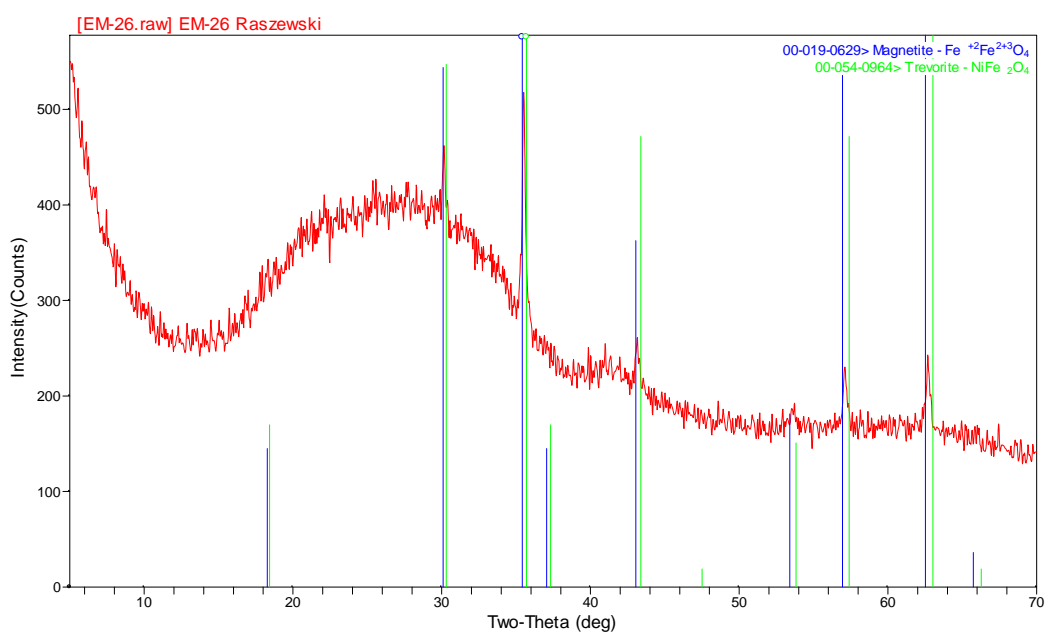

(a)

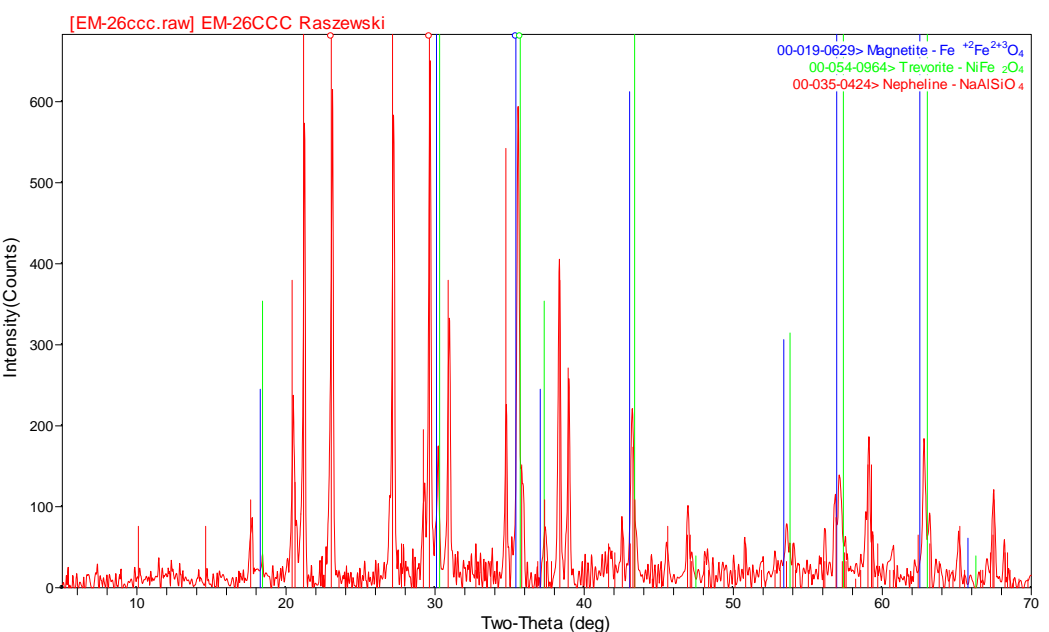

(b)

Figure 45. XRD patterns of (a) quenched and (b) cec EM-26. 
SRNL-STI-2009-00778, REVISION 0

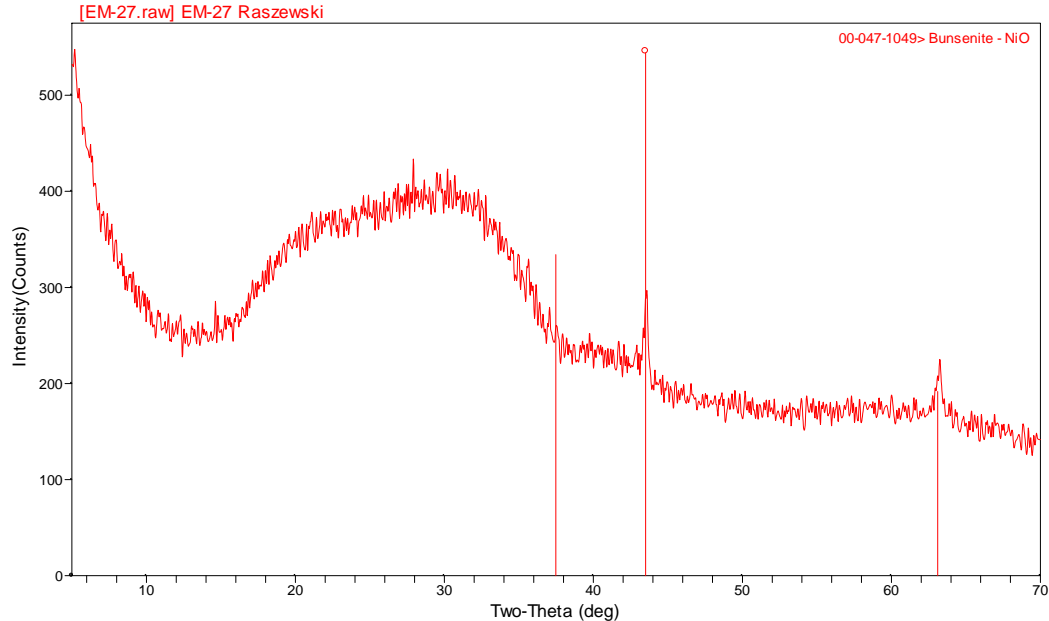

(a)

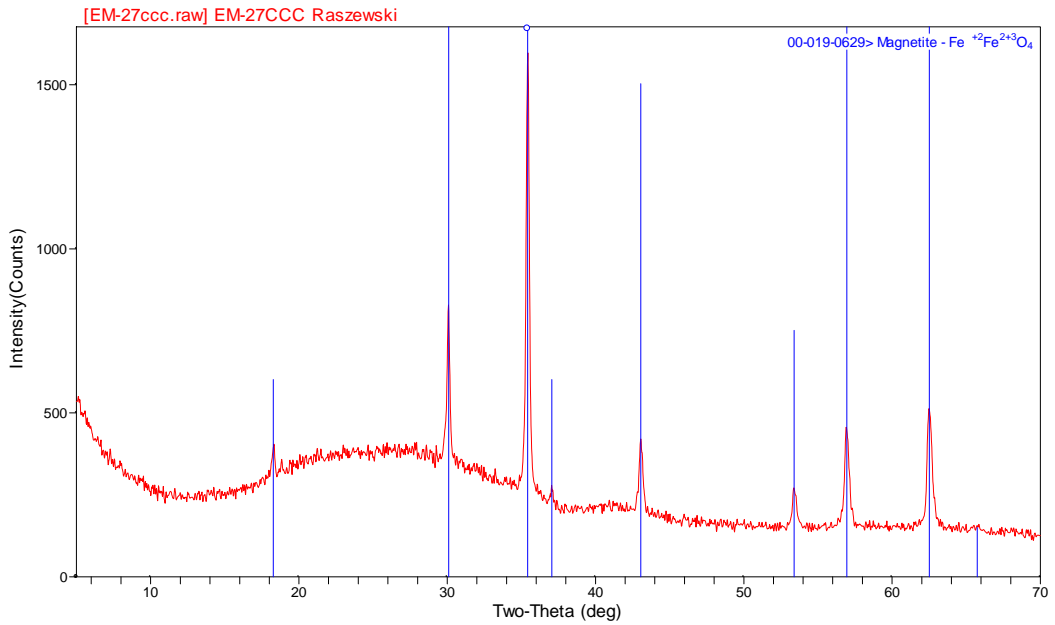

(b)

Figure 46. XRD patterns of (a) quenched and (b) cec EM-27.

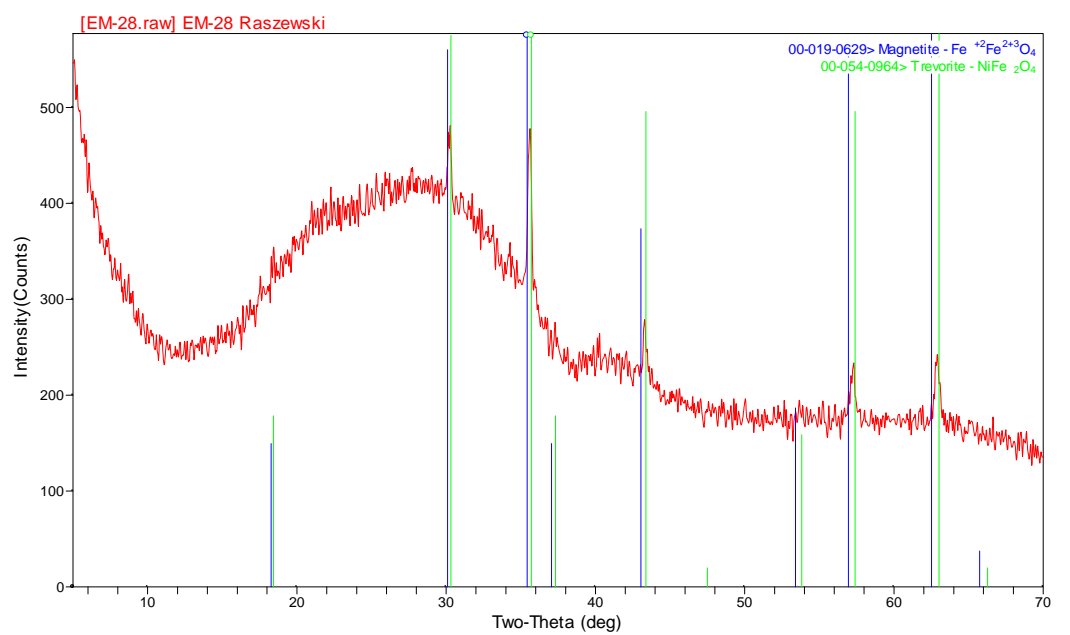

(a)

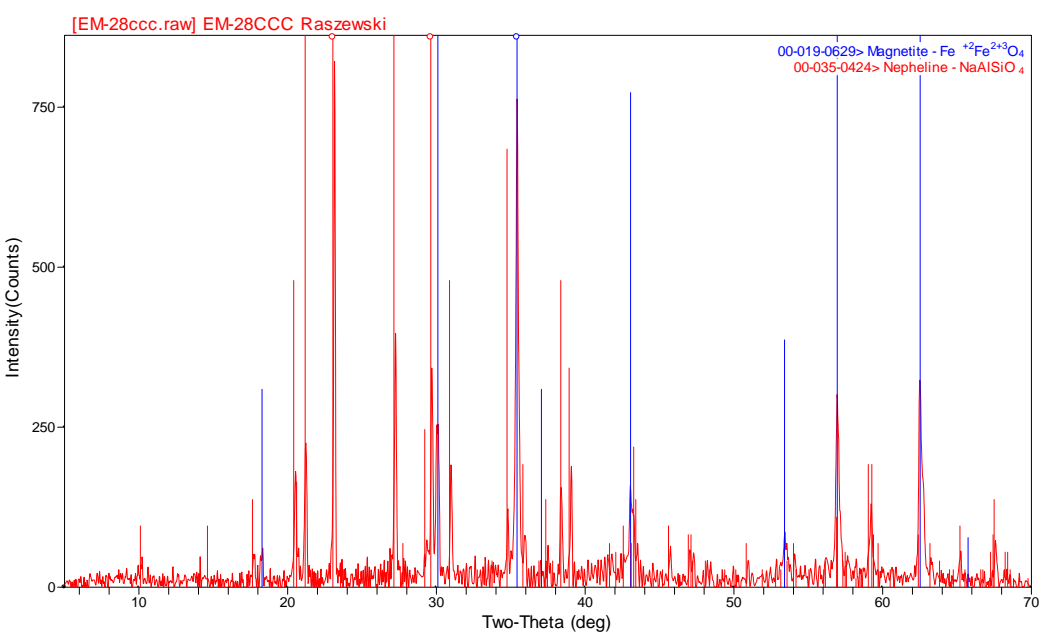

(b)

Figure 47. XRD patterns of (a) quenched and (b) ccc EM-28. 
SRNL-STI-2009-00778, REVISION 0

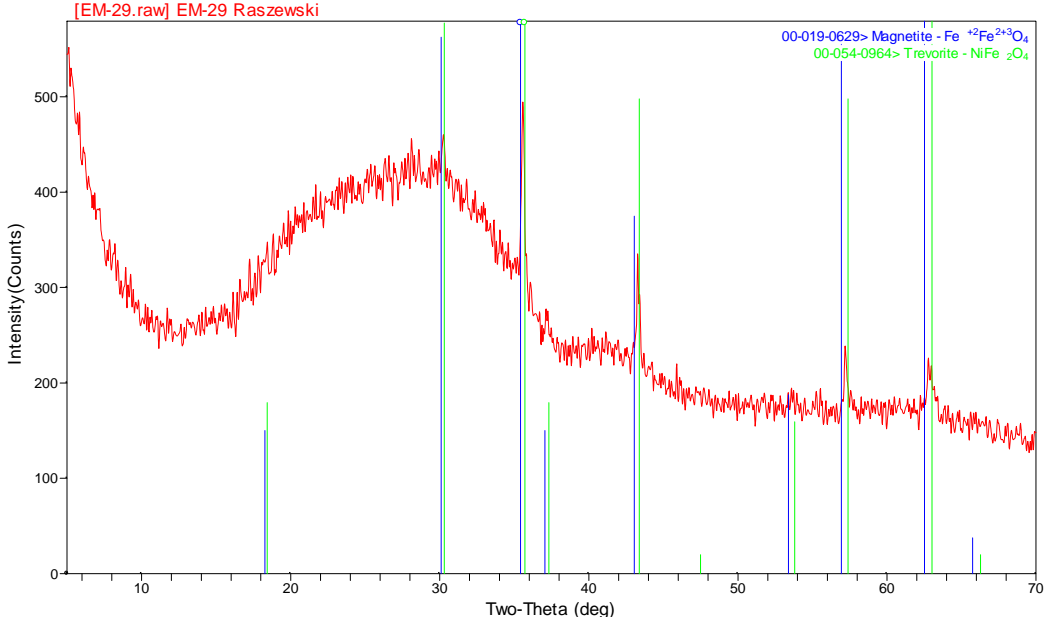

(a)

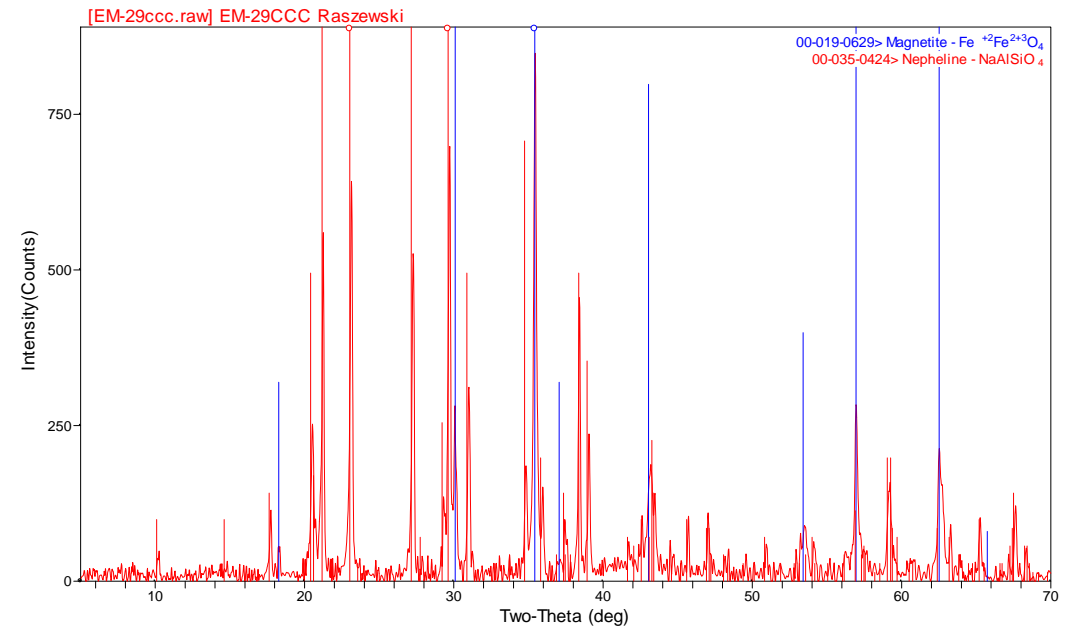

(b)

Figure 48. XRD patterns of (a) quenched and (b) cec EM-29.

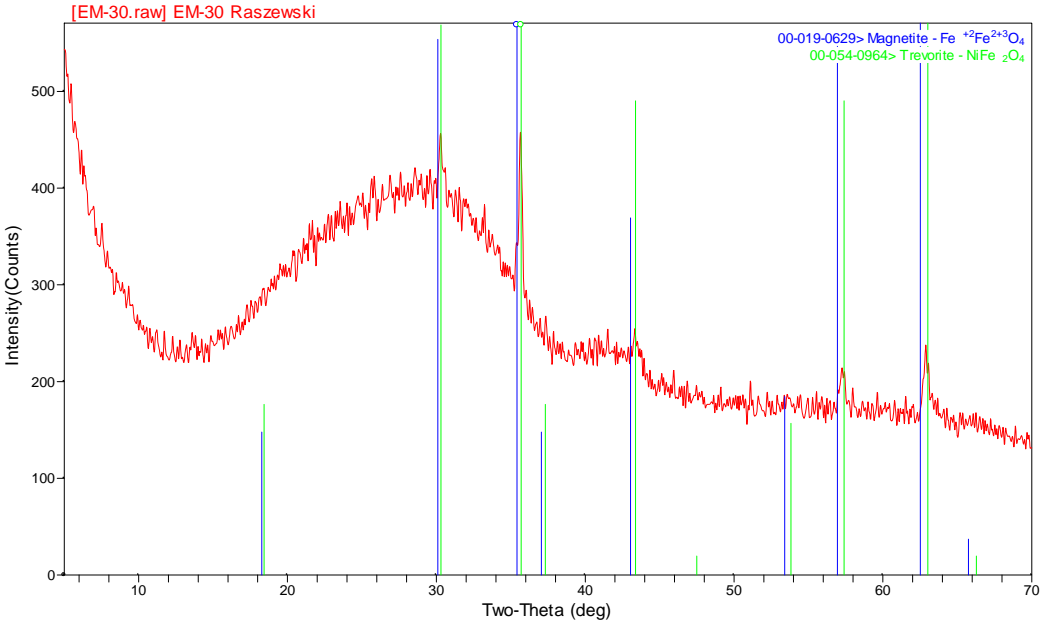

(a)

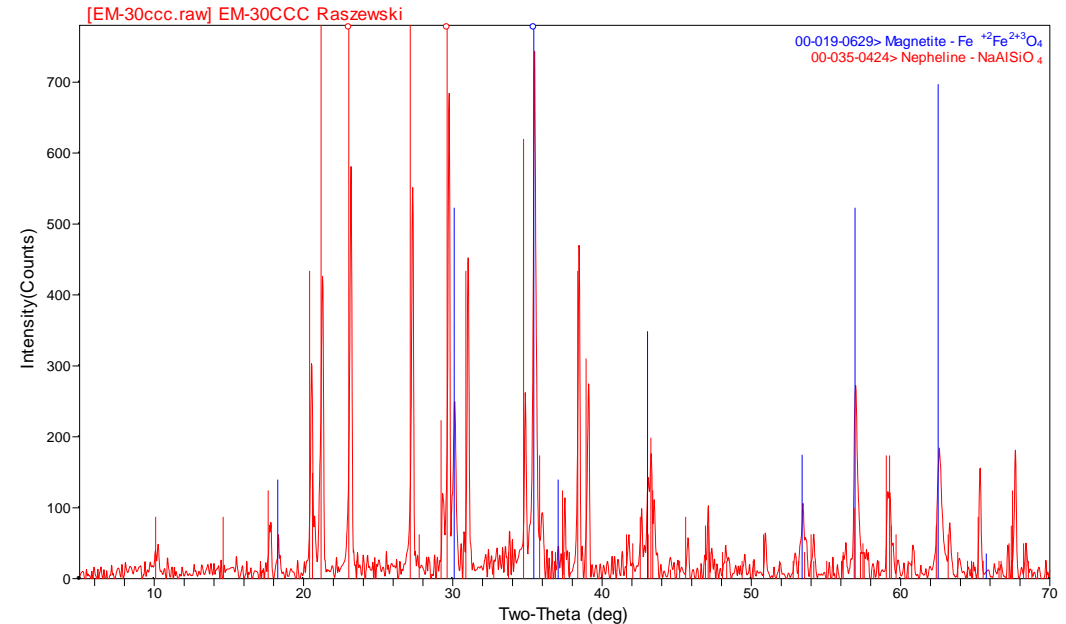

(b)

Figure 49. XRD patterns of (a) quenched and (b) cce EM-30. 


\subsubsection{A Statistical Review of the Matrix 2A PCT Results}

Table E1 in Appendix E provides the elemental leachate concentration measurements determined by the PSAL for the solution samples generated by the PCTs. Adjustments for detection limits and dilution factors are discussed in Section 5.1.4. None of the PCTs from these glasses indicated a solution-weight loss problem.

\subsubsection{Measurements in Analytical Sequence}

Exhibit E1 in Appendix E provides plots of the leachate concentrations in analytical sequence as generated by the PSAL for all of the data from all three sets of PCTs. No issues are observed in these plots.

\subsubsection{Results of the Multi-Element Solution Standard Samples}

Exhibit E2 in Appendix E provides analyses of measurements of the multi-element solution standard by analytical set/ICP-AES calibration block and an ANOVA investigation for each element of interest. A statistically significant (at a 5\% level) difference among the averages of these measurements was indicated for $\mathrm{Li}$ and $\mathrm{Na}$. No attempt was made to bias correct for these effects since averaging the ppm values for each set of triplicates helps to minimize the impact of any potential effects of the instrument.

Table 21 summarizes the average measurements and the reference values for the four elements of interest. The results indicate consistent and accurate measurements from the PSAL processes used to conduct these analyses. 
Table 21. Multi-Element Solution Standard Results of the Matrix 2A Glasses

\begin{tabular}{|c|c|c|c|c|c|}
\hline Set & Block & B (ppm) & Li (ppm) & $\mathrm{Na}(\mathrm{ppm})$ & Si (ppm) \\
\hline 1 & 1 & 21.5 & 10.0 & 82.7 & 52.7 \\
\hline 1 & 1 & 20.5 & 10.0 & 81.9 & 51.5 \\
\hline 1 & 1 & 19.9 & 10.0 & 84.0 & 52.1 \\
\hline 1 & 2 & 20.4 & 9.9 & 84.4 & 52.5 \\
\hline 1 & 2 & 19.2 & 10.0 & 85.1 & 52.6 \\
\hline 1 & 2 & 18.2 & 9.6 & 79.9 & 51.3 \\
\hline 1 & 3 & 22.0 & 10.2 & 83.4 & 51.9 \\
\hline 1 & 3 & 19.9 & 9.9 & 77.7 & 50.6 \\
\hline 1 & 3 & 20.4 & 10.1 & 80.6 & 51.5 \\
\hline \multicolumn{2}{|c|}{ Average } & 20.2 & 10.0 & 82.2 & 51.9 \\
\hline 2 & 1 & 21.4 & 9.8 & 81.7 & 50.1 \\
\hline 2 & 1 & 20.5 & 9.9 & 81.4 & 50.5 \\
\hline 2 & 1 & 20.1 & 9.6 & 78.7 & 49.3 \\
\hline 2 & 2 & 21.6 & 10.0 & 82.4 & 50.4 \\
\hline 2 & 2 & 19.9 & 9.9 & 80.7 & 50.2 \\
\hline 2 & 2 & 20.0 & 9.9 & 81.3 & 50.6 \\
\hline 2 & 3 & 21.3 & 9.9 & 83.0 & 51.6 \\
\hline 2 & 3 & 20.3 & 9.9 & 82.5 & 51.7 \\
\hline 2 & 3 & 20.1 & 9.9 & 82.7 & 51.6 \\
\hline \multicolumn{2}{|c|}{ Average } & 20.6 & 9.9 & 81.6 & 50.7 \\
\hline 3 & 1 & 21.2 & 10.1 & 83.1 & 51.1 \\
\hline 3 & 1 & 20.2 & 9.9 & 82.7 & 49.5 \\
\hline 3 & 1 & 20.7 & 10.2 & 86.4 & 50.1 \\
\hline 3 & 2 & 21.0 & 10.2 & 81.9 & 51.3 \\
\hline 3 & 2 & 20.2 & 10.1 & 80.8 & 50.8 \\
\hline 3 & 2 & 20.4 & 10.2 & 81.6 & 51.6 \\
\hline 3 & 3 & 20.3 & 10.0 & 82.8 & 50.4 \\
\hline 3 & 3 & 20.2 & 10.1 & 83.8 & 50.6 \\
\hline 3 & 3 & 22.9 & 10.1 & 82.1 & 50.8 \\
\hline \multicolumn{2}{|c|}{ Average } & 20.8 & 10.1 & 82.8 & 50.7 \\
\hline \multicolumn{2}{|c|}{ Grand Average } & 20.5 & 10.0 & 82.2 & 51.1 \\
\hline \multicolumn{2}{|c|}{ Reference Value } & 20 & 10 & 81 & 50 \\
\hline \multicolumn{2}{|c|}{$\%$ difference } & $2.7 \%$ & $-0.2 \%$ & $1.5 \%$ & $2.1 \%$ \\
\hline
\end{tabular}

\subsubsection{Measurements by Glass Identifier}

Exhibit E3 in Appendix E provides plots of the leachate concentrations for each type of submitted sample by analytical set: the study glasses by heat treatment and the standards (EA, ARM, the multi-element solution standard, and blanks). Limited scatter is observed in the triplicate values for the analytes for almost all of the glasses. An exception is the scatter in the replicate Na PCTs for the quenched version of EM-06.

\subsubsection{Normalized PCT Results}

PCT leachate concentrations were normalized using the method described in Section 5.1.4.4. Exhibit E4 in Appendix E provides scatter plots for these results and offers an opportunity to investigate the consistency in the leaching across the elements for the glasses of this study. All combinations of the normalizations of the PCTs (i.e., those generated using the target, measured, and bias-corrected compositional views) and both heat treatments are represented in the series of 
scatter plots. Consistency in the leaching across the elements is typically demonstrated by a high degree of linear correlation among the values for pairs of these elements. The smallest correlation in this plot is that for $\mathrm{Li}$ and $\mathrm{Si}$, with a value of $\sim 88.7 \%$. It should be noted that this value is lower than values typically observed.

Table 22 summarizes the normalized PCTs for the glasses of this study. The PCTs are listed by heat treatment and compositional view for each glass. 
SRNL-STI-2009-00778, REVISION 0

Table 22. Normalized PCT Results of the Matrix 2A Glasses

\begin{tabular}{|c|c|c|c|c|c|c|c|c|c|c|c|}
\hline Set & Glass ID & $\begin{array}{c}\text { Heat } \\
\text { Treatment }\end{array}$ & $\begin{array}{l}\text { Comp } \\
\text { View }\end{array}$ & $\begin{array}{c}\log \mathrm{NL} \\
{[\mathrm{B}(\mathrm{g} / \mathrm{L})]}\end{array}$ & $\begin{array}{c}\log \mathrm{NL} \\
{[\mathrm{Li}(\mathrm{g} / \mathrm{L})]}\end{array}$ & \begin{tabular}{|c|}
$\log \mathrm{NL}$ \\
{$[\mathrm{Na}(\mathrm{g} / \mathrm{L})]$}
\end{tabular} & \begin{tabular}{|c|}
$\log \mathrm{NL}$ \\
{$[\mathrm{Si}(\mathrm{g} / \mathrm{L})]$}
\end{tabular} & $\begin{array}{c}\mathrm{NL} \\
\mathrm{B}(\mathrm{g} / \mathrm{L})\end{array}$ & $\begin{array}{c}\mathrm{NL} \\
\mathrm{Li}(\mathrm{g} / \mathrm{L})\end{array}$ & \begin{tabular}{|c|}
$\mathrm{NL}$ \\
$\mathrm{Na}(\mathrm{g} / \mathrm{L})$ \\
\end{tabular} & $\begin{array}{c}\mathrm{NL} \\
\mathrm{Si}(\mathrm{g} / \mathrm{L}) \\
\end{array}$ \\
\hline 1 & ARM & ref & reference & -0.35 & -0.28 & -0.34 & -0.57 & 0.45 & 0.53 & 0.45 & 0.27 \\
\hline 2 & ARM & ref & reference & -0.33 & -0.27 & -0.33 & -0.57 & 0.47 & 0.54 & 0.47 & 0.27 \\
\hline 3 & ARM & ref & reference & -0.29 & -0.23 & -0.31 & -0.56 & 0.51 & 0.58 & 0.50 & 0.28 \\
\hline 1 & EA & ref & reference & 1.21 & 0.94 & 1.09 & 0.58 & 16.06 & 8.76 & 12.29 & 3.80 \\
\hline 2 & EA & ref & reference & 1.19 & 0.94 & 1.08 & 0.57 & 15.47 & 8.79 & 11.94 & 3.76 \\
\hline 3 & EA & ref & reference & 1.24 & 0.96 & 1.12 & 0.59 & 17.49 & 9.21 & 13.16 & 3.86 \\
\hline 1 & \multirow{6}{*}{ EM-01 } & \multirow{3}{*}{ quenched } & Measured & -0.05 & -0.12 & 0.00 & -0.27 & 0.89 & 0.76 & 0.99 & 0.53 \\
\hline 1 & & & \begin{tabular}{|l|} 
Measured bc \\
\end{tabular} & -0.03 & -0.11 & 0.00 & -0.30 & 0.93 & 0.78 & 1.01 & 0.50 \\
\hline 1 & & & Target & -0.04 & -0.12 & 0.00 & -0.27 & 0.92 & 0.76 & 0.99 & 0.54 \\
\hline 1 & & \multirow{3}{*}{$\mathrm{ccc}$} & Measured & -0.06 & -0.08 & 0.00 & -0.25 & 0.86 & 0.82 & 1.01 & 0.56 \\
\hline 1 & & & Measured bc & -0.04 & -0.07 & 0.01 & -0.27 & 0.90 & 0.84 & 1.03 & 0.53 \\
\hline 1 & & & Target & -0.05 & -0.08 & 0.00 & -0.24 & 0.90 & 0.83 & 1.01 & 0.57 \\
\hline 1 & \multirow{6}{*}{ EM-02 } & \multirow{3}{*}{ quenched } & Measured & -0.20 & -0.19 & -0.14 & -0.35 & 0.63 & 0.65 & 0.73 & 0.45 \\
\hline 1 & & & \begin{tabular}{|l|} 
Measured bc \\
\end{tabular} & -0.18 & -0.18 & -0.13 & -0.37 & 0.66 & 0.67 & 0.74 & 0.42 \\
\hline 1 & & & Target & -0.19 & -0.19 & -0.14 & -0.35 & 0.65 & 0.65 & 0.73 & 0.45 \\
\hline 1 & & \multirow{3}{*}{$\mathrm{ccc}$} & Measured & 0.37 & 0.35 & 0.12 & -0.12 & 2.32 & 2.21 & 1.32 & 0.76 \\
\hline 1 & & & Measured bc & 0.38 & 0.36 & 0.13 & -0.14 & 2.42 & 2.27 & 1.34 & 0.72 \\
\hline 1 & & & Target & 0.38 & 0.34 & 0.12 & -0.12 & 2.38 & 2.19 & 1.32 & 0.77 \\
\hline 1 & \multirow{6}{*}{ EM-03 } & \multirow{3}{*}{ quenched } & Measured & 0.04 & -0.02 & 0.15 & -0.16 & 1.11 & 0.96 & 1.40 & 0.69 \\
\hline 1 & & & \begin{tabular}{|l|} 
Measured bc \\
\end{tabular} & 0.06 & -0.01 & 0.15 & -0.18 & 1.15 & 0.98 & 1.42 & 0.66 \\
\hline 1 & & & \begin{tabular}{|l|} 
Target \\
\end{tabular} & 0.06 & -0.02 & 0.14 & -0.16 & 1.14 & 0.95 & 1.38 & 0.69 \\
\hline 1 & & \multirow{3}{*}{$\mathrm{ccc}$} & Measured & 0.00 & 0.04 & 0.18 & -0.11 & 1.01 & 1.09 & 1.52 & 0.78 \\
\hline 1 & & & Measured bc & 0.02 & 0.05 & 0.19 & -0.13 & 1.05 & 1.12 & 1.53 & 0.74 \\
\hline 1 & & & Target & 0.02 & 0.03 & 0.17 & -0.11 & 1.04 & 1.08 & 1.49 & 0.77 \\
\hline 1 & \multirow{6}{*}{ EM-04 } & \multirow{3}{*}{ quenched } & Measured & 0.12 & 0.06 & 0.21 & -0.08 & 1.32 & 1.14 & 1.62 & 0.84 \\
\hline 1 & & & \begin{tabular}{|l|} 
Measured bc \\
\end{tabular} & 0.13 & 0.06 & 0.21 & -0.11 & 1.34 & 1.14 & 1.64 & 0.78 \\
\hline 1 & & & Target & 0.13 & 0.05 & 0.20 & -0.09 & 1.36 & 1.12 & 1.58 & 0.82 \\
\hline 1 & & \multirow{3}{*}{$\mathrm{ccc}$} & Measured & 0.05 & 0.06 & 0.19 & -0.08 & 1.13 & 1.15 & 1.55 & 0.83 \\
\hline 1 & & & \begin{tabular}{|l|} 
Measured bc \\
\end{tabular} & 0.06 & 0.06 & 0.20 & -0.11 & 1.14 & 1.15 & 1.57 & 0.78 \\
\hline 1 & & & Target & 0.06 & 0.05 & 0.18 & -0.09 & 1.16 & 1.12 & 1.52 & 0.81 \\
\hline 1 & \multirow{6}{*}{ EM-05 } & & Measured & 0.12 & 0.04 & 0.15 & -0.13 & 1.30 & 1.10 & 1.42 & 0.75 \\
\hline 1 & & quenched & Measured bc & 0.12 & 0.04 & 0.16 & -0.15 & 1.32 & 1.10 & 1.44 & 0.70 \\
\hline 1 & & & Target & 0.12 & 0.04 & 0.14 & -0.13 & 1.33 & 1.09 & 1.40 & 0.74 \\
\hline 1 & & & Measured & 0.05 & 0.05 & 0.15 & -0.10 & 1.13 & 1.13 & 1.42 & 0.79 \\
\hline 1 & & $\mathrm{ccc}$ & \begin{tabular}{|l|} 
Measured bc \\
\end{tabular} & 0.06 & 0.06 & 0.16 & -0.13 & 1.14 & 1.14 & 1.44 & 0.74 \\
\hline 1 & & & Target & 0.06 & 0.05 & 0.14 & -0.11 & 1.16 & 1.12 & 1.39 & 0.78 \\
\hline 1 & & & Measured & 0.05 & 0.01 & -0.29 & -0.19 & 1.13 & 1.02 & 0.52 & 0.64 \\
\hline 1 & & quenched & \begin{tabular}{|l|} 
Measured bc \\
\end{tabular} & 0.06 & 0.01 & -0.28 & -0.22 & 1.14 & 1.03 & 0.53 & 0.60 \\
\hline 1 & FM-O & & Target & 0.05 & 0.00 & -0.28 & -0.20 & 1.13 & 1.01 & 0.53 & 0.64 \\
\hline 1 & EM-06 & & Measured & -0.05 & -0.06 & 0.01 & -0.21 & 0.88 & 0.87 & 1.03 & 0.61 \\
\hline 1 & & $\mathrm{ccc}$ & Measured bc & -0.05 & -0.06 & 0.02 & -0.24 & 0.89 & 0.88 & 1.04 & 0.58 \\
\hline 1 & & & \begin{tabular}{|l|} 
Target \\
\end{tabular} & -0.05 & -0.07 & 0.02 & -0.22 & 0.88 & 0.86 & 1.04 & 0.61 \\
\hline 1 & & & Measured & 0.17 & 0.12 & 0.18 & -0.17 & 1.49 & 1.33 & 1.50 & 0.68 \\
\hline 1 & & quenched & \begin{tabular}{|l|} 
Measured bc \\
\end{tabular} & 0.18 & 0.12 & 0.18 & -0.19 & 1.50 & 1.33 & 1.51 & 0.64 \\
\hline 1 & F 07 & & Target & 0.19 & 0.12 & 0.17 & -0.17 & 1.54 & 1.33 & 1.49 & 0.68 \\
\hline 1 & EM-07 & & Measured & -0.05 & -0.01 & 0.06 & -0.24 & 0.89 & 0.99 & 1.15 & 0.57 \\
\hline 1 & & $\mathrm{ccc}$ & \begin{tabular}{|l|} 
Measured bc \\
\end{tabular} & -0.05 & 0.00 & 0.07 & -0.27 & 0.90 & 0.99 & 1.17 & 0.54 \\
\hline 1 & & & Target & -0.04 & 0.00 & 0.06 & -0.24 & 0.92 & 0.99 & 1.15 & 0.57 \\
\hline 1 & & & Measured & 0.17 & 0.15 & 0.19 & -0.18 & 1.49 & 1.41 & 1.53 & 0.66 \\
\hline 1 & & quenched & Measured bc & 0.18 & 0.15 & 0.19 & -0.21 & 1.50 & 1.42 & 1.56 & 0.62 \\
\hline 1 & EM-08 & & Target & 0.18 & 0.15 & 0.19 & -0.19 & 1.51 & 1.40 & 1.56 & 0.65 \\
\hline 1 & EM-08 & & Measured & -0.02 & 0.05 & 0.08 & -0.26 & 0.96 & 1.12 & 1.21 & 0.56 \\
\hline 1 & & $\mathrm{ccc}$ & Measured bc & -0.02 & 0.05 & 0.09 & -0.28 & 0.97 & 1.12 & 1.23 & 0.52 \\
\hline 1 & & & Target & -0.01 & 0.04 & 0.09 & -0.26 & 0.97 & 1.11 & 1.23 & 0.55 \\
\hline 1 & & & Measured & -0.11 & -0.13 & -0.08 & -0.30 & 0.77 & 0.75 & 0.84 & 0.51 \\
\hline 1 & & quenched & Measured bc & -0.11 & -0.12 & -0.07 & -0.32 & 0.78 & 0.75 & 0.85 & 0.48 \\
\hline 1 & FM- 0 & & \begin{tabular}{|c|} 
Target \\
\end{tabular} & -0.11 & -0.13 & -0.07 & -0.30 & 0.77 & 0.74 & 0.85 & 0.50 \\
\hline 1 & EM-09 & & Measured & -0.09 & -0.05 & -0.06 & -0.27 & 0.81 & 0.90 & 0.86 & 0.54 \\
\hline 1 & & $\mathrm{ccc}$ & Measured bc & -0.09 & -0.05 & -0.06 & -0.30 & 0.82 & 0.90 & 0.88 & 0.51 \\
\hline 1 & & & Target & -0.09 & -0.05 & -0.06 & -0.27 & 0.81 & 0.89 & 0.88 & 0.53 \\
\hline
\end{tabular}


SRNL-STI-2009-00778, REVISION 0

Table 22 continued.

\begin{tabular}{|c|c|c|c|c|c|c|c|c|c|c|c|}
\hline Set & Glass ID & $\begin{array}{c}\text { Heat } \\
\text { Treatment }\end{array}$ & $\begin{array}{l}\text { Comp } \\
\text { View }\end{array}$ & $\begin{array}{c}\log N L \\
{[B(g / L)]}\end{array}$ & $\begin{array}{c}\log \mathbf{N L} \\
{[\mathrm{Li}(\mathrm{g} / \mathrm{L})]}\end{array}$ & \begin{tabular}{|c|}
$\log \mathrm{NL}$ \\
{$[\mathrm{Na}(\mathrm{g} / \mathrm{L})]$}
\end{tabular} & $\begin{array}{c}\log \mathrm{NL} \\
{[\mathrm{Si}(\mathrm{g} / \mathrm{L})]}\end{array}$ & $\begin{array}{c}\text { NL } \\
B(g / L)\end{array}$ & $\begin{array}{c}\mathrm{NL} \\
\mathrm{Li}(\mathrm{g} / \mathrm{L})\end{array}$ & \begin{tabular}{c|}
$\mathrm{NL}$ \\
$\mathrm{Na}(\mathrm{g} / \mathrm{L})$
\end{tabular} & $\begin{array}{c}\mathrm{NL} \\
\mathrm{Si}(\mathrm{g} / \mathrm{L})\end{array}$ \\
\hline 1 & \multirow{6}{*}{ EM-10 } & \multirow{3}{*}{ quenched } & Measured & -0.05 & -0.10 & 0.07 & -0.22 & 0.90 & 0.80 & 1.17 & 0.60 \\
\hline 1 & & & Measured bc & -0.04 & -0.10 & 0.08 & -0.25 & 0.91 & 0.80 & 1.19 & 0.57 \\
\hline 1 & & & Target & -0.04 & -0.11 & 0.06 & -0.23 & 0.91 & 0.77 & 1.16 & 0.59 \\
\hline 1 & & \multirow{3}{*}{$\operatorname{ccc}$} & Measured & 1.05 & 0.95 & 0.77 & 0.42 & 11.33 & 8.87 & 5.83 & 2.65 \\
\hline 1 & & & \begin{tabular}{|l|} 
Measured bc \\
\end{tabular} & 1.06 & 0.95 & 0.77 & 0.40 & 11.44 & 8.90 & 5.92 & 2.49 \\
\hline 1 & & & Target & 1.06 & 0.93 & 0.76 & 0.41 & 11.42 & 8.59 & 5.76 & 2.57 \\
\hline 2 & \multirow{6}{*}{ EM-11 } & \multirow{3}{*}{ quenched } & Measured & -0.13 & -0.15 & -0.08 & -0.34 & 0.74 & 0.72 & 0.82 & 0.45 \\
\hline 2 & & & Measured bc & -0.11 & -0.14 & -0.08 & -0.37 & 0.77 & 0.73 & 0.83 & 0.43 \\
\hline 2 & & & Target & -0.12 & -0.14 & -0.09 & -0.34 & 0.76 & 0.72 & 0.81 & 0.46 \\
\hline 2 & & \multirow{3}{*}{$\operatorname{ccc}$} & Measured & -0.23 & -0.19 & -0.08 & -0.35 & 0.60 & 0.65 & 0.82 & 0.45 \\
\hline 2 & & & Measured bc & -0.21 & -0.18 & -0.08 & -0.37 & 0.62 & 0.66 & 0.83 & 0.42 \\
\hline 2 & & & \begin{tabular}{|c|} 
Target \\
\end{tabular} & -0.21 & -0.19 & -0.09 & -0.34 & 0.62 & 0.65 & 0.81 & 0.46 \\
\hline 2 & \multirow{6}{*}{ EM-12 } & \multirow{3}{*}{ quenched } & Measured & -0.01 & -0.09 & 0.13 & -0.20 & 0.98 & 0.82 & 1.35 & 0.64 \\
\hline 2 & & & Measured bc & 0.01 & -0.08 & 0.14 & -0.22 & 1.02 & 0.84 & 1.37 & 0.60 \\
\hline 2 & & & Target & 0.00 & -0.09 & 0.13 & -0.20 & 1.01 & 0.82 & 1.35 & 0.64 \\
\hline 2 & & \multirow{3}{*}{$\operatorname{ccc}$} & Measured & 0.97 & 0.87 & 0.74 & 0.38 & 9.44 & 7.37 & 5.51 & 2.38 \\
\hline 2 & & & Measured bc & 0.99 & 0.88 & 0.75 & 0.35 & 9.85 & 7.55 & 5.59 & 2.26 \\
\hline 2 & & & Target & 0.99 & 0.87 & 0.74 & 0.38 & 9.74 & 7.37 & 5.50 & 2.38 \\
\hline 2 & \multirow{6}{*}{ EM-13 } & & Measured & -0.16 & -0.17 & -0.06 & -0.37 & 0.69 & 0.67 & 0.87 & 0.43 \\
\hline 2 & & quenched & Measured bc & -0.14 & -0.16 & -0.06 & -0.39 & 0.72 & 0.69 & 0.88 & 0.41 \\
\hline 2 & & & \begin{tabular}{|c|} 
Target \\
\end{tabular} & -0.14 & -0.17 & -0.06 & -0.36 & 0.72 & 0.67 & 0.88 & 0.43 \\
\hline 2 & & & Measured & 0.32 & 0.41 & 0.15 & -0.10 & 2.10 & 2.60 & 1.41 & 0.80 \\
\hline 2 & & $\operatorname{ccc}$ & Measured bc & 0.34 & 0.42 & 0.15 & -0.12 & 2.19 & 2.66 & 1.43 & 0.76 \\
\hline 2 & & & Target & 0.34 & 0.41 & 0.15 & -0.10 & 2.17 & 2.59 & 1.43 & 0.80 \\
\hline 2 & & & Measured & -0.15 & -0.15 & -0.07 & -0.40 & 0.70 & 0.71 & 0.85 & 0.40 \\
\hline 2 & & quenched & Measured bc & -0.14 & -0.14 & -0.06 & -0.42 & 0.73 & 0.73 & 0.86 & 0.38 \\
\hline 2 & FM-14 & & Target & -0.14 & -0.15 & -0.07 & -0.40 & 0.72 & 0.70 & 0.86 & 0.40 \\
\hline 2 & EM-14 & & Measured & -0.12 & 0.12 & -0.06 & -0.37 & 0.76 & 1.33 & 0.88 & 0.43 \\
\hline 2 & & $\mathrm{ccc}$ & Measured bc & -0.10 & 0.13 & -0.05 & -0.39 & 0.79 & 1.36 & 0.89 & 0.41 \\
\hline 2 & & & Target & -0.11 & 0.12 & -0.06 & -0.37 & 0.78 & 1.32 & 0.88 & 0.43 \\
\hline 2 & & & Measured & -0.21 & -0.15 & -0.07 & -0.46 & 0.62 & 0.71 & 0.85 & 0.35 \\
\hline 2 & & quenched & Measured bc & -0.19 & -0.14 & -0.06 & -0.48 & 0.65 & 0.73 & 0.86 & 0.33 \\
\hline 2 & EM-15 & & Target & -0.18 & -0.14 & -0.07 & -0.45 & 0.67 & 0.73 & 0.86 & 0.36 \\
\hline 2 & & & Measured & -0.25 & 0.13 & -0.12 & -0.47 & 0.56 & 1.34 & 0.77 & 0.34 \\
\hline 2 & & $\operatorname{ccc}$ & Measured bc & -0.23 & 0.14 & -0.11 & -0.49 & 0.59 & 1.38 & 0.77 & 0.32 \\
\hline 2 & & & Target & -0.22 & 0.14 & -0.11 & -0.45 & 0.60 & 1.38 & 0.77 & 0.35 \\
\hline 2 & & & Measured & -0.01 & -0.05 & 0.05 & -0.26 & 0.99 & 0.89 & 1.13 & 0.54 \\
\hline 2 & & quenched & Measured bc & -0.01 & -0.05 & 0.05 & -0.30 & 0.98 & 0.89 & 1.12 & 0.51 \\
\hline 2 & FM-16 & & Target & -0.01 & -0.05 & 0.04 & -0.27 & 0.99 & 0.88 & 1.09 & 0.54 \\
\hline 2 & EM-10 & & Measured & 0.26 & 0.19 & 0.16 & -0.15 & 1.80 & 1.54 & 1.44 & 0.71 \\
\hline 2 & & $\mathrm{ccc}$ & Measured bc & 0.25 & 0.19 & 0.16 & -0.18 & 1.79 & 1.55 & 1.44 & 0.66 \\
\hline 2 & & & Target & 0.26 & 0.18 & 0.15 & -0.15 & 1.80 & 1.53 & 1.40 & 0.70 \\
\hline 2 & & & Measured & -0.11 & -0.12 & -0.08 & -0.34 & 0.78 & 0.76 & 0.84 & 0.46 \\
\hline 2 & & quenched & Measured bc & -0.11 & -0.12 & -0.08 & -0.37 & 0.77 & 0.76 & 0.83 & 0.43 \\
\hline 2 & ЕM-17 & & Target & -0.12 & -0.13 & -0.10 & -0.35 & 0.76 & 0.74 & 0.80 & 0.45 \\
\hline 2 & EM-17 & & Measured & 0.50 & 0.42 & 0.23 & -0.09 & 3.13 & 2.65 & 1.69 & 0.81 \\
\hline 2 & & $\operatorname{ccc}$ & Measured bc & 0.49 & 0.42 & 0.23 & -0.13 & 3.11 & 2.66 & 1.69 & 0.75 \\
\hline 2 & & & Target & 0.49 & 0.41 & 0.21 & -0.10 & 3.07 & 2.60 & 1.63 & 0.79 \\
\hline 2 & & & Measured & 0.14 & 0.03 & 0.18 & -0.16 & 1.39 & 1.08 & 1.51 & 0.69 \\
\hline 2 & & quenched & \begin{tabular}{|l|} 
Measured bc \\
\end{tabular} & 0.14 & 0.04 & 0.19 & -0.19 & 1.38 & 1.09 & 1.55 & 0.64 \\
\hline 2 & FM-18 & & Target & 0.13 & 0.03 & 0.17 & -0.17 & 1.35 & 1.06 & 1.48 & 0.67 \\
\hline 2 & & & Measured & 0.53 & 0.43 & 0.41 & 0.04 & 3.36 & 2.67 & 2.55 & 1.10 \\
\hline 2 & & $\operatorname{ccc}$ & Measured bc & 0.52 & 0.43 & 0.42 & 0.01 & 3.35 & 2.69 & 2.62 & 1.02 \\
\hline 2 & & & Target & 0.52 & 0.42 & 0.40 & 0.03 & 3.29 & 2.62 & 2.50 & 1.07 \\
\hline 2 & & & Measured & 0.17 & 0.07 & 0.23 & -0.11 & 1.47 & 1.17 & 1.71 & 0.78 \\
\hline 2 & & quenched & Measured bc & 0.16 & 0.07 & 0.23 & -0.14 & 1.46 & 1.17 & 1.70 & 0.73 \\
\hline 2 & FM-19 & & Target & 0.19 & 0.08 & 0.21 & -0.12 & 1.55 & 1.20 & 1.63 & 0.77 \\
\hline 2 & EM-19 & & Measured & 0.09 & 0.04 & 0.19 & -0.12 & 1.23 & 1.09 & 1.54 & 0.75 \\
\hline 2 & & $\operatorname{ccc}$ & Measured bc & 0.09 & 0.04 & 0.19 & -0.15 & 1.22 & 1.10 & 1.53 & 0.70 \\
\hline 2 & & & Target & 0.11 & 0.05 & 0.17 & -0.13 & 1.29 & 1.12 & 1.47 & 0.74 \\
\hline 2 & & & Measured & 0.23 & 0.14 & 0.21 & -0.12 & 1.69 & 1.38 & 1.61 & 0.76 \\
\hline 2 & & quenched & Measured bc & 0.22 & 0.13 & 0.22 & -0.15 & 1.68 & 1.35 & 1.66 & 0.71 \\
\hline 2 & FM-20 & & Target & 0.22 & 0.12 & 0.20 & -0.13 & 1.64 & 1.31 & 1.59 & 0.74 \\
\hline 2 & EIVI-20 & & Measured & 0.14 & 0.10 & 0.15 & -0.13 & 1.38 & 1.26 & 1.41 & 0.75 \\
\hline 2 & & $\operatorname{ccc}$ & Measured bc & 0.14 & 0.09 & 0.16 & -0.16 & 1.37 & 1.24 & 1.45 & 0.69 \\
\hline 2 & & & Target & 0.13 & 0.08 & 0.14 & -0.14 & 1.34 & 1.20 & 1.39 & 0.72 \\
\hline
\end{tabular}


SRNL-STI-2009-00778, REVISION 0

Table 22 continued.

\begin{tabular}{|c|c|c|c|c|c|c|c|c|c|c|c|}
\hline Set & Glass ID & $\begin{array}{c}\text { Heat } \\
\text { Treatment }\end{array}$ & $\begin{array}{l}\text { Comp } \\
\text { View }\end{array}$ & $\begin{array}{c}\log \mathbf{N L} \\
{[B(g / L)]}\end{array}$ & $\begin{array}{c}\log \mathrm{NL} \\
{[\mathrm{Li}(\mathrm{g} / \mathrm{L})]}\end{array}$ & $\begin{array}{c}\log \mathrm{NL} \\
{[\mathrm{Na}(\mathrm{g} / \mathrm{L})]}\end{array}$ & $\begin{array}{c}\log \mathbf{N L} \\
{[\mathrm{Si}(\mathrm{g} / \mathrm{L})]}\end{array}$ & $\begin{array}{c}N L \\
B(g / L)\end{array}$ & \begin{tabular}{c|} 
NL \\
$\mathrm{Li}(\mathrm{g} / \mathrm{L})$ \\
\end{tabular} & $\begin{array}{c}\mathrm{NL} \\
\mathrm{Na}(\mathrm{g} / \mathrm{L}) \\
\end{array}$ & $\begin{array}{c}N L \\
\mathrm{Si}(\mathrm{g} / \mathrm{L})\end{array}$ \\
\hline 3 & \multirow{6}{*}{ EM-21 } & \multirow{3}{*}{ quenched } & Measured & 0.21 & 0.13 & 0.14 & -0.17 & 1.62 & 1.36 & 1.38 & 0.67 \\
\hline 3 & & & Measured bc & 0.21 & 0.12 & 0.15 & -0.21 & 1.61 & 1.33 & 1.42 & 0.62 \\
\hline 3 & & & Target & 0.21 & 0.12 & 0.15 & $\begin{array}{l}-0.18 \\
\end{array}$ & 1.62 & 1.32 & 1.41 & 0.66 \\
\hline 3 & & \multirow{3}{*}{$\mathrm{ccc}$} & Measured & -0.05 & -0.05 & -0.03 & -0.25 & 0.89 & 0.88 & 0.93 & 0.56 \\
\hline 3 & & & Measured bc & -0.05 & -0.06 & -0.02 & -0.29 & 0.88 & 0.87 & 0.96 & 0.52 \\
\hline 3 & & & Target & -0.05 & -0.07 & -0.02 & -0.26 & 0.89 & 0.86 & 0.95 & 0.55 \\
\hline 3 & \multirow{6}{*}{ EM-22 } & \multirow{3}{*}{ quenched } & Measured & 0.44 & 0.36 & 0.36 & -0.07 & 2.77 & 2.30 & 2.27 & 0.84 \\
\hline 3 & & & Measured bc & 0.44 & 0.35 & 0.37 & -0.11 & 2.75 & 2.25 & 2.33 & 0.78 \\
\hline 3 & & & Target & 0.45 & 0.35 & 0.36 & -0.08 & 2.79 & 2.24 & 2.30 & 0.83 \\
\hline 3 & & \multirow{3}{*}{$\mathrm{ccc}$} & Measured & 0.03 & 0.06 & 0.08 & -0.23 & 1.07 & 1.14 & 1.19 & 0.59 \\
\hline 3 & & & Measured bc & 0.03 & 0.05 & 0.09 & -0.27 & 1.07 & 1.12 & 1.23 & 0.54 \\
\hline 3 & & & Target & 0.03 & 0.05 & 0.08 & -0.24 & 1.08 & 1.11 & 1.21 & 0.58 \\
\hline 3 & \multirow{6}{*}{ EM-23 } & \multirow{3}{*}{ quenched } & Measured & 0.59 & 0.51 & 0.57 & -0.01 & 3.93 & 3.25 & 3.70 & 0.97 \\
\hline 3 & & & Measured bc & 0.59 & 0.50 & 0.57 & -0.05 & 3.91 & 3.18 & 3.68 & 0.90 \\
\hline 3 & & & Target & 0.60 & 0.51 & 0.56 & 0.00 & 3.96 & 3.20 & 3.62 & 1.00 \\
\hline 3 & & \multirow{3}{*}{$\operatorname{ccc}$} & Measured & 0.09 & 0.11 & 0.15 & -0.23 & 1.23 & 1.29 & 1.41 & 0.59 \\
\hline 3 & & & Measured bc & 0.09 & 0.10 & 0.15 & -0.27 & 1.22 & 1.26 & 1.40 & 0.54 \\
\hline 3 & & & \begin{tabular}{|l|} 
Target \\
\end{tabular} & 0.09 & 0.10 & 0.14 & -0.22 & 1.24 & 1.27 & 1.38 & 0.61 \\
\hline 3 & \multirow{6}{*}{ EM-24 } & \multirow{3}{*}{ quenched } & Measured & -0.03 & -0.05 & -0.02 & -0.30 & 0.93 & 0.88 & 0.95 & 0.50 \\
\hline 3 & & & Measured bc & -0.04 & -0.05 & -0.01 & -0.33 & 0.92 & 0.89 & 0.98 & 0.46 \\
\hline 3 & & & Target & -0.03 & -0.05 & -0.02 & -0.30 & 0.94 & 0.89 & 0.95 & 0.51 \\
\hline 3 & & \multirow{3}{*}{$\mathrm{ccc}$} & Measured & 0.11 & 0.05 & 0.02 & -0.24 & 1.30 & 1.14 & 1.04 & 0.58 \\
\hline 3 & & & Measured bc & 0.11 & 0.06 & 0.03 & -0.27 & 1.29 & 1.14 & 1.07 & 0.54 \\
\hline 3 & & & \begin{tabular}{|l|} 
Target \\
\end{tabular} & 0.12 & 0.06 & 0.02 & -0.23 & 1.31 & 1.14 & 1.04 & 0.59 \\
\hline 3 & \multirow{6}{*}{ EM-25 } & & Measured & 0.08 & -0.03 & 0.13 & -0.21 & 1.21 & 0.93 & 1.36 & 0.61 \\
\hline 3 & & quenched & Measured bc & 0.08 & -0.04 & 0.15 & -0.25 & 1.20 & 0.91 & 1.40 & 0.57 \\
\hline 3 & & & Target & 0.08 & -0.05 & 0.13 & -0.22 & 1.20 & 0.90 & 1.33 & 0.60 \\
\hline 3 & & & Measured & 1.09 & 0.99 & 0.82 & 0.40 & 12.42 & 9.82 & 6.65 & 2.49 \\
\hline 3 & & $\operatorname{ccc}$ & Measured bc & 1.09 & 0.98 & 0.84 & 0.36 & 12.35 & 9.62 & 6.84 & 2.30 \\
\hline 3 & & & Target & 1.09 & 0.98 & 0.81 & 0.39 & 12.36 & 9.45 & 6.53 & 2.43 \\
\hline 3 & & & Measured & -0.01 & -0.04 & 0.00 & -0.30 & 0.98 & 0.91 & 0.99 & 0.51 \\
\hline 3 & & quenched & Measured bc & -0.01 & -0.05 & -0.01 & -0.33 & 0.97 & 0.89 & 0.99 & 0.47 \\
\hline 3 & FM-26 & & \begin{tabular}{|l|} 
Target \\
\end{tabular} & -0.02 & -0.06 & -0.02 & -0.30 & 0.96 & 0.88 & 0.96 & 0.50 \\
\hline 3 & EM-26 & & Measured & 1.33 & 1.19 & 1.13 & 0.44 & 21.45 & 15.58 & 13.56 & 2.75 \\
\hline 3 & & $\mathrm{ccc}$ & Measured bc & 1.33 & 1.18 & 1.13 & 0.40 & 21.33 & 15.26 & 13.50 & 2.54 \\
\hline 3 & & & \begin{tabular}{|l|} 
Target \\
\end{tabular} & 1.33 & 1.18 & 1.12 & 0.43 & 21.17 & 15.03 & 13.17 & 2.70 \\
\hline 3 & & & Measured & 0.10 & 0.00 & 0.19 & -0.18 & 1.25 & 0.99 & 1.55 & 0.66 \\
\hline 3 & & quenched & Measured bc & 0.10 & 0.00 & 0.19 & -0.21 & 1.25 & 1.00 & 1.54 & 0.61 \\
\hline 3 & & & Target & 0.09 & -0.01 & 0.17 & -0.19 & 1.24 & 0.99 & 1.49 & 0.65 \\
\hline 3 & EM-27 & & Measured & -0.05 & -0.03 & -0.14 & -0.25 & 0.89 & 0.93 & 0.73 & 0.56 \\
\hline 3 & & $\mathrm{ccc}$ & Measured bc & -0.05 & -0.03 & -0.14 & -0.28 & 0.89 & 0.93 & 0.72 & 0.52 \\
\hline 3 & & & Target & -0.05 & -0.04 & -0.15 & -0.26 & 0.88 & 0.92 & 0.70 & 0.56 \\
\hline 3 & & & Measured & -0.01 & -0.04 & 0.02 & -0.31 & 0.97 & 0.92 & 1.05 & 0.49 \\
\hline 3 & & quenched & Measured bc & -0.02 & -0.04 & 0.03 & -0.34 & 0.96 & 0.90 & 1.08 & 0.46 \\
\hline 3 & FM 20 & & Target & -0.02 & -0.05 & 0.02 & -0.32 & 0.96 & 0.88 & 1.06 & 0.48 \\
\hline 3 & ElM- 28 & & Measured & 0.91 & 0.83 & 0.54 & 0.08 & 8.22 & 6.72 & 3.49 & 1.19 \\
\hline 3 & & $\mathrm{ccc}$ & Measured bc & 0.91 & 0.82 & 0.55 & 0.04 & 8.17 & 6.58 & 3.59 & 1.10 \\
\hline 3 & & & \begin{tabular}{|l|} 
Target \\
\end{tabular} & 0.91 & 0.81 & 0.55 & 0.07 & 8.12 & 6.44 & 3.52 & 1.17 \\
\hline 3 & & & Measured & 0.01 & -0.01 & 0.05 & -0.35 & 1.03 & 0.97 & 1.11 & 0.45 \\
\hline 3 & & quenched & Measured bc & 0.01 & -0.02 & 0.04 & -0.38 & 1.02 & 0.95 & 1.10 & 0.41 \\
\hline 3 & FM-20 & & \begin{tabular}{|l|} 
Target \\
\end{tabular} & 0.02 & -0.02 & 0.03 & -0.35 & 1.05 & 0.95 & 1.08 & 0.45 \\
\hline 3 & EM-29 & & Measured & 0.57 & 0.51 & 0.29 & -0.15 & 3.67 & 3.26 & 1.97 & 0.72 \\
\hline 3 & & $\mathrm{ccc}$ & Measured bc & 0.56 & 0.50 & 0.29 & -0.18 & 3.65 & 3.19 & 1.96 & 0.66 \\
\hline 3 & & & Target & 0.57 & 0.50 & 0.28 & -0.14 & 3.74 & 3.19 & 1.92 & 0.72 \\
\hline 3 & & & Measured & -0.01 & 0.01 & 0.06 & -0.37 & 0.98 & 1.03 & 1.14 & 0.42 \\
\hline 3 & & quenched & Measured bc & -0.01 & 0.02 & 0.07 & -0.40 & 0.98 & 1.04 & 1.17 & 0.39 \\
\hline 3 & EM-30 & & Target & -0.01 & 0.01 & 0.06 & -0.37 & 0.98 & 1.03 & 1.14 & 0.43 \\
\hline 3 & EM-30 & & Measured & 0.27 & 0.27 & 0.07 & -0.34 & 1.87 & 1.84 & 1.18 & 0.46 \\
\hline 3 & & $\mathrm{ccc}$ & Measured bc & 0.27 & 0.27 & 0.08 & -0.37 & 1.86 & 1.85 & 1.22 & 0.42 \\
\hline 3 & & & \begin{tabular}{l|} 
Target \\
\end{tabular} & 0.27 & 0.26 & 0.07 & -0.34 & 1.86 & 1.83 & 1.19 & 0.46 \\
\hline
\end{tabular}




\subsubsection{Effects of Heat Treatment}

Exhibit E5 in Appendix E provides a series of plots and statistical comparisons that show the effects of heat treatment on the common logarithm ppm-responses of interest of the triplicate PCTs for each element for each study glass. The quenched version of a given glass yielded measurements indicating a significantly (at the 5\% significance level) different mean $\log (\mathrm{ppm})$ response than the ccc version of the glass for a given element if the Prob $>|\mathbf{t}|$ value in the exhibit is 0.05 or smaller. Table 23 summaries the comparisons between the quenched and ccc versions of the study glasses for the four primary elements of the PCTs.

Many of these glasses showed a statistically significant difference between the ccc versus the quenched versions for one or more of the PCT elements. In the cases where the ccc version of the glasses were determined to contain nepheline by XRD, the ccc versions of the glasses were statistically less durable than the quenched versions for all four elements ( $\mathrm{B}, \mathrm{Li}, \mathrm{Na}$ and $\mathrm{Si}$ ). Exhibit E6 in Appendix E provides plots of the normalized PCT responses between the two heat treatments. These plots provide a basis for judging the practical impact of differences in the PCT response due to the heat treatment of the glass.

The NL [B] values range from $0.65 \mathrm{~g} / \mathrm{L}(\mathrm{EM}-15)$ to $3.91 \mathrm{~g} / \mathrm{L}(\mathrm{EM}-23)$ for the quenched glasses and $0.59 \mathrm{~g} / \mathrm{L}$ (EM-15) to $21.33 \mathrm{~g} / \mathrm{L}$ (EM-26) for the ccc glasses. Three of the slow cooled (ccc) glasses (EM-10, -25 and -26) are unacceptable; however, this behavior is expected as all of these glasses contain nepheline. It is interesting to note that some of the ccc samples (EM-13, -14, -15, -29 and -30) completely crystallized with both magnetite and nepheline, but still had extremely low NL [B] values. These particular glasses have more $\mathrm{CaO}$ present than any of the other glasses in the matrix. It appears that while all of the glasses contain nepheline, the NL [B] values decrease as the $\mathrm{CaO}$ concentration increases from $2.3 \mathrm{wt} \%$ to $4.3 \mathrm{wt} \%$.

\subsubsection{Predicted versus Measured}

Exhibits E7 through E10 in Appendix E provide plots of the DWPF models for B, Li, Na, and Si that relate the logarithm of the normalized PCT (for each element of interest) to a linear function of a free energy of hydration term $\left(\Delta \mathrm{G}_{\mathrm{p}}, \mathrm{kcal} / 100 \mathrm{~g}\right.$ glass $)$ derived for each of the compositional views and heat treatments. ${ }^{27}$ Prediction limits (at a 95\% confidence) for an individual PCT result are also plotted along with the linear fit. The EA and ARM results are also indicated on these plots.

Exhibit E7 is repeated below as Figure 50. Note that there are some points that fall above the confidence limits for the study glasses. A majority of these points correspond to glasses containing nepheline. These points have been removed in Figure 51. While some of the glasses that still remain outside of the bounds are unpredictable, they are still acceptable; none of the glasses have NL [B] values greater than $4 \mathrm{~g} / \mathrm{L} .^{22}$

\footnotetext{
${ }^{22}$ Target EM-17, -23 and -24. Measured EM-17, -23 and -24. Measured bc EM-02, -17, -22, -23 and -24.
} 
Table 23. Samples Exhibiting a Statistically Significant Difference Between the Quenched and cec Versions of the Matrix 2A Glasses

\begin{tabular}{|c|c|c|c|c|c|}
\hline Glass ID & $\mathbf{B}$ & $\mathbf{L i}$ & $\mathbf{N a}$ & $\mathbf{S i}$ & Nepheline for cce Heat \\
\hline EM-01 & & $\mathrm{ccc}$ & & & \\
\hline EM-02 & $\mathrm{ccc}$ & $\mathrm{ccc}$ & $\mathrm{ccc}$ & $\operatorname{ccc}$ & $\mathrm{X}$ \\
\hline EM-03 & $\mathrm{Q}$ & $\mathrm{ccc}$ & $\mathrm{ccc}$ & $\operatorname{ccc}$ & \\
\hline EM-04 & $\mathrm{Q}$ & & $\mathrm{Q}$ & & \\
\hline EM-05 & $\mathrm{Q}$ & $\mathrm{ccc}$ & & & \\
\hline EM-06 & $\mathrm{Q}$ & $\mathrm{Q}$ & & $\mathrm{Q}$ & \\
\hline EM-07 & Q & $\mathrm{Q}$ & $\mathrm{Q}$ & Q & \\
\hline EM-08 & $\mathrm{Q}$ & $\mathrm{Q}$ & Q & $\mathrm{Q}$ & \\
\hline EM-09 & & $\mathrm{ccc}$ & & $\mathrm{ccc}$ & $\mathrm{X}$ \\
\hline EM-10 & $\mathrm{ccc}$ & $\mathrm{ccc}$ & $\mathrm{ccc}$ & $\operatorname{ccc}$ & $\mathrm{X}$ \\
\hline EM-11 & $\mathrm{Q}$ & $\mathrm{Q}$ & & & \\
\hline EM-12 & $\mathrm{ccc}$ & $\mathrm{ccc}$ & $\mathrm{ccc}$ & $\operatorname{ccc}$ & $\mathrm{X}$ \\
\hline EM-13 & $\mathrm{ccc}$ & $\mathrm{ccc}$ & $\mathrm{ccc}$ & $\operatorname{ccc}$ & $\mathrm{X}$ \\
\hline EM-14 & & $\mathrm{ccc}$ & & $\operatorname{ccc}$ & $\mathrm{X}$ \\
\hline EM-15 & & $\mathrm{ccc}$ & $\mathrm{Q}$ & & $\mathrm{X}$ \\
\hline EM-16 & $\mathrm{ccc}$ & $\mathrm{ccc}$ & $\mathrm{ccc}$ & $\mathrm{ccc}$ & \\
\hline EM-17 & $\mathrm{ccc}$ & $\mathrm{ccc}$ & $\mathrm{ccc}$ & $\operatorname{ccc}$ & \\
\hline EM-18 & $\mathrm{ccc}$ & $\mathrm{ccc}$ & $\mathrm{ccc}$ & $\operatorname{ccc}$ & \\
\hline EM-19 & $\mathrm{Q}$ & & Q & & \\
\hline EM-20 & $\mathrm{Q}$ & $\mathrm{Q}$ & $\mathrm{Q}$ & & \\
\hline EM-21 & $\mathrm{Q}$ & $\mathrm{Q}$ & $\mathrm{Q}$ & $\mathrm{Q}$ & \\
\hline EM-22 & $\mathrm{Q}$ & $\mathrm{Q}$ & $\mathrm{Q}$ & $\mathrm{Q}$ & \\
\hline EM-23 & $\mathrm{Q}$ & $\mathrm{Q}$ & $\mathrm{Q}$ & $\mathrm{Q}$ & \\
\hline EM-24 & $\mathrm{ccc}$ & $\mathrm{ccc}$ & $\mathrm{ccc}$ & $\mathrm{ccc}$ & \\
\hline EM-25 & $\mathrm{ccc}$ & $\mathrm{ccc}$ & $\mathrm{ccc}$ & $\mathrm{ccc}$ & $X$ \\
\hline EM-26 & $\mathrm{ccc}$ & $\mathrm{ccc}$ & $\mathrm{ccc}$ & $\operatorname{ccc}$ & $\mathrm{X}$ \\
\hline EM-27 & $\mathrm{Q}$ & $\mathrm{Q}$ & $\mathrm{Q}$ & $\mathrm{Q}$ & \\
\hline EM-28 & $\mathrm{ccc}$ & $\mathrm{ccc}$ & $\mathrm{ccc}$ & ccc & $\mathrm{X}$ \\
\hline EM-29 & $\mathrm{ccc}$ & $\mathrm{ccc}$ & $\mathrm{ccc}$ & ccc & $\mathrm{X}$ \\
\hline EM-30 & ccc & ccc & & ccc & $\mathrm{X}$ \\
\hline
\end{tabular}


SRNL-STI-2009-00778, REVISION 0

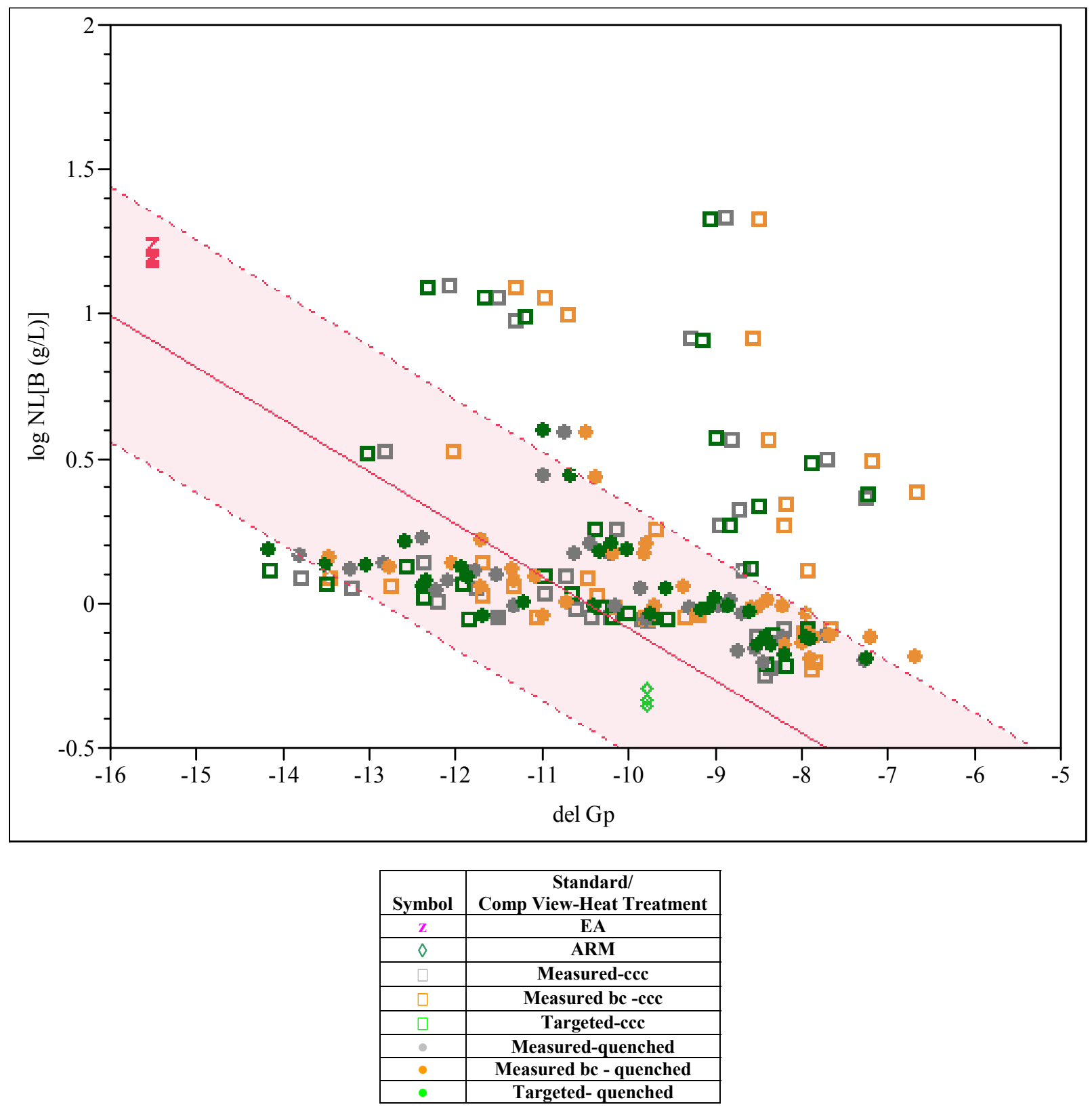

Figure 50. $\log$ NL [B (g/L)] versus del Gp model with $95 \%$ confidence interval for individual PCTs. 
SRNL-STI-2009-00778, REVISION 0

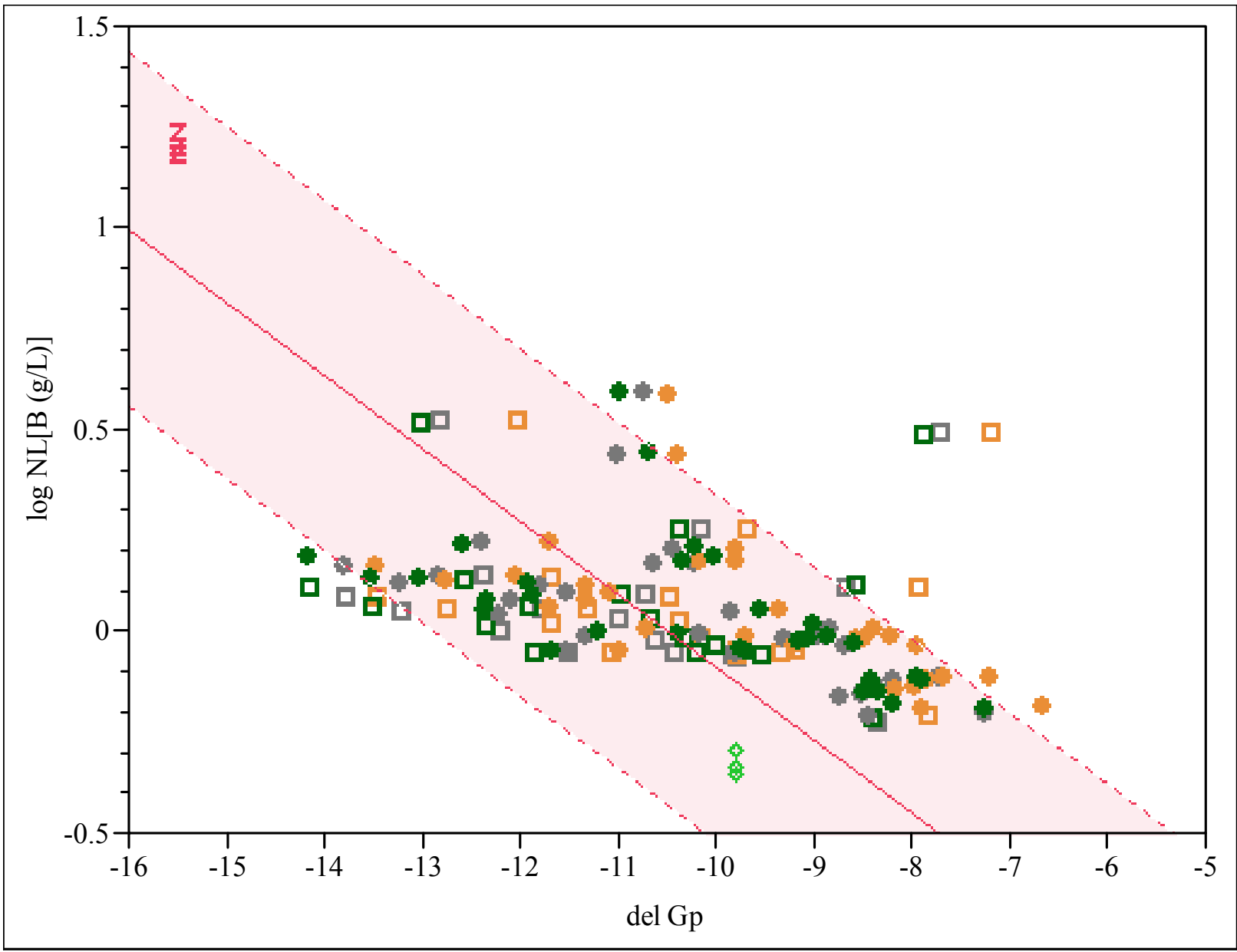

\begin{tabular}{|c|c|}
\hline Symbol & $\begin{array}{c}\text { Standard/ } \\
\text { Comp View-Heat Treatment }\end{array}$ \\
\hline $\mathrm{Z}$ & EA \\
\hline$\diamond$ & ARM \\
\hline$\square$ & Measured-ccc \\
\hline$\square$ & Measured bc -ccc \\
\hline$\square$ & Targeted-ccc \\
\hline$\bullet$ & Measured-quenched \\
\hline$\bullet$ & Measured bc - quenched \\
\hline$\bullet$ & Targeted- quenched \\
\hline
\end{tabular}

Figure 51. $\log$ NL [B (g/L)] versus del Gp model with $95 \%$ confidence interval for individual PCTs. Data points corresponding to glasses containing nepheline were removed. 


\subsubsection{Matrix 2A $\mathrm{T}_{\mathrm{L}}$ Data}

A comparison of the predicted and measured $T_{L}$ values is shown in Table 24 . There are only five samples because all of the other glasses contained crystals upon quenching (see Section 5.2.3). The measured $\mathrm{T}_{\mathrm{L}}$ values for these glasses seem a bit high compared to the Matrix 2 glasses, which were based off of the same compositional projections. It is possible that these glasses contained a small volume percent of crystals, which was not detected by XRD. The presence of any pre-existing crystals in a liquidus sample will significantly affect the results. A sample from each glass should be viewed with optical microscopy to determine if any crystals are present. If crystals are observed, then the analyses need to be repeated.

Table 24. Matrix 2A $T_{L}$ Data

\begin{tabular}{|c|c|c|c|}
\hline Glass ID & $\mathbf{T}_{\mathbf{L}}$ Predicted $\left({ }^{\circ} \mathbf{C}\right)$ & $\mathbf{T}_{\mathbf{L}}$ Measured & Measured - Predicted $\left({ }^{\circ} \mathbf{C}\right)$ \\
\hline EM-03 & 997 & 1157 & 160 \\
\hline EM-04 & 959 & 1096 & 137 \\
\hline EM-05 & 1012 & 1148 & 136 \\
\hline EM-18 & 1002 & 1148 & 146 \\
\hline EM-19 & 962 & 1147 & 185 \\
\hline
\end{tabular}




\subsection{COMPARISON OF DATA TO MODEL PREDICTIONS}

In this section, data collected in the FY07 and FY09 tasks will be compared to the various models for $\mathrm{T}_{\mathrm{L}}$, viscosity and chemical durability. Note that all predicted data are with respect to the measured composition.

\section{$6.1 T_{L}$}

In general, the $T_{L}$ model appears to be under-predicting for most of the glasses from FY07 and FY09 (Figure 52). Any glasses containing crystals other than spinel have been removed from the plot. A majority of these glasses are outside of the compositional region used to develop the current model; thus, it would not be expected to accurately predict the $\mathrm{T}_{\mathrm{L}}$ values of higher waste loading glasses. It also appears that glasses with higher $\mathrm{TiO}_{2}$ concentrations form crystals other than spinels, i.e. iron titanate. If future processing of these glasses is pursued, then the current $T_{L}$ model will need to be modified to include crystals other than spinels. Some of the glasses from FY07 and FY09 should be re-measured and care should be taken to ensure that no crystals are present in the as-fabricated glass.

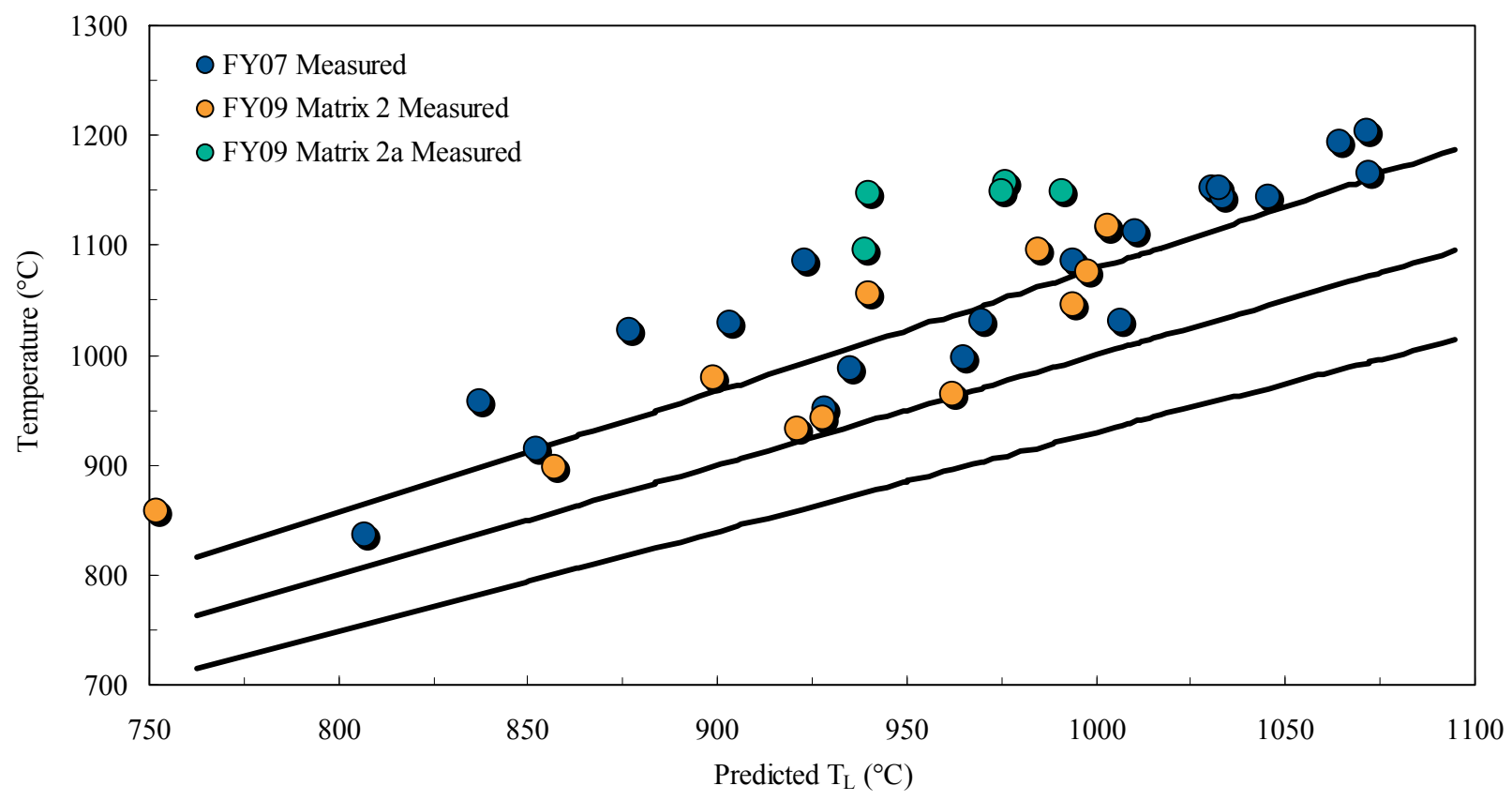

Figure 52. FY07 and FY09 $T_{L}$ data. 


\subsection{VISCOSITY}

The FY07 glasses are extremely predictable by the model. ${ }^{23}$ There are a number of glasses from the FY09 matrix, which have much lower measured viscosities than the model development region. A calculation of the NBO ratio indicates that these glasses have much higher values of this ratio than historical glasses. These glasses also have high $\mathrm{Fe}_{2} \mathrm{O}_{3}$ concentrations compared to other glasses in the study, which accounts for the increased NBO concentration. Additional glasses would be required to determine the applicability of the viscosity model if processing were desired at lower values of viscosity.

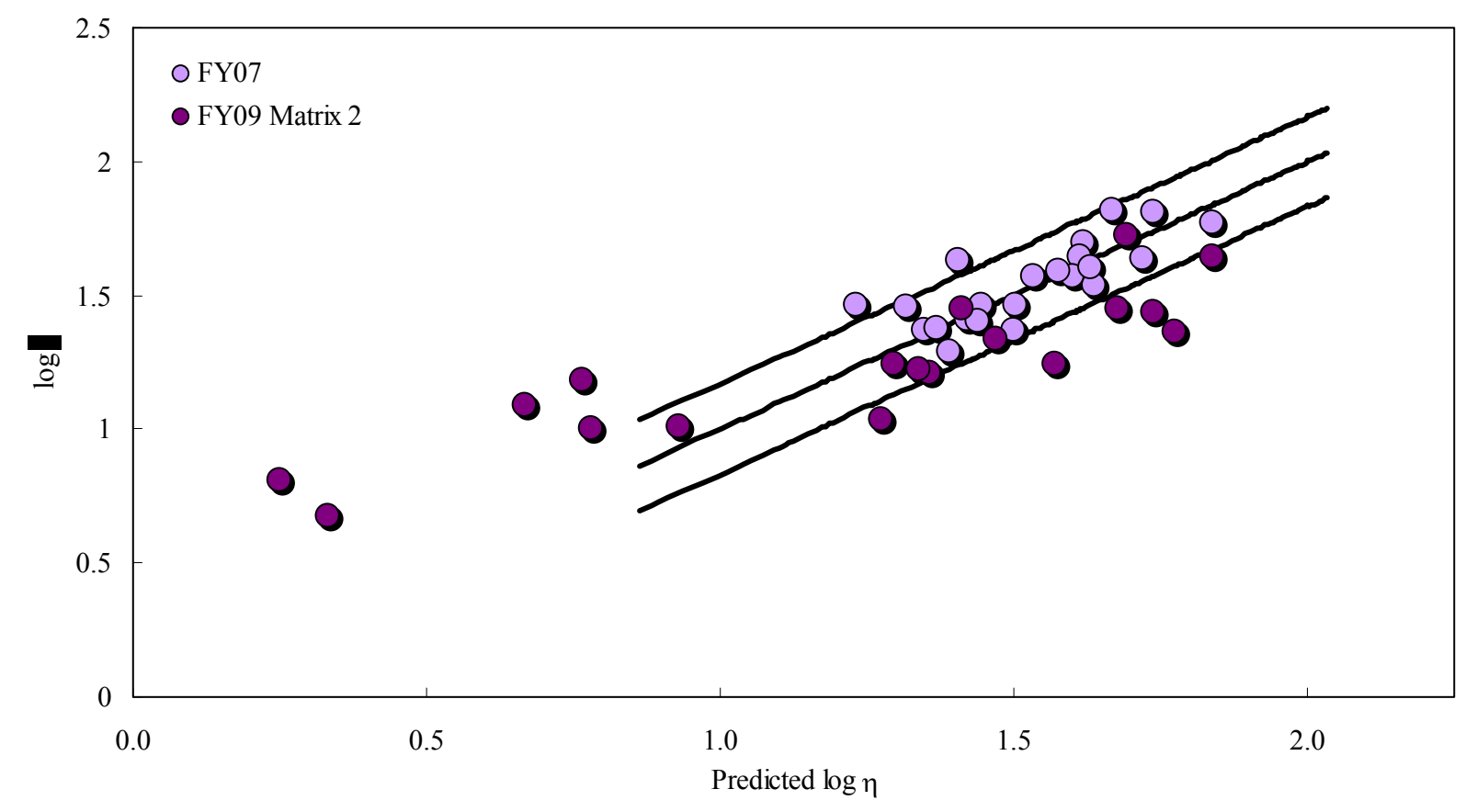

Figure 53. FY07 and FY09 viscosity data.

\footnotetext{
${ }^{23}$ Since the FY07 data was not presented in the FY07 report (SRNS-STI-2008-00055), the fitted data as well as the values at $1150^{\circ} \mathrm{C}$ are presented in Exhibits F1 through F26 in Appendix F.
} 


\subsection{CHEMICAL DURABILITY}

Note that glasses containing nepheline have been removed from Figure 54 as the model applies only to homogeneous samples. In general, the FY07 glasses are well-predicted by the durability model. A small number of glasses extend beyond the prediction limits around $\Delta \mathrm{G}_{\mathrm{p}}$ values of -6 to -8 , but the NL [B] values are so low that these glasses are of little concern. As stated in Section 5.1.4.5, some of the quenched glasses from the FY09 Matrix $2^{24}$ with $\Delta G_{p}$ values between -14 and -12 exhibited anomalous behavior and thus have unexplained poor durabilities at this time. Further characterization of these glasses would be required to clarify these results. The questionable glasses from Matrix 2 (FY09EM21-02, -03, -07 and -21) were removed and the quenched and ccc durabilities were re-plotted in Figures Figure 55 and Figure 56. Since XRD of the ccc versions of these same glasses could not be completed, the presence of nepheline could not be verified. If nepheline is present, then the model would not be expected to predict the durabilities of these glasses and the points would be removed from this plot. A few other glasses are also under predicted by the model, but they are still acceptable with respect to the $10 \mathrm{~g} / \mathrm{L}$ limit for boron release. There are a number of points that are outside of the prediction bounds at less negative $\Delta \mathrm{G}_{\mathrm{p}}$ values. These glasses are of low concern as the NL [B] values are so low. Almost all of the FY09 Matrix 2A glasses are predicted by the model, which is encouraging as all of these glasses are at 50\% WL. Two of the glasses (shown in light and dark purple) are under predicted by the model, but they are still acceptable.

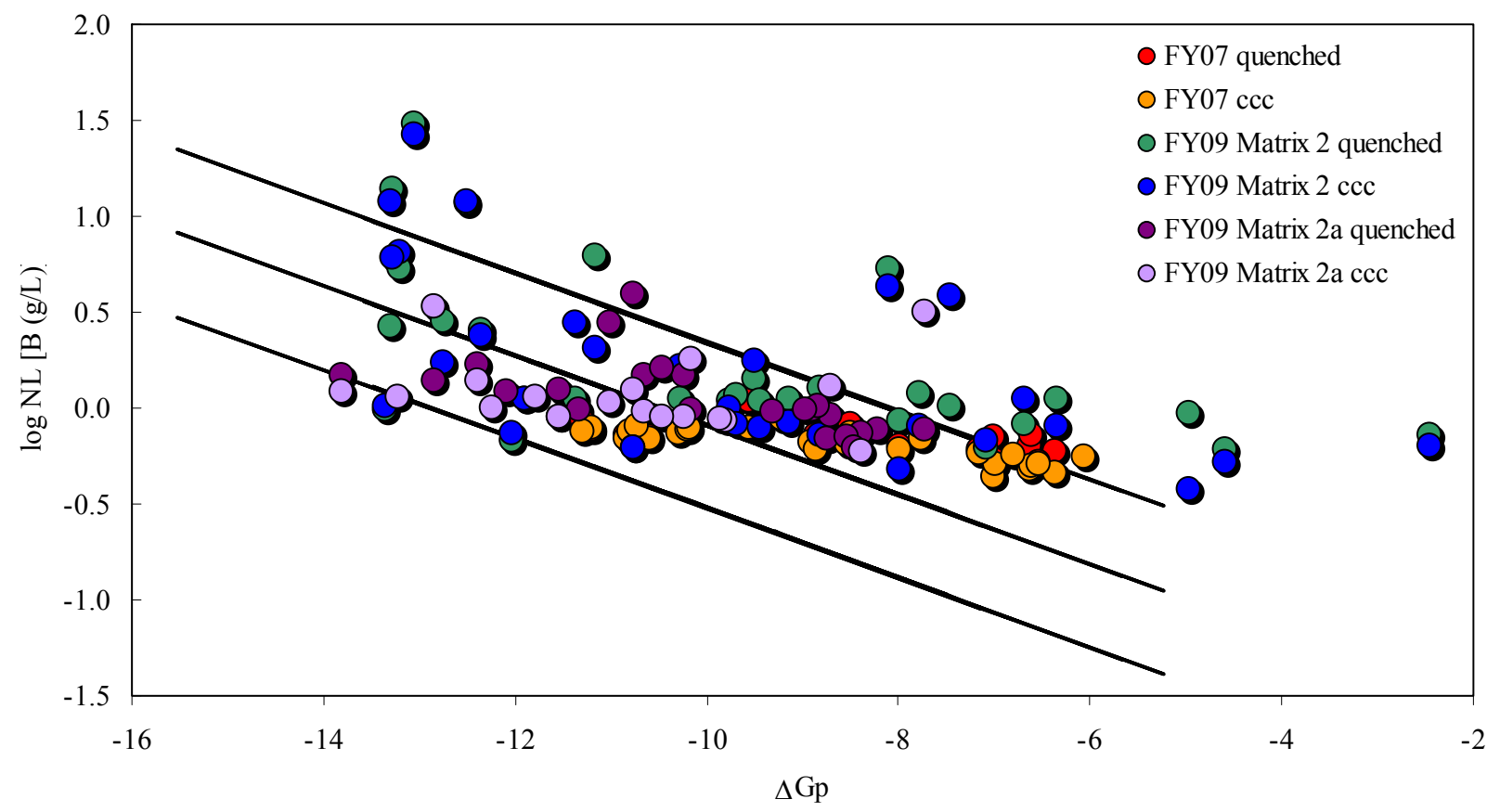

Figure 54. Comparison of durability data from the FY07 and FY09 tasks to the model predictions. Glasses containing nepheline have been removed from the plot.

\footnotetext{
${ }^{24}$ FY09EM21-02, -07 and -21.
} 


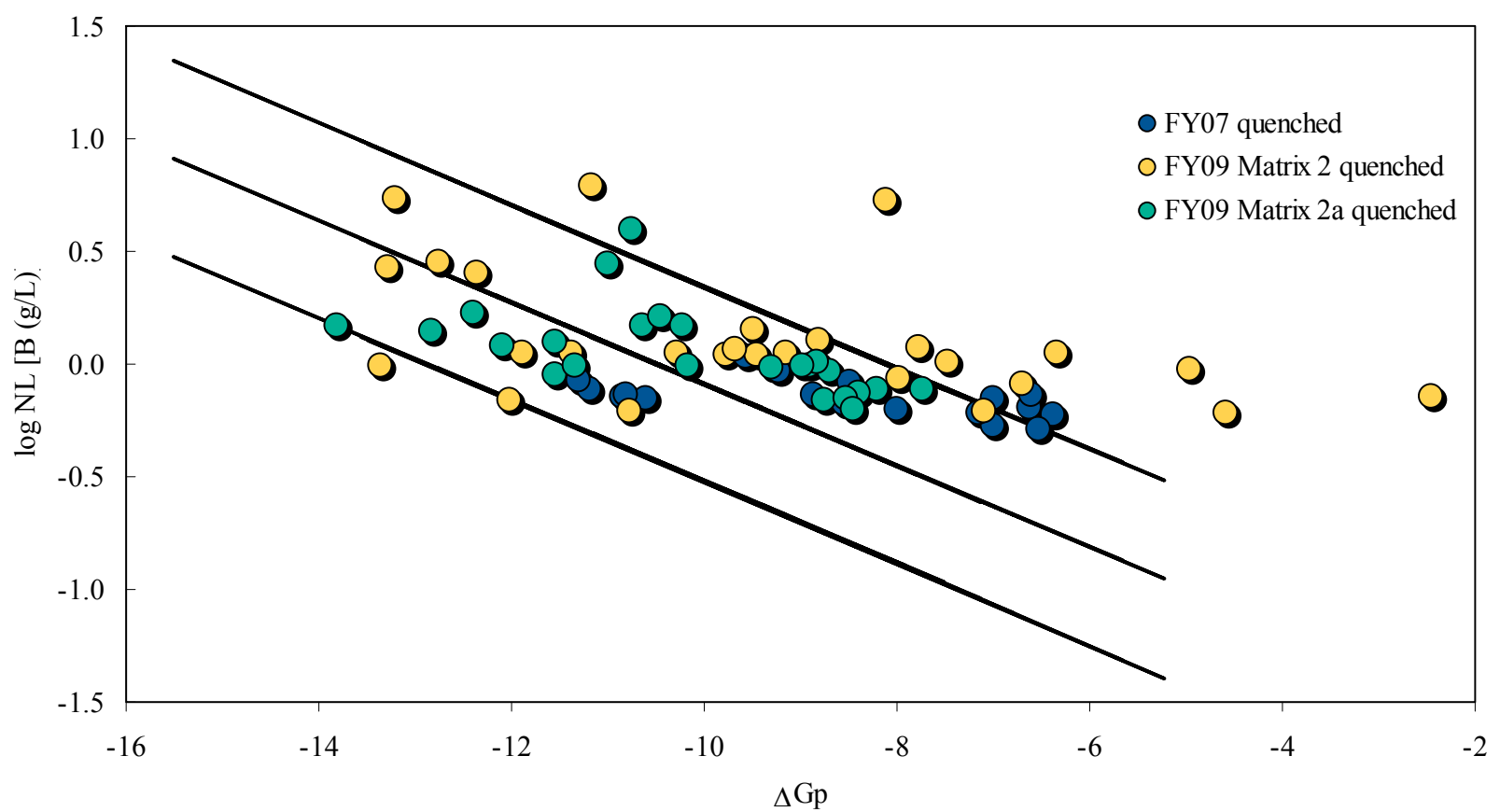

Figure 55. Comparison of quenched durability data from the FY07 and FY09 tasks to the durability model. Questionable glasses from Matrix 2 have been removed from the plot (FY09EM21-02, -03, -07 and -21).

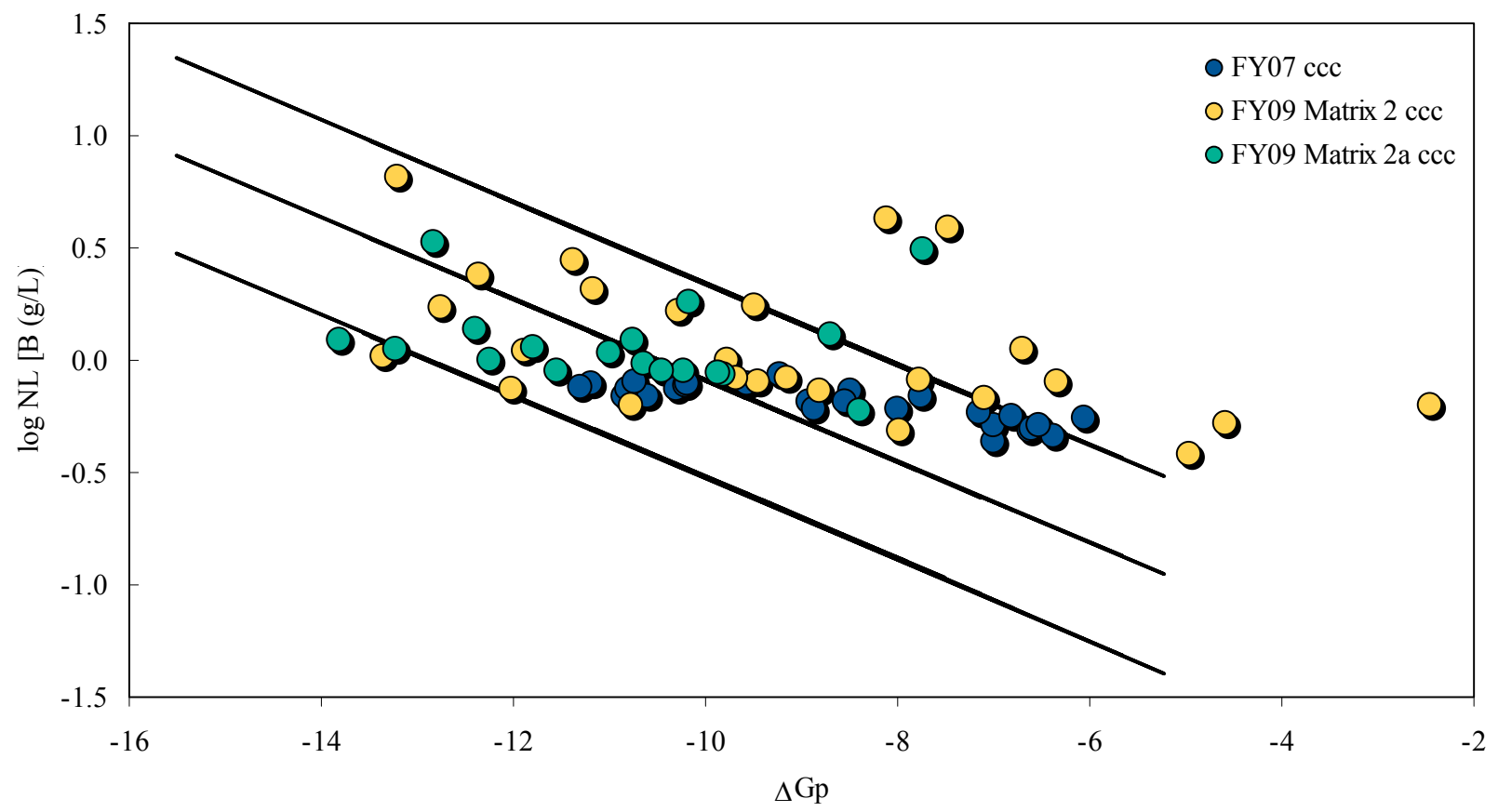

Figure 56. Figure 57. Comparison of ccc durability data from the FY07 and FY09 tasks to the durability model. Questionable glasses from Matrix 2 have been removed from the plot (FY09EM21-02, -03, -07 and -21). 


\subsection{SUMMARY}

Sixty glass compositions were fabricated and characterized in order to generate supplemental property data (viscosity, $\mathrm{T}_{\mathrm{L}}$ and durability) to be used to determine the applicability of the current DWPF process control models for higher waste loading glasses. Two series of glasses were developed using the HLW Revision 13 sludge projections.

The results of this study are summarized below:

- In general, the current durability model predicts the durabilities of higher waste loading glasses quite well. A few of the glasses exhibited poorer durability than predicted.

- Some of the glasses exhibited anomalous behavior with respect to durability (normalized leachate for boron (NL [B])). The quenched samples of FY09EM21-02, -07 and -21 contained no nepheline or other wasteform affecting crystals, but have unacceptable NL [B] values (>10 g/L). The ccc sample of FY09EM21-07 has a NL [B] value that is more than one half the value of the quenched sample. These glasses also have lower concentrations of $\mathrm{Al}_{2} \mathrm{O}_{3}$ and $\mathrm{SiO}_{2}$.

- Five of the ccc samples (EM-13, -14, -15, -29 and -30) completely crystallized with both magnetite and nepheline, and still had extremely low NL [B] values. These particular glasses have more $\mathrm{CaO}$ present than any of the other glasses in the matrix. It appears that while all of the glasses contain nepheline, the NL [B] values decrease as the $\mathrm{CaO}$ concentration increases from $2.3 \mathrm{wt} \%$ to $4.3 \mathrm{wt} \%$. A different form of nepheline may be created at higher concentrations of $\mathrm{CaO}$ that does not significantly reduce glass durability.

- The $\mathrm{T}_{\mathrm{L}}$ model appears to be under-predicting the measured values of higher waste loading glasses. Trends in $\mathrm{T}_{\mathrm{L}}$ with composition are not evident in the data from these studies.

- A small number of glasses in the FY09 matrix have measured viscosities that are much lower than the viscosity range over which the current model was developed. The decrease in viscosity is due to a higher concentration of non-bridging oxygens (NBO). A high iron concentration is the cause of the increase in NBO.

Durability, viscosity and $\mathrm{T}_{\mathrm{L}}$ data collected during FY07 and FY09 was compiled and assessed. It appears that additional data would be required to refit the $\mathrm{T}_{\mathrm{L}}$ and viscosity models. In general, the compositional regions of the higher waste loading glasses are very different than those used to develop these models. On the other hand, the current durability model seems to be very applicable to the new data. At this time, there is no evidence to modify this model.

\subsection{RECOMMENDATIONS/PATH FORWARD}

In order to determine the cause of some of the durability anomalies and $T_{L}$ issues observed in the glasses of these matrices, some additional experimental work is recommended.

- Further characterization of FY09EM21-02, -07 and -21 is recommended using scanning electron microscopy and various spectroscopy techniques (infrared, Raman and Mossbauer). 
- Study the influence of increased $\mathrm{CaO}$ concentration on PCT response and the formation of nepheline. Determine if a different form of nepheline is being created at higher concentrations of $\mathrm{CaO}$ that does not significantly decrease the durability of the glass. It has been previously shown that not all nepheline is detrimental to glass durability.

- Before any $T_{L}$ measurements, several fragments of each as-fabricated sample should be examined by optical microscopy to determine if crystals are present. XRD does not appear to be sensitive enough for some samples.

- Fabricate glasses to generate additional $\mathrm{T}_{\mathrm{L}}$ data including those glasses with increased $\mathrm{TiO}_{2}$ concentrations. Determine the crystalline phase(s) formed at the liquidus temperature and if the current model needs to be revised to include crystalline phases other than spinel.

\subsection{REFERENCES}

1. K.G. Brown, R.L. Postles, and T.B. Edwards, "SME Acceptability Determination for DWPF Process Control," Savannah River National Laboratory, Aiken, SC, WSRC-TR95-00364, Rev. 5, 2006.

2. F.C. Raszewski, T.B. Edwards, and D.K. Peeler, "Matrix 2 Results of the FY07 Enhanced DOE High-Level Waste Melter Throughput Studies at SRNL," Savannah River National Laboratory, Aiken, SC, SRNS-STI-2008-00055, 2008.

3. K. Gerdes, "Enhanced DOE High-Level Waste Melter Throughput Studies [Work Authorization / Task Change Request (TCR)]," Project Manager at the U.S. Department of Energy Headquarters, Washington, D.C., Project Number SR071401, 2007.

4. K. Gerdes, "Enhanced DOE High-Level Waste Melter Throughput Studies [Work Authorization / Task Change Request (TCR)]," Project Manager at the U.S. Department of Energy Headquarters, Washington, D.C., Project Number RV071301, 2008.

5. D. Kim, "EM-21 HLW Glass Studies Test Plan: Crystal Accumulation Modeling Task Glass Matrix Testing,” Pacific Northwest National Laboratory (PNNL), Richland, WA, EMHLW-TP-07-01, Rev. 0,

6. F.C. Raszewski, A.S. Choi, T.B. Edwards, K.M. Fox, and M.E. Smith, "Enhanced DOE High-Level Waste Melter Throughput Studies: Task Plan," Savannah River National Laboratory, Aiken, SC, WSRC-STI-2007-00483, Rev. 0, 2007.

7. F.C. Raszewski, T.B. Edwards, and D.K. Peeler, "Enhanced DOE High-Level Waste Melter Throughput Studies: FY09 Test Plan," Savannah River National Laboratory, Aiken, SC, SRNL-STI-2008-00530, 2009.

8. T.B. Caldwell, D.P. Chew, H.H. Elder, M.J. Mahoney, K.B. Way, W.A. Wilson, and F.E. Wise, "Savannah River Site High Level Waste System Plan: Waste Immobilization (Revision 13)," Washington Savannah River Company, Aiken, SC, HLW-2002-00025, 2002. 
9. $\quad \mathrm{JMP}^{\mathrm{TM}}$ Version 7.0.2, SAS Institute Inc., Cary, NC, 2008.

10. "Glass Batching," Savannah River National Laboratory, Aiken, SC, ITS-0001, Rev. 1, 2007.

11. "Glass Melting," Savannah River National Laboratory, Aiken, SC, ITS-0003, Rev. 2, 2007.

12. S.L. Marra and C.M. Jantzen, "Characterization of Projected DWPF Glass Heat Treated to Simulate Canister Centerline Cooling," U.S. Department of Energy - Westinghouse Savannah River Company, Aiken, SC, WSRC-TR-92-142, Rev. 1,

13. T.B. Edwards, "Analytical Plans for Measuring the Chemical Compositions of the Matrix 2 Glasses Supporting the EM-21 FY09 Task," Savannah River National Laboratory, Aiken, SC, SRNL-L5200-2009-00007, 2009.

14. T.B. Edwards, "Analytical Plans for Measuring the Chemical Compositions of the Matrix 2a Glasses Supporting the EM-21 FY09 Task," Savannah River National Laboratory, Aiken, SC, SRNL-L5200-2009-00025, 2009.

15. T.B. Edwards, "An Analytical Plan for Measuring the Chemical Compositions of the Second Set of ROC Glasses and Three Glasses from the EM-21 FY09 Task," Savannah River National Laboratory, Aiken, SC, SRNL-L5100-2009-00003, 2009.

16. "Statistical Analysis of Ground-Water Monitoring Data at RCRA Facilities," United States Environmental Protection Agency, Washington, D.C., 530-SW-89-026, 1989.

17. "Standard Test Methods for Determining Chemical Durability of Nuclear, Hazardous, and Mixed Waste Glasses and Multiphase Glass Ceramics: The Product Consistency Test (PCT),” ASTM International, West Conshohocken, PA, ASTM C 1285-02(2008), 2008.

18. C.M. Jantzen, N.E. Bibler, D.C. Beam, C.L. Crawford, and M.A. Pickett, "Characterization of the Defense Waste Processing Facility (DWPF) Environmental Assessment (EA) Glass Standard Reference Material," Westinghouse Savannah River Company, Aiken, SC, WSRC-TR-92-346, Rev. 1, 1993.

19. "Nuclear Waste Glass and Glass-Ceramic Product Consistency Test (PCT) Methods (ASTM C1285 Latest Revision)," Savannah River National Laboratory, Aiken, SC, ITS0009, Rev. 2, 2006.

20. T.B. Edwards, "Analytical Plans for Measuring the PCT Solutions of Matrix 2 Glasses from the EM-21 FY09 Task," Savannah River National Laboratory, Aiken, SC, SRNLL5200-2009-00008

2009. 
21. T.B. Edwards, "Analytical Plans for Measuring the PCT Solutions of Matrix 2a Glasses from the EM-21 FY09 Task," Savannah River National Laboratory, Aiken, SC, SRNLL5200-2009-00024, 2009.

22. T.B. Edwards, "An Analytical Plan for Measuring the PCTs for the Third Set of ROC Glasses and Three Glasses from the EM-21 FY09 Task," Savannah River National Laboratory, Aiken, SC, SRNL-L5200-2009-00006, 2009.

23. "Operation of Scintag Pad-V X-Ray Diffractometer," Pacific Northwest National Laboratory (PNNL), Richland, WA, Safe Operating Procedure APEL-PAD-V, Rev. 2, 2002.

24. "Quantitative and Semi-Quantitative Analysis Using X-Ray Diffraction," Pacific Northwest National Laboratory (PNNL), Richland, WA, GDL-XRD, Rev. 1, 2007.

25. "Standard Test Methods for Determining the Liquidus Temperature $\left(\mathrm{T}_{1}\right)$ of Waste Glasses and Simulated Waste Glasses," Pacific Northwest National Laboratory (PNNL), Richland, WA, GDL-LQT, Rev. 4, 2007.

26. "Standard Practice for Measuring Viscosity of Glass above the Softening Point," ASTM International, West Conshohocken, PA, ASTM C965-81, 1990.

27. C.M. Jantzen, J.B. Pickett, K.G. Brown, T.B. Edwards, and D.C. Beam, "Process/Product Models for the Defense Waste Processing Facility (DWPF): Part I. Predicting Glass Durability from Composition Using a Thermodynamic Hydration Energy Reaction Model (THERMO)," Westinghouse Savannah River Company, Aiken, SC, WSRC-TR93-672, Rev. 1, 1995.

28. T.B. Edwards and K.G. Brown, "Evaluating the Glasses Batched for the Tank 42 Variability Study," Westinghouse Savannah River Company, Aiken, SC, SRT-SCS-98017, Rev. 0, 1998.

29. K.M. Fox and D.K. Peeler, "Demonstration of Very High Aluminum Retention in Simulated HLW Glass," Savannah River National Laboratory, Aiken, SC, SRNL-PSE2007-00231 Rev. 0, 2007. 


\section{Appendix A:}

\section{Tables and Exhibits Supporting the Analysis of the Chemical Composition Measurements of the Matrix 2 Study Glasses}


SRNL-STI-2009-00778, Revision 0

This page intentionally left blank. 
SRNL-STI-2009-00778, Revision 0

Table A1. Targeted Oxide Concentrations (wt\%) for the Non-Rad Matrix 2 Study Glasses (part 1)

\begin{tabular}{|c|c|c|c|c|c|c|c|c|c|c|c|c|c|}
\hline Glass ID & Al2O3 wt\% & B2O3 wt\% & BaO wt \% & $\mathrm{CaO} w \mathrm{wt} \%$ & CdO wt $\%$ & Ce203wt\% & Cr2O3 wt $\%$ & CuOwt\% & Fe2O3 wt $\%$ & K2O wt\% & La2O3 wt $\%$ & Li2O wt \% & MgO wt $\%$ \\
\hline FY09EM21-01 & 9.969 & 4.500 & 0.000 & 0.000 & 0 & 0.000 & 0.200 & 0.000 & 18.355 & 0.000 & 0.000 & 4.000 & 0.000 \\
\hline FY09EM21-02 & 4.007 & 5.066 & 0.080 & 0.000 & 0.296474 & 0.361 & 0.000 & 0.128 & 20.631 & 0.000 & 0.098 & 4.000 & 1.500 \\
\hline FY09EM21-03 & 6.970 & 4.500 & 0.000 & 0.000 & 0 & 0.000 & 0.000 & 0.000 & 12.519 & 0.000 & 0.000 & 7.000 & 1.500 \\
\hline FY09EM21-04 & 7.027 & 14.000 & 0.000 & 4.000 & 0 & 0.000 & 0.000 & 0.000 & \begin{tabular}{l|}
16.487 \\
\end{tabular} & 0.000 & 0.000 & 7.000 & 1.500 \\
\hline FY09EM21-05 & 3.604 & 4.500 & 0.000 & 4.000 & 0 & 0.000 & 0.000 & 0.000 & 13.771 & 0.000 & 0.000 & 4.001 & 0.000 \\
\hline FY09EM21-06 & 4.390 & 11.492 & 0.080 & 0.000 & 0.296474 & 0.361 & 0.200 & 0.128 & 5.297 & 0.000 & 0.098 & 4.000 & 0.000 \\
\hline \begin{tabular}{|l} 
FY09EM21-07 \\
\end{tabular} & 4.866 & 13.924 & 0.000 & 3.922 & 0 & 0.000 & 0.000 & 0.000 & 17.984 & 0.000 & 0.000 & 4.000 & 1.500 \\
\hline FY09EM21-08 & 3.250 & 4.974 & 0.000 & 0.505 & 0 & 0.000 & 0.200 & 0.000 & 20.479 & 0.000 & 0.000 & 7.000 & 1.500 \\
\hline \begin{tabular}{|l} 
FY09EM21-09 \\
\end{tabular} & 12.973 & 9.484 & 0.000 & 3.027 & 0 & 0.000 & 0.200 & 0.000 & 10.960 & 0.000 & 0.000 & 7.000 & 0.000 \\
\hline FY09EM21-10 & 6.395 & 4.720 & 0.080 & 4.000 & 0.296474 & 0.361 & 0.000 & 0.128 & 17.731 & 0.000 & 0.098 & 4.000 & 0.000 \\
\hline FY09EM21-11 & 3.250 & 4.578 & 0.080 & 4.000 & 0.296474 & 0.361 & 0.000 & 0.128 & 6.040 & 0.000 & 0.098 & 5.627 & 1.500 \\
\hline FY09EM21-12 & 4.554 & 5.291 & 0.000 & 0.000 & 0 & 0.000 & 0.000 & 0.000 & 16.865 & 0.000 & 0.000 & 6.794 & 0.000 \\
\hline FY09EM21-13 & 10.378 & 5.883 & 0.080 & 4.000 & 0.296474 & 0.361 & 0.200 & 0.128 & \begin{tabular}{|l|}
14.078 \\
\end{tabular} & 0.000 & 0.098 & 7.000 & 0.000 \\
\hline FY09EM21-14 & 3.250 & 13.647 & 0.080 & 0.000 & 0.296474 & 0.361 & 0.200 & 0.128 & 20.544 & 0.000 & 0.098 & 4.000 & 0.000 \\
\hline FY09EM21-15 & 5.323 & 4.500 & 0.080 & 3.190 & 0.296474 & 0.361 & 0.200 & 0.128 & 8.290 & 0.000 & 0.098 & 6.002 & 1.500 \\
\hline FY09EM21-16 & 3.677 & 6.427 & 0.080 & 3.821 & 0.296474 & 0.361 & 0.200 & 0.128 & 9.046 & 0.000 & 0.098 & 4.000 & 1.500 \\
\hline \begin{tabular}{|l} 
FY09EM21-17 \\
\end{tabular} & 7.193 & 4.500 & 0.080 & 0.104 & 0.296474 & 0.361 & 0.000 & 0.128 & 13.913 & 0.000 & 0.098 & 6.740 & 0.000 \\
\hline FY09EM21-18 & 10.916 & 4.844 & 0.080 & 0.439 & 0.296474 & 0.361 & 0.000 & 0.128 & 6.791 & 0.000 & 0.098 & 5.493 & 0.000 \\
\hline FY09EM21-19 & 5.719 & 4.501 & 0.000 & 4.000 & 0 & 0.000 & 0.000 & 0.000 & 5.000 & 0.000 & 0.000 & 4.002 & 0.000 \\
\hline FY09EM21-20 & 6.024 & 5.311 & 0.000 & 0.000 & 0 & 0.000 & 0.200 & 0.000 & 11.830 & 0.000 & 0.000 & 4.000 & 1.269 \\
\hline FY09EM21-21 & 4.857 & 9.010 & 0.080 & 0.000 & 0.296474 & 0.361 & 0.200 & 0.128 & 19.951 & 0.000 & 0.098 & 7.000 & 0.000 \\
\hline FY09EM21-22 & 14.041 & 13.199 & 0.080 & 0.000 & 0.296474 & 0.361 & 0.000 & 0.128 & 9.177 & 0.000 & 0.098 & 4.000 & 0.000 \\
\hline FY09EM21-23 & 13.959 & 5.781 & 0.080 & 0.000 & 0.296474 & 0.361 & 0.000 & 0.128 & $\begin{array}{l}12.109 \\
\end{array}$ & 0.000 & 0.098 & 4.000 & 1.500 \\
\hline FY09EM21-24 & 10.506 & 4.500 & 0.080 & 4.000 & 0.296474 & 0.361 & 0.200 & 0.128 & 20.560 & 0.000 & 0.098 & 4.000 & 1.500 \\
\hline FY09EM21-25 & 6.243 & 4.730 & 0.000 & 4.000 & 0 & 0.000 & 0.200 & 0.000 & 7.830 & 0.000 & 0.000 & 4.000 & 0.000 \\
\hline FY09EM21-26 & 13.656 & 7.576 & 0.000 & 0.000 & 0 & 0.000 & 0.200 & 0.000 & 5.000 & 0.000 & 0.000 & 4.001 & 1.500 \\
\hline FY09EM21-27 & 7.192 & 6.978 & 0.043 & 1.808 & 0.15964 & 0.194 & 0.100 & 0.069 & 13.125 & 0.000 & 0.053 & 5.102 & 0.683 \\
\hline
\end{tabular}


SRNL-STI-2009-00778, Revision 0

Table A1. Targeted Oxide Concentrations (wt\%) for the Non-Rad Matrix 2 Study Glasses (part 2)

\begin{tabular}{|c|c|c|c|c|c|c|c|c|c|c|c|c|}
\hline Glass ID & MnO wt $\%$ & $\mathrm{Na2O}$ wt $\%$ & $\mathrm{NiO}$ wt\% & P2O5 wt \% & PbO wt \% & $\begin{array}{l}\text { SO4 } \mathrm{wt} \% \\
\end{array}$ & $\begin{array}{l}\mathrm{SiO} 2 \mathrm{wt} \% \\
\end{array}$ & ThO2 (wt\%) & $\begin{array}{l}\text { TiO2 wt } \% \\
\end{array}$ & U308 wt\% & $\begin{array}{l}\mathrm{ZnO} \text { wt } \% \\
\end{array}$ & $\mathrm{ZrO2}(\mathrm{wt} \%)$ \\
\hline FY09EM21-01 & 5.500 & 11.390 & 2.500 & 0.000 & $\begin{array}{l}0.000 \\
\end{array}$ & 0 & 37.988 & 0.000 & 5.599 & 0.000 & 0.000 & 0.000 \\
\hline FY09EM21-02 & 0.300 & 17.977 & 2.500 & 0.000 & 0.216 & 0.48077 & 40.019 & 0.000 & 2.000 & 0.000 & 0.135 & 0.205 \\
\hline FY09EM21-03 & 5.500 & 14.131 & 2.500 & 0.000 & 0.000 & 0 & 40.650 & 0.000 & 4.730 & 0.000 & 0.000 & 0.000 \\
\hline FY09EM21-04 & 0.300 & 10.737 & 2.500 & 0.000 & 0.000 & 0 & \begin{tabular}{|l|}
32.449 \\
\end{tabular} & 0.000 & 4.000 & 0.000 & 0.000 & 0.000 \\
\hline FY09EM21-05 & 0.300 & 10.000 & 2.500 & 0.000 & 0.000 & 0 & \begin{tabular}{|l|}
51.497 \\
\end{tabular} & 0.000 & 5.827 & 0.000 & 0.000 & 0.000 \\
\hline \begin{tabular}{|l|l|} 
FY99M21-06 \\
\end{tabular} & 5.500 & 14.784 & 2.500 & 0.000 & 0.216 & 0.48077 & 43.836 & 0.000 & 6.000 & 0.000 & 0.135 & 0.205 \\
\hline \begin{tabular}{|l|l|} 
FY09EM21-07 \\
\end{tabular} & 5.500 & 12.706 & 0.000 & 0.000 & 0.000 & 0 & \begin{tabular}{|l|}
33.598 \\
\end{tabular} & 0.000 & 2.000 & 0.000 & 0.000 & 0.000 \\
\hline FY09EM21-08 & 1.386 & 10.000 & 0.000 & 0.000 & 0.000 & 0 & 44.706 & 0.000 & 6.000 & 0.000 & 0.000 & 0.000 \\
\hline FY09EM21-09 & 2.013 & 10.001 & 0.685 & 0.000 & 0.000 & 0 & 41.188 & 0.000 & 2.469 & 0.000 & 0.000 & 0.000 \\
\hline FY09EM21-10 & 5.464 & 10.389 & 2.500 & 0.000 & 0.216 & 0.48077 & 40.404 & 0.000 & 2.397 & 0.000 & 0.135 & 0.205 \\
\hline FY09EM21-11 & 5.500 & 12.208 & 0.000 & 0.000 & \begin{tabular}{l|l}
0.216 \\
\end{tabular} & 0.48077 & 49.297 & 0.000 & 6.000 & 0.000 & 0.135 & 0.205 \\
\hline FY09EM21-12 & 4.674 & 10.000 & 1.907 & 0.000 & 0.000 & 0 & 47.916 & 0.000 & 2.000 & 0.000 & 0.000 & 0.000 \\
\hline FY09EM21-13 & 0.300 & 10.516 & 2.500 & 0.000 & 0.216 & 0.48077 & 37.145 & 0.000 & 6.000 & 0.000 & 0.135 & 0.205 \\
\hline FY09EM21-14 & 0.300 & 10.000 & 0.000 & 0.000 & 0.216 & 0.48077 & 40.354 & 0.000 & 5.706 & 0.000 & 0.135 & 0.205 \\
\hline FY09EM21-15 & 5.359 & 10.000 & 2.500 & 0.000 & 0.216 & 0.48077 & 49.135 & 0.000 & 2.000 & 0.000 & 0.135 & 0.205 \\
\hline FY09EM21-16 & 0.300 & 15.820 & 2.500 & 0.000 & 0.216 & 0.48077 & \begin{tabular}{|l|}
48.708 \\
\end{tabular} & 0.000 & 2.000 & 0.000 & 0.135 & 0.205 \\
\hline \begin{tabular}{|l|l|} 
FY09EM21-17 \\
\end{tabular} & 5.500 & 14.855 & 0.000 & 0.000 & \begin{tabular}{l|l}
0.216 \\
\end{tabular} & 0.48077 & \begin{tabular}{|l|}
39.196 \\
\end{tabular} & 0.000 & 6.000 & 0.000 & 0.135 & 0.205 \\
\hline FY09EM21-18 & 0.835 & 17.019 & 0.000 & 0.000 & 0.216 & 0.48077 & 49.663 & 0.000 & 2.000 & 0.000 & 0.135 & 0.205 \\
\hline FY09EM21-19 & 0.300 & 18.000 & 0.981 & 0.000 & 0.000 & 0 & 51.497 & 0.000 & 6.000 & 0.000 & 0.000 & 0.000 \\
\hline FY09EM21-20 & 0.300 & 18.000 & 2.500 & 0.000 & 0.000 & 0 & $\begin{array}{l}48.565 \\
\end{array}$ & 0.000 & 2.000 & 0.000 & 0.000 & 0.000 \\
\hline FY09EM21-21 & 0.997 & 14.667 & 0.000 & 0.000 & 0.216 & 0.48077 & $\begin{array}{ll}39.306 \\
\end{array}$ & 0.000 & 2.013 & 0.000 & 0.135 & 0.205 \\
\hline FY09EM21-22 & 0.300 & 10.000 & 2.500 & 0.000 & 0.216 & 0.48077 & \begin{tabular}{|l|l|}
42.740 \\
\end{tabular} & 0.000 & 2.042 & 0.000 & 0.135 & 0.205 \\
\hline FY09EM21-23 & 0.300 & 10.000 & 0.000 & 0.000 & \begin{tabular}{l|l}
0.216 \\
\end{tabular} & 0.48077 & $\begin{array}{l}44.352 \\
\end{array}$ & 0.000 & 6.000 & 0.000 & 0.135 & 0.205 \\
\hline FY09EM21-24 & 0.917 & 10.001 & 0.000 & 0.000 & 0.216 & 0.48077 & 37.880 & 0.000 & 3.936 & 0.000 & 0.135 & 0.205 \\
\hline FY09EM21-25 & 5.500 & 15.464 & 0.000 & 0.000 & 0.000 & 0 & 50.034 & 0.000 & 2.000 & 0.000 & 0.000 & 0.000 \\
\hline FY09EM21-26 & 5.051 & 11.956 & 0.000 & 0.000 & 0.000 & 0 & \begin{tabular}{|l|}
45.703 \\
\end{tabular} & 0.000 & 5.357 & 0.000 & 0.000 & 0.000 \\
\hline FY09EM21-27 & 2.623 & 12.716 & 1.291 & 0.000 & 0.116 & 0.258876 & 43.378 & 0.000 & 3.926 & 0.000 & 0.072 & 0.110 \\
\hline
\end{tabular}


SRNL-STI-2009-00778, Revision 0

Table A2. Measured Elemental Concentrations (wt\%) for the Non-Rad Matrix 2 Glasses Prepared Using Lithium Metaborate (part 1)

\begin{tabular}{|c|c|c|c|c|c|c|c|c|c|c|c|c|c|c|c|}
\hline Set & Glass ID & Block & \begin{tabular}{|l|} 
Sub-Block \\
\end{tabular} & Sequence 1 & Lab ID & \begin{tabular}{|l|l|}
$\mathrm{Al}(\mathrm{wt} \%)$ \\
\end{tabular} & Ba (wt\%) & Ca (wt\%) & Cd (wt\%) & \begin{tabular}{|l|}
$\mathrm{Ce}(\mathrm{wt} \%)$ \\
\end{tabular} & \begin{tabular}{|l|} 
Cr (wt\%) \\
\end{tabular} & Cu (wt\%) & \begin{tabular}{|l|l}
$\mathrm{Fe}$ \\
\end{tabular} & La (wt\%) & $\operatorname{Mg}(\mathrm{wt} \%)$ \\
\hline 1 & Batch 1 & 1 & 1 & \begin{tabular}{l|l}
1 & 1 \\
\end{tabular} & BCHLM1111 & 2.45 & 0.125 & 0.818 & $<0.010$ & $<0.010$ & 0.080 & 0.304 & 8.62 & $<0.010$ & 0.796 \\
\hline 1 & FY09EM21-05 & 1 & 1 & 211 & \begin{tabular}{|l|} 
F12LM21 \\
\end{tabular} & 1.98 & $<0.010$ & 2.94 & $<0.010$ & $<0.010$ & 0.015 & $<0.010$ & 9.39 & $<0.010$ & $<0.010$ \\
\hline 1 & FY09EM21-12 & 1 & 1 & 31 & \begin{tabular}{|l|} 
F05LM21 \\
\end{tabular} & 2.45 & $<0.010$ & $<0.010$ & $<0.010$ & 0.011 & 0.015 & $<0.010$ & 10.7 & $<0.010$ & $<0.010$ \\
\hline 1 & FY09EM21-12 & 1 & 1 & $4 \sqrt{1}$ & \begin{tabular}{|l|} 
F05LM11 \\
\end{tabular} & 2.44 & $<0.010$ & $<0.010$ & $<0.010$ & 0.011 & 0.014 & $<0.010$ & 10.9 & $<0.010$ & $<0.010$ \\
\hline 1 & FY09EM21-01 & 1 & 1 & 51 & \begin{tabular}{|l|} 
F02LM11 \\
\end{tabular} & 5.41 & $<0.010$ & $<0.010$ & $<0.010$ & $<0.010$ & 0.135 & $<0.010$ & 12.5 & $<0.010$ & $<0.010$ \\
\hline 1 & FY09EM21-14 & 1 & 1 & 61 & \begin{tabular}{|l|} 
F09LM11 \\
\end{tabular} & 1.70 & 0.071 & 0.024 & 0.223 & 0.292 & 0.101 & 0.102 & 13.8 & 0.073 & 0.000 \\
\hline 1 & FY09EM21-13 & 1 & 1 & 71 & \begin{tabular}{|l|} 
F13LM11 \\
\end{tabular} & 5.40 & 0.067 & 2.83 & 0.226 & 0.286 & 0.119 & 0.097 & 9.28 & 0.073 & $<0.010$ \\
\hline 1 & FY09EM21-02 & 1 & 1 & 81 & \begin{tabular}{|l|} 
F03LM11 \\
\end{tabular} & 2.17 & 0.075 & $<0.010$ & 0.221 & 0.309 & 0.013 & 0.103 & 14.1 & 0.077 & 0.873 \\
\hline 1 & Batch 1 & 1 & 1 & 911 & BCHLM1112 & 2.42 & 0.127 & 0.829 & $<0.010$ & $<0.010$ & 0.081 & 0.305 & 8.70 & $<0.010$ & 0.807 \\
\hline 1 & FY09EM21-05 & 1 & 1 & 101 & \begin{tabular}{|l|} 
F12LM11 \\
\end{tabular} & 1.90 & $<0.010$ & 2.88 & $<0.010$ & $<0.010$ & 0.015 & $<0.010$ & 9.25 & $<0.010$ & $<0.010$ \\
\hline 1 & FY09EM21-01 & 1 & 1 & $11 \mid 1$ & \begin{tabular}{|l|} 
F02LM21 \\
\end{tabular} & 5.25 & $<0.010$ & $<0.010$ & $<0.010$ & $<0.010$ & 0.136 & $<0.010$ & 12.4 & $<0.010$ & $<0.010$ \\
\hline 1 & FY09EM21-13 & 1 & 1 & \begin{tabular}{l|l}
12 & 1 \\
\end{tabular} & \begin{tabular}{|l|} 
F13LM21 \\
\end{tabular} & 5.43 & 0.069 & 2.85 & 0.229 & 0.293 & \begin{tabular}{|l|}
0.124 \\
\end{tabular} & 0.098 & 9.41 & 0.074 & $<0.010$ \\
\hline 1 & FY09EM21-02 & 1 & 1 & 13 i & \begin{tabular}{|l|} 
F03LM21 \\
\end{tabular} & 2.17 & 0.077 & $<0.010$ & 0.224 & 0.311 & 0.013 & 0.103 & 14.1 & 0.078 & 0.892 \\
\hline 1 & FY09EM21-08 & 1 & 1 & $14 \mathrm{l}$ & \begin{tabular}{|l|} 
F01LM21 \\
\end{tabular} & 1.72 & $<0.010$ & 0.373 & $<0.010$ & 0.012 & 0.109 & $<0.010$ & 14.1 & $<0.010$ & 0.832 \\
\hline 1 & FY09EM21-14 & 1 & 1 & $15 \mid 1$ & \begin{tabular}{|l|} 
F09LM21 \\
\end{tabular} & 1.69 & 0.071 & 0.022 & 0.222 & 0.299 & 0.100 & 0.104 & 13.6 & 0.075 & $<0.010$ \\
\hline 1 & FY09EM21-08 & 1 & 1 & 16 1 & \begin{tabular}{|l|} 
F01LM11 \\
\end{tabular} & 1.70 & $<0.010$ & 0.372 & $<0.010$ & 0.011 & \begin{tabular}{|l|}
0.109 \\
\end{tabular} & $<0.010$ & 13.9 & $<0.010$ & 0.832 \\
\hline 1 & Batch 1 & 1 & 1 & \begin{tabular}{l|l}
17 & 1 \\
\end{tabular} & BCHLM1113 & 2.41 & 0.126 & 0.848 & $<0.010$ & $<0.010$ & 0.080 & 0.306 & 8.60 & $<0.010$ & 0.792 \\
\hline 1 & Batch 1 & 1 & 2 & $\begin{array}{lll} & 1 & \\
\end{array}$ & BCHLM1121 & 2.50 & 0.123 & 0.836 & $<0.010$ & $<0.010$ & 0.076 & 0.306 & 8.75 & $<0.010$ & \begin{tabular}{|l|l|}
0.784 \\
\end{tabular} \\
\hline 1 & FY09EM21-13 & 1 & 2 & 2 - 1 & \begin{tabular}{|l|} 
F13LM12 \\
\end{tabular} & 5.54 & 0.065 & 2.95 & 0.216 & 0.283 & 0.114 & 0.098 & 9.19 & 0.072 & $<0.010$ \\
\hline 1 & FY09EM21-01 & 1 & 2 & 311 & \begin{tabular}{|l|} 
F02LM12 \\
\end{tabular} & 5.57 & $<0.010$ & $<0.010$ & $<0.010$ & $<0.010$ & 0.132 & $<0.010$ & 12.5 & $<0.010$ & $<0.010$ \\
\hline 1 & FY09EM21-05 & 1 & 2 & $4 \sqrt{1}$ & \begin{tabular}{|l|} 
F12LM22 \\
\end{tabular} & 1.98 & $<0.010$ & 2.96 & $<0.010$ & $<0.010$ & 0.011 & $<0.010$ & 9.43 & $<0.010$ & $<0.010$ \\
\hline 1 & FY09EM21-12 & 1 & 2 & 5 - 1 & \begin{tabular}{|l|} 
F05LM12 \\
\end{tabular} & 2.41 & $<0.010$ & $<0.010$ & $<0.010$ & $<0.010$ & 0.010 & $<0.010$ & 10.9 & $<0.010$ & $<0.010$ \\
\hline 1 & FY09EM21-14 & 1 & 2 & 6 - 1 & \begin{tabular}{|l|} 
F09LM12 \\
\end{tabular} & 1.68 & 0.069 & 0.029 & 0.215 & 0.290 & 0.096 & 0.103 & 13.8 & 0.073 & $<0.010$ \\
\hline 1 & FY09EM21-08 & 1 & 2 & $7 \sqrt{7}$ & \begin{tabular}{|l|} 
F01LM12 \\
\end{tabular} & 1.71 & $<0.010$ & 0.366 & $<0.010$ & $<0.010$ & 0.105 & $<0.010$ & \begin{tabular}{|l|l|}
14.1 & \\
\end{tabular} & $<0.010$ & 0.841 \\
\hline 1 & FY09EM21-13 & 1 & 2 & $8 \sqrt{1}$ & \begin{tabular}{|l|} 
F13LM22 \\
\end{tabular} & 5.46 & 0.066 & 2.88 & 0.221 & 0.288 & \begin{tabular}{|l|}
0.119 \\
\end{tabular} & 0.099 & 9.51 & 0.073 & $<0.010$ \\
\hline 1 & Batch 1 & 1 & 2 & 91 & BCHLM1122 & 2.47 & 0.127 & 0.822 & $<0.010$ & $<0.010$ & 0.078 & 0.301 & 8.91 & $<0.010$ & 0.814 \\
\hline 1 & FY09EM21-08 & 1 & 2 & 101 & \begin{tabular}{|l|l} 
F01LM22 \\
\end{tabular} & 1.76 & $<0.010$ & 0.361 & $<0.010$ & 0.010 & 0.104 & $<0.010$ & 14.3 & $<0.010$ & 0.830 \\
\hline 1 & FY09EM21-01 & 1 & 2 & $11 \mid 1$ & \begin{tabular}{|l|} 
F02LM22 \\
\end{tabular} & 5.46 & $<0.010$ & $<0.010$ & $<0.010$ & $<0.010$ & 0.131 & $<0.010$ & 12.5 & $<0.010$ & $<0.010$ \\
\hline 1 & FY09EM21-14 & 1 & 2 & $12 \mathrm{l}$ & \begin{tabular}{|l|} 
F09LM22 \\
\end{tabular} & 1.74 & 0.069 & 0.025 & 0.215 & 0.289 & 0.095 & 0.103 & 13.7 & 0.073 & $<0.010$ \\
\hline 1 & FY09EM21-05 & 1 & 2 & $\begin{array}{lll}13 & 1 \\
\end{array}$ & \begin{tabular}{|l|} 
F12LM12 \\
\end{tabular} & 1.95 & $<0.010$ & 2.97 & $<0.010$ & $<0.010$ & 0.011 & $<0.010$ & 9.27 & $<0.010$ & $<0.010$ \\
\hline 1 & FY09EM21-02 & 1 & 2 & $14 \mathrm{l}$ & \begin{tabular}{|l|} 
F03LM22 \\
\end{tabular} & 2.22 & 0.073 & $<0.010$ & 0.215 & 0.305 & $<0.010$ & 0.103 & 14.2 & 0.076 & 0.875 \\
\hline 1 & FY09EM21-12 & 1 & 2 & $15 \mid 1$ & \begin{tabular}{|l|} 
F05LM22 \\
\end{tabular} & 2.37 & $<0.010$ & $<0.010$ & $<0.010$ & $<0.010$ & 0.010 & $<0.010$ & 10.5 & $<0.010$ & $<0.010$ \\
\hline 1 & FY09EM21-02 & 1 & 2 & $16 \mathrm{I}$ & \begin{tabular}{|l|} 
F03LM12 \\
\end{tabular} & 2.15 & 0.072 & $<0.010$ & 0.214 & 0.306 & $<0.010$ & 0.103 & 14.2 & 0.076 & 0.879 \\
\hline 1 & Batch 1 & 1 & 2 & $17 \sqrt{17}$ & BCHLM1123 & 2.43 & 0.125 & 0.838 & $<0.010$ & $<0.010$ & 0.076 & 0.304 & 8.68 & $<0.010$ & 0.806 \\
\hline 1 & Batch 1 & 2 & 1 & \begin{tabular}{l|l}
1 & 1 \\
\end{tabular} & BCHLM1211 & 2.45 & 0.121 & 0.843 & $<0.010$ & $<0.010$ & 0.076 & 0.302 & 8.85 & $<0.010$ & 0.786 \\
\hline 1 & FY09EM21-04 & 2 & 1 & 211 & \begin{tabular}{|l|} 
F14LM21 \\
\end{tabular} & 3.71 & $<0.010$ & 2.85 & $<0.010$ & $<0.010$ & $<0.010$ & $<0.010$ & 11.0 & $<0.010$ & 0.817 \\
\hline 1 & FY09EM21-09 & 2 & 1 & $3 \sqrt{3}$ & \begin{tabular}{|l|} 
F10LM21 \\
\end{tabular} & 6.92 & $<0.010$ & 2.15 & $<0.010$ & $<0.010$ & 0.092 & $<0.010$ & 7.38 & $<0.010$ & $<0.010$ \\
\hline 1 & FY09EM21-10 & 2 & 1 & $4 \sqrt{1}$ & \begin{tabular}{|l|} 
F11LM11 \\
\end{tabular} & 3.41 & 0.065 & 2.95 & 0.215 & 0.290 & 0.010 & 0.098 & 11.5 & 0.070 & $<0.010$ \\
\hline 1 & FY09EM21-10 & 2 & 1 & 51 & \begin{tabular}{|l|} 
F11LM21 \\
\end{tabular} & 3.44 & 0.064 & 2.93 & 0.212 & 0.285 & 0.011 & 0.097 & 11.4 & 0.069 & $<0.010$ \\
\hline 1 & FY09EM21-09 & 2 & 1 & 61 & \begin{tabular}{|l|} 
F10LM11 \\
\end{tabular} & 7.03 & $<0.010$ & 2.21 & $<0.010$ & $<0.010$ & 0.094 & $<0.010$ & 7.23 & $<0.010$ & $<0.010$ \\
\hline 1 & FY09EM21-06 & 2 & 1 & 71 & \begin{tabular}{|l|} 
F08LM21 \\
\end{tabular} & 2.38 & 0.066 & 0.013 & 0.214 & 0.291 & 0.130 & 0.099 & 3.59 & 0.078 & $<0.010$ \\
\hline 1 & FY09EM21-07 & 2 & 1 & 81 & \begin{tabular}{|l|} 
F04LM11 \\
\end{tabular} & 2.55 & $<0.010$ & 2.82 & $<0.010$ & $<0.010$ & $<0.010$ & $<0.010$ & 11.4 & $<0.010$ & 0.802 \\
\hline 1 & Batch 1 & 2 & 1 & 911 & BCHLM1212 & 2.52 & 0.122 & 0.833 & $<0.010$ & $<0.010$ & 0.076 & 0.301 & 8.71 & $<0.010$ & 0.783 \\
\hline 1 & FY09EM21-11 & 2 & 1 & \begin{tabular}{l|l}
10 & 1 \\
1
\end{tabular} & \begin{tabular}{|l|} 
F06LM21 \\
\end{tabular} & 1.73 & 0.065 & 2.92 & 0.217 & 0.285 & 0.012 & \begin{tabular}{|l|}
0.099 \\
\end{tabular} & 4.21 & 0.070 & 0.809 \\
\hline 1 & FY09EM21-07 & 2 & 1 & $11 \mathrm{l}$ & \begin{tabular}{|l|} 
F04LM21 \\
\end{tabular} & 2.61 & $<0.010$ & 2.93 & $<0.010$ & $<0.010$ & $<0.010$ & $<0.010$ & 11.8 & $<0.010$ & 0.839 \\
\hline 1 & FY09EM21-03 & 2 & 1 & $12 \mathrm{l}$ & \begin{tabular}{|l|} 
F07LM11 \\
\end{tabular} & 3.75 & $<0.010$ & 0.018 & $<0.010$ & $<0.010$ & \begin{tabular}{|l|l|}
0.011 \\
\end{tabular} & $<0.010$ & 8.99 & $<0.010$ & 0.842 \\
\hline 1 & FY09EM21-04 & 2 & 1 & $\begin{array}{lll}13 & 1 \\
\end{array}$ & \begin{tabular}{|l|} 
F14LM11 \\
\end{tabular} & 3.89 & $<0.010$ & 3.03 & $<0.010$ & $<0.010$ & $<0.010$ & $<0.010$ & 10.6 & $<0.010$ & 0.820 \\
\hline 1 & FY09EM21-06 & 2 & 1 & $14 \mid 1$ & \begin{tabular}{|l|} 
F08LM11 \\
\end{tabular} & 2.44 & 0.064 & 0.032 & 0.205 & 0.284 & \begin{tabular}{|l|l|}
0.127 \\
\end{tabular} & 0.100 & 3.56 & 0.076 & $<0.010$ \\
\hline
\end{tabular}


SRNL-STI-2009-00778, Revision 0

Table A2. Measured Elemental Concentrations (wt\%) for the Non-Rad Matrix 2 Glasses Prepared Using Lithium Metaborate (part 1)

\begin{tabular}{|c|c|c|c|c|c|c|c|c|c|c|c|c|c|c|c|}
\hline Set & Glass ID & Block & \begin{tabular}{|l|} 
Sub-Block \\
\end{tabular} & Sequence & Lab ID & Al (wt\%) & Ba (wt\%) & Ca (wt\%) & Cd (wt\%) & Ce (wt\%) & Cr (wt \%) & Cu (wt\%) & $\mathrm{Fe}(\mathrm{wt} \%)$ & La (wt\%) & Mg (wt\%) \\
\hline 1 & FY09EM21-03 & 2 & 1 & 15 & \begin{tabular}{|l|} 
F07LM21 \\
\end{tabular} & 3.76 & $<0.010$ & 0.012 & $<0.010$ & $<0.010$ & 0.011 & $<0.010$ & 9.11 & $<0.010$ & 0.845 \\
\hline 1 & FY09EM21-11 & 2 & 1 & \begin{tabular}{l|l}
16 & 1 \\
\end{tabular} & \begin{tabular}{|l|} 
F06LM11 \\
\end{tabular} & 1.76 & 0.064 & 2.98 & 0.216 & \begin{tabular}{|l|}
0.283 \\
\end{tabular} & \begin{tabular}{|l|}
0.012 \\
\end{tabular} & 0.097 & 4.11 & 0.070 & \begin{tabular}{|l|}
0.798 \\
\end{tabular} \\
\hline 1 & Batch 1 & 2 & 1 & \begin{tabular}{|l|l}
17 & 1 \\
\end{tabular} & BCHLM1213 & 2.60 & 0.125 & $\begin{array}{l}0.819 \\
\end{array}$ & $<0.010$ & $<0.010$ & \begin{tabular}{|l|}
0.077 \\
\end{tabular} & 0.300 & \begin{tabular}{|l|}
8.61 \\
\end{tabular} & $<0.010$ & \begin{tabular}{|l|}
0.789 \\
\end{tabular} \\
\hline 1 & Batch 1 & 2 & 2 & $1 / 1$ & \begin{tabular}{|l} 
BCHLM1221 \\
\end{tabular} & 2.50 & 0.125 & \begin{tabular}{|l|}
0.839 \\
\end{tabular} & $<0.010$ & $<0.010$ & \begin{tabular}{|l|}
0.079 \\
\end{tabular} & 0.306 & \begin{tabular}{|l|}
8.82 \\
\end{tabular} & $<0.010$ & \begin{tabular}{|l|}
0.807 \\
\end{tabular} \\
\hline 1 & \begin{tabular}{|l|} 
FY09EM21-03 \\
\end{tabular} & 2 & 2 & 21 & \begin{tabular}{|l|} 
F07LM22 \\
\end{tabular} & 3.63 & $<0.010$ & \begin{tabular}{|l|}
0.011 \\
\end{tabular} & $<0.010$ & $<0.010$ & \begin{tabular}{|l|}
0.010 \\
\end{tabular} & $<0.010$ & \begin{tabular}{|l|}
9.39 \\
\end{tabular} & $<0.010$ & \begin{tabular}{|l|}
0.866 \\
\end{tabular} \\
\hline 1 & \begin{tabular}{|l} 
FY09EM21-07 \\
\end{tabular} & 2 & 2 & 31 & \begin{tabular}{|l|} 
F04LM22 \\
\end{tabular} & 2.52 & $<0.010$ & \begin{tabular}{|l|}
2.80 \\
\end{tabular} & $<0.010$ & $<0.010$ & \begin{tabular}{|l|}
$<0.010$ \\
\end{tabular} & $<0.010$ & \begin{tabular}{|l|}
12.2 \\
\end{tabular} & $<0.010$ & \begin{tabular}{|l|}
0.838 \\
\end{tabular} \\
\hline 1 & FY09EM21-09 & 2 & 2 & $4 \mathrm{I}$ & \begin{tabular}{|l|} 
F10LM12 \\
\end{tabular} & 6.84 & $<0.010$ & 2.17 & $<0.010$ & $<0.010$ & \begin{tabular}{|l|l|}
0.095 \\
\end{tabular} & $<0.010$ & 7.41 & $<0.010$ & $<0.010$ \\
\hline 1 & FY09EM21-11 & 2 & 2 & 5 & \begin{tabular}{|l|} 
F06LM22 \\
\end{tabular} & 1.67 & 0.064 & 2.80 & 0.221 & 0.286 & \begin{tabular}{|l|}
0.012 \\
\end{tabular} & 0.099 & 4.22 & \begin{tabular}{|l|l|}
0.072 \\
\end{tabular} & \begin{tabular}{|l|l|}
0.817 \\
\end{tabular} \\
\hline 1 & FY09EM21-04 & 2 & 2 & $6 \mathrm{i}$ & \begin{tabular}{|l|} 
F14LM12 \\
\end{tabular} & 3.58 & $<0.010$ & 2.77 & $<0.010$ & $<0.010$ & $<0.010$ & $<0.010$ & 11.0 & $<0.010$ & 0.850 \\
\hline 1 & FY09EM21-09 & 2 & 2 & 7 & \begin{tabular}{|l|} 
F10LM22 \\
\end{tabular} & 6.66 & $<0.010$ & 2.08 & $<0.010$ & $<0.010$ & \begin{tabular}{|l|l|}
0.094 \\
\end{tabular} & $<0.010$ & 7.43 & $<0.010$ & $<0.010$ \\
\hline 1 & \begin{tabular}{|l} 
FY09EM21-06 \\
\end{tabular} & 2 & 2 & 81 & \begin{tabular}{|l|} 
F08LM22 \\
\end{tabular} & 2.27 & \begin{tabular}{|l|l|}
0.067 \\
\end{tabular} & 0.012 & 0.221 & 0.296 & \begin{tabular}{|l|}
0.133 \\
\end{tabular} & 0.100 & \begin{tabular}{|l|}
3.66 \\
\end{tabular} & \begin{tabular}{|l|l|}
0.081 \\
\end{tabular} & $<0.010$ \\
\hline 1 & Batch 1 & 2 & 2 & $9 \sqrt{1}$ & BCHLM1222 & 2.43 & 0.123 & 0.836 & $<0.010$ & $<0.010$ & \begin{tabular}{|l|}
0.078 \\
\end{tabular} & 0.304 & 8.97 & $<0.010$ & 0.793 \\
\hline 1 & FY09EM21-10 & 2 & 2 & $10[1$ & \begin{tabular}{|l|} 
F11LM22 \\
\end{tabular} & 3.35 & 0.063 & 2.84 & 0.211 & 0.286 & 0.010 & 0.098 & 11.9 & 0.070 & $<0.010$ \\
\hline 1 & FY09EM21-03 & 2 & 2 & \begin{tabular}{l|l}
11 & 1 \\
\end{tabular} & \begin{tabular}{|l|} 
F07LM12 \\
\end{tabular} & 3.54 & $<0.010$ & \begin{tabular}{|l|}
0.017 \\
\end{tabular} & $<0.010$ & $<0.010$ & \begin{tabular}{|l|}
0.010 \\
\end{tabular} & $<0.010$ & 9.49 & $<0.010$ & \begin{tabular}{|l|l|}
0.861 \\
\end{tabular} \\
\hline 1 & FY09EM21-06 & 2 & 2 & \begin{tabular}{l|l}
12 & 1 \\
\end{tabular} & \begin{tabular}{|l|} 
F08LM12 \\
\end{tabular} & 2.31 & 0.066 & 0.030 & 0.218 & \begin{tabular}{|l|}
0.297 \\
\end{tabular} & \begin{tabular}{|l|}
0.132 \\
\end{tabular} & 0.101 & 3.71 & 0.079 & $<0.010$ \\
\hline 1 & FY09EM21-11 & 2 & 2 & 13 i & \begin{tabular}{|l|} 
F06LM12 \\
\end{tabular} & 1.68 & 0.065 & 2.82 & 0.223 & 0.285 & \begin{tabular}{|l|}
0.013 \\
\end{tabular} & 0.098 & 4.21 & \begin{tabular}{|l|}
0.072 \\
\end{tabular} & 0.823 \\
\hline 1 & FY09EM21-04 & 2 & 2 & \begin{tabular}{l|l}
14 & 1 \\
\end{tabular} & \begin{tabular}{|l|} 
F14LM22 \\
\end{tabular} & 3.66 & $<0.010$ & \begin{tabular}{|l|}
2.86 \\
\end{tabular} & $<0.010$ & $<0.010$ & $<0.010$ & $<0.010$ & 11.1 & $<0.010$ & \begin{tabular}{|l|}
0.851 \\
\end{tabular} \\
\hline 1 & FY09EM21-10 & 2 & 2 & 15 & \begin{tabular}{|l|} 
F11LM12 \\
\end{tabular} & 3.32 & 0.064 & 2.89 & 0.216 & 0.269 & \begin{tabular}{|l|l|}
0.010 \\
\end{tabular} & 0.098 & \begin{tabular}{|l|}
11.7 \\
\end{tabular} & \begin{tabular}{|l|l|}
0.071 \\
\end{tabular} & $<0.010$ \\
\hline 1 & FY09EM21-07 & 2 & 2 & $16 \mid$ & \begin{tabular}{|l|} 
F04LM12 \\
\end{tabular} & 2.51 & $<0.010$ & 2.80 & $<0.010$ & \begin{tabular}{|l|l|}
$<0.010$ \\
\end{tabular} & \begin{tabular}{|l|}
$<0.010$ \\
\end{tabular} & $<0.010$ & 11.7 & $<0.010$ & 0.829 \\
\hline 1 & Batch 1 & 2 & 2 & \begin{tabular}{l|l}
17 & 1 \\
\end{tabular} & BCHLM1223 & 2.47 & 0.125 & 0.825 & $<0.010$ & $<0.010$ & \begin{tabular}{|l|l|}
0.078 \\
\end{tabular} & 0.303 & 8.89 & $<0.010$ & \begin{tabular}{|l|}
0.806 \\
\end{tabular} \\
\hline 2 & Batch 1 & 1 & 1 & \begin{tabular}{l|l}
1 & 1 \\
\end{tabular} & BCHLM2111 & 2.50 & 0.125 & 0.850 & $<0.010$ & $<0.010$ & \begin{tabular}{|l|}
0.078 \\
\end{tabular} & 0.303 & 8.72 & $<0.010$ & 0.803 \\
\hline 2 & FY09EM21-16 & 1 & 1 & 2 & \begin{tabular}{|l|} 
G01LM11 \\
\end{tabular} & 1.96 & 0.066 & 2.73 & 0.188 & 0.297 & 0.102 & 0.102 & 6.05 & 0.073 & 0.819 \\
\hline 2 & FY09EM21-23 & 1 & 1 & 3 & \begin{tabular}{|l|} 
G06LM11 \\
\end{tabular} & 7.33 & 0.069 & $\begin{array}{l}<<0.010 \\
\end{array}$ & 0.229 & \begin{tabular}{ll|}
0.304 \\
\end{tabular} & \begin{tabular}{|l|}
0.011 \\
\end{tabular} & 0.102 & 7.94 & \begin{tabular}{|l|}
0.075 \\
\end{tabular} & 0.844 \\
\hline 2 & FY09EM21-26 & 1 & 1 & 4 & \begin{tabular}{|l|} 
G11LM11 \\
\end{tabular} & 7.19 & $<0.010$ & 0.006 & $<0.010$ & $<0.010$ & 0.094 & $<0.010$ & 3.36 & $<0.010$ & 0.878 \\
\hline 2 & FY09EM21-22 & 1 & 1 & 5 & \begin{tabular}{|l|} 
G12LM21 \\
\end{tabular} & 7.51 & 0.070 & $<0.010$ & 0.236 & 0.302 & 0.013 & 0.104 & 6.04 & 0.075 & $<0.010$ \\
\hline 2 & FY09EM21-21 & 1 & 1 & 6 & \begin{tabular}{|l|} 
G09LM21 \\
\end{tabular} & 2.54 & 0.076 & $<0.010$ & 0.260 & \begin{tabular}{|l|}
0.316 \\
\end{tabular} & \begin{tabular}{|l|}
0.137 \\
\end{tabular} & 0.104 & 13.1 & \begin{tabular}{|l|l|}
0.077 \\
\end{tabular} & $<0.010$ \\
\hline 2 & FY09EM21-16 & 1 & 1 & 7 & \begin{tabular}{|l|} 
G01LM21 \\
\end{tabular} & 1.97 & 0.072 & 2.74 & 0.210 & \begin{tabular}{|l|}
0.311 \\
\end{tabular} & \begin{tabular}{|l|}
0.110 \\
\end{tabular} & 0.104 & 6.01 & \begin{tabular}{|l|l|}
0.077 \\
\end{tabular} & \begin{tabular}{|l|l|}
0.884 \\
\end{tabular} \\
\hline 2 & FY09EM21-21 & 1 & 1 & 8 & \begin{tabular}{|l|} 
G09LM11 \\
\end{tabular} & 2.58 & 0.072 & $\begin{array}{l}<0.010 \\
\end{array}$ & 0.243 & 0.307 & 0.129 & 0.106 & 13.1 & 0.075 & $<0.010$ \\
\hline 2 & Batch 1 & 1 & 1 & 91 & BCHLM2112 & 2.53 & 0.126 & \begin{tabular}{|l|} 
\\
0.871
\end{tabular} & $<0.010$ & $<0.010$ & \begin{tabular}{|l|}
0.079 \\
\end{tabular} & 0.305 & 8.63 & $<0.010$ & 0.812 \\
\hline 2 & \begin{tabular}{|l} 
FY09EM21-25 \\
\end{tabular} & 1 & 1 & 10 & \begin{tabular}{|l|} 
G04LM21 \\
\end{tabular} & 3.36 & $<0.010$ & \begin{tabular}{|l|}
2.90 \\
\end{tabular} & $<0.010$ & $<0.010$ & \begin{tabular}{|l|}
0.116 \\
\end{tabular} & 0.013 & 5.27 & $<0.010$ & $<0.010$ \\
\hline 2 & FY09EM21-23 & 1 & 1 & 11 & \begin{tabular}{|l|} 
G06LM21 \\
\end{tabular} & 7.35 & 0.069 & $\begin{array}{l}<0.010 \\
\end{array}$ & 0.235 & \begin{tabular}{|l|}
0.298 \\
\end{tabular} & \begin{tabular}{|l|}
0.012 \\
\end{tabular} & 0.098 & \begin{tabular}{|l|l|}
7.89 \\
\end{tabular} & \begin{tabular}{|l|l|}
0.075 \\
\end{tabular} & \begin{tabular}{|l|l|}
0.862 \\
\end{tabular} \\
\hline 2 & FY09EM21-17 & 1 & 1 & 12 & \begin{tabular}{|l|} 
G05LM21 \\
\end{tabular} & 4.04 & 0.096 & 0.078 & 0.242 & 0.335 & \begin{tabular}{|l|}
0.011 \\
\end{tabular} & 0.109 & 9.72 & 0.095 & $<0.010$ \\
\hline 2 & FY09EM21-22 & 1 & 1 & 13 & \begin{tabular}{|l|} 
G12LM11 \\
\end{tabular} & 7.56 & 0.069 & $<0.010$ & 0.236 & \begin{tabular}{|l|}
0.304 \\
\end{tabular} & \begin{tabular}{|l|}
0.013 \\
\end{tabular} & 0.102 & 5.96 & \begin{tabular}{|l|}
0.075 \\
\end{tabular} & $<0.010$ \\
\hline 2 & FY09EM21-17 & 1 & 1 & 14 & \begin{tabular}{|l|} 
G05LM11 \\
\end{tabular} & 4.03 & 0.066 & 0.089 & 0.243 & \begin{tabular}{|l|}
0.315 \\
\end{tabular} & \begin{tabular}{|l|}
0.011 \\
\end{tabular} & 0.118 & 9.58 & \begin{tabular}{|l|}
0.078 \\
\end{tabular} & $<0.010$ \\
\hline 2 & FY09EM21-25 & 1 & 1 & 15 & \begin{tabular}{|l|} 
G04LM11 \\
\end{tabular} & 3.38 & $<0.010$ & \begin{tabular}{|l|}
2.95 \\
\end{tabular} & $<0.010$ & $\begin{array}{l}<0.010 \\
\end{array}$ & 0.112 & 0.011 & 5.22 & $<0.010$ & $<0.010$ \\
\hline 2 & FY09EM21-26 & 1 & 1 & 16 & \begin{tabular}{|l|} 
G11LM21 \\
\end{tabular} & 7.31 & $<0.010$ & $\begin{array}{l}<0.010 \\
\end{array}$ & $<0.010$ & $<0.010$ & \begin{tabular}{|l|}
0.094 \\
\end{tabular} & $<0.010$ & 3.30 & $<0.010$ & \begin{tabular}{|l|l|}
0.877 \\
\end{tabular} \\
\hline 2 & Batch 1 & 1 & 1 & \begin{tabular}{l|l}
17 & 1 \\
\end{tabular} & BCHLM2113 & 2.56 & 0.128 & 0.872 & $<0.010$ & $<0.010$ & 0.080 & 0.304 & 8.62 & $<0.010$ & 0.814 \\
\hline 2 & Batch 1 & 1 & 2 & \begin{tabular}{l|l}
1 & 1 \\
\end{tabular} & BCHLM2121 & 2.50 & 0.124 & 0.803 & $<0.010$ & $<0.010$ & 0.076 & 0.290 & 8.74 & $<0.010$ & 0.812 \\
\hline 2 & FY09EM21-26 & 1 & 2 & 2 & \begin{tabular}{|l|} 
G11LM22 \\
\end{tabular} & 7.29 & $<0.010$ & $<0.010$ & $<0.010$ & $<0.010$ & \begin{tabular}{|l|}
0.091 \\
\end{tabular} & $<0.010$ & 3.41 & $<0.010$ & \begin{tabular}{|l|l|}
0.888 \\
\end{tabular} \\
\hline 2 & FY09EM21-21 & 1 & 2 & 3 & \begin{tabular}{|l|} 
G09LM222 \\
\end{tabular} & 2.58 & 0.071 & $<0.010$ & 0.250 & \begin{tabular}{|l|}
0.308 \\
\end{tabular} & \begin{tabular}{|l|}
0.130 \\
\end{tabular} & 0.098 & 13.4 & \begin{tabular}{|l|l|}
0.073 \\
\end{tabular} & $<0.010$ \\
\hline 2 & FY09EM21-23 & 1 & 2 & 4 & \begin{tabular}{|l|} 
G06LM22 \\
\end{tabular} & 7.45 & 0.066 & $<0.010$ & 0.227 & 0.284 & $<0.010$ & 0.093 & 7.94 & 0.070 & 0.851 \\
\hline 2 & FY09EM21-16 & 1 & 2 & 5 & \begin{tabular}{|l|} 
G01LM22 \\
\end{tabular} & 2.00 & \begin{tabular}{ll|}
0.068 \\
\end{tabular} & 2.77 & 0.202 & \begin{tabular}{|l|}
0.296 \\
\end{tabular} & \begin{tabular}{|l|l|}
0.104 \\
\end{tabular} & 0.099 & 6.19 & \begin{tabular}{|l|}
0.073 \\
\end{tabular} & \begin{tabular}{|l|}
0.858 \\
\end{tabular} \\
\hline 2 & FY09EM21-16 & 1 & 2 & 6 & \begin{tabular}{|l|} 
G01LM12 \\
\end{tabular} & 1.98 & 0.065 & 2.75 & 0.194 & \begin{tabular}{|l|}
0.284 \\
\end{tabular} & \begin{tabular}{|l|}
0.101 \\
\end{tabular} & 0.097 & 6.20 & \begin{tabular}{|l|}
0.071 \\
\end{tabular} & \begin{tabular}{|l|}
0.838 \\
\end{tabular} \\
\hline 2 & FY09EM21-23 & 1 & 2 & 7 & \begin{tabular}{|l|} 
G06LM12 \\
\end{tabular} & 7.47 & \begin{tabular}{|l|}
0.069 \\
\end{tabular} & $<0.010$ & 0.242 & 0.306 & \begin{tabular}{|l|}
$<0.010$ \\
\end{tabular} & $\begin{array}{l}0.097 \\
\end{array}$ & 8.01 & \begin{tabular}{|l|}
0.073 \\
\end{tabular} & \begin{tabular}{|l|}
0.898 \\
\end{tabular} \\
\hline 2 & FY09EM21-22 & 1 & 2 & 8 & \begin{tabular}{|l|} 
G12LM12 \\
\end{tabular} & 7.52 & \begin{tabular}{|l|}
0.066 \\
\end{tabular} & $<0.010$ & 0.231 & 0.294 & $<0.010$ & \begin{tabular}{|l|}
0.097 \\
\end{tabular} & \begin{tabular}{|l|}
6.14 \\
\end{tabular} & \begin{tabular}{|l|}
0.071 \\
\end{tabular} & $<0.010$ \\
\hline 2 & \begin{tabular}{|l} 
Batch 1 \\
\end{tabular} & 1 & 2 & 9 & \begin{tabular}{|l} 
BCHLM2122 \\
\end{tabular} & 2.53 & \begin{tabular}{|l|}
0.122 \\
\end{tabular} & \begin{tabular}{|l|l|}
0.826 \\
\end{tabular} & $<0.010$ & $<0.010$ & \begin{tabular}{|l|l|}
0.075 \\
\end{tabular} & 0.296 & \begin{tabular}{|l|}
8.68 \\
\end{tabular} & $<0.010$ & \begin{tabular}{|l|l|}
0.798 \\
\end{tabular} \\
\hline 2 & \begin{tabular}{|l|} 
FY09EM21-17 \\
\end{tabular} & 1 & 2 & \begin{tabular}{l|l}
10 & ( \\
\end{tabular} & \begin{tabular}{|l|} 
G05LM22 \\
\end{tabular} & 4.09 & 0.090 & 0.075 & 0.230 & 0.324 & $<0.010$ & 0.103 & 9.58 & \begin{tabular}{|l|l|}
0.089 \\
\end{tabular} & $<0.010$ \\
\hline 2 & FY09EM21-22 & 1 & 2 & 11 & \begin{tabular}{|l|} 
G12LM22 \\
\end{tabular} & 7.60 & 0.065 & \begin{tabular}{|l|}
$<0.010$ \\
\end{tabular} & 0.228 & $\begin{array}{l}0.289 \\
\end{array}$ & $<0.010$ & \begin{tabular}{|l|}
0.097 \\
\end{tabular} & 5.98 & \begin{tabular}{|l|}
0.069 \\
\end{tabular} & $<0.010$ \\
\hline
\end{tabular}


SRNL-STI-2009-00778, Revision 0

Table A2. Measured Elemental Concentrations (wt\%) for the Non-Rad Matrix 2 Glasses Prepared Using Lithium Metaborate (part 1)

\begin{tabular}{|c|c|c|c|c|c|c|c|c|c|c|c|c|c|c|c|}
\hline Set & Glass ID & Block & \begin{tabular}{|l|} 
Sub-Block \\
\end{tabular} & Sequence & Lab ID & Al (wt\%) & Ba (wt\%) & Ca (wt\%) & Cd (wt\%) & Ce (wt\%) & Cr (wt \%) & Cu (wt\%) & $\mathrm{Fe}(\mathrm{wt} \%)$ & La (wt\%) & Mg (wt\%) \\
\hline 2 & FY09EM21-25 & 1 & 2 & 12 & \begin{tabular}{|l|} 
G04LM12 \\
\end{tabular} & 3.42 & $<0.010$ & \begin{tabular}{|l|}
3.00 \\
\end{tabular} & $<0.010$ & $\begin{array}{l}<0.010 \\
\end{array}$ & \begin{tabular}{|l|}
0.109 \\
\end{tabular} & $<0.010$ & 5.33 & $<0.010$ & $<0.010$ \\
\hline 2 & FY09EM21-17 & 1 & 2 & 13 & \begin{tabular}{|l|} 
G05LM12 \\
\end{tabular} & 4.07 & 0.063 & \begin{tabular}{|l|}
0.084 \\
\end{tabular} & 0.243 & 0.302 & $<0.010$ & 0.111 & 9.77 & \begin{tabular}{|l|l|}
0.074 \\
\end{tabular} & $<0.010$ \\
\hline 2 & FY09EM21-26 & 1 & 2 & 14 & \begin{tabular}{|l|} 
G11LM12 \\
\end{tabular} & 7.28 & $<0.010$ & $<0.010$ & $<0.010$ & $\mid<0.010$ & \begin{tabular}{|l|l|}
0.091 \\
\end{tabular} & $<0.010$ & 3.33 & $<0.010$ & \begin{tabular}{|l|l|}
0.877 \\
\end{tabular} \\
\hline 2 & FY09EM21-25 & 1 & 2 & 15 & \begin{tabular}{|l|} 
G04LM22 \\
\end{tabular} & 3.41 & $<0.010$ & 2.97 & $<0.010$ & $<0.010$ & 0.113 & 0.011 & 5.24 & $<0.010$ & $<0.010$ \\
\hline 2 & FY09EM21-21 & 1 & 2 & 16 & \begin{tabular}{|l|} 
G09LM12 \\
\end{tabular} & 2.59 & 0.068 & $<0.010$ & 0.241 & 0.292 & 0.125 & 0.099 & 13.1 & 0.070 & $<0.010$ \\
\hline 2 & Batch 1 & 1 & 2 & 17 & \begin{tabular}{|l} 
BCHLM2123 \\
\end{tabular} & 2.55 & 0.122 & \begin{tabular}{|l|}
0.830 \\
\end{tabular} & $<0.010$ & $<0.010$ & \begin{tabular}{|l|}
0.075 \\
\end{tabular} & 0.293 & 8.68 & $<0.010$ & \begin{tabular}{|l|}
0.803 \\
\end{tabular} \\
\hline 2 & Batch 1 & 2 & 1 & 1 & BCHLM2211 & 2.49 & 0.127 & \begin{tabular}{|l|}
0.827 \\
\end{tabular} & $<0.010$ & $<0.010$ & \begin{tabular}{|l|}
0.079 \\
\end{tabular} & 0.298 & \begin{tabular}{|l|l|}
8.74 & \\
\end{tabular} & $<0.010$ & \begin{tabular}{|l|}
0.812 \\
\end{tabular} \\
\hline 2 & FY09EM21-19 & 2 & 1 & 2 & \begin{tabular}{|l|} 
G07LM21 \\
\end{tabular} & 3.11 & $<0.010$ & 2.95 & $<0.010$ & $<0.010$ & 0.013 & $<0.010$ & 3.47 & $<0.010$ & $<0.010$ \\
\hline 2 & FY09EM21-15 & 2 & 1 & 3 & \begin{tabular}{|l|} 
G03LM11 \\
\end{tabular} & 2.87 & 0.079 & 2.30 & 0.190 & 0.302 & \begin{tabular}{|l|l|}
0.098 \\
\end{tabular} & 0.098 & 5.49 & 0.078 & 0.810 \\
\hline 2 & FY09EM21-27 & 2 & 1 & 4 & \begin{tabular}{|l|} 
G13LM11 \\
\end{tabular} & 3.83 & 0.035 & 1.30 & 0.119 & 0.159 & 0.063 & 0.053 & 8.83 & 0.040 & 0.406 \\
\hline 2 & FY09EM21-20 & 2 & 1 & 5 & \begin{tabular}{|l|} 
G02LM21 \\
\end{tabular} & 3.24 & $<0.010$ & $<0.010$ & $<0.010$ & $<0.010$ & 0.110 & $<0.010$ & 7.96 & $<0.010$ & 0.713 \\
\hline 2 & FY09EM21-18 & 2 & 1 & 6 & \begin{tabular}{|l|} 
G10LM11 \\
\end{tabular} & 5.79 & 0.075 & 0.287 & 0.182 & 0.297 & 0.013 & 0.099 & 4.60 & 0.073 & $<0.010$ \\
\hline 2 & FY09EM21-24 & 2 & 1 & 7 & \begin{tabular}{|l|} 
G08LM11 \\
\end{tabular} & 5.55 & 0.069 & 2.88 & 0.225 & 0.293 & 0.105 & 0.095 & 13.1 & \begin{tabular}{|l|l|}
0.074 \\
\end{tabular} & 0.831 \\
\hline 2 & FY09EM21-18 & 2 & 1 & 8 & \begin{tabular}{|l|} 
G10LM21 \\
\end{tabular} & 5.87 & 0.075 & 0.293 & 0.180 & \begin{tabular}{|l|}
0.298 \\
\end{tabular} & \begin{tabular}{|l|}
0.013 \\
\end{tabular} & 0.100 & 4.66 & 0.073 & $<0.010$ \\
\hline 2 & Batch 1 & 2 & 1 & 9 & BCHLM2212 & 2.56 & 0.125 & 0.824 & $<0.010$ & $<0.010$ & 0.077 & 0.304 & 9.01 & $<0.010$ & 0.791 \\
\hline 2 & FY09EM21-27 & 2 & 1 & 10 & \begin{tabular}{|l|} 
G13LM21 \\
\end{tabular} & 3.80 & 0.035 & 1.26 & 0.116 & 0.158 & 0.062 & 0.053 & 8.99 & 0.040 & 0.399 \\
\hline 2 & FY09EM21-20 & 2 & 1 & 11 & \begin{tabular}{|l|} 
G02LM11 \\
\end{tabular} & 3.21 & $<0.010$ & $<0.010$ & $<0.010$ & $<0.010$ & \begin{tabular}{|l|}
0.109 \\
\end{tabular} & $<0.010$ & 8.00 & $<0.010$ & 0.708 \\
\hline 2 & FY09EM21-24 & 2 & 1 & 12 & \begin{tabular}{|l|} 
G08LM21 \\
\end{tabular} & 5.49 & 0.069 & 2.85 & 0.222 & 0.292 & 0.136 & 0.099 & 13.5 & 0.073 & 0.826 \\
\hline 2 & FY09EM21-19 & 2 & 1 & 13 & \begin{tabular}{|l|} 
G07LM11 \\
\end{tabular} & 3.07 & $<0.010$ & 2.88 & $<0.010$ & $<0.010$ & 0.013 & $<0.010$ & 3.45 & $<0.010$ & $<0.010$ \\
\hline 2 & FY09EM21-15 & 2 & 1 & 14 & \begin{tabular}{|l|} 
G03LM21 \\
\end{tabular} & 2.84 & \begin{tabular}{|l|}
0.079 \\
\end{tabular} & 2.25 & 0.185 & 0.299 & \begin{tabular}{|l|}
0.096 \\
\end{tabular} & 0.105 & 5.51 & 0.078 & 0.799 \\
\hline 2 & Batch 1 & 2 & 1 & 15 & BCHLM2213 & 2.46 & 0.124 & 0.829 & $<0.010$ & $<0.010$ & 0.077 & 0.304 & 8.87 & $<0.010$ & 0.783 \\
\hline 2 & Batch 1 & 2 & 2 & 1 & BCHLM2221 & 2.43 & 0.122 & 0.820 & $<0.010$ & $<0.010$ & 0.075 & 0.302 & 8.79 & $<0.010$ & 0.782 \\
\hline 2 & FY09EM21-19 & 2 & 2 & 2 & \begin{tabular}{|l|} 
G07LM22 \\
\end{tabular} & 3.08 & $<0.010$ & 2.89 & $<0.010$ & $<0.010$ & 0.012 & $<0.010$ & 3.53 & $<0.010$ & $<0.010$ \\
\hline 2 & FY09EM21-24 & 2 & 2 & 3 & \begin{tabular}{|l|} 
G08LM22 \\
\end{tabular} & 5.56 & 0.069 & 2.87 & 0.220 & 0.288 & 0.134 & 0.098 & 13.7 & 0.072 & 0.822 \\
\hline 2 & FY09EM21-20 & 2 & 2 & 4 & \begin{tabular}{|l|} 
G02LM12 \\
\end{tabular} & 3.29 & $<0.010$ & $<0.010$ & $<0.010$ & $<0.010$ & \begin{tabular}{|l|}
0.108 \\
\end{tabular} & $<0.010$ & 7.88 & $<0.010$ & 0.709 \\
\hline 2 & FY09EM21-27 & 2 & 2 & 5 & \begin{tabular}{|l|} 
G13LM22 \\
\end{tabular} & 3.90 & 0.037 & 1.29 & 0.120 & 0.156 & \begin{tabular}{|l|}
0.063 \\
\end{tabular} & 0.052 & 8.80 & 0.040 & 0.410 \\
\hline 2 & FY09EM21-18 & 2 & 2 & 6 & \begin{tabular}{|l|} 
G10LM22 \\
\end{tabular} & 5.93 & 0.074 & 0.286 & 0.178 & \begin{tabular}{|l|}
0.291 \\
\end{tabular} & \begin{tabular}{|l|}
0.013 \\
\end{tabular} & 0.099 & 4.70 & \begin{tabular}{|l|}
0.072 \\
\end{tabular} & $<0.010$ \\
\hline 2 & FY09EM21-27 & 2 & 2 & 7 & G13LM12 & 3.90 & 0.036 & 1.28 & 0.118 & 0.157 & 0.063 & 0.053 & 8.87 & 0.040 & 0.405 \\
\hline 2 & FY09EM21-18 & 2 & 2 & 8 & \begin{tabular}{|l|} 
G10LM12 \\
\end{tabular} & 5.84 & 0.073 & 0.286 & 0.175 & 0.293 & 0.012 & 0.099 & 4.70 & 0.072 & $<0.010$ \\
\hline 2 & Batch 1 & 2 & 2 & 9 & BCHLM2222 & 2.57 & 0.121 & \begin{tabular}{|l|l|}
0.807 \\
\end{tabular} & $<0.010$ & $<0.010$ & 0.076 & 0.301 & 8.99 & $<0.010$ & 0.779 \\
\hline 2 & FY09EM21-15 & 2 & 2 & 10 & \begin{tabular}{|l|} 
G03LM22 \\
\end{tabular} & 2.81 & 0.078 & 2.19 & 0.184 & 0.282 & 0.094 & 0.103 & 5.49 & 0.076 & 0.798 \\
\hline 2 & FY09EM21-15 & 2 & 2 & 11 & \begin{tabular}{|l|} 
G03LM12 \\
\end{tabular} & 2.84 & 0.079 & 2.22 & 0.189 & 0.297 & \begin{tabular}{|l|}
0.097 \\
\end{tabular} & 0.098 & 5.52 & \begin{tabular}{|l|}
0.077 \\
\end{tabular} & 0.815 \\
\hline 2 & FY09EM21-20 & 2 & 2 & 12 & \begin{tabular}{|l|} 
G02LM22 \\
\end{tabular} & 3.30 & $<0.010$ & $<0.010$ & $<0.010$ & $<0.010$ & 0.109 & $<0.010$ & 8.02 & $<0.010$ & 0.712 \\
\hline 2 & FY09EM21-19 & 2 & 2 & 13 & \begin{tabular}{|l|} 
G07LM12 \\
\end{tabular} & 3.10 & $<0.010$ & 2.90 & $<0.010$ & $<0.010$ & 0.012 & $<0.010$ & 3.49 & $<0.010$ & $<0.010$ \\
\hline 2 & FY09EM21-24 & 2 & 2 & 14 & \begin{tabular}{|l|} 
G08LM12 \\
\end{tabular} & 5.68 & 0.067 & 2.92 & 0.219 & 0.293 & 0.102 & 0.096 & 13.1 & 0.073 & 0.816 \\
\hline 2 & Batch 1 & 2 & 2 & 15 & BCHLM2223 & 2.58 & 0.123 & 0.813 & $<0.010$ & $<0.010$ & 0.076 & 0.303 & 9.12 & $<0.010$ & 0.783 \\
\hline
\end{tabular}


SRNL-STI-2009-00778, Revision 0

Table A2. Measured Elemental Concentrations (wt\%) for the Non-Rad Matrix 2 Glasses Prepared Using Lithium Metaborate (part 2)

\begin{tabular}{|c|c|c|c|c|c|c|c|c|c|c|c|c|c|c|}
\hline Set & \begin{tabular}{|l|} 
Glass ID \\
\end{tabular} & Block & \begin{tabular}{|l|} 
Sub-Block \\
\end{tabular} & Sequence & Lab ID & Mn (wt\%) & \begin{tabular}{|l|}
$\mathrm{Na}$ (wt\%) \\
\end{tabular} & \begin{tabular}{|l|l}
$\mathrm{Ni}(\mathrm{wt} \%)$ \\
\end{tabular} & Pb (wt\%) & S (wt\%) & Si (wt\%) & Ti (wt\%) & Zn (wt\%) & Zr (wt\%) \\
\hline 1 & Batch 1 & 1 & 1 & 1 & BCHLM1111 & 1.31 & 6.56 & 0.524 & $<0.010$ & $<0.050$ & 22.9 & 0.382 & $<0.010$ & 0.064 \\
\hline 1 & \begin{tabular}{|l|l|} 
FY09EM21-05 \\
\end{tabular} & 1 & 1 & 2 & F12LM21 & 0.244 & 7.46 & 1.83 & $<0.010$ & $<0.050$ & 23.5 & 3.52 & $<0.010$ & $<0.010$ \\
\hline 1 & \begin{tabular}{|l|} 
FY09EM21-12 \\
\end{tabular} & 1 & 1 & 3 & \begin{tabular}{|l|} 
F05LM21 \\
\end{tabular} & 3.60 & 7.39 & 1.33 & $<0.010$ & $<0.050$ & 21.8 & 1.20 & $<0.010$ & $<0.010$ \\
\hline 1 & \begin{tabular}{|l|} 
FY09EM21-12 \\
\end{tabular} & 1 & 1 & 4 & \begin{tabular}{|l|} 
F05LM11 \\
\end{tabular} & 3.68 & 7.36 & 1.36 & $<0.010$ & $<0.050$ & 22.0 & 1.21 & $<0.010$ & $<0.010$ \\
\hline 1 & FY09EM21-01 & 1 & 1 & 5 & \begin{tabular}{|l|} 
F02LM11 \\
\end{tabular} & 4.40 & 8.66 & $<0.010$ & $<0.010$ & $<0.050$ & 18.0 & 3.38 & $<0.010$ & $<0.010$ \\
\hline 1 & FY09EM21-14 & 1 & 1 & 6 & \begin{tabular}{|l|} 
F09LM11 \\
\end{tabular} & 0.234 & 7.23 & $<0.010$ & 0.174 & 0.142 & 18.5 & 3.38 & 0.105 & 0.143 \\
\hline 1 & \begin{tabular}{|l|l|} 
FY09EM21-13 \\
\end{tabular} & 1 & 1 & 7 & \begin{tabular}{|l|} 
F13LM11 \\
\end{tabular} & 0.225 & 7.62 & 1.75 & 0.171 & 0.151 & 17.1 & 3.55 & 0.110 & 0.143 \\
\hline 1 & \begin{tabular}{|l|l|} 
FY09EM21-02 \\
\end{tabular} & 1 & 1 & 8 & F03LM11 & 0.244 & 12.63 & $<0.010$ & 0.172 & 0.163 & 18.8 & 1.23 & 0.109 & 0.148 \\
\hline 1 & Batch 1 & 1 & 1 & 9 & BCHLM1112 & 1.33 & 6.46 & 0.533 & $<0.010$ & $<0.050$ & 22.8 & 0.380 & $<0.010$ & 0.065 \\
\hline 1 & \begin{tabular}{|l|l|} 
FY09EM21-05 \\
\end{tabular} & 1 & 1 & 10 & \begin{tabular}{|l|} 
F12LM11 \\
\end{tabular} & 0.241 & 7.21 & 1.80 & $<0.010$ & $<0.050$ & 23.1 & 3.48 & $<0.010$ & $<0.010$ \\
\hline 1 & \begin{tabular}{|l|} 
FY09EM21-01 \\
\end{tabular} & 1 & 1 & 11 & \begin{tabular}{|l|} 
F02LM21 \\
\end{tabular} & 4.36 & 8.31 & $<0.010$ & $<0.010$ & $<0.050$ & 17.7 & 3.34 & $<0.010$ & $<0.010$ \\
\hline 1 & \begin{tabular}{|l|} 
FY09EM21-13 \\
\end{tabular} & 1 & 1 & 12 & \begin{tabular}{|l|} 
F13LM21 \\
\end{tabular} & 0.230 & 7.72 & 1.78 & 0.177 & 0.151 & 17.2 & 3.59 & 0.111 & 0.145 \\
\hline 1 & \begin{tabular}{|l|l|} 
FY09EM21-02 \\
\end{tabular} & 1 & 1 & 13 & \begin{tabular}{|l|} 
F03LM21 \\
\end{tabular} & 0.247 & 12.59 & $<0.010$ & 0.175 & 0.168 & 19.0 & 1.24 & 0.110 & 0.152 \\
\hline 1 & \begin{tabular}{|l|} 
FY09EM21-08 \\
\end{tabular} & 1 & 1 & 14 & \begin{tabular}{|l|} 
F01LM21 \\
\end{tabular} & 1.11 & 7.14 & 0.014 & $<0.010$ & $<0.050$ & 20.0 & 3.56 & $<0.010$ & $<0.010$ \\
\hline 1 & \begin{tabular}{|l|} 
FY09EM21-14 \\
\end{tabular} & 1 & 1 & 15 & F09LM21 & 0.235 & 7.17 & $<0.010$ & 0.175 & 0.144 & 18.3 & 3.34 & 0.104 & 0.146 \\
\hline 1 & \begin{tabular}{|l|} 
FY09EM21-08 \\
\end{tabular} & 1 & 1 & 16 & \begin{tabular}{|l|} 
F01LM11 \\
\end{tabular} & 1.10 & 7.06 & 0.014 & $<0.010$ & $<0.050$ & 19.8 & 3.55 & $<0.010$ & $<0.010$ \\
\hline 1 & \begin{tabular}{|l|} 
Batch 1 \\
\end{tabular} & 1 & 1 & 17 & BCHLM1113 & 1.32 & 6.45 & 0.526 & $<0.010$ & $<0.050$ & 22.7 & 0.390 & $<0.010$ & 0.065 \\
\hline 1 & Batch 1 & 1 & 2 & 1 & BCHLM1121 & 1.32 & 6.76 & 0.517 & $<0.010$ & $<0.050$ & 23.3 & 0.386 & $<0.010$ & 0.063 \\
\hline 1 & FY09EM21-13 & 1 & 2 & 2 & F13LM12 & 0.220 & 7.91 & 1.71 & 0.165 & 0.146 & 17.1 & 3.56 & 0.107 & 0.142 \\
\hline 1 & \begin{tabular}{|l|} 
FY09EM21-01 \\
\end{tabular} & 1 & 2 & 3 & \begin{tabular}{|l|} 
F02LM12 \\
\end{tabular} & 4.41 & 8.97 & $<0.010$ & $<0.010$ & $<0.050$ & 18.2 & 3.39 & $<0.010$ & $<0.010$ \\
\hline 1 & \begin{tabular}{|l|l|} 
FY09EM21-05 \\
\end{tabular} & 1 & 2 & 4 & \begin{tabular}{|l} 
F12LM22 \\
\end{tabular} & 0.239 & 7.46 & 1.82 & $<0.010$ & $<0.050$ & 23.3 & 3.51 & $<0.010$ & $<0.010$ \\
\hline 1 & \begin{tabular}{|l|} 
FY09EM21-12 \\
\end{tabular} & 1 & 2 & 5 & \begin{tabular}{|l|l|} 
F05LM12 \\
\end{tabular} & 3.650 & 7.27 & 1.33 & $<0.010$ & $<0.050$ & 21.7 & 1.20 & $<0.010$ & $<0.010$ \\
\hline 1 & \begin{tabular}{|l|l|} 
FY09EM21-14 \\
\end{tabular} & 1 & 2 & 6 & \begin{tabular}{|l|} 
F09LM12 \\
\end{tabular} & 0.229 & 7.19 & $<0.010$ & 0.171 & 0.147 & 18.3 & 3.33 & 0.101 & 0.143 \\
\hline 1 & \begin{tabular}{|l|} 
FY09EM21-08 \\
\end{tabular} & 1 & 2 & 7 & \begin{tabular}{|l|} 
F01LM12 \\
\end{tabular} & 1.09 & 7.11 & 0.010 & $<0.010$ & $<0.050$ & 19.8 & 3.55 & $<0.010$ & $<0.010$ \\
\hline 1 & \begin{tabular}{|l|} 
FY09EM21-13 \\
\end{tabular} & 1 & 2 & 8 & \begin{tabular}{|l|} 
F13LM22 \\
\end{tabular} & 0.224 & 7.78 & 1.77 & 0.171 & 0.144 & 17.2 & 3.61 & 0.107 & 0.144 \\
\hline 1 & Batch 1 & 1 & 2 & 9 & BCHLM1122 & 1.34 & 6.58 & 0.531 & $<0.010$ & $<0.050$ & 23.2 & 0.386 & $<0.010$ & 0.064 \\
\hline 1 & \begin{tabular}{|l|} 
FY09EM21-08 \\
\end{tabular} & 1 & 2 & 10 & F01LM22 & 1.11 & 7.35 & 0.010 & $<0.010$ & $<0.050$ & 20.3 & 3.61 & $<0.010$ & $<0.010$ \\
\hline 1 & \begin{tabular}{|l|} 
FY09EM21-01 \\
\end{tabular} & 1 & 2 & 11 & \begin{tabular}{|l|} 
F02LM22 \\
\end{tabular} & 4.40 & 8.68 & $<0.010$ & $<0.010$ & $<0.050$ & 18.0 & 3.37 & $<0.010$ & $<0.010$ \\
\hline 1 & \begin{tabular}{|l|} 
FY09EM21-14 \\
\end{tabular} & 1 & 2 & 12 & F09LM22 & 0.229 & 7.41 & $<0.010$ & 0.171 & 0.140 & 18.6 & 3.37 & 0.101 & 0.143 \\
\hline 1 & \begin{tabular}{|l|} 
FY09EM21-05 \\
\end{tabular} & 1 & 2 & 13 & \begin{tabular}{|l|} 
F12LM12 \\
\end{tabular} & 0.239 & 7.39 & 1.76 & $<0.010$ & $<0.050$ & 23.1 & 3.46 & $<0.010$ & $<0.010$ \\
\hline 1 & FY09EM21-02 & 1 & 2 & 14 & F03LM22 & 0.240 & 12.79 & $<0.010$ & 0.169 & 0.161 & 19.0 & 1.24 & 0.107 & 0.149 \\
\hline 1 & FY09EM21-12 & 1 & 2 & 15 & \begin{tabular}{|l|} 
F05LM22 \\
\end{tabular} & 3.54 & 7.08 & 1.29 & $<0.010$ & $<0.050$ & 21.0 & 1.17 & $<0.010$ & $<0.010$ \\
\hline 1 & \begin{tabular}{|l|l|} 
FY09EM21-02 \\
\end{tabular} & 1 & 2 & 16 & \begin{tabular}{|l} 
F03LM12 \\
\end{tabular} & 0.240 & 12.59 & $<0.010$ & 0.169 & 0.156 & 18.7 & 1.23 & 0.106 & 0.146 \\
\hline 1 & Batch 1 & 1 & 2 & 17 & BCHLM1123 & 1.31 & 6.48 & 0.523 & $<0.010$ & $<0.050$ & 22.7 & 0.382 & $<0.010$ & 0.064 \\
\hline 1 & Batch 1 & 2 & 1 & 1 & BCHLM1211 & 1.35 & 6.60 & 0.511 & $<0.010$ & $<0.050$ & 23.3 & 0.378 & $<0.010$ & 0.063 \\
\hline 1 & \begin{tabular}{|l|l|} 
FY09EM21-04 \\
\end{tabular} & 2 & 1 & 2 & \begin{tabular}{|l|} 
F14LM21 \\
\end{tabular} & 0.225 & 7.89 & 1.80 & $<0.010$ & $<0.050$ & 15.0 & 2.380 & $<0.010$ & $<0.010$ \\
\hline 1 & \begin{tabular}{|l|} 
FY09EM21-09 \\
\end{tabular} & 2 & 1 & 3 & \begin{tabular}{|l|} 
F10LM21 \\
\end{tabular} & 1.60 & 7.51 & 0.449 & $<0.010$ & $<0.050$ & 19.0 & 1.50 & $<0.010$ & $<0.010$ \\
\hline 1 & \begin{tabular}{|l|} 
FY09EM21-10 \\
\end{tabular} & 2 & 1 & 4 & \begin{tabular}{|l|} 
F11LM11 \\
\end{tabular} & 4.19 & 8.02 & 1.72 & 0.173 & 0.267 & 18.6 & 1.45 & 0.111 & 0.142 \\
\hline 1 & \begin{tabular}{|l|l|} 
FY09EM21-10 \\
\end{tabular} & 2 & 1 & 5 & \begin{tabular}{|l|} 
F11LM21 \\
\end{tabular} & 4.16 & 8.07 & 1.72 & 0.171 & 0.263 & 18.6 & 1.45 & 0.110 & 0.141 \\
\hline 1 & \begin{tabular}{|l|l|} 
FY09EM21-09 \\
\end{tabular} & 2 & 1 & 6 & F10LM11 & 1.58 & 7.65 & 0.462 & 0.003 & $<0.050$ & 19.0 & 1.49 & $<0.010$ & $<0.010$ \\
\hline 1 & \begin{tabular}{|l|} 
FY09EM21-06 \\
\end{tabular} & 2 & 1 & 7 & \begin{tabular}{|l|} 
F08LM21 \\
\end{tabular} & 4.25 & 11.07 & 1.86 & 0.173 & 0.148 & 20.1 & 3.52 & 0.108 & 0.143 \\
\hline 1 & \begin{tabular}{|l|} 
FY09EM21-07 \\
\end{tabular} & 2 & 1 & 8 & \begin{tabular}{|l|} 
F04LM11 \\
\end{tabular} & 4.10 & 9.50 & 0.091 & $<0.010$ & $<0.050$ & 15.5 & 1.17 & $<0.010$ & $<0.010$ \\
\hline 1 & Batch 1 & 2 & 1 & 9 & BCHLM1212 & 1.34 & 6.77 & 0.514 & $<0.010$ & $<0.050$ & 23.4 & 0.373 & $<0.010$ & 0.063 \\
\hline 1 & FY09EM21-11 & 2 & 1 & 10 & F06LM21 & 4.20 & 9.24 & $<0.010$ & 0.164 & 0.154 & 22.6 & 4.08 & 0.108 & 0.141 \\
\hline 1 & \begin{tabular}{|l|} 
FY09EM21-07 \\
\end{tabular} & 2 & 1 & 11 & \begin{tabular}{|l|} 
F04LM21 \\
\end{tabular} & 4.22 & 9.84 & $<0.010$ & $<0.010$ & $<0.050$ & 15.6 & 1.20 & $<0.010$ & $<0.010$ \\
\hline 1 & \begin{tabular}{|l|l|} 
FY09EM21-03 \\
\end{tabular} & 2 & 1 & 12 & \begin{tabular}{|l|} 
F07LM11 \\
\end{tabular} & 4.27 & 11.11 & $<0.010$ & $<0.010$ & $<0.050$ & 18.7 & 2.96 & $<0.010$ & $<0.010$ \\
\hline 1 & \begin{tabular}{|l|} 
FY09EM21-04 \\
\end{tabular} & 2 & 1 & 13 & \begin{tabular}{|l} 
F14LM11 \\
\end{tabular} & 0.223 & 8.35 & 1.72 & $<0.010$ & $<0.050$ & 15.2 & 2.37 & $<0.010$ & $<0.010$ \\
\hline 1 & \begin{tabular}{|l|} 
FY09EM21-06 \\
\end{tabular} & 2 & 1 & 14 & \begin{tabular}{|l|} 
F08LM11 \\
\end{tabular} & 4.22 & 11.49 & 1.85 & 0.165 & 0.141 & 20.3 & 3.54 & 0.109 & 0.140 \\
\hline
\end{tabular}


SRNL-STI-2009-00778, Revision 0

Table A2. Measured Elemental Concentrations (wt\%) for the Non-Rad Matrix 2 Glasses Prepared Using Lithium Metaborate (part 2)

\begin{tabular}{|c|c|c|c|c|c|c|c|c|c|c|c|c|c|c|}
\hline Set & Glass ID & Block & \begin{tabular}{|l|} 
Sub-Block \\
\end{tabular} & Sequence & Lab ID & \begin{tabular}{|l|l} 
Mn (wt\%) \\
\end{tabular} & \begin{tabular}{|l|}
$\mathrm{Na}(\mathrm{wt} \%)$ \\
\end{tabular} & \begin{tabular}{|l|}
$\mathrm{Ni}(\mathrm{wt} \%)$ \\
\end{tabular} & Pb (wt\%) & S (wt\%) & Si (wt\%) & Ti (wt\%) & Zn (wt\%) & $\mathrm{Zr}(\mathrm{wt} \%)$ \\
\hline 1 & FY09EM21-03 & 2 & 1 & 15 & \begin{tabular}{|l|} 
F07LM21 \\
\end{tabular} & 4.30 & 11.17 & $<0.010$ & $<0.010$ & $<0.050$ & 18.9 & 2.98 & $<0.010$ & $<0.010$ \\
\hline 1 & FY09EM21-11 & 2 & 1 & 16 & \begin{tabular}{|l|} 
F06LM11 \\
\end{tabular} & 4.14 & 9.45 & $<0.010$ & \begin{tabular}{|l|}
0.161 \\
\end{tabular} & 0.149 & 22.5 & \begin{tabular}{|l|l|}
4.04 \\
\end{tabular} & 0.105 & 0.140 \\
\hline 1 & Batch 1 & 2 & 1 & 17 & BCHLM1213 & 1.33 & 7.08 & 0.522 & $<0.010$ & $<0.050$ & 23.8 & 0.376 & $<0.010$ & 0.064 \\
\hline 1 & Batch 1 & 2 & 2 & 1 & BCHLM1221 & 1.34 & 6.81 & \begin{tabular}{|l|}
0.529 \\
\end{tabular} & $<0.010$ & $<0.050$ & 23.4 & \begin{tabular}{|l|}
0.383 \\
\end{tabular} & $<0.010$ & \begin{tabular}{|l|}
0.064 \\
\end{tabular} \\
\hline \begin{tabular}{|l|l|}
1 \\
\end{tabular} & FY09EM21-03 & 2 & 2 & 2 & \begin{tabular}{|l|} 
F07LM22 \\
\end{tabular} & 4.42 & \begin{tabular}{|l|}
10.59 \\
\end{tabular} & $<0.010$ & $<0.010$ & $<0.050$ & $\mid 18.8$ & 3.00 & $<0.010$ & $<0.010$ \\
\hline \begin{tabular}{|l|l|}
1 \\
\end{tabular} & FY09EM21-07 & 2 & 2 & 3 & \begin{tabular}{|l|} 
F04LM22 \\
\end{tabular} & 4.34 & \begin{tabular}{|l|}
9.40 \\
\end{tabular} & $<0.010$ & $<0.010$ & $<0.050$ & 15.5 & 1.21 & $<0.010$ & $<0.010$ \\
\hline \begin{tabular}{|l|}
1 \\
\end{tabular} & FY09EM21-09 & 2 & 2 & 4 & \begin{tabular}{|l|} 
F10LM12 \\
\end{tabular} & 1.60 & 7.50 & 0.462 & $<0.010$ & $<0.050$ & 18.9 & 1.50 & $<0.010$ & $<0.010$ \\
\hline \begin{tabular}{|l|}
1 \\
\end{tabular} & FY09EM21-11 & 2 & 2 & 5 & \begin{tabular}{|l|} 
F06LM22 \\
\end{tabular} & 4.21 & \begin{tabular}{|l|}
8.90 \\
\end{tabular} & $<0.010$ & 0.166 & 0.149 & \begin{tabular}{|l|}
22.0 \\
\end{tabular} & \begin{tabular}{|l|}
4.05 \\
\end{tabular} & $\mid 0.109$ & 0.142 \\
\hline 1 & FY09EM21-04 & 2 & 2 & 6 & F14LM12 & 0.231 & 7.66 & 1.78 & $<0.010$ & $<0.050$ & 14.8 & 2.35 & $<0.010$ & $<0.010$ \\
\hline \begin{tabular}{|l|l|}
1 & \\
\end{tabular} & FY09EM21-09 & 2 & 2 & 7 & \begin{tabular}{|l|} 
F10LM22 \\
\end{tabular} & 1.61 & 7.24 & \begin{tabular}{|l|l|}
0.468 \\
\end{tabular} & $<0.010$ & $<0.050$ & \begin{tabular}{|l|l|}
18.7 \\
\end{tabular} & 1.49 & $<0.010$ & $<0.010$ \\
\hline 1 & FY09EM21-06 & 2 & 2 & 8 & \begin{tabular}{|l|} 
F08LM22 \\
\end{tabular} & 4.31 & 10.52 & 1.90 & \begin{tabular}{|l|l|}
0.178 \\
\end{tabular} & 0.142 & 19.8 & 3.51 & 0.112 & 0.146 \\
\hline 1 & Batch 1 & 2 & 2 & 9 & BCHLM1222 & 1.36 & 6.54 & 0.520 & $<0.010$ & $<0.050$ & 23.2 & 0.382 & $<0.010$ & 0.064 \\
\hline 1 & FY09EM21-10 & 2 & 2 & 10 & F11LM22 & 4.32 & 7.81 & 1.79 & 0.169 & 0.262 & 18.7 & 1.48 & 0.109 & \begin{tabular}{|l|}
0.141 \\
\end{tabular} \\
\hline 1 & FY09EM21-03 & 2 & 2 & 11 & \begin{tabular}{|l} 
F07LM12 \\
\end{tabular} & 4.41 & 10.38 & 0.003 & $<0.010$ & $<0.050$ & 18.6 & 3.01 & $<0.010$ & $<0.010$ \\
\hline 1 & FY09EM21-06 & 2 & 2 & 12 & \begin{tabular}{|l|} 
F08LM12 \\
\end{tabular} & 4.35 & 10.85 & 1.91 & \begin{tabular}{|l|}
0.172 \\
\end{tabular} & 0.145 & 20.1 & \begin{tabular}{|l|}
3.57 \\
\end{tabular} & 0.114 & 0.143 \\
\hline 1 & FY09EM21-11 & 2 & 2 & 13 & \begin{tabular}{|l|} 
F06LM12 \\
\end{tabular} & 4.20 & 8.89 & $<0.010$ & 0.166 & 0.147 & 22.1 & 4.04 & 0.107 & 0.142 \\
\hline \begin{tabular}{|l|l|}
1 \\
\end{tabular} & FY09EM21-04 & 2 & 2 & 14 & \begin{tabular}{|l|} 
F14LM22 \\
\end{tabular} & 0.232 & 7.81 & 1.80 & 0.005 & $<0.050$ & 14.9 & 2.38 & $<0.010$ & $<0.010$ \\
\hline 1 & FY09EM21-10 & 2 & 2 & 15 & \begin{tabular}{|l|} 
F11LM12 \\
\end{tabular} & 4.24 & 7.83 & 1.75 & \begin{tabular}{|l|}
0.173 \\
\end{tabular} & 0.269 & 18.5 & 1.46 & 0.111 & 0.143 \\
\hline 1 & FY09EM21-07 & 2 & 2 & 16 & \begin{tabular}{|l|} 
F04LM12 \\
\end{tabular} & 4.17 & 9.33 & 0.094 & $<0.010$ & $<0.050$ & 15.4 & 1.19 & $<0.010$ & $<0.010$ \\
\hline \begin{tabular}{|l|}
1 \\
\end{tabular} & Batch 1 & 2 & \begin{tabular}{|l|}
2 \\
\end{tabular} & 17 & BCHLM1223 & 1.35 & \begin{tabular}{|l|}
6.65 \\
\end{tabular} & \begin{tabular}{|l|}
0.528 \\
\end{tabular} & $<0.010$ & $<0.050$ & 23.3 & \begin{tabular}{|l|}
0.386 \\
\end{tabular} & $<0.010$ & 0.064 \\
\hline 2 & \begin{tabular}{|l|} 
Batch 1 \\
\end{tabular} & 1 & $\mid 1$ & 1 & BCHLM2111 & 1.32 & \begin{tabular}{|l|l|}
6.49 \\
\end{tabular} & \begin{tabular}{|l|}
0.520 \\
\end{tabular} & $<0.010$ & $<0.050$ & \begin{tabular}{|l|}
23.5 \\
\end{tabular} & \begin{tabular}{|l|}
0.377 \\
\end{tabular} & $<0.010$ & \begin{tabular}{|l|}
0.063 \\
\end{tabular} \\
\hline 2 & FY09EM21-16 & 1 & 1 & 2 & G01LM11 & 0.230 & 11.1 & 1.82 & 0.166 & 0.144 & 22.4 & 1.16 & \begin{tabular}{|l|}
0.109 \\
\end{tabular} & 0.142 \\
\hline 2 & FY09EM21-23 & 1 & 1 & 3 & G06LM11 & 0.234 & 7.13 & $<0.010$ & \begin{tabular}{|l|}
0.181 \\
\end{tabular} & \begin{tabular}{|l|}
0.128 \\
\end{tabular} & 20.3 & 3.40 & 0.102 & 0.146 \\
\hline 2 & FY09EM21-26 & 1 & 1 & 4 & G11LM11 & 4.01 & 8.62 & $<0.010$ & $<0.010$ & $<0.050$ & 21.0 & 2.90 & $<0.010$ & $<0.010$ \\
\hline 2 & FY09EM21-22 & 1 & 1 & 5 & G12LM21 & 0.242 & 7.27 & 1.74 & 0.184 & 0.141 & 19.8 & 1.20 & 0.115 & \begin{tabular}{|l|}
0.148 \\
\end{tabular} \\
\hline 2 & FY09EM21-21 & 1 & 1 & 6 & G09LM21 & \begin{tabular}{|l|}
0.808 \\
\end{tabular} & \begin{tabular}{|l|l|}
10.4 \\
\end{tabular} & $<0.010$ & 0.205 & 0.165 & \begin{tabular}{|l|l|}
17.8 \\
\end{tabular} & 1.17 & 0.110 & \begin{tabular}{|l|}
0.152 \\
\end{tabular} \\
\hline 2 & FY09EM21-16 & 1 & 1 & 7 & G01LM21 & \begin{tabular}{|l|}
0.249 \\
\end{tabular} & 11.1 & 1.80 & \begin{tabular}{|l|}
0.181 \\
\end{tabular} & 0.155 & 22.2 & 1.16 & 0.117 & \begin{tabular}{|l|}
0.149 \\
\end{tabular} \\
\hline 2 & FY09EM21-21 & 1 & 1 & 8 & G09LM11 & 0.770 & 10.5 & $<0.010$ & 0.193 & 0.165 & 17.9 & 1.18 & 0.107 & 0.147 \\
\hline 2 & Batch 1 & 1 & 1 & 9 & BCHLM2112 & 1.31 & 6.61 & 0.530 & $<0.010$ & $<0.050$ & 23.6 & 0.390 & $<0.010$ & 0.063 \\
\hline 2 & FY09EM21-25 & 1 & 1 & 10 & G04LM21 & 4.36 & 11.1 & $<0.010$ & $<0.010$ & $<0.050$ & 22.1 & 1.19 & $<0.010$ & 0.015 \\
\hline 2 & FY09EM21-23 & 1 & 1 & 11 & G06LM21 & \begin{tabular}{|l|}
0.237 \\
\end{tabular} & 7.13 & $<0.010$ & 0.179 & 0.130 & 20.1 & 3.37 & \begin{tabular}{|l|}
0.107 \\
\end{tabular} & \begin{tabular}{|l|}
0.142 \\
\end{tabular} \\
\hline 2 & FY09EM21-17 & 1 & 1 & 12 & G05LM21 & 0.457 & 10.9 & $<0.010$ & 0.192 & 0.170 & 19.1 & 3.65 & 0.115 & 0.156 \\
\hline \begin{tabular}{|l|}
2 \\
\end{tabular} & FY09EM21-22 & 1 & 1 & 13 & G12LM11 & 0.243 & 7.36 & 1.73 & \begin{tabular}{ll|}
0.184 \\
\end{tabular} & 0.142 & \begin{tabular}{|l|l|}
19.7 \\
\end{tabular} & 1.18 & 0.115 & \begin{tabular}{|l|}
0.149 \\
\end{tabular} \\
\hline 2 & FY09EM21-17 & 1 & \begin{tabular}{|l|l|}
1 \\
\end{tabular} & 14 & G05LM11 & \begin{tabular}{|l|}
0.466 \\
\end{tabular} & 11.0 & $<0.010$ & \begin{tabular}{|l|}
0.195 \\
\end{tabular} & \begin{tabular}{|l|}
0.173 \\
\end{tabular} & \begin{tabular}{|l|}
18.9 \\
\end{tabular} & \begin{tabular}{|l|}
3.64 \\
\end{tabular} & 0.114 & \begin{tabular}{|l|}
0.155 \\
\end{tabular} \\
\hline 2 & FY09EM21-25 & 1 & 1 & 15 & G04LM11 & 4.34 & 11.2 & $<0.010$ & $<0.010$ & $<0.050$ & 22.2 & 1.19 & $<0.010$ & $<0.010$ \\
\hline \begin{tabular}{|l|}
2 \\
\end{tabular} & FY09EM21-26 & 1 & 1 & 16 & G11LM21 & 3.97 & 8.73 & $<0.010$ & $<0.010$ & $<0.050$ & 21.0 & 2.87 & $<0.010$ & $<0.010$ \\
\hline 2 & \begin{tabular}{|l|} 
Batch 1 \\
\end{tabular} & 1 & 1 & 17 & BCHLM2113 & 1.31 & 6.63 & 0.540 & $<0.010$ & $<0.050$ & 23.6 & 0.385 & $<0.010$ & 0.063 \\
\hline 2 & Batch 1 & 1 & 2 & 1 & BCHLM2121 & 1.33 & 6.65 & 0.530 & $<0.010$ & $<0.050$ & 23.1 & 0.374 & $<0.010$ & 0.060 \\
\hline \begin{tabular}{|l|}
2 \\
\end{tabular} & FY09EM21-26 & 1 & 2 & 2 & G11LM22 & 4.08 & \begin{tabular}{|l|}
8.97 \\
\end{tabular} & $<0.010$ & $<0.010$ & $<0.050$ & 20.9 & 2.96 & $<0.010$ & $<0.010$ \\
\hline 2 & FY09EM21-21 & 1 & 2 & 3 & G09LM22 & 0.790 & 10.9 & $<0.010$ & \begin{tabular}{|l|l|}
0.197 \\
\end{tabular} & 0.150 & 17.9 & 1.20 & 0.104 & 0.146 \\
\hline 2 & FY09EM21-23 & 1 & 2 & 4 & G06LM22 & 0.229 & 7.49 & $<0.010$ & 0.172 & 0.119 & 20.0 & 3.43 & 0.100 & 0.137 \\
\hline 2 & FY09EM21-16 & 1 & 2 & 5 & G01LM22 & 0.241 & 11.5 & 1.87 & \begin{tabular}{|l|}
0.175 \\
\end{tabular} & \begin{tabular}{|l|}
0.138 \\
\end{tabular} & 22.4 & 1.20 & 0.111 & 0.144 \\
\hline 2 & FY09EM21-16 & 1 & 2 & 6 & G01LM12 & 0.233 & 11.6 & 1.87 & \begin{tabular}{|l|l|}
0.167 \\
\end{tabular} & 0.139 & 22.4 & 1.20 & 0.108 & \begin{tabular}{|l|}
0.141 \\
\end{tabular} \\
\hline 2 & FY09EM21-23 & 1 & 2 & 7 & G06LM12 & 0.240 & 7.46 & $<0.010$ & 0.185 & 0.126 & 20.3 & 3.48 & 0.104 & 0.146 \\
\hline 2 & FY09EM21-22 & 1 & 2 & 8 & \begin{tabular}{|l|} 
G12LM12 \\
\end{tabular} & \begin{tabular}{|l|} 
\\
\end{tabular} & 7.55 & 1.79 & \begin{tabular}{|l|}
0.178 \\
\end{tabular} & 0.130 & 19.6 & 1.21 & $\begin{array}{l}0.109 \\
\end{array}$ & \begin{tabular}{|l|}
0.144 \\
\end{tabular} \\
\hline \begin{tabular}{|l|}
2 \\
\end{tabular} & Batch 1 & 1 & \begin{tabular}{|l|}
2 \\
\end{tabular} & 9 & BCHLM2122 & 1.33 & 6.83 & 0.522 & $<0.010$ & $<0.050$ & 23.3 & \begin{tabular}{|l|}
0.379 \\
\end{tabular} & $<0.010$ & \begin{tabular}{|l|}
0.061 \\
\end{tabular} \\
\hline 2 & FY09EM21-17 & 1 & \begin{tabular}{|l|l|}
2 \\
\end{tabular} & 10 & G05LM22 & \begin{tabular}{|l|}
0.442 \\
\end{tabular} & \begin{tabular}{|l|}
11.3 \\
\end{tabular} & $<0.010$ & \begin{tabular}{|l|l|}
0.183 \\
\end{tabular} & 0.155 & \begin{tabular}{|l|l|}
18.8 \\
\end{tabular} & \begin{tabular}{|l|}
3.68 \\
\end{tabular} & 0.106 & \begin{tabular}{|l|}
0.150 \\
\end{tabular} \\
\hline 2 & FY09EM21-22 & 1 & 2 & 11 & G12LM22 & 0.233 & 7.66 & 1.75 & 0.176 & 0.129 & 19.6 & 1.21 & 0.108 & 0.141 \\
\hline
\end{tabular}


SRNL-STI-2009-00778, Revision 0

Table A2. Measured Elemental Concentrations (wt\%) for the Non-Rad Matrix 2 Glasses Prepared Using Lithium Metaborate (part 2)

\begin{tabular}{|c|c|c|c|c|c|c|c|c|c|c|c|c|c|c|}
\hline Set & Glass ID & Block & \begin{tabular}{|l|} 
Sub-Block \\
\end{tabular} & Sequence & Lab ID & Mn (wt\%) & \begin{tabular}{|l|}
$\mathrm{Na}(\mathrm{wt} \%)$ \\
\end{tabular} & Ni (wt\%) & Pb (wt\%) & S (wt\%) & Si (wt\%) & Ti (wt\%) & Zn (wt\%) & $\mathrm{Zr}(\mathrm{wt} \%)$ \\
\hline 2 & FY09EM21-25 & 1 & 2 & 12 & G04LM12 & 4.43 & \begin{tabular}{|l|}
11.8 \\
\end{tabular} & $<0.010$ & $<0.010$ & $<0.050$ & 22.3 & 1.22 & $<0.010$ & $<0.010$ \\
\hline 2 & FY09EM21-17 & 1 & 2 & 13 & G05LM12 & 0.463 & \begin{tabular}{|l|l|}
11.4 \\
\end{tabular} & $<0.010$ & \begin{tabular}{|l|l|}
0.192 \\
\end{tabular} & \begin{tabular}{|l|l|}
0.157 \\
\end{tabular} & 19.0 & 3.72 & 0.111 & 0.150 \\
\hline 2 & FY09EM21-26 & 1 & 2 & 14 & G11LM12 & 4.00 & \begin{tabular}{|l|}
9.07 \\
\end{tabular} & $<0.010$ & $<0.010$ & $<0.050$ & 20.8 & \begin{tabular}{|l|}
2.92 \\
\end{tabular} & $<0.010$ & $<0.010$ \\
\hline 2 & FY09EM21-25 & 1 & 2 & 15 & G04LM22 & 4.36 & 11.6 & $<0.010$ & $<0.010$ & $<0.050$ & 21.9 & 1.21 & $<0.010$ & 0.013 \\
\hline 2 & FY09EM21-21 & 1 & 2 & 16 & G09LM12 & 0.760 & 11.0 & $<0.010$ & \begin{tabular}{|l|l|}
0.188 \\
\end{tabular} & 0.153 & 17.8 & 1.19 & 0.102 & 0.140 \\
\hline 2 & Batch 1 & 1 & 2 & 17 & BCHLM2123 & 1.33 & 6.85 & 0.524 & $<0.010$ & $<0.050$ & 23.4 & \begin{tabular}{|l|}
0.381 \\
\end{tabular} & $<0.010$ & 0.060 \\
\hline 2 & Batch 1 & 2 & 1 & 1 & BCHLM2211 & 1.32 & 6.60 & 0.533 & $<0.010$ & $<0.050$ & 23.1 & 0.384 & $<0.010$ & 0.064 \\
\hline 2 & FY09EM21-19 & 2 & 1 & 2 & G07LM21 & 0.238 & 12.4 & 0.712 & $<0.010$ & $<0.050$ & 23.7 & 3.52 & $<0.010$ & $<0.010$ \\
\hline 2 & FY09EM21-15 & 2 & 1 & 3 & G03LM11 & 4.13 & 7.44 & 1.77 & 0.170 & 0.147 & 22.8 & 1.12 & 0.107 & 0.140 \\
\hline 2 & FY09EM21-27 & 2 & 1 & 4 & G13LM11 & 2.07 & 9.35 & 0.884 & 0.097 & 0.086 & 19.9 & 2.31 & 0.055 & 0.081 \\
\hline 2 & FY09EM21-20 & 2 & 1 & 5 & G02LM21 & 0.233 & 12.5 & 1.80 & $<0.010$ & $<0.050$ & 22.2 & 1.18 & $<0.010$ & $<0.010$ \\
\hline 2 & FY09EM21-18 & 2 & 1 & 6 & G10LM11 & 0.629 & 11.9 & $<0.010$ & \begin{tabular}{|l|}
0.179 \\
\end{tabular} & 0.147 & 22.8 & 1.16 & \begin{tabular}{|l|}
0.100 \\
\end{tabular} & 0.144 \\
\hline 2 & FY09EM21-24 & 2 & 1 & 7 & G08LM11 & 0.651 & 7.39 & $<0.010$ & \begin{tabular}{|l|}
0.178 \\
\end{tabular} & 0.133 & 17.6 & 2.11 & 0.101 & 0.142 \\
\hline 2 & FY09EM21-18 & 2 & 1 & 8 & G10LM21 & 0.624 & 11.9 & $<0.010$ & \begin{tabular}{|l|l|}
0.177 \\
\end{tabular} & 0.148 & 22.9 & \begin{tabular}{|l|l|}
1.17 \\
\end{tabular} & 0.102 & \begin{tabular}{|l|}
0.153 \\
\end{tabular} \\
\hline 2 & Batch 1 & 2 & 1 & 9 & BCHLM2212 & 1.35 & 6.77 & 0.519 & $<0.010$ & $<0.050$ & 23.8 & 0.386 & $<0.010$ & 0.064 \\
\hline 2 & FY09EM21-27 & 2 & 1 & 10 & G13LM21 & 2.11 & 9.12 & 0.869 & 0.094 & 0.085 & 19.9 & 2.34 & 0.054 & 0.080 \\
\hline 2 & FY09EM21-20 & 2 & 1 & 11 & G02LM11 & 0.230 & 12.3 & 1.82 & $<0.010$ & $<0.050$ & 22.1 & 1.18 & $<0.010$ & $<0.010$ \\
\hline 2 & FY09EM21-24 & 2 & 1 & 12 & G08LM21 & 0.664 & 7.28 & $<0.010$ & 0.176 & 0.133 & 17.3 & 2.11 & 0.104 & 0.141 \\
\hline 2 & FY09EM21-19 & 2 & 1 & 13 & G07LM11 & 0.237 & 12.3 & 0.711 & $<0.010$ & $<0.050$ & 23.5 & 3.49 & $<0.010$ & $<0.010$ \\
\hline 2 & FY09EM21-15 & 2 & 1 & 14 & G03LM21 & 4.13 & 7.38 & 1.78 & 0.166 & 0.149 & 22.7 & 1.11 & 0.107 & 0.138 \\
\hline 2 & Batch 1 & 2 & 1 & 15 & BCHLM2213 & 1.33 & 6.49 & 0.518 & $<0.010$ & $<0.050$ & 23.1 & 0.383 & $<0.010$ & 0.063 \\
\hline 2 & Batch 1 & 2 & 2 & 1 & BCHLM2221 & 1.33 & 6.43 & 0.511 & $<0.010$ & $<0.050$ & 23.0 & 0.384 & $<0.010$ & 0.062 \\
\hline 2 & FY09EM21-19 & 2 & 2 & 2 & G07LM22 & 0.231 & 12.5 & \begin{tabular}{|l|l|}
0.688 \\
\end{tabular} & $<0.010$ & $<0.050$ & 23.8 & 3.52 & $<0.010$ & $<0.010$ \\
\hline 2 & FY09EM21-24 & 2 & 2 & 3 & G08LM22 & 0.661 & 7.39 & $<0.010$ & 0.174 & 0.129 & 17.5 & 2.14 & 0.103 & 0.141 \\
\hline 2 & FY09EM21-20 & 2 & 2 & 4 & G02LM12 & 0.231 & 12.7 & 1.81 & $<0.010$ & $<0.050$ & 22.3 & 1.18 & $<0.010$ & $<0.010$ \\
\hline 2 & FY09EM21-27 & 2 & 2 & 5 & G13LM22 & 2.08 & 9.52 & 0.892 & 0.098 & 0.081 & 20.1 & 2.34 & 0.055 & 0.080 \\
\hline 2 & FY09EM21-18 & 2 & 2 & 6 & G10LM22 & 0.620 & 12.0 & $<0.010$ & \begin{tabular}{|l|}
0.175 \\
\end{tabular} & 0.140 & 23.1 & 1.19 & 0.100 & \begin{tabular}{|l|}
0.152 \\
\end{tabular} \\
\hline 2 & FY09EM21-27 & 2 & 2 & 7 & G13LM12 & 2.10 & 9.49 & 0.892 & 0.097 & 0.078 & 20.1 & 2.34 & 0.054 & 0.081 \\
\hline 2 & FY09EM21-18 & 2 & 2 & 8 & G10LM12 & 0.610 & 12.0 & $<0.010$ & 0.174 & 0.142 & 23.0 & 1.18 & 0.097 & 0.143 \\
\hline 2 & Batch 1 & 2 & 2 & 9 & BCHLM2222 & 1.36 & 6.83 & \begin{tabular}{|l|}
0.509 \\
\end{tabular} & $<0.010$ & $<0.050$ & 23.9 & 0.383 & $<0.010$ & 0.062 \\
\hline 2 & FY09EM21-15 & 2 & 2 & 10 & G03LM22 & 4.12 & 7.28 & 1.79 & \begin{tabular}{|l|}
0.165 \\
\end{tabular} & 0.144 & 22.6 & 1.12 & 0.106 & \begin{tabular}{|l|}
0.137 \\
\end{tabular} \\
\hline 2 & FY09EM21-15 & 2 & 2 & 11 & G03LM12 & 4.13 & 7.30 & 1.80 & 0.171 & 0.142 & 22.7 & 1.12 & 0.105 & 0.139 \\
\hline 2 & FY09EM21-20 & 2 & 2 & 12 & G02LM22 & 0.236 & 12.8 & 1.84 & $<0.010$ & $<0.050$ & 22.6 & 1.20 & $<0.010$ & $<0.010$ \\
\hline 2 & FY09EM21-19 & 2 & 2 & 13 & G07LM12 & 0.234 & 12.4 & 0.705 & $<0.010$ & $<0.050$ & 23.8 & 3.50 & $<0.010$ & $<0.010$ \\
\hline 2 & FY09EM21-24 & 2 & 2 & 14 & G08LM12 & 0.646 & 7.54 & $<0.010$ & 0.175 & 0.134 & 17.8 & 2.13 & \begin{tabular}{|l|}
0.098 \\
\end{tabular} & 0.142 \\
\hline 2 & Batch 1 & 2 & 2 & 15 & BCHLM2223 & 1.38 & 6.82 & 0.513 & $<0.010$ & $<0.050$ & 24.1 & 0.380 & $<0.010$ & 0.063 \\
\hline
\end{tabular}


SRNL-STI-2009-00778, Revision 0

Table A3. Measured Elemental Concentrations (wt\%) for the Non-Rad Matrix 2 Glasses Prepared Using Peroxide Fusion

\begin{tabular}{|c|c|c|c|c|c|c|c|c|}
\hline Set & Glass ID & Block & Sub-Block & Sequence & Lab ID & B (wt\%) & Li (wt\%) & Si (wt\%) \\
\hline 1 & Batch 1 & 1 & 1 & 1 & BCHPF1111 & 2.57 & 2.04 & 23.3 \\
\hline 1 & FY09EM21-14 & 1 & 1 & 2 & F09PF11 & 4.25 & 1.80 & 19.1 \\
\hline 1 & FY09EM21-11 & 1 & 1 & 3 & F06PF21 & 1.46 & 2.55 & 22.6 \\
\hline 1 & FY09EM21-14 & 1 & 1 & 4 & F09PF21 & 5.08 & 2.23 & 18.9 \\
\hline 1 & \begin{tabular}{|l|} 
FY09EM21-02 \\
\end{tabular} & 1 & 1 & 5 & F03PF11 & 1.64 & 1.88 & 18.9 \\
\hline 1 & FY09EM21-03 & 1 & 1 & 6 & F07PF11 & 1.47 & 3.18 & 19.0 \\
\hline 1 & FY09EM21-11 & 1 & 1 & 7 & F06PF11 & 1.29 & 2.53 & 21.0 \\
\hline 1 & FY09EM21-06 & 1 & 1 & 8 & F08PF21 & 3.48 & 1.78 & 19.8 \\
\hline 1 & Batch 1 & 1 & 1 & 9 & BCHPF1112 & 2.38 & 2.05 & 23.2 \\
\hline 1 & \begin{tabular}{|l|} 
FY09EM21-02 \\
\end{tabular} & 1 & 1 & 10 & F03PF21 & 1.70 & 1.87 & 19.2 \\
\hline 1 & FY09EM21-04 & 1 & 1 & 11 & F14PF11 & 4.40 & 3.23 & 15.1 \\
\hline 1 & FY09EM21-06 & 1 & 1 & 12 & F08PF11 & 3.60 & 1.77 & 20.1 \\
\hline 1 & FY09EM21-13 & 1 & 1 & 13 & F13PF21 & 1.88 & 3.20 & 17.6 \\
\hline 1 & FY09EM21-13 & 1 & 1 & 14 & F13PF11 & 1.83 & 3.20 & 17.2 \\
\hline 1 & FY09EM21-04 & 1 & 1 & 15 & F14PF21 & 4.25 & 3.18 & 14.8 \\
\hline 1 & FY09EM21-03 & 1 & 1 & 16 & F07PF21 & 1.38 & 3.06 & 18.5 \\
\hline 1 & Batch 1 & 1 & 1 & 17 & BCHPF1113 & 2.36 & 2.02 & 23.3 \\
\hline 1 & Batch 1 & 1 & 2 & 1 & BCHPF1121 & 2.61 & 2.02 & 24.2 \\
\hline 1 & FY09EM21-03 & 1 & 2 & 2 & F07PF22 & 1.43 & 3.13 & 18.8 \\
\hline 1 & FY09EM21-02 & 1 & 2 & 3 & F03PF12 & 1.61 & 1.89 & 19.8 \\
\hline 1 & FY09EM21-14 & 1 & 2 & 4 & F09PF12 & 4.27 & 1.84 & 19.3 \\
\hline 1 & FY09EM21-13 & 1 & 2 & 5 & F13PF22 & 1.84 & 3.26 & 18.1 \\
\hline 1 & FY09EM21-13 & 1 & 2 & 6 & F13PF12 & 1.76 & 3.18 & 17.5 \\
\hline 1 & FY09EM21-11 & 1 & 2 & 7 & F06PF22 & 1.27 & 2.54 & 22.3 \\
\hline 1 & FY09EM21-02 & 1 & 2 & 8 & F03PF22 & 1.49 & 1.84 & 19.1 \\
\hline 1 & Batch 1 & 1 & 2 & 9 & BCHPF1122 & 2.30 & 2.03 & 23.8 \\
\hline 1 & FY09EM21-03 & 1 & 2 & 10 & F07PF12 & 1.47 & 3.16 & 19.2 \\
\hline 1 & FY09EM21-04 & 1 & 2 & 11 & F14PF12 & 4.37 & 3.25 & 15.5 \\
\hline 1 & FY09EM21-06 & 1 & 2 & 12 & F08PF12 & 3.60 & 1.81 & 20.7 \\
\hline 1 & FY09EM21-06 & 1 & 2 & 13 & F08PF22 & 3.58 & 1.80 & 20.8 \\
\hline 1 & FY09EM21-11 & 1 & 2 & 14 & F06PF12 & 1.30 & 2.57 & 22.3 \\
\hline 1 & FY09EM21-04 & 1 & 2 & 15 & F14PF22 & 4.36 & 3.24 & 15.6 \\
\hline 1 & FY09EM21-14 & 1 & 2 & 16 & F09PF22 & 5.00 & 2.20 & 19.1 \\
\hline 1 & Batch 1 & 1 & 2 & 17 & BCHPF1123 & 2.39 & 2.04 & 24.0 \\
\hline 1 & Batch 1 & 2 & 1 & 1 & BCHPF1211 & 2.70 & 2.05 & 23.6 \\
\hline 1 & FY09EM21-10 & 2 & 1 & 2 & F11PF21 & 1.63 & 1.79 & 18.6 \\
\hline 1 & FY09EM21-12 & 2 & 1 & 3 & F05PF21 & 1.75 & 3.06 & 21.8 \\
\hline 1 & FY09EM21-08 & 2 & 1 & 4 & F01PF21 & 1.58 & 3.20 & 19.8 \\
\hline 1 & FY09EM21-05 & 2 & 1 & 5 & F12PF21 & 1.43 & 1.85 & 22.9 \\
\hline 1 & FY09EM21-01 & 2 & 1 & 6 & F02PF11 & 1.43 & 1.86 & 17.4 \\
\hline 1 & FY09EM21-05 & 2 & 1 & 7 & F12PF11 & 1.40 & 1.83 & 23.0 \\
\hline 1 & FY09EM21-08 & 2 & 1 & 8 & F01PF11 & 1.52 & 3.07 & 19.5 \\
\hline 1 & Batch 1 & 2 & 1 & 9 & BCHPF2112 & 2.36 & 2.04 & 22.9 \\
\hline 1 & FY09EM21-09 & 2 & 1 & 10 & F10PF11 & 2.95 & 3.15 & 18.3 \\
\hline 1 & FY09EM21-01 & 2 & 1 & 11 & F02PF21 & 1.50 & 1.84 & 17.5 \\
\hline 1 & FY09EM21-09 & 2 & 1 & 12 & F10PF21 & 2.95 & 3.10 & 18.6 \\
\hline 1 & FY09EM21-10 & 2 & 1 & 13 & F11PF11 & 1.52 & 1.80 & 18.5 \\
\hline 1 & FY09EM21-12 & 2 & 1 & 14 & F05PF11 & 1.68 & 3.11 & 21.6 \\
\hline 1 & FY09EM21-07 & 2 & 1 & 15 & F04PF21 & 4.30 & 1.82 & 15.3 \\
\hline 1 & FY09EM21-07 & 2 & 1 & 16 & F04PF11 & 4.30 & 1.77 & 15.2 \\
\hline 1 & Batch 1 & 2 & 1 & 17 & BCHPF1213 & 2.40 & 2.05 & 22.6 \\
\hline 1 & Batch 1 & 2 & 2 & 1 & BCHPF1221 & 2.59 & 2.05 & 24.0 \\
\hline 1 & FY09EM21-09 & 2 & 2 & 2 & F10PF12 & 3.02 & 3.18 & 19.5 \\
\hline 1 & FY09EM21-09 & 2 & 2 & 3 & F10PF22 & 2.93 & 3.19 & 19.3 \\
\hline 1 & FY09EM21-01 & 2 & 2 & 4 & F02PF12 & 1.37 & 1.84 & 18.2 \\
\hline 1 & FY09EM21-08 & 2 & 2 & 5 & F01PF22 & 1.47 & 3.14 & 20.8 \\
\hline 1 & FY09EM21-12 & 2 & 2 & 6 & F05PF22 & 1.56 & 3.06 & 22.7 \\
\hline 1 & \begin{tabular}{|l|} 
FY09EM21-08 \\
\end{tabular} & 2 & 2 & 7 & F01PF12 & 1.43 & 3.09 & 20.5 \\
\hline 1 & FY09EM21-07 & 2 & 2 & 8 & F04PF12 & 4.22 & 1.81 & 15.9 \\
\hline 1 & Batch 1 & 2 & 2 & 9 & BCHPF1222 & 2.36 & 2.05 & 24.1 \\
\hline 1 & FY09EM21-01 & 2 & 2 & 10 & F02PF22 & 1.43 & 1.88 & 18.5 \\
\hline 1 & FY09EM21-05 & 2 & 2 & 11 & F12PF12 & 1.30 & 1.81 & 23.8 \\
\hline 1 & FY09EM21-12 & 2 & 2 & 12 & F05PF12 & 1.51 & 3.06 & 22.0 \\
\hline 1 & FY09EM21-10 & 2 & 2 & 13 & F11PF12 & 1.33 & 1.81 & 19.1 \\
\hline 1 & FY09EM21-10 & 2 & 2 & 14 & F11PF22 & 1.31 & 1.79 & 19.0 \\
\hline 1 & FY09EM21-05 & 2 & 2 & 15 & F12PF22 & 1.25 & 1.85 & 24.1 \\
\hline 1 & \begin{tabular}{|l|} 
FY09EM21-07 \\
\end{tabular} & 2 & 2 & 16 & F04PF22 & 4.20 & 1.79 & 15.9 \\
\hline 1 & Batch 1 & 2 & 2 & 17 & BCHPF2113 & 2.29 & 2.04 & 23.8 \\
\hline 2 & Batch 1 & 1 & 1 & 1 & BCHPF2111 & 2.57 & 2.04 & 23.6 \\
\hline 2 & FY09EM21-23 & 1 & 1 & 2 & G06PF21 & 1.91 & 1.82 & 21.5 \\
\hline 2 & FY09EM21-24 & 1 & 1 & 3 & G08PF11 & 1.48 & 1.85 & 18.5 \\
\hline 2 & FY09EM21-21 & 1 & 1 & 4 & G09PF11 & 2.85 & 3.24 & 18.8 \\
\hline 2 & FY09EM21-19 & 1 & 1 & 5 & G07PF21 & 1.46 & 1.88 & 25.1 \\
\hline
\end{tabular}


SRNL-STI-2009-00778, Revision 0

Table A3. Measured Elemental Concentrations (wt\%) for the Non-Rad Matrix 2 Glasses Prepared Using Peroxide Fusion

\begin{tabular}{|c|c|c|c|c|c|c|c|c|}
\hline Set & Glass ID & Block & Sub-Block & Sequence & Lab ID & B (wt\%) & $\mathrm{Li}(\mathrm{wt} \%)$ & Si (wt\%) \\
\hline 2 & FY09EM21-19 & 1 & 1 & 6 & G07PF11 & 1.41 & 1.84 & 24.3 \\
\hline 2 & FY09EM21-21 & 1 & 1 & 7 & G09PF21 & 2.83 & 3.17 & 18.5 \\
\hline 2 & FY09EM21-18 & 1 & 1 & 8 & G10PF11 & 1.53 & 2.46 & 23.5 \\
\hline 2 & Batch 1 & 1 & 1 & 9 & BCHPF2112 & 2.40 & 2.02 & 23.9 \\
\hline 2 & FY09EM21-24 & 1 & 1 & 10 & G08PF21 & 1.49 & 1.83 & 18.3 \\
\hline 2 & FY09EM21-23 & 1 & 1 & 11 & G06PF11 & 1.83 & 1.82 & 21.1 \\
\hline 2 & FY09EM21-18 & 1 & 1 & 12 & G10PF21 & 1.57 & 2.50 & 23.9 \\
\hline 2 & FY09EM21-27 & 1 & 1 & 13 & G13PF11 & 2.18 & 2.32 & 20.5 \\
\hline 2 & FY09EM21-15 & 1 & 1 & 14 & G03PF21 & 1.46 & 2.69 & 23.3 \\
\hline 2 & FY09EM21-27 & 1 & 1 & 15 & G13PF21 & 2.16 & 2.30 & 20.3 \\
\hline 2 & FY09EM21-15 & 1 & 1 & 16 & G03PF11 & 1.48 & 2.69 & 23.6 \\
\hline 2 & Batch 1 & 1 & 1 & 17 & BCHPF2113 & 2.41 & 2.04 & 24.0 \\
\hline 2 & Batch 1 & 1 & 2 & 1 & BCHPF2121 & 2.46 & 2.02 & 22.7 \\
\hline 2 & FY09EM21-19 & 1 & 2 & 2 & G07PF22 & 1.44 & 1.82 & 23.5 \\
\hline 2 & FY09EM21-23 & 1 & 2 & 3 & G06PF22 & 1.79 & 1.80 & 20.2 \\
\hline 2 & FY09EM21-21 & 1 & 2 & 4 & G09PF22 & 2.76 & 3.17 & 17.9 \\
\hline 2 & FY09EM21-19 & 1 & 2 & 5 & G07PF12 & 1.38 & 1.83 & 23.3 \\
\hline 2 & FY09EM21-18 & 1 & 2 & 6 & G10PF22 & 1.48 & 2.48 & 22.8 \\
\hline 2 & FY09EM21-18 & 1 & 2 & 7 & G10PF12 & 1.46 & 2.46 & 22.8 \\
\hline 2 & FY09EM21-21 & 1 & 2 & 8 & G09PF12 & 2.68 & 3.21 & 18.0 \\
\hline 2 & Batch 1 & 1 & 2 & 9 & BCHPF2122 & 2.35 & 2.04 & 23.6 \\
\hline 2 & FY09EM21-24 & 1 & 2 & 10 & G08PF12 & 1.43 & 1.79 & 17.5 \\
\hline 2 & FY09EM21-27 & 1 & 2 & 11 & G13PF12 & 2.09 & 2.28 & 19.6 \\
\hline 2 & FY09EM21-23 & 1 & 2 & 12 & G06PF12 & 1.69 & 1.77 & 19.6 \\
\hline 2 & FY09EM21-24 & 1 & 2 & 13 & G08PF22 & 1.34 & 1.80 & 17.5 \\
\hline 2 & FY09EM21-15 & 1 & 2 & 14 & G03PF12 & 1.39 & 2.71 & 22.8 \\
\hline 2 & FY09EM21-27 & 1 & 2 & 15 & G13PF22 & 2.10 & 2.29 & 20.1 \\
\hline 2 & FY09EM21-15 & 1 & 2 & 16 & G03PF22 & 1.39 & 2.70 & 22.8 \\
\hline 2 & Batch 1 & 1 & 2 & 17 & BCHPF2123 & 2.30 & 2.02 & 23.4 \\
\hline 2 & Batch 1 & 2 & 1 & 1 & BCHPF2211 & 2.58 & 2.03 & 23.8 \\
\hline 2 & FY09EM21-16 & 2 & 1 & 2 & G01PF11 & 2.04 & 1.85 & 22.4 \\
\hline 2 & FY09EM21-25 & 2 & 1 & 3 & G04PF21 & 1.52 & 1.85 & 23.1 \\
\hline 2 & FY09EM21-20 & 2 & 1 & 4 & G02PF21 & 1.64 & 1.88 & 22.5 \\
\hline 2 & FY09EM21-26 & 2 & 1 & 5 & G11PF11 & 2.30 & 1.90 & 21.3 \\
\hline 2 & FY09EM21-17 & 2 & 1 & 6 & G05PF11 & 1.43 & 3.28 & 19.6 \\
\hline 2 & FY09EM21-22 & 2 & 1 & 7 & G12PF21 & 4.08 & 1.89 & 20.7 \\
\hline 2 & FY09EM21-17 & 2 & 1 & 8 & G05PF21 & 1.43 & 3.26 & 19.3 \\
\hline 2 & Batch 1 & 2 & 1 & 9 & BCHPF2212 & 2.32 & 2.10 & 23.8 \\
\hline 2 & FY09EM21-20 & 2 & 1 & 10 & G02PF11 & 1.67 & 1.87 & 22.6 \\
\hline 2 & FY09EM21-22 & 2 & 1 & 11 & G12PF11 & 4.09 & 1.85 & 20.4 \\
\hline 2 & FY09EM21-16 & 2 & 1 & 12 & G01PF21 & 2.01 & 1.84 & 22.9 \\
\hline 2 & FY09EM21-25 & 2 & 1 & 13 & G04PF11 & 1.49 & 1.88 & 23.2 \\
\hline 2 & FY09EM21-26 & 2 & 1 & 14 & G11PF21 & 2.30 & 1.90 & 21.5 \\
\hline 2 & Batch 1 & 2 & 1 & 15 & BCHPF2213 & 2.37 & 2.09 & 24.1 \\
\hline 2 & Batch 1 & 2 & 2 & 1 & BCHPF2221 & 2.66 & 2.09 & 23.7 \\
\hline 2 & FY09EM21-16 & 2 & 2 & 2 & G01PF22 & 2.11 & 1.86 & 22.4 \\
\hline 2 & FY09EM21-17 & 2 & 2 & 3 & G05PF22 & 1.56 & 3.24 & 19.2 \\
\hline 2 & FY09EM21-17 & 2 & 2 & 4 & G05PF12 & 1.54 & 3.22 & 19.1 \\
\hline 2 & FY09EM21-26 & 2 & 2 & 5 & G11PF22 & 2.40 & 1.91 & 21.2 \\
\hline 2 & FY09EM21-22 & 2 & 2 & 6 & G12PF12 & 4.20 & 1.88 & 20.3 \\
\hline 2 & FY09EM21-16 & 2 & 2 & 7 & G01PF12 & 2.06 & 1.86 & 22.2 \\
\hline 2 & FY09EM21-26 & 2 & 2 & 8 & G11PF12 & 2.36 & 1.89 & 20.9 \\
\hline 2 & Batch 1 & 2 & 2 & 9 & BCHPF2212 & 2.40 & 2.09 & 23.2 \\
\hline 2 & FY09EM21-25 & 2 & 2 & 10 & G04PF12 & 1.63 & 1.86 & 22.3 \\
\hline 2 & FY09EM21-20 & 2 & 2 & 11 & G02PF22 & 1.72 & 1.86 & 22.2 \\
\hline 2 & FY09EM21-25 & 2 & 2 & 12 & G04PF22 & 1.55 & 1.86 & 22.7 \\
\hline 2 & FY09EM21-20 & 2 & 2 & 13 & G02PF12 & 1.69 & 1.85 & 22.2 \\
\hline 2 & FY09EM21-22 & 2 & 2 & 14 & G12PF22 & 4.14 & 1.92 & 20.2 \\
\hline 2 & Batch 1 & 2 & 2 & 15 & BCHPF2223 & 2.46 & 2.10 & 23.8 \\
\hline
\end{tabular}


SRNL-STI-2009-00778, Revision 0

Table A4. Average Measured and Bias-Corrected Chemical Compositions Versus Targeted Compositions by Oxide by Glass ID for the Non-Rad Matrix 2 Study

\begin{tabular}{|c|c|c|c|c|c|c|c|c|c|}
\hline & & & & Measured & & Difference & Difference & $\%$ & $\%$ \\
\hline & & & Measured & Bias-Corrected & Targeted & of & of & Difference & Difference \\
\hline Set & Glass \# & Oxide & $(\mathrm{wt} \%)$ & (BC) (wt\%) & (wt\%) & Measured & Meas BC & Measured & Meas BC \\
\hline 1 & Batch 1 & $\mathrm{Al} 2 \mathrm{O} 3$ & 4.6686 & 4.8770 & 4.8770 & -0.2084 & 0.0000 & $-4.3 \%$ & $0.0 \%$ \\
\hline 1 & Batch 1 & $\mathrm{~B} 2 \mathrm{O} 3$ & 7.8646 & 7.7770 & 7.7770 & 0.0876 & 0.0000 & $1.1 \%$ & $0.0 \%$ \\
\hline 1 & Batch 1 & $\mathrm{BaO}$ & 0.1390 & 0.1510 & 0.1510 & -0.0120 & 0.0000 & $-7.9 \%$ & $0.0 \%$ \\
\hline 1 & Batch 1 & $\mathrm{CaO}$ & 1.1644 & 1.2200 & 1.2200 & -0.0556 & 0.0000 & $-4.6 \%$ & $0.0 \%$ \\
\hline 1 & Batch 1 & $\mathrm{CdO}$ & 0.0057 & 0.0057 & 0.0000 & 0.0057 & 0.0057 & & \\
\hline 1 & Batch 1 & Ce2O3 & 0.0059 & 0.0059 & 0.0000 & 0.0059 & 0.0059 & & \\
\hline 1 & Batch 1 & Cr2O3 & 0.1139 & 0.1070 & 0.1070 & 0.0069 & 0.0000 & $6.4 \%$ & $0.0 \%$ \\
\hline 1 & Batch 1 & $\mathrm{CuO}$ & 0.3799 & 0.3990 & 0.3990 & -0.0191 & 0.0000 & $-4.8 \%$ & $0.0 \%$ \\
\hline 1 & Batch 1 & $\mathrm{Fe} 2 \mathrm{O} 3$ & 12.5230 & 12.8390 & 12.8390 & -0.3160 & 0.0000 & $-2.5 \%$ & $0.0 \%$ \\
\hline 1 & Batch 1 & La2O3 & 0.0059 & 0.0059 & 0.0000 & 0.0059 & 0.0059 & & \\
\hline 1 & Batch 1 & $\mathrm{Li2O}$ & 4.3919 & 4.4290 & 4.4290 & -0.0371 & 0.0000 & $-0.8 \%$ & $0.0 \%$ \\
\hline 1 & Batch 1 & $\mathrm{MgO}$ & 1.3215 & 1.4190 & 1.4190 & -0.0975 & 0.0000 & $-6.9 \%$ & $0.0 \%$ \\
\hline 1 & Batch 1 & $\mathrm{MnO}$ & 1.7216 & 1.7260 & 1.7260 & -0.0044 & 0.0000 & $-0.3 \%$ & $0.0 \%$ \\
\hline 1 & Batch 1 & $\mathrm{Na} 2 \mathrm{O}$ & 8.9575 & 9.0030 & 9.0030 & -0.0455 & 0.0000 & $-0.5 \%$ & $0.0 \%$ \\
\hline 1 & Batch 1 & $\mathrm{NiO}$ & 0.6657 & 0.7510 & 0.7510 & -0.0853 & 0.0000 & $-11.4 \%$ & $0.0 \%$ \\
\hline 1 & Batch 1 & $\mathrm{PbO}$ & 0.0054 & 0.0054 & 0.0000 & 0.0054 & 0.0054 & & \\
\hline 1 & Batch 1 & $\mathrm{SiO} 2$ & 49.5605 & 50.2200 & 50.2200 & -0.6595 & 0.0000 & $-1.3 \%$ & $0.0 \%$ \\
\hline 1 & Batch 1 & $\mathrm{SO} 4$ & 0.0749 & 0.0749 & 0.0000 & 0.0749 & 0.0749 & & \\
\hline 1 & Batch 1 & $\mathrm{TiO} 2$ & 0.6372 & 0.6770 & 0.6770 & -0.0398 & 0.0000 & $-5.9 \%$ & $0.0 \%$ \\
\hline 1 & Batch 1 & $\mathrm{ZnO}$ & 0.0062 & 0.0062 & 0.0000 & 0.0062 & 0.0062 & & \\
\hline 1 & Batch 1 & $\mathrm{ZrO} 2$ & 0.0863 & 0.0863 & 0.0980 & -0.0117 & -0.0117 & $-11.9 \%$ & $-11.9 \%$ \\
\hline 1 & Batch 1 & Sum & 94.2995 & 95.7853 & 95.6930 & -1.3935 & 0.0923 & $-1.5 \%$ & $0.1 \%$ \\
\hline 1 & FY09EM21-01 & $\mathrm{Al} 2 \mathrm{O} 3$ & 10.2458 & 10.8080 & 9.9690 & 0.2768 & 0.8390 & $2.8 \%$ & $8.4 \%$ \\
\hline 1 & FY09EM21-01 & $\mathrm{B} 2 \mathrm{O} 3$ & 4.6125 & 4.5466 & 4.5000 & 0.1125 & 0.0466 & $2.5 \%$ & $1.0 \%$ \\
\hline 1 & FY09EM21-01 & $\mathrm{BaO}$ & 0.0056 & 0.0060 & 0.0000 & 0.0056 & 0.0060 & & \\
\hline 1 & FY09EM21-01 & $\mathrm{CaO}$ & 0.0070 & 0.0073 & 0.0000 & 0.0070 & 0.0073 & & \\
\hline 1 & FY09EM21-01 & $\mathrm{CdO}$ & 0.0057 & 0.0057 & 0.0000 & 0.0057 & 0.0057 & & \\
\hline 1 & FY09EM21-01 & $\mathrm{Ce} 2 \mathrm{O} 3$ & 0.0059 & 0.0059 & 0.0000 & 0.0059 & 0.0059 & & \\
\hline 1 & FY09EM21-01 & Cr2O3 & 0.1951 & 0.1820 & 0.2000 & -0.0049 & -0.0180 & $-2.4 \%$ & $-9.0 \%$ \\
\hline 1 & FY09EM21-01 & $\mathrm{CuO}$ & 0.0063 & 0.0066 & 0.0000 & 0.0063 & 0.0066 & & \\
\hline 1 & FY09EM21-01 & $\mathrm{Fe} 2 \mathrm{O} 3$ & 17.8355 & 18.3897 & 18.3546 & -0.5191 & 0.0351 & $-2.8 \%$ & $0.2 \%$ \\
\hline 1 & FY09EM21-01 & La2O3 & 0.0059 & 0.0059 & 0.0000 & 0.0059 & 0.0059 & & \\
\hline 1 & FY09EM21-01 & $\mathrm{Li} 2 \mathrm{O}$ & 3.9936 & 4.0142 & 4.0000 & -0.0064 & 0.0142 & $-0.2 \%$ & $0.4 \%$ \\
\hline 1 & FY09EM21-01 & $\mathrm{MgO}$ & 0.0083 & 0.0089 & 0.0000 & 0.0083 & 0.0089 & & \\
\hline 1 & FY09EM21-01 & $\mathrm{MnO}$ & 5.6716 & 5.7363 & 5.5000 & 0.1716 & 0.2363 & $3.1 \%$ & $4.3 \%$ \\
\hline 1 & FY09EM21-01 & $\mathrm{Na} 2 \mathrm{O}$ & 11.6669 & 11.8982 & 11.3900 & 0.2769 & 0.5082 & $2.4 \%$ & $4.5 \%$ \\
\hline 1 & FY09EM21-01 & $\mathrm{NiO}$ & 0.0064 & 0.0071 & 2.5000 & -2.4936 & -2.4929 & $-99.7 \%$ & $-99.7 \%$ \\
\hline 1 & FY09EM21-01 & $\mathrm{PbO}$ & 0.0054 & 0.0054 & 0.0000 & 0.0054 & 0.0054 & & \\
\hline 1 & FY09EM21-01 & $\mathrm{SiO} 2$ & 38.4539 & 39.3619 & 37.9878 & 0.4661 & 1.3741 & $1.2 \%$ & $3.6 \%$ \\
\hline 1 & FY09EM21-01 & $\mathrm{SO} 4$ & 0.0749 & 0.0749 & 0.0000 & 0.0749 & 0.0749 & & \\
\hline 1 & FY09EM21-01 & $\mathrm{TiO} 2$ & 5.6212 & 5.9362 & 5.5985 & 0.0226 & 0.3377 & $0.4 \%$ & $6.0 \%$ \\
\hline 1 & FY09EM21-01 & $\mathrm{ZnO}$ & 0.0062 & 0.0062 & 0.0000 & 0.0062 & 0.0062 & & \\
\hline 1 & FY09EM21-01 & $\mathrm{ZrO} 2$ & 0.0068 & 0.0068 & 0.0000 & 0.0068 & 0.0068 & & \\
\hline 1 & FY09EM21-01 & Sum & 98.4404 & 101.0198 & 100.0000 & -1.5596 & 1.0198 & $-1.6 \%$ & $1.0 \%$ \\
\hline 1 & FY09EM21-02 & $\mathrm{Al} 2 \mathrm{O} 3$ & 4.1144 & 4.3406 & 4.0071 & 0.1073 & 0.3336 & $2.7 \%$ & $8.3 \%$ \\
\hline 1 & FY09EM21-02 & $\mathrm{B} 2 \mathrm{O} 3$ & 5.1840 & 5.1420 & 5.0660 & 0.1180 & 0.0760 & $2.3 \%$ & $1.5 \%$ \\
\hline 1 & FY09EM21-02 & $\mathrm{BaO}$ & 0.0829 & 0.0893 & 0.0801 & 0.0028 & 0.0092 & $3.5 \%$ & $11.5 \%$ \\
\hline 1 & FY09EM21-02 & $\mathrm{CaO}$ & 0.0070 & 0.0073 & 0.0000 & 0.0070 & 0.0073 & & \\
\hline 1 & FY09EM21-02 & $\mathrm{CdO}$ & 0.2496 & 0.2496 & 0.2965 & -0.0469 & -0.0469 & $-15.8 \%$ & $-15.8 \%$ \\
\hline 1 & FY09EM21-02 & $\mathrm{Ce} 2 \mathrm{O} 3$ & 0.3605 & 0.3605 & 0.3606 & -0.0001 & -0.0001 & $0.0 \%$ & $0.0 \%$ \\
\hline 1 & FY09EM21-02 & Cr2O3 & 0.0132 & 0.0121 & 0.0000 & 0.0132 & 0.0121 & & \\
\hline 1 & FY09EM21-02 & $\mathrm{CuO}$ & 0.1289 & 0.1350 & 0.1282 & 0.0007 & 0.0068 & $0.6 \%$ & $5.3 \%$ \\
\hline 1 & FY09EM21-02 & $\mathrm{Fe} 2 \mathrm{O} 3$ & 20.2303 & 20.8586 & 20.6311 & -0.4008 & 0.2275 & $-1.9 \%$ & $1.1 \%$ \\
\hline 1 & FY09EM21-02 & La2O3 & 0.0900 & 0.0900 & 0.0978 & -0.0077 & -0.0077 & $-7.9 \%$ & $-7.9 \%$ \\
\hline 1 & FY09EM21-02 & $\mathrm{Li} 2 \mathrm{O}$ & 4.0259 & 4.0732 & 4.0000 & 0.0259 & 0.0732 & $0.6 \%$ & $1.8 \%$ \\
\hline 1 & FY09EM21-02 & $\mathrm{MgO}$ & 1.4589 & 1.5608 & 1.5000 & -0.0411 & 0.0608 & $-2.7 \%$ & $4.1 \%$ \\
\hline 1 & FY09EM21-02 & $\mathrm{MnO}$ & 0.3134 & 0.3170 & 0.3000 & 0.0134 & 0.0170 & $4.5 \%$ & $5.7 \%$ \\
\hline 1 & FY09EM21-02 & $\mathrm{Na} 2 \mathrm{O}$ & 17.0522 & 17.3928 & 17.9769 & -0.9247 & -0.5841 & $-5.1 \%$ & $-3.2 \%$ \\
\hline 1 & FY09EM21-02 & $\mathrm{NiO}$ & 0.0064 & 0.0071 & 2.5000 & -2.4936 & -2.4929 & $-99.7 \%$ & $-99.7 \%$ \\
\hline 1 & FY09EM21-02 & $\mathrm{PbO}$ & 0.1845 & 0.1845 & 0.2163 & -0.0319 & -0.0319 & $-14.7 \%$ & $-14.7 \%$ \\
\hline
\end{tabular}


SRNL-STI-2009-00778, Revision 0

Table A4. Average Measured and Bias-Corrected Chemical Compositions Versus Targeted Compositions by Oxide by Glass ID for the Non-Rad Matrix 2 Study

\begin{tabular}{|c|c|c|c|c|c|c|c|c|c|}
\hline & & & & Measured & & Difference & Difference & $\%$ & $\%$ \\
\hline & & & Measured & Bias-Corrected & Targeted & of & of & Difference & Difference \\
\hline Set & Glass \# & Oxide & $(\mathrm{wt} \%)$ & $(\mathrm{BC})(\mathrm{wt} \%)$ & $(\mathrm{wt} \%)$ & Measured & Meas BC & Measured & Meas BC \\
\hline 1 & FY09EM21-02 & $\mathrm{SiO} 2$ & 40.3793 & 41.3347 & 40.0190 & 0.3603 & 1.3157 & $0.9 \%$ & $3.3 \%$ \\
\hline 1 & FY09EM21-02 & $\mathrm{SO} 4$ & 0.4853 & 0.4853 & 0.4808 & 0.0046 & 0.0046 & $0.9 \%$ & $0.9 \%$ \\
\hline 1 & FY09EM21-02 & $\mathrm{TiO} 2$ & 2.0600 & 2.1754 & 2.0000 & 0.0600 & 0.1754 & $3.0 \%$ & $8.8 \%$ \\
\hline 1 & FY09EM21-02 & $\mathrm{ZnO}$ & 0.1344 & 0.1344 & 0.1346 & -0.0002 & -0.0002 & $-0.1 \%$ & $-0.1 \%$ \\
\hline 1 & FY09EM21-02 & $\mathrm{ZrO} 2$ & 0.2009 & 0.2009 & 0.2051 & -0.0042 & -0.0042 & $-2.0 \%$ & $-2.0 \%$ \\
\hline 1 & FY09EM21-02 & Sum & 96.7620 & 99.1514 & 100.0000 & -3.2380 & -0.8486 & $-3.2 \%$ & $-0.8 \%$ \\
\hline 1 & FY09EM21-03 & $\mathrm{Al} 2 \mathrm{O} 3$ & 6.9345 & 7.1728 & 6.9696 & -0.0351 & 0.2032 & $-0.5 \%$ & $2.9 \%$ \\
\hline 1 & FY09EM21-03 & B2O3 & 4.6286 & 4.5912 & 4.5000 & 0.1286 & 0.0912 & $2.9 \%$ & $2.0 \%$ \\
\hline 1 & FY09EM21-03 & $\mathrm{BaO}$ & 0.0056 & 0.0061 & 0.0000 & 0.0056 & 0.0061 & & \\
\hline 1 & FY09EM21-03 & $\mathrm{CaO}$ & 0.0203 & 0.0212 & 0.0000 & 0.0203 & 0.0212 & & \\
\hline 1 & FY09EM21-03 & $\mathrm{CdO}$ & 0.0057 & 0.0057 & 0.0000 & 0.0057 & 0.0057 & & \\
\hline 1 & FY09EM21-03 & $\mathrm{Ce} 2 \mathrm{O} 3$ & 0.0059 & 0.0059 & 0.0000 & 0.0059 & 0.0059 & & \\
\hline 1 & FY09EM21-03 & Cr2O3 & 0.0153 & 0.0145 & 0.0000 & 0.0153 & 0.0145 & & \\
\hline 1 & FY09EM21-03 & $\mathrm{CuO}$ & 0.0063 & 0.0066 & 0.0000 & 0.0063 & 0.0066 & & \\
\hline 1 & FY09EM21-03 & $\mathrm{Fe} 2 \mathrm{O} 3$ & 13.2176 & 13.4740 & 12.5192 & 0.6984 & 0.9549 & $5.6 \%$ & $7.6 \%$ \\
\hline 1 & FY09EM21-03 & La2O3 & 0.0059 & 0.0059 & 0.0000 & 0.0059 & 0.0059 & & \\
\hline 1 & FY09EM21-03 & $\mathrm{Li2O}$ & 6.7440 & 6.8233 & 7.0000 & -0.2560 & -0.1767 & $-3.7 \%$ & $-2.5 \%$ \\
\hline 1 & FY09EM21-03 & $\mathrm{MgO}$ & 1.4154 & 1.5253 & 1.5000 & -0.0846 & 0.0253 & $-5.6 \%$ & $1.7 \%$ \\
\hline 1 & FY09EM21-03 & $\mathrm{MnO}$ & 5.6167 & 5.5820 & 5.5000 & 0.1167 & 0.0820 & $2.1 \%$ & $1.5 \%$ \\
\hline 1 & FY09EM21-03 & $\mathrm{Na} 2 \mathrm{O}$ & 14.5753 & 14.4362 & 14.1314 & 0.4438 & 0.3048 & $3.1 \%$ & $2.2 \%$ \\
\hline 1 & FY09EM21-03 & $\mathrm{NiO}$ & 0.0057 & 0.0065 & 2.5000 & -2.4943 & -2.4935 & $-99.8 \%$ & $-99.7 \%$ \\
\hline 1 & FY09EM21-03 & $\mathrm{PbO}$ & 0.0054 & 0.0054 & 0.0000 & 0.0054 & 0.0054 & & \\
\hline 1 & FY09EM21-03 & $\mathrm{SiO} 2$ & 40.1119 & 40.2406 & 40.6502 & -0.5383 & -0.4095 & $-1.3 \%$ & $-1.0 \%$ \\
\hline 1 & FY09EM21-03 & $\mathrm{SO} 4$ & 0.0749 & 0.0749 & 0.0000 & 0.0749 & 0.0749 & & \\
\hline 1 & FY09EM21-03 & $\mathrm{TiO} 2$ & 4.9832 & 5.3274 & 4.7297 & 0.2535 & 0.5977 & $5.4 \%$ & $12.6 \%$ \\
\hline 1 & FY09EM21-03 & $\mathrm{ZnO}$ & 0.0062 & 0.0062 & 0.0000 & 0.0062 & 0.0062 & & \\
\hline 1 & FY09EM21-03 & $\mathrm{ZrO} 2$ & 0.0068 & 0.0068 & 0.0000 & 0.0068 & 0.0068 & & \\
\hline 1 & FY09EM21-03 & Sum & 98.3909 & 99.3385 & 100.0000 & -1.6091 & -0.6615 & $-1.6 \%$ & $-0.7 \%$ \\
\hline 1 & FY09EM21-04 & $\mathrm{Al} 2 \mathrm{O} 3$ & 7.0100 & 7.2509 & 7.0273 & -0.0172 & 0.2236 & $-0.2 \%$ & $3.2 \%$ \\
\hline 1 & FY09EM21-04 & $\mathrm{B} 2 \mathrm{O} 3$ & 13.9905 & 13.8773 & 14.0000 & -0.0095 & -0.1227 & $-0.1 \%$ & $-0.9 \%$ \\
\hline 1 & FY09EM21-04 & $\mathrm{BaO}$ & 0.0056 & 0.0061 & 0.0000 & 0.0056 & 0.0061 & & \\
\hline 1 & FY09EM21-04 & $\mathrm{CaO}$ & 4.0262 & 4.2170 & 4.0000 & 0.0262 & 0.2170 & $0.7 \%$ & $5.4 \%$ \\
\hline 1 & FY09EM21-04 & $\mathrm{CdO}$ & 0.0057 & 0.0057 & 0.0000 & 0.0057 & 0.0057 & & \\
\hline 1 & FY09EM21-04 & Ce2O3 & 0.0059 & 0.0059 & 0.0000 & 0.0059 & 0.0059 & & \\
\hline 1 & FY09EM21-04 & $\mathrm{Cr} 2 \mathrm{O} 3$ & 0.0073 & 0.0069 & 0.0000 & 0.0073 & 0.0069 & & \\
\hline 1 & FY09EM21-04 & $\mathrm{CuO}$ & 0.0063 & 0.0066 & 0.0000 & 0.0063 & 0.0066 & & \\
\hline 1 & FY09EM21-04 & $\mathrm{Fe} 2 \mathrm{O} 3$ & 15.6195 & 15.9240 & 16.4866 & -0.8671 & -0.5626 & $-5.3 \%$ & $-3.4 \%$ \\
\hline 1 & FY09EM21-04 & $\mathrm{La} 2 \mathrm{O} 3$ & 0.0059 & 0.0059 & 0.0000 & 0.0059 & 0.0059 & & \\
\hline 1 & FY09EM21-04 & $\mathrm{Li2O}$ & 6.9431 & 7.0248 & 7.0000 & -0.0569 & 0.0248 & $-0.8 \%$ & $0.4 \%$ \\
\hline 1 & FY09EM21-04 & $\mathrm{MgO}$ & 1.3839 & 1.4912 & 1.5000 & -0.1161 & -0.0088 & $-7.7 \%$ & $-0.6 \%$ \\
\hline 1 & FY09EM21-04 & $\mathrm{MnO}$ & 0.2941 & 0.2923 & 0.3000 & -0.0059 & -0.0077 & $-2.0 \%$ & $-2.6 \%$ \\
\hline 1 & FY09EM21-04 & $\mathrm{Na} 2 \mathrm{O}$ & 10.6863 & 10.5850 & 10.7374 & -0.0512 & -0.1524 & $-0.5 \%$ & $-1.4 \%$ \\
\hline 1 & FY09EM21-04 & $\mathrm{NiO}$ & 2.2587 & 2.5603 & 2.5000 & -0.2413 & 0.0603 & $-9.7 \%$ & $2.4 \%$ \\
\hline 1 & FY09EM21-04 & $\mathrm{PbO}$ & 0.0054 & 0.0054 & 0.0000 & 0.0054 & 0.0054 & & \\
\hline 1 & FY09EM21-04 & $\mathrm{SiO} 2$ & 32.0360 & 32.1381 & 32.4487 & -0.4127 & -0.3107 & $-1.3 \%$ & $-1.0 \%$ \\
\hline 1 & FY09EM21-04 & $\mathrm{SO} 4$ & 0.0749 & 0.0749 & 0.0000 & 0.0749 & 0.0749 & & \\
\hline 1 & FY09EM21-04 & $\mathrm{TiO} 2$ & 3.9532 & 4.2266 & 4.0000 & -0.0468 & 0.2266 & $-1.2 \%$ & $5.7 \%$ \\
\hline 1 & FY09EM21-04 & $\mathrm{ZnO}$ & 0.0062 & 0.0062 & 0.0000 & 0.0062 & 0.0062 & & \\
\hline 1 & FY09EM21-04 & $\mathrm{ZrO} 2$ & 0.0068 & 0.0068 & 0.0000 & 0.0068 & 0.0068 & & \\
\hline 1 & FY09EM21-04 & Sum & 98.3312 & 99.7177 & 100.0000 & -1.6688 & -0.2823 & $-1.7 \%$ & $-0.3 \%$ \\
\hline 1 & FY09EM21-05 & $\mathrm{Al} 2 \mathrm{O} 3$ & 3.6892 & 3.8920 & 3.6040 & 0.0852 & 0.2880 & $2.4 \%$ & $8.0 \%$ \\
\hline 1 & FY09EM21-05 & $\mathrm{B} 2 \mathrm{O} 3$ & 4.3308 & 4.2670 & 4.5000 & -0.1692 & -0.2330 & $-3.8 \%$ & $-5.2 \%$ \\
\hline 1 & FY09EM21-05 & $\mathrm{BaO}$ & 0.0056 & 0.0060 & 0.0000 & 0.0056 & 0.0060 & & \\
\hline 1 & FY09EM21-05 & $\mathrm{CaO}$ & 4.1102 & 4.3083 & 4.0000 & 0.1102 & 0.3083 & $2.8 \%$ & $7.7 \%$ \\
\hline 1 & FY09EM21-05 & $\mathrm{CdO}$ & 0.0057 & 0.0057 & 0.0000 & 0.0057 & 0.0057 & & \\
\hline 1 & FY09EM21-05 & $\mathrm{Ce} 2 \mathrm{O} 3$ & 0.0059 & 0.0059 & 0.0000 & 0.0059 & 0.0059 & & \\
\hline 1 & FY09EM21-05 & Cr2O3 & 0.0190 & 0.0177 & 0.0000 & 0.0190 & 0.0177 & & \\
\hline 1 & FY09EM21-05 & $\mathrm{CuO}$ & 0.0063 & 0.0066 & 0.0000 & 0.0063 & 0.0066 & & \\
\hline 1 & FY09EM21-05 & $\mathrm{Fe} 2 \mathrm{O} 3$ & 13.3462 & 13.7610 & 13.7706 & -0.4244 & -0.0096 & $-3.1 \%$ & $-0.1 \%$ \\
\hline 1 & FY09EM21-05 & $\mathrm{La} 2 \mathrm{O} 3$ & 0.0059 & 0.0059 & 0.0000 & 0.0059 & 0.0059 & & \\
\hline
\end{tabular}


SRNL-STI-2009-00778, Revision 0

Table A4. Average Measured and Bias-Corrected Chemical Compositions Versus Targeted Compositions by Oxide by Glass ID for the Non-Rad Matrix 2 Study

\begin{tabular}{|c|c|c|c|c|c|c|c|c|c|}
\hline & & & & Measured & & Difference & Difference & $\%$ & $\%$ \\
\hline & & & Measured & Bias-Corrected & Targeted & of & of & \begin{tabular}{|l|} 
Difference \\
\end{tabular} & Difference \\
\hline Set & Glass \# & Oxide & (wt\%) & $(\mathrm{BC})(\mathrm{wt} \%)$ & (wt\%) & Measured & Meas BC & Measured & Meas BC \\
\hline 1 & FY09EM21-05 & Li2O & 3.9506 & 3.9710 & 4.0010 & -0.0504 & -0.0300 & $-1.3 \%$ & $-0.8 \%$ \\
\hline 1 & FY09EM21-05 & $\mathrm{MgO}$ & 0.0083 & 0.0089 & 0.0000 & 0.0083 & 0.0089 & & \\
\hline 1 & FY09EM21-05 & $\mathrm{MnO}$ & 0.3109 & 0.3144 & 0.3000 & 0.0109 & 0.0144 & $3.6 \%$ & $4.8 \%$ \\
\hline 1 & FY09EM21-05 & $\mathrm{Na} 2 \mathrm{O}$ & 9.9482 & 10.1467 & 10.0000 & -0.0518 & 0.1467 & $-0.5 \%$ & $1.5 \%$ \\
\hline 1 & FY09EM21-05 & $\mathrm{NiO}$ & 2.2937 & 2.5751 & 2.5000 & -0.2063 & 0.0751 & $-8.3 \%$ & $3.0 \%$ \\
\hline 1 & FY09EM21-05 & $\mathrm{PbO}$ & 0.0054 & 0.0054 & 0.0000 & 0.0054 & 0.0054 & & \\
\hline 1 & FY09EM21-05 & $\mathrm{SiO} 2$ & 49.7387 & 50.9158 & 51.4971 & -1.7584 & -0.5813 & $-3.4 \%$ & $-1.1 \%$ \\
\hline 1 & FY09EM21-05 & $\mathrm{SO} 4$ & 0.0749 & 0.0749 & 0.0000 & 0.0749 & 0.0749 & & \\
\hline 1 & FY09EM21-05 & $\mathrm{TiO2}$ & 5.8255 & 6.1520 & 5.8273 & -0.0018 & 0.3248 & $0.0 \%$ & $5.6 \%$ \\
\hline 1 & FY09EM21-05 & $\mathrm{ZnO}$ & 0.0062 & 0.0062 & 0.0000 & 0.0062 & 0.0062 & & \\
\hline 1 & FY09EM21-05 & $\mathrm{ZrO} 2$ & 0.0068 & 0.0068 & 0.0000 & 0.0068 & 0.0068 & & \\
\hline 1 & FY09EM21-05 & Sum & 97.6938 & 100.4531 & 100.0000 & -2.3062 & 0.4531 & $-2.3 \%$ & $0.5 \%$ \\
\hline 1 & "FY09EM21-06 & $\mathrm{Al} 2 \mathrm{O} 3$ & "4.4403 & $\begin{array}{l}4.5928 \\
\end{array}$ & 4.3905 & 0.0498 & 0.2023 & $1.1 \%$ & "4.6\% \\
\hline 1 & FY09EM21-06 & $\mathrm{B} 2 \mathrm{O} 3$ & 11.4789 & 11.3861 & 11.4921 & -0.0132 & -0.1060 & $-0.1 \%$ & $-0.9 \%$ \\
\hline 1 & FY09EM21-06 & $\mathrm{BaO}$ & 0.0734 & 0.0804 & 0.0801 & -0.0067 & 0.0003 & $-8.4 \%$ & $0.3 \%$ \\
\hline 1 & FY09EM21-06 & $\mathrm{CaO}$ & 0.0304 & 0.0319 & 0.0000 & 0.0304 & 0.0319 & & \\
\hline 1 & FY09EM21-06 & $\mathrm{CdO}$ & 0.2450 & 0.2450 & 0.2965 & -0.0515 & -0.0515 & $-17.4 \%$ & $-17.4 \%$ \\
\hline 1 & FY09EM21-06 & $\mathrm{Ce} 2 \mathrm{O} 3$ & 0.3420 & 0.3420 & 0.3606 & -0.0186 & -0.0186 & $-5.1 \%$ & $-5.1 \%$ \\
\hline 1 & FY09EM21-06 & $\mathrm{Cr} 2 \mathrm{O} 3$ & 0.1907 & 0.1806 & 0.2000 & -0.0093 & -0.0194 & $-4.6 \%$ & $-9.7 \%$ \\
\hline 1 & FY09EM21-06 & $\mathrm{CuO}$ & 0.1252 & 0.1318 & 0.1282 & -0.0030 & 0.0036 & $-2.4 \%$ & $2.8 \%$ \\
\hline 1 & FY09EM21-06 & $\mathrm{Fe} 2 \mathrm{O} 3$ & 5.1898 & 5.2908 & 5.2967 & -0.1069 & $\begin{array}{l}-0.0059 \\
\end{array}$ & $-2.0 \%$ & $-0.1 \%$ \\
\hline 1 & FY09EM21-06 & $\mathrm{La} 2 \mathrm{O} 3$ & 0.0921 & 0.0921 & 0.0978 & -0.0057 & -0.0057 & $-5.8 \%$ & $-5.8 \%$ \\
\hline 1 & FY09EM21-06 & $\mathrm{Li} 2 \mathrm{O}$ & 3.8537 & 3.8990 & 4.0000 & -0.1463 & -0.1010 & $-3.7 \%$ & $-2.5 \%$ \\
\hline 1 & FY09EM21-06 & $\mathrm{MgO}$ & 0.0083 & 0.0089 & 0.0000 & 0.0083 & 0.0089 & & \\
\hline 1 & FY09EM21-06 & $\mathrm{MnO}$ & 5.5296 & 5.4955 & 5.5000 & 0.0296 & -0.0045 & $0.5 \%$ & $-0.1 \%$ \\
\hline 1 & FY09EM21-06 & $\mathrm{Na} 2 \mathrm{O}$ & 14.8044 & 14.6637 & 14.7842 & 0.0202 & -0.1205 & $0.1 \%$ & $-0.8 \%$ \\
\hline 1 & FY09EM21-06 & $\mathrm{NiO}$ & 2.3923 & 2.7116 & 2.5000 & -0.1077 & 0.2116 & $-4.3 \%$ & $8.5 \%$ \\
\hline 1 & FY09EM21-06 & $\mathrm{PbO}$ & 0.1853 & 0.1853 & 0.2163 & -0.0311 & -0.0311 & $-14.4 \%$ & $-14.4 \%$ \\
\hline 1 & FY09EM21-06 & $\mathrm{SiO} 2$ & 42.9464 & 43.0837 & 43.8365 & -0.8900 & $\begin{array}{l}-0.7528 \\
\end{array}$ & $-2.0 \%$ & $-1.7 \%$ \\
\hline 1 & FY09EM21-06 & $\mathrm{SO} 4$ & 0.4314 & 0.4314 & 0.4808 & -0.0494 & -0.0494 & $-10.3 \%$ & $-10.3 \%$ \\
\hline 1 & FY09EM21-06 & $\mathrm{TiO} 2$ & 5.8964 & 6.3040 & 6.0000 & -0.1036 & 0.3040 & $-1.7 \%$ & $5.1 \%$ \\
\hline 1 & FY09EM21-06 & $\mathrm{ZnO}$ & 0.1379 & 0.1379 & 0.1346 & 0.0032 & 0.0032 & $2.4 \%$ & $2.4 \%$ \\
\hline 1 & FY09EM21-06 & $\mathrm{ZrO2}$ & 0.1932 & 0.1932 & 0.2051 & -0.0120 & -0.0120 & $-5.8 \%$ & $-5.8 \%$ \\
\hline 1 & FY09EM21-06 & Sum & 98.5867 & 99.4876 & 100.0000 & -1.4133 & -0.5124 & $-1.4 \%$ & $-0.5 \%$ \\
\hline 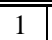 & "FY09EM21-07 & Al2O3 & "4.8135 & 4.9795 & 4.8660 & $\begin{array}{c}-0.0525 \\
\end{array}$ & "0.1135 & "-1.1\% & $2.3 \%$ \\
\hline 1 & FY09EM21-07 & B2O3 & 13.7007 & 13.5075 & 13.9244 & -0.2237 & -0.4169 & $-1.6 \%$ & $-3.0 \%$ \\
\hline 1 & FY09EM21-07 & $\mathrm{BaO}$ & 0.0056 & 0.0061 & 0.0000 & 0.0056 & 0.0061 & & \\
\hline 1 & FY09EM21-07 & $\mathrm{CaO}$ & 3.9702 & 4.1583 & 3.9217 & 0.0485 & 0.2366 & $1.2 \%$ & $6.0 \%$ \\
\hline 1 & FY09EM21-07 & $\mathrm{CdO}$ & 0.0057 & 0.0057 & 0.0000 & 0.0057 & 0.0057 & & \\
\hline 1 & FY09EM21-07 & Ce2O3 & 0.0059 & 0.0059 & 0.0000 & 0.0059 & 0.0059 & & \\
\hline 1 & FY09EM21-07 & Cr2O3 & 0.0073 & 0.0069 & 0.0000 & 0.0073 & 0.0069 & & \\
\hline 1 & FY09EM21-07 & $\mathrm{CuO}$ & 0.0063 & 0.0066 & 0.0000 & 0.0063 & 0.0066 & & \\
\hline 1 & FY09EM21-07 & $\mathrm{Fe} 2 \mathrm{O} 3$ & 16.8347 & 17.1624 & 17.9841 & -1.1494 & -0.8217 & $-6.4 \%$ & $-4.6 \%$ \\
\hline 1 & FY09EM21-07 & La2O3 & 0.0059 & 0.0059 & 0.0000 & 0.0059 & 0.0059 & & \\
\hline 1 & FY09EM21-07 & $\mathrm{Li2O}$ & 3.8698 & 3.8898 & 4.0000 & -0.1302 & -0.1102 & $-3.3 \%$ & $-2.8 \%$ \\
\hline 1 & FY09EM21-07 & $\mathrm{MgO}$ & 1.3714 & 1.4780 & 1.5000 & -0.1286 & -0.0220 & $-8.6 \%$ & $-1.5 \%$ \\
\hline 1 & FY09EM21-07 & $\mathrm{MnO}$ & 5.4327 & 5.3992 & 5.5000 & -0.0673 & -0.1008 & $-1.2 \%$ & $-1.8 \%$ \\
\hline 1 & FY09EM21-07 & $\mathrm{Na} 2 \mathrm{O}$ & 12.8296 & 12.7092 & 12.7062 & 0.1234 & 0.0030 & $1.0 \%$ & $0.0 \%$ \\
\hline 1 & FY09EM21-07 & $\mathrm{NiO}$ & 0.0620 & 0.0703 & 0.0000 & 0.0620 & 0.0703 & & \\
\hline 1 & FY09EM21-07 & $\mathrm{PbO}$ & 0.0054 & 0.0054 & 0.0000 & 0.0054 & 0.0054 & & \\
\hline 1 & FY09EM21-07 & $\mathrm{SiO} 2$ & 33.1592 & 33.2655 & 33.5976 & -0.4385 & -0.3321 & $-1.3 \%$ & $-1.0 \%$ \\
\hline 1 & FY09EM21-07 & SO4 & 0.0749 & 0.0749 & 0.0000 & 0.0749 & 0.0749 & & \\
\hline 1 & FY09EM21-07 & $\mathrm{TiO} 2$ & 1.9891 & 2.1265 & 2.0000 & -0.0109 & 0.1265 & $-0.5 \%$ & $6.3 \%$ \\
\hline 1 & FY09EM21-07 & $\mathrm{ZnO}$ & 0.0062 & 0.0062 & 0.0000 & 0.0062 & 0.0062 & & \\
\hline 1 & FY09EM21-07 & $\mathrm{ZrO} 2$ & 0.0068 & 0.0068 & 0.0000 & 0.0068 & 0.0068 & & \\
\hline 1 & FY09EM21-07 & Sum & 98.1628 & 98.8766 & 100.0000 & -1.8372 & -1.1234 & $-1.8 \%$ & $-1.1 \%$ \\
\hline 1 & "FY09EM21-08 & Al2O3 & 3.2547 & 3.4335 & 3.2500 & 0.0047 & 0.1835 & $0.1 \%$ & $5.6 \%$ \\
\hline 1 & FY09EM21-08 & $\mathrm{B} 2 \mathrm{O} 3$ & 4.8299 & 4.7601 & 4.9741 & -0.1443 & -0.2140 & $-2.9 \%$ & $-4.3 \%$ \\
\hline 1 & FY09EM21-08 & $\mathrm{BaO}$ & 0.0056 & 0.0060 & 0.0000 & 0.0056 & 0.0060 & & \\
\hline 1 & FY09EM21-08 & $\mathrm{CaO}$ & 0.5149 & 0.5397 & 0.5047 & 0.0102 & 0.0350 & $2.0 \%$ & $6.9 \%$ \\
\hline
\end{tabular}


SRNL-STI-2009-00778, Revision 0

Table A4. Average Measured and Bias-Corrected Chemical Compositions Versus Targeted Compositions by Oxide by Glass ID for the Non-Rad Matrix 2 Study

\begin{tabular}{|c|c|c|c|c|c|c|c|c|c|}
\hline & & & & Measured & & Difference & Difference & $\%$ & $\%$ \\
\hline & & & Measured & Bias-Corrected & Targeted & of & of & Difference & Difference \\
\hline Set & Glass \# & Oxide & (wt\%) & $(\mathrm{BC})(\mathrm{wt} \%)$ & (wt\%) & Measured & Meas BC & Measured & Meas BC \\
\hline 1 & FY09EM21-08 & $\mathrm{CdO}$ & 0.0057 & 0.0057 & 0.0000 & 0.0057 & 0.0057 & & \\
\hline 1 & FY09EM21-08 & $\mathrm{Ce} 2 \mathrm{O} 3$ & 0.0111 & 0.0111 & 0.0000 & 0.0111 & 0.0111 & & \\
\hline 1 & FY09EM21-08 & Cr2O3 & 0.1560 & 0.1455 & 0.2000 & -0.0440 & -0.0545 & $-22.0 \%$ & $-27.2 \%$ \\
\hline 1 & FY09EM21-08 & $\mathrm{CuO}$ & 0.0063 & 0.0066 & 0.0000 & 0.0063 & 0.0066 & & \\
\hline 1 & FY09EM21-08 & $\mathrm{Fe} 2 \mathrm{O} 3$ & 20.1588 & 20.7843 & 20.4787 & -0.3200 & 0.3056 & $-1.6 \%$ & $1.5 \%$ \\
\hline 1 & FY09EM21-08 & $\mathrm{La} 2 \mathrm{O} 3$ & 0.0059 & 0.0059 & 0.0000 & 0.0059 & 0.0059 & & \\
\hline 1 & FY09EM21-08 & $\mathrm{Li} 2 \mathrm{O}$ & 6.7278 & 6.7625 & 7.0000 & -0.2722 & -0.2375 & $-3.9 \%$ & $-3.4 \%$ \\
\hline 1 & FY09EM21-08 & $\mathrm{MgO}$ & 1.3826 & 1.4792 & 1.5000 & -0.1174 & -0.0208 & $-7.8 \%$ & $-1.4 \%$ \\
\hline 1 & FY09EM21-08 & $\mathrm{MnO}$ & 1.4235 & 1.4398 & 1.3863 & 0.0373 & 0.0535 & $2.7 \%$ & $3.9 \%$ \\
\hline 1 & FY09EM21-08 & $\mathrm{Na} 2 \mathrm{O}$ & 9.6584 & 9.8508 & 10.0000 & -0.3416 & -0.1492 & $-3.4 \%$ & $-1.5 \%$ \\
\hline 1 & FY09EM21-08 & $\mathrm{NiO}$ & 0.0153 & 0.0171 & 0.0000 & 0.0153 & 0.0171 & & \\
\hline 1 & FY09EM21-08 & $\mathrm{PbO}$ & 0.0054 & 0.0054 & 0.0000 & 0.0054 & 0.0054 & & \\
\hline 1 & FY09EM21-08 & $\mathrm{SiO} 2$ & 42.7325 & 43.7423 & 44.7062 & -1.9737 & -0.9639 & $-4.4 \%$ & $-2.2 \%$ \\
\hline 1 & FY09EM21-08 & SO4 & 0.0749 & 0.0749 & 0.0000 & 0.0749 & 0.0749 & & \\
\hline 1 & FY09EM21-08 & $\mathrm{TiO} 2$ & 5.9506 & 6.2841 & 6.0000 & -0.0494 & 0.2841 & $-0.8 \%$ & $4.7 \%$ \\
\hline 1 & FY09EM21-08 & $\mathrm{ZnO}$ & 0.0062 & 0.0062 & 0.0000 & 0.0062 & 0.0062 & & \\
\hline 1 & FY09EM21-08 & $\mathrm{ZrO} 2$ & 0.0068 & 0.0068 & 0.0000 & 0.0068 & 0.0068 & & \\
\hline 1 & FY09EM21-08 & Sum & 96.9328 & 99.3675 & 100.0000 & -3.0672 & -0.6325 & $-3.1 \%$ & $-0.6 \%$ \\
\hline 1 & FY09EM21-09 & $\mathrm{Al} 2 \mathrm{O} 3$ & 12.9667 & 13.4134 & 12.9733 & -0.0066 & 0.4401 & $-0.1 \%$ & $3.4 \%$ \\
\hline 1 & FY09EM21-09 & $\mathrm{B} 2 \mathrm{O} 3$ & 9.5390 & 9.4065 & 9.4842 & 0.0548 & -0.0777 & $0.6 \%$ & $-0.8 \%$ \\
\hline 1 & FY09EM21-09 & $\mathrm{BaO}$ & 0.0056 & 0.0061 & 0.0000 & 0.0056 & 0.0061 & & \\
\hline 1 & FY09EM21-09 & $\mathrm{CaO}$ & 3.0118 & 3.1545 & 3.0266 & -0.0148 & 0.1278 & $-0.5 \%$ & $4.2 \%$ \\
\hline 1 & FY09EM21-09 & $\mathrm{CdO}$ & 0.0057 & 0.0057 & 0.0000 & 0.0057 & 0.0057 & & \\
\hline 1 & FY09EM21-09 & $\mathrm{Ce} 2 \mathrm{O} 3$ & 0.0059 & 0.0059 & 0.0000 & 0.0059 & 0.0059 & & \\
\hline 1 & FY09EM21-09 & Cr2O3 & 0.1370 & 0.1297 & 0.2000 & -0.0630 & -0.0703 & $-31.5 \%$ & $-35.1 \%$ \\
\hline 1 & FY09EM21-09 & $\mathrm{CuO}$ & 0.0063 & 0.0066 & 0.0000 & 0.0063 & 0.0066 & & \\
\hline 1 & FY09EM21-09 & $\mathrm{Fe} 2 \mathrm{O} 3$ & 10.5262 & 10.7318 & 10.9602 & -0.4341 & -0.2285 & $-4.0 \%$ & $-2.1 \%$ \\
\hline 1 & FY09EM21-09 & $\mathrm{La} 2 \mathrm{O} 3$ & 0.0059 & 0.0059 & 0.0000 & 0.0059 & 0.0059 & & \\
\hline 1 & FY09EM21-09 & $\mathrm{Li2O}$ & 6.7924 & 6.8274 & 7.0000 & -0.2076 & -0.1726 & $-3.0 \%$ & $-2.5 \%$ \\
\hline 1 & FY09EM21-09 & $\mathrm{MgO}$ & 0.0083 & 0.0089 & 0.0000 & 0.0083 & 0.0089 & & \\
\hline 1 & FY09EM21-09 & $\mathrm{MnO}$ & 2.0627 & 2.0500 & 2.0133 & 0.0494 & 0.0367 & $2.5 \%$ & $1.8 \%$ \\
\hline 1 & FY09EM21-09 & $\mathrm{Na} 2 \mathrm{O}$ & 10.0763 & 9.9820 & 10.0010 & 0.0753 & -0.0190 & $0.8 \%$ & $-0.2 \%$ \\
\hline 1 & FY09EM21-09 & $\mathrm{NiO}$ & 0.5857 & 0.6639 & 0.6848 & -0.0992 & -0.0210 & $-14.5 \%$ & $-3.1 \%$ \\
\hline 1 & FY09EM21-09 & $\mathrm{PbO}$ & 0.0048 & 0.0048 & 0.0000 & 0.0048 & 0.0048 & & \\
\hline 1 & FY09EM21-09 & $\mathrm{SiO} 2$ & 40.4328 & 40.5621 & 41.1878 & -0.7550 & -0.6257 & $-1.8 \%$ & $-1.5 \%$ \\
\hline 1 & FY09EM21-09 & SO4 & 0.0749 & 0.0749 & 0.0000 & 0.0749 & 0.0749 & & \\
\hline 1 & FY09EM21-09 & TiO2 & 2.4937 & 2.6661 & 2.4687 & 0.0250 & 0.1974 & $1.0 \%$ & $8.0 \%$ \\
\hline 1 & FY09EM21-09 & $\mathrm{ZnO}$ & 0.0062 & 0.0062 & 0.0000 & 0.0062 & 0.0062 & & \\
\hline 1 & FY09EM21-09 & $\mathrm{ZrO} 2$ & 0.0068 & 0.0068 & 0.0000 & 0.0068 & 0.0068 & & \\
\hline 1 & FY09EM21-09 & Sum & 98.7544 & 99.7192 & 100.0000 & -1.2456 & -0.2808 & $-1.2 \%$ & $-0.3 \%$ \\
\hline 1 & FY09EM21-10 & $\mathrm{Al} 2 \mathrm{O} 3$ & 6.3865 & 6.6068 & 6.3955 & -0.0090 & 0.2113 & $-0.1 \%$ & $3.3 \%$ \\
\hline 1 & FY09EM21-10 & $\mathrm{B} 2 \mathrm{O} 3$ & 4.6608 & 4.5897 & 4.7196 & -0.0588 & -0.1299 & $-1.2 \%$ & $-2.8 \%$ \\
\hline 1 & FY09EM21-10 & $\mathrm{BaO}$ & 0.0715 & 0.0783 & 0.0801 & -0.0087 & -0.0019 & $-10.8 \%$ & $-2.3 \%$ \\
\hline 1 & FY09EM21-10 & $\mathrm{CaO}$ & 4.0612 & 4.2536 & 4.0000 & 0.0612 & 0.2536 & $1.5 \%$ & $6.3 \%$ \\
\hline 1 & FY09EM21-10 & $\mathrm{CdO}$ & 0.2439 & 0.2439 & 0.2965 & -0.0526 & -0.0526 & $-17.7 \%$ & $-17.7 \%$ \\
\hline 1 & FY09EM21-10 & $\mathrm{Ce} 2 \mathrm{O} 3$ & 0.3309 & 0.3309 & 0.3606 & -0.0297 & -0.0297 & $-8.2 \%$ & $-8.2 \%$ \\
\hline 1 & FY09EM21-10 & $\mathrm{Cr} 2 \mathrm{O} 3$ & 0.0150 & 0.0142 & 0.0000 & 0.0150 & 0.0142 & & \\
\hline 1 & FY09EM21-10 & $\mathrm{CuO}$ & 0.1224 & 0.1289 & 0.1282 & -0.0058 & 0.0007 & $-4.6 \%$ & $0.5 \%$ \\
\hline 1 & FY09EM21-10 & $\mathrm{Fe} 2 \mathrm{O} 3$ & 16.6203 & 16.9437 & 17.7314 & -1.1111 & -0.7877 & $-6.3 \%$ & $-4.4 \%$ \\
\hline 1 & FY09EM21-10 & $\mathrm{La} 2 \mathrm{O} 3$ & 0.0821 & 0.0821 & 0.0978 & -0.0157 & -0.0157 & $-16.0 \%$ & $-16.0 \%$ \\
\hline 1 & FY09EM21-10 & $\mathrm{Li} 2 \mathrm{O}$ & 3.8698 & 3.8898 & 4.0000 & -0.1302 & -0.1102 & $-3.3 \%$ & $-2.8 \%$ \\
\hline 1 & FY09EM21-10 & $\mathrm{MgO}$ & 0.0083 & 0.0089 & 0.0000 & 0.0083 & 0.0089 & & \\
\hline 1 & FY09EM21-10 & $\mathrm{MnO}$ & 5.4585 & 5.4249 & 5.4644 & -0.0059 & -0.0396 & $-0.1 \%$ & $-0.7 \%$ \\
\hline 1 & FY09EM21-10 & $\mathrm{Na} 2 \mathrm{O}$ & 10.6930 & 10.5929 & 10.3889 & 0.3041 & 0.2040 & $2.9 \%$ & $2.0 \%$ \\
\hline 1 & FY09EM21-10 & $\mathrm{NiO}$ & 2.2205 & 2.5168 & 2.5000 & -0.2795 & 0.0168 & $-11.2 \%$ & $0.7 \%$ \\
\hline 1 & FY09EM21-10 & $\mathrm{PbO}$ & 0.1847 & 0.1847 & 0.2163 & -0.0316 & -0.0316 & $-14.6 \%$ & $-14.6 \%$ \\
\hline 1 & FY09EM21-10 & $\mathrm{SiO} 2$ & 39.7910 & 39.9192 & 40.4036 & -0.6126 & -0.4845 & $-1.5 \%$ & $-1.2 \%$ \\
\hline 1 & FY09EM21-10 & $\mathrm{SO} 4$ & 0.7947 & 0.7947 & 0.4808 & 0.3139 & 0.3139 & $65.3 \%$ & $65.3 \%$ \\
\hline 1 & FY09EM21-10 & $\mathrm{TiO} 2$ & 2.4353 & 2.6035 & 2.3966 & 0.0386 & 0.2069 & $1.6 \%$ & $8.6 \%$ \\
\hline 1 & FY09EM21-10 & $\mathrm{ZnO}$ & 0.1372 & 0.1372 & 0.1346 & 0.0026 & 0.0026 & $1.9 \%$ & $1.9 \%$ \\
\hline
\end{tabular}


SRNL-STI-2009-00778, Revision 0

Table A4. Average Measured and Bias-Corrected Chemical Compositions Versus Targeted Compositions by Oxide by Glass ID for the Non-Rad Matrix 2 Study

\begin{tabular}{|c|c|c|c|c|c|c|c|c|c|}
\hline & & & & Measured & & Difference & Difference & $\%$ & $\%$ \\
\hline & & & Measured & Bias-Corrected & Targeted & of & of & Difference & Difference \\
\hline Set & Glass \# & Oxide & (wt\%) & (BC) (wt\%) & (wt\%) & Measured & Meas BC & Measured & Meas BC \\
\hline 1 & "FY09EM21-10 & $\mathrm{ZrO} 2$ & 0.1915 & 0.1915 & 0.2051 & -0.0137 & $\begin{array}{l}-0.0137 \\
\end{array}$ & $-6.7 \%$ & $-6.7 \%$ \\
\hline 1 & FY09EM21-10 & Sum & 98.3790 & 99.5361 & 100.0000 & -1.6210 & -0.4640 & $-1.6 \%$ & $-0.5 \%$ \\
\hline 1 & FY09EM21-11 & $\mathrm{Al} 2 \mathrm{O} 3$ & 3.2310 & 3.3422 & 3.2500 & -0.0190 & 0.0922 & $-0.6 \%$ & $2.8 \%$ \\
\hline 1 & FY09EM21-11 & B2O3 & 4.2825 & 4.2477 & 4.5777 & -0.2952 & -0.3300 & $-6.4 \%$ & $-7.2 \%$ \\
\hline 1 & FY09EM21-11 & $\mathrm{BaO}$ & 0.0720 & 0.0789 & 0.0801 & -0.0081 & $\begin{array}{l}-0.0013 \\
\end{array}$ & $-10.1 \%$ & $-1.6 \%$ \\
\hline 1 & FY09EM21-11 & $\mathrm{CaO}$ & 4.0297 & 4.2206 & 4.0000 & 0.0297 & 0.2206 & $0.7 \%$ & $5.5 \%$ \\
\hline 1 & FY09EM21-11 & $\mathrm{CdO}$ & 0.2504 & 0.2504 & 0.2965 & -0.0460 & -0.0460 & $-15.5 \%$ & $-15.5 \%$ \\
\hline 1 & FY09EM21-11 & Ce2O3 & 0.3335 & 0.3335 & 0.3606 & -0.0270 & -0.0270 & $-7.5 \%$ & $-7.5 \%$ \\
\hline 1 & FY09EM21-11 & $\mathrm{Cr} 2 \mathrm{O} 3$ & 0.0179 & 0.0169 & 0.0000 & 0.0179 & 0.0169 & & \\
\hline 1 & FY09EM21-11 & $\mathrm{CuO}$ & 0.1230 & 0.1295 & 0.1282 & -0.0052 & 0.0013 & $-4.1 \%$ & $1.0 \%$ \\
\hline 1 & FY09EM21-11 & $\mathrm{Fe} 2 \mathrm{O} 3$ & 5.9869 & 6.1039 & 6.0404 & -0.0535 & 0.0635 & $-0.9 \%$ & $1.1 \%$ \\
\hline 1 & FY09EM21-11 & $\mathrm{La} 2 \mathrm{O} 3$ & 0.0833 & 0.0833 & 0.0978 & -0.0145 & -0.0145 & $-14.8 \%$ & $-14.8 \%$ \\
\hline 1 & FY09EM21-11 & $\mathrm{Li} 2 \mathrm{O}$ & 5.4845 & 5.5490 & 5.6270 & -0.1425 & -0.0780 & $-2.5 \%$ & $-1.4 \%$ \\
\hline 1 & FY09EM21-11 & $\mathrm{MgO}$ & 1.3461 & 1.4507 & 1.5000 & -0.1539 & $\begin{array}{l}-0.0493 \\
\end{array}$ & $-10.3 \%$ & $-3.3 \%$ \\
\hline 1 & FY09EM21-11 & $\mathrm{MnO}$ & 5.4069 & 5.3737 & 5.5000 & -0.0931 & $\begin{array}{l}-0.1263 \\
\end{array}$ & $-1.7 \%$ & $-2.3 \%$ \\
\hline 1 & FY09EM21-11 & $\mathrm{Na} 2 \mathrm{O}$ & 12.2938 & 12.1772 & 12.2079 & 0.0859 & -0.0307 & $0.7 \%$ & $-0.3 \%$ \\
\hline 1 & FY09EM21-11 & $\mathrm{NiO}$ & 0.0064 & 0.0072 & 0.0000 & 0.0064 & 0.0072 & & \\
\hline 1 & FY09EM21-11 & $\mathrm{PbO}$ & 0.1769 & 0.1769 & 0.2163 & -0.0394 & -0.0394 & $-18.2 \%$ & $-18.2 \%$ \\
\hline 1 & FY09EM21-11 & $\mathrm{SiO} 2$ & 47.7064 & 47.8578 & 49.2970 & -1.5906 & -1.4392 & $-3.2 \%$ & $-2.9 \%$ \\
\hline 1 & FY09EM21-11 & $\mathrm{SO} 4$ & 0.4486 & 0.4486 & 0.4808 & -0.0321 & $\begin{array}{l}-0.0321 \\
\end{array}$ & $-6.7 \%$ & $-6.7 \%$ \\
\hline 1 & FY09EM21-11 & $\mathrm{TiO} 2$ & 6.7596 & 7.2271 & 6.0000 & 0.7596 & 1.2271 & $12.7 \%$ & $20.5 \%$ \\
\hline 1 & FY09EM21-11 & $\mathrm{ZnO}$ & 0.1335 & 0.1335 & 0.1346 & -0.0011 & -0.0011 & $-0.8 \%$ & $-0.8 \%$ \\
\hline 1 & FY09EM21-11 & $\mathrm{ZrO} 2$ & 0.1908 & 0.1908 & 0.2051 & -0.0143 & -0.0143 & $-7.0 \%$ & $-7.0 \%$ \\
\hline 1 & FY09EM21-11 & Sum & 98.3637 & 99.3997 & 100.0000 & -1.6363 & -0.6003 & $-1.6 \%$ & $-0.6 \%$ \\
\hline 1 & FY09EM21-12 & $\mathrm{Al} 2 \mathrm{O} 3$ & 4.5679 & 4.8196 & 4.5542 & 0.0136 & 0.2654 & $0.3 \%$ & $5.8 \%$ \\
\hline 1 & FY09EM21-12 & B2O3 & 5.2323 & 5.1551 & 5.2908 & -0.0585 & -0.1357 & $-1.1 \%$ & $-2.6 \%$ \\
\hline 1 & FY09EM21-12 & $\mathrm{BaO}$ & 0.0056 & 0.0060 & 0.0000 & 0.0056 & 0.0060 & & \\
\hline 1 & FY09EM21-12 & $\mathrm{CaO}$ & 0.0070 & 0.0073 & 0.0000 & 0.0070 & 0.0073 & & \\
\hline 1 & FY09EM21-12 & $\mathrm{CdO}$ & 0.0057 & 0.0057 & 0.0000 & 0.0057 & 0.0057 & & \\
\hline 1 & FY09EM21-12 & $\mathrm{Ce} 2 \mathrm{O} 3$ & 0.0094 & 0.0094 & 0.0000 & 0.0094 & 0.0094 & & \\
\hline 1 & FY09EM21-12 & $\mathrm{Cr} 2 \mathrm{O} 3$ & 0.0179 & 0.0166 & 0.0000 & 0.0179 & 0.0166 & & \\
\hline 1 & FY09EM21-12 & $\mathrm{CuO}$ & 0.0063 & 0.0066 & 0.0000 & 0.0063 & 0.0066 & & \\
\hline 1 & FY09EM21-12 & $\mathrm{Fe} 2 \mathrm{O} 3$ & 15.3693 & 15.8477 & 16.8649 & -1.4957 & -1.0173 & $-8.9 \%$ & $-6.0 \%$ \\
\hline 1 & FY09EM21-12 & $\mathrm{La} 2 \mathrm{O} 3$ & 0.0059 & 0.0059 & 0.0000 & 0.0059 & 0.0059 & & \\
\hline 1 & FY09EM21-12 & $\mathrm{Li} 2 \mathrm{O}$ & 6.6148 & 6.6489 & 6.7937 & $\begin{array}{l}-0.1789 \\
\end{array}$ & -0.1448 & $-2.6 \%$ & $-2.1 \%$ \\
\hline 1 & FY09EM21-12 & $\mathrm{MgO}$ & 0.0083 & 0.0089 & 0.0000 & 0.0083 & 0.0089 & & \\
\hline 1 & FY09EM21-12 & $\mathrm{MnO}$ & 4.6709 & 4.7242 & 4.6737 & -0.0028 & 0.0506 & $-0.1 \%$ & $1.1 \%$ \\
\hline 1 & FY09EM21-12 & $\mathrm{Na} 2 \mathrm{O}$ & 9.8067 & 10.0041 & 10.0000 & -0.1933 & 0.0041 & $-1.9 \%$ & $0.0 \%$ \\
\hline 1 & FY09EM21-12 & $\mathrm{NiO}$ & 1.6892 & 1.8965 & 1.9071 & -0.2179 & -0.0106 & $-11.4 \%$ & $-0.6 \%$ \\
\hline 1 & FY09EM21-12 & $\mathrm{PbO}$ & 0.0054 & 0.0054 & 0.0000 & 0.0054 & 0.0054 & & \\
\hline 1 & FY09EM21-12 & $\mathrm{SiO} 2$ & 46.2624 & 47.3601 & 47.9155 & -1.6532 & -0.5554 & $-3.5 \%$ & $-1.2 \%$ \\
\hline 1 & FY09EM21-12 & SO4 & 0.0749 & 0.0749 & 0.0000 & 0.0749 & 0.0749 & & \\
\hline 1 & FY09EM21-12 & $\mathrm{TiO} 2$ & 1.9933 & 2.1050 & 2.0000 & -0.0067 & 0.1050 & $-0.3 \%$ & $5.2 \%$ \\
\hline 1 & FY09EM21-12 & $\mathrm{ZnO}$ & 0.0062 & 0.0062 & 0.0000 & 0.0062 & 0.0062 & & \\
\hline 1 & FY09EM21-12 & $\mathrm{ZrO} 2$ & 0.0068 & 0.0068 & 0.0000 & 0.0068 & 0.0068 & & \\
\hline 1 & FY09EM21-12 & Sum & 96.3660 & 98.7208 & 100.0000 & -3.6340 & -1.2792 & $-3.6 \%$ & $-1.3 \%$ \\
\hline 1 & FY09EM21-13 & $\mathrm{Al} 2 \mathrm{O} 3$ & 10.3119 & 10.8786 & 10.3780 & -0.0661 & 0.5006 & $-0.6 \%$ & $4.8 \%$ \\
\hline 1 & FY09EM21-13 & B2O3 & 5.8844 & 5.8367 & 5.8833 & 0.0011 & -0.0466 & $0.0 \%$ & $-0.8 \%$ \\
\hline 1 & FY09EM21-13 & $\mathrm{BaO}$ & 0.0745 & 0.0803 & 0.0801 & $\begin{array}{l}-0.0056 \\
\end{array}$ & 0.0002 & $-7.0 \%$ & $0.2 \%$ \\
\hline 1 & FY09EM21-13 & $\mathrm{CaO}$ & 4.0262 & 4.2203 & 4.0000 & 0.0262 & 0.2203 & $0.7 \%$ & $5.5 \%$ \\
\hline 1 & FY09EM21-13 & $\mathrm{CdO}$ & 0.2547 & 0.2547 & 0.2965 & -0.0417 & -0.0417 & $-14.1 \%$ & $-14.1 \%$ \\
\hline 1 & FY09EM21-13 & Ce2O3 & 0.3367 & 0.3367 & 0.3606 & $\begin{array}{l}-0.0238 \\
\end{array}$ & -0.0238 & $-6.6 \%$ & $-6.6 \%$ \\
\hline 1 & FY09EM21-13 & $\mathrm{Cr} 2 \mathrm{O} 3$ & 0.1739 & 0.1622 & 0.2000 & -0.0261 & -0.0378 & $-13.0 \%$ & $-18.9 \%$ \\
\hline 1 & FY09EM21-13 & $\mathrm{CuO}$ & 0.1227 & 0.1285 & 0.1282 & -0.0055 & 0.0003 & $-4.3 \%$ & $0.2 \%$ \\
\hline 1 & FY09EM21-13 & $\mathrm{Fe} 2 \mathrm{O} 3$ & 13.3641 & 13.7796 & 14.0780 & -0.7139 & -0.2985 & $-5.1 \%$ & $-2.1 \%$ \\
\hline 1 & FY09EM21-13 & $\mathrm{La} 2 \mathrm{O} 3$ & 0.0856 & 0.0856 & 0.0978 & -0.0121 & -0.0121 & $-12.4 \%$ & $-12.4 \%$ \\
\hline 1 & FY09EM21-13 & $\mathrm{Li} 2 \mathrm{O}$ & 6.9108 & 6.9921 & 7.0000 & -0.0892 & -0.0079 & $-1.3 \%$ & $-0.1 \%$ \\
\hline 1 & FY09EM21-13 & $\mathrm{MgO}$ & 0.0083 & 0.0089 & 0.0000 & 0.0083 & 0.0089 & & \\
\hline 1 & FY09EM21-13 & $\mathrm{MnO}$ & 0.2902 & 0.2935 & 0.3000 & -0.0098 & -0.0065 & $-3.3 \%$ & $-2.2 \%$ \\
\hline 1 & FY09EM21-13 & $\mathrm{Na} 2 \mathrm{O}$ & 10.4571 & 10.6652 & 10.5159 & -0.0588 & 0.1493 & $-0.6 \%$ & $1.4 \%$ \\
\hline
\end{tabular}


SRNL-STI-2009-00778, Revision 0

Table A4. Average Measured and Bias-Corrected Chemical Compositions Versus Targeted Compositions by Oxide by Glass ID for the Non-Rad Matrix 2 Study

\begin{tabular}{|c|c|c|c|c|c|c|c|c|c|}
\hline & & & & Measured & & Difference & \begin{tabular}{|l|} 
Difference \\
\end{tabular} & $\%$ & $\%$ \\
\hline & & & Measured & Bias-Corrected & Targeted & of & of & Difference & Difference \\
\hline Set & Glass \# & Oxide & $(\mathrm{wt} \%)$ & (BC) (wt\%) & (wt\%) & Measured & Meas BC & Measured & Meas BC \\
\hline 1 & FY09EM21-13 & $\mathrm{NiO}$ & 2.2301 & 2.5037 & 2.5000 & $\begin{array}{l}-0.2699 \\
\end{array}$ & 0.0037 & $-10.8 \%$ & $0.1 \%$ \\
\hline 1 & FY09EM21-13 & $\mathrm{PbO}$ & 0.1842 & 0.1842 & 0.2163 & -0.0321 & -0.0321 & $-14.9 \%$ & $-14.9 \%$ \\
\hline 1 & FY09EM21-13 & $\mathrm{SiO} 2$ & 36.6890 & 37.5568 & 37.1447 & -0.4557 & 0.4121 & $-1.2 \%$ & $1.1 \%$ \\
\hline 1 & FY09EM21-13 & $\mathrm{SO} 4$ & 0.4434 & 0.4434 & 0.4808 & -0.0374 & -0.0374 & $-7.8 \%$ & $-7.8 \%$ \\
\hline 1 & FY09EM21-13 & $\mathrm{TiO} 2$ & 5.9673 & 6.3017 & 6.0000 & -0.0327 & 0.3017 & $-0.5 \%$ & $5.0 \%$ \\
\hline 1 & FY09EM21-13 & $\mathrm{ZnO}$ & 0.1354 & 0.1354 & 0.1346 & 0.0008 & 0.0008 & $0.6 \%$ & $0.6 \%$ \\
\hline 1 & FY09EM21-13 & $\mathrm{ZrO} 2$ & 0.1938 & 0.1938 & 0.2051 & -0.0113 & -0.0113 & $-5.5 \%$ & $-5.5 \%$ \\
\hline 1 & FY09EM21-13 & Sum & 98.1444 & 101.0419 & 100.0000 & -1.8556 & 1.0419 & $-1.9 \%$ & $1.0 \%$ \\
\hline 1 & $\begin{array}{l}\text { FY09EM21-14 } \\
\end{array}$ & $\mathrm{Al} 2 \mathrm{O} 3$ & 3.2169 & 3.3937 & 3.2500 & $\begin{array}{c}-0.0331 \\
\end{array}$ & 0.1437 & $-1.0 \%$ & $4.4 \%$ \\
\hline 1 & FY09EM21-14 & $\mathrm{B} 2 \mathrm{O} 3$ & 14.9725 & 14.8513 & 13.6465 & 1.3260 & 1.2048 & $9.7 \%$ & $8.8 \%$ \\
\hline 1 & FY09EM21-14 & $\mathrm{BaO}$ & 0.0782 & 0.0842 & 0.0801 & -0.0020 & 0.0041 & $-2.5 \%$ & $5.1 \%$ \\
\hline 1 & FY09EM21-14 & $\mathrm{CaO}$ & 0.0350 & 0.0367 & 0.0000 & 0.0350 & 0.0367 & & \\
\hline 1 & FY09EM21-14 & $\mathrm{CdO}$ & 0.2499 & 0.2499 & 0.2965 & -0.0466 & -0.0466 & $-15.7 \%$ & $-15.7 \%$ \\
\hline 1 & FY09EM21-14 & $\mathrm{Ce} 2 \mathrm{O} 3$ & 0.3426 & 0.3426 & 0.3606 & -0.0180 & -0.0180 & $-5.0 \%$ & $-5.0 \%$ \\
\hline 1 & FY09EM21-14 & $\mathrm{Cr} 2 \mathrm{O} 3$ & 0.1432 & 0.1336 & 0.2000 & $\begin{array}{l}-0.0568 \\
\end{array}$ & $\begin{array}{l}-0.0664 \\
\end{array}$ & $-28.4 \%$ & $-33.2 \%$ \\
\hline 1 & FY09EM21-14 & $\mathrm{CuO}$ & 0.1289 & 0.1350 & 0.1282 & 0.0007 & 0.0068 & $0.6 \%$ & $5.3 \%$ \\
\hline 1 & FY09EM21-14 & $\mathrm{Fe} 2 \mathrm{O} 3$ & 19.6226 & 20.2324 & 20.5438 & -0.9212 & -0.3114 & $-4.5 \%$ & $-1.5 \%$ \\
\hline 1 & FY09EM21-14 & $\mathrm{La} 2 \mathrm{O} 3$ & 0.0862 & 0.0862 & 0.0978 & -0.0116 & -0.0116 & $-11.8 \%$ & $-11.8 \%$ \\
\hline 1 & FY09EM21-14 & $\mathrm{Li} 2 \mathrm{O}$ & 4.3435 & 4.3945 & 4.0000 & 0.3435 & 0.3945 & $8.6 \%$ & $9.9 \%$ \\
\hline 1 & FY09EM21-14 & $\mathrm{MgO}$ & 0.0062 & 0.0066 & 0.0000 & 0.0062 & 0.0066 & & \\
\hline 1 & FY09EM21-14 & $\mathrm{MnO}$ & 0.2992 & 0.3027 & 0.3000 & -0.0008 & 0.0027 & $-0.3 \%$ & $0.9 \%$ \\
\hline 1 & FY09EM21-14 & $\mathrm{Na} 2 \mathrm{O}$ & 9.7730 & 9.9679 & 10.0000 & -0.2270 & -0.0321 & $-2.3 \%$ & $-0.3 \%$ \\
\hline 1 & FY09EM21-14 & $\mathrm{NiO}$ & 0.0064 & 0.0071 & 0.0000 & 0.0064 & 0.0071 & & \\
\hline 1 & FY09EM21-14 & $\mathrm{PbO}$ & 0.1861 & 0.1861 & 0.2163 & -0.0303 & -0.0303 & $-14.0 \%$ & $-14.0 \%$ \\
\hline 1 & FY09EM21-14 & $\mathrm{SiO} 2$ & 39.4166 & 40.3486 & 40.3535 & -0.9369 & -0.0049 & $-2.3 \%$ & $0.0 \%$ \\
\hline 1 & FY09EM21-14 & SO4 & 0.4292 & 0.4292 & 0.4808 & -0.0516 & -0.0516 & $-10.7 \%$ & $-10.7 \%$ \\
\hline 1 & FY09EM21-14 & $\mathrm{TiO} 2$ & 5.5961 & 5.9098 & 5.7062 & -0.1101 & 0.2036 & $-1.9 \%$ & $3.6 \%$ \\
\hline 1 & FY09EM21-14 & $\mathrm{ZnO}$ & 0.1279 & 0.1279 & 0.1346 & -0.0067 & -0.0067 & $-5.0 \%$ & $-5.0 \%$ \\
\hline 1 & FY09EM21-14 & $\mathrm{ZrO} 2$ & 0.1942 & 0.1942 & 0.2051 & -0.0110 & -0.0110 & $-5.3 \%$ & $-5.3 \%$ \\
\hline 1 & FY09EM21-14 & Sum & 99.2544 & 101.4202 & 100.0000 & -0.7456 & 1.4202 & $-0.7 \%$ & $1.4 \%$ \\
\hline 2 & "Batch 1 & $\mathrm{Al} 2 \mathrm{O} 3$ & $\begin{array}{c}4.7647 \\
\end{array}$ & 4.8770 & 4.8770 & $\begin{array}{c}-0.1123 \\
\end{array}$ & 0.0000 & $-2.3 \%$ & $0.0 \%$ \\
\hline 2 & Batch 1 & $\mathrm{~B} 2 \mathrm{O} 3$ & 7.8566 & 7.7770 & 7.7770 & 0.0796 & 0.0000 & $1.0 \%$ & $0.0 \%$ \\
\hline 2 & Batch 1 & $\mathrm{BaO}$ & 0.1385 & 0.1510 & 0.1510 & -0.0125 & 0.0000 & $-8.3 \%$ & $0.0 \%$ \\
\hline 2 & Batch 1 & $\mathrm{CaO}$ & 1.1627 & 1.2200 & 1.2200 & -0.0573 & 0.0000 & $-4.7 \%$ & $0.0 \%$ \\
\hline 2 & Batch 1 & $\mathrm{CdO}$ & 0.0057 & 0.0057 & 0.0000 & 0.0057 & 0.0057 & & \\
\hline 2 & Batch 1 & Ce2O3 & 0.0059 & 0.0059 & 0.0000 & 0.0059 & 0.0059 & & \\
\hline 2 & Batch 1 & Cr2O3 & 0.1124 & 0.1079 & 0.1070 & 0.0054 & 0.0009 & $5.1 \%$ & $0.9 \%$ \\
\hline 2 & Batch 1 & $\mathrm{CuO}$ & 0.3759 & 0.3990 & 0.3990 & -0.0231 & 0.0000 & $-5.8 \%$ & $0.0 \%$ \\
\hline 2 & Batch 1 & $\mathrm{Fe} 2 \mathrm{O} 3$ & 12.5802 & 12.8390 & 12.8390 & -0.2588 & 0.0000 & $-2.0 \%$ & $0.0 \%$ \\
\hline 2 & Batch 1 & La2O3 & 0.0059 & 0.0059 & 0.0000 & 0.0059 & 0.0059 & & \\
\hline 2 & Batch 1 & $\mathrm{Li} 2 \mathrm{O}$ & 4.4278 & 4.4290 & 4.4290 & -0.0012 & 0.0000 & $0.0 \%$ & $0.0 \%$ \\
\hline 2 & Batch 1 & $\mathrm{MgO}$ & 1.3228 & 1.4190 & 1.4190 & -0.0962 & 0.0000 & $-6.8 \%$ & $0.0 \%$ \\
\hline 2 & Batch 1 & $\mathrm{MnO}$ & 1.7216 & 1.7260 & 1.7260 & -0.0044 & 0.0000 & $-0.3 \%$ & $0.0 \%$ \\
\hline 2 & Batch 1 & $\mathrm{Na} 2 \mathrm{O}$ & 8.9867 & 9.0030 & 9.0030 & -0.0163 & 0.0000 & $-0.2 \%$ & $0.0 \%$ \\
\hline 2 & Batch 1 & $\mathrm{NiO}$ & 0.6648 & 0.7510 & 0.7510 & -0.0862 & 0.0000 & $-11.5 \%$ & $0.0 \%$ \\
\hline 2 & Batch 1 & $\mathrm{PbO}$ & 0.0054 & 0.0054 & 0.0000 & 0.0054 & 0.0054 & & \\
\hline 2 & Batch 1 & $\mathrm{SiO} 2$ & 50.5588 & 50.2200 & 50.2200 & 0.3388 & 0.0000 & $0.7 \%$ & $0.0 \%$ \\
\hline 2 & Batch 1 & $\mathrm{SO} 4$ & 0.0749 & 0.0749 & 0.0000 & 0.0749 & 0.0749 & & \\
\hline 2 & Batch 1 & $\mathrm{TiO} 2$ & 0.6375 & 0.6770 & 0.6770 & -0.0395 & 0.0000 & $-5.8 \%$ & $0.0 \%$ \\
\hline 2 & Batch 1 & $\mathrm{ZnO}$ & 0.0062 & 0.0062 & 0.0000 & 0.0062 & 0.0062 & & \\
\hline 2 & Batch 1 & $\mathrm{ZrO} 2$ & 0.0842 & 0.0842 & 0.0980 & -0.0138 & -0.0138 & $-14.1 \%$ & $-14.1 \%$ \\
\hline 2 & Batch 1 & Sum & 95.4990 & 95.7840 & 95.6930 & -0.1940 & 0.0910 & $-0.2 \%$ & $0.1 \%$ \\
\hline 2 & FY09EM21-15 & Al2O3 & 5.3662 & 5.5075 & 5.3229 & 0.0433 & 0.1846 & $0.8 \%$ & $3.5 \%$ \\
\hline 2 & FY09EM21-15 & $\mathrm{B} 2 \mathrm{O} 3$ & 4.6045 & 4.6042 & 4.5000 & 0.1045 & 0.1042 & $2.3 \%$ & $2.3 \%$ \\
\hline 2 & FY09EM21-15 & $\mathrm{BaO}$ & 0.0879 & 0.0962 & 0.0801 & 0.0078 & 0.0160 & $9.7 \%$ & $20.0 \%$ \\
\hline 2 & FY09EM21-15 & $\mathrm{CaO}$ & 3.1342 & 3.3325 & 3.1904 & -0.0562 & 0.1421 & $-1.8 \%$ & $4.5 \%$ \\
\hline 2 & FY09EM21-15 & $\mathrm{CdO}$ & 0.2136 & 0.2136 & 0.2965 & -0.0829 & -0.0829 & $-27.9 \%$ & $-27.9 \%$ \\
\hline 2 & FY09EM21-15 & Ce2O3 & 0.3455 & 0.3455 & 0.3606 & -0.0150 & -0.0150 & $-4.2 \%$ & $-4.2 \%$ \\
\hline 2 & FY09EM21-15 & Cr2O3 & 0.1407 & 0.1343 & 0.2000 & -0.0593 & -0.0657 & $-29.7 \%$ & $-32.8 \%$ \\
\hline 2 & FY09EM21-15 & $\mathrm{CuO}$ & 0.1264 & 0.1334 & 0.1282 & -0.0018 & 0.0052 & $-1.4 \%$ & $4.1 \%$ \\
\hline
\end{tabular}


SRNL-STI-2009-00778, Revision 0

Table A4. Average Measured and Bias-Corrected Chemical Compositions Versus Targeted Compositions by Oxide by Glass ID for the Non-Rad Matrix 2 Study

\begin{tabular}{|c|c|c|c|c|c|c|c|c|c|}
\hline & & & & Measured & & Difference & Difference & $\%$ & $\%$ \\
\hline & & & Measured & Bias-Corrected & Targeted & of & of & Difference & Difference \\
\hline Set & Glass \# & Oxide & (wt\%) & (BC) (wt\%) & $(w t \%)$ & Measured & Meas BC & Measured & Meas BC \\
\hline 2 & FY09EM21-15 & Fe2O3 & 7.8669 & 7.9202 & 8.2902 & -0.4232 & -0.3699 & $-5.1 \%$ & $-4.5 \%$ \\
\hline 2 & FY09EM21-15 & $\mathrm{La} 2 \mathrm{O} 3$ & 0.0906 & 0.0906 & 0.0978 & -0.0072 & -0.0072 & $-7.3 \%$ & $-7.3 \%$ \\
\hline 2 & FY09EM21-15 & $\mathrm{Li} 2 \mathrm{O}$ & 5.8074 & 5.8854 & 6.0020 & -0.1946 & -0.1166 & $-3.2 \%$ & $-1.9 \%$ \\
\hline 2 & FY09EM21-15 & $\mathrm{MgO}$ & 1.3358 & 1.4500 & 1.5000 & -0.1642 & -0.0500 & $-10.9 \%$ & $-3.3 \%$ \\
\hline 2 & FY09EM21-15 & $\mathrm{MnO}$ & 5.3294 & 5.2971 & 5.3591 & -0.0296 & -0.0619 & $-0.6 \%$ & $-1.2 \%$ \\
\hline 2 & FY09EM21-15 & $\mathrm{Na} 2 \mathrm{O}$ & 9.9078 & 9.9415 & 10.0000 & -0.0922 & -0.0585 & $-0.9 \%$ & $-0.6 \%$ \\
\hline 2 & FY09EM21-15 & $\mathrm{NiO}$ & 2.2714 & 2.5926 & 2.5000 & -0.2286 & 0.0926 & $-9.1 \%$ & $3.7 \%$ \\
\hline 2 & FY09EM21-15 & $\mathrm{PbO}$ & 0.1810 & 0.1810 & 0.2163 & -0.0354 & -0.0354 & $-16.4 \%$ & $-16.4 \%$ \\
\hline 2 & FY09EM21-15 & $\mathrm{SiO} 2$ & 49.4713 & 49.3478 & 49.1354 & 0.3359 & 0.2124 & $0.7 \%$ & $0.4 \%$ \\
\hline 2 & FY09EM21-15 & $\mathrm{SO} 4$ & 0.4359 & 0.4359 & 0.4808 & -0.0449 & -0.0449 & $-9.3 \%$ & $-9.3 \%$ \\
\hline 2 & FY09EM21-15 & $\mathrm{TiO} 2$ & 1.8640 & 1.9736 & 2.0000 & -0.1360 & -0.0264 & $-6.8 \%$ & $-1.3 \%$ \\
\hline 2 & FY09EM21-15 & $\mathrm{ZnO}$ & 0.1323 & 0.1323 & 0.1346 & -0.0024 & -0.0024 & $-1.8 \%$ & $-1.8 \%$ \\
\hline 2 & FY09EM21-15 & $\mathrm{ZrO} 2$ & 0.1871 & 0.1871 & 0.2051 & -0.0180 & -0.0180 & $-8.8 \%$ & $-8.8 \%$ \\
\hline 2 & FY09EM21-15 & Sum & 98.8999 & 99.8023 & 100.0000 & -1.1001 & -0.1977 & $-1.1 \%$ & $-0.2 \%$ \\
\hline 2 & FY09EM21-16 & $\mathrm{Al} 2 \mathrm{O} 3$ & 3.7365 & 3.8145 & 3.6772 & 0.0593 & 0.1373 & $1.6 \%$ & $3.7 \%$ \\
\hline 2 & FY09EM21-16 & $\mathrm{B} 2 \mathrm{O} 3$ & 6.6169 & 6.4837 & 6.4270 & 0.1899 & 0.0567 & $3.0 \%$ & $0.9 \%$ \\
\hline 2 & FY09EM21-16 & $\mathrm{BaO}$ & 0.0756 & 0.0822 & 0.0801 & -0.0045 & 0.0020 & $-5.6 \%$ & $2.5 \%$ \\
\hline 2 & FY09EM21-16 & $\mathrm{CaO}$ & 3.8443 & 3.9842 & 3.8213 & 0.0230 & 0.1629 & $0.6 \%$ & $4.3 \%$ \\
\hline 2 & FY09EM21-16 & $\mathrm{CdO}$ & 0.2267 & 0.2267 & 0.2965 & -0.0697 & -0.0697 & $-23.5 \%$ & $-23.5 \%$ \\
\hline 2 & FY09EM21-16 & Ce2O3 & 0.3479 & 0.3479 & 0.3606 & -0.0127 & -0.0127 & $-3.5 \%$ & $-3.5 \%$ \\
\hline 2 & FY09EM21-16 & $\mathrm{Cr} 2 \mathrm{O} 3$ & 0.1524 & 0.1471 & 0.2000 & -0.0476 & -0.0529 & $-23.8 \%$ & $-26.4 \%$ \\
\hline 2 & FY09EM21-16 & $\mathrm{CuO}$ & 0.1258 & 0.1343 & 0.1282 & -0.0024 & 0.0061 & $-1.9 \%$ & $4.8 \%$ \\
\hline 2 & FY09EM21-16 & $\mathrm{Fe} 2 \mathrm{O} 3$ & 8.7390 & 9.0428 & 9.0461 & -0.3070 & -0.0033 & $-3.4 \%$ & $0.0 \%$ \\
\hline 2 & FY09EM21-16 & $\mathrm{La} 2 \mathrm{O} 3$ & 0.0862 & 0.0862 & 0.0978 & -0.0116 & -0.0116 & $-11.8 \%$ & $-11.8 \%$ \\
\hline 2 & FY09EM21-16 & $\mathrm{Li} 2 \mathrm{O}$ & 3.9882 & 3.9383 & 4.0000 & -0.0118 & -0.0617 & $-0.3 \%$ & $-1.5 \%$ \\
\hline 2 & FY09EM21-16 & $\mathrm{MgO}$ & 1.4091 & 1.4942 & 1.5000 & -0.0909 & -0.0058 & $-6.1 \%$ & $-0.4 \%$ \\
\hline 2 & FY09EM21-16 & $\mathrm{MnO}$ & 0.3076 & 0.3112 & 0.3000 & 0.0076 & 0.0112 & $2.5 \%$ & $3.7 \%$ \\
\hline 2 & FY09EM21-16 & $\mathrm{Na} 2 \mathrm{O}$ & 15.2661 & 15.2698 & 15.8200 & -0.5539 & -0.5502 & $-3.5 \%$ & $-3.5 \%$ \\
\hline 2 & FY09EM21-16 & $\mathrm{NiO}$ & 2.3414 & 2.6190 & 2.5000 & -0.1586 & 0.1190 & $-6.3 \%$ & $4.8 \%$ \\
\hline 2 & FY09EM21-16 & $\mathrm{PbO}$ & 0.1855 & 0.1855 & 0.2163 & -0.0308 & -0.0308 & $-14.2 \%$ & $-14.2 \%$ \\
\hline 2 & FY09EM21-16 & $\mathrm{SiO} 2$ & 48.0808 & 47.5571 & 48.7083 & -0.6275 & -1.1512 & $-1.3 \%$ & $-2.4 \%$ \\
\hline 2 & FY09EM21-16 & SO4 & 0.4314 & 0.4314 & 0.4808 & -0.0494 & -0.0494 & $-10.3 \%$ & $-10.3 \%$ \\
\hline 2 & FY09EM21-16 & $\mathrm{TiO} 2$ & 1.9682 & 2.0972 & 2.0000 & -0.0318 & 0.0972 & $-1.6 \%$ & $4.9 \%$ \\
\hline 2 & FY09EM21-16 & $\mathrm{ZnO}$ & 0.1385 & 0.1385 & 0.1346 & 0.0039 & 0.0039 & $2.9 \%$ & $2.9 \%$ \\
\hline 2 & FY09EM21-16 & $\mathrm{ZrO} 2$ & 0.1945 & 0.1945 & 0.2051 & -0.0106 & -0.0106 & $-5.2 \%$ & $-5.2 \%$ \\
\hline 2 & FY09EM21-16 & Sum & 98.2628 & 98.5862 & 99.9999 & -1.7371 & -1.4137 & $-1.7 \%$ & $-1.4 \%$ \\
\hline 2 & FY09EM21-17 & $\mathrm{Al} 2 \mathrm{O} 3$ & 7.6666 & 7.8267 & 7.1927 & 0.4739 & 0.6340 & $6.6 \%$ & $8.8 \%$ \\
\hline 2 & FY09EM21-17 & $\mathrm{B} 2 \mathrm{O} 3$ & 4.7977 & 4.6990 & 4.5000 & 0.2977 & 0.1990 & $6.6 \%$ & $4.4 \%$ \\
\hline 2 & FY09EM21-17 & $\mathrm{BaO}$ & 0.0879 & 0.0955 & 0.0801 & 0.0078 & 0.0154 & $9.7 \%$ & $19.2 \%$ \\
\hline 2 & FY09EM21-17 & $\mathrm{CaO}$ & 0.1140 & 0.1181 & 0.1041 & 0.0100 & 0.0140 & $9.6 \%$ & $13.5 \%$ \\
\hline 2 & FY09EM21-17 & $\mathrm{CdO}$ & 0.2736 & 0.2736 & 0.2965 & -0.0229 & -0.0229 & $-7.7 \%$ & $-7.7 \%$ \\
\hline 2 & FY09EM21-17 & $\mathrm{Ce} 2 \mathrm{O} 3$ & 0.3736 & 0.3736 & 0.3606 & 0.0131 & 0.0131 & $3.6 \%$ & $3.6 \%$ \\
\hline 2 & FY09EM21-17 & $\mathrm{Cr} 2 \mathrm{O} 3$ & 0.0117 & 0.0113 & 0.0000 & 0.0117 & 0.0113 & & \\
\hline 2 & FY09EM21-17 & $\mathrm{CuO}$ & 0.1380 & 0.1473 & 0.1282 & 0.0098 & 0.0191 & $7.6 \%$ & $14.9 \%$ \\
\hline 2 & FY09EM21-17 & $\mathrm{Fe} 2 \mathrm{O} 3$ & 13.8145 & 14.2951 & 13.9129 & -0.0985 & 0.3821 & $-0.7 \%$ & $2.7 \%$ \\
\hline 2 & FY09EM21-17 & $\mathrm{La} 2 \mathrm{O} 3$ & 0.0985 & 0.0985 & 0.0978 & 0.0008 & 0.0008 & $0.8 \%$ & $0.8 \%$ \\
\hline 2 & FY09EM21-17 & $\mathrm{Li} 2 \mathrm{O}$ & 6.9969 & 6.9096 & 6.7401 & 0.2568 & 0.1695 & $3.8 \%$ & $2.5 \%$ \\
\hline 2 & FY09EM21-17 & $\mathrm{MgO}$ & 0.0083 & 0.0088 & 0.0000 & 0.0083 & 0.0088 & & \\
\hline 2 & FY09EM21-17 & $\mathrm{MnO}$ & 0.5901 & 0.5969 & 5.5000 & -4.9099 & -4.9031 & $-89.3 \%$ & $-89.1 \%$ \\
\hline 2 & FY09EM21-17 & $\mathrm{Na} 2 \mathrm{O}$ & 15.0302 & 15.0343 & 14.8547 & 0.1755 & 0.1796 & $1.2 \%$ & $1.2 \%$ \\
\hline 2 & FY09EM21-17 & $\mathrm{NiO}$ & 0.0064 & 0.0071 & 0.0000 & 0.0064 & 0.0071 & & \\
\hline 2 & FY09EM21-17 & $\mathrm{PbO}$ & 0.2052 & 0.2052 & 0.2163 & -0.0111 & -0.0111 & $-5.1 \%$ & $-5.1 \%$ \\
\hline 2 & FY09EM21-17 & $\mathrm{SiO} 2$ & 41.2885 & 40.8388 & 39.1955 & 2.0930 & 1.6433 & $5.3 \%$ & $4.2 \%$ \\
\hline 2 & FY09EM21-17 & $\mathrm{SO} 4$ & 0.4906 & 0.4906 & 0.4808 & 0.0098 & 0.0098 & $2.0 \%$ & $2.0 \%$ \\
\hline 2 & FY09EM21-17 & $\mathrm{TiO} 2$ & 6.1257 & 6.5265 & 6.0000 & 0.1257 & 0.5265 & $2.1 \%$ & $8.8 \%$ \\
\hline 2 & FY09EM21-17 & $\mathrm{ZnO}$ & 0.1388 & 0.1388 & 0.1346 & 0.0042 & 0.0042 & $3.1 \%$ & $3.1 \%$ \\
\hline 2 & FY09EM21-17 & $\mathrm{ZrO} 2$ & 0.2063 & 0.2063 & 0.2051 & 0.0012 & 0.0012 & $0.6 \%$ & $0.6 \%$ \\
\hline 2 & FY09EM21-17 & Sum & 98.4632 & 98.9016 & 100.0000 & -1.5369 & -1.0985 & $-1.5 \%$ & $-1.1 \%$ \\
\hline 2 & FY09EM21-18 & $\mathrm{Al} 2 \mathrm{O} 3$ & 11.0677 & 11.3587 & 10.9157 & 0.1521 & 0.4430 & $1.4 \%$ & $4.1 \%$ \\
\hline 2 & FY09EM21-18 & $\mathrm{B} 2 \mathrm{O} 3$ & 4.8620 & 4.8619 & 4.8441 & 0.0179 & 0.0178 & $0.4 \%$ & $0.4 \%$ \\
\hline
\end{tabular}


SRNL-STI-2009-00778, Revision 0

Table A4. Average Measured and Bias-Corrected Chemical Compositions Versus Targeted Compositions by Oxide by Glass ID for the Non-Rad Matrix 2 Study

\begin{tabular}{|c|c|c|c|c|c|c|c|c|c|}
\hline & & & & Measured & & Difference & Difference & $\%$ & $\%$ \\
\hline & & & Measured & Bias-Corrected & Targeted & of & of & Difference & Difference \\
\hline Set & Glass \# & Oxide & $(\mathrm{wt} \%)$ & $(\mathrm{BC})(\mathrm{wt} \%)$ & (wt\%) & Measured & Meas BC & Measured & Meas BC \\
\hline 2 & FY09EM21-18 & $\overline{\mathrm{BaO}}$ & 0.0829 & 0.0907 & 0.0801 & 0.0028 & 0.0105 & $3.5 \%$ & $13.2 \%$ \\
\hline 2 & FY09EM21-18 & $\mathrm{CaO}$ & 0.4030 & 0.4285 & 0.4385 & -0.0355 & -0.0100 & $-8.1 \%$ & $-2.3 \%$ \\
\hline 2 & FY09EM21-18 & $\mathrm{CdO}$ & 0.2042 & 0.2042 & 0.2965 & -0.0923 & -0.0923 & $-31.1 \%$ & $-31.1 \%$ \\
\hline 2 & FY09EM21-18 & Ce2O3 & 0.3452 & 0.3452 & 0.3606 & -0.0153 & -0.0153 & $-4.3 \%$ & $-4.3 \%$ \\
\hline 2 & FY09EM21-18 & Cr2O3 & 0.0186 & 0.0178 & 0.0000 & 0.0186 & 0.0178 & & \\
\hline 2 & FY09EM21-18 & $\mathrm{CuO}$ & 0.1242 & 0.1311 & 0.1282 & -0.0040 & 0.0029 & $-3.1 \%$ & $2.3 \%$ \\
\hline 2 & FY09EM21-18 & Fe2O3 & 6.6696 & 6.7145 & 6.7914 & -0.1218 & -0.0769 & $-1.8 \%$ & $-1.1 \%$ \\
\hline 2 & FY09EM21-18 & $\mathrm{La} 2 \mathrm{O} 3$ & 0.0850 & 0.0850 & 0.0978 & -0.0127 & -0.0127 & $-13.0 \%$ & $-13.0 \%$ \\
\hline 2 & FY09EM21-18 & Li2O & 5.3284 & 5.3999 & 5.4934 & -0.1650 & -0.0935 & $-3.0 \%$ & $-1.7 \%$ \\
\hline 2 & FY09EM21-18 & $\mathrm{MgO}$ & 0.0083 & 0.0090 & 0.0000 & 0.0083 & 0.0090 & & \\
\hline 2 & FY09EM21-18 & $\mathrm{MnO}$ & 0.8015 & 0.7967 & 0.8350 & -0.0335 & -0.0383 & $-4.0 \%$ & $-4.6 \%$ \\
\hline 2 & FY09EM21-18 & $\mathrm{Na} 2 \mathrm{O}$ & 16.1086 & 16.1622 & 17.0195 & -0.9109 & -0.8572 & $-5.4 \%$ & $-5.0 \%$ \\
\hline 2 & FY09EM21-18 & $\mathrm{NiO}$ & 0.0064 & 0.0073 & 0.0000 & 0.0064 & 0.0073 & & \\
\hline 2 & FY09EM21-18 & $\mathrm{PbO}$ & 0.1899 & 0.1899 & 0.2163 & -0.0265 & -0.0265 & $-12.2 \%$ & $-12.2 \%$ \\
\hline 2 & FY09EM21-18 & $\mathrm{SiO} 2$ & 49.7387 & 49.6111 & 49.6625 & 0.0762 & -0.0514 & $0.2 \%$ & $-0.1 \%$ \\
\hline 2 & FY09EM21-18 & SO4 & 0.4322 & 0.4322 & 0.4808 & -0.0486 & -0.0486 & $-10.1 \%$ & $-10.1 \%$ \\
\hline 2 & FY09EM21-18 & $\mathrm{TiO} 2$ & 1.9599 & 2.0752 & 2.0000 & -0.0401 & 0.0752 & $-2.0 \%$ & $3.8 \%$ \\
\hline 2 & FY09EM21-18 & $\mathrm{ZnO}$ & 0.1242 & 0.1242 & 0.1346 & -0.0104 & -0.0104 & $-7.8 \%$ & $-7.8 \%$ \\
\hline 2 & FY09EM21-18 & $\mathrm{ZrO} 2$ & 0.1999 & 0.1999 & 0.2051 & -0.0052 & -0.0052 & $-2.5 \%$ & $-2.5 \%$ \\
\hline 2 & FY09EM21-18 & Sum & 98.7605 & 99.2452 & 100.0000 & -1.2395 & -0.7548 & $-1.2 \%$ & $-0.8 \%$ \\
\hline 2 & FY09EM21-19 & $\overline{\mathrm{Al} 2 \mathrm{O} 3}$ & 5.8386 & 5.9921 & 5.7191 & 0.1194 & 0.2730 & $2.1 \%$ & $4.8 \%$ \\
\hline 2 & FY09EM21-19 & $\mathrm{B} 2 \mathrm{O} 3$ & 4.5803 & 4.5817 & 4.5009 & 0.0794 & 0.0808 & $1.8 \%$ & $1.8 \%$ \\
\hline 2 & FY09EM21-19 & $\mathrm{BaO}$ & 0.0056 & 0.0061 & 0.0000 & 0.0056 & 0.0061 & & \\
\hline 2 & FY09EM21-19 & $\mathrm{CaO}$ & 4.0647 & 4.3222 & 4.0000 & 0.0647 & 0.3222 & $1.6 \%$ & $8.1 \%$ \\
\hline 2 & FY09EM21-19 & $\mathrm{CdO}$ & 0.0057 & 0.0057 & 0.0000 & 0.0057 & 0.0057 & & \\
\hline 2 & FY09EM21-19 & Ce2O3 & 0.0059 & 0.0059 & 0.0000 & 0.0059 & 0.0059 & & \\
\hline 2 & FY09EM21-19 & Cr2O3 & 0.0183 & 0.0174 & 0.0000 & 0.0183 & 0.0174 & & \\
\hline 2 & FY09EM21-19 & $\mathrm{CuO}$ & 0.0063 & 0.0066 & 0.0000 & 0.0063 & 0.0066 & & \\
\hline 2 & FY09EM21-19 & $\mathrm{Fe} 2 \mathrm{O} 3$ & 4.9825 & 5.0161 & 5.0000 & -0.0175 & 0.0161 & $-0.3 \%$ & $0.3 \%$ \\
\hline 2 & FY09EM21-19 & La2O3 & 0.0059 & 0.0059 & 0.0000 & 0.0059 & 0.0059 & & \\
\hline 2 & FY09EM21-19 & Li2O & 3.9667 & 4.0199 & 4.0021 & -0.0354 & 0.0178 & $-0.9 \%$ & $0.4 \%$ \\
\hline 2 & FY09EM21-19 & $\mathrm{MgO}$ & 0.0083 & 0.0090 & 0.0000 & 0.0083 & 0.0090 & & \\
\hline 2 & FY09EM21-19 & $\mathrm{MnO}$ & 0.3034 & 0.3016 & 0.3000 & 0.0034 & 0.0016 & $1.1 \%$ & $0.5 \%$ \\
\hline 2 & FY09EM21-19 & $\mathrm{Na} 2 \mathrm{O}$ & 16.7152 & 16.7709 & 18.0000 & -1.2848 & -1.2291 & $-7.1 \%$ & $-6.8 \%$ \\
\hline 2 & FY09EM21-19 & $\mathrm{NiO}$ & 0.8958 & 1.0223 & 0.9809 & -0.0850 & 0.0415 & $-8.7 \%$ & $4.2 \%$ \\
\hline 2 & FY09EM21-19 & $\mathrm{PbO}$ & 0.0054 & 0.0054 & 0.0000 & 0.0054 & 0.0054 & & \\
\hline 2 & FY09EM21-19 & $\mathrm{SiO} 2$ & 51.4502 & 51.3132 & 51.4970 & -0.0468 & -0.1838 & $-0.1 \%$ & $-0.4 \%$ \\
\hline 2 & FY09EM21-19 & SO4 & 0.0749 & 0.0749 & 0.0000 & 0.0749 & 0.0749 & & \\
\hline 2 & FY09EM21-19 & $\mathrm{TiO} 2$ & 5.8505 & 6.1946 & 6.0000 & -0.1495 & 0.1946 & $-2.5 \%$ & $3.2 \%$ \\
\hline 2 & FY09EM21-19 & $\mathrm{ZnO}$ & 0.0062 & 0.0062 & 0.0000 & 0.0062 & 0.0062 & & \\
\hline 2 & FY09EM21-19 & $\mathrm{ZrO} 2$ & 0.0068 & 0.0068 & 0.0000 & 0.0068 & 0.0068 & & \\
\hline 2 & FY09EM21-19 & Sum & 98.7970 & 99.6845 & 100.0000 & -1.2030 & -0.3155 & $-1.2 \%$ & $-0.3 \%$ \\
\hline 2 & FY09EM21-20 & $\mathrm{Al} \mathrm{AlO3}$ & 6.1598 & 6.3215 & 6.0242 & 0.1356 & 0.2973 & $2.3 \%$ & $4.9 \%$ \\
\hline 2 & FY09EM21-20 & $\mathrm{B} 2 \mathrm{O} 3$ & 5.4094 & 5.3005 & 5.3114 & 0.0980 & -0.0109 & $1.8 \%$ & $-0.2 \%$ \\
\hline 2 & FY09EM21-20 & $\mathrm{BaO}$ & 0.0056 & 0.0061 & 0.0000 & 0.0056 & 0.0061 & & \\
\hline 2 & FY09EM21-20 & $\mathrm{CaO}$ & 0.0070 & 0.0074 & 0.0000 & 0.0070 & 0.0074 & & \\
\hline 2 & FY09EM21-20 & $\mathrm{CdO}$ & 0.0057 & 0.0057 & 0.0000 & 0.0057 & 0.0057 & & \\
\hline 2 & FY09EM21-20 & Ce2O3 & 0.0059 & 0.0059 & 0.0000 & 0.0059 & 0.0059 & & \\
\hline 2 & FY09EM21-20 & $\mathrm{Cr} 2 \mathrm{O} 3$ & 0.1593 & 0.1521 & 0.2000 & -0.0407 & -0.0479 & $-20.3 \%$ & $-23.9 \%$ \\
\hline 2 & FY09EM21-20 & $\mathrm{CuO}$ & 0.0063 & 0.0066 & 0.0000 & 0.0063 & 0.0066 & & \\
\hline 2 & FY09EM21-20 & $\mathrm{Fe} 2 \mathrm{O} 3$ & 11.3876 & 11.4649 & 11.8301 & -0.4426 & -0.3652 & $-3.7 \%$ & $-3.1 \%$ \\
\hline 2 & FY09EM21-20 & La2O3 & 0.0059 & 0.0059 & 0.0000 & 0.0059 & 0.0059 & & \\
\hline 2 & FY09EM21-20 & $\mathrm{Li2O}$ & 4.0152 & 3.9650 & 4.0000 & 0.0152 & -0.0350 & $0.4 \%$ & $-0.9 \%$ \\
\hline 2 & FY09EM21-20 & $\mathrm{MgO}$ & 1.1782 & 1.2790 & 1.2691 & -0.0908 & 0.0099 & $-7.2 \%$ & $0.8 \%$ \\
\hline 2 & FY09EM21-20 & $\mathrm{MnO}$ & 0.3002 & 0.2984 & 0.3000 & 0.0002 & -0.0016 & $0.1 \%$ & $-0.5 \%$ \\
\hline 2 & FY09EM21-20 & $\mathrm{Na} 2 \mathrm{O}$ & 16.9511 & 17.0066 & 18.0000 & -1.0489 & -0.9934 & $-5.8 \%$ & $-5.5 \%$ \\
\hline 2 & FY09EM21-20 & $\mathrm{NiO}$ & 2.3128 & 2.6398 & 2.5000 & -0.1872 & 0.1398 & $-7.5 \%$ & $5.6 \%$ \\
\hline 2 & FY09EM21-20 & $\mathrm{PbO}$ & 0.0054 & 0.0054 & 0.0000 & 0.0054 & 0.0054 & & \\
\hline 2 & FY09EM21-20 & $\mathrm{SiO} 2$ & 47.8668 & 47.3455 & 48.5652 & -0.6984 & -1.2197 & $-1.4 \%$ & $-2.5 \%$ \\
\hline 2 & FY09EM21-20 & SO4 & 0.0749 & 0.0749 & 0.0000 & 0.0749 & 0.0749 & & \\
\hline
\end{tabular}


SRNL-STI-2009-00778, Revision 0

Table A4. Average Measured and Bias-Corrected Chemical Compositions Versus Targeted Compositions by Oxide by Glass ID for the Non-Rad Matrix 2 Study

\begin{tabular}{|c|c|c|c|c|c|c|c|c|c|}
\hline & & & & Measured & & Difference & Difference & $\%$ & $\%$ \\
\hline & & & Measured & Bias-Corrected & Targeted & of & of & Difference & Difference \\
\hline Set & Glass \# & Oxide & (wt\%) & (BC) (wt\%) & (wt\%) & Measured & Meas BC & Measured & Meas BC \\
\hline 2 & FY09EM21-20 & $\mathrm{TiO} 2$ & 1.9766 & 2.0929 & 2.0000 & -0.0234 & 0.0929 & $-1.2 \%$ & $4.6 \%$ \\
\hline 2 & FY09EM21-20 & $\mathrm{ZnO}$ & 0.0062 & 0.0062 & 0.0000 & 0.0062 & 0.0062 & & \\
\hline 2 & FY09EM21-20 & $\mathrm{ZrO} 2$ & 0.0068 & 0.0068 & 0.0000 & 0.0068 & 0.0068 & & \\
\hline 2 & FY09EM21-20 & Sum & 97.8465 & 97.9970 & 99.9999 & -2.1534 & -2.0029 & $-2.2 \%$ & $-2.0 \%$ \\
\hline 2 & FY09EM21-21 & $\mathrm{Al} 2 \mathrm{O} 3$ & 4.8607 & 4.9622 & 4.8572 & 0.0035 & 0.1050 & $0.1 \%$ & $2.2 \%$ \\
\hline 2 & FY09EM21-21 & $\mathrm{B} 2 \mathrm{O} 3$ & 8.9513 & 8.9519 & 9.0097 & -0.0584 & -0.0578 & $-0.6 \%$ & $-0.6 \%$ \\
\hline 2 & FY09EM21-21 & $\mathrm{BaO}$ & 0.0801 & 0.0870 & 0.0801 & 0.0000 & 0.0069 & $0.0 \%$ & $8.6 \%$ \\
\hline 2 & FY09EM21-21 & $\mathrm{CaO}$ & 0.0070 & 0.0072 & 0.0000 & 0.0070 & 0.0072 & & \\
\hline 2 & FY09EM21-21 & $\mathrm{CdO}$ & 0.2839 & 0.2839 & 0.2965 & -0.0126 & -0.0126 & $-4.3 \%$ & $-4.3 \%$ \\
\hline 2 & FY09EM21-21 & $\mathrm{Ce} 2 \mathrm{O} 3$ & 0.3581 & 0.3581 & 0.3606 & -0.0025 & -0.0025 & $-0.7 \%$ & $-0.7 \%$ \\
\hline 2 & FY09EM21-21 & $\mathrm{Cr} 2 \mathrm{O} 3$ & 0.1904 & 0.1838 & 0.2000 & -0.0096 & $\begin{array}{l}-0.0162 \\
\end{array}$ & $-4.8 \%$ & $-8.1 \%$ \\
\hline 2 & FY09EM21-21 & $\mathrm{CuO}$ & 0.1274 & 0.1360 & 0.1282 & -0.0008 & 0.0078 & $-0.7 \%$ & $6.1 \%$ \\
\hline 2 & FY09EM21-21 & $\mathrm{Fe} 2 \mathrm{O} 3$ & 18.8363 & 19.4914 & 19.9511 & -1.1148 & -0.4597 & $-5.6 \%$ & $-2.3 \%$ \\
\hline 2 & FY09EM21-21 & $\mathrm{La} 2 \mathrm{O} 3$ & 0.0865 & 0.0865 & 0.0978 & -0.0113 & -0.0113 & $-11.5 \%$ & $-11.5 \%$ \\
\hline 2 & FY09EM21-21 & $\mathrm{Li2O}$ & 6.8839 & 6.9762 & 7.0000 & -0.1161 & -0.0238 & $-1.7 \%$ & $-0.3 \%$ \\
\hline 2 & FY09EM21-21 & $\mathrm{MgO}$ & 0.0083 & 0.0088 & 0.0000 & 0.0083 & 0.0088 & & \\
\hline 2 & FY09EM21-21 & $\mathrm{MnO}$ & 1.0097 & 1.0213 & 0.9970 & 0.0127 & 0.0243 & $1.3 \%$ & $2.4 \%$ \\
\hline 2 & FY09EM21-21 & $\mathrm{Na} 2 \mathrm{O}$ & 14.4236 & 14.4264 & 14.6666 & -0.2430 & -0.2403 & $-1.7 \%$ & $-1.6 \%$ \\
\hline 2 & FY09EM21-21 & $\mathrm{NiO}$ & 0.0064 & 0.0071 & 0.0000 & 0.0064 & 0.0071 & & \\
\hline 2 & FY09EM21-21 & $\mathrm{PbO}$ & 0.2109 & 0.2109 & 0.2163 & -0.0055 & -0.0055 & $-2.5 \%$ & $-2.5 \%$ \\
\hline 2 & FY09EM21-21 & $\mathrm{SiO} 2$ & 39.1492 & 39.0489 & 39.3055 & -0.1563 & $\begin{array}{l}-0.2566 \\
\end{array}$ & $-0.4 \%$ & $-0.7 \%$ \\
\hline 2 & FY09EM21-21 & $\mathrm{SO} 4$ & 0.4741 & 0.4741 & 0.4808 & -0.0067 & -0.0067 & $-1.4 \%$ & $-1.4 \%$ \\
\hline 2 & FY09EM21-21 & $\mathrm{TiO} 2$ & 1.9766 & 2.1059 & 2.0129 & -0.0363 & 0.0930 & $-1.8 \%$ & $4.6 \%$ \\
\hline 2 & FY09EM21-21 & $\mathrm{ZnO}$ & 0.1316 & 0.1316 & 0.1346 & -0.0030 & -0.0030 & $-2.2 \%$ & $-2.2 \%$ \\
\hline 2 & FY09EM21-21 & $\mathrm{ZrO} 2$ & 0.1976 & 0.1976 & 0.2051 & -0.0076 & -0.0076 & $-3.7 \%$ & $-3.7 \%$ \\
\hline 2 & FY09EM21-21 & Sum & 98.2535 & 99.1567 & 100.0000 & -1.7465 & -0.8433 & $-1.7 \%$ & $-0.8 \%$ \\
\hline 2 & "FY09EM21-22 & Al2O3 & 14.2610 & 14.5587 & 1414.0414 & 0.2196 & 0.5172 & $1.6 \%$ & $3.7 \%$ \\
\hline 2 & FY09EM21-22 & $\mathrm{B} 2 \mathrm{O} 3$ & 13.2901 & 13.0236 & 13.1992 & 0.0909 & -0.1756 & $0.7 \%$ & $-1.3 \%$ \\
\hline 2 & FY09EM21-22 & $\mathrm{BaO}$ & 0.0754 & 0.0818 & 0.0801 & -0.0048 & 0.0017 & $-5.9 \%$ & $2.1 \%$ \\
\hline 2 & FY09EM21-22 & $\mathrm{CaO}$ & 0.0070 & 0.0072 & 0.0000 & 0.0070 & 0.0072 & & \\
\hline 2 & FY09EM21-22 & $\mathrm{CdO}$ & 0.2659 & 0.2659 & 0.2965 & -0.0306 & -0.0306 & $-10.3 \%$ & $-10.3 \%$ \\
\hline 2 & FY09EM21-22 & Ce2O3 & 0.3482 & 0.3482 & 0.3606 & -0.0124 & -0.0124 & $-3.4 \%$ & $-3.4 \%$ \\
\hline 2 & FY09EM21-22 & Cr2O3 & 0.0132 & 0.0127 & 0.0000 & 0.0132 & 0.0127 & & \\
\hline 2 & FY09EM21-22 & $\mathrm{CuO}$ & 0.1252 & 0.1336 & 0.1282 & -0.0030 & 0.0054 & $-2.4 \%$ & $4.2 \%$ \\
\hline 2 & FY09EM21-22 & $\mathrm{Fe} 2 \mathrm{O} 3$ & 8.6211 & 8.9209 & 9.1773 & -0.5563 & -0.2564 & $-6.1 \%$ & $-2.8 \%$ \\
\hline 2 & FY09EM21-22 & $\mathrm{La} 2 \mathrm{O} 3$ & 0.0850 & 0.0850 & 0.0978 & -0.0127 & -0.0127 & $-13.0 \%$ & $-13.0 \%$ \\
\hline 2 & FY09EM21-22 & $\mathrm{Li} 2 \mathrm{O}$ & 4.0582 & 4.0073 & 4.0000 & 0.0582 & 0.0073 & $1.5 \%$ & $0.2 \%$ \\
\hline 2 & FY09EM21-22 & $\mathrm{MgO}$ & 0.0083 & 0.0088 & 0.0000 & 0.0083 & 0.0088 & & \\
\hline 2 & FY09EM21-22 & $\mathrm{MnO}$ & 0.3086 & 0.3122 & 0.3000 & 0.0086 & 0.0122 & $2.9 \%$ & $4.1 \%$ \\
\hline 2 & FY09EM21-22 & $\mathrm{Na} 2 \mathrm{O}$ & 10.0561 & 10.0586 & 10.0000 & 0.0561 & 0.0586 & $0.6 \%$ & $0.6 \%$ \\
\hline 2 & FY09EM21-22 & $\mathrm{NiO}$ & 2.2301 & 2.4944 & 2.5000 & -0.2699 & -0.0056 & $-10.8 \%$ & $-0.2 \%$ \\
\hline 2 & FY09EM21-22 & $\mathrm{PbO}$ & 0.1944 & 0.1944 & 0.2163 & -0.0219 & -0.0219 & $-10.1 \%$ & $-10.1 \%$ \\
\hline 2 & FY09EM21-22 & $\mathrm{SiO} 2$ & 43.6417 & 43.1665 & 42.7403 & 0.9014 & 0.4262 & $2.1 \%$ & $1.0 \%$ \\
\hline 2 & FY09EM21-22 & SO4 & 0.4059 & 0.4059 & 0.4808 & -0.0748 & -0.0748 & $-15.6 \%$ & $-15.6 \%$ \\
\hline 2 & FY09EM21-22 & $\mathrm{TiO} 2$ & 2.0016 & 2.1326 & 2.0417 & -0.0401 & 0.0908 & $-2.0 \%$ & $4.4 \%$ \\
\hline 2 & FY09EM21-22 & $\mathrm{ZnO}$ & 0.1391 & 0.1391 & 0.1346 & 0.0045 & 0.0045 & $3.3 \%$ & $3.3 \%$ \\
\hline 2 & FY09EM21-22 & $\mathrm{ZrO} 2$ & 0.1965 & 0.1965 & 0.2051 & -0.0086 & -0.0086 & $-4.2 \%$ & $-4.2 \%$ \\
\hline 2 & FY09EM21-22 & Sum & 100.3326 & 100.5540 & 100.0000 & 0.3326 & 0.5540 & $0.3 \%$ & $0.6 \%$ \\
\hline 2 & "FY09EM21-23 & $\mathrm{Al2O} 3$ & 13.9823 & 14.2742 & 13.9588 & 0.0235 & 0.3154 & $0.2 \%$ & $2.3 \%$ \\
\hline 2 & FY09EM21-23 & $\mathrm{B} 2 \mathrm{O} 3$ & 5.8119 & 5.8107 & 5.7805 & 0.0314 & 0.0302 & $0.5 \%$ & $0.5 \%$ \\
\hline 2 & FY09EM21-23 & $\mathrm{BaO}$ & 0.0762 & 0.0828 & 0.0801 & -0.0039 & 0.0027 & $-4.9 \%$ & $3.3 \%$ \\
\hline 2 & FY09EM21-23 & $\mathrm{CaO}$ & 0.0070 & 0.0072 & 0.0000 & 0.0070 & 0.0072 & & \\
\hline 2 & FY09EM21-23 & $\mathrm{CdO}$ & 0.2664 & 0.2664 & 0.2965 & -0.0300 & -0.0300 & $-10.1 \%$ & $-10.1 \%$ \\
\hline 2 & FY09EM21-23 & $\mathrm{Ce} 2 \mathrm{O} 3$ & 0.3490 & 0.3490 & 0.3606 & -0.0115 & -0.0115 & $-3.2 \%$ & $-3.2 \%$ \\
\hline 2 & FY09EM21-23 & Cr2O3 & 0.0121 & 0.0116 & 0.0000 & 0.0121 & 0.0116 & & \\
\hline 2 & FY09EM21-23 & $\mathrm{CuO}$ & 0.1221 & 0.1303 & 0.1282 & -0.0062 & 0.0021 & $-4.8 \%$ & $1.6 \%$ \\
\hline 2 & FY09EM21-23 & $\mathrm{Fe} 2 \mathrm{O} 3$ & 11.3590 & 11.7541 & 12.1089 & -0.7500 & -0.3549 & $-6.2 \%$ & $-2.9 \%$ \\
\hline 2 & FY09EM21-23 & La2O3 & 0.0859 & 0.0859 & 0.0978 & -0.0118 & -0.0118 & $-12.1 \%$ & $-12.1 \%$ \\
\hline 2 & FY09EM21-23 & Li2O & 3.8806 & 3.9326 & 4.0000 & -0.1194 & -0.0674 & $-3.0 \%$ & $-1.7 \%$ \\
\hline 2 & FY09EM21-23 & $\mathrm{MgO}$ & 1.4324 & 1.5189 & 1.5000 & -0.0676 & 0.0189 & $-4.5 \%$ & $1.3 \%$ \\
\hline
\end{tabular}


SRNL-STI-2009-00778, Revision 0

Table A4. Average Measured and Bias-Corrected Chemical Compositions Versus Targeted Compositions by Oxide by Glass ID for the Non-Rad Matrix 2 Study

\begin{tabular}{|c|c|c|c|c|c|c|c|c|c|}
\hline & & & & Measured & & Difference & Difference & $\%$ & $\%$ \\
\hline & & & Measured & Bias-Corrected & Targeted & of & of & Difference & Difference \\
\hline Set & Glass \# & Oxide & $(\mathrm{wt} \%)$ & $(\mathrm{BC})(\mathrm{wt} \%)$ & (wt\%) & Measured & Meas BC & Measured & Meas BC \\
\hline 2 & FY09EM21-23 & $\mathrm{MnO}$ & 0.3034 & 0.3069 & 0.3000 & 0.0034 & 0.0069 & $1.1 \%$ & $2.3 \%$ \\
\hline 2 & FY09EM21-23 & $\mathrm{Na} 2 \mathrm{O}$ & 9.8438 & 9.8456 & 10.0000 & -0.1562 & -0.1544 & $-1.6 \%$ & $-1.5 \%$ \\
\hline 2 & FY09EM21-23 & $\mathrm{NiO}$ & 0.0064 & 0.0071 & 0.0000 & 0.0064 & 0.0071 & & \\
\hline 2 & FY09EM21-23 & $\mathrm{PbO}$ & 0.1931 & 0.1931 & 0.2163 & -0.0233 & -0.0233 & $-10.8 \%$ & $-10.8 \%$ \\
\hline 2 & FY09EM21-23 & $\mathrm{SiO} 2$ & 44.0696 & 43.9483 & 44.3518 & -0.2822 & -0.4035 & $-0.6 \%$ & $-0.9 \%$ \\
\hline 2 & FY09EM21-23 & $\mathrm{SO} 4$ & 0.3767 & 0.3767 & 0.4808 & -0.1040 & -0.1040 & $-21.6 \%$ & $-21.6 \%$ \\
\hline 2 & FY09EM21-23 & $\mathrm{TiO} 2$ & 5.7046 & 6.0779 & 6.0000 & -0.2954 & 0.0779 & $-4.9 \%$ & $1.3 \%$ \\
\hline 2 & FY09EM21-23 & $\mathrm{ZnO}$ & 0.1285 & 0.1285 & 0.1346 & -0.0061 & -0.0061 & $-4.5 \%$ & $-4.5 \%$ \\
\hline 2 & FY09EM21-23 & $\mathrm{ZrO} 2$ & 0.1928 & 0.1928 & 0.2051 & -0.0123 & -0.0123 & $-6.0 \%$ & $-6.0 \%$ \\
\hline 2 & FY09EM21-23 & Sum & 98.2037 & 99.3008 & 100.0000 & -1.7963 & -0.6992 & $-1.8 \%$ & $-0.7 \%$ \\
\hline 2 & FY09EM21-24 & $\mathrm{Al} 2 \mathrm{O} 3$ & 10.5245 & 10.8009 & 10.5061 & 0.0184 & 0.2948 & $0.2 \%$ & $2.8 \%$ \\
\hline 2 & FY09EM21-24 & $\mathrm{B} 2 \mathrm{O} 3$ & 4.6206 & 4.6197 & 4.5000 & 0.1206 & 0.1197 & $2.7 \%$ & $2.7 \%$ \\
\hline 2 & FY09EM21-24 & $\mathrm{BaO}$ & 0.0765 & 0.0836 & 0.0801 & -0.0036 & 0.0035 & $-4.6 \%$ & $4.4 \%$ \\
\hline 2 & FY09EM21-24 & $\mathrm{CaO}$ & 4.0297 & 4.2853 & 4.0000 & 0.0297 & 0.2853 & $0.7 \%$ & $7.1 \%$ \\
\hline 2 & FY09EM21-24 & $\mathrm{CdO}$ & 0.2530 & 0.2530 & 0.2965 & -0.0435 & -0.0435 & $-14.7 \%$ & $-14.7 \%$ \\
\hline 2 & FY09EM21-24 & $\mathrm{Ce} 2 \mathrm{O} 3$ & 0.3414 & 0.3414 & 0.3606 & -0.0191 & -0.0191 & $-5.3 \%$ & $-5.3 \%$ \\
\hline 2 & FY09EM21-24 & $\mathrm{Cr} 2 \mathrm{O} 3$ & 0.1743 & 0.1664 & 0.2000 & -0.0257 & -0.0336 & $-12.9 \%$ & $-16.8 \%$ \\
\hline 2 & FY09EM21-24 & $\mathrm{CuO}$ & 0.1214 & 0.1282 & 0.1282 & -0.0068 & -0.0001 & $-5.3 \%$ & $0.0 \%$ \\
\hline 2 & FY09EM21-24 & $\mathrm{Fe} 2 \mathrm{O} 3$ & 19.0865 & 19.2155 & 20.5597 & -1.4732 & -1.3442 & $-7.2 \%$ & $-6.5 \%$ \\
\hline 2 & FY09EM21-24 & La2O3 & 0.0856 & 0.0856 & 0.0978 & -0.0121 & -0.0121 & $-12.4 \%$ & $-12.4 \%$ \\
\hline 2 & FY09EM21-24 & $\mathrm{Li} 2 \mathrm{O}$ & 3.9129 & 3.9653 & 4.0000 & -0.0871 & -0.0347 & $-2.2 \%$ & $-0.9 \%$ \\
\hline 2 & FY09EM21-24 & $\mathrm{MgO}$ & 1.3660 & 1.4828 & 1.5000 & -0.1340 & -0.0172 & $-8.9 \%$ & $-1.1 \%$ \\
\hline 2 & FY09EM21-24 & $\mathrm{MnO}$ & 0.8464 & 0.8413 & 0.9172 & -0.0708 & -0.0759 & $-7.7 \%$ & $-8.3 \%$ \\
\hline 2 & FY09EM21-24 & $\mathrm{Na} 2 \mathrm{O}$ & 9.9752 & 10.0082 & 10.0010 & -0.0258 & 0.0072 & $-0.3 \%$ & $0.1 \%$ \\
\hline 2 & FY09EM21-24 & $\mathrm{NiO}$ & 0.0064 & 0.0073 & 0.0000 & 0.0064 & 0.0073 & & \\
\hline 2 & FY09EM21-24 & $\mathrm{PbO}$ & 0.1893 & 0.1893 & 0.2163 & -0.0270 & -0.0270 & $-12.5 \%$ & $-12.5 \%$ \\
\hline 2 & FY09EM21-24 & $\mathrm{SiO} 2$ & 38.4004 & 38.2992 & 37.8796 & 0.5208 & 0.4196 & $1.4 \%$ & $1.1 \%$ \\
\hline 2 & FY09EM21-24 & $\mathrm{SO} 4$ & 0.3962 & 0.3962 & 0.4808 & -0.0846 & -0.0846 & $-17.6 \%$ & $-17.6 \%$ \\
\hline 2 & FY09EM21-24 & $\mathrm{TiO} 2$ & 3.5403 & 3.7486 & 3.9364 & -0.3961 & -0.1878 & $-10.1 \%$ & $-4.8 \%$ \\
\hline 2 & FY09EM21-24 & $\mathrm{ZnO}$ & 0.1263 & 0.1263 & 0.1346 & -0.0083 & -0.0083 & $-6.1 \%$ & $-6.1 \%$ \\
\hline 2 & FY09EM21-24 & $\mathrm{ZrO} 2$ & 0.1911 & 0.1911 & 0.2051 & -0.0140 & -0.0140 & $-6.8 \%$ & $-6.8 \%$ \\
\hline 2 & FY09EM21-24 & Sum & 98.2642 & 99.2353 & 100.0000 & -1.7358 & -0.7647 & $-1.7 \%$ & $-0.8 \%$ \\
\hline 2 & FY09EM21-25 & $\mathrm{Al} 2 \mathrm{O} 3$ & 6.4101 & 6.5440 & 6.2430 & 0.1671 & 0.3009 & $2.7 \%$ & $4.8 \%$ \\
\hline 2 & FY09EM21-25 & $\mathrm{B} 2 \mathrm{O} 3$ & 4.9828 & 4.8814 & 4.7295 & 0.2533 & 0.1519 & $5.4 \%$ & $3.2 \%$ \\
\hline 2 & FY09EM21-25 & $\mathrm{BaO}$ & 0.0056 & 0.0061 & 0.0000 & 0.0056 & 0.0061 & & \\
\hline 2 & FY09EM21-25 & $\mathrm{CaO}$ & 4.1346 & 4.2857 & 4.0000 & 0.1346 & 0.2857 & $3.4 \%$ & $7.1 \%$ \\
\hline 2 & FY09EM21-25 & $\mathrm{CdO}$ & 0.0057 & 0.0057 & 0.0000 & 0.0057 & 0.0057 & & \\
\hline 2 & FY09EM21-25 & $\mathrm{Ce} 2 \mathrm{O} 3$ & 0.0059 & 0.0059 & 0.0000 & 0.0059 & 0.0059 & & \\
\hline 2 & FY09EM21-25 & Cr2O3 & 0.1644 & 0.1587 & 0.2000 & -0.0356 & -0.0413 & $-17.8 \%$ & $-20.6 \%$ \\
\hline 2 & FY09EM21-25 & $\mathrm{CuO}$ & 0.0125 & 0.0133 & 0.0000 & 0.0125 & 0.0133 & & \\
\hline 2 & FY09EM21-25 & $\mathrm{Fe} 2 \mathrm{O} 3$ & 7.5274 & 7.7892 & 7.8302 & -0.3028 & -0.0410 & $-3.9 \%$ & $-0.5 \%$ \\
\hline 2 & FY09EM21-25 & $\mathrm{La} 2 \mathrm{O} 3$ & 0.0059 & 0.0059 & 0.0000 & 0.0059 & 0.0059 & & \\
\hline 2 & FY09EM21-25 & $\mathrm{Li} 2 \mathrm{O}$ & 4.0098 & 3.9596 & 4.0000 & 0.0098 & -0.0404 & $0.2 \%$ & $-1.0 \%$ \\
\hline 2 & FY09EM21-25 & $\mathrm{MgO}$ & 0.0083 & 0.0088 & 0.0000 & 0.0083 & 0.0088 & & \\
\hline 2 & FY09EM21-25 & $\mathrm{MnO}$ & 5.6458 & 5.7102 & 5.5000 & 0.1458 & 0.2102 & $2.7 \%$ & $3.8 \%$ \\
\hline 2 & FY09EM21-25 & $\mathrm{Na} 2 \mathrm{O}$ & 15.4009 & 15.4037 & 15.4638 & -0.0629 & -0.0601 & $-0.4 \%$ & $-0.4 \%$ \\
\hline 2 & FY09EM21-25 & $\mathrm{NiO}$ & 0.0064 & 0.0071 & 0.0000 & 0.0064 & 0.0071 & & \\
\hline 2 & FY09EM21-25 & $\mathrm{PbO}$ & 0.0054 & 0.0054 & 0.0000 & 0.0054 & 0.0054 & & \\
\hline 2 & FY09EM21-25 & $\mathrm{SiO} 2$ & 48.8295 & 48.2955 & 50.0335 & -1.2040 & -1.7380 & $-2.4 \%$ & $-3.5 \%$ \\
\hline 2 & FY09EM21-25 & $\mathrm{SO} 4$ & 0.0749 & 0.0749 & 0.0000 & 0.0749 & 0.0749 & & \\
\hline 2 & FY09EM21-25 & $\mathrm{TiO} 2$ & 2.0058 & 2.1370 & 2.0000 & 0.0058 & 0.1370 & $0.3 \%$ & $6.9 \%$ \\
\hline 2 & FY09EM21-25 & $\mathrm{ZnO}$ & 0.0062 & 0.0062 & 0.0000 & 0.0062 & 0.0062 & & \\
\hline 2 & FY09EM21-25 & $\mathrm{ZrO} 2$ & 0.0128 & 0.0128 & 0.0000 & 0.0128 & 0.0128 & & \\
\hline 2 & FY09EM21-25 & Sum & 99.2606 & 99.3172 & 100.0000 & -0.7394 & -0.6828 & $-0.7 \%$ & $-0.7 \%$ \\
\hline 2 & FY09EM21-26 & $\mathrm{Al} 2 \mathrm{O} 3$ & 13.7319 & 14.0186 & 13.6557 & 0.0762 & 0.3628 & $0.6 \%$ & $2.7 \%$ \\
\hline 2 & FY09EM21-26 & $\mathrm{B} 2 \mathrm{O} 3$ & 7.5346 & 7.3826 & 7.5763 & -0.0417 & -0.1937 & $-0.6 \%$ & $-2.6 \%$ \\
\hline 2 & FY09EM21-26 & $\mathrm{BaO}$ & 0.0056 & 0.0061 & 0.0000 & 0.0056 & 0.0061 & & \\
\hline 2 & FY09EM21-26 & $\mathrm{CaO}$ & 0.0073 & 0.0076 & 0.0000 & 0.0073 & 0.0076 & & \\
\hline 2 & FY09EM21-26 & $\mathrm{CdO}$ & 0.0057 & 0.0057 & 0.0000 & 0.0057 & 0.0057 & & \\
\hline 2 & FY09EM21-26 & $\mathrm{Ce} 2 \mathrm{O} 3$ & 0.0059 & 0.0059 & 0.0000 & 0.0059 & 0.0059 & & \\
\hline
\end{tabular}


SRNL-STI-2009-00778, Revision 0

Table A4. Average Measured and Bias-Corrected Chemical Compositions Versus Targeted Compositions by Oxide by Glass ID for the Non-Rad Matrix 2 Study

\begin{tabular}{|c|c|c|c|c|c|c|c|c|c|}
\hline & & & & Measured & & Difference & Difference & $\%$ & $\%$ \\
\hline & & & Measured & Bias-Corrected & Targeted & of & of & Difference & Difference \\
\hline Set & Glass \# & Oxide & (wt\%) & (BC) (wt\%) & (wt\%) & Measured & Meas BC & Measured & Meas BC \\
\hline 2 & FY09EM21-26 & Cr2O3 & 0.1352 & 0.1305 & 0.2000 & -0.0648 & -0.0695 & $-32.4 \%$ & $-34.7 \%$ \\
\hline 2 & FY09EM21-26 & $\mathrm{CuO}$ & 0.0063 & 0.0067 & 0.0000 & 0.0063 & 0.0067 & & \\
\hline 2 & FY09EM21-26 & $\mathrm{Fe} 2 \mathrm{O} 3$ & 4.7895 & 4.9561 & 5.0000 & -0.2105 & -0.0439 & $-4.2 \%$ & $-0.9 \%$ \\
\hline 2 & FY09EM21-26 & $\mathrm{La} 2 \mathrm{O} 3$ & 0.0059 & 0.0059 & 0.0000 & 0.0059 & 0.0059 & & \\
\hline 2 & FY09EM21-26 & $\mathrm{Li} 2 \mathrm{O}$ & 4.0905 & 4.0393 & 4.0010 & 0.0895 & 0.0383 & $2.2 \%$ & $1.0 \%$ \\
\hline 2 & FY09EM21-26 & $\mathrm{MgO}$ & 1.4593 & 1.5474 & 1.5000 & -0.0407 & 0.0474 & $-2.7 \%$ & $3.2 \%$ \\
\hline 2 & FY09EM21-26 & $\mathrm{MnO}$ & 5.1842 & 5.2433 & 5.0509 & 0.1333 & 0.1924 & $2.6 \%$ & $3.8 \%$ \\
\hline 2 & FY09EM21-26 & $\mathrm{Na} 2 \mathrm{O}$ & 11.9264 & 11.9294 & 11.9564 & -0.0299 & -0.0270 & $-0.3 \%$ & $-0.2 \%$ \\
\hline 2 & FY09EM21-26 & $\mathrm{NiO}$ & 0.0064 & 0.0071 & 0.0000 & 0.0064 & 0.0071 & & \\
\hline 2 & FY09EM21-26 & $\mathrm{PbO}$ & 0.0054 & 0.0054 & 0.0000 & 0.0054 & 0.0054 & & \\
\hline 2 & FY09EM21-26 & $\mathrm{SiO} 2$ & 45.4066 & 44.9119 & 45.7031 & -0.2965 & -0.7912 & $-0.6 \%$ & $-1.7 \%$ \\
\hline 2 & FY09EM21-26 & SO4 & 0.0749 & 0.0749 & 0.0000 & 0.0749 & 0.0749 & & \\
\hline 2 & FY09EM21-26 & TiO2 & 4.8581 & 5.1759 & 5.3566 & -0.4985 & -0.1806 & $-9.3 \%$ & $-3.4 \%$ \\
\hline 2 & FY09EM21-26 & $\mathrm{ZnO}$ & 0.0062 & 0.0062 & 0.0000 & 0.0062 & 0.0062 & & \\
\hline 2 & FY09EM21-26 & $\mathrm{ZrO} 2$ & 0.0068 & 0.0068 & 0.0000 & 0.0068 & 0.0068 & & \\
\hline 2 & FY09EM21-26 & Sum & 99.2525 & 99.4732 & 100.0000 & -0.7474 & -0.5268 & $-0.7 \%$ & $-0.5 \%$ \\
\hline 2 & FY09EM21-27 & $\mathrm{Al} 2 \mathrm{O} 3$ & 7.2887 & 7.4801 & 7.1923 & 0.0965 & 0.2878 & $1.3 \%$ & $4.0 \%$ \\
\hline 2 & FY09EM21-27 & $\mathrm{B} 2 \mathrm{O} 3$ & 6.8664 & 6.8674 & 6.9784 & -0.1120 & -0.1110 & $-1.6 \%$ & $-1.6 \%$ \\
\hline 2 & FY09EM21-27 & $\mathrm{BaO}$ & 0.0399 & 0.0437 & 0.0431 & -0.0032 & 0.0005 & $-7.5 \%$ & $1.2 \%$ \\
\hline 2 & FY09EM21-27 & $\mathrm{CaO}$ & 1.7945 & 1.9083 & 1.8080 & -0.0135 & 0.1003 & $-0.7 \%$ & $5.5 \%$ \\
\hline 2 & FY09EM21-27 & $\mathrm{CdO}$ & 0.1351 & 0.1351 & 0.1596 & -0.0246 & -0.0246 & $-15.4 \%$ & $-15.4 \%$ \\
\hline 2 & FY09EM21-27 & $\mathrm{Ce} 2 \mathrm{O} 3$ & 0.1845 & 0.1845 & 0.1942 & -0.0097 & -0.0097 & $-5.0 \%$ & $-5.0 \%$ \\
\hline 2 & FY09EM21-27 & Cr2O3 & 0.0917 & 0.0876 & 0.1000 & -0.0083 & -0.0124 & $-8.3 \%$ & $-12.4 \%$ \\
\hline 2 & FY09EM21-27 & $\mathrm{CuO}$ & 0.0660 & 0.0697 & 0.0690 & -0.0030 & 0.0007 & $-4.3 \%$ & $1.0 \%$ \\
\hline 2 & FY09EM21-27 & $\mathrm{Fe} 2 \mathrm{O} 3$ & 12.6850 & 12.7713 & 13.1246 & -0.4395 & -0.3533 & $-3.3 \%$ & $-2.7 \%$ \\
\hline 2 & FY09EM21-27 & $\mathrm{La} 2 \mathrm{O} 3$ & 0.0469 & 0.0469 & 0.0526 & -0.0057 & -0.0057 & $-10.9 \%$ & $-10.9 \%$ \\
\hline 2 & FY09EM21-27 & $\mathrm{Li} 2 \mathrm{O}$ & 4.9463 & 5.0126 & 5.1023 & -0.1560 & -0.0897 & $-3.1 \%$ & $-1.8 \%$ \\
\hline 2 & FY09EM21-27 & $\mathrm{MgO}$ & 0.6716 & 0.7291 & 0.6834 & -0.0118 & 0.0457 & $-1.7 \%$ & $6.7 \%$ \\
\hline 2 & FY09EM21-27 & $\mathrm{MnO}$ & 2.6986 & 2.6822 & 2.6230 & 0.0757 & 0.0593 & $2.9 \%$ & $2.3 \%$ \\
\hline 2 & FY09EM21-27 & $\mathrm{Na} 2 \mathrm{O}$ & 12.6308 & 12.6721 & 12.7162 & -0.0855 & -0.0441 & $-0.7 \%$ & $-0.3 \%$ \\
\hline 2 & FY09EM21-27 & $\mathrm{NiO}$ & 1.1252 & 1.2844 & 1.2913 & -0.1661 & -0.0069 & $-12.9 \%$ & $-0.5 \%$ \\
\hline 2 & FY09EM21-27 & $\mathrm{PbO}$ & 0.1039 & 0.1039 & 0.1165 & -0.0125 & -0.0125 & $-10.8 \%$ & $-10.8 \%$ \\
\hline 2 & FY09EM21-27 & $\mathrm{SiO} 2$ & 43.0534 & 42.9461 & 43.3778 & -0.3244 & -0.4317 & $-0.7 \%$ & $-1.0 \%$ \\
\hline 2 & FY09EM21-27 & $\mathrm{SO} 4$ & 0.2472 & 0.2472 & 0.2589 & -0.0117 & -0.0117 & $-4.5 \%$ & $-4.5 \%$ \\
\hline 2 & FY09EM21-27 & $\mathrm{TiO} 2$ & 3.8906 & 4.1195 & 3.9259 & -0.0353 & 0.1935 & $-0.9 \%$ & $4.9 \%$ \\
\hline 2 & FY09EM21-27 & $\mathrm{ZnO}$ & 0.0678 & 0.0678 & 0.0725 & -0.0046 & -0.0046 & $-6.4 \%$ & $-6.4 \%$ \\
\hline 2 & FY09EM21-27 & $\mathrm{ZrO} 2$ & 0.1087 & 0.1087 & 0.1105 & -0.0017 & -0.0017 & $-1.6 \%$ & $-1.6 \%$ \\
\hline 2 & FY09EM21-27 & Sum & 98.7430 & 99.5681 & 100.0000 & -1.2570 & -0.4319 & $-1.3 \%$ & $-0.4 \%$ \\
\hline
\end{tabular}


SRNL-STI-2009-00778, Revision 0

Table A5. Measured, Measured Bias-Corrected (BC), and Targeted Chemical Compositions for the Samples of the Rad Glasses of the Matrix 2 Study

\begin{tabular}{|c|c|c|c|c|c|c|c|}
\hline Set & Prep Method & Glass ID & Lab ID & Analyte & Measured & Measured bc & Targeted \\
\hline 2 & LM & FY09EM21-28 & b04LM11 & $\mathrm{BaO}$ (wt\%) & 0.0391 & 0.0401 & 0.0423 \\
\hline 2 & LM & FY09EM21-28 & b04LM12 & $\mathrm{BaO}$ (wt\%) & 0.0391 & 0.0399 & 0.0423 \\
\hline 2 & LM & FY09EM21-28 & b04LM21 & $\mathrm{BaO}$ (wt\%) & 0.0435 & 0.0447 & 0.0423 \\
\hline 2 & LM & FY09EM21-28 & b04LM22 & $\mathrm{BaO}$ (wt\%) & 0.0424 & 0.0434 & 0.0423 \\
\hline 2 & LM & FY09EM21-28 & b04LM11 & $\mathrm{CaO}(\mathrm{wt} \%)$ & 1.6231 & 1.6348 & 1.7718 \\
\hline 2 & LM & FY09EM21-28 & b04LM12 & $\mathrm{CaO}(\mathrm{wt} \%)$ & 1.6231 & 1.6380 & 1.7718 \\
\hline 2 & LM & FY09EM21-28 & b04LM21 & $\mathrm{CaO}(\mathrm{wt} \%)$ & 1.8050 & 1.8180 & 1.7718 \\
\hline 2 & LM & FY09EM21-28 & b04LM22 & $\mathrm{CaO}(\mathrm{wt} \%)$ & 1.8050 & 1.8215 & 1.7718 \\
\hline 2 & LM & FY09EM21-28 & b04LM11 & $\mathrm{CdO}(\mathrm{wt} \%)$ & 0.1257 & 0.1257 & 0.1564 \\
\hline 2 & LM & FY09EM21-28 & b04LM12 & CdO (wt\%) & 0.1211 & 0.1211 & 0.1564 \\
\hline 2 & LM & FY09EM21-28 & b04LM21 & $\mathrm{CdO}(\mathrm{wt} \%)$ & 0.1382 & 0.1382 & 0.1564 \\
\hline 2 & LM & FY09EM21-28 & b04LM22 & $\mathrm{CdO}(\mathrm{wt} \%)$ & 0.1348 & 0.1348 & 0.1564 \\
\hline 2 & LM & FY09EM21-28 & b04LM11 & Ce2O3 (wt\%) & 0.1722 & 0.1722 & 0.1903 \\
\hline 2 & LM & FY09EM21-28 & b04LM12 & Ce2O3 (wt\%) & 0.1652 & 0.1652 & 0.1903 \\
\hline 2 & LM & FY09EM21-28 & b04LM21 & Ce2O3 (wt\%) & 0.1839 & 0.1839 & 0.1903 \\
\hline 2 & LM & FY09EM21-28 & b04LM22 & Ce2O3 (wt\%) & 0.1816 & 0.1816 & 0.1903 \\
\hline 2 & LM & FY09EM21-28 & b04LM11 & Cr2O3 (wt\%) & 0.0935 & 0.0942 & 0.0980 \\
\hline 2 & LM & FY09EM21-28 & b04LM12 & Cr2O3 (wt\%) & 0.0950 & 0.0927 & 0.0980 \\
\hline 2 & LM & FY09EM21-28 & b04LM21 & $\mathrm{Cr} 2 \mathrm{O}$ & 0.0994 & 0.1001 & 0.0980 \\
\hline 2 & LM & FY09EM21-28 & b04LM22 & (wt\%) & 38 & & 980 \\
\hline 2 & LM & FY09EM21-28 & b04LM11 & $\mathrm{CuO}(\mathrm{wt} \%)$ & 0.0688 & 0.0693 & 0.0677 \\
\hline 2 & LM & FY09EM21-28 & b04LM12 & $\mathrm{CuO}$ (wt\%) & 0.0776 & 0.0793 & 0.0677 \\
\hline 2 & LM & FY09EM21-28 & b04LM21 & $\mathrm{CuO}(\mathrm{wt} \%)$ & 0.0814 & & 0.0677 \\
\hline 2 & LM & FY09EM21-28 & b04LM22 & $\mathrm{CuO}(\mathrm{wt} \%)$ & 0.0876 & 95 & 0.0677 \\
\hline 2 & LM & FY09EM21-28 & b04LM11 & K2O (wt\%) & 0.0301 & 0.0309 & 0.0000 \\
\hline 2 & LM & FY09EM21-28 & b04LM12 & K2O (wt\%) & 0.0687 & & 000 \\
\hline 2 & LM & FY09EM21-28 & b04LM21 & $v t \%)$ & 301 & & 000 \\
\hline 2 & LM & FY09EM21-28 & b04LM22 & K2O (wt\%) & 0.0301 & 0.0312 & 0.0000 \\
\hline 2 & LM & FY09EM21-28 & b04LM11 & La2O3 (wt\%) & 0.0387 & 0.0387 & 0.0516 \\
\hline 2 & LM & FY09EM21-28 & b04LM12 & La2O3 (wt\%) & 0.0387 & 387 & 0.0516 \\
\hline 2 & LM & FY09EM21-28 & b04LM21 & La2O3 (wt\%) & 410 & 110 & 0.0516 \\
\hline 2 & LM & FY09EM21-28 & b04LM22 & La2O3 (wt\%) & 0.0422 & 0.0422 & 0.0516 \\
\hline 2 & LM & FY09EM21-28 & b04LM11 & $\mathrm{MgO}(\mathrm{wt} \%)$ & 202 & & 698 \\
\hline 2 & LM & \begin{tabular}{|l|} 
FY09EM21-28 \\
\end{tabular} & b04LM12 & $\mathrm{MgO}$ & 119 & 172 & 698 \\
\hline 2 & LM & FY09EM21-28 & b04LM21 & $\mathrm{MgO}(\mathrm{wt} \%)$ & 0.6832 & & 0.6698 \\
\hline 2 & LM & FY09EM21-28 & b04LM22 & $\mathrm{MgO}$ (wt\%) & 0.6816 & 0.6875 & 0.6698 \\
\hline 2 & LM & FY09EM21-28 & b04LM11 & $\mathrm{MnO}$ (wt\%) & 2.2209 & 2.3133 & 2.5705 \\
\hline 2 & LM & FY09EM21-28 & b04LM12 & $\mathrm{MnO}$ (wt\%) & 2.2338 & 2.2969 & 2.5705 \\
\hline 2 & LM & FY09EM21-28 & b04LM21 & $\mathrm{MnO}(\mathrm{wt} \%)$ & & 2.5688 & 2.5705 \\
\hline 2 & LM & FY09EM21-28 & b04LM22 & $\mathrm{MnO}$ (wt\%) & & & 2.5705 \\
\hline 2 & LM & FY09EM21-28 & b04LM11 & Na2O (wt\%) & 11.8759 & 622 & 12.4619 \\
\hline 2 & LM & FY09EM21-28 & b04LM12 & $\mathrm{Na} 2 \mathrm{O}(\mathrm{wt} \%)$ & 11.2154 & 4592 & 12.4619 \\
\hline 2 & LM & FY09EM21-28 & b04LM21 & Na2O (wt\%) & 12.9947 & 12.6514 & 12.4619 \\
\hline 2 & LM & FY09EM21-28 & b04LM22 & $\mathrm{Na} 2 \mathrm{O}$ (wt\%) & 12.3612 & 12.6299 & 12.4619 \\
\hline 2 & LM & FY09EM21-28 & b04LM11 & $\mathrm{NiO}(\mathrm{wt} \%)$ & 1.0651 & 1.0471 & 1.2654 \\
\hline 2 & LM & FY09EM21-28 & b04LM12 & $\mathrm{NiO}(\mathrm{wt} \%)$ & 320 & 101 & 1.2654 \\
\hline 2 & LM & FY09EM21-28 & b04LM21 & & & 96 & 1.2654 \\
\hline 2 & LM & FY09EM21-28 & b04LM22 & $\mathrm{NiO}(\mathrm{wt} \%)$ & 567 & 321 & 1.2654 \\
\hline 2 & LM & FY09EM21-28 & b04LM11 & $\mathrm{PbO}(\mathrm{wt} \%)$ & 0.1002 & 0.1002 & 0.1142 \\
\hline 2 & LM & FY09EM21-28 & b04LM12 & PbO (wt\%) & 969 & 0.0969 & 0.1142 \\
\hline 2 & LM & FY09EM21-28 & b04LM21 & $\mathrm{PbO}$ (wt\%) & 0.1056 & 0.1056 & 0.1142 \\
\hline 2 & LM & FY09EM21-28 & b04LM22 & $\mathrm{PbO}$ (wt\%) & 0.1088 & 0.1088 & 0.1142 \\
\hline 2 & LM & FY09EM21-28 & b04LM11 & $\mathrm{SiO} 2$ (wt\%) & 38.7213 & 39.1803 & 42.5103 \\
\hline 2 & LM & FY09EM21-28 & b04LM12 & $w t \%)$ & 37.8656 & 359 & 103 \\
\hline 2 & LM & FY09EM21-28 & b04LM21 & SiO2 (wt\%) & 42.7860 & 43.2931 & 42.5103 \\
\hline 2 & LM & FY09EM21-28 & b04LM22 & $\mathrm{SiO} 2(\mathrm{wt} \%)$ & 42.1442 & 42.8902 & 42.5103 \\
\hline 2 & LM & FY09EM21-28 & b04LM11 & SO4 (wt\%) & 0.2247 & 0.2247 & 0.2537 \\
\hline 2 & LM & FY09EM21-28 & b04LM12 & SO4 (wt\%) & 0.2247 & 0.2247 & 0.2537 \\
\hline 2 & LM & FY09EM21-28 & b04LM21 & SO4 (wt\%) & 0.2247 & 0.2247 & 0.2537 \\
\hline 2 & LM & FY09EM21-28 & b04LM22 & SO4 (wt\%) & 0.2247 & 0.2247 & 0.2537 \\
\hline 2 & LM & FY09EM21-28 & b04LM11 & $\mathrm{TiO} 2$ (wt\%) & & & 3.8474 \\
\hline 2 & LM & FY09EM21-28 & b04LM12 & TiO2 (wt\%) & 3.2359 & 3.4143 & 3.8474 \\
\hline 2 & LM & FY09EM21-28 & b04LM21 & $\mathrm{TiO} 2$ (wt\%) & 3.5695 & 3.7827 & 3.8474 \\
\hline 2 & LM & FY09EM21-28 & b04LM22 & $\mathrm{TiO} 2$ (wt\%) & 3.5862 & 3.7839 & 3.8474 \\
\hline 2 & LM & FY09EM21-28 & b04LM11 & U3O8 (wt\%) & 1.8631 & 1.8727 & 2.0000 \\
\hline 2 & LM & FY09EM21-28 & b04LM12 & U3O8 (wt\%) & 1.9811 & 1.9248 & 2.0000 \\
\hline 2 & LM & FY09EM21-28 & b04LM21 & U3O8 (wt\%) & 2.2051 & 2.2164 & 2.0000 \\
\hline 2 & LM & FY09EM21-28 & b04LM22 & U3O8 (wt\%) & 2.2051 & 2.1425 & 2.0000 \\
\hline 2 & LM & \begin{tabular}{|l|} 
FY09EM21-28 \\
\end{tabular} & b04LM11 & $\mathrm{ZnO}$ (wt\%) & 0.0747 & 0.0747 & 0.0710 \\
\hline 2 & LM & FY09EM21-28 & b04LM12 & $\mathrm{ZnO}$ (wt\%) & 0.0759 & 0.0759 & 0.0710 \\
\hline 2 & LM & FY09EM21-28 & b04LM21 & $\mathrm{ZnO}$ (wt\%) & 0.0809 & 0.0809 & 0.0710 \\
\hline
\end{tabular}


SRNL-STI-2009-00778, Revision 0

Table A5. Measured, Measured Bias-Corrected (BC), and Targeted Chemical Compositions for the Samples of the Rad Glasses of the Matrix 2 Study

\begin{tabular}{|c|c|c|c|c|c|c|c|}
\hline Set & Prep Method & Glass ID & Lab ID & Analyte & Measured & Measured bc & Targeted \\
\hline 2 & LM & FY09EM21-28 & b04LM22 & $\mathrm{ZnO}$ (wt\%) & 0.0846 & 0.0846 & 0.0710 \\
\hline 2 & LM & FY09EM21-28 & b04LM11 & $\mathrm{ZrO} 2$ (wt\%) & 0.0946 & 0.0946 & 0.1082 \\
\hline 2 & LM & FY09EM21-28 & b04LM12 & $\mathrm{ZrO} 2$ (wt\%) & 0.0905 & 0.0905 & 0.1082 \\
\hline 2 & LM & FY09EM21-28 & b04LM21 & $\mathrm{ZrO} 2$ (wt\%) & 0.1040 & 0.1040 & 0.1082 \\
\hline 2 & LM & FY09EM21-28 & b04LM22 & ZrO2 (wt\%) & 0.1054 & 0.1054 & 0.1082 \\
\hline 2 & PF & FY09EM21-28 & b04PF11 & $\mathrm{Al} 2 \mathrm{O} 3(\mathrm{wt} \%)$ & 7.2179 & 7.5732 & 7.0484 \\
\hline 2 & $\mathrm{PF}$ & FY09EM21-28 & b04PF12 & Al2O3 (wt\%) & 7.1423 & 7.2962 & 7.0484 \\
\hline 2 & $\mathrm{PF}$ & FY09EM21-28 & b04PF21 & $\mathrm{Al} 2 \mathrm{O} 3$ (wt\%) & 7.0856 & 7.4345 & 7.0484 \\
\hline 2 & $\mathrm{PF}$ & FY09EM21-28 & b04PF22 & $\mathrm{Al} 2 \mathrm{O} 3$ (wt\%) & 7.2368 & 7.3927 & 7.0484 \\
\hline 2 & $\mathrm{PF}$ & FY09EM21-28 & b04PF11 & B2O3 (wt\%) & 6.7940 & 7.2501 & 6.8388 \\
\hline 2 & $\mathrm{PF}$ & FY09EM21-28 & b04PF12 & B2O3 (wt\%) & 6.7296 & 7.1185 & 6.8388 \\
\hline 2 & $\mathrm{PF}$ & FY09EM21-28 & b04PF21 & B2O3 (wt\%) & 6.6652 & 7.1127 & 6.8388 \\
\hline 2 & PF & FY09EM21-28 & b04PF22 & B2O3 (wt\%) & 6.7940 & 7.1866 & 6.8388 \\
\hline 2 & PF & FY09EM21-28 & b04PF11 & Fe2O3 (wt\%) & 12.6243 & 13.0159 & 12.8621 \\
\hline 2 & $\mathrm{PF}$ & FY09EM21-28 & b04PF12 & Fe2O3 (wt\%) & 12.3526 & 12.6343 & 12.8621 \\
\hline 2 & $\mathrm{PF}$ & FY09EM21-28 & b04PF21 & Fe2O3 (wt $\%)$ & 12.4098 & 12.7948 & 12.8621 \\
\hline 2 & $\mathrm{PF}$ & FY09EM21-28 & b04PF22 & Fe2O3 (wt\%) & 12.4813 & 12.7659 & 12.8621 \\
\hline 2 & $\mathrm{PF}$ & FY09EM21-28 & b04PF11 & Li2O (wt\%) & 4.7149 & 5.0961 & 5.0003 \\
\hline 2 & $\mathrm{PF}$ & FY09EM21-28 & b04PF12 & Li2O (wt\%) & 579 & & 003 \\
\hline 2 & $\mathrm{PF}$ & FY09EM21-28 & b04PF21 & $\mathrm{vt} \%)$ & 4.7579 & 126 & 003 \\
\hline 2 & $\mathrm{PF}$ & FY09EM21-28 & b04PF22 & Li2O (wt\%) & 4.8225 & 5.0190 & 5.0003 \\
\hline 2 & LM & FY09EM21-29 & b15LM11 & $\mathrm{BaO}(\mathrm{wt} \%)$ & 0.0447 & 0.0459 & 0.0414 \\
\hline 2 & LM & FY09EM21-29 & b15LM12 & $\mathrm{BaO}$ (wt\%) & 0.0447 & 156 & 414 \\
\hline 2 & LM & \begin{tabular}{|l|} 
FY09EM21-29 \\
\end{tabular} & b15LM21 & $\mathrm{BaO}$ (wt\%) & 0.0435 & 147 & 414 \\
\hline 2 & LM & FY09EM21-29 & b15LM22 & $\mathrm{BaO}$ (wt\%) & 0.0447 & 0.0456 & 0.0414 \\
\hline 2 & LM & FY09EM & b15L & $\mathrm{CaO}$ & & & 357 \\
\hline 2 & LM & \begin{tabular}{|l|} 
FY09EM21-29 \\
\end{tabular} & b15LM12 & t\%) & 1.7630 & 992 & 357 \\
\hline 2 & LM & FY09EM21-29 & b15LM21 & $\mathrm{CaO}$ (wt\%) & 1.7350 & 1.7476 & 1.7357 \\
\hline 2 & LM & FY09EM21-29 & b15LM22 & $\mathrm{CaO}$ (wt\%) & 1.7490 & 1.7650 & 1.7357 \\
\hline 2 & LM & FY09EM21-29 & b15LM11 & $\%)$ & & & 0.1533 \\
\hline 2 & LM & FY09EM21-29 & b15LM12 & CdO (wt\%) & 0.1462 & 62 & 0.1533 \\
\hline 2 & LM & FY09EM21-29 & b15LM21 & $\mathrm{CdO}(\mathrm{wt} \%)$ & 0.1474 & 474 & 0.1533 \\
\hline 2 & LM & FY09EM21-29 & b15LM22 & & & & \\
\hline 2 & LM & \begin{tabular}{|l|} 
FY09EM21-29 \\
\end{tabular} & b15LM11 & Ce2O3 (wt\%) & 398 & 98 & 864 \\
\hline 2 & LM & FY09EM21-29 & b15LM12 & Ce2O3 (wt\%) & 0.1816 & & 0.1864 \\
\hline 2 & LM & FY09EM21-29 & b15LM21 & Ce2O3 (wt\%) & 0.1862 & 62 & 0.1864 \\
\hline 2 & LM & FY09EM21-29 & b15I & $\mathrm{Ce} 2 \mathrm{C}$ & 0.1804 & & 0.1864 \\
\hline 2 & LM & FY09EM21-29 & b15LM11 & Cr2O3 (wt\%) & 0.0935 & 42 & 0.0960 \\
\hline 2 & LM & FY09EM21-29 & b15LM12 & Cr2O3 (wt\%) & 79 & 56 & 0.0960 \\
\hline 2 & LM & FY09EM21-29 & b15LM21 & Cr2O3 (wt\%) & & & 960 \\
\hline 2 & LM & FY09EM21-29 & b15LM22 & Cr2O3 (wt\%) & 50 & 27 & 0.0960 \\
\hline 2 & LM & FY09EM21-29 & b15LM11 & $\mathrm{CuO}(\mathrm{wt} \%)$ & 0.0864 & 369 & 0.0663 \\
\hline 2 & LM & FY09EM21-29 & b15LM12 & $\mathrm{CuO}$ (wt\%) & 0.0839 & 0.0857 & 0.0663 \\
\hline 2 & LM & FY09EM21-29 & b15LM21 & $\mathrm{CuO}(\mathrm{wt} \%)$ & 0.0776 & 0.0781 & 0.0663 \\
\hline 2 & LM & FY09EM21-29 & b15LM22 & $\mathrm{CuO}(\mathrm{wt} \%)$ & 0.0864 & 0.0882 & 0.0663 \\
\hline 2 & LM & FY09EM21-29 & b15LM11 & K2O (wt\%) & 0.0301 & 0.0309 & 0.0000 \\
\hline 2 & LM & FY09EM21-29 & b15LM12 & K2O (wt\%) & & & \\
\hline 2 & LM & FY09EM21-29 & b15LM21 & K2O (wt\%) & 301 & 309 & 000 \\
\hline 2 & LM & FY09EM21-29 & b15LM22 & K2O (wt\%) & 0.0301 & 0.0312 & 0.0000 \\
\hline 2 & LM & FY09EM21-29 & b15LM11 & La2O3 (wt\%) & 0.0446 & 0.0446 & 0.0505 \\
\hline 2 & LM & FY09EM21-29 & b15LM12 & La2O3 (wt\%) & 0.0446 & 0.0446 & 0.0505 \\
\hline 2 & LM & FY09EM21-29 & b15LM21 & La2O3 (wt\%) & 0.0434 & 0.0434 & 0.0505 \\
\hline 2 & LM & FY09EM21-29 & b15LM22 & La2O3 (wt\%) & 0.0446 & 0.0446 & 0.0505 \\
\hline 2 & LM & FY09EM21-29 & b15LM11 & $\mathrm{MgO}$ & & & 0.6561 \\
\hline 2 & LM & FY09EM21-29 & b15LM12 & $\mathrm{MgO}(\mathrm{wt} \%)$ & 583 & 0.6641 & 0.6561 \\
\hline 2 & LM & FY09EM21-29 & b15LM21 & $\mathrm{MgO}(\mathrm{wt} \%)$ & 0.6583 & 0.6604 & 0.6561 \\
\hline 2 & LM & FY09EM21-29 & b15LM22 & $\mathrm{MgO}$ (wt\%) & 0.6534 & 0.6590 & 0.6561 \\
\hline 2 & LM & FY09EM21-29 & b15LM11 & $\mathrm{MnO}$ (wt\%) & 2.3758 & 2.4747 & 2.5180 \\
\hline 2 & LM & FY09EM21-29 & b15LM12 & $\mathrm{MnO}$ (wt\%) & 2.4145 & 2.4828 & 2.5180 \\
\hline 2 & LM & FY09EM21-29 & b15LM21 & $\mathrm{MnO}$ (wt\%) & 2.3629 & 2.4612 & 2.5180 \\
\hline 2 & LM & FY09EM21-29 & b15LM22 & $\mathrm{MnO}(\mathrm{wt} \%)$ & & 2.4695 & 2.5180 \\
\hline 2 & LM & FY09EM21-29 & b15LM11 & Na2O (wt\%) & 12.8869 & 12.5465 & 12.2076 \\
\hline 2 & LM & FY09EM21-29 & b15LM12 & $\mathrm{Na} 2 \mathrm{O}$ (wt\%) & 11.9298 & 12.1892 & 12.2076 \\
\hline 2 & LM & FY09EM21-29 & b15LM21 & $\mathrm{Na} 2 \mathrm{O}(\mathrm{wt} \%)$ & 12.6847 & 12.3496 & 12.2076 \\
\hline 2 & LM & FY09EM21-29 & b15LM22 & Na2O (wt\%) & 11.9028 & 12.1616 & 12.2076 \\
\hline 2 & LM & FY09EM21-29 & b15LM11 & $\mathrm{NiO}(\mathrm{wt} \%)$ & 1.0753 & 1.0571 & 1.2396 \\
\hline 2 & LM & FY09EM21-29 & b15LM12 & $\mathrm{NiO}(\mathrm{wt} \%)$ & 1.0676 & 1.0449 & 1.2396 \\
\hline 2 & LM & FY09EM21-29 & b15LM21 & $\mathrm{NiO}(\mathrm{wt} \%)$ & 1.0816 & 1.0633 & 1.2396 \\
\hline 2 & LM & FY09EM21-29 & b15LM22 & $\mathrm{NiO}(\mathrm{wt} \%)$ & 1.0880 & 1.0649 & 1.2396 \\
\hline 2 & LM & FY09EM21-29 & b15LM11 & $\mathrm{PbO}$ (wt\%) & 0.1282 & 0.1282 & 0.1118 \\
\hline 2 & LM & FY09EM21-29 & b15LM12 & PbO (wt\%) & 0.1325 & 0.1325 & 0.1118 \\
\hline
\end{tabular}


SRNL-STI-2009-00778, Revision 0

Table A5. Measured, Measured Bias-Corrected (BC), and Targeted Chemical Compositions for the Samples of the Rad Glasses of the Matrix 2 Study

\begin{tabular}{|c|c|c|c|c|c|c|c|}
\hline Set & Prep Method & Glass ID & Lab ID & Analyte & Measured & Measured bc & Targeted \\
\hline 2 & LM & FY09EM21-29 & b15LM21 & $\mathrm{PbO}$ (wt\%) & 0.1260 & 0.1260 & 0.1118 \\
\hline 2 & LM & FY09EM21-29 & b15LM22 & $\mathrm{PbO}$ (wt\%) & 0.1325 & 0.1325 & 0.1118 \\
\hline 2 & LM & FY09EM21-29 & b15LM11 & SiO2 (wt\%) & 41.7164 & 42.2108 & 41.6427 \\
\hline 2 & LM & FY09EM21-29 & b15LM12 & SiO2 (wt\%) & 41.5024 & 42.2371 & 41.6427 \\
\hline 2 & LM & FY09EM21-29 & b15LM21 & $\mathrm{SiO} 2$ (wt\%) & 40.6467 & 41.1284 & 41.6427 \\
\hline 2 & LM & FY09EM21-29 & b15LM22 & $\mathrm{SiO} 2$ (wt\%) & 40.6467 & 41.3662 & 41.6427 \\
\hline 2 & LM & FY09EM21-29 & b15LM11 & SO4 (wt\%) & 0.2247 & 0.2247 & 0.2485 \\
\hline 2 & LM & FY09EM21-29 & b15LM12 & SO4 (wt\%) & 0.2247 & 0.2247 & 0.2485 \\
\hline 2 & LM & FY09EM21-29 & b15LM21 & SO4 (wt\%) & 0.2247 & 0.2247 & 0.2485 \\
\hline 2 & LM & FY09EM21-29 & b15LM22 & SO4 (wt\%) & 0.2247 & 0.2247 & 0.2485 \\
\hline 2 & LM & FY09EM21-29 & b15LM11 & $\mathrm{TiO} 2$ (wt\%) & 3.5528 & 3.7650 & 3.7689 \\
\hline 2 & LM & FY09EM21-29 & b15LM12 & TiO2 (wt\%) & 3.6029 & 3.8015 & 3.7689 \\
\hline 2 & LM & FY09EM21-29 & b15LM21 & TiO2 (wt\%) & 3.5362 & 3.7474 & 3.7689 \\
\hline 2 & LM & FY09EM21-29 & b15LM22 & TiO2 (wt\%) & 3.5862 & 3.7839 & 3.7689 \\
\hline 2 & LM & FY09EM21-29 & b15LM11 & U3O8 (wt\%) & 3.9975 & 4.0179 & 4.0000 \\
\hline 2 & LM & FY09EM21-29 & b15LM12 & U3O8 (wt\%) & 4.0564 & 3.9413 & 4.0000 \\
\hline 2 & LM & FY09EM21-29 & b15LM21 & U3O8 (wt\%) & 3.9739 & 3.9942 & 000 \\
\hline 2 & LM & FY09EM21-29 & b15LM22 & U3O8 (wt\%) & 4.0329 & 3.9183 & 4.0000 \\
\hline 2 & LM & FY09EM21-29 & b15LM11 & $\mathrm{ZnO}$ (wt\%) & 0.0747 & 747 & 696 \\
\hline 2 & LM & FY09EM21-29 & b15LM12 & $\mathrm{ZnO}$ (wt\%) & 0.0772 & 772 & 596 \\
\hline 2 & LM & FY09EM21-29 & b15LM21 & $\mathrm{ZnO}$ (wt\%) & 0.0734 & 0.0734 & 0.0696 \\
\hline 2 & LM & FY09EM21-29 & b15LM22 & $\mathrm{ZnO}$ (wt\%) & 0.0772 & 0.0772 & 0.0696 \\
\hline 2 & LM & FY09EM21-29 & b15LM11 & ZrO2 (wt\%) & 0.1040 & & 060 \\
\hline 2 & LM & \begin{tabular}{|l|} 
FY09EM21-29 \\
\end{tabular} & b15LM12 & ZrO2 (wt\%) & 0.1000 & 00 & 060 \\
\hline 2 & LM & FY09EM21-29 & b15LM21 & $\mathrm{ZrO} 2$ (wt\%) & 0.0837 & 0.0837 & 0.1060 \\
\hline 2 & LM & FY09EM21-29 & b15LM22 & $\mathrm{ZrO}$ & & & \\
\hline 2 & PF & \begin{tabular}{|l|} 
FY09EM21-29 \\
\end{tabular} & b15PF11 & $\mathrm{Al} 2 \mathrm{O} 3(\mathrm{wt} \%)$ & 179 & 991 & 046 \\
\hline 2 & $\mathrm{PF}$ & FY09EM21-29 & b15PF12 & $\mathrm{Al} 2 \mathrm{O} 3$ (wt\%) & 7.2557 & 7.2588 & 6.9046 \\
\hline 2 & $\mathrm{PF}$ & FY09EM21-29 & b15PF21 & $\mathrm{Al} 2 \mathrm{O} 3$ (wt\%) & 35 & & 6.9046 \\
\hline 2 & PF & FY09EM21-29 & b15PF22 & $\mathrm{Al} 2 \mathrm{O} 3(\mathrm{wt} \%)$ & & & 6.9046 \\
\hline 2 & $\mathrm{PF}$ & FY09EM21-29 & b15PF11 & B2O3 (wt\%) & 6.3754 & 6.8948 & 6.6992 \\
\hline 2 & $\mathrm{PF}$ & FY09EM21-29 & b15PF12 & $\mathrm{B} 2 \mathrm{O} 3(\mathrm{wt} \%)$ & 6.6652 & 290 & 6.6992 \\
\hline 2 & PF & FY09EM21-29 & \begin{tabular}{|l|} 
b15PF21 \\
\end{tabular} & wt\%) & & & \\
\hline 2 & PF & \begin{tabular}{|l|} 
FY09EM21-29 \\
\end{tabular} & b15PF22 & wt\%) & 6.6008 & & 6.6992 \\
\hline 2 & $\mathrm{PF}$ & FY09EM21-29 & b15PF11 & Fe2O3 (wt\%) & 12.2239 & 789 & 12.5996 \\
\hline 2 & $\mathrm{PF}$ & FY09EM21-29 & b15PF12 & Fe2O3 (wt\%) & 12.3669 & 12.1907 & 12.5996 \\
\hline 2 & $\mathrm{PF}$ & FY09EM21-29 & b15PF21 & Fe2O3 (wt \%) & & & 12.5996 \\
\hline 2 & $\mathrm{PF}$ & FY09EM21-29 & b15PF22 & Fe2O3 (wt\%) & 12.8101 & 12.6276 & 12.5996 \\
\hline 2 & $\mathrm{PF}$ & FY09EM21-29 & b15PF11 & $\mathrm{Li} 2 \mathrm{O}(\mathrm{wt} \%)$ & 4.8656 & 4.9881 & 4.8982 \\
\hline 2 & PF & FY09EM21-29 & \begin{tabular}{|l|} 
b15PF12 \\
\end{tabular} & Li2O (wt\%) & 440 & & 4.8982 \\
\hline 2 & PF & FY09EM21-29 & b15PF21 & Li2O (wt\%) & 301 & 844 & 4.8982 \\
\hline 2 & $\mathrm{PF}$ & FY09EM21-29 & b15PF22 & Li2O (wt\%) & 086 & 156 & 4.8982 \\
\hline 2 & LM & FY09EM21-30 & b18LM11 & $\mathrm{BaO}$ (wt\%) & 0.0424 & 430 & 0.0406 \\
\hline 2 & LM & FY09EM21-30 & b18LM12 & $\mathrm{BaO}$ (wt\%) & 0.0424 & 0.0431 & 0.0406 \\
\hline 2 & LM & FY09EM21-30 & b18LM21 & $\mathrm{BaO}$ (wt\%) & 0.0435 & 442 & 0.0406 \\
\hline 2 & LM & FY09EM21-30 & b18LM22 & $\mathrm{BaO}(\mathrm{wt} \%)$ & 0.0424 & 431 & 0.0406 \\
\hline 2 & LM & FY09EM21-30 & b18LM11 & t\%) & & & 995 \\
\hline 2 & LM & FY09EM21-30 & b18LM12 & $\mathrm{CaO}$ (wt\%) & 91 & 10 & 1.6995 \\
\hline 2 & LM & FY09EM21-30 & b18LM21 & $\mathrm{CaO}(\mathrm{wt} \%)$ & 1.6790 & 1.6795 & 1.6995 \\
\hline 2 & LM & FY09EM21-30 & b18LM22 & $\mathrm{CaO}$ (wt\%) & 1.6511 & 1.7043 & 1.6995 \\
\hline 2 & LM & FY09EM21-30 & b18LM11 & CdO (wt\%) & 0.1371 & 0.1371 & 0.1501 \\
\hline 2 & LM & FY09EM21-30 & b18LM12 & CdO (wt\%) & 0.1359 & 0.1359 & 0.1501 \\
\hline 2 & LM & FY09EM21-30 & b18LM21 & $\mathrm{CdO}$ (wt\%) & 451 & 451 & 0.1501 \\
\hline 2 & LM & FY09EM21-30 & b18LM22 & CdO (wt\%) & & & 0.1501 \\
\hline 2 & LM & FY09EM21-30 & b18LM11 & Ce2O3 (wt\%) & 0.1722 & 0.1722 & 0.1825 \\
\hline 2 & LM & FY09EM21-30 & b18LM12 & Ce2O3 (wt\%) & 0.1734 & 0.1734 & 0.1825 \\
\hline 2 & LM & FY09EM21-30 & b18LM21 & Ce2O3 (wt\%) & 0.1745 & 0.1745 & 0.1825 \\
\hline 2 & LM & FY09EM21-30 & b18LM22 & Ce2O3 (wt\%) & 0.1839 & 0.1839 & 0.1825 \\
\hline 2 & LM & FY09EM21-30 & b18LM11 & Cr2O3 (wt \%) & 0.0848 & 0.0846 & 0.0940 \\
\hline 2 & LM & FY09EM21-30 & b18LM12 & Cr2O3 (wt\%) & 0.0837 & 0.0849 & 0.0940 \\
\hline 2 & LM & FY09EM21-30 & b18LM21 & Cr2O3 (wt \%) & 0.0892 & & 0.0940 \\
\hline 2 & LM & FY09EM21-30 & b18LM22 & Cr2O3 (wt \%) & 0.0862 & 0.0874 & 0.0940 \\
\hline 2 & LM & FY09EM21-30 & b18LM11 & $\mathrm{CuO}$ (wt\%) & 0.0851 & 0.0872 & 0.0649 \\
\hline 2 & LM & FY09EM21-30 & b18LM12 & $\mathrm{CuO}$ (wt\%) & 0.0739 & 0.0773 & 0.0649 \\
\hline 2 & LM & FY09EM21-30 & b18LM21 & $\mathrm{CuO}(\mathrm{wt} \%)$ & 0.0839 & 0.0860 & 0.0649 \\
\hline 2 & LM & FY09EM21-30 & b18LM22 & $\mathrm{CuO}$ (wt\%) & 0.0789 & 0.0825 & 0.0649 \\
\hline 2 & LM & FY09EM21-30 & b18LM11 & K2O (wt\%) & 0.0301 & 0.0304 & 0.0000 \\
\hline 2 & LM & FY09EM21-30 & b18LM12 & K2O (wt\%) & 0.0301 & 0.0304 & 0.0000 \\
\hline 2 & LM & FY09EM21-30 & b18LM21 & K2O (wt\%) & 0.0301 & 0.0304 & 0.0000 \\
\hline 2 & LM & FY09EM21-30 & b18LM22 & K2O (wt\%) & 0.0301 & 0.0304 & 0.0000 \\
\hline 2 & LM & FY09EM21-30 & b18LM11 & La2O3 (wt\%) & 0.0273 & 0.0273 & 0.0495 \\
\hline
\end{tabular}


SRNL-STI-2009-00778, Revision 0

Table A5. Measured, Measured Bias-Corrected (BC), and Targeted Chemical Compositions for the Samples of the Rad Glasses of the Matrix 2 Study

\begin{tabular}{|c|c|c|c|c|c|c|c|}
\hline Set & Prep Method & Glass ID & Lab ID & Analyte & Measured & Measured bc & Targeted \\
\hline 2 & LM & FY09EM21-30 & b18LM12 & La2O3 (wt\%) & 0.0276 & 0.0276 & 0.0495 \\
\hline 2 & LM & FY09EM21-30 & b18LM21 & La2O3 (wt\%) & 0.0359 & 0.0359 & 0.0495 \\
\hline 2 & LM & FY09EM21-30 & b18LM22 & La2O3 (wt\%) & 0.0367 & 0.0367 & 0.0495 \\
\hline 2 & LM & FY09EM21-30 & b18LM11 & $\mathrm{MgO}(\mathrm{wt} \%)$ & 0.6318 & 0.6358 & 0.6424 \\
\hline 2 & LM & FY09EM21-30 & b18LM12 & $\mathrm{MgO}(\mathrm{wt} \%)$ & 0.6384 & 0.6340 & 0.6424 \\
\hline 2 & LM & FY09EM21-30 & b18LM21 & $\mathrm{MgO}(\mathrm{wt} \%)$ & 0.6517 & 0.6558 & 0.6424 \\
\hline 2 & LM & FY09EM21-30 & b18LM22 & $\mathrm{MgO}(\mathrm{wt} \%)$ & 0.6550 & 0.6505 & 0.6424 \\
\hline 2 & LM & FY09EM21-30 & b18LM11 & $\mathrm{MnO}$ (wt\%) & 2.2983 & 2.3694 & 2.4656 \\
\hline 2 & LM & FY09EM21-30 & b18LM12 & $\mathrm{MnO}(\mathrm{wt} \%)$ & 2.3242 & 2.3656 & 2.4656 \\
\hline 2 & LM & FY09EM21-30 & b18LM21 & $\mathrm{MnO}(\mathrm{wt} \%)$ & 2.3758 & 2.4492 & 2.4656 \\
\hline 2 & LM & FY09EM21-30 & b18LM22 & $\mathrm{MnO}(\mathrm{wt} \%)$ & 2.4016 & 2.4444 & 2.4656 \\
\hline 2 & LM & FY09EM21-30 & b18LM11 & $\mathrm{Na} 2 \mathrm{O}$ (wt\%) & 11.5254 & 11.5348 & 11.9532 \\
\hline 2 & LM & FY09EM21-30 & b18LM12 & $\mathrm{Na} 2 \mathrm{O}$ (wt\%) & 11.5658 & 11.5695 & 11.9532 \\
\hline 2 & LM & FY09EM21-30 & b18LM21 & $\mathrm{Na} 2 \mathrm{O}(\mathrm{wt} \%)$ & 11.8894 & 11.8991 & 11.9532 \\
\hline 2 & LM & FY09EM21-30 & b18LM22 & $\mathrm{Na} 2 \mathrm{O}$ (wt\%) & 11.7276 & 11.7313 & 11.9532 \\
\hline 2 & LM & FY09EM21-30 & b18LM11 & $\mathrm{NiO}(\mathrm{wt} \%)$ & 1.0256 & 1.0055 & 1.2138 \\
\hline 2 & LM & FY09EM21-30 & b18LM12 & $\mathrm{NiO}(\mathrm{wt} \%)$ & 1.0218 & 1.0233 & 1.2138 \\
\hline 2 & LM & FY09EM21-30 & b18LM21 & $\mathrm{NiO}$ (wt\%) & 1.0765 & 1.0554 & 1.2138 \\
\hline 2 & LM & FY09EM21-30 & b18LM22 & $\mathrm{NiO}(\mathrm{wt} \%)$ & 1.0562 & 1.0577 & 1.2138 \\
\hline 2 & LM & FY09EM21-30 & b18LM11 & $\mathrm{PbO}$ (wt\%) & 0.0840 & & 095 \\
\hline 2 & LM & FY09EM21-30 & b18LM12 & PbO (wt\%) & 0.0808 & 0.0808 & 095 \\
\hline 2 & LM & FY09EM21-30 & b18LM21 & $\mathrm{PbO}$ (wt\%) & 0.0894 & 0.0894 & 0.1095 \\
\hline 2 & LM & FY09EM21-30 & b18LM22 & $\mathrm{PbO}$ (wt\%) & 0.0829 & 0.0829 & 0.1095 \\
\hline 2 & LM & FY09EM21-30 & b18LM11 & $\mathrm{SiO} 2$ (wt\%) & 38.2935 & 39.3122 & 40.7751 \\
\hline 2 & LM & FY09EM21-30 & b18LM12 & $\mathrm{SiO} 2$ (wt\%) & 38.2935 & 39.1410 & 40.7751 \\
\hline 2 & LM & FY09EM21-30 & b18LM21 & $\mathrm{SiO} 2$ (wt\%) & 39.7910 & 40.8495 & 40.7751 \\
\hline 2 & LM & FY09EM21-30 & b18LM22 & SiO2 (wt\%) & 39.5771 & 40.4530 & 40.7751 \\
\hline 2 & LM & FY09EM21-30 & b18LM11 & SO4 (wt\%) & 0.2247 & 0.2247 & 0.2433 \\
\hline 2 & LM & FY09EM21-30 & b18LM12 & SO4 (wt\%) & 0.2247 & 0.2247 & 0.2433 \\
\hline 2 & LM & FY09EM21-30 & b18LM21 & SO4 (wt\%) & 0.2247 & 0.2247 & 0.2433 \\
\hline 2 & LM & FY09EM21-30 & b18LM22 & $\mathrm{wt} \%)$ & 0.2247 & 0.2247 & 0.2433 \\
\hline 2 & LM & FY09EM21-30 & b18LM11 & TiO2 (wt\%) & 3.5695 & 3.6740 & 3.6904 \\
\hline 2 & LM & FY09EM21-30 & b18LM12 & TiO2 (wt\%) & 3.5528 & 3.6568 & 3.6904 \\
\hline 2 & LM & \begin{tabular}{|l|} 
FY09EM21-30 \\
\end{tabular} & b18LM21 & TiO2 (wt\%) & 3.6529 & 3.7598 & 3.6904 \\
\hline 2 & LM & FY09EM21-30 & b18LM22 & TiO2 (wt\%) & 3.6696 & 3.7770 & 3.6904 \\
\hline 2 & LM & FY09EM21-30 & b18LM11 & U3O8 (wt\%) & 5.5069 & 5.5808 & 6.0000 \\
\hline 2 & LM & FY09EM21-30 & b18LM12 & U3O8 (wt\%) & 5.5422 & 5.5432 & 6.0000 \\
\hline 2 & LM & FY09EM21-30 & b18LM21 & U3O8 & & & 6.0000 \\
\hline 2 & LM & FY09EM21-30 & b18LM22 & U3O8 (wt\%) & 5.7899 & 5.7909 & 6.0000 \\
\hline 2 & LM & FY09EM21-30 & b18LM11 & $\mathrm{ZnO}(\mathrm{wt} \%)$ & 0.0622 & 0.0622 & 0.0681 \\
\hline 2 & LM & FY09EM21-30 & b18LM12 & $\mathrm{ZnO}$ (wt\%) & & 889 & 0.0681 \\
\hline 2 & LM & FY09EM21-30 & b18LM21 & $\mathrm{ZnO}$ (wt\%) & 0.0647 & 0.0647 & 0.0681 \\
\hline 2 & LM & FY09EM21-30 & b18LM22 & $\mathrm{ZnO}$ (wt\%) & 0.0598 & 0.0598 & 0.0681 \\
\hline 2 & LM & FY09EM21-30 & b18LM11 & ZrO2 (wt\%) & 0.0959 & 0.0959 & 0.1038 \\
\hline 2 & LM & FY09EM21-30 & b18LM12 & ZrO2 (wt\%) & 0.0959 & 0.0959 & 0.1038 \\
\hline 2 & LM & FY09EM21-30 & b18LM21 & $\mathrm{ZrO} 2$ (wt\%) & 0.0973 & 0.0973 & 0.1038 \\
\hline 2 & LM & FY09EM21-30 & b18LM22 & $\mathrm{ZrO} 2(\mathrm{wt} \%)$ & 0.0973 & 0.0973 & 0.1038 \\
\hline 2 & PF & FY09EM21-30 & b18PF11 & $\mathrm{Al} 2 \mathrm{O} 3(\mathrm{wt} \%)$ & 6.8022 & 7.1371 & 6.7607 \\
\hline 2 & $\mathrm{PF}$ & FY09EM21-30 & b18PF12 & $\mathrm{Al} 2 \mathrm{O} 3(\mathrm{wt} \%)$ & 6.9723 & 7.1225 & 6.7607 \\
\hline 2 & PF & FY09EM21-30 & \begin{tabular}{|l|} 
b18PF21 \\
\end{tabular} & $\mathrm{Al} 2 \mathrm{O} 3(\mathrm{wt} \%)$ & 6.9534 & 7.2957 & 6.7607 \\
\hline 2 & PF & FY09EM21-30 & b18PF22 & $\mathrm{Al} 2 \mathrm{O} 3(\mathrm{wt} \%)$ & 7.0100 & 7.1611 & 6.7607 \\
\hline 2 & PF & FY09EM21-30 & b18PF11 & $\mathrm{B} 2 \mathrm{O} 3(\mathrm{wt} \%)$ & 6.3754 & 6.8034 & 6.5597 \\
\hline 2 & $\mathrm{PF}$ & FY09EM21-30 & b18PF12 & B2O3 (wt\%) & 6.5364 & 6.9142 & 6.5597 \\
\hline 2 & $\mathrm{PF}$ & FY09EM21-30 & b18PF21 & B2O3 (wt\%) & 6.5364 & 6.9752 & 6.5597 \\
\hline 2 & $\mathrm{PF}$ & FY09EM21-30 & b18PF22 & B2O3 (wt\%) & 6.5686 & 6.9482 & 6.5597 \\
\hline 2 & PF & FY09EM21-30 & b18PF11 & $\mathrm{Fe} 2 \mathrm{O} 3(\mathrm{wt} \%)$ & 12.2811 & 12.6621 & 12.3371 \\
\hline 2 & $\mathrm{PF}$ & FY09EM21-30 & b18PF12 & Fe2O3 (wt $\%)$ & 12.6385 & 12.9267 & 12.3371 \\
\hline 2 & PF & FY09EM21-30 & b18PF21 & $\mathrm{Fe} 2 \mathrm{O} 3(\mathrm{wt} \%)$ & 12.5385 & 12.9274 & 12.3371 \\
\hline 2 & PF & FY09EM21-30 & b18PF22 & $\mathrm{Fe} 2 \mathrm{O} 3(\mathrm{wt} \%)$ & 12.4384 & 12.7220 & 12.3371 \\
\hline 2 & $\mathrm{PF}$ & \begin{tabular}{|l|} 
FY09EM21-30 \\
\end{tabular} & b18PF11 & Li2O (wt\%) & 4.4996 & 4.8634 & 4.7962 \\
\hline 2 & $\mathrm{PF}$ & FY09EM21-30 & b18PF12 & Li2O (wt\%) & 4.8225 & 5.0190 & 4.7962 \\
\hline 2 & $\mathrm{PF}$ & FY09EM21-30 & b18PF21 & Li2O (wt\%) & 4.5426 & 4.9099 & 4.7962 \\
\hline 2 & PF & FY09EM21-30 & b18PF22 & Li2O (wt\%) & 4.6718 & 4.8622 & 4.7962 \\
\hline
\end{tabular}


SRNL-STI-2009-00778, Revision 0

Table A6. Average Measured and Bias-Corrected Chemical Compositions Versus Targeted Compositions by Oxide by Glass ID for Rad Glasses of the Matrix 2 Study

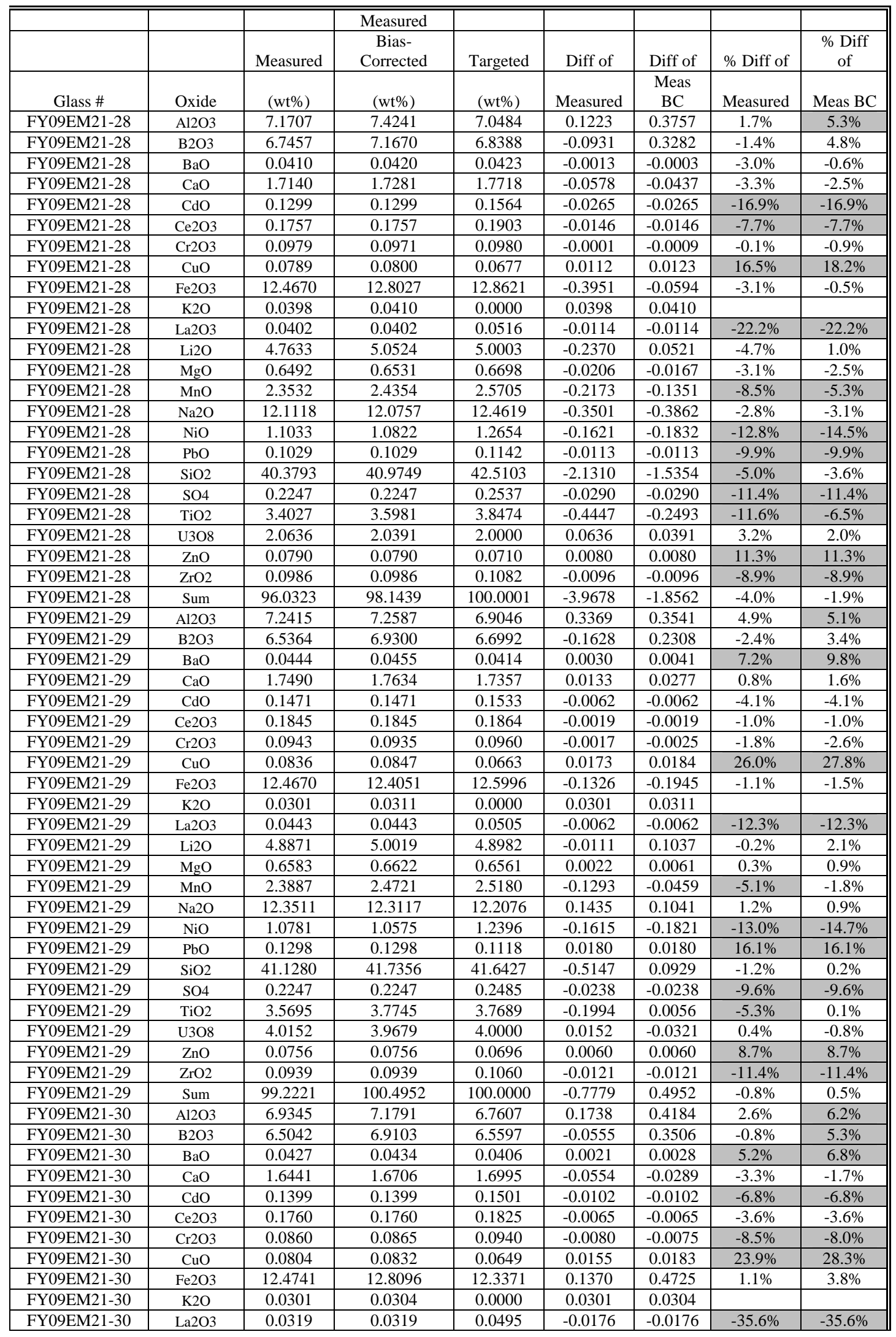


SRNL-STI-2009-00778, Revision 0

Table A6. Average Measured and Bias-Corrected Chemical Compositions Versus Targeted Compositions by Oxide by Glass ID for Rad Glasses of the Matrix 2 Study

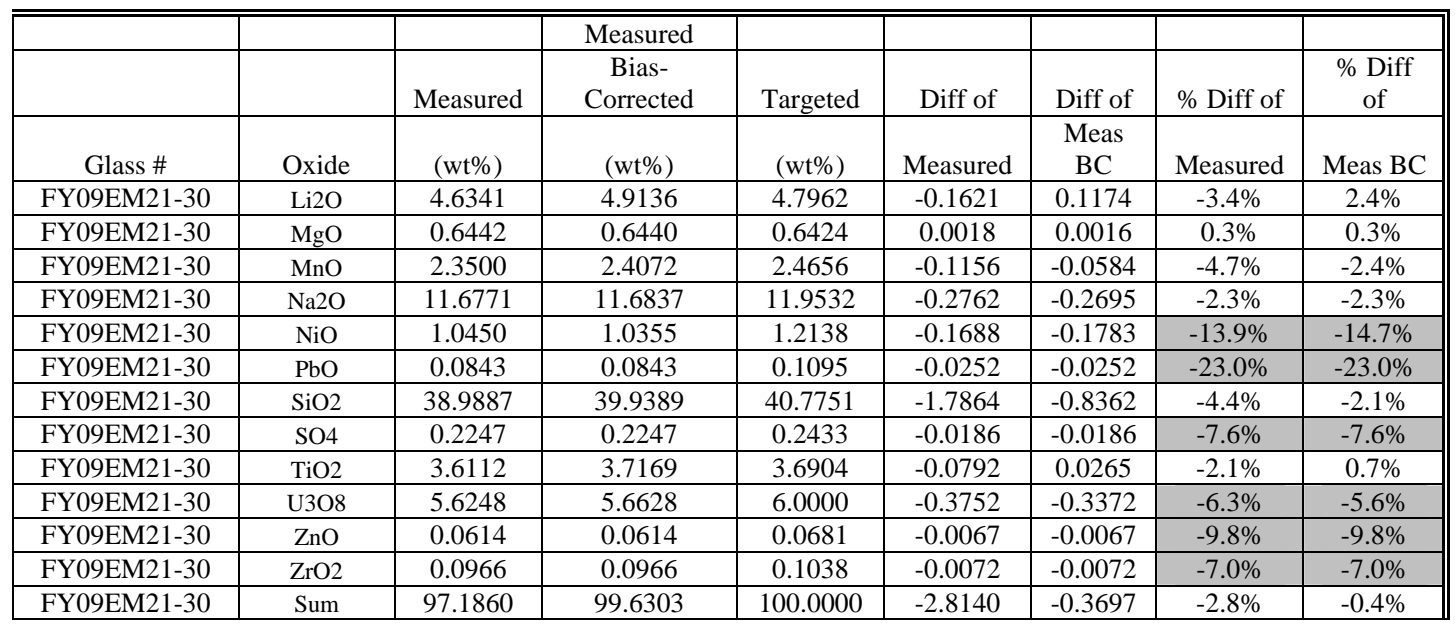




\section{Exhibit A1. Non-Rad Sample Measurements in Analytical Sequence by Oxide for the Matrix 2 Study}

Measured By Analytical Sequence Oxide $=\mathbf{A l} 203$ (wt\%)

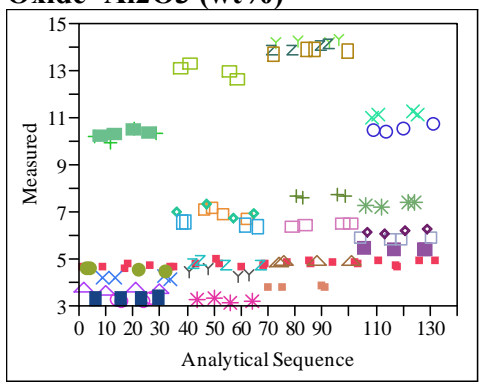

Measured By Analytical Sequence Oxide $=$ B2O3 (wt\%)

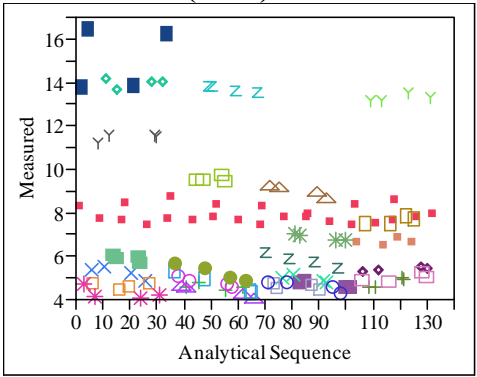

Measured By Analytical Sequence Oxide $=\mathrm{BaO}($ wt $\%)$

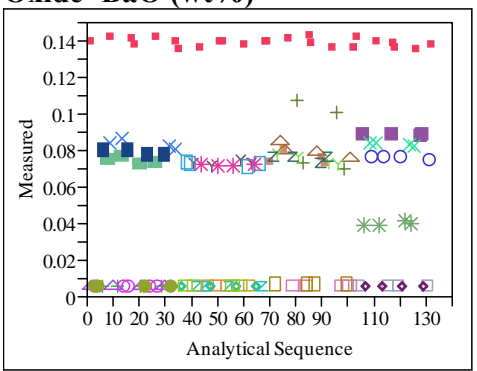

Measured By Analytical Sequence Oxide $=\mathrm{CaO}(\mathrm{wt} \%)$

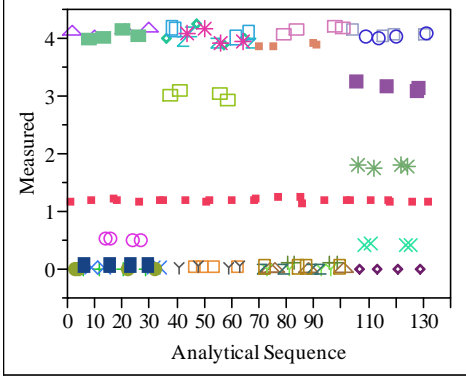

Measured By Analytical Sequence Oxide $=\mathbf{C d O}($ wt $\%)$

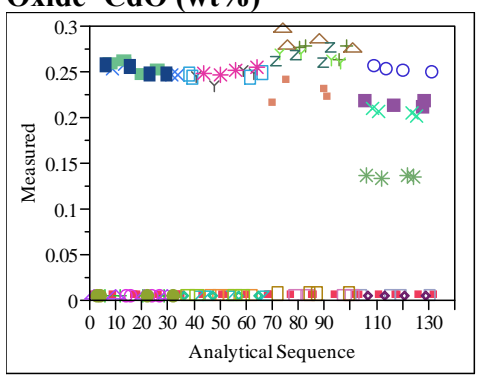

Measured By Analytical Sequence Oxide $=\mathrm{Ce} 2 \mathrm{O3}(\mathrm{wt} \%)$

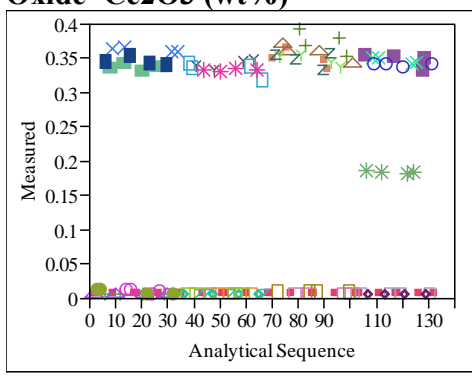

Measured By Analytical Sequence Oxide $=$ Cr2O3 (wt\%)

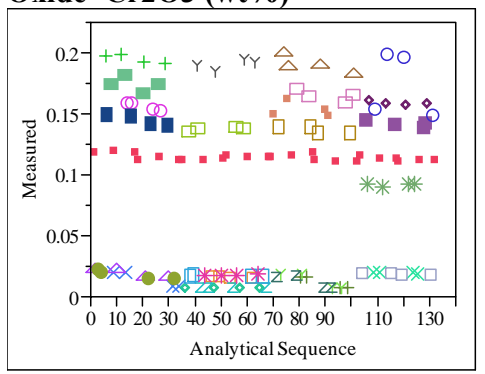

Measured By Analytical Sequence Oxide $=\mathrm{CuO}(\mathrm{wt} \%)$

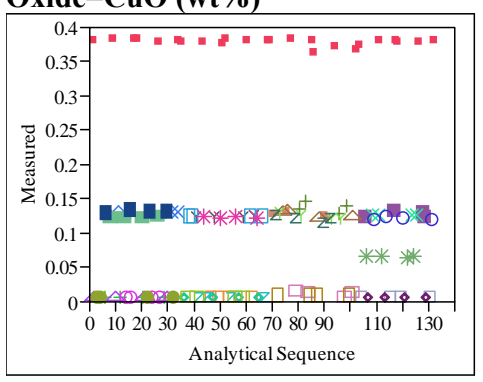

Measured By Analytical Sequence Oxide $=$ Fe2O3 $(w t \%)$

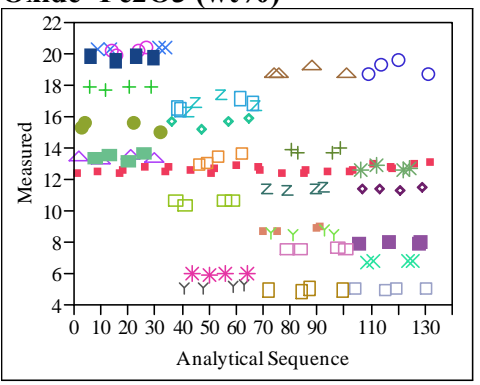

Measured By Analytical Sequence Oxide $=$ La2O3 (wt\%)

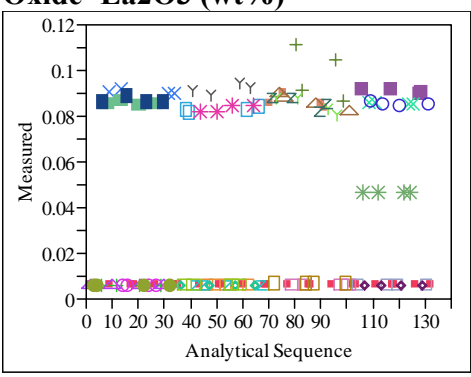

Measured By Analytical Sequence Oxide $=\mathbf{L i 2 O}(w t \%)$

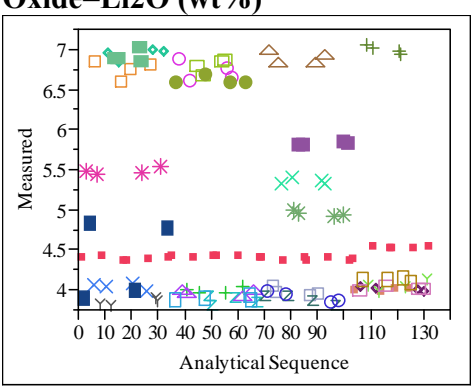

Measured By Analytical Sequence Oxide $=\operatorname{MgO}(\mathbf{w t} \%)$

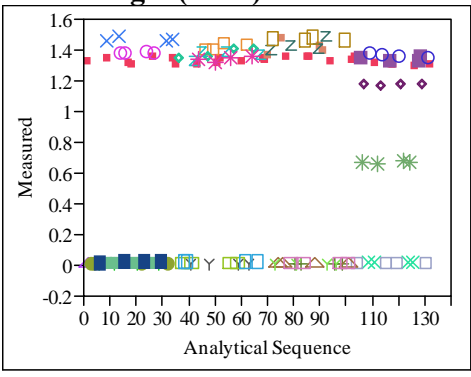




\section{Exhibit A1. Non-Rad Sample Measurements in Analytical Sequence by Oxide for the Matrix 2 Study}

Measured By Analytical Sequence Oxide $=\mathrm{MnO}(\mathbf{w t} \%)$

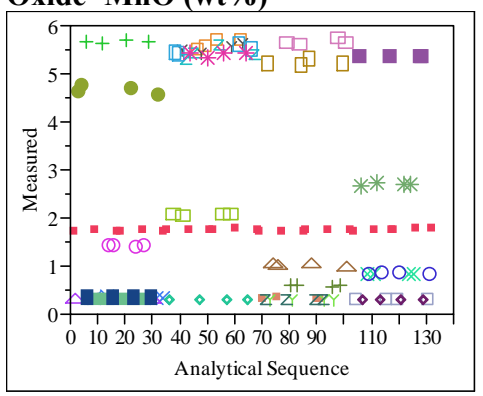

Measured By Analytical Sequence Oxide $=\mathrm{Na2O}(\mathrm{wt} \%)$

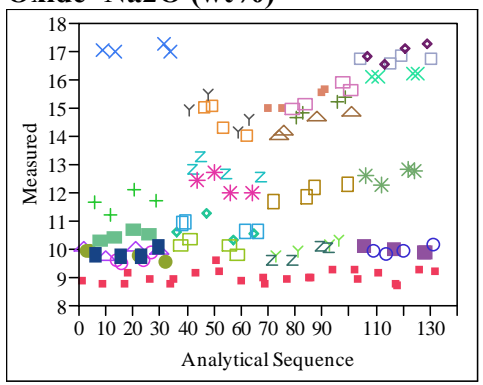

Measured By Analytical Sequence Oxide $=\mathrm{NiO}(\mathbf{w t} \%)$

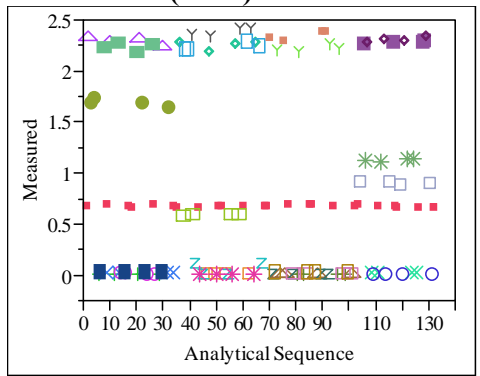

Measured By Analytical Sequence Oxide $=\mathbf{P b O}(\mathbf{w t} \%)$

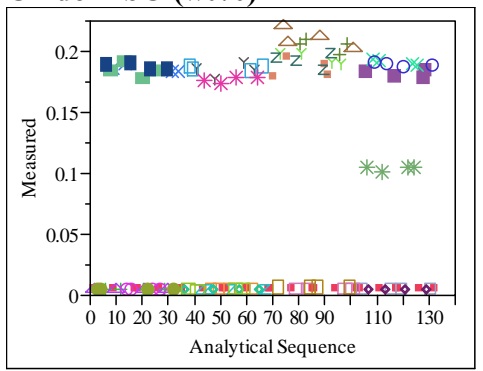

Measured By Analytical Sequence Oxide $=\mathrm{SiO} 2(\mathbf{w t} \%)$

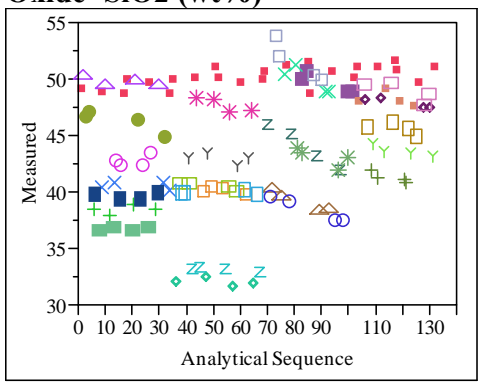

Measured By Analytical Sequence Oxide $=\mathrm{SO4}(\mathbf{w t} \%)$

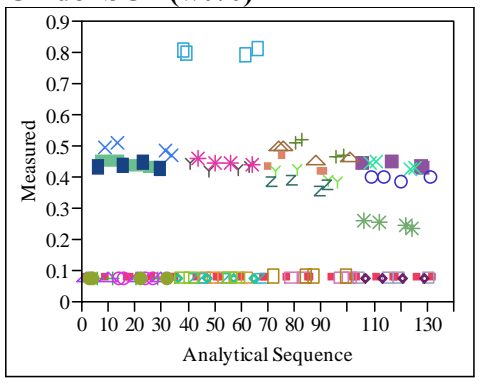

Measured By Analytical Sequence Oxide $=\mathrm{TiO} 2(\mathbf{w t} \%)$

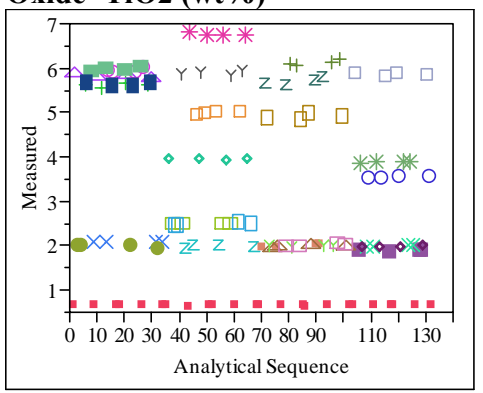

Measured By Analytical Sequence Oxide $=\mathbf{Z n O}(\mathbf{w t} \%)$

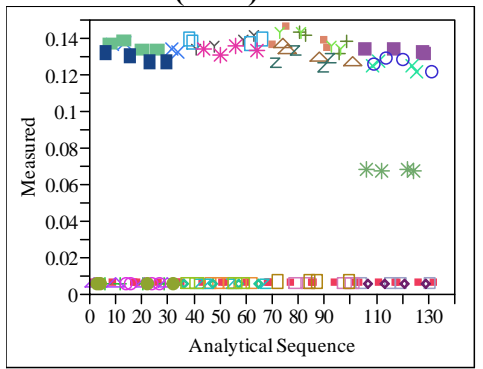

Measured By Analytical Sequence Oxide $=\mathrm{ZrO2}(\mathrm{wt} \%)$

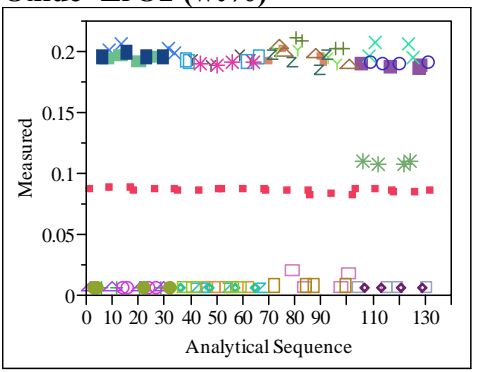


Exhibit A2. Non-Rad Sample Measurements by Lab ID within Glass ID for the Matrix 2 Study by Oxide by Analytical Block

Set $=1$, Oxide $=\mathbf{A l 2 O} 3(\mathrm{wt} \%)$

Variability Chart for Measured

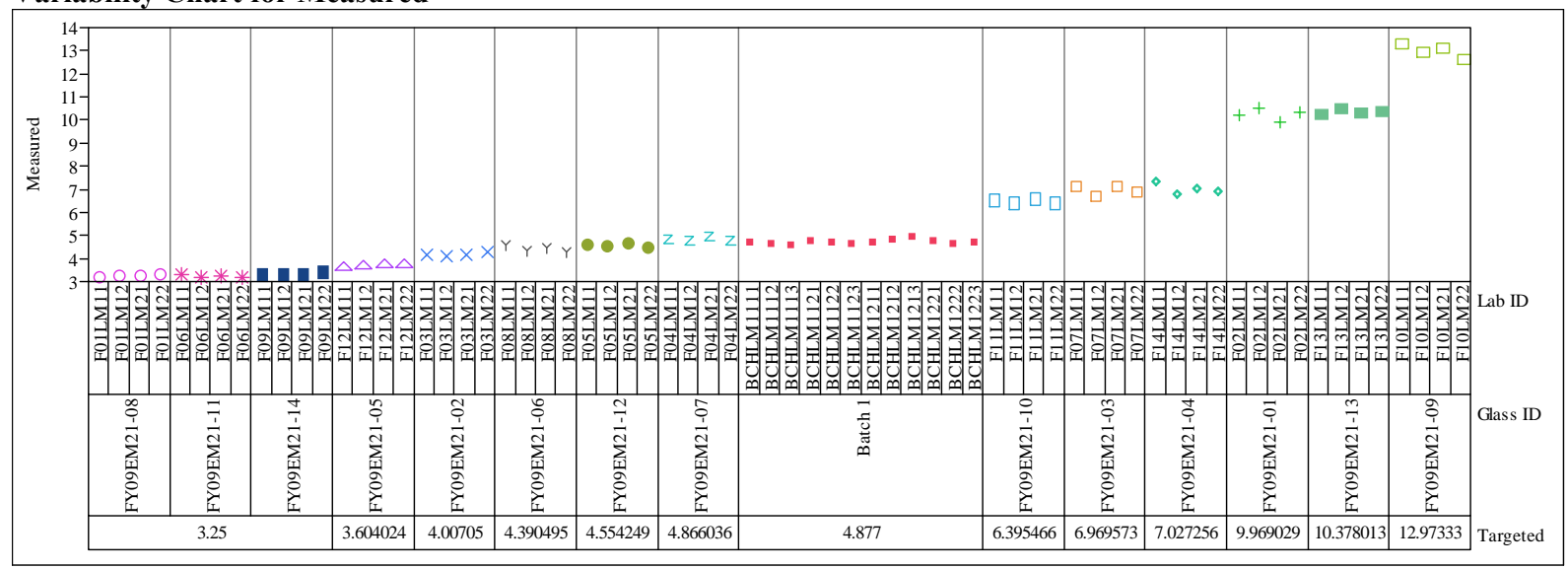

Set $=1$, Oxide $=$ B2O3 $($ wt\%)

Variability Chart for Measured

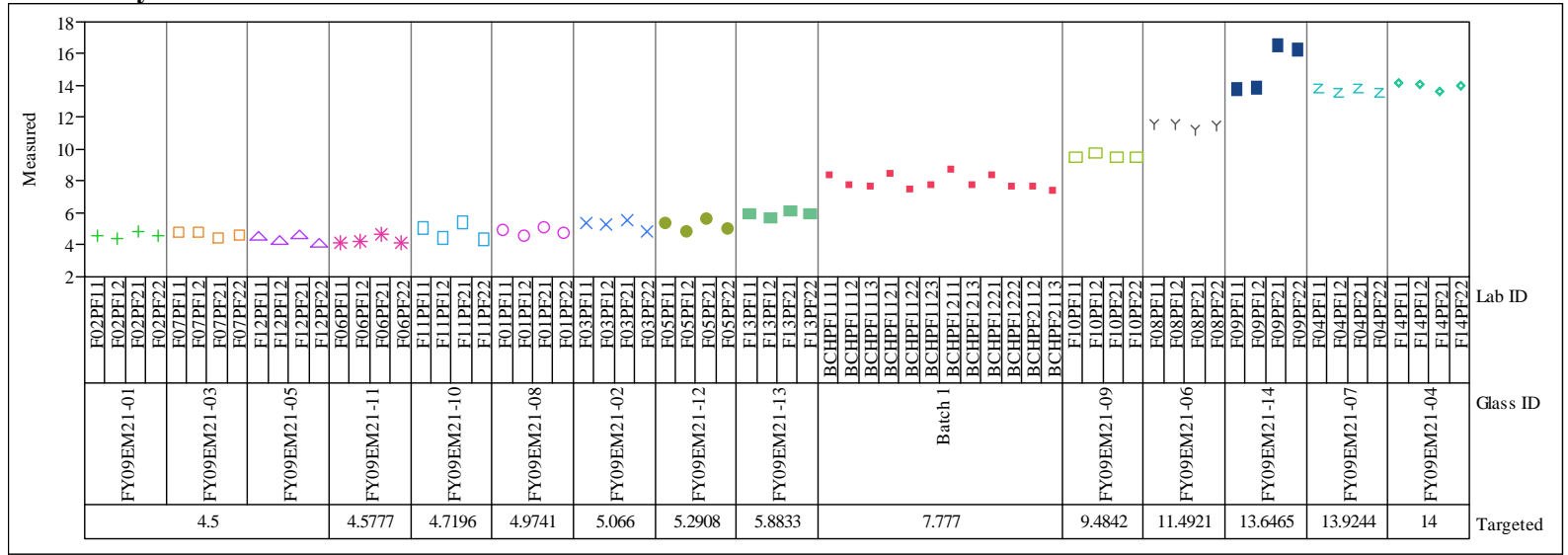

Set $=1$, Oxide $=$ BaO $(w t \%)$

Variability Chart for Measured

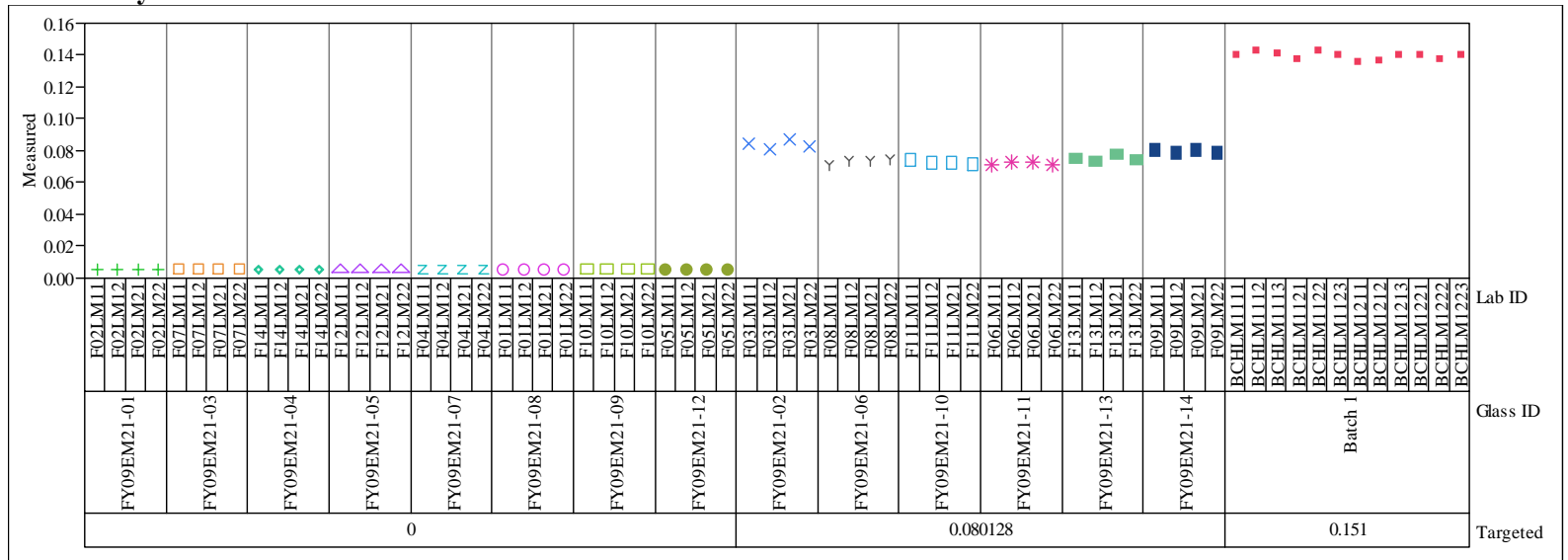


Exhibit A2. Non-Rad Sample Measurements by Lab ID within Glass ID for the Matrix 2 Study by Oxide by Analytical Block

Set $=1$, Oxide $=\mathrm{CaO}(\mathrm{wt} \%)$

Variability Chart for Measured

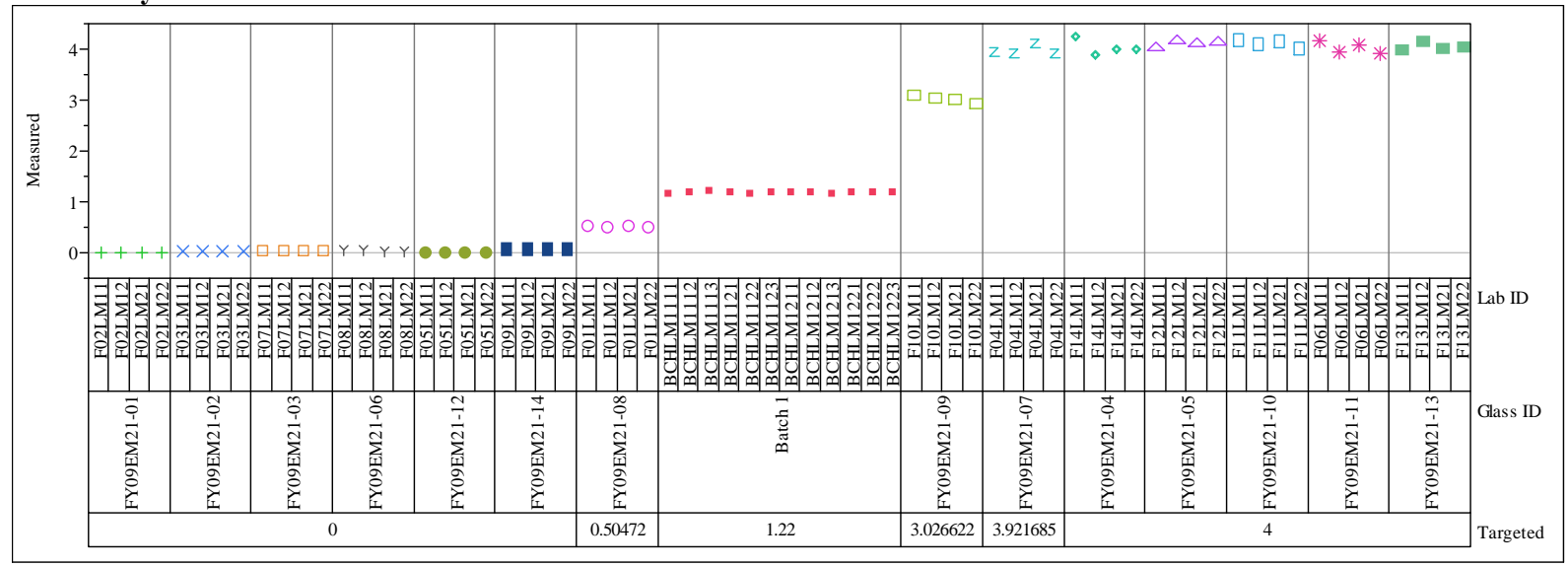

Set $=1$, Oxide $=$ CdO $(w t \%)$

Variability Chart for Measured

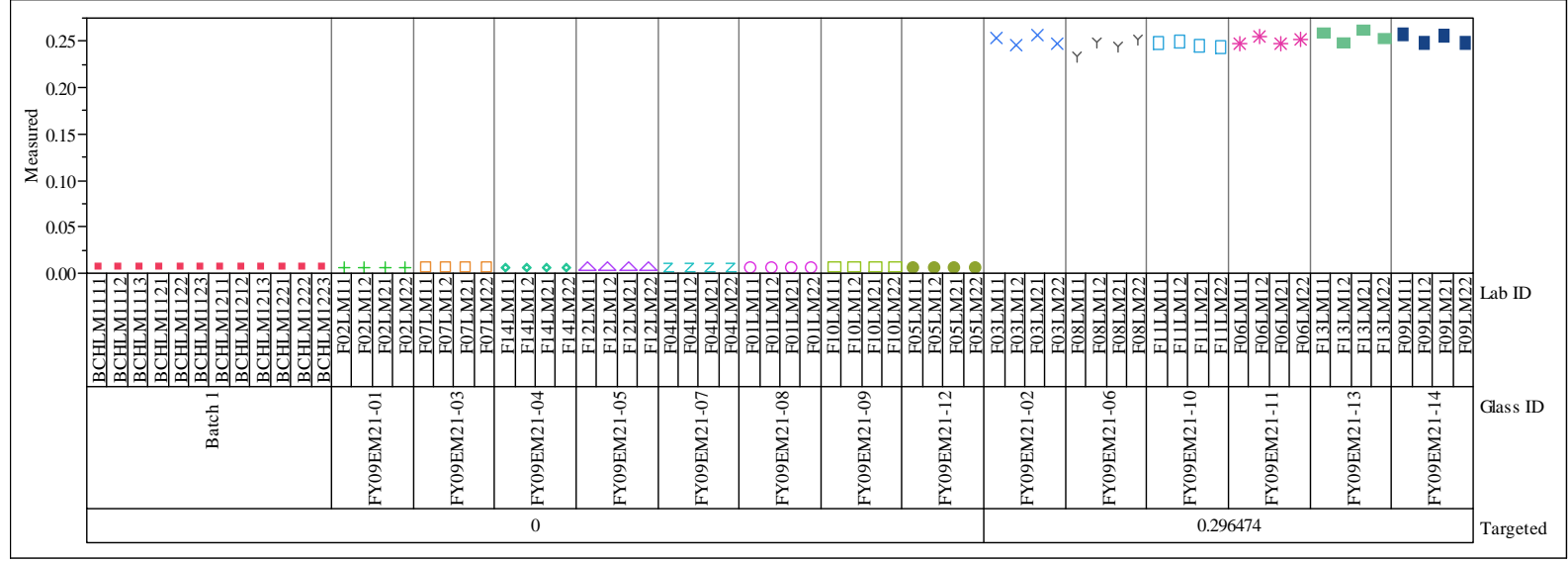

Set $=1$, Oxide $=$ Ce2O3 $($ wt $\%)$

Variability Chart for Measured

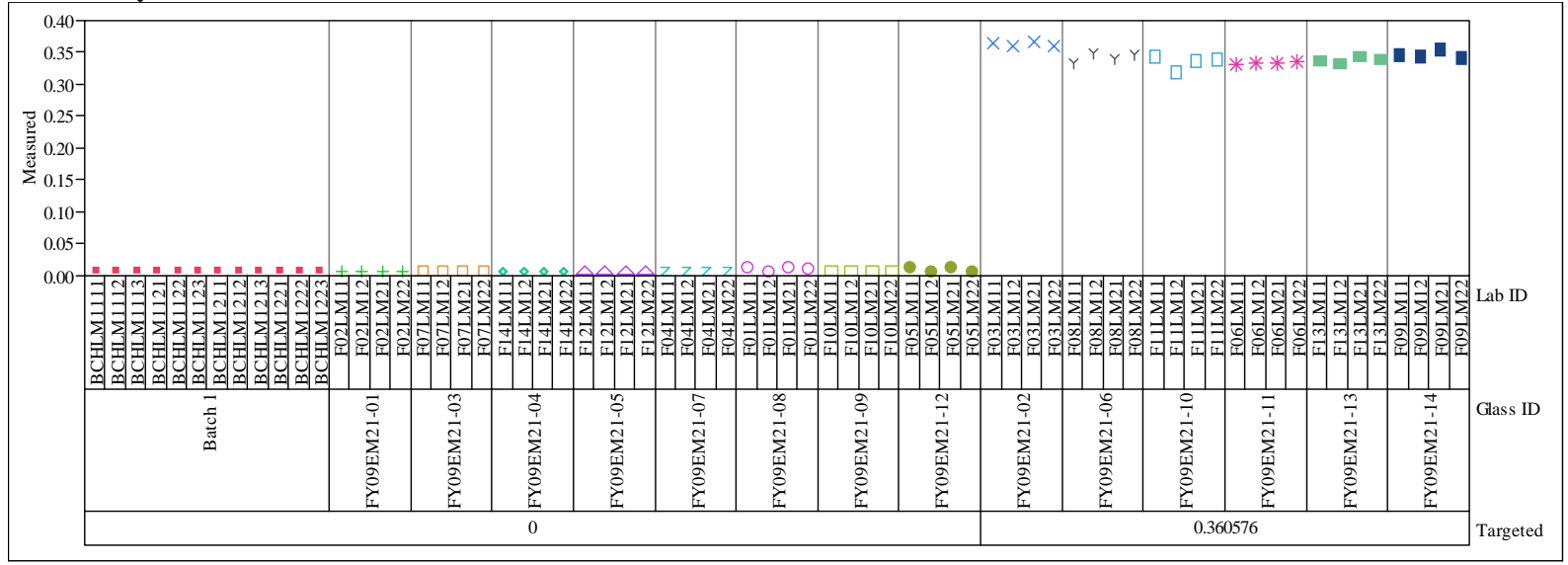


Exhibit A2. Non-Rad Sample Measurements by Lab ID within Glass ID for the Matrix 2 Study by Oxide by Analytical Block

Set $=1$, Oxide $=\mathbf{C r 2 O 3}(\mathrm{wt} \%)$

Variability Chart for Measured

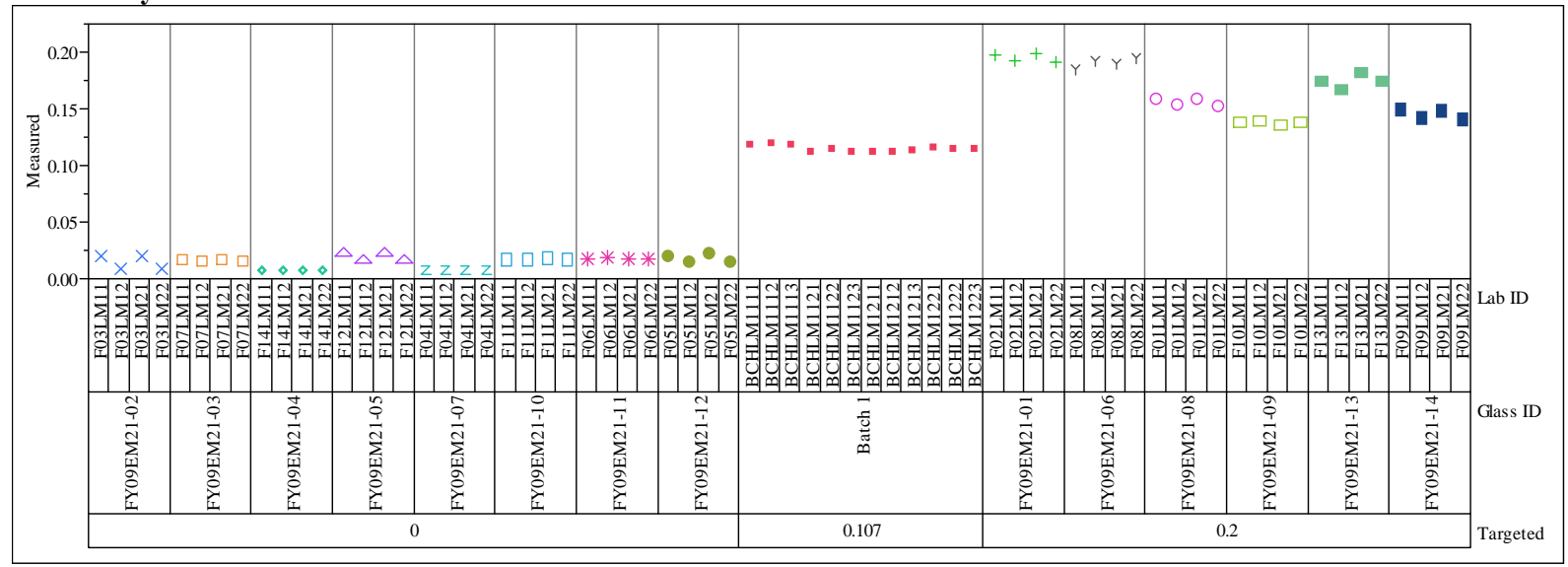

Set $=1$, Oxide $=\mathrm{CuO}($ wt $\%)$

Variability Chart for Measured

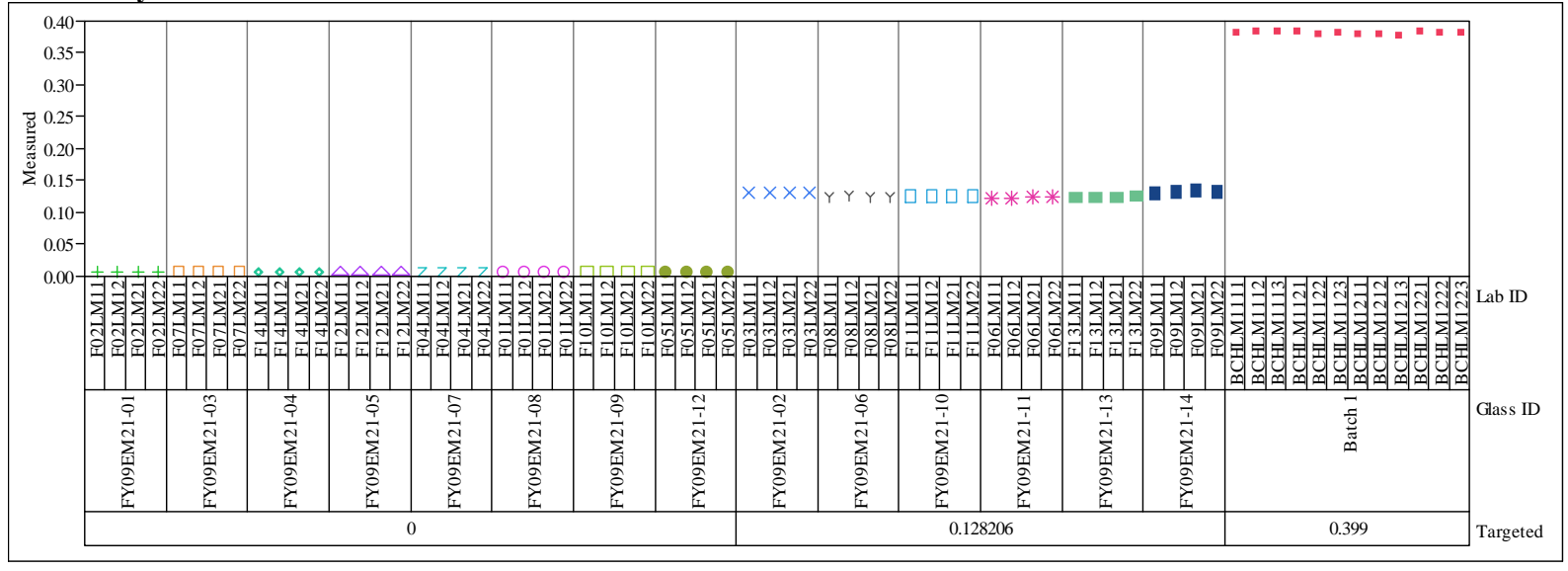

Set $=1$, Oxide $=$ Fe2O3 $($ wt $\%)$

Variability Chart for Measured

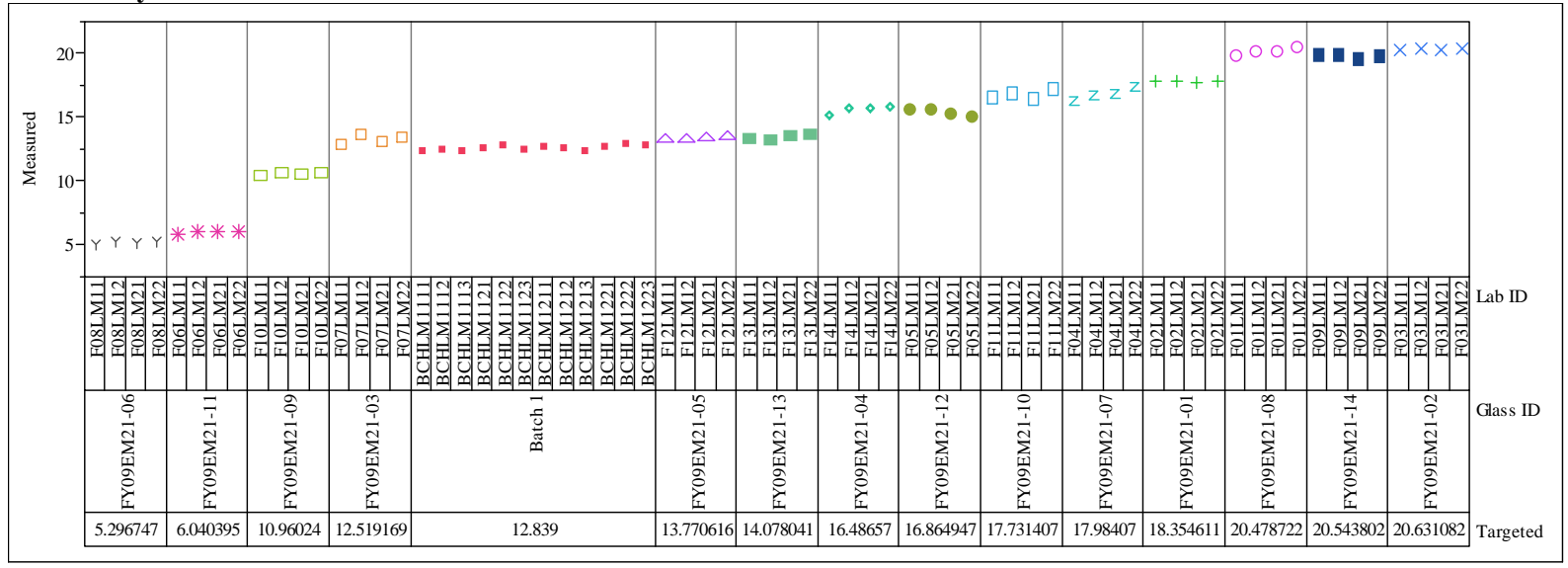


Exhibit A2. Non-Rad Sample Measurements by Lab ID within Glass ID for the Matrix 2 Study by Oxide by Analytical Block

Set $=1$, Oxide $=$ La2O3 $(w t \%)$

Variability Chart for Measured

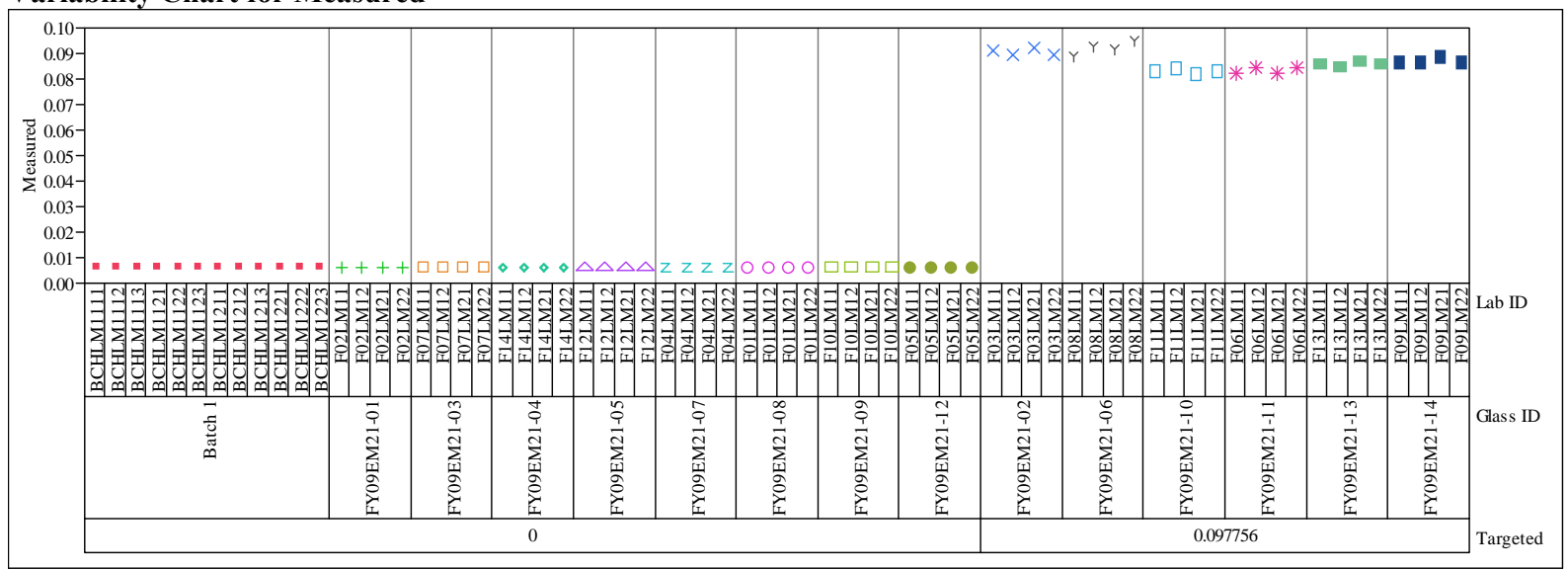

Set $=1$, Oxide $=$ Li2O $($ wt $\%)$

Variability Chart for Measured

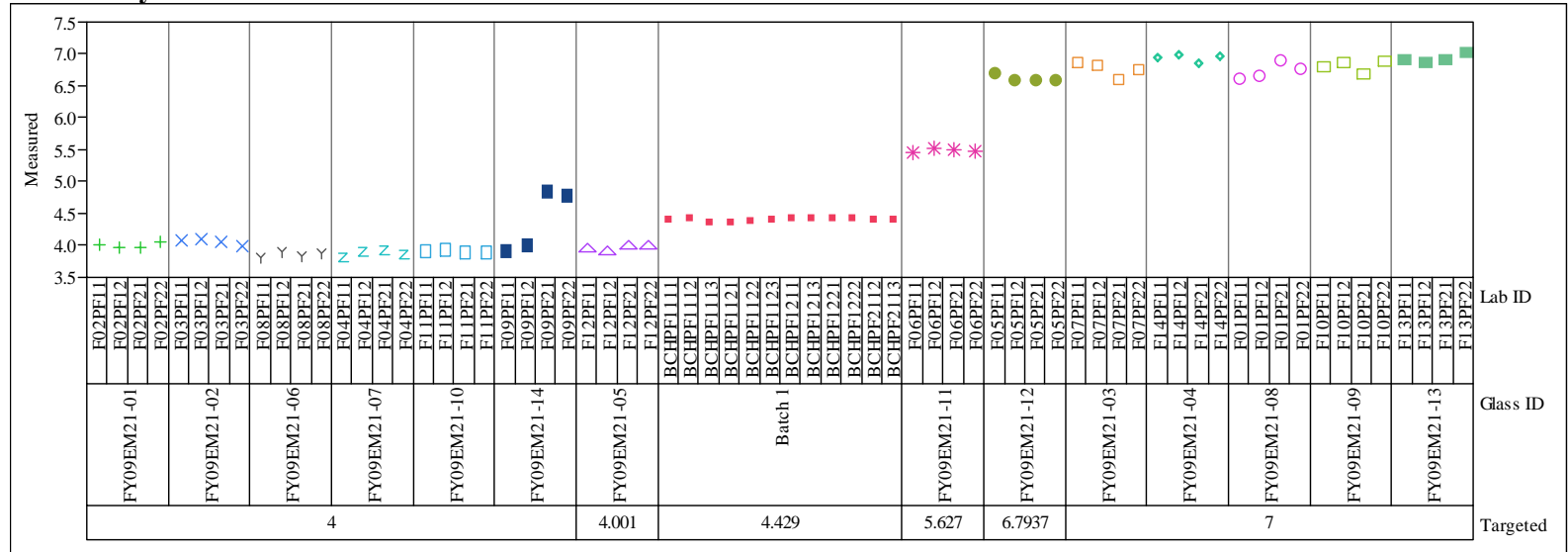

Set $=1$, Oxide $=\operatorname{MgO}($ wt $\%)$

Variability Chart for Measured

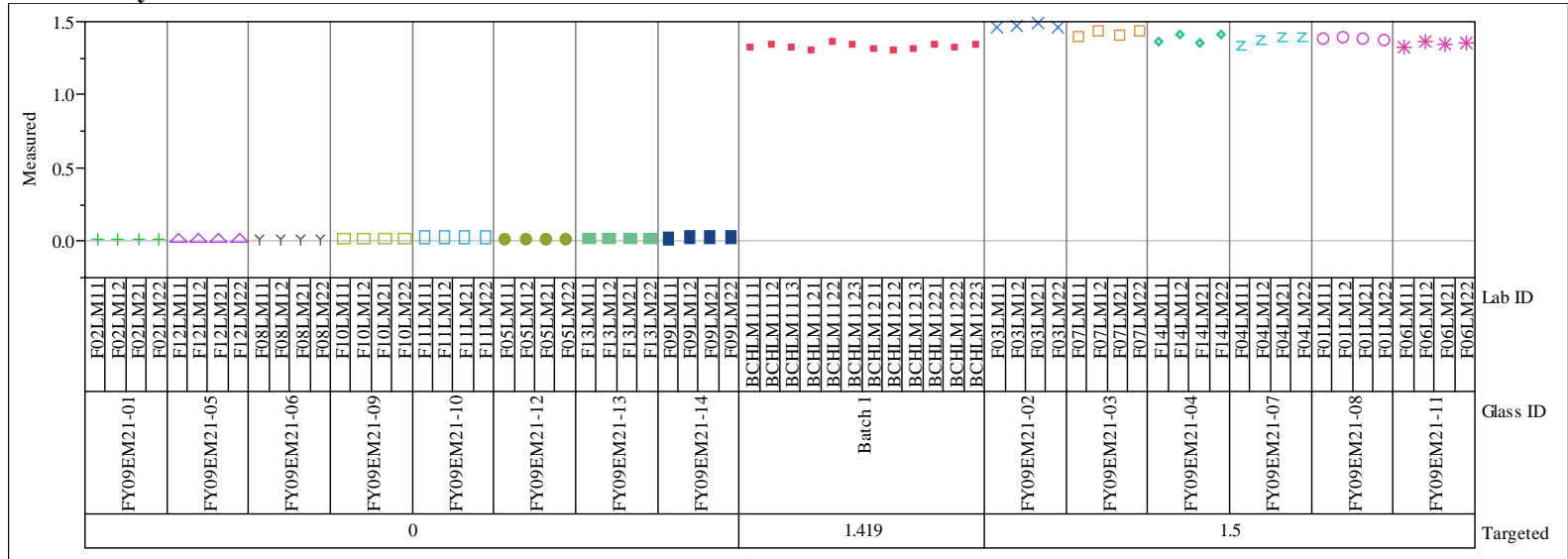


Exhibit A2. Non-Rad Sample Measurements by Lab ID within Glass ID for the Matrix 2 Study by Oxide by Analytical Block

Set $=1$, Oxide $=\mathrm{MnO}(\mathrm{wt} \%)$

Variability Chart for Measured

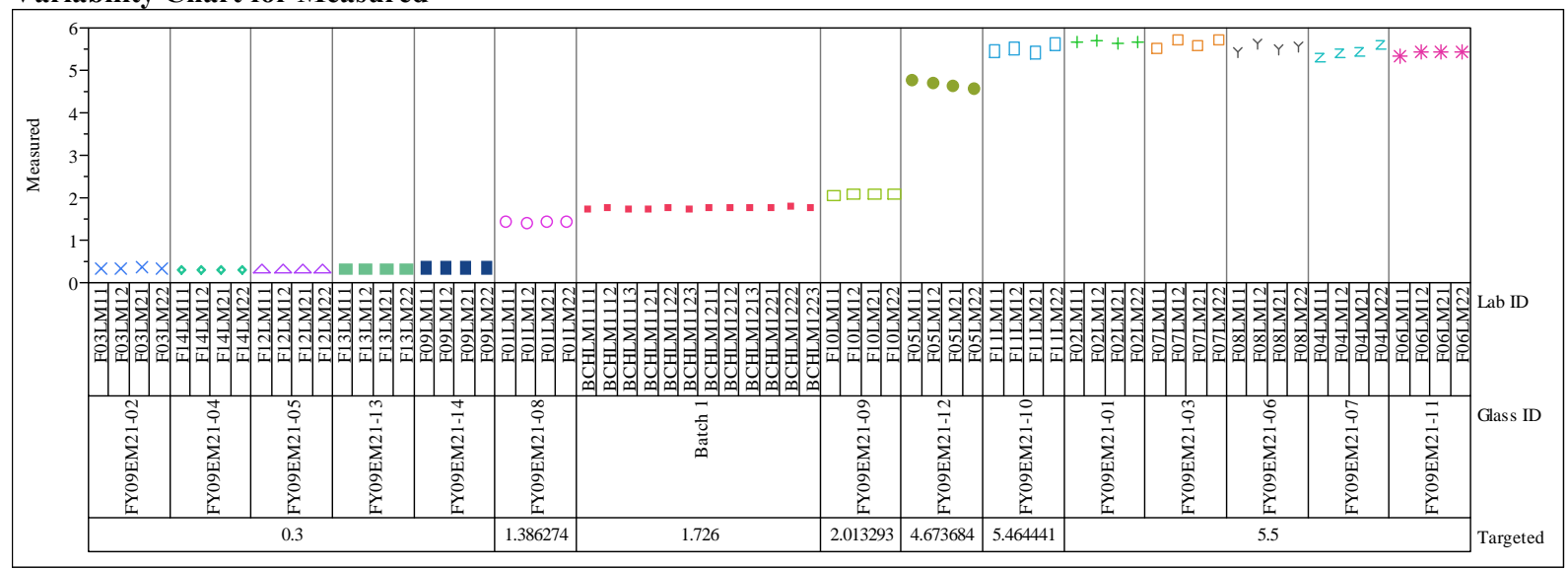

Set $=1$, Oxide $=\mathrm{Na2O}(\mathrm{wt} \%)$

Variability Chart for Measured

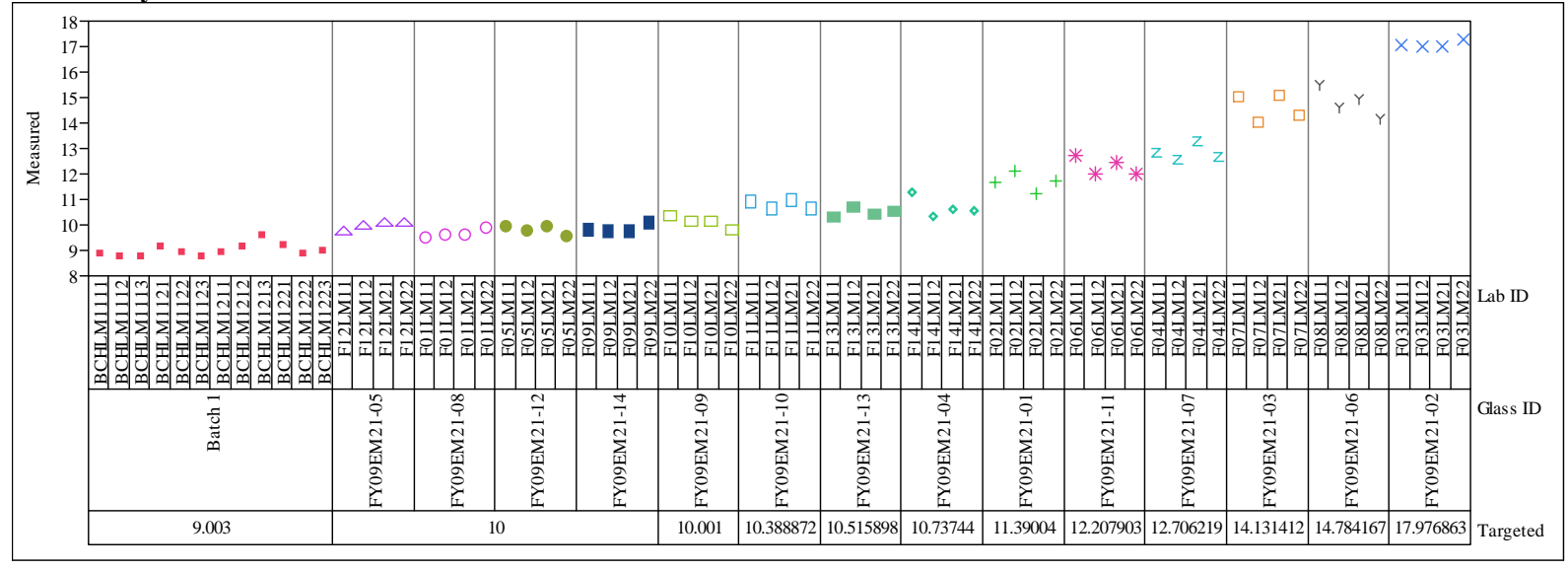

Set $=1$, Oxide $=\mathrm{NiO}(\mathrm{wt} \%)$

Variability Chart for Measured

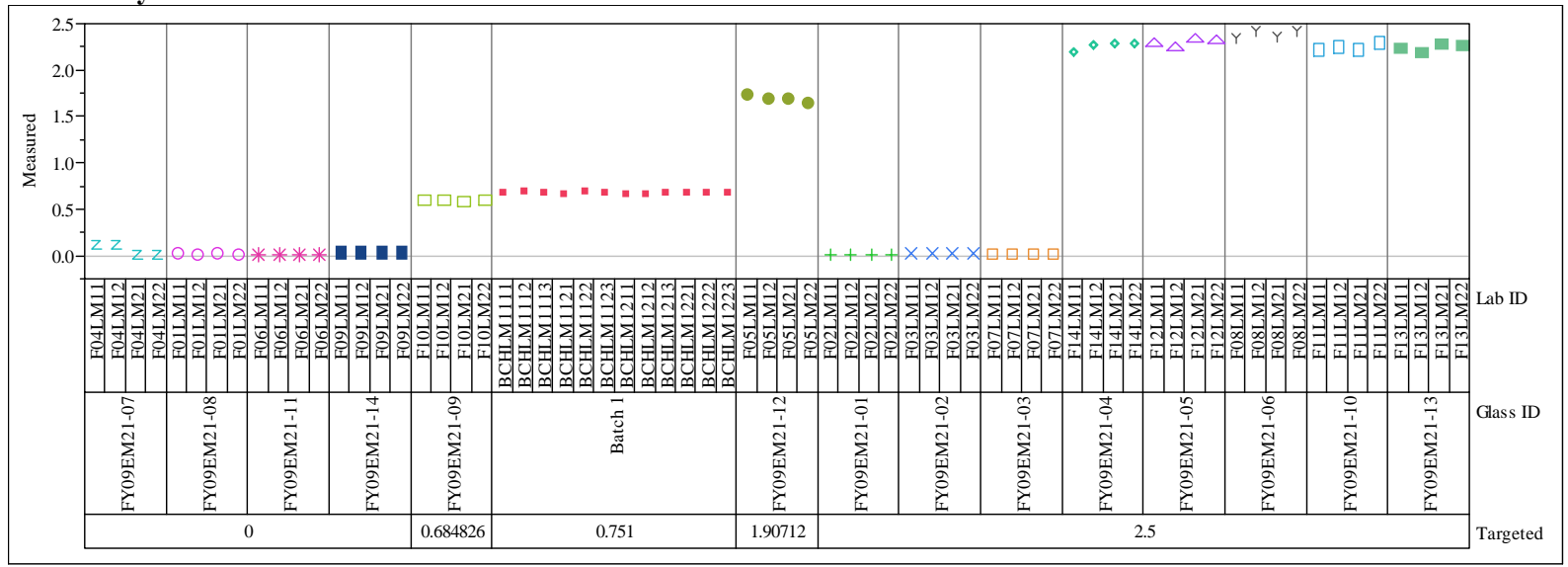


SRNL-STI-2009-00778, Revision 0

Exhibit A2. Non-Rad Sample Measurements by Lab ID within Glass ID for the Matrix 2 Study by Oxide by Analytical Block

Set $=1$, Oxide $=$ PbO $(w t \%)$

Variability Chart for Measured

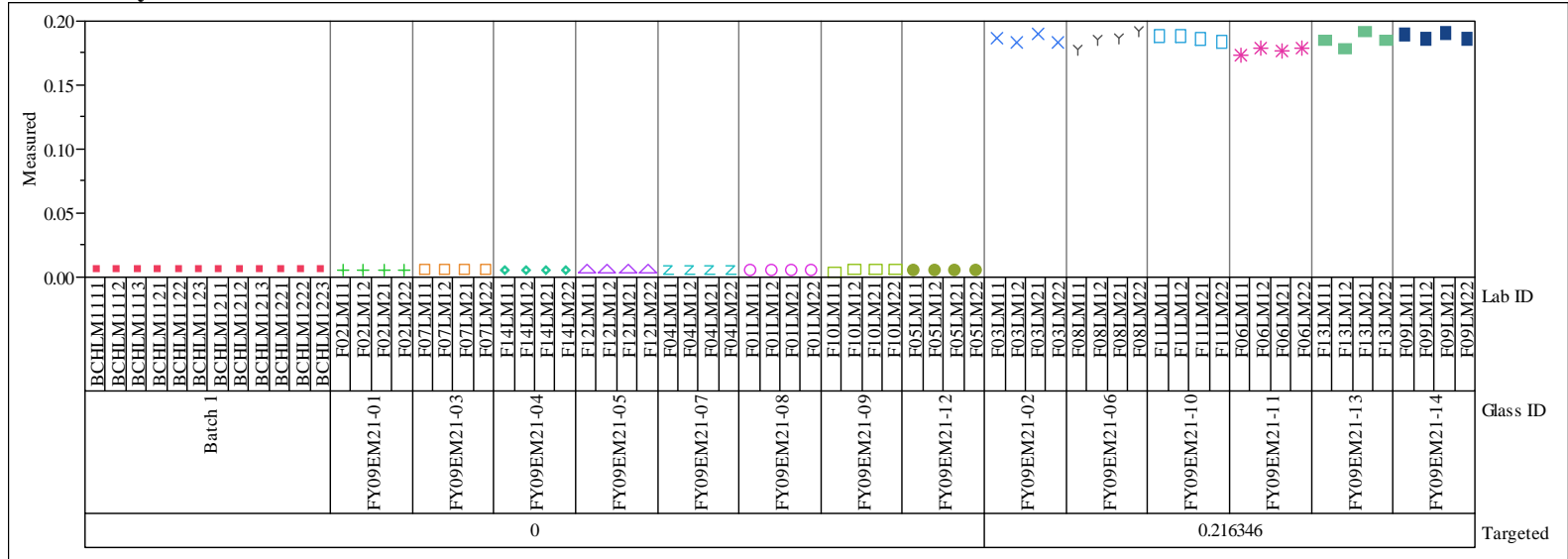

Set $=1$, Oxide $=\mathrm{SiO} 2(w t \%)$

Variability Chart for Measured

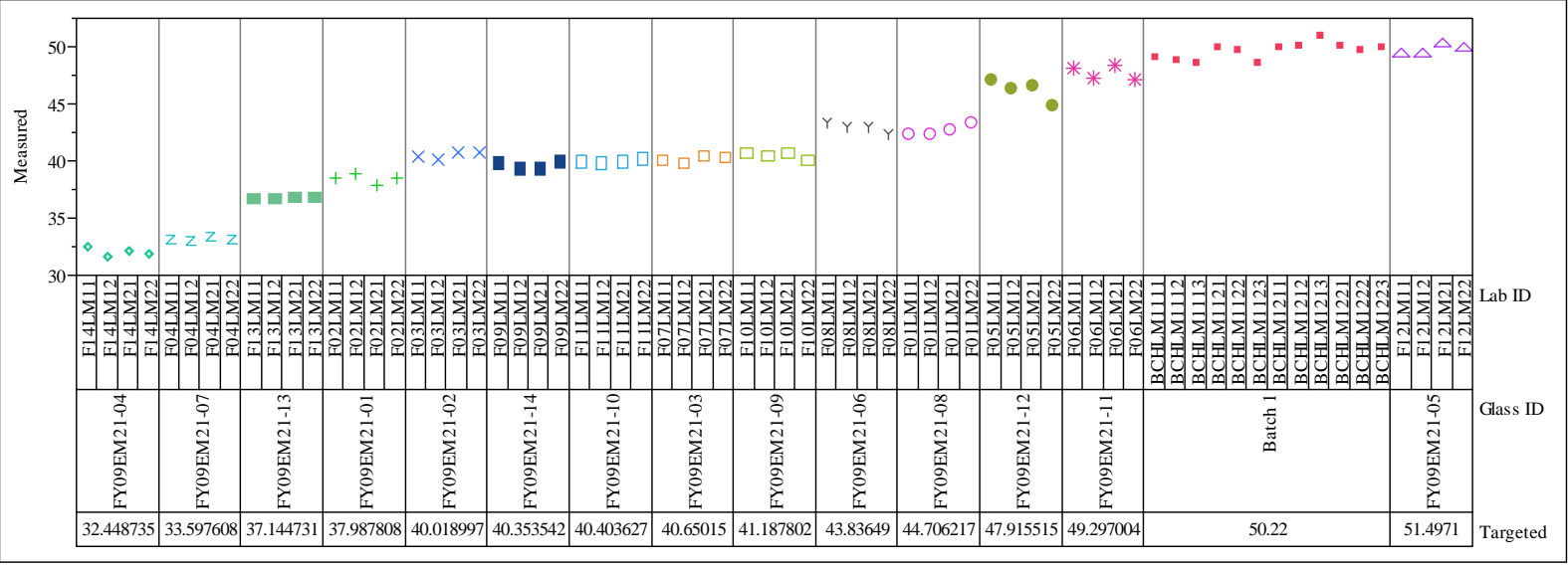

Set $=1$, Oxide $=\mathrm{SO} 4(\mathrm{wt} \%)$

Variability Chart for Measured

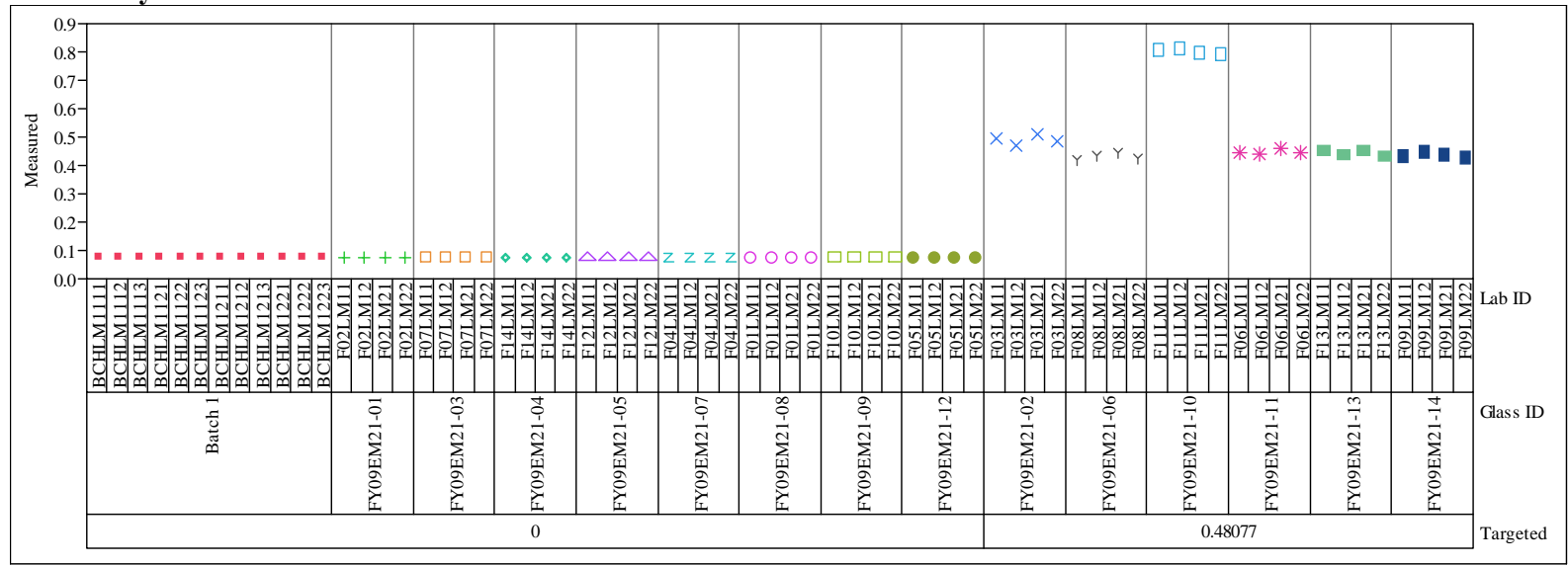


Exhibit A2. Non-Rad Sample Measurements by Lab ID within Glass ID for the Matrix 2 Study by Oxide by Analytical Block

Set $=1$, Oxide $=\mathrm{TiO} 2(\mathrm{wt} \%)$

Variability Chart for Measured

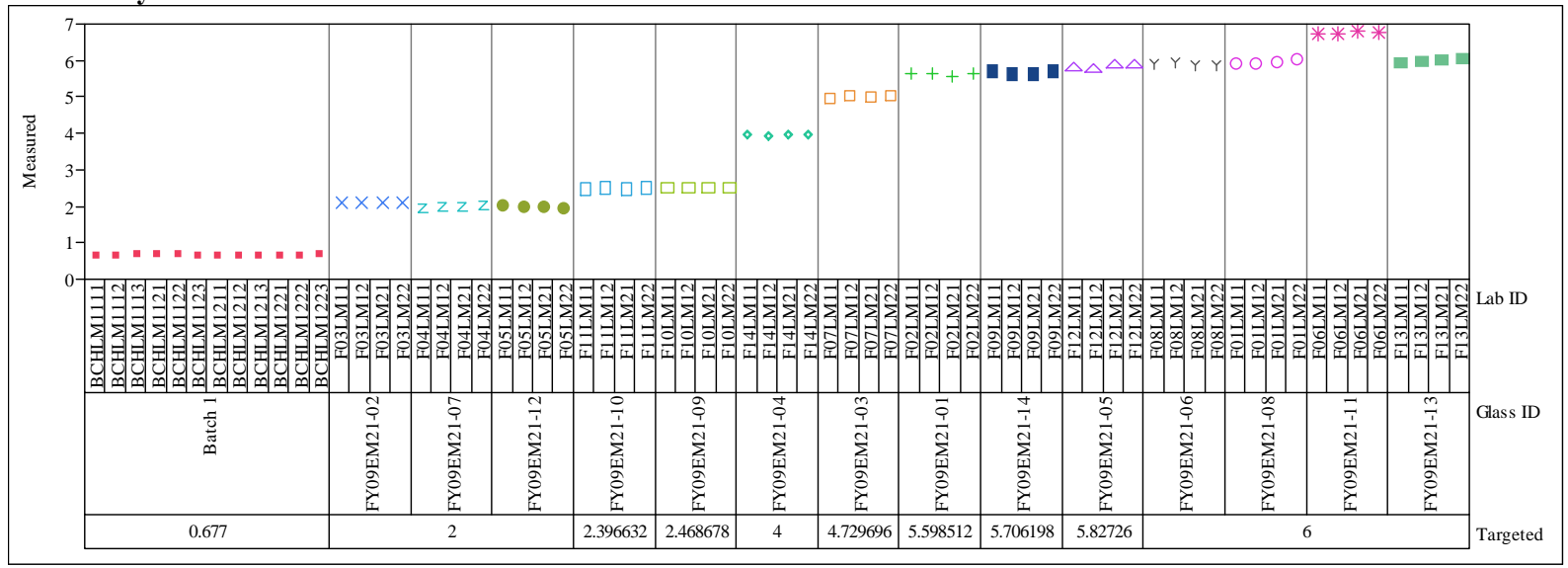

Set $=1$, Oxide $=\mathrm{ZnO}(\mathrm{wt} \%)$

Variability Chart for Measured

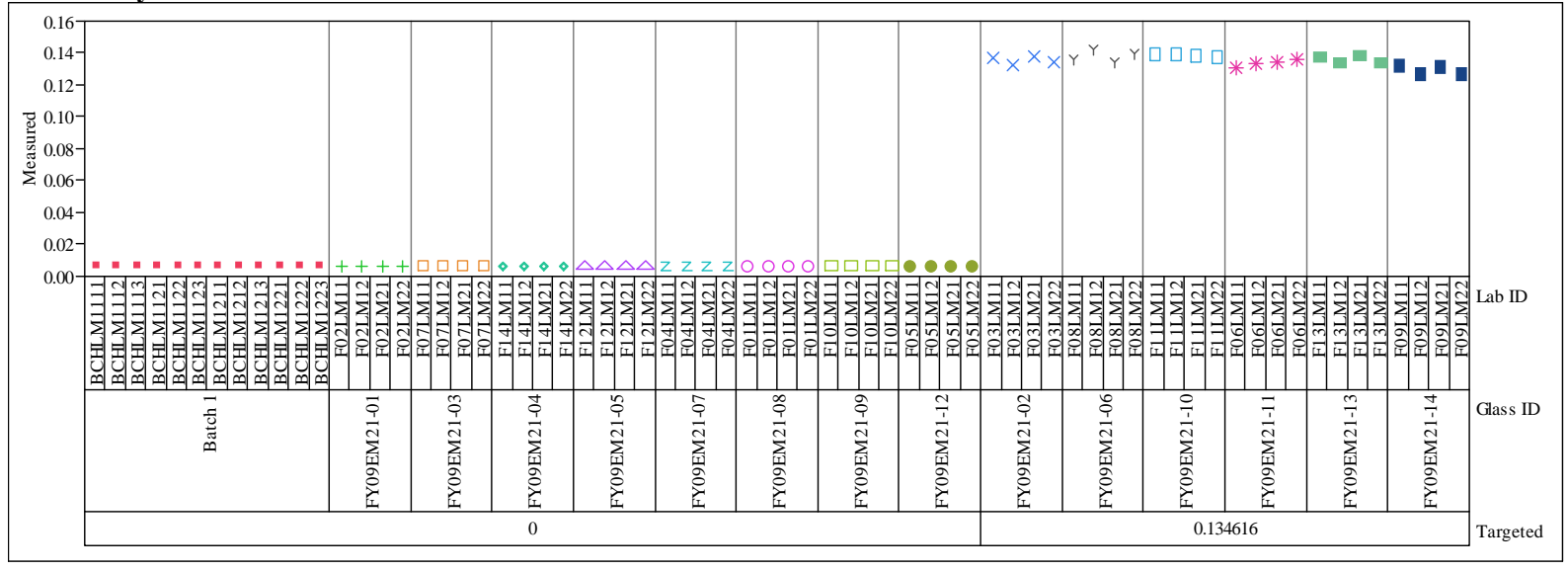

Set $=1$, Oxide $=\mathrm{ZrO2}(\mathrm{wt} \%)$

Variability Chart for Measured

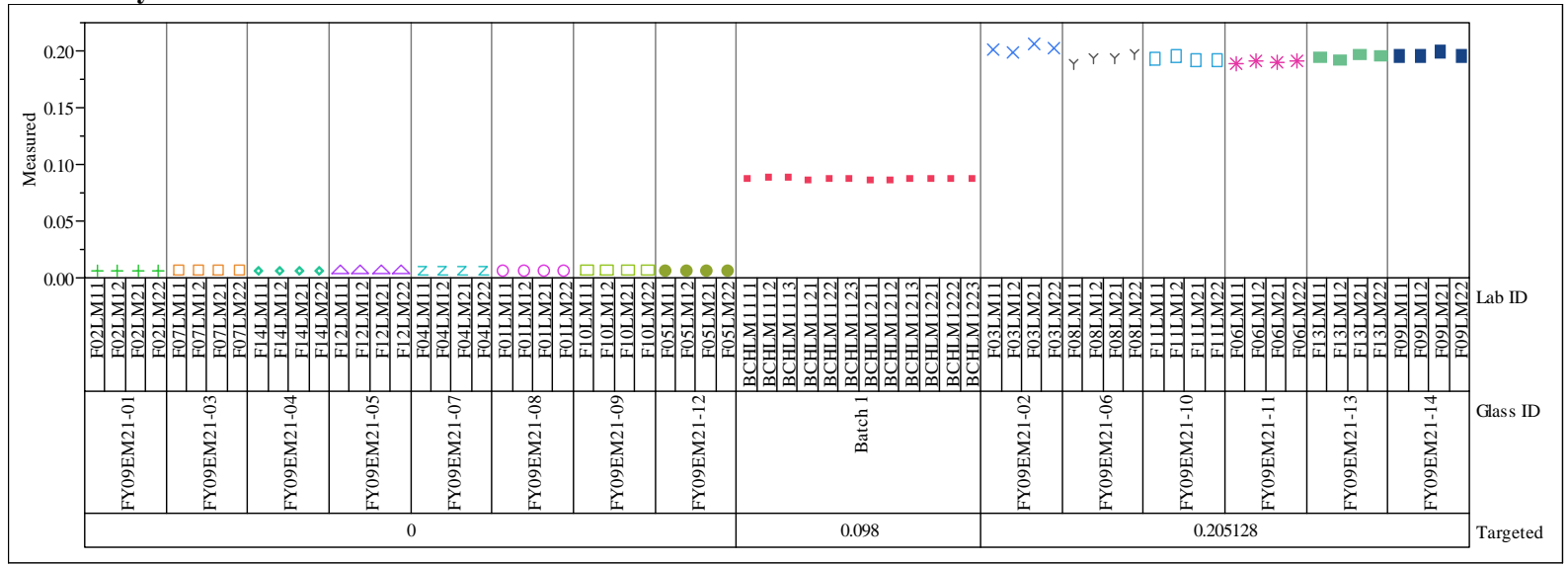


Exhibit A2. Non-Rad Sample Measurements by Lab ID within Glass ID for the Matrix 2 Study by Oxide by Analytical Block

Set $=2$, Oxide $=A 12 O 3(w t \%)$

Variability Chart for Measured

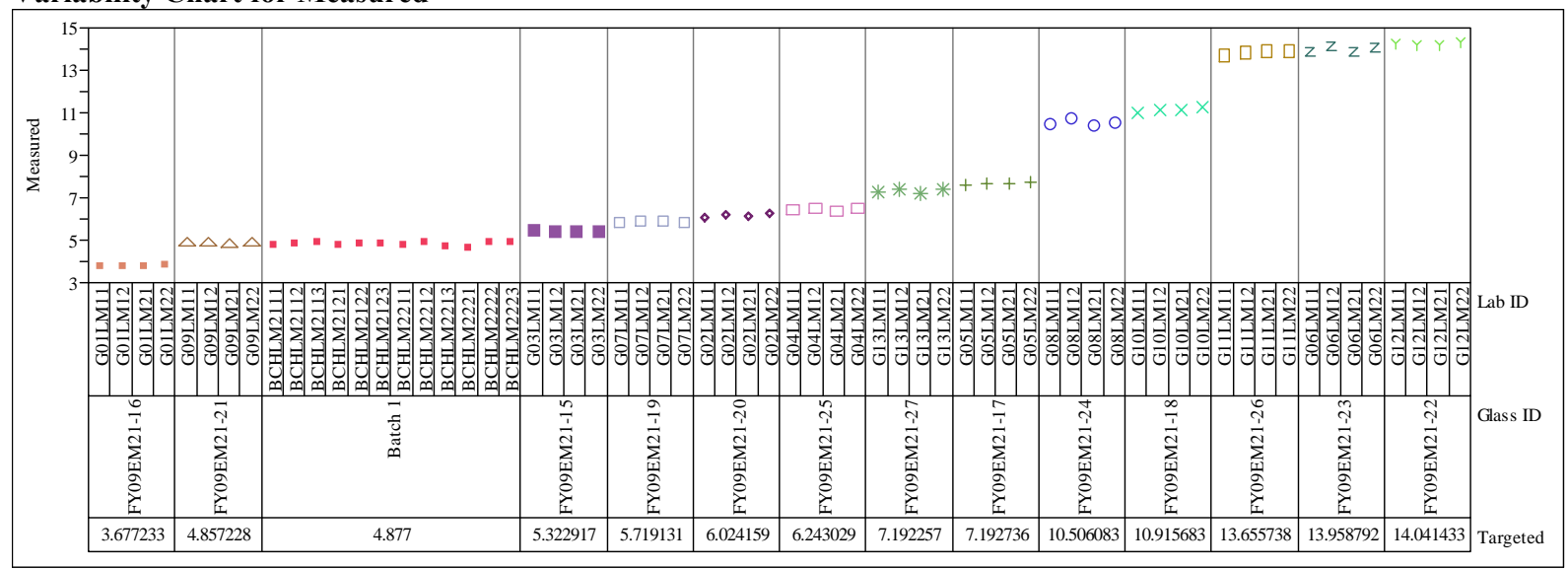

Set $=2$, Oxide $=\mathrm{B} 2 \mathrm{O} 3(\mathrm{wt} \%)$

Variability Chart for Measured

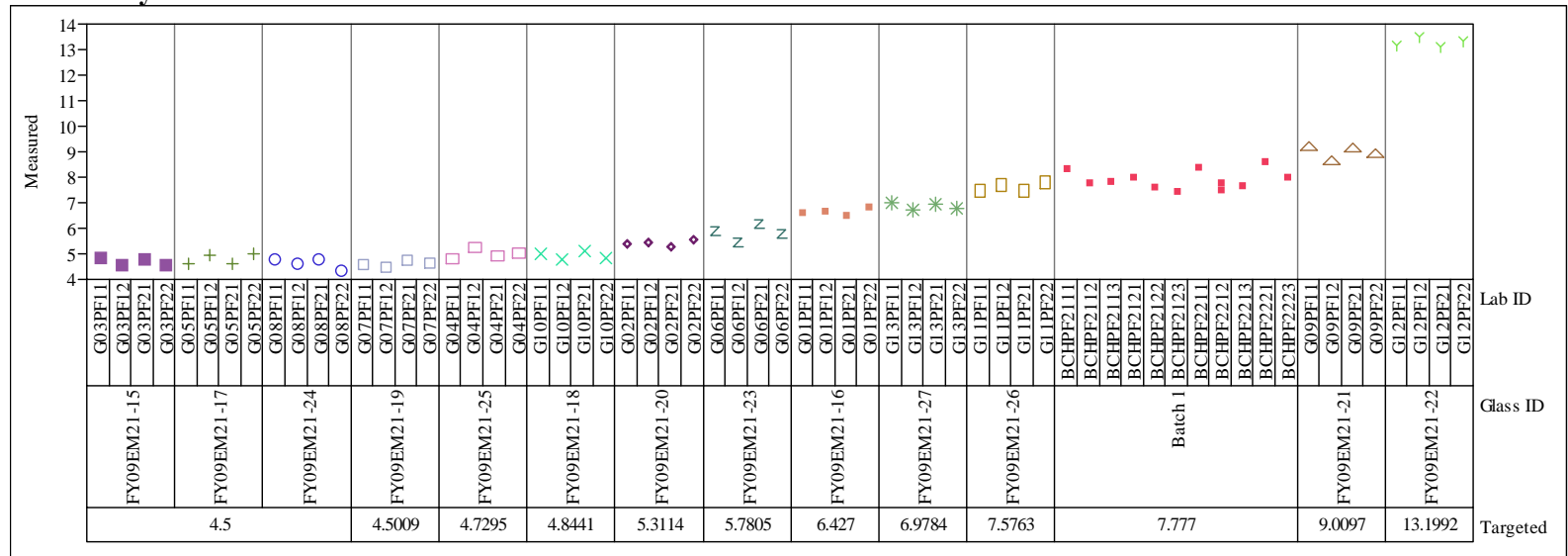

Set $=2$, Oxide $=\mathrm{BaO}(\mathrm{wt} \%)$

Variability Chart for Measured

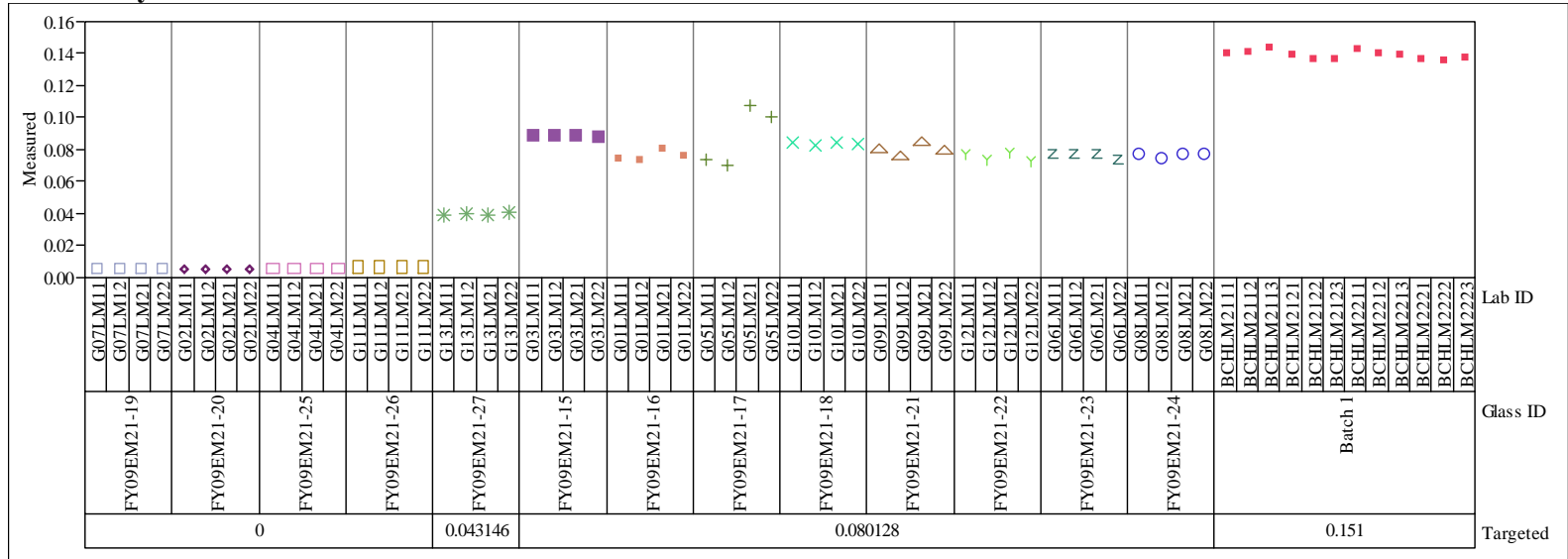


Exhibit A2. Non-Rad Sample Measurements by Lab ID within Glass ID for the Matrix 2 Study by Oxide by Analytical Block

Set $=2$, Oxide $=\mathrm{CaO}\left(\mathrm{wt}_{\mathrm{t}} \%\right)$

Variability Chart for Measured

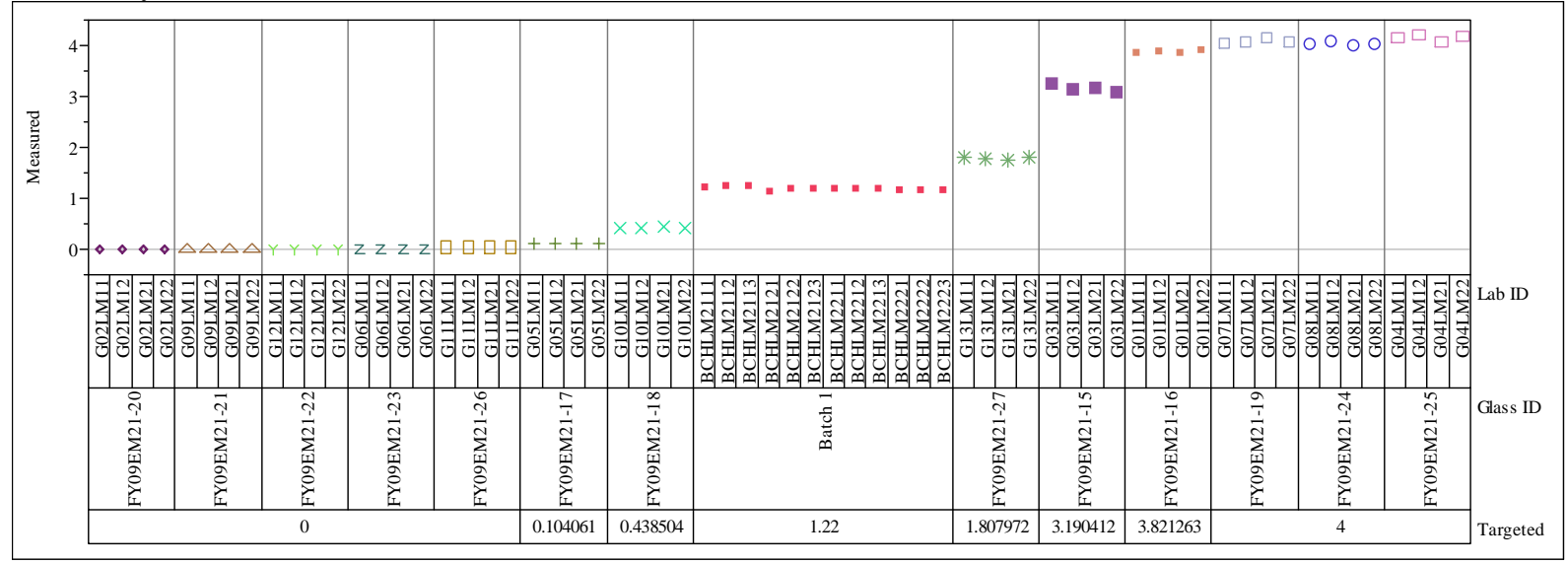

Set $=2$, Oxide $=$ CdO $($ wt $\%)$

Variability Chart for Measured

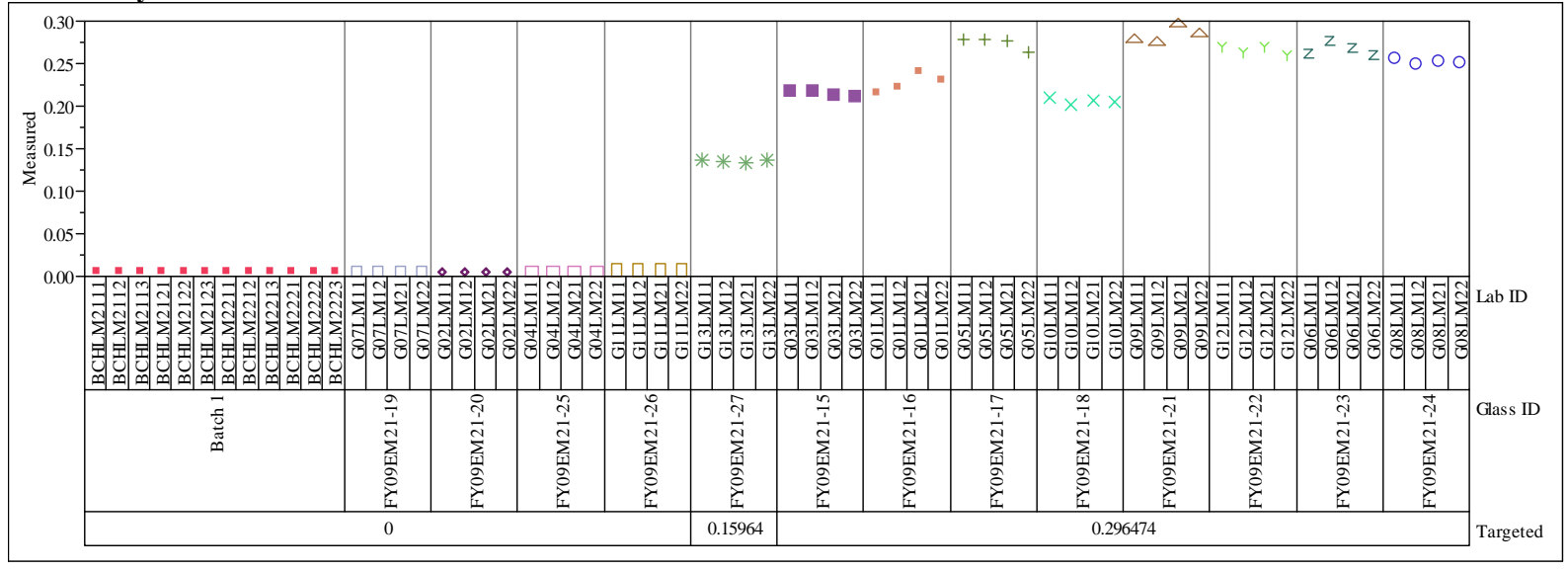

Set $=2$, Oxide $=$ Ce2O3 $($ wt $\%)$

Variability Chart for Measured

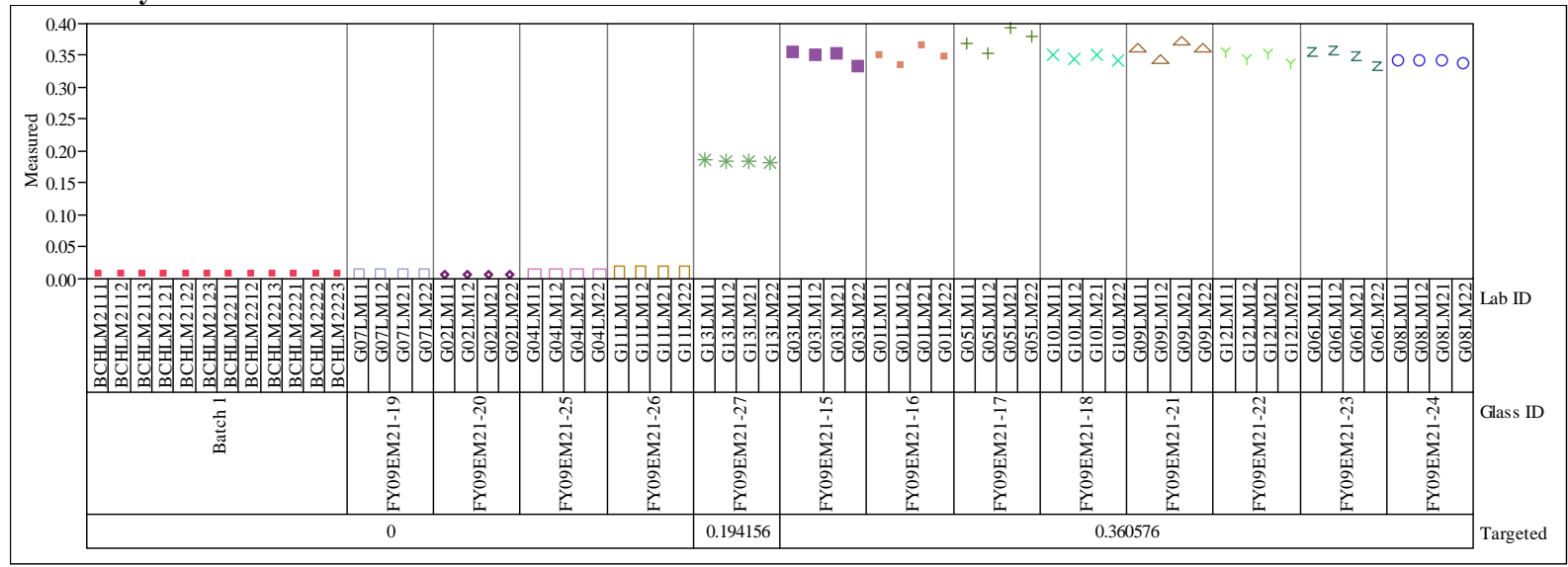


Exhibit A2. Non-Rad Sample Measurements by Lab ID within Glass ID for the Matrix 2 Study by Oxide by Analytical Block

Set $=2$, Oxide $=\operatorname{Cr2O3}(\mathbf{w t} \%)$

Variability Chart for Measured

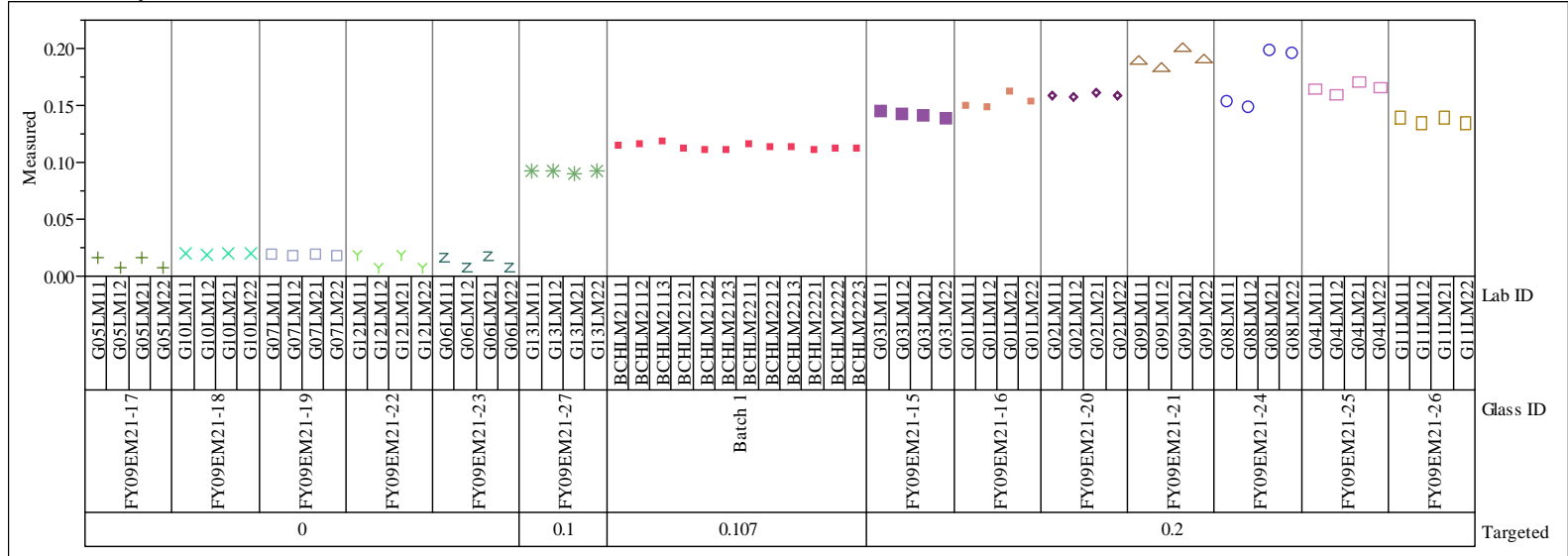

Set $=2$, Oxide $=\mathrm{CuO}(\mathbf{w t} \%)$

Variability Chart for Measured

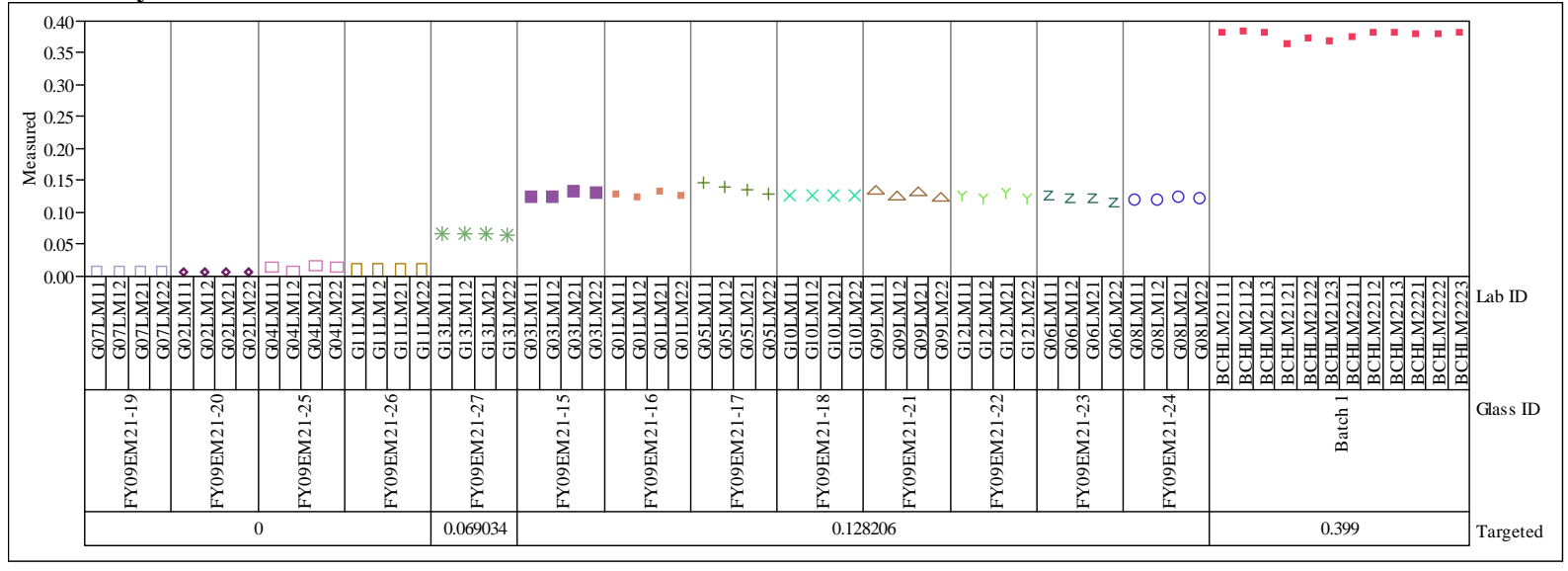

Set $=2$, Oxide $=$ Fe2O3 $($ wt $\%)$

Variability Chart for Measured

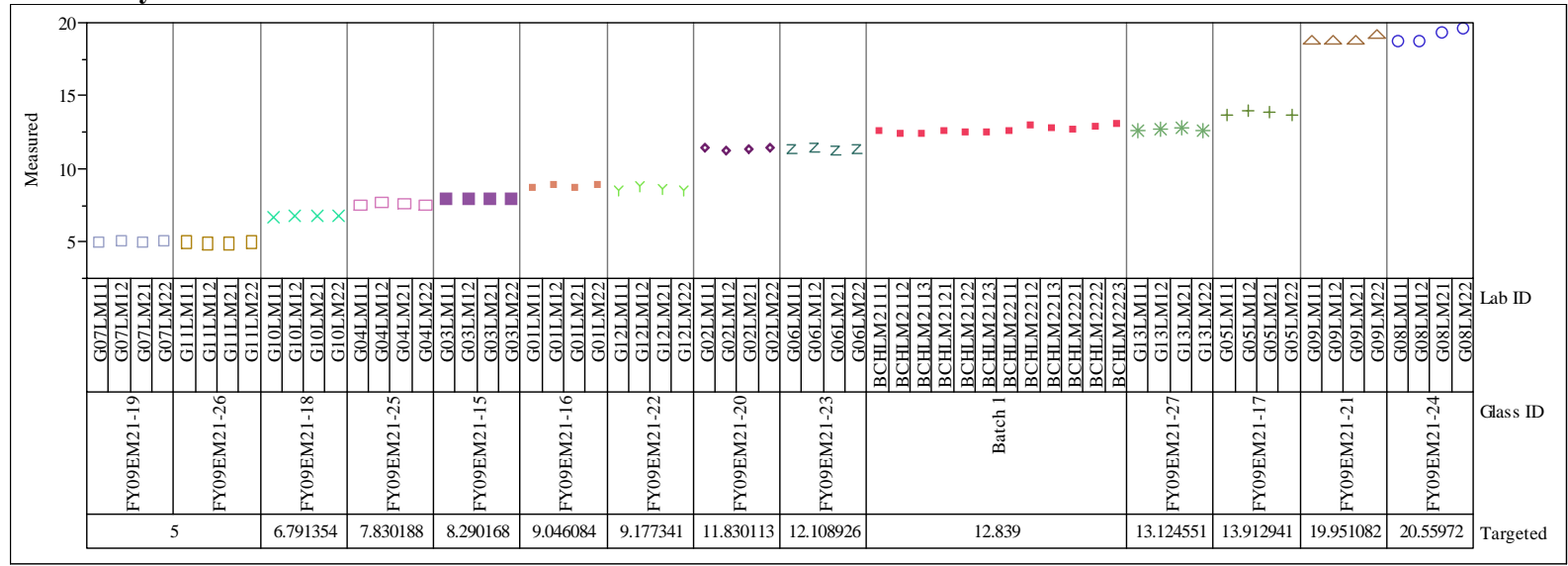


Exhibit A2. Non-Rad Sample Measurements by Lab ID within Glass ID for the Matrix 2 Study by Oxide by Analytical Block

Set $=2$, Oxide $=$ La2O3 $($ wt $\%)$

Variability Chart for Measured

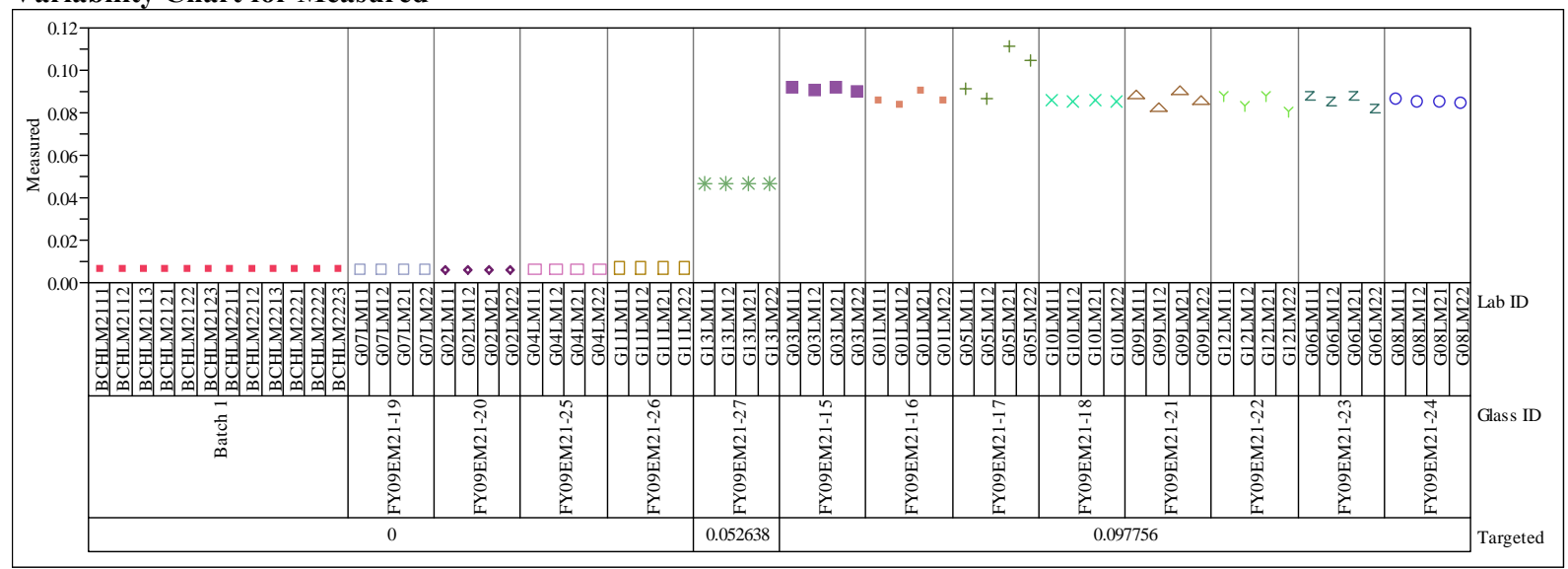

Set $=2$, Oxide $=$ Li2O $(w t \%)$

Variability Chart for Measured

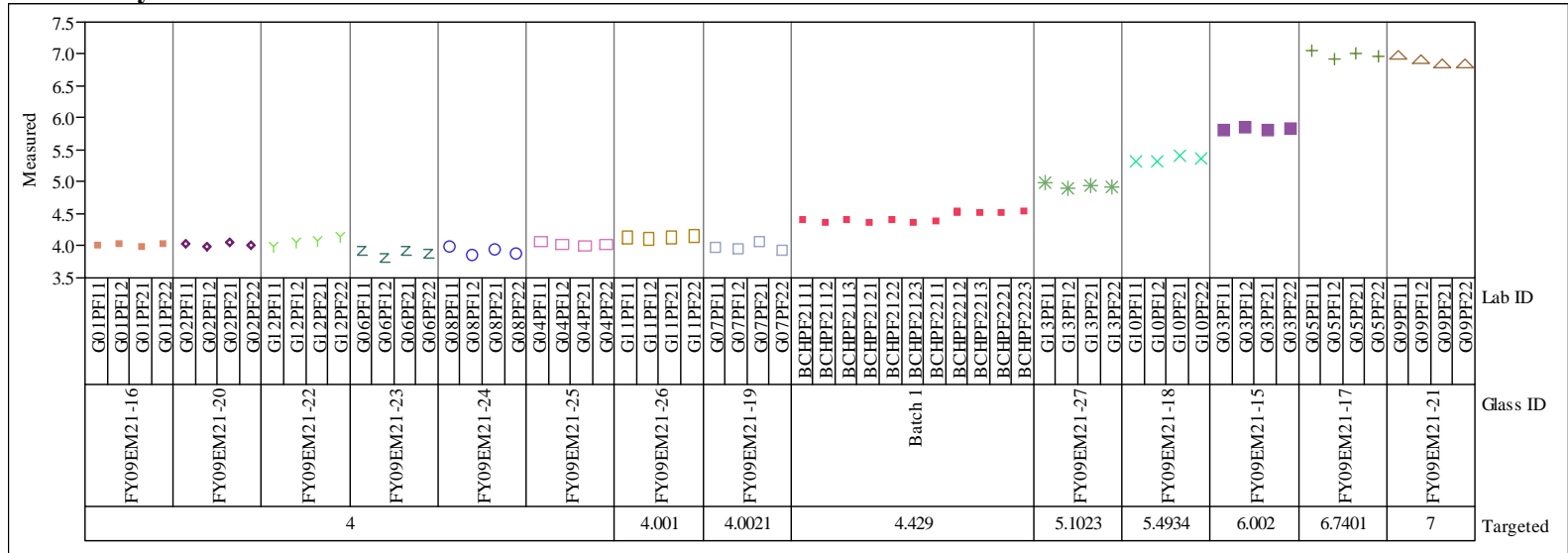

Set $=2$, Oxide $=\operatorname{MgO}($ wt $\%)$

Variability Chart for Measured

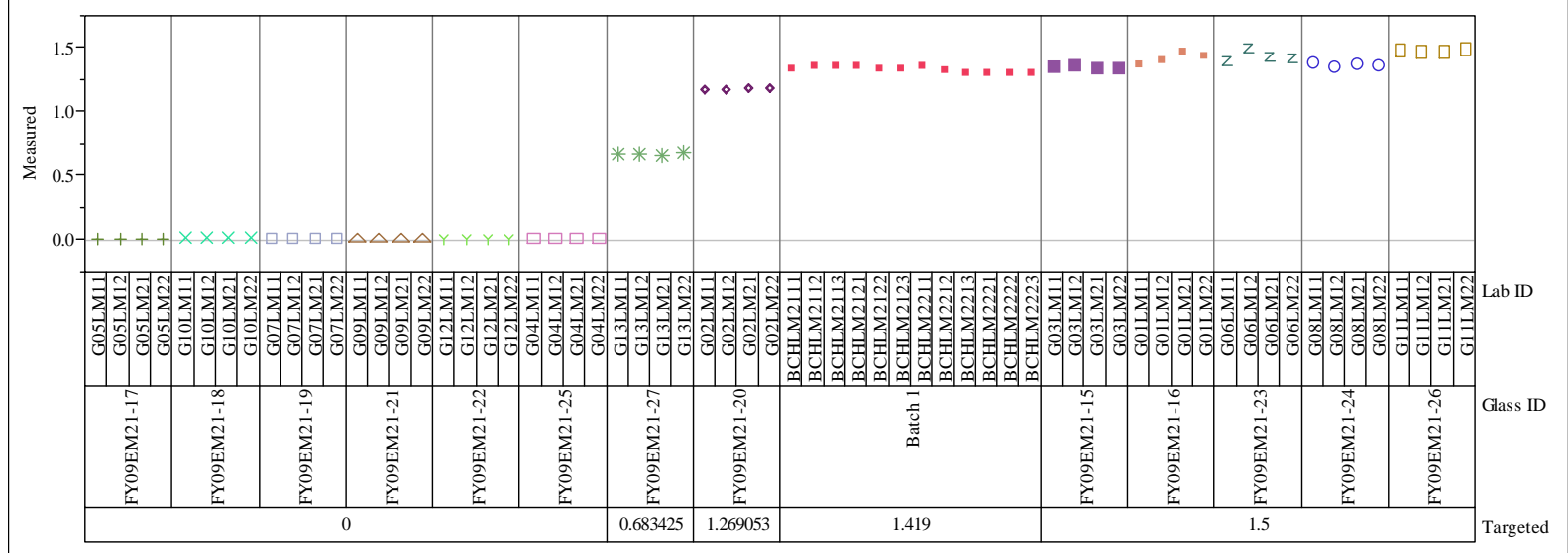


Exhibit A2. Non-Rad Sample Measurements by Lab ID within Glass ID for the Matrix 2 Study by Oxide by Analytical Block

Set $=2$, Oxide $=\mathrm{MnO}(\mathrm{wt} \%)$

Variability Chart for Measured

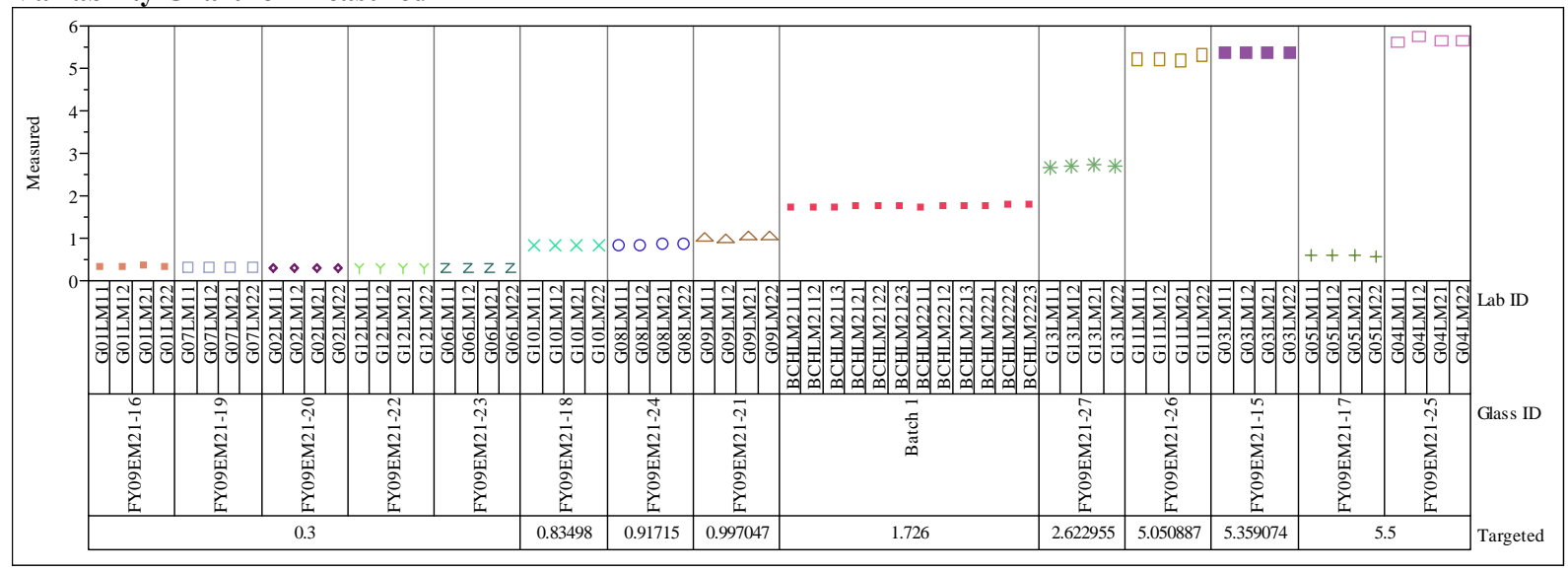

Set $=2$, Oxide $=\mathrm{Na2O}(\mathrm{wt} \%)$

Variability Chart for Measured

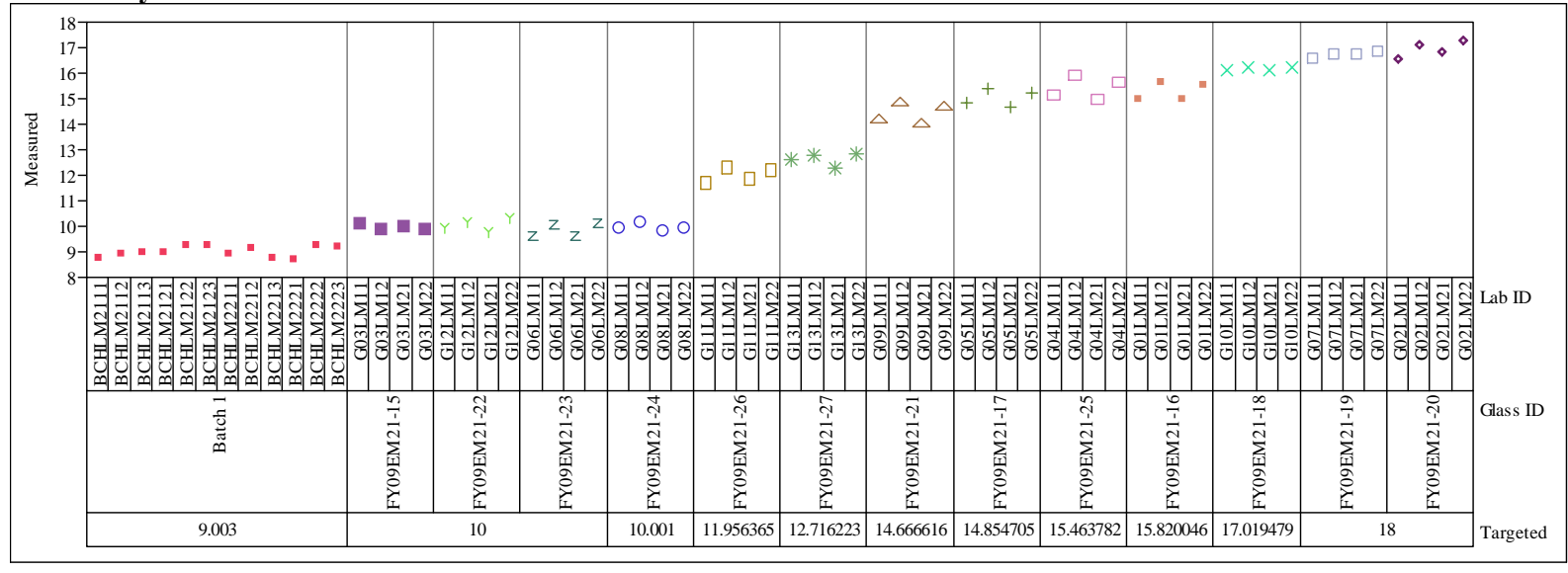

Set $=2$, Oxide $=\mathrm{NiO}(\mathrm{wt} \%)$

Variability Chart for Measured

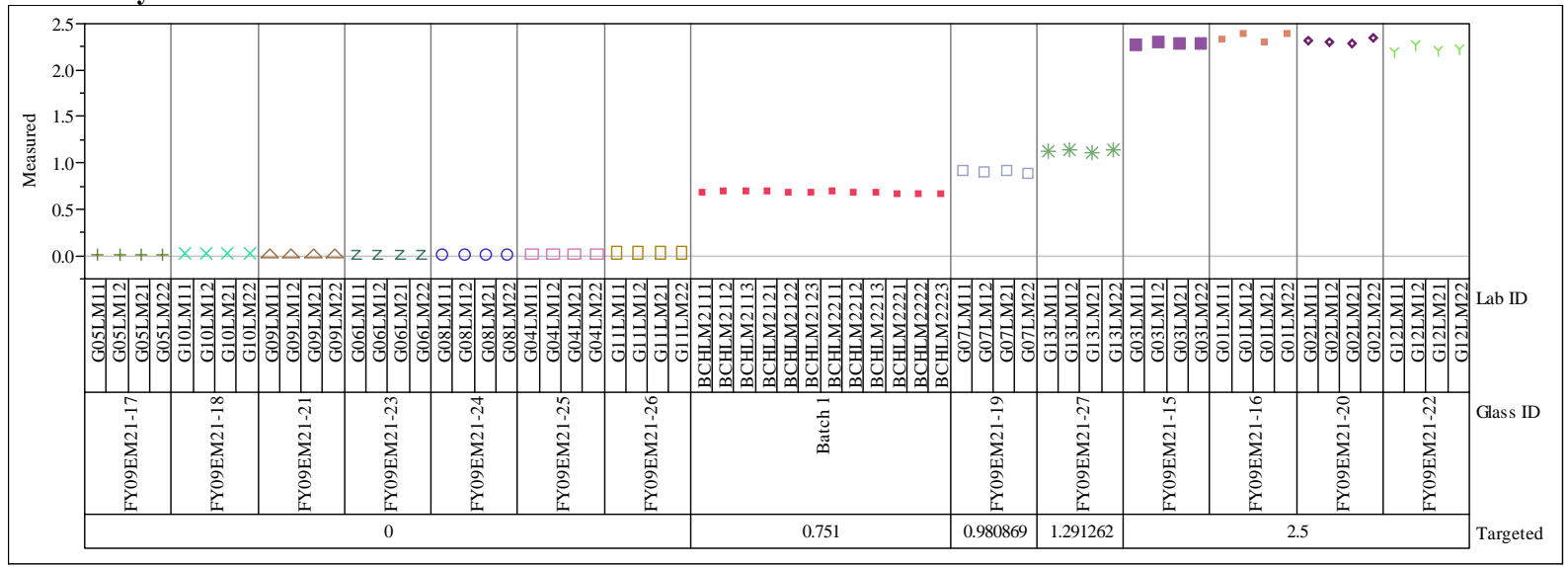


Exhibit A2. Non-Rad Sample Measurements by Lab ID within Glass ID for the Matrix 2 Study by Oxide by Analytical Block

Set $=2$, Oxide $=$ PbO $($ wt $\%)$

Variability Chart for Measured

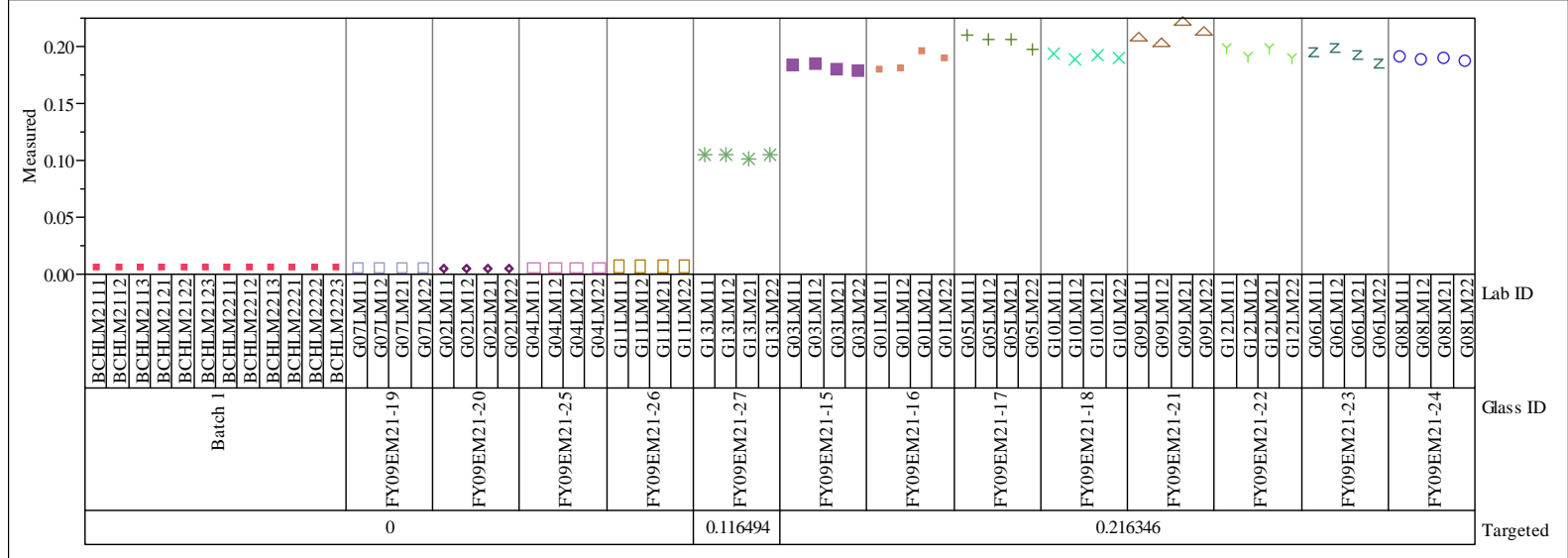

Set $=2$, Oxide $=\mathrm{SiO} 2($ wt $\%)$

Variability Chart for Measured

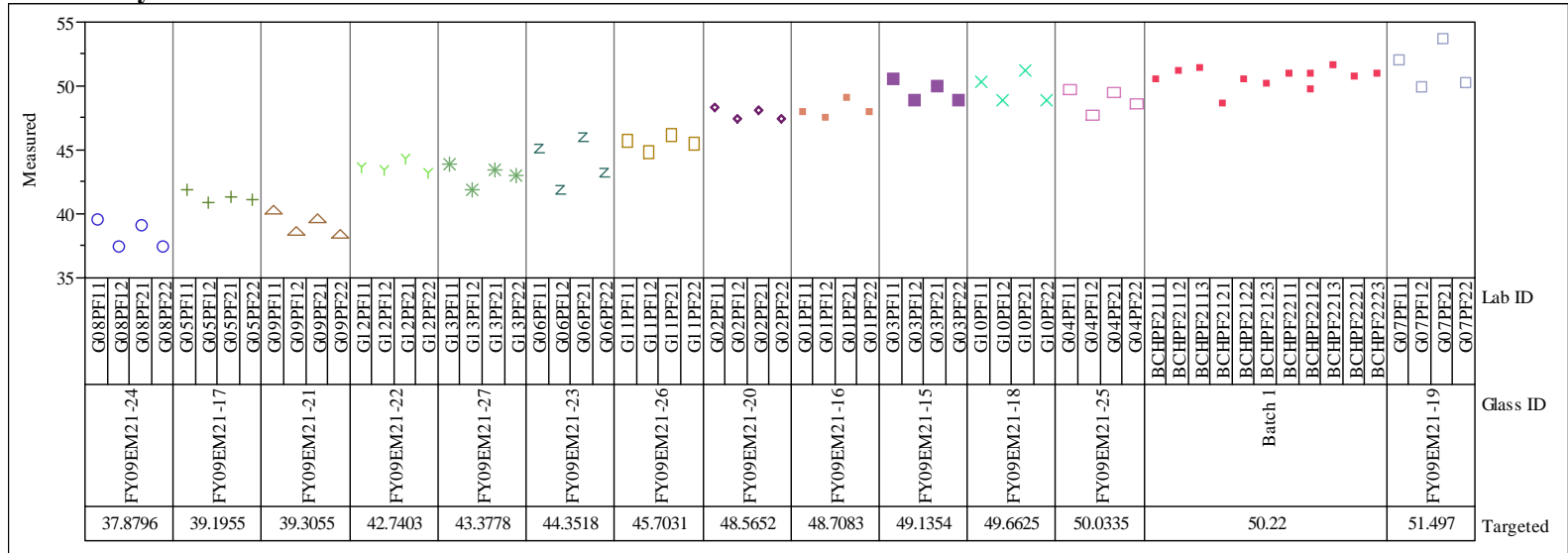

Set $=2$, Oxide $=$ SO4 $($ wt $\%)$

Variability Chart for Measured
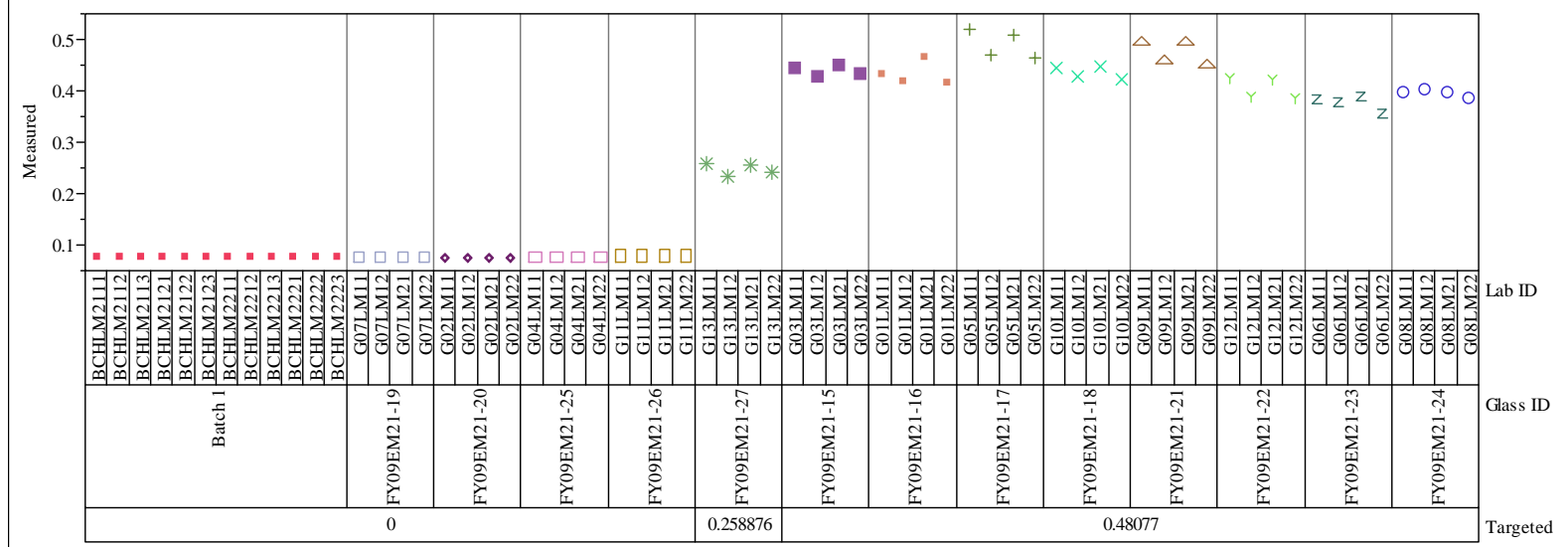
Exhibit A2. Non-Rad Sample Measurements by Lab ID within Glass ID for the Matrix 2 Study by Oxide by Analytical Block

Set $=2$, Oxide $=\mathrm{TiO} 2(\mathrm{wt} \%)$

Variability Chart for Measured

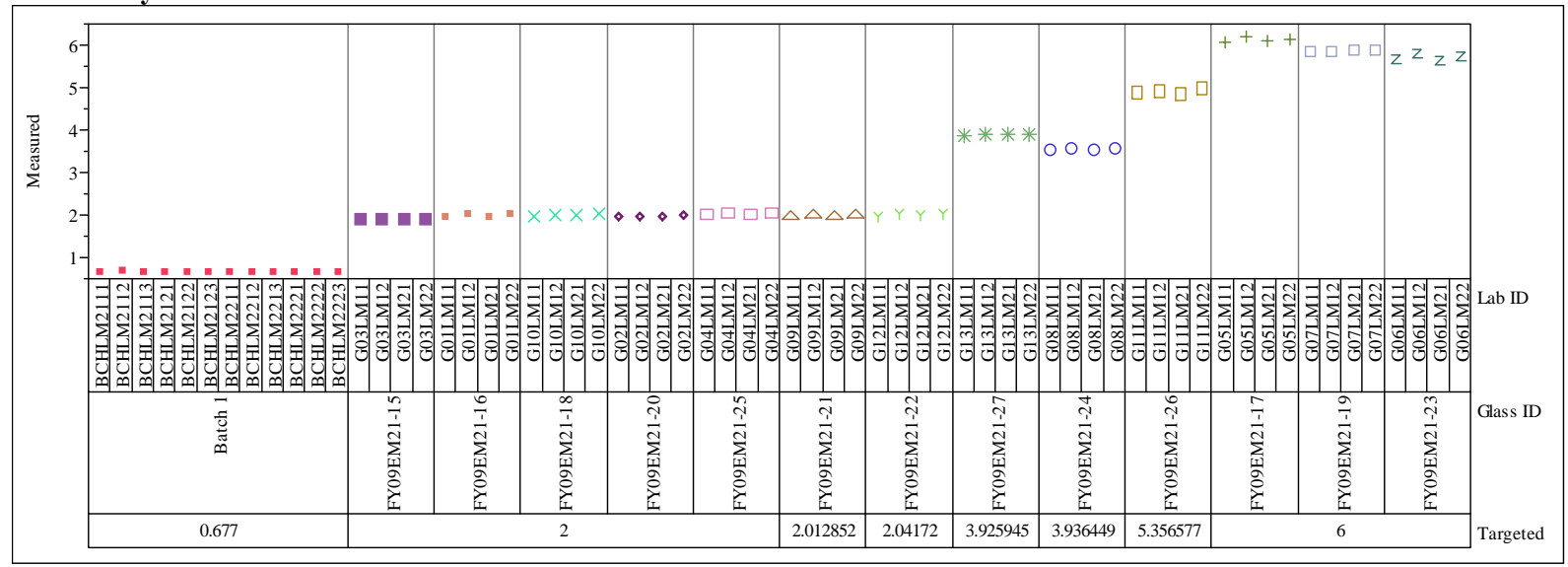

Set $=2$, Oxide $=\mathrm{ZnO}(\mathrm{wt} \%)$

Variability Chart for Measured

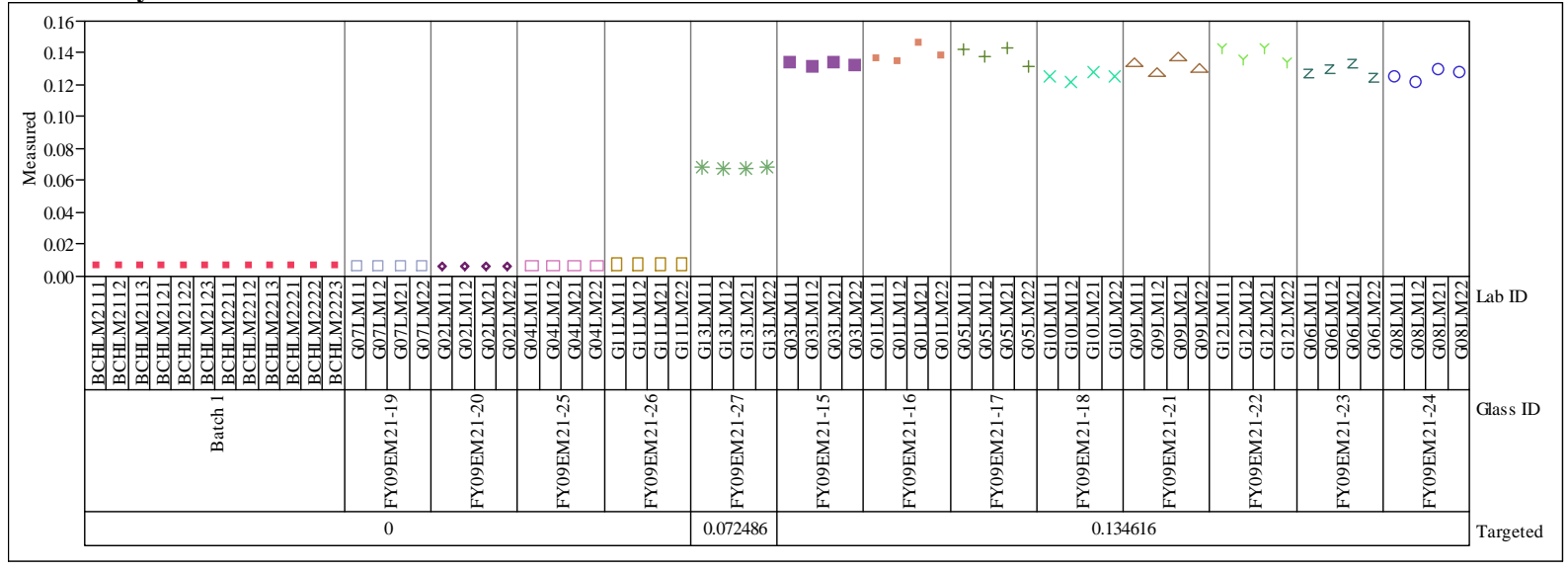

Set $=2$, Oxide $=\mathrm{ZrO} 2(\mathrm{wt} \%)$

Variability Chart for Measured

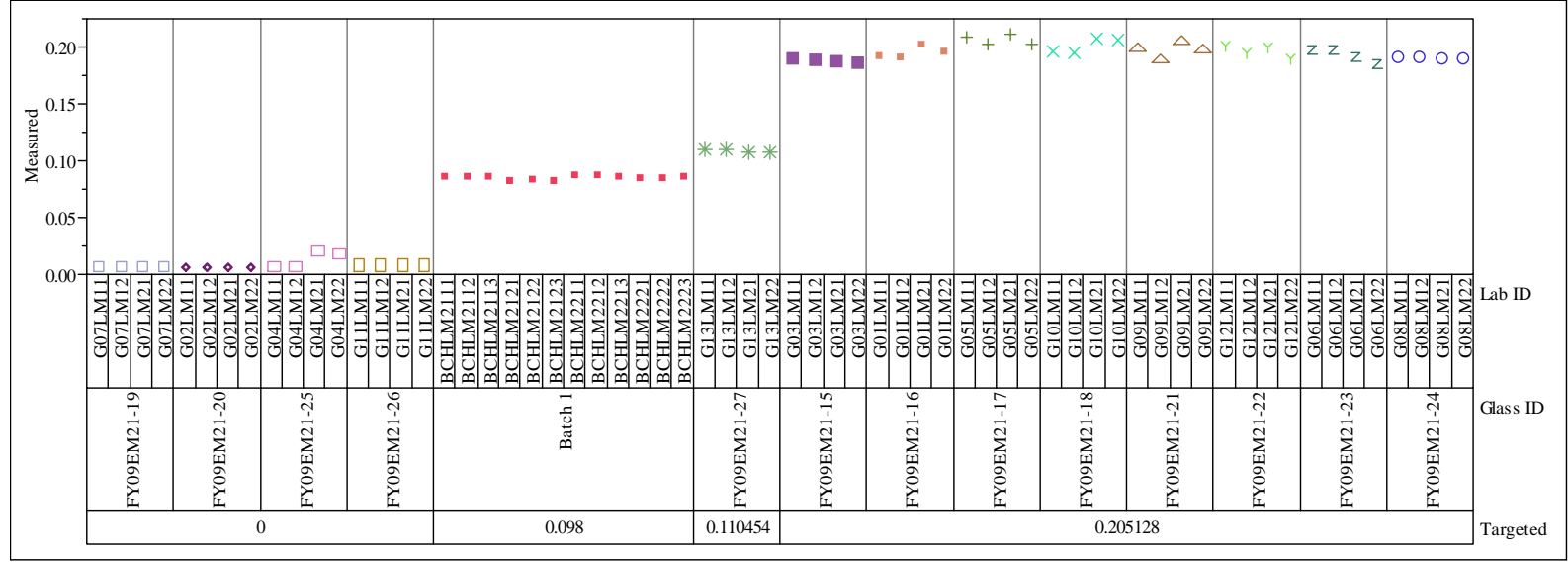


SRNL-STI-2009-00778, Revision 0

Exhibit A3. Batch 1 Sample Measurements by Block and Sub-Block

by Oxide for Both Preparation Methods for the Non-Rad Matrix 2 Study

Oneway Analysis of Measured By Set/Block/Sub-Block

Reference Value $=4.877 \mathrm{wt} \%$

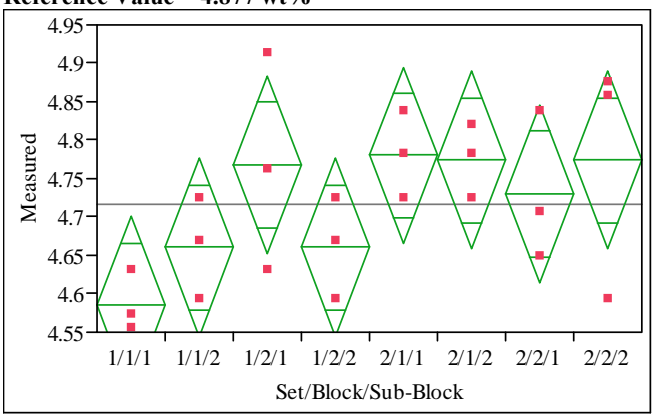

Oneway Anova

Summary of Fit

$\begin{array}{lr}\text { Rsquare } & 0.440685 \\ \text { Adj Rsquare } & 0.195985 \\ \text { Root Mean Square Error } & 0.093843 \\ \text { Mean of Response } & 4.716664 \\ \text { Observations (or Sum Wgts) } & 24\end{array}$

Analysis of Variance

Source DF Sum of

Set/Block/Sub- $\quad 7 \quad \begin{array}{r}\text { Squares } \\ \text { - }\end{array}$

Block $\quad 16 \quad 0.14090430$

$\begin{array}{lll}\text { Error } & 16 & 0.14090430 \\ \text { C. Total } & 23 & 0.25192296\end{array}$

Mean

Square Ratio $\begin{array}{rrr}\text { Ratio } & \text { F } \\ 0.015860 & 1.8009 & 0.156\end{array}$

0.008807

Means for Oneway Anova

Level Number Mean Std Error Lower 95\% Upper 95\%

$\begin{array}{llllrr}1 / 1 / 1 & 3 & 4.58519 & 0.05418 & 4.4703 & 4.7000\end{array}$

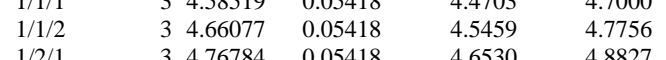

$\begin{array}{llllll}1 / 2 / 1 & 3 & 4.76784 & 0.05418 & 4.6530 & 4.8827 \\ 1 / 2 / 2 & 3 & 4.66077 & 0.05418 & 4.5459 & 4.7756\end{array}$

$\begin{array}{llllll}2 / 1 / 1 & 3 & 4.78044 & 0.05418 & 4.6656 & 4.8953\end{array}$

$\begin{array}{llllll}2 / 1 / 2 & 3 & 4.77414 & 0.05418 & 4.6593 & 4.8890\end{array}$

$\begin{array}{llllll}2 / 2 / 1 & 3 & 4.73005 & 0.05418 & 4.6152 & 4.8449 \\ 2 / 2 / 2 & 3 & 4.77414 & 0.05418 & 4.6593 & 4.8890\end{array}$

Std Error uses a pooled estimate of error variance
Oneway Analysis of Measured By Set/Block/Sub-Block

Reference Value $=7.777 \mathrm{wt} \%$

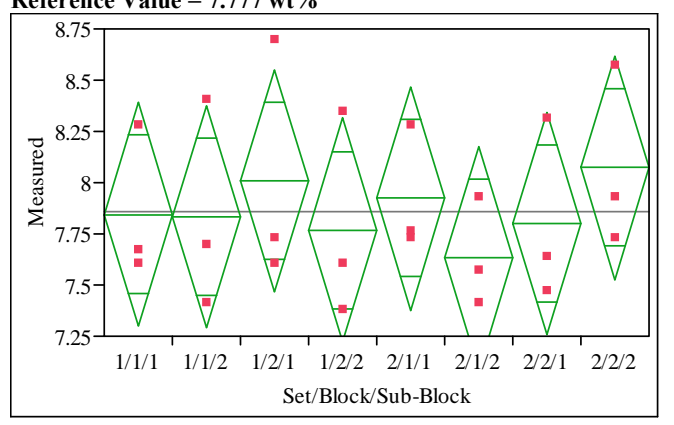

Oneway Anova

Summary of Fit

Rsquare $\quad 0.113745$

$\begin{array}{ll}\text { Adj Rsquare } & -0.27399 \\ \text { Rot Mean Square Error } & 0.442956\end{array}$

Mean of Response 7.860581

Observations (or Sum Wgts)

Analysis of Variance

Source

Set/Block/Sub- 7

Block

C. Tota

16
23

16
23

\begin{tabular}{r}
$\begin{array}{r}\text { Sum of } \\
\text { Squares }\end{array}$ \\
0.4029169 \\
\hline \\
\hline .1393565 \\
3.5422734
\end{tabular}

Means for Oneway Anova

Level Number Mean Std Error Lower 95\% Upper 95\%

$\begin{array}{llllrr}1 / 1 / 1 & 3 & 7.84582 & 0.25574 & 7.3037 & 8.3880\end{array}$

$\begin{array}{llllll}1 / 1 / 2 & 3 & 7.83509 & 0.25574 & 7.2929 & 8.3772\end{array}$

$\begin{array}{llllll}1 / 2 / 1 & 3 & 8.00682 & 0.25574 & 7.4647 & 8.5490\end{array}$

$\begin{array}{llllll}1 / 2 / 2 & 3 & 7.77069 & 0.25574 & 7.2285 & 8.3128 \\ 2 & 3 & 7.92095 & 0.25574 & 7.3788 & 8.4631\end{array}$

\begin{tabular}{llllll}
$2 / 1 / 1$ & 3 & 7.92095 & 0.25574 & 7.3788 & 8.4631 \\
\hline & 3 & 7.63116 & 0.25574 & 7.0890 & 8.1733
\end{tabular}

$\begin{array}{llllll}2 / 1 / 2 & 3 & 7.63116 & 0.25574 & 7.0890 & 8.1733 \\ 2 / 2 / 1 & 3 & 7.80289 & 0.25574 & 7.2607 & 8.3450 \\ 2 / 2 / 2 & 3 & 8.07122 & 0.25574 & 7.5291 & 8.6134\end{array}$

$\begin{array}{llllll}2 / 2 / 2 & 3 & 8.07122 & 0.25574 & 7.5291 & 8.6134\end{array}$

Std Error uses a pooled estimate of error variance
Oneway Analysis of Measured By Set/Block/Sub-Block Oxide $=\mathrm{BaO}\left(\mathrm{wt}_{\mathrm{t}} \%\right)$

Reference Value $=0.151 \mathrm{wt} \%$

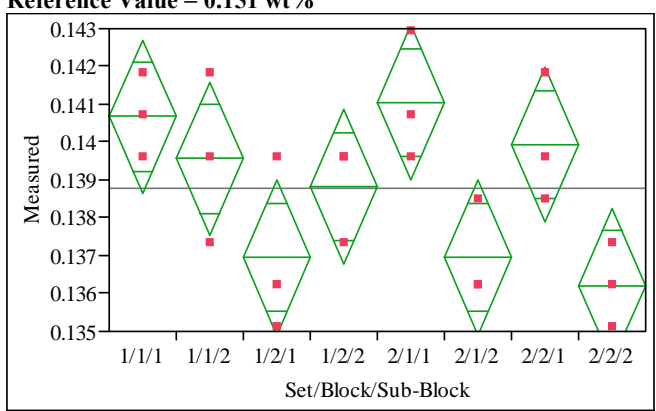

Oneway Anova

Summary of Fit

$\begin{array}{ll} & 0.619901 \\ \text { Adj Rsquare } & 0.453608\end{array}$

Root Mean Square Error $\quad 0.001659$

Mean of Response $\quad 0.138772$

Observations (or Sum Wgts) $\quad 24$

Analysis of Variance

Source DF Sum of

$\begin{array}{lrr}\text { Set/Block/Sub- } & 7 & 0.00007183\end{array}$

$\begin{array}{rrr}\text { Mean } & \mathbf{F} & \text { Prob }> \\ \text { Square } & \text { Ratio } & \text { F }\end{array}$

Block

C. Total

$16 \quad 0.00004405$

0.000011588

0.0139

Means for Oneway Anova

Level Number Mean Std Error Lower 95\% Upper 95\%

$\begin{array}{lllrrr}1 / 1 / 1 & 3 & 0.140679 & 0.00096 & 0.13865 & 0.1427\end{array}$

$\begin{array}{llllll}1 / 1 / 2 & 3 & 0.139563 & 0.00096 & 0.13753 & 0.14159\end{array}$

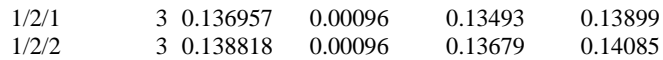

$\begin{array}{lllllll}1 / 2 / 2 & 3 & 0.138818 & 0.00096 & 0.13679 & 0.14085 \\ 2 / 1 / 1 & & 3 & 0.141051 & 0.00096 & 0.13902 & 0.14308\end{array}$

$\begin{array}{llllll}2 / 1 / 2 & 3 & 0.136957 & 0.00096 & 0.13493 & 0.13899\end{array}$

$\begin{array}{llllll}2 / 2 / 1 & 3 & 0.139935 & 0.00096 & 0.13790 & 0.14197 \\ 2 / 2 / 2 & 3 & 0.136213 & 0.00096 & 0.13418 & 0.13824\end{array}$

Std Error uses a pooled estimate of error variance 
SRNL-STI-2009-00778, Revision 0

Exhibit A3. Batch 1 Sample Measurements by Block and Sub-Block

by Oxide for Both Preparation Methods for the Non-Rad Matrix 2 Study

Oneway Analysis of Measured By Set/Block/Sub-Block

Reference Value $=1.220 \mathrm{wt} \%$

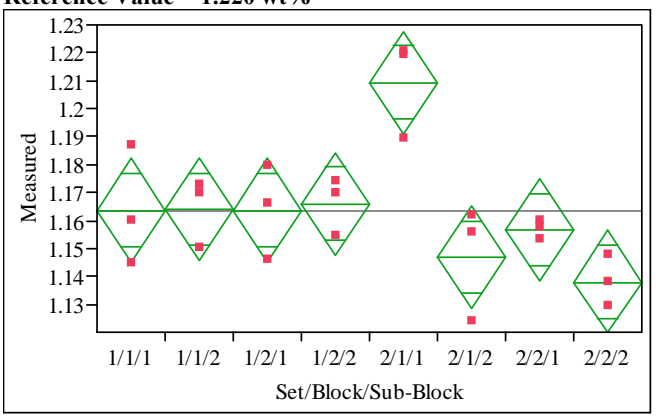

Oneway Anova

Summary of Fit

$\begin{array}{lr}\text { Rsquare } & 0.719441 \\ \text { Adj Rsquare } & 0.596696 \\ \text { Root Mean Square Error } & 0.015016 \\ \text { Mean of Response } & 1.163551 \\ \text { Observations (or Sum Wgts) } & 24\end{array}$

Observations (or Sum Wgts) 24

Analysis of Variance

Source DF Sum of

Set/Block/Sub- $\quad 7 \quad \begin{array}{rr}\text { Squares } \\ \text { - }\end{array}$

Block

Error

C. Total

$\begin{array}{ll}16 & 0.00360750 \\ 23 & 0.01285825\end{array}$

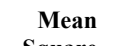

$\begin{array}{rr}\text { Mean } & \mathbf{F} \\ \text { Square } & \text { Ratio } \\ 0.001322 & 5.8613\end{array}$

Prob $>$ Source D

0.000225

Means for Oneway Anova

Level Number Mean Std Error Lower 95\% Upper 95\%

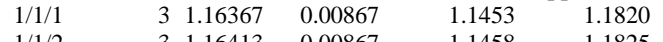

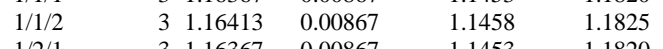

$\begin{array}{llllll}1 / 2 / 1 & 3 & 1.16367 & 0.00867 & 1.1453 & 1.1820 \\ 1 / 2 & 3 & 1.1660 & 0.00867 & 1.1476 & 1.1844\end{array}$

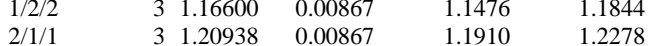

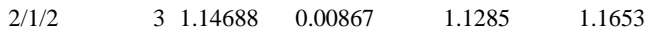

$\begin{array}{llllll}2 / 2 / 1 & 3 & 1.15667 & 0.00867 & 1.1383 & 1.1751 \\ 2 / 2 / 2 & 3 & 1.13802 & 0.00867 & 1.1196 & 1.1564\end{array}$

Std Error uses a pooled estimate of error variance
Oneway Analysis of Measured By Set/Block/Sub-Block

Oxide $=\mathbf{C d O}(\mathbf{w t} \%)$

Reference Value $=0.0 \mathrm{wt} \%$

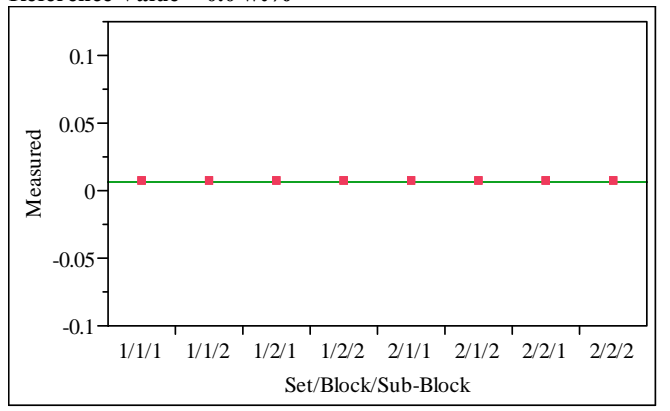

Oneway Anova

Summary of Fi

Rsquare

Adj Rsquare

Root Mean Square Error

Mean of Respons

Observations (or Sum Wgts) $\quad 24$

Analysis of Variance

Set/Block/Sub- 7

Block

C. Total

16
23

Sum of
Squares
0
0
0

Means for Oneway Anova

Level Number Mean Std Error Lower 95\% Upper 95\%

$\begin{array}{lllllr}1 / 1 / 1 & 3 & 0.005712 & 0 & 0.00571 & 0.00571\end{array}$

$\begin{array}{llllll}1 / 1 / 2 & 3 & 0.005712 & 0 & 0.00571 & 0.00571\end{array}$

$\begin{array}{lll}1 / 2 / 1 & 3 & 0.005712\end{array}$

$\begin{array}{llll}1 / 2 / 2 & 3 & 0.005712\end{array}$

$\begin{array}{lll}2 / 1 / 1 & 3 & 0.005712\end{array}$

$2 / 1 / 2 \quad 30.005712$

$\begin{array}{lll}2 / 2 / 1 & 3 & 0.005712 \\ 2 / 2 / 2 & 3 & 0.005712\end{array}$

$0 \quad 0.00571$

$\begin{array}{ll}0.00571 & 0.00571 \\ 0.00571 & 0.00571\end{array}$

$\begin{array}{lll}0 & 0.00571 & 0.00571\end{array}$

$\begin{array}{lll}0 & 0.00571 & 0.00571 \\ 0 & 0.00571 & 0.00571\end{array}$

$\begin{array}{llll}0.005712 & 0 & 0.00571 & 0.00571 \\ 0.005712 & 0 & 0.00571 & 0.00571 \\ & & \end{array}$

Std Error uses a pooled estimate of error variance
Oneway Analysis of Measured By Set/Block/Sub-Block

Reference Value $=0.0 \mathrm{wt} \%$

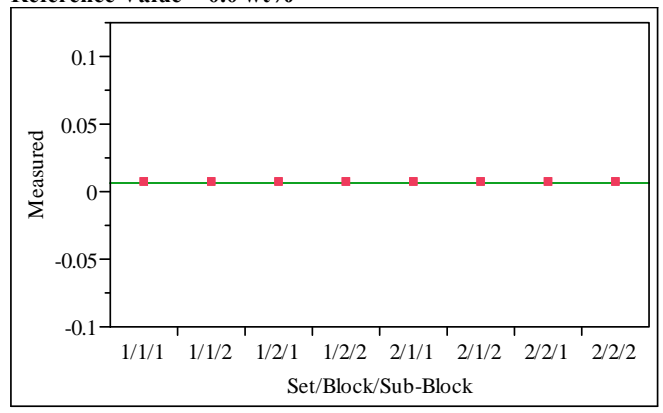

Oneway Anova

Summary of Fit

Rsquare

Adj Rsquare

$\begin{array}{lr}\text { Root Mean Square Error } & 0 \\ \text { Mean of Response } & 0.005857\end{array}$

Observations (or Sum Wgts) $\quad 24$

Analysis of Variance

$\begin{array}{lrrrrr}\text { Source } & \text { DF } & \begin{array}{r}\text { Sum of } \\ \text { Squares }\end{array} & \begin{array}{r}\text { Mean } \\ \text { Square }\end{array} & \begin{array}{r}\text { R } \\ \text { Ratio }\end{array} & \begin{array}{r}\text { Prob }> \\ \text { Set/Block/Sub- }\end{array} \\ \text { Block } & 7 & 0 & 0 & \cdot & \text {. } \\ \text { Error } & 16 & 0 & 0 & & \\ \text { C. Total } & 23 & 0 & & & \end{array}$

Means for Oneway Anova

Level Number Mean Std Error Lower 95\% Upper 95\%

$\begin{array}{lrrrrr}1 / 1 / 1 & 3 & 0.005857 & 0 & 0.00586 & 0.00586\end{array}$

$\begin{array}{llllll}1 / 1 / 1 & 3 & 0.005857 & 0 & 0.00586 & 0.00586 \\ 1 / 21 & 3 & 0.005857 & 0 & 0.00586 & 0.00586\end{array}$

$\begin{array}{llllll}1 / 2 / 1 & 3 & 0.005857 & 0 & 0.00586 & 0.00586 \\ 1 / 2 / 2 & 3 & 0.005857 & 0 & 0.00586 & 0.00586\end{array}$

$\begin{array}{llllll}2 / 1 / 1 & 3 & 0.005857 & 0 & 0.00586 & 0.00586\end{array}$

$\begin{array}{llllll}2 / 1 / 2 & 3 & 0.005857 & 0 & 0.00586 & 0.00586\end{array}$

$\begin{array}{llllll}2 / 2 / 1 & 3 & 0.005857 & 0 & 0.00586 & 0.00586 \\ 2 / 2 / 2 & 3 & 0.005857 & 0 & 0.00586 & 0.00586\end{array}$

Std Error uses a pooled estimate of error variance 
SRNL-STI-2009-00778, Revision 0

Exhibit A3. Batch 1 Sample Measurements by Block and Sub-Block

by Oxide for Both Preparation Methods for the Non-Rad Matrix 2 Study

Oneway Analysis of Measured By Set/Block/Sub-Block

Reference Value $=0.107 \mathrm{wt} \%$

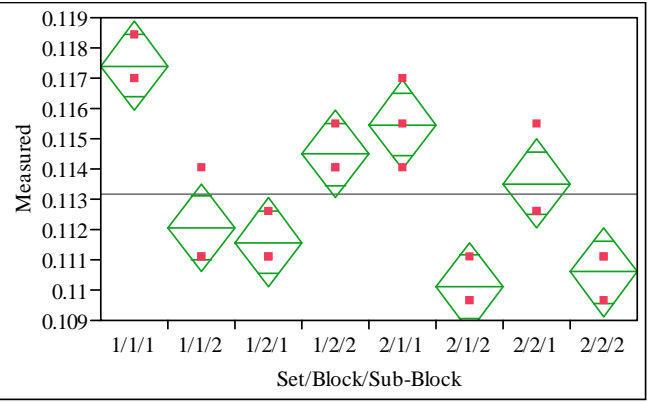

Oneway Anova

Summary of Fit

Adj Rsquare

Root Mean Square Error 0.792325

Mean of Response

0.001193

0.113152

Analysis of Variance

$\begin{array}{llr}\text { Source DF } & \begin{array}{r}\text { Sum of } \\ \text { Squares }\end{array}\end{array}$

Set/Block/Sub- $\quad 7 \quad \begin{array}{rr}\text { Squares } \\ \text { S }\end{array}$

Block
Error

Error
C. Total

$\begin{array}{ll}16 & 0.00002279\end{array}$

$23 \quad 0.00015773$

Means for Oneway Anova

Level Number Mean Std Error Lower 95\% Upper 95\%

$\begin{array}{lllllr}1 / 1 / 1 & 3 & 0.117415 & 0.00069 & 0.11595 & 0.11888\end{array}$

$\begin{array}{llllll}1 / 1 / 2 & 3 & 0.112056 & 0.00069 & 0.11060 & 0.11352\end{array}$

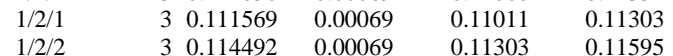

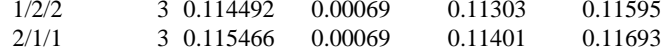

$\begin{array}{llllll}2 / 1 / 2 & 3 & 0.110107 & 0.00069 & 0.10865 & 0.11157\end{array}$

$\begin{array}{llllll}2 / 2 / 1 & 3 & 0.113518 & 0.00069 & 0.11206 & 0.11498\end{array}$

$\begin{array}{llllll}2 / 2 / 2 & 3 & 0.110594 & 0.00069 & 0.10913 & 0.11206\end{array}$

Std Error uses a pooled estimate of error variance
Oneway Analysis of Measured By Set/Block/Sub-Block

Oxide $=\mathrm{CuO}($ wt $\%)$

Reference Value $=0.399 \mathrm{wt} \%$

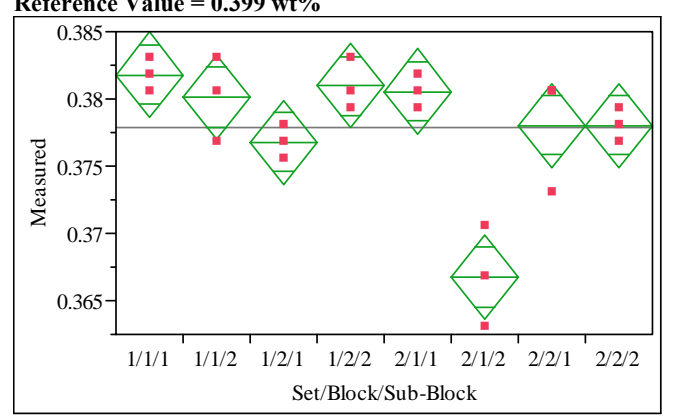

Oneway Anova

Summary of Fit

Rsquare

Adj Rsquare

Root Mean Square

Mean of Response

Observations (or Sum Wgts) 0.377887

Analysis of Variance

Source DF Sum of

$\begin{array}{rlrr}\text { F } & & & \begin{array}{r}\text { Squares } \\ \text { Set/Block/Sub- }\end{array} \\ & 7 & 0.00048466\end{array}$

Error

C. Total

$\begin{array}{ll}16 & 0.00010551 \\ 23 & 0.00059017\end{array}$

Means for Oneway Anova

Level Number Mean Std Error Lower 95\% Upper 95\%

\begin{tabular}{lll|rrr}
$1 / 1 / 1$ & 3 & 0.381799 & 0.00148 & 0.37866 & 0.38494
\end{tabular}

$\begin{array}{llllll}1 / 1 / 2 & 3 & 0.380130 & 0.00148 & 0.37699 & 0.38327\end{array}$

$\begin{array}{lllllll}1 / 2 / 1 & & 3 & 0.376792 & 0.00148 & 0.37365 & 0.37993 \\ 1 / 2 / 2 & & 3 & 0.380964 & 0.00148 & 0.37782 & 0.38411\end{array}$

$\begin{array}{lllllll}1 / 2 / 2 & & 3 & 0.380964 & 0.00148 & 0.37782 & 0.38411 \\ 2 / 1 / 1 & & 3 & 0.380547 & 0.00148 & 0.37740 & 0.38369\end{array}$

$\begin{array}{llllll}2 / 1 / 2 & 3 & 0.366777 & 0.00148 & 0.36363 & 0.36992\end{array}$

$\begin{array}{lllllll}2 / 2 / 1 & 3 & 0.378044 & 0.00148 & 0.37490 & 0.38119 \\ 2 / 2 / 2 & & 3 & 0.378044 & 0.00148 & 0.37490 & 0.38119\end{array}$

$2 / 2 / 2$

0.37490

0.38119

Std Error uses a pooled estimate of error variance
Oneway Analysis of Measured By Set/Block/Sub-Block Oxide $=\mathrm{Fe} 2 \mathrm{O} 3(\mathrm{wt} \%)$

Reference Value $=12.839 \mathrm{wt} \%$

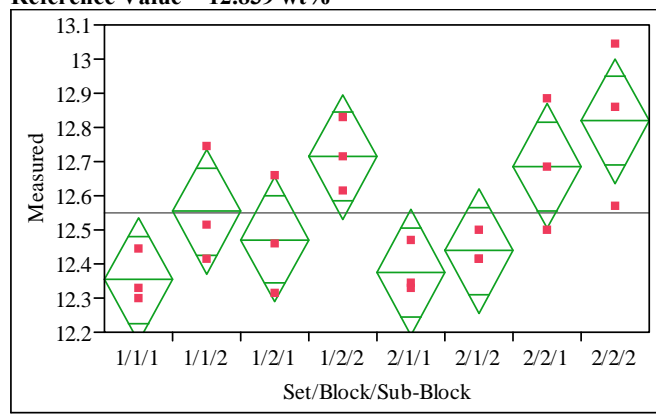

Oneway Anova

Summary of Fit

$\begin{array}{lr}\text { Rsquare } & 0.634888 \\ \text { Adj Rsquare } & 0.475151 \\ \text { Root Mean Square Error } & 0.149065 \\ \text { Mean of Response } & 12.55157 \\ \text { Observations (or Sum Wgts) } & 24\end{array}$

Analysis of Variance

Source DF Sum of

$\begin{array}{lrr}\text { Set/Block/Sub- } & 7 & 0.61822053\end{array}$

$\begin{array}{lll}\text { Block } & & \\ \text { Error } & 16 & 0.35552705\end{array}$

$\begin{array}{lll}\text { C. Total } & 23 & 0.97374758\end{array}$

$\begin{array}{rrr}\text { Mean } & \text { F } & \text { Prob }> \\ \text { Square } & \text { Ratio } & \text { F }\end{array}$ $\begin{array}{rrr}0.088317 & 3.9746 & 0.0106\end{array}$ 0.022220

Means for Oneway Anova

Level Number Mean Std Error Lower 95\% Upper 95\%

$\begin{array}{llllrr}1 / 1 / 1 & 3 & 12.3526 & 0.08606 & 12.170 & 12.535\end{array}$

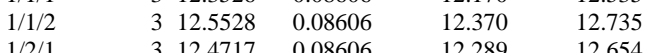

$\begin{array}{llllll}1 / 2 / 1 & 3 & 12.4717 & 0.08606 & 12.289 & 12.654 \\ 1 / 2 / 2 & 3 & 12.7148 & 0.08606 & 12.532 & 12.897\end{array}$

$\begin{array}{llllll}1 / 2 / 2 & 3 & 12.7148 & 0.08606 & 12.532 & 12.897 \\ 2 / 1 / 1 & 3 & 12.3764 & 0.08606 & 12.194 & 12.559\end{array}$

$\begin{array}{llllll}2 / 1 / 2 & 3 & 12.4384 & 0.08606 & 12.256 & 12.621\end{array}$

$\begin{array}{llllll}2 / 2 / 1 & 3 & 12.6862 & 0.08606 & 12.504 & 12.869\end{array}$

$\begin{array}{llllll}2 / 2 / 2 & 3 & 12.8196 & 0.08606 & 12.637 & 13.002\end{array}$

Std Error uses a pooled estimate of error variance 
SRNL-STI-2009-00778, Revision 0

Exhibit A3. Batch 1 Sample Measurements by Block and Sub-Block

by Oxide for Both Preparation Methods for the Non-Rad Matrix 2 Study

Oneway Analysis of Measured By Set/Block/Sub-Block

Reference Value $=0.0 \mathrm{wt} \%$

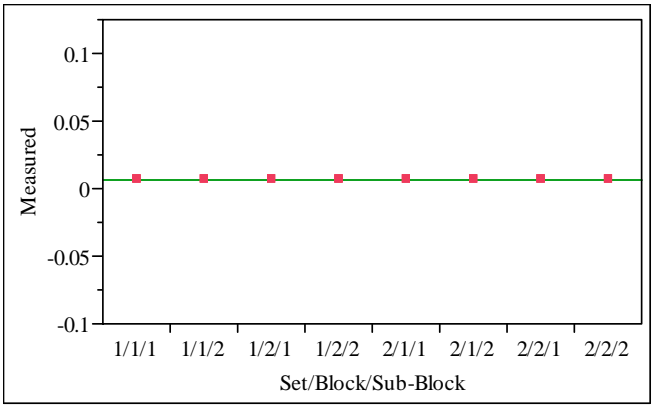

Oneway Anova

Summary of Fit

Rsquare

Adj Rsquare

$\begin{array}{lr}\text { Root Mean Square Error } & 0 \\ \text { Mean of Response } & 0.005864\end{array}$

Observations (or Sum Wgts) $\quad 24$

Analysis of Variance

$\begin{array}{lr}\text { Source } & \text { DF } \\ \text { Set/Block/Sub- } & 7\end{array}$

Block

Error
C. Total

Sum of
Squares

Squares

\section{Mean}

F
Ratio

Prob $>>$
$F$

16
23

0

Means for Oneway Anova

$\begin{array}{lrrrrr}\text { Level Number } & \text { Mean } & \text { Std Error } & \text { Lower 95\% } & \text { Upper 95\% } \\ 1 / 1 / 1 & 3 & 0.005864 & 0 & 0.00586 & 0.00586\end{array}$

$\begin{array}{lll}1 / 1 / 2 & 3 & 0.005864 \\ 1 / 2 / 1 & 3 & 0.005864\end{array}$

$\begin{array}{lll}1 / 2 / 1 & 3 & 0.005864 \\ 1 / 2 / 2 & 3 & 0.005864\end{array}$

$\begin{array}{lll}2 / 1 / 1 & 3 & 0.005864\end{array}$

$\begin{array}{llll}2 / 1 / 2 & 3 & 0.00586\end{array}$

$2 / 2 / 1 \quad 3 \quad 0.00586$

$2 / 2 / 2 \quad 3 \quad 0.005864$

$\begin{array}{rrr}\text { ror } & \text { Lower } \mathbf{9 5 \%} & \text { Uper } \mathbf{9 5 \%} \\ 0 & 0.00586 & 0.00586 \\ 0 & 0.00586 & 0.00586 \\ 0 & 0.00586 & 0.00586 \\ 0 & 0.00586 & 0.00586 \\ 0 & 0.00586 & 0.00586 \\ 0 & 0.00586 & 0.00586 \\ 0 & 0.00586 & 0.00586 \\ 0 & 0.00586 & 0.00586\end{array}$

Std Error uses a pooled estimate of error variance
Oneway Analysis of Measured By Set/Block/Sub-Block

Oxide $=\mathrm{Li2O}(\mathrm{wt} \%)$

Reference Value $=4.429 \mathrm{wt} \%$

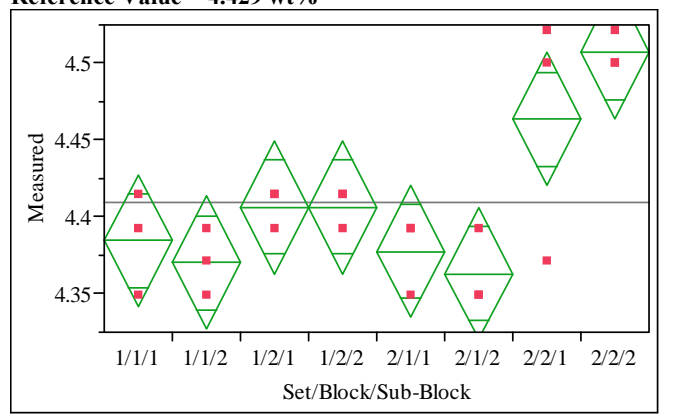

Oneway Anova

Summary of Fit

$\begin{array}{lr}\text { Rsquare } & 0.728814 \\ \text { Adj Rsquare } & 0.610169 \\ \text { Root Mean Square Error } & 0.035157 \\ \text { Mean of Response } & 4.409857 \\ \text { Observations (or Sum Wgts) } & 24\end{array}$

Observations (or Sum Wgts) $\quad 24$

Analysis of Variance

Square Ratio $\begin{array}{ll}0.007593 & 6.1429\end{array}$

0.001236

Means for Oneway Anova

Level Number Mean Std Error Lower 95\% Upper 95\%

$\begin{array}{llllll}1 / 1 / 1 & 3 & 4.38474 & 0.02030 & 4.3417 & 4.4278\end{array}$

$\begin{array}{llllll}1 / 1 / 2 & 3 & 4.37039 & 0.02030 & 4.3274 & 4.4134\end{array}$

$\begin{array}{llllll}1 / 2 / 1 & 3 & 4.40627 & 0.02030 & 4.3632 & 4.4493\end{array}$

$\begin{array}{llllll}1 / 2 / 2 & 3 & 4.40627 & 0.02030 & 4.3632 & 4.4493\end{array}$

$\begin{array}{llllll}2 / 1 / 1 & 3 & 4.37756 & 0.02030 & 4.3345 & 4.4206\end{array}$

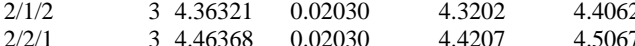

$\begin{array}{llllll}2 / 2 / 2 & 3 & 4.50674 & 0.02030 & 4.4637 & 4.5498\end{array}$

Std Error uses a pooled estimate of error variance
Oneway Analysis of Measured By Set/Block/Sub-Block Oxide= $=$

Reference Value $=1.419 \mathrm{wt} \%$

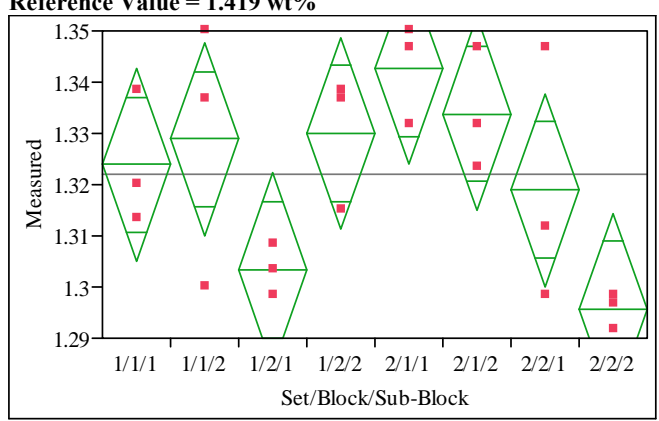

Oneway Anova

Summary of Fit

$\begin{array}{ll}\text { Rsquare } & 0.579104 \\ \text { Adj Rsquare } & 0.394962\end{array}$

Root Mean Square Error $\quad 0.015345$

$\begin{array}{lr}\text { Mean of Response } & 1.322149 \\ \text { Observations (or Sum Wgts) } & 24\end{array}$

Analysis of Variance

Source DF Sum of

Set/Block/Sub-

Block

C. Total

Squares
0.00518356

$\begin{array}{rrr}\text { Mean } & \text { F } & \text { Prob }> \\ \text { Square } & \text { Ratio } & \text { F }\end{array}$ $\begin{array}{lll}0.000741 & 3.1449 & 0.0273\end{array}$

0.000235

Means for Oneway Anova

Level Number Mean Std Error Lower 95\% Upper 95\%

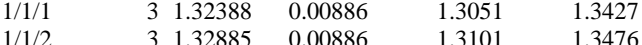

$\begin{array}{llllll}1 / 1 / 2 & 3 & 1.32885 & 0.00886 & 1.3101 & 1.3476 \\ 1 / 2 / 1 & 3 & 1.30342 & 0.00886 & 1.2846 & 1.3222\end{array}$

$\begin{array}{llllll}1 / 2 / 2 & 3 & 1.32996 & 0.00886 & 1.3112 & 1.3487\end{array}$

$\begin{array}{llllll}2 / 1 / 1 & 3 & 1.34267 & 0.00886 & 1.3239 & 1.3615 \\ 2 / 1 / 2 & 3 & 1.33383 & 0.0088 & 1.3150 & 1.3526\end{array}$

$\begin{array}{llllll}2 / 1 / 2 & 3 & 1.33383 & 0.00886 & 1.3150 & 1.3526 \\ 2 / 2 / 1 & 3 & 1.31890 & 0.00886 & 1.3001 & 1.3377\end{array}$

$\begin{array}{llllll}2 / 2 / 2 & 3 & 1.29569 & 0.00886 & 1.2769 & 1.3145\end{array}$

Std Error uses a pooled estimate of error variance 
SRNL-STI-2009-00778, Revision 0

Exhibit A3. Batch 1 Sample Measurements by Block and Sub-Block

by Oxide for Both Preparation Methods for the Non-Rad Matrix 2 Study

Oneway Analysis of Measured By Set/Block/Sub-Block

Reference Value $=1.726 \mathrm{wt} \%$

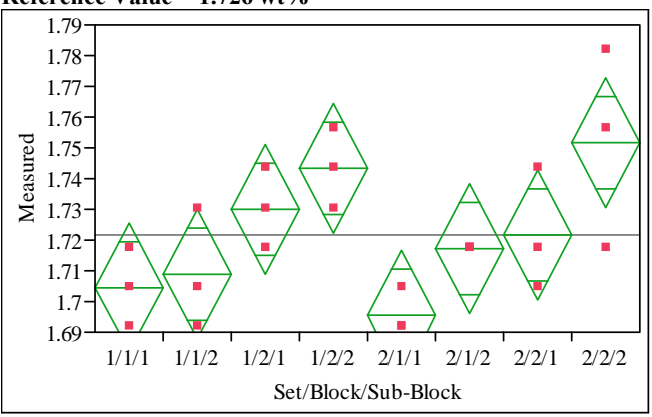

\section{Oneway Anova}

ummary of Fit

$\begin{array}{lr}\text { Rsquare } & 0.619469 \\ \text { Adj Rsquare } & 0.452987 \\ \text { Root Mean Square Error } & 0.017283 \\ \text { Mean of Response } & 1.7216 \\ \text { Observations (or Sum Wgts) } & 24\end{array}$

Observations (or Sum Wgts)

Analysis of Variance
Source

$\begin{array}{llr}\text { Set/Block/Sub- } & 7 & 0.00778025\end{array}$

Block

Error $\quad 16 \quad 0.00477930$

C. Total

$23 \quad 0.01255955$

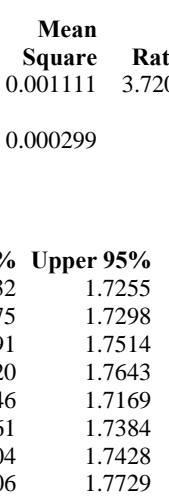

Means for Oneway Anova

$\begin{array}{lrrrrr}\text { Level } & \text { Number } & \text { Mean } & \text { Std Error } & \text { Lower 95\% } & \text { Upper 95\% } \\ 1 / 1 / 1 & 3 & 1.70438 & 0.00998 & 1.6832 & 1.7255 \\ 1 / 1 / 2 & 3 & 1.70869 & 0.00998 & 1.6875 & 1.7298 \\ 1 / 2 / 1 & 3 & 1.73021 & 0.00998 & 1.7091 & 1.7514 \\ 1 / 2 / 2 & 3 & 1.74312 & 0.00998 & 1.7220 & 1.7643 \\ 2 / 1 / 1 & 3 & 1.69578 & 0.00998 & 1.6746 & 1.7169 \\ 2 / 1 / 2 & 3 & 1.71730 & 0.00998 & 1.6961 & 1.7384 \\ 2 / 2 / 1 & 3 & 1.72160 & 0.00998 & 1.7004 & 1.7428 \\ 2 / 2 / 2 & 3 & 1.75173 & 0.00998 & 1.7306 & 1.7729\end{array}$

Std Error uses a pooled estimate of error variance
Oneway Analysis of Measured By Set/Block/Sub-Block Oxide $=\mathrm{Na} 2 \mathrm{O}(\mathrm{wt} \%)$

Reference Value $=9.003 \mathrm{wt} \%$

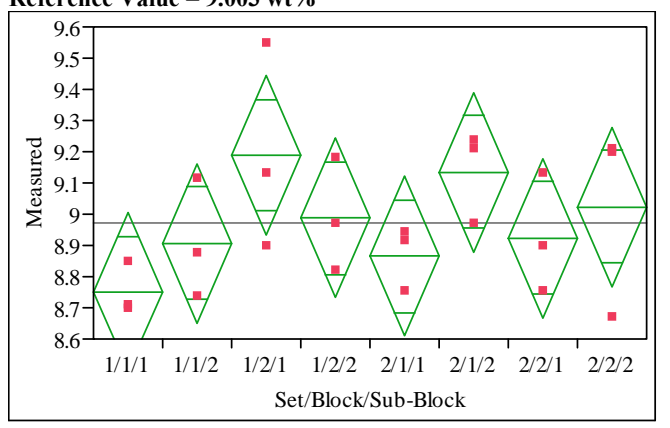

Oneway Anova

Summary of Fit

Rsquare

$\begin{array}{lr}\text { Adj Rsquare } & 0.114328 \\ \text { Root Mean Square Error } & 0.20845\end{array}$

Mean of Response $\quad 0.20845$

Observations (or Sum Wots)

Analysis of Variance

Prob $>$ Source DF Dariance
$F$

Set/Block/Sub-
Block

Block

C. Total

Squares
0.4331673

$\begin{array}{ll}16 & 0.6952240 \\ 23 & 1.1283913\end{array}$

Means for Oneway Anova

Level Number Mean Std Error Lower 95\% Upper 95\%

$\begin{array}{llllrr}1 / 1 / 1 & 3 & 8.74852 & 0.12035 & 8.4934 & 9.0036\end{array}$

$\begin{array}{llllll}1 / 1 / 1 & 3 & 8.74852 & 0.12035 & 8.4934 & 9.0036 \\ 1 / 2 & 3 & 8.90579 & 0.12035 & 8.6507 & 9.1609\end{array}$

$\begin{array}{llllll}1 / 2 / 1 & 3 & 9.18887 & 0.12035 & 8.9337 & 9.4440\end{array}$

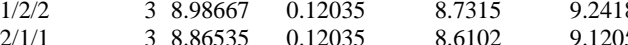

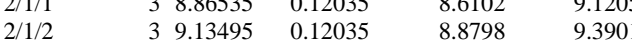

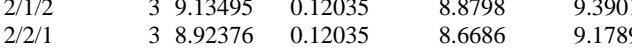

$\begin{array}{llllll}2 / 2 / 2 & 3 & 9.02261 & 0.12035 & 8.6685 & 9.1789 \\ & & & & & \end{array}$

Std Error uses a pooled estimate of error variance
Oneway Analysis of Measured By Set/Block/Sub-Block Oxide $=\mathrm{NiO}(\mathrm{wt} \%)$

Reference Value $=0.751 \mathrm{wt} \%$

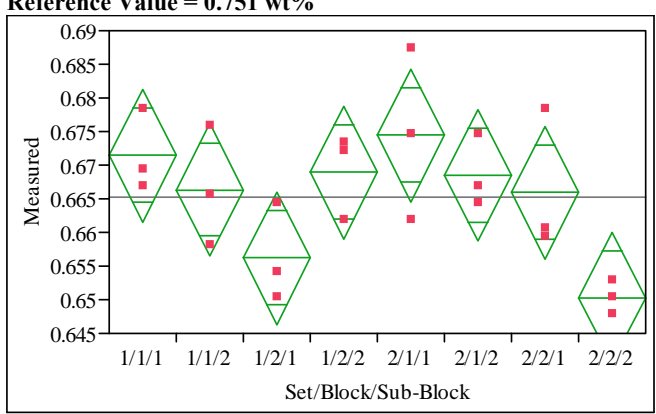

Oneway Anova

Summary of Fit

$\begin{array}{lr}\text { Rsquare } & 0.568721 \\ \text { Adj Rsquare } & 0.380037 \\ \text { Root Mean Square Error } & 0.008048 \\ \text { Mean of Response } & 0.665252 \\ \text { Observations (or Sum Wgts) } & 24\end{array}$

Analysis of Variance

Source DF Sum of

$\begin{array}{lrr}\text { Squares } \\ \text { Set/Block/Sub- } & 7 & 0.00136658\end{array}$

$\begin{array}{rrr}\text { Mean } & \mathbf{F} & \text { Prob > } \\ \text { Square } & \text { Ratio } & \text { F }\end{array}$

Block

\begin{tabular}{lll} 
Error & 16 & 0.00103632 \\
\hline & & 0.0230291
\end{tabular}

C. Total

0.00240291

0.000065

Means for Oneway Anova

Level Number Mean Std Error Lower 95\% Upper 95\%

$\begin{array}{llllll}1 / 1 / 1 & 3 & 0.671456 & 0.00465 & 0.66161 & 0.68131\end{array}$

$\begin{array}{llllll}1 / 1 / 2 & 3 & 0.666366 & 0.00465 & 0.65652 & 0.67622 \\ 1 / 2 / 1 & 3 & 0.656186 & 0.00655 & 0.64634 & 0.66604\end{array}$

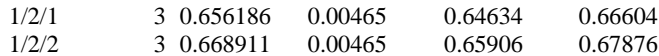

$\begin{array}{llllll}2 / 1 / 1 & 3 & 0.674425 & 0.00465 & 0.66457 & 0.68428\end{array}$

$\begin{array}{llllll}2 / 1 / 2 & 3 & 0.668487 & 0.00465 & 0.65864 & 0.67834\end{array}$

$\begin{array}{llllll}2 / 2 / 1 & 3 & 0.665942 & 0.00465 & 0.65609 & 0.67579 \\ 2 / 2 / 2 & 3 & 0.650248 & 0.00465 & 0.64040 & 0.66010\end{array}$

$\begin{array}{llllll}2 / 2 / 2 & 3 & 0.650248 & 0.00465 & 0.64040 & 0.66010\end{array}$

Std Error uses a pooled estimate of error variance 
SRNL-STI-2009-00778, Revision 0

Exhibit A3. Batch 1 Sample Measurements by Block and Sub-Block

by Oxide for Both Preparation Methods for the Non-Rad Matrix 2 Study

Oneway Analysis of Measured By Set/Block/Sub-Block

Reference Value $=0.0 \mathrm{wt} \%$

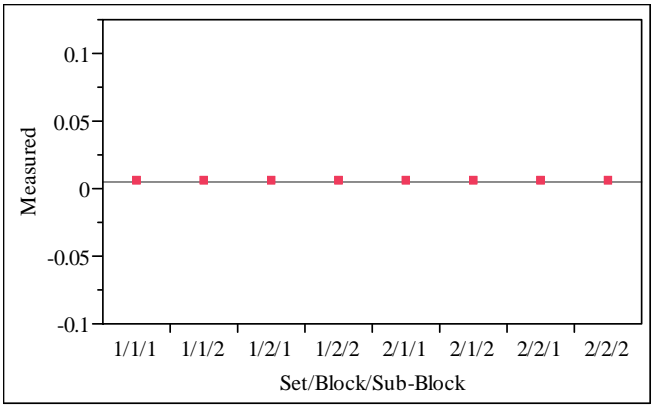

Oneway Anova

Summary of Fit

$\begin{array}{lr}\text { Rsquare } & 4 \\ \text { Adj Rsquare } & 5.3125 \\ \text { Root Mean Square Error } & \text {. } \\ \text { Mean of Response } & 0.005386 \\ \text { Observations (or Sum Wgts) } & 24\end{array}$

Analysis of Variance
Source

Set/Block/Sub- $\quad 7 \quad \begin{array}{r}\text { Squares } \\ 7.2222 \mathrm{e}-35\end{array}$

Block

Error
C. Total

$\begin{array}{ll}16 & -5.417 \mathrm{e}-35 \\ 23 & 1.8056 \mathrm{e}-35\end{array}$

Mean F Ratio
Square

$-3.39 \mathrm{e}-36$

Means for Oneway Anova

Level Number Mean Std Error Lower 95\% Upper 95\%

$\begin{array}{lll}1 / 1 / 1 & 3 & 0.005386\end{array}$

$\begin{array}{llll}1 / 1 / 2 & 3 & 0.005386\end{array}$

$\begin{array}{lll}1 / 2 / 1 & 3 & 0.005386 \\ 1 / 2 / 2 & 3 & 0.005386\end{array}$

$\begin{array}{lll}1 / 2 / 2 & 3 & 0.005386 \\ 2 / 1 / 1 & 3 & 0.005386\end{array}$

$\begin{array}{lll}2 / 1 / 1 & 3 & 0.005386 \\ 2 / 1 / 2 & 3 & 0.005386 \\ 2 / 2 / 1 & 3 & 0.005386\end{array}$

$2 / 2 / 1 \quad 3 \quad 30.005386$

$2 / 2 / 2 \quad 30.005386$

Std Error uses a pooled estimate of error variance
Oneway Analysis of Measured By Set/Block/Sub-Block
Oxide $=\mathrm{SiO2}(\mathrm{wt} \%)$

Reference Value $=50.22 \mathrm{wt} \%$

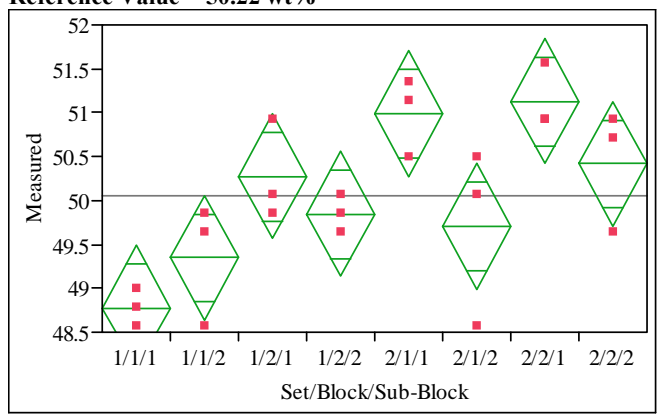

Oneway Anova

Summary of Fit

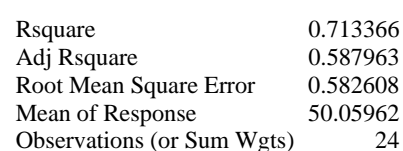

Observations (or Sum Wgts)

Analysis of Variance

Source DF Sum of

$\begin{array}{lrr}\text { Squares } \\ \text { Set/Block/Sub- } & 7 & 13.516239\end{array}$

Block

$\begin{array}{llr}\text { Block } & 16 & 5.430904 \\ \text { Error } & & \end{array}$

C. Total

23

18.947143

Means for Oneway Anova

Level Number Mean Std Error Lower 95\% Upper 95\%

$\begin{array}{llllll}1 / 1 / 1 & 3 & 48.7760 & 0.33637 & 48.063 & 49.489\end{array}$

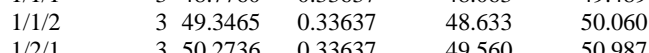

$\begin{array}{llllll}1 / 2 / 1 & 3 & 50.2736 & 0.33637 & 49.560 & 50.987 \\ 1 / 2 / 2 & 3 & 49.8457 & 0.33637 & 49.133 & 50.559\end{array}$

$\begin{array}{llllll}1 / 2 & 3 & 49.8457 & 0.33637 & 4.563 & 50.987 \\ 2 / 1 / 1 & 3 & 50.9867 & 0.33637 & 50.274 & 51.700\end{array}$

$\begin{array}{llllll}2 / 1 / 2 & 3 & 49.7031 & 0.33637 & 48.990 & 50.416\end{array}$

$\begin{array}{llllll}2 / 2 / 1 & 3 & 51.1293 & 0.33637 & 50.416 & 51.842\end{array}$

$\begin{array}{llllll}2 / 2 / 2 & 3 & 50.4162 & 0.33637 & 49.703 & 51.129\end{array}$

Std Error uses a pooled estimate of error variance
Oneway Analysis of Measured By Set/Block/Sub-Block Oxide $=\mathrm{SO} 4($ wt $\%)$

Reference Value $=0.0 \mathbf{w t}^{\circ} \%$

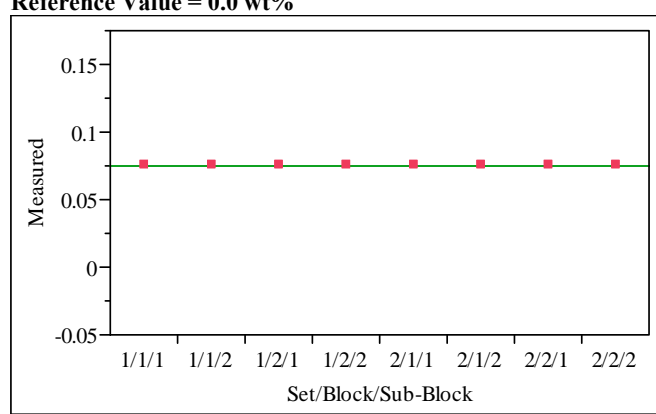

Oneway Anova

Summary of Fit

Rsquare

Adj Rsquare

$\begin{array}{lr}\text { Root Mean Square Error } & 0 \\ \text { Mean of Response } & 0.074898\end{array}$

Observations (or Sum Wgts) $\quad 24$

Analysis of Variance

Source DF Sum of

(1)

Source

7

Squares

Mean
Square Square
0

F Prob >

Block

Error
C. Total

16
23

0

Means for Oneway Anova

Level Number Mean Std Error Lower 95\% Upper 95\%

$\begin{array}{lllrrr}1 / 1 / 1 & 3 & 0.074898 & 0 & 0.07490 & 0.07490\end{array}$

$\begin{array}{llllll}1 / 1 / 2 & 3 & 0.074898 & 0 & 0.07490 & 0.07490\end{array}$

$\begin{array}{llllll}1 / 2 / 1 & 3 & 0.074898 & 0 & 0.07490 & 0.07490 \\ 1 / 2 / 2 & 3 & 0.074898 & 0 & 0.07490 & 0.07490\end{array}$

$\begin{array}{llllll}2 / 1 / 1 & 3 & 0.074898 & 0 & 0.07490 & 0.07490\end{array}$

$\begin{array}{llllll}2 / 1 / 2 & 3 & 0.074898 & 0 & 0.07490 & 0.07490\end{array}$

$\begin{array}{llllll}2 / 2 / 1 & 3 & 0.074898 & 0 & 0.07490 & 0.07490\end{array}$

$\begin{array}{llllll}2 / 2 / 2 & 3 & 0.074898 & 0 & 0.07490 & 0.07490\end{array}$

Std Error uses a pooled estimate of error variance 
SRNL-STI-2009-00778, Revision 0

Exhibit A3. Batch 1 Sample Measurements by Block and Sub-Block

by Oxide for Both Preparation Methods for the Non-Rad Matrix 2 Study

Oneway Analysis of Measured By Set/Block/Sub-Block

Reference Value $=0.677 \mathrm{wt} \%$

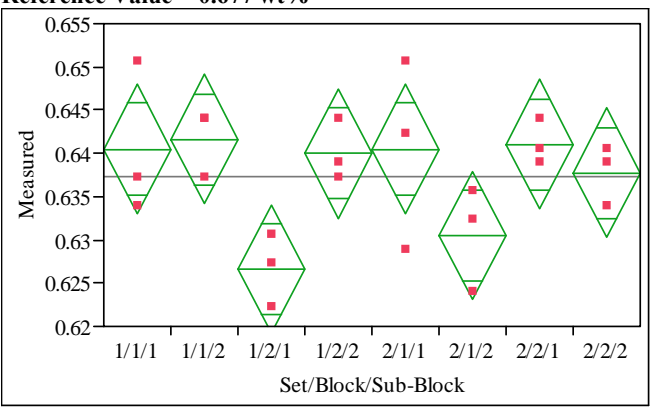

Oneway Anova

Summary of Fit

$\begin{array}{lr}\text { Rsquare } & 0.52785 \\ \text { Adj Rsquare } & 0.321284 \\ \text { Root Mean Square Error } & 0.006091 \\ \text { Mean of Response } & 0.637315 \\ \text { Observations (or Sum Wgts) } & 24\end{array}$

Analysis of Variance

$\begin{array}{lrrrr}\text { Source } & \text { DF } & \begin{array}{r}\text { Sum of } \\ \text { Squares }\end{array} & \begin{array}{r}\text { Mean } \\ \text { Square }\end{array} & \begin{array}{r}\text { F } \\ \text { Ratio }\end{array} \\ \text { Set/Block/Sub- } & 7 & 0.00066356 & 0.000095 & 2.5554 \\ \text { Block } & & & & \\ \text { Error } & 16 & 0.00059354 & 0.000037 & \\ \text { C. Total } & 23 & 0.00125710 & & \end{array}$

Means for Oneway Anova

Level Number Mean Std Error Lower 95\% Upper 95\%

$\begin{array}{lllllr}1 / 1 / 1 & 3 & 0.640512 & 0.00352 & 0.63306 & 0.64797\end{array}$

$\begin{array}{llllll}1 / 1 / 2 & 3 & 0.641624 & 0.00352 & 0.63417 & 0.64908\end{array}$

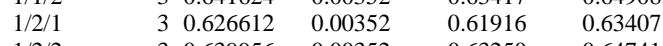

$\begin{array}{llllll}1 / 2 / 2 & 3 & 0.639956 & 0.00352 & 0.63250 & 0.64741 \\ 2 / 1 / 1 & 3 & 0.640512 & 0.00352 & 0.63306 & 0.64797\end{array}$

$\begin{array}{llllll}2 / 1 / 2 & 3 & 0.630504 & 0.00352 & 0.62305 & 0.63796\end{array}$

$\begin{array}{llllll}2 / 2 / 1 & 3 & 0.641068 & 0.00352 & 0.63361 & 0.64852\end{array}$

$\begin{array}{llllll}2 / 2 / 2 & 3 & 0.637732 & 0.00352 & 0.63028 & 0.64519\end{array}$

Std Error uses a pooled estimate of error variance
Oneway Analysis of Measured By Set/Block/Sub-Block
Oxide $=\mathrm{ZnO}\left(\right.$ wt $\left.^{\circ}\right)$

Reference Value $=0.0 \mathrm{wt} \%$

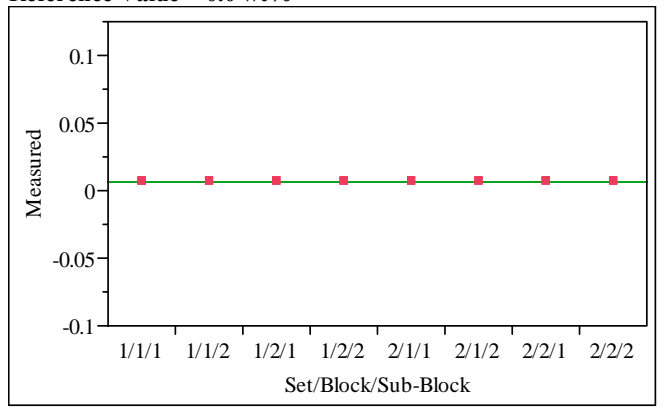

Oneway Anova

Summary of Fit

Rsquare

Adj Rsquare

Root Mean Square Error

Mean of Response $\quad 0.006224$

Observations (or Sum Wgts) $\quad 24$

Analysis of Variance

Set/Block/Sub- 7

Block
Error

C. Total

7
16
23

\section{Sum of}

Squares
0

0

Means for Oneway Anova

Level Number Mean Std Error Lower 95\% Upper 95\%

\begin{tabular}{lllrrr}
$1 / 1 / 1$ & 3 & 0.006224 & 0 & 0.00622 & 0.00622 \\
\hline
\end{tabular}

$\begin{array}{llll}1 / 1 / 2 & 3 & 0.006224 \\ 1 / 2 & 3 & 0.006224\end{array}$

$\begin{array}{llll}1 / 2 / 1 & 3 & 0.006224 \\ 1 / 2 / 2 & 3 & 0.00624\end{array}$

$\begin{array}{llll}1 / 2 / 2 & 3 & 0.006224\end{array}$

$\begin{array}{lll}2 / 1 / 1 & 3 & 0.006224 \\ 2 / 1 / 2 & 3 & 0.006224\end{array}$

$\begin{array}{lll}2 / 1 / 2 & 3 & 0.006224 \\ 2 / 2 / 1 & 3 & 0.006224\end{array}$

$\begin{array}{lll}2 / 2 / 2 & 3 & 0.006224\end{array}$
$\begin{array}{lll}0 & 0.00622 & 0.00622 \\ 0 & 0.00622 & 0.00622\end{array}$
$\begin{array}{lll}0 & 0.00622 & 0.00622\end{array}$
$\begin{array}{lll}0 & 0.00622 & 0.00622\end{array}$
$\begin{array}{lll}0 & 0.00622 & 0.00622 \\ 0 & 0.00622 & 0.00622\end{array}$
$\begin{array}{lll}0 & 0.00622 & 0.00622 \\ 0 & 0.00622 & 0.00622\end{array}$

Std Error uses a pooled estimate of error variance
Oneway Analysis of Measured By Set/Block/Sub-Block Oxide $=\mathbf{Z r O 2}(\mathbf{w t} \%)$

Reference Value $=0.098 \mathrm{wt} \%$

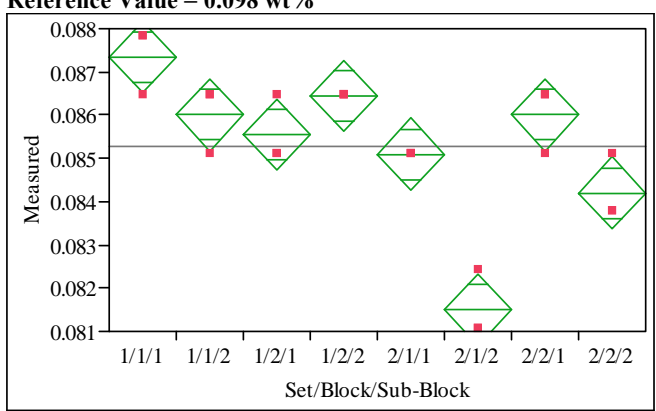

Oneway Anova

Summary of Fit

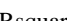

Rsquare

0.901538
0.858462

Root Mean Square Error $\quad 0.000675$

Mean of Response $\quad 0.085269$

Observations (or Sum Wgts) $\quad 24$

Analysis of Variance

$\begin{array}{lrrrr}\text { Source } & \text { DF } & \begin{array}{r}\text { Sum of } \\ \text { Squares }\end{array} & \begin{array}{r}\text { Mean F Ratio } \\ \text { Square }\end{array} & \begin{array}{r}\text { Prob }> \\ \text { F }\end{array} \\ \text {. Set/Block/Sub- } & 7 & 0.00006683 & 9.5469 \mathrm{e}-620.9286 & <.0001 \\ \text { Block } & & & & \\ \text { Error } & 16 & 0.00000730 & 4.5617 \mathrm{e}-7 & \\ \text { C. Total } & 23 & 0.00007413 & & \end{array}$

C. Total

0.00007413

Means for Oneway Anova

\begin{tabular}{|rrrrrr} 
Level Number & \multicolumn{2}{c}{ Mean } & Std Error & Lower 95\% & Upper 95\% \\
$1 / 1 / 1$ & 3 & 0.087352 & 0.00039 & 0.08653 & 0.08818
\end{tabular}

$\begin{array}{llllll}1 / 1 / 2 & 3 & 0.086001 & 0.00039 & 0.08517 & 0.08683\end{array}$

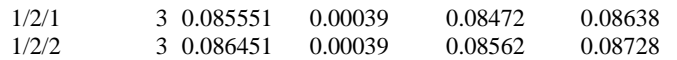

$\begin{array}{llllll}1 / 2 / 2 & 3 & 0.086451 & 0.00039 & 0.08562 & 0.08728 \\ 2 / 1 / 1 & 3 & 0.085100 & 0.00039 & 0.08427 & 0.08593\end{array}$

$\begin{array}{llllll}2 / 1 / 2 & 3 & 0.081498 & 0.00039 & 0.08067 & 0.08232\end{array}$

$\begin{array}{llllll}2 / 2 / 1 & 3 & 0.086001 & 0.00039 & 0.08517 & 0.08683 \\ 2 / 2 / 2 & 3 & 0.084200 & 0.00039 & 0.08337 & 0.08503\end{array}$

$\begin{array}{llllll}2 / 2 / 2 & 3 & 0.084200 & 0.00039 & 0.08337 & 0.08503\end{array}$

Std Error uses a pooled estimate of error variance 
SRNL-STI-2009-00778, Revision 0

Exhibit A4. Average Measured and Bias-Corrected (bc) Versus Targeted Compositions by Glass ID by Oxide for the Non-Rad Matrix 2 Study

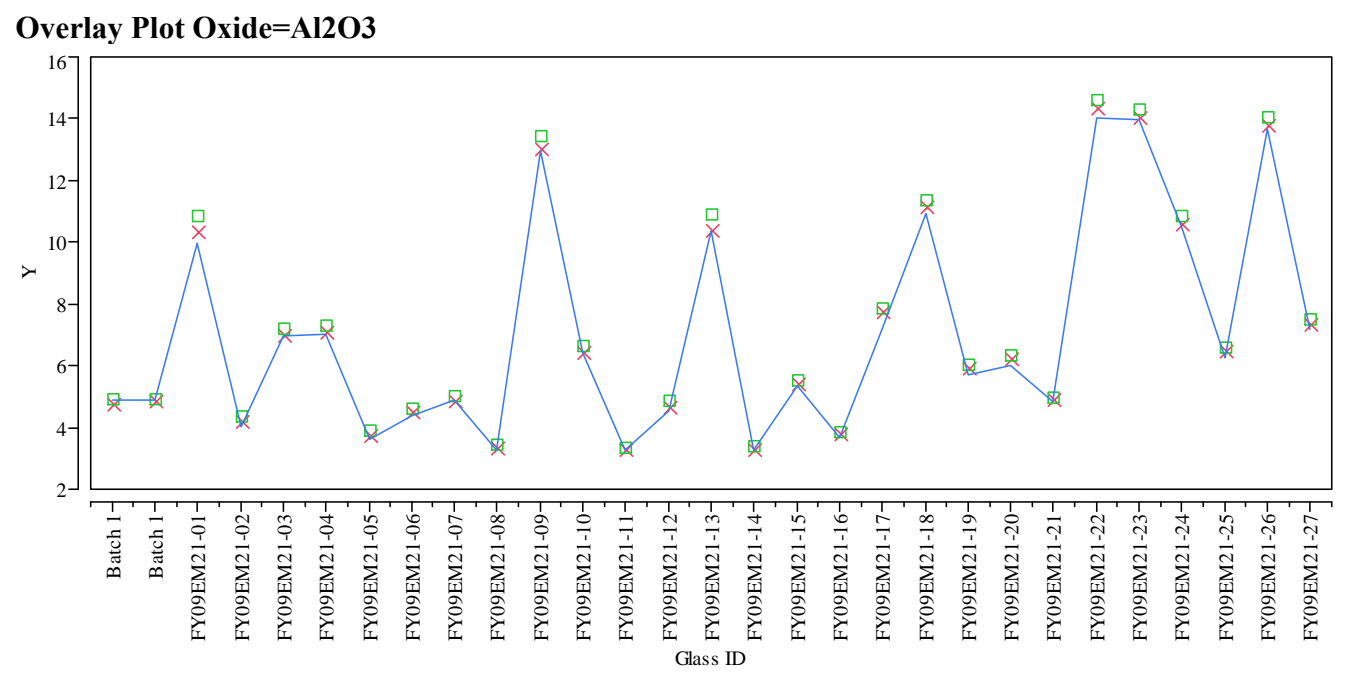

Overlay Plot Oxide=B2O3

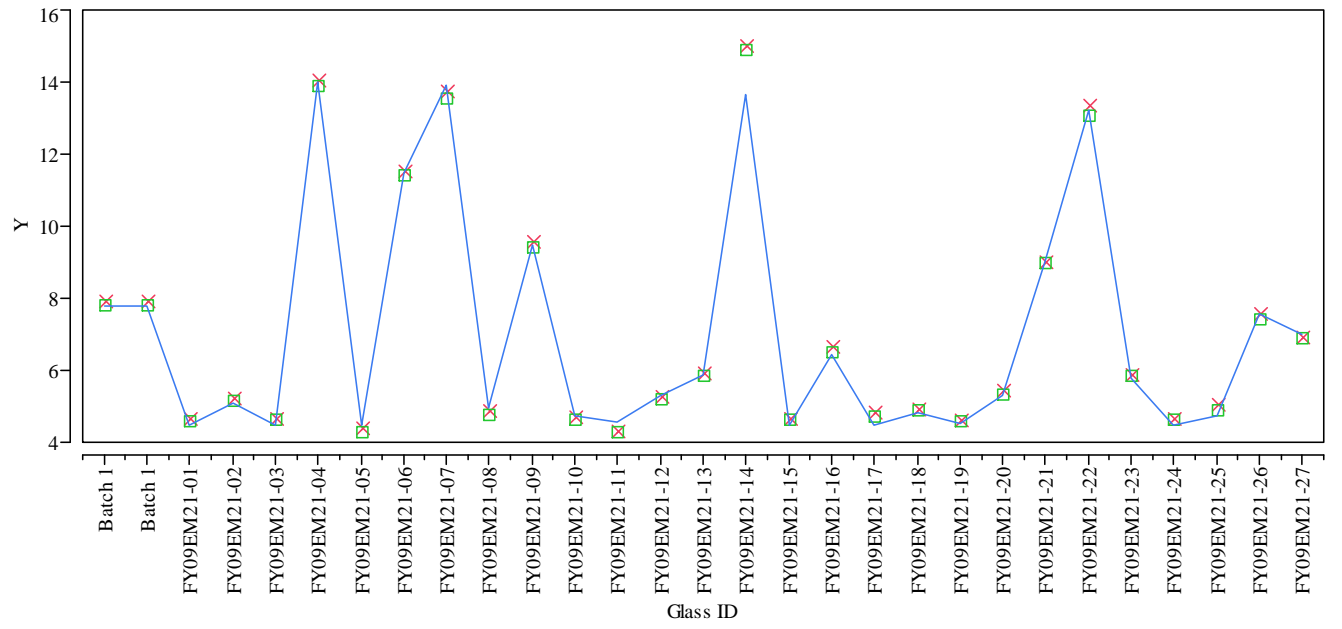

Overlay Plot Oxide $=\mathrm{BaO}$

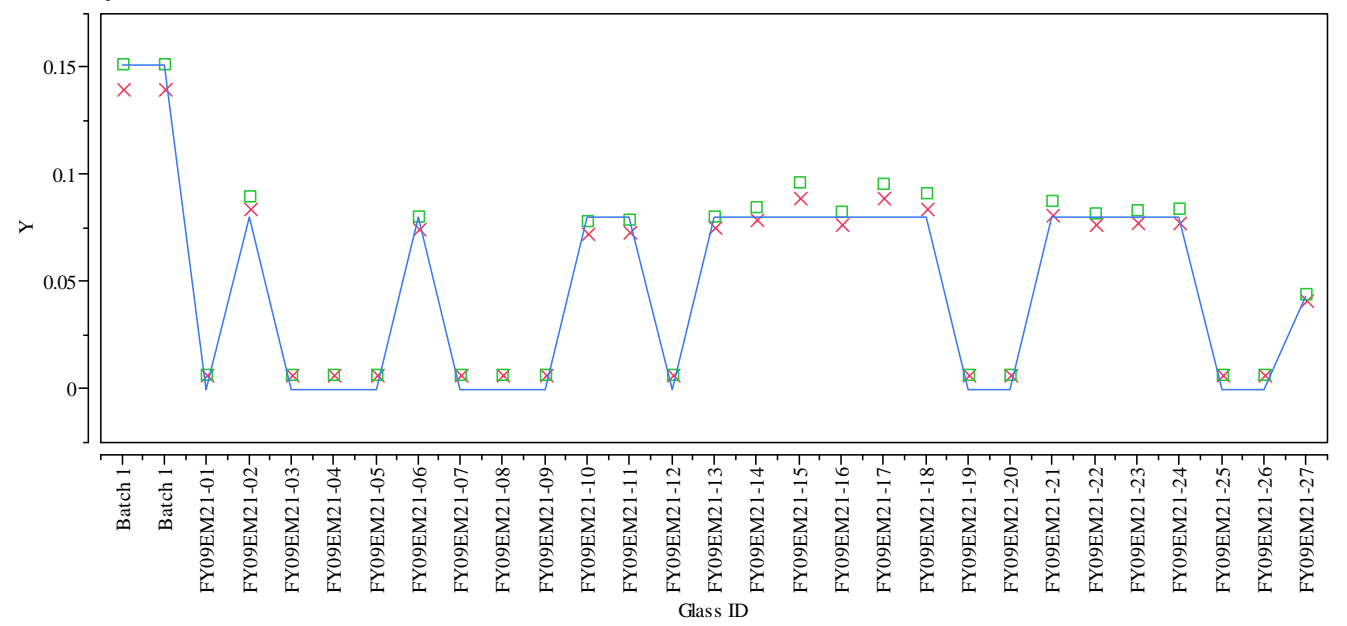

$\mathrm{Y} \times$ Measured Measured bc — Targeted 
SRNL-STI-2009-00778, Revision 0

Exhibit A4. Average Measured and Bias-Corrected (bc) Versus Targeted Compositions by Glass ID by Oxide for the Non-Rad Matrix 2 Study

\section{Overlay Plot Oxide $=\mathrm{CaO}$}

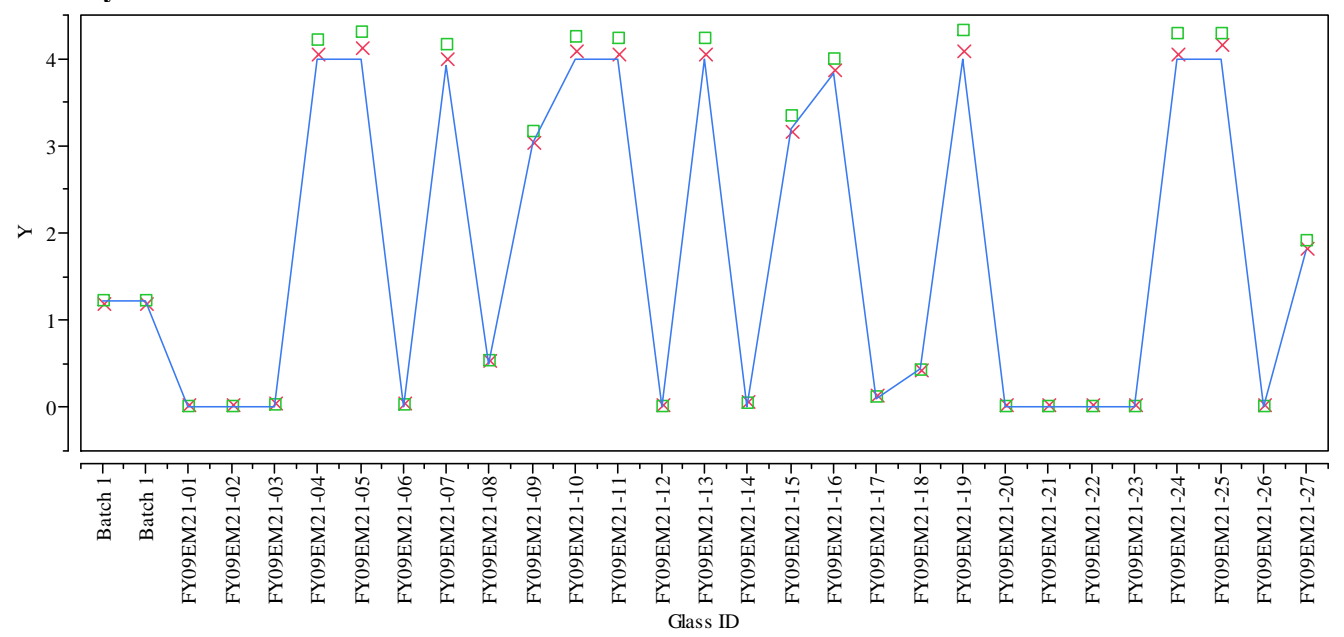

Overlay Plot Oxide $=\mathbf{C d O}$

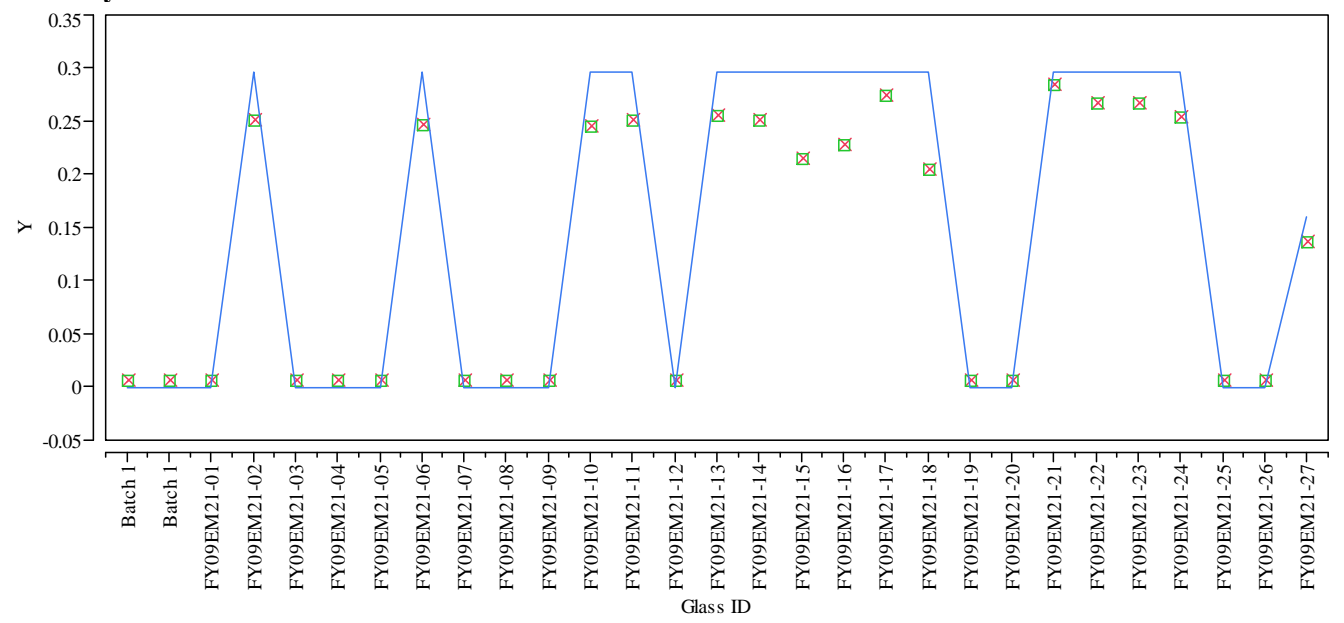

Overlay Plot Oxide $=\mathrm{Ce} 2 \mathrm{O3}$

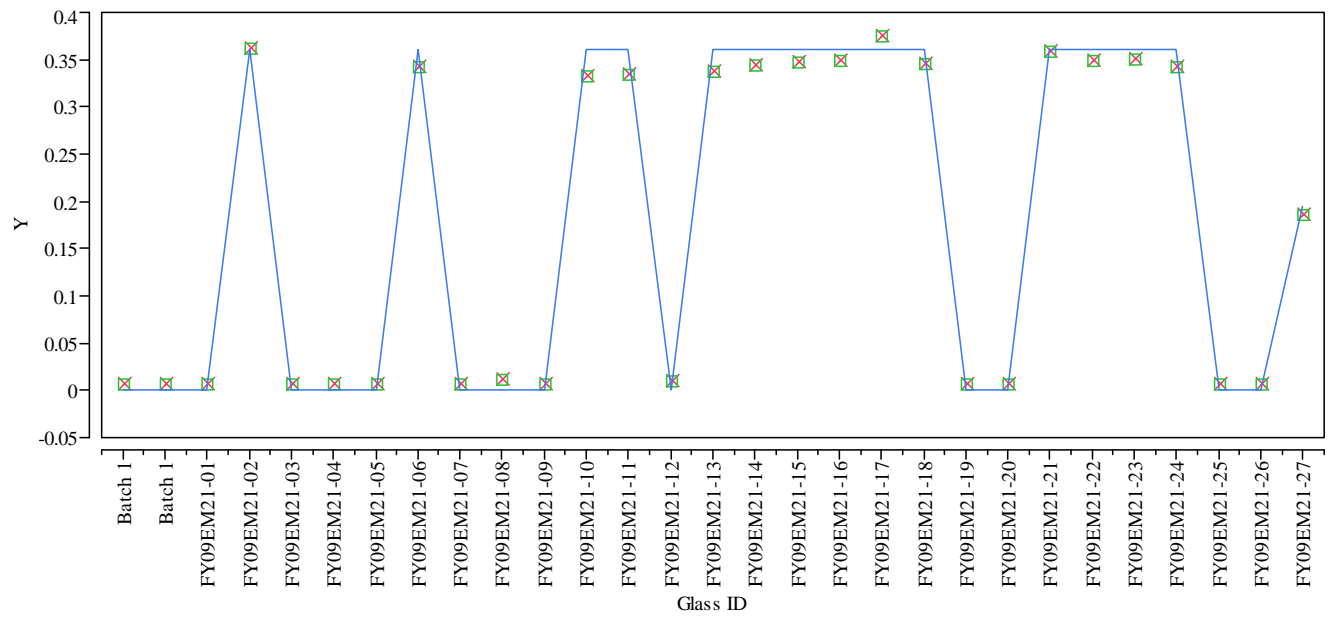

$\mathrm{Y} \times$ Measured $\square$ Measured bc - Targeted 
Exhibit A4. Average Measured and Bias-Corrected (bc) Versus Targeted Compositions by Glass ID by Oxide for the Non-Rad Matrix 2 Study

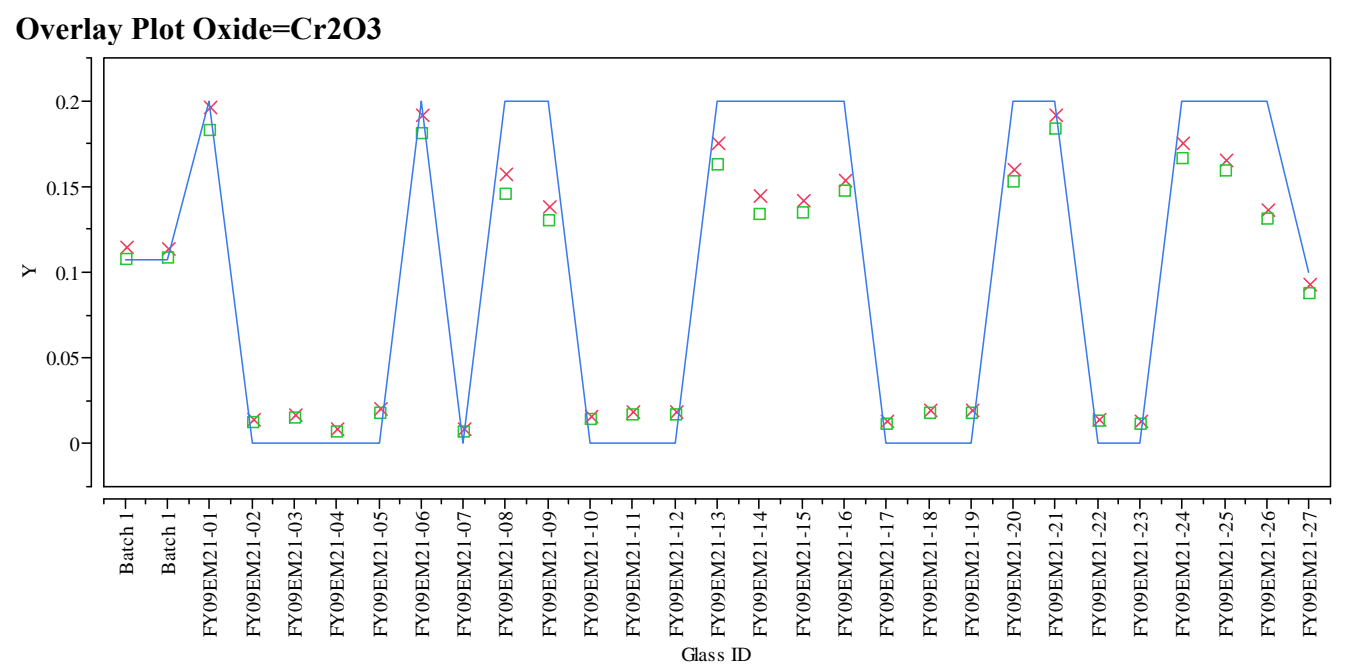

Overlay Plot Oxide $=\mathrm{CuO}$

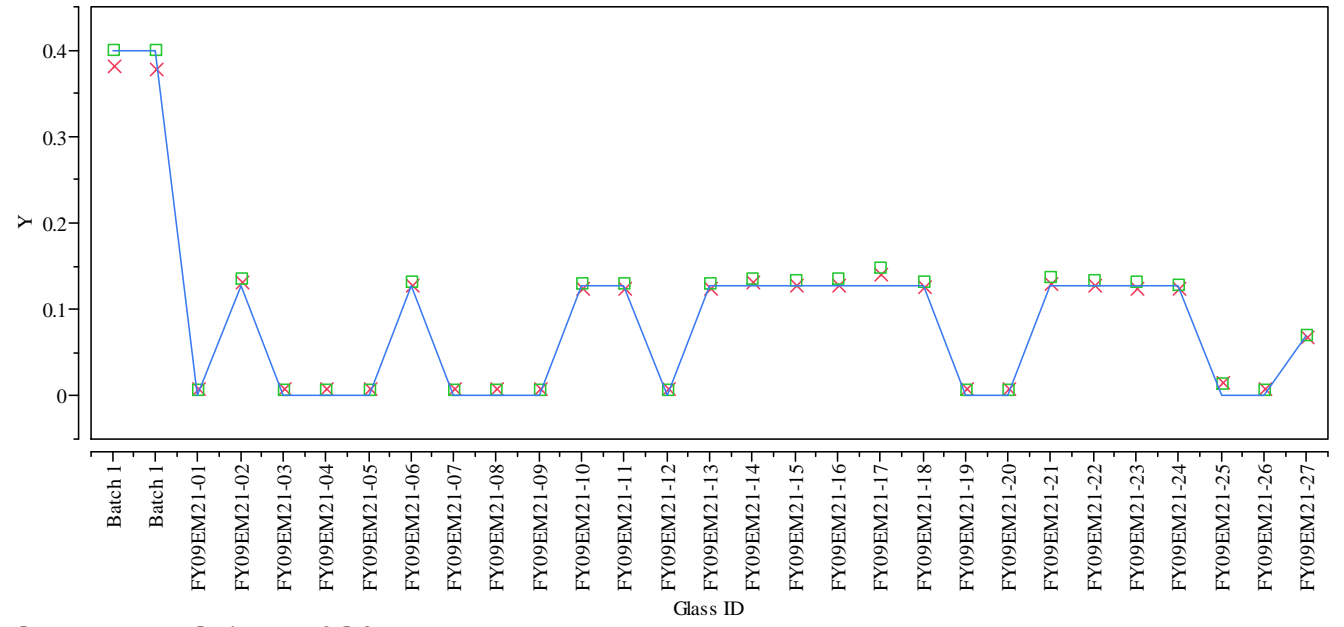

Overlay Plot Oxide $=\mathrm{Fe} 2 \mathrm{O3}$

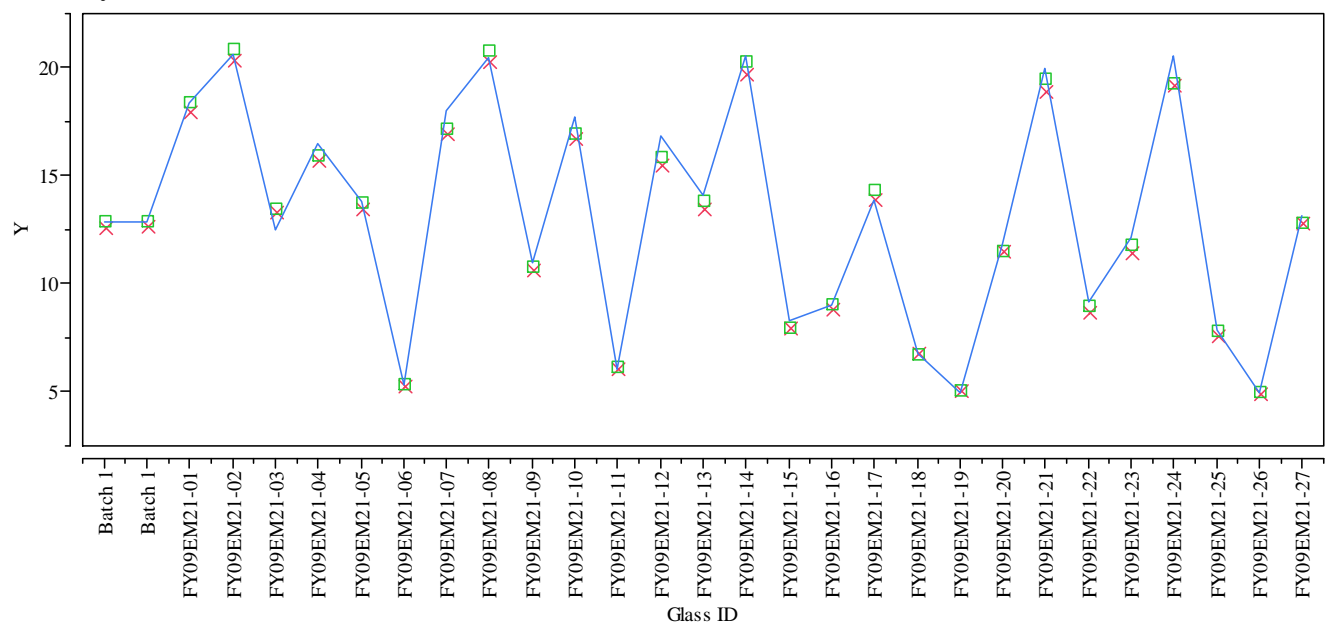

$\mathrm{Y} \times$ Measured $\square$ Measured bc - Targeted 
Exhibit A4. Average Measured and Bias-Corrected (bc) Versus Targeted Compositions by Glass ID by Oxide for the Non-Rad Matrix 2 Study

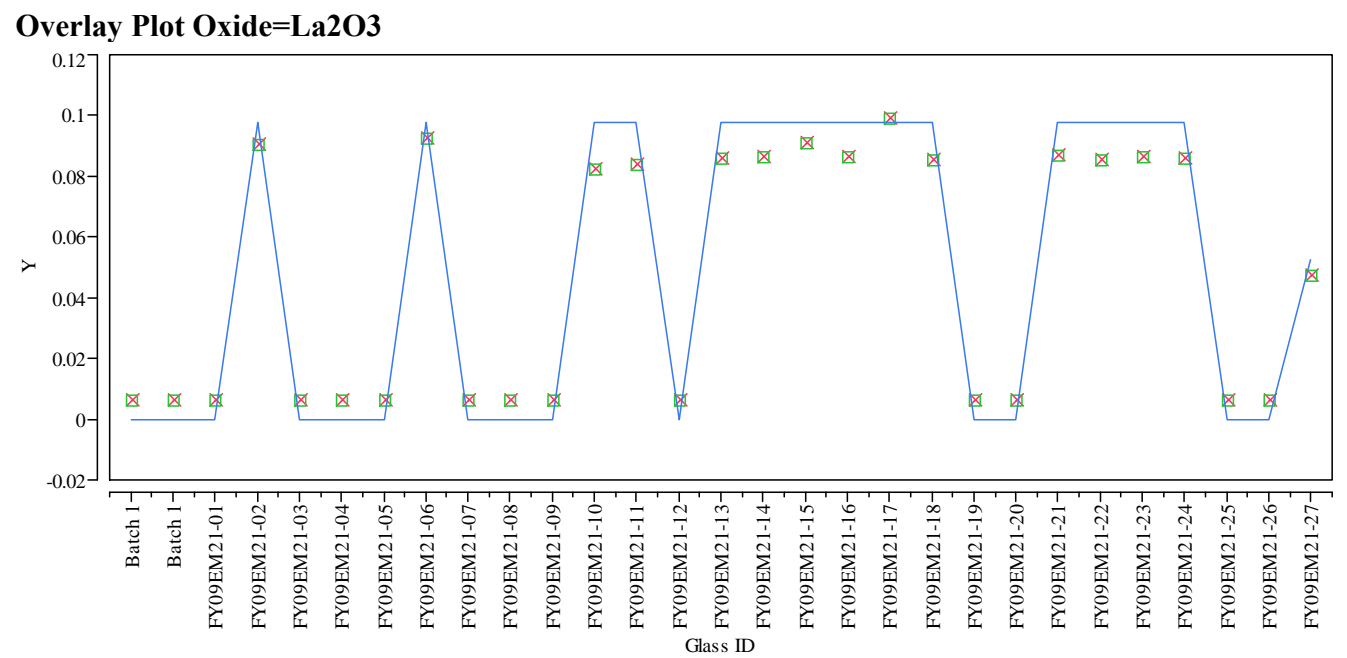

Overlay Plot Oxide $=$ Li2O

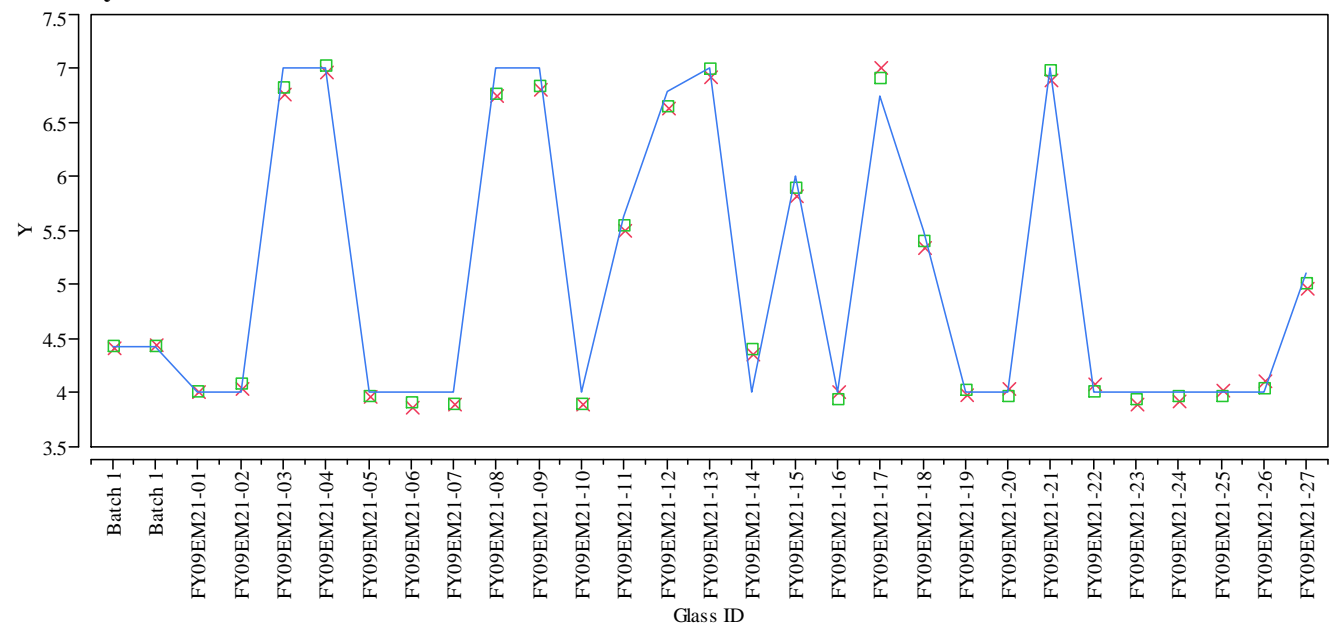

Overlay Plot Oxide $=$ MgO

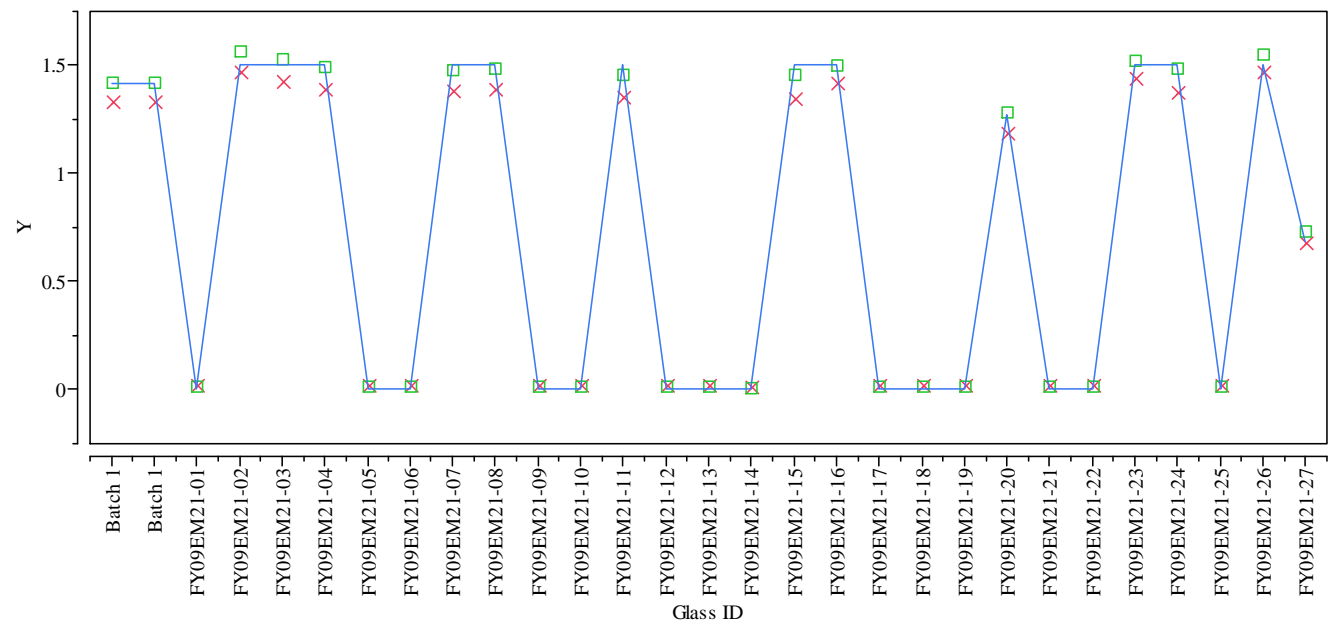

$\mathrm{Y} \times$ Measured $\square$ Measured bc — Targeted 
Exhibit A4. Average Measured and Bias-Corrected (bc) Versus Targeted Compositions by Glass ID by Oxide for the Non-Rad Matrix 2 Study

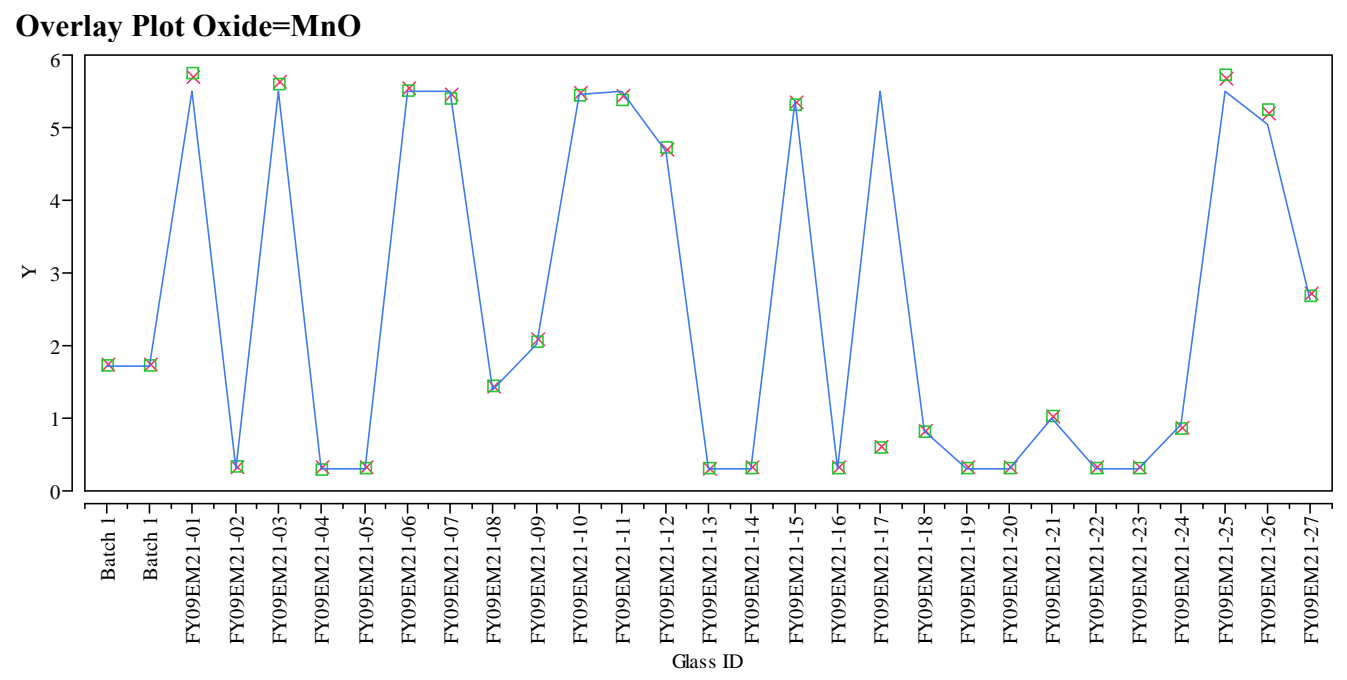

Overlay Plot Oxide $=\mathrm{Na} 2 \mathrm{O}$

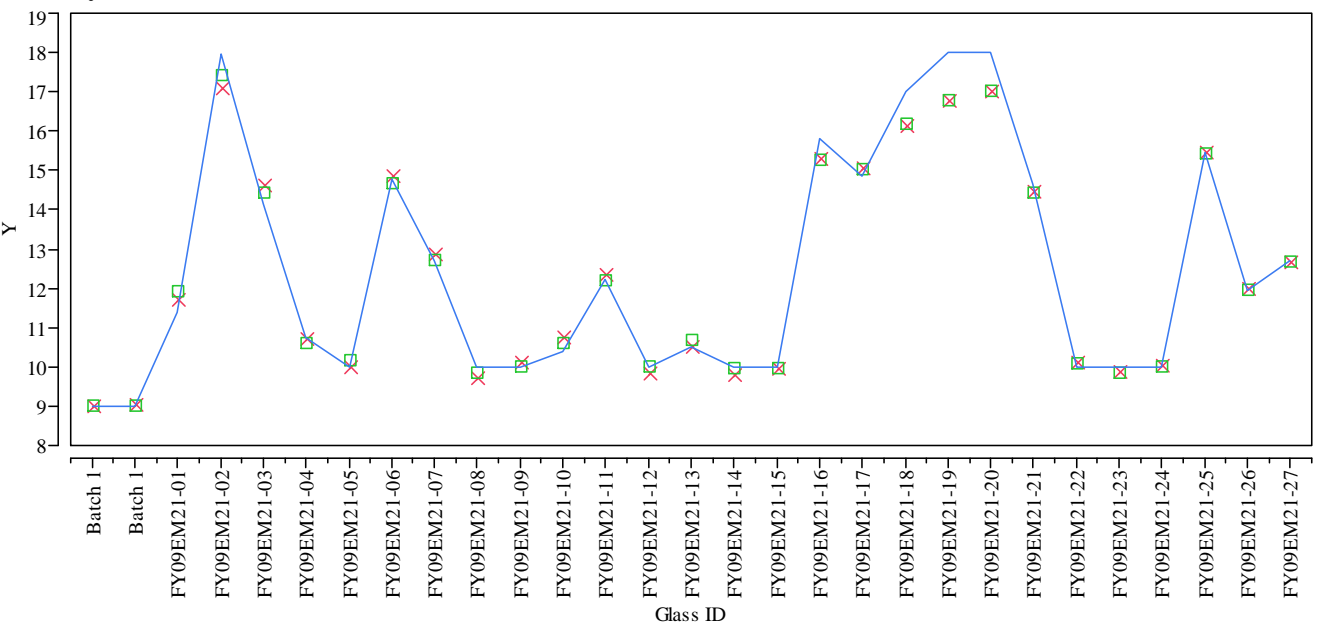

Overlay Plot Oxide $=\mathrm{NiO}$

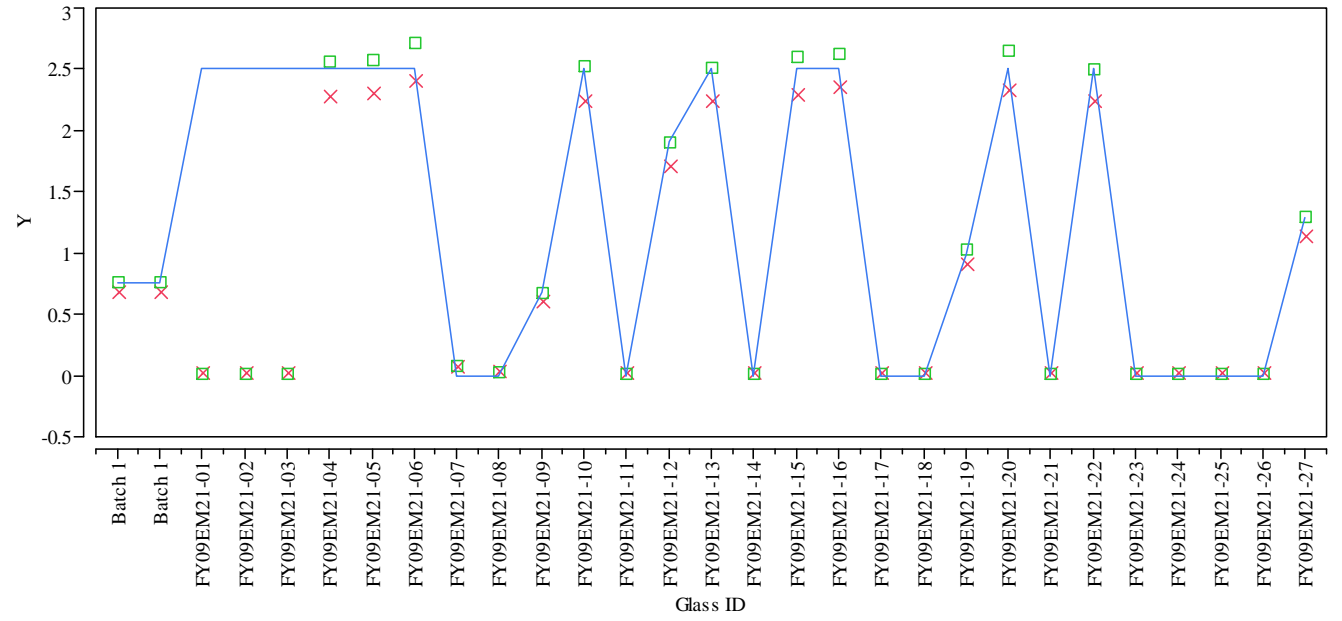

$\mathrm{Y} \times$ Measured Measured bc —Targeted 
Exhibit A4. Average Measured and Bias-Corrected (bc) Versus Targeted Compositions by Glass ID by Oxide for the Non-Rad Matrix 2 Study

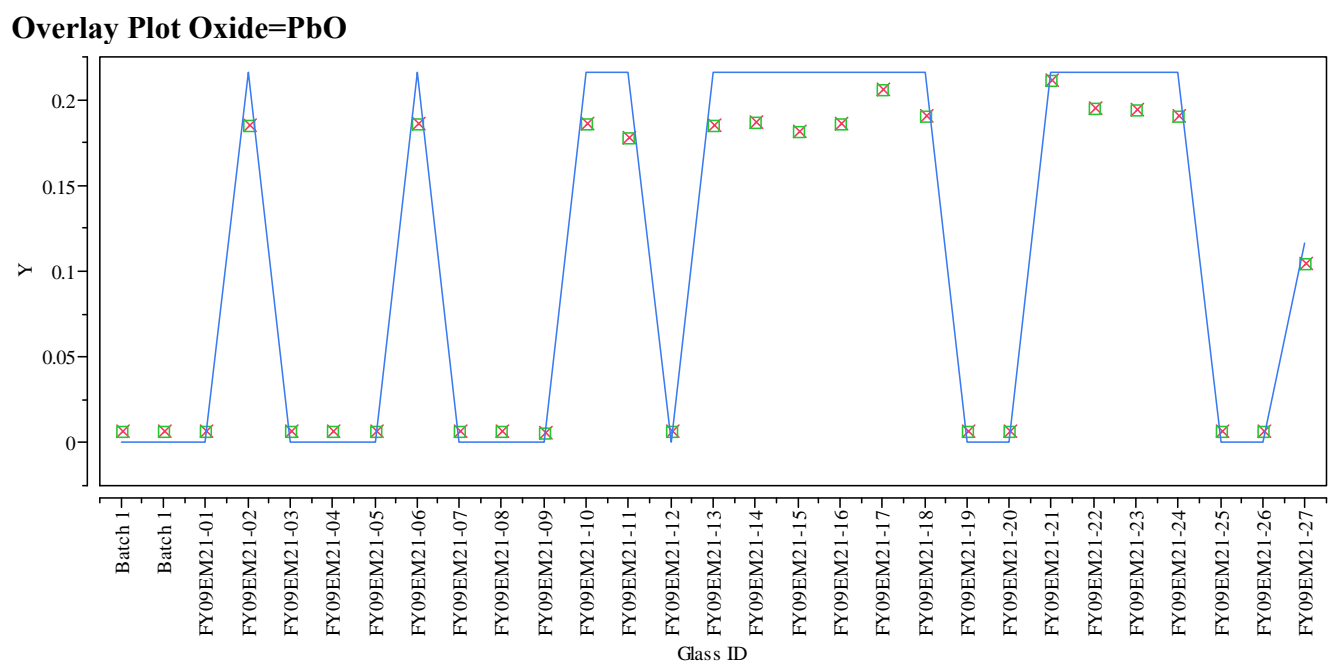

Overlay Plot Oxide $=\mathrm{SiO} 2$

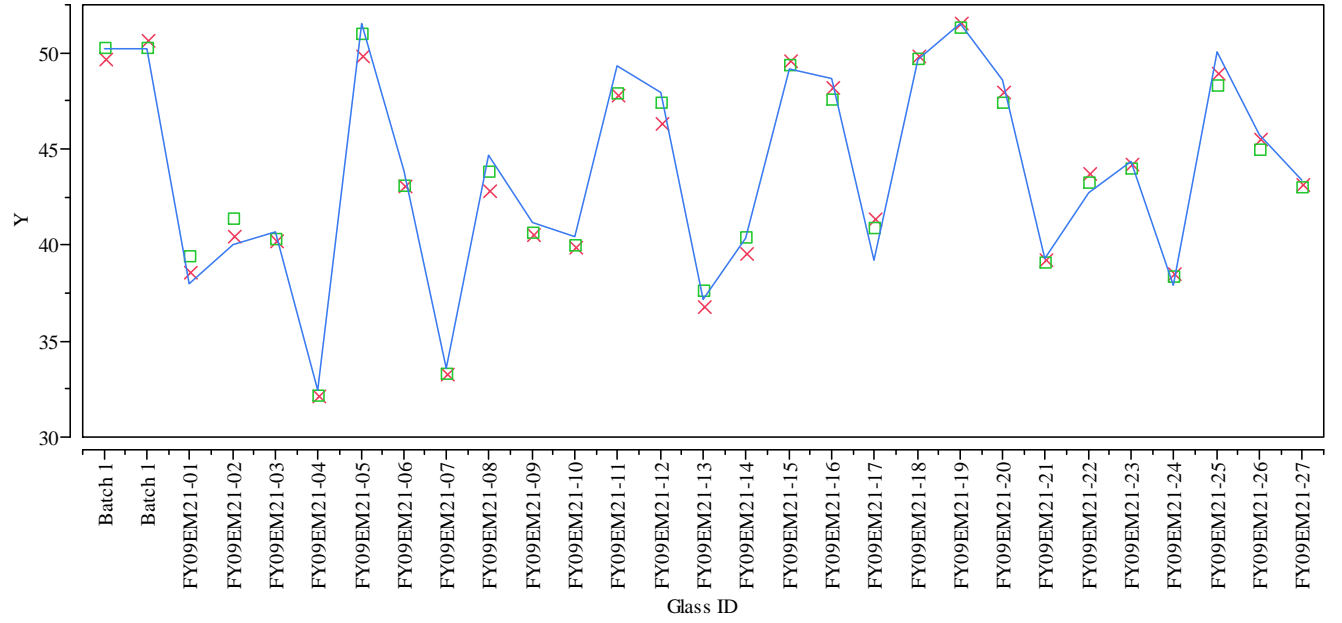

Overlay Plot Oxide $=\mathrm{SO} 4$

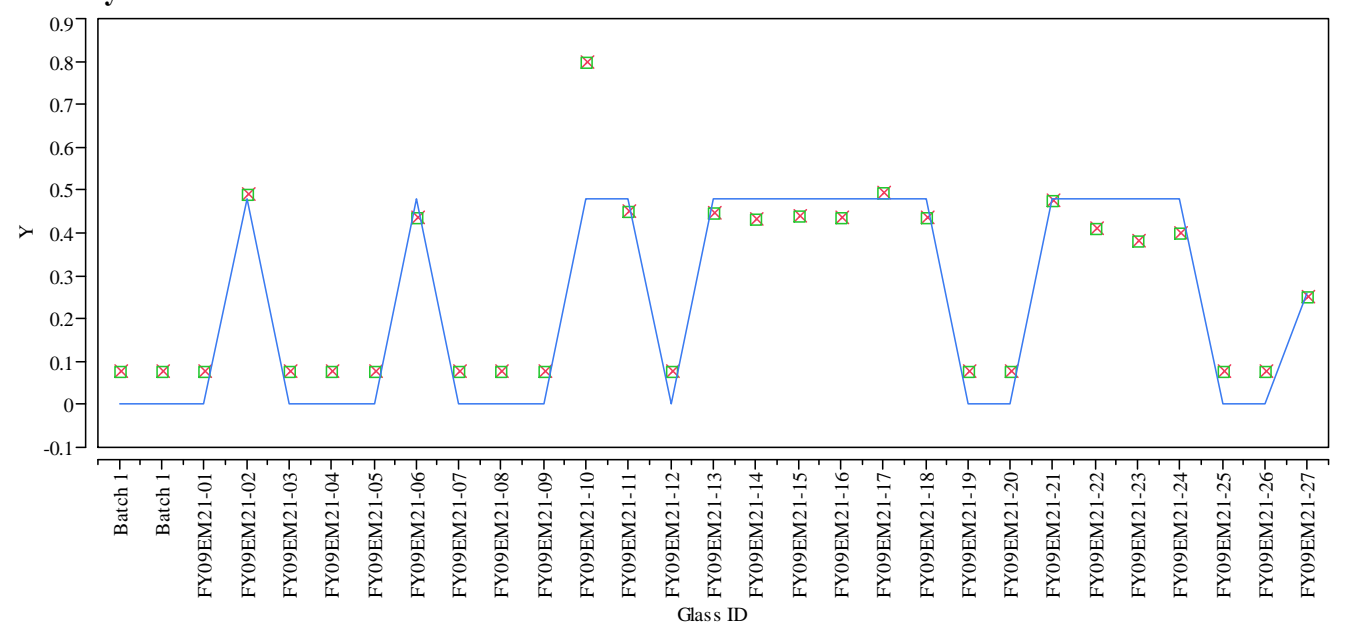

$\mathrm{Y} \times$ Measured $\square$ Measured bc - Targeted 
Exhibit A4. Average Measured and Bias-Corrected (bc) Versus Targeted Compositions by Glass ID by Oxide for the Non-Rad Matrix 2 Study

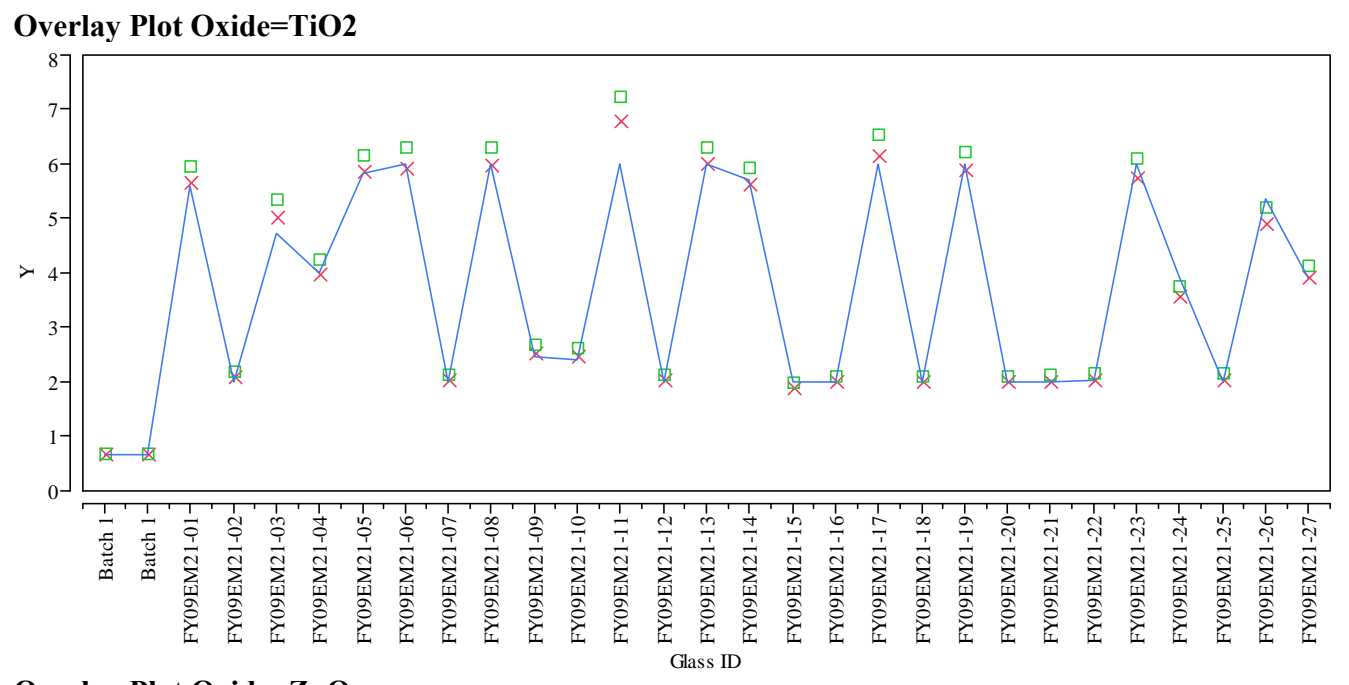

Overlay Plot Oxide $=\mathbf{Z n O}$

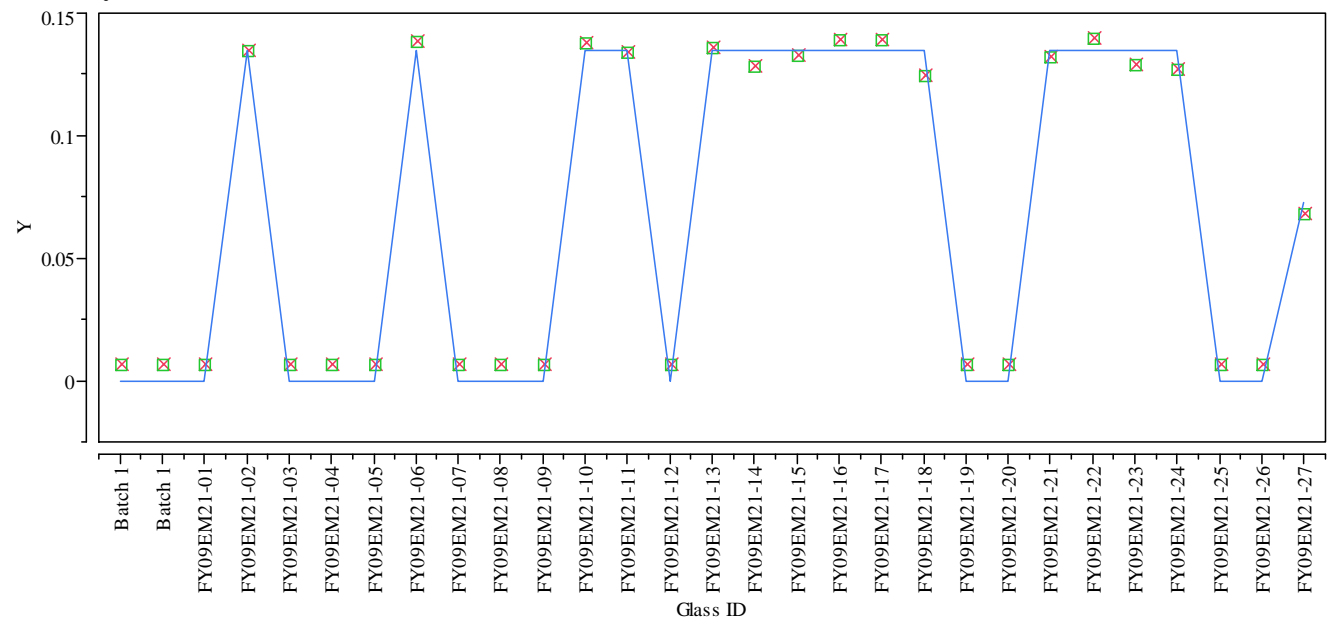

Overlay Plot Oxide $=\mathrm{ZrO2}$

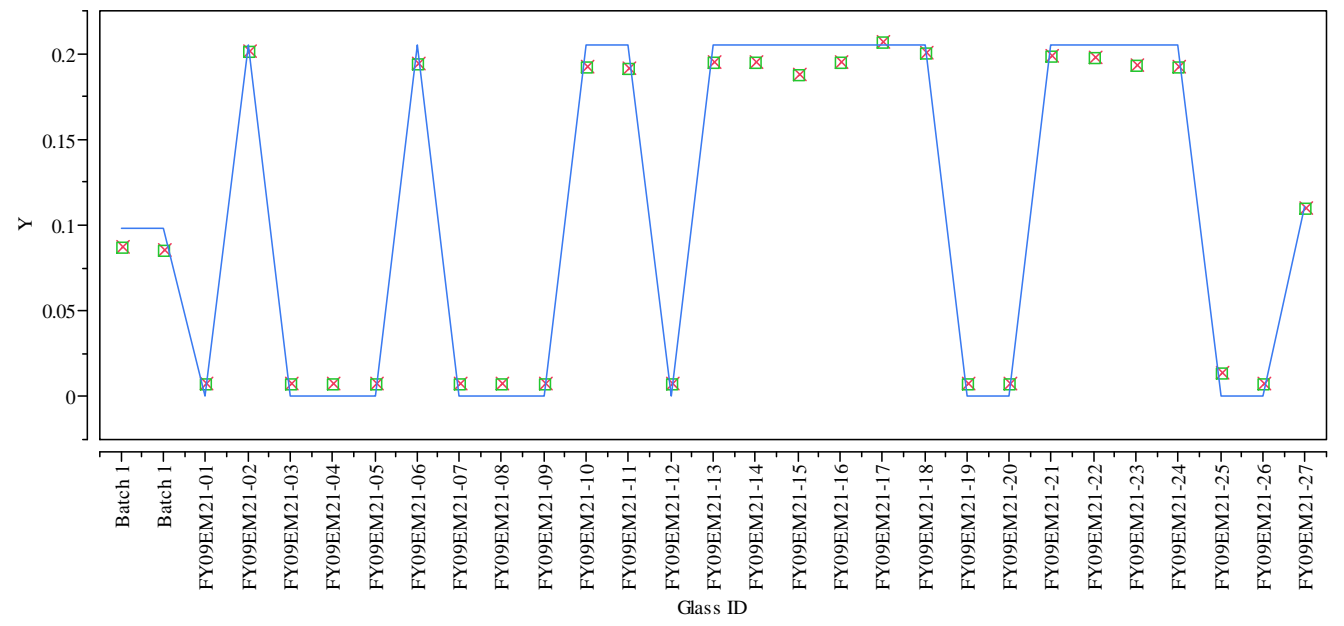

$\mathrm{Y} \times$ Measured $\square$ Measured bc — Targeted 
SRNL-STI-2009-00778, Revision 0

Exhibit A4. Average Measured and Bias-Corrected (bc) Versus Targeted Compositions by Glass ID by Oxide for the Non-Rad Matrix 2 Study

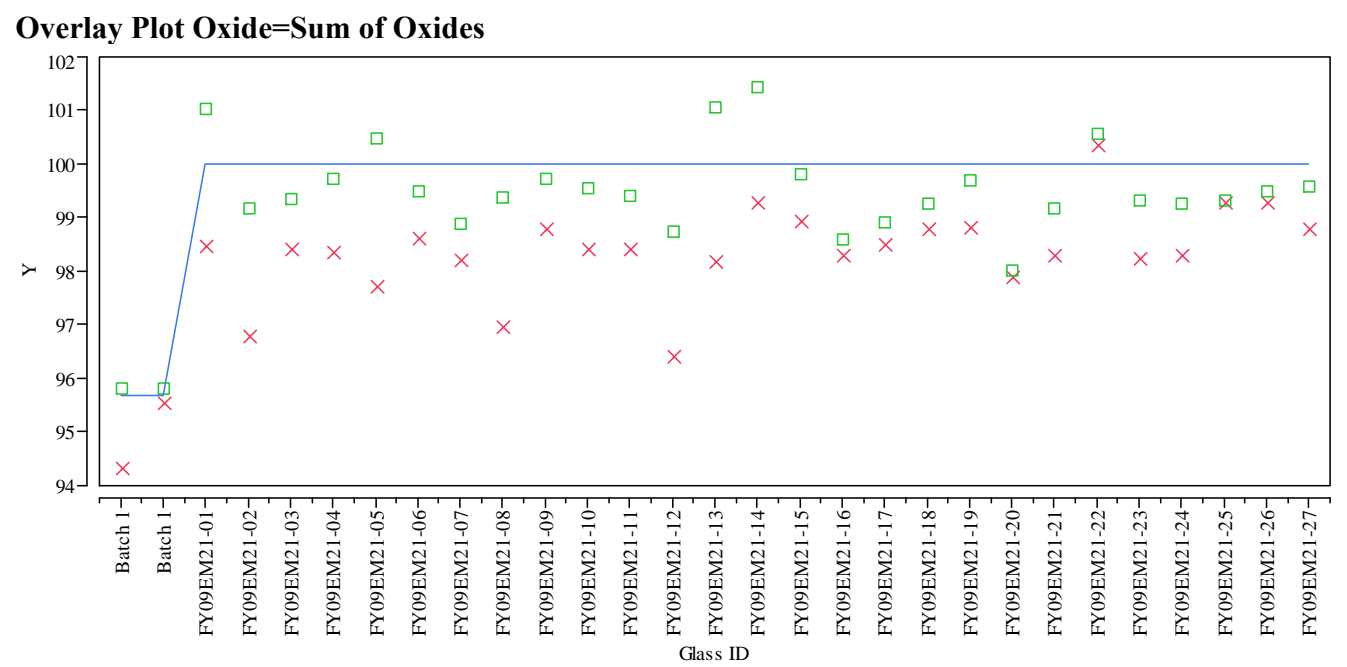

$\mathrm{Y} \times$ Measured $\square$ Measured bc - Targeted 
Exhibit A5. Sample Measurements by Lab ID within Glass ID for the Rad Glasses of the Matrix 2 Study by Oxide by Analytical Block

Analyte=Al2O3 (wt\%)

Variability Chart for Measured

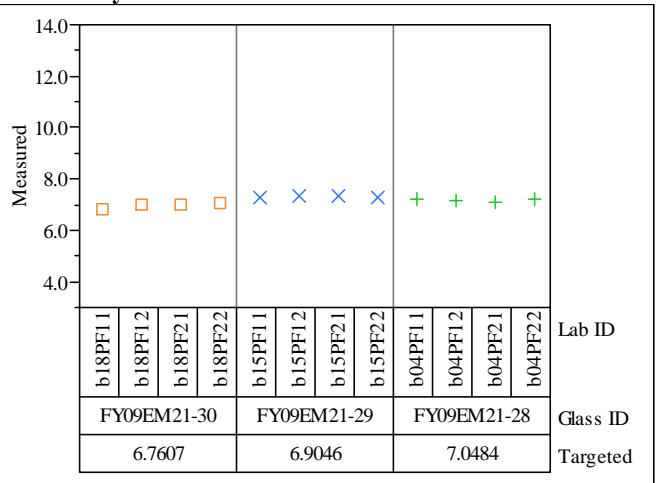

Analyte $=\mathrm{B} 2 \mathrm{O} 3(\mathrm{wt} \%)$

Variability Chart for Measured

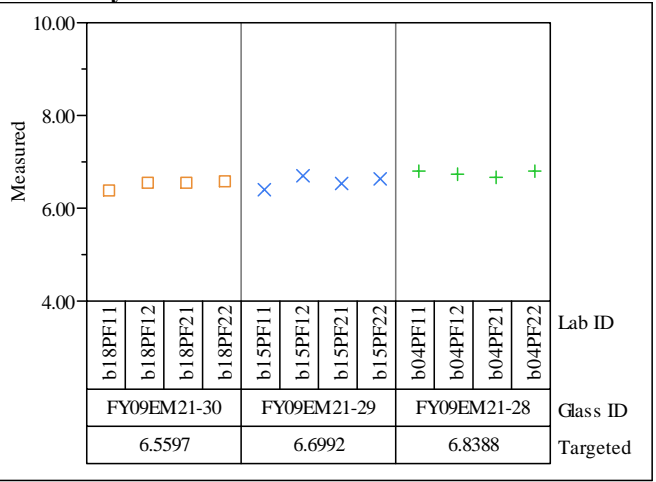

Analyte $=\mathrm{BaO}(\mathbf{w t} \%)$

Variability Chart for Measured

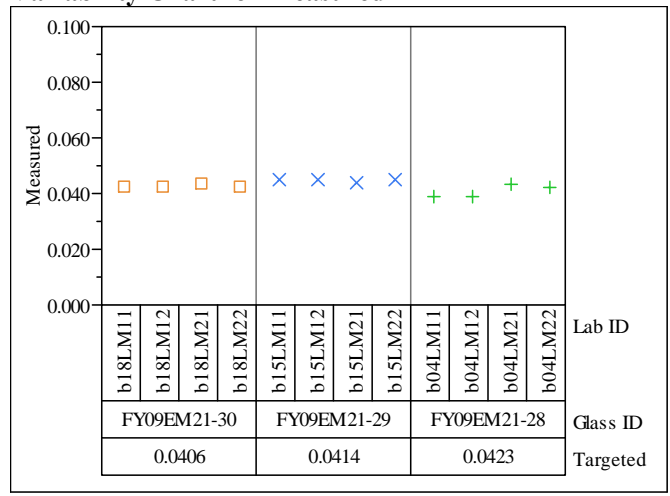

Analyte $=\mathrm{CaO}(\mathrm{wt} \%)$

Variability Chart for Measured

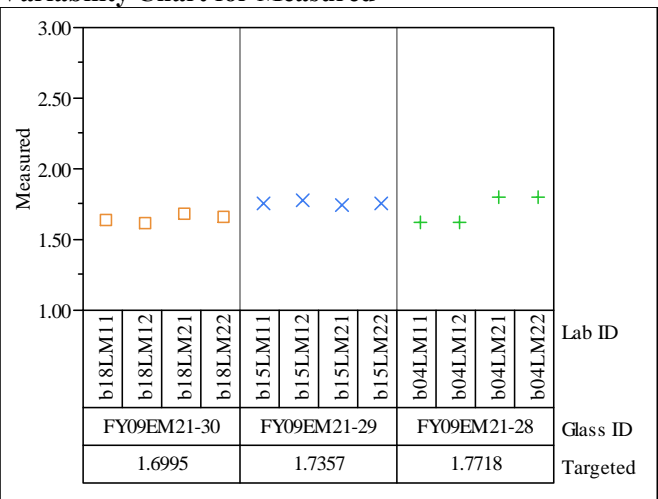

Analyte $=\mathrm{CdO}(\mathrm{wt} \%)$

Variability Chart for Measured

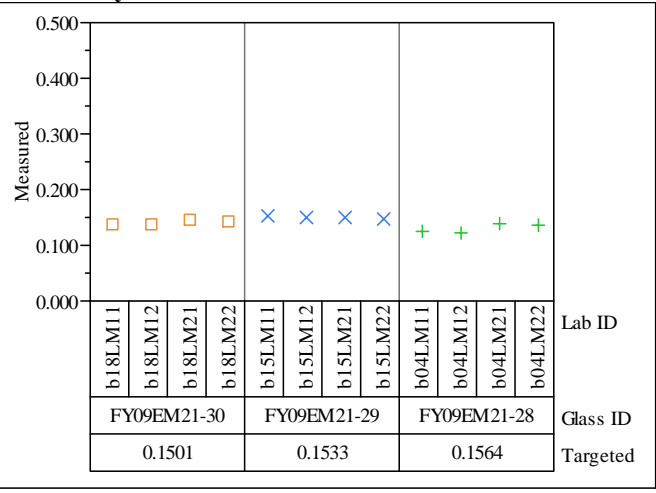

Analyte $=\mathrm{Ce} 2 \mathrm{O} 3(\mathrm{wt} \%)$

Variability Chart for Measured

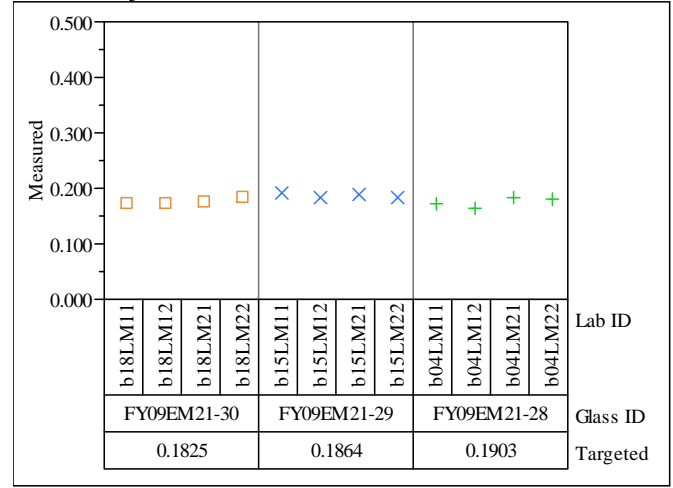


Exhibit A5. Sample Measurements by Lab ID within Glass ID for the Rad Glasses of the Matrix 2 Study by Oxide by Analytical Block

Analyte $=\mathrm{Cr} 2 \mathrm{O3}(\mathrm{wt} \%)$

Variability Chart for Measured

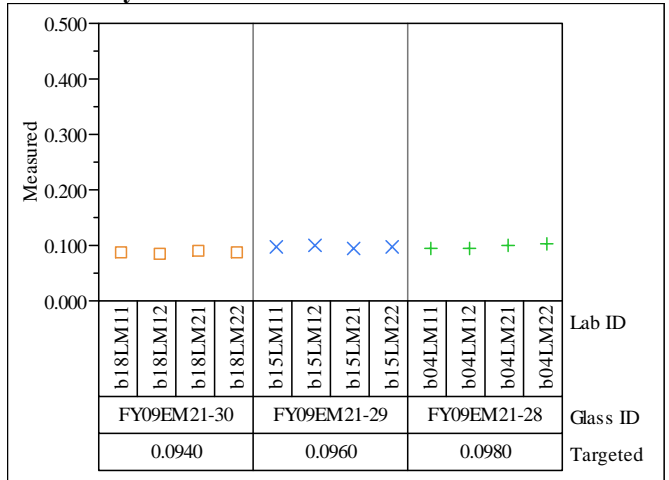

Analyte $=\mathrm{CuO}(\mathrm{wt} \%)$

Variability Chart for Measured

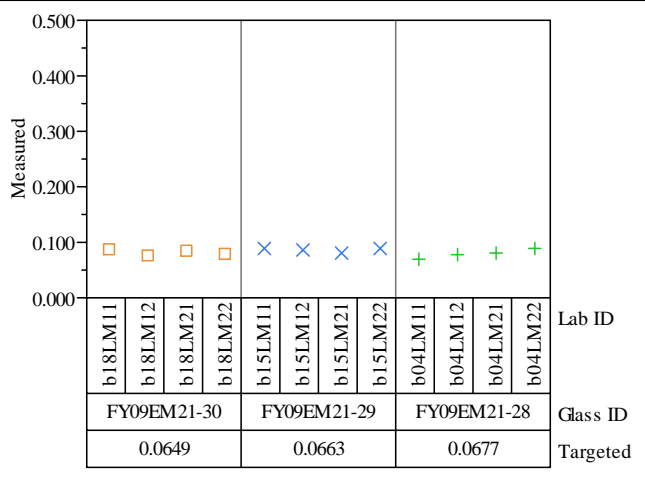

Analyte $=\mathrm{Fe} 2 \mathrm{O3}(\mathrm{wt} \%)$

Variability Chart for Measured

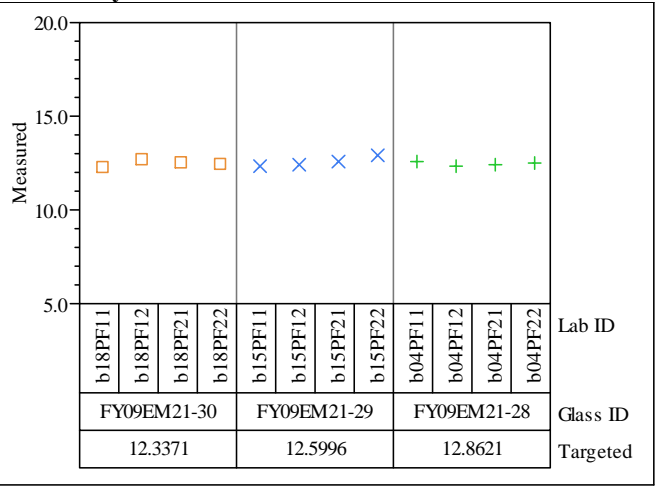

Analyte $=$ K2O (wt\%)

Variability Chart for Measured

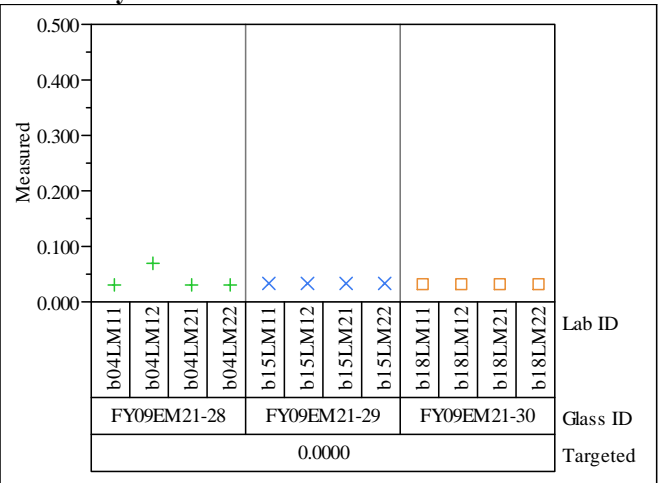

Analyte $=$ La2O3 (wt\%)

Variability Chart for Measured

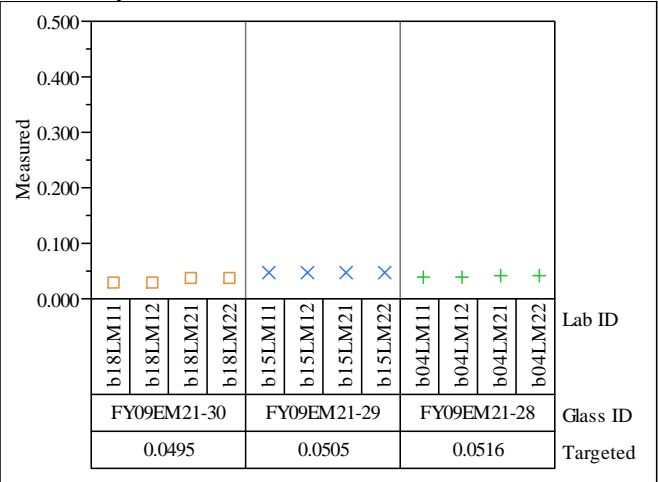

Analyte $=\mathbf{L i 2 O}(w t \%)$

Variability Chart for Measured

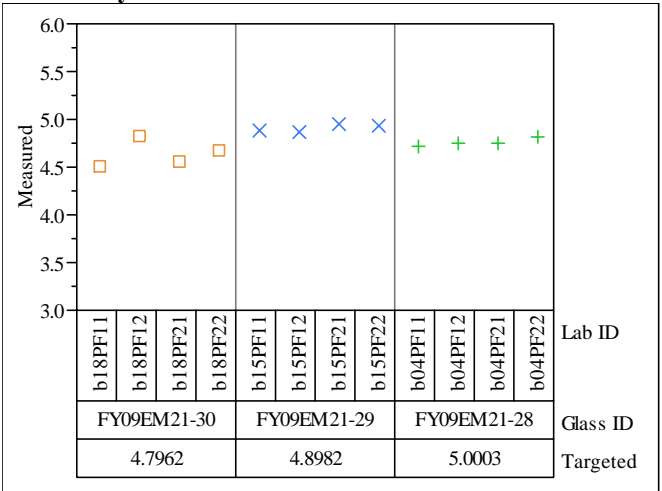


Exhibit A5. Sample Measurements by Lab ID within Glass ID for the Rad Glasses of the Matrix 2 Study by Oxide by Analytical Block

Analyte $=\operatorname{MgO}(w t \%)$

Variability Chart for Measured

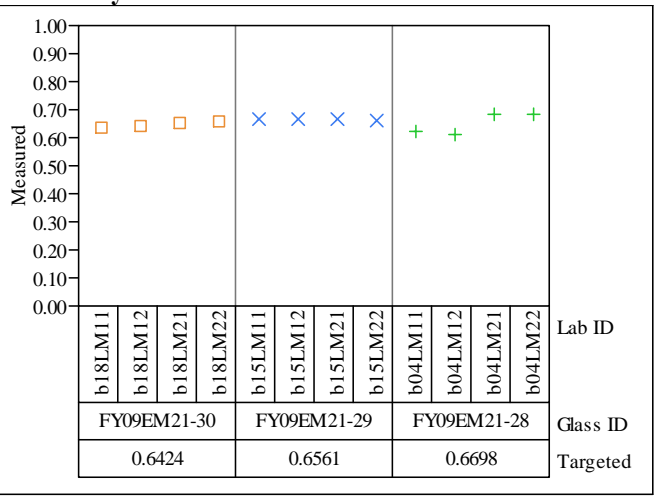

Analyte $=\mathrm{MnO}(\mathrm{wt} \%)$

Variability Chart for Measured

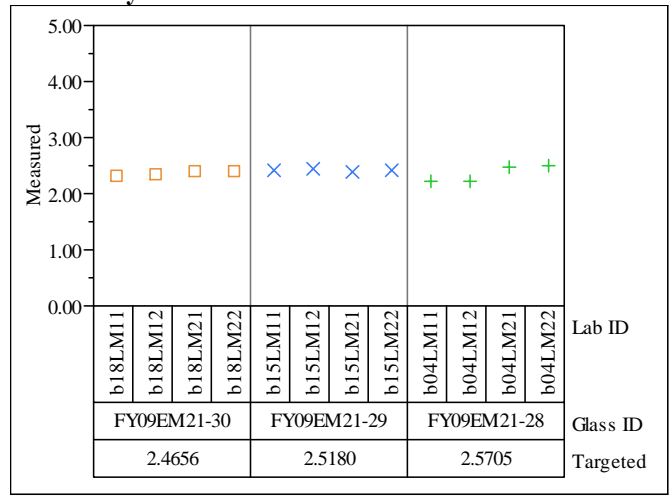

Analyte $=\mathrm{Na2O}(\mathrm{wt} \%)$

Variability Chart for Measured

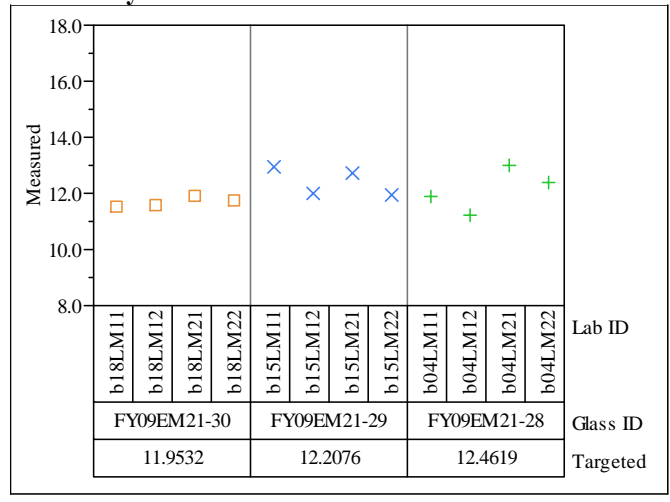

Analyte $=\mathrm{NiO}(\mathbf{w t} \%)$

Variability Chart for Measured

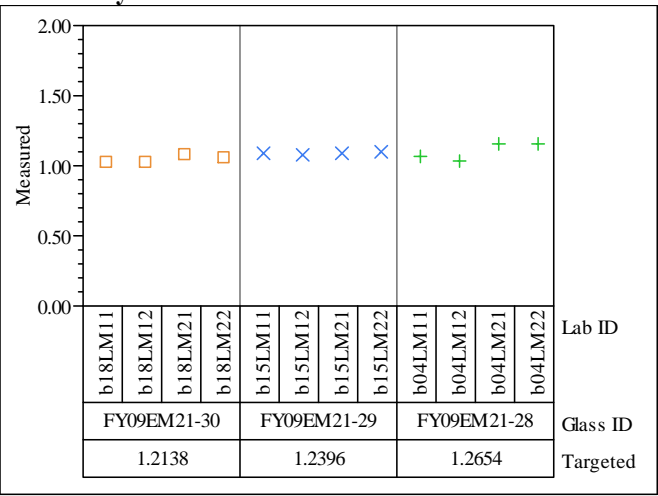

Analyte $=\mathrm{PbO}(\mathrm{wt} \%)$

Variability Chart for Measured

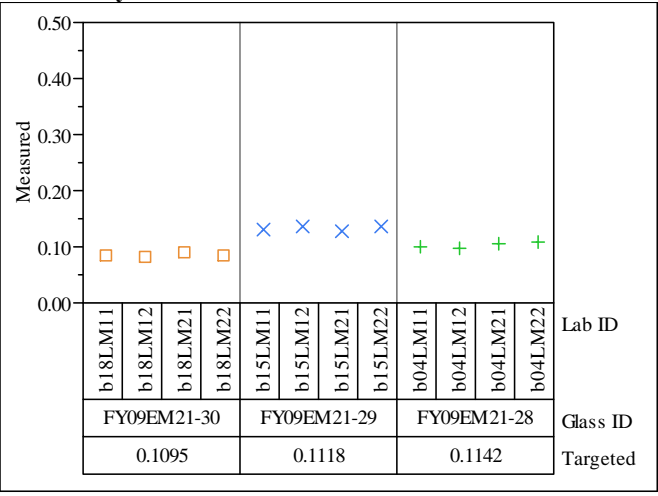

Analyte $=\mathrm{SiO} 2(\mathrm{wt} \%)$

Variability Chart for Measured

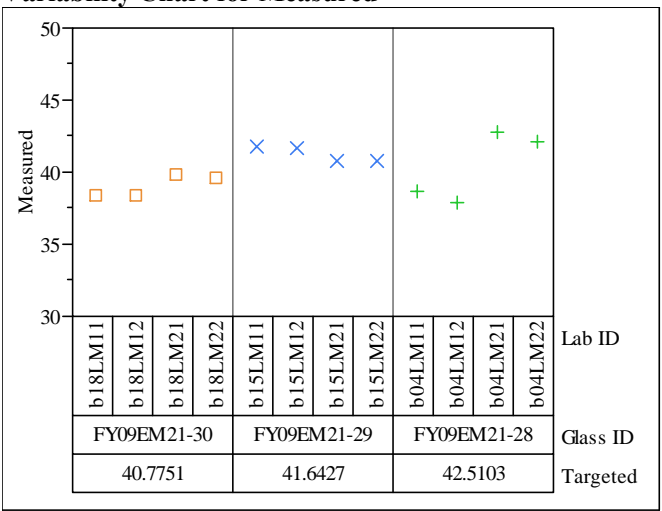


Exhibit A5. Sample Measurements by Lab ID within Glass ID for the Rad Glasses of the Matrix 2 Study by Oxide by Analytical Block

Analyte=SO4 (wt\%)

Variability Chart for Measured

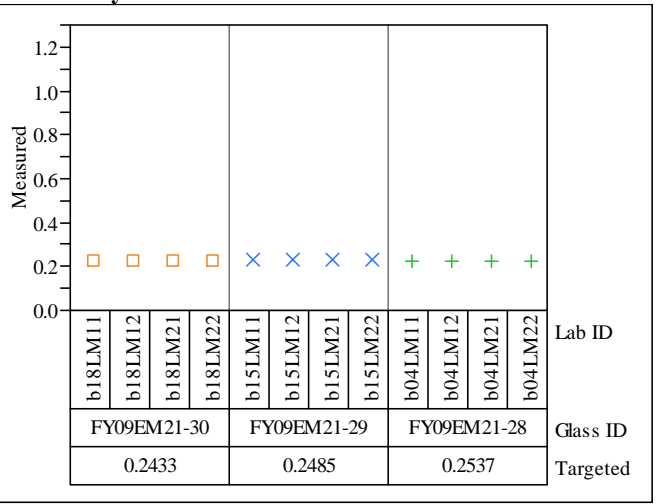

Analyte $=\mathrm{TiO} 2(\mathrm{wt} \%)$

Variability Chart for Measured

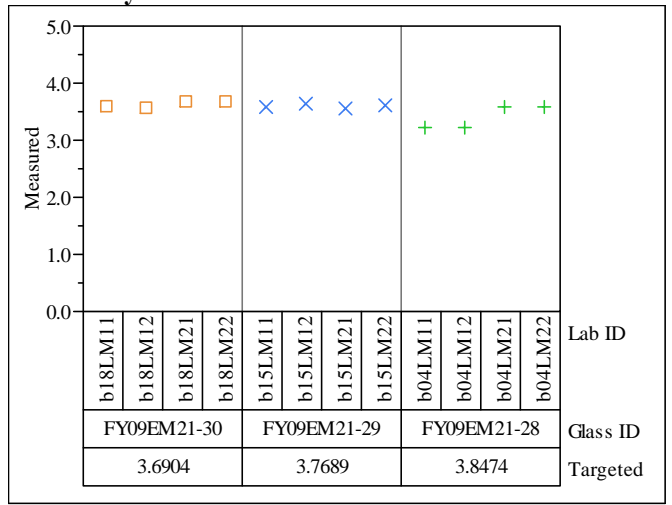

Analyte $=$ U308 (wt\%)

Variability Chart for Measured

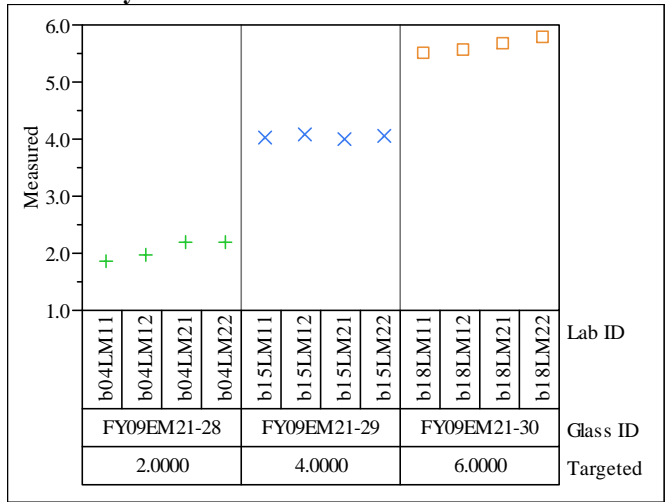

Analyte $=\mathrm{ZnO}(\mathrm{wt} \%)$

Variability Chart for Measured

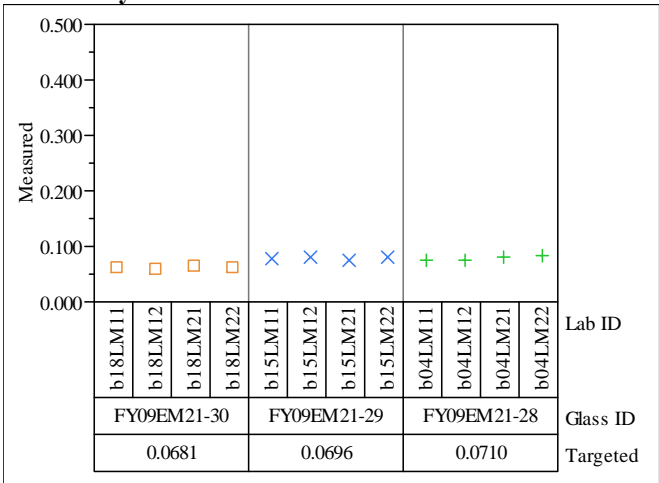

Analyte $=\mathrm{ZrO2}(\mathrm{wt} \%)$

Variability Chart for Measured

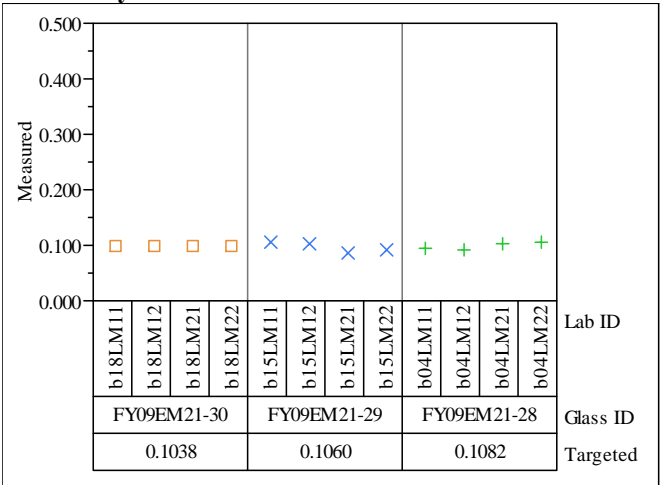


SRNL-STI-2009-00778, Revision 0

Exhibit A6. Average Measured and Bias-Corrected (bc) Versus Targeted Compositions by Glass ID by Oxide for the Rad Glasses of the Matrix 2 Study
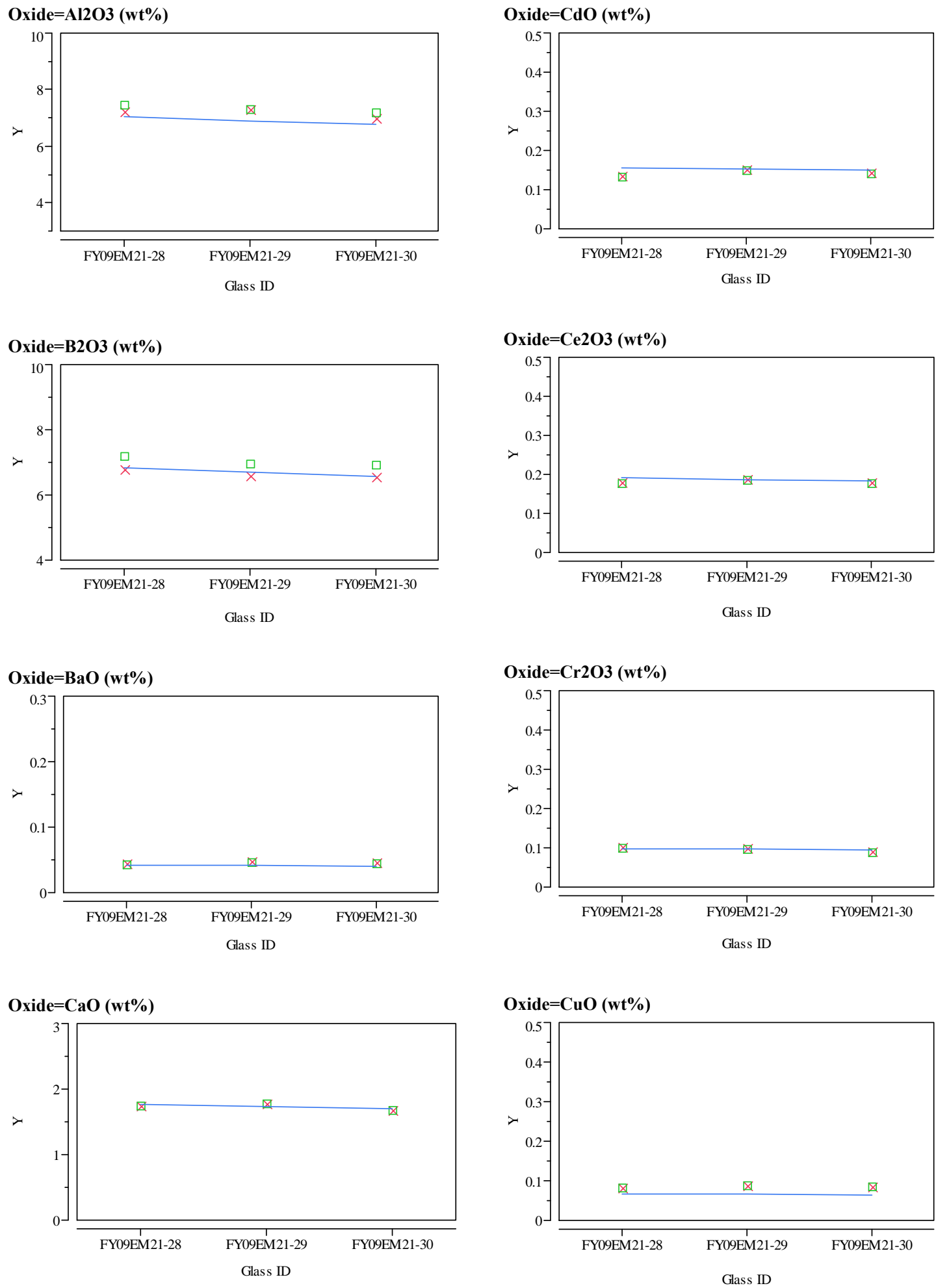

$\mathrm{Y} \times$ Measured Measured bc - Targeted

$\mathrm{Y} \times$ Measured $\square$ Measured bc — Targeted 
SRNL-STI-2009-00778, Revision 0

Exhibit A6. Average Measured and Bias-Corrected (bc) Versus Targeted Compositions by Glass ID by Oxide for the Rad Glasses of the Matrix 2 Study

Oxide=Fe2O3 (wt\%)

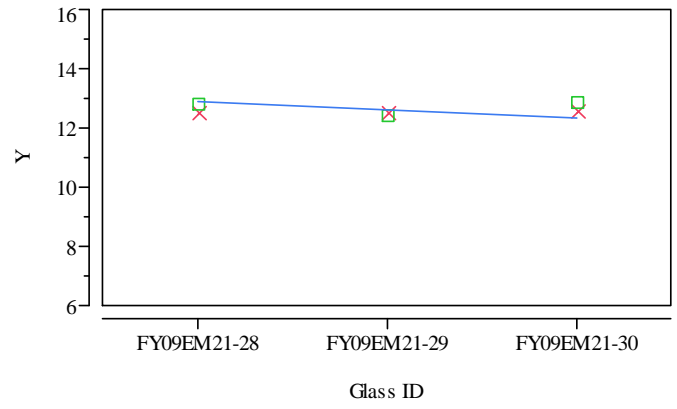

Oxide $=\mathrm{K2O}(\mathrm{wt} \%)$

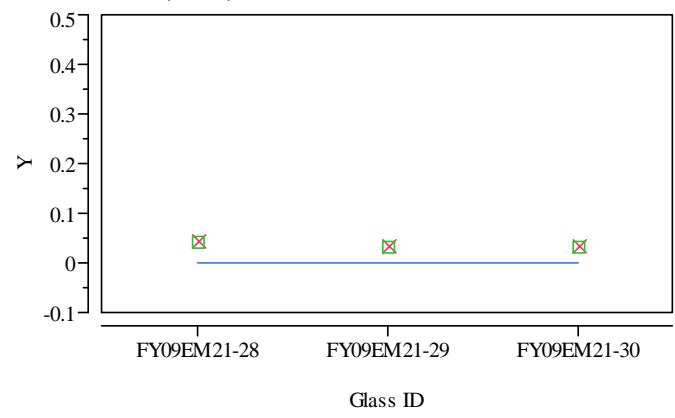

Oxide $=\mathbf{L a 2 O 3}(\mathbf{w t} \%)$

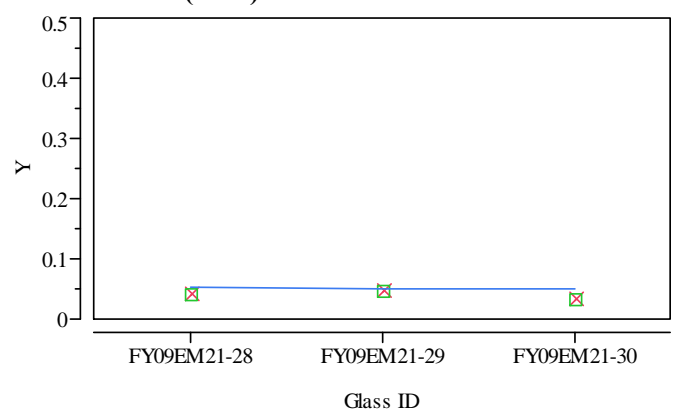

Oxide $=$ Li2O $($ wt $\%)$

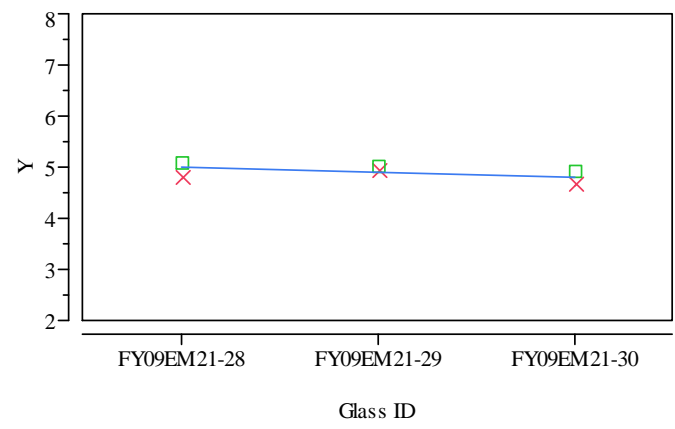

$\mathrm{Y} \times$ Measured $\square$ Measured bc —Targeted
Oxide $=\operatorname{MgO}(\mathbf{w t} \%)$

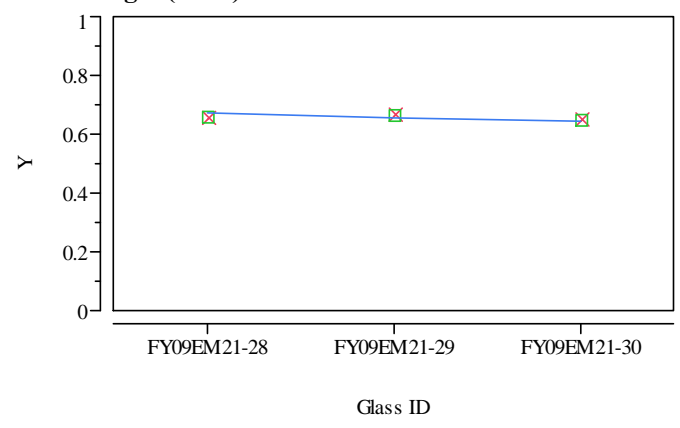

Oxide $=\operatorname{MnO}(\mathbf{w t} \%)$

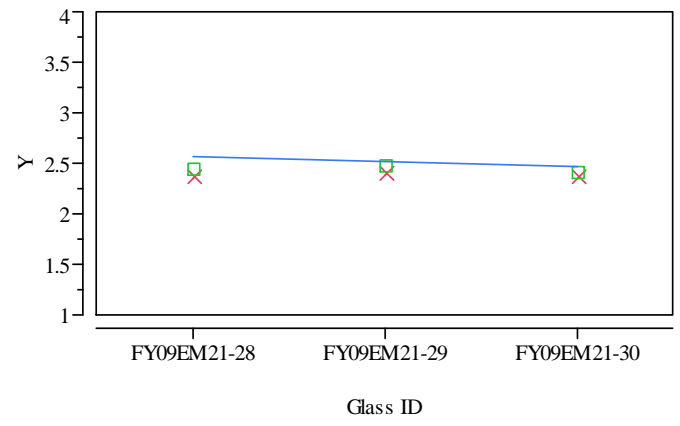

Oxide $=\mathrm{Na2O}(\mathbf{w t} \%)$

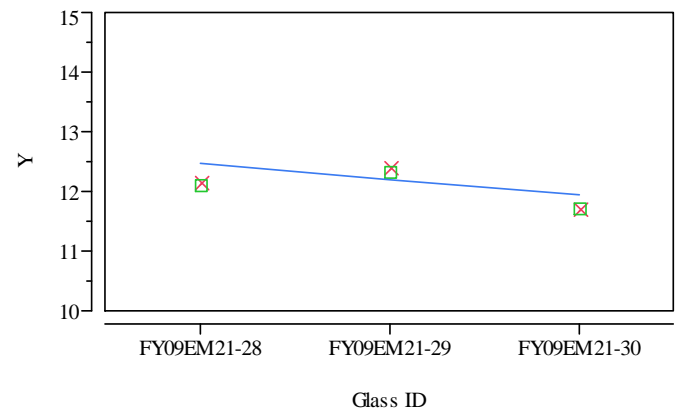

Oxide $=\mathrm{NiO}(\mathbf{w t} \%)$

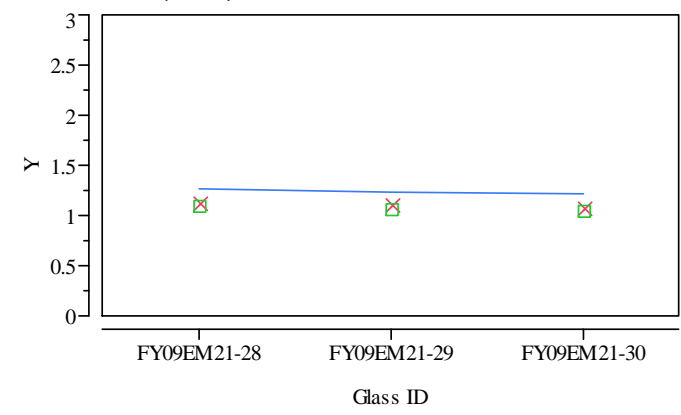

$\mathrm{Y} \times$ Measured $\square$ Measured bc — Targeted 
SRNL-STI-2009-00778, Revision 0

Exhibit A6. Average Measured and Bias-Corrected (bc) Versus Targeted Compositions by Glass ID by Oxide for the Rad Glasses of the Matrix 2 Study

Oxide $=\mathbf{P b O}(\mathbf{w t} \%)$
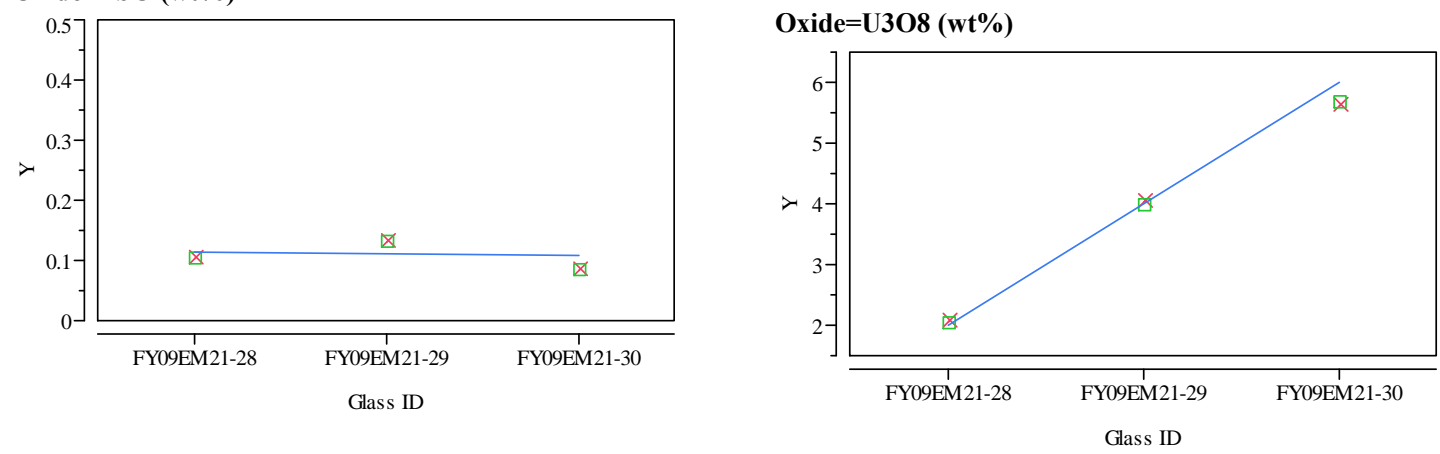

Oxide $=\mathrm{SiO} 2(\mathbf{w t} \%)$
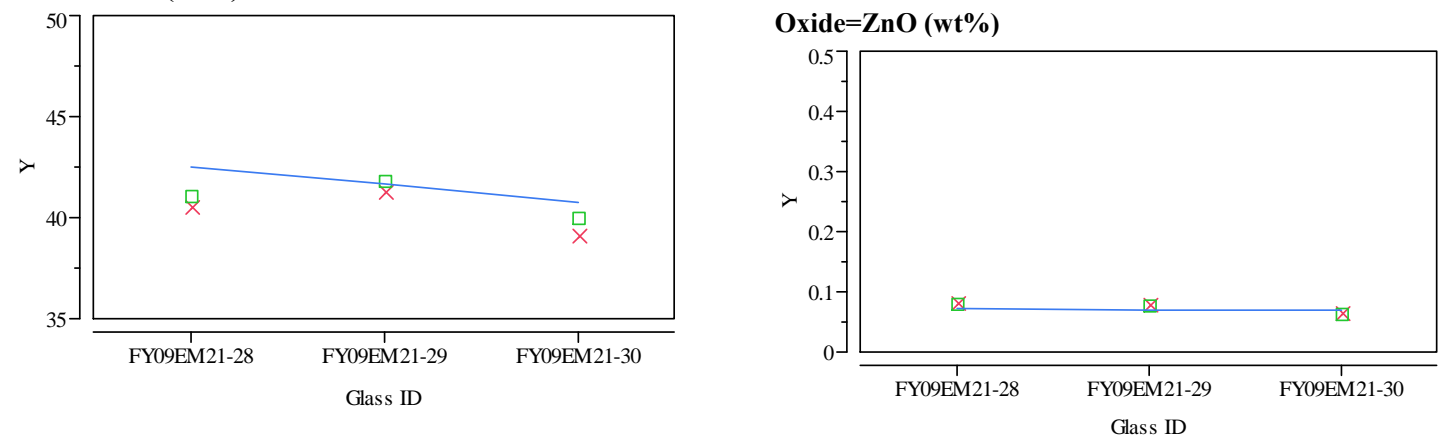

Oxide=SO4 (wt\%)
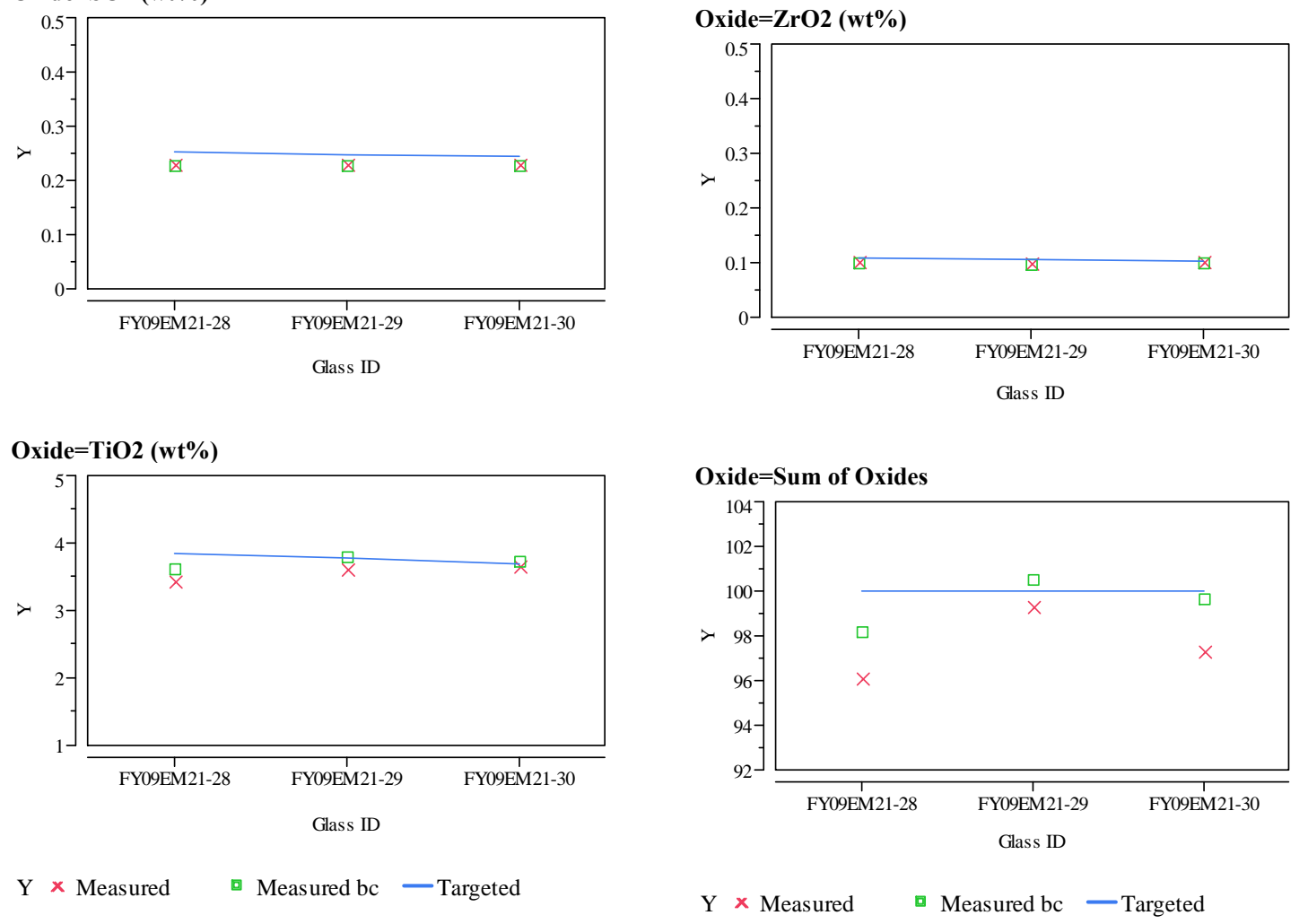


\section{Appendix B:}

\section{Tables and Exhibits Supporting the Analysis of the PCT Results for the Matrix 2 Study \\ Glasses}


SRNL-STI-2009-00778, Revision 0

This page intentionally left blank. 
SRNL-STI-2009-00778, Revision 0

Table B1. PSAL's Measurements of the PCT Solutions for the Matrix 2 Non-Rad Glasses As-Received (ar) and After Appropriate Adjustments (in ppm)

\begin{tabular}{|c|c|c|c|c|c|c|c|c|c|c|c|c|}
\hline Set & Glass ID (w HT) & Block & Seq & \begin{tabular}{|l|} 
Lab ID \\
\end{tabular} & B ar & Li ar & Na ar & Si ar & B (ppm) & Li (ppm) & $\mathrm{Na}(\mathrm{ppm})$ & Si (ppm) \\
\hline 1 & Soln Std & 1 & 1 & std-11-1 & 21.5 & 10.0 & 82.7 & 52.7 & 21.50 & 10.00 & 82.70 & 52.70 \\
\hline 1 & FY09EM21-09 & 1 & 3 & H35 & 13.9 & 16.4 & 31.9 & 52.0 & 23.17 & 27.33 & 53.17 & 86.67 \\
\hline 1 & FY09EM21-07 & 1 & 5 & H39 & 357 & 122 & 572 & 102 & 595.01 & 203.34 & 953.35 & 170.00 \\
\hline 1 & FY09EM21-04 & 1 & 6 & H32 & 166 & 101 & 238 & 104 & 276.67 & 168.34 & 396.67 & 173.34 \\
\hline 1 & FY09EM21-06 & 1 & 7 & $\mathrm{H} 55$ & 119 & 45.9 & 241 & 163 & 198.34 & 76.50 & 401.67 & 271.67 \\
\hline 1 & FY09EM21-08 & 1 & 10 & $\mathrm{H} 54$ & 48.8 & 71.0 & 160 & 215 & 81.33 & 118.34 & 266.67 & 358.34 \\
\hline 1 & FY09EM21-09сcс & 1 & 11 & $\mathrm{H} 48$ & 9.49 & 11.1 & 22.4 & 40.8 & 15.82 & 18.50 & 37.33 & 68.00 \\
\hline 1 & FY09EM21-04ccc & 1 & 12 & H37 & 55.2 & 39.6 & 97.8 & 78.4 & 92.00 & 66.00 & 163.00 & 130.67 \\
\hline 1 & Soln Std & 1 & 13 & std-11-2 & 20.5 & 9.99 & 81.9 & 51.5 & 20.50 & 9.99 & 81.90 & 51.50 \\
\hline 1 & blank & 1 & 14 & H07 & 1.01 & $<0.100$ & $<0.100$ & $<0.100$ & 1.68 & 0.08 & 0.08 & 0.08 \\
\hline 1 & ARM-1 & 1 & 15 & H10 & 10.7 & 7.75 & 20.7 & 38.1 & 17.83 & 12.92 & 34.50 & 63.50 \\
\hline 1 & FY09EM21-07сcс & 1 & 20 & $\mathrm{H} 04$ & 165 & 57.6 & 261 & 93.3 & 275.01 & 96.00 & 435.01 & 155.50 \\
\hline 1 & FY09EM21-08ccc & 1 & 21 & $\mathrm{H} 40$ & 41.2 & 90.4 & 162 & 278 & 68.67 & 150.67 & 270.01 & 463.34 \\
\hline 1 & FY09EM21-06сcс & 1 & 22 & H31 & 157 & 61.7 & 320 & 209 & 261.67 & 102.84 & 533.34 & 348.34 \\
\hline 1 & FY09EM21-05сcс & 1 & 23 & $\mathrm{H} 47$ & 8.30 & 8.39 & 28.3 & 54.7 & 13.83 & 13.98 & 47.17 & 91.17 \\
\hline 1 & Soln Std & 1 & 24 & \begin{tabular}{|l|} 
std-11-3 \\
\end{tabular} & 19.9 & 10.0 & 84.0 & 52.1 & 19.90 & 10.00 & 84.00 & 52.10 \\
\hline 1 & Soln Std & 2 & 1 & \begin{tabular}{|l|} 
std-12-1 \\
\end{tabular} & 20.4 & 9.91 & 84.4 & 52.5 & 20.40 & 9.91 & 84.40 & 52.50 \\
\hline 1 & FY09EM21-04ccc & 2 & 2 & $\mathrm{H} 58$ & 50.3 & 36.5 & 83.9 & 74.5 & 83.84 & 60.83 & 139.84 & 124.17 \\
\hline 1 & FY09EM21-05сcC & 2 & 3 & H19 & 5.37 & 8.61 & 27.3 & 54.0 & 8.95 & 14.35 & 45.50 & 90.00 \\
\hline 1 & EA & 2 & 4 & H30 & 35.7 & 10.9 & 94.6 & 55.3 & 595.00 & 181.67 & 1576.67 & 921.67 \\
\hline 1 & FY09EM21-06 & 2 & 5 & $\mathrm{H} 13$ & 106 & 45.6 & 259 & 172 & 176.67 & 76.00 & 431.68 & 286.67 \\
\hline 1 & FY09EM21-01 & 2 & 6 & H17 & 7.06 & 8.41 & 35.7 & 53.6 & 11.77 & 14.02 & 59.50 & 89.34 \\
\hline 1 & ARM-1 & 2 & 15 & H36 & 12.4 & 7.61 & 19.5 & 36.0 & 20.67 & 12.68 & 32.50 & 60.00 \\
\hline 1 & FY09EM21-03ссс & 2 & 16 & H29 & 91.1 & 109 & 474 & 324 & 151.84 & 181.67 & 790.02 & 540.01 \\
\hline 1 & FY09EM21-06сcс & 2 & 17 & H61 & 124 & 56.0 & 311 & 210 & 206.67 & 93.34 & 518.34 & 350.01 \\
\hline 1 & FY09EM21-07ccc & 2 & 18 & H18 & 150 & 56.6 & 296 & 90.8 & 250.01 & 94.34 & 493.34 & 151.34 \\
\hline 1 & FY09EM21-01сCC & 2 & 19 & $\mathrm{H} 60$ & 10.2 & 9.00 & 38.0 & 65.3 & 17.00 & 15.00 & 63.33 & 108.84 \\
\hline 1 & FY09EM21-08 & 2 & 20 & $\mathrm{H} 01$ & 47.4 & 67.6 & 151 & 205 & 79.00 & 112.67 & 251.67 & 341.67 \\
\hline 1 & FY09EM21-05 & 2 & 21 & H57 & 8.63 & 10.5 & 38.2 & 71.9 & 14.38 & 17.50 & 63.67 & 119.84 \\
\hline 1 & FY09EM21-03 & 2 & 22 & $\mathrm{H} 49$ & 22.4 & 42.5 & 138 & 141 & 37.33 & 70.83 & 230.00 & 235.00 \\
\hline 1 & Soln Std & 2 & 23 & \begin{tabular}{|l|} 
std-12-3 \\
\end{tabular} & 18.2 & 9.55 & 79.9 & 51.3 & 18.20 & 9.55 & 79.90 & 51.30 \\
\hline 1 & Soln Std & 3 & 1 & \begin{tabular}{|l|} 
std13-1 \\
\end{tabular} & 22.0 & 10.2 & 83.4 & 51.9 & 22.00 & 10.20 & 83.40 & 51.90 \\
\hline 1 & FY09EM21-04 & 3 & 2 & H33 & 167 & 100 & 236 & 107 & 278.34 & 166.67 & 393.34 & 178.34 \\
\hline 1 & ARM-1 & 3 & 3 & H46 & 11.1 & 7.61 & 21.2 & 36.1 & 18.50 & 12.68 & 35.33 & 60.17 \\
\hline 1 & FY09EM21-07сcC & 3 & 4 & $\mathrm{H} 41$ & 147 & 52.2 & 251 & 88.3 & 245.00 & 87.00 & 418.34 & 147.17 \\
\hline 1 & FY09EM21-08 & 3 & 5 & H15 & 46.5 & 64.7 & 152 & 213 & 77.50 & 107.84 & 253.34 & 355.01 \\
\hline 1 & FY09EM21-03ссC & 3 & 6 & $\mathrm{H} 20$ & 104 & 106 & 445 & 314 & 173.34 & 176.67 & 741.68 & 523.34 \\
\hline 1 & FY09EM21-01ccc & 3 & 7 & $\mathrm{H} 28$ & 8.23 & 9.34 & 21.2 & 66.1 & 13.72 & 15.57 & 35.33 & 110.17 \\
\hline 1 & FY09EM21-02 & 3 & 8 & $\mathrm{H} 03$ & 118 & 64.6 & 757 & 324 & 196.67 & 107.67 & 1261.69 & 540.01 \\
\hline 1 & FY09EM21-04ccc & 3 & 9 & $\mathrm{H} 24$ & 54.8 & 38.2 & 88.8 & 78.3 & 91.34 & 63.67 & 148.00 & 130.50 \\
\hline 1 & FY09EM21-08ccC & 3 & 10 & $\mathrm{H} 51$ & 37.3 & 82.2 & 129 & 304 & 62.17 & 137.00 & 215.00 & 506.68 \\
\hline 1 & FY09EM21-06ссC & 3 & 11 & $\mathrm{H} 42$ & 138 & 54.6 & 274 & 204 & 230.00 & 91.00 & 456.68 & 340.01 \\
\hline 1 & FY09EM21-05 & 3 & 12 & $\mathrm{H} 23$ & 9.05 & 10.4 & 36.3 & 71.6 & 15.08 & 17.33 & 60.50 & 119.34 \\
\hline 1 & Soln Std & 3 & 13 & \begin{tabular}{|l|} 
std-13-2 \\
\end{tabular} & 19.9 & 9.89 & 77.7 & 50.6 & 19.90 & 9.89 & 77.70 & 50.60 \\
\hline 1 & FY09EM21-09ccc & 3 & 14 & H34 & 8.23 & 11.4 & 22.9 & 40.4 & 13.72 & 19.00 & 38.17 & 67.33 \\
\hline 1 & FY09EM21-06 & 3 & 15 & H14 & 121 & 49.5 & 242 & 172 & 201.67 & 82.50 & 403.34 & 286.67 \\
\hline
\end{tabular}


SRNL-STI-2009-00778, Revision 0

Table B1. PSAL's Measurements of the PCT Solutions for the Matrix 2 Non-Rad Glasses As-Received (ar) and After Appropriate Adjustments (in ppm)

\begin{tabular}{|c|c|c|c|c|c|c|c|c|c|c|c|c|}
\hline Set & Glass ID (w HT) & Block & Seq & Lab ID & B ar & Li ar & Na ar & Si ar & B (ppm) & Li (ppm) & $\mathrm{Na}$ (ppm) & Si (ppm) \\
\hline 2 & FY09EM21-10ccc & 1 & 3 & I03 & 6.68 & 11.7 & 40.6 & 53.6 & 11.13 & 19.50 & 67.67 & 89.34 \\
\hline 2 & FY09EM21-18 & 1 & 5 & I52 & 5.76 & 11.1 & 82.8 & 78.6 & 9.60 & 18.50 & 138.00 & 131.00 \\
\hline 2 & FY09EM21-15сcC & 1 & 6 & I43 & 14.1 & 38.1 & 63.8 & 128 & 23.50 & 63.50 & 106.34 & 213.34 \\
\hline 2 & FY09EM21-17сcC & 1 & 7 & I09 & 24.7 & 69.2 & 152 & 211 & 41.17 & 115.34 & 253.34 & 351.67 \\
\hline 2 & FY09EM21-14ccC & 1 & 10 & I21 & 108 & 40.7 & 119 & 109 & 180.00 & 67.83 & 198.34 & 181.67 \\
\hline 2 & FY09EM21-15 & 1 & 11 & I37 & 11.1 & 18.2 & 48.2 & 81.3 & 18.50 & 30.33 & 80.33 & 135.50 \\
\hline 2 & blank & 1 & 12 & I14 & 0.867 & $<0.100$ & $<0.100$ & $<0.100$ & 1.45 & 0.08 & 0.08 & 0.08 \\
\hline 2 & Soln Std & 1 & 13 & std-21-2 & 20.5 & 9.87 & 81.4 & 50.5 & 20.50 & 9.87 & 81.40 & 50.50 \\
\hline 2 & EA & 1 & 14 & I44 & 22.8 & 7.82 & 61.6 & 38.2 & 380.00 & 130.33 & 1026.67 & 636.67 \\
\hline 2 & FY09EM21-16 & 1 & 15 & I41 & 34.4 & 25.6 & 200 & 174 & 57.33 & 42.67 & 333.34 & 290.01 \\
\hline 2 & FY09EM21-11 & 1 & 20 & I51 & 19.4 & 32.3 & 122 & 161 & 32.33 & 53.83 & 203.34 & 268.34 \\
\hline 2 & FY09EM21-17 & 1 & 21 & I45 & 10.2 & 23.7 & 107 & 105 & 17.00 & 39.50 & 178.34 & 175.00 \\
\hline 2 & FY09EM21-12ccc & 1 & 22 & $\mathrm{I} 05$ & 17.0 & 58.2 & 67.4 & 171 & 28.33 & 97.00 & 112.34 & 285.01 \\
\hline 2 & FY09EM21-16сcс & 1 & 23 & $\mathrm{I} 20$ & 21.2 & 17.3 & 134 & 121 & 35.33 & 28.83 & 223.34 & 201.67 \\
\hline 2 & Soln Std & 1 & 24 & std-21-3 & 20.1 & 9.64 & 78.7 & 49.3 & 20.10 & 9.64 & 78.70 & 49.30 \\
\hline 2 & Soln Std & 2 & 1 & \begin{tabular}{|l|} 
std-22-1 \\
\end{tabular} & 21.6 & 10.0 & 82.4 & 50.4 & 21.60 & 10.00 & 82.40 & 50.40 \\
\hline 2 & FY09EM21-13ссс & 2 & 2 & I19 & 9.67 & 24.5 & 43.5 & 49.6 & 16.12 & 40.83 & 72.50 & 82.67 \\
\hline 2 & FY09EM21-16 & 2 & 3 & I46 & 34.2 & 25.8 & 190 & 168 & 57.00 & 43.00 & 316.67 & 280.01 \\
\hline 2 & FY09EM21-17 & 2 & 4 & I57 & 10.4 & 24.7 & 114 & 107 & 17.33 & 41.17 & 190.00 & 178.34 \\
\hline 2 & FY09EM21-10сcC & 2 & 5 & I38 & 6.45 & 11.6 & 41.2 & 52.9 & 10.75 & 19.33 & 68.67 & 88.17 \\
\hline 2 & FY09EM21-11ccc & 2 & 6 & I47 & 19.5 & 33.0 & 112 & 157 & 32.50 & 55.00 & 186.67 & 261.67 \\
\hline 2 & FY09EM21-18сcс & 2 & 7 & I48 & 5.58 & 12.0 & 78.3 & 79.8 & 9.30 & 20.00 & 130.50 & 133.00 \\
\hline 2 & FY09EM21-14ccC & 2 & 15 & I23 & 107 & 40.1 & 117 & 108 & 178.34 & 66.83 & 195.00 & 180.00 \\
\hline 2 & FY09EM21-11 & 2 & 16 & I61 & 21.3 & 33.3 & 117 & 164 & 35.50 & 55.50 & 195.00 & 273.34 \\
\hline 2 & FY09EM21-10 & 2 & 17 & I01 & 12.1 & 14.3 & 61.0 & 66.3 & 20.17 & 23.83 & 101.67 & 110.50 \\
\hline 2 & FY09EM21-13 & 2 & 18 & I15 & 12.5 & 25.5 & 61.1 & 64.2 & 20.83 & 42.50 & 101.84 & 107.00 \\
\hline 2 & FY09EM21-15сcC & 2 & 19 & I39 & 14.9 & 39.0 & 66.7 & 125 & 24.83 & 65.00 & 111.17 & 208.34 \\
\hline 2 & FY09EM21-15 & 2 & 20 & I35 & 9.20 & 18.2 & 48.3 & 81.0 & 15.33 & 30.33 & 80.50 & 135.00 \\
\hline 2 & ARM-1 & 2 & 21 & I62 & 9.04 & 7.56 & 19.4 & 34.2 & 15.07 & 12.60 & 32.33 & 57.00 \\
\hline 2 & FY09EM21-12 & 2 & 22 & I54 & 13.6 & 24.9 & 54.5 & 109 & 22.67 & 41.50 & 90.84 & 181.67 \\
\hline 2 & Soln Std & 2 & 23 & std-22-3 & 20.0 & 9.94 & 81.3 & 50.6 & 20.00 & 9.94 & 81.30 & 50.60 \\
\hline 2 & Soln Std & 3 & 1 & \begin{tabular}{|l|} 
std 23-1 \\
\end{tabular} & 21.3 & 9.89 & 83.0 & 51.6 & 21.30 & 9.89 & 83.00 & 51.60 \\
\hline 2 & FY09EM21-16 & 3 & 2 & I53 & 35.8 & 26.3 & 197 & 170 & 59.67 & 43.83 & 328.34 & 283.34 \\
\hline 2 & FY09EM21-13 & 3 & 3 & I06 & 13.2 & 25.9 & 62.2 & 66.0 & 22.00 & 43.17 & 103.67 & 110.00 \\
\hline 2 & FY09EM21-16сcс & 3 & 4 & I36 & 20.7 & 16.9 & 130 & 115 & 34.50 & 28.17 & 216.67 & 191.67 \\
\hline 2 & FY09EM21-11 & 3 & 5 & I22 & 19.9 & 33.2 & 124 & 158 & 33.17 & 55.33 & 206.67 & 263.34 \\
\hline 2 & FY09EM21-18 & 3 & 6 & I07 & 5.39 & 11.2 & 85.6 & 79.6 & 8.98 & 18.67 & 142.67 & 132.67 \\
\hline 2 & FY09EM21-10 & 3 & 7 & I08 & 10.2 & 13.3 & 57.8 & 63.1 & 17.00 & 22.17 & 96.34 & 105.17 \\
\hline 2 & FY09EM21-17 & 3 & 8 & I16 & 9.32 & 23.9 & 109 & 106 & 15.53 & 39.83 & 181.67 & 176.67 \\
\hline 2 & FY09EM21-13ссс & 3 & 9 & I13 & 8.00 & 23.3 & 41.4 & 47.6 & 13.33 & 38.83 & 69.00 & 79.33 \\
\hline 2 & FY09EM21-14 & 3 & 10 & I32 & 27.9 & 12.2 & 32.9 & 51.5 & 46.50 & 20.33 & 54.83 & 85.84 \\
\hline 2 & EA & 3 & 11 & $\mathrm{I} 04$ & 22.6 & 7.89 & 64.4 & 40.4 & 376.67 & 131.50 & 1073.34 & 673.33 \\
\hline 2 & FY09EM21-12ccc & 3 & 12 & I33 & 16.8 & 58.3 & 68.8 & 162 & 28.00 & 97.17 & 114.67 & 270.01 \\
\hline 2 & Soln Std & 3 & 13 & \begin{tabular}{|l|} 
std-23-2 \\
\end{tabular} & 20.3 & 9.90 & 82.5 & 51.7 & 20.30 & 9.90 & 82.50 & 51.70 \\
\hline 2 & FY09EM21-18сcс & 3 & 14 & I17 & 5.56 & 11.6 & 74.6 & 80.3 & 9.27 & 19.33 & 124.34 & 133.84 \\
\hline 2 & blank & 3 & 15 & $\mathrm{I} 12$ & $<0.100$ & $<0.100$ & $<0.100$ & $<0.100$ & 0.08 & 0.08 & 0.08 & 0.08 \\
\hline
\end{tabular}


SRNL-STI-2009-00778, Revision 0

Table B1. PSAL's Measurements of the PCT Solutions for the Matrix 2 Non-Rad Glasses As-Received (ar) and After Appropriate Adjustments (in ppm)

\begin{tabular}{|c|c|c|c|c|c|c|c|c|c|c|c|c|}
\hline Set & Glass ID (w HT) & Block & Seq & Lab ID & B ar & Li ar & Na ar & Si ar & B (ppm) & Li (ppm) & $\mathrm{Na}$ (ppm) & Si (ppm) \\
\hline 3 & FY09EM21-19 & 1 & 3 & J05 & 5.92 & 11.4 & 108 & 78.6 & 9.87 & 19.00 & 180.00 & 131.00 \\
\hline 3 & FY09EM21-27сcс & 1 & 5 & J33 & 12.9 & 14.3 & 57.7 & 71.7 & 21.50 & 23.83 & 96.17 & 119.50 \\
\hline 3 & ARM-1 & 1 & 6 & J60 & 11.2 & 8.9 & 23.4 & 37.9 & 18.67 & 14.83 & 39.00 & 63.17 \\
\hline 3 & FY09EM21-20 & 1 & 7 & $\mathrm{~J} 10$ & 11.6 & 11.1 & 121 & 119 & 19.33 & 18.50 & 201.67 & 198.34 \\
\hline 3 & FY09EM21-21 & 1 & 10 & $\mathrm{~J} 25$ & 498 & 272 & 1380 & 409 & 830.02 & 453.34 & 2300.05 & 681.68 \\
\hline 3 & FY09EM21-23 & 1 & 11 & J03 & 10.7 & 9.89 & 13.3 & 48.1 & 17.83 & 16.48 & 22.17 & 80.17 \\
\hline 3 & FY09EM21-21ccc & 1 & 12 & J58 & 409 & 247 & 1160 & 368 & 681.68 & 411.67 & 1933.37 & 613.35 \\
\hline 3 & Soln Std & 1 & 13 & std-31-2 & 20.2 & 9.92 & 82.7 & 49.5 & 20.20 & 9.92 & 82.70 & 49.50 \\
\hline 3 & EA & 1 & 14 & J19 & 41.8 & 11.2 & 104 & 54.1 & 696.67 & 186.67 & 1733.34 & 901.67 \\
\hline 3 & FY09EM21-22 & 1 & 15 & $\mathrm{~J} 40$ & 17.3 & 9.91 & 13.2 & 51.5 & 28.83 & 16.52 & 22.00 & 85.84 \\
\hline 3 & FY09EM21-26сcс & 1 & 20 & $\mathrm{~J} 24$ & 9.36 & 7.92 & 30.4 & 55.8 & 15.60 & 13.20 & 50.67 & 93.00 \\
\hline 3 & FY09EM21-20ссс & 1 & 21 & J52 & 11.5 & 11.5 & 114 & 114 & 19.17 & 19.17 & 190.00 & 190.00 \\
\hline 3 & FY09EM21-19сcс & 1 & 22 & J06 & 6.87 & 12.6 & 117 & 79.6 & 11.45 & 21.00 & 195.00 & 132.67 \\
\hline 3 & blank & 1 & 23 & J47 & $<0.100$ & $<0.500$ & $<0.100$ & $<0.100$ & 0.08 & 0.42 & 0.08 & 0.08 \\
\hline 3 & Soln Std & 1 & 24 & std-31-3 & 20.7 & 10.2 & 86.4 & 50.1 & 20.70 & 10.20 & 86.40 & 50.10 \\
\hline 3 & Soln Std & 2 & 1 & \begin{tabular}{|l|} 
std-32-1 \\
\end{tabular} & 21.0 & 10.2 & 81.9 & 51.3 & 21.00 & 10.20 & 81.90 & 51.30 \\
\hline 3 & FY09EM21-22ссс & 2 & 2 & \begin{tabular}{|l|} 
J31 \\
\end{tabular} & 13.1 & 8.34 & 14.2 & 50.9 & 21.83 & 13.90 & 23.67 & 84.84 \\
\hline 3 & FY09EM21-19cсc & 2 & 3 & $\mathrm{~J} 50$ & 6.27 & 12.5 & 111 & 81.2 & 10.45 & 20.83 & 185.00 & 135.34 \\
\hline 3 & EA & 2 & 4 & J27 & 35.8 & 11.1 & 97.9 & 54.8 & 596.67 & 185.00 & 1631.67 & 913.34 \\
\hline 3 & FY09EM21-25сcC & 2 & 5 & J38 & 9.29 & 14.1 & 86.4 & 85.9 & 15.48 & 23.50 & 144.00 & 143.17 \\
\hline 3 & FY09EM21-23ссс & 2 & 6 & J09 & 6.11 & 9.50 & 19.0 & 60.9 & 10.18 & 15.83 & 31.67 & 101.50 \\
\hline 3 & FY09EM21-26ссс & 2 & 7 & $\mathrm{~J} 23$ & 7.27 & 7.24 & 26.7 & 51.5 & 12.12 & 12.07 & 44.50 & 85.84 \\
\hline 3 & FY09EM21-23 & 2 & 15 & J26 & 7.29 & 10.1 & 12.9 & 50.2 & 12.15 & 16.83 & 21.50 & 83.67 \\
\hline 3 & FY09EM21-20сcс & 2 & 16 & J55 & 11.8 & 10.6 & 125 & 115 & 19.67 & 17.67 & 208.34 & 191.67 \\
\hline 3 & FY09EM21-27 & 2 & 17 & J32 & 14.7 & 14.8 & 59.1 & 72.7 & 24.50 & 24.67 & 98.50 & 121.17 \\
\hline 3 & FY09EM21-25 & 2 & 18 & J07 & 9.73 & 13.7 & 88.1 & 86.4 & 16.22 & 22.83 & 146.84 & 144.00 \\
\hline 3 & FY09EM21-24 & 2 & 19 & J48 & 8.43 & 11.2 & 41.9 & 43.7 & 14.05 & 18.67 & 69.83 & 72.83 \\
\hline 3 & FY09EM21-20 & 2 & 20 & J04 & 11.6 & 10.9 & 120 & 120 & 19.33 & 18.17 & 200.00 & 200.00 \\
\hline 3 & FY09EM21-19 & 2 & 21 & J59 & 6.11 & 11.7 & 122 & 81.5 & 10.18 & 19.50 & 203.34 & 135.84 \\
\hline 3 & FY09EM21-24cCC & 2 & 22 & $\mathrm{~J} 21$ & 3.55 & 6.29 & 24.2 & 29.4 & 5.92 & 10.48 & 40.33 & 49.00 \\
\hline 3 & Soln Std & 2 & 23 & std-32-3 & 20.4 & 10.2 & 81.6 & 51.6 & 20.40 & 10.20 & 81.60 & 51.60 \\
\hline 3 & Soln Std & 3 & 1 & \begin{tabular}{|l|} 
std 33-1 \\
\end{tabular} & 20.3 & 9.95 & 82.8 & 50.4 & 20.30 & 9.95 & 82.80 & 50.40 \\
\hline 3 & FY09EM21-20сcс & 3 & 2 & \begin{tabular}{|l|}
$\mathrm{J} 46$ \\
\end{tabular} & 10.2 & 10.9 & 107 & 110 & 17.00 & 18.17 & 178.34 & 183.34 \\
\hline 3 & FY09EM21-25сcC & 3 & 3 & J18 & 8.93 & 13.9 & 85.9 & 84.3 & 14.88 & 23.17 & 143.17 & 140.50 \\
\hline 3 & FY09EM21-22 & 3 & 4 & J51 & 13.6 & 9.60 & 12.2 & 49.9 & 22.67 & 16.00 & 20.33 & 83.17 \\
\hline 3 & FY09EM21-22сcс & 3 & 5 & J41 & 11.7 & 7.67 & 13.7 & 46.7 & 19.50 & 12.78 & 22.83 & 77.83 \\
\hline 3 & FY09EM21-25 & 3 & 6 & J14 & 8.62 & 13.3 & 87.7 & 83.2 & 14.37 & 22.17 & 146.17 & 138.67 \\
\hline 3 & FY09EM21-24 & 3 & 7 & J37 & 7.46 & 10.8 & 41.4 & 42.3 & 12.43 & 18.00 & 69.00 & 70.50 \\
\hline 3 & FY09EM21-19 & 3 & 8 & $\mathrm{~J} 17$ & 5.49 & 11.8 & 114 & 81.4 & 9.15 & 19.67 & 190.00 & 135.67 \\
\hline 3 & FY09EM21-24cсc & 3 & 9 & $\mathrm{~J} 44$ & 2.84 & 6.32 & 24.9 & 30.0 & 4.73 & 10.53 & 41.50 & 50.00 \\
\hline 3 & FY09EM21-20 & 3 & 10 & $\mathrm{~J} 43$ & 10.7 & 10.9 & 119 & 115 & 17.83 & 18.17 & 198.34 & 191.67 \\
\hline 3 & FY09EM21-27 & 3 & 11 & J49 & 13.5 & 14.8 & 61.1 & 71.2 & 22.50 & 24.67 & 101.84 & 118.67 \\
\hline 3 & FY09EM21-21 & 3 & 12 & J08 & 508 & 277 & 1420 & 423 & 846.68 & 461.68 & 2366.71 & 705.01 \\
\hline 3 & Soln Std & 3 & 13 & std-33-2 & 20.2 & 10.1 & 83.8 & 50.6 & 20.20 & 10.10 & 83.80 & 50.60 \\
\hline 3 & ARM-1 & 3 & 14 & J12 & 12.4 & 8.85 & 23.1 & 38.2 & 20.67 & 14.75 & 38.50 & 63.67 \\
\hline 3 & blank & 3 & 15 & J28 & $<0.100$ & $<0.500$ & 0.717 & $<0.100$ & 0.08 & 0.42 & 1.20 & 0.08 \\
\hline
\end{tabular}


SRNL-STI-2009-00778, Revision 0

Table B2. Analytical Development's Measurements (in ppm) of the PCT Solutions for the Rad Glasses from the Matrix 2 Study

\begin{tabular}{|c|c|c|c|c|c|c|}
\hline Glass ID & $\begin{array}{c}\text { Heat } \\
\text { Treatment }\end{array}$ & Lab ID & $\begin{array}{c}\text { B } \\
\text { (ppm) }\end{array}$ & $\begin{array}{c}\mathbf{L i} \\
(\mathrm{ppm})\end{array}$ & $\begin{array}{c}\text { Na } \\
(\mathrm{ppm})\end{array}$ & $\begin{array}{c}\mathbf{S i} \\
(\mathrm{ppm})\end{array}$ \\
\hline FY09EM21-28 & CCC & $\mathrm{e} 72$ & 17.50 & 22.00 & 85.67 & 114.50 \\
\hline FY09EM21-28 & quenched & $\mathrm{e} 74$ & 22.33 & 24.33 & 95.67 & 114.84 \\
\hline FY09EM21-28 & CCC & $\mathrm{e} 76$ & 16.40 & 20.83 & 80.83 & 111.17 \\
\hline FY09EM21-28 & quenched & $\mathrm{e} 79$ & 23.17 & 24.83 & 98.00 & 119.34 \\
\hline FY09EM21-28 & CCC & e80 & 16.13 & 20.67 & 78.33 & 110.84 \\
\hline FY09EM21-28 & quenched & e85 & 23.00 & 25.33 & 96.50 & 120.67 \\
\hline FY09EM21-29 & quenched & e73 & 24.33 & 26.83 & 102.34 & 127.67 \\
\hline FY09EM21-29 & quenched & e77 & 23.83 & 26.00 & 99.17 & 124.17 \\
\hline FY09EM21-29 & $\mathrm{CCC}$ & $\mathrm{e} 78$ & 16.52 & 20.67 & 81.83 & 110.84 \\
\hline FY09EM21-29 & CCC & e81 & 18.00 & 21.83 & 84.17 & 118.17 \\
\hline FY09EM21-29 & quenched & e83 & 23.00 & 25.17 & 97.00 & 121.67 \\
\hline FY09EM21-29 & $\mathrm{CCC}$ & e84 & 15.83 & 19.83 & 78.17 & 109.00 \\
\hline FY09EM21-30 & quenched & e69 & 22.17 & 24.33 & 91.00 & 114.67 \\
\hline FY09EM21-30 & CCC & $\mathrm{e} 70$ & 16.63 & 21.17 & 78.50 & 111.84 \\
\hline FY09EM21-30 & quenched & e71 & 22.50 & 24.50 & 92.84 & 115.34 \\
\hline FY09EM21-30 & CCC & e75 & 17.17 & 21.50 & 78.00 & 115.34 \\
\hline FY09EM21-30 & CCC & e82 & 16.63 & 21.67 & 78.83 & 114.34 \\
\hline FY09EM21-30 & quenched & e86 & 23.33 & 25.50 & 96.17 & 121.17 \\
\hline
\end{tabular}


SRNL-STI-2009-00778, Revision 0

\section{Exhibit B1. PCT Measurements in Analytical Sequence over All of the Analytical Plans for the Matrix 2 Non-Rad Glasses}

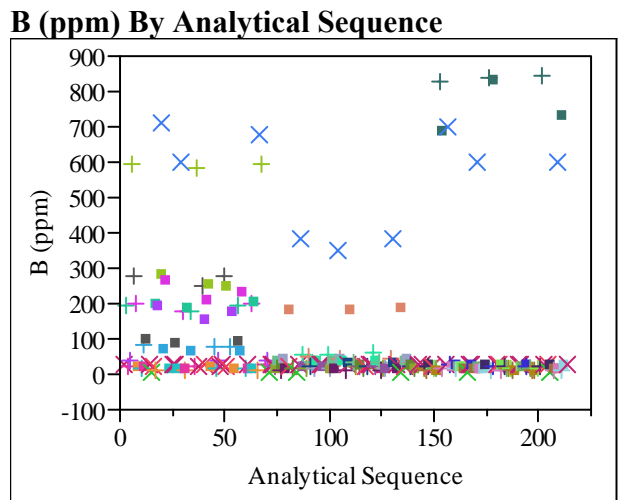

$\log [B$ ppm] By Analytical Sequence
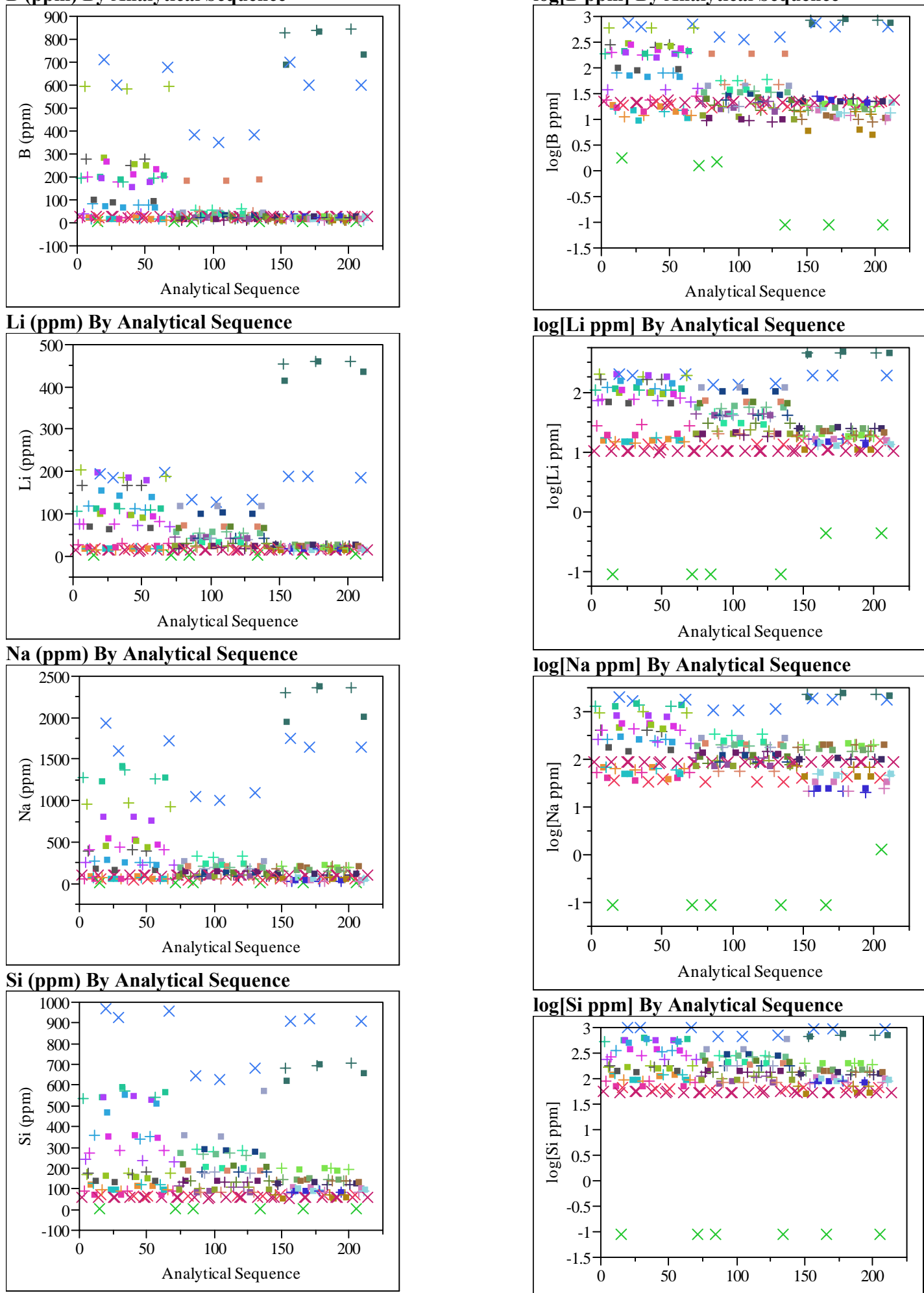

$\log [\mathrm{Li}$ ppm] By Analytical Sequence

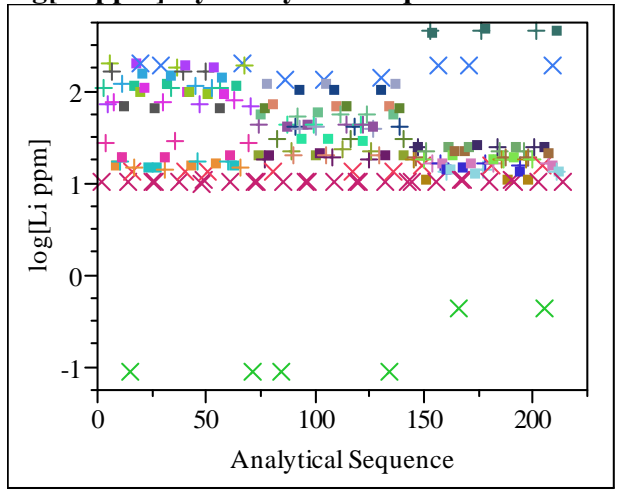

$\log [$ Na ppm] By Analytical Sequence

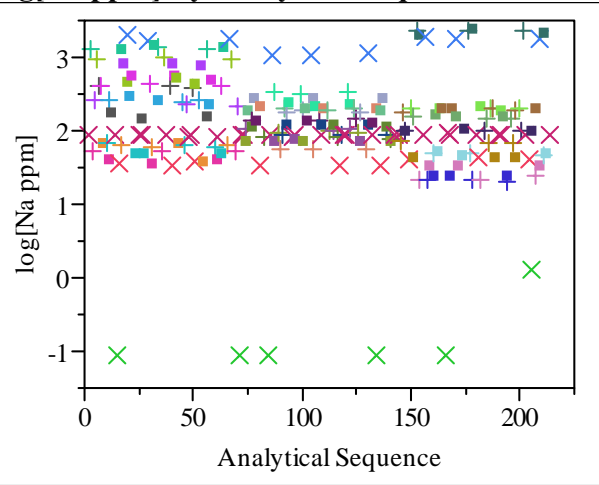

$\log [$ Si ppm] By Analytical Sequence

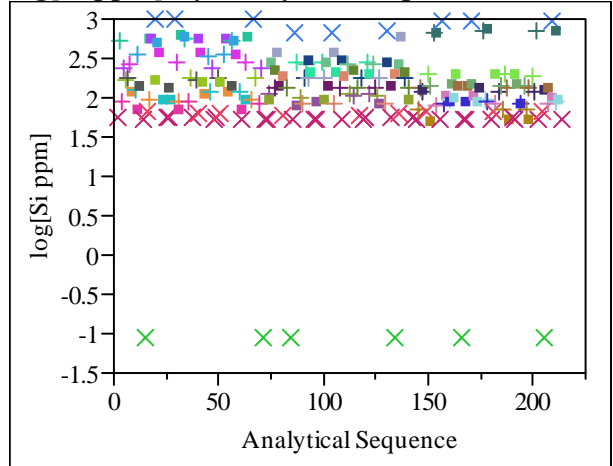


SRNL-STI-2009-00778, Revision 0

\section{Exhibit B2. Measurements of the Multi-Element Solution Standard by ICP Block for the Non-Rad Matrix 2 Study}

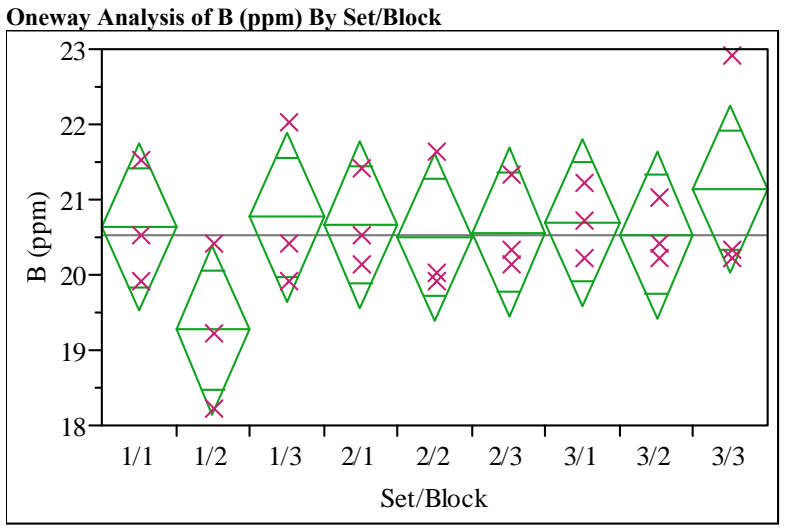

Oneway Anova

Summary of Fit

$\begin{array}{lr}\text { Rsquare } & 0.290611 \\ \text { Adj Rsquare } & -0.02467 \\ \text { Root Mean Square Error } & 0.919138 \\ \text { Mean of Response } & 20.52963 \\ \text { Observations (or Sum Wgts) } & 27\end{array}$

Analysis of Variance

Source DF Sum of Squares Mean Square F Ratio Prob $>$ F

$\begin{array}{llllll}\text { Set/Block } & 8 & 6.229630 & 0.778704 & 0.9217 & 0.5216\end{array}$

$\begin{array}{llll}\text { Error } & 18 & 15.206667 & 0.844815\end{array}$

Means for Oneway Anova

Level Number Mean Std Error Lower 95\% Upper 95\%

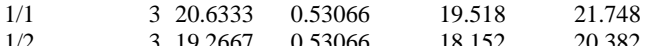

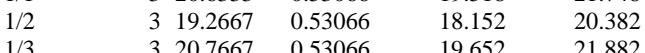

$\begin{array}{llllll}1 / 3 & 3 & 20.7667 & 0.53066 & 19.652 & 21.882 \\ 2 & 3 & 20.6667 & 0.53066 & 19.552 & 21.782\end{array}$

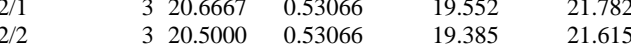

$\begin{array}{llllll}2 / 3 & 3 & 20.5667 & 0.53066 & 19.452 & 21.682 \\ 3 / 1 & 3 & 20.7000 & 0.53066 & 19.585 & 21.815\end{array}$

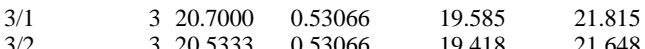

$\begin{array}{llllll}3 / 2 & 3 & 20.5333 & 0.53066 & 19.418 & 21.648 \\ 3 / 3 & 3 & 21.1333 & 0.53066 & 20.018 & 22.248\end{array}$

Std Error uses a pooled estimate of error variance

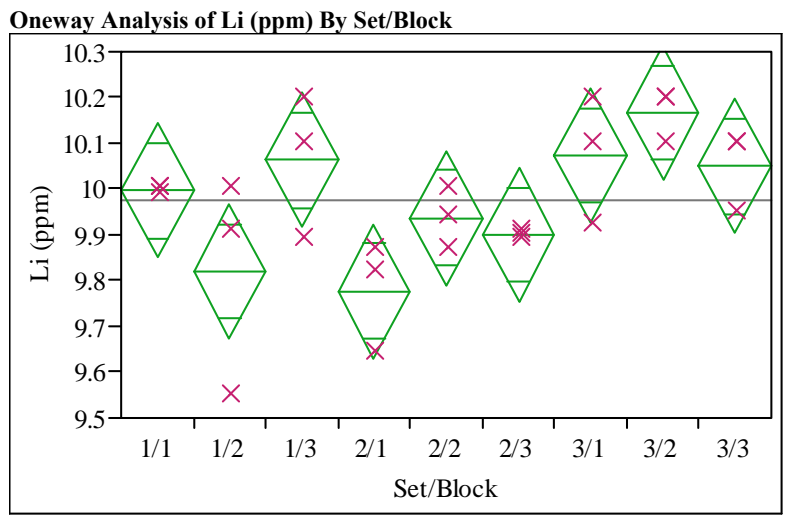

Oneway Anova

Summary of Fit

$\begin{array}{ll}\text { Rsquare } & 0.598262 \\ \text { Adj Rsquare } & 0.419712\end{array}$

$\begin{array}{ll}\text { Adj Rsquare } & 0.419712 \\ \text { Root Mean Square Error } & 0.120968\end{array}$

Mean of Response $\quad 9.975926$

Observations (or Sum Wgts)

Analysis of Variance

Source DF Sum of Squares Mean Square F Ratio Prob $>$ F

$\begin{array}{llllll}\text { Set/Block } & 0.39225185 & 0.049031 & 3.3507 & 0.0158\end{array}$

$\begin{array}{lll}\text { Error } & 18 & 0.26340000 \\ \text { C. Total } & 26 & 0.65565185\end{array}$

Means for Oneway Anova

Level Number Mean Std Error Lower 95\% Upper 95\%

$\begin{array}{llllrr}1 / 1 & 3 & 9.9967 & 0.06984 & 9.850 & 10.143\end{array}$

$\begin{array}{rrrrrr}1 / 2 & 3 & 9.8200 & 0.06984 & 9.673 & 9.967 \\ 1 / 3 & 3 & 10.0633 & 0.06984 & 9.917 & 10.210\end{array}$

$\begin{array}{rrrrrr}2 / 1 & 3 & 9.7767 & 0.06984 & 9.630 & 9.923\end{array}$

$\begin{array}{llllll}2 / 2 & 3 & 9.9367 & 0.06984 & 9.790 & 10.083 \\ 2 / 3 & 3 & 9.9000 & 0.06984 & 9.753 & 10.047\end{array}$

$\begin{array}{rrrrrr}3 / 1 & 3 & 10.0733 & 0.06984 & 9.927 & 10.220\end{array}$

Std Error uses a pooled estimate of error variance 
SRNL-STI-2009-00778, Revision 0

Exhibit B2. Measurements of the Multi-Element Solution Standard by ICP Block for the Non-Rad Matrix 2 Study

Oneway Analysis of Na (ppm) By Set/Block

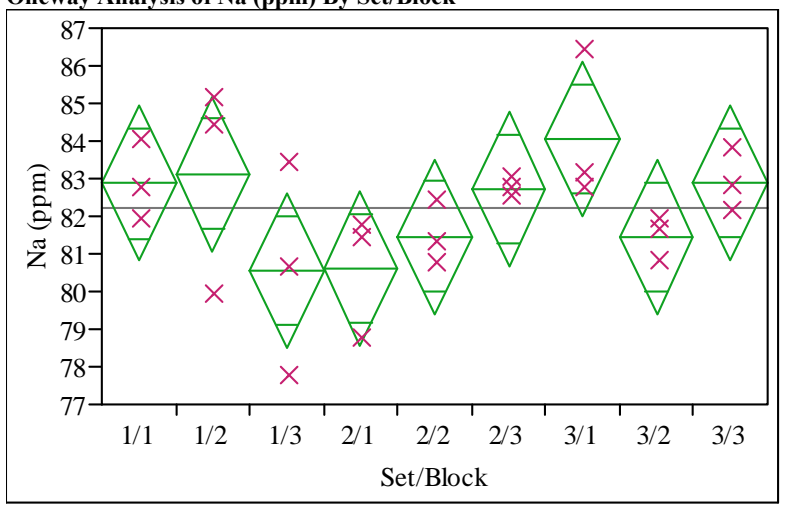

Oneway Anova
Summary of Fit

$\begin{array}{lr}\text { Rsquare } & 0.408343 \\ \text { Adj Rsquare } & 0.145385 \\ \text { Root Mean Square Error } & 1.697165 \\ \text { Mean of Response } & 82.1963 \\ \text { Observations (or Sum Wgts) } & 27\end{array}$

Observations (or Sum Wgts) $\begin{array}{r}82.1963 \\ \hline\end{array}$

\section{Analysis of Variance}

Source DF Sum of Squares Mean Square F Ratio Prob $>$ F

$\begin{array}{lrrrrr}\text { Set/Block } & 8 & 35.782963 & 4.47287 & 1.5529 & 0.2080 \\ \text { Error } & 18 & 51.846667 & 2.88037 & & \end{array}$

$\begin{array}{llr}\text { Crror } & 18 & 51.846667 \\ \text { Total } & 26 & 87.629630\end{array}$

Means for Oneway Anova

Level Number Mean Std Error Lower 95\% Upper 95\%

$\begin{array}{llllll}1 / 1 & 3 & 82.8667 & 0.97986 & 80.808 & 84.925\end{array}$

$\begin{array}{llllll}1 / 2 & 3 & 83.1333 & 0.97986 & 81.075 & 85.192\end{array}$

$\begin{array}{llllll}1 / 3 & 3 & 80.5667 & 0.97986 & 78.508 & 82.625 \\ 2 / 1 & 3 & 80.6000 & 0.97986 & 78.541 & 82.659\end{array}$

$\begin{array}{llllll}1 / 1 & 3 & 80.6000 & 0.97986 & 78.541 & 82.659 \\ 2 / 2 & 3 & 81.4667 & 0.97986 & 79.408 & 83.525\end{array}$

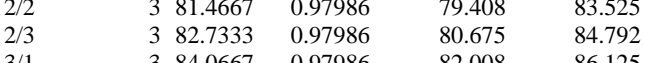

$\begin{array}{llllll}3 / 1 & 3 & 84.0667 & 0.97986 & 82.008 & 86.125 \\ 3 / 2 & 3 & 81.4333 & 0.97986 & 79.375 & 83.492\end{array}$

$\begin{array}{llllll}3 / 2 & 3 & 81.4333 & 0.97986 & 79.375 & 83.492 \\ 3 / 3 & 3 & 82.9000 & 0.97986 & 80.841 & 84.959\end{array}$

Std Error uses a pooled estimate of error variance

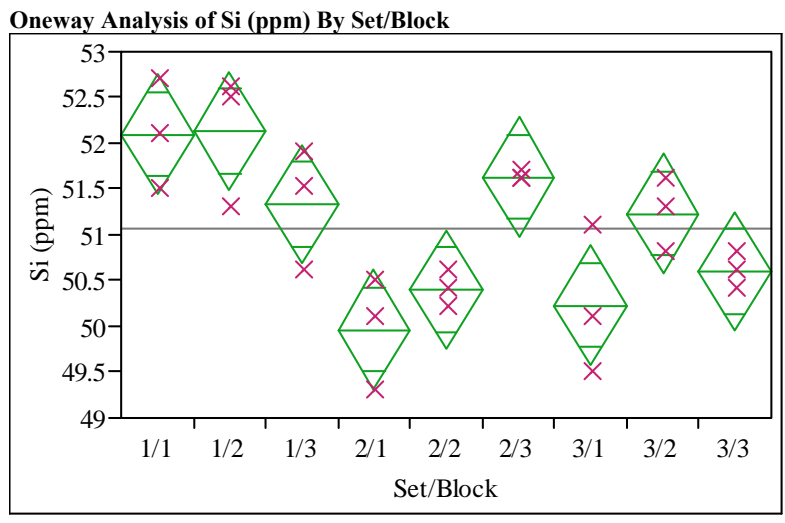

Oneway Anova

Summary of Fi

$\begin{array}{lr}\text { Rsquare } & 0.74971 \\ \text { Adj Rsquare } & 0.638469 \\ \text { Root Mean Square Error } & 0.537499 \\ \text { Mean of Response } & 51.0704 \\ \text { Observations (or Sum Wgts) } & 27\end{array}$

Analysis of Variance

Source DF Sum of Squares Mean Square F Ratio Prob $>$ F

$\begin{array}{lll}1.94710 & 6.7396 & 0.0004\end{array}$

$\begin{array}{lrr}\text { Error } & 18 & 5.200301 \\ \text { C. Total } & 26 & 20.777070\end{array}$

\section{Means for Oneway Anova}

Level Number Mean Std Error Lower 95\% Upper 95\%

$\begin{array}{llrrrr}1 / 1 & 3 & 52.1000 & 0.31033 & 51.448 & 52.752 \\ 1 / 2 & 3 & 52.1333 & 0.31033 & 51.481 & 52.785\end{array}$

$\begin{array}{llllll}1 / 2 & 3 & 52.1333 & 0.31033 & 51.481 & 52.785 \\ 1 / 3 & 3 & 51.3336 & 0.31033 & 50.682 & 51.986\end{array}$

$\begin{array}{llllll}2 / 1 & 3 & 49.9667 & 0.31033 & 49.315 & 50.619 \\ 2 / 2 & 3 & 50.4000 & 0.31033 & 49.748 & 51.052\end{array}$

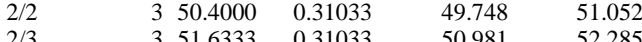

$\begin{array}{llllll}2 / 3 & 3 & 51.6333 & 0.31033 & 50.981 & 52.285 \\ 3 / 1 & 3 & 50.2333 & 0.31033 & 49.581 & 50.885\end{array}$

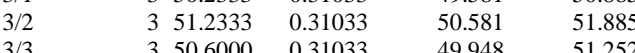

Std Error uses a pooled estimate of error variance 
SRNL-STI-2009-00778, Revision 0

\section{Exhibit B3. Laboratory PCT Measurements by Glass Identifier for Matrix 2 Non-Rad Glasses and Standards}

Set=1

Variability Chart for B (ppm)

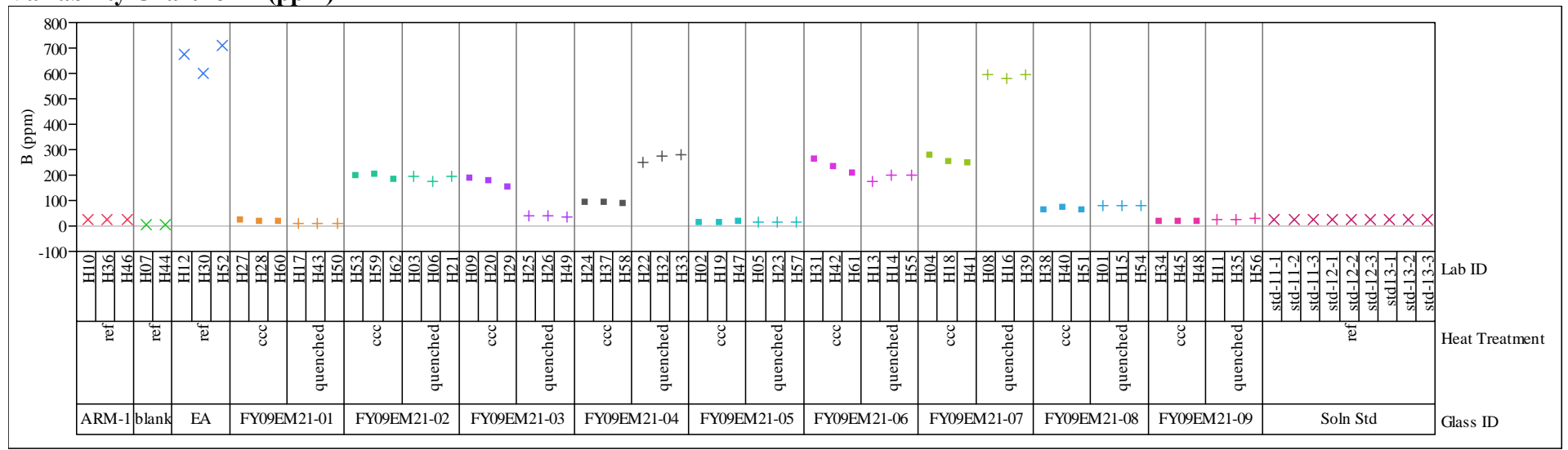

Set=1

Variability Chart for Li (ppm)

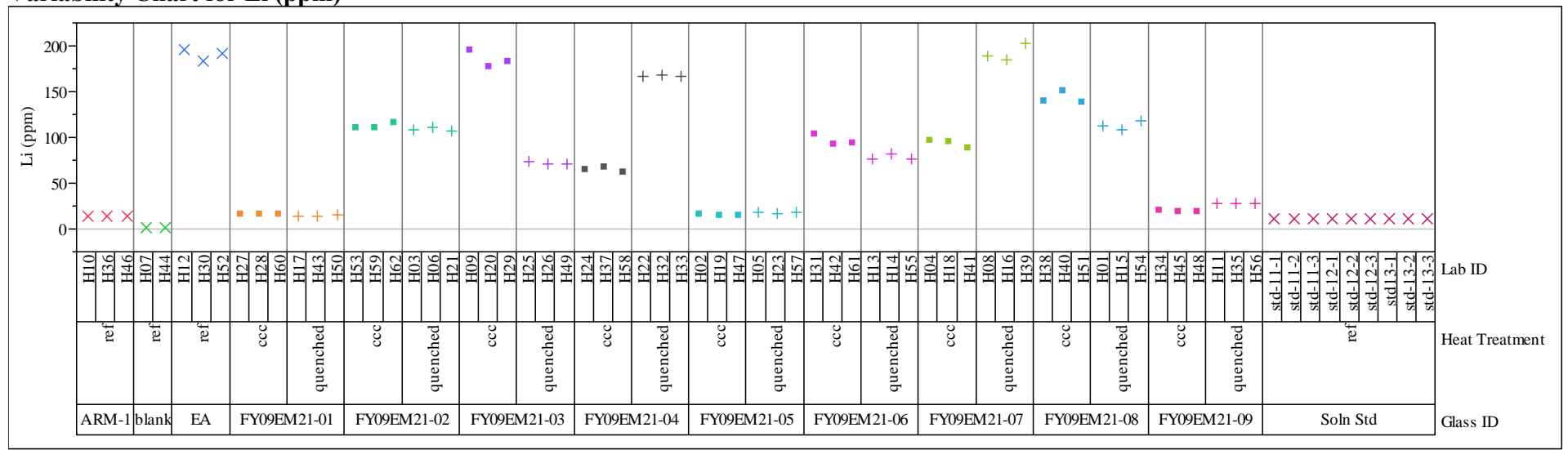




\section{Exhibit B3. Laboratory PCT Measurements by Glass Identifier for Matrix 2 Non-Rad Glasses and Standards}

Set=1

Variability Chart for Na (ppm)

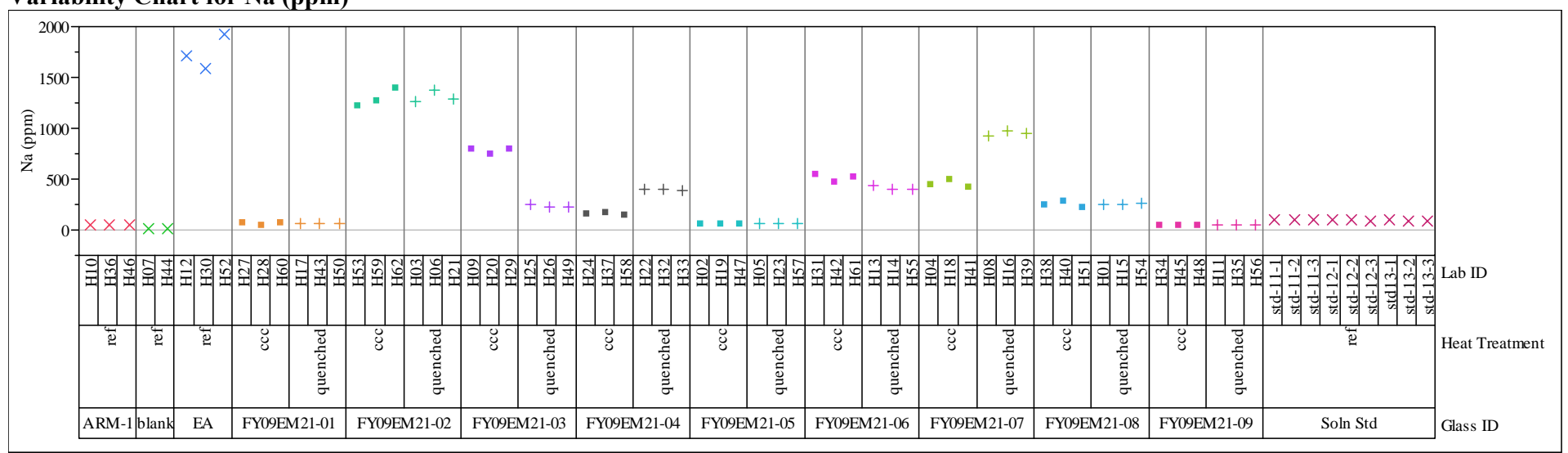

Set=1

Variability Chart for Si (ppm)

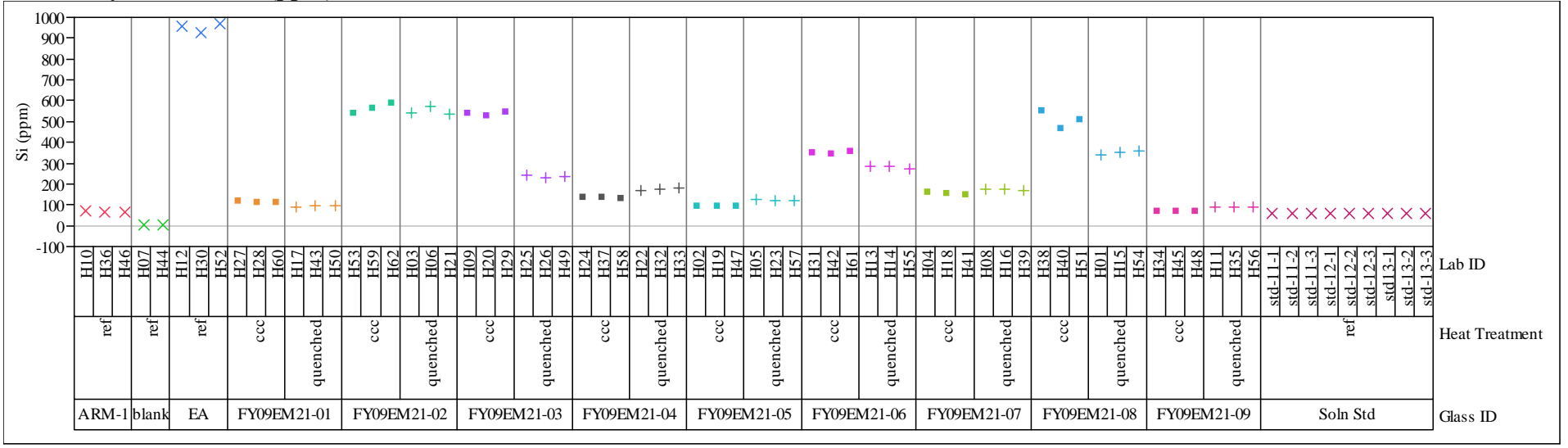


SRNL-STI-2009-00778, Revision 0

\section{Exhibit B3. Laboratory PCT Measurements by Glass Identifier for Matrix 2 Non-Rad Glasses and Standards}

Set=1

Variability Chart for $\log [\mathrm{B}$ ppm]

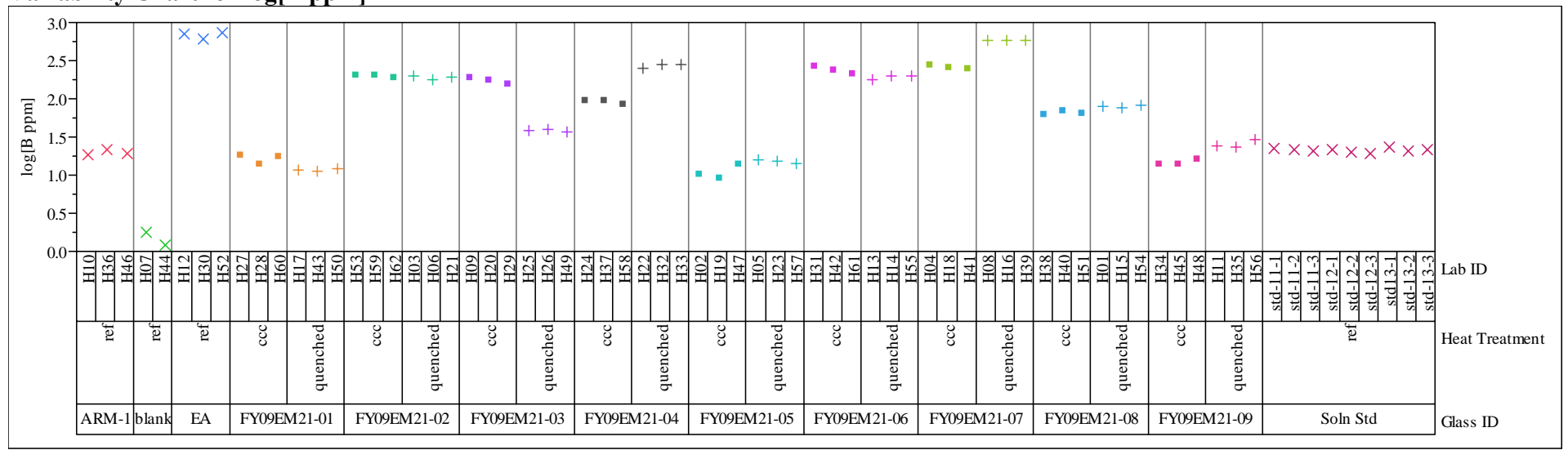

Set=1

Variability Chart for $\log [\mathrm{Li}$ ppm]

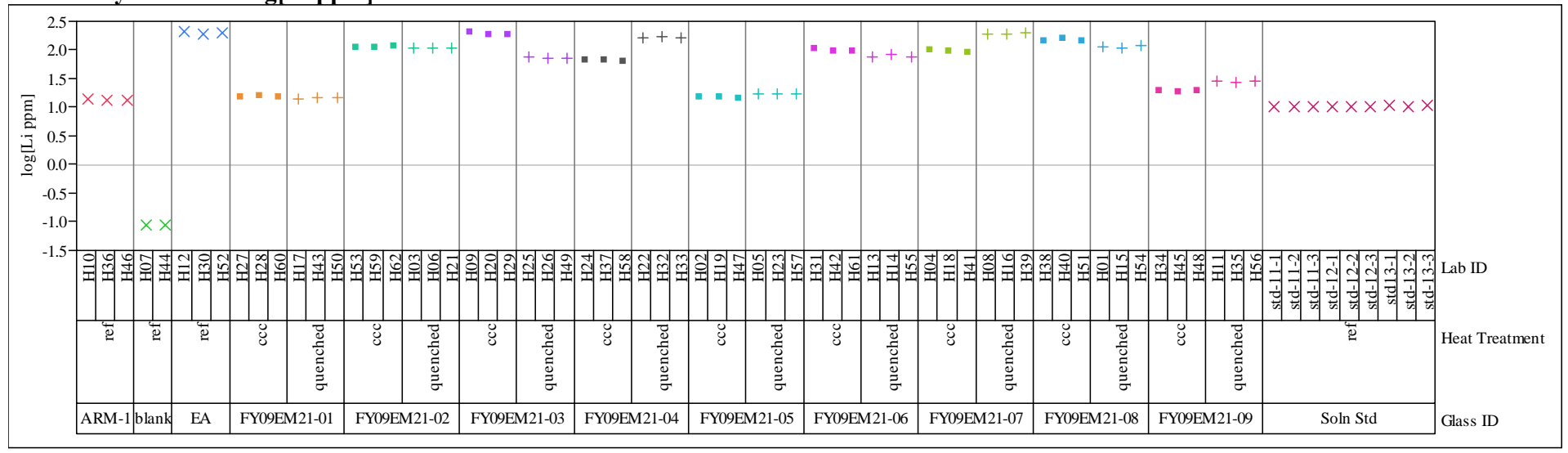


SRNL-STI-2009-00778, Revision 0

\section{Exhibit B3. Laboratory PCT Measurements by Glass Identifier for Matrix 2 Non-Rad Glasses and Standards}

Set=1

Variability Chart for log[Na ppm]

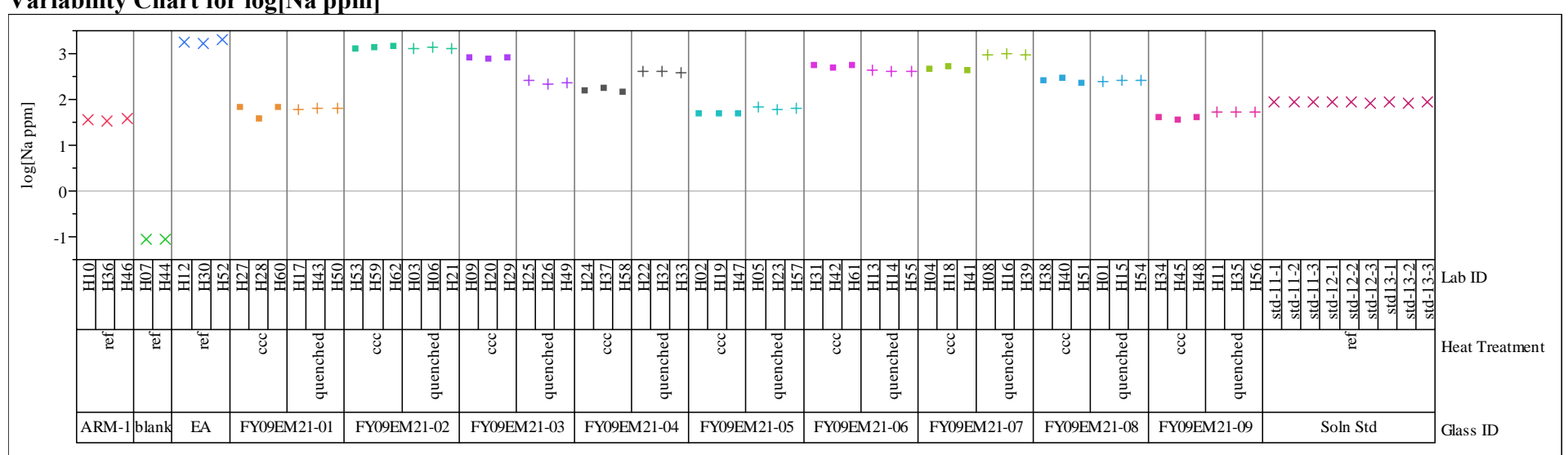

Set $=1$

Variability Chart for $\log [\mathrm{Si}$ ppm

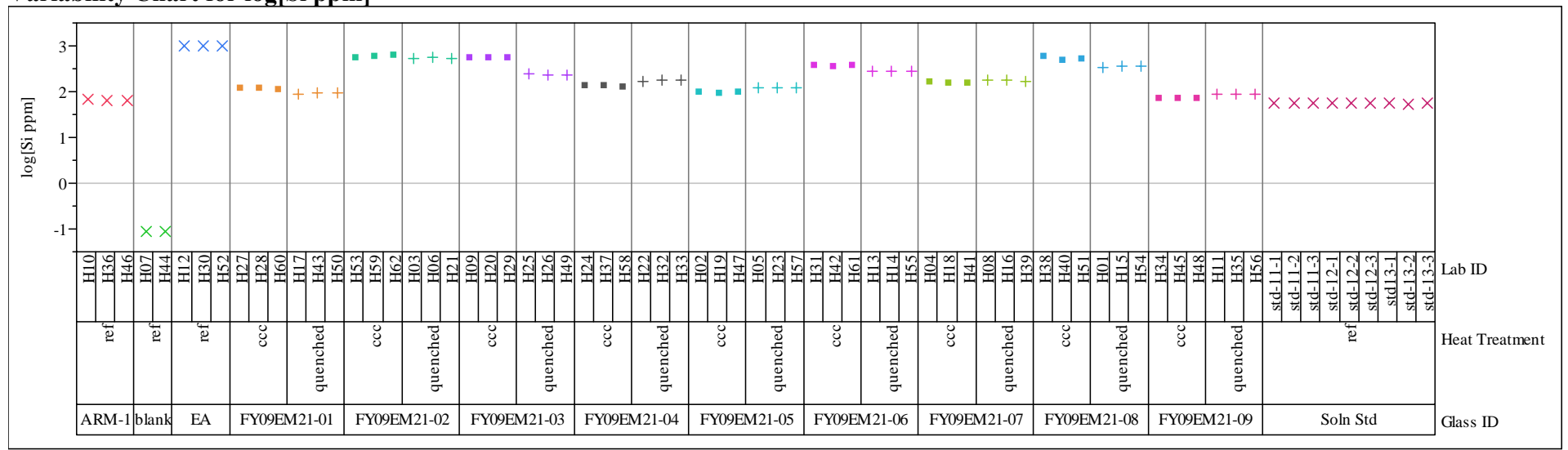


SRNL-STI-2009-00778, Revision 0

\section{Exhibit B3. Laboratory PCT Measurements by Glass Identifier for Matrix 2 Non-Rad Glasses and Standards}

Set $=2$

Variability Chart for B (ppm)

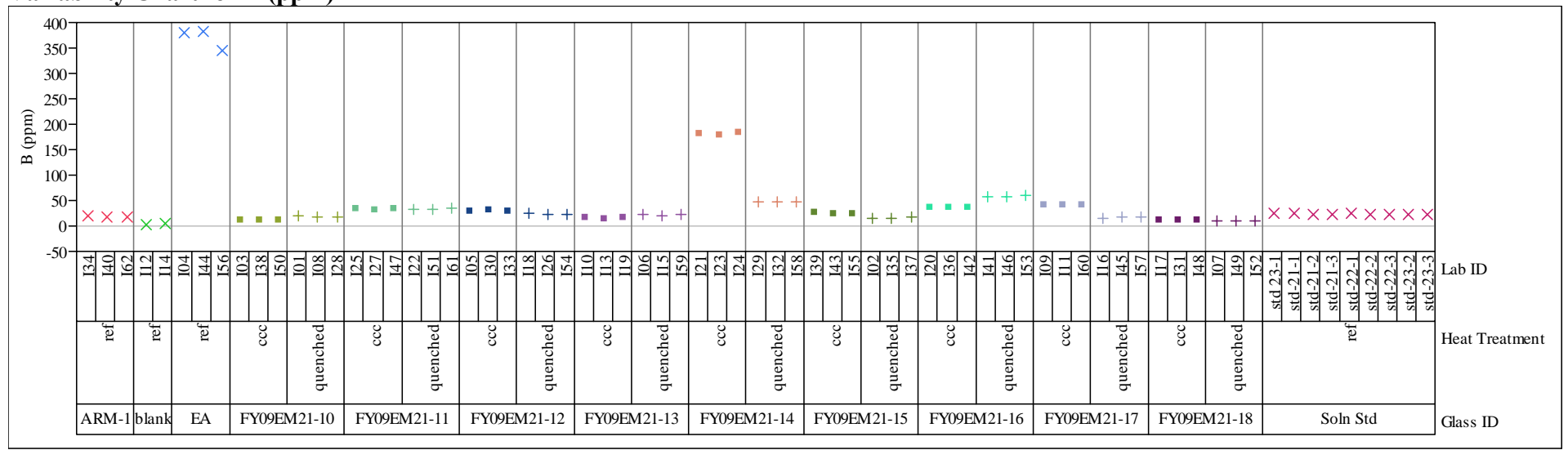

Set $=2$

Variability Chart for Li (ppm)

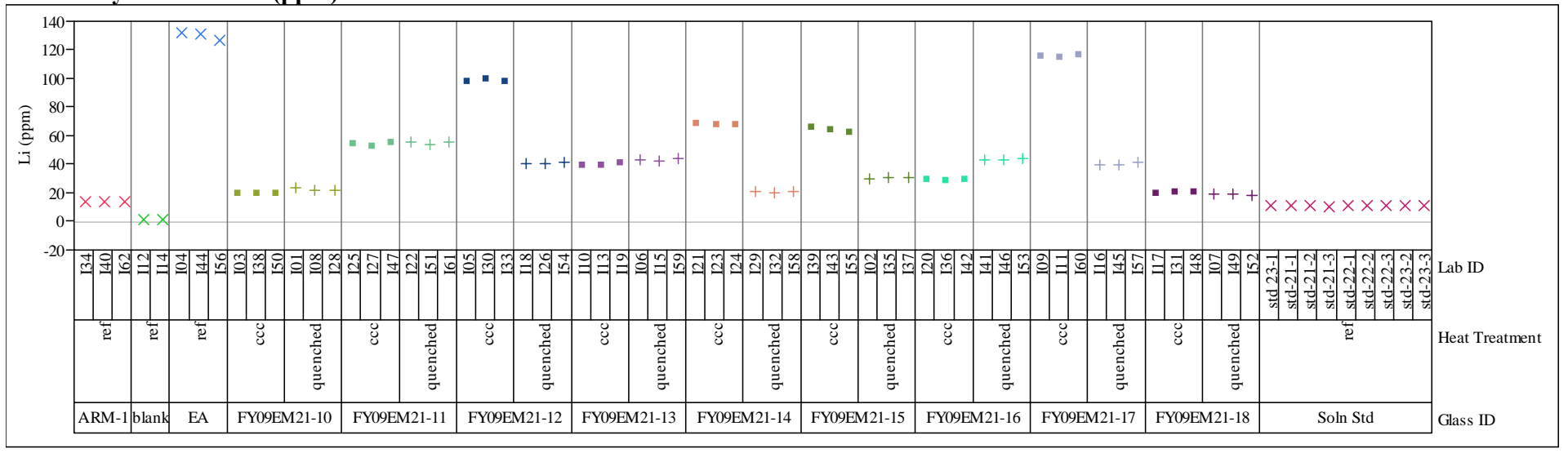


SRNL-STI-2009-00778, Revision 0

\section{Exhibit B3. Laboratory PCT Measurements by Glass Identifier for Matrix 2 Non-Rad Glasses and Standards}

Set $=2$

Variability Chart for Na (ppm)

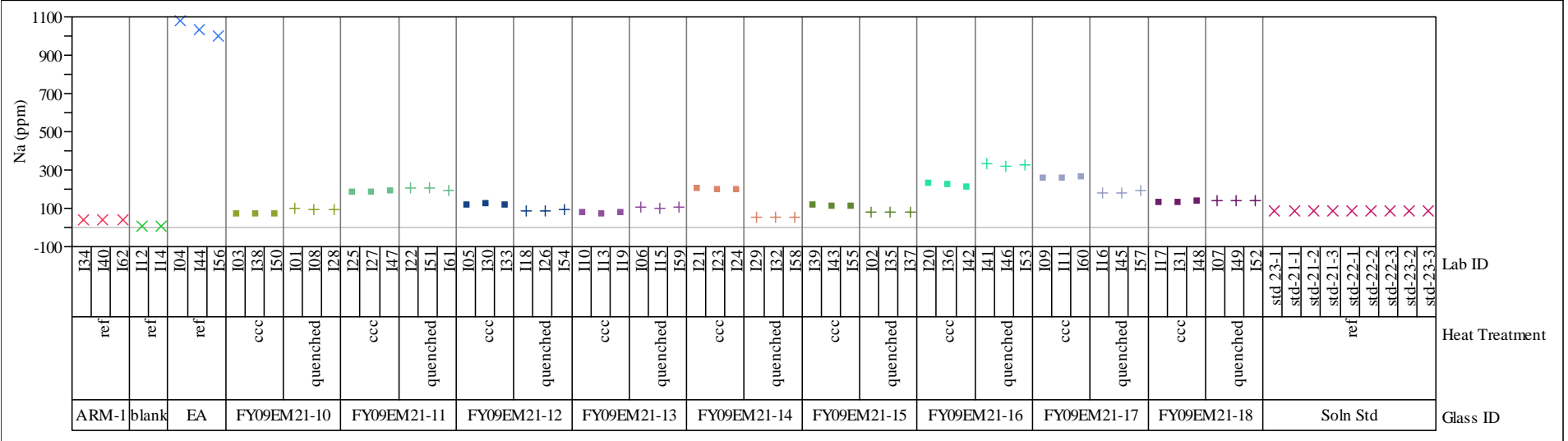

Set $=2$

Variability Chart for Si (ppm)

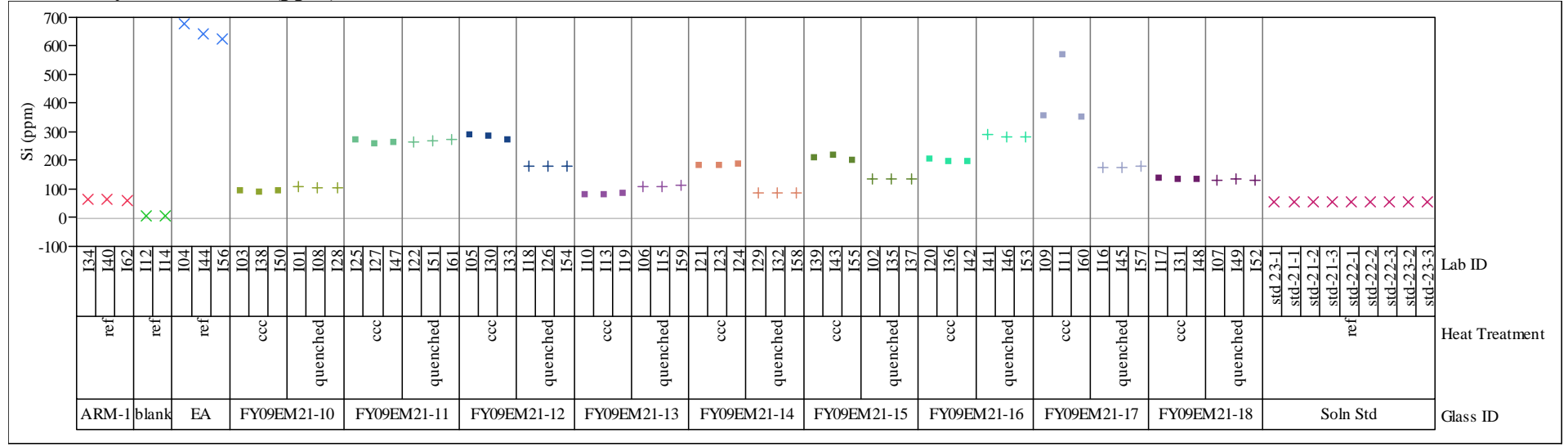


SRNL-STI-2009-00778, Revision 0

\section{Exhibit B3. Laboratory PCT Measurements by Glass Identifier for Matrix 2 Non-Rad Glasses and Standards}

Set $=2$

Variability Chart for $\log [\mathrm{B}$ ppm]

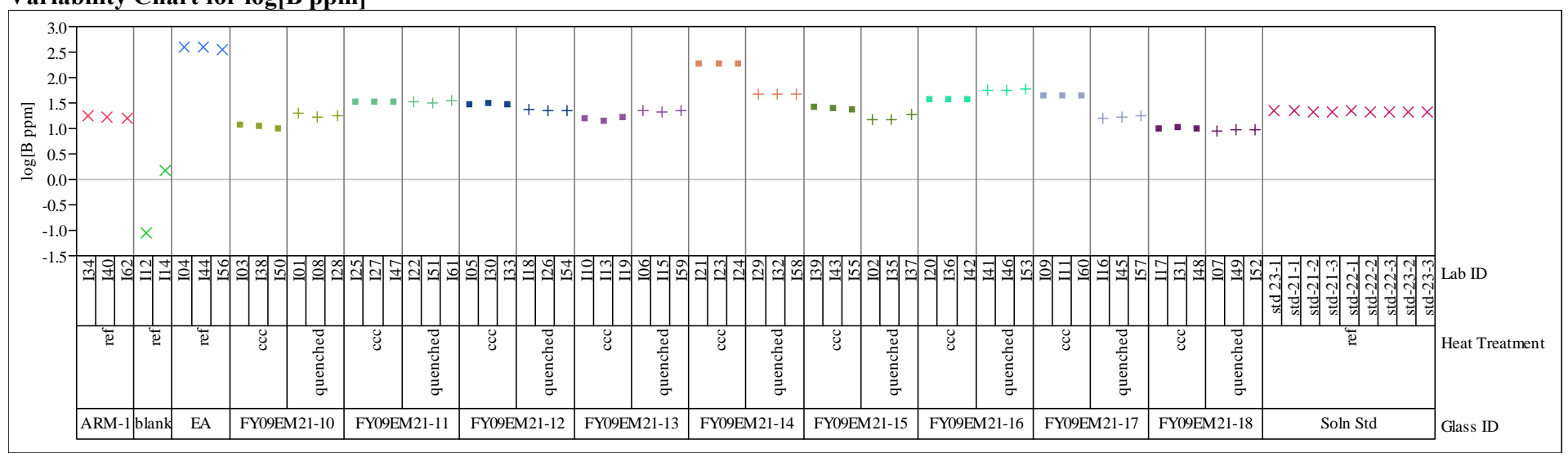

Set $=2$

Variability Chart for $\log [\mathrm{Li}$ ppm]

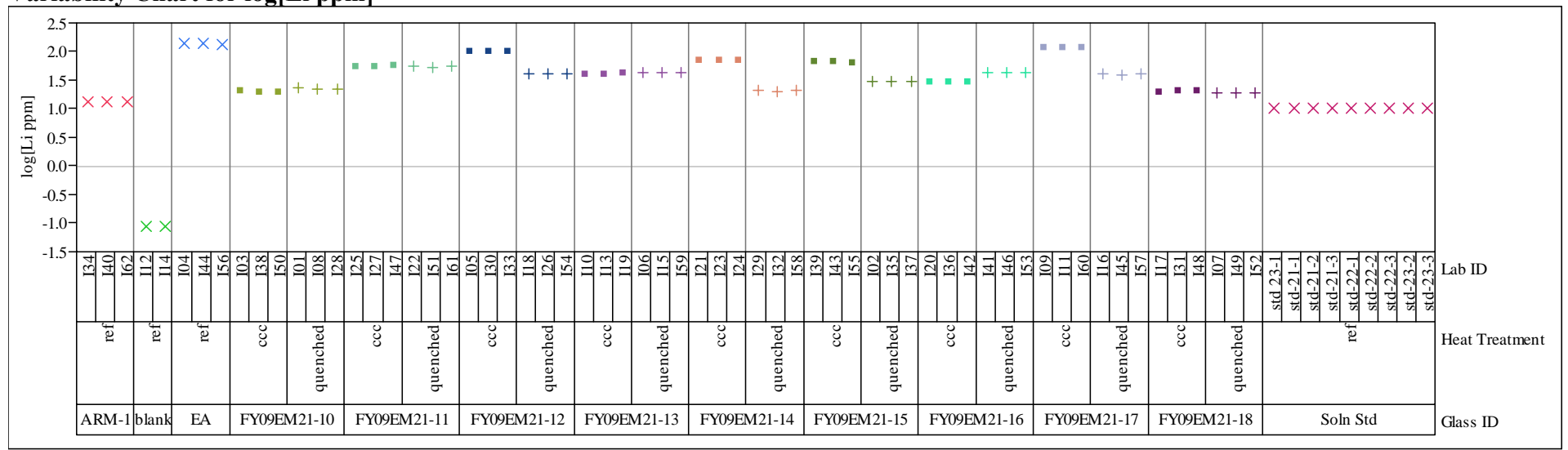


SRNL-STI-2009-00778, Revision 0

\section{Exhibit B3. Laboratory PCT Measurements by Glass Identifier for Matrix 2 Non-Rad Glasses and Standards}

Set $=2$

Variability Chart for $\log [\mathrm{Na}$ ppm]

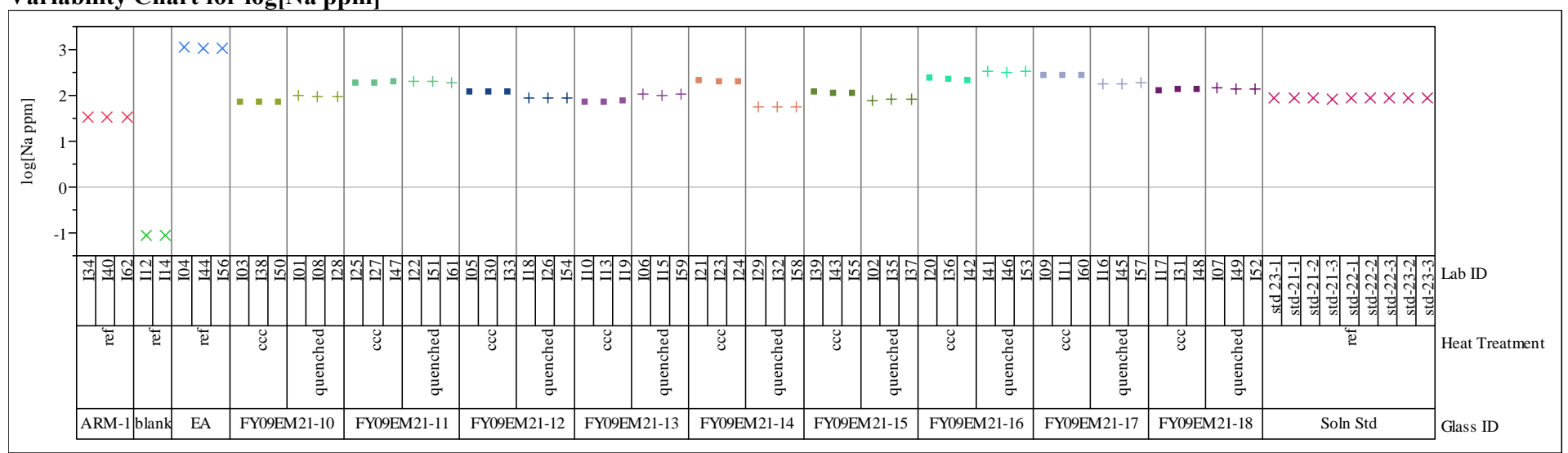

Set $=2$

Variability Chart for $\log [\mathrm{Si}$ ppm

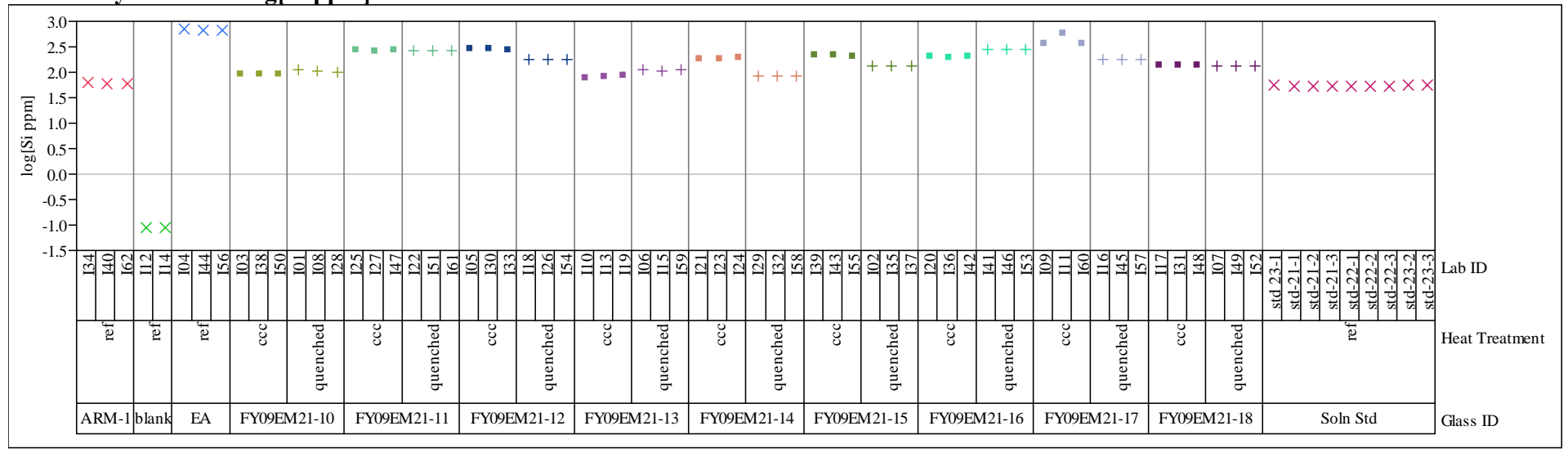




\section{Exhibit B3. Laboratory PCT Measurements by Glass Identifier for Matrix 2 Non-Rad Glasses and Standards}

Set $=3$

Variability Chart for B (ppm)

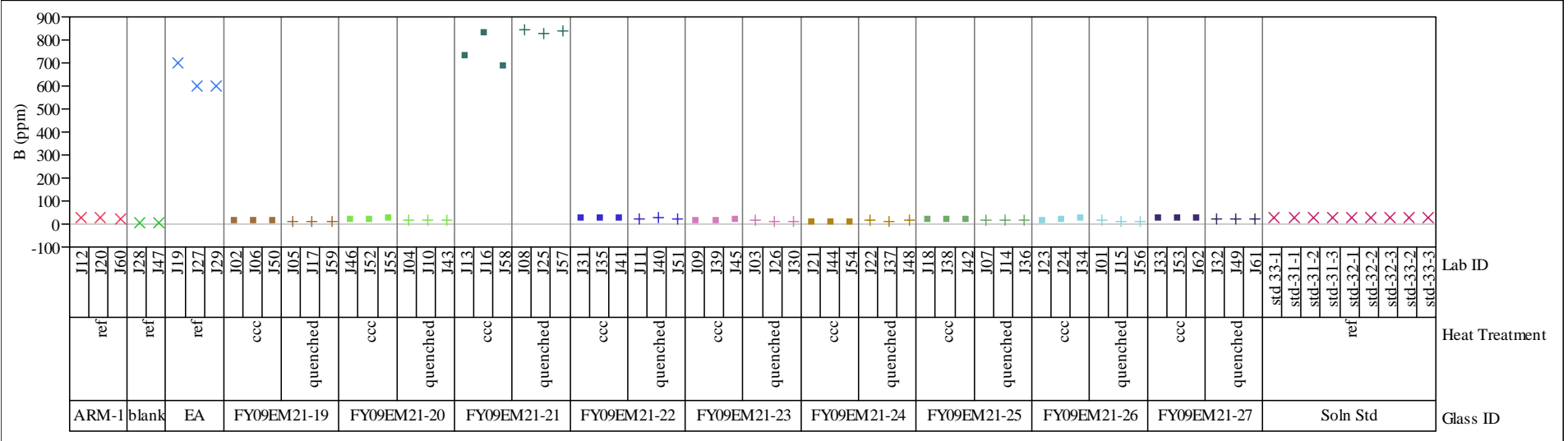

Set $=3$

Variability Chart for Li (ppm)

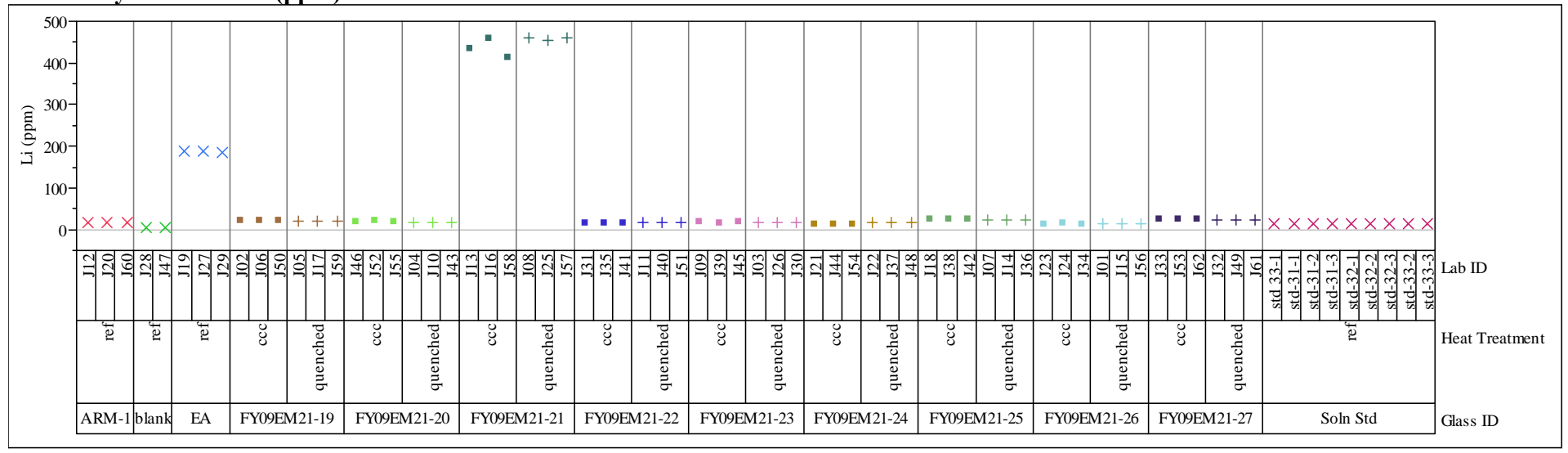




\section{Exhibit B3. Laboratory PCT Measurements by Glass Identifier for Matrix 2 Non-Rad Glasses and Standards}

Set $=3$

Variability Chart for Na (ppm)

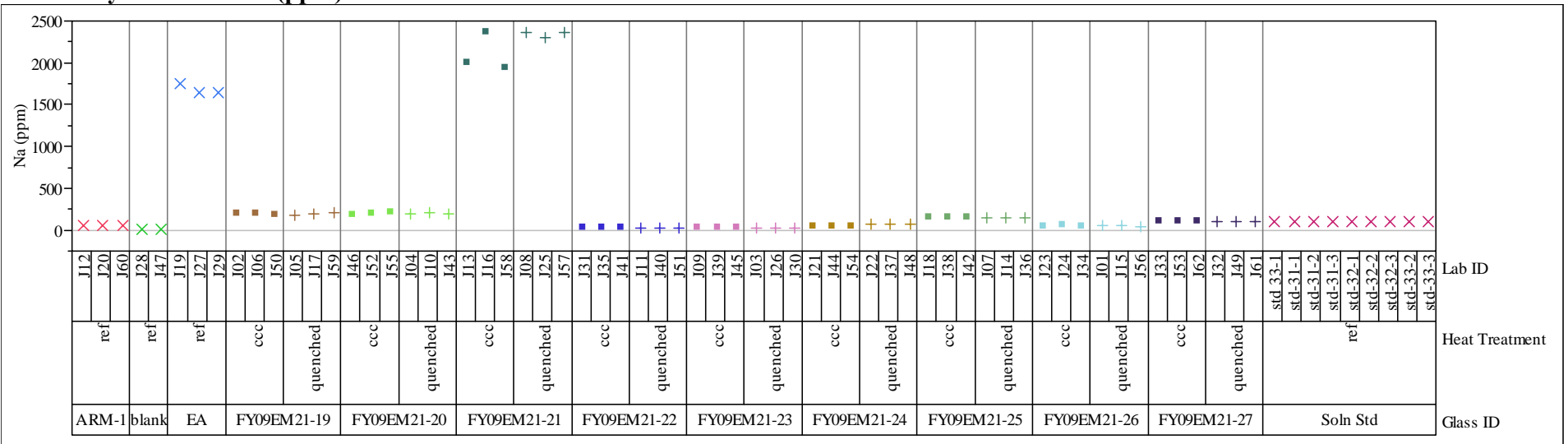

Set $=3$

Variability Chart for Si (ppm)

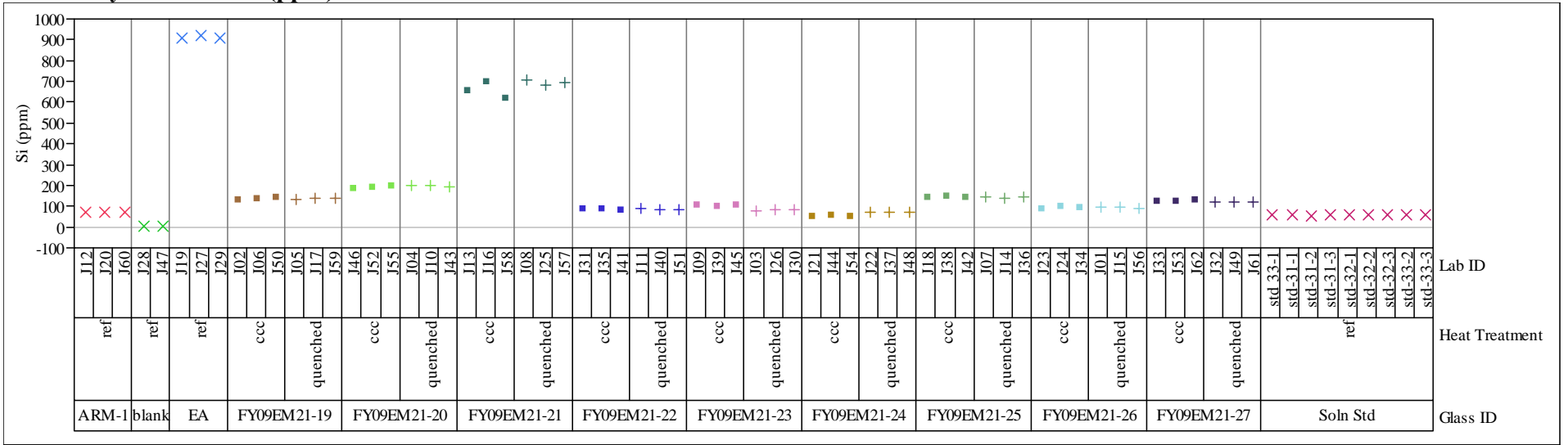


SRNL-STI-2009-00778, Revision 0

\section{Exhibit B3. Laboratory PCT Measurements by Glass Identifier for Matrix 2 Non-Rad Glasses and Standards}

Set $=3$

Variability Chart for $\log [\mathrm{B}$ ppm]

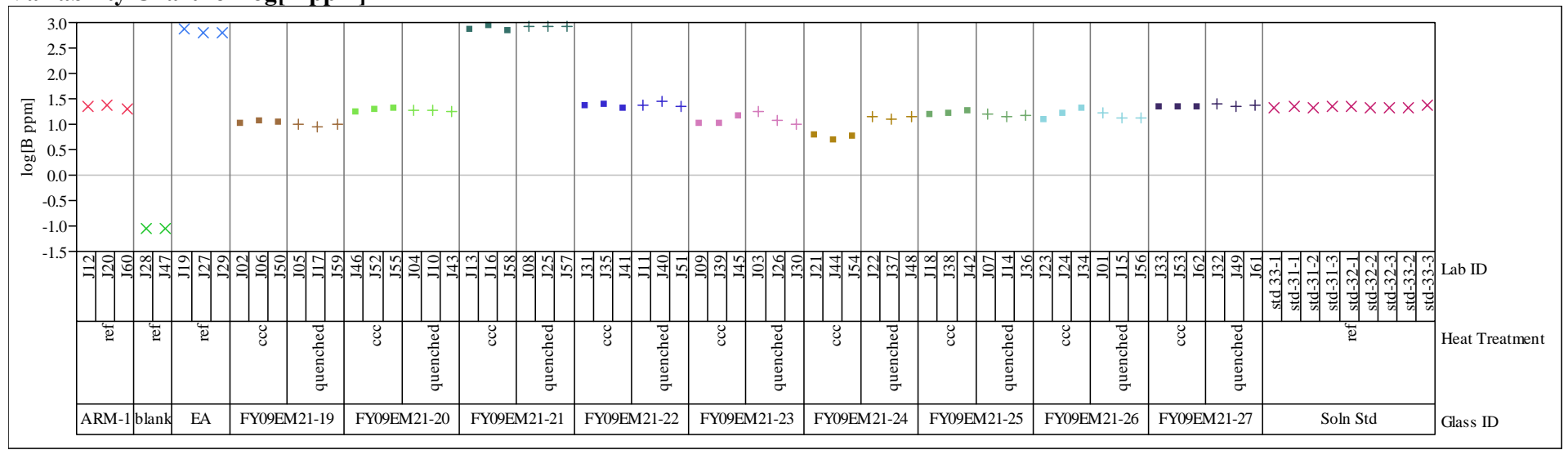

Set $=3$

Variability Chart for $\log [\mathrm{Li}$ ppm]

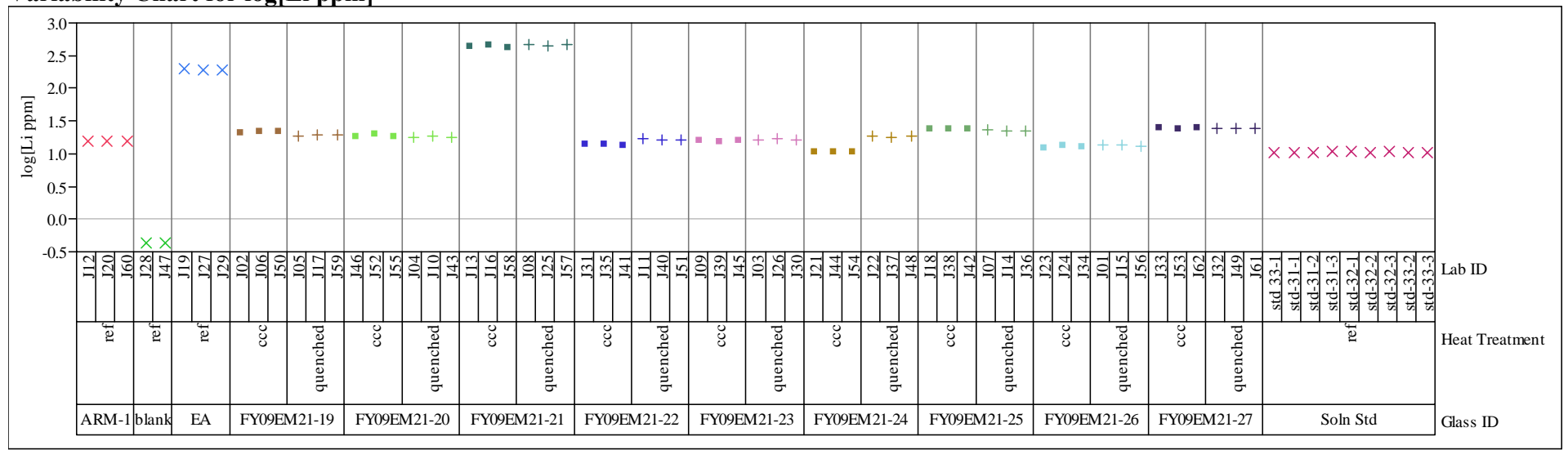


SRNL-STI-2009-00778, Revision 0

\section{Exhibit B3. Laboratory PCT Measurements by Glass Identifier for Matrix 2 Non-Rad Glasses and Standards}

Set $=3$

Variability Chart for $\log [\mathrm{Na}$ ppm]

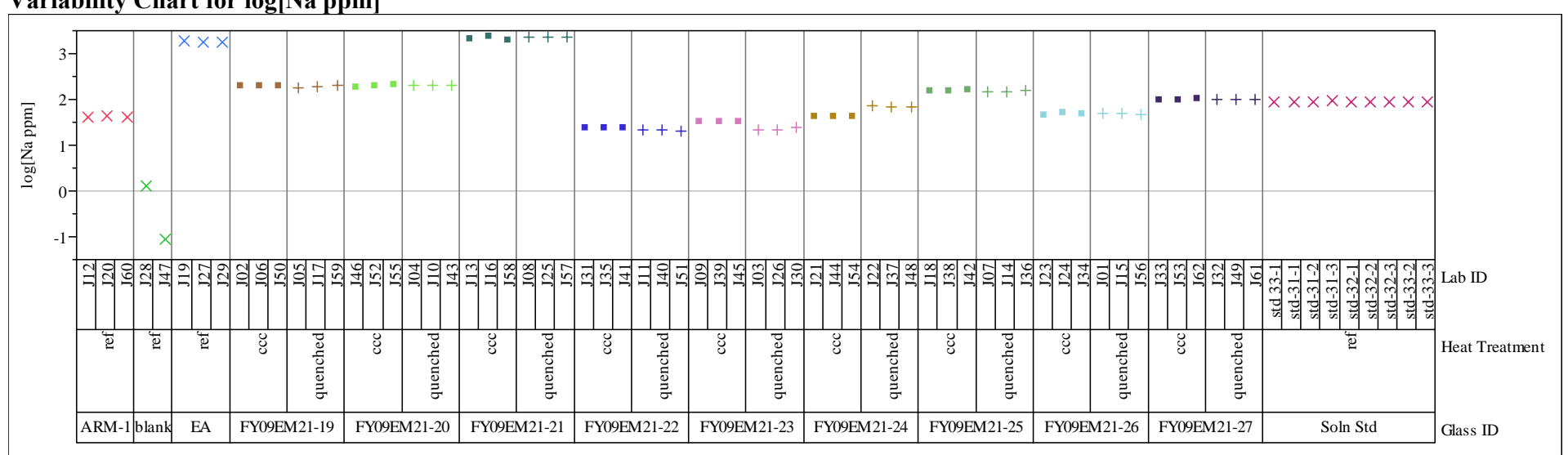

Set $=3$

Variability Chart for $\log [\mathrm{Si}$ ppm]

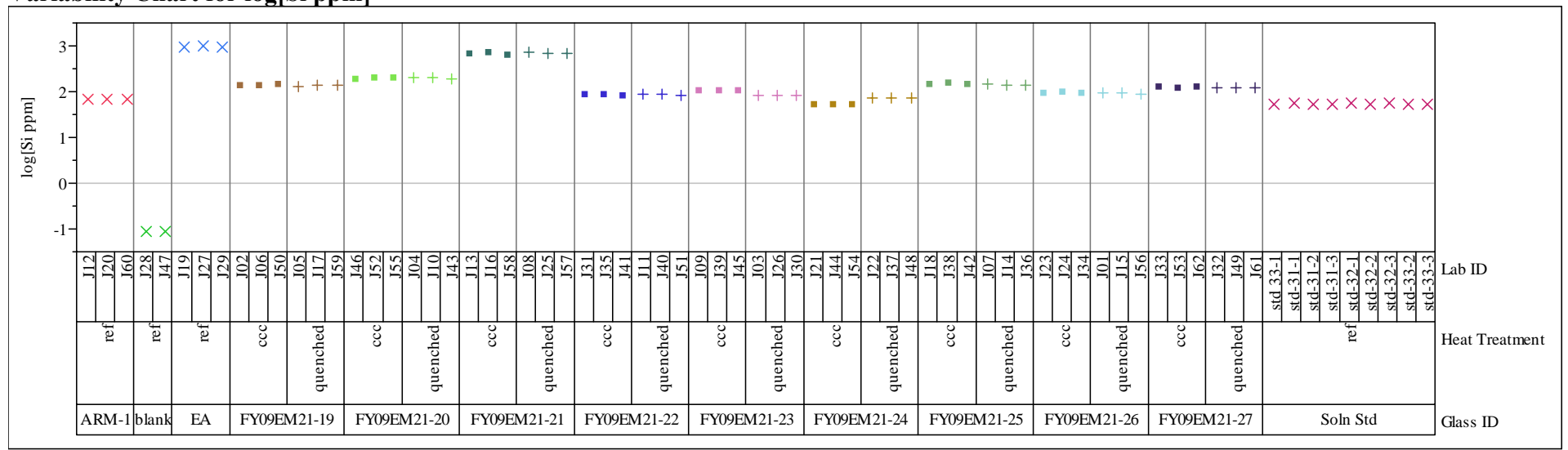


Exhibit B4. Correlations and Scatter Plots of Normalized PCTs Over All Compositional Views and Heat Treatments for the Non-Rad Matrix 2 Study

\section{Multivariate \\ Correlations}

$\log \mathrm{NL}[\mathrm{B}(\mathrm{g} / \mathrm{L})]$

$\log \mathrm{NL}[\mathrm{Li}(\mathrm{g} / \mathrm{L})]$

$\log \mathrm{NL}[\mathrm{Na}(\mathrm{g} / \mathrm{L})]$

$\log \mathrm{NL}[\mathrm{Si}(\mathrm{g} / \mathrm{L})]$ $\log \mathbf{N L}[B(g / L)]$

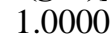

0.9770

0.9547

0.9226 $\log \mathrm{NL}[\mathrm{Li}$

$$
\begin{aligned}
& 0.9770 \\
& 1.0000 \\
& 0.9422
\end{aligned}
$$

0.9132
(g/L)]

0.9547

0.9422

1.0000

0.9128 $\log \mathbf{N L}[\mathbf{S i}(\mathrm{g} / \mathrm{L})]$

0.9226

0.9132

0.9128

1.0000

\section{Scatterplot Matrix}

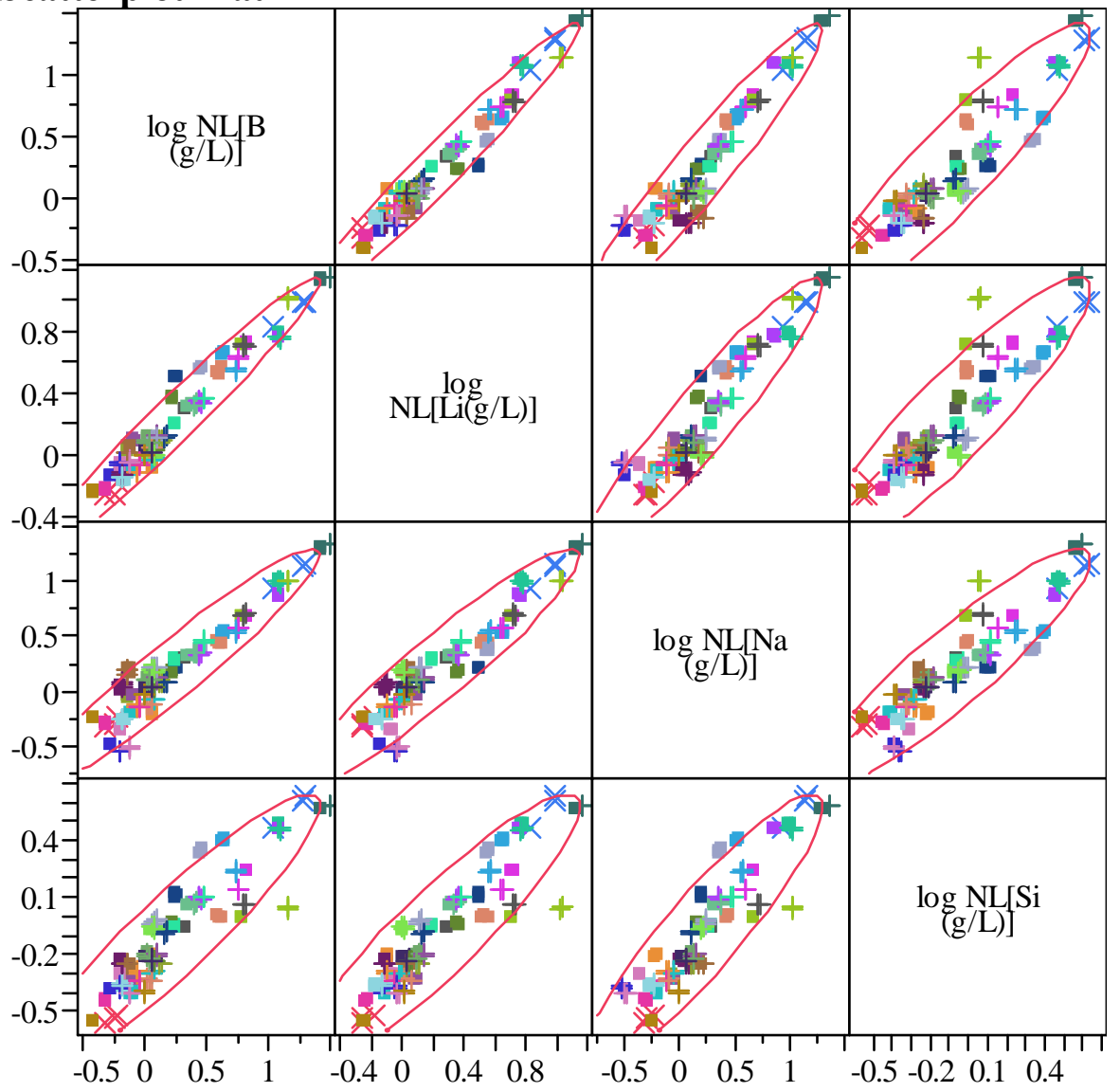


SRNL-STI-2009-00778, Revision 0

\section{Exhibit B5. Effects of Heat Treatment (HT) on PCT $\log (\mathrm{ppm})$ Response of Matrix 2 Non-Rad Glasses}

Oneway Analysis of $\log [\mathrm{B}$ ppm] By Heat Treatment Glass ID=FY09EM21-01

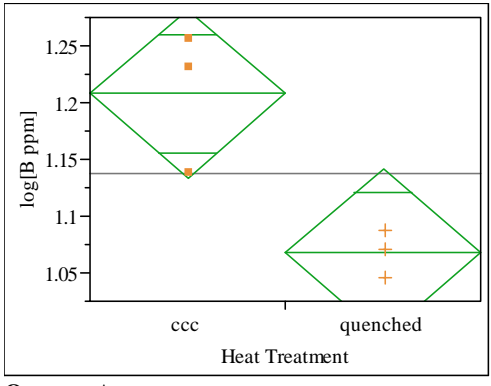

Oneway Anova

Summary of Fit

0.773269

0.716586

1.13756

1.137767
0

t Test

quenched-ccc

Assuming equal variances

Difference $\quad-0.13980$ t Ratio $\quad-3.69351$

Std Err Dif 0.03785 DF

Upper CLL Dif -0.03478 Prob $>|t| \quad 0.0210$

Lower CL Dif -0.24488 Prob $>t \quad 0.9895$

Confidence $\quad 0.95$ Prob $<t \quad 0.0105$

Analysis of Variance

Source DF Sum of Squares Mean Square F Ratio Prob $>$ F

$\begin{array}{llllll}\text { Heat Treatment } & 1 & 0.02931456 & 0.029315 & 13.6420 & 0.0210\end{array}$

0.03790992

0.002149

Means for Oneway Anova

Level Number Mean Std Error Lower 95\% Upper 95\%

$\begin{array}{llllll}\text { ccc } & 3 & 1.20767 & 0.02676 & 1.1334 & 1.2820 \\ \text { quenched } & 3 & 1.06787 & 0.02676 & 0.9936 & 1.1422\end{array}$

Std Error uses a pooled estimate of error variance
Oneway Analysis of $\log [\mathrm{Li}$ ppm] By Heat Treatment Glass

ID=FY09EM21-01

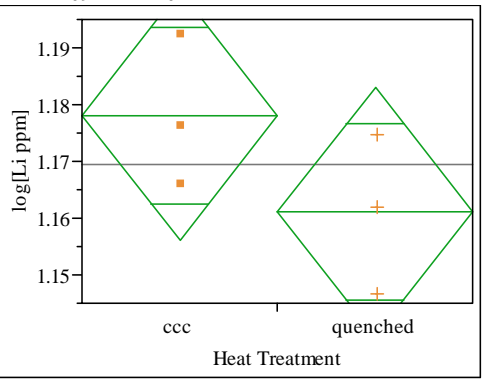

Oneway Anova

Summary of Fit

Rsquare

Adj Rsquare

\subsection{8}

Root Mean Square Error $\quad 0.013656$

Mean of Response 1.169555

t Test

quenched-ccc

Assuming equal variances

Difference $\quad-0.01699$ t Ratio $\quad-1.52377$

Std Err Dif $\quad 0.01115$ DF

4
0.2022

Lower CL Dif -0.04795 Prob $>t \quad 0.898$

Confidence $\quad 0.95$ Prob $<\mathrm{t} \quad 0.101$

Analysis of Variance

Source DF Sum of Squares Mean Square F Ratio Prob $>$ F

$\begin{array}{lll}\text { Heat Treatment } & 1 & 0.00043302 \\ \text { Cut }\end{array}$

$\begin{array}{lll}\text { Error } & 4 & 0.00074598 \\ \text { C. Total } & 5 & 0.00117899\end{array}$

$\begin{array}{lll}0.000433 & 2.3219 \quad 0.2022\end{array}$

00018

Means for Oneway Anova

Level Number Mean Std Error Lower 95\% Upper 95\%

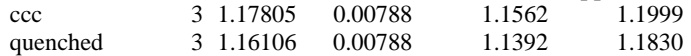

Std Error uses a pooled estimate of error variance
Oneway Analysis of $\log [\mathrm{Na} \mathrm{ppm}] \mathrm{By}$ Heat Treatment Glass

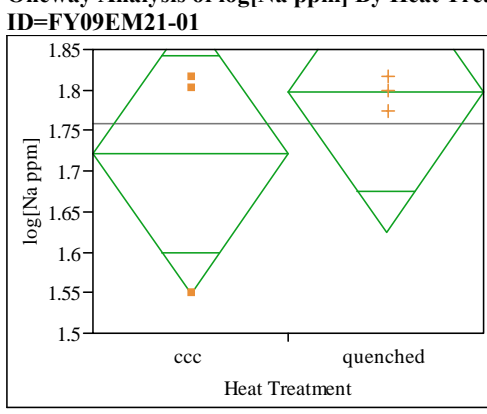

Oneway Anova

Summary of Fit

Rsquare

0.156743

Root Men Square Error $\quad-0.05407$

Mear of Reprose Eror 0.107123

Observations (or Sum Wgts) $\begin{array}{r}1.758999 \\ \hline\end{array}$

\section{Test}

quenched-ccc

Assuming equal variances

Difference $\quad 0.07542$ t Ratio $\quad 0.86227$ Std Err Dif 0.08747 DF

Upper CL Dif 0.31874 Prob $>|t| \quad 0.4372$ Lower CL Dif -0.16742 Prob $>t \quad 0.2186$ $\begin{array}{lll}\text { Confidence } & 0.95 \text { Prob }<\mathrm{t} \quad 0.7814\end{array}$

\section{Analysis of Variance}

DF Sum of Squares Mean Square F Ratio Prob $>$ $\begin{array}{llllll}\text { Heat Treatment } & 1 & 0.00853199 & 0.008532 & 0.7435 & 0.4372\end{array}$ $\begin{array}{lll}\text { Error } & 4 & 0.04590116 \\ \text { C. Total } & 5 & 0.05443315\end{array}$

Means for Oneway Anova

Level Number Mean Std Error Lower 95\% Upper 95\% $\begin{array}{llllll}\text { ccc } & 3 & 1.72129 & 0.06185 & 1.5496 & 1.8930 \\ \text { quenched } & 3 & 1.79671 & 0.06185 & 1.6250 & 1.9684\end{array}$

Std Error uses a pooled estimate of error variance 
SRNL-STI-2009-00778, Revision 0

\section{Exhibit B5. Effects of Heat Treatment (HT) on PCT $\log (\mathrm{ppm})$ Response of Matrix 2 Non-Rad Glasses}

Oneway Analysis of $\log [\mathrm{Si}$ ppm] By Heat Treatment Glass

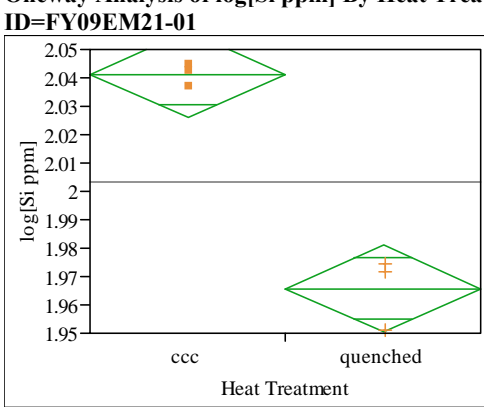

\section{Oneway Anova}

Summary of Fit

Adj Rsquare

0.948952

Square

Observations (or

2.003466
6

Test

quenched-ccc

Assuming equal variances

Difference $\quad-0.07541$ t Ratio $\quad-9.69259$ Std Err Dif 0.00778 DF

Upper CL Dif -0.05381 Prob $>|t| \quad 0.0006$

Lower CL Dif -0.09701 Prob $>t \quad 0.9997$

Confidence $\quad 0.95$ Prob $<t \quad 0.0003$

Analysis of Variance

Source DF Sum of Squares Mean Square F Ratio Prob $>$ F

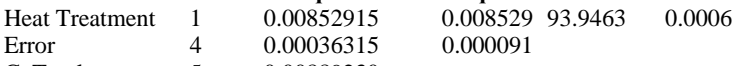

Error

0.00036315

Means for Oneway Anova

Level Number Mean Std Error Lower 95\% Upper 95\%

$\begin{array}{llllll}\text { cCC } & 3 & 2.04117 & 0.00550 & 2.0259 & 2.0564\end{array}$

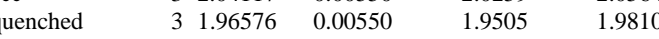

Std Error uses a pooled estimate of error variance
Oneway Analysis of $\log [\mathrm{B}$ ppm] By Heat Treatment Glass

ID=FY09EM21-02

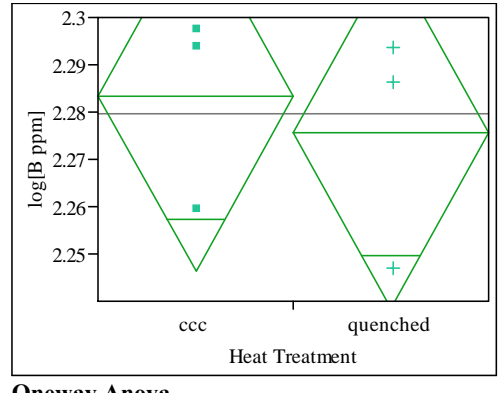

Oneway Anova

Summary of Fit

Rsquare

Adj Rsquare

Root Mean Square $\mathrm{E}$

0.040318

Mean of Respons

.023114

Observations (or Sum Wgts) 2.279608

t Test

quenched-ccc

Assuming equal variances

Difference $\quad-0.00774$ t Ratio $\quad-0.40994$

Std Err Dif $\quad 0.01887$ DF

Upper CL Dif 0.04466 Prob $>\mid 10.7029$

Lower CL Dif -0.06014 Prob $>t \quad 0.648$

Confidence $\quad 0.95$ Prob $<\mathrm{t} \quad 0.3514$

Analysis of Variance

Source DF Sum of Squares Mean Square F Ratio Prob $>$ $\begin{array}{llllll}\text { Heat Treatment } & 1 & 0.00008978 & 0.000090 & 0.1680 & 0.7029\end{array}$

Eror 0.00213704
0.00222682

Means for Oneway Anova

Level Number Mean Std Error Lower 95\% Upper 95\%

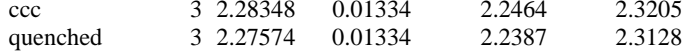

Std Error uses a pooled estimate of error variance
Oneway Analysis of $\log [\mathrm{Li} \mathrm{ppm]} \mathrm{By} \mathrm{Heat} \mathrm{Treatment} \mathrm{Glass}$

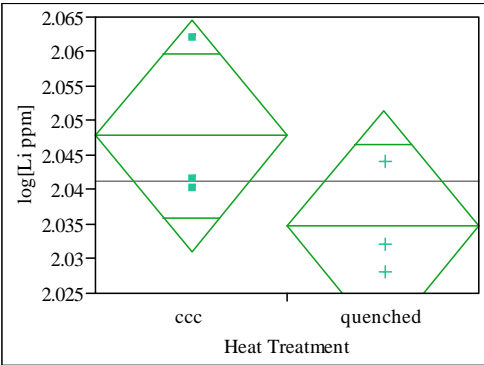

Oneway Anova

Summary of Fit

Rsquare

Root Mean Square Error $\quad 0.010479$

Mean of Response 2.041267

2.041267
6

t Test

quenched-ccc

Assuming equal variances

Difference $\quad-0.01310$ t Ratio -1.53086

Std Err Dif $\quad 0.00856$ DF

Upper CL Dif 0.01066 Prob $>|t| 0.2006$

Lower CL Dif -0.03685 Prob $>t \quad 0.8997$

$\quad 0.95$ Prob $<\mathrm{t} \quad 0.1003$

Analysis of Variance

Source DF Sum of Squares Mean Square F Ratio Prob $>$ F

$\begin{array}{lllllll}\text { Heat Treatment } & 1 & 0.00025735 & 0.000257 & 2.3435 & 0.2006\end{array}$

$\begin{array}{lll}\text { Error } & 4 & 0.00043926\end{array}$ 0.000110

Means for Oneway Anova

Level Number Mean Std Error Lower 95\% Upper 95\%

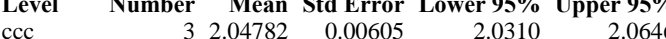

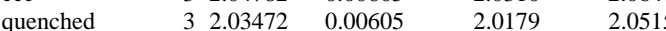

Std Error uses a pooled estimate of error variance 
SRNL-STI-2009-00778, Revision 0

\section{Exhibit B5. Effects of Heat Treatment (HT) on PCT $\log (\mathrm{ppm})$ Response of Matrix 2 Non-Rad Glasses}

Oneway Analysis of $\log [\mathbf{N a}$ ppm] By Heat Treatment Glass

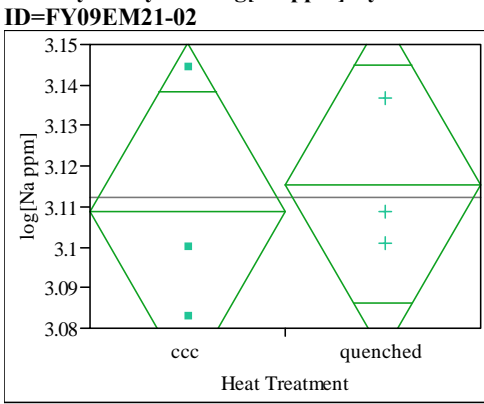

Oneway Anova

Heat Treatmen

\section{Summary of Fit}

Rsquare

Adj Rsquare $\quad 0.023882$

Root Mean Square Error $\quad \begin{array}{r}-0.22015 \\ 0.026012\end{array}$

Mean of Respon 3.112209
6

t Test

quenched-ccc

Assuming equal variances

Difference $\quad 0.00664$ t Ratio 0.312833 Std Err Dif 0.02124 DF

Upper CL Dif 0.06561 Prob $>|t| \quad 0.7700$

Lower CL Dif -0.05232 Prob $>t \quad 0.3850$

$\begin{array}{lll}\text { Confidence } & 0.95 \text { Prob }<\mathrm{t} \quad 0.6150\end{array}$

Analysis of Variance

Source DF Sum of Squares Mean Square F Ratio Prob $>$ F $\begin{array}{llllll}\text { Heat Treatment } & 1 & 0.00006622 & 0.000066 & 0.0979 & 0.7700\end{array}$ $4 \quad 0.00270643$ 0.000677

Means for Oneway Anova

Level Number Mean Std Error Lower 95\% Upper 95\% $\begin{array}{llllll}\text { сCC } & 3 & 3.10889 & 0.01502 & 3.0672 & 3.1506\end{array}$

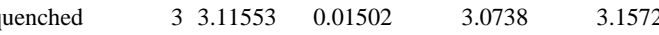

Std Error uses a pooled estimate of error variance
Oneway Analysis of $\log [\mathrm{Si}$ ppm $]$ By Heat Treatment Glass

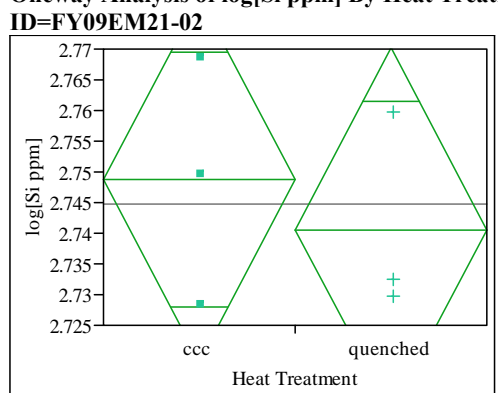

\section{Oneway Anova}

Summary of Fit

\section{Rsquare}

\section{Adj Rsquare}

Mean of Response

Observations (or Sum Wgts)$$
-0.16415
$$

-0.16415
0.018384

2.744674

t Test

quenched-ccc

Assuming equal variances

Difference $\quad-0.00815$ t Ratio $\quad-0.54311$

$0.01501 \mathrm{DF} \quad-0.54311$

$\begin{array}{lll}0.03352 & \text { Prob }>|t| & 0.6159 \\ \text { Lower CL Dif }-0.04983 & \text { Prob }>t & 0.6920\end{array}$

Confidence $\quad 0.95$ Prob $<t \quad 0.3080$

Analysis of Variance

Source DF Sum of Squares Mean Square F Ratio Prob $>$ F $\begin{array}{lrrrrr}\text { Source } & \text { DF Sum of Squares } & \text { Mean Square } & \text { Fatio } & \text { Prob }>\text { Feat Treatment } \\ \text { Heat } & 1 & 0.00009970 & 0.000100 & 0.2950 & 0.6159\end{array}$ Error $4 \quad 0.00135196$

C. Total 0.00145165

0.000338

Means for Oneway Anova

Level Number Mean Std Error Lower 95\% Upper 95\%

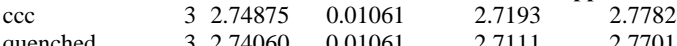

Std Error uses a pooled estimate of error variance
Oneway Analysis of $\log [\mathrm{B}$ ppm] By Heat Treatment Glass ID=FY09EM21-03

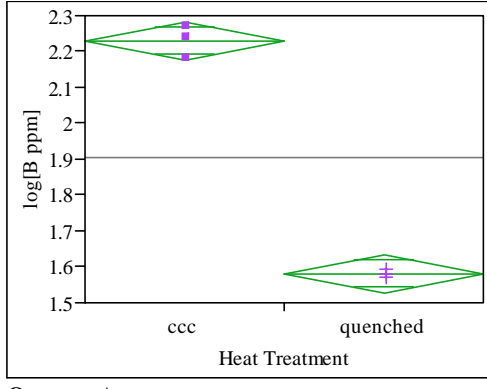

Oneway Anova

Root Mean Square Error $\quad 0.033194$

Mean of Response 1.906017

Observations (or Sum Wgts) 6

t Test

quenched-ccc

Assuming equal variance

Difference -0.64886 t Ratio -23.9408

Std Err Dif 0.02710 DF $>-23.9408$

Upper CL Dif -0.57361 Prob $>|\mathrm{t}| \quad<.0001$

Lower CL Dif -0.72411 Prob $>t \quad 1.0000$

$\begin{array}{lr}\text { Confidence } & 0.95 \text { Prob }<\mathrm{t} \quad<.000\end{array}$

Analysis of Variance

Source DF Sum of Squares Mean Square F Ratio Prob $>$ $\begin{array}{llllll}\text { Heat Treatment } & 1 & 0.63153145 & 0.631531 & 573.1628 & <.0001\end{array}$ $\begin{array}{lll}\text { Error } & 4 & 0.00440734\end{array}$ 0.63593880

Means for Oneway Anova

Level Number Mean Std Error Lower 95\% Upper 95\% $\begin{array}{llllll}\text { ccC } & 3 & 2.23045 & 0.01916 & 2.1772 & 2.2837\end{array}$

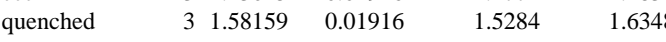

Std Error uses a pooled estimate of error variance 
SRNL-STI-2009-00778, Revision 0

\section{Exhibit B5. Effects of Heat Treatment (HT) on PCT $\log (\mathrm{ppm})$ Response of Matrix 2 Non-Rad Glasses}

Oneway Analysis of $\log [\mathrm{Li}$ ppm] By Heat Treatment Glass ID=FY09EM21-03

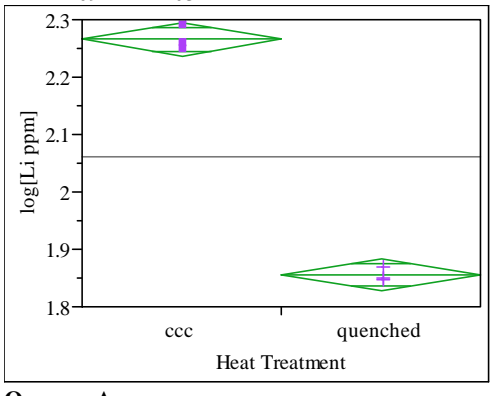

Oneway Anova

Summary of Fit

Rsquare

Adj Rsquare

Error 0.017585

Mean of Response

2.060361
6

t Test

quenched-ccc

Assuming equal variances

Difference $\quad-0.41027$ t Ratio $\quad-28.57$

Std Err Dif 0.01436 DF $>14<.0001$

Upper CL Dif -0.37041 Prob $>|\mathrm{t}|<.0001$

Lower CL Dif -0.45013 Prob $>t \quad 1.0000$

$\begin{array}{lll}\text { Confidence } \quad 0.95 \mathrm{Prob}<\mathrm{t} & <.000 \\ & & \end{array}$

Analysis of Variance

$\begin{array}{lrrrrr}\text { Source } & \text { DF } & \text { Sum of Squares } & \text { Mean Square } & \text { F Ratio } & \text { Prob }>\text { F } \\ \text { Heat Treatment } & 1 & 0.25248349 & 0.252483 & 816.5301 & <.0001\end{array}$

Error $\quad 4 \quad 0.25248349$

C. Total

0.25372035 0.000309

Means for Oneway Anova

Level Number Mean Std Error Lower 95\% Upper 95\%

$\begin{array}{lllllr}\text { ccc } & 3 & 2.26550 & 0.01015 & 2.2373 & 2.2937\end{array}$

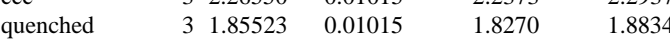

Std Error uses a pooled estimate of error variance
Oneway Analysis of log[Na ppm] By Heat Treatment Glass ID=FY09EM21-03

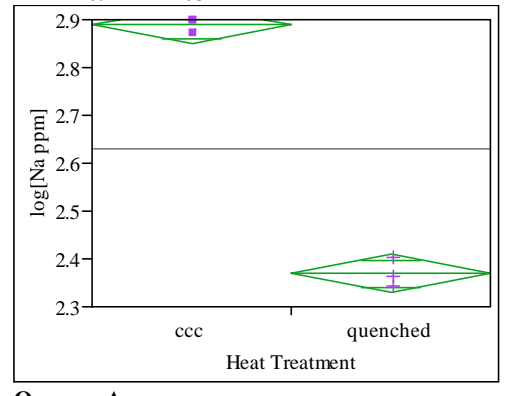

Oneway Anova

Rsquare

Adj Rsquare

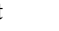

Root Mean Square

\subsection{4}

0.99243

Observations (or Sum Wgts)

t Test

quenched-ccc

Assuming equal variances

Difference $\quad-0.51921$ t Ratio $\quad-25.6223$

Std Err Dif 0.02026 DF

4
$<.0001$

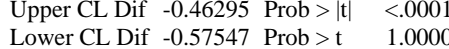

$\begin{array}{lrr}\text { Lower CL Dif } & -0.57547 \text { Prob }>t & 1.0000 \\ \text { Confidence } & 0.95 \text { Prob }<t & <.0001\end{array}$

Analysis of Variance

Source DF Sum of Squares Mean Square F Ratio Prob $>$ F Heat Treatment $1 \quad 0.40436339$

Erro

Tot

0.00246374

$\begin{array}{lll}0.404363 & 656.5043 & <.0001 \\ 0.000616 & \end{array}$

Means for Oneway Anova

Level Number Mean Std Error Lower 95\% Upper 95\%

$\begin{array}{llllll}\text { CCC } & 3 & 2.88850 & 0.01433 & 2.8487 & 2.9283\end{array}$

$\begin{array}{llllll}\text { quenched } & 3 & 2.36929 & 0.01433 & 2.3295 & 2.4091\end{array}$

Std Error uses a pooled estimate of error variance
Oneway Analysis of $\log [\mathrm{Si}$ ppm] By Heat Treatment Glass

ID=FY09EM21-03

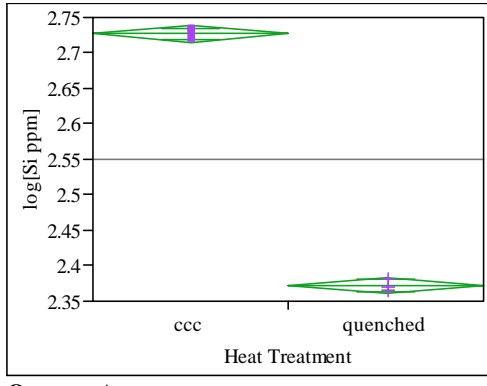

Oneway Anova

Summary of Fit

$\begin{array}{lr}\text { Rsquare } & 0.99882 \\ \text { Adj Rsquare } & 0.998525 \\ \text { Root Mean Square Error } & 0.00747 \\ \text { Mean of Response } & 2.549512 \\ \text { Observations (or Sum Wgts) } & 6\end{array}$

Test

quenched-ccc

Assuming equal variances

Difference $\quad-0.35491$ t Ratio $\quad-58.1883$

Std Err Dif $\quad 0.00610$ D

Upper CL Dif -0.33798 Prob $>|t|<.000$

Lower CL Dif -0.37185 Prob $>t \quad 1.0000$

$\begin{array}{lll}\text { Confidence } & 0.95 \text { Prob }<\mathrm{t} \quad<.0001\end{array}$

Analysis of Variance

Source DF Sum of Squares Mean Square F Ratio Prob $>$ F $\begin{array}{lllllll}\text { Heat Treatment } & 1 & 0.18894313 & 0.188943 & 3385.883 & <.0001\end{array}$ $\begin{array}{lll}\text { Error } & 4 & 0.00022321 \\ \text { C. } & 5 & 0.1896534\end{array}$ 0.000056

\section{Means for Oneway Anova}

Level Number Mean Std Error Lower 95\% Upper 95\%

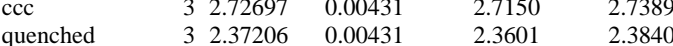

Std Error uses a pooled estimate of error variance 
SRNL-STI-2009-00778, Revision 0

\section{Exhibit B5. Effects of Heat Treatment (HT) on PCT $\log (\mathrm{ppm})$ Response of Matrix 2 Non-Rad Glasses}

Oneway Analysis of $\log [\mathrm{B}$ ppm] By Heat Treatment Glass ID=FY09EM21-04

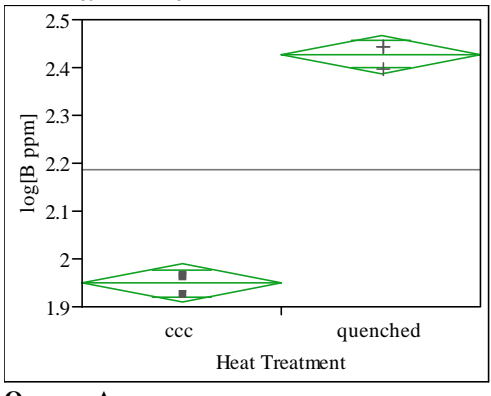

Oneway Anova

\section{Rsquare}

Adj Rsquare

Mean of Response

0.025309

2.188241
6

t Test

quenched-ccc

Assuming equal variances

Std Err Dif 0.020664 DF

Upper CL Dif 0.535281 Prob $>|t|<.0001$

Confidence $\quad 0.95$ Prob $<\mathrm{t} \quad 1.0000$
Difference $\quad 0.477908$ t Ratio $\quad 23.12716$

Lower CL Dif 0.420534 Prob $>t \quad<.0001$

Oneway Analysis of $\log [\mathrm{Li}$ ppm] By Heat Treatment Glass ID=FY09EM21-04

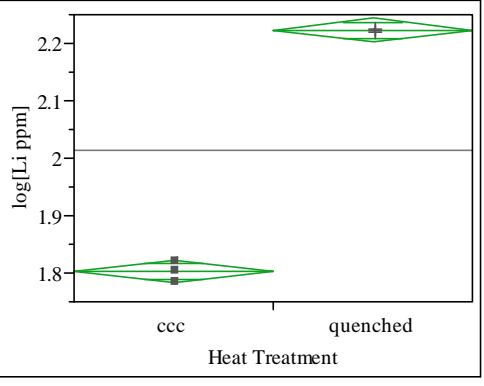

Oneway Anova

Rsquare

Adj Rsquare

Root Mean Square Enror

Mean of Response

0.99755

0.012738

t Test

quenched-ccc

Assuming equal variances

Difference $\quad 0.420176$ t Ratio $\quad 40.4003$

Std Err Dif 0.010400 DF

4
$<.0001$

Upper CL Dif 0.449

Lower CL Dif 0.391300 Prob $>t<<000$

$\begin{array}{lll}\text { Confidence } & 0.95 \text { Prob }<\mathrm{t} \quad 1.0000\end{array}$

Analysis of Variance

Source DF Sum of Squares Mean Square F Ratio Prob $>$ F $\begin{array}{lll}\text { Heat Treatment } & 1 & 0.26482235 \\ \text { C. }\end{array}$ $\begin{array}{lll}\text { Error } & 4 & 0.00064900 \\ \text { C. Total } & 5 & 0.26547135\end{array}$ $0.2648221632 .184<.000$

$\begin{array}{lrrrrr}\text { Source } & \text { DF } & \text { Sum of Squares } & \text { Mean Square } & \text { F Ratio } & \text { Prob }>\text { F } \\ \text { Heat Treatment } & 1 & 0.34259371 & 0.342594 & 534.8654 & <.0001\end{array}$ Heat Treatment 110.34259371

Eror 0.34515580

Means for Oneway Anova

Level Number Mean Std Error Lower 95\% Upper 95\% $\begin{array}{lllllr}\text { ccc } & 3 & 1.94929 & 0.01461 & 1.9087 & 1.9899\end{array}$ $\begin{array}{llllll}\text { quenched } & 3 & 2.42719 & 0.01461 & 2.3866 & 2.4678\end{array}$

Std Error uses a pooled estimate of error variance
Means for Oneway Anova

Level Number Mean Std Error Lower 95\% Upper 95\%

$\begin{array}{llllll}\text { ccC } & 3 & 1.80254 & 0.00735 & 1.7821 & 1.8230\end{array}$

Std Error uses a pooled estimate of error variance $\begin{array}{llllll}\text { quenched } & 3 & 2.22272 & 0.00735 & 2.2023 & 2.2431\end{array}$
Oneway Analysis of $\log [\mathrm{Na}$ ppm] By Heat Treatment Glass ID=FY09EM21-04

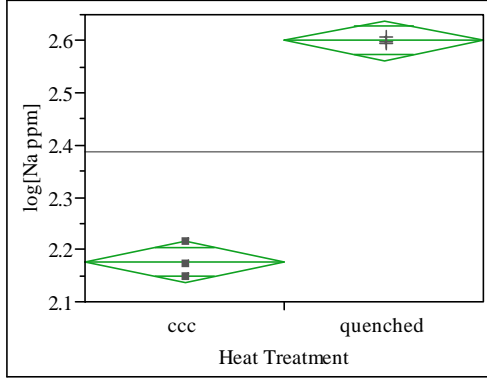

Oneway Anova

Rsquare $\quad 0.991364$

Adj Rsquare $\quad 0.989205$

Root Mean Square Error $\quad 0.024245$

$\begin{array}{lr}\text { Mean of Response } & 2.388126 \\ \text { Observations (or Sum Wgts) } & 6\end{array}$

t Test

quenched-ccc

Assuming equal variances

Difference $\quad 0.424194$ t Ratio $\quad 21.428$

Std Err Dif 0.019796 DF

Lower CL Dif 0.369231 Prob $>t<.000$

Confidence $\quad 0.95$ Prob $<\mathrm{t} \quad 1.0000$

Analysis of Variance

Source DF Sum of Squares Mean Square F Ratio Prob $>$ F $\begin{array}{llllll}\text { Heat Treatment } & 1 & 0.26991059 & 0.269911 & 459.1592 & <.0001\end{array}$ \begin{tabular}{lll} 
Error & 4 & 0.00235135 \\
\hline
\end{tabular} 0.000588

Means for Oneway Anova

Level Number Mean Std Error Lower 95\% Upper 95\%

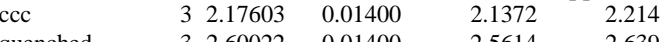
$\begin{array}{llllll}\text { quenched } & 32.60022 & 0.01400 & 2.5614 & 2.6391\end{array}$

Std Error uses a pooled estimate of error variance 
SRNL-STI-2009-00778, Revision 0

\section{Exhibit B5. Effects of Heat Treatment (HT) on PCT $\log (\mathrm{ppm})$ Response of Matrix 2 Non-Rad Glasses}

Oneway Analysis of $\log [\mathrm{Si}$ ppm] By Heat Treatment Glass ID=FY09EM21-04

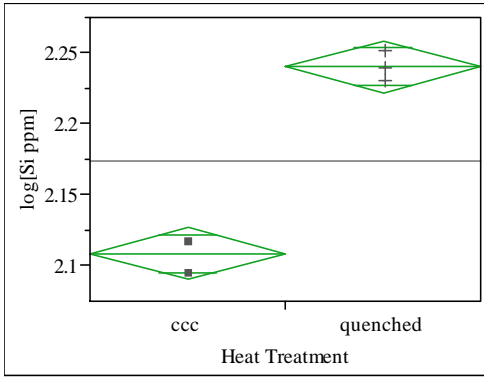

\section{Oneway Anova}

Summary of Fit

$\begin{array}{lr}\text { Rsquare } & 0.979711 \\ \text { Adj Rsquare } & 0.974639 \\ \text { Root Mean Square Error } & 0.011597 \\ \text { Mean of Response } & 2.174399 \\ \text { Observations (or Sum Wgts) } & 6\end{array}$

Observations (or Sum Wgts)

Test

quenched-ccc

Assuming equal variances

Difference $\quad 0.131594$ t Ratio 13.89801 Std Err Dif 0.009469 DF

Upper CL Dif $0.157883 \mathrm{Prob}>|\mathrm{t}| \quad 0.0002$

Lower CL Dif 0.105305 Prob $>t \quad<.0001$

Confidence $\quad 0.95$ Prob $<\mathrm{t} \quad 0.9999$
Oneway Analysis of $\log [\mathrm{B}$ ppm] By Heat Treatment Glass

ID=FY09EM21-05

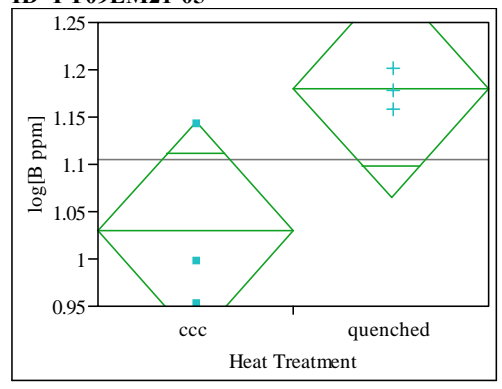

Oneway Anova

Summary of Fit

Rsquare

Adj Rsquare

Root Mean of Response

0.526531

t Test

quenched-ccc

Assuming equal variances

Difference $\quad 0.14985$ t Ratio $\quad 2.561317$

Std Err Dif $\quad 0.05850$ DF

4
0.0626

Lower CL Dif -0.01259 Prob $>t \quad 0.0313$

$\begin{array}{lrr} & \\ \text { Confidence } & 0.95 \text { Prob }<\mathrm{t} & 0.9687\end{array}$

Analysis of Variance

Analysis of Variance

Source DF Sum of Squares Mean Square F Ratio Prob $>$ F

$\begin{array}{llllll}\text { Heat Treatment } & 1 & 0.02597563 & 0.025976 & 193.1548 & 0.0002 \\ \text { Error } & 4 & 0.00053792 & 0.000134 & & \end{array}$

Error

0.00053792

Means for Oneway Anova

Level Number Mean Std Error Lower 95\% Upper 95\%

$\begin{array}{llllll}\text { ccc } & 3 & 2.10860 & 0.00670 & 2.0900 & 2.1272 \\ \text { quenched } & 3 & 2.24020 & 0.00670 & 2.2216 & 2.2588\end{array}$

Std Error uses a pooled estimate of error variance
Source DF Sum of Squares Mean Square F Ratio Prob $>$ F $\begin{array}{lll}\text { Error } & 4 & 0.02053686 \\ \text { C. Total } & 5 & 0.05421908\end{array}$ $\begin{array}{lll}0.033682 & 6.5603 & 0.0626\end{array}$

Means for Oneway Anova

Level Number Mean Std Error Lower 95\% Upper 95\%

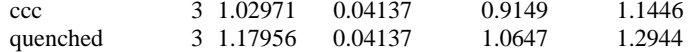

Std Error uses a pooled estimate of error variance $\begin{array}{lll}\text { Heat Treatment } & 1 & 0.03368222 \\ \text { Error } & 4 & 0.02053686\end{array}$
Oneway Analysis of $\log [\mathrm{Li}$ ppm] By Heat Treatment Glass D=FY09EM21-05

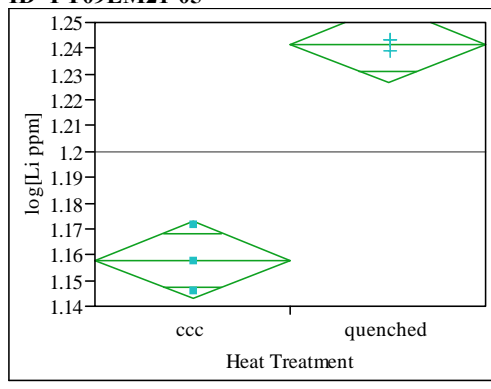

\section{Oneway Anova}

Summary of Fit

Rsquare

$\begin{array}{ll}\text { Adj Rsquare } & 0.960697 \\ \text { Root Mean Square Error } & 0.009241\end{array}$

0.968558

Mean of Respon

1.199785

Observations (or Sum Wgts)

Test

quenched-ccc

Assuming equal variance

Difference $\quad 0.083752$ t Ratio $\quad 11.10037$

Std Err Dif 0.007545 DF

Upper CL Dif 0.104701 Prob $>|\uparrow| \quad 0.0004$

Lower CL Dif 0.062804 Prob $>t \quad 0.0002$

Confidence $\quad 0.95$ Prob $<\mathrm{t} \quad 0.9998$

Analysis of Variance

Source DF Sum of Squares Mean Square F Ratio Prob $>$ F $\begin{array}{lrrrrr}\text { Heat Treatment } & 1 & 0.01052166 & 0.010522 & 123.2181 & 0.0004\end{array}$ $\begin{array}{lll}\text { Error } & 4 & 0.00034156\end{array}$

\section{Means for Oneway Anova}

Level Number Mean Std Error Lower 95\% Upper 95\%

$\begin{array}{lrrrrr}\text { ccc } & 3 & 1.15791 & 0.00534 & 1.1431 & 1.1727 \\ \text { quenched } & 3 & 1.24166 & 0.00534 & 1.2268 & 1.2565\end{array}$

3 1.24166 $0.00534-1$.

Std Error uses a pooled estimate of error variance 
SRNL-STI-2009-00778, Revision 0

\section{Exhibit B5. Effects of Heat Treatment (HT) on PCT $\log (\mathrm{ppm})$ Response of Matrix 2 Non-Rad Glasses}

Oneway Analysis of $\log [\mathrm{Na}$ ppm] By Heat Treatment Glass

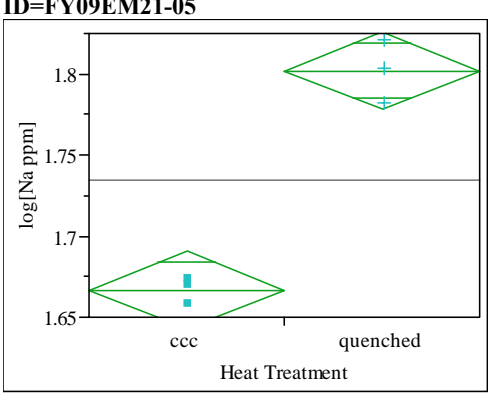

\section{Oneway Anova}

Summary of Fit

Rsquare

Adj Rsquare

0.968579

Root Mean Sc

0.960724

Mean of Respon 1.734502

t Test

quenched-ccc

Assuming equal variances

Difference $\quad 0.135218$ t Ratio 11.10421 Std Err Dif 0.012177 DF

Upper CL Dif 0.169027 Prob $>|t| \quad 0.0004$

Lower CL Dif 0.101409 Prob $>t \quad 0.0002$

$\begin{array}{lll}\text { Confidence } & 0.95 \text { Prob }<\mathrm{t} \quad 0.9998\end{array}$
Oneway Analysis of $\log [\mathrm{Si}$ ppm] By Heat Treatment Glass ID=FY09EM21-05

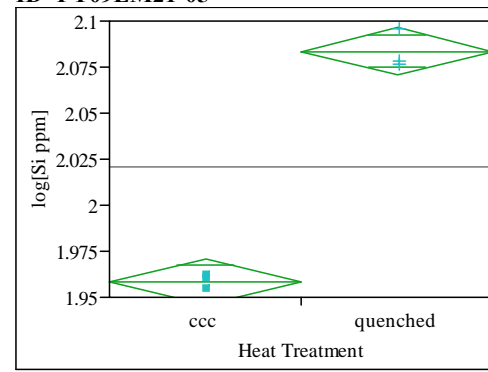

Oneway Anova

Summary of Fit

Rsquare

Adj Rsquare

Mean of Resquare

Observations (or Sum Wgts)

\subsection{6}

0.986951
0.007875 2.021107

Test

quenched-ccc

Assuming equal variances

Difference $\quad 0.125197$ t Ratio $\quad 19.47216$

Prob $>|t|<0001$

Upper CL Dif 0.143048 Prob $>|t|<.0001$

Analysis of Variance

Source DF Sum of Squares Mean Square F Ratio Prob $>$ F $\begin{array}{llllll}\text { Heat Treatment } & 1 & 0.02742578 & 0.027426 & 123.3036 & 0.0004 \\ \text { Error } & 4 & 0.00088970 & 0.000222 & & \end{array}$ 0.00088970

$$
0.000222
$$

Means for Oneway Anova

Level Number Mean Std Error Lower 95\% Upper 95\% $\begin{array}{llllll}\text { сCC } & 3 & 1.66689 & 0.00861 & 1.6430 & 1.6908\end{array}$

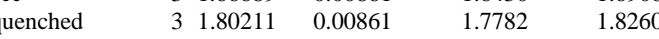

Std Error uses a pooled estimate of error variance $\begin{array}{llll}\text { Error } & 4 & 0.00024803\end{array}$

C. Total 0.02375935

0.000062

Means for Oneway Anova

Std Error uses a pooled estimate of error variance
Confidence $\quad 0.95 \mathrm{Prob}<\mathrm{t} \quad 1.0000$

Oneway Analysis of $\log [\mathrm{B}$ ppm] By Heat Treatment Glass ID=FY09EM21-06

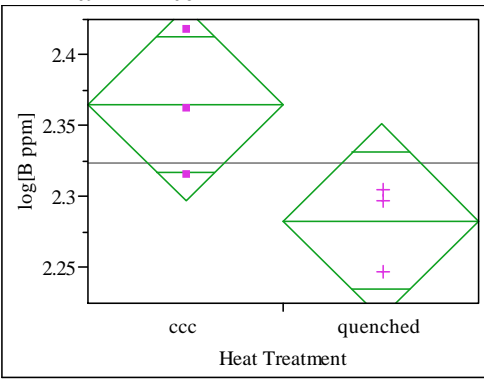

Oneway Anova

Summary of Fit

$\begin{array}{lr}\text { Rsquare } & 0.581726 \\ \text { Adj Rsquare } & 0.477158 \\ \text { Root Mean Square Error } & 0.042504 \\ \text { Mean of Response } & 2.323997 \\ \text { Observations (or Sum Wgts) } & 6\end{array}$

Test

quenched-ccc

Assuming equal variance

Difference $\quad-0.08185$ t Ratio $\quad-2.35863$ Std Err Dif 0.03470 DF

Upper CL Dif 0.01450 Prob $>|t| \quad 0.0778$ Lower CL Dif -0.17821 Prob $>$ t 0.961 $\begin{array}{lll}\text { Confidence } & 0.95 \text { Prob }<\mathrm{t} \quad 0.0389\end{array}$

\section{Analysis of Variance}

Source DF Sum of Squares Mean Square F Ratio Prob $>$ F $\begin{array}{llllll}\text { Heat Treatment } & 1 & 0.01005013 & 0.010050 & 5.5631 & 0.0778\end{array}$ $\begin{array}{lll}\text { Error } & 4 & 0.00722626 \\ \text { C. Total } & 5 & 0.01727640\end{array}$

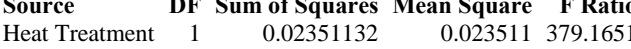

Level Number Mean Std Error Lower 95\% Upper 95\%

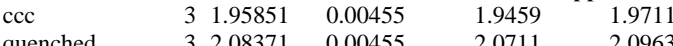

Means for Oneway Anova

Level Number Mean Std Error Lower 95\% Upper 95\%

\begin{tabular}{lll|lll} 
ccc & 3 & 2.36492 & 0.02454 & 2.2968 & 2.433
\end{tabular}

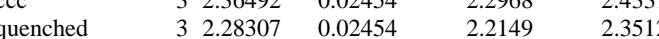

Std Error uses a pooled estimate of error variance 
SRNL-STI-2009-00778, Revision 0

\section{Exhibit B5. Effects of Heat Treatment (HT) on PCT $\log (\mathrm{ppm})$ Response of Matrix 2 Non-Rad Glasses}

Oneway Analysis of $\log [\mathrm{Li}$ ppm] By Heat Treatment Glass

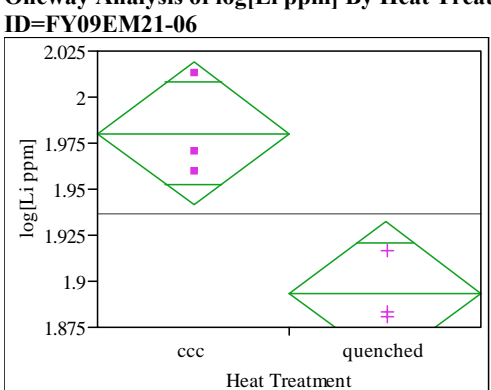

Oneway Anova

Summary of Fit

$\begin{array}{lr}\text { Rsquare } & 0.827413 \\ \text { Adj Rsquare } & 0.784266 \\ \text { Root Mean Square Error } & 0.024265 \\ \text { Mean of Response } & 1.937032 \\ \text { Observations (or Sum Wgts) } & 6\end{array}$

t Test

quenched-ccc

Assuming equal variances

Difference $\quad-0.08676$ t Ratio $\quad-4.37912$

$\begin{array}{llll}\text { Std Err Dif } \quad 0.01981 \text { DF } & 4\end{array}$

Upper CL Dif -0.03175 Prob $>|t| 0.0119$

Lower CL Dif -0.14177 Prob $>t \quad 0.9941$
Cong

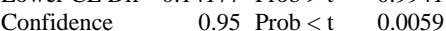

Analysis of Variance

Source DF Sum of Squares Mean Square F Ratio Prob $>$ F $\begin{array}{llllll}\text { Heat Treatment } & 1 & 0.01129122 & 0.011291 & 19.1767 & 0.0119\end{array}$

Error $4 \quad 4 \quad 0.00235519$

C. Tot

0.01364641 0.000589

Means for Oneway Anova

Level Number Mean Std Error Lower 95\% Upper 95\%

$\begin{array}{llllll}\text { CCC } & 3 & 1.98041 & 0.01401 & 1.9415 & 2.0193\end{array}$

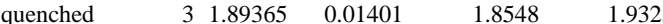

Std Error uses a pooled estimate of error variance
Oneway Analysis of $\log [\mathrm{Na}$ ppm] By Heat Treatment Glass

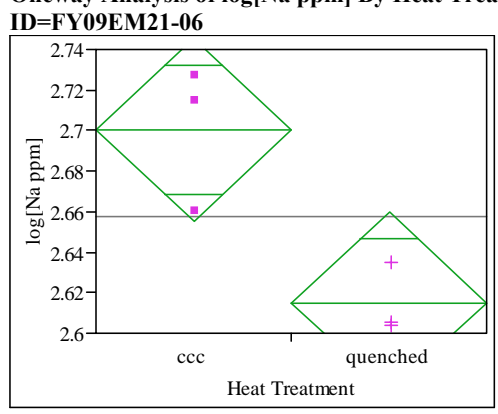

Oneway Anova

Summary of Fit

Rsquare

Adj Rsquare

Root Mean Square Error $\quad 0.028245$

Mean of Response 2.657656

t Test

quenched-ccc

Assuming equal variances

Difference $\quad-0.08551$ t Ratio $\quad-3.7078$

Std Err Dif 0.02306 DF

Upper CL Dif -0.02148 PFb $>100.0207$

Lower CL Dif $-0.14954 \mathrm{Prob}>\mathrm{t} \quad 0.9897$

Confidence -0.95 Prob $<t \quad 0.0103$

Analysis of Variance

Source DF Sum of Squares Mean Square F Ratio Prob $>$ F

$\begin{array}{lll}\text { Heat Treatment } & 1 & 0.01096783 \\ \text { Eror } & 4 & 0.0031915\end{array}$

$\begin{array}{lll}\text { Error } & 4 & 0.00319115 \\ \text { C. Total } & 5 & 0.01415898\end{array}$

$0.01096813 .7478 \quad 0.0207$

Level Number Mean Std Error Lower 95\% Upper 95\%

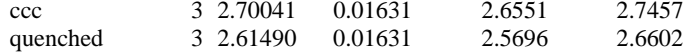

Std Error uses a pooled estimate of error variance
Oneway Analysis of $\log [\mathrm{Si}$ ppm] By Heat Treatment Glass ID=FY09EM21-06

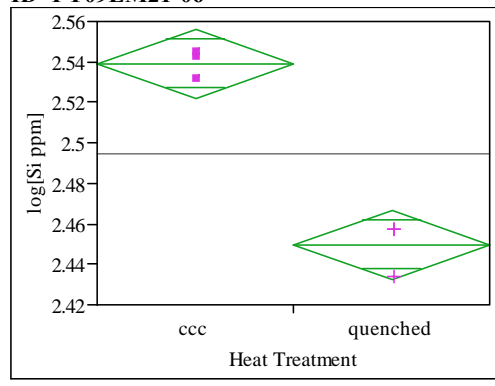

\section{Oneway Anova}

Summary of Fit

0.963632

0.95454
Root Mern Square Eror $\quad 0.010657$

Mect Men Square Eror 0.010657

Mean of Response 2.494397

quenched-ccc

Assuming equal variances

Difference $\quad-0.08958$ t Ratio $\quad-10.2949$ Std Err Dif $\quad 0.00870$ DF

Upper CL Dif -0.06542 Prob $>|t| \quad 0.0005$

Lower CL Dif -0.11374 Prob $>t \quad 0.9997$

Confidence $\quad 0.95 \mathrm{Prob}<\mathrm{t} \quad 0.0003$

Analysis of Variance

Source DF Sum of Squares Mean Square F Ratio Prob $>$ F $\begin{array}{llllll}\text { Heat Treatment } & 1 & 0.01203787 & 0.012038 & 105.9857 & 0.0005\end{array}$ $\begin{array}{lll}\text { Error } & 4 & 0.00045432\end{array}$ 000114

\section{Means for Oneway Anova}

Number Mean Std Error Lower 95\% Upper 95\%

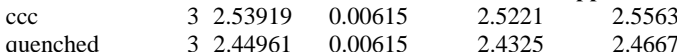

Std Error uses a pooled estimate of error variance 
SRNL-STI-2009-00778, Revision 0

\section{Exhibit B5. Effects of Heat Treatment (HT) on PCT $\log (\mathrm{ppm})$ Response of Matrix 2 Non-Rad Glasses}

Oneway Analysis of $\log [\mathrm{B}$ ppm] By Heat Treatment Glass

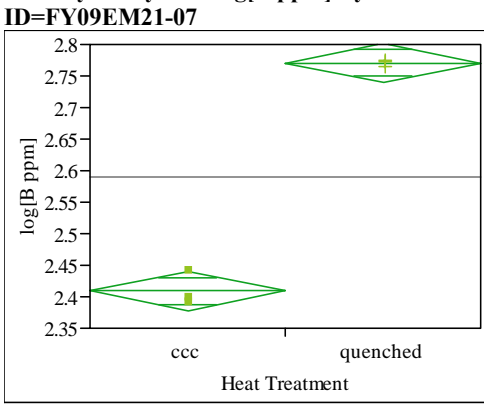

Oneway Anova

Summary of Fit

Rsquare

Adj Rsquare

0.992461

Root Me

0.990577

Mean of Response

2.58983

Observations (or Sum Wgts)

Test

quenched-ccc

Assuming equal variances

Difference $\quad 0.362017$ t Ratio $\quad 22.9476$

Std Err Dif 0.015776 DF

4
$<.0001$

$\begin{array}{lll}\text { Upper CL Dif } 0.405818 \text { Prob }>|t| & <.0001 \\ \text { Lower CL Dif } 0.318216 \text { Prob }>t & <.0001\end{array}$

$\begin{array}{lrr}\text { Lower CL Dif } & 0.318216 \text { Prob }>t & <.0001 \\ \text { Confidence } & 0.95 \text { Prob }<t & 1.0000\end{array}$

Source DF Sum of Squares Mean Square F Ratio Prob $>$ F $\begin{array}{llllll}\text { Heat Treatment } & 1 & 0.19658450 & 0.196584 & 526.5924 & <.0001 \\ \text { Error } & 4 & 0.00149326 & 0.000373 & & \end{array}$ 0.19807776 0.000373

Means for Oneway Anova

Level Number Mean Std Error Lower 95\% Upper 95\%

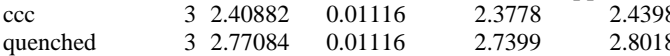

Std Error uses a pooled estimate of error variance
Oneway Analysis of $\log [\mathrm{Li}$ ppm] By Heat Treatment Glass ID=FY09EM21-07

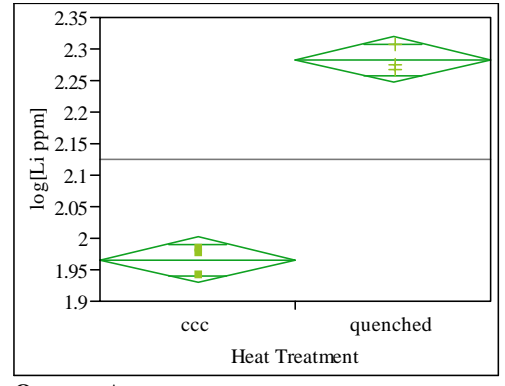

Oneway Anova

Summary of Fit

Rsquare

Adj Rsquare

Root Mean Square E

0.987041

ean of Response

0.983801

t Test

quenched-ccc

Assuming equal variances

Difference $\quad 0.317951$ t Ratio $\quad 17.45471$

Std Err Dif 0.018216 DF

4

Upper CL Dif 0.368526 Prob $>|1|<.0001$

$\begin{array}{lll}\text { Confidence } \quad 0.95 \text { Prob }<\mathrm{t} \quad 1.0000 & \end{array}$

Analysis of Variance

Source DF Sum of Squares Mean Square F Ratio Prob $>$ F

$\begin{array}{lll}\text { Heat Treatment } & 1 & 0.15163889 \\ \text { Error } & 4 & 0.00199088\end{array}$

$\begin{array}{lll}\text { Error } & 4 & 0.00199088 \\ \text { C. Total } & 5 & 0.15362977\end{array}$

0.151639304 .6670

Means for Oneway Anova

Level Number Mean Std Error Lower 95\% Upper 95\%

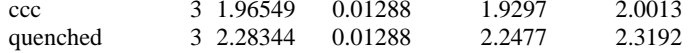

Std Error uses a pooled estimate of error variance
Oneway Analysis of $\log [\mathrm{Na}$ ppm] By Heat Treatment Glass

ID=FY09EM21-07

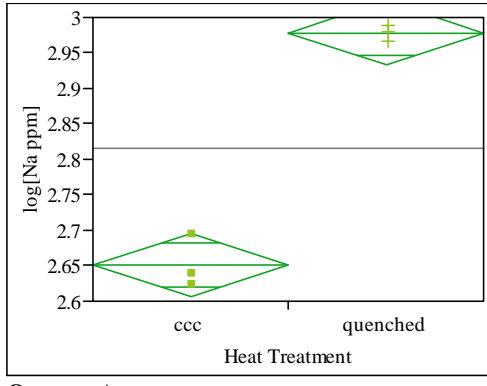

Oneway Anova

Summary of Fit

Rsquare

0.98119

Root Mesh Square Error $\quad 0.976488$

0.027753

Observations (or Sum Wgts) $\quad 6$

Test

quenched-ccc

Assuming equal variance

Difference $\quad 0.327327$ t Ratio $\quad 14.4448$

Std Err Dif $\quad 0.022660$ DF

Upper CL Dif $0.390242 \mathrm{Prob}>|\mathrm{t}| \quad 0.0001$

Lower CL Dif 0.264411 Prob $>$ t $\quad<.0001$

Confidence $\quad 0.95$ Prob $<\mathrm{t} \quad 0.9999$

Analysis of Variance

Source DF Sum of Squares Mean Square F Ratio Prob $>$ F

$\begin{array}{llllll}\text { Heat Treatment } & 1 & 0.16071435 & 0.160714 & 208.6541 & 0.0001 \\ \text { Error } & 4 & 0.00308097 & 0.000770 & & \end{array}$

$\begin{array}{lll}\text { Error } & 4 & 0.00308097\end{array}$ 0.000770

Means for Oneway Anova

Level Number Mean Std Error Lower 95\% Upper 95\%

ccC $\quad 32.65106 \quad 0.01602 \quad 2.6066 \quad 2.6955$

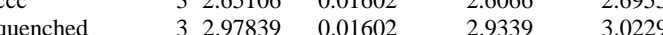

Std Error uses a pooled estimate of error variance 


\section{Exhibit B5. Effects of Heat Treatment (HT) on PCT $\log (\mathrm{ppm})$ Response of Matrix 2 Non-Rad Glasses}

Oneway Analysis of $\log [\mathrm{Si}$ ppm] By Heat Treatment Glass D=FY09EM21-07

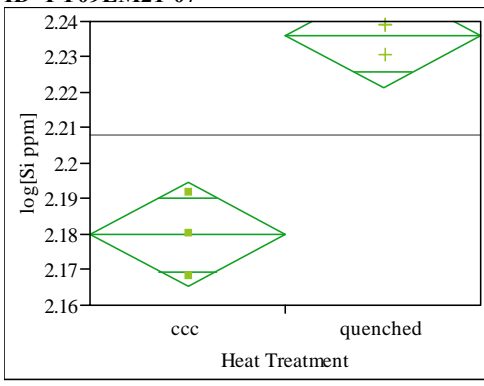

\section{Oneway Anova}

Summary of Fit

Rsquare

Adj Rsquare

0.93433

Root Mean Sc

0.917913

Mean of Response

2.207957

Observations (or Sum Wgts)

Test

quenched-ccc

Assuming equal variances

Difference $\quad 0.056246$ t Ratio $\quad 7.543929$ Std Err Dif 0.007456 DF

Upper CL Dif 0.076947 Prob $>|t| \quad 0.0017$

Lower CL Dif 0.035546 Prob $>t \quad 0.0008$

Confidence $\quad 0.95$ Prob $<\mathrm{t} \quad 0.9992$

Analysis of Variance

Source DF Sum of Squares Mean Square F Ratio Prob $>$ F

$\begin{array}{llllll}\text { Heat Treatment } & 1 & 0.00474546 & 0.004745 & 56.9109 & 0.0017 \\ \text { Error } & 4 & 0.00033354 & 0.000083 & & \end{array}$

Error

0.00033354

Means for Oneway Anova

Level Number Mean Std Error Lower 95\% Upper 95\%

$\begin{array}{llllll}\text { ccc } & 3 & 2.17983 & 0.00527 & 2.1652 & 2.1945 \\ \text { quenched } & 3 & 2.23608 & 0.00527 & 2.2214 & 2.2507\end{array}$

Std Error uses a pooled estimate of error variance
Oneway Analysis of $\log [\mathrm{B}$ ppm] By Heat Treatment Glass

ID=FY09EM21-08

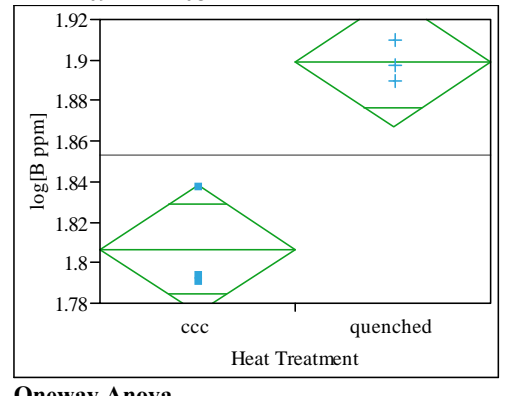

Oneway Anova

Summary of Fit

Rsquare

Adj Rsquare

0.890198

Root Mean Square Error $\quad 0.019847$

Mean of Response

0.019847
1.852934

Observations (or Sum Wgts)

t Test

quenched-ccc

Assuming equal variances

Difference $\quad 0.092281$ t Ratio $\quad 5.694669$

Std Err Dif $\quad 0.016205$ DF

Upper CL Dif 0.137273 Prob $>|t| \quad 0.0047$

$\begin{array}{llll} & \text { Lower CL Dif } 0.04728 \text { Prob }>t & 0.0023\end{array}$

$\begin{array}{lll}\text { Confidence } & 0.95 \text { Prob }<\mathrm{t} \quad 0.9977\end{array}$

Analysis of Variance

Source DF Sum of Squares Mean Square F Ratio Prob $>$ F $\begin{array}{lllllll}\text { Heat Treatment } & 1 & 0.01277371 & 0.012774 & 32.4293 & 0.0047\end{array}$ Error 0.01434928 0.000394

\section{Means for Oneway Anova}

Level Number Mean Std Error Lower 95\% Upper 95\%

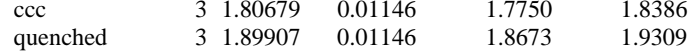

Std Error uses a pooled estimate of error variance
Oneway Analysis of $\log [\mathrm{Li}$ ppm] By Heat Treatment Glass ID=FY09EM21-08

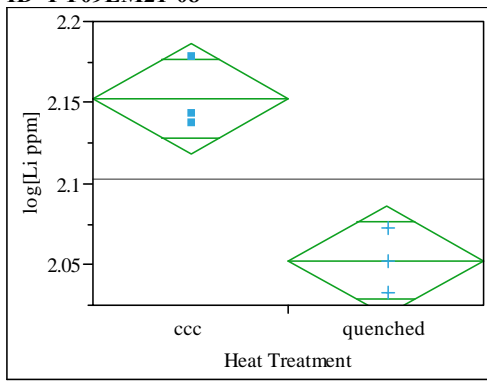

Oneway Anova

Summary of Fit

Adj Rsquere 0.892646

0.865807

Root Men Square Error $\quad 0.021244$

Mean of Response 2.102577

Test

quenched-ccc

Assuming equal variance

Difference $\quad-0.10003$ t Ratio $\quad-5.76713$ Std Err Dif 0.01735 DF

Upper CL Dif -0.05187 Prob $>|t| \quad 0.004$ Lower CL Dif -0.14819 Prob $>t \quad 0.9978$ $\begin{array}{lll}\text { Confidence } & 0.95 \text { Prob }<\mathrm{t} \quad 0.0022\end{array}$

Analysis of Variance

DF Sum of Squares Mean Square F Ratio Prob $>$ F $\begin{array}{llllll}\text { Heat Treatment } & 1 & 0.01500970 & 0.015010 & 33.2598 & 0.0045\end{array}$ $\begin{array}{lll}\text { Error } & 4 & 0.00180515 \\ \text { C. Total } & 5 & 0.01681485\end{array}$

Means for Oneway Anova

Level Number Mean Std Error Lower 95\% Upper 95\% $\begin{array}{llllll}\text { ccC } & 3 & 2.15259 & 0.01226 & 2.1185 & 2.186\end{array}$ 2.0866

Std Error uses a pooled estimate of error variance 
SRNL-STI-2009-00778, Revision 0

\section{Exhibit B5. Effects of Heat Treatment (HT) on PCT $\log (\mathrm{ppm})$ Response of Matrix 2 Non-Rad Glasses}

Oneway Analysis of log[Na ppm] By Heat Treatment Glass ID=FY09EM21-08

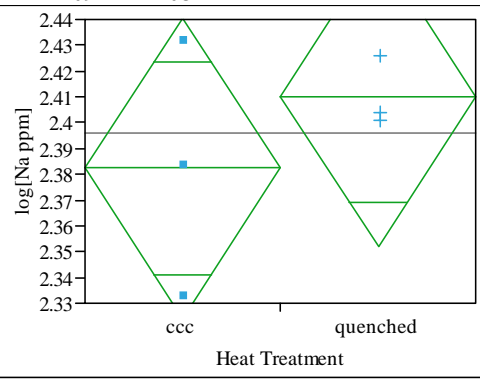

Oneway Anova

Summary of Fit

Adj Rsquare

0.180462

Root Mean Sc

$-0.02442$

Mean of Respons

2.39626

Observations (or Sum Wgts)

6

Test

quenched-ccc

Assuming equal variances

Difference $\quad 0.02782$ t Ratio $\quad 0.9385$

Std Err Dif 0.02965 DF

Upper CL Dif 0.11013 Prob $>|t| 0.401$

Lower CL Dif -0.05449 Prob $>$ t 0.2006

Confidence $\quad 0.95$ Prob $<t \quad 0.7994$

Analysis of Variance

Source DF Sum of Squares Mean Square F Ratio Prob $>$ F

$\begin{array}{llllll}\text { Heat Treatment } & 1 & 0.00116115 & 0.001161 & 0.8808 & 0.4011\end{array}$

0.00527314

0.001318

Means for Oneway Anova

Level Number Mean Std Error Lower 95\% Upper 95\%

$\begin{array}{llllll}\text { cCC } & 3 & 2.38235 & 0.02096 & 2.3241 & 2.4405\end{array}$

$\begin{array}{lllll}3 & 2.41017 & 0.02096 & 2.3520 & 2.4684\end{array}$

Std Error uses a pooled estimate of error variance
Oneway Analysis of $\log [\mathrm{Si}$ ppm] By Heat Treatment Glass

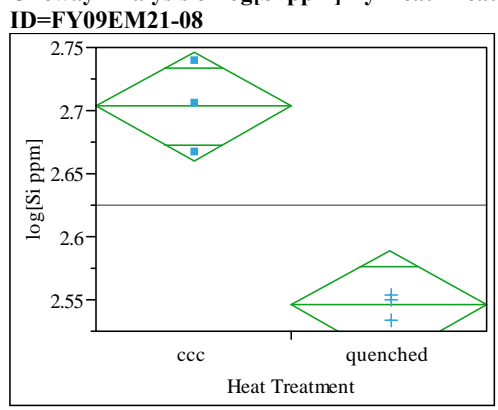

\section{Oneway Anova}

Rsquare

Adj Rsquare

Root Mean of Response

0.908725

bservations (or Sum Wgts) 2.624638

t Test

quenched-ccc

Assuming equal variances

Difference $\quad-0.15718$ t Ratio $\quad-7.1259$

Std Err Dif $\quad 0.02206$ DF

4
0.0021

Lower CL Dif -0.21842 Prob $>t \quad 0.9990$

Confide Dif 0.21842 Prob $>t \quad 0.9090$

Analysis of Variance

Source DF Sum of Squares Mean Square F Ratio Prob $>$ F $\begin{array}{llllll}\text { Heat Treatment } & 1 & 0.03705870 & 0.037059 & 50.7797 & 0.002\end{array}$ Eror $4 \quad 0.00291917$

\section{Means for Oneway Anova}

Level Number Mean Std Error Lower 95\% Upper 95\% $\begin{array}{llllll}\text { ccc } & 3 & 2.70323 & 0.01560 & 2.6599 & 2.7465 \\ \text { quenched } & 3 & 2.54605 & 0.01560 & 2.5027 & 2.5894\end{array}$

Std Error uses a pooled estimate of error variance
Oneway Analysis of $\log [\mathrm{B}$ ppm] By Heat Treatment Glass ID=FY09EM21-09

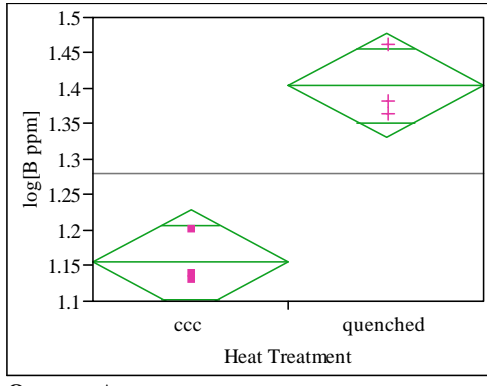

\section{Oneway Anova}

Summary of Fit

Rsquare

Adj Rsquare $\quad 0.917365$

\begin{tabular}{ll}
0.896707 \\
\hline
\end{tabular}

Eror $\quad 0.045699$ 1.27918

\section{Test}

quenched-ccc

Assuming equal variance

Difference $\quad 0.248644$ t Ratio $\quad 6.663774$ Std Err Dif 0.037313 D

Upper CL Dif 0.352240 Prob $>|t| \quad 0.0026$ Lower CL Dif 0.145047 Prob $>t \quad 0.0013$ $\begin{array}{lll}\text { Confidence } & 0.95 \text { Prob }<\mathrm{t} \quad 0.9987\end{array}$

\section{Analysis of Variance}

DF Sum of Squares Mean Square F Ratio Prob $>$ F $\begin{array}{llllll}\text { Heat Treatment } & 1 & 0.09273550 & 0.092735 & 44.4059 & 0.0026\end{array}$ $\begin{array}{lll}\text { Error } & 4 & 0.00835344\end{array}$ 0.002088

\section{Means for Oneway Anova}

Level Number Mean Std Error Lower 95\% Upper 95\% $\begin{array}{llllll}\text { ccc } & 3 & 1.15486 & 0.02638 & 1.0816 & 1.2281 \\ \text { quenched } & 3 & 1.40350 & 0.02638 & 1.3302 & 1.4768\end{array}$

Std Error uses a pooled estimate of error variance 
SRNL-STI-2009-00778, Revision 0

\section{Exhibit B5. Effects of Heat Treatment (HT) on PCT $\log (\mathrm{ppm})$ Response of Matrix 2 Non-Rad Glasses}

Oneway Analysis of $\log [\mathrm{Li}$ ppm] By Heat Treatment Glass

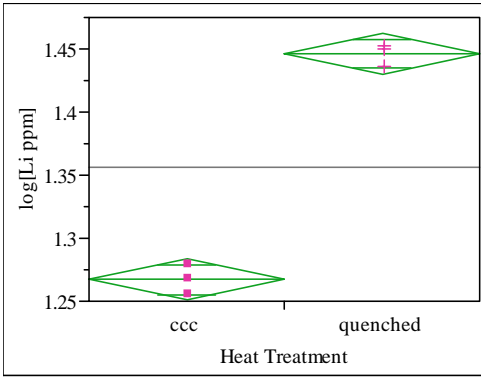

Oneway Anova

Summary of Fit

Adj Rsquare

\begin{tabular}{rr}
0.010195 \\
\hline
\end{tabular}

Mean of Response 1.356663

Observations (or Sum Wgts)

6

t Test

quenched-ccc

Assuming equal variances

Difference $\quad 0.179176$ t Ratio $\quad 21.52444$

Std Err Dif 0.008324 DF

Upper CL Dif 0.202288 Prob $>|t| \quad<.0001$

Lower CL Dif 0.156064 Prob $>$ t $\quad<.000$

Confidence $\quad 0.95$ Prob $<\mathrm{t} \quad 1.0000$

Analysis of Variance

Source DF Sum of Squares Mean Square F Ratio Prob $>$ F

$\begin{array}{llllll}\text { Heat Treatment } & 1 & 0.04815604 & 0.048156 & 463.3015 & <.0001 \\ \text { Error } & 4 & 0.00041576 & 0.000104 & & \end{array}$

Means for Oneway Anova

Level Number Mean Std Error Lower 95\% Upper 95\%

$\begin{array}{llllll}\text { CCC } & 3 & 1.26707 & 0.00589 & 1.2507 & 1.283\end{array}$

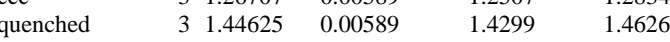

Std Error uses a pooled estimate of error variance
Oneway Analysis of $\log [\mathrm{Na}$ ppm] By Heat Treatment Glass

ID=FY09EM21-09

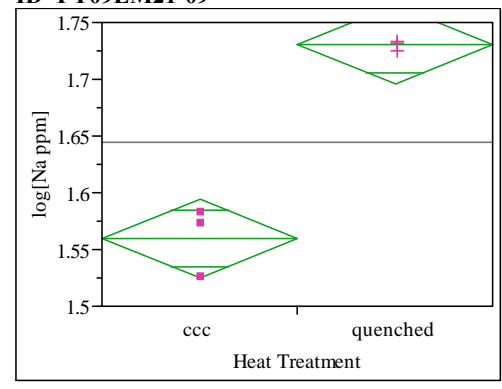

Oneway Anova

Summary of Fit

Adj Rsquare

0.958983

0.948729
0.021654

Mean of Response

Observations (or Sum Wgts)

t Test

quenched-ccc

Assuming equal variances

Difference $\quad 0.170980$ t Ratio $\quad 9.670589$

Std Err Dif $\quad 0.017680$ DF

Upper CL Dif 0.220068 Prob $>|t| \quad 0.0006$

$\begin{array}{lll} & \text { Lower CL Dif } 0.121891 \mathrm{Prob}>\mathrm{t} & 0.0003\end{array}$

Confidence $\quad 0.95$ Prob $<\mathrm{t} \quad 0.9997$

Analysis of Variance

Source DF Sum of Squares Mean Square F Ratio Prob $>$ F

$\begin{array}{llllll}\text { Heat Treatment } & 1 & 0.04385115 & 0.04385193 .5203 & 0.0006\end{array}$

Error

0.00187558

Means for Oneway Anova

Level Number Mean Std Error Lower 95\% Upper 95\%

$\begin{array}{llllll}\text { ccc } & 3 & 1.55962 & 0.01250 & 1.5249 & 1.5943 \\ \text { quenched } & 3 & 1.73060 & 0.01250 & 1.6959 & 1.7653\end{array}$

Std Error uses a pooled estimate of error variance
Oneway Analysis of $\log [\mathrm{Si}$ ppm] By Heat Treatment Glass ID=FY09EM21-09

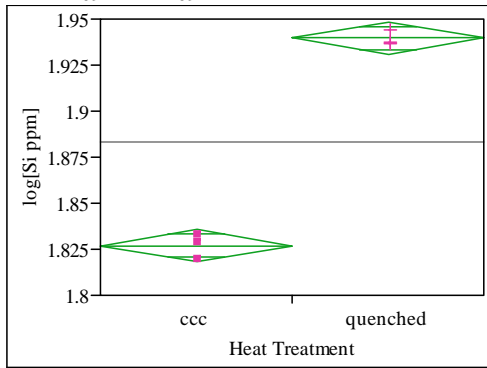

Oneway Anova

Summary of Fit

Rsquare

Root Mean Square Error $\quad 0.005494$

Mean of Response 1.883281

1.883281

t Test

quenched-ccc

Assuming equal variances

Difference $\quad 0.113023$ t Ratio 25.1936

$0.004486 \mathrm{DF}$

Upper CL Dif 0.125478 Prob $>|t|<.0001$

Lower CL Dif 0.100567 Prob $>t \quad<.001$

Analysis of Variance

Source DF Sum of Squares Mean Square F Ratio Prob $>$ F

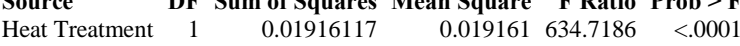

$\begin{array}{llll}\text { Error } & 4 & 0.00012075 & 0.000030\end{array}$

C. Total

0.01928193

Means for Oneway Anova

Level Number Mean Std Error Lower 95\% Upper 95\%

$\begin{array}{llllll}\text { CCC } & 3 & 1.82677 & 0.00317 & 1.8180 & 1.8356\end{array}$

$\begin{array}{lllll}3 & 1.93979 & 0.00317 & 1.9310 & 1.9486\end{array}$

Std Error uses a pooled estimate of error variance 
SRNL-STI-2009-00778, Revision 0

\section{Exhibit B5. Effects of Heat Treatment (HT) on PCT $\log (\mathrm{ppm})$ Response of Matrix 2 Non-Rad Glasses}

Oneway Analysis of $\log [\mathrm{B}$ ppm] By Heat Treatment Glass

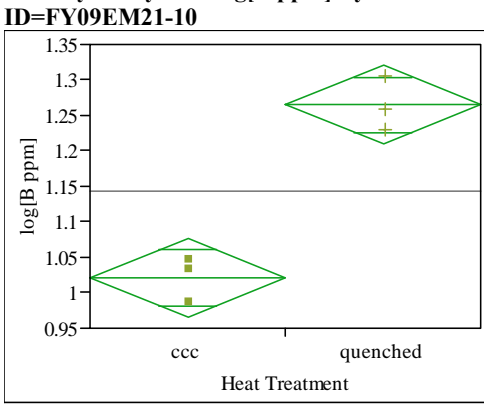

\section{Oneway Anova}

Summary of Fit

Rsquare

Adj Rsquare

0.948479

Root Mean Sq

0.034779

$\begin{array}{lr} & 1.142953 \\ \text { Observations (or Sum Wgts) } & 6\end{array}$

t Test

quenched-ccc

Assuming equal variances

Difference $\quad 0.243683$ t Ratio 8.581241 Std Err Dif 0.028397 DF

Upper CL Dif 0.322526 Prob $>|t| \quad 0.0010$

Lower CL Dif 0.164840 Prob $>t \quad 0.0005$

Confidence $\quad 0.95$ Prob $<\mathrm{t} \quad 0.9995$

Analysis of Variance

Source DF Sum of Squares Mean Square F Ratio Prob $>$ F

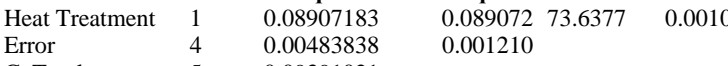

Error

0.00483838

Means for Oneway Anova

Level Number Mean Std Error Lower 95\% Upper 95\%

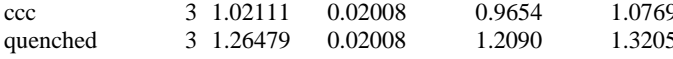

Std Error uses a pooled estimate of error variance
Oneway Analysis of $\log [\mathrm{Li}$ ppm] By Heat Treatment Glass

ID=FY09EM21-10

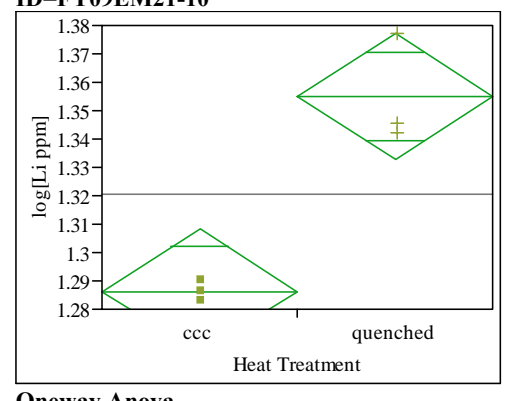

Oneway Anova

Summary of Fit

Rsquare

Adj Rsquare

Root Mean Square

0.902771

0.878464

t Test

quenched-ccc

Assuming equal variances

Difference $\quad 0.068807$ t Ratio $\quad 6.094255$

Std Err Dif 0.011290 DF

Upper CL Dif 0.100154 Prob $>|t| \quad 0.0037$

Lower CL Dif 0.037459 Prob $>t \quad 0.0018$

Confidence $\quad 0.95$ Prob $<\mathrm{t} \quad 0.9982$

Analysis of Variance

Source DF Sum of Squares Mean Square F Ratio Prob $>$ F $\begin{array}{lllllll}\text { Heat Treatment } & 1 & 0.00710153 & 0.007102 & 37.1399 & 0.0037\end{array}$

Eror

0.00076484 0.00019

Means for Oneway Anova

Level Number Mean Std Error Lower 95\% Upper 95\%

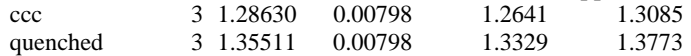

Std Error uses a pooled estimate of error variance
Oneway Analysis of $\log [\mathrm{Na}$ ppm] By Heat Treatment Glass D=FY09EM21-10

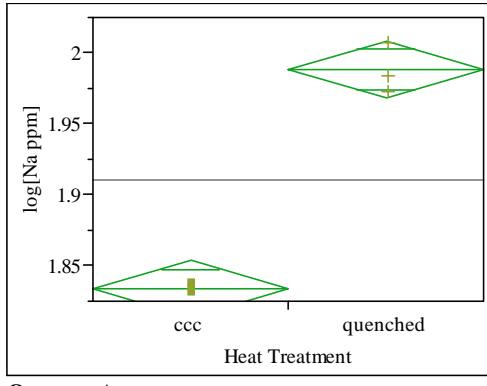

Oneway Anova

Summary of Fi

Rsquare

Adj Rsquare $\quad 0.982779$

0.978473

1.910805

Observations (or Sum Wgts) $\begin{array}{r}1.910805 \\ \hline\end{array}$

t Test

quenched-ccc

Assuming equal variance

Difference $\quad 0.154463$ t Ratio $\quad 15.10854$ Std Err Dif $\quad 0.010224$ DF

Upper CL Dif 0.182849 Prob $>|t| \quad 0.0001$

Lower CL Dif 0.126078 Prob $>t \quad<.000$

$\begin{array}{lll}\text { Confidence } & 0.95 \mathrm{Prob}<\mathrm{t} \quad 0.9999\end{array}$

Analysis of Variance

DF Sum of Squares Mean Square F Ratio Prob $>$ F $\begin{array}{llllll}\text { Heat Treatment } & 1 & 0.03578842 & 0.035788 & 228.2680 & 0.000\end{array}$ $\begin{array}{lll}\text { Error } & 4 & 0.00062713\end{array}$ 0.00015

\section{Means for Oneway Anova}

Level Number Mean Std Error Lower 95\% Upper 95\% $\begin{array}{llllll}\text { ccc } & 3 & 1.83357 & 0.00723 & 1.8135 & 1.8536 \\ \text { quenched } & 3 & 1.98804 & 0.00723 & 1.9680 & 2.0081\end{array}$

Std Error uses a pooled estimate of error variance 
SRNL-STI-2009-00778, Revision 0

\section{Exhibit B5. Effects of Heat Treatment (HT) on PCT $\log (\mathrm{ppm})$ Response of Matrix 2 Non-Rad Glasses}

Oneway Analysis of $\log [\mathrm{Si}$ ppm] By Heat Treatment Glass

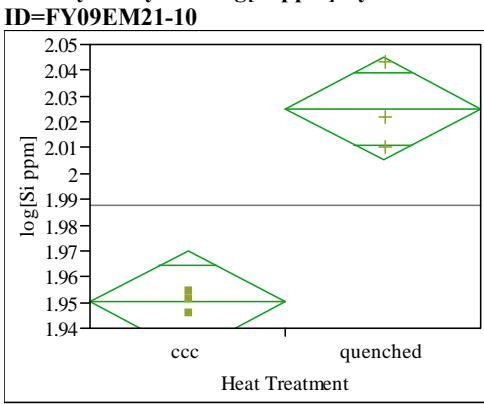

\section{Oneway Anova}

Summary of Fit

Rsquare

Adj Rsquare

0.915207

0.012373

Observations (or

1.987645
6

t Test

quenched-ccc

Assuming equal variances

Difference $\quad 0.074899$ t Ratio $\quad 7.413964$ Std Err Dif 0.010102 DF

Upper CL Dif 0.102948 Prob $>|t| \quad 0.0018$

Lower CL Dif 0.046850 Prob $>t \quad 0.0009$

$\begin{array}{lll} & & \\ \text { Confidence } & 0.95 \text { Prob }<\mathrm{t} \quad 0.9991\end{array}$

Analysis of Variance

Source DF Sum of Squares Mean Square F Ratio Prob $>$ F

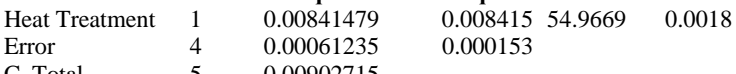

Means for Oneway Anova

Level Number Mean Std Error Lower 95\% Upper 95\% $\begin{array}{llllll}\text { ccc } & 3 & 1.95020 & 0.00714 & 1.9304 & 1.9700 \\ \text { quenched } & 3 & 2.02509 & 0.00714 & 2.0053 & 2.0449\end{array}$

Std Error uses a pooled estimate of error variance
Oneway Analysis of $\log [\mathrm{B}$ ppm] By Heat Treatment Glass ID=FY09EM21-11

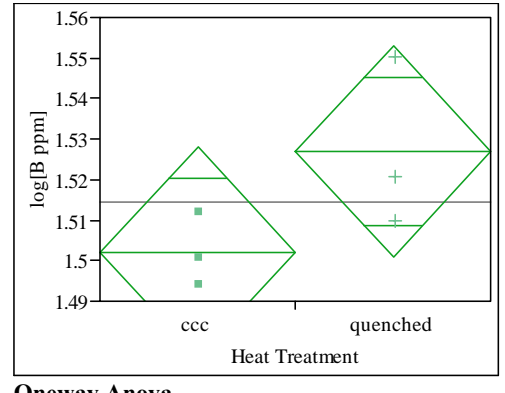

Oneway Anova

Summary of Fit

Adj Rsquare

\subsection{2}

Reot Mean Square Error $\quad 0.016193$

Mean of Response 1.514468

\section{t Test}

quenched-ccc

Assuming equal variances

Difference $\quad 0.02480$ t Ratio $\quad 1.875852$

Std Err Dif $\quad 0.01322$ DF

Upper CL Dif $0.06151 \mathrm{PFb}>|\mathrm{t}|-0.1339$

Lower CL Dif -0.01191 Prob $>t \quad 0.0670$

Confidence $\quad 0.95$ Prob $<\mathrm{t} \quad 0.9330$

Analysis of Variance

Source DF Sum of Squares Mean Square F Ratio Prob $>$ $\begin{array}{llllll}\text { Heat Treatment } & 1 & 0.00092267 & 0.000923 & 3.5188 & 0.1339\end{array}$ Error 0.00104884

\section{Means for Oneway Anova}

Level Number Mean Std Error Lower 95\% Upper 95\%

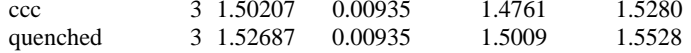

Std Error uses a pooled estimate of error variance
Oneway Analysis of $\log [\mathrm{Li}$ ppm] By Heat Treatment Glass ID=FY09EM21-11

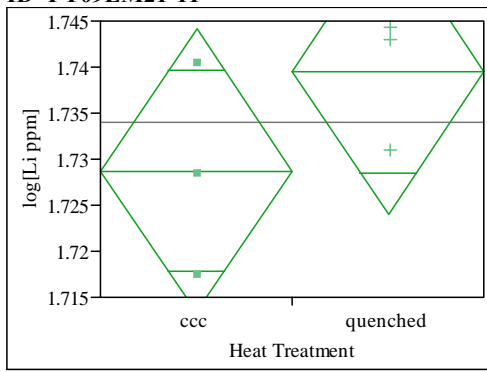

Oneway Anova

Summary of Fit

Rsquare

Root Mean Square Error $\quad 0.009624$

1.734082

6

t Test

quenched-ccc

Assuming equal variances

Difference $\quad 0.01074$ t Ratio 1.366829 Dif $0.00786 \mathrm{DF}$

Upper CL Dif 0.03256 Prob $>|t| \quad 0.2435$

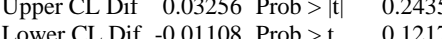

Confidence $\quad 0.95$ Prob $<\mathrm{t} \quad 0.8783$

Analysis of Variance

Source DF Sum of Squares Mean Square F Ratio Prob $>$ F

$\begin{array}{lrrrrr}\text { Heat Treatment } & 1 & 0.00017304 & 0.000173 & 1.8682 & 0.2435\end{array}$ $\begin{array}{llll}\text { Error } & 4 & 0.00037049 & 0.000093\end{array}$

C. Total $\quad 5 \quad 0.00054352$

Means for Oneway Anova

Level Number Mean Std Error Lower 95\% Upper 95\%

$\begin{array}{llllll}\text { CCC } & 3 & 1.72871 & 0.00556 & 1.7133 & 1.7441\end{array}$

$\begin{array}{lllll}3 & 1.73945 & 0.00556 & 1.7240 & 1.7549\end{array}$

Std Error uses a pooled estimate of error variance 
SRNL-STI-2009-00778, Revision 0

\section{Exhibit B5. Effects of Heat Treatment (HT) on PCT $\log (\mathrm{ppm})$ Response of Matrix 2 Non-Rad Glasses}

Oneway Analysis of $\log [\mathbf{N a}$ ppm] By Heat Treatment Glass

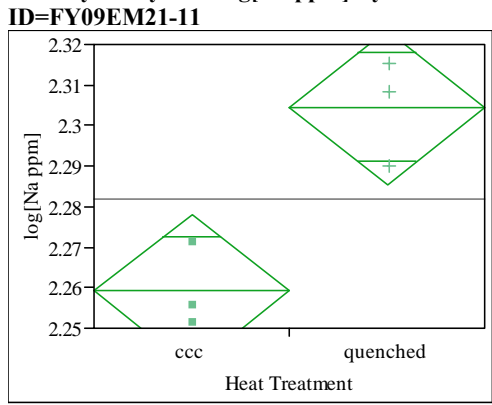

\section{Oneway Anova}

Summary of Fit

Rsquare

0.846451

Root Men Square Error $\quad 0.808063$

Root Mean Square Error $\quad 0.011819$

Observations (or

2.281856
6

t Test

quenched-ccc

Assuming equal variances

Difference $\quad 0.045314$ t Ratio $\quad 4.69577$ Std Err Dif 0.009650 DF

Upper CL DI 0.072106 Prob > |t 0.0093 Lower CL Dif 0.018521 Prob $>$ t 0.0047 Confidence $\quad 0.95$ Prob $<t \quad 0.9953$

Analysis of Variance

Source DF Sum of Squares Mean Square F Ratio Prob $>$ F $\begin{array}{llllll}\text { Heat Treatment } & 1 & 0.00308003 & 0.003080 & 22.0503 & 0.0093\end{array}$ 0.00363876

Means for Oneway Anova

Level Number Mean Std Error Lower 95\% Upper 95\%

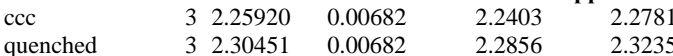

Std Error uses a pooled estimate of error variance
Oneway Analysis of $\log [\mathrm{Si}$ ppm] By Heat Treatment Glas ID=FY09EM21-11

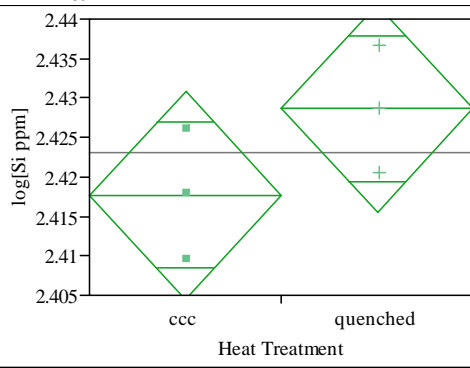

Oneway Anova

Summary of Fit

Rsquare

Adj Rsquare

Root Mean Square

0.249952
0.008197

Observations (or Sum Wgts)

23169

t Test

quenched-ccc

Assuming equal variances

Difference $\quad 0.01093$ t Ratio 1.632861

$\begin{array}{lll}0.00669 \mathrm{DF} & 1.632861 \\ & & \end{array}$

Lower CL Dif -0.00765 Prob $>t \quad 0.1778$

Confidence $\quad 0.95$ Prob $<\mathrm{t} \quad 0.9111$

Analysis of Variance

Source DF Sum of Squares Mean Square F Ratio Prob $>$ F Error $\quad 4-0.00026878$ 0.00026878
0.00044794

0.000067

Means for Oneway Anova

Level Number Mean Std Error Lower 95\% Upper 95\%

$\begin{array}{llllll} & 3 & 2.41770 & 0.00473 & 2.4046 & 2.4308\end{array}$

Std Error uses a pooled estimate of error variance
Oneway Analysis of $\log [\mathrm{B}$ ppm] By Heat Treatment Glass

ID=FY09EM21-12

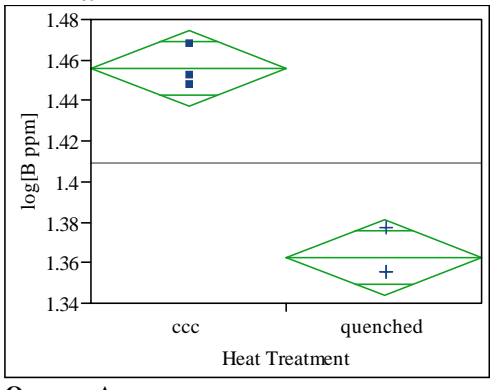

Oneway Anova

Summary of Fit

Rsquare

0.960196

0.950246

Ret Meris Square Error 0.011589

$\begin{array}{lr} & 1.409138 \\ \text { Observations (or Sum Wgts) } & 6\end{array}$

\section{Test}

quenched-ccc

Assuming equal variance

Difference $\quad-0.09295$ t Ratio $\quad-9.82312$ Std Err Dif 0.00946 DF

Upper CL Dif -0.06668 Prob $>|t| \quad 0.0006$

Lower CL Dif -0.11922 Prob $>t \quad 0.9997$

Confidence $\quad 0.95$ Prob $<\mathrm{t} \quad 0.0003$

Analysis of Variance

Source DF Sum of Squares Mean Square F Ratio Prob $>$ F $\begin{array}{llllll}\text { Heat Treatment } & 1 & 0.01296021 & 0.012960 & 96.4936 & 0.0006\end{array}$ $\begin{array}{lll}\text { Error } & 4 & 0.00053725 \\ \text { C. Total } & 5 & 0.01349745\end{array}$

\section{Means for Oneway Anova}

Level Number Mean Std Error Lower 95\% Upper 95\%

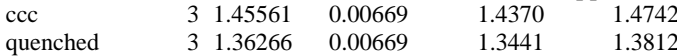

Std Error uses a pooled estimate of error variance 
SRNL-STI-2009-00778, Revision 0

\section{Exhibit B5. Effects of Heat Treatment (HT) on PCT $\log (\mathrm{ppm})$ Response of Matrix 2 Non-Rad Glasses}

Oneway Analysis of $\log [\mathrm{Li}$ ppm] By Heat Treatment Glass ID=FY09EM21-12

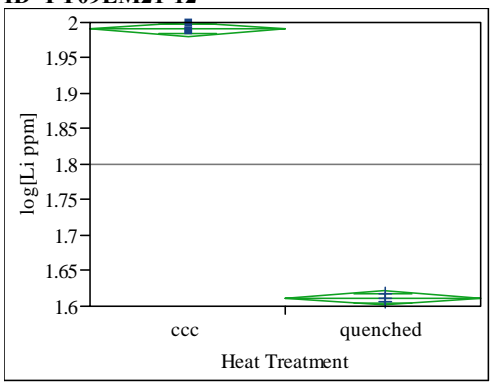

Oneway Anova

Summary of Fit

Adj Rsquare

0.999334

0.999168

Root Mean Square Error $\quad 0.005988$

Observations (or

1.801027

t Test

quenched-ccc

Assuming equal variances

Difference $\quad-0.37889$ t Ratio $\quad-77.4907$

$\begin{array}{lrr}\text { Std Err Dif } & 0.00489 \text { DF } & 4 \\ \text { Upper CL Dif } & -0.36531 \text { Prob }>|t| & <.0001\end{array}$

$\begin{array}{lll}\text { Upper CL Dif }-0.36531 & \text { Prob }>|t| & <.0001 \\ \text { Lower CL Dif }-0.39246 \text { Prob }>t & 1.0000\end{array}$

$\begin{array}{lrr}\text { Lower CL Dif } & -0.39246 \text { Prob }>t & 1.0000 \\ \text { Confidence } & 0.95 \text { Prob }<\mathrm{t} & <.0001\end{array}$

Analysis of Variance

Source DF Sum of Squares Mean Square F Ratio Prob $>$ F

$\begin{array}{llllll}\text { Heat Treatment } & 1 & 0.21533152 & 0.215332 & 6004.807 & <.0001 \\ \text { Error } & 4 & 0.00014344 & 0.000036 & & \end{array}$

Means for Oneway Anova

Level Number Mean Std Error Lower 95\% Upper 95\%

$\begin{array}{lllllr}\text { ccc } & 3 & 1.99047 & 0.00346 & 1.9809 & 2.000\end{array}$

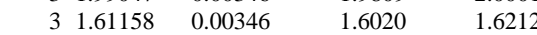

Std Error uses a pooled estimate of error variance
Oneway Analysis of log[Na ppm] By Heat Treatment Glass

ID=FY09EM21-12

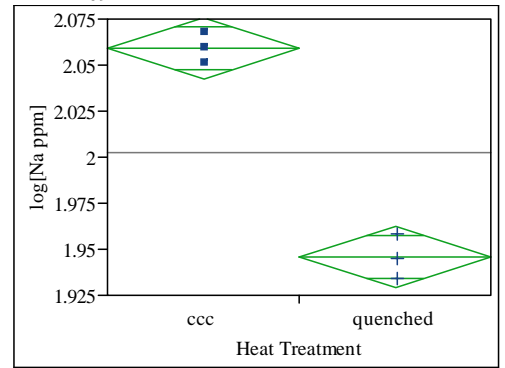

Oneway Anova

Summary of Fi

Rsquare

Adj Rsquare

Root Mean Square

0.972732
0.010348
2.002602

2.002602

t Test

quenched-ccc

Assuming equal variances

Difference $\quad-0.11315$ t Ratio $\quad-13.3927$

Upper CL Dif -0.08970 Prob $>1 t \quad 0.0002$

Lower CL Dif -0.13661 Prob $>t \quad 0.9999$

Confidence $\quad 0.95$ Prob $<\mathrm{t}<0001$

Analysis of Variance
Source DF Sum of Squares Mean Square $\quad$ F Ratio Prob $>$ F $\begin{array}{lrrrrr}\text { Heat Treatment } & 1 & 0.01920602 & 0.019206 & 179.3652 & 0.0002\end{array}$

C. Tota 0.01963433

0.000107

Means for Oneway Anova

Level Number Mean Std Error Lower 95\% Upper 95\%

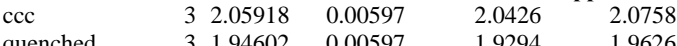

Std Error uses a pooled estimate of error variance
Oneway Analysis of $\log [\mathrm{Si}$ ppm] By Heat Treatment Glass ID=FY09EM21-12

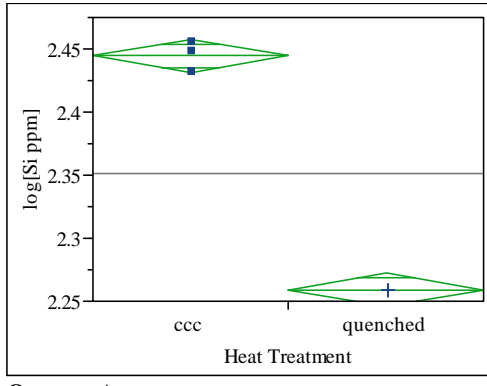

Oneway Anova

Summary of Fit

0.994458

Root Men Square Error $\quad 0.993073$

Roor Mean Square Error 0.008465

Observations (or Sum Wgts) $\begin{array}{r}2.351874 \\ \hline\end{array}$

Test

quenched-ccc

Assuming equal variance

Difference $\quad-0.18518$ t Ratio $\quad-26.792$

Std Err Dif 0.00691 DF -26.792

Upper CL Dif -0.16599 Prob $>\mid \pi<.0001$

Lower CL Dif -0.20437 Prob $>t \quad 1.0000$

Confidence $\quad 0.95$ Prob $<t \quad<0001$

Analysis of Variance

Source DF Sum of Squares Mean Square F Ratio Prob $>$ F $\begin{array}{llllll}\text { Heat Treatment } & 1 & 0.05143762 & 0.051438 & 717.8109 & <.0001\end{array}$ $\begin{array}{lll}\text { Error } & 4 & 0.00028664\end{array}$

\section{Means for Oneway Anova}

Level Number Mean Std Error Lower 95\% Upper 95\%

$\begin{array}{lrrrrr}\text { ccc } & 3 & 2.44446 & 0.00489 & 2.4309 & 2.4580\end{array}$

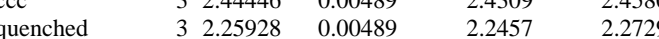

Std Error uses a pooled estimate of error variance 
SRNL-STI-2009-00778, Revision 0

\section{Exhibit B5. Effects of Heat Treatment (HT) on PCT $\log (\mathrm{ppm})$ Response of Matrix 2 Non-Rad Glasses}

Oneway Analysis of $\log [\mathrm{B}$ ppm] By Heat Treatment Glass

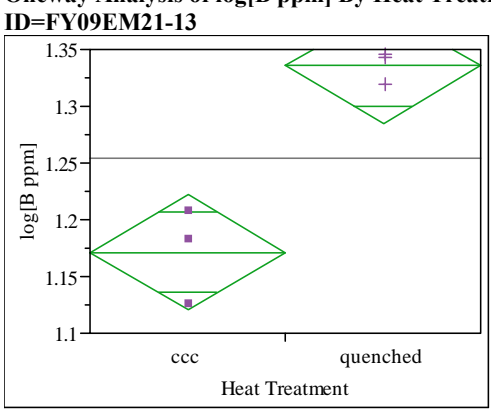

Oneway Anova

Summary of Fit

Rsquare

Adj Rsquare

0.910338

Root Mean Sc

0.031571

Observations (or Su

1.253499
6

t Test

quenched-ccc

Assuming equal variances

Difference $\quad 0.164275$ t Ratio 6.372751

Std Err Dif 0.025778 DF

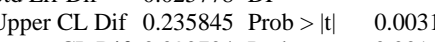

Lower CL Dif 0.092704 Prob $>$ t $\quad 0.001$

$\begin{array}{lll}\text { Confidence } & 0.95 \mathrm{Prob}<\mathrm{t} \quad 0.9984\end{array}$

Analysis of Variance

Source DF Sum of Squares Mean Square F Ratio Prob $>$ F

$\begin{array}{llllll}\text { Heat Treatment } & 1 & 0.04047930 & 0.040479 & 40.6120 & 0.0031\end{array}$

Error

0.00398693

0.000997

Means for Oneway Anova

Level Number Mean Std Error Lower 95\% Upper 95\% $\begin{array}{llllll}\text { ccc } & 3 & 1.17136 & 0.01823 & 1.1208 & 1.2220 \\ \text { quenched } & 3 & 1.33564 & 0.01823 & 1.2850 & 1.3862\end{array}$

Std Error uses a pooled estimate of error variance
Oneway Analysis of $\log [\mathrm{Li}$ ppm] By Heat Treatment Glass

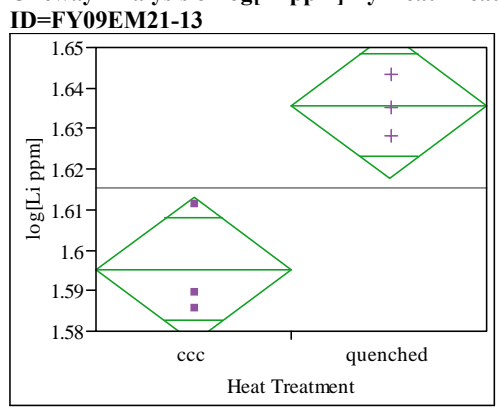

\section{Oneway Anova}

Rsquare

Adj Rsquare

Root Mean of Response

0.832139

0.790174

t Test

quenched-ccc

Assuming equal variances

Difference $\quad 0.040437$ t Ratio $\quad 4.453006$

Std Err Dif 0.009081 DF

4
0.0112

Lower CL Dif 0.015224 Prob $>\mathrm{t} \quad 0.0056$

$\begin{array}{lrr}\text { Confidence } & 0.95 \text { Prob }<\mathrm{t} & 0.9944\end{array}$

Analysis of Variance

Source DF Sum of Squares Mean Square F Ratio Prob $>$ F $\begin{array}{llllll}\text { Heat Treatment } & 1 & 0.00245268 & 0.002453 & 19.8293 & 0.0112\end{array}$ Eror 0.00049476

\section{Means for Oneway Anova}

Level Number Mean Std Error Lower 95\% Upper 95\%

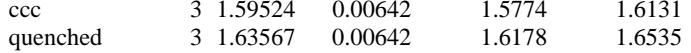

Std Error uses a pooled estimate of error variance
Oneway Analysis of $\log [\mathrm{Na}$ ppm] By Heat Treatment Glass ID=FY09EM21-13

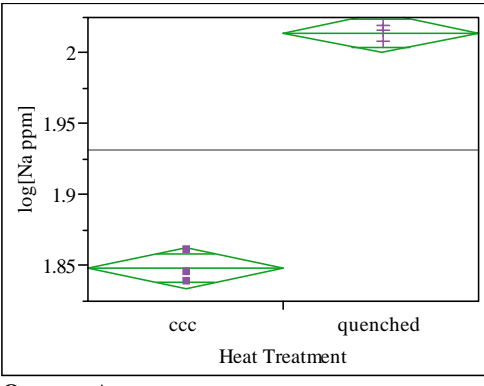

Oneway Anova

Summary of Fit

Rsquare

.992557

Adj Rsquare

0.990696

Root Mean Square Error $\quad 0.008809$

Mean of Respons

1.931164

Observations (or Sum Wgts)

Test

quenched-ccc

Assuming equal variances

Difference $\quad 0.166120$ t Ratio $\quad 23.09538$

Std Err Dif $\quad 0.007193$ DF

Upper CL Dif 0.186090 Prob $>|t|<.000$

Lower CL Dif 0.146150 Prob $>\mathrm{t} \quad<.0001$

Confidence $\quad 0.95$ Prob $<\mathrm{t} \quad 1.0000$

Analysis of Variance

DF Sum of Squares Mean Square F Ratio Prob $>$ F $\begin{array}{llllll}\text { Heat Treatment } & 1 & 0.04139382 & 0.041394 & 533.3968 & <.0001\end{array}$ $\begin{array}{lll}\text { Error } & 4 & 0.00031042\end{array}$

\section{Means for Oneway Anova}

Level Number Mean Std Error Lower 95\% Upper 95\%

$\begin{array}{lrrrrr}\text { ccc } & 3 & 1.84810 & 0.00509 & 1.8340 & 1.862\end{array}$

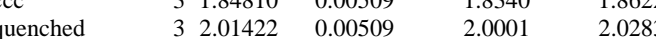

Std Error uses a pooled estimate of error variance 
SRNL-STI-2009-00778, Revision 0

Exhibit B5. Effects of Heat Treatment (HT) on PCT $\log (\mathrm{ppm})$ Response of Matrix 2 Non-Rad Glasses

Oneway Analysis of $\log [\mathrm{Si}$ ppm] By Heat Treatment Glass

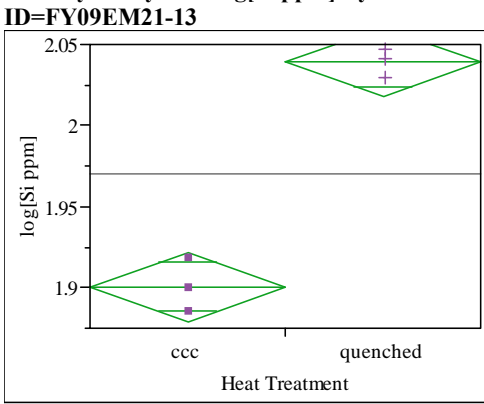

\section{Oneway Anova}

Heat Treatment

Summary of Fit

Rsquare

Adj Rsquare

0.97653

Root Mean Squ

0.970662

Mean of Respons

1.969808

Observations (or Sum Wgts)

6

Test

quenched-ccc

Assuming equal variances

Difference $\quad 0.138670$ t Ratio $\quad 12.90078$ Std Err Dif 0.010749 DF

Upper CL Dif 0.168513 Prob $>|t| \quad 0.0002$

Lower CL Dif 0.108826 Prob $>t \quad 0.000$

$\begin{array}{lll}\text { Confidence } & 0.95 \text { Prob }<\mathrm{t} \quad 0.9999\end{array}$

Analysis of Variance

Source DF Sum of Squares Mean Square F Ratio Prob > F

$\begin{array}{llllll}\text { Heat Treatment } & 1 & 0.02884391 & 0.028844 & 166.4302 & 0.0002 \\ \text { Error } & 4 & 0.00069324 & 0.000173 & & \end{array}$

Means for Oneway Anova

Level Number Mean Std Error Lower 95\% Upper 95\%

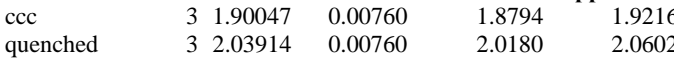

Std Error uses a pooled estimate of error variance
Oneway Analysis of $\log [\mathrm{B}$ ppm] By Heat Treatment Glass ID=FY09EM21-14

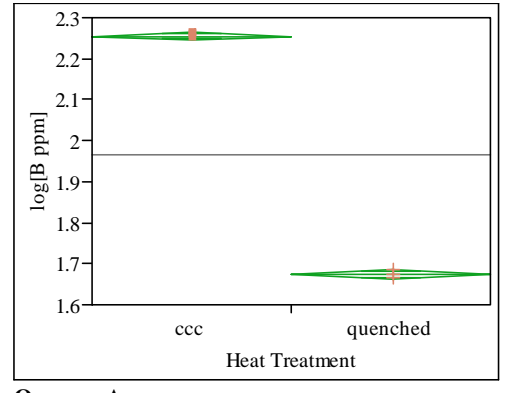

Oneway Anova

Rsquare

Adj Rsquare

Root Mean Square Error

Mean of Response

0.999639

0.999548

Observations (or Sum Wgts)

6

t Test

quenched-ccc

Assuming equal variances

Difference $\quad-0.58066$ t Ratio $\quad-105.215$

Std Err Dif 0.00552 DF $>$ -

Upper CL Dif $-0.56534 \mathrm{Prob}>\mid \mathrm{|l}<.0001$

Lower CL Dif -0.59598 Prob $>t \quad 1.0000$

$\begin{array}{lll}\text { Confidence } & 0.95 \text { Prob }<\mathrm{t} \quad<.0001\end{array}$

Analysis of Variance

$\begin{array}{lrrrrr}\text { Source } & \text { DF } & \text { Sum of Squares } & \text { Mean Square } & \text { F Ratio } & \text { Prob }>\text { F } \\ \text { Heat Treatment } & 1 & 0.50575063 & 0.505751 & 11070.17 & <.0001\end{array}$

Eror 0.50575063

$\begin{array}{lll}\text { C. Total } & 4 & 0.00018274 \\ & 5 & 0.5059338\end{array}$

0.00004

Means for Oneway Anova

Level Number Mean Std Error Lower 95\% Upper 95\%

$\begin{array}{llllll}\text { CCC } & 3 & 2.25527 & 0.00390 & 2.2444 & 2.2661\end{array}$

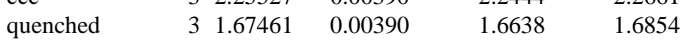

Std Error uses a pooled estimate of error variance
Oneway Analysis of $\log [\mathrm{Li}$ ppm] By Heat Treatment Glass ID=FY09EM21-14

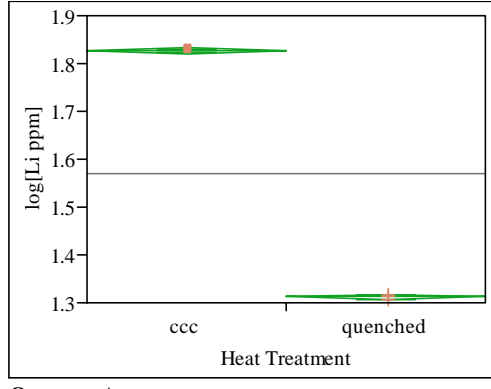

Oneway Anova

Adj Rsquare $\quad 0.999868$

Root Mean Square Error $\quad 0.003629$

Mean of Response 1.569452

Observations (or Sum Wgts) 6

t Test

quenched-ccc

Assuming equal variances

Difference -0.51540 t Ratio -173.95

Std Err Dif 0.00296 DF $>0.173 .955$

Upper CL Dif -0.50717 Prob $>|t|<.0001$

Lower CL Dif -0.52362 Prob $>t \quad 1.0000$

$\begin{array}{lll}\text { Confidence } & 0.95 \text { Prob }<\mathrm{t} \quad<.000\end{array}$

Analysis of Variance

Source DF Sum of Squares Mean Square F Ratio Prob $>$ F

$\begin{array}{lllllll}\text { Heat Treatment } & 1 & 0.39845395 & 0.398454 & 30260.21 & <.0001\end{array}$

$\begin{array}{lll}\text { Error } & 4 & 0.00005267\end{array}$ 0.000013

Means for Oneway Anova

Level Number Mean Std Error Lower 95\% Upper 95\%

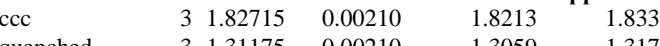

$\begin{array}{llllll}\text { quenched } & 3 & 1.31175 & 0.00210 & 1.3059 & 1.3176\end{array}$

Std Error uses a pooled estimate of error variance 
SRNL-STI-2009-00778, Revision 0

\section{Exhibit B5. Effects of Heat Treatment (HT) on PCT $\log (\mathrm{ppm})$ Response of Matrix 2 Non-Rad Glasses}

Oneway Analysis of log[Na ppm] By Heat Treatment Glass ID=FY09EM21-14

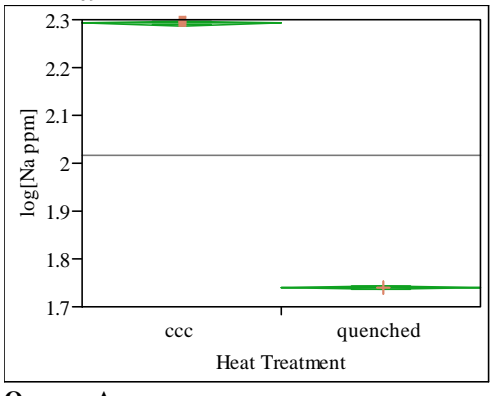

Oneway Anova

Summary of Fit

Rsquare

Adj Rsquare $\quad 0.999919$

\begin{tabular}{ll}
0.003053 \\
\hline
\end{tabular}

Mean of Response

2.016215

Observations (or Sum Wgts)

t Test

quenched-ccc

Assuming equal variances

Difference $\quad-0.55256$ t Ratio $\quad-221.671$

Std Err Dif 0.00249 DF $>0.0001$

Upper CL Dif -0.54564 Prob $>|t|<.0001$

Lower CL Dif -0.55949 Prob $>t \quad 1.0000$

$\begin{array}{lll}\text { Confidence } & 0.95 \text { Prob }<\mathrm{t} \quad<.0001\end{array}$

Analysis of Variance

$\begin{array}{lrrrrr}\text { Source } & \text { DF } & \text { Sum of Squares } & \text { Mean Square } & \text { F Ratio } & \text { Prob }>\text { F } \\ \text { Heat Treatment } & 1 & 0.45799207 & 0.457992 & 49138.12 & <.0001\end{array}$

0.00003728

0.45802935

9.321e-6

Means for Oneway Anova

Level Number Mean Std Error Lower 95\% Upper 95\%

$\begin{array}{lllllr}\text { CCC } & 3 & 2.29250 & 0.00176 & 2.2876 & 2.2974\end{array}$

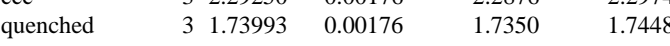

Std Error uses a pooled estimate of error varianc
Oneway Analysis of $\log [\mathrm{Si}$ ppm] By Heat Treatment Glass ID=FY09EM21-14

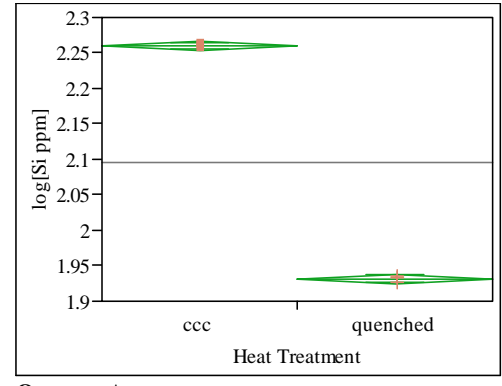

Oneway Anova

Summary of Fit

Rsquare

Adj Rsquare

Root Mean Square Er

0.999552

Mean of Respons

0.999439

Observations (or Sum Wgts)

t Test

quenched-ccc

Assuming equal variances

Difference $\quad-0.32731$ t Ratio $\quad-94.427$

Std Err Dif 0.00347 DF

Upper CL Dif -0.31769 Prob $>\mid 0<<<000$

Lower CL Dif -0.33694 Prob $>t \quad 1.0000$

Confidence $\quad 0.95$ Prob $<\mathrm{t} \quad<.000$

Analysis of Variance

Source DF Sum of Squares Mean Square F Ratio Prob $>$ F

$\begin{array}{lll}\text { Heat Treatment } & 1 & 0.16070011 \\ \text { Error } & 4 & 0.00007209\end{array}$

Error

0.00007209

0.160700

0.000018

Means for Oneway Anova

Level Number Mean Std Error Lower 95\% Upper 95\%

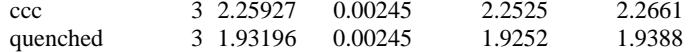

Std Error uses a pooled estimate of error variance
Oneway Analysis of $\log [\mathrm{B}$ ppm] By Heat Treatment Glass

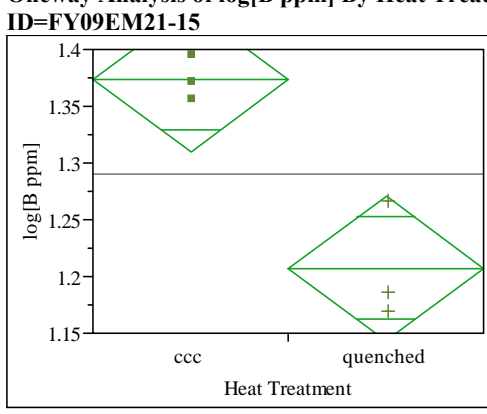

Oneway Anova

Summary of Fit

Rsquare

0.86799

Root Men Square Error $\quad 0.834988$

Ret Re 0.039794 1.290524

Test

quenched-ccc

Assuming equal variances

Difference $\quad-0.16663$ t Ratio $\quad-5.12843$ Std Err Dif 0.03249 DF

Upper CL Dif -0.07642 Prob $>|t| \quad 0.0068$

Lower CL Dif -0.25684 Prob $>t \quad 0.996$

$\begin{array}{lll}\text { Confidence } & 0.95 \text { Prob }<\mathrm{t} \quad 0.0034\end{array}$

Analysis of Variance

Source DF Sum of Squares Mean Square F Ratio Prob $>$ F $\begin{array}{llllll}\text { Heat Treatment } & 1 & 0.04164837 & 0.041648 & 26.3008 & 0.0068\end{array}$ $\begin{array}{lll}\text { Error } & 4 & 0.00633416 \\ \text { C. Total } & 5 & 0.04798254\end{array}$

\section{Means for Oneway Anova}

Level Number Mean Std Error Lower 95\% Upper 95\%

$\begin{array}{llllll}\text { ccc } & 3 & 1.37384 & 0.02297 & 1.3101 & 1.4376 \\ \text { quenched } & 3 & 1.20721 & 0.02297 & 1.1434 & 1.2710\end{array}$

Std Error uses a pooled estimate of error variance 
SRNL-STI-2009-00778, Revision 0

\section{Exhibit B5. Effects of Heat Treatment (HT) on PCT $\log (\mathrm{ppm})$ Response of Matrix 2 Non-Rad Glasses}

Oneway Analysis of $\log [\mathrm{Li}$ ppm] By Heat Treatment Glass

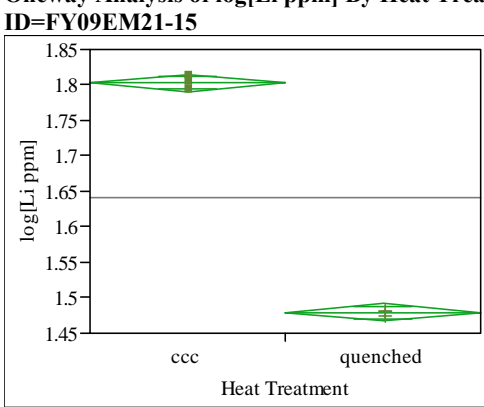

\section{Oneway Anova}

Summary of Fit

Rsquare

Adj Rsquare

0.998437

0.998046

Reot Mean squ

Observations (orse 1.641112

t Test

quenched-ccc

Assuming equal variances

Difference $\quad-0.32318$ t Ratio $\quad-50.5424$ Std Err Dif 0.00639 DF

Upper CL Dif -0.30543 Prob $>|t| \quad<.0001$

Lower CL Dif -0.34093 Prob $>t \quad 1.0000$

$\begin{array}{lll}\text { Confidence } & 0.95 \text { Prob }<t \quad<.0001\end{array}$

Analysis of Variance

Source DF Sum of Squares Mean Square F Ratio Prob $>$ F

$\begin{array}{llllll}\text { Heat Treatment } & 1 & 0.15666691 & 0.156667 & 2554.536 & <.0001 \\ \text { Error } & 4 & 0.00024532 & 0.000061 & & \end{array}$

0.15691222

Means for Oneway Anova

Level Number Mean Std Error Lower 95\% Upper 95\%

$\begin{array}{llllll}\text { ccc } & 3 & 1.80270 & 0.00452 & 1.7901 & 1.8153 \\ \text { quenched } & 3 & 1.47952 & 0.00452 & 1.4670 & 1.4921\end{array}$

Std Error uses a pooled estimate of error variance
Oneway Analysis of $\log [\mathrm{Na}$ ppm] By Heat Treatment Glass

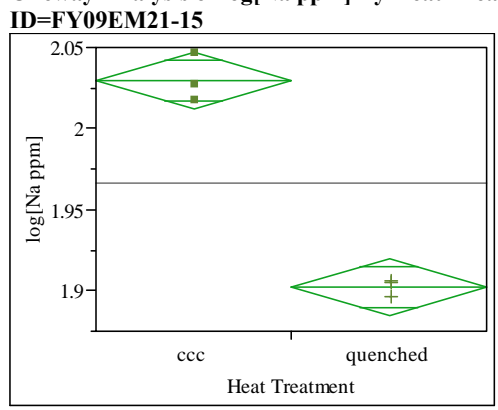

Oneway Anova

Summary of Fit

Rsquare

$\begin{array}{ll}\text { Adj Rsquare } & 0.975619 \\ \text { Root Mean Square Error } & 0.011006\end{array}$

Mean of Repuare Error 0.011006

Observations (or Sum Wgts) 6

t Test

quenched-ccc

Assuming equal variances

Difference $\quad-0.12743$ t Ratio $\quad-14.1802$

Std Err Dif $\quad 0.00899$ DF

Lower CL Dif -0.15237 Prob $>t \quad 0.9999$

Confidence $\quad 0.95$ Prob $<\mathrm{t} \quad<.000$

Analysis of Variance

Source DF Sum of Squares Mean Square F Ratio Prob $>$ F Heat Treatment $1 \quad 0.02435580$

Error 0.00048450

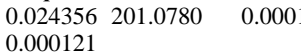

Means for Oneway Anova

Level Number Mean Std Error Lower 95\% Upper 95\%

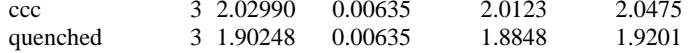

Std Error uses a pooled estimate of error variance
Oneway Analysis of $\log [\mathrm{Si}$ ppm] By Heat Treatment Glass ID=FY09EM21-15

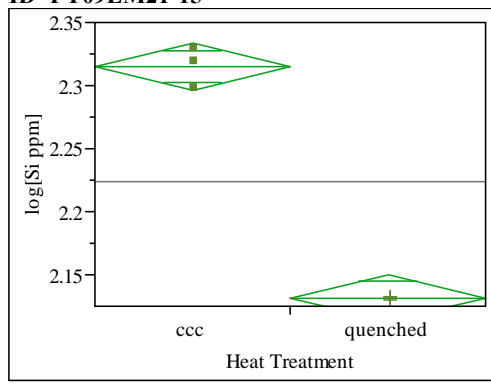

Oneway Anova

Summary of Fit

0.989728

0.98716

Root Mean Square Error $\quad 0.011447$

Mean of Response

2.223335

t Test

quenched-ccc

Assuming equal variance

Difference $\quad-0.18349$ t Ratio $\quad-19.6317$ Std Err Dif 0.00935 DF

Upper CL Dif -0.15754 Prob $>|t| \quad<.000$

Lower CL Dif -0.20944 Prob $>t \quad 1.0000$

$\begin{array}{lll}\text { Confidence } & 0.95 \mathrm{Prob}<\mathrm{t} & <.0001\end{array}$

Analysis of Variance

Source DF Sum of Squares Mean Square F Ratio Prob $>$ F $\begin{array}{llllll}\text { Heat Treatment } & 1 & 0.05050232 & 0.050502 & 385.4023 & <.0001\end{array}$ $\begin{array}{lll}\text { Error } & 4 & 0.00052415 \\ \text { C. } & 5 & 0.05102647\end{array}$ 0.000131

\section{Means for Oneway Anova}

Level Number Mean Std Error Lower 95\% Upper 95\%

$\begin{array}{llllll}\text { ccc } & 3 & 2.31508 & 0.00661 & 2.2967 & 2.3334 \\ \text { quenched } & 3 & 2.13159 & 0.00661 & 2.1132 & 2.1499\end{array}$

Std Error uses a pooled estimate of error variance 
SRNL-STI-2009-00778, Revision 0

\section{Exhibit B5. Effects of Heat Treatment (HT) on PCT $\log (\mathrm{ppm})$ Response of Matrix 2 Non-Rad Glasses}

Oneway Analysis of $\log [\mathrm{B}$ ppm] By Heat Treatment Glass

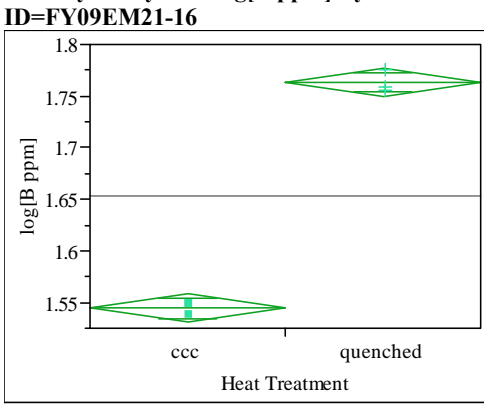

\section{Oneway Anova}

Heat Treatment

$\begin{array}{lr} & 0.99576 \\ \text { Adj Rsquare } & 0.9947\end{array}$

Root Mean Square Error $\quad 0.008736$

Mean of Response 1.654042

Observations (or Sum Wgts)

t Test

quenched-ccc

Assuming equal variances

Difference $\quad 0.218609$ t Ratio 30.64908 Std Err Dif 0.007133 DF

Upper CL Dif 0.238412 Prob $>|t| \quad<.0001$

Confidence $\quad 0.95$ Prob $<\mathrm{t} \quad 1.0000$
Summary of Fit

Lower CL Dif 0.198805 Prob $>t \quad<.000$

Oneway Analysis of $\log [\mathrm{Li}$ ppm] By Heat Treatment Glass

ID=FY09EM21-16

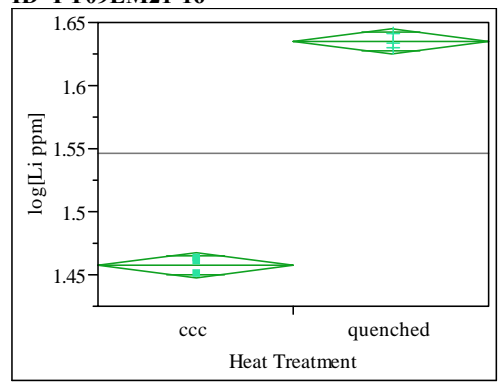

Oneway Anova

Summary of Fit

Rsquare

Adj Rsquare

$0.9+20$

0.995726

0.006377

Mean of Response

1.54624

t Test

quenched-ccc

Assuming equal variances

Difference $\quad 0.177778$ t Ratio $\quad 34.14349$

Std Err Dif $\quad 0.005207$ DF

4
$<.0001$

0.192234 Prob $>|k|<.0001$

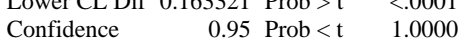

Analysis of Variance

Analysis of Variance

Source DF Sum of Squares Mean Square F Ratio Prob $>$ F

$\begin{array}{llllll}\text { Heat Treatment } & 1 & 0.07168452 & 0.071685 & 939.3661 & <.0001 \\ \text { Error } & 4 & 0.00030525 & 0.000076 & & \end{array}$

Means for Oneway Anova

Level Number Mean Std Error Lower 95\% Upper 95\%

$\begin{array}{lllllr}\text { ccc } & 3 & 1.54474 & 0.00504 & 1.5307 & 1.5587\end{array}$

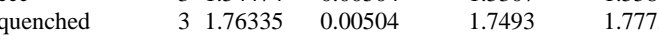

Std Error uses a pooled estimate of error variance
DF Sum of Squares Mean Square F Ratio Prob > F $\begin{array}{lll}\text { Heat Treatment } & 1 & 0.04740741 \\ \text { Error } & 4 & 0.000162608\end{array}$ $\begin{array}{lll}\text { Error } & 4 & 0.00016266 \\ \text { C. Total } & 5 & 0.04757008\end{array}$ $0.0474071165 .778<000$

Means for Oneway Anov

Level Number Mean Std Error Lower 95\% Upper 95\%

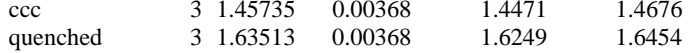

Std Error uses a pooled estimate of error variance
Oneway Analysis of $\log [\mathrm{Na}$ ppm] By Heat Treatment Glass ID=FY09EM21-16

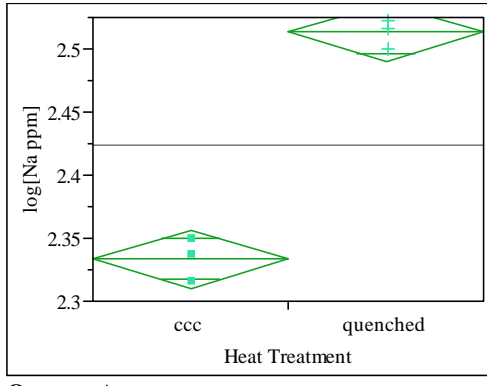

Oneway Anova

Summary of Fit

Rsquare

0.983028

Root Men Square Error $\quad 0.978786$

0.014477 2.423311

t Test

quenched-ccc

Assuming equal variance

Difference $\quad 0.179927$ t Ratio $\quad 15.22132$

Std Err Dif 0.011821 DF

Upper CL Dif 0.212746 Prob $>|t| \quad 0.0001$

Lower CL Dif 0.147107 Prob $>t \quad<.0001$

$\begin{array}{lll}\text { Confidence } & 0.95 \text { Prob }<\mathrm{t} \quad 0.9999\end{array}$

Analysis of Variance

Source DF Sum of Squares Mean Square F Ratio Prob $>$ F $\begin{array}{llllll}\text { Heat Treatment } & 1 & 0.04856041 & 0.048560 & 231.6885 & 0.000\end{array}$ $\begin{array}{lll}\text { Error } & 4 & 0.00083837\end{array}$ 0.000210

\section{Means for Oneway Anova}

Level Number Mean Std Error Lower 95\% Upper 95\%

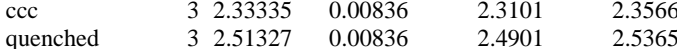

Std Error uses a pooled estimate of error variance 
SRNL-STI-2009-00778, Revision 0

\section{Exhibit B5. Effects of Heat Treatment (HT) on PCT $\log (\mathrm{ppm})$ Response of Matrix 2 Non-Rad Glasses}

Oneway Analysis of $\log [\mathrm{Si}$ ppm] By Heat Treatment Glass ID=FY09EM21-16

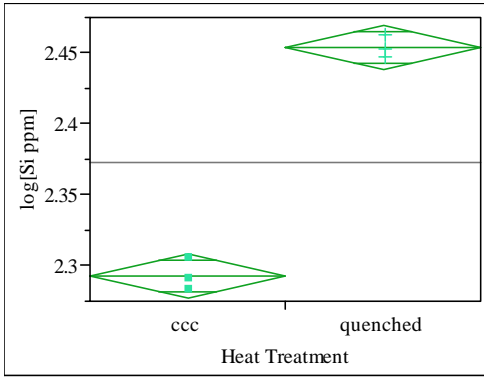

\section{Oneway Anova}

Summary of Fit

Rsquare

Adj Rsquare

0.988215

0.009651

2.373187
$\quad 6$

t Test

quenched-ccc

Assuming equal variances

Difference $\quad 0.161546$ t Ratio $\quad 20.50018$ Std Err Dif $\quad 0.007880$ DF

Upper CL Dif 0.183425 Prob $>|t| \quad<.0001$

Lower CL Dif 0.139667 Prob $>t \quad<.0001$

Confidence $\quad 0.95$ Prob $<\mathrm{t} \quad 1.0000$
Oneway Analysis of $\log [\mathrm{B}$ ppm] By Heat Treatment Glass ID=FY09EM21-17

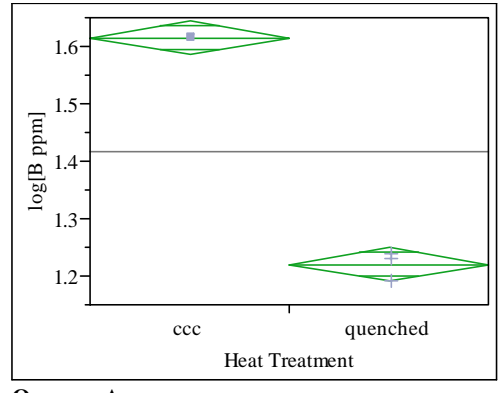

Oneway Anova

Rsquare

Adj Rsquare

Root Mean Square E

Mean of Response

0.994495

0.993118

1.41738

Observations (or Sum Wgts)

t Test

quenched-ccc

Assuming equal variances

Difference $\quad-0.39435$ t Ratio $\quad-26.8806$

Std Err Dif $\quad 0.01467$ DF

Prob $>|t|<.000$

Lower CL Dif -0.43508 Prob $>t \quad 1.0000$

$\begin{array}{lr}\text { Confidence } & 0.95 \text { Prob }<\mathrm{t} \quad<.0001\end{array}$

Analysis of Variance

Analysis of Variance

Source DF Sum of Squares Mean Square F Ratio Prob $>$ F

$\begin{array}{llllll}\text { Heat Treatment } & 1 & 0.03914573 & 0.039146 & 420.2574 & <.0001 \\ \text { Error } & 4 & 0.00037259 & 0.000093 & & \end{array}$ 0.000093

Means for Oneway Anova

Level Number Mean Std Error Lower 95\% Upper 95\%

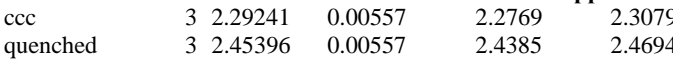

Std Error uses a pooled estimate of error variance $\begin{array}{lrrrrr}\text { Analysis of Variance } & & & & \\ \text { Source } & \text { DF } & \text { Sum of Squares } & \text { Mean Square } & \text { F Ratio } & \text { Prob }>\text { F } \\ \text { Heat Treatment } & 1 & 0.23326451 & 0.233265 & 722.5644 & <.0001\end{array}$

Erro

Tot 0.23455582

Means for Oneway Anova

Level Number Mean Std Error Lower 95\% Upper 95\%

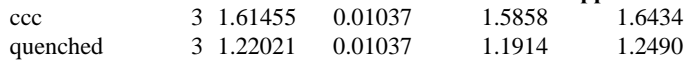

Std Error uses a pooled estimate of error variance
Oneway Analysis of $\log [\mathrm{Li}$ ppm] By Heat Treatment Glass ID=FY09EM21-17

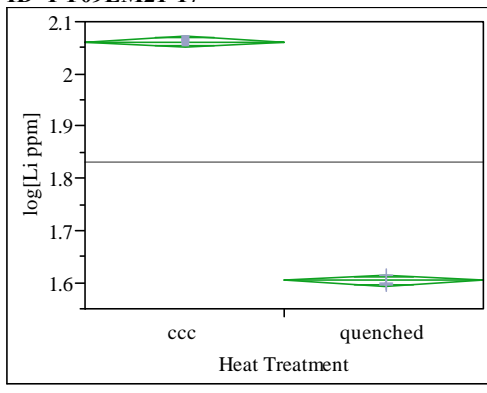

Oneway Anova

Adj Rsquare $\quad 0.999229$

Root Mean Square Error 0.006959

$\begin{array}{lr}\text { Mean of Response } & 1.832463 \\ \text { Observations (or Sum Wgts) } & 6\end{array}$

t Test

quenched-ccc

Assuming equal variances

Difference $\quad-0.45732$ t Ratio $\quad-80.4828$

Upper CL Dif -0.44154 Prob $>|t|<.000$

Lower CL Dif -0.47309 Prob $>t \quad 1.0000$

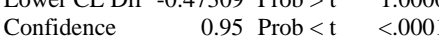

Analysis of Variance

Source DF Sum of Squares Mean Square F Ratio Prob $>$ F

$\begin{array}{lrrrrr}\text { Heat Treatment } & 1 & 0.31370619 & 0.313706 & 6477.476 & <.0001\end{array}$

$\begin{array}{lll}\text { Error } & 4 & 0.00019372\end{array}$ 0.000048

Means for Oneway Anova

Level Number Mean Std Error Lower 95\% Upper 95\%

$\begin{array}{llllll}\text { ccC } & 3 & 2.06112 & 0.00402 & 2.0500 & 2.0723\end{array}$

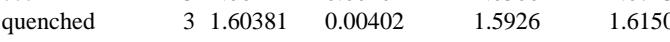

Std Error uses a pooled estimate of error variance 
SRNL-STI-2009-00778, Revision 0

\section{Exhibit B5. Effects of Heat Treatment (HT) on PCT $\log (\mathrm{ppm})$ Response of Matrix 2 Non-Rad Glasses}

Oneway Analysis of $\log [\mathrm{Na}$ ppm] By Heat Treatment Glass

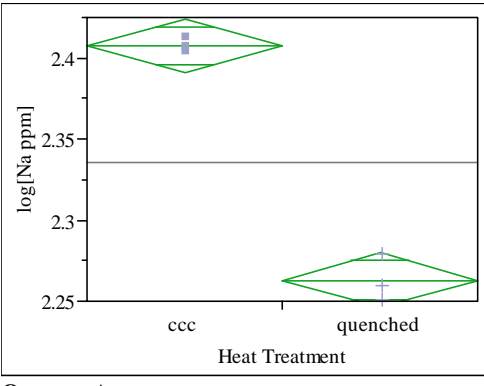

Oneway Anova

Summary of Fit

Rsquare

Adj Rsquare $\quad 0.986192$

Root Mean Square Error $\quad 0.010462$

Mean of Response 2.335288

Observations (or Sum Wgts)

6

t Test

quenched-ccc

Assuming equal variances

Difference $\quad-0.14438$ t Ratio -16.9022

$\begin{array}{lrr}\text { Std Err Dif } & 0.00854 \text { DF } & 4 \\ \text { Upper CL Dif } & -0.12067 \text { Prob }>|t| & <.0001\end{array}$

$\begin{array}{lll}\text { Upper CL Dif }-0.12067 & \text { Prob }>|t| & <.0001 \\ \text { Lower CL Dif }-0.16810 & \text { Prob }>t & 1.0000\end{array}$

$\begin{array}{rrr} & \\ \text { Confidence } & 0.95 \text { Prob }<\mathrm{t} & <.0001\end{array}$

Analysis of Variance

Source DF Sum of Squares Mean Square F Ratio Prob $>$ F

$\begin{array}{llllll}\text { Heat Treatment } & 1 & 0.03127005 & 0.031270 & 285.6860 & <.0001 \\ \text { Error } & 4 & 0.00043782 & 0.000109 & & \end{array}$$$
0.000109
$$

Means for Oneway Anova

Level Number Mean Std Error Lower 95\% Upper 95\%

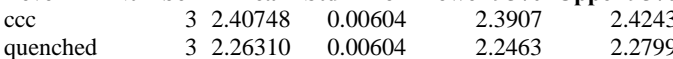

Std Error uses a pooled estimate of error variance
Oneway Analysis of $\log [\mathrm{Si}$ ppm] By Heat Treatment Glas ID=FY09EM21-17

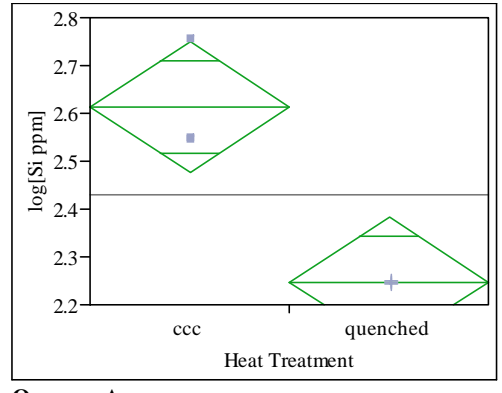

Oneway Anova

Rsquare

Adj Rsquare

(n)

0.84173

Root Mean Square Error $\quad 0.085494$

Observations (or Sum Wgts)

t Test

quenched-ccc

Assuming equal variances

Difference $\quad-0.36668$ t Ratio $\quad-5.25285$

Std Err Dif 0.06981 DF

4
0.0063

$\begin{array}{lll}\text { Lower CL Dif }-0.56049 \text { Prob }>t & 0.9969\end{array}$

$\begin{array}{lrl} & \\ \text { Confidence } & 0.95 \text { Prob }<\mathrm{t} & 0.0031\end{array}$

Analysis of Variance

Source DF Sum of Squares Mean Square F Ratio Prob $>$ F

$\begin{array}{llllll}\text { Heat Treatment } & 1 & 0.20167720 & 0.201677 & 27.5925 & 0.0063\end{array}$

Error

0.02923656

0.007309

Means for Oneway Anova

Level Number Mean Std Error Lower 95\% Upper 95\%

$\begin{array}{llllll}\text { ccC } & 3 & 2.61383 & 0.04936 & 2.4768 & 2.7509 \\ & 3 & 2.24715 & 0.04936 & 2.1101 & 2.3842\end{array}$

$\begin{array}{llllll}\text { quenched } & 3 & 2.24715 & 0.04936 & 2.1101 & 2.3842\end{array}$

Std Error uses a pooled estimate of error variance
Oneway Analysis of $\log [\mathrm{B}$ ppm] By Heat Treatment Glass

ID=FY09EM21-18

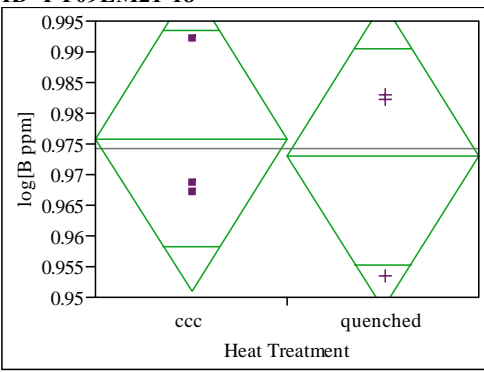

Oneway Anova

Summary of Fit

Rsquare

Root Mean Square Error $\quad 0.015514$

Mean of Response 0.974359

t Test

quenched-ccc

Assuming equal variances

Difference $\quad-0.00288$ t Ratio $\quad-0.22729$

Std Err Dif 0.01267 DF $\quad 4$

Upper CL Dif 0.03229 Prob $>|t| \quad 0.8313$

Lower CL Dif -0.03805 Prob $>t \quad 0.5843$

Analysis of Variance

Source DF Sum of Squares Mean Square F Ratio Prob $>$ F

$\begin{array}{lrrrrr}\text { Source } & \text { DF } & \text { Sum of Squares } & \text { Mean Square } & & \\ \text { Heat Treatment Ratio } & \text { Prob }>\text { F } \\ & 1 & 0.00001243 & 0.000012 & 0.0517 & 0.8313\end{array}$

$\begin{array}{llll}\text { Error } & 4 & 0.00096272 & 0.000241\end{array}$

$5 \quad 0.00097516$

Means for Oneway Anova

Level Number Mean Std Error Lower 95\% Upper 95\%

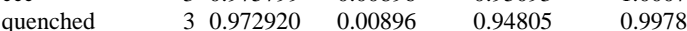

Std Error uses a pooled estimate of error variance 
SRNL-STI-2009-00778, Revision 0

\section{Exhibit B5. Effects of Heat Treatment (HT) on PCT $\log (\mathrm{ppm})$ Response of Matrix 2 Non-Rad Glasses}

Oneway Analysis of $\log [\mathrm{Li}$ ppm] By Heat Treatment Glass

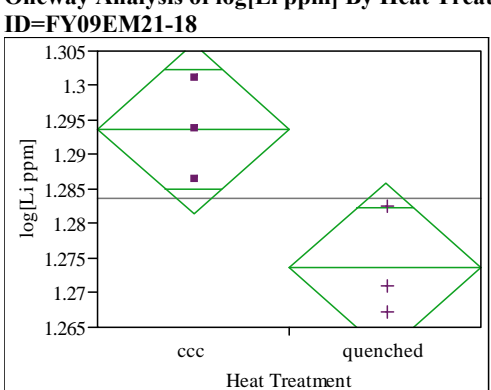

Oneway Anova

Summary of Fit

$\begin{array}{lr}\text { Rsquare } & 0.719456 \\ \text { Adj Rsquare } & 0.64932 \\ \text { Root Mean Square Error } & 0.007684 \\ \text { Mean of Response } & 1.283651\end{array}$

Root Mean Square Error $\quad 0.007684$

\section{t Test}

quenched-ccc

Assuming equal variances

Difference $\quad-0.02009$ t Ratio $\quad-3.20281$

$\begin{array}{lll}\text { Std Err Dif } \quad 0.00627 \text { DF } & 4\end{array}$

Upper CL Dif -0.00267 Prob $>|t| 0.0328$

Confidence

0.95 Prob $<\mathrm{t} \quad 0.0164$

Analysis of Variance

Source DF Sum of Squares Mean Square F Ratio Prob $>$ F $\begin{array}{lllllll}\text { Heat Treatment } & 1 & 0.00060566 & 0.000606 & 10.2580 & 0.0328\end{array}$

$\begin{array}{lll}\text { Error } & 4 & 0.00023617\end{array}$

C. Total

0.00023617 0.000059

Means for Oneway Anova

Level Number Mean Std Error Lower 95\% Upper 95\%

\begin{tabular}{lrrrrr} 
ccc & Number & Mean & Std Error & Lower 95\% & Upper 95\% \\
\hline & 3 & 1.29370 & 0.00444 & 1.2814 & 1.3060
\end{tabular}

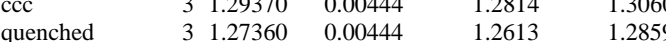

Std Error uses a pooled estimate of error variance

Oneway Analysis of $\log [\mathrm{Na}$ ppm] By Heat Treatment Glass

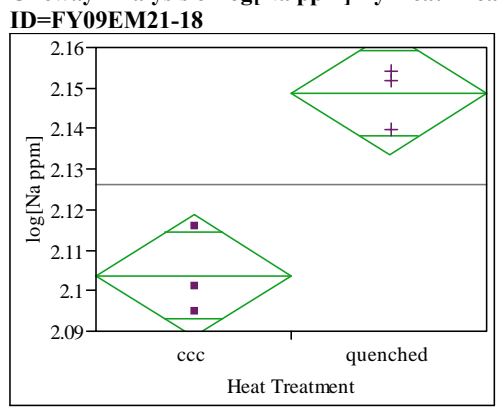

\section{Oneway Anova}

Summary of Fit

Adj Rsquare

Root Mean Square En

0.896089

Mean of Respons

0.870112

Observations (or Sum Wgts) 26196

t Test

quenched-ccc

Assuming equal variances

Difference $\quad 0.044946$ t Ratio $\quad 5.873207$

Std Err Dif 0.007653 DF

Upper CL Dif 0.066193 Prob $>|t| \quad 0.0042$

$\begin{array}{lll}\text { Lower CL Dif } 0.023639 \mathrm{Prob}>\mathrm{t} & 0.0021\end{array}$

$\begin{array}{lll}\text { Confidence } & 0.95 \text { Prob }<t \quad 0.9979\end{array}$

Analysis of Variance

Source DF Sum of Squares Mean Square F Ratio Prob $>$ F $\begin{array}{llllll}\text { Heat Treatment } & 1 & 0.00303018 & 0.003030 & 34.4946 & 0.0042\end{array}$

Error 0.00035138

Means for Oneway Anova

Level Number Mean Std Error Lower 95\% Upper 95\%

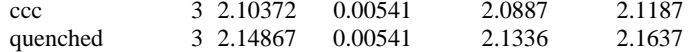

Std Error uses a pooled estimate of error variance
Oneway Analysis of $\log [\mathrm{Si}$ ppm] By Heat Treatment Glass

ID=FY09EM21-18

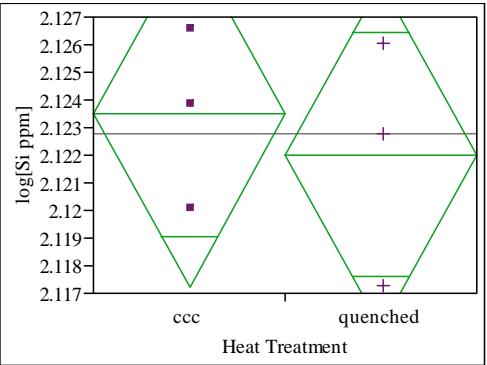

Oneway Anova

Summary of Fit

Root Mean Square Error $\quad 0.003896$

Mean of Response 0.003896

6

t Test

quenched-ccc

Assuming equal variances

-0.00146 t Ratio -0.4595

$\begin{array}{lrr}\text { Std Err Dif } & 0.00318 \text { DF } & 4 \\ \end{array}$

Lower CL Dif -0.01029 Prob $>t \quad 0.6651$

Confidence $\quad 0.95$ Prob $<t \quad 0.3349$

Analysis of Variance

Source DF Sum of Squares Mean Square F Ratio Prob $>$ F

$\begin{array}{llllll}\text { Heat Treatment } & 1 & 0.00000320 & 3.205 \mathrm{e}-6 & 0.2111 & 0.6697\end{array}$

$\begin{array}{lll}\text { Error } & 4 & 0.00006071\end{array}$

0.000015

Means for Oneway Anova

Level Number Mean Std Error Lower 95\% Upper 95\%

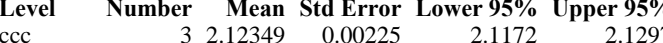

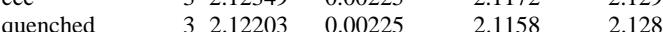

Std Error uses a pooled estimate of error variance 
SRNL-STI-2009-00778, Revision 0

\section{Exhibit B5. Effects of Heat Treatment (HT) on PCT $\log (\mathrm{ppm})$ Response of Matrix 2 Non-Rad Glasses}

Oneway Analysis of $\log [\mathrm{B}$ ppm] By Heat Treatment Glass

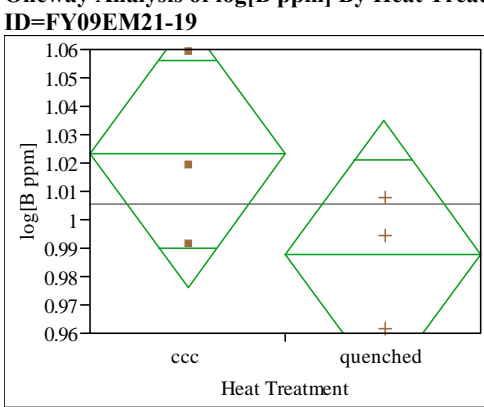

Oneway Anova

Heat Treatmen

Summary of Fit

Adj Rsquare $\quad 0.350608$

Root Mean Square Error $\quad 0.029354$

Mean of Response 1.005447

Observations (or Sum Wgts)

t Test

quenched-ccc

Assuming equal variances

Difference $\quad-0.03522$ t Ratio $\quad-1.46956$

Std Err Dif 0.02397 DF

Upper CL Dif 0.03132 Prob $>|t| \quad 0.2156$

Lower CL Dif -0.1017 Prob $>\mathrm{t} \quad 0.8922$

$\begin{array}{lll}\text { Confidence } & 0.95 \mathrm{Prob}<\mathrm{t} \quad 0.1078\end{array}$

Analysis of Variance

Source DF Sum of Squares Mean Square F Ratio Prob $>$ F

$\begin{array}{llllll}\text { Heat Treatment } & 1 & 0.00186090 & 0.001861 & 2.1596 & 0.2156\end{array}$

Error

0.00344672

0.000862

Means for Oneway Anova

Level Number Mean Std Error Lower 95\% Upper 95\%

$\begin{array}{llllll}\text { ccc } & 3 & 1.02306 & 0.01695 & 0.97600 & 1.0701 \\ \text { quenched } & 3 & 0.98784 & 0.01695 & 0.94078 & 1.0349\end{array}$

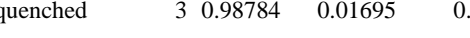

Std Error uses a pooled estimate of error variance
Oneway Analysis of $\log [\mathrm{Li}$ ppm] By Heat Treatment Glass

ID=FY09EM21-19

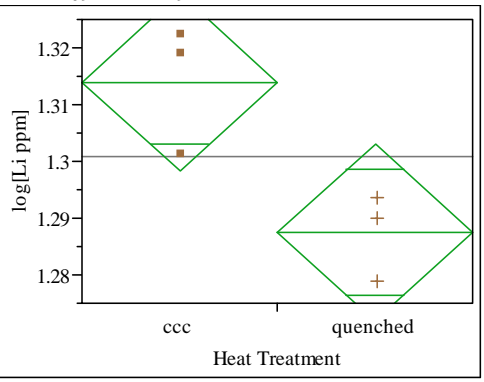

Oneway Anova

Summary of Fit

Rsquare

Adj Rsquare

\subsection{1}

Root Mean Square Error $\quad 0.009749$

Mean of Response 1.300763

t Test

quenched-ccc

Assuming equal variances

Difference $\quad-0.02650$ t Ratio $\quad-3.32869$

Std Err Dif 0.00796 DF 4
0.0291

$\begin{array}{lll}\text { Upper CL Dif } & -0.00440 \text { Prob }>|t| & 0.0291 \\ \text { Lower CL Dif }-0.04860 \text { Prob }>t & 0.9854\end{array}$

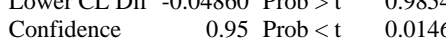

Analysis of Variance

Source DF Sum of Squares Mean Square F Ratio Prob $>$ F Heat Treatment 1

Error

0.00105309

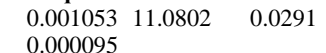

0.00143326

Means for Oneway Anova

Level Number Mean Std Error Lower 95\% Upper 95\%

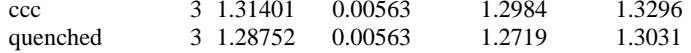

Std Error uses a pooled estimate of error variance
Oneway Analysis of $\log [\mathrm{Na} \mathrm{ppm}] \mathrm{By}$ Heat Treatment Glass

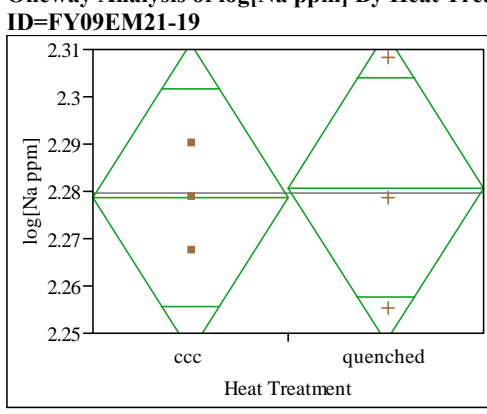

Oneway Anova

Summary of Fit

0.003918

-0.2451
Ropt Rere

Root Mean Square Error $\quad 0.020423$

Mean of Response 2.279708

quenched-ccc

Assuming equal variances

Difference $\quad 0.00209$ t Ratio $\quad 0.125428$ Std Err Dif 0.01668 DF

Upper CL Dif 0.04839 Prob $>|t| \quad 0.9062$ Lower CL Dif -0.04421 Prob $>t \quad 0.453$ $\begin{array}{lll}\text { Confidence } & 0.95 \text { Prob }<t \quad 0.5469\end{array}$

Analysis of Variance

DF Sum of Squares Mean Square F Ratio Prob $>$ $\begin{array}{llllll}\text { Heat Treatment } & 1 & 0.00000656 & 6.562 \mathrm{e}-6 & 0.0157 & 0.9062\end{array}$ $\begin{array}{lll}\text { Error } & 4 & 0.00166843 \\ \text { C. Total } & 5 & 0.00167499\end{array}$

\section{Means for Oneway Anova}

Level Number Mean Std Error Lower 95\% Upper 95\% $\begin{array}{llllll}\text { ccc } & 3 & 2.27866 & 0.01179 & 2.2459 & 2.3114 \\ \text { quenched } & 3 & 2.28075 & 0.01179 & 2.2480 & 2.3135\end{array}$

Std Error uses a pooled estimate of error variance 
SRNL-STI-2009-00778, Revision 0

\section{Exhibit B5. Effects of Heat Treatment (HT) on PCT $\log (\mathrm{ppm})$ Response of Matrix 2 Non-Rad Glasses}

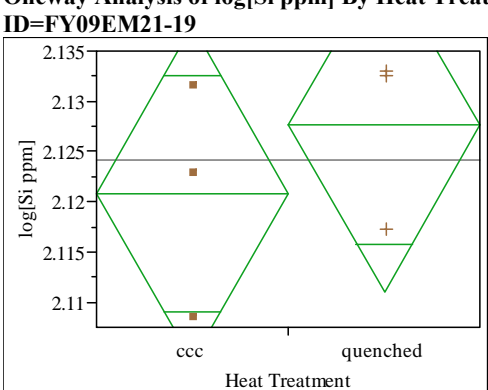

Oneway Anova

Summary of Fit

$\begin{array}{ll}\text { Rsquare } & 0.136743 \\ \text { Adj Rsquare } & -0.07907 \\ \text { Root Mean Square Error } & 0.010383 \\ \text { Mean of Response } & 2.124218 \\ \text { Obe }\end{array}$

Observations (or Sum Wgts)

t Test

quenched-ccc

Assuming equal variances

Difference $\quad 0.00675$ t Ratio $\quad 0.795999$

Std Err Dif $\quad 0.00848$ DF

Upper CL Dif 0.03029 Prob $>|t| \quad 0.4706$

Lower CL Dif -0.01679 Prob $>t \quad 0.2353$

$\begin{array}{lll}\text { Confidence } & 0.95 \text { Prob }<\mathrm{t} \quad 0.7647\end{array}$

Analysis of Variance

Source DF Sum of Squares Mean Square F Ratio Prob $>$ F $\begin{array}{llllll}\text { Heat Treatment } & 1 & 0.00006831 & 0.000068 & 0.6336 & 0.4706\end{array}$

Error 40.00043123 0.000108

0.0004995

Means for Oneway Anova

Level Number Mean Std Error Lower 95\% Upper 95\%

$\begin{array}{llllll} & 3 & 2.12084 & 0.00599 & 2.1042 & 2.1375 \\ \text { quenched } & 3 & 2.12759 & 0.00599 & 2.1109 & \end{array}$

Std Error uses a pooled estimate of error variance
Oneway Analysis of $\log [\mathrm{B}$ ppm] By Heat Treatment Glass

ID=FY09EM21-20

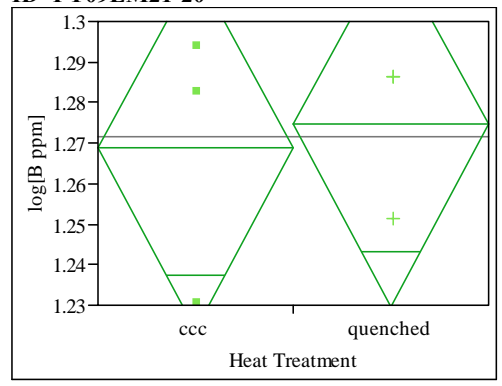

Oneway Anova

Summary of Fit

Adj Rsquare

$\begin{array}{ll}\text { Adj Rsquare } & -0.23062 \\ \text { Root Mean Square Error } & 0.027845\end{array}$

Mean of Response

0.015506

Observations (or Sum Wgts)

6

t Test

quenched-ccc

Assuming equal variances

Difference $\quad 0.00571$ t Ratio $\quad 0.25099$

Std Err Dif $\quad 0.02274$ DF

Upper CL Dif 0.06883 Prob $>|\uparrow| \quad 0.8142$

Lower CL Dif -0.05742 Prob $>\mathrm{t} \quad 0.4071$

0.95 Prob $<\mathrm{t} \quad 0.5929$

Analysis of Variance

Source DF Sum of Squares Mean Square F Ratio Prob $>$

$\begin{array}{llllll}\text { Heat Treatment } & 1 & 0.00004885 & 0.000049 & 0.0630 & 0.8142\end{array}$

Error $4 \quad-5=.00310141$

Means for Oneway Anova

Level Number Mean Std Error Lower 95\% Upper 95\%

$\begin{array}{llllll}\text { ccc } & 3 & 1.26892 & 0.01608 & 1.2243 & 1.3136 \\ \text { quenched } & 3 & 1.27462 & 0.01608 & 1.2300 & 1.3193\end{array}$

Std Error uses a pooled estimate of error variance
Oneway Analysis of $\log [\mathrm{Li}$ ppm] By Heat Treatment Glass

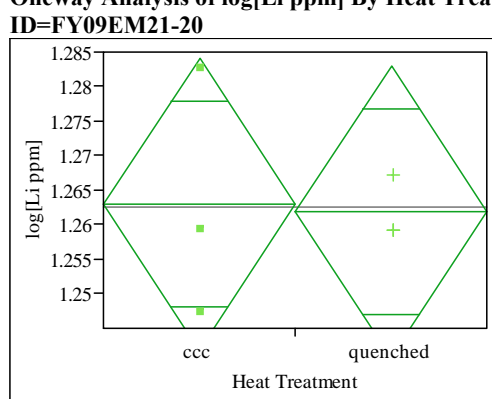

Oneway Anova

Summary of Fit

$\begin{array}{lr}\text { Rsquare } & 0.002557 \\ \text { Adj Rsquare } & -0.2468 \\ \text { Root Mean Square Error } & 0.01312 \\ \text { Mean of Response } & 1.262458 \\ \text { Observations (or Sum Wgts) } & 6\end{array}$

t Test

quenched-ccc

Assuming equal variances

Difference $\quad-0.00108$ t Ratio $\quad-0.10126$

Std Err Dif $\quad 0.01071$ DF

Upper CL Dif 0.02866 Prob $>|t| \quad 0.9242$

Lower CL Dif -0.03083 Prob $>$ t 0.537

$\quad 0.95$ Prob $<\mathrm{t} \quad 0.4621$

Analysis of Varianc

Source DF Sum of Squares Mean Square F Ratio Prob $>$ F

$\begin{array}{lrrrrr}\text { Heat Treatment } & 1 & 0.00000177 & 1.765 \mathrm{e}-6 & 0.0103 & 0.9242\end{array}$

$\begin{array}{lll}\text { Error } & 4 & 0.00068859\end{array}$

0.000172

Means for Oneway Anova

Level Number Mean Std Error Lower 95\% Upper 95\%

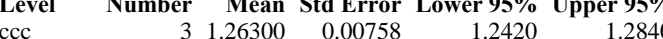

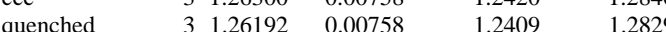

Std Error uses a pooled estimate of error variance 
SRNL-STI-2009-00778, Revision 0

\section{Exhibit B5. Effects of Heat Treatment (HT) on PCT $\log (\mathrm{ppm})$ Response of Matrix 2 Non-Rad Glasses}

Oneway Analysis of $\log [\mathbf{N a}$ ppm] By Heat Treatment Glass

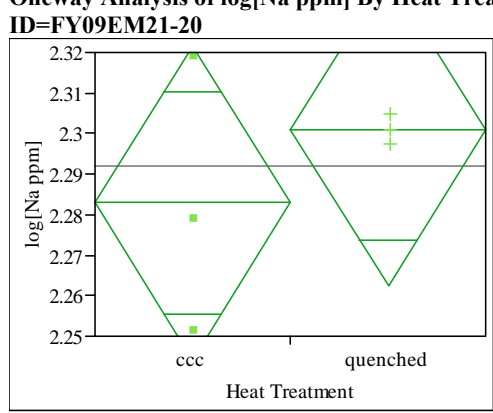

\section{Oneway Anova}

Summary of Fit

Rsquare

Adj Rsquare

0.174125

Root Mean Squ

0.024146

Observations (or Sum Wgts) $\begin{array}{r}2.291976 \\ \hline\end{array}$

t Test

quenched-ccc

Assuming equal variances

Difference $\quad 0.01810$ t Ratio $\quad 0.918339$ Std Err Dif 0.01971 DF

Upper CL Dif 0.07284 Prob $>|t| \quad 0.4104$

Lower CL Dif -0.03663 Prob $>t \quad 0.2052$

$\begin{array}{lll}\text { Confidence } & 0.95 \text { Prob }<\mathrm{t} \quad 0.7948\end{array}$

Analysis of Variance

Source DF Sum of Squares Mean Square F Ratio Prob $>$ F

$\begin{array}{llllll}\text { Heat Treatment } & 1 & 0.00049169 & 0.000492 & 0.8433 & 0.4104\end{array}$

C. Total

0.00233207

0.000583

Means for Oneway Anova

Level Number Mean Std Error Lower 95\% Upper 95\%

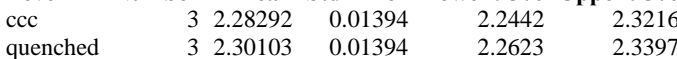

Std Error uses a pooled estimate of error variance
Oneway Analysis of $\log [\mathrm{Si}$ ppm] By Heat Treatment Glass ID=FY09EM21-20

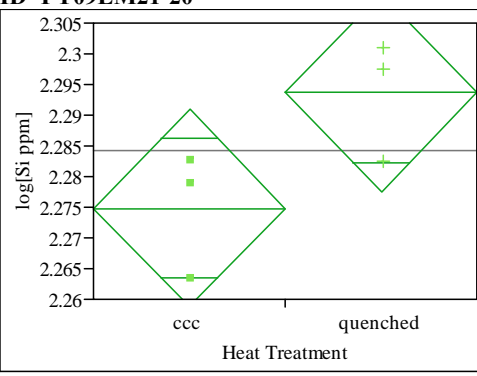

Oneway Anova

Summary of Fit

\section{Rsquare}

Adj Rsquare

Root Mean Square
Mean of Response

0.56612

0.46201

0.01013

Observations (or Sum Wgts)

t Test

quenched-ccc

Assuming equal variances

Difference $\quad 0.01881$ t Ratio 2.300856

Std Err Dif $\quad 0.00818$ DF

Upper CL Dif 0.04151 Prob $>|t| \quad 0.0829$

$\begin{array}{lll}\text { Confidence } & 0.95 \text { Prob }<t \quad 0.9586\end{array}$

Analysis of Variance

Source DF Sum of Squares Mean Square F Ratio Prob $>$ F $\begin{array}{lllllll}\text { Heat Treatment } & 1 & 0.00053074 & 0.000531 & 5.2939 & 0.0829\end{array}$ Error $\quad 4-0.00040101$ 0.00040101

0.000100

Means for Oneway Anova

Level Number Mean Std Error Lower 95\% Upper 95\%

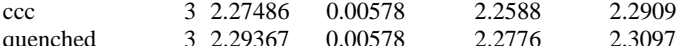

32.293670 .00578

Std Error uses a pooled estimate of error variance
Oneway Analysis of $\log [\mathrm{B}$ ppm] By Heat Treatment Glass

ID=FY09EM21-21

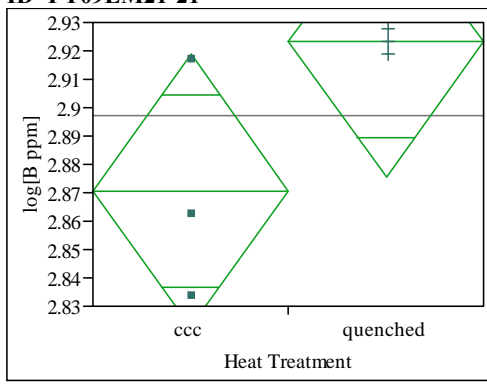

Oneway Anova

Summary of Fit

$\begin{array}{lr}\text { Rsquare } & 0.537093 \\ \text { Adj Rsquare } & 0.421366 \\ \text { Root Mean Square Error } & 0.029913 \\ \text { Mean of Response } & 2.897103 \\ \text { Observations (or Sum Wgts) } & 6\end{array}$

Test

quenched-ccc

Assuming equal variance

Difference $\quad 0.05262$ t Ratio $\quad 2.154309$ Std Err Dif 0.02442 DF

Upper CL Dif 0.12043 Prob $>|t| \quad 0.0975$ Lower CL Dif -0.01520 Prob $>t \quad 0.0488$ $\begin{array}{lll}\text { Confidence } & 0.95 \text { Prob }<\mathrm{t} \quad 0.9512\end{array}$

Analysis of Variance

Source DF Sum of Squares Mean Square F Ratio Prob $>$ F $\begin{array}{lllrlr}\text { Heat Treatment } & 1 & 0.00415283 & 0.004153 & 4.6410 & 0.0975\end{array}$ $\begin{array}{lll}\text { Error } & 4 & 0.00357922\end{array}$

\section{Means for Oneway Anova}

Level Number Mean Std Error Lower 95\% Upper 95\% $\begin{array}{llllll}\text { ccc } & 3 & 2.87079 & 0.01727 & 2.8228 & 2.9187 \\ \text { quenched } & 3 & 2.92341 & 0.01727 & 2.8755 & 2.9714\end{array}$

Std Error uses a pooled estimate of error variance 
SRNL-STI-2009-00778, Revision 0

\section{Exhibit B5. Effects of Heat Treatment (HT) on PCT $\log (\mathrm{ppm})$ Response of Matrix 2 Non-Rad Glasses}

Oneway Analysis of $\log [\mathrm{Li}$ ppm] By Heat Treatment Glass

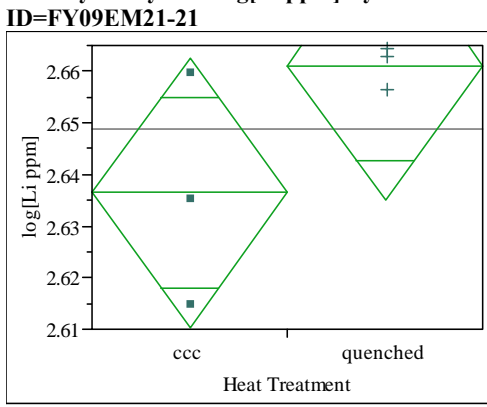

Oneway Anova

Summary of Fit

$\begin{array}{ll} & 0.465844 \\ \text { Adj Rsquare } & 0.332305\end{array}$

Root Mean Square Error $\quad 0.016221$

Mean of Response 2.648808

Observations (or Sum Wgts)

t Test

quenched-ccc

Assuming equal variances

Difference $\quad 0.02474$ t Ratio $\quad 1.867738$ Std Err Dif 0.01324 DF

Upper CL Dif 0.06151 Prob $>|t| \quad 0.1352$

Lower CL Dif -0.01204 Prob $>t \quad 0.0676$

Confidence $\quad 0.95$ Prob $<t \quad 0.9324$

Analysis of Variance

Source DF Sum of Squares Mean Square F Ratio Prob $>$ F

$\begin{array}{llllll}\text { Heat Treatment } & 1 & 0.00091787 & 0.000918 & 3.4884 & 0.1352 \\ \text { Error } & 4 & 0.00105246 & 0.000263 & & \end{array}$

0.000263

Means for Oneway Anova

Level Number Mean Std Error Lower 95\% Upper 95\%

$\begin{array}{llllll}\text { ccc } & 3 & 2.63644 & 0.00937 & 2.6104 & 2.6624 \\ \text { quenched } & 3 & 2.66118 & 0.00937 & 2.6352 & 2.6872\end{array}$

Std Error uses a pooled estimate of error variance
Oneway Analysis of $\log [\mathrm{Na}$ ppm] By Heat Treatment Glass

ID=FY09EM21-21

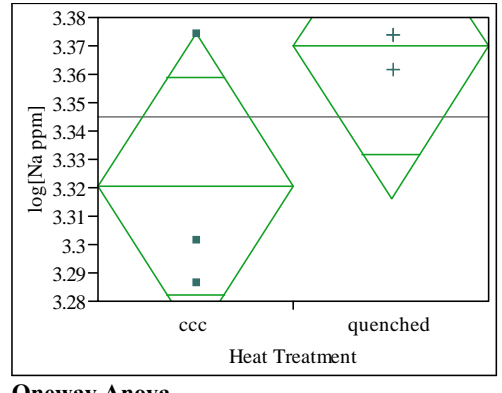

Oneway Anova

Summary of Fit

Rsquare

Adj Rsquare

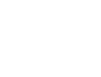

0.448134

Root Mean Square Error $\quad 0.033645$

Mean of Response
Observations (or Sum Wgts)

0.033645
3.345255

t Test

quenched-ccc

Assuming equal variances

Difference $\quad 0.04951$ t Ratio 1.802258

Std Err Dif $\quad 0.02747$ DF

4
0.1459

Lower CL Dif -0.02676 Prob $>t \quad 0.0729$

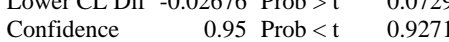

Analysis of Variance

Source DF Sum of Squares Mean Square F Ratio Prob $>$ F

Heat Treatment 1

Error

10.00367677

$\begin{array}{lll}0.003677 & 3.2481 & 0.1459 \\ 0.001132 & & \end{array}$

0.00820463

Means for Oneway Anova

Level Number Mean Std Error Lower 95\% Upper 95\%

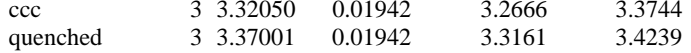

Std Error uses a pooled estimate of error variance
Oneway Analysis of $\log [\mathrm{Si}$ ppm] By Heat Treatment Glass

ID=FY09EM21-21

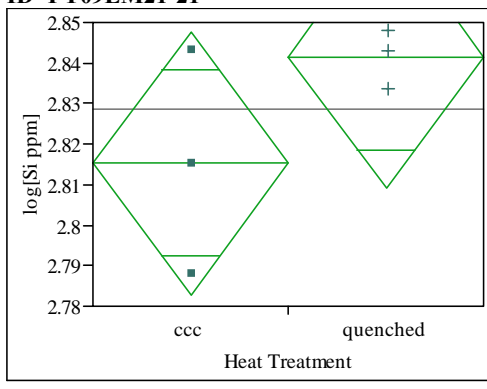

Oneway Anova

Summary of Fit

Rsquare

Adj Rsquare $\quad 0.387596$

Root Men Square Eror $\quad 0.234495$

Square Error 0.020252

Test

quenched-ccc

Assuming equal variances

Difference $\quad 0.02631$ t Ratio 1.591112 Std Err Dif $\quad 0.01654$ DF

Upper CL Dif 0.07222 Prob $>|t| \quad 0.1868$

Lower CL Dif -0.01960 Prob $>t \quad 0.0934$

$\begin{array}{lll}\text { Confidence } & 0.95 \text { Prob }<\mathrm{t} \quad 0.9066\end{array}$

Analysis of Variance

Source DF Sum of Squares Mean Square F Ratio Prob $>$ F $\begin{array}{lllrlr}\text { Heat Treatment } & 1 & 0.00103832 & 0.001038 & 2.5316 & 0.1868\end{array}$ $\begin{array}{lll}\text { Error } & 4 & 0.00164055 \\ & 5 & 0.002688\end{array}$ 0.000410

\section{Means for Oneway Anova}

Level Number Mean Std Error Lower 95\% Upper 95\% $\begin{array}{llllll}\text { ccc } & 3 & 2.81529 & 0.01169 & 2.7828 & 2.8478 \\ \text { quenched } & 3 & 2.84160 & 0.01169 & 2.8091 & 2.8741\end{array}$

Std Error uses a pooled estimate of error variance 
SRNL-STI-2009-00778, Revision 0

\section{Exhibit B5. Effects of Heat Treatment (HT) on PCT $\log (\mathrm{ppm})$ Response of Matrix 2 Non-Rad Glasses}

Oneway Analysis of $\log [\mathrm{B}$ ppm] By Heat Treatment Glass ID=FY09EM21-22

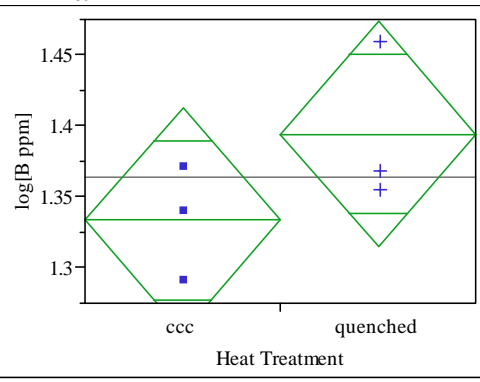

\section{Oneway Anova}

Summary of Fit

Rsquare

$\begin{array}{ll} & 0.361979 \\ \text { Adj Rsquare } & 0.202473\end{array}$

Root Mean Square Error $\quad 0.049603$

Mean of Respons 1.363922
6

\section{t Test}

quenched-ccc

Assuming equal variances

Difference $\quad 0.06101$ t Ratio $\quad 1.506447$ Std Err Dif 0.04050 DF

Upper CL Dif 0.17346 Prob $>|t| \quad 0.2064$

Lower CL Dif -0.05144 Prob $>t \quad 0.1032$

$\begin{array}{lll} & \text { Confidence } & 0.95 \text { Prob }<\mathrm{t} \quad 0.8968\end{array}$

Analysis of Variance

Source DF Sum of Squares Mean Square F Ratio Prob $>$ F $\begin{array}{llllll}\text { Heat Treatment } & 1 & 0.00558374 & 0.005584 & 2.2694 & 0.2064 \\ \text { Error } & 4 & 0.00984187 & 0.002460 & & \end{array}$

Error 0.00984187

Means for Oneway Anova

Level Number Mean Std Error Lower 95\% Upper 95\%

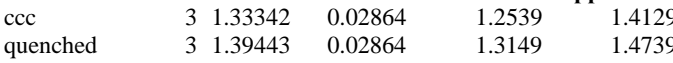

Std Error uses a pooled estimate of error variance
Oneway Analysis of $\log [\mathrm{Li}$ ppm] By Heat Treatment Glass

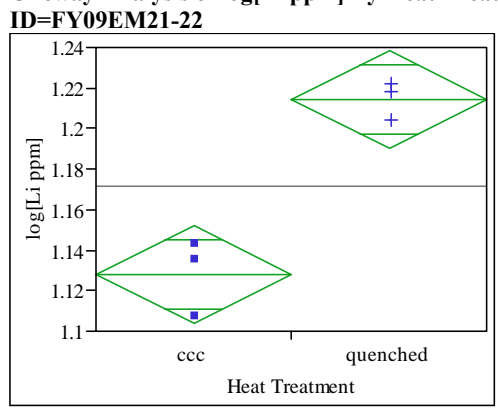

\section{Oneway Anova}

Rsquare

Adj Rsquare

Root Mean of Response

0.925123

0.906403 171456

t Test

quenched-ccc

Assuming equal variances

Difference $\quad 0.086367$ t Ratio $\quad 7.029981$

Std Err Dif 0.012285 DF

4
0.0022

Lower CL Dif 0.052257 Prob $>t \quad 0.0011$

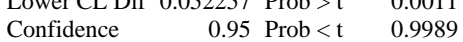

Analysis of Variance

Source DF Sum of Squares Mean Square F Ratio Prob $>$ F Heat Treatment $1 \quad 0.01118876$ 0.00090559 $0.01118949 .4206 \quad 0.0022$

Means for Oneway Anova

Level Number Mean Std Error Lower 95\% Upper 95\%

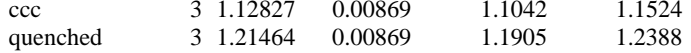

Std Error uses a pooled estimate of error variance
Oneway Analysis of $\log [\mathrm{Na} \mathrm{ppm}] \mathrm{By}$ Heat Treatment Glass ID=FY09EM21-22

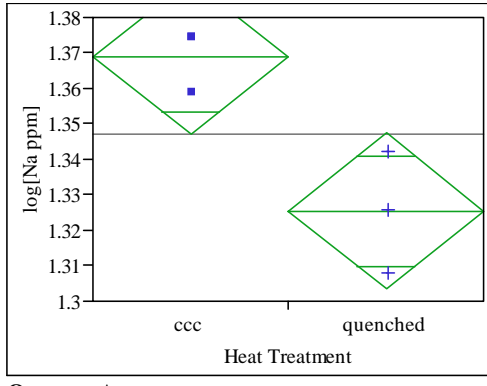

Oneway Anova

Summary of Fit

$\begin{array}{lr}\text { Rsquare } & 0.791816 \\ \text { Adj Rsquare } & 0.73977 \\ \text { Root Mean Square Error } & 0.013665 \\ \text { Mean of Response } & 1.347197 \\ \text { Observations (or Sum Wgts) } & 6\end{array}$

quenched-ccc

Assuming equal variances

Difference $\quad-0.04352$ t Ratio $\quad-3.90048$ Std Err Dif $\quad 0.01116$ DF

Upper CL Dif -0.01254 Prob $>|t| \quad 0.0175$

Lower CL Dif -0.07450 Prob $>t \quad 0.9912$

Confidence $\quad 0.95$ Prob $<\mathrm{t} \quad 0.0088$

Analysis of Variance

DF Sum of Squares Mean Square F Ratio Prob $>$ F $\begin{array}{llrrrr}\text { Heat Treatment } & 1 & 0.00284098 & 0.002841 & 15.2138 & 0.0175\end{array}$ $\begin{array}{lll}\text { Error } & 4 & 0.00074695 \\ \text { C. } & 5 & 0.0035892\end{array}$ 0.00018

\section{Means for Oneway Anova}

Level Number Mean Std Error Lower 95\% Upper 95\% $\begin{array}{llllll}\text { ccc } & 3 & 1.36896 & 0.00789 & 1.3471 & 1.3909 \\ \text { quenched } & 3 & 1.32544 & 0.00789 & 1.3035 & 1.3473\end{array}$

Std Error uses a pooled estimate of error variance 
SRNL-STI-2009-00778, Revision 0

\section{Exhibit B5. Effects of Heat Treatment (HT) on PCT $\log (\mathrm{ppm})$ Response of Matrix 2 Non-Rad Glasses}

Oneway Analysis of $\log [\mathrm{Si}$ ppm] By Heat Treatment Glass

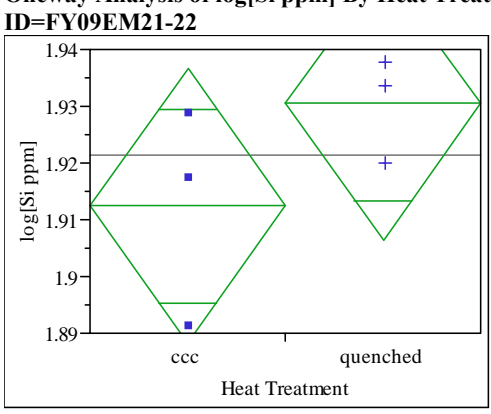

Oneway Anova

Summary of Fit

Adj Rsquare

\begin{tabular}{ll}
0.35079 & 015099 \\
\hline
\end{tabular}

Mean of Response

1.921429

t Test

quenched-ccc

Assuming equal variances

Difference $\quad 0.01813$ t Ratio 1.470756 Std Err Dif $\quad 0.01233$ DF

Upper CL Dif 0.05236 Prob $>|t| \quad 0.2153$

Lower CL Dif -0.01610 Prob $>\mathrm{t} \quad 0.1077$

$\begin{array}{lll}\text { Confidence } & 0.95 \text { Prob }<\mathrm{t} \quad 0.8923\end{array}$

Analysis of Variance

Source DF Sum of Squares Mean Square F Ratio Prob $>$ F

$\begin{array}{llllll}\text { Heat Treatment } & 1 & 0.00049313 & 0.000493 & 2.1631 & 0.2153\end{array}$

Error

0.00091189

0.000228

Means for Oneway Anova

Level Number Mean Std Error Lower 95\% Upper 95\%

$\begin{array}{lllllr}\text { ccc } & 3 & 1.91236 & 0.00872 & 1.8882 & 1.9366\end{array}$

$\begin{array}{llllll}\text { quenched } & 3 & 1.93049 & 0.00872 & 1.9063 & 1.9547\end{array}$

Std Error uses a pooled estimate of error variance
Oneway Analysis of $\log [\mathrm{B}$ ppm] By Heat Treatment Glass ID=FY09EM21-23

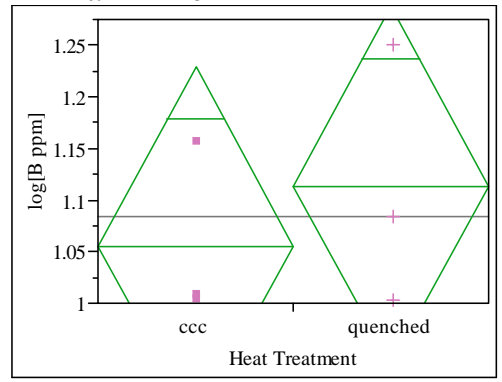

\section{Oneway Anova}

Rsquare

Adj Rsquare

Root Mean Square Enor

0.09507

ean of Response 1.084431

t Test

quenched-ccc

Assuming equal variances

Difference $\quad 0.05743$ t Ratio $\quad 0.648272$

Std Err Dif 0.08859 DF

Upper CL Dif $0.30339 \mathrm{Prob}>\mathrm{Ml} \quad 0.552$

Lower CL Dif -0.18853 Prob $>t \quad 0.2761$

Confidence $\quad 0.95$ Prob $<\mathrm{t} \quad 0.7239$

Analysis of Variance

Source DF Sum of Squares Mean Square F Ratio Prob $>$ F $\begin{array}{llllll}\text { Heat Treatment } & 1 & 0.00494731 & 0.004947 & 0.4203 & 0.5521\end{array}$ Error 0.04708855

Means for Oneway Anova

Level Number Mean Std Error Lower 95\% Upper 95\%

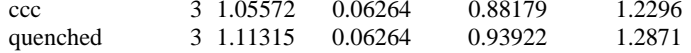

Std Error uses a pooled estimate of error variance
Oneway Analysis of $\log [\mathrm{Li}$ ppm] By Heat Treatment Glass ID=FY09EM21-23

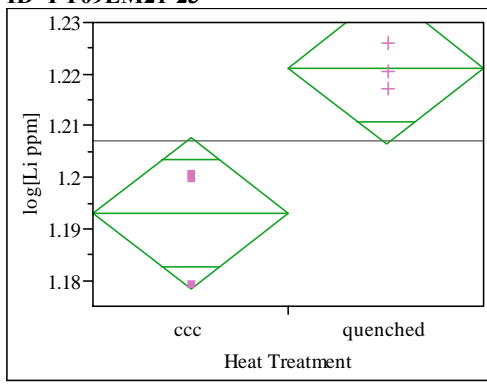

Oneway Anova

Summary of Fit

Rsquare

Adj Rsquare $\quad 0.779578$

0.724473

0.009196 1.207141

\section{Test}

quenched-ccc

Assuming equal variance

Difference $\quad 0.028241$ t Ratio 3.761254 Std Err Dif 0.007508 DF

Upper CL Dif 0.049088 Prob $>|t| \quad 0.0198$ Lower CL Dif 0.007394 Prob $>t \quad 0.009$ Confidence $\quad 0.95$ Prob $<\mathrm{t} \quad 0.990$

Analysis of Variance

DF Sum of Squares Mean Square F Ratio Prob $>$ F $\begin{array}{lrrrrr}\text { Heat Treatment } & 1 & 0.00119636 & 0.001196 & 14.1470 & 0.0198\end{array}$ Error $\quad 4 \quad 0.00033826$

\section{Means for Oneway Anova}

Level Number Mean Std Error Lower 95\% Upper 95\% $\begin{array}{llrrrr}\text { ccc } & 3 & 1.19302 & 0.00531 & 1.1783 & 1.2078\end{array}$

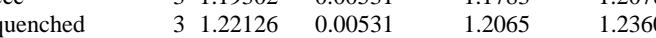

Std Error uses a pooled estimate of error variance 
SRNL-STI-2009-00778, Revision 0

\section{Exhibit B5. Effects of Heat Treatment (HT) on PCT $\log (\mathrm{ppm})$ Response of Matrix 2 Non-Rad Glasses}

Oneway Analysis of $\log [\mathrm{Na}$ ppm] By Heat Treatment Glass ID=FY09EM21-23

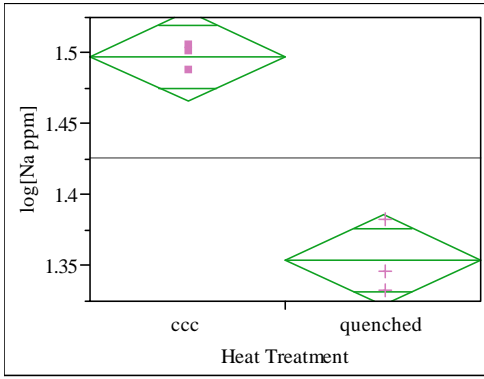

\section{Oneway Anova}

Summary of Fit

Rsquare

Root Men Square Error $\quad 0.939587$

Root Mean Square Error $\quad 0.019829$

1.425638
6

t Test

quenched-ccc

Assuming equal variances

Difference $\quad-0.14369$ t Ratio $\quad-8.87488$ Std Err Dif 0.01619 DF

\begin{tabular}{ll}
0.0009 \\
\hline pper CL Dif -0.09874 Prob $>|t|$ & 0.009
\end{tabular} Lower CL Dif -0.18864 Prob $>$ t 0.9996 Confidence $\quad 0.95$ Prob $<t \quad 0.0004$

Analysis of Variance

Source DF Sum of Squares Mean Square F Ratio Prob $>$ F

$\begin{array}{llllll}\text { Heat Treatment } & 1 & 0.03096926 & 0.030969 & 78.7635 & 0.0009 \\ \text { Error } & 4 & 0.00157277 & 0.000393 & & \end{array}$ 0.00157277

Means for Oneway Anova

Level Number Mean Std Error Lower 95\% Upper 95\% $\begin{array}{lllllr}\text { ccC } & 3 & 1.49748 & 0.01145 & 1.4657 & 1.5293\end{array}$

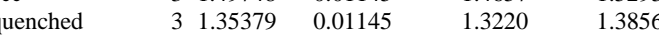

Std Error uses a pooled estimate of error variance
Oneway Analysis of $\log [\mathrm{Si}$ ppm] By Heat Treatment Glass

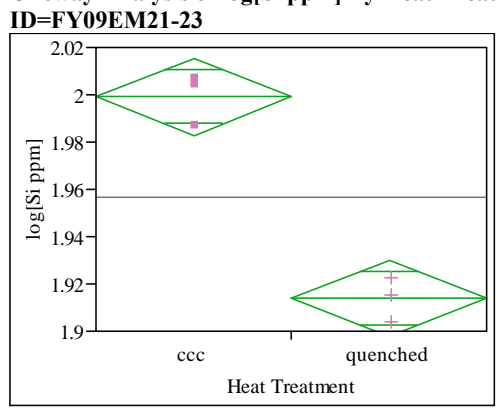

Oneway Anova

Summary of Fit

Rsquare

Adj Rsquare

0.96

Square Error $\quad 0.010115$ 1.956622

t Test

quenched-ccc

Assuming equal variances

Difference $\quad-0.08515$ t Ratio $\quad-10.3099$

Std Err Dif 0.00826 DF

Upper CL Dif $0.06222 \mathrm{PF}>/ \mathrm{l}-0.0005$

Lower CL Dif -0.10808 Prob $>t \quad 0.9998$

Confidence $\quad 0.95$ Prob $<\mathrm{t} \quad 0.0002$

Analysis of Variance

Source DF Sum of Squares Mean Square F Ratio Prob $>$ F

Heat Treatment $1 \quad 0.01087469$

Error

0.00040923
0.01128392

$\begin{array}{lll}0.010875 & 106.2939 & 0.0005\end{array}$

Means for Oneway Anova

Level Number Mean Std Error Lower 95\% Upper 95\%

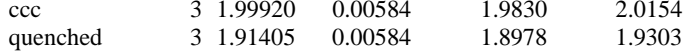

Std Error uses a pooled estimate of error variance
Oneway Analysis of $\log [\mathrm{B}$ ppm] By Heat Treatment Glass ID=FY09EM21-24

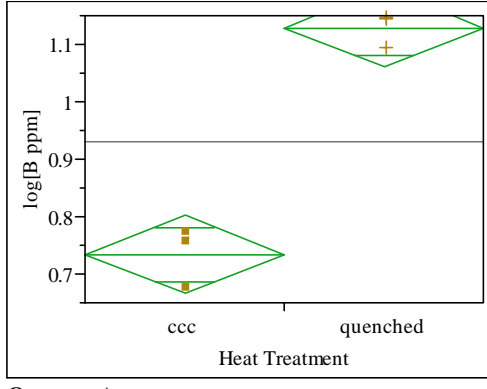

Oneway Anova

Adj Rsquare $\quad 0.963138$

Root Mean Square Error $\quad 0.042164$

Mean of Response 0.931456

Observations (or Sum Wgts) 6

Test

quenched-ccc

Assuming equal variances

Difference $\quad 0.394997$ t Ratio 11.47353 Std Err Dif 0.034427 DF

$\begin{array}{ll} & \end{array}$

Lower CL Dif 0.299413 Prob $>t \quad 0.0002$

$\begin{array}{lrl}\text { Confidence } & 0.95 \text { Prob }<\mathrm{t} & 0.9998\end{array}$

Analysis of Variance

Source DF Sum of Squares Mean Square F Ratio Prob $>$ F

$\begin{array}{lllrrr}\text { Heat Treatment } & 1 & 0.23403386 & 0.234034 & 131.6419 & 0.0003\end{array}$

$\begin{array}{lll}\text { Error } & 4 & 0.00711123\end{array}$ 0.001778

Means for Oneway Anova

Level Number Mean Std Error Lower 95\% Upper 95\%

$\begin{array}{llllll}\text { cCC } & 3 & 0.73396 & 0.02434 & 0.6664 & 0.8015\end{array}$

$\begin{array}{llllll}\text { quenched } & 3 & 1.12895 & 0.02434 & 1.0614 & 1.1965\end{array}$

Std Error uses a pooled estimate of error variance 
SRNL-STI-2009-00778, Revision 0

\section{Exhibit B5. Effects of Heat Treatment (HT) on PCT $\log (\mathrm{ppm})$ Response of Matrix 2 Non-Rad Glasses}

Oneway Analysis of $\log [\mathrm{Li}$ ppm] By Heat Treatment Glass

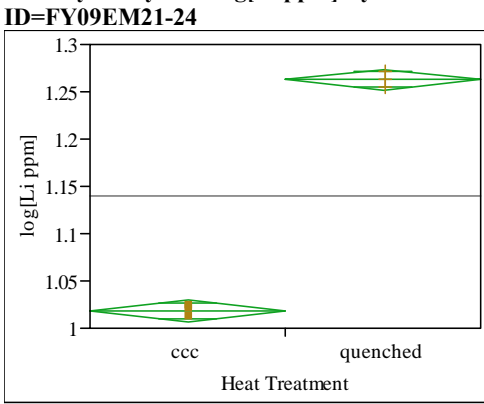

\section{Oneway Anova}

Heat Treatment

Summary of Fit

Rsquare

Adj Rsquare 0.997938

$\begin{array}{ll}\text { Adj Rsquare } & 0.997422 \\ \text { Root Mean Square Error } & 0.006815\end{array}$

Mean of Response

Observations (or Sum Wgts)

6

t Test

quenched-ccc

Assuming equal variances

Difference $\quad 0.244794$ t Ratio $\quad 43.99487$ Std Err Dif 0.005564 DF

Upper CL Dif 0.260242 Prob $>|t|<.0001$

Lower CL Dif 0.229345 Prob $>t \quad<.0001$

Confidence $\quad 0.95$ Prob $<\mathrm{t} \quad 1.0000$
Oneway Analysis of $\log [\mathrm{Na}$ ppm] By Heat Treatment Glass ID=FY09EM21-24

Analysis of Variance

Source DF Sum of Squares Mean Square F Ratio Prob $>$ F $\begin{array}{llllll}\text { Heat Treatment } & 1 & 0.08988605 & 0.089886 & 1935.549 & <.0001 \\ \text { Error } & 4 & 0.00018576 & 0.000046 & & \end{array}$ Error 0.09007181

Means for Oneway Anova

Level Number Mean Std Error Lower 95\% Upper 95\% $\begin{array}{llllll}\text { ccc } & 3 & 1.01841 & 0.00393 & 1.0075 & 1.0293 \\ \text { quenched } & 3 & 1.26320 & 0.00393 & 1.2523 & 1.2741\end{array}$

Std Error uses a pooled estimate of error variance

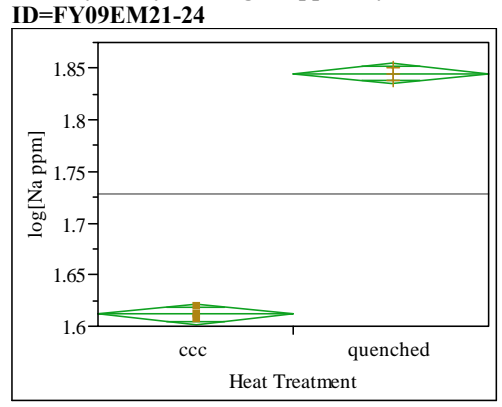

\section{Oneway Anova}

Summary of Fit

Rsquare

Adj Rsquare

Mean of Response

0.998105

0.597631 1.728158

t Test

quenched-ccc

Assuming equal variances

Difference $\quad 0.233148$ t Ratio $\quad 45.9005$

Std Err Dif 0.005079 DF

$\begin{array}{r}4 \\ <.0001 \\ \hline\end{array}$

Lower CL Dif 0.219045 Prob $>t<0.0001$

$\begin{array}{lrl}\text { Confidence } & 0.95 \text { Prob }<\mathrm{t} & 1.0000\end{array}$

Analysis of Variance

Source DF Sum of Squares Mean Square F Ratio Prob $>$ F $\begin{array}{lll}\text { Heat Treatment } & 1 & 0.08153679 \\ & 4 & 0.00015480\end{array}$ $\begin{array}{lll}\text { Error } & 4 & 0.00015480 \\ \text { C. Total } & 5 & 0.08169159\end{array}$ $0.0815372106 .856<0001$

Means for Oneway Anova

Level Number Mean Std Error Lower 95\% Upper 95\%

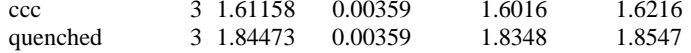

Std Error uses a pooled estimate of error variance
Oneway Analysis of $\log [\mathrm{Si}$ ppm] By Heat Treatment Glass ID=FY09EM21-24

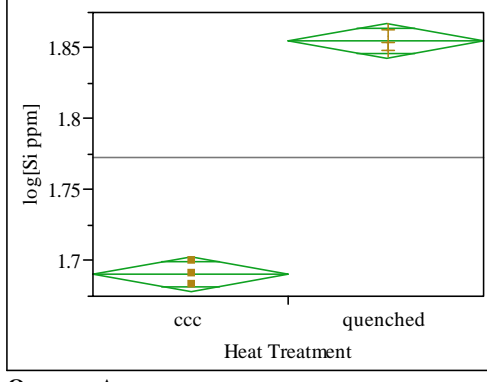

Oneway Anova

Summary of Fit

Rsquare

Adj Rsquare $\quad 0.994292$

0.992864

0.992864
$\quad$ Square Error $\quad 0.007624$

Observations (or Sum Wgts) $\quad 6$

t Test

quenched-ccc

Assuming equal variance

Difference $\quad 0.164304$ t Ratio $\quad 26.39543$

Std Err Dif $\quad 0.006225$ DF

Upper CL Dif 0.181587 Prob $>|t| \quad<.0001$

Lower CL Dif 0.147022 Prob $>t \quad<.0001$

Confidence $\quad 0.95$ Prob $<\mathrm{t} \quad 1.0000$

Analysis of Variance

DF Sum of Squares Mean Square F Ratio Prob $>$ F $\begin{array}{llllll}\text { Heat Treatment } & 1 & 0.04049382 & 0.040494 & 696.7185 & <.0001\end{array}$ $\begin{array}{lll}\text { Error } & 4 & 0.00023248\end{array}$ 0.000058

\section{Means for Oneway Anova}

Level Number Mean Std Error Lower 95\% Upper 95\% $\begin{array}{llllll}\text { ccc } & 3 & 1.69065 & 0.00440 & 1.6784 & 1.7029 \\ \text { quenched } & 3 & 1.85495 & 0.00440 & 1.8427 & 1.8672\end{array}$

Std Error uses a pooled estimate of error variance 
SRNL-STI-2009-00778, Revision 0

\section{Exhibit B5. Effects of Heat Treatment (HT) on PCT $\log (\mathrm{ppm})$ Response of Matrix 2 Non-Rad Glasses}

Oneway Analysis of $\log [\mathrm{B}$ ppm] By Heat Treatment Glass

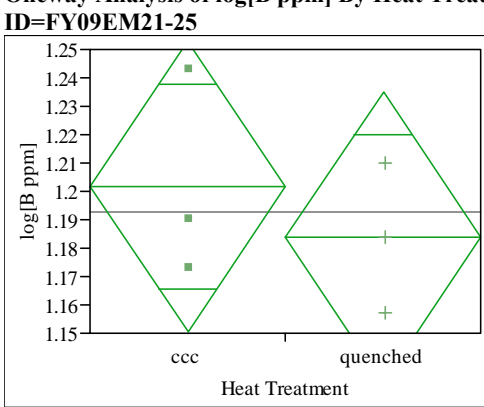

Oneway Anova

Heat Treatmen

Summary of Fit

Adj Rsquare

$\begin{array}{lr}\text { Mean of Response } & 1.192786 \\ \text { Observations (or Sum Wgts) } & 6\end{array}$

t Test

quenched-ccc

Assuming equal variances

Difference $\quad-0.01818$ t Ratio $\quad-0.69774$ Std Err Dif 0.02606 DF

Upper CL Dif 0.05416 Prob $>|t| \quad 0.5238$

Lower CL Dif -0.09052 Prob $>$ t 0.7381

$\begin{array}{lrl}\text { Confidence } & 0.95 \text { Prob }<\mathrm{t} \quad 0.2619\end{array}$

Analysis of Variance

Source DF Sum of Squares Mean Square F Ratio Prob $>$ F

$\begin{array}{llllll}\text { Heat Treatment } & 1 & 0.00049578 & 0.000496 & 0.4868 & 0.5238\end{array}$

C. Total

0.00407350 0.001018

Means for Oneway Anova

Level Number Mean Std Error Lower 95\% Upper 95\% $\begin{array}{llllll}\text { cCC } & 3 & 1.20188 & 0.01842 & 1.1507 & 1.2530\end{array}$ $\begin{array}{lllll}3 & 1.18370 & 0.01842 & 1.1325 & 1.2349\end{array}$

Std Error uses a pooled estimate of error variance
Oneway Analysis of $\log [\mathrm{Li}$ ppm] By Heat Treatment Glass ID=FY09EM21-25

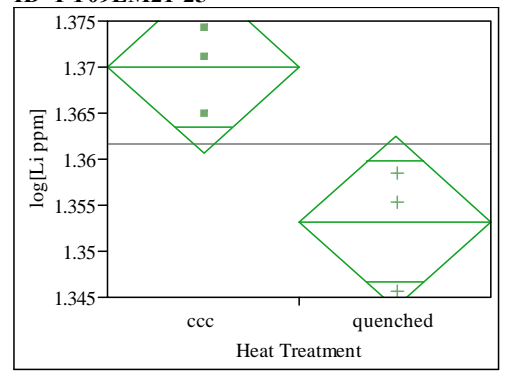

Oneway Anova

Summary of Fit

Rsquare

Adj Rsquare

Root Mean Square

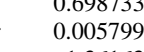

Observations (or Sum Wgts)

(1)

t Test

quenched-ccc

Assuming equal variances

Difference $\quad-0.01680$ t Ratio $\quad-3.54917$

$\begin{array}{ll}0.00473 \mathrm{DF} & 4.547 \\ 0.0238\end{array}$

$\begin{array}{lll}0.00473 & \text { PF }>|t| & 0.0238 \\ \text { Lower CL Dif }-0.003695 & \text { Prob }>t & 0.9881\end{array}$

Confidence $\quad 0.95$ Prob $<t \quad 0.0119$

Analysis of Variance

Source DF Sum of Squares Mean Square F Ratio Prob $>$ F

$\begin{array}{lllllll}\text { Heat Treatment } & 1 & 0.00042355 & 0.000424 & 12.5966 & 0.0238\end{array}$

Error

C. Total

0.00013450

Means for Oneway Anova

Level Number Mean Std Error Lower 95\% Upper 95\%

$\begin{array}{llllll} & 3 & 1.37003 & 0.00335 & 1.3607 & 1.3793 \\ \text { quenched } & 3 & 1.35323 & 0.00335 & 1.3439 & 1.3625\end{array}$

Std Error uses a pooled estimate of error variance
Oneway Analysis of $\log [\mathrm{Na}$ ppm] By Heat Treatment Glass ID=FY09EM21-25

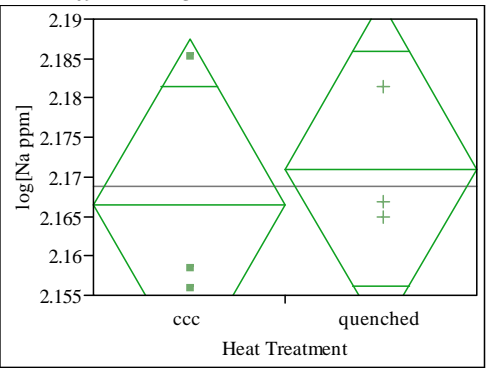

Oneway Anova

Summary of Fit

Rsquare

Root Mean Square Error $\quad-0.19607$

Mean of Response 2.168743

Observations (or Sum Wgts) 2.168743

t Test

quenched-ccc

Assuming equal variances

Difference $\quad 0.00456$ t Ratio $\quad 0.424675$

Std Err Dif $\quad 0.01073$ DF

$\begin{array}{rrr}\text { Upper CL Dif } & 0.03435 \text { Prob }>|t| & 0.6929 \\ \text { Lower CL Dif }-0.02524 \text { Prob }>t & 0.3465\end{array}$

Confidence $\quad 0.95$ Prob $<\mathrm{t} \quad 0.6535$

Analysis of Variance

Source DF Sum of Squares Mean Square F Ratio Prob $>$ F

$\begin{array}{llllll}\text { Heat Treatment } & 1 & 0.00003115 & 0.000031 & 0.1803 & 0.6929\end{array}$

$\begin{array}{lll}\text { Error } & 4 & 0.00069092\end{array}$

0.000173

Means for Oneway Anova

Level Number Mean Std Error Lower 95\% Upper 95\%

$\begin{array}{llllll}\text { cCC } & 3 & 2.16646 & 0.00759 & 2.1454 & 2.1875\end{array}$

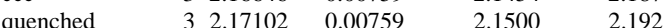

Std Error uses a pooled estimate of error variance 
SRNL-STI-2009-00778, Revision 0

\section{Exhibit B5. Effects of Heat Treatment (HT) on PCT $\log (\mathrm{ppm})$ Response of Matrix 2 Non-Rad Glasses}

Oneway Analysis of $\log [\mathrm{Si}$ ppm] By Heat Treatment Glass

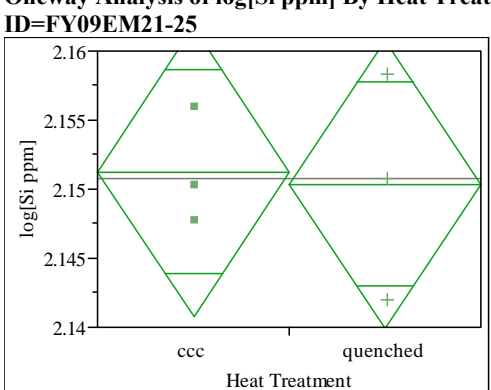

Oneway Anova

Summary of Fit

Rsquare

Root Mean Square Error $\quad-0.24128$

(2.150818

2.150818

t Test

quenched-ccc

Assuming equal variances

Difference $\quad-0.00089$ t Ratio $\quad-0.1676$

Std Err Dif $0.00531 \mathrm{DF} \quad-0.1676$

Upper CL Dif 0.01386 Prob $>|t| \quad 0.8750$

$\begin{array}{lll}\text { Lower CL Dif }-0.01564 \text { Prob }>t & 0.5625 \\ \text { Con }\end{array}$

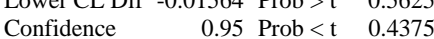

Analysis of Variance

Source DF Sum of Squares Mean Square F Ratio Prob $>$ F

$\begin{array}{lrrrrr}\text { Heat Treatment } & 1 & 0.00000119 & 1.19 \mathrm{e}-6 & 0.0281 & 0.8750 \\ \text { Error } & 4 & 0.00016942 & 0.000042 & & \end{array}$

C. Total

0.0001706

0.000042

Means for Oneway Anova

Level Number Mean Std Error Lower 95\% Upper 95\%

$\begin{array}{lrrrrr}\text { Level } & \text { Number } & \text { Mean } & \text { Std Error } & \text { Lower 95\% } & \text { Upper 95\% } \\ \text { ccc } & 3 & 2.15126 & 0.00376 & 2.1408 & 2.1617 \\ \text { quenched } & 3 & 2.15037 & 0.00376 & 2.1399 & 2.1608\end{array}$

Std Error uses a pooled estimate of error variance
Oneway Analysis of $\log [\mathrm{B}$ ppm] By Heat Treatment Glass

ID=FY09EM21-26

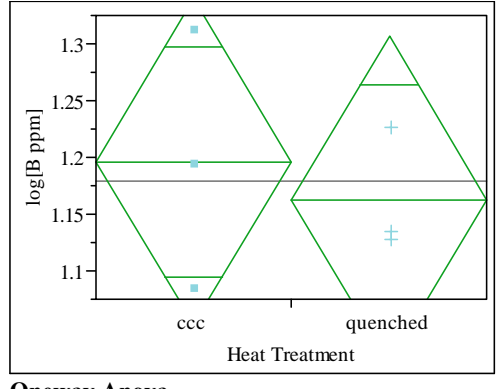

Oneway Anova

Summary of Fit

Rsquare

Adj Rsquare

Root Mean Square Er

0.04871

Mean of Respons

$-0.18911$

0.089581

Observations (or Sum Wgts)

6

t Test

quenched-ccc

Assuming equal variances

Difference $\quad-0.03310$ t Ratio $\quad-0.45257$

Std Err Dif 0.07314 DF

Upper CL Dif $0.16997 \mathrm{Prob}>\mathrm{HI} \quad 0.6743$

Lower CL Dif -0.23618 Prob $>$ t $\quad 0.6628$

Confidence $\quad 0.95$ Prob $<\mathrm{t} \quad 0.3372$

Analysis of Variance

Source DF Sum of Squares Mean Square F Ratio Prob $>$ F

Heat Treatment $\quad 1 \quad 0.00164360$

$\begin{array}{lll}\text { Error } & 4 & 0.03209883 \\ \text { C. Total } & 5 & 0.03374243\end{array}$

$\begin{array}{lll}0.001644 & 0.2048 \quad 0.6743\end{array}$

Means for Oneway Anova

Level Number Mean Std Error Lower 95\% Upper 95\%

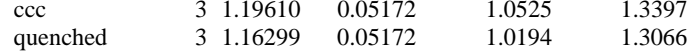

Std Error uses a pooled estimate of error variance
Oneway Analysis of $\log [\mathrm{Li}$ ppm] By Heat Treatment Glass

ID=FY09EM21-26

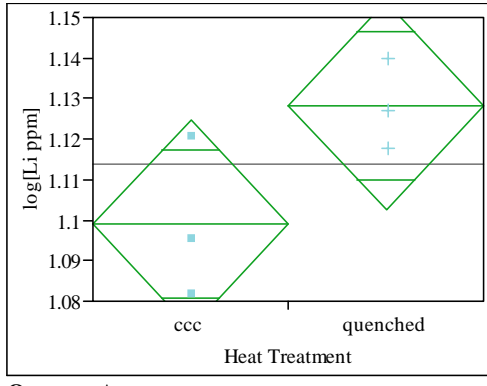

Oneway Anova

Summary of Fit

0.553568

Adj Rsquare $\quad 0.441961$

Root Mean Square Error $\quad 0.016035$

Mean of Response

1.113698

Observations (or Sum Wgts)

t Test

quenched-ccc

Assuming equal variances

Difference $\quad 0.02916$ t Ratio $\quad 2.227092$ Std Err Dif $\quad 0.01309$ DF

Upper CL Dif 0.06551 Prob $>|t| \quad 0.0899$

Lower CL Dif -0.00719 Prob $>$ t 0.0450

$\begin{array}{lll}\text { Confidence } & 0.95 \text { Prob }<\mathrm{t} \quad 0.9550\end{array}$

Analysis of Variance

Source DF Sum of Squares Mean Square F Ratio Prob $>$ F $\begin{array}{lllrlrl}\text { Heat Treatment } & 1 & 0.00127536 & 0.001275 & 4.9599 & 0.0899\end{array}$ $\begin{array}{lll}\text { Error } & 4 & 0.00102853\end{array}$

Means for Oneway Anova

Level Number Mean Std Error Lower 95\% Upper 95\%

$\begin{array}{llrrrr}\text { ccC } & 3 & 1.09912 & 0.00926 & 1.0734 & 1.1248\end{array}$

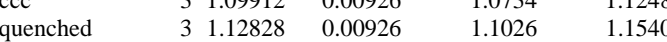

Std Error uses a pooled estimate of error variance 
SRNL-STI-2009-00778, Revision 0

\section{Exhibit B5. Effects of Heat Treatment (HT) on PCT $\log (\mathrm{ppm})$ Response of Matrix 2 Non-Rad Glasses}

Oneway Analysis of $\log [\mathbf{N a}$ ppm] By Heat Treatment Glass

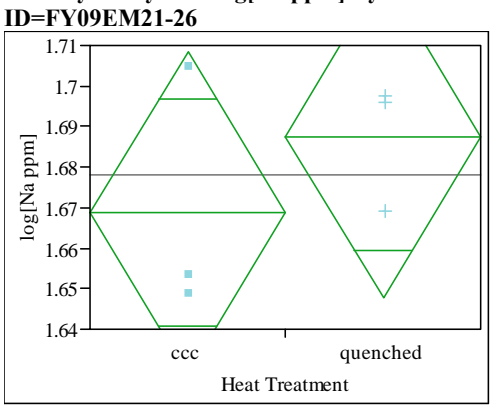

Oneway Anova

Summary of Fit

Rsquare

$\begin{array}{ll} & 0.176359 \\ \text { Adj Rsquare } & -0.02955\end{array}$

Root Mean Square Error $\quad 0.024834$

Mean of Response $\quad 1.678156$

Observations (or Sum Wgts)

t Test

quenched-ccc

Assuming equal variances

Difference $\quad 0.01877$ t Ratio $\quad 0.925466$ Std Err Dif 0.02028 DF

Upper CL Dif 0.07506 Prob $>|t| \quad 0.407$

Lower CL Dif -0.03753 Prob $>t \quad 0.2036$

Confidence $\quad 0.95$ Prob $<t \quad 0.7964$

Analysis of Variance

Source DF Sum of Squares Mean Square F Ratio Prob $>$ F

$\begin{array}{llllll}\text { Heat Treatment } & 1 & 0.00052822 & 0.000528 & 0.8565 & 0.4071 \\ \text { Error } & 4 & 0.00246693 & 0.000617 & & \end{array}$

0.00246693

Means for Oneway Anova

Level Number Mean Std Error Lower 95\% Upper 95\%

$\begin{array}{lllllr}\text { ccc } & 3 & 1.66877 & 0.01434 & 1.6290 & 1.7086\end{array}$

$\begin{array}{llllll}\text { quenched } & 3 & 1.68754 & 0.01434 & 1.6477 & 1.7273\end{array}$

Std Error uses a pooled estimate of error variance
Oneway Analysis of $\log [\mathrm{Si}$ ppm] By Heat Treatment Glass

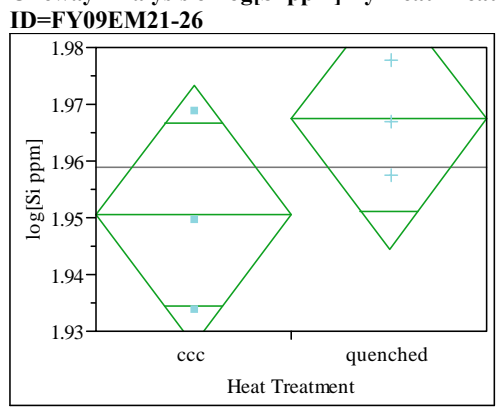

Oneway Anova

Summary of Fit

Rsquare

Adj Rsquare

oot Men Square Error $\quad 0.17947$

Mean of Respone Eror 0.014267

Observations (or Sum Wgts)

t Test

quenched-ccc

Assuming equal variances

Difference $\quad 0.01686$ t Ratio $\quad 1.446936$

Std Err Dif $\quad 0.01165$ DF

4
0.2215

$\begin{array}{lll}\text { Lower CL Dif }-0.01549 \text { Prob }>t & 0.1107\end{array}$

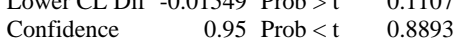

Analysis of Variance

Source DF Sum of Squares Mean Square F Ratio Prob $>$ $\begin{array}{llllll}\text { Heat Treatment } & 1 & 0.00042615 & 0.000426 & 2.0936 & 0.2215\end{array}$ Error 0.00081419
0.00124034

Means for Oneway Anova

Level Number Mean Std Error Lower 95\% Upper 95\%

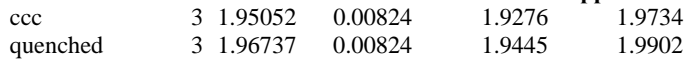

Std Error uses a pooled estimate of error variance
Oneway Analysis of $\log [\mathrm{B}$ ppm] By Heat Treatment Glass

ID=FY09EM21-27

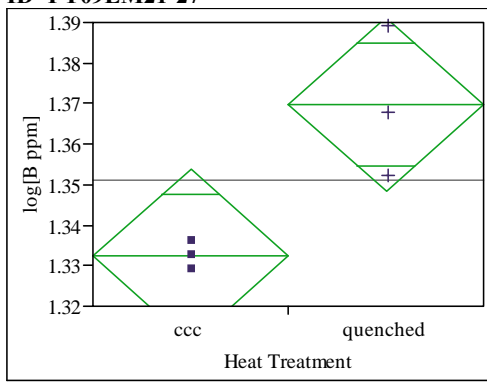

Oneway Anova

Summary of Fit

0.746235

0.682793

Root Mean Square Error 0.013336

Mean of Response

1.351111

t Test

quenched-ccc

Assuming equal variances

Difference $\quad 0.037345$ t Ratio $\quad 3.429665$

Std Err Dif 0.010859 t DF

Upper DL D 0.010889 DF $>0.078$ Pro 0.0265

Upe CL Dif 0.067578 Prob $>|1| \quad 0.0265$

$\begin{array}{lrr}\text { Lower CL Dif } & 0.007113 \text { Prob }>t & 0.0133 \\ \text { Confidence } & 0.95 \text { Prob }<\mathrm{t} & 0.9867\end{array}$

Analysis of Variance

Source DF Sum of Squares Mean Square F Ratio Prob > F $\begin{array}{lrrrrr}\text { Heat Treatment } & 1 & 0.00209201 & 0.002092 & 11.7626 & 0.0265\end{array}$ $\begin{array}{lll}\text { Error } & 4 & 0.00071141 \\ & 5 & 0.0028343\end{array}$ 0.000178

\section{Means for Oneway Anova}

Level Number Mean Std Error Lower 95\% Upper 95\%

$\begin{array}{llllll}\text { ccc } & 3 & 1.33244 & 0.00770 & 1.3111 & 1.3538 \\ \text { quenched } & 3 & 1.36978 & 0.00770 & 1.3484 & 1.3912\end{array}$

$\begin{array}{llll}3 & 1.36978 & 0.00770 & 1.348\end{array}$

Std Error uses a pooled estimate of error variance 
SRNL-STI-2009-00778, Revision 0

\section{Exhibit B5. Effects of Heat Treatment (HT) on PCT $\log (\mathrm{ppm})$ Response of Matrix 2 Non-Rad Glasses}

Oneway Analysis of $\log [\mathrm{Li}$ ppm] By Heat Treatment Glass

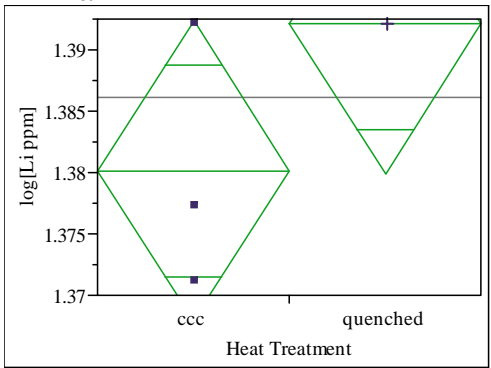

Oneway Anova

Summary of Fit

$\begin{array}{lr}\text { Rsquare } & 0.47921 \\ \text { Adj Rsquare } & 0.349013 \\ \text { Root Mean Square Error } & 0.007654 \\ \text { Mean of Response } & 1.386124 \\ \text { Obse }\end{array}$

Observations (or Sum Wgts)

t Test

quenched-ccc

Assuming equal variances

Difference $\quad 0.01199$ t Ratio 1.918501

Std Err Dif $\quad 0.00625$ DF

Upper CL Dif 0.02934 Prob $>|t| \quad 0.1275$

wer CL Dif -0.00536 Prob $>$

Confidence $\quad 0.95$ Prob $<\mathrm{t} \quad 0.9363$

Analysis of Variance

Source DF Sum of Squares Mean Square F Ratio Prob $>$ F

$\begin{array}{llllll}\text { Heat Treatment } & 1 & 0.00021562 & 0.000216 & 3.6806 & 0.1275\end{array}$

Error 0.000059

0.00044995

Means for Oneway Anova

Level Number Mean Std Error Lower 95\% Upper 95\%

\begin{tabular}{llllll}
\hline CC & 3 & 1.38013 & 0.00442 & 1.3679 & 1.3924
\end{tabular}

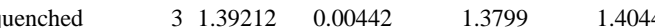

Std Error uses a pooled estimate of error variance
Oneway Analysis of $\log [\mathrm{Na}$ ppm] By Heat Treatment Glass

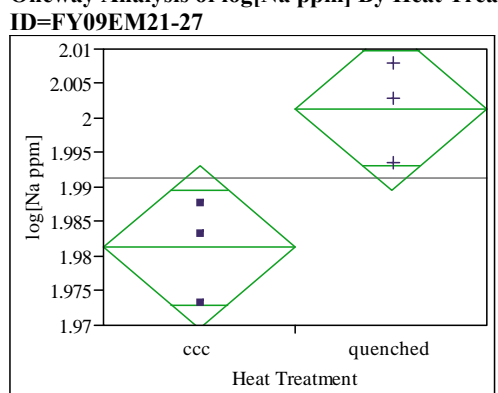

\section{Oneway Anova}

Summary of Fit

Rsquare

Adj Rsquare

Root Mean Square

\subsection{3}

0.673316
0.007351

Mean of Response

1.991322

Observations (or Sum Wgts)

t Test

quenched-ccc

Assuming equal variances

Difference $\quad 0.020181$ t Ratio 3.362335

$\begin{array}{llr}\text { Std Err Dif } & 0.006002 \text { DF } & 4\end{array}$

Upper CL Dif 0.036845 Prob $>|t| \quad 0.0282$

$\begin{array}{lll}\text { Lower CL Dif } & 0.003517 \text { Prob }>t & 0.0141\end{array}$

Confidence $\quad 0.95$ Prob $<\mathrm{t} \quad 0.9859$

Analysis of Variance

Source DF Sum of Squares Mean Square F Ratio Prob $>$ F $\begin{array}{lllrlll}\text { Heat Treatment } & 1 & 0.00061089 & 0.000611 & 11.3053 & 0.0282\end{array}$ Error 450.00021614 0.00021614 0.000054

Means for Oneway Anova

Level Number Mean Std Error Lower 95\% Upper 95\%

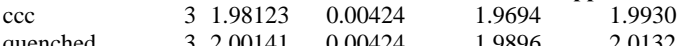

Std Error uses a pooled estimate of error variance
Oneway Analysis of $\log [\mathrm{Si}$ ppm] By Heat Treatment Glass ID=FY09EM21-27

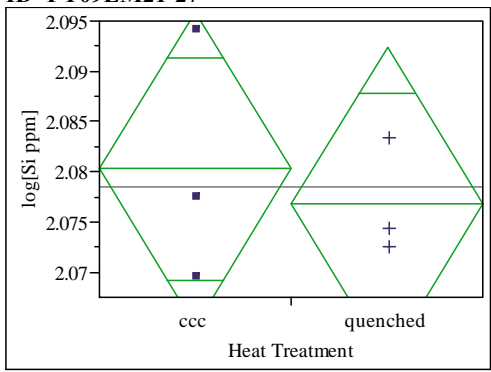

Oneway Anova

Summary of Fit

$\begin{array}{lr}\text { Rsquare } & 0.046541 \\ \text { Adj Rsquare } & -0.19182 \\ \text { Root Mean Square Error } & 0.009782 \\ \text { Mean of Response } & 2.078509 \\ \text { Observations (or Sum Wgts) } & 6\end{array}$

t Test

quenched-ccc

Assuming equal variances

Difference $\quad-0.00353$ t Ratio $\quad-0.44187$

Std Err Dif $\quad 0.00799$ DF $\quad 4$

Upper CL Dif 0.01865 Prob $>|t| 0.6814$

Lower CL Dif -0.02570 Prob $>t \quad 0.6593$

Confidence $\quad 0.95$ Prob $<\mathrm{t} \quad 0.3407$

\section{Analysis of Variance}

Source DF Sum of Squares Mean Square F Ratio Prob $>$ F $\begin{array}{llllll}\text { Heat Treatment } & 1 & 0.00001868 & 0.000019 & 0.1953 & 0.6814\end{array}$ $\begin{array}{lll}\text { Error } & 4 & 0.00038273\end{array}$ 0.000096

Means for Oneway Anova

Level Number Mean Std Error Lower 95\% Upper 95\%

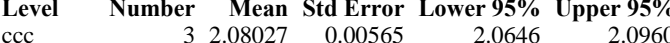

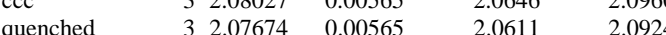

Std Error uses a pooled estimate of error variance 


\section{Exhibit B6. Effects of Heat Treatment for the Matrix 2 Non-Rad Glasses by Compositional View}

Comp View $=$ Measured

Variability Chart for $\log$ NL[B (g/L)]

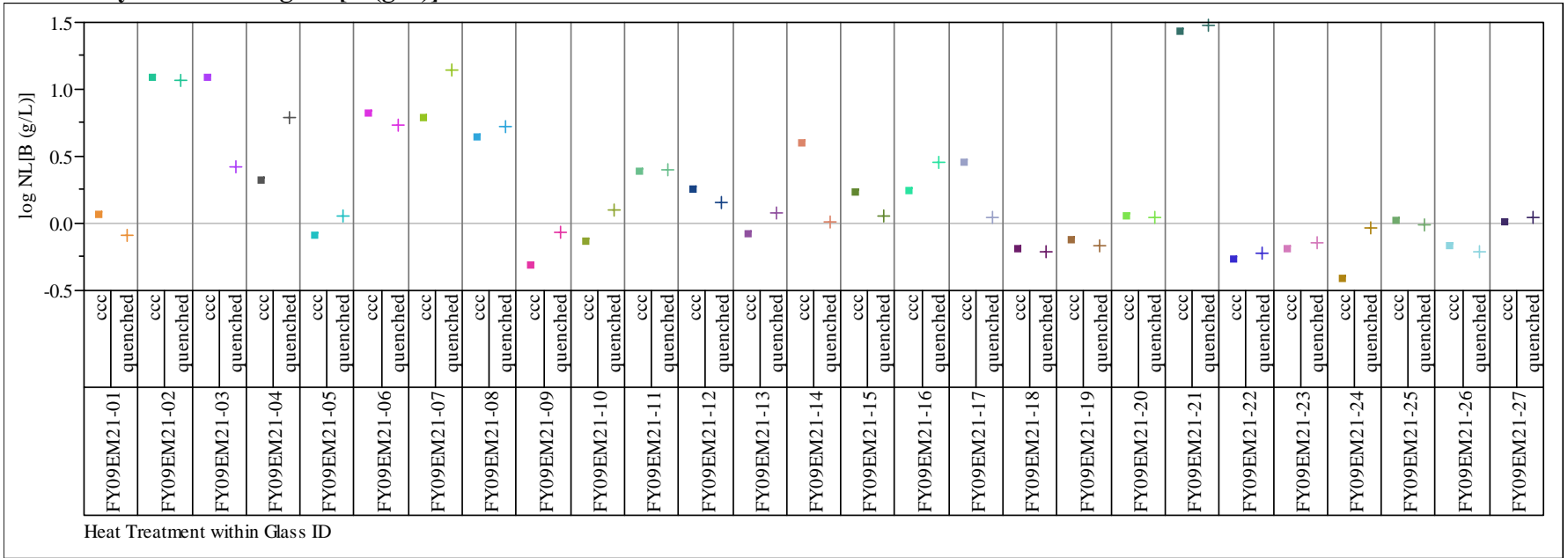

Comp View $=$ Measured

Variability Chart for $\log \mathrm{NL}[\mathrm{Li}(\mathrm{g} / \mathrm{L})]$

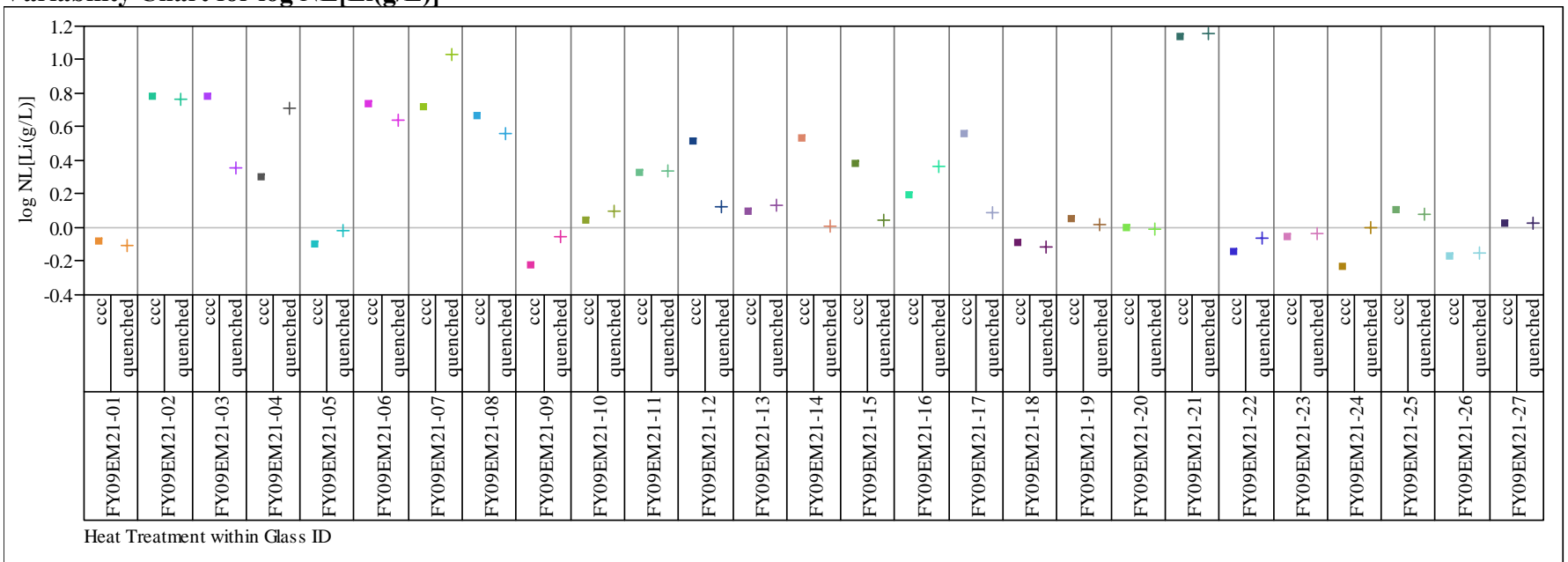


Exhibit B6. Effects of Heat Treatment for the Matrix 2 Non-Rad Glasses by Compositional View

Comp View $=$ Measured

Variability Chart for log NL[Na (g/L)]

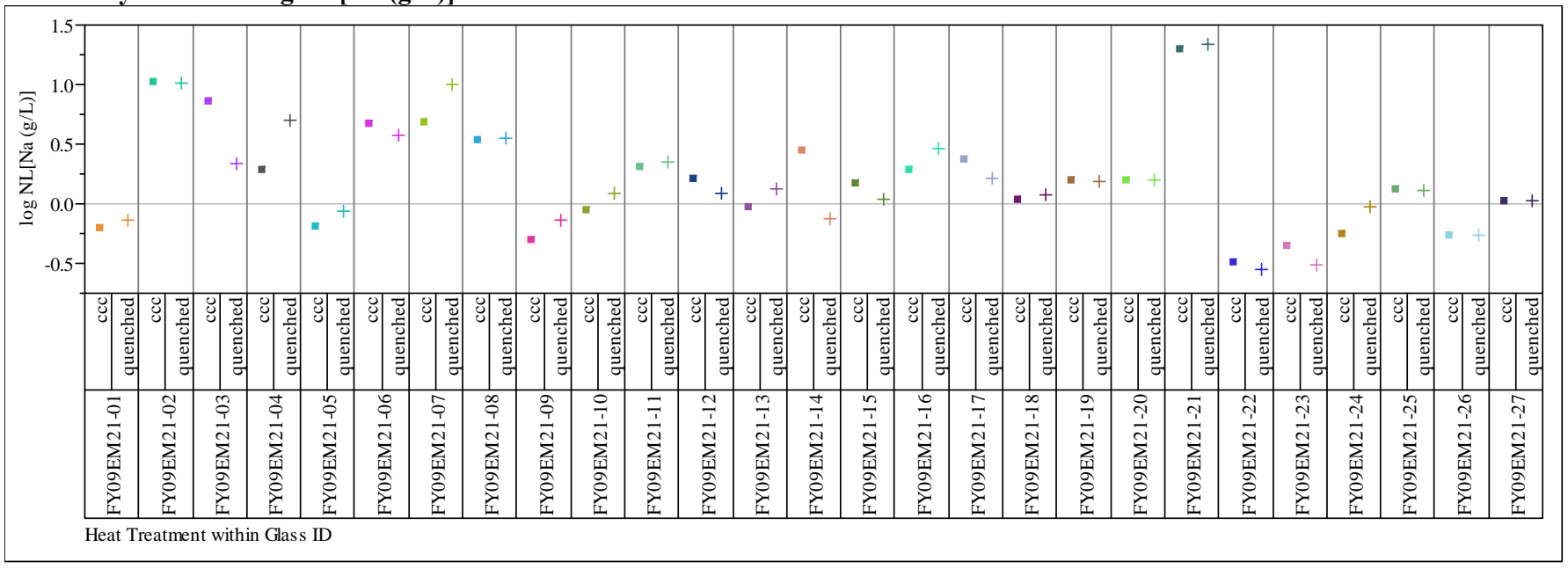

Comp View $=$ Measured

Variability Chart for $\log \mathrm{NL}[\mathrm{Si}(\mathrm{g} / \mathrm{L})]$

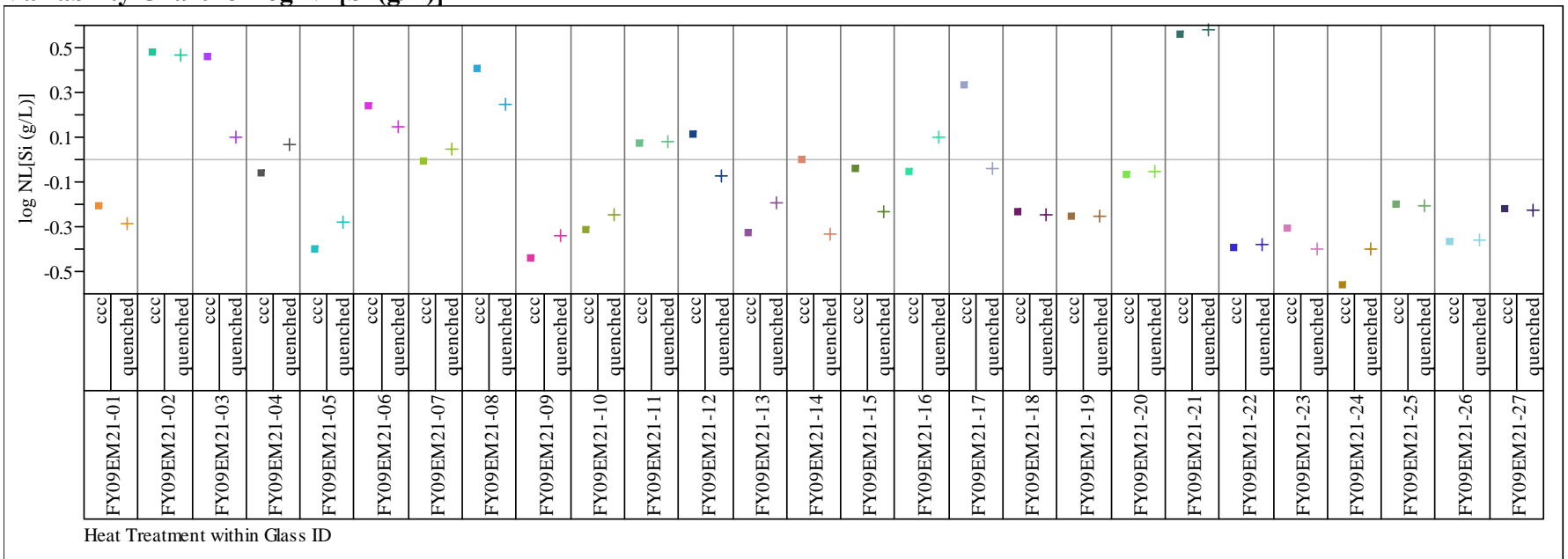




\section{Exhibit B6. Effects of Heat Treatment for the Matrix 2 Non-Rad Glasses by Compositional View}

Comp View=Measured bc

Variability Chart for $\log$ NL[B (g/L)]

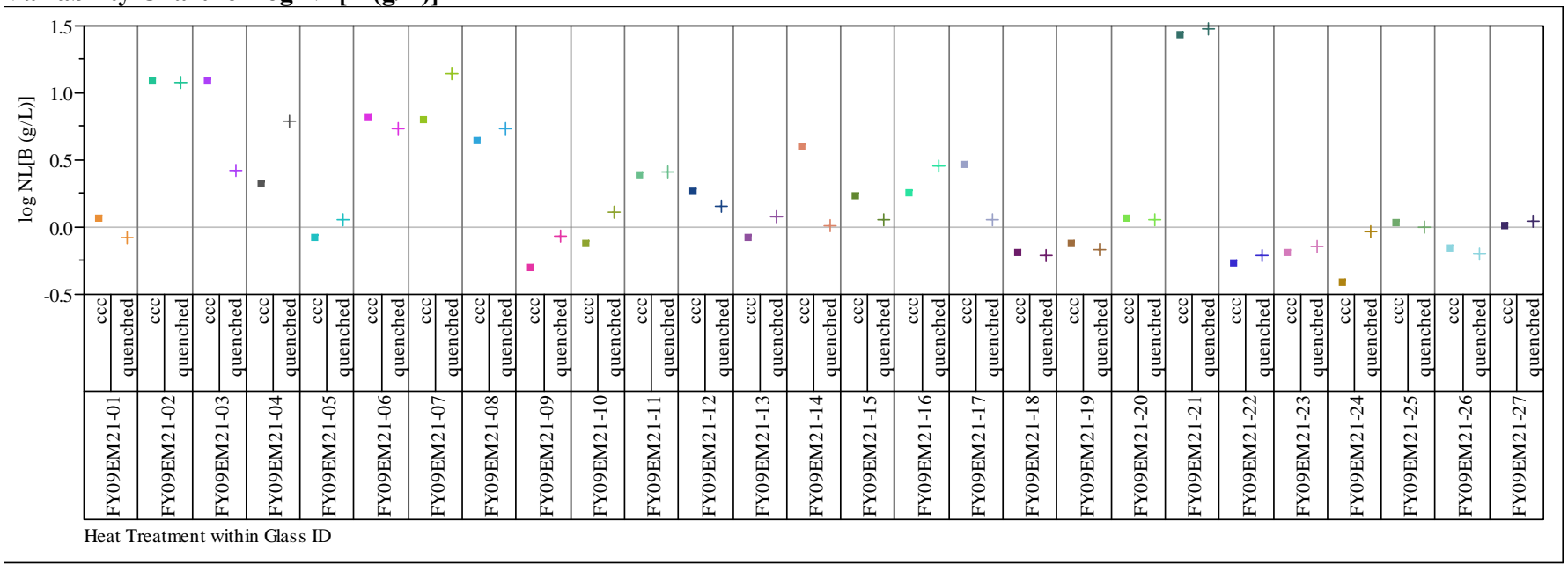

Comp View $=$ Measured bc

Variability Chart for $\log \mathrm{NL}[\mathrm{Li}(\mathrm{g} / \mathrm{L})]$

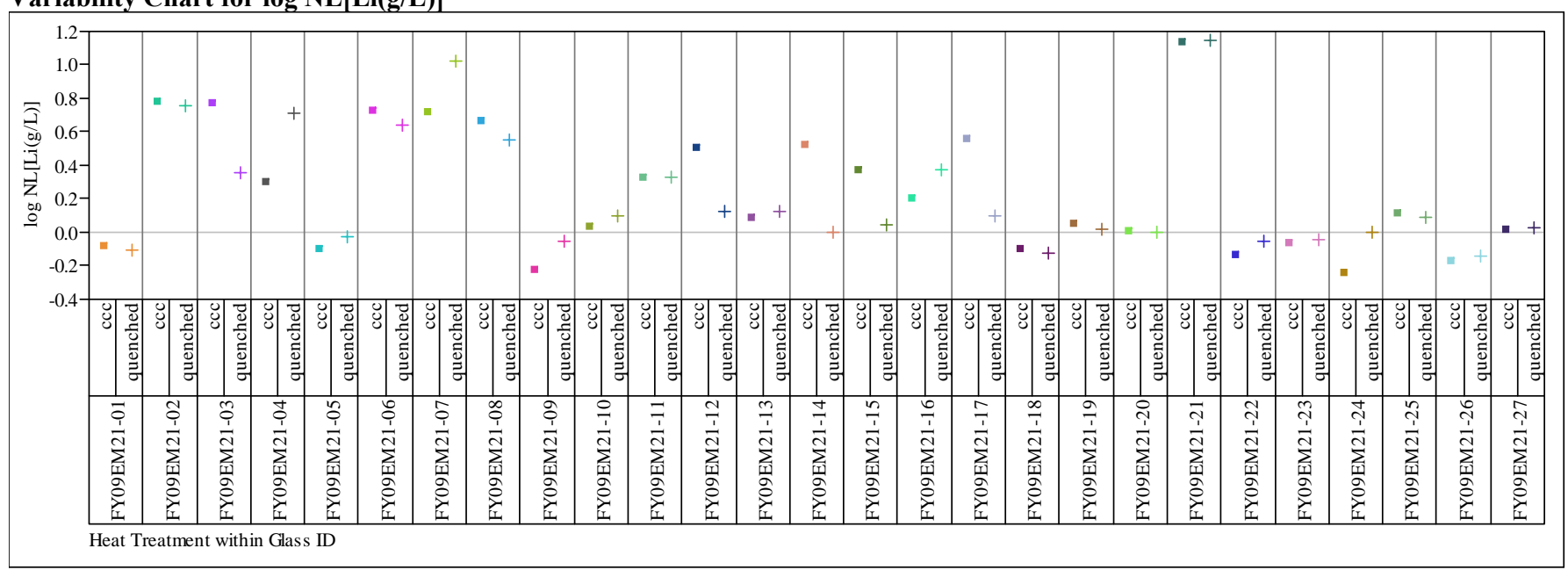




\section{Exhibit B6. Effects of Heat Treatment for the Matrix 2 Non-Rad Glasses by Compositional View}

Comp View $=$ Measured bc

Variability Chart for log NL[Na (g/L)]

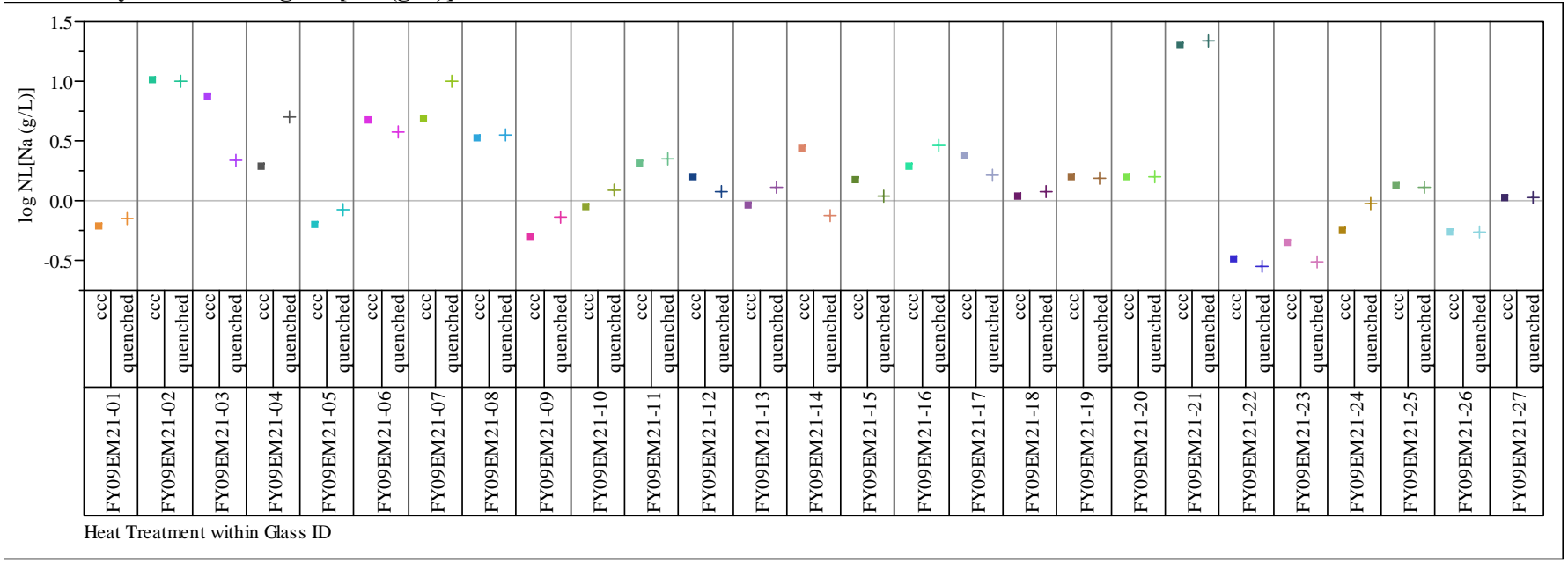

Comp View $=$ Measured bc

Variability Chart for $\log \mathrm{NL}[\mathrm{Si}(\mathrm{g} / \mathrm{L})]$

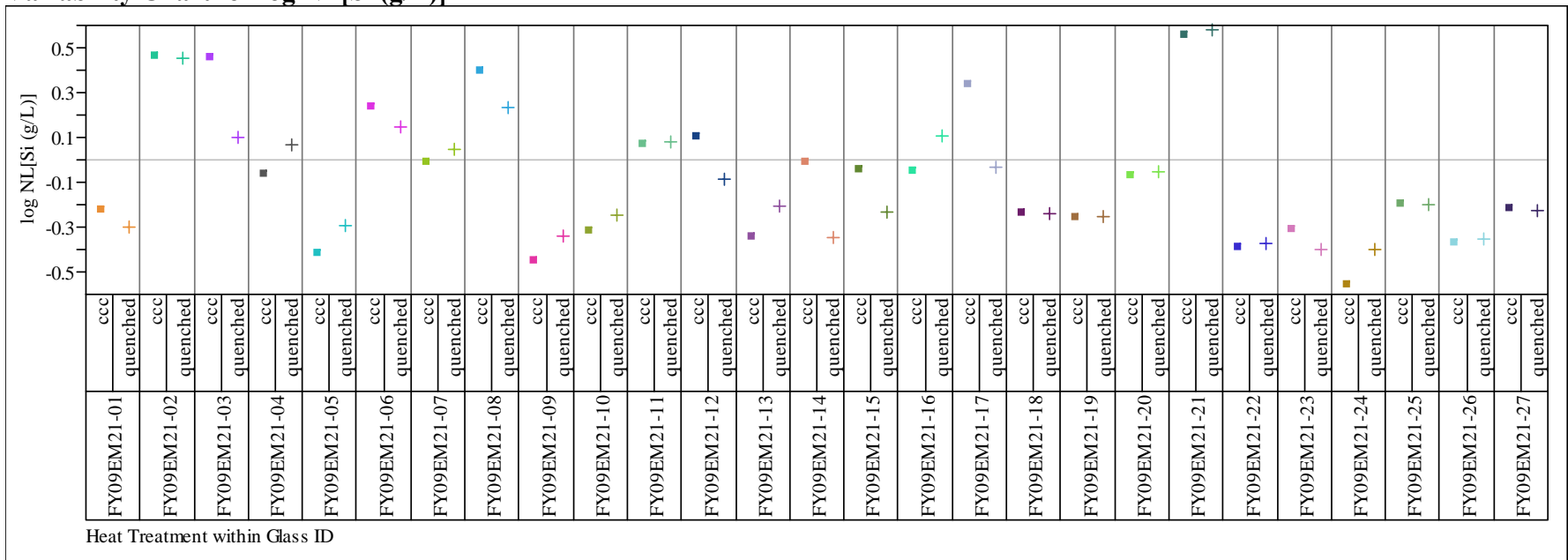


Exhibit B6. Effects of Heat Treatment for the Matrix 2 Non-Rad Glasses by Compositional View

Comp View $=$ Targeted

Variability Chart for $\log$ NL[B (g/L)]

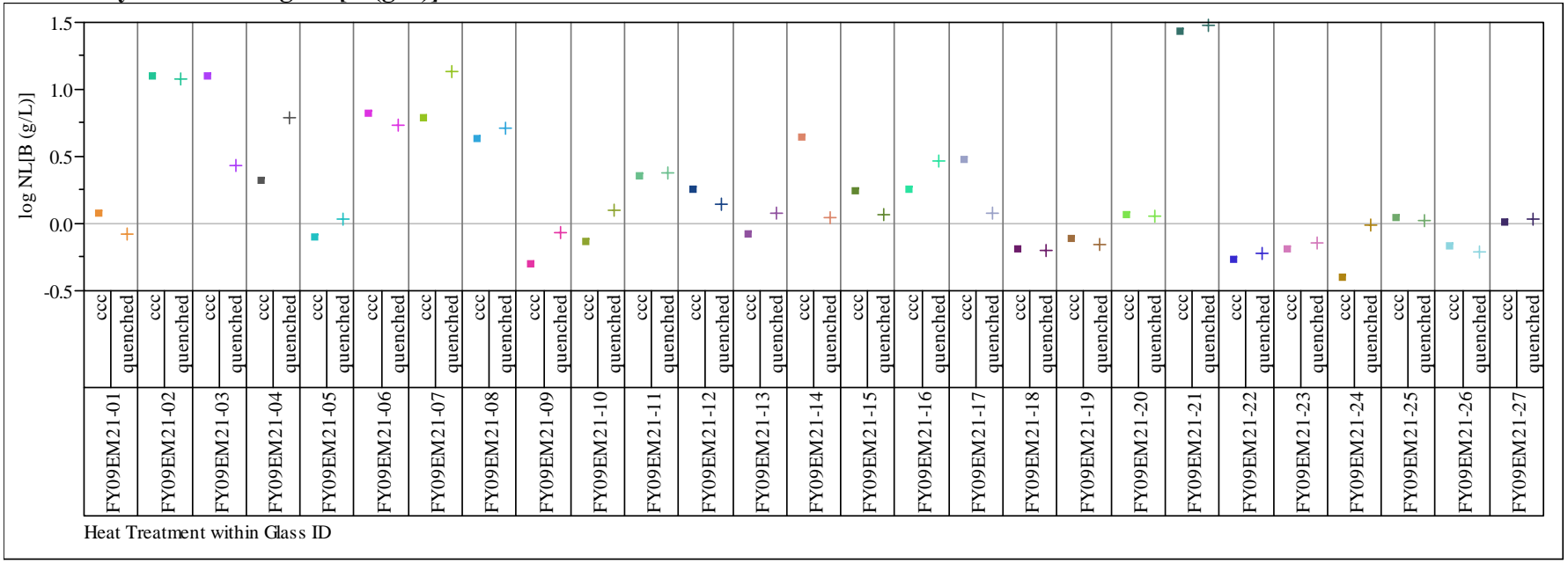

Comp View=Targeted

Variability Chart for $\log \mathrm{NL}[\mathrm{Li}(\mathrm{g} / \mathrm{L})]$

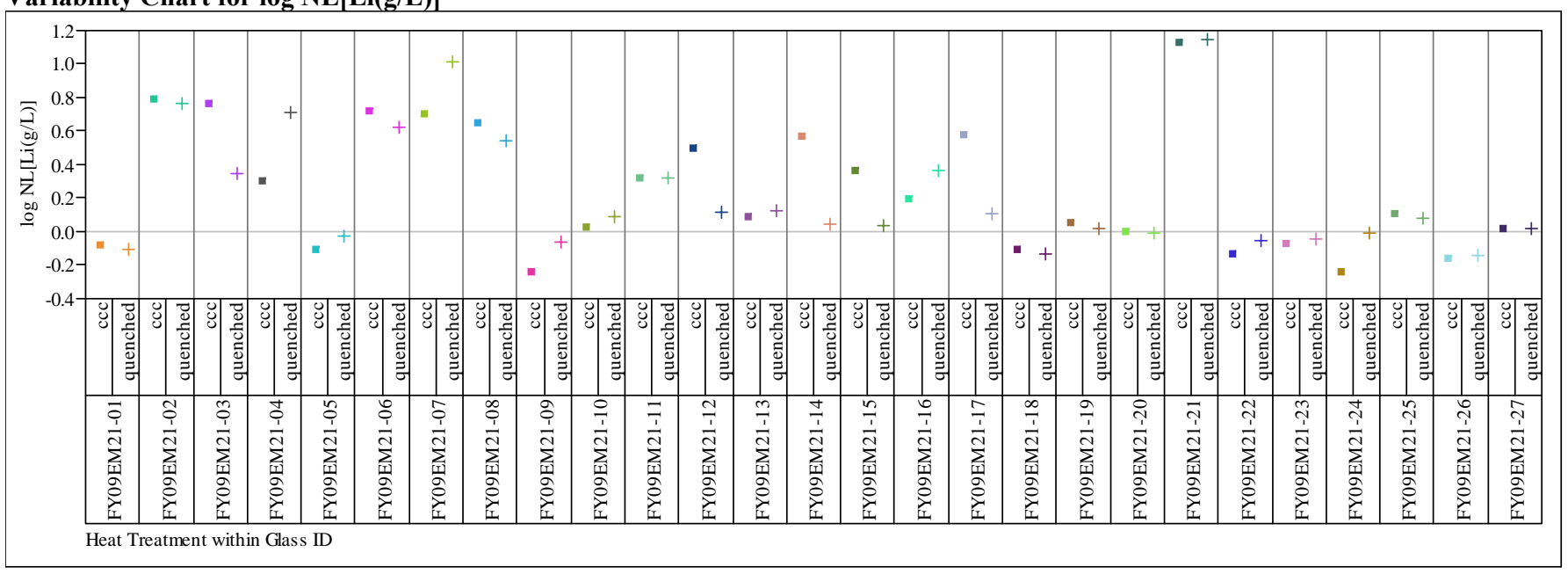


Exhibit B6. Effects of Heat Treatment for the Matrix 2 Non-Rad Glasses by Compositional View

Comp View=Targeted

Variability Chart for log NL[Na (g/L)]

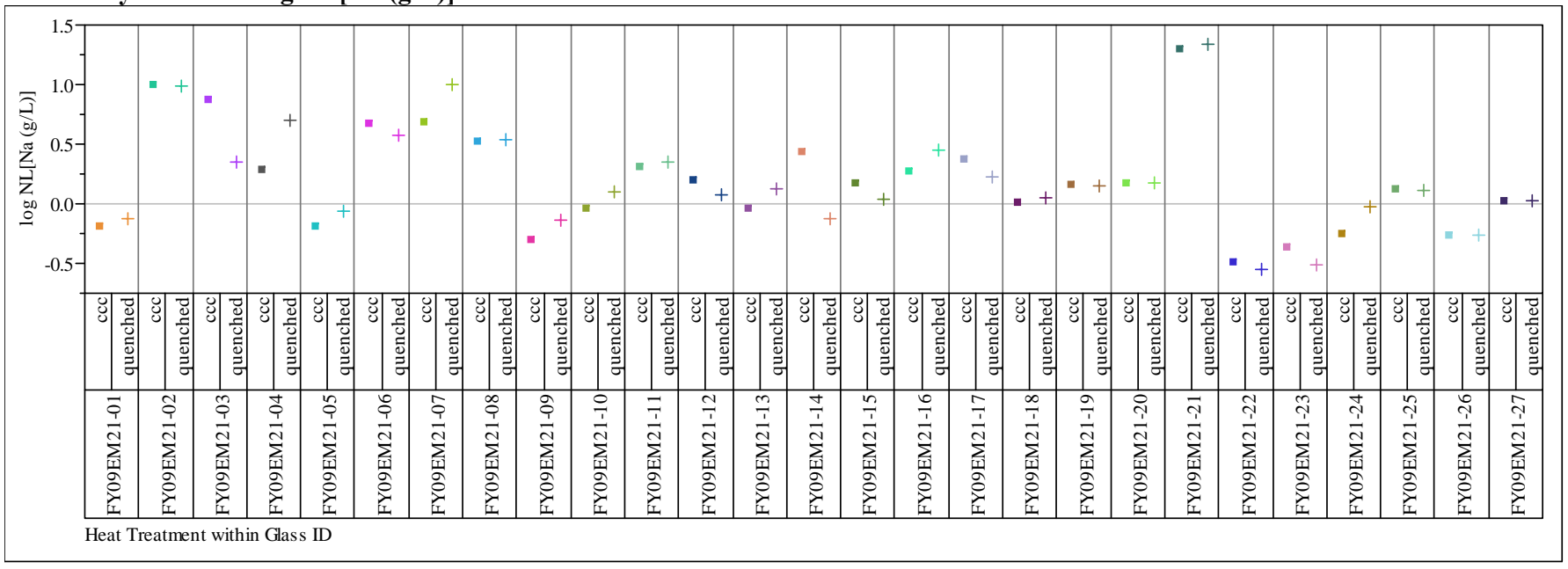

Comp View=Targeted

Variability Chart for $\log \mathrm{NL}[\mathrm{Si}(\mathrm{g} / \mathrm{L})]$

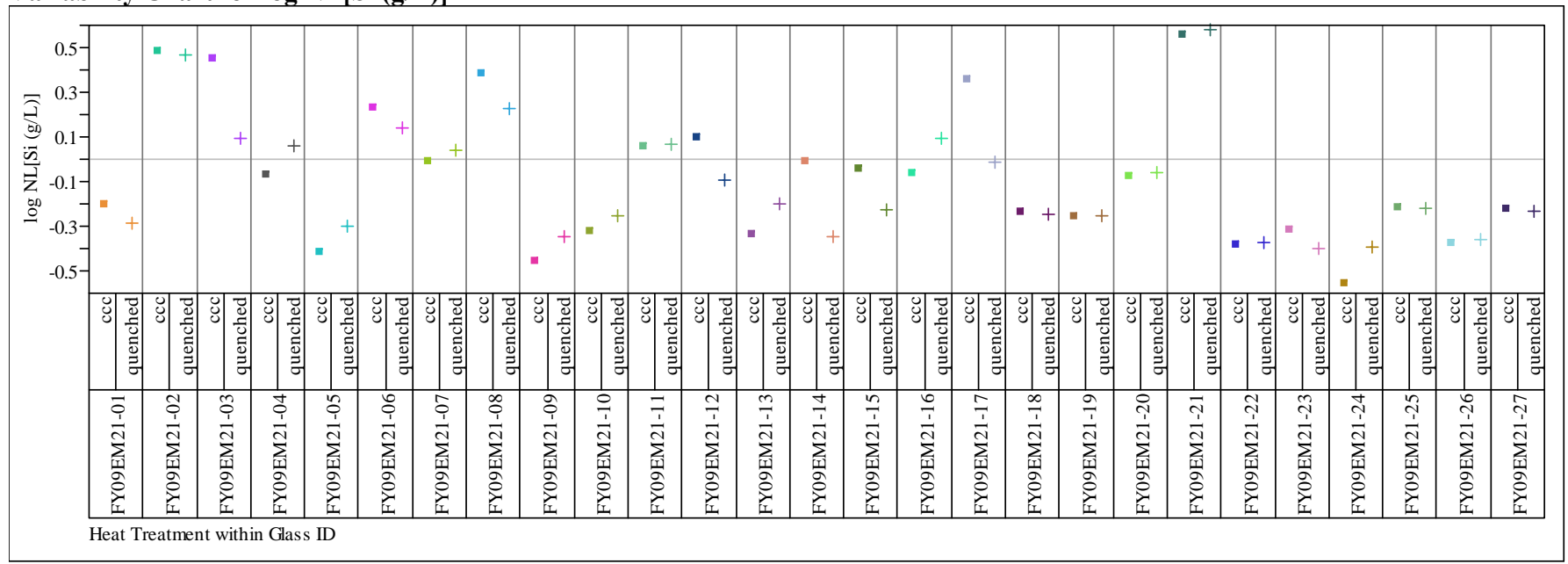


Exhibit B7. del $G p\left(\Delta G_{p}\right)$ Predictions versus Common Logarithm Normalized Leachate (log NL[.]) for B over All Compositional Views and Heat Treatments for the Non-Rad Glasses from the Matrix 2 Study

\begin{tabular}{|c|c|}
\multicolumn{1}{c}{ Legend } \\
\hline Symbol & $\begin{array}{c}\text { Standard/ } \\
\text { Comp View-Heat Treatment }\end{array}$ \\
\hline $\mathrm{z}$ & EA \\
\hline$\diamond$ & ARM \\
\hline$\square$ & Measured-ccc \\
\hline$\square$ & Measured bc -ccc \\
\hline$\square$ & Targeted-ccc \\
\hline$\bullet$ & Measured-quenched \\
\hline$\bullet$ & Measured bc - quenched \\
\hline$\bullet$ & Targeted- quenched \\
\hline
\end{tabular}

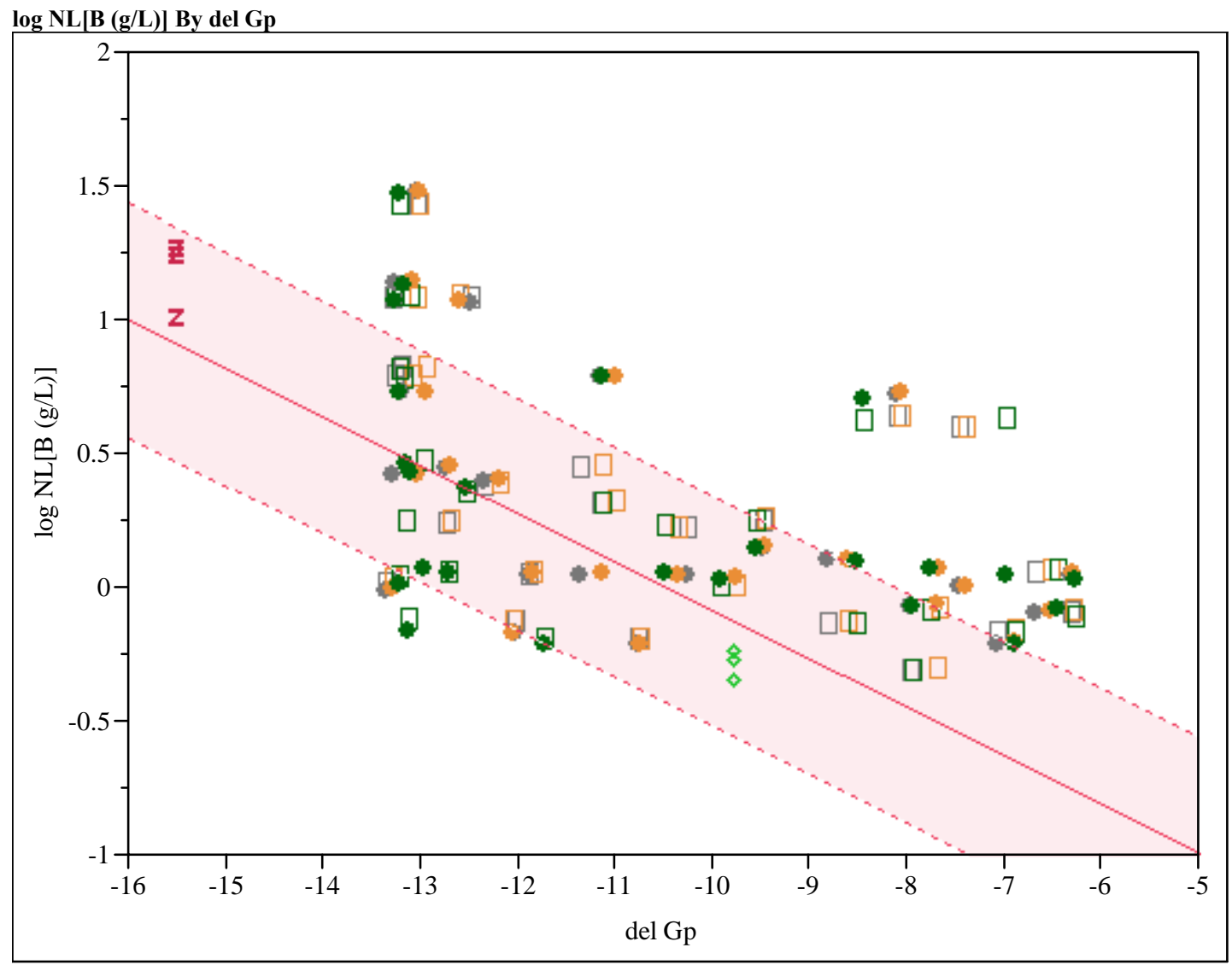


Exhibit B8. del Gp $\left(\Delta G_{p}\right)$ Predictions versus Common Logarithm Normalized Leachate $(\log \mathrm{NL}[]$.$) for Li over All Compositional Views and Heat Treatments for$ the Non-Rad Glasses from the Matrix 2 Study

\begin{tabular}{|c|c|}
\multicolumn{1}{c}{ Legend } \\
\hline Symbol & $\begin{array}{c}\text { Standard/ } \\
\text { Comp View-Heat Treatment }\end{array}$ \\
\hline $\mathrm{z}$ & EA \\
\hline$\triangleright$ & ARM \\
\hline$\square$ & Measured-ccc \\
\hline$\square$ & Measured bc -ccc \\
\hline$\square$ & Targeted-ccc \\
\hline$\bullet$ & Measured-quenched \\
\hline$\bullet$ & Measured bc - quenched \\
\hline$\bullet$ & Targeted- quenched \\
\hline
\end{tabular}

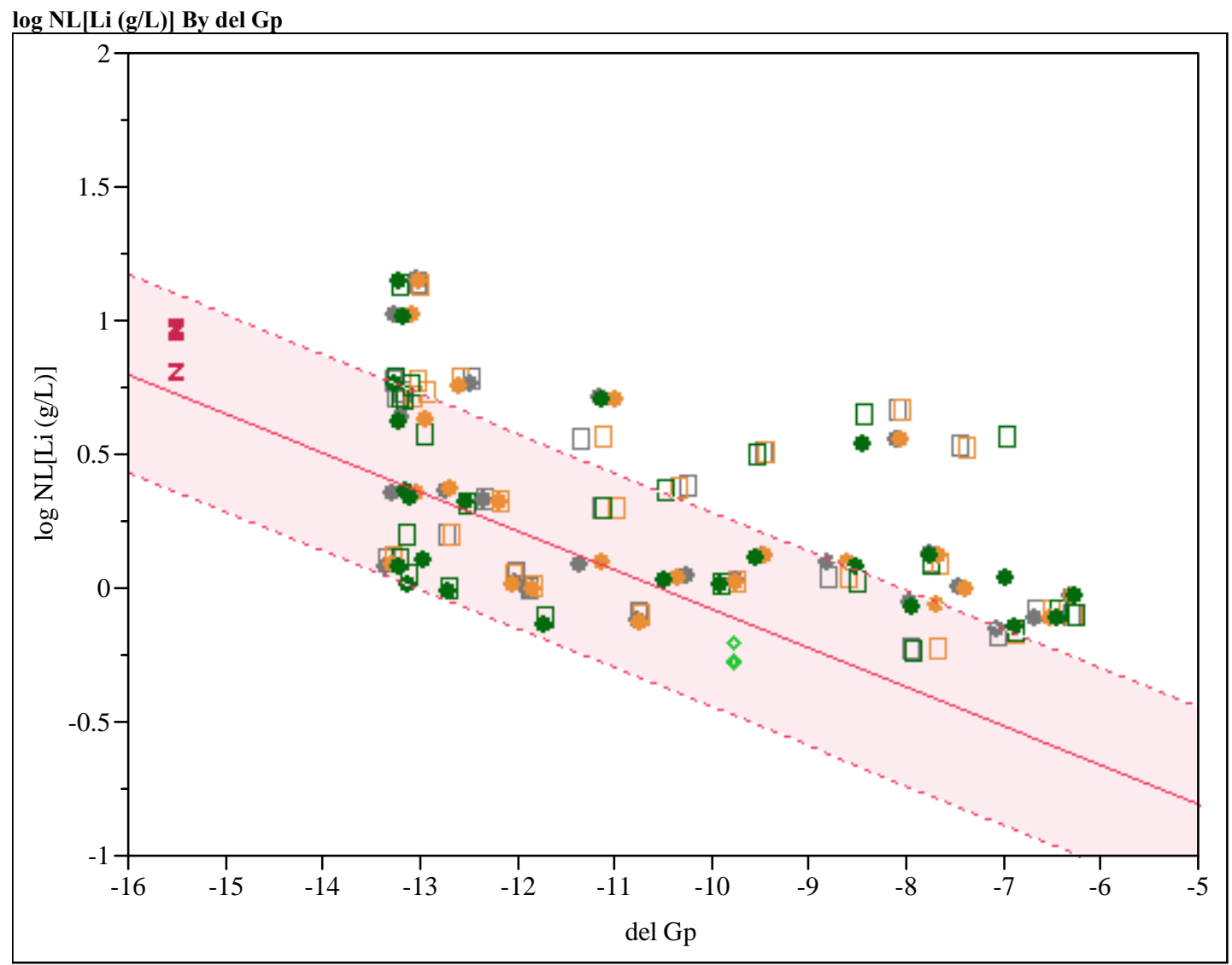


Exhibit B9. del $G p\left(\Delta G_{p}\right)$ Predictions versus Common Logarithm Normalized Leachate $(\log$ NL[.]) for Na over All Compositional Views and Heat Treatments for the Non-Rad Glasses from the Matrix 2 Study

\begin{tabular}{|c|c|}
\multicolumn{1}{c}{ Legend } \\
\hline Symbol & $\begin{array}{c}\text { Standard/ } \\
\text { Comp View-Heat Treatment }\end{array}$ \\
\hline $\mathrm{z}$ & EA \\
\hline$\triangleright$ & ARM \\
\hline$\square$ & Measured-ccc \\
\hline$\square$ & Measured bc -ccc \\
\hline$\square$ & Targeted-ccc \\
\hline$\bullet$ & Measured-quenched \\
\hline$\bullet$ & Measured bc - quenched \\
\hline$\bullet$ & Targeted- quenched \\
\hline
\end{tabular}

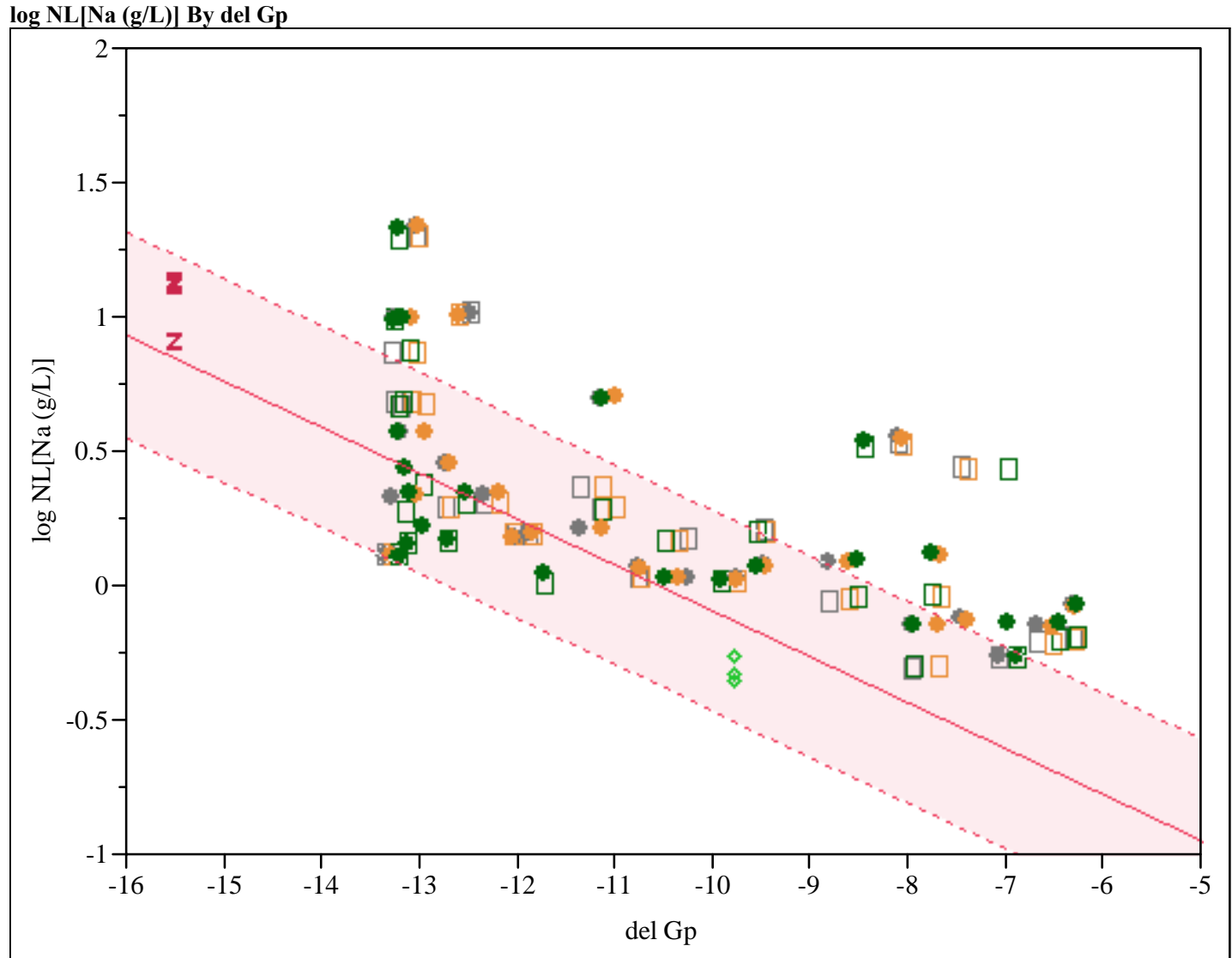


Exhibit B10. del $G p\left(\Delta G_{p}\right)$ Predictions versus Common Logarithm Normalized Leachate $(\log$ NL[.]) for Si over All Compositional Views and Heat Treatments for the Non-Rad Glasses from the Matrix 2 Study

\begin{tabular}{|c|c|}
\multicolumn{2}{c}{ Legend } \\
\hline Symbol & $\begin{array}{c}\text { Standard/ } \\
\text { Comp View-Heat Treatment }\end{array}$ \\
\hline $\mathrm{Z}$ & EA \\
\hline$\triangleright$ & ARM \\
\hline$\square$ & Measured-ccc \\
\hline$\square$ & Measured bc -ccc \\
\hline$\square$ & Targeted-ccc \\
\hline$\bullet$ & Measured-quenched \\
\hline$\bullet$ & Measured bc - quenched \\
\hline$\bullet$ & Targeted- quenched \\
\hline
\end{tabular}

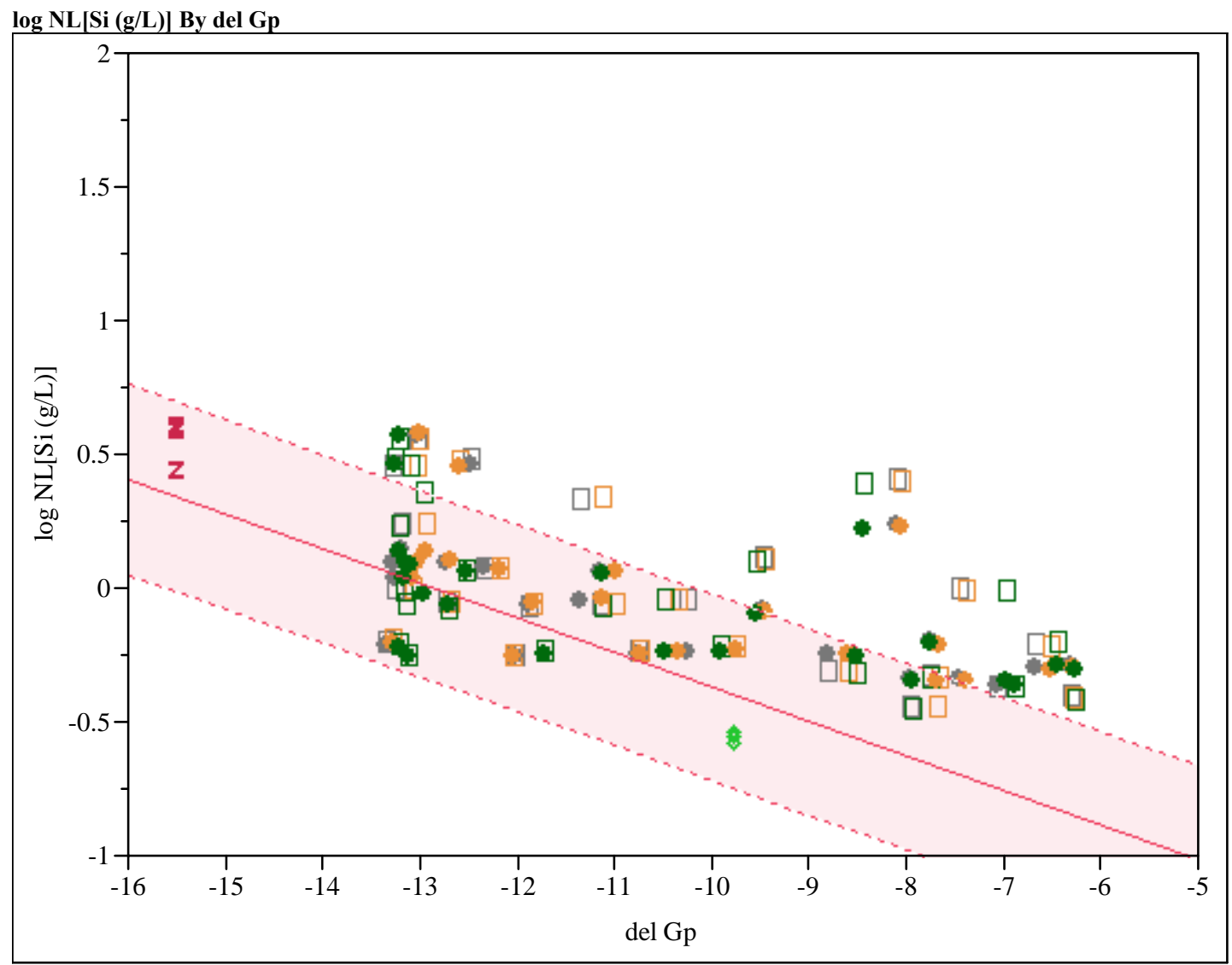


SRNL-STI-2009-00778, Revision 0

\section{Exhibit B11. Effects of Heat Treatment for the Matrix 2 Rad Glasses}

Oneway Analysis of $\log [\mathrm{B}$ ppm] By Heat Treatment Glass ID=FY09EM21-28

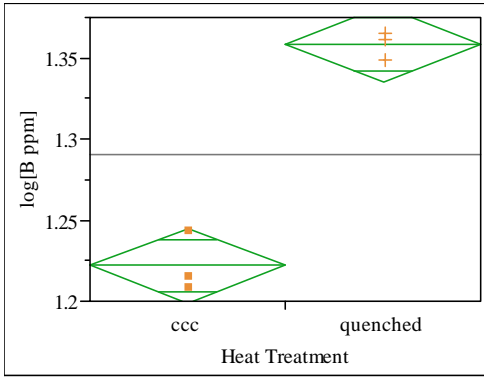

Oneway Anova

Summary of Fit

Adj Rsquare

\begin{tabular}{ll}
0.974488 \\
\hline
\end{tabular}

Mean of Respons 1.290201
6

Test

quenched-ccc

Assuming equal variances

Difference $\quad 0.136646$ t Ratio $\quad 11.55142$ Std Err Dif $\quad 0.011829$ DF

Upper CL Dif 0.169490 Prob $>|t| \quad 0.0003$

Lower CL Dif 0.103803 Prob $>$ t $\quad 0.0002$

$\begin{array}{lll}\text { Confidence } & 0.95 \text { Prob }<\mathrm{t} \quad 0.9998\end{array}$

Analysis of Variance

Source DF Sum of Squares Mean Square F Ratio Prob $>$ F $\begin{array}{llllll}\text { Heat Treatment } & 1 & 0.02800832 & 0.028008 & 133.4354 & 0.0003 \\ \text { Error } & 4 & 0.00083961 & 0.000210 & & \end{array}$ 0.02884793

Means for Oneway Anova

Level Number Mean Std Error Lower 95\% Upper 95\% $\begin{array}{llllll}\text { ccc } & 3 & 1.22188 & 0.00836 & 1.1987 & 1.2451 \\ \text { quenched } & 3 & 1.35852 & 0.00836 & 1.3353 & 1.3817\end{array}$

Std Error uses a pooled estimate of error variance
Oneway Analysis of $\log [\mathrm{Li}$ ppm] By Heat Treatment Glass ID=FY09EM21-28

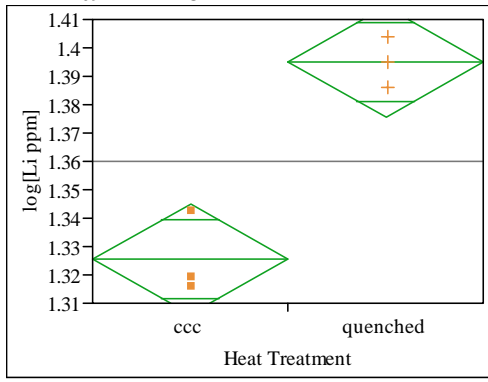

\section{Oneway Anova}

Summary of Fit

Rsquare

Adj Rsquare

Root Mean Square Error $\quad 0.012139$

Mean of Response 1.360239

Observations (or Sum Wgts)

6

t Test

quenched-ccc

Assuming equal variances

Difference $\quad 0.069492$ t Ratio $\quad 7.011252$

Std Err Dif 0.009912 DF

Upper CL Dif 0.097011 Prob $>|t| \quad 0.0022$

Lower CL Dif 0.041973 Prob $>t \quad 0.001$

$\begin{array}{lll}\text { Confidence } & 0.95 \mathrm{Prob}<\mathrm{t} \quad 0.9989\end{array}$

Analysis of Variance

Source DF Sum of Squares Mean Square F Ratio Prob > F $\begin{array}{llllll}\text { Heat Treatment } & 1 & 0.00724378 & 0.007244 & 49.1577 & 0.0022\end{array}$ Erro 0.00058943 0.000147

Means for Oneway Anova

Level Number Mean Std Error Lower 95\% Upper 95\%

$\begin{array}{llllll}\text { ccc } & 3 & 1.32549 & 0.00701 & 1.3060 & 1.3450 \\ \text { quenched } & 3 & 1.39499 & 0.00701 & 1.3755 & 1.4144\end{array}$

Std Error uses a pooled estimate of error variance
Oneway Analysis of $\log [\mathrm{Na}$ ppm] By Heat Treatment Glass ID=FY09EM21-28

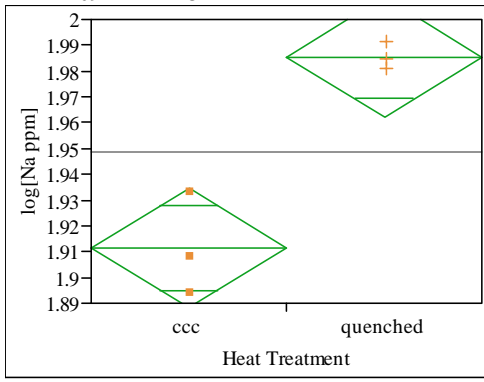

Oneway Anova

Summary of Fit

Rsquare

Adj Rsquare $\quad 0.90797$

Root Mean Square Enror $\quad 0.884963$

0.014438

Mean of Response 1.948486

\section{t Test}

quenched-ccc

Assuming equal variances

Difference $\quad 0.074055$ t Ratio $\quad 6.282058$ Std Err Dif 0.011788 DF

Upper CL Dif 0.106785 Prob $>|t| \quad 0.0033$

Lower CL Dif 0.041325 Prob $>$ t $\quad 0.001$

$\begin{array}{lll}\text { Confidence } & 0.95 \mathrm{Prob}<\mathrm{t} \quad 0.9984\end{array}$

\section{Analysis of Variance}

Dource DF Sum of Squares Mean Square F Ratio Prob $>$ F $\begin{array}{lllllll}\text { Heat Treatment } & 1 & 0.00822622 & 0.008226 & 39.4642 & 0.0033\end{array}$ $\begin{array}{lll}\text { Error } & 4 & 0.00083379 \\ \text { C. Total } & 5 & 0.00906001\end{array}$

Means for Oneway Anova

Level Number Mean Std Error Lower 95\% Upper 95\%

$\begin{array}{llllll}\text { ccc } & 3 & 1.91146 & 0.00834 & 1.8883 & 1.9346\end{array}$

$\begin{array}{llll}3 & 1.98551 & 0.00834 & 1.9624\end{array} 2.0087$

Std Error uses a pooled estimate of error variance 
SRNL-STI-2009-00778, Revision 0

\section{Exhibit B11. Effects of Heat Treatment for the Matrix 2 Rad Glasses}

Oneway Analysis of $\log [\mathrm{Si}$ ppm] By Heat Treatment Glass

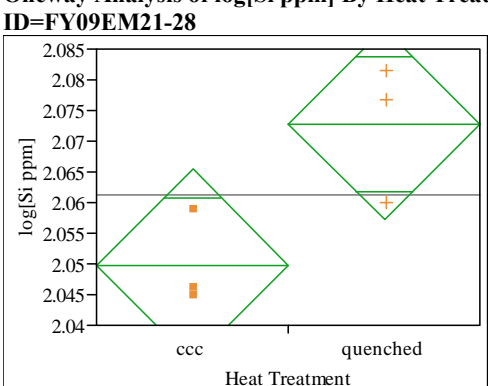

Oneway Anova

Summary of Fit

Rsquare

Ad Rs 0.009709

(U)

2.06132
6

\section{t Test}

quenched-ccc

Assuming equal variances

Difference $\quad 0.022989$ t Ratio 2.899927

Std Err Dif $\quad 0.007927$ DF

2.899927
4

Upper CL Dif 0.044999 Prob $>|t| \quad 0.0441$

Lower CL Dif 0.000979 Prob $>t \quad 0.0221$

$\begin{array}{lll}\text { Confidence } & 0.95 \text { Prob }<\mathrm{t} \quad 0.9779\end{array}$

\section{Analysis of Variance}

Source DF Sum of Squares Mean Square F Ratio Prob $>$ F $\begin{array}{lllllll}\text { Heat Treatment } & 1 & 0.00079273 & 0.000793 & 8.4096 & 0.0441\end{array}$ Error

$$
0.00079273
$$$$
0.000094
$$

C. Total

0.00116979

Means for Oneway Anova

Level Number Mean Std Error Lower 95\% Upper 95\%

$\begin{array}{llrrrr}\text { cCC } & 3 & 2.04983 & 0.00561 & 2.0343 & 2.0654\end{array}$

$\begin{array}{llllll} & 3 & 2.07281 & 0.00561 & 2.0573 & 2.0884\end{array}$

Std Error uses a pooled estimate of error variance
Oneway Analysis of $\log [\mathrm{B}$ ppm] By Heat Treatment Glass

ID=FY09EM21-29

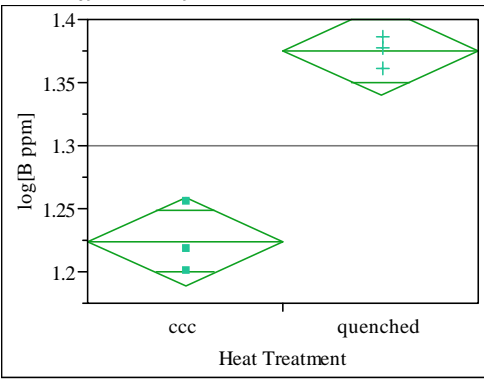

\section{Oneway Anova}

Summary of Fit

Adj Rsquare

Root Mean Square Error $\quad 0.021896$

Mean of Response 1.299656

Observations (or Sum Wgts)

6

Test

quenched-ccc

Assuming equal variances

Difference $\quad 0.150782$ t Ratio 8.433787 Std Err Dif $\quad 0.017878$ D

Upper CL Dif 0.200421 Prob $>|t| \quad 0.0011$

Lower CL Dif 0.101144 Prob $>t \quad 0.000$

$\begin{array}{lll}\text { Confidence } & 0.95 \text { Prob }<\mathrm{t} \quad 0.9995\end{array}$

Analysis of Variance

Source DF Sum of Squares Mean Square F Ratio Prob > $\begin{array}{lrrrrr}\text { Heat Treatment } & 1 & 0.03410296 & 0.034103 & 71.1288 & 0.0011\end{array}$ Error 0.00191782 0.000479

\section{Means for Oneway Anova}

Level Number Mean Std Error Lower 95\% Upper 95\%

$\begin{array}{llllll}\text { ccc } & 3 & 1.22426 & 0.01264 & 1.1892 & 1.2594 \\ \text { quenched } & 3 & 1.37505 & 0.01264 & 1.3399 & 1.4101\end{array}$

Std Error uses a pooled estimate of error variance
Oneway Analysis of $\log [\mathrm{Li}$ ppm] By Heat Treatment Glass ID=FY09EM21-29

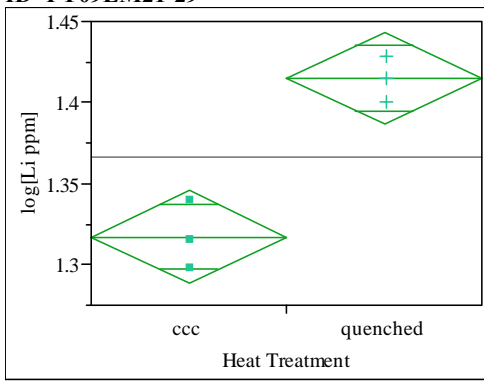

Oneway Anova

Summary of Fit

Rsquare

0.918657

Root Mean Square Enror $\quad 0.898321$

Reot Mean Square En

0.017778

Mean of Response

1.366052
6

Test

quenched-ccc

Assuming equal variance

Difference $\quad 0.097562$ t Ratio $\quad 6.721194$ Std Err Dif 0.014516 DF

Upper CL Dif 0.137864 Prob $>|t| \quad 0.002$

Lower CL Dif 0.057261 Prob $>$ t $\quad 0.0013$

$\begin{array}{lrl}\text { Confidence } & 0.95 \mathrm{Prob}<\mathrm{t} \quad 0.9987\end{array}$

\section{Analysis of Variance}

Source DF Sum of Squares Mean Square F Ratio Prob $>$ F $\begin{array}{lllllll}\text { Heat Treatment } & 1 & 0.01427766 & 0.014278 & 45.1744 & 0.0026\end{array}$ $\begin{array}{lll}\text { Error } & 4 & 0.00126422 \\ \text { C. Total } & 5 & 0.01554188\end{array}$

Means for Oneway Anova

Level Number Mean Std Error Lower 95\% Upper 95\%

$\begin{array}{llllll}\text { ccc } & 3 & 1.31727 & 0.01026 & 1.2888 & 1.3458 \\ \text { quenched } & 3 & 1.41483 & 0.01026 & 1.3863 & 1.4433\end{array}$

Std Error uses a pooled estimate of error variance 
SRNL-STI-2009-00778, Revision 0

\section{Exhibit B11. Effects of Heat Treatment for the Matrix 2 Rad Glasses}

Oneway Analysis of $\log [\mathrm{Na}$ ppm] By Heat Treatment Glass

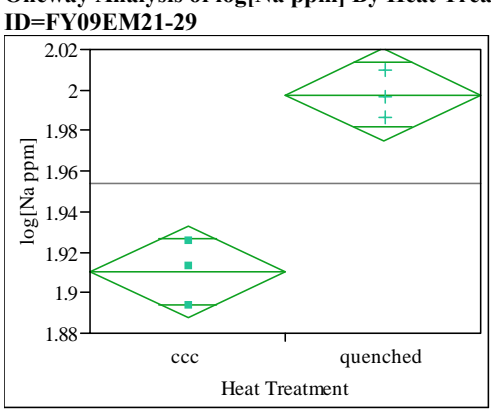

Oneway Anova

Summary of Fit

Rsquare

Adj Rsquare

0.93478

0.918476

Root Mean Square Error $\quad 0.01413$

Observations (or

1.95405
6

Test

uenched-ccc

Assuming equal variances

Difference $\quad 0.087354$ t Ratio 7.571741

Std Err Dif $\quad 0.011537$ DF

Upper CL Dif 0.119386 Prob $>|t| \quad 0.0016$

$\begin{array}{lrll}\text { Lower CL Dif } & 0.055323 & \text { Prob }>t & 0.0008 \\ \text { Confidence } & 0.95 & \text { Prob }<\mathrm{t} & 0.9992\end{array}$ 0.95 Prob $<$

Analysis of Variance

Source DF Sum of Squares Mean Square F Ratio Prob $>$ F $\begin{array}{llllll}\text { Heat Treatment } & 1 & 0.01144614 & 0.011446 & 57.3313 & 0.0016\end{array}$ 0.00079860 0.000200

Means for Oneway Anova

Level Number Mean Std Error Lower 95\% Upper 95\% $\begin{array}{llllll}\text { ccC } & 3 & 1.91037 & 0.00816 & 1.8877 & 1.933\end{array}$ $\begin{array}{lllll}\text { quenched } & 31.99773 & 0.00816 & 1.9751 & 2.0204\end{array}$

Std Error uses a pooled estimate of error variance
Oneway Analysis of $\log [\mathrm{Si}$ ppm] By Heat Treatment Glass ID=FY09EM21-29

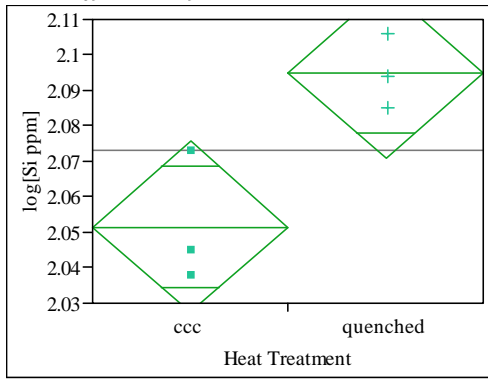

\section{Oneway Anova}

Summary of Fit

Rsquare

.758539

Adj Rsquare

0.698174

Root Mean Square Error $\quad 0.015048$

Mean of Respons

2.073316

Observations (or Sum Wgts)

Test

quenched-ccc

Assuming equal variances

Difference $\quad 0.043554$ t Ratio 3.54483 Std Err Dif 0.012287 D

Upper CL Dif 0.077667 Prob $>|t| 0.0239$ Lower CL Dif 0.009441 Prob $>t \quad 0.0120$ $\begin{array}{lll}\text { Confidence } & 0.95 \text { Prob }<\mathrm{t} \quad 0.9880\end{array}$

\section{Analysis of Variance}

DF Sum of Squares Mean Square F Ratio Prob $>$ F $\begin{array}{llllll}\text { Heat Treatment } & 1 & 0.00284544 & 0.002845 & 12.5658 & 0.0239\end{array}$ Erro 0.00090577 0.000226

Means for Oneway Anova

Level Number Mean Std Error Lower 95\% Upper 95\% $\begin{array}{llllll}\text { ccc } & 3 & 2.05154 & 0.00869 & 2.0274 & 2.0757 \\ \text { quenched } & 3 & 2.09509 & 0.00869 & 2.0710 & 2.1192\end{array}$

$$
\begin{array}{lll}
3 & 2.09509 & 0.00869
\end{array}
$$$$
2.119
$$

Std Error uses a pooled estimate of error variance
Oneway Analysis of $\log [\mathrm{B}$ ppm] By Heat Treatment Glass ID=FY09EM21-30

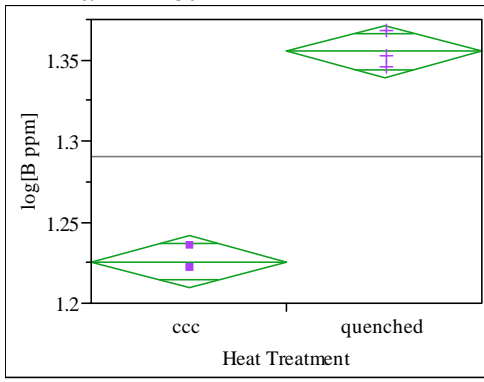

Oneway Anova

Summary of

Rsquare

0.984872

Root Mean Square Error $\quad 0.009847$

Mean of Response 1.290426
6

Observations (or Sum Wgts)

Test

quenched-ccc

Assuming equal variance

Difference $\quad 0.129738$ t Ratio $\quad 16.13722$ Std Err Dif 0.008040 DF

Upper CL Dif 0.152060 Prob $>|t| \quad<.0001$

Lower CL Dif 0.107417 Prob $>t \quad<.0001$

Confidence $\quad 0.95$ Prob $<\mathrm{t} \quad 1.0000$

Analysis of Variance

DF Sum of Squares Mean Square F Ratio Prob $>$ F $\begin{array}{lrrrrr}\text { Heat Treatment } & 1 & 0.02524807 & 0.025248 & 260.4100 & <.000\end{array}$ $\begin{array}{lll}\text { Error } & 4 & 0.00038782 \\ \text { C. Total } & 5 & 0.02563589\end{array}$

Means for Oneway Anova

Level Number Mean Std Error Lower 95\% Upper 95\%

$\begin{array}{lrrrrr}\text { ccC } & 3 & 1.22556 & 0.00568 & 1.2098 & 1.2413\end{array}$

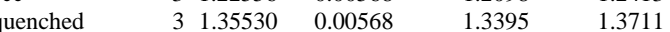

Std Error uses a pooled estimate of error variance 
SRNL-STI-2009-00778, Revision 0

\section{Exhibit B11. Effects of Heat Treatment for the Matrix 2 Rad Glasses}

Oneway Analysis of $\log [\mathrm{Li}$ ppm] By Heat Treatment Glass DD=FY09EM21-30

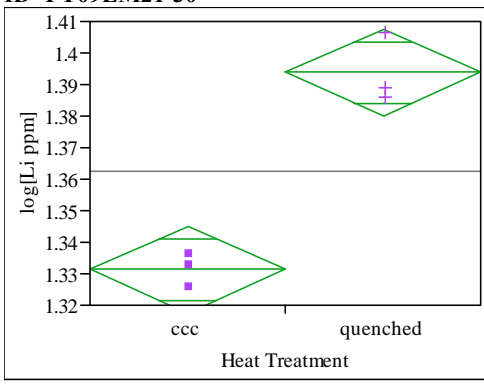

\section{Oneway Anova}

Summary of Fit

Rsquare

Adj Rsquare

Eror -0.008585

Mean of Respon 1.362641
6

t Test

quenched-ccc

Assuming equal variances

Difference $\quad 0.062675$ t Ratio $\quad 8.941338$ Std Err Dif $\quad 0.007010$ DF

Upper CL Dif 0.082137 Prob $>|t| \quad 0.0009$ Lower CL Dif 0.043213 Prob $>$ t 0.0004

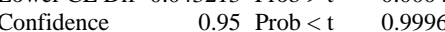

Analysis of Variance

Source DF Sum of Squares Mean Square F Ratio Prob $>$ F $\begin{array}{llllll}\text { Heat Treatment } & 1 & 0.00589222 & 0.005892 & 79.9475 & 0.0009\end{array}$ Error 0.00618703

Means for Oneway Anova

Level Number Mean Std Error Lower 95\% Upper 95\%

$\begin{array}{lllllr}\text { ccc } & 3 & 1.33130 & 0.00496 & 1.3175 & 1.3451 \\ \text { quenched } & 3 & 1.39398 & 0.00496 & 1.3802 & 1.4077\end{array}$

Std Error uses a pooled estimate of error variance
Oneway Analysis of $\log [\mathrm{Na}$ ppm] By Heat Treatment Glass

ID=FY09EM21-30

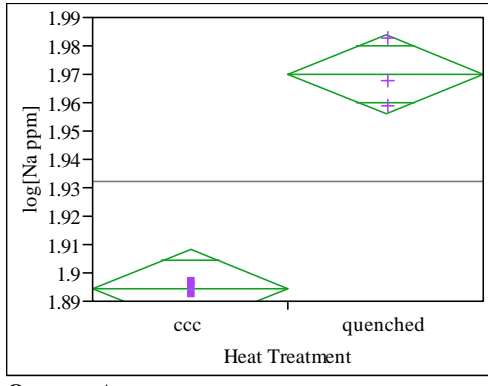

\section{Oneway Anova}

Summary of Fit

Adj Rsquare

Root Mean Square Error 0.956692

Square Error 0.008743

Observations (onse 1.932249

\section{Test}

quenched-ccc

Assuming equal variances

Difference $\quad 0.075365$ t Ratio $\quad 10.55703$

Std Err Dif 0.007139 D

Upper CL Dif 0.095186 Prob $>|t| \quad 0.0005$

Lower CL Dif 0.055545 Prob $>t \quad 0.0002$

$\begin{array}{lrl}\text { Confidence } & 0.95 \text { Prob }<\mathrm{t} \quad 0.9998\end{array}$

Analysis of Variance

Source DF Sum of Squares Mean Square F Ratio Prob $>$ F $\begin{array}{llllll}\text { Heat Treatment } & 1 & 0.00851988 & 0.008520 & 111.4509 & 0.0005 \\ \text { Error } & 4 & 0.00030578 & 0.000076 & & \end{array}$ 0.000076

\section{Means for Oneway Anova}

Level Number Mean Std Error Lower 95\% Upper 95\%

$\begin{array}{lllllr}\text { ccc } & 3 & 1.89457 & 0.00505 & 1.8806 & 1.9086 \\ \text { quenched } & 3 & 1.96993 & 0.00505 & 1.9559 & 1.9839\end{array}$

$$
\begin{array}{lll}
3 & 1.96993 & 0.00505
\end{array}
$$$$
1.955
$$

Std Error uses a pooled estimate of error variance
Oneway Analysis of $\log [\mathrm{Si}$ ppm] By Heat Treatment Glass ID=FY09EM21-30

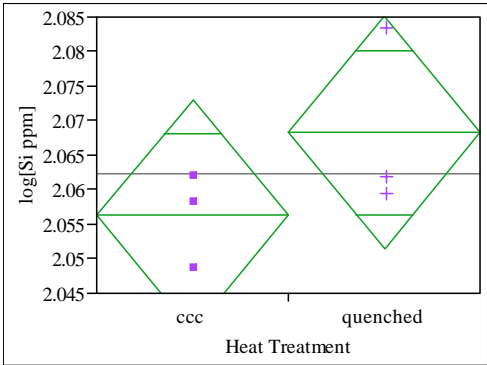

Oneway Anova

Summary of Fit

Rsquare

Root Mean Square Error $\quad 0.161806$

0.161806

2.062254

t Test

quenched-ccc

Assuming equal variances

Difference $\quad 0.01203$ t Ratio 1.401859

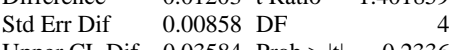

Upper CL Dif $\quad 0.03584$ Prob $>|t| \quad 0.2336$

Lower CL Dif -0.01179 Prob $>t \quad 0.1168$

Analysis of Variance

Source DF Sum of Squares Mean Square F Ratio Prob $>$ F $\begin{array}{lrrrrr}\text { Source } & \text { DF Sum of Squares } & \text { Mean Square } & & \\ \text { Heat Treatment } & 1 & 0.00021692 & 0.000217 & 1.9652 & 0.2336\end{array}$ $\begin{array}{llll}\text { Error } & 4 & 0.00044151 & 0.000110\end{array}$

C. Total

0.00044151

Means for Oneway Anova

Level Number Mean Std Error Lower 95\% Upper 95\%

$\begin{array}{llllll}\text { ccc } & 3 & 2.05624 & 0.00607 & 2.0394 & 2.073 \\ \text { quenched } & 3 & 2.06827 & 0.00607 & 2.0514 & 2.0851\end{array}$

Std Error uses a pooled estimate of error variance 
Exhibit B12. Effects of Heat Treatment for the Matrix 2 Rad Glasses by Compositional View

Variability Chart for $\log \mathrm{NL}[\mathrm{B}(\mathrm{g} / \mathrm{L})]$

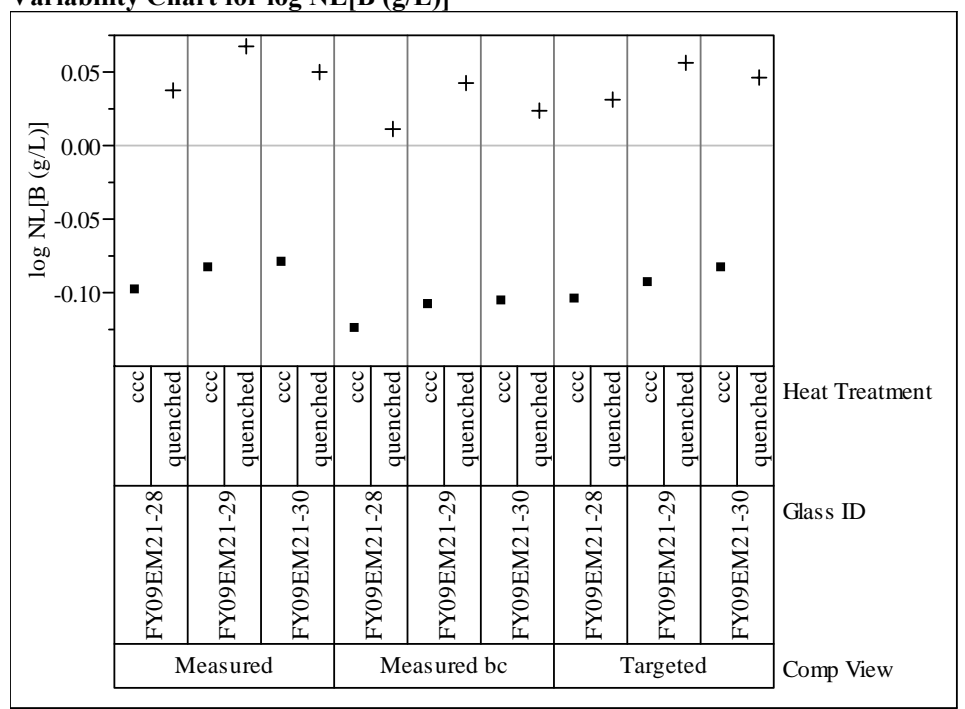

Variability Chart for $\log \mathrm{NL}[\mathrm{Li}(\mathrm{g} / \mathrm{L})]$

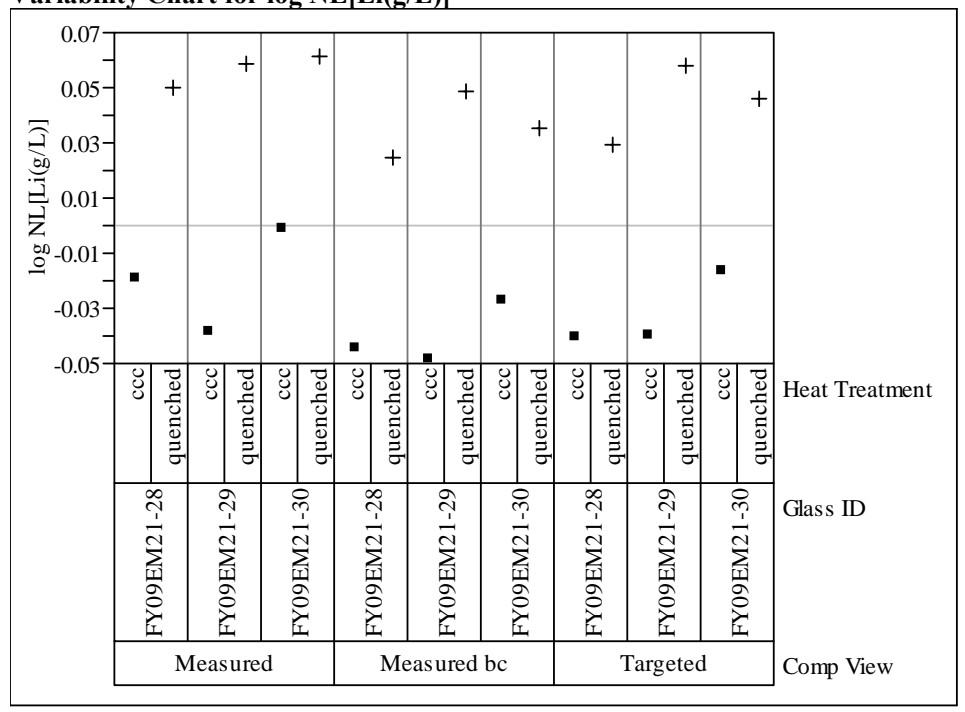

Variability Chart for log NL[Na (g/L)]
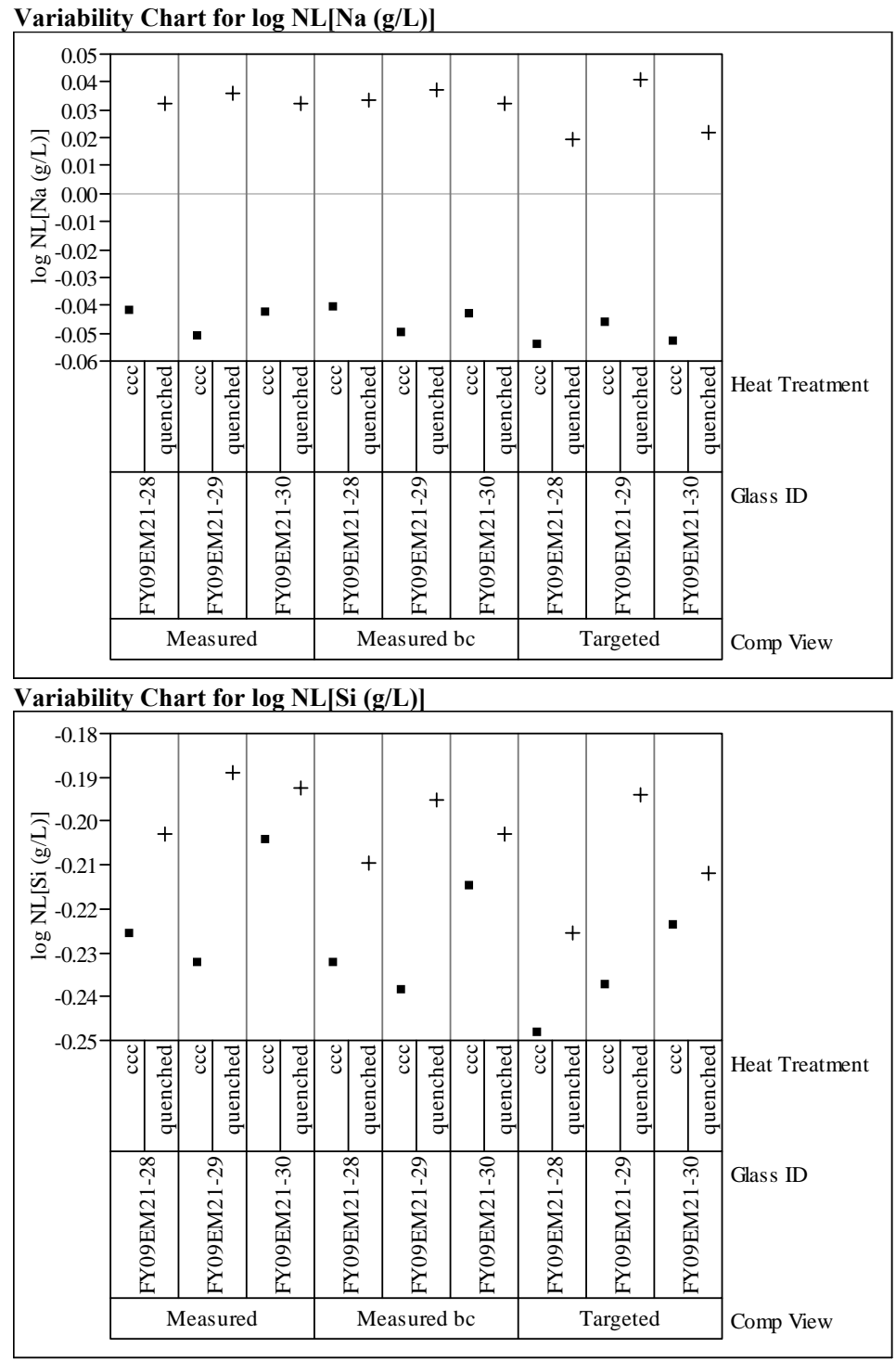
Exhibit B13. del $G p\left(\Delta G_{p}\right)$ Predictions versus Common Logarithm Normalized Leachate Concentrations over All Compositional Views and Heat Treatments for the Rad Glasses from the Matrix 2 Study

$\log \mathrm{NL}[\mathrm{B}(\mathrm{g} / \mathrm{L})]$ By del Gp

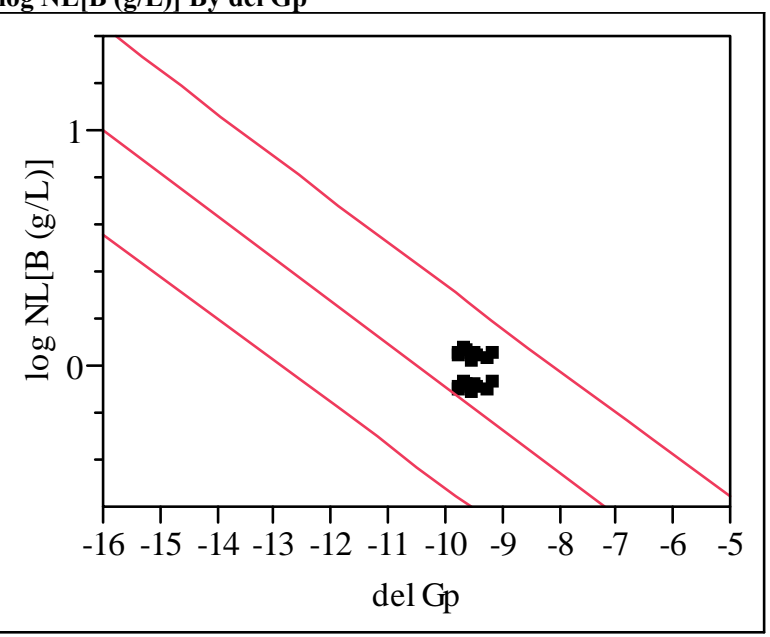

$\log \mathrm{NL}[\mathrm{Li}(\mathrm{g} / \mathrm{L})]$ By del Gp

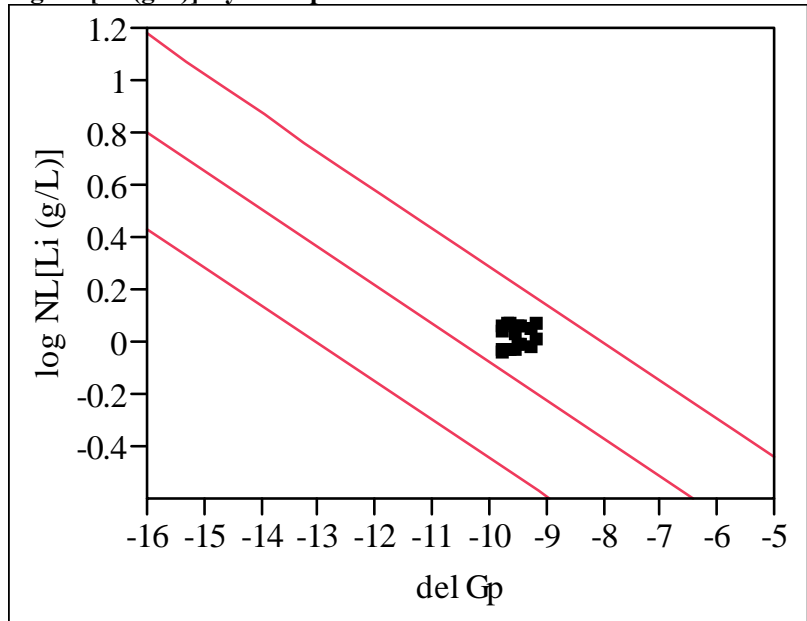

$\log \mathrm{NL}[\mathrm{Na}(\mathrm{g} / \mathrm{L})]$ By del Gp

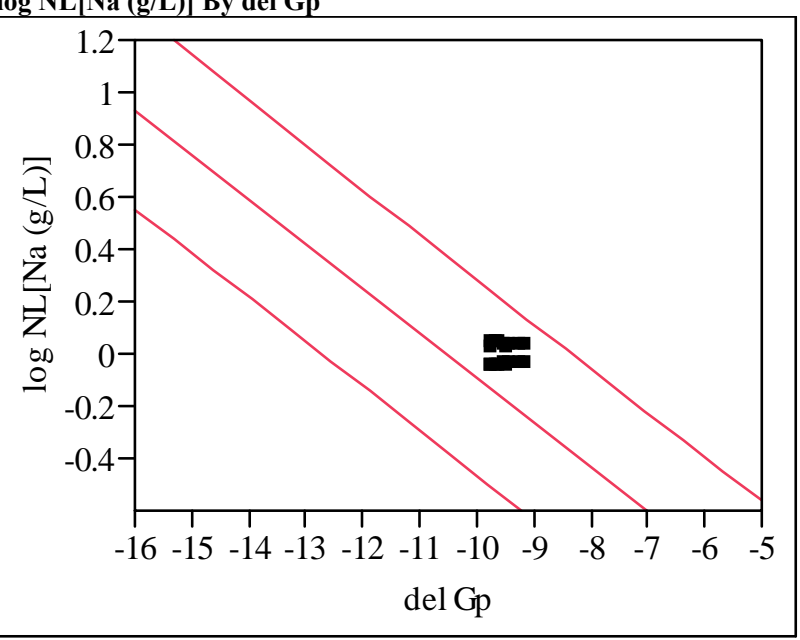

$\log \mathbf{N L}[\mathrm{Si}(\mathrm{g} / \mathrm{L})]$ By del Gp

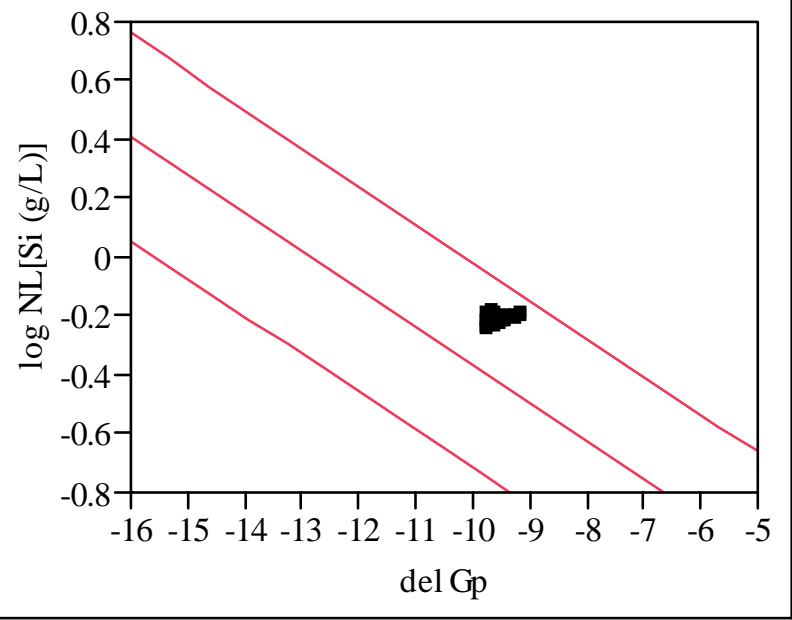




\section{Appendix C:}

\section{Exhibits Supporting the Analysis of the Viscosity Measurements of the Matrix 2 Study Glasses}


Exhibit C1. VFT fit of FY09EM21-02. Nonlinear Fit Glass ID=FY09EM21-02

Response: $\ln (n$; poise), Predictor: $\ln (n$; VTF)

Control Panel

Converged in Gradient

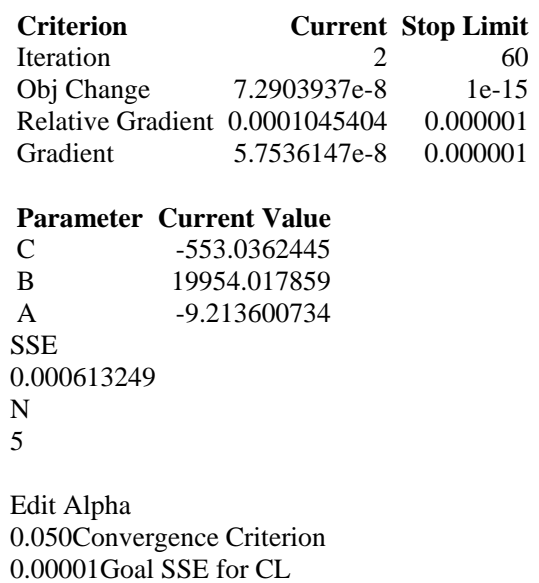

Plot

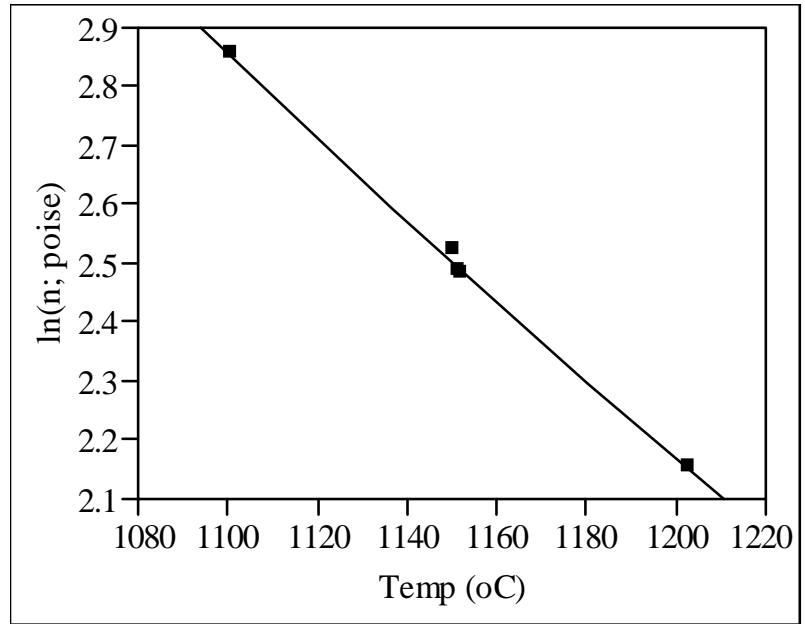

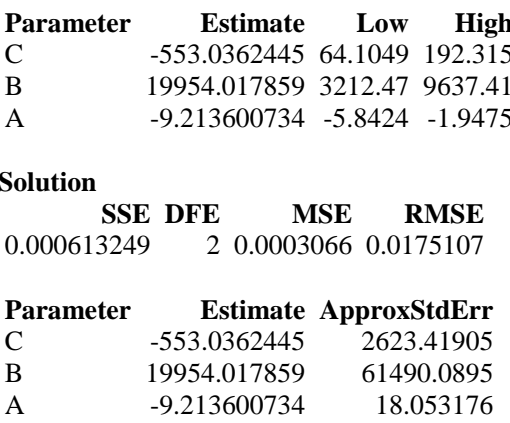

Solved By:

Analytic NR
Exhibit C2. VFT fit of FY09EM21-05.

Nonlinear Fit Glass ID=FY09EM21-05

Response: $\ln (n$; poise), Predictor: $\ln (\mathrm{n}$; VTF)

Control Panel

Converged in Gradient

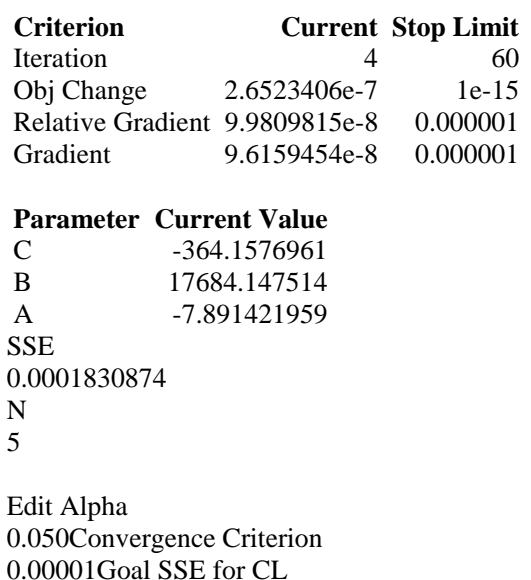

Plot

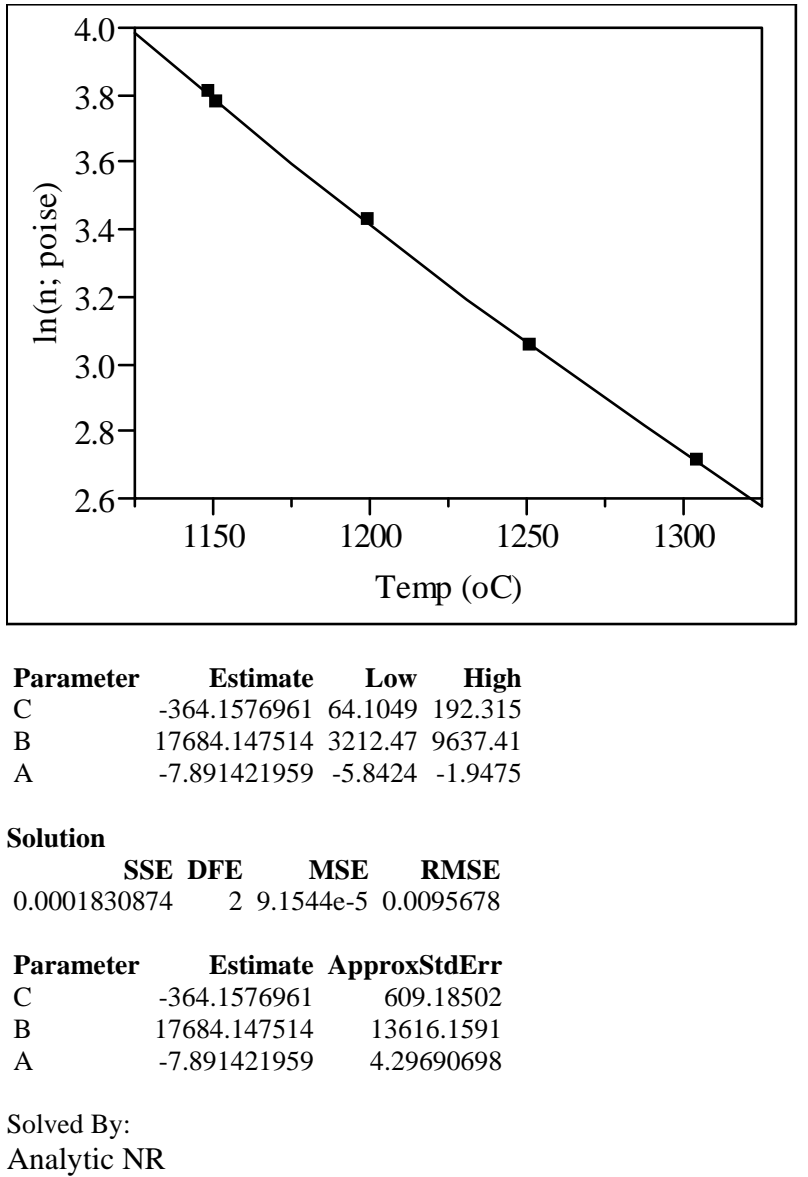




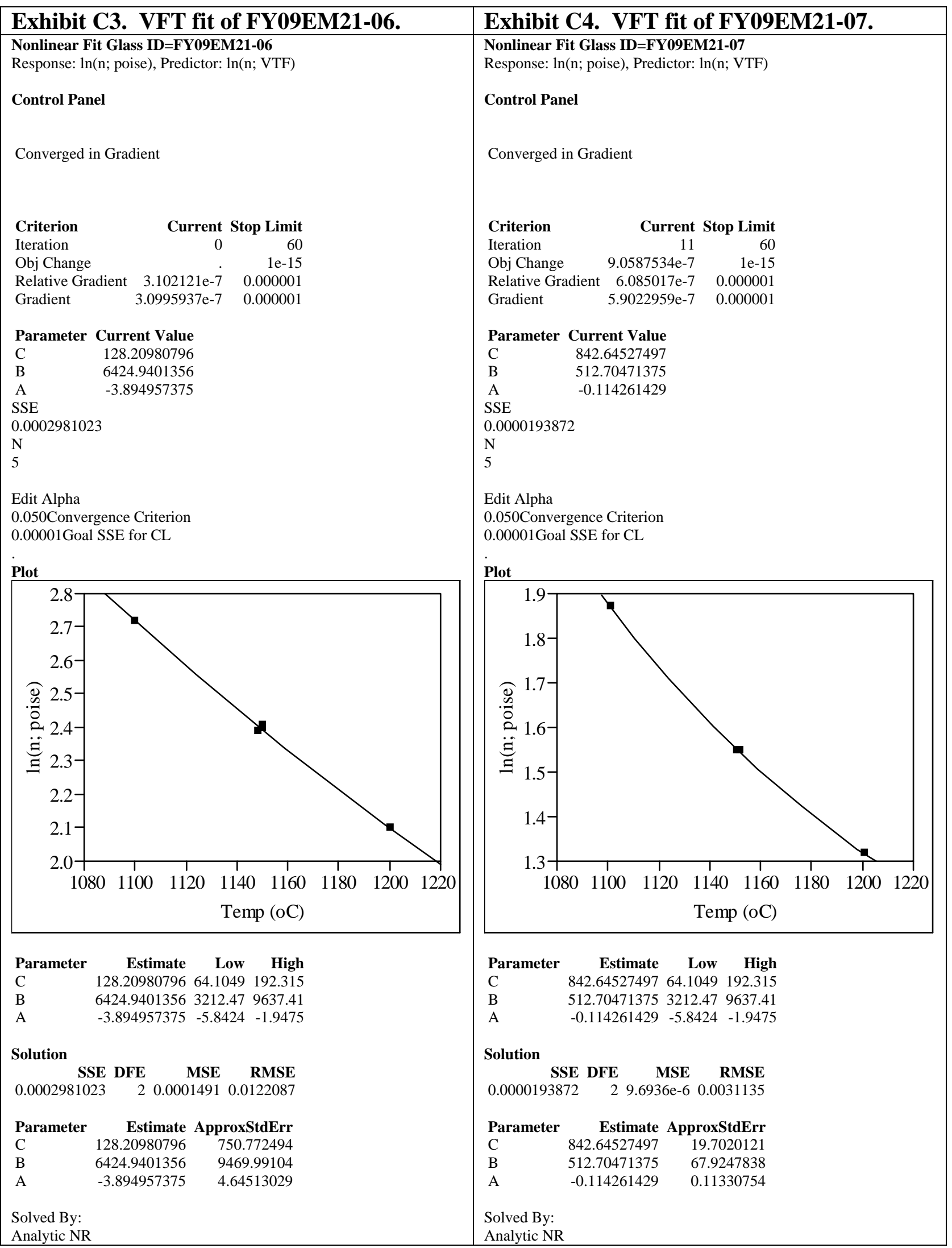


Exhibit C5. VFT fit of FY09EM21-08. Nonlinear Fit Glass ID=FY09EM21-08

Response: $\ln (n$; poise), Predictor: $\ln (n$; VTF)

Control Panel

Converged in Gradient

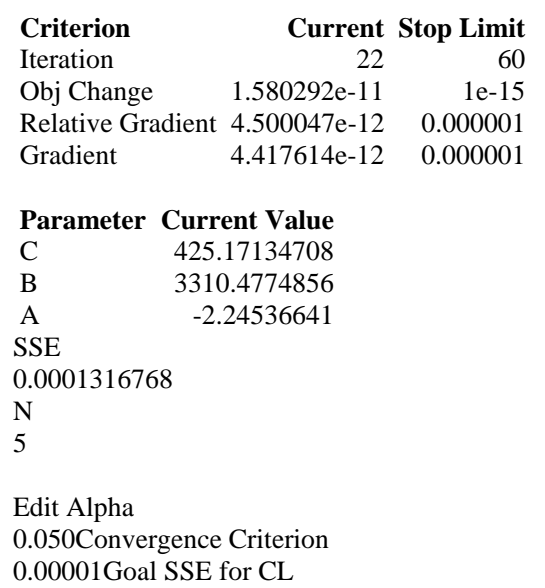

Plot

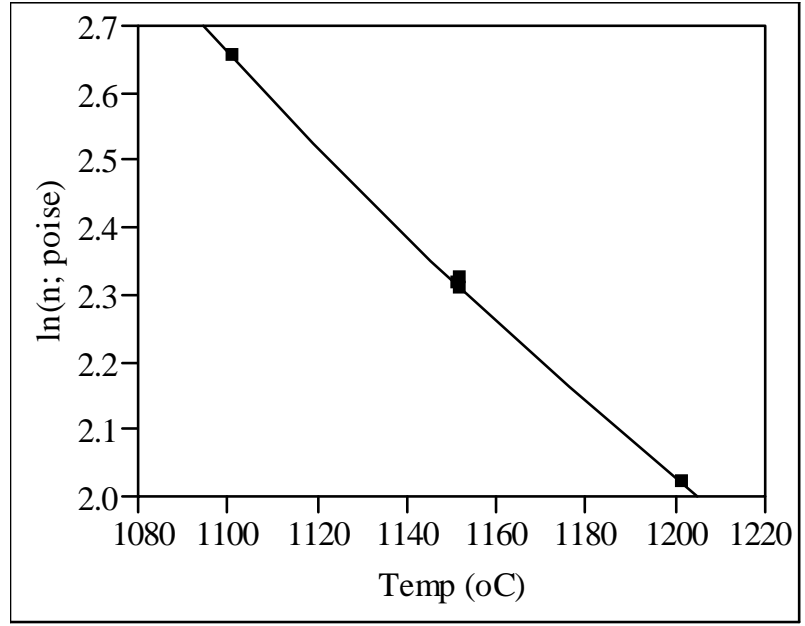

\begin{tabular}{|c|c|c|c|}
\hline & \multicolumn{3}{|c|}{ Parameter } \\
\hline \multicolumn{4}{|c|}{$\begin{array}{llll}\text { C } & 425.17134708 & 64.1049 & 192.315\end{array}$} \\
\hline 3 & 3310.4774856 & 3212.47 & 963 \\
\hline \multicolumn{4}{|c|}{$-2.24536641-5.8424$} \\
\hline \multicolumn{4}{|l|}{ Solution } \\
\hline SSE & E DFE & MSE & RMS \\
\hline 0.0001316768 & 26.5838 & $8 e-5 \quad 0.00$ & 08114 \\
\hline \multirow{2}{*}{$\begin{array}{l}\text { Parameter } \\
\text { C }\end{array}$} & Estimate & \multirow{2}{*}{\multicolumn{2}{|c|}{ ApproxStdErr }} \\
\hline & 425.17134708 & & \\
\hline & 3310.4774856 & \multicolumn{2}{|c|}{2261.24789} \\
\hline & -2.24536641 & \multicolumn{2}{|c|}{1.56529602} \\
\hline
\end{tabular}

Solved By:

Analytic NR
Exhibit C6. VFT fit of FY09EM21-09.

Nonlinear Fit Glass ID=FY09EM21-09

Response: $\ln (n$; poise), Predictor: $\ln (\mathrm{n}$; VTF)

Control Panel

Converged in Gradient

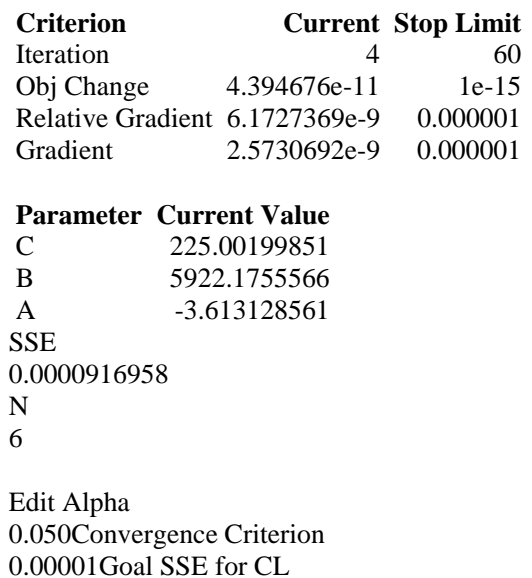

Plot

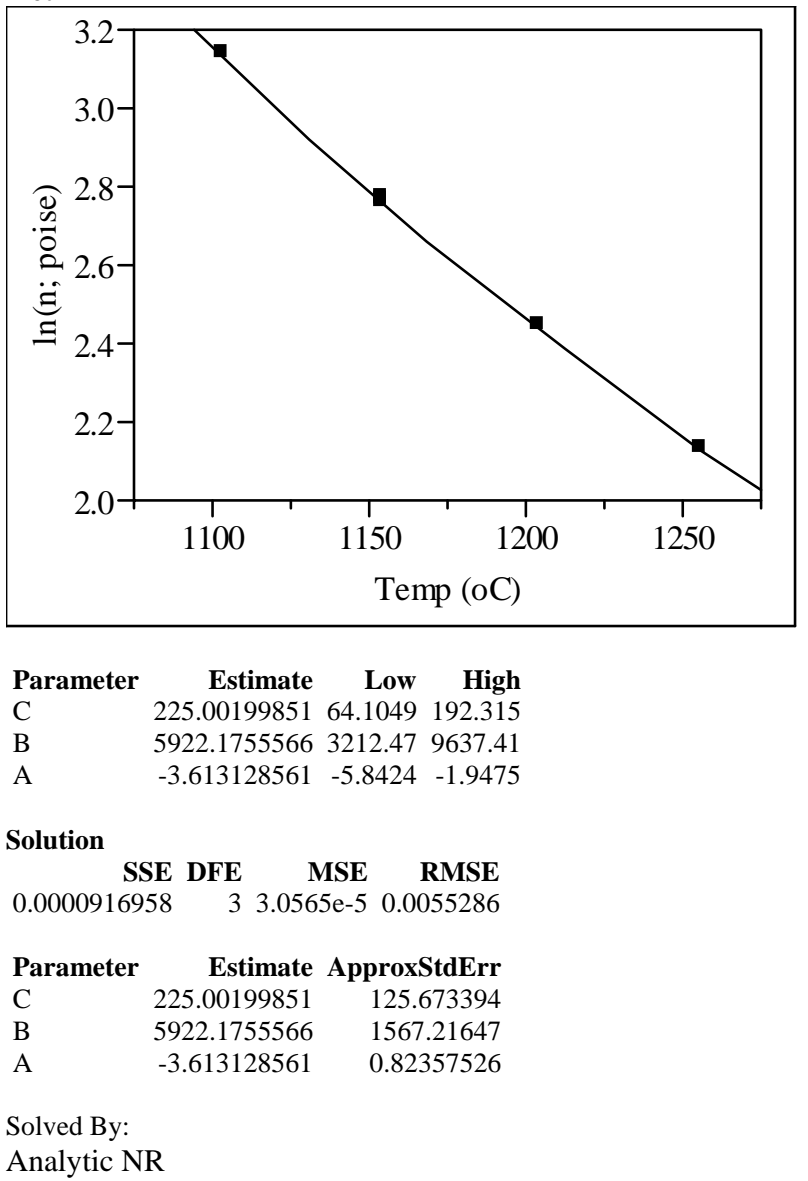


Exhibit C7. VFT fit of FY09EM21-11. Nonlinear Fit Glass ID=FY09EM21-11

Response: $\ln (n$; poise), Predictor: $\ln (n$; VTF)

Control Panel

Converged in Gradient

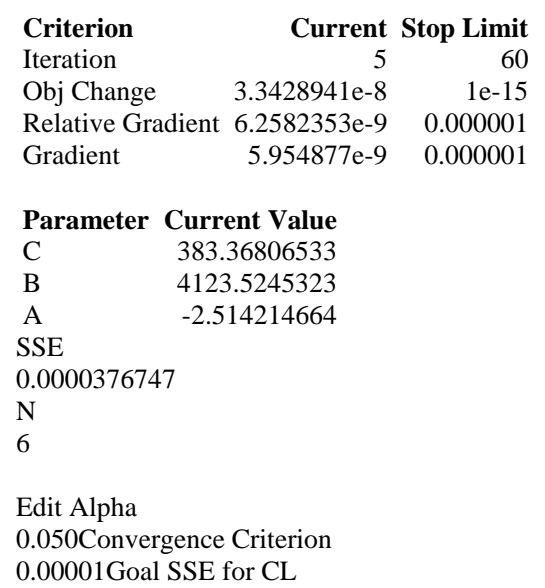

Plot

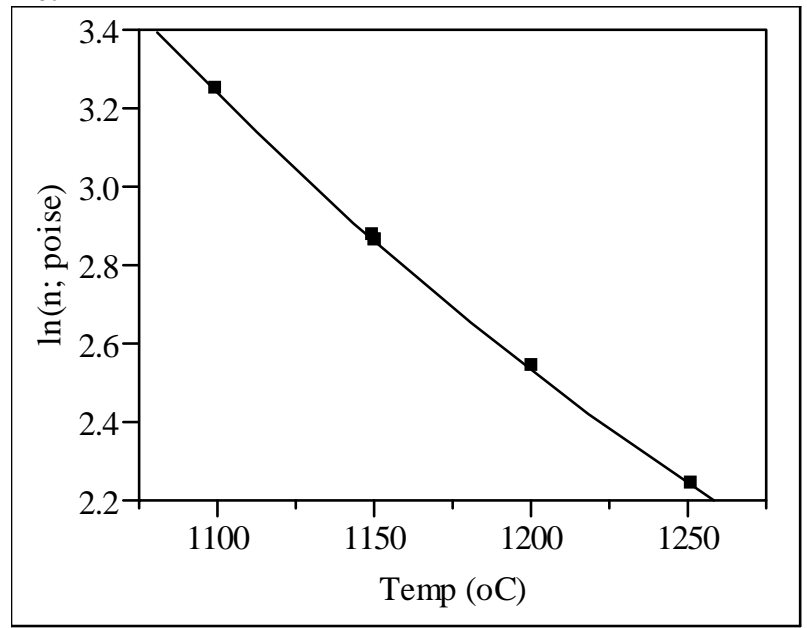

\begin{tabular}{|c|c|c|c|}
\hline Parameter & Estimate & Low & High \\
\hline C & 383.36806533 & 64.1049 & 192.315 \\
\hline B & 4123.5245323 & 3212.47 & 963 \\
\hline A & -2.514214664 & -5.8424 & \\
\hline \multicolumn{4}{|l|}{ Solution } \\
\hline \multirow{2}{*}{$\begin{array}{r}\text { SSE } \\
0.0000376747\end{array}$} & E DFE & ISE & RMs \\
\hline & 31. & $8 e-5 \quad 0.00$ & 0354 \\
\hline Par & Estimate & ApproxS & Std \\
\hline $\mathrm{C}$ & 383.36806533 & & \\
\hline B & 4123.524 & 571.2 & 2 \\
\hline A & -2.514214664 & 0.362 & 22733 \\
\hline
\end{tabular}

Solved By:

Analytic NR
Exhibit C8. VFT fit of FY09EM21-12.

Nonlinear Fit Glass ID=FY09EM21-12

Response: $\ln (\mathrm{n}$; poise), Predictor: $\ln (\mathrm{n}$; VTF)

Control Panel

Converged in Gradient

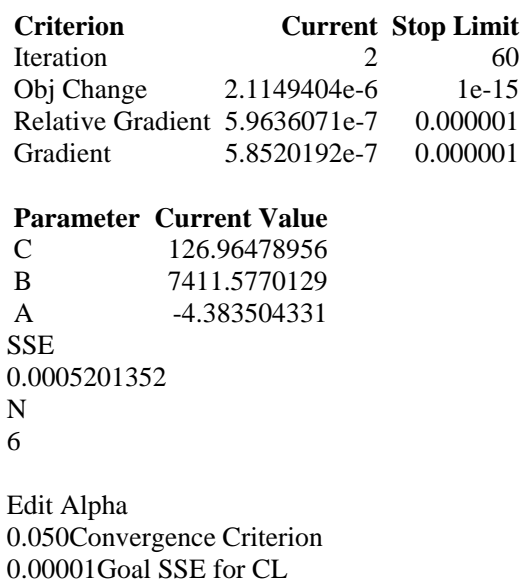

Plot

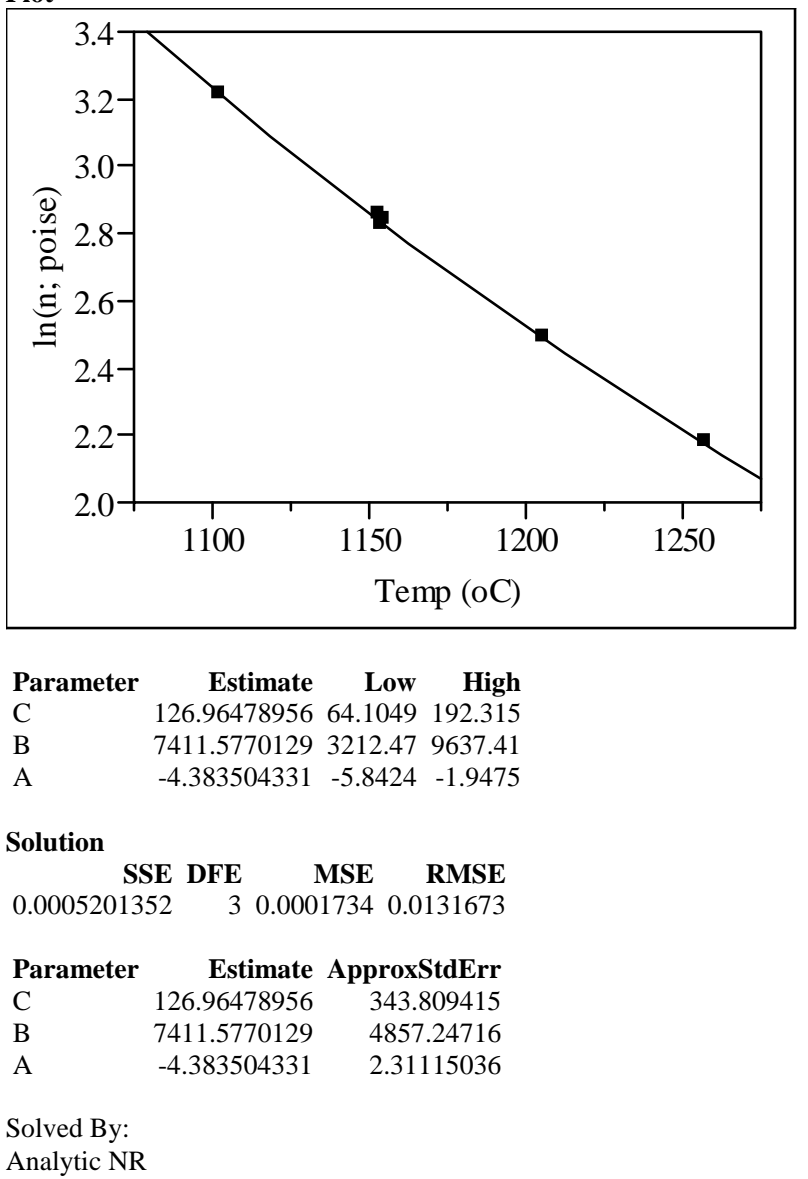


Exhibit C9. VFT fit of FY09EM21-14.

Nonlinear Fit Glass ID=FY09EM21-14

Response: $\ln (n$; poise), Predictor: $\ln (n$; VTF)

Control Panel

Converged in Gradient

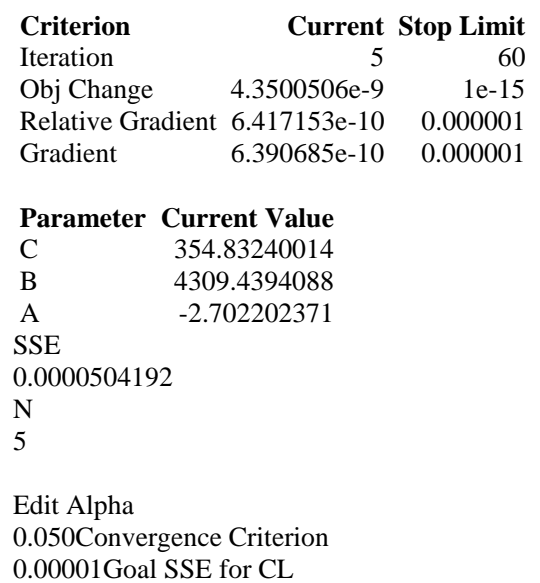

Plot

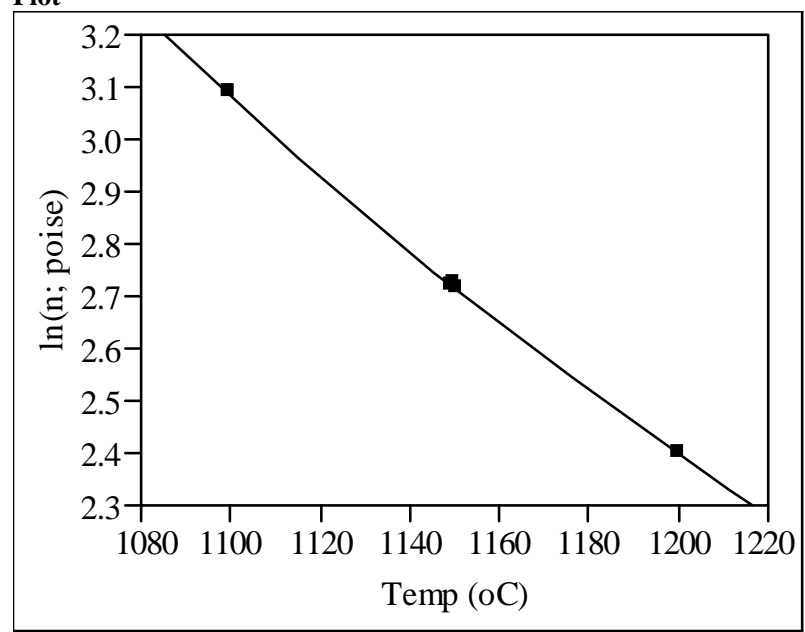

\begin{tabular}{|c|c|c|c|}
\hline Parameter & Estimate & Low & High \\
\hline C & 354.83240014 & 64.1049 & 192.3 \\
\hline B & 4309.4394088 & 3212.47 & 7963 \\
\hline A & -2.702202371 & -5.8424 & \\
\hline \multicolumn{4}{|l|}{ Solution } \\
\hline \multirow{2}{*}{$\begin{array}{r}\text { SSF } \\
0.000050419\end{array}$} & E DFE & MSE & RMSE \\
\hline & 20.000 & 02520.0 & $005020 s$ \\
\hline Para & ate & Approx & xStdI \\
\hline C & 354.83240014 & 167. & .383678 \\
\hline B & 4309.4394088 & 1826 & 6.09644 \\
\hline A & -2.702202371 & & 1545323 \\
\hline
\end{tabular}

Solved By:

Analytic NR
Exhibit C10. VFT fit of FY09EM21-15.

Nonlinear Fit Glass ID=FY09EM21-15

Response: $\ln (\mathrm{n}$; poise), Predictor: $\ln (\mathrm{n}$; VTF)

Control Panel

Converged in Gradient

\begin{tabular}{|c|c|c|c|}
\hline Criterion & & Current & Stop Limit \\
\hline \multicolumn{2}{|l|}{ Iteration } & 4 & 60 \\
\hline Obj Change & & $5.7405127 e-9$ & 1e-15 \\
\hline \multicolumn{2}{|c|}{ Relative Gradient } & $1.531954 \mathrm{e}-9$ & 0.000001 \\
\hline \multicolumn{2}{|l|}{ Gradient } & $9.362284 \mathrm{e}-10$ & 0.000001 \\
\hline \multicolumn{4}{|c|}{ Parameter Current Value } \\
\hline C & \multicolumn{2}{|c|}{249.64447364} & \\
\hline B & \multicolumn{2}{|c|}{6062.7150125} & \\
\hline $\mathrm{A}$ & \multicolumn{2}{|c|}{-3.591171912} & \\
\hline \multicolumn{4}{|l|}{ SSE } \\
\hline \multicolumn{4}{|l|}{0.0000884628} \\
\hline \multicolumn{4}{|l|}{$\mathrm{N}$} \\
\hline \multicolumn{4}{|c|}{6} \\
\hline Edit Alpha & & & \\
\hline 0.050Converger & ence $C$ & Criterion & \\
\hline
\end{tabular}

Plot

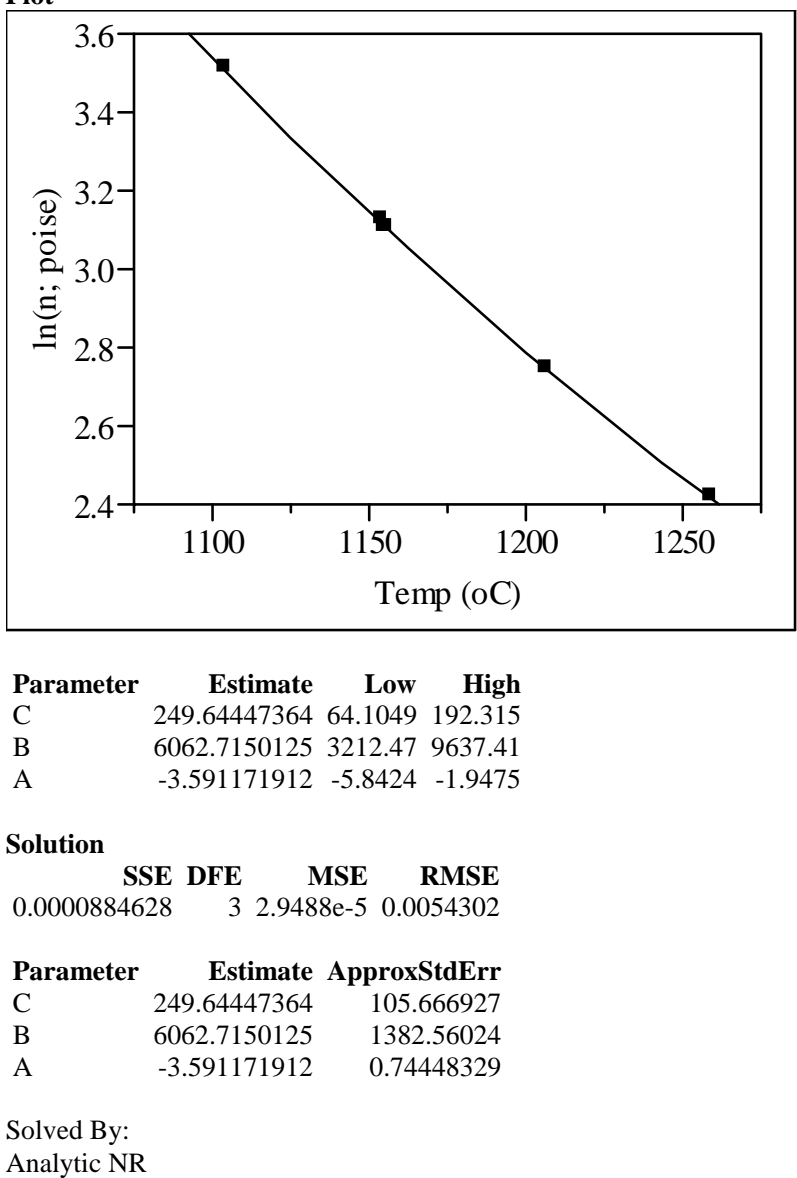


Exhibit C13. VFT fit of FY09EM21-18. Nonlinear Fit Glass ID=FY09EM21-18

Response: $\ln (n$; poise), Predictor: $\ln (n$; VTF)

Control Panel

Converged in Gradient

$\begin{array}{lrr}\text { Criterion } & \text { Current } & \text { Stop Limit } \\ \text { Iteration } & 15 & 60 \\ \text { Obj Change } & 6.164005 \mathrm{e}-10 & 1 \mathrm{e}-15 \\ \text { Relative Gradient } & & 0.000001 \\ \text { Gradient } & 3.2366588 \mathrm{e}-7 & 0.000001\end{array}$

Parameter Current Value

$-1079.690952$

B $\quad 32182.060002$

A $\quad-10.46855138$

SSE

0.042426804

$\mathrm{N}$

5

Edit Alpha

0.050Convergence Criterion

0.00001Goal SSE for CL

Plot

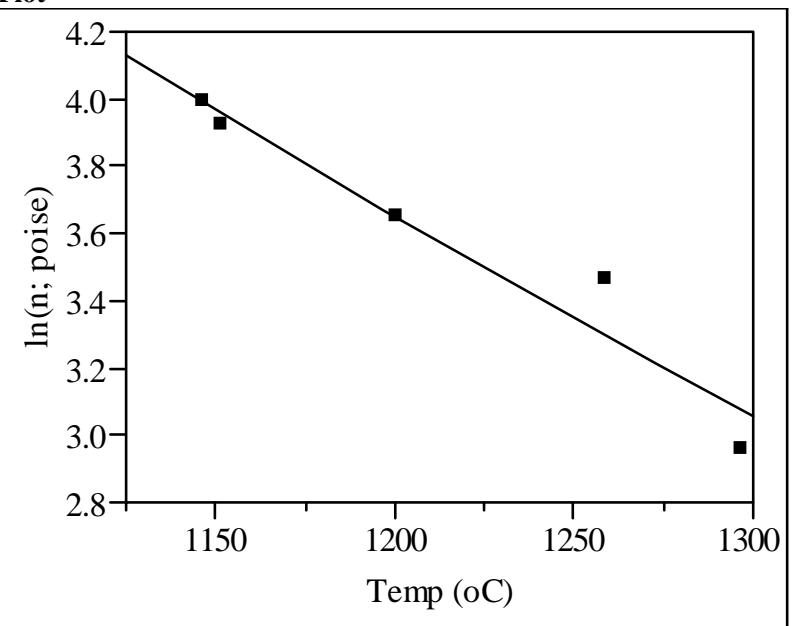

Parameter Estimate Low High

C $\quad-1079.69095264 .1049 \quad 192.315$

B $\quad 32182.060002 \quad 3212.479637 .41$

$\begin{array}{llll}\text { A } & -10.46855138 & -5.8424 & -1.9475\end{array}$

Solution

SSE DFE MSE RMSE

$\begin{array}{llll}0.042426804 & 2 & 0.0212134 & 0.1456482\end{array}$

$\begin{array}{lrr}\text { Parameter } & \text { Estimate } & \text { ApproxStdErr } \\ \text { C } & -1079.690952 & 4243.08542 \\ \text { B } & 32182.060002 & 118122.459 \\ \text { A } & -10.46855138 & 25.587628\end{array}$

Solved By:

Analytic NR
Exhibit C14. VFT fit of FY09EM21-19.

Nonlinear Fit Glass ID=FY09EM21-19

Response: $\ln (\mathrm{n}$; poise), Predictor: $\ln (\mathrm{n}$; VTF)

Control Panel

Converged in Gradient

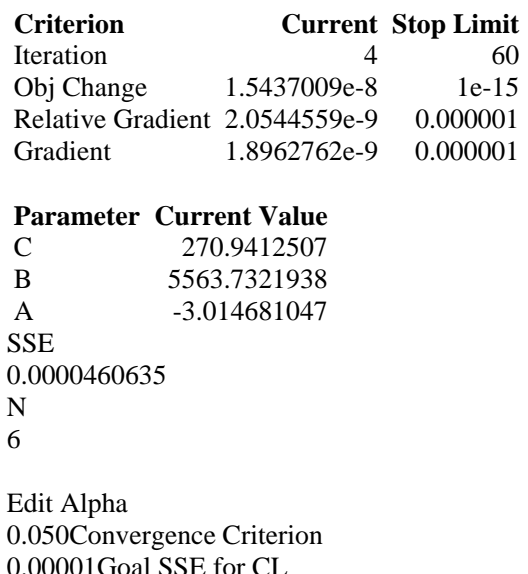

Plot

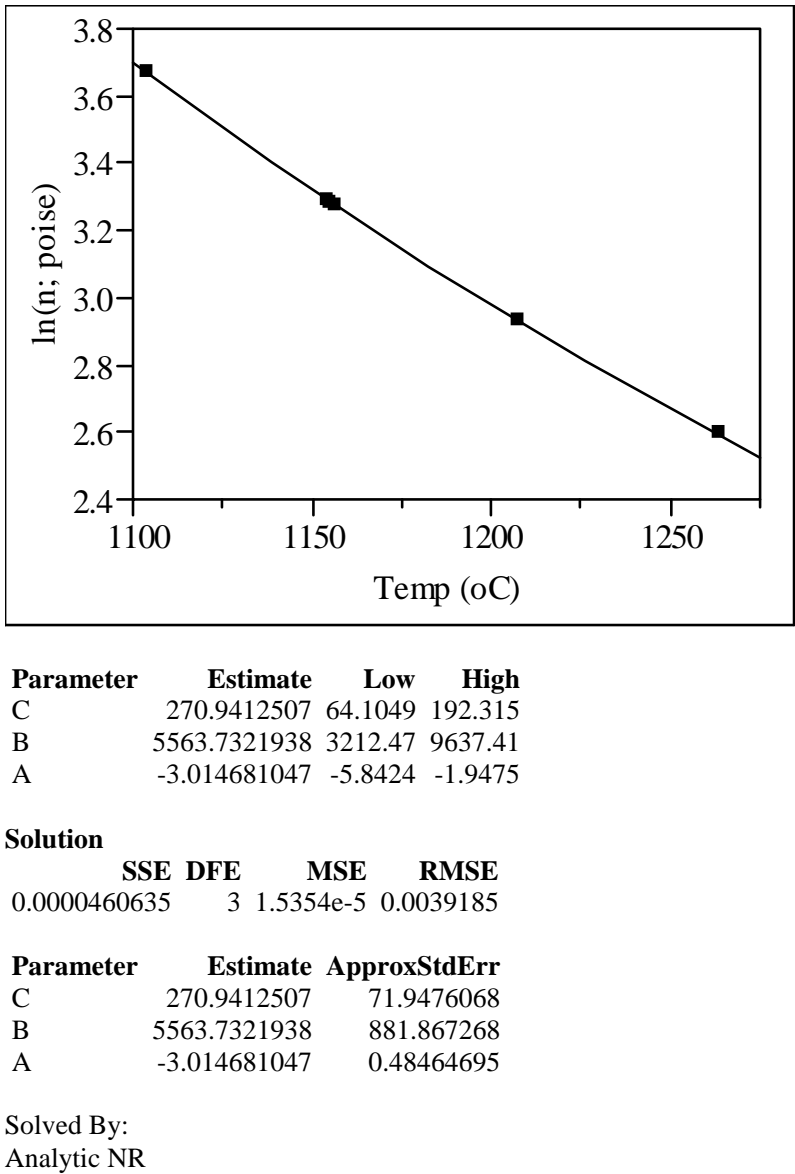


Exhibit C15. VFT fit of FY09EM21-20. Nonlinear Fit Glass ID=FY09EM21-20

Response: $\ln (n$; poise), Predictor: $\ln (n$; VTF)

Control Panel

Converged in Gradient

\begin{tabular}{|c|c|c|}
\hline Criterion & Current & Stop Limit \\
\hline Iteration & 3 & 60 \\
\hline Obj Change & $1.3081083 \mathrm{e}-6$ & $1 e-15$ \\
\hline Relative Gradi & lient $2.8569496 \mathrm{e}-7$ & 0.000001 \\
\hline Gradient & $2.7224125 \mathrm{e}-7$ & 0.000001 \\
\hline \multicolumn{3}{|c|}{ Parameter Current Value } \\
\hline C & 54.212644118 & \\
\hline B & 8661.4175821 & \\
\hline A & -4.565140805 & \\
\hline \multicolumn{3}{|l|}{ SSE } \\
\hline \multicolumn{3}{|l|}{0.0000761407} \\
\hline \multicolumn{3}{|l|}{$\mathrm{N}$} \\
\hline \multicolumn{3}{|l|}{6} \\
\hline \multicolumn{3}{|l|}{ Edit Alpha } \\
\hline \multicolumn{3}{|c|}{$\begin{array}{l}\text { 0.050Convergence Criterion } \\
\text { 0.00001Goal SSE for CL }\end{array}$} \\
\hline
\end{tabular}

Plot

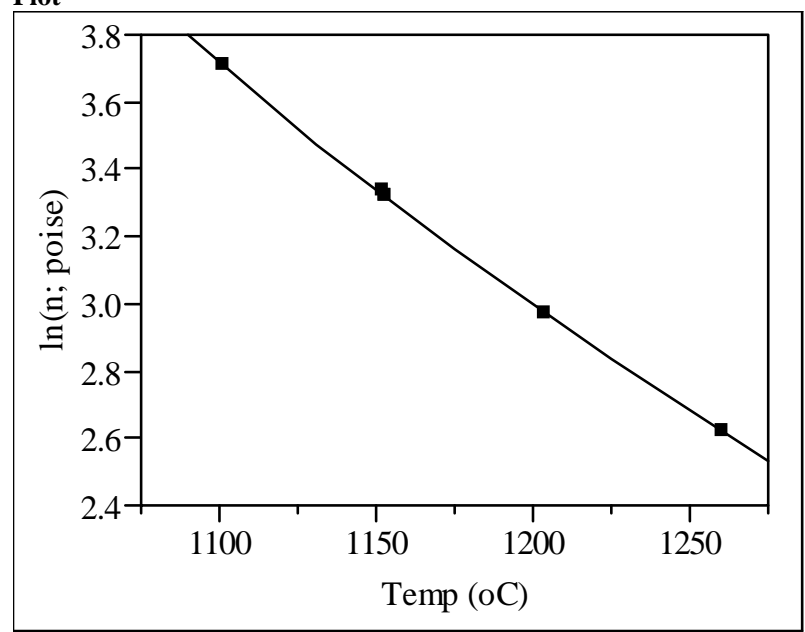

\begin{tabular}{|c|c|c|}
\hline Parameter & Estimate & Low \\
\hline C & 54.212644118 & 64.1049192 .315 \\
\hline 8 & 8661.4175821 & 3212.479637 .4 \\
\hline- & -4.565140805 & $-5.8424-1$ \\
\hline \multicolumn{3}{|l|}{ Solution } \\
\hline SSE & E DFE & RMSE \\
\hline \multicolumn{3}{|l|}{0.0000761407} \\
\hline arameter & Estimate & ApproxStdEr \\
\hline 5 & 54.212644118 & 141.025648 \\
\hline B & 8661.4175821 & 2174.72464 \\
\hline$A$ & -4.565140805 & 0.96659439 \\
\hline
\end{tabular}

Solved By:

Analytic NR
Exhibit C16. VFT fit of FY09EM21-21.

Nonlinear Fit Glass ID=FY09EM21-21

Response: $\ln (\mathrm{n}$; poise), Predictor: $\ln (\mathrm{n}$; VTF)

Control Panel

Converged in Gradient

\begin{tabular}{|c|c|c|c|}
\hline \multicolumn{2}{|l|}{ Criterion } & Current & Stop Limit \\
\hline \multicolumn{2}{|l|}{ Iteration } & 3 & 60 \\
\hline \multicolumn{2}{|l|}{ Obj Change } & $2.3640854 \mathrm{e}-6$ & 1e-15 \\
\hline \multicolumn{2}{|c|}{ Relative Gradient } & $7.0755497 \mathrm{e}-7$ & 0.000001 \\
\hline \multicolumn{2}{|l|}{ Gradient } & 7.0472487e-7 & 0.000001 \\
\hline \multicolumn{4}{|c|}{ Parameter Current Value } \\
\hline $\mathrm{C}$ & \multicolumn{2}{|c|}{239.5386168} & \\
\hline $\mathrm{B}$ & \multicolumn{2}{|c|}{4797.7500663} & \\
\hline A & \multicolumn{2}{|c|}{-3.414128589} & \\
\hline \multicolumn{4}{|l|}{ SSE } \\
\hline \multicolumn{4}{|l|}{0.0004897508} \\
\hline \multicolumn{4}{|l|}{$\mathrm{N}$} \\
\hline \multicolumn{4}{|l|}{5} \\
\hline Edit Alpha & & & \\
\hline $\begin{array}{l}\text { 0.050Convergen } \\
0.00001 \mathrm{G} a l \text { SS }\end{array}$ & ence $\mathrm{C}$ & Criterion & \\
\hline
\end{tabular}

Plot

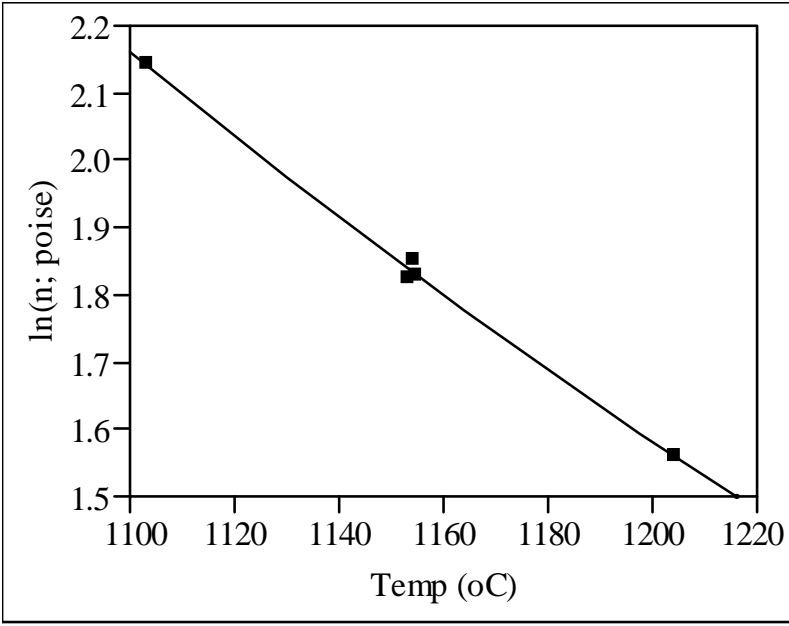

Parameter Estimate Low High

C $\quad 239.538616864 .1049 \quad 192.315$

$\begin{array}{lllll}\text { B } & 4797.7500663 & 3212.47 & 9637.41\end{array}$

A $\quad-3.414128589 \quad-5.8424 \quad-1.9475$

Solution

SSE DFE MSE RMSE $\begin{array}{llll}0.0004897508 & 2 & 0.0002449 & 0.0156485\end{array}$

$\begin{array}{lrr}\text { Parameter } & \text { Estimate } & \text { ApproxStdErr } \\ \text { C } & 239.5386168 & 814.014125 \\ \text { B } & 4797.7500663 & 8583.15162 \\ \text { A } & -3.414128589 & 4.71219599\end{array}$

Solved By:

Analytic NR 
Exhibit C17. VFT fit of FY09EM21-25. Nonlinear Fit Glass ID=FY09EM21-25

Response: $\ln (n$; poise), Predictor: $\ln (n$; VTF)

Control Panel

Converged in Gradient

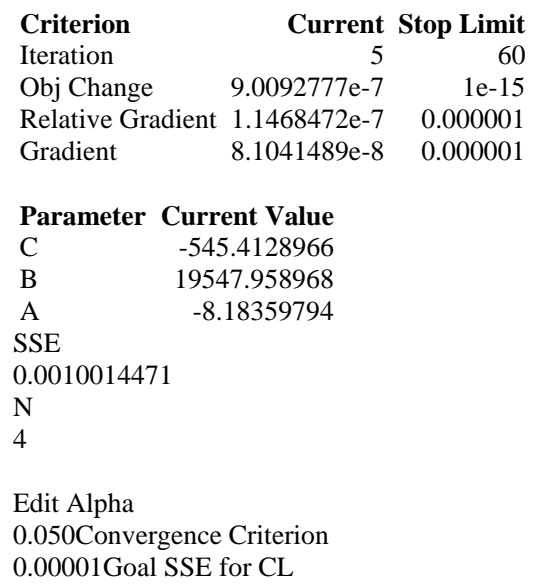

Plot

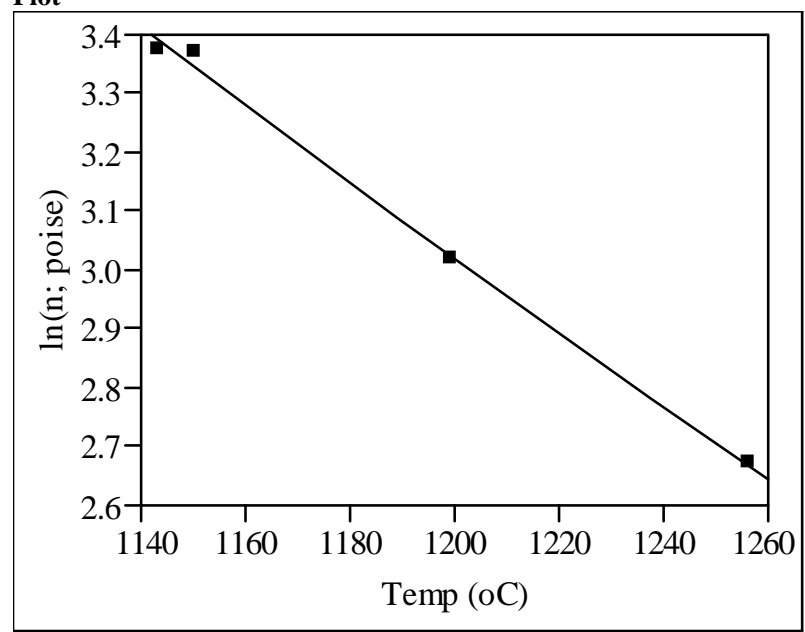

\begin{tabular}{|c|c|c|c|}
\hline Parameter & Estimate & Low & High \\
\hline C & -545.4128966 & 64.1049 & 192.315 \\
\hline 19 & 19547.958968 & 3212.47 & 9637.41 \\
\hline A & -8.18359794 & -5.8424 & \\
\hline \multicolumn{4}{|l|}{ Solution } \\
\hline SSE & E DFE & MSE & RMSE \\
\hline 0.0010014471 & 10.001 & 00140.03 & 316456 \\
\hline arameter & Estimate & ApproxS & StdE \\
\hline-5 & -545.4128966 & 5733. & .2884 \\
\hline B & 19547.958968 & 12865 & 56.272 \\
\hline 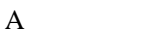 & -8.18359794 & 36.89 & 99152 \\
\hline
\end{tabular}

Solved By:

Analytic NR
Exhibit C18. VFT fit of FY09EM21-27.

Nonlinear Fit Glass ID=FY09EM21-27

Response: $\ln (\mathrm{n}$; poise), Predictor: $\ln (\mathrm{n}$; VTF)

Control Panel

Converged in Gradient

\begin{tabular}{lrr} 
Criterion & \multicolumn{1}{r}{ Current } & Stop Limit \\
Iteration & 5 & 60 \\
Obj Change & $2.8163382 \mathrm{e}-7$ & $1 \mathrm{e}-15$ \\
Relative Gradient & $5.2615069 \mathrm{e}-8$ & 0.000001 \\
Gradient & $5.2348983 \mathrm{e}-8$ & 0.000001 \\
\multicolumn{4}{c}{} \\
Parameter & Current Value \\
C & 418.63075019 \\
B & 3785.1845033 \\
A & -2.359939853 \\
SSE & \\
0.0002613861 \\
N \\
5
\end{tabular}

Plot

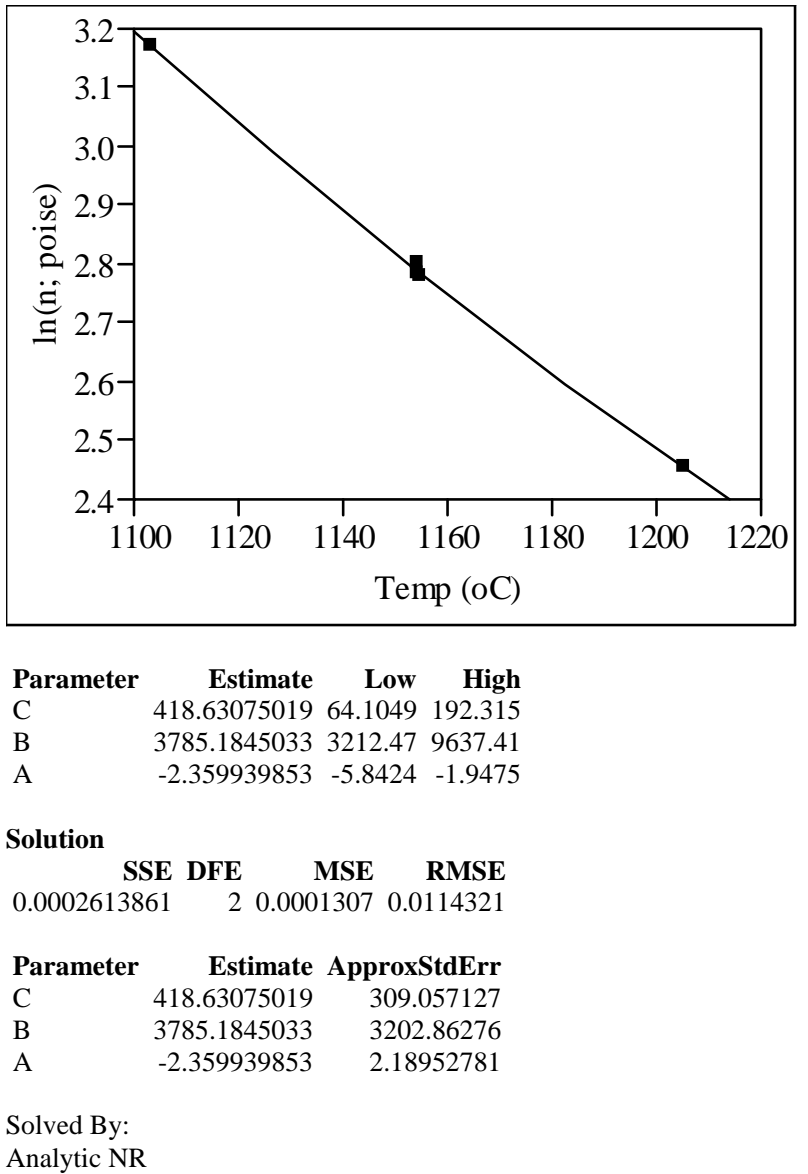




\section{Appendix D:}

\section{Tables and Exhibits Supporting the Analysis of the Chemical Composition Measurements of the Matrix 2A Study Glasses}


SRNL-STI-2009-00778, Revision 0

This page intentionally left blank. 
SRNL-STI-2009-00778, Revision 0

\section{Table D1. Measured Elemental Concentrations (wt\%) for the Matrix 2A Study Glasses Prepared Using Lithium Metaborate (part 1)}

\begin{tabular}{|c|c|c|c|c|c|c|c|c|c|c|c|c|c|c|}
\hline Set & Glass ID & Block & $\begin{array}{c}\text { Sub- } \\
\text { Block }\end{array}$ & Sequence & Lab ID & $\begin{array}{c}\text { Al } \\
(w t \%)\end{array}$ & $\begin{array}{c}\text { Ba } \\
(\mathbf{w t} \%)\end{array}$ & $\begin{array}{c}\mathrm{Ca} \\
(\mathrm{wt} \%)\end{array}$ & $\begin{array}{c}\mathrm{Cd} \\
(\mathrm{wt} \%)\end{array}$ & $\begin{array}{c}\mathrm{Ce} \\
(\mathrm{wt} \%)\end{array}$ & $\begin{array}{c}\mathrm{Cr} \\
(\mathrm{wt} \%)\end{array}$ & $\begin{array}{c}\mathrm{Cu} \\
(\mathrm{wt} \%)\end{array}$ & $\begin{array}{c}\mathrm{Fe} \\
(\mathrm{wt} \%)\end{array}$ & $\begin{array}{c}\text { La } \\
\text { (wt\%) }\end{array}$ \\
\hline 1 & Batch 1 & 1 & 1 & 1 & BCHLM1111 & 2.48 & 0.123 & 0.864 & $<0.010$ & $<0.010$ & 0.072 & 0.308 & 8.82 & $<0.010$ \\
\hline 1 & EM-15 & 1 & 1 & 2 & r10LM21 & 7.69 & 0.062 & 3.26 & $<0.010$ & 0.198 & 0.056 & 0.036 & 7.93 & 0.059 \\
\hline 1 & EM-13 & 1 & 1 & 3 & r02LM21 & 7.54 & 0.057 & 1.74 & $<0.010$ & 0.197 & 0.064 & 0.040 & 8.04 & 0.057 \\
\hline 1 & EM-04 & 1 & 1 & 4 & r07LM11 & 3.51 & 0.062 & 0.939 & $<0.010$ & 0.193 & 0.082 & 0.045 & 9.98 & 0.055 \\
\hline 1 & EM-15 & 1 & 1 & 5 & r10LM11 & 7.49 & 0.063 & 3.14 & $<0.010$ & 0.202 & 0.058 & 0.036 & 7.95 & 0.060 \\
\hline 1 & EM-05 & 1 & 1 & 6 & r11LM11 & 3.44 & 0.063 & 0.927 & $<0.010$ & 0.200 & 0.072 & 0.035 & 11.3 & 0.060 \\
\hline 1 & EM-11 & 1 & 1 & 7 & r15LM21 & 5.98 & 0.063 & 0.929 & $<0.010$ & 0.204 & 0.064 & 0.038 & 9.48 & 0.060 \\
\hline 1 & EM-07 & 1 & 1 & 8 & r09LM21 & 3.45 & 0.062 & 2.36 & $<0.010$ & 0.201 & 0.065 & 0.045 & 12.5 & 0.059 \\
\hline 1 & EM-04 & 1 & 1 & 9 & r07LM21 & 3.40 & 0.062 & 0.920 & $<0.010$ & 0.195 & 0.081 & 0.042 & 9.87 & 0.055 \\
\hline 1 & Batch 1 & 1 & 1 & 10 & BCHLM1112 & 2.48 & 0.119 & 0.842 & $<0.010$ & $<0.010$ & 0.070 & 0.308 & 8.65 & $<0.010$ \\
\hline 1 & EM-05 & 1 & 1 & 11 & r11LM21 & 3.56 & 0.063 & 0.989 & $<0.010$ & 0.200 & 0.074 & 0.035 & 11.0 & 0.060 \\
\hline 1 & EM-03 & 1 & 1 & 12 & r04LM21 & 4.78 & 0.063 & 0.974 & $<0.010$ & 0.196 & 0.080 & 0.048 & 9.69 & 0.059 \\
\hline 1 & EM-11 & 1 & 1 & 13 & r15LM11 & 6.10 & 0.064 & 0.943 & $<0.010$ & 0.203 & 0.064 & 0.037 & 9.34 & 0.061 \\
\hline 1 & EM-13 & 1 & 1 & 14 & r02LM11 & 7.61 & 0.055 & 1.77 & $<0.010$ & 0.191 & 0.063 & 0.041 & 8.07 & 0.055 \\
\hline 1 & EM-03 & 1 & 1 & 15 & r04LM11 & 4.85 & 0.062 & 0.992 & $<0.010$ & 0.196 & 0.079 & 0.040 & 9.66 & 0.059 \\
\hline 1 & EM-14 & 1 & 1 & 16 & r08LM11 & 7.63 & 0.063 & 2.50 & $<0.010$ & 0.194 & 0.063 & 0.042 & 8.02 & 0.059 \\
\hline 1 & EM-07 & 1 & 1 & 17 & r09LM11 & 3.59 & 0.060 & 2.49 & $<0.010$ & 0.196 & 0.063 & 0.059 & 12.1 & 0.057 \\
\hline 1 & EM-14 & 1 & 1 & 18 & r08LM21 & 7.55 & 0.060 & 2.95 & $<0.010$ & 0.190 & 0.060 & 0.043 & 8.08 & 0.057 \\
\hline 1 & Batch 1 & 1 & 1 & 19 & BCHLM1113 & 2.52 & 0.119 & 0.871 & $<0.010$ & $<0.010$ & 0.070 & 0.302 & 8.62 & $<0.010$ \\
\hline 1 & Batch 1 & 1 & 2 & 1 & BCHLM1121 & 2.50 & 0.119 & 0.857 & $<0.010$ & $<0.010$ & 0.072 & 0.308 & 8.91 & $<0.010$ \\
\hline 1 & EM-07 & 1 & 2 & 2 & r09LM22 & 3.48 & 0.063 & 2.42 & $<0.010$ & 0.203 & 0.067 & 0.043 & 12.7 & 0.060 \\
\hline 1 & EM-15 & 1 & 2 & 3 & r10LM12 & 7.60 & 0.063 & 3.21 & $<0.010$ & 0.204 & 0.059 & 0.035 & 8.04 & 0.059 \\
\hline 1 & EM-11 & 1 & 2 & 4 & r15LM12 & 6.16 & 0.062 & 0.969 & $<0.010$ & 0.206 & 0.064 & 0.037 & 9.48 & 0.061 \\
\hline 1 & EM-07 & 1 & 2 & 5 & r09LM12 & 3.59 & 0.060 & 2.50 & $<0.010$ & 0.200 & 0.063 & 0.058 & 12.6 & 0.058 \\
\hline 1 & EM-04 & 1 & 2 & 6 & r07LM22 & 3.48 & 0.062 & 0.955 & $<0.010$ & 0.198 & 0.082 & 0.041 & 9.98 & 0.055 \\
\hline 1 & EM-14 & 1 & 2 & 7 & r08LM12 & 7.56 & 0.062 & 2.47 & $<0.010$ & 0.196 & 0.063 & 0.041 & 8.41 & 0.059 \\
\hline 1 & EM-03 & 1 & 2 & 8 & r04LM12 & 4.86 & 0.063 & 0.984 & $<0.010$ & 0.200 & 0.081 & 0.039 & 10.1 & 0.059 \\
\hline 1 & EM-05 & 1 & 2 & 9 & r11LM12 & 3.48 & 0.063 & 0.944 & $<0.010$ & 0.202 & 0.072 & 0.034 & 11.5 & 0.059 \\
\hline 1 & Batch 1 & 1 & 2 & 10 & BCHLM1122 & 2.53 & 0.118 & 0.876 & $<0.010$ & $<0.010$ & 0.071 & 0.324 & 8.77 & $<0.010$ \\
\hline 1 & EM-15 & 1 & 2 & 11 & r10LM22 & 7.51 & 0.061 & 3.17 & $<0.010$ & 0.202 & 0.057 & 0.035 & 7.95 & 0.058 \\
\hline 1 & EM-03 & 1 & 2 & 12 & r04LM22 & 4.75 & 0.061 & 0.965 & $<0.010$ & 0.200 & 0.078 & 0.048 & 9.96 & 0.059 \\
\hline 1 & EM-14 & 1 & 2 & 13 & r08LM22 & 7.43 & 0.063 & 2.87 & $<0.010$ & 0.198 & 0.064 & 0.043 & 8.49 & 0.059 \\
\hline 1 & EM-05 & 1 & 2 & 14 & r11LM22 & 3.47 & 0.062 & 0.946 & $<0.010$ & 0.203 & 0.073 & 0.034 & 11.7 & 0.060 \\
\hline 1 & EM-11 & 1 & 2 & 15 & r15LM22 & 6.09 & 0.062 & 0.966 & $<0.010$ & 0.206 & 0.064 & 0.037 & 9.44 & 0.060 \\
\hline 1 & EM-04 & 1 & 2 & 16 & r07LM12 & 3.53 & 0.061 & 0.953 & $<0.010$ & 0.194 & 0.080 & 0.044 & 9.93 & 0.054 \\
\hline 1 & EM-13 & 1 & 2 & 17 & r02LM12 & 7.59 & 0.055 & 1.77 & $<0.010$ & 0.194 & 0.063 & 0.040 & 8.14 & 0.055 \\
\hline 1 & EM-13 & 1 & 2 & 18 & r02LM22 & 7.53 & 0.057 & 1.74 & $<0.010$ & 0.197 & 0.064 & 0.039 & 8.10 & 0.057 \\
\hline 1 & Batch 1 & 1 & 2 & 19 & BCHLM1123 & 2.52 & 0.118 & 0.881 & $<0.010$ & $<0.010$ & 0.071 & 0.319 & 8.88 & $<0.010$ \\
\hline 1 & Batch 1 & 2 & 1 & 1 & BCHLM1211 & 2.53 & 0.121 & 0.864 & $<0.010$ & $<0.010$ & 0.072 & 0.309 & 8.86 & $<0.010$ \\
\hline 1 & EM-02 & 2 & 1 & 2 & r05LM11 & 7.68 & 0.060 & 0.997 & $<0.010$ & 0.194 & 0.076 & 0.039 & 9.75 & 0.060 \\
\hline 1 & EM-06 & 2 & 1 & 3 & r13LM21 & 3.58 & 0.061 & 0.976 & $<0.010$ & 0.198 & 0.071 & 0.037 & 12.6 & 0.058 \\
\hline 1 & EM-09 & 2 & 1 & 4 & r06LM11 & 6.22 & 0.059 & 0.976 & $<0.010$ & 0.195 & 0.063 & 0.048 & 11.2 & 0.057 \\
\hline 1 & EM-12 & 2 & 1 & 5 & r14LM11 & 6.09 & 0.063 & 0.938 & $<0.010$ & 0.198 & 0.084 & 0.039 & 10.0 & 0.061 \\
\hline 1 & EM-10 & 2 & 1 & 6 & r01LM21 & 6.12 & 0.058 & 0.962 & $<0.010$ & 0.191 & 0.068 & 0.045 & 8.27 & 0.058 \\
\hline 1 & EM-12 & 2 & 1 & 7 & r14LM21 & 6.12 & 0.063 & 0.997 & $<0.010$ & 0.196 & 0.077 & 0.037 & 9.42 & 0.060 \\
\hline 1 & EM-08 & 2 & 1 & 8 & r03LM11 & 3.52 & 0.054 & 3.24 & $<0.010$ & 0.197 & 0.059 & 0.033 & 12.6 & 0.058 \\
\hline 1 & Batch 1 & 2 & 1 & 9 & BCHLM1212 & 2.52 & 0.121 & 0.868 & $<0.010$ & $<0.010$ & 0.072 & 0.309 & 8.68 & $<0.010$ \\
\hline 1 & EM-10 & 2 & 1 & 10 & r01LM11 & 6.06 & 0.059 & 0.954 & $<0.010$ & 0.189 & 0.069 & 0.048 & 8.28 & 0.058 \\
\hline 1 & EM-02 & 2 & 1 & 11 & r05LM21 & 7.45 & 0.060 & 0.956 & $<0.010$ & 0.193 & 0.074 & 0.039 & 9.74 & 0.060 \\
\hline 1 & EM-08 & 2 & 1 & 12 & r03LM21 & 3.56 & 0.054 & 3.25 & $<0.010$ & 0.197 & 0.059 & 0.033 & 12.7 & 0.058 \\
\hline 1 & EM-01 & 2 & 1 & 13 & r12LM21 & 6.12 & 0.060 & 0.964 & $<0.010$ & 0.192 & 0.069 & 0.037 & 9.72 & 0.058 \\
\hline 1 & EM-06 & 2 & 1 & 14 & r13LM11 & 3.59 & 0.063 & 0.999 & $<0.010$ & 0.202 & 0.072 & 0.035 & 12.6 & 0.059 \\
\hline 1 & EM-09 & 2 & 1 & 15 & r06LM21 & 6.26 & 0.060 & 1.01 & $<0.010$ & 0.195 & 0.065 & 0.041 & 10.9 & 0.057 \\
\hline 1 & EM-01 & 2 & 1 & 16 & r12LM11 & 6.17 & 0.061 & 0.997 & $<0.010$ & 0.195 & 0.070 & 0.036 & 9.65 & 0.059 \\
\hline 1 & Batch 1 & 2 & 1 & 17 & BCHLM1213 & 2.52 & 0.119 & 0.870 & $<0.010$ & $<0.010$ & 0.070 & 0.305 & 8.65 & $<0.010$ \\
\hline 1 & Batch 1 & 2 & 2 & 1 & BCHLM1221 & 2.50 & 0.122 & 0.841 & $<0.010$ & $<0.010$ & 0.072 & 0.308 & 8.96 & $<0.010$ \\
\hline 1 & EM-01 & 2 & 2 & 2 & r12LM22 & 6.04 & 0.062 & 0.925 & $<0.010$ & 0.191 & 0.071 & 0.035 & 10.1 & 0.059 \\
\hline 1 & EM-10 & 2 & 2 & 3 & r01LM22 & 6.09 & 0.061 & 0.944 & $<0.010$ & 0.188 & 0.071 & 0.043 & 8.43 & 0.059 \\
\hline 1 & EM-08 & 2 & 2 & 4 & r03LM12 & 3.51 & 0.056 & 3.21 & $<0.010$ & 0.194 & 0.060 & 0.032 & 12.8 & 0.057 \\
\hline 1 & EM-10 & 2 & 2 & 5 & r01LM12 & 6.09 & 0.061 & 0.954 & $<0.010$ & 0.186 & 0.071 & 0.046 & 8.35 & 0.058 \\
\hline 1 & EM-08 & 2 & 2 & 6 & r03LM22 & 3.47 & 0.055 & 3.14 & $<0.010$ & 0.195 & 0.060 & 0.032 & 12.9 & 0.058 \\
\hline 1 & EM-02 & 2 & 2 & 7 & r05LM12 & 7.43 & 0.061 & 0.934 & $<0.010$ & 0.190 & 0.075 & 0.037 & 9.93 & 0.059 \\
\hline 1 & EM-02 & 2 & 2 & 8 & r05LM22 & 7.37 & 0.063 & 0.926 & $<0.010$ & 0.193 & 0.078 & 0.037 & 9.97 & 0.061 \\
\hline 1 & Batch 1 & 2 & 2 & 9 & BCHLM1222 & 2.48 & 0.123 & 0.855 & $<0.010$ & $<0.010$ & 0.073 & 0.302 & 8.95 & $<0.010$ \\
\hline 1 & EM-12 & 2 & 2 & 10 & r14LM22 & 6.06 & 0.065 & 0.977 & $<0.010$ & 0.194 & 0.078 & 0.036 & 9.52 & 0.061 \\
\hline 1 & EM-09 & 2 & 2 & 11 & r06LM22 & 6.18 & 0.061 & 0.976 & $<0.010$ & 0.192 & 0.066 & 0.039 & 11.2 & 0.058 \\
\hline 1 & EM-06 & 2 & 2 & 12 & r13LM12 & 3.50 & 0.063 & 0.951 & $<0.010$ & 0.200 & 0.072 & 0.034 & 12.9 & 0.059 \\
\hline 1 & EM-09 & 2 & 2 & 13 & r06LM12 & 6.05 & 0.061 & 0.923 & $<0.010$ & 0.192 & 0.064 & 0.046 & 11.4 & 0.057 \\
\hline 1 & EM-01 & 2 & 2 & 14 & r12LM12 & 5.99 & 0.062 & 0.931 & $<0.010$ & 0.195 & 0.071 & 0.035 & 10.1 & 0.060 \\
\hline 1 & EM-12 & 2 & 2 & 15 & r14LM12 & 6.05 & 0.065 & 0.926 & $<0.010$ & 0.195 & 0.087 & 0.037 & 9.98 & 0.061 \\
\hline 1 & EM-06 & 2 & 2 & 16 & r13LM22 & 3.53 & 0.062 & 0.950 & $<0.010$ & 0.196 & 0.073 & 0.035 & 12.7 & 0.058 \\
\hline
\end{tabular}


SRNL-STI-2009-00778, Revision 0

\section{Table D1. Measured Elemental Concentrations (wt\%) for the Matrix 2A Study Glasses Prepared Using Lithium Metaborate (part 1)}

\begin{tabular}{|c|c|c|c|c|c|c|c|c|c|c|c|c|c|c|}
\hline Set & Glass ID & Block & $\begin{array}{c}\text { Sub- } \\
\text { Block }\end{array}$ & Sequence & Lab ID & $\begin{array}{c}\text { Al } \\
(w t \%)\end{array}$ & $\begin{array}{c}\text { Ba } \\
(w t \%)\end{array}$ & $\begin{array}{c}\mathrm{Ca} \\
(\mathrm{wt} \%)\end{array}$ & $\begin{array}{c}\text { Cd } \\
(w t \%)\end{array}$ & $\begin{array}{c}\mathrm{Ce} \\
(\mathrm{wt} \%)\end{array}$ & $\begin{array}{c}\mathrm{Cr} \\
(w t \%)\end{array}$ & $\begin{array}{c}\mathrm{Cu} \\
(\mathrm{wt} \%)\end{array}$ & $\begin{array}{c}\begin{array}{c}\mathrm{Fe} \\
(\mathrm{wt} \%)\end{array} \\
\end{array}$ & $\begin{array}{c}\text { La } \\
(w t \%) \\
\end{array}$ \\
\hline 1 & Batch 1 & 2 & 2 & 17 & BCHLM1223 & 2.51 & 0.120 & 0.854 & $<0.010$ & $<0.010$ & 0.072 & 0.304 & 8.70 & $<0.010$ \\
\hline 2 & Batch 1 & 1 & 1 & 1 & BCHLM2111 & 2.46 & 0.119 & 0.836 & $<0.010$ & $<0.010$ & 0.070 & 0.305 & 8.79 & $<0.010$ \\
\hline 2 & EM-21 & 1 & 1 & 2 & s03LM11 & 3.55 & 0.061 & 0.987 & $<0.010$ & 0.194 & 0.075 & 0.038 & 12.9 & 0.058 \\
\hline 2 & EM-30 & 1 & 1 & 3 & s13LM11 & 7.44 & 0.058 & 3.18 & $<0.010$ & 0.186 & 0.060 & 0.036 & 8.28 & 0.059 \\
\hline 2 & EM-22 & 1 & 1 & 4 & s02LM21 & 3.44 & 0.065 & 2.44 & $<0.010$ & 0.202 & 0.074 & 0.041 & 12.8 & 0.073 \\
\hline 2 & EM-25 & 1 & 1 & 5 & s14LM11 & 6.08 & 0.058 & 0.968 & $<0.010$ & 0.190 & 0.071 & 0.036 & 8.26 & 0.060 \\
\hline 2 & EM-18 & 1 & 1 & 6 & s08LM21 & 4.72 & 0.059 & 0.947 & $<0.010$ & 0.190 & 0.070 & 0.035 & 9.52 & 0.060 \\
\hline 2 & EM-25 & 1 & 1 & 7 & s14LM21 & 6.08 & 0.060 & 0.975 & $<0.010$ & 0.194 & 0.072 & 0.036 & 8.02 & 0.060 \\
\hline 2 & EM-30 & 1 & 1 & 8 & s13LM21 & 7.49 & 0.057 & 3.23 & $<0.010$ & 0.183 & 0.061 & 0.034 & 8.12 & 0.058 \\
\hline 2 & EM-24 & 1 & 1 & 9 & s05LM21 & 6.30 & 0.059 & 0.982 & $<0.010$ & 0.191 & 0.062 & 0.040 & 11.0 & 0.060 \\
\hline 2 & Batch 1 & 1 & 1 & 10 & BCHLM2112 & 2.50 & 0.119 & 0.886 & $<0.010$ & $<0.010$ & 0.071 & 0.302 & 8.51 & $<0.010$ \\
\hline 2 & EM-21 & 1 & 1 & 11 & s03LM21 & 3.82 & 0.060 & 1.00 & $<0.010$ & 0.196 & 0.075 & 0.036 & 13.2 & 0.058 \\
\hline 2 & EM-18 & 1 & 1 & 12 & s08LM11 & 4.78 & 0.061 & 0.977 & $<0.010$ & 0.198 & 0.072 & 0.037 & 9.82 & 0.061 \\
\hline 2 & EM-20 & 1 & 1 & 13 & s04LM11 & 3.51 & 0.066 & 0.973 & $<0.010$ & 0.196 & 0.077 & 0.042 & 11.2 & 0.069 \\
\hline 2 & EM-20 & 1 & 1 & 14 & s04LM21 & 3.45 & 0.066 & 0.914 & $<0.010$ & 0.190 & 0.076 & 0.032 & 11.2 & 0.066 \\
\hline 2 & EM-24 & 1 & 1 & 15 & s05LM11 & 6.11 & 0.062 & 0.945 & $<0.010$ & 0.196 & 0.062 & 0.032 & 10.9 & 0.059 \\
\hline 2 & EM-28 & 1 & 1 & 16 & s01LM21 & 7.43 & 0.059 & 1.72 & $<0.010$ & 0.187 & 0.070 & 0.040 & 8.22 & 0.061 \\
\hline 2 & EM-22 & 1 & 1 & 17 & s02LM11 & 3.48 & 0.062 & 2.47 & $<0.010$ & 0.190 & 0.070 & 0.036 & 12.9 & 0.067 \\
\hline 2 & EM-28 & 1 & 1 & 18 & s01LM11 & 7.36 & 0.058 & 1.71 & $<0.010$ & 0.185 & 0.062 & 0.040 & 7.83 & 0.059 \\
\hline 2 & Batch 1 & 1 & 1 & 19 & BCHLM2113 & 2.47 & 0.118 & 0.863 & $<0.010$ & $<0.010$ & 0.071 & 0.303 & 8.37 & $<0.010$ \\
\hline 2 & Batch 1 & 1 & 2 & 1 & BCHLM2121 & 2.44 & 0.121 & 0.848 & $<0.010$ & $<0.010$ & 0.074 & 0.307 & 8.79 & $<0.010$ \\
\hline 2 & EM-30 & 1 & 2 & 2 & s13LM22 & 7.43 & 0.061 & 3.17 & $<0.010$ & 0.184 & 0.064 & 0.033 & 8.44 & 0.060 \\
\hline 2 & EM-24 & 1 & 2 & 3 & s05LM12 & 6.10 & 0.066 & 0.930 & $<0.010$ & 0.195 & 0.064 & 0.031 & 11.2 & 0.063 \\
\hline 2 & EM-22 & 1 & 2 & 4 & s02LM12 & 3.48 & 0.064 & 2.46 & $<0.010$ & 0.189 & 0.071 & 0.035 & 13.2 & 0.070 \\
\hline 2 & EM-25 & 1 & 2 & 5 & s14LM22 & 6.07 & 0.064 & 0.959 & $<0.010$ & 0.194 & 0.075 & 0.035 & 8.31 & 0.062 \\
\hline 2 & EM-28 & 1 & 2 & 6 & s01LM22 & 7.46 & 0.062 & 1.71 & $<0.010$ & 0.186 & 0.071 & 0.040 & 8.32 & 0.064 \\
\hline 2 & EM-20 & 1 & 2 & 7 & s04LM22 & 3.46 & 0.071 & 0.910 & $<0.010$ & 0.193 & 0.078 & 0.031 & 11.3 & 0.069 \\
\hline 2 & EM-24 & 1 & 2 & 8 & s05LM22 & 6.32 & 0.063 & 0.970 & $<0.010$ & 0.190 & 0.064 & 0.040 & 11.3 & 0.062 \\
\hline 2 & EM-21 & 1 & 2 & 9 & s03LM12 & 3.60 & 0.066 & 1.01 & $<0.010$ & 0.194 & 0.077 & 0.037 & 13.1 & 0.059 \\
\hline 2 & Batch 1 & 1 & 2 & 10 & BCHLM2122 & 2.51 & 0.124 & 0.853 & $<0.010$ & $<0.010$ & 0.072 & 0.306 & 8.68 & $<0.010$ \\
\hline 2 & EM-18 & 1 & 2 & 11 & s08LM12 & 4.77 & 0.065 & 0.971 & $<0.010$ & 0.197 & 0.074 & 0.036 & 9.57 & 0.063 \\
\hline 2 & EM-30 & 1 & 2 & 12 & s13LM12 & 7.46 & 0.061 & 3.21 & $<0.010$ & 0.185 & 0.061 & 0.035 & 8.11 & 0.058 \\
\hline 2 & EM-22 & 1 & 2 & 13 & s02LM22 & 3.43 & 0.068 & 2.44 & $<0.010$ & 0.201 & 0.076 & 0.040 & 12.6 & 0.072 \\
\hline 2 & EM-25 & 1 & 2 & 14 & s14LM12 & 6.10 & 0.063 & 0.969 & $<0.010$ & 0.190 & 0.074 & 0.036 & 8.18 & 0.059 \\
\hline 2 & EM-21 & 1 & 2 & 15 & s03LM22 & 3.77 & 0.065 & 0.984 & $<0.010$ & 0.196 & 0.078 & 0.035 & 12.9 & 0.067 \\
\hline 2 & EM-18 & 1 & 2 & 16 & s08LM22 & 4.72 & 0.063 & 0.943 & $<0.010$ & 0.190 & 0.071 & 0.034 & 9.64 & 0.068 \\
\hline 2 & EM-28 & 1 & 2 & 17 & s01LM12 & 7.42 & 0.060 & 1.72 & $<0.010$ & 0.183 & 0.063 & 0.040 & 7.89 & 0.068 \\
\hline 2 & EM-20 & 1 & 2 & 18 & s04LM12 & 3.52 & 0.069 & 0.975 & $<0.010$ & 0.195 & 0.077 & 0.041 & 11.1 & 0.078 \\
\hline 2 & Batch 1 & 1 & 2 & 19 & BCHLM2123 & 2.47 & 0.120 & 0.867 & $<0.010$ & $<0.010$ & 0.072 & 0.305 & 8.45 & $<0.010$ \\
\hline 2 & Batch 1 & 2 & 1 & 1 & BCHLM2211 & 2.44 & 0.120 & 0.841 & $<0.010$ & $<0.010$ & 0.071 & 0.305 & 8.77 & $<0.010$ \\
\hline 2 & EM-17 & 2 & 1 & 2 & s10LM21 & 7.28 & 0.062 & 0.910 & $<0.010$ & 0.188 & 0.068 & 0.039 & 9.77 & 0.064 \\
\hline 2 & EM-19 & 2 & 1 & 3 & s06LM21 & 3.52 & 0.059 & 0.979 & $<0.010$ & 0.184 & 0.081 & 0.033 & 9.98 & 0.062 \\
\hline 2 & EM-26 & 2 & 1 & 4 & s07LM11 & 6.03 & 0.058 & 0.955 & $<0.010$ & 0.191 & 0.064 & 0.038 & 9.60 & 0.064 \\
\hline 2 & EM-27 & 2 & 1 & 5 & s15LM21 & 5.88 & 0.061 & 0.912 & $<0.010$ & 0.191 & 0.073 & 0.038 & 9.83 & 0.066 \\
\hline 2 & EM-16 & 2 & 1 & 6 & s11LM11 & 6.03 & 0.063 & 0.917 & $<0.010$ & 0.199 & 0.061 & 0.041 & 9.61 & 0.069 \\
\hline 2 & EM-29 & 2 & 1 & 7 & s09LM21 & 7.29 & 0.063 & 2.36 & $<0.010$ & 0.193 & 0.063 & 0.036 & 8.32 & 0.063 \\
\hline 2 & EM-16 & 2 & 1 & 8 & s11LM21 & 6.01 & 0.064 & 0.927 & $<0.010$ & 0.200 & 0.062 & 0.033 & 9.82 & 0.068 \\
\hline 2 & Batch 1 & 2 & 1 & 9 & BCHLM2212 & 2.41 & 0.122 & 0.840 & $<0.010$ & $<0.010$ & 0.073 & 0.308 & 8.81 & $<0.010$ \\
\hline 2 & EM-29 & 2 & 1 & 10 & s09LM11 & 7.37 & 0.061 & 2.43 & $<0.010$ & 0.191 & 0.065 & 0.035 & 8.33 & 0.064 \\
\hline 2 & EM-23 & 2 & 1 & 11 & s12LM21 & 3.60 & 0.060 & 3.08 & $<0.010$ & 0.188 & 0.068 & 0.036 & 13.0 & 0.066 \\
\hline 2 & EM-23 & 2 & 1 & 12 & s12LM11 & 3.36 & 0.061 & 3.00 & $<0.010$ & 0.193 & 0.069 & 0.038 & 12.9 & 0.067 \\
\hline 2 & EM-26 & 2 & 1 & 13 & s07LM21 & 5.94 & 0.060 & 0.907 & $<0.010$ & 0.196 & 0.058 & 0.032 & 9.45 & 0.066 \\
\hline 2 & EM-17 & 2 & 1 & 14 & s10LM11 & 7.21 & 0.063 & 0.897 & $<0.010$ & 0.194 & 0.067 & 0.043 & 9.54 & 0.067 \\
\hline 2 & EM-19 & 2 & 1 & 15 & s06LM11 & 3.46 & 0.063 & 0.916 & $<0.010$ & 0.191 & 0.087 & 0.033 & 9.91 & 0.066 \\
\hline 2 & EM-27 & 2 & 1 & 16 & s15LM11 & 5.94 & 0.061 & 0.912 & $<0.010$ & 0.189 & 0.076 & 0.045 & 9.86 & 0.067 \\
\hline 2 & Batch 1 & 2 & 1 & 17 & BCHLM2213 & 2.41 & 0.124 & 0.829 & $<0.010$ & $<0.010$ & 0.071 & 0.303 & 8.70 & $<0.010$ \\
\hline 2 & Batch 1 & 2 & 2 & 1 & BCHLM2221 & 2.47 & 0.116 & 0.852 & $<0.010$ & $<0.010$ & 0.071 & 0.311 & 8.49 & $<0.010$ \\
\hline 2 & EM-23 & 2 & 2 & 2 & s12LM12 & 3.45 & 0.061 & 3.10 & $<0.010$ & 0.195 & 0.068 & 0.038 & 12.5 & 0.069 \\
\hline 2 & EM-16 & 2 & 2 & 3 & s11LM22 & 6.02 & 0.065 & 0.920 & $<0.010$ & 0.200 & 0.061 & 0.032 & 9.45 & 0.070 \\
\hline 2 & EM-26 & 2 & 2 & 4 & s07LM22 & 5.99 & 0.060 & 0.912 & $<0.010$ & 0.198 & 0.058 & 0.032 & 9.11 & 0.069 \\
\hline 2 & EM-16 & 2 & 2 & 5 & s11LM12 & 6.05 & 0.064 & 0.903 & $<0.010$ & 0.202 & 0.063 & 0.040 & 9.49 & 0.073 \\
\hline 2 & EM-23 & 2 & 2 & 6 & s12LM22 & 3.65 & 0.059 & 3.08 & $<0.010$ & 0.190 & 0.068 & 0.035 & 12.5 & 0.068 \\
\hline 2 & EM-27 & 2 & 2 & 7 & s15LM12 & 5.91 & 0.059 & 0.894 & $<0.010$ & 0.191 & 0.074 & 0.045 & 9.61 & 0.068 \\
\hline 2 & EM-27 & 2 & 2 & 8 & s15LM22 & 5.84 & 0.060 & 0.895 & $<0.010$ & 0.192 & 0.073 & 0.037 & 9.55 & 0.068 \\
\hline 2 & Batch 1 & 2 & 2 & 9 & BCHLM2222 & 2.40 & 0.118 & 0.803 & $<0.010$ & $<0.010$ & 0.072 & 0.305 & 8.35 & $<0.010$ \\
\hline 2 & EM-29 & 2 & 2 & 10 & s09LM22 & 7.26 & 0.065 & 2.36 & $<0.010$ & 0.196 & 0.064 & 0.035 & 7.95 & 0.066 \\
\hline 2 & EM-17 & 2 & 2 & 11 & s10LM22 & 7.16 & 0.061 & 0.879 & $<0.010$ & 0.189 & 0.067 & 0.038 & 9.33 & 0.067 \\
\hline 2 & EM-26 & 2 & 2 & 12 & s07LM12 & 5.85 & 0.057 & 0.902 & $<0.010$ & 0.192 & 0.062 & 0.037 & 9.30 & 0.067 \\
\hline 2 & EM-29 & 2 & 2 & 13 & s09LM12 & 7.23 & 0.060 & 2.37 & $<0.010$ & 0.190 & 0.062 & 0.034 & 8.05 & 0.066 \\
\hline 2 & EM-19 & 2 & 2 & 14 & s06LM12 & 3.41 & 0.062 & 0.886 & $<0.010$ & 0.193 & 0.084 & 0.032 & 9.48 & 0.068 \\
\hline 2 & EM-19 & 2 & 2 & 15 & s06LM22 & 3.43 & 0.058 & 0.931 & $<0.010$ & 0.182 & 0.082 & 0.031 & 9.39 & 0.065 \\
\hline
\end{tabular}


SRNL-STI-2009-00778, Revision 0

Table D1. Measured Elemental Concentrations (wt\%) for the Matrix 2A Study Glasses Prepared Using Lithium Metaborate (part 1)

\begin{tabular}{|c|c|c|c|c|c|c|c|c|c|c|c|c|c|c|}
\hline Set & Glass ID & Block & $\begin{array}{l}\text { Sub- } \\
\text { Block }\end{array}$ & Sequence & Lab ID & $\begin{array}{c}\mathrm{Al} \\
(\mathrm{wt} \%)\end{array}$ & $\begin{array}{c}\mathrm{Ba} \\
(\mathrm{wt} \%)\end{array}$ & $\begin{array}{c}\mathrm{Ca} \\
(\mathrm{wt} \%)\end{array}$ & $\begin{array}{c}\mathrm{Cd} \\
(\mathbf{w t} \%)\end{array}$ & $\begin{array}{c}\mathrm{Ce} \\
(\mathrm{wt} \%)\end{array}$ & $\begin{array}{c}\mathbf{C r} \\
(w t \%)\end{array}$ & $\begin{array}{c}\mathrm{Cu} \\
(\mathrm{wt} \%)\end{array}$ & $\begin{array}{c}\mathrm{Fe} \\
(\mathrm{wt} \%)\end{array}$ & $\begin{array}{c}\text { La } \\
(w t \%)\end{array}$ \\
\hline 2 & EM-1? & 2 & 2 & 16 & 112 & 7.09 & 0.062 & 0.879 & $<0.010$ & 0.195 & 0.067 & 0.042 & 9.05 & 0.069 \\
\hline 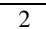 & Batch 1 & 2 & 2 & 17 & BCHLM2223 & 2.36 & 0.119 & 0.798 & $<0.010$ & $<0.010$ & 0.072 & 0.306 & 8.39 & $<0.010$ \\
\hline
\end{tabular}


SRNL-STI-2009-00778, Revision 0

\section{Table D1. Measured Elemental Concentrations (wt\%) for the Matrix 2A Study Glasses Prepared Using Lithium Metaborate (part 2)}

\begin{tabular}{|c|c|c|c|c|c|c|c|c|c|c|c|c|c|c|}
\hline Set & $\begin{array}{c}\text { Glass } \\
\text { ID }\end{array}$ & Block & $\begin{array}{c}\text { Sub- } \\
\text { Block }\end{array}$ & Sequence & Lab ID & $\begin{array}{c}\mathrm{Mg} \\
(\mathrm{wt} \%)\end{array}$ & $\begin{array}{c}\text { Mn } \\
(\mathbf{w t} \%)\end{array}$ & $\begin{array}{c}\mathrm{Na} \\
(\mathrm{wt} \%)\end{array}$ & $\begin{array}{c}\mathrm{Ni} \\
(\mathrm{wt} \%)\end{array}$ & $\begin{array}{c}\mathrm{Pb} \\
(\mathrm{wt} \%)\end{array}$ & $\begin{array}{c}S \\
(w t \%)\end{array}$ & $\begin{array}{c}\mathbf{T i} \\
(w t \%)\end{array}$ & $\begin{array}{c}\mathrm{Zn} \\
(w t \%)\end{array}$ & $\begin{array}{c}\mathrm{Zr} \\
(\mathrm{wt} \%)\end{array}$ \\
\hline 1 & Batch 1 & 1 & 1 & 1 & BCHLM1111 & 0.789 & 1.35 & 6.80 & 0.522 & $<0.010$ & $<0.100$ & 0.399 & $<0.010$ & 0.064 \\
\hline 1 & EM-15 & 1 & 1 & 2 & r10LM21 & 0.420 & 2.29 & 10.2 & 0.704 & 0.082 & 0.146 & 1.13 & 0.049 & 0.128 \\
\hline 1 & EM-13 & 1 & 1 & 3 & r02LM21 & 0.420 & 2.26 & 10.8 & 0.741 & 0.081 & 0.144 & 1.14 & 0.052 & 0.129 \\
\hline 1 & EM-04 & 1 & 1 & 4 & r07LM11 & 0.419 & 2.29 & 12.9 & 0.783 & 0.078 & 0.152 & 1.19 & 0.052 & 0.126 \\
\hline 1 & EM-15 & 1 & 1 & 5 & r10LM11 & 0.427 & 2.30 & 9.89 & 0.721 & 0.086 & 0.150 & 1.12 & 0.047 & 0.133 \\
\hline 1 & EM-05 & 1 & 1 & 6 & r11LM11 & 0.422 & 2.29 & 11.6 & 0.772 & 0.085 & 0.151 & 1.17 & 0.048 & 0.127 \\
\hline 1 & EM-11 & 1 & 1 & 7 & r15LM21 & 0.415 & 3.04 & 9.65 & 0.738 & 0.084 & 0.143 & 1.17 & 0.049 & 0.127 \\
\hline 1 & EM-07 & 1 & 1 & 8 & r09LM21 & 0.416 & 2.29 & 9.82 & 0.714 & 0.082 & 0.147 & 1.13 & 0.051 & 0.127 \\
\hline 1 & EM-04 & 1 & 1 & 9 & r07LM21 & 0.413 & 2.27 & 12.6 & 0.785 & 0.078 & 0.149 & 1.16 & 0.050 & 0.127 \\
\hline 1 & Batch 1 & 1 & 1 & 10 & BCHLM1112 & 0.774 & 1.32 & 6.49 & 0.514 & $<0.010$ & $<0.100$ & 0.386 & $<0.010$ & 0.064 \\
\hline 1 & EM-05 & 1 & 1 & 11 & r11LM21 & 0.435 & 2.25 & 11.9 & 0.792 & 0.087 & 0.158 & 1.17 & 0.051 & 0.128 \\
\hline 1 & EM-03 & 1 & 1 & 12 & r04LM21 & 0.422 & 2.27 & 12.5 & 0.752 & 0.082 & 0.150 & 1.18 & 0.053 & 0.127 \\
\hline 1 & EM-11 & 1 & 1 & 13 & r15LM11 & 0.422 & 3.02 & 9.72 & 0.751 & 0.085 & 0.148 & 1.16 & 0.047 & 0.129 \\
\hline 1 & EM-13 & 1 & 1 & 14 & r02LM11 & 0.401 & 2.26 & 10.9 & 0.709 & 0.077 & 0.140 & 1.15 & 0.051 & 0.125 \\
\hline 1 & EM-03 & 1 & 1 & 15 & r04LM11 & 0.416 & 2.26 & 12.8 & 0.763 & 0.080 & 0.150 & 1.18 & 0.051 & 0.126 \\
\hline 1 & EM-14 & 1 & 1 & 16 & r08LM11 & 0.410 & 2.21 & 10.5 & 0.696 & 0.078 & 0.142 & 1.18 & 0.049 & 0.127 \\
\hline 1 & EM-07 & 1 & 1 & 17 & r09LM11 & 0.408 & 2.23 & 10.0 & 0.690 & 0.081 & 0.152 & 1.12 & 0.049 & 0.126 \\
\hline 1 & EM-14 & 1 & 1 & 18 & r08LM21 & 0.401 & 2.21 & 10.3 & 0.676 & 0.077 & 0.136 & 1.18 & 0.044 & 0.122 \\
\hline 1 & Batch 1 & 1 & 1 & 19 & BCHLM1113 & 0.773 & 1.32 & 6.63 & 0.512 & $<0.010$ & $<0.100$ & 0.388 & $<0.010$ & 0.063 \\
\hline 1 & Batch 1 & 1 & 2 & 1 & BCHLM1121 & 0.770 & 1.35 & 6.78 & 0.512 & $<0.010$ & $<0.100$ & 0.398 & $<0.010$ & 0.064 \\
\hline 1 & EM-07 & 1 & 2 & 2 & r09LM22 & 0.418 & 2.31 & 10.1 & 0.713 & 0.084 & 0.152 & 1.16 & 0.054 & 0.128 \\
\hline 1 & EM-15 & 1 & 2 & 3 & r10LM12 & 0.419 & 2.30 & 10.3 & 0.699 & 0.087 & 0.141 & 1.14 & 0.049 & 0.133 \\
\hline 1 & EM-11 & 1 & 2 & 4 & r15LM12 & 0.408 & 3.03 & 10.2 & 0.718 & 0.083 & 0.140 & 1.20 & 0.048 & 0.129 \\
\hline 1 & EM-07 & 1 & 2 & 5 & r09LM12 & 0.397 & 2.29 & 10.3 & 0.663 & 0.081 & 0.141 & 1.17 & 0.051 & 0.127 \\
\hline 1 & EM-04 & 1 & 2 & 6 & r07LM22 & 0.403 & 2.27 & 13.2 & 0.760 & 0.080 & 0.140 & 1.20 & 0.052 & 0.128 \\
\hline 1 & EM-14 & 1 & 2 & 7 & r08LM12 & 0.397 & 2.28 & 10.6 & 0.673 & 0.079 & 0.138 & 1.24 & 0.050 & 0.127 \\
\hline 1 & EM-03 & 1 & 2 & 8 & r04LM12 & 0.414 & 2.34 & 13.1 & 0.752 & 0.083 & 0.145 & 1.23 & 0.054 & 0.128 \\
\hline 1 & EM-05 & 1 & 2 & 9 & r11LM12 & 0.412 & 2.33 & 12.0 & 0.751 & 0.086 & 0.144 & 1.21 & 0.050 & 0.127 \\
\hline 1 & Batch 1 & 1 & 2 & 10 & BCHLM1122 & 0.751 & 1.33 & 6.86 & 0.499 & $<0.010$ & $<0.100$ & 0.395 & $<0.010$ & 0.064 \\
\hline 1 & EM-15 & 1 & 2 & 11 & r10LM22 & 0.400 & 2.27 & 10.1 & 0.666 & 0.082 & 0.137 & 1.13 & 0.050 & 0.128 \\
\hline 1 & EM-03 & 1 & 2 & 12 & r04LM22 & 0.403 & 2.31 & 12.7 & 0.712 & 0.081 & 0.147 & 1.21 & 0.052 & 0.128 \\
\hline 1 & EM-14 & 1 & 2 & 13 & r08LM22 & 0.403 & 2.30 & 10.4 & 0.675 & 0.080 & 0.140 & 1.23 & 0.048 & 0.127 \\
\hline 1 & EM-05 & 1 & 2 & 14 & r11LM22 & 0.420 & 2.35 & 11.8 & 0.759 & 0.086 & 0.143 & 1.21 & 0.051 & 0.129 \\
\hline 1 & EM-11 & 1 & 2 & 15 & r15LM22 & 0.405 & 3.03 & 10.1 & 0.712 & 0.082 & 0.138 & 1.19 & 0.050 & 0.128 \\
\hline 1 & EM-04 & 1 & 2 & 16 & r07LM12 & 0.396 & 2.28 & 13.2 & 0.735 & 0.077 & 0.139 & 1.20 & 0.052 & 0.125 \\
\hline 1 & EM-13 & 1 & 2 & 17 & r02LM12 & 0.393 & 2.27 & 11.1 & 0.684 & 0.077 & 0.134 & 1.17 & 0.053 & 0.126 \\
\hline 1 & EM-13 & 1 & 2 & 18 & r02LM22 & 0.404 & 2.26 & 10.9 & 0.705 & 0.081 & 0.136 & 1.16 & 0.053 & 0.128 \\
\hline 1 & Batch 1 & 1 & 2 & 19 & BCHLM1123 & 0.758 & 1.35 & 6.93 & 0.500 & $<0.010$ & $<0.100$ & 0.397 & $<0.010$ & 0.064 \\
\hline 1 & Batch 1 & 2 & 1 & 1 & BCHLM1211 & 0.803 & 1.35 & 6.82 & 0.523 & $<0.010$ & $<0.100$ & 0.395 & $<0.010$ & 0.064 \\
\hline 1 & EM-02 & 2 & 1 & 2 & \begin{tabular}{|l|} 
r05LM11 \\
\end{tabular} & 0.420 & 2.28 & 10.4 & 0.757 & 0.078 & 0.138 & 1.25 & 0.049 & 0.130 \\
\hline 1 & EM-06 & 2 & 1 & 3 & r13LM21 & 0.427 & 2.29 & 10.4 & 0.740 & 0.081 & 0.138 & 1.22 & 0.049 & 0.128 \\
\hline 1 & EM-09 & 2 & 1 & 4 & r06LM11 & 0.415 & 2.27 & 10.4 & 0.720 & 0.081 & 0.132 & 1.26 & 0.050 & 0.128 \\
\hline 1 & EM-12 & 2 & 1 & 5 & r14LM11 & 0.423 & 1.51 & 12.7 & 0.733 & 0.081 & 0.143 & 1.23 & 0.050 & 0.130 \\
\hline 1 & EM-10 & 2 & 1 & 6 & r01LM21 & 0.409 & 2.24 & 12.9 & 0.722 & 0.077 & 0.141 & 1.25 & 0.057 & 0.125 \\
\hline 1 & EM-12 & 2 & 1 & 7 & r14LM21 & 0.426 & 1.43 & 13.4 & 0.607 & 0.080 & 0.137 & 1.21 & 0.049 & 0.130 \\
\hline 1 & EM-08 & 2 & 1 & 8 & r03LM11 & 0.421 & 2.23 & 10.4 & 0.693 & 0.080 & 0.143 & 1.35 & 0.048 & 0.129 \\
\hline 1 & Batch 1 & 2 & 1 & 9 & BCHLM1212 & 0.797 & 1.33 & 6.79 & 0.521 & $<0.010$ & $<0.100$ & 0.395 & $<0.010$ & 0.065 \\
\hline 1 & EM-10 & 2 & 1 & 10 & r01LM11 & 0.413 & 2.24 & 12.8 & 0.723 & 0.079 & 0.136 & 1.24 & 0.057 & 0.124 \\
\hline 1 & EM-02 & 2 & 1 & 11 & r05LM21 & 0.422 & 2.27 & 10.1 & 0.767 & 0.080 & 0.138 & 1.23 & 0.047 & 0.128 \\
\hline 1 & EM-08 & 2 & 1 & 12 & r03LM21 & 0.418 & 2.26 & 10.3 & 0.690 & 0.080 & 0.141 & 1.36 & 0.049 & 0.129 \\
\hline 1 & EM-01 & 2 & 1 & 13 & r12LM21 & 0.419 & 2.25 & 11.5 & 0.746 & 0.082 & 0.136 & 1.24 & 0.045 & 0.124 \\
\hline 1 & EM-06 & 2 & 1 & 14 & r13LM11 & 0.442 & 2.28 & 10.6 & 0.767 & 0.087 & 0.138 & 1.22 & 0.050 & 0.131 \\
\hline 1 & EM-09 & 2 & 1 & 15 & r06LM21 & 0.418 & 2.23 & 10.4 & 0.722 & 0.082 & 0.128 & 1.24 & 0.049 & 0.129 \\
\hline 1 & EM-01 & 2 & 1 & 16 & r12LM11 & 0.421 & 2.24 & 11.8 & 0.743 & 0.084 & 0.142 & 1.24 & 0.046 & 0.125 \\
\hline 1 & Batch 1 & 2 & 1 & 17 & BCHLM1213 & 0.778 & 1.33 & 6.81 & 0.514 & $<0.010$ & $<0.100$ & 0.389 & $<0.010$ & 0.064 \\
\hline 1 & Batch 1 & 2 & 2 & 1 & BCHLM1221 & 0.794 & 1.35 & 6.64 & 0.521 & $<0.010$ & $<0.100$ & 0.39 & $<0.010$ & 0.067 \\
\hline 1 & EM-01 & 2 & 2 & 2 & \begin{tabular}{|l|} 
r12LM22 \\
\end{tabular} & 0.425 & 2.31 & 11.2 & 0.757 & 0.085 & 0.147 & 1.25 & 0.048 & 0.125 \\
\hline 1 & EM-10 & 2 & 2 & 3 & r01LM22 & 0.420 & 2.27 & 12.7 & 0.733 & 0.083 & 0.143 & 1.24 & 0.060 & 0.127 \\
\hline 1 & EM-08 & 2 & 2 & 4 & r03LM12 & 0.419 & 2.25 & 10.3 & 0.690 & 0.081 & 0.146 & 1.35 & 0.049 & 0.130 \\
\hline 1 & EM-10 & 2 & 2 & 5 & r01LM12 & 0.417 & 2.25 & 12.9 & 0.732 & 0.081 & 0.140 & 1.24 & 0.060 & 0.126 \\
\hline 1 & EM-08 & 2 & 2 & 6 & r03LM22 & 0.420 & 2.27 & 9.96 & 0.692 & 0.082 & 0.149 & 1.34 & 0.051 & 0.129 \\
\hline 1 & EM-02 & 2 & 2 & 7 & r05LM12 & 0.416 & 2.30 & 9.91 & 0.746 & 0.080 & 0.144 & 1.23 & 0.050 & 0.130 \\
\hline 1 & EM-02 & 2 & 2 & 8 & r05LM22 & 0.434 & 2.31 & 9.92 & 0.787 & 0.084 & 0.144 & 1.23 & 0.051 & 0.130 \\
\hline 1 & Batch 1 & 2 & 2 & 9 & BCHLM1222 & 0.807 & 1.35 & 6.83 & 0.528 & $<0.010$ & $<0.100$ & 0.39 & $<0.010$ & 0.067 \\
\hline 1 & EM-12 & 2 & 2 & 10 & r14LM22 & 0.424 & 1.43 & 13.1 & 0.602 & 0.082 & 0.143 & 1.19 & 0.051 & 0.132 \\
\hline 1 & EM-09 & 2 & 2 & 11 & r06LM22 & 0.422 & 2.26 & 10.2 & 0.733 & 0.083 & 0.140 & 1.24 & 0.051 & 0.130 \\
\hline 1 & EM-06 & 2 & 2 & 12 & r13LM12 & 0.438 & 2.31 & 10.3 & 0.761 & 0.088 & 0.148 & 1.21 & 0.051 & 0.132 \\
\hline 1 & EM-09 & 2 & 2 & 13 & r06LM12 & 0.419 & 2.30 & 9.93 & 0.727 & 0.085 & 0.136 & 1.25 & 0.051 & 0.129 \\
\hline 1 & EM-01 & 2 & 2 & 14 & r12LM12 & 0.427 & 2.30 & 11.3 & 0.757 & 0.086 & 0.145 & 1.24 & 0.048 & 0.127 \\
\hline 1 & EM-12 & 2 & 2 & 15 & r14LM12 & 0.437 & 1.48 & 12.7 & 0.749 & 0.084 & 0.139 & 1.21 & 0.052 & 0.132 \\
\hline 1 & EM-06 & 2 & 2 & 16 & r13LM22 & 0.427 & 2.29 & 10.2 & 0.744 & 0.083 & 0.146 & 1.21 & 0.051 & 0.130 \\
\hline
\end{tabular}


SRNL-STI-2009-00778, Revision 0

\section{Table D1. Measured Elemental Concentrations (wt\%) for the Matrix 2A Study Glasses Prepared Using Lithium Metaborate (part 2)}

\begin{tabular}{|c|c|c|c|c|c|c|c|c|c|c|c|c|c|c|}
\hline Set & $\begin{array}{c}\text { Glass } \\
\text { ID }\end{array}$ & Block & $\begin{array}{c}\text { Sub- } \\
\text { Block }\end{array}$ & Sequence & Lab ID & $\begin{array}{c}\mathrm{Mg} \\
(\mathbf{w t} \%)\end{array}$ & $\begin{array}{c}\text { Mn } \\
(\mathbf{w t} \%)\end{array}$ & $\begin{array}{c}\mathrm{Na} \\
(\mathbf{w t} \%)\end{array}$ & $\begin{array}{c}\mathrm{Ni} \\
(\mathrm{wt} \%)\end{array}$ & $\begin{array}{c}\mathrm{Pb} \\
(\mathbf{w t} \%)\end{array}$ & $\begin{array}{c}\mathrm{S} \\
(\mathrm{wt} \%)\end{array}$ & $\begin{array}{c}\mathbf{T i} \\
(\mathbf{w t} \%)\end{array}$ & $\begin{array}{c}\mathrm{Zn} \\
(\mathbf{w t} \%)\end{array}$ & $\begin{array}{c}\mathrm{Zr} \\
(\mathrm{wt} \%)\end{array}$ \\
\hline 1 & Batch 1 & 2 & 2 & 17 & BCHLM1223 & 0.786 & 1.31 & 6.79 & 0.516 & $<0.010$ & $<0.100$ & 0.38 & $<0.010$ & 0.066 \\
\hline 2 & Batch 1 & 1 & 1 & 1 & BCHLM2111 & 0.796 & 1.32 & 6.61 & 0.522 & $<0.010$ & $<0.100$ & 0.393 & $<0.010$ & 0.066 \\
\hline 2 & EM-21 & 1 & 1 & 2 & s03LM11 & 0.428 & 2.28 & 10.2 & 0.740 & 0.081 & 0.146 & 1.13 & 0.049 & 0.128 \\
\hline 2 & EM-30 & 1 & 1 & 3 & s13LM11 & 0.408 & 2.20 & 10.1 & 0.640 & 0.071 & 0.142 & 1.20 & 0.045 & 0.124 \\
\hline 2 & EM-22 & 1 & 1 & 4 & s02LM21 & 0.434 & 2.22 & 10.0 & 0.664 & 0.083 & 0.142 & 1.18 & 0.050 & 0.135 \\
\hline 2 & EM-25 & 1 & 1 & 5 & s14LM11 & 0.416 & 2.25 & 12.7 & 0.749 & 0.073 & 0.142 & 1.20 & 0.047 & 0.126 \\
\hline 2 & EM-18 & 1 & 1 & 6 & s08LM21 & 0.415 & 2.19 & 12.5 & 0.741 & 0.076 & 0.138 & 1.17 & 0.043 & 0.126 \\
\hline 2 & EM-25 & 1 & 1 & 7 & s14LM21 & 0.421 & 2.19 & 12.7 & 0.765 & 0.075 & 0.139 & 1.18 & 0.047 & 0.128 \\
\hline 2 & EM-30 & 1 & 1 & 8 & s13LM21 & 0.402 & 2.16 & 10.1 & 0.627 & 0.069 & 0.135 & 1.19 & 0.045 & 0.123 \\
\hline 2 & EM-24 & 1 & 1 & 9 & s05LM21 & 0.426 & 2.19 & 10.1 & 0.690 & 0.080 & 0.144 & 1.21 & 0.044 & 0.152 \\
\hline 2 & Batch 1 & 1 & 1 & 10 & BCHLM2112 & 0.799 & 1.28 & 7.08 & 0.527 & $<0.010$ & $<0.100$ & 0.386 & $<0.010$ & 0.065 \\
\hline 2 & EM-21 & 1 & 1 & 11 & s03LM21 & 0.433 & 2.33 & 10.4 & 0.757 & 0.082 & 0.148 & 1.15 & 0.048 & 0.129 \\
\hline 2 & EM-18 & 1 & 1 & 12 & s08LM11 & 0.425 & 2.26 & 12.8 & 0.764 & 0.080 & 0.142 & 1.20 & 0.044 & 0.130 \\
\hline 2 & EM-20 & 1 & 1 & 13 & s04LM11 & 0.428 & 2.20 & 11.9 & 0.698 & 0.081 & 0.143 & 1.19 & 0.045 & 0.130 \\
\hline 2 & EM-20 & 1 & 1 & 14 & s04LM21 & 0.406 & 2.19 & 11.6 & 0.699 & 0.075 & 0.140 & 1.18 & 0.044 & 0.125 \\
\hline 2 & EM-24 & 1 & 1 & 15 & s05LM11 & 0.431 & 2.17 & 9.98 & 0.699 & 0.082 & 0.143 & 1.19 & 0.044 & 0.129 \\
\hline 2 & EM-28 & 1 & 1 & 16 & s01LM21 & 0.427 & 2.21 & 10.9 & 0.715 & 0.081 & 0.144 & 1.23 & 0.048 & 0.130 \\
\hline 2 & EM-22 & 1 & 1 & 17 & s02LM11 & 0.407 & 2.21 & 10.4 & 0.610 & 0.072 & 0.150 & 1.17 & 0.048 & 0.127 \\
\hline 2 & EM-28 & 1 & 1 & 18 & s01LM11 & 0.407 & 2.13 & 10.7 & 0.602 & 0.077 & 0.139 & 1.20 & 0.047 & 0.124 \\
\hline 2 & Batch 1 & 1 & 1 & 19 & BCHLM2113 & 0.795 & 1.26 & 6.71 & 0.521 & $<0.010$ & $<0.100$ & 0.383 & $<0.010$ & 0.066 \\
\hline 2 & Batch 1 & 1 & 2 & 1 & BCHLM2121 & 0.808 & 1.33 & 6.76 & 0.536 & $<0.010$ & $<0.100$ & 0.389 & $<0.010$ & 0.068 \\
\hline 2 & EM-30 & 1 & 2 & 2 & s13LM22 & 0.406 & 2.24 & 10.0 & 0.637 & 0.073 & 0.139 & 1.22 & 0.048 & 0.124 \\
\hline 2 & EM-24 & 1 & 2 & 3 & s05LM12 & 0.430 & 2.23 & 10.1 & 0.700 & 0.085 & 0.141 & 1.21 & 0.047 & 0.130 \\
\hline 2 & EM-22 & 1 & 2 & 4 & s02LM12 & 0.399 & 2.26 & 10.5 & 0.600 & 0.073 & 0.135 & 1.19 & 0.050 & 0.127 \\
\hline 2 & EM-25 & 1 & 2 & 5 & s14LM22 & 0.421 & 2.28 & 12.8 & 0.765 & 0.079 & 0.137 & 1.21 & 0.051 & 0.129 \\
\hline 2 & EM-28 & 1 & 2 & 6 & s01LM22 & 0.417 & 2.24 & 11.1 & 0.704 & 0.084 & 0.137 & 1.23 & 0.050 & 0.130 \\
\hline 2 & EM-20 & 1 & 2 & 7 & s04LM22 & 0.406 & 2.22 & 11.9 & 0.701 & 0.078 & 0.138 & 1.18 & 0.047 & 0.128 \\
\hline 2 & EM-24 & 1 & 2 & 8 & s05LM22 & 0.425 & 2.25 & 10.3 & 0.689 & 0.084 & 0.142 & 1.23 & 0.047 & 0.152 \\
\hline 2 & EM-21 & 1 & 2 & 9 & s03LM12 & 0.426 & 2.31 & 10.7 & 0.736 & 0.086 & 0.144 & 1.15 & 0.051 & 0.128 \\
\hline 2 & Batch 1 & 1 & 2 & 10 & BCHLM2122 & 0.785 & 1.31 & 6.91 & 0.519 & $<0.010$ & $<0.100$ & 0.386 & $<0.010$ & 0.067 \\
\hline 2 & EM-18 & 1 & 2 & 11 & s08LM12 & 0.424 & 2.22 & 12.9 & 0.764 & 0.083 & 0.137 & 1.19 & 0.047 & 0.130 \\
\hline 2 & EM-30 & 1 & 2 & 12 & s13LM12 & 0.398 & 2.17 & 10.2 & 0.628 & 0.072 & 0.132 & 1.19 & 0.046 & 0.123 \\
\hline 2 & EM-22 & 1 & 2 & 13 & s02LM22 & 0.428 & 2.22 & 10.1 & 0.658 & 0.085 & 0.147 & 1.18 & 0.052 & 0.135 \\
\hline 2 & EM-25 & 1 & 2 & 14 & s14LM12 & 0.419 & 2.25 & 12.8 & 0.755 & 0.076 & 0.143 & 1.19 & 0.051 & 0.127 \\
\hline 2 & EM-21 & 1 & 2 & 15 & s03LM22 & 0.433 & 2.29 & 10.4 & 0.761 & 0.088 & 0.142 & 1.13 & 0.051 & 0.130 \\
\hline 2 & EM-18 & 1 & 2 & 16 & s08LM22 & 0.413 & 2.23 & 12.7 & 0.738 & 0.079 & 0.140 & 1.18 & 0.045 & 0.127 \\
\hline 2 & EM-28 & 1 & 2 & 17 & s01LM12 & 0.397 & 2.16 & 10.9 & 0.586 & 0.078 & 0.133 & 1.21 & 0.048 & 0.123 \\
\hline 2 & EM-20 & 1 & 2 & 18 & s04LM12 & 0.418 & 2.20 & 12.1 & 0.685 & 0.084 & 0.138 & 1.19 & 0.047 & 0.129 \\
\hline 2 & Batch 1 & 1 & 2 & 19 & BCHLM2123 & 0.785 & 1.28 & 7.16 & 0.515 & $<0.010$ & $<0.100$ & 0.379 & $<0.010$ & 0.065 \\
\hline 2 & Batch 1 & 2 & 1 & 1 & BCHLM2211 & 0.798 & 1.31 & 6.69 & 0.524 & $<0.010$ & $<0.100$ & 0.383 & $<0.010$ & 0.067 \\
\hline 2 & EM-17 & 2 & 1 & 2 & s10LM21 & 0.424 & 2.25 & 9.85 & 0.733 & 0.080 & 0.148 & 1.13 & 0.049 & 0.127 \\
\hline 2 & EM-19 & 2 & 1 & 3 & s06LM21 & 0.410 & 2.27 & 12.9 & 0.735 & 0.073 & 0.144 & 1.22 & 0.047 & 0.125 \\
\hline 2 & EM-26 & 2 & 1 & 4 & s07LM11 & 0.414 & 2.98 & 10.0 & 0.618 & 0.075 & 0.138 & 1.18 & 0.044 & 0.126 \\
\hline 2 & EM-27 & 2 & 1 & 5 & s15LM21 & 0.422 & 1.45 & 12.5 & 0.707 & 0.080 & 0.148 & 1.22 & 0.054 & 0.129 \\
\hline 2 & EM-16 & 2 & 1 & 6 & s11LM11 & 0.433 & 2.20 & 11.1 & 0.701 & 0.085 & 0.145 & 1.21 & 0.048 & 0.132 \\
\hline 2 & EM-29 & 2 & 1 & 7 & s09LM21 & 0.422 & 2.22 & 10.1 & 0.671 & 0.078 & 0.141 & 1.20 & 0.046 & 0.129 \\
\hline 2 & EM-16 & 2 & 1 & 8 & s11LM21 & 0.433 & 2.25 & 11.0 & 0.694 & 0.082 & 0.143 & 1.22 & 0.049 & 0.134 \\
\hline 2 & Batch 1 & 2 & 1 & 9 & BCHLM2212 & 0.820 & 1.32 & 6.65 & 0.540 & $<0.010$ & $<0.100$ & 0.380 & $<0.010$ & 0.069 \\
\hline 2 & EM-29 & 2 & 1 & 10 & s09LM11 & 0.433 & 2.21 & 10.3 & 0.679 & 0.083 & 0.143 & 1.21 & 0.047 & 0.131 \\
\hline 2 & EM-23 & 2 & 1 & 11 & s12LM21 & 0.428 & 2.24 & 9.88 & 0.619 & 0.084 & 0.151 & 1.17 & 0.046 & 0.128 \\
\hline 2 & EM-23 & 2 & 1 & 12 & s12LM11 & 0.435 & 2.23 & 9.62 & 0.619 & 0.088 & 0.152 & 1.16 & 0.048 & 0.130 \\
\hline 2 & EM-26 & 2 & 1 & 13 & s07LM21 & 0.435 & 2.96 & 9.68 & 0.588 & 0.080 & 0.139 & 1.17 & 0.046 & 0.129 \\
\hline 2 & EM-17 & 2 & 1 & 14 & s10LM11 & 0.437 & 2.20 & 9.66 & 0.744 & 0.086 & 0.143 & 1.11 & 0.049 & 0.130 \\
\hline 2 & EM-19 & 2 & 1 & 15 & s06LM11 & 0.440 & 2.27 & 12.7 & 0.808 & 0.083 & 0.142 & 1.22 & 0.048 & 0.128 \\
\hline 2 & EM-27 & 2 & 1 & 16 & s15LM11 & 0.433 & 1.45 & 12.4 & 0.724 & 0.083 & 0.149 & 1.21 & 0.053 & 0.128 \\
\hline 2 & Batch 1 & 2 & 1 & 17 & BCHLM2213 & 0.797 & 1.30 & 6.55 & 0.521 & $<0.010$ & $<0.100$ & 0.376 & $<0.010$ & 0.067 \\
\hline 2 & Batch 1 & 2 & 2 & 1 & BCHLM2221 & 0.762 & 1.28 & 7.04 & 0.505 & $<0.010$ & $<0.100$ & 0.381 & $<0.010$ & 0.065 \\
\hline 2 & EM-23 & 2 & 2 & 2 & s12LM12 & 0.419 & 2.18 & 10.0 & 0.600 & 0.086 & 0.141 & 1.16 & 0.048 & 0.130 \\
\hline 2 & EM-16 & 2 & 2 & 3 & s11LM22 & 0.421 & 2.19 & 11.3 & 0.677 & 0.081 & 0.142 & 1.21 & 0.049 & 0.134 \\
\hline 2 & EM-26 & 2 & 2 & 4 & s07LM22 & 0.425 & 2.88 & 9.81 & 0.578 & 0.081 & 0.140 & 1.17 & 0.046 & 0.129 \\
\hline 2 & EM-16 & 2 & 2 & 5 & s11LM12 & 0.434 & 2.20 & 11.2 & 0.701 & 0.086 & 0.140 & 1.21 & 0.050 & 0.133 \\
\hline 2 & EM-23 & 2 & 2 & 6 & s12LM22 & 0.417 & 2.19 & 10.0 & 0.601 & 0.083 & 0.142 & 1.16 & 0.046 & 0.127 \\
\hline 2 & EM-27 & 2 & 2 & 7 & s15LM12 & 0.414 & 1.43 & 12.6 & 0.696 & 0.081 & 0.143 & 1.21 & 0.052 & 0.127 \\
\hline 2 & EM-27 & 2 & 2 & 8 & s15LM22 & 0.411 & 1.42 & 12.6 & 0.692 & 0.080 & 0.137 & 1.21 & 0.054 & 0.128 \\
\hline 2 & Batch 1 & 2 & 2 & 9 & BCHLM2222 & 0.774 & 1.26 & 6.50 & 0.512 & $<0.010$ & $<0.100$ & 0.372 & $<0.010$ & 0.067 \\
\hline 2 & EM-29 & 2 & 2 & 10 & s09LM22 & 0.420 & 2.15 & 10.1 & 0.666 & 0.080 & 0.137 & 1.18 & 0.047 & 0.128 \\
\hline 2 & EM-17 & 2 & 2 & 11 & s10LM22 & 0.412 & 2.18 & 9.70 & 0.713 & 0.078 & 0.137 & 1.10 & 0.049 & 0.125 \\
\hline 2 & EM-26 & 2 & 2 & 12 & s07LM12 & 0.401 & 2.94 & 9.72 & 0.600 & 0.073 & 0.134 & 1.15 & 0.044 & 0.125 \\
\hline 2 & EM-29 & 2 & 2 & 13 & s09LM12 & 0.411 & 2.17 & 10.1 & 0.648 & 0.079 & 0.138 & 1.19 & 0.046 & 0.128 \\
\hline 2 & EM-19 & 2 & 2 & 14 & s06LM12 & 0.423 & 2.19 & 12.7 & 0.781 & 0.081 & 0.144 & 1.19 & 0.048 & 0.127 \\
\hline 2 & EM-19 & 2 & 2 & 15 & s06LM22 & 0.406 & 2.17 & 12.6 & 0.727 & 0.072 & 0.138 & 1.18 & 0.047 & 0.123 \\
\hline
\end{tabular}


SRNL-STI-2009-00778, Revision 0

Table D1. Measured Elemental Concentrations (wt\%) for the Matrix 2A Study Glasses Prepared Using Lithium Metaborate (part 2)

\begin{tabular}{|c|c|c|c|c|c|c|c|c|c|c|c|c|c|c|}
\hline Set & $\begin{array}{l}\text { Glass } \\
\text { ID }\end{array}$ & Block & $\begin{array}{l}\text { Sub- } \\
\text { Block }\end{array}$ & Sequence & Lab ID & $\begin{array}{c}\mathrm{Mg} \\
(\mathbf{w t} \%)\end{array}$ & $\begin{array}{c}\text { Mn } \\
(w t \%)\end{array}$ & $\begin{array}{c}\mathrm{Na} \\
(\mathrm{wt} \%)\end{array}$ & $\begin{array}{c}\mathrm{Ni} \\
(w t \%)\end{array}$ & $\begin{array}{c}\mathrm{Pb} \\
(\mathrm{wt} \%)\end{array}$ & $\underset{(w t \%)}{S}$ & $\begin{array}{c}\mathrm{Ti} \\
(\mathrm{wt} \%)\end{array}$ & $\begin{array}{c}\mathbf{Z n} \\
(\mathbf{w t} \%)\end{array}$ & $\begin{array}{c}\mathrm{Zr} \\
(\mathrm{wt} \%)\end{array}$ \\
\hline 2 & EM-17 & 2 & 2 & 16 & s10LM12 & 0.428 & 2.12 & 9.60 & 0.732 & 0.084 & 0.138 & 1.08 & 0.049 & 0.129 \\
\hline 2 & Batch 1 & 2 & 2 & 17 & BCHLM2223 & 0.798 & 1.27 & 6.47 & 0.529 & $<0.010$ & $<0.100$ & 0.369 & $<0.010$ & 0.068 \\
\hline
\end{tabular}


SRNL-STI-2009-00778, Revision 0

Table D2. Measured Elemental Concentrations (wt\%) for the Matrix 2A Study Glasses Prepared Using Peroxide Fusion

\begin{tabular}{|c|c|c|c|c|c|c|c|c|}
\hline Set & Glass ID & Block & Sub-Block & Sequence & Lab ID & B (wt\%) & Li (wt\%) & Si (wt\%) \\
\hline 1 & Batch 1 & 1 & 1 & 1 & BCHPF1111 & 2.68 & 2.14 & 22.9 \\
\hline 1 & EM-15 & 1 & 1 & 2 & r10PF21 & 1.41 & 1.95 & 19.4 \\
\hline 1 & EM-01 & 1 & 1 & 3 & r12PF21 & 1.30 & 1.87 & 19.3 \\
\hline 1 & EM-01 & 1 & 1 & 4 & r12PF11 & 1.30 & 1.90 & 19.8 \\
\hline 1 & EM-12 & 1 & 1 & 5 & r14PF21 & 1.30 & 1.92 & 19.1 \\
\hline 1 & EM-14 & 1 & 1 & 6 & r08PF11 & 1.27 & 1.90 & 18.8 \\
\hline 1 & EM-11 & 1 & 1 & 7 & r15PF21 & 1.28 & 1.91 & 20.1 \\
\hline 1 & EM-15 & 1 & 1 & 8 & r10PF11 & 1.28 & 1.93 & 18.8 \\
\hline 1 & EM-12 & 1 & 1 & 9 & r14PF11 & 1.24 & 1.88 & 18.5 \\
\hline 1 & Batch 1 & 1 & 1 & 10 & BCHPF1112 & 2.50 & 2.16 & 22.7 \\
\hline 1 & EM-13 & 1 & 1 & 11 & r02PF21 & 1.33 & 1.91 & 19.3 \\
\hline 1 & EM-14 & 1 & 1 & 12 & r08PF21 & 1.29 & 1.89 & 18.8 \\
\hline 1 & EM-02 & 1 & 1 & 13 & r05PF11 & 1.29 & 1.89 & 19.3 \\
\hline 1 & EM-03 & 1 & 1 & 14 & r04PF11 & 1.27 & 1.87 & 19.5 \\
\hline 1 & EM-13 & 1 & 1 & 15 & r02PF11 & 1.27 & 1.89 & 19.1 \\
\hline 1 & EM-11 & 1 & 1 & 16 & r15PF11 & 1.27 & 1.89 & 20.0 \\
\hline 1 & EM-03 & 1 & 1 & 17 & r04PF21 & 1.24 & 1.86 & 19.3 \\
\hline 1 & EM-02 & 1 & 1 & 18 & r05PF21 & 1.24 & 1.87 & 19.0 \\
\hline 1 & Batch 1 & 1 & 1 & 19 & BCHPF1113 & 2.49 & 2.15 & 22.8 \\
\hline 1 & Batch 1 & 1 & 2 & 1 & BCHPF1121 & 2.58 & 2.07 & 21.9 \\
\hline 1 & EM-03 & 1 & 2 & 2 & r04PF12 & 1.34 & 1.81 & 18.6 \\
\hline 1 & EM-02 & 1 & 2 & 3 & r05PF22 & 1.30 & 1.78 & 18.3 \\
\hline 1 & EM-15 & 1 & 2 & 4 & r10PF12 & 1.32 & 1.85 & 18.3 \\
\hline 1 & EM-01 & 1 & 2 & 5 & r12PF22 & 1.29 & 1.83 & 18.7 \\
\hline 1 & EM-14 & 1 & 2 & 6 & r08PF22 & 1.26 & 1.80 & 17.8 \\
\hline 1 & EM-12 & 1 & 2 & 7 & r14PF22 & 1.28 & 1.83 & 18.2 \\
\hline 1 & EM-14 & 1 & 2 & 8 & r08PF12 & 1.26 & 1.80 & 17.9 \\
\hline 1 & EM-15 & 1 & 2 & 9 & r10PF22 & 1.31 & 1.89 & 18.6 \\
\hline 1 & Batch 1 & 1 & 2 & 10 & BCHPF1122 & 2.45 & 2.07 & 21.8 \\
\hline 1 & EM-12 & 1 & 2 & 11 & r14PF12 & 1.31 & 1.80 & 17.9 \\
\hline 1 & EM-11 & 1 & 2 & 12 & r15PF12 & 1.30 & 1.82 & 19.1 \\
\hline 1 & EM-13 & 1 & 2 & 13 & r02PF22 & 1.29 & 1.82 & 18.4 \\
\hline 1 & EM-03 & 1 & 2 & 14 & r04PF22 & 1.27 & 1.80 & 18.5 \\
\hline 1 & EM-11 & 1 & 2 & 15 & r15PF22 & 1.30 & 1.85 & 19.5 \\
\hline 1 & EM-13 & 1 & 2 & 16 & r02PF12 & 1.26 & 1.81 & 18.2 \\
\hline 1 & EM-01 & 1 & 2 & 17 & r12PF12 & 1.27 & 1.85 & 19.1 \\
\hline 1 & EM-02 & 1 & 2 & 18 & r05PF12 & 1.26 & 1.82 & 18.4 \\
\hline 1 & Batch 1 & 1 & 2 & 19 & BCHPF1123 & 2.43 & 2.05 & 21.6 \\
\hline 1 & Batch 1 & 2 & 1 & 1 & BCHPF1211 & 2.51 & 2.04 & 22.2 \\
\hline 1 & EM-04 & 2 & 1 & 2 & r07PF21 & 1.28 & 1.80 & 19.6 \\
\hline 1 & EM-05 & 2 & 1 & 3 & r11PF11 & 1.27 & 1.82 & 19.6 \\
\hline 1 & EM-07 & 2 & 1 & 4 & r09PF11 & 1.28 & 1.88 & 19.2 \\
\hline 1 & EM-08 & 2 & 1 & 5 & r03PF21 & 1.26 & 1.83 & 18.6 \\
\hline 1 & EM-10 & 2 & 1 & 6 & r01PF11 & 1.22 & 1.79 & 18.5 \\
\hline 1 & EM-05 & 2 & 1 & 7 & r11PF21 & 1.22 & 1.84 & 19.6 \\
\hline 1 & EM-06 & 2 & 1 & 8 & r13PF21 & 1.21 & 1.81 & 19.6 \\
\hline 1 & Batch 1 & 2 & 1 & 9 & BCHPF1212 & 2.41 & 2.08 & 22.1 \\
\hline 1 & EM-09 & 2 & 1 & 10 & r06PF21 & 1.27 & 1.82 & 18.5 \\
\hline 1 & EM-10 & 2 & 1 & 11 & r01PF21 & 1.22 & 1.79 & 18.6 \\
\hline 1 & EM-06 & 2 & 1 & 12 & r13PF11 & 1.23 & 1.84 & 19.7 \\
\hline 1 & EM-09 & 2 & 1 & 13 & r06PF11 & 1.21 & 1.84 & 18.5 \\
\hline 1 & EM-04 & 2 & 1 & 14 & r07PF11 & 1.23 & 1.81 & 19.9 \\
\hline 1 & EM-08 & 2 & 1 & 15 & r03PF11 & 1.22 & 1.85 & 18.3 \\
\hline 1 & EM-07 & 2 & 1 & 16 & r09PF21 & 1.22 & 1.86 & 18.9 \\
\hline 1 & Batch 1 & 2 & 1 & 17 & BCHPF1213 & 2.35 & 2.05 & 21.7 \\
\hline 1 & Batch 1 & 2 & 2 & 1 & BCHPF1221 & 2.56 & 2.09 & 22.1 \\
\hline 1 & EM-07 & 2 & 2 & 2 & r09PF22 & 1.35 & 1.85 & 18.6 \\
\hline 1 & EM-05 & 2 & 2 & 3 & r11PF22 & 1.31 & 1.85 & 19.4 \\
\hline 1 & EM-10 & 2 & 2 & 4 & r01PF22 & 1.29 & 1.81 & 18.6 \\
\hline 1 & EM-08 & 2 & 2 & 5 & r03PF22 & 1.29 & 1.83 & 18.2 \\
\hline 1 & EM-05 & 2 & 2 & 6 & r11PF12 & 1.27 & 1.86 & 19.5 \\
\hline 1 & EM-07 & 2 & 2 & 7 & r09PF12 & 1.29 & 1.86 & 18.8 \\
\hline 1 & EM-09 & 2 & 2 & 8 & r06PF22 & 1.27 & 1.84 & 18.3 \\
\hline 1 & Batch 1 & 2 & 2 & 9 & BCHPF1222 & 2.40 & 2.06 & 22.0 \\
\hline
\end{tabular}


SRNL-STI-2009-00778, Revision 0

Table D2. Measured Elemental Concentrations (wt\%) for the Matrix 2A Study Glasses Prepared Using Peroxide Fusion

\begin{tabular}{|c|c|c|c|c|c|c|c|c|}
\hline Set & Glass ID & Block & Sub-Block & Sequence & Lab ID & B (wt\%) & Li (wt\%) & Si (wt\%) \\
\hline 1 & EM-04 & 2 & 2 & 10 & r07PF12 & 1.36 & 1.85 & 19.9 \\
\hline 1 & EM-10 & 2 & 2 & 11 & r01PF12 & 1.28 & 1.81 & 18.5 \\
\hline 1 & EM-06 & 2 & 2 & 12 & r13PF12 & 1.28 & 1.85 & 19.5 \\
\hline 1 & EM-08 & 2 & 2 & 13 & r03PF12 & 1.27 & 1.85 & 17.8 \\
\hline 1 & EM-04 & 2 & 2 & 14 & r07PF22 & 1.24 & 1.81 & 19.4 \\
\hline 1 & EM-06 & 2 & 2 & 15 & r13PF22 & 1.25 & 1.82 & 19.3 \\
\hline 1 & EM-09 & 2 & 2 & 16 & r06PF12 & 1.24 & 1.84 & 18.2 \\
\hline 1 & Batch 1 & 2 & 2 & 17 & BCHPF1223 & 2.40 & 2.07 & 22.1 \\
\hline 2 & Batch 1 & 1 & 1 & 1 & BCHPF2111 & 2.50 & 2.01 & 21.8 \\
\hline 2 & EM-23 & 1 & 1 & 2 & s12PF21 & 2.25 & 1.83 & 17.6 \\
\hline 2 & EM-23 & 1 & 1 & 3 & s12PF11 & 2.20 & 1.81 & 17.4 \\
\hline 2 & EM-26 & 1 & 1 & 4 & s07PF11 & 2.18 & 1.81 & 17.6 \\
\hline 2 & EM-22 & 1 & 1 & 5 & s02PF21 & 2.19 & 1.79 & 17.2 \\
\hline 2 & EM-28 & 1 & 1 & 6 & s01PF21 & 2.20 & 1.78 & 17.1 \\
\hline 2 & EM-29 & 1 & 1 & 7 & s09PF21 & 2.22 & 1.80 & 17.0 \\
\hline 2 & EM-25 & 1 & 1 & 8 & s14PF21 & 2.17 & 1.77 & 17.3 \\
\hline 2 & EM-20 & 1 & 1 & 9 & s04PF21 & 2.14 & 1.75 & 17.6 \\
\hline 2 & Batch 1 & 1 & 1 & 10 & BCHPF2112 & 2.40 & 2.02 & 21.6 \\
\hline 2 & EM-25 & 1 & 1 & 11 & s14PF11 & 2.22 & 1.77 & 17.3 \\
\hline 2 & EM-22 & 1 & 1 & 12 & s02PF11 & 2.23 & 1.81 & 17.3 \\
\hline 2 & EM-28 & 1 & 1 & 13 & s01PF11 & 2.20 & 1.78 & 17.0 \\
\hline 2 & EM-21 & 1 & 1 & 14 & s03PF11 & 2.23 & 1.83 & 18.2 \\
\hline 2 & EM-20 & 1 & 1 & 15 & s04PF11 & 2.20 & 1.79 & 18.0 \\
\hline 2 & EM-29 & 1 & 1 & 16 & s09PF11 & 2.27 & 1.85 & 17.3 \\
\hline 2 & EM-21 & 1 & 1 & 17 & s03PF21 & 2.18 & 1.79 & 18.0 \\
\hline 2 & EM-26 & 1 & 1 & 18 & s07PF21 & 2.16 & 1.77 & 17.5 \\
\hline 2 & Batch 1 & 1 & 1 & 19 & BCHPF2113 & 2.41 & 2.02 & 21.8 \\
\hline 2 & Batch 1 & 1 & 2 & 1 & BCHPF2121 & 2.45 & 2.01 & 21.5 \\
\hline 2 & EM-22 & 1 & 2 & 2 & s02PF22 & 2.20 & 1.83 & 17.3 \\
\hline 2 & EM-26 & 1 & 2 & 3 & s07PF12 & 2.14 & 1.80 & 17.6 \\
\hline 2 & EM-29 & 1 & 2 & 4 & s09PF22 & 2.19 & 1.82 & 17.1 \\
\hline 2 & EM-21 & 1 & 2 & 5 & s03PF12 & 2.14 & 1.81 & 18.0 \\
\hline 2 & EM-25 & 1 & 2 & 6 & s14PF12 & 2.12 & 1.80 & 17.3 \\
\hline 2 & EM-23 & 1 & 2 & 7 & s12PF22 & 2.20 & 1.88 & 17.9 \\
\hline 2 & EM-25 & 1 & 2 & 8 & s14PF22 & 2.14 & 1.81 & 17.4 \\
\hline 2 & EM-23 & 1 & 2 & 9 & s12PF12 & 2.12 & 1.81 & 17.3 \\
\hline 2 & Batch 1 & 1 & 2 & 10 & BCHPF2122 & 2.33 & 2.02 & 21.7 \\
\hline 2 & EM-21 & 1 & 2 & 11 & s03PF22 & 2.18 & 1.81 & 18.0 \\
\hline 2 & EM-29 & 1 & 2 & 12 & s09PF12 & 2.17 & 1.81 & 16.9 \\
\hline 2 & EM-22 & 1 & 2 & 13 & s02PF12 & 2.15 & 1.81 & 17.2 \\
\hline 2 & EM-26 & 1 & 2 & 14 & s07PF22 & 2.10 & 1.79 & 17.5 \\
\hline 2 & EM-28 & 1 & 2 & 15 & s01PF22 & 2.11 & 1.79 & 16.9 \\
\hline 2 & EM-20 & 1 & 2 & 16 & s04PF12 & 2.08 & 1.78 & 17.6 \\
\hline 2 & EM-28 & 1 & 2 & 17 & s01PF12 & 2.08 & 1.77 & 16.6 \\
\hline 2 & EM-20 & 1 & 2 & 18 & s04PF22 & 2.06 & 1.76 & 17.4 \\
\hline 2 & Batch 1 & 1 & 2 & 19 & BCHPF2123 & 2.32 & 2.01 & 21.5 \\
\hline 2 & Batch 1 & 2 & 1 & 1 & BCHPF2211 & 2.50 & 2.07 & 22.0 \\
\hline 2 & EM-24 & 2 & 1 & 2 & s05PF11 & 2.27 & 1.88 & 17.7 \\
\hline 2 & EM-30 & 2 & 1 & 3 & s13PF11 & 2.20 & 1.86 & 17.1 \\
\hline 2 & EM-19 & 2 & 1 & 4 & s06PF21 & 2.40 & 1.97 & 18.6 \\
\hline 2 & EM-27 & 2 & 1 & 5 & s15PF11 & 2.20 & 1.86 & 17.0 \\
\hline 2 & EM-30 & 2 & 1 & 6 & s13PF21 & 2.17 & 1.83 & 16.8 \\
\hline 2 & EM-27 & 2 & 1 & 7 & s15PF21 & 2.17 & 1.84 & 17.0 \\
\hline 2 & EM-17 & 2 & 1 & 8 & s10PF11 & 2.14 & 1.82 & 17.0 \\
\hline 2 & Batch 1 & 2 & 1 & 9 & BCHPF2212 & 2.39 & 2.10 & 22.0 \\
\hline 2 & EM-16 & 2 & 1 & 10 & s11PF11 & 2.21 & 1.86 & 17.6 \\
\hline 2 & EM-19 & 2 & 1 & 11 & s06PF11 & 2.20 & 1.84 & 18.1 \\
\hline 2 & EM-18 & 2 & 1 & 12 & s08PF21 & 2.12 & 1.81 & 17.4 \\
\hline 2 & EM-18 & 2 & 1 & 13 & s08PF11 & 2.13 & 1.83 & 17.5 \\
\hline 2 & EM-24 & 2 & 1 & 14 & s05PF21 & 2.17 & 1.85 & 17.4 \\
\hline 2 & EM-17 & 2 & 1 & 15 & s10PF21 & 2.13 & 1.83 & 17.1 \\
\hline 2 & EM-16 & 2 & 1 & 16 & s11PF21 & 2.10 & 1.82 & 17.3 \\
\hline 2 & Batch 1 & 2 & 1 & 17 & BCHPF2213 & 2.37 & 2.07 & 22.0 \\
\hline 2 & Batch 1 & 2 & 2 & 1 & BCHPF2221 & 2.47 & 2.06 & 21.6 \\
\hline
\end{tabular}


SRNL-STI-2009-00778, Revision 0

Table D2. Measured Elemental Concentrations (wt\%) for the Matrix 2A Study Glasses Prepared Using Peroxide Fusion

\begin{tabular}{|c|c|c|c|c|c|c|c|c|}
\hline Set & Glass ID & Block & Sub-Block & Sequence & Lab ID & B (wt\%) & Li (wt\%) & Si (wt\%) \\
\hline 2 & EM-16 & 2 & 2 & 2 & s11PF22 & 2.18 & 1.85 & 17.2 \\
\hline 2 & EM-19 & 2 & 2 & 3 & s06PF12 & 2.22 & 1.87 & 18.2 \\
\hline 2 & EM-18 & 2 & 2 & 4 & s08PF22 & 2.12 & 1.81 & 17.2 \\
\hline 2 & EM-18 & 2 & 2 & 5 & s08PF12 & 2.13 & 1.83 & 17.3 \\
\hline 2 & EM-24 & 2 & 2 & 6 & s05PF22 & 2.17 & 1.86 & 17.2 \\
\hline 2 & EM-27 & 2 & 2 & 7 & s15PF22 & 2.12 & 1.84 & 16.7 \\
\hline 2 & EM-27 & 2 & 2 & 8 & s15PF12 & 2.12 & 1.84 & 16.8 \\
\hline 2 & Batch 1 & 2 & 2 & 9 & BCHPF2222 & 2.33 & 2.04 & 21.5 \\
\hline 2 & EM-16 & 2 & 2 & 10 & s11PF12 & 2.19 & 1.85 & 17.4 \\
\hline 2 & EM-17 & 2 & 2 & 11 & s10PF12 & 2.11 & 1.81 & 16.8 \\
\hline 2 & EM-19 & 2 & 2 & 12 & s06PF22 & 2.35 & 1.97 & 18.4 \\
\hline 2 & EM-17 & 2 & 2 & 13 & s10PF22 & 2.14 & 1.83 & 16.9 \\
\hline 2 & EM-30 & 2 & 2 & 14 & s13PF12 & 2.14 & 1.84 & 16.7 \\
\hline 2 & EM-30 & 2 & 2 & 15 & s13PF22 & 2.15 & 1.84 & 16.8 \\
\hline 2 & EM-24 & 2 & 2 & 16 & s05PF12 & 2.18 & 1.87 & 17.4 \\
\hline 2 & Batch 1 & 2 & 2 & 17 & BCHPF2223 & 2.36 & 2.07 & 21.9 \\
\hline
\end{tabular}


SRNL-STI-2009-00778, Revision 0

Table D3. Average Measured and Bias-Corrected Chemical Compositions Versus Targeted Compositions by Oxide by Glass ID for the Matrix 2A Study

\begin{tabular}{|c|c|c|c|c|c|c|c|c|c|}
\hline & & & & Measured & & & & & \\
\hline & & & Measured & Bias-Corrected & Targeted & Diff of & Diff of & \% Diff of & $\%$ Diff of \\
\hline Set & Glass \# & Oxide & (wt\%) & (wt\%) & $(\mathrm{wt} \%)$ & Measured & Meas BC & Measured & Meas BC \\
\hline 1 & Batch 1 & $\mathrm{Al} 2 \mathrm{O} 3$ (wt\%) & 4.7379 & 4.8770 & 4.8770 & -0.1391 & 0.0000 & $-2.9 \%$ & $0.0 \%$ \\
\hline 1 & Batch 1 & B2O3 (wt\%) & 7.9854 & 7.7770 & 7.7770 & 0.2084 & 0.0000 & $2.7 \%$ & $0.0 \%$ \\
\hline 1 & Batch 1 & $\mathrm{BaO}(\mathrm{wt} \%)$ & 0.1342 & 0.1510 & 0.1510 & -0.0168 & 0.0000 & $-11.1 \%$ & $0.0 \%$ \\
\hline 1 & Batch 1 & $\mathrm{CaO}$ (wt\%) & 1.2060 & 1.2200 & 1.2200 & -0.0140 & 0.0000 & $-1.1 \%$ & $0.0 \%$ \\
\hline 1 & Batch 1 & Ce2O3 (wt\%) & 0.0059 & 0.0059 & 0.0000 & 0.0059 & 0.0059 & & \\
\hline 1 & Batch 1 & Cr2O3 (wt\%) & 0.1044 & 0.1070 & 0.1070 & -0.0026 & 0.0000 & $-2.4 \%$ & $0.0 \%$ \\
\hline 1 & Batch 1 & $\mathrm{CuO}$ (wt\%) & 0.3866 & 0.3990 & 0.3990 & -0.0124 & 0.0000 & $-3.1 \%$ & $0.0 \%$ \\
\hline 1 & Batch 1 & $\mathrm{Fe} 2 \mathrm{O} 3(\mathrm{wt} \%)$ & 12.5635 & 12.8390 & 12.8390 & -0.2755 & 0.0000 & $-2.1 \%$ & $0.0 \%$ \\
\hline 1 & Batch 1 & La2O3 (wt\%) & 0.0059 & 0.0059 & 0.0000 & 0.0059 & 0.0059 & & \\
\hline 1 & Batch 1 & $\mathrm{Li} 2 \mathrm{O}$ (wt\%) & 4.4906 & 4.4290 & 4.4290 & 0.0616 & 0.0000 & $1.4 \%$ & $0.0 \%$ \\
\hline 1 & Batch 1 & $\mathrm{MgO}$ (wt\%) & 1.2962 & 1.4190 & 1.4190 & -0.1228 & 0.0000 & $-8.7 \%$ & $0.0 \%$ \\
\hline 1 & Batch 1 & $\mathrm{MnO}$ (wt\%) & 1.7259 & 1.7260 & 1.7260 & -0.0001 & 0.0000 & $0.0 \%$ & $0.0 \%$ \\
\hline 1 & Batch 1 & $\mathrm{Na} 2 \mathrm{O}$ (wt\%) & 9.1181 & 9.0030 & 9.0030 & 0.1151 & 0.0000 & $1.3 \%$ & $0.0 \%$ \\
\hline 1 & Batch 1 & $\mathrm{NiO}$ (wt\%) & 0.6555 & 0.7510 & 0.7510 & -0.0955 & 0.0000 & $-12.7 \%$ & $0.0 \%$ \\
\hline 1 & Batch 1 & $\mathrm{PbO}(\mathrm{wt} \%)$ & 0.0054 & 0.0054 & 0.0000 & 0.0054 & 0.0054 & & \\
\hline 1 & Batch 1 & $\mathrm{SiO} 2$ (wt\%) & 47.4033 & 50.2200 & 50.2200 & -2.8167 & 0.0000 & $-5.6 \%$ & $0.0 \%$ \\
\hline 1 & Batch 1 & SO4 (wt\%) & 0.1498 & 0.1498 & 0.0000 & 0.1498 & 0.1498 & & \\
\hline 1 & Batch 1 & $\mathrm{TiO} 2$ (wt\%) & 0.6536 & 0.6770 & 0.6770 & -0.0234 & 0.0000 & $-3.5 \%$ & $0.0 \%$ \\
\hline 1 & Batch 1 & $\mathrm{ZnO}$ (wt\%) & 0.0062 & 0.0062 & 0.0000 & 0.0062 & 0.0062 & & \\
\hline 1 & Batch 1 & ZrO2 (wt\%) & 0.0874 & 0.0874 & 0.0980 & -0.0106 & -0.0106 & $-10.9 \%$ & $-10.9 \%$ \\
\hline 1 & Batch 1 & $\mathrm{ZZ} \mathrm{sum}$ & 92.7217 & 95.8555 & 95.6930 & -2.9713 & 0.1625 & $-3.1 \%$ & $0.2 \%$ \\
\hline 1 & EM-01 & "Al2O3 (wt\%) & 111.4882 & 111.8133 & 111.5900 & -0.1018 & 0.2233 & $-0.9 \%$ & $1.9 \%$ \\
\hline 1 & EM-01 & B2O3 (wt\%) & 4.1537 & 3.9788 & 4.0000 & 0.1537 & -0.0212 & $3.8 \%$ & $-0.5 \%$ \\
\hline 1 & EM-01 & $\mathrm{BaO}(\mathrm{wt} \%)$ & 0.0684 & 0.0764 & 0.0800 & -0.0116 & -0.0036 & $-14.5 \%$ & $-4.5 \%$ \\
\hline 1 & EM-01 & $\mathrm{CaO}$ (wt\%) & 1.3352 & 1.3556 & 1.3200 & 0.0152 & 0.0356 & $1.2 \%$ & $2.7 \%$ \\
\hline 1 & EM-01 & Ce2O3 (wt\%) & 0.2264 & 0.2264 & 0.2400 & -0.0136 & -0.0136 & $-5.7 \%$ & $-5.7 \%$ \\
\hline 1 & EM-01 & & 0.1027 & 0.1046 & 0.1400 & -0.0373 & -0.0354 & $-26.7 \%$ & $-25.3 \%$ \\
\hline 1 & EM-01 & $\mathrm{CuO}$ (wt\%) & 0.0448 & 0.0466 & 0.0400 & 0.0048 & 0.0066 & $11.9 \%$ & $16.5 \%$ \\
\hline 1 & EM-01 & $\mathrm{Fe} 2 \mathrm{O} 3$ (wt\%) & 14.1433 & 14.4314 & 14.9500 & -0.8067 & -0.5186 & $-5.4 \%$ & $-3.5 \%$ \\
\hline 1 & EM-01 & La2O3 (wt\%) & 0.0692 & 0.0692 & 0.0800 & -0.0108 & -0.0108 & $-13.5 \%$ & $-13.5 \%$ \\
\hline 1 & EM-01 & $\mathrm{Li} 2 \mathrm{O}$ (wt\%) & 4.0098 & 3.9164 & 4.0000 & 0.0098 & -0.0836 & $0.2 \%$ & $-2.1 \%$ \\
\hline 1 & EM-01 & MgO (wt\%) & 0.7015 & 0.7558 & 0.7100 & -0.0085 & 0.0458 & $-1.2 \%$ & $6.5 \%$ \\
\hline 1 & EM-01 & $\mathrm{MnO}(\mathrm{wt} \%)$ & 2.9375 & 2.9376 & 2.9200 & 0.0175 & 0.0176 & $0.6 \%$ & $0.6 \%$ \\
\hline 1 & EM-01 & $\mathrm{Na} 2 \mathrm{O}$ (wt\%) & 15.4346 & 15.2034 & 15.4800 & -0.0454 & -0.2766 & $-0.3 \%$ & $-1.8 \%$ \\
\hline 1 & EM-01 & $\mathrm{NiO}$ (wt\%) & 0.9553 & 1.0832 & 1.1400 & -0.1847 & -0.0568 & $-16.2 \%$ & $-5.0 \%$ \\
\hline 1 & EM-01 & $\mathrm{PbO}$ (wt\%) & 0.0908 & 0.0908 & 0.1000 & -0.0092 & -0.0092 & $-9.2 \%$ & $-9.2 \%$ \\
\hline 1 & EM-01 & $\mathrm{SiO} 2$ (wt\%) & 41.1280 & 43.3337 & 40.4200 & 0.7080 & 2.9137 & $1.8 \%$ & $7.2 \%$ \\
\hline 1 & EM-01 & SO4 (wt\%) & 0.4269 & 0.4269 & 0.4500 & -0.0231 & -0.0231 & $-5.1 \%$ & $-5.1 \%$ \\
\hline 1 & EM-01 & TiO2 (wt\%) & 2.0725 & 2.1580 & 2.0000 & 0.0725 & 0.1580 & $3.6 \%$ & $7.9 \%$ \\
\hline 1 & EM-01 & $\mathrm{ZnO}$ (wt\%) & 0.0582 & & 0.0700 & -0.0118 & -0.0118 & $-16.9 \%$ & $-16.9 \%$ \\
\hline 1 & EM-01 & ZrO2 (wt\%) & 0.1692 & 0.1692 & 0.1700 & -0.0008 & -0.0008 & $-0.5 \%$ & $-0.5 \%$ \\
\hline 1 & EM-01 & ZZ sum & 99.6159 & 102.2354 & 99.9000 & -0.2841 & 2.3354 & $-0.3 \%$ & $2.3 \%$ \\
\hline 1 & EM-02 & $\mathrm{Al} 2 \mathrm{O} 3$ (wt\%) & 14.1382 & 14.5383 & 14.0900 & 0.0482 & 0.4483 & $0.3 \%$ & $3.2 \%$ \\
\hline 1 & EM-02 & B2O3 (wt\%) & 4.0973 & 3.9256 & 4.0000 & 0.0973 & -0.0744 & $2.4 \%$ & $-1.9 \%$ \\
\hline 1 & EM-02 & $\mathrm{BaO}$ (wt\%) & 0.0681 & 0.0761 & 0.0800 & -0.0119 & -0.0039 & $-14.9 \%$ & $-4.9 \%$ \\
\hline 1 & EM-02 & $\mathrm{CaO}$ (wt\%) & 1.3338 & 1.3542 & 1.3200 & 0.0138 & 0.0342 & $1.0 \%$ & $2.6 \%$ \\
\hline 1 & EM-02 & Ce2O3 (wt\%) & 0.2255 & 0.2255 & 0.2400 & -0.0145 & -0.0145 & $-6.1 \%$ & $-6.1 \%$ \\
\hline 1 & EM-02 & Cr2O3 (wt\%) & 0.1107 & 0.1128 & 0.1400 & -0.0293 & -0.0272 & $-20.9 \%$ & $-19.4 \%$ \\
\hline 1 & EM-02 & $\mathrm{CuO}$ (wt\%) & 0.0476 & 0.0495 & 0.0400 & 0.0076 & 0.0095 & $18.9 \%$ & $23.8 \%$ \\
\hline 1 & EM-02 & $\mathrm{Fe} 2 \mathrm{O} 3(\mathrm{wt} \%)$ & 14.0790 & 14.3670 & 14.9500 & -0.8710 & -0.5830 & $-5.8 \%$ & $-3.9 \%$ \\
\hline 1 & EM-02 & La2O3 (wt\%) & 0.0704 & 0.0704 & 0.0800 & -0.0096 & -0.0096 & $-12.0 \%$ & $-12.0 \%$ \\
\hline 1 & EM-02 & $\mathrm{Li} 2 \mathrm{O}$ (wt\%) & 3.9613 & 3.8683 & 4.0000 & -0.0387 & -0.1317 & $-1.0 \%$ & $-3.3 \%$ \\
\hline 1 & EM-02 & $\mathrm{MgO}(\mathrm{wt} \%)$ & 0.7015 & 0.7558 & 0.7100 & -0.0085 & 0.0458 & $-1.2 \%$ & $6.5 \%$ \\
\hline 1 & EM-02 & $\mathrm{MnO}(\mathrm{wt} \%)$ & 2.9568 & 2.9570 & 2.9200 & 0.0368 & 0.0370 & $1.3 \%$ & $1.3 \%$ \\
\hline 1 & EM-02 & $\mathrm{Na} 2 \mathrm{O}$ (wt\%) & 13.5912 & 13.3876 & 13.6000 & -0.0088 & -0.2124 & $-0.1 \%$ & $-1.6 \%$ \\
\hline 1 & EM-02 & $\mathrm{NiO}$ (wt\%) & 0.9725 & 1.1027 & 1.1400 & -0.1675 & -0.0373 & $-14.7 \%$ & $-3.3 \%$ \\
\hline 1 & EM-02 & $\mathrm{PbO}$ (wt\%) & 0.0867 & 0.0867 & 0.1000 & -0.0133 & -0.0133 & $-13.3 \%$ & $-13.3 \%$ \\
\hline 1 & EM-02 & $\mathrm{SiO} 2$ (wt\%) & 40.1119 & 42.2587 & 39.8100 & 0.3019 & 2.4487 & $0.8 \%$ & $6.2 \%$ \\
\hline
\end{tabular}


SRNL-STI-2009-00778, Revision 0

Table D3. Average Measured and Bias-Corrected Chemical Compositions Versus Targeted Compositions by Oxide by Glass ID for the Matrix 2A Study

\begin{tabular}{|c|c|c|c|c|c|c|c|c|c|}
\hline & & & & Measured & & & & & \\
\hline & & & Measured & Bias-Corrected & Targeted & Diff of & Diff of & \% Diff of & \% Diff of \\
\hline Set & Glass \# & Oxide & $(w t \%)$ & (wt\%) & (wt\%) & Measured & Meas BC & Measured & Meas BC \\
\hline 1 & EM-02 & " SO4 (wt\%) & 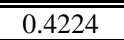 & 0.4224 & "0.4500 & -0.0276 & -0.0276 & $=-6.1 \%$ & "-6.1\% \\
\hline 1 & EM-02 & TiO2 (wt\%) & 2.0600 & 2.1448 & 2.0000 & 0.0600 & 0.1448 & $3.0 \%$ & $7.2 \%$ \\
\hline 1 & EM-02 & $\mathrm{ZnO}(\mathrm{wt} \%)$ & 0.0613 & 0.0613 & 0.0700 & -0.0087 & -0.0087 & $-12.4 \%$ & $-12.4 \%$ \\
\hline 1 & EM-02 & $\mathrm{ZrO} 2$ (wt\%) & 0.1749 & 0.1749 & 0.1700 & 0.0049 & 0.0049 & $2.9 \%$ & $2.9 \%$ \\
\hline 1 & EM-02 & $\mathrm{ZZ} \mathrm{sum}$ & 99.2711 & 101.9397 & 99.9100 & $\begin{array}{l}-0.6389 \\
\end{array}$ & 2.0297 & $-0.6 \%$ & $2.0 \%$ \\
\hline 1 & EM-03 & Al2O3 (wt\%) & 9.0885 & 9.3649 & 9.0900 & -0.0015 & 0.2749 & $0.0 \%$ & $3.0 \%$ \\
\hline 1 & EM-03 & B2O3 (wt\%) & 4.1215 & 3.9494 & 4.0000 & 0.1215 & -0.0506 & $3.0 \%$ & $-1.3 \%$ \\
\hline 1 & EM-03 & $\mathrm{BaO}$ (wt\%) & 0.0695 & 0.0788 & 0.0800 & -0.0105 & -0.0012 & $-13.1 \%$ & $-1.5 \%$ \\
\hline 1 & EM-03 & $\mathrm{CaO}(\mathrm{wt} \%)$ & 1.3695 & 1.3803 & 1.3200 & 0.0495 & 0.0603 & $3.7 \%$ & $4.6 \%$ \\
\hline 1 & EM-03 & Ce2O3 (wt\%) & 0.2319 & 0.2319 & 0.2400 & -0.0081 & $\begin{array}{l}-0.0081 \\
\end{array}$ & $-3.4 \%$ & $-3.4 \%$ \\
\hline 1 & EM-03 & Cr2O3 (wt\%) & 0.1162 & 0.1198 & 0.1400 & -0.0238 & -0.0202 & $-17.0 \%$ & $-14.4 \%$ \\
\hline 1 & EM-03 & $\mathrm{CuO}(\mathrm{wt} \%)$ & 0.0548 & 0.0561 & 0.0400 & 0.0148 & 0.0161 & $36.9 \%$ & $40.2 \%$ \\
\hline 1 & EM-03 & $\mathrm{Fe} 2 \mathrm{O} 3(\mathrm{wt} \%)$ & 14.0861 & 14.4144 & 14.9500 & -0.8639 & -0.5356 & $-5.8 \%$ & $-3.6 \%$ \\
\hline 1 & EM-03 & La2O3 (wt\%) & 0.0692 & 0.0692 & 0.0800 & -0.0108 & -0.0108 & $-13.5 \%$ & $-13.5 \%$ \\
\hline 1 & EM-03 & Li2O (wt\%) & 3.9506 & 3.8582 & 4.0000 & -0.0494 & -0.1418 & $-1.2 \%$ & $-3.5 \%$ \\
\hline 1 & EM-03 & $\mathrm{MgO}(\mathrm{wt} \%)$ & 0.6861 & 0.7633 & 0.7100 & -0.0239 & 0.0533 & $-3.4 \%$ & $7.5 \%$ \\
\hline 1 & EM-03 & $\mathrm{MnO}$ (wt\%) & 2.9633 & 2.9633 & 2.9200 & 0.0433 & 0.0433 & $1.5 \%$ & $1.5 \%$ \\
\hline 1 & EM-03 & $\mathrm{Na} 2 \mathrm{O}$ (wt\%) & 17.2207 & 17.0449 & 17.5100 & -0.2893 & -0.4651 & $-1.7 \%$ & $-2.7 \%$ \\
\hline 1 & EM-03 & $\mathrm{NiO}$ (wt\%) & 0.9477 & 1.0970 & 1.1400 & -0.1923 & -0.0430 & $-16.9 \%$ & $-3.8 \%$ \\
\hline 1 & EM-03 & $\mathrm{PbO}$ (wt\%) & 0.0878 & 0.0878 & 0.1000 & -0.0122 & -0.0122 & $-12.2 \%$ & $-12.2 \%$ \\
\hline 1 & EM-03 & $\mathrm{SiO} 2$ (wt\%) & 40.5932 & 42.7648 & 40.9000 & -0.3068 & 1.8648 & $-0.8 \%$ & $4.6 \%$ \\
\hline 1 & EM-03 & SO4 (wt\%) & 0.4434 & 0.4434 & 0.4500 & -0.0066 & -0.0066 & $-1.5 \%$ & $-1.5 \%$ \\
\hline 1 & EM-03 & & 2.0016 & 2.0627 & 2.0000 & 0.0016 & 0.0627 & $0.1 \%$ & $3.1 \%$ \\
\hline 1 & EM-03 & $\mathrm{ZnO}$ (wt\%) & 0.0654 & 0.0654 & 0.0700 & -0.0046 & -0.0046 & $-6.6 \%$ & $-6.6 \%$ \\
\hline 1 & EM-03 & $\mathrm{ZrO2}$ (wt\%) & 0.1719 & 0.1719 & 0.1700 & 0.0019 & 0.0019 & $1.1 \%$ & $1.1 \%$ \\
\hline 1 & EM-03 & $\mathrm{ZZ}$ sum & 98.3388 & 100.9873 & 99.9100 & -1.5712 & 1.0773 & $-1.6 \%$ & $1.1 \%$ \\
\hline$\overline{1} 1$ & EM-04 & Al2O3 (wt\%) & 6.5755 & 6.7752 & 6.5900 & -0.0145 & 0.1852 & $-0.2 \%$ & $2.8 \%$ \\
\hline 1 & EM-04 & $2 \mathrm{O}$ (wt\%) & 4.1134 & 4.0743 & 4.0000 & 0.1134 & 0.0743 & $2.8 \%$ & $1.9 \%$ \\
\hline 1 & EM-04 & $\mathrm{BaO}$ (wt\%) & 0.0689 & 0.0781 & 0.0800 & -0.0111 & -0.0019 & $-13.8 \%$ & $-2.3 \%$ \\
\hline 1 & EM-04 & $\mathrm{CaO}$ (wt\%) & 1.3177 & 1.3279 & 1.3200 & -0.0023 & 0.0079 & $-0.2 \%$ & $0.6 \%$ \\
\hline 1 & EM-04 & Ce2O3 (wt\%) & 0.2284 & 0.2284 & 0.2400 & -0.0116 & -0.0116 & $-4.8 \%$ & $-4.8 \%$ \\
\hline 1 & EM-04 & Cr2O3 (wt\%) & 0.1188 & 0.1225 & 0.1400 & -0.0212 & -0.0175 & $-15.2 \%$ & $-12.5 \%$ \\
\hline 1 & EM-04 & $\mathrm{CuO}$ (wt\%) & 0.0538 & 0.0551 & 0.0400 & 0.0138 & 0.0151 & $34.6 \%$ & $37.8 \%$ \\
\hline 1 & EM-04 & Fe2O3 (wt\%) & 14.2112 & 14.5445 & 14.9500 & -0.7388 & -0.4055 & $-4.9 \%$ & $-2.7 \%$ \\
\hline 1 & EM-04 & La2O3 (wt\%) & 0.0642 & 0.0642 & 0.0800 & -0.0158 & -0.0158 & $-19.7 \%$ & $-19.7 \%$ \\
\hline 1 & EM-04 & Li2O (wt\%) & 3.9129 & 3.8981 & 4.0000 & -0.0871 & -0.1019 & $-2.2 \%$ & $-2.5 \%$ \\
\hline 1 & EM-04 & & 0.6762 & 0.7522 & 0.7100 & -0.0338 & 0.0422 & $-4.8 \%$ & $5.9 \%$ \\
\hline 1 & EM-04 & $\mathrm{MnO}$ (wt\%) & 2.9407 & & 2.9200 & 0.0207 & 0.0210 & $0.7 \%$ & $0.7 \%$ \\
\hline 1 & EM-04 & $\mathrm{Na} 2 \mathrm{O}$ (wt\%) & 17.4903 & 17.3097 & 17.9300 & -0.4397 & -0.6203 & $-2.5 \%$ & $-3.5 \%$ \\
\hline 1 & EM-04 & $\mathrm{NiO}$ (wt\%) & 0.9744 & 1.1278 & 1.1400 & -0.1656 & -0.0122 & $-14.5 \%$ & $-1.1 \%$ \\
\hline 1 & EM-04 & $\mathrm{PbO}(\mathrm{wt} \%)$ & 0.0843 & 0.0843 & 0.1000 & -0.0157 & -0.0157 & $-15.7 \%$ & $-15.7 \%$ \\
\hline 1 & EM-04 & $\mathrm{SiO} 2$ (wt\%) & 42.1442 & 44.9020 & 42.9800 & -0.8358 & 1.9220 & $-1.9 \%$ & $4.5 \%$ \\
\hline 1 & EM-04 & SO4 (wt\%) & 0.4344 & 0.4344 & 0.4500 & -0.0156 & -0.0156 & $-3.5 \%$ & $-3.5 \%$ \\
\hline 1 & EM-04 & TiO2 (wt\%) & 1.9808 & 2.0413 & 2.0000 & -0.0193 & 0.0413 & $-1.0 \%$ & $2.1 \%$ \\
\hline 1 & EM-04 & $\mathrm{ZnO}$ (wt\%) & 0.0641 & 0.0641 & 0.0700 & -0.0059 & -0.0059 & $-8.4 \%$ & $-8.4 \%$ \\
\hline 1 & EM-04 & ZrO2 (wt\%) & 0.1709 & 0.1709 & 0.1700 & 0.0009 & 0.0009 & $0.5 \%$ & $0.5 \%$ \\
\hline 1 & EM-04 & ZZ sum & 97.6251 & 100.9958 & 99.9100 & -2.2849 & 1.0858 & $-2.3 \%$ & $1.1 \%$ \\
\hline 1 & EM-05 & Al2O3 (wt\%) & 6.5896 & "6.7901 & "6.5900 & -0.0004 & 0.2001 & $0.0 \%$ & $3.0 \%$ \\
\hline 1 & EM-05 & $\mathrm{B} 2 \mathrm{O} 3(\mathrm{wt} \%)$ & 4.0812 & 4.0424 & 4.0000 & 0.0812 & 0.0424 & $2.0 \%$ & $1.1 \%$ \\
\hline 1 & EM-05 & $\mathrm{BaO}$ (wt\%) & 0.0701 & 0.0794 & 0.0800 & -0.0099 & -0.0006 & $-12.4 \%$ & $-0.7 \%$ \\
\hline 1 & EM-05 & $\mathrm{CaO}$ (wt\%) & 1.3313 & 1.3419 & 1.3200 & 0.0113 & 0.0219 & $0.9 \%$ & $1.7 \%$ \\
\hline 1 & EM-05 & Ce2O3 (wt\%) & 0.2357 & 0.2357 & 0.2400 & -0.0043 & -0.0043 & $-1.8 \%$ & $-1.8 \%$ \\
\hline 1 & EM-05 & Cr2O3 (wt\%) & 0.1063 & 0.1096 & 0.1400 & -0.0337 & -0.0304 & $-24.0 \%$ & $-21.7 \%$ \\
\hline 1 & EM-05 & $\mathrm{CuO}(\mathrm{wt} \%)$ & 0.0432 & 0.0442 & 0.0400 & 0.0032 & 0.0042 & $8.0 \%$ & $10.5 \%$ \\
\hline 1 & EM-05 & Fe2O3 (wt\%) & 16.2628 & 16.6415 & 17.4500 & -1.1872 & -0.8085 & $-6.8 \%$ & $-4.6 \%$ \\
\hline 1 & EM-05 & La2O3 (wt\%) & 0.0701 & 0.0701 & 0.0800 & -0.0099 & -0.0099 & $-12.4 \%$ & $-12.4 \%$ \\
\hline 1 & EM-05 & Li2O (wt\%) & 3.9667 & 3.9517 & 4.0000 & -0.0333 & -0.0483 & $-0.8 \%$ & $-1.2 \%$ \\
\hline 1 & EM-05 & $\mathrm{MgO}$ (wt\%) & 0.7002 & 0.7790 & 0.7100 & -0.0098 & 0.0690 & $-1.4 \%$ & $9.7 \%$ \\
\hline
\end{tabular}


SRNL-STI-2009-00778, Revision 0

Table D3. Average Measured and Bias-Corrected Chemical Compositions Versus Targeted Compositions by Oxide by Glass ID for the Matrix 2A Study

\begin{tabular}{|c|c|c|c|c|c|c|c|c|c|}
\hline & & & & Measured & & & & & \\
\hline & & & Measured & Bias-Corrected & Targeted & Diff of & Diff of & $\%$ Diff of & \% Diff of \\
\hline Set & Glass \# & Oxide & (wt\%) & (wt\%) & $(\mathrm{wt} \%)$ & Measured & Meas BC & Measured & Meas BC \\
\hline 1 & EM-05 & $\mathrm{MnO}$ (wt\%) & 2.9762 & 2.9762 & 2.9200 & 0.0562 & 0.0562 & $1.9 \%$ & $1.9 \%$ \\
\hline 1 & EM-05 & $\mathrm{Na} 2 \mathrm{O}$ (wt\%) & 15.9401 & 15.7783 & 16.2700 & -0.3299 & -0.4917 & $-2.0 \%$ & $-3.0 \%$ \\
\hline 1 & EM-05 & $\mathrm{NiO}$ (wt\%) & 0.9779 & 1.1319 & 1.1400 & -0.1621 & -0.0081 & $-14.2 \%$ & $-0.7 \%$ \\
\hline 1 & EM-05 & $\mathrm{PbO}(\mathrm{wt} \%)$ & 0.0926 & 0.0926 & 0.1000 & -0.0074 & -0.0074 & $-7.4 \%$ & $-7.4 \%$ \\
\hline 1 & EM-05 & $\mathrm{SiO2}$ (wt\%) & 41.7698 & 44.5032 & 42.1400 & $\begin{array}{l}-0.3702 \\
\end{array}$ & 2.3632 & $-0.9 \%$ & $5.6 \%$ \\
\hline 1 & EM-05 & SO4 (wt\%) & 0.4464 & 0.4464 & 0.4500 & -0.0036 & -0.0036 & $-0.8 \%$ & $-0.8 \%$ \\
\hline 1 & EM-05 & $\mathrm{TiO} 2$ (wt\%) & 1.9849 & 2.0455 & 2.0000 & -0.0151 & 0.0455 & $-0.8 \%$ & $2.3 \%$ \\
\hline 1 & EM-05 & $\mathrm{ZnO}$ (wt\%) & 0.0622 & 0.0622 & 0.0700 & -0.0078 & -0.0078 & $-11.1 \%$ & $-11.1 \%$ \\
\hline 1 & EM-05 & $\mathrm{ZrO2}$ (wt\%) & 0.1726 & 0.1726 & 0.1700 & 0.0026 & 0.0026 & $1.5 \%$ & $1.5 \%$ \\
\hline 1 & EM-05 & $\mathrm{ZZ}$ sum & 97.8802 & 101.2946 & 99.9100 & -2.0298 & 1.3846 & $-2.0 \%$ & $1.4 \%$ \\
\hline 1 & EM-06 & Al2O3 (wt\%) & 6.7077 & 6.8976 & 6.5900 & 0.1177 & 0.3076 & $1.8 \%$ & $4.7 \%$ \\
\hline 1 & EM-06 & B2O3 (wt\%) & 4.0007 & 3.9626 & 4.0000 & 0.0007 & -0.0374 & $0.0 \%$ & $-0.9 \%$ \\
\hline 1 & EM-06 & $\mathrm{BaO}$ (wt\%) & 0.0695 & 0.0777 & 0.0800 & -0.0105 & -0.0023 & $-13.1 \%$ & $-2.9 \%$ \\
\hline 1 & EM-06 & $\mathrm{CaO}(\mathrm{wt} \%)$ & 1.3558 & 1.3766 & 1.3200 & 0.0358 & 0.0566 & $2.7 \%$ & $4.3 \%$ \\
\hline 1 & EM-06 & Ce2O3 (wt\%) & 0.2331 & 0.2331 & 0.2400 & -0.0069 & -0.0069 & $-2.9 \%$ & $-2.9 \%$ \\
\hline 1 & EM-06 & Cr2O3 (wt\%) & 0.1052 & 0.1072 & 0.1400 & -0.0348 & -0.0328 & $-24.8 \%$ & $-23.4 \%$ \\
\hline 1 & EM-06 & $\mathrm{CuO}(\mathrm{wt} \%)$ & 0.0441 & 0.0459 & 0.0400 & 0.0041 & 0.0059 & $10.3 \%$ & $14.8 \%$ \\
\hline 1 & EM-06 & $\mathrm{Fe} 2 \mathrm{O} 3(\mathrm{wt} \%)$ & 18.1572 & 18.5290 & 19.9500 & -1.7928 & -1.4210 & $-9.0 \%$ & $-7.1 \%$ \\
\hline 1 & EM-06 & La2O3 (wt\%) & 0.0686 & 0.0686 & 0.0800 & -0.0114 & -0.0114 & $-14.2 \%$ & $-14.2 \%$ \\
\hline 1 & EM-06 & Li2O (wt\%) & 3.9398 & 3.9250 & 4.0000 & -0.0602 & -0.0750 & $-1.5 \%$ & $-1.9 \%$ \\
\hline 1 & EM-06 & $\mathrm{MgO}(\mathrm{wt} \%)$ & 0.7189 & 0.7746 & 0.7100 & 0.0089 & 0.0646 & $1.2 \%$ & $9.1 \%$ \\
\hline 1 & EM-06 & $\mathrm{MnO}$ (wt\%) & 2.9601 & 2.9602 & 2.9200 & 0.0401 & 0.0402 & $1.4 \%$ & $1.4 \%$ \\
\hline 1 & EM-06 & $\mathrm{Na} 2 \mathrm{O}(\mathrm{wt} \%)$ & 13.9855 & 13.7763 & 13.7700 & 0.2155 & 0.0063 & $1.6 \%$ & $0.0 \%$ \\
\hline 1 & EM-06 & $\mathrm{NiO}$ (wt\%) & 0.9582 & 1.0865 & 1.1400 & -0.1818 & -0.0535 & $-15.9 \%$ & $-4.7 \%$ \\
\hline 1 & EM-06 & $\mathrm{PbO}$ (wt\%) & 0.0913 & 0.0913 & 0.1000 & -0.0087 & -0.0087 & $-8.7 \%$ & $-8.7 \%$ \\
\hline 1 & EM-06 & SiO2 (wt\%) & 41.7698 & 44.5034 & 42.1400 & -0.3702 & 2.3634 & $-0.9 \%$ & $5.6 \%$ \\
\hline 1 & EM-06 & SO4 (wt\%) & 0.4269 & 0.4269 & 0.4500 & -0.0231 & -0.0231 & $-5.1 \%$ & $-5.1 \%$ \\
\hline 1 & EM-06 & $\mathrm{TiO} 2$ (wt\%) & 2.0266 & 2.1101 & 2.0000 & 0.0266 & 0.1101 & $1.3 \%$ & $5.5 \%$ \\
\hline 1 & EM-06 & $\mathrm{ZnO}$ (wt\%) & 0.0626 & 0.0626 & 0.0700 & -0.0074 & -0.0074 & $-10.6 \%$ & $-10.6 \%$ \\
\hline 1 & EM-06 & ZrO2 (wt\%) & 0.1759 & 0.1759 & 0.1700 & 0.0059 & 0.0059 & $3.5 \%$ & $3.5 \%$ \\
\hline 1 & EM-06 & $\mathrm{ZZ}$ sum & 97.8576 & 101.1911 & 99.9100 & -2.0524 & 1.2811 & $-2.1 \%$ & $1.3 \%$ \\
\hline 1 & EM-07 & Al2O3 (wt\%) & 6.6652 & 6.8678 & 6.5900 & 0.0752 & 0.2778 & $1.1 \%$ & $4.2 \%$ \\
\hline 1 & EM-07 & B2O3 (wt\%) & 4.1376 & 4.0979 & 4.0000 & 0.1376 & 0.0979 & $3.4 \%$ & $2.4 \%$ \\
\hline 1 & EM-07 & & 0.0684 & 0.0775 & 0.0800 & -0.0116 & -0.0025 & $-14.5 \%$ & $-3.1 \%$ \\
\hline 1 & EM-07 & $\mathrm{CaO}(\mathrm{wt} \%)$ & 3.4175 & 3.4442 & 3.3200 & 0.0975 & 0.1242 & $2.9 \%$ & $3.7 \%$ \\
\hline 1 & EM-07 & Ce2O3 (wt\%) & 0.2343 & 0.2343 & 0.2400 & -0.0057 & -0.0057 & $-2.4 \%$ & $-2.4 \%$ \\
\hline 1 & EM-07 & Cr2O3 (wt\%) & 0.0943 & 0.0972 & 0.1400 & -0.0457 & -0.0428 & $-32.7 \%$ & $-30.6 \%$ \\
\hline 1 & EM-07 & $\mathrm{CuO}$ (wt\%) & 0.0642 & 0.0657 & 0.0400 & 0.0242 & 0.0257 & $60.4 \%$ & $64.2 \%$ \\
\hline 1 & EM-07 & $\mathrm{Fe} 2 \mathrm{O} 3(\mathrm{wt} \%)$ & 17.8355 & 18.2518 & 19.9500 & -2.1145 & -1.6982 & $-10.6 \%$ & $-8.5 \%$ \\
\hline 1 & EM-07 & La2O3 (wt $\%)$ & 0.0686 & 0.0686 & 0.0800 & -0.0114 & -0.0114 & $-14.2 \%$ & $-14.2 \%$ \\
\hline 1 & EM-07 & Li2O (wt\%) & 4.0098 & 3.9948 & 4.0000 & 0.0098 & -0.0052 & $0.2 \%$ & $-0.1 \%$ \\
\hline 1 & EM-07 & $\mathrm{MgO}(\mathrm{wt} \%)$ & 0.6795 & 0.7560 & 0.7100 & -0.0305 & 0.0460 & $-4.3 \%$ & $6.5 \%$ \\
\hline 1 & EM-07 & $\mathrm{MnO}$ (wt\%) & 2.9439 & 2.9440 & 2.9200 & 0.0239 & 0.0240 & $0.8 \%$ & $0.8 \%$ \\
\hline 1 & EM-07 & $\mathrm{Na2O}$ (wt\%) & 13.5541 & 13.4148 & 13.6000 & -0.0459 & -0.1852 & $-0.3 \%$ & $-1.4 \%$ \\
\hline 1 & EM-07 & $\mathrm{NiO}$ (wt\%) & 0.8844 & 1.0238 & 1.1400 & -0.2556 & -0.1162 & $-22.4 \%$ & $-10.2 \%$ \\
\hline 1 & EM-07 & & 0.0883 & & 0.1000 & -0.0117 & -0.0117 & $-11.7 \%$ & $-11.7 \%$ \\
\hline 1 & EM-07 & & 40.3793 & & 40.3100 & 0.0693 & 2.7120 & $0.2 \%$ & $6.7 \%$ \\
\hline 1 & EM-07 & SO4 (wt\%) & 0.4434 & 0.4434 & 0.4500 & -0.0066 & -0.0066 & $-1.5 \%$ & $-1.5 \%$ \\
\hline 1 & EM-07 & TiO2 (wt\%) & 1.9099 & 1.9681 & 2.0000 & -0.0901 & -0.0319 & $-4.5 \%$ & $-1.6 \%$ \\
\hline 1 & EM-07 & $\mathrm{ZnO}$ (wt\%) & 0.0638 & 0.0638 & 0.0700 & -0.0062 & -0.0062 & $-8.9 \%$ & $-8.9 \%$ \\
\hline 1 & EM-07 & $\mathrm{ZrO2}(\mathrm{wt} \%)$ & 0.1716 & 0.1716 & 0.1700 & 0.0016 & 0.0016 & $0.9 \%$ & $0.9 \%$ \\
\hline 1 & EM-07 & $\mathrm{ZZ}$ sum & 97.7135 & 101.0956 & 99.9100 & $\begin{array}{l}-2.1965 \\
\end{array}$ & 1.1856 & $-2.2 \%$ & $1.2 \%$ \\
\hline 1 & EM-08 & Al2O3 (wt\%) & 6.6416 & 6.8297 & 6.5900 & 0.0516 & 0.2397 & $0.8 \%$ & $3.6 \%$ \\
\hline 1 & EM-08 & B2O3 (wt\%) & 4.0571 & 4.0185 & 4.0000 & 0.0571 & 0.0185 & $1.4 \%$ & $0.5 \%$ \\
\hline 1 & EM-08 & $\mathrm{BaO}$ (wt\%) & 0.0611 & 0.0683 & 0.0800 & -0.0189 & -0.0117 & $-23.6 \%$ & $-14.6 \%$ \\
\hline 1 & EM-08 & $\mathrm{CaO}$ (wt\%) & 4.4914 & 4.5608 & 4.3200 & 0.1714 & 0.2408 & $4.0 \%$ & $5.6 \%$ \\
\hline 1 & EM-08 & Ce2O3 (wt\%) & 0.2293 & 0.2293 & 0.2400 & -0.0107 & -0.0107 & $-4.5 \%$ & $-4.5 \%$ \\
\hline 1 & EM-08 & Cr2O3 (wt\%) & 0.0870 & 0.0886 & 0.1400 & -0.0530 & -0.0514 & $-37.9 \%$ & $-36.7 \%$ \\
\hline
\end{tabular}


SRNL-STI-2009-00778, Revision 0

Table D3. Average Measured and Bias-Corrected Chemical Compositions Versus Targeted Compositions by Oxide by Glass ID for the Matrix 2A Study

\begin{tabular}{|c|c|c|c|c|c|c|c|c|c|}
\hline & & & & Measured & & & & & \\
\hline & & & Measured & Bias-Corrected & Targeted & Diff of & Diff of & \% Diff of & $\%$ Diff of \\
\hline Set & Glass \# & Oxide & (wt\%) & (wt\%) & (wt\%) & Measured & Meas BC & Measured & Meas BC \\
\hline 1 & EM-08 & 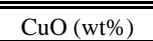 & 0.0407 & 0.0424 & 0.0400 & 0.0007 & 0.0024 & $1.7 \%$ & 5.9\% \\
\hline 1 & EM-08 & Fe2O3 (wt\%) & 18.2287 & 18.6020 & 19.9500 & -1.7213 & -1.3480 & $-8.6 \%$ & $-6.8 \%$ \\
\hline 1 & EM-08 & La2O3 (wt\%) & 0.0677 & 0.0677 & 0.0800 & -0.0123 & -0.0123 & $-15.3 \%$ & $-15.3 \%$ \\
\hline 1 & EM-08 & Li2O (wt\%) & 3.9613 & 3.9465 & 4.0000 & -0.0387 & -0.0535 & $-1.0 \%$ & $-1.3 \%$ \\
\hline 1 & EM-08 & $\mathrm{MgO}$ (wt\%) & 0.6957 & 0.7496 & 0.7100 & -0.0143 & 0.0396 & $-2.0 \%$ & $5.6 \%$ \\
\hline 1 & EM-08 & $\mathrm{MnO}$ (wt\%) & 2.9084 & 2.9086 & 2.9200 & -0.0116 & -0.0114 & $-0.4 \%$ & $-0.4 \%$ \\
\hline 1 & EM-08 & $\mathrm{Na} 2 \mathrm{O}$ (wt\%) & 13.8035 & 13.5971 & 13.6000 & 0.2035 & -0.0029 & $1.5 \%$ & $0.0 \%$ \\
\hline 1 & EM-08 & $\mathrm{NiO}$ (wt\%) & 0.8796 & 0.9974 & 1.1400 & -0.2604 & -0.1426 & $-22.8 \%$ & $-12.5 \%$ \\
\hline 1 & EM-08 & $\mathrm{PbO}$ (wt\%) & 0.0870 & 0.0870 & 0.1000 & -0.0130 & -0.0130 & $-13.0 \%$ & $-13.0 \%$ \\
\hline 1 & EM-08 & $\mathrm{SiO} 2$ (wt\%) & 38.9887 & 41.5406 & 39.3100 & -0.3213 & 2.2306 & $-0.8 \%$ & $5.7 \%$ \\
\hline 1 & EM-08 & SO4 (wt\%) & 0.4337 & 0.4337 & 0.4500 & -0.0163 & -0.0163 & $-3.6 \%$ & $-3.6 \%$ \\
\hline 1 & EM-08 & TiO2 (wt\%) & 2.2518 & 2.3445 & 2.0000 & 0.2518 & 0.3445 & $12.6 \%$ & $17.2 \%$ \\
\hline 1 & EM-08 & $\mathrm{ZnO}(\mathrm{wt} \%)$ & 0.0613 & 0.0613 & 0.0700 & -0.0087 & -0.0087 & $-12.4 \%$ & $-12.4 \%$ \\
\hline 1 & EM-08 & ZrO2 (wt\%) & 0.1746 & 0.1746 & 0.1700 & 0.0046 & 0.0046 & $2.7 \%$ & $2.7 \%$ \\
\hline 1 & EM-08 & ZZ sum & 98.1502 & 101.3480 & 99.9100 & -1.7598 & 1.4380 & $-1.8 \%$ & $1.4 \%$ \\
\hline 1 & EM-09 & Al2O3 (wt\%) & 1011.6724 & ב12.0027 & 11.5900 & $\begin{array}{l}0.0824 \\
\end{array}$ & 0.4127 & $0.7 \%$ & $3.6 \%$ \\
\hline 1 & EM-09 & B2O3 (wt\%) & 4.0168 & 3.9789 & 4.0000 & 0.0168 & -0.0211 & $0.4 \%$ & $-0.5 \%$ \\
\hline 1 & EM-09 & $\mathrm{BaO}$ (wt\%) & 0.0673 & 0.0752 & 0.0800 & -0.0127 & -0.0048 & $-15.9 \%$ & $-6.0 \%$ \\
\hline 1 & EM-09 & & 1.3590 & 1.3798 & 1.3200 & 0.0390 & 0.0598 & $3.0 \%$ & $4.5 \%$ \\
\hline 1 & EM-09 & & 0.2266 & 0.2 & 0.2400 & -0.0134 & -0.0134 & $-5.6 \%$ & $-5.6 \%$ \\
\hline 1 & EM-09 & (wt\%) & 0.0943 & & 0.1400 & -0.0457 & -0.0439 & $-32.7 \%$ & $-31.4 \%$ \\
\hline 1 & EM-09 & (wt\%) & 0.0545 & 0.0567 & 0.0400 & 0.0145 & 0.0167 & $36.1 \%$ & $41.7 \%$ \\
\hline 1 & EM-09 & Fe2O3 (wt\%) & 15.9769 & 16.3037 & 17.4500 & -1.4731 & -1.1463 & $-8.4 \%$ & $-6.6 \%$ \\
\hline 1 & EM-09 & $\mathrm{La} 2 \mathrm{O} 3$ (wt\%) & 0.0671 & 0.0671 & 0.0800 & -0.0129 & -0.0129 & $-16.1 \%$ & $-16.1 \%$ \\
\hline 1 & EM-09 & Li2O (wt\%) & 3.9506 & 3.9357 & 4.0000 & -0.0494 & -0.0643 & $-1.2 \%$ & $-1.6 \%$ \\
\hline 1 & EM-09 & $\mathrm{MgO}(\mathrm{wt} \%)$ & 0.6940 & 0.7478 & 0.7100 & -0.0160 & 0.0378 & $-2.3 \%$ & $5.3 \%$ \\
\hline 1 & EM-09 & $\mathrm{MnO}$ (wt\%) & 2.9246 & 2.9247 & 2.9200 & 0.0046 & 0.0047 & $0.2 \%$ & $0.2 \%$ \\
\hline 1 & EM-09 & $\mathrm{Na} 2 \mathrm{O}$ (wt\%) & 13.7934 & 13.5868 & 13.6000 & 0.1934 & -0.0132 & $1.4 \%$ & $-0.1 \%$ \\
\hline 1 & EM-09 & $\mathrm{NiO}$ (wt\%) & 0.9232 & 1.0468 & 1.1400 & -0.2168 & -0.0932 & $-19.0 \%$ & $-8.2 \%$ \\
\hline 1 & EM-09 & $\mathrm{PbO}$ (wt\%) & 0.0891 & 0.0891 & 0.1000 & -0.0109 & -0.0109 & $-10.9 \%$ & $-10.9 \%$ \\
\hline 1 & EM-09 & $\mathrm{SiO} 2$ (wt\%) & 39.3096 & 41.8822 & 39.8100 & -0.5004 & 2.0722 & $-1.3 \%$ & $5.2 \%$ \\
\hline 1 & EM-09 & & 0.4015 & & 0.4500 & -0.0485 & -0.0485 & $-10.8 \%$ & $-10.8 \%$ \\
\hline 1 & EM-09 & & 2.0808 & & 2.0000 & 0.0808 & 0.1666 & $4.0 \%$ & $8.3 \%$ \\
\hline 1 & EM-09 & (wt\%) & 0.0626 & & 0.0700 & -0.0074 & -0.0074 & $-10.6 \%$ & $-10.6 \%$ \\
\hline 1 & EM-09 & ZrO2 (wt\%) & 0.1743 & 0.1743 & 0.1700 & 0.0043 & 0.0043 & $2.5 \%$ & $2.5 \%$ \\
\hline 1 & EM-09 & ZZ sum & 97.9385 & 101.2048 & 99.9100 & -1.9715 & 1.2948 & $-2.0 \%$ & $1.3 \%$ \\
\hline 1 & EM-10 & OO3 (wt\%) & 11.5071 & 111.8334 & 11.5900 & -0.0829 & 0.2434 & $-0.7 \%$ & $2.1 \%$ \\
\hline 1 & EM-10 & $\mathrm{B} 2 \mathrm{O} 3$ (wt\%) & 4.0329 & 3.9943 & 4.0000 & 0.0329 & -0.0057 & $0.8 \%$ & $-0.1 \%$ \\
\hline 1 & EM-10 & $\mathrm{BaO}(\mathrm{wt} \%)$ & 0.0667 & 0.0746 & 0.0800 & -0.0133 & -0.0054 & $-16.6 \%$ & $-6.8 \%$ \\
\hline 1 & EM-10 & $\mathrm{CaO}$ (wt\%) & 1.3341 & 1.3548 & 1.3200 & 0.0141 & 0.0348 & $1.1 \%$ & $2.6 \%$ \\
\hline 1 & EM-10 & Ce2O3 (wt\%) & 0.2208 & 0.2208 & 0.2400 & -0.0192 & -0.0192 & $-8.0 \%$ & $-8.0 \%$ \\
\hline 1 & EM-10 & Cr2O3 (wt\%) & 0.1019 & 0.1039 & 0.1400 & -0.0381 & -0.0361 & $-27.2 \%$ & $-25.8 \%$ \\
\hline 1 & EM-10 & $\mathrm{CuO}(\mathrm{wt} \%)$ & 0.0570 & 0.0593 & 0.0400 & 0.0170 & 0.0193 & $42.4 \%$ & $48.2 \%$ \\
\hline 1 & EM-10 & Fe2O3 (wt\%) & 11.9130 & 12.1570 & 12.4500 & -0.5370 & -0.2930 & $-4.3 \%$ & $-2.4 \%$ \\
\hline 1 & EM-10 & La2O3 (wt\%) & 0.0683 & 0.0683 & 0.0800 & -0.0117 & -0.0117 & $-14.6 \%$ & $-14.6 \%$ \\
\hline 1 & EM-10 & & 3.8752 & 3.8606 & 4.0000 & -0.1248 & -0.1394 & $-3.1 \%$ & $-3.5 \%$ \\
\hline 1 & EM-10 & & & & 0.7100 & -0.0222 & 0.0311 & $-3.1 \%$ & $4.4 \%$ \\
\hline 1 & EM-10 & $\mathrm{MnO}(\mathrm{wt} \%)$ & 2.9052 & 2.9054 & 2.9200 & -0.0148 & -0.0146 & $-0.5 \%$ & $-0.5 \%$ \\
\hline 1 & EM-10 & $\mathrm{Na} 2 \mathrm{O}$ (wt\%) & 17.2881 & 17.0301 & 17.5100 & -0.2219 & -0.4799 & $-1.3 \%$ & $-2.7 \%$ \\
\hline 1 & EM-10 & $\mathrm{NiO}$ (wt\%) & 0.9257 & 1.0497 & 1.1400 & -0.2143 & -0.0903 & $-18.8 \%$ & $-7.9 \%$ \\
\hline 1 & EM-10 & $\mathrm{PbO}$ (wt\%) & 0.0862 & 0.0862 & 0.1000 & -0.0138 & -0.0138 & $-13.8 \%$ & $-13.8 \%$ \\
\hline 1 & EM-10 & $\mathrm{SiO} 2$ (wt\%) & 39.6840 & 42.2806 & 40.9000 & -1.2160 & 1.3806 & $-3.0 \%$ & $3.4 \%$ \\
\hline 1 & EM-10 & SO4 (wt\%) & 0.4194 & 0.4194 & 0.4500 & -0.0306 & -0.0306 & $-6.8 \%$ & $-6.8 \%$ \\
\hline 1 & EM-10 & TiO2 (wt\%) & 2.0725 & 2.1579 & 2.0000 & 0.0725 & 0.1579 & $3.6 \%$ & $7.9 \%$ \\
\hline 1 & EM-10 & $\mathrm{ZnO}$ (wt\%) & 0.0728 & 0.0728 & 0.0700 & 0.0028 & 0.0028 & $4.0 \%$ & $4.0 \%$ \\
\hline 1 & EM-10 & $\mathrm{ZrO} 2(\mathrm{wt} \%)$ & 0.1695 & 0.1695 & 0.1700 & -0.0005 & -0.0005 & $-0.3 \%$ & $-0.3 \%$ \\
\hline 1 & EM-10 & ZZ sum & 97.4883 & 100.6397 & 99.9100 & -2.4217 & 0.7297 & $-2.4 \%$ & $0.7 \%$ \\
\hline 1 & EM-11 & Al2O3 (wt\%) & 11.4929 & 11.8419 & 11.5900 & -0.0971 & 0.2519 & $-0.8 \%$ & $2.2 \%$ \\
\hline
\end{tabular}


SRNL-STI-2009-00778, Revision 0

Table D3. Average Measured and Bias-Corrected Chemical Compositions Versus Targeted Compositions by Oxide by Glass ID for the Matrix 2A Study

\begin{tabular}{|c|c|c|c|c|c|c|c|c|c|}
\hline & & & & "Measured & & & & & \\
\hline & & & Measured & Bias-Corrected & Targeted & Diff of & Diff of & \% Diff of & \% Diff of \\
\hline Set & Glass \# & Oxide & (wt\%) & (wt\%) & (wt\%) & Measured & Meas BC & Measured & Meas BC \\
\hline 1 & EM-11 & B2O3 (wt $\%)$ & 4.1456 & 3.9720 & 4.0000 & 0.1456 & -0.0280 & $3.6 \%$ & $-0.7 \%$ \\
\hline 1 & EM-11 & $\mathrm{BaO}(\mathrm{wt} \%)$ & 0.0701 & 0.0794 & 0.0800 & -0.0099 & -0.0006 & $-12.4 \%$ & $-0.8 \%$ \\
\hline 1 & EM-11 & $\mathrm{CaO}(\mathrm{wt} \%)$ & 1.3317 & 1.3420 & 1.3200 & 0.0117 & 0.0220 & $0.9 \%$ & $1.7 \%$ \\
\hline 1 & EM-11 & Ce2O3 (wt\%) & 0.2398 & 0.2398 & 0.2400 & -0.0002 & -0.0002 & $-0.1 \%$ & $-0.1 \%$ \\
\hline 1 & EM-11 & Cr2O3 (wt\%) & 0.0935 & 0.0965 & 0.1400 & -0.0465 & -0.0435 & $-33.2 \%$ & $-31.1 \%$ \\
\hline 1 & EM-11 & $\mathrm{CuO}$ (wt\%) & 0.0466 & 0.0477 & 0.0400 & 0.0066 & 0.0077 & $16.6 \%$ & $19.3 \%$ \\
\hline 1 & EM-11 & $\mathrm{Fe} 2 \mathrm{O} 3(\mathrm{wt} \%)$ & 13.4892 & 13.8054 & 14.9500 & -1.4608 & -1.1446 & $-9.8 \%$ & $-7.7 \%$ \\
\hline 1 & EM-11 & La2O3 (wt\%) & 0.0710 & 0.0710 & 0.0800 & $\begin{array}{l}-0.0090 \\
\end{array}$ & -0.0090 & $-11.3 \%$ & $-11.3 \%$ \\
\hline 1 & EM-11 & Li2O (wt\%) & 4.0205 & 3.9264 & 4.0000 & 0.0205 & -0.0736 & $0.5 \%$ & $-1.8 \%$ \\
\hline 1 & EM-11 & $\mathrm{MgO}(\mathrm{wt} \%)$ & 0.6840 & 0.7610 & 0.7100 & -0.0260 & 0.0510 & $-3.7 \%$ & $7.2 \%$ \\
\hline 1 & EM-11 & $\mathrm{MnO}(\mathrm{wt} \%)$ & 3.9123 & 3.9127 & 3.9200 & -0.0077 & -0.0073 & $-0.2 \%$ & $-0.2 \%$ \\
\hline 1 & EM-11 & $\mathrm{Na2O}$ (wt\%) & 13.3688 & 13.2294 & 13.6000 & -0.2312 & -0.3706 & $-1.7 \%$ & $-2.7 \%$ \\
\hline 1 & EM-11 & $\mathrm{NiO}(\mathrm{wt} \%)$ & 0.9286 & 1.0748 & 1.1400 & -0.2114 & -0.0652 & $-18.5 \%$ & $-5.7 \%$ \\
\hline 1 & EM-11 & $\mathrm{PbO}$ (wt\%) & 0.0899 & 0.0899 & 0.1000 & -0.0101 & -0.0101 & $-10.1 \%$ & $-10.1 \%$ \\
\hline 1 & EM-11 & $\mathrm{SiO} 2$ (wt\%) & 42.0907 & 44.3458 & 41.3100 & 0.7807 & 3.0358 & $1.9 \%$ & $7.3 \%$ \\
\hline 1 & EM-11 & SO4 (wt\%) & 0.4262 & 0.4262 & 0.4500 & -0.0238 & -0.0238 & $-5.3 \%$ & $-5.3 \%$ \\
\hline 1 & EM-11 & TiO2 (wt\%) & 1.9682 & 2.0283 & 2.0000 & -0.0318 & 0.0283 & $-1.6 \%$ & $1.4 \%$ \\
\hline 1 & EM-11 & $\mathrm{ZnO}$ (wt\%) & 0.0604 & 0.0604 & 0.0700 & -0.0096 & -0.0096 & $-13.8 \%$ & $-13.8 \%$ \\
\hline 1 & EM-11 & ZrO2 (wt\%) & 0.1732 & 0.1732 & 0.1700 & 0.0032 & 0.0032 & $1.9 \%$ & $1.9 \%$ \\
\hline 1 & EM-11 & ZZ sum & 98.7034 & 101.5240 & 99.9100 & -1.2066 & 1.6140 & $-1.2 \%$ & $1.6 \%$ \\
\hline 1 & EM-12 & Al2O3 (wt\%) & 11.4882 & 11.8137 & 11.5900 & -0.1018 & 0.2237 & $-0.9 \%$ & $1.9 \%$ \\
\hline 1 & EM-12 & B2O3 (wt\%) & 4.1295 & 3.9566 & 4.0000 & 0.1295 & -0.0434 & $3.2 \%$ & $-1.1 \%$ \\
\hline 1 & EM-12 & $\mathrm{BaO}$ (wt\%) & 0.0715 & 0.0799 & 0.0800 & -0.0085 & -0.0001 & $-10.7 \%$ & $-0.2 \%$ \\
\hline 1 & EM-12 & $\mathrm{CaO}$ (wt\%) & 1.3425 & 1.3633 & 1.3200 & 0.0225 & 0.0433 & $1.7 \%$ & $3.3 \%$ \\
\hline 1 & EM-12 & Ce2O3 (wt\%) & 0.2293 & 0.2293 & 0.2400 & -0.0107 & -0.0107 & $-4.5 \%$ & $-4.5 \%$ \\
\hline 1 & EM-12 & Cr2O3 (wt\%) & 0.1191 & 0.1214 & 0.1400 & -0.0209 & -0.0186 & $-14.9 \%$ & $-13.3 \%$ \\
\hline 1 & EM-12 & $\mathrm{CuO}$ (wt\%) & 0.0466 & 0.0485 & 0.0400 & 0.0066 & 0.0085 & $16.6 \%$ & $21.4 \%$ \\
\hline 1 & EM-12 & $\mathrm{Fe} 2 \mathrm{O} 3(\mathrm{wt} \%)$ & 13.9110 & 14.1965 & 14.9500 & -1.0390 & -0.7535 & $-6.9 \%$ & $-5.0 \%$ \\
\hline 1 & EM-12 & La2O3 (wt\%) & 0.0712 & 0.0712 & 0.0800 & -0.0088 & -0.0088 & $-10.9 \%$ & $-10.9 \%$ \\
\hline 1 & EM-12 & Li2O (wt\%) & 3.9990 & 3.9050 & 4.0000 & -0.0010 & -0.0950 & $0.0 \%$ & $-2.4 \%$ \\
\hline 1 & EM-12 & $\mathrm{MgO}(\mathrm{wt} \%)$ & 0.7089 & 0.7638 & 0.7100 & -0.0011 & 0.0538 & $-0.2 \%$ & $7.6 \%$ \\
\hline 1 & EM-12 & $\mathrm{MnO}(\mathrm{wt} \%)$ & 1.8884 & 1.8885 & 1.9200 & -0.0316 & -0.0315 & $-1.6 \%$ & $-1.6 \%$ \\
\hline 1 & EM-12 & $\mathrm{Na} 2 \mathrm{O}$ (wt\%) & 17.4903 & 17.2291 & 17.5100 & -0.0197 & -0.2809 & $-0.1 \%$ & $-1.6 \%$ \\
\hline 1 & EM-12 & $\mathrm{NiO}$ (wt\%) & 0.8561 & 0.9707 & 1.1400 & -0.2839 & -0.1693 & $-24.9 \%$ & $-14.9 \%$ \\
\hline 1 & EM-12 & $\mathrm{PbO}$ (wt\%) & 0.0881 & 0.0881 & 0.1000 & -0.0119 & -0.0119 & $-11.9 \%$ & $-11.9 \%$ \\
\hline 1 & EM-12 & $\mathrm{SiO} 2$ (wt\%) & 39.4166 & 41.5272 & 39.4000 & 0.0166 & 2.1272 & $0.0 \%$ & $5.4 \%$ \\
\hline 1 & EM-12 & SO4 (wt\%) & 0.4209 & 0.4209 & 0.4500 & -0.0291 & -0.0291 & $-6.5 \%$ & $-6.5 \%$ \\
\hline 1 & EM-12 & $\mathrm{TiO} 2$ (wt\%) & 2.0183 & 2.1013 & 2.0000 & 0.0183 & 0.1013 & $0.9 \%$ & $5.1 \%$ \\
\hline 1 & EM-12 & $\mathrm{ZnO}$ (wt\%) & 0.0629 & 0.0629 & 0.0700 & -0.0071 & -0.0071 & $-10.2 \%$ & $-10.2 \%$ \\
\hline 1 & EM-12 & $\mathrm{ZrO} 2(\mathrm{wt} \%)$ & 0.1770 & 0.1770 & 0.1700 & 0.0070 & 0.0070 & $4.1 \%$ & $4.1 \%$ \\
\hline 1 & EM-12 & $\mathrm{ZZ} \mathrm{sum}$ & 98.5353 & 101.0148 & 99.9100 & -1.3747 & 1.1048 & $-1.4 \%$ & $1.1 \%$ \\
\hline 1 & EM-13 & Al2O3 (wt\%) & 14.2988 & 14.7336 & 14.0900 & 0.2088 & 0.6436 & $1.5 \%$ & $4.6 \%$ \\
\hline 1 & EM-13 & B2O3 (wt\%) & 4.1456 & 3.9710 & 4.0000 & 0.1456 & -0.0290 & $3.6 \%$ & $-0.7 \%$ \\
\hline 1 & EM-13 & $\mathrm{BaO}$ (wt\%) & 0.0625 & 0.0709 & 0.0800 & -0.0175 & -0.0091 & $-21.8 \%$ & $-11.4 \%$ \\
\hline 1 & EM-13 & $\mathrm{CaO}$ (wt\%) & 2.4556 & 2.4749 & 2.3200 & 0.1356 & 0.1549 & $5.8 \%$ & $6.7 \%$ \\
\hline 1 & EM-13 & Ce2O3 (wt\%) & 0.2281 & 0.2281 & 0.2400 & -0.0119 & -0.0119 & $-5.0 \%$ & $-5.0 \%$ \\
\hline 1 & EM-13 & Cr2O3 (wt\%) & 0.0928 & 0.0957 & 0.1400 & -0.0472 & -0.0443 & $-33.7 \%$ & $-31.6 \%$ \\
\hline 1 & EM-13 & $\mathrm{CuO}$ (wt\%) & 0.0501 & 0.0513 & 0.0400 & 0.0101 & 0.0113 & $25.2 \%$ & $28.2 \%$ \\
\hline 1 & EM-13 & Fe2O3 (wt\%) & 11.5627 & 11.8336 & 12.9500 & -1.3873 & -1.1164 & $-10.7 \%$ & $-8.6 \%$ \\
\hline \begin{tabular}{|l|}
1 \\
\end{tabular} & EM-13 & La2O3 (wt\%) & 0.0657 & 0.0657 & 0.0800 & -0.0143 & -0.0143 & $-17.9 \%$ & $-17.9 \%$ \\
\hline 1 & EM-13 & Li2O (wt\%) & 3.9990 & 3.9050 & 4.0000 & -0.0010 & -0.0950 & $0.0 \%$ & $-2.4 \%$ \\
\hline 1 & EM-13 & MgO (wt\%) & 0.6708 & 0.7462 & 0.7100 & -0.0392 & 0.0362 & $-5.5 \%$ & $5.1 \%$ \\
\hline 1 & EM-13 & $\mathrm{MnO}(\mathrm{wt} \%)$ & 2.9213 & 2.9216 & 2.9200 & 0.0013 & 0.0016 & $0.0 \%$ & $0.1 \%$ \\
\hline 1 & EM-13 & $\mathrm{Na2O}$ (wt\%) & 14.7269 & 14.5773 & 14.5600 & 0.1669 & 0.0173 & $1.1 \%$ & $0.1 \%$ \\
\hline 1 & EM-13 & $\mathrm{NiO}(\mathrm{wt} \%)$ & 0.9032 & 1.0454 & 1.1400 & -0.2368 & -0.0946 & $-20.8 \%$ & $-8.3 \%$ \\
\hline 1 & EM-13 & $\mathrm{PbO}$ (wt\%) & 0.0851 & 0.0851 & 0.1000 & -0.0149 & -0.0149 & $-14.9 \%$ & $-14.9 \%$ \\
\hline 1 & EM-13 & $\mathrm{SiO} 2$ (wt\%) & 40.1119 & 42.2561 & 39.8500 & 0.2619 & 2.4061 & $0.7 \%$ & $6.0 \%$ \\
\hline 1 & EM-13 & SO4 (wt\%) & 0.4149 & 0.4149 & 0.4500 & $\begin{array}{l}-0.0351 \\
\end{array}$ & -0.0351 & $-7.8 \%$ & $-7.8 \%$ \\
\hline
\end{tabular}


SRNL-STI-2009-00778, Revision 0

Table D3. Average Measured and Bias-Corrected Chemical Compositions Versus Targeted Compositions by Oxide by Glass ID for the Matrix 2A Study

\begin{tabular}{|c|c|c|c|c|c|c|c|c|c|}
\hline & & & & Measured & & & & & \\
\hline & & & Measured & Bias-Corrected & Targeted & Diff of & Diff of & \% Diff of & $\%$ Diff of \\
\hline Set & Glass \# & Oxide & (wt\%) & (wt\%) & (wt\%) & Measured & Meas BC & Measured & Meas BC \\
\hline 1 & EM-13 & "TiO2 (wt\%) & 1.9265 & "1.9854 & 2.0000 & -0.0735 & -0.0146 & $-3.7 \%$ & $-0.7 \%$ \\
\hline 1 & EM-13 & $\mathrm{ZnO}$ (wt\%) & 0.0650 & 0.0650 & 0.0700 & -0.0050 & -0.0050 & $-7.1 \%$ & $-7.1 \%$ \\
\hline 1 & EM-13 & ZrO2 (wt\%) & 0.1716 & 0.1716 & 0.1700 & 0.0016 & 0.0016 & $0.9 \%$ & $0.9 \%$ \\
\hline 1 & EM-13 & $\mathrm{ZZ}$ sum & 98.9581 & 101.6983 & 99.9100 & -0.9519 & 1.7883 & $-1.0 \%$ & $1.8 \%$ \\
\hline 1 & EM-14 & Al2O3 (wt\%) & 14.2516 & 14.6853 & 14.0900 & 0.1616 & 0.5953 & $1.1 \%$ & $4.2 \%$ \\
\hline 1 & EM-14 & B2O3 (wt\%) & 4.0893 & 3.9171 & 4.0000 & 0.0893 & -0.0829 & $2.2 \%$ & $-2.1 \%$ \\
\hline 1 & EM-14 & $\mathrm{BaO}$ (wt\%) & 0.0692 & 0.0785 & 0.0800 & -0.0108 & -0.0015 & $-13.5 \%$ & $-1.9 \%$ \\
\hline 1 & EM-14 & $\mathrm{CaO}$ (wt\%) & 3.7743 & 3.8043 & 3.3200 & 0.4543 & 0.4843 & $13.7 \%$ & $14.6 \%$ \\
\hline 1 & EM-14 & Ce2O3 (wt\%) & 0.2278 & 0.2278 & 0.2400 & -0.0122 & -0.0122 & $-5.1 \%$ & $-5.1 \%$ \\
\hline 1 & EM-14 & Cr2O3 (wt\%) & 0.0914 & 0.0942 & 0.1400 & -0.0487 & -0.0458 & $-34.8 \%$ & $-32.7 \%$ \\
\hline 1 & EM-14 & $\mathrm{CuO}$ (wt\%) & 0.0529 & 0.0541 & 0.0400 & 0.0129 & 0.0141 & $32.2 \%$ & $35.4 \%$ \\
\hline 1 & EM-14 & $\mathrm{Fe} 2 \mathrm{O} 3(\mathrm{wt} \%)$ & 11.7950 & 12.0692 & 12.9500 & -1.1550 & -0.8808 & $-8.9 \%$ & $-6.8 \%$ \\
\hline 1 & EM-14 & La2O3 (wt\%) & 0.0686 & 0.0686 & 0.0800 & -0.0114 & -0.0114 & $-14.2 \%$ & $-14.2 \%$ \\
\hline 1 & EM-14 & Li2O (wt\%) & 3.9775 & 3.8837 & 4.0000 & -0.0225 & -0.1163 & $-0.6 \%$ & $-2.9 \%$ \\
\hline 1 & EM-14 & $\mathrm{MgO}$ (wt\%) & 0.6679 & 0.7431 & 0.7100 & -0.0421 & 0.0331 & $-5.9 \%$ & $4.7 \%$ \\
\hline 1 & EM-14 & $\mathrm{MnO}$ (wt\%) & 2.9052 & 2.9052 & 2.9200 & -0.0148 & -0.0148 & $-0.5 \%$ & $-0.5 \%$ \\
\hline 1 & EM-14 & $\mathrm{Na} 2 \mathrm{O}$ (wt\%) & 14.0866 & 13.9439 & 14.0600 & 0.0266 & -0.1161 & $0.2 \%$ & $-0.8 \%$ \\
\hline 1 & EM-14 & $\mathrm{NiO}$ (wt\%) & 0.8653 & 1.0017 & 1.1400 & -0.2747 & -0.1383 & $-24.1 \%$ & $-12.1 \%$ \\
\hline 1 & EM-14 & & 0.0846 & 0.0 & 0.1000 & -0.0154 & -0.0154 & $-15.4 \%$ & $-15.4 \%$ \\
\hline 1 & EM-14 & $\mathrm{wt} \%)$ & 39.2027 & 41.2965 & 39.3500 & -0.1473 & 1.9465 & $-0.4 \%$ & $4.9 \%$ \\
\hline 1 & EM-14 & SO4 (wt\%) & 0.4164 & 0.4164 & 0.4500 & -0.0336 & -0.0336 & $-7.5 \%$ & $-7.5 \%$ \\
\hline 1 & EM-14 & $\mathrm{TiO} 2$ (wt\%) & 2.0141 & 2.0755 & 2.0000 & 0.0141 & 0.0755 & $0.7 \%$ & $3.8 \%$ \\
\hline 1 & EM-14 & $\mathrm{ZnO}(\mathrm{wt} \%)$ & 0.0594 & 0.0594 & 0.0700 & -0.0106 & -0.0106 & $-15.1 \%$ & $-15.1 \%$ \\
\hline 1 & EM-14 & ZrO2 (wt\%) & 0.1699 & 0.1699 & 0.1700 & -0.0001 & -0.0001 & $-0.1 \%$ & $-0.1 \%$ \\
\hline 1 & EM-14 & $\mathrm{ZZ}$ sum & 98.8696 & 101.5789 & 99.9100 & -1.0404 & 1.6689 & $-1.0 \%$ & $1.7 \%$ \\
\hline 1 & EM-15 & Al2O3 (wt\%) & 14.3082 & 14.7434 & 14.0900 & 0.2182 & 0.6534 & $1.5 \%$ & "4.6\% \\
\hline 1 & EM-15 & B2O3 (wt\%) & 4.2825 & 4.1020 & 4.0000 & 0.2825 & 0.1020 & $7.1 \%$ & $2.5 \%$ \\
\hline 1 & EM-15 & $\mathrm{BaO}$ (wt\%) & 0.0695 & 0.0788 & 0.0800 & -0.0105 & -0.0012 & $-13.1 \%$ & $-1.5 \%$ \\
\hline 1 & EM-15 & $\mathrm{CaO}$ (wt\%) & 4.4704 & 4.5057 & 4.3200 & 0.1504 & 0.1857 & $3.5 \%$ & $4.3 \%$ \\
\hline 1 & EM-15 & Ce2O3 (wt\%) & 0.2360 & 0.2360 & 0.2400 & -0.0040 & -0.0040 & $-1.7 \%$ & $-1.7 \%$ \\
\hline 1 & EM-15 & Cr2O3 (wt\%) & 0.0840 & 0.0867 & 0.1400 & -0.0560 & -0.0533 & $-40.0 \%$ & $-38.1 \%$ \\
\hline 1 & EM-15 & $\mathrm{CuO}$ (wt\%) & 0.0444 & 0.0455 & 0.0400 & 0.0044 & 0.0055 & $11.1 \%$ & $13.7 \%$ \\
\hline 1 & EM-15 & $\mathrm{Fe} 2 \mathrm{O} 3(\mathrm{wt} \%)$ & 11.3911 & & 12.9500 & -1.5589 & -1.2919 & $-12.0 \%$ & $-10.0 \%$ \\
\hline 1 & EM-15 & 3 (wt\%) & 0.0692 & & 0.0800 & -0.0108 & -0.0108 & $-13.5 \%$ & $-13.5 \%$ \\
\hline 1 & EM-15 & Li2O (wt\%) & 4.1013 & 4.0052 & 4.0000 & 0.1013 & 0.0052 & $2.5 \%$ & $0.1 \%$ \\
\hline 1 & EM-15 & $\mathrm{MgO}(\mathrm{wt} \%)$ & 0.6907 & 0.7683 & 0.7100 & -0.0193 & 0.0583 & $-2.7 \%$ & $8.2 \%$ \\
\hline 1 & EM-15 & $\mathrm{MnO}$ (wt\%) & 2.9568 & 2.9571 & 2.9200 & 0.0368 & 0.0371 & $1.3 \%$ & $1.3 \%$ \\
\hline 1 & EM-15 & $\mathrm{Na} 2 \mathrm{O}$ (wt\%) & 13.6451 & 13.5063 & 13.5600 & 0.0851 & -0.0537 & $0.6 \%$ & $-0.4 \%$ \\
\hline 1 & EM-15 & $\mathrm{NiO}$ (wt\%) & 0.8876 & 1.0273 & 1.1400 & -0.2524 & -0.1127 & $-22.1 \%$ & $-9.9 \%$ \\
\hline 1 & EM-15 & $\mathrm{PbO}$ (wt\%) & 0.0908 & 0.0908 & 0.1000 & -0.0092 & -0.0092 & $-9.2 \%$ & $-9.2 \%$ \\
\hline 1 & EM-15 & $\mathrm{SiO} 2$ (wt\%) & 40.1654 & 42.3190 & 38.8500 & 1.3154 & 3.4690 & $3.4 \%$ & $8.9 \%$ \\
\hline 1 & EM-15 & SO4 (wt $\%)$ & 0.4299 & 0.4299 & 0.4500 & -0.0201 & -0.0201 & $-4.5 \%$ & $-4.5 \%$ \\
\hline 1 & EM-15 & TiO2 (wt\%) & 1.8848 & 1.9425 & 2.0000 & -0.1152 & -0.0575 & $-5.8 \%$ & $-2.9 \%$ \\
\hline 1 & EM-15 & & 0.0607 & 0.0607 & 0.0700 & -0.0093 & -0.0093 & $-13.3 \%$ & $-13.3 \%$ \\
\hline 1 & EM-15 & ZrO2 (wt\%) & 0.1763 & 0.1763 & 0.1700 & 0.0063 & 0.0063 & $3.7 \%$ & $3.7 \%$ \\
\hline 1 & EM-15 & $\mathrm{ZZ}$ sum & 100.0448 & 102.8087 & 99.9100 & 0.1348 & 2.8987 & $0.1 \%$ & $2.9 \%$ \\
\hline 2 & Batch 1 & Al2O3 (wt\%) & 4.6198 & 4.8770 & 4.8770 & -0.2572 & 0.0000 & $-5.3 \%$ & $0.0 \%$ \\
\hline 2 & Batch 1 & B2O3 (wt\%) & 7.7358 & & 7.7770 & -0.0412 & 0.0000 & $-0.5 \%$ & $0.0 \%$ \\
\hline 2 & Batch 1 & $\mathrm{BaO}$ (wt\%) & 0.1340 & 0.1510 & 0.1510 & -0.0170 & 0.0000 & $-11.3 \%$ & $0.0 \%$ \\
\hline 2 & Batch 1 & $\mathrm{CaO}$ (wt\%) & 1.1795 & 1.2200 & 1.2200 & -0.0405 & 0.0000 & $-3.3 \%$ & $0.0 \%$ \\
\hline 2 & Batch 1 & Ce2O3 (wt\%) & 0.0059 & 0.0059 & 0.0000 & 0.0059 & 0.0059 & & \\
\hline 2 & Batch 1 & Cr2O3 (wt\%) & 0.1047 & 0.1070 & 0.1070 & -0.0023 & 0.0000 & $-2.1 \%$ & $0.0 \%$ \\
\hline 2 & Batch 1 & $\mathrm{CuO}$ (wt\%) & 0.3824 & 0.3990 & 0.3990 & -0.0166 & 0.0000 & $-4.2 \%$ & $0.0 \%$ \\
\hline 2 & Batch 1 & $\mathrm{Fe} 2 \mathrm{O} 3(\mathrm{wt} \%)$ & 12.2835 & 12.8390 & 12.8390 & -0.5555 & 0.0000 & $-4.3 \%$ & $0.0 \%$ \\
\hline 2 & Batch 1 & La2O3 (wt\%) & 0.0059 & 0.0059 & 0.0000 & 0.0059 & 0.0059 & & \\
\hline 2 & Batch 1 & $\mathrm{Li} 2 \mathrm{O}$ (wt\%) & 4.3955 & 4.4290 & 4.4290 & -0.0335 & 0.0000 & $-0.8 \%$ & $0.0 \%$ \\
\hline 2 & Batch 1 & $\mathrm{MgO}$ (wt\%) & 1.3152 & 1.4190 & 1.4190 & -0.1038 & 0.0000 & $-7.3 \%$ & $0.0 \%$ \\
\hline 2 & Batch 1 & $\mathrm{MnO}$ (wt\%) & 1.6700 & 1.7260 & 1.7260 & -0.0560 & 0.0000 & $-3.2 \%$ & $0.0 \%$ \\
\hline
\end{tabular}


SRNL-STI-2009-00778, Revision 0

Table D3. Average Measured and Bias-Corrected Chemical Compositions Versus Targeted Compositions by Oxide by Glass ID for the Matrix 2A Study

\begin{tabular}{|c|c|c|c|c|c|c|c|c|c|}
\hline & & & & Measured & & & & & \\
\hline & & & Measured & Bias-Corrected & Targeted & Diff of & Diff of & \% Diff of & $\%$ Diff of \\
\hline Set & Glass \# & Oxide & (wt\%) & $(\mathrm{wt} \%)$ & $(w t \%)$ & Measured & Meas BC & Measured & Meas BC \\
\hline 2 & Batch 1 & Na2O (wt\%) & 9.1136 & 9.0030 & 9.0030 & 0.1106 & 0.0000 & $1.2 \%$ & $0.0 \%$ \\
\hline 2 & Batch 1 & $\mathrm{NiO}(\mathrm{wt} \%)$ & 0.6650 & 0.7510 & 0.7510 & -0.0860 & 0.0000 & $-11.5 \%$ & $0.0 \%$ \\
\hline 2 & Batch 1 & $\mathrm{PbO}(\mathrm{wt} \%)$ & 0.0054 & 0.0054 & 0.0000 & 0.0054 & 0.0054 & & \\
\hline 2 & Batch 1 & $\mathrm{SiO} 2(\mathrm{wt} \%)$ & 46.5119 & 50.2200 & 50.2200 & -3.7081 & 0.0000 & $-7.4 \%$ & $0.0 \%$ \\
\hline 2 & Batch 1 & SO4 (wt\%) & 0.1498 & 0.1498 & 0.0000 & 0.1498 & 0.1498 & & \\
\hline 2 & Batch 1 & TiO2 (wt\%) & 0.6362 & 0.6770 & 0.6770 & -0.0408 & 0.0000 & $-6.0 \%$ & $0.0 \%$ \\
\hline 2 & Batch 1 & $\mathrm{ZnO}(\mathrm{wt} \%)$ & 0.0062 & 0.0062 & 0.0000 & 0.0062 & 0.0062 & & \\
\hline 2 & Batch 1 & $\mathrm{ZrO} 2$ (wt\%) & 0.0901 & 0.0901 & 0.0980 & -0.0079 & -0.0079 & $-8.1 \%$ & $-8.1 \%$ \\
\hline 2 & Batch 1 & ZZ sum & 91.0104 & 95.8582 & 95.6930 & -4.6826 & 0.1652 & $-4.9 \%$ & $0.2 \%$ \\
\hline 2 & EM-16 & $\mathrm{Al} 2 \mathrm{O} 3$ (wt\%) & 11.3890 & 12.1724 & 11.5900 & -0.2010 & 0.5824 & $-1.7 \%$ & $5.0 \%$ \\
\hline 2 & EM-16 & B2O3 (wt\%) & 6.9872 & 7.0226 & 7.0000 & -0.0128 & 0.0226 & $-0.2 \%$ & $0.3 \%$ \\
\hline 2 & EM-16 & $\mathrm{BaO}$ (wt\%) & 0.0715 & 0.0807 & 0.0800 & -0.0085 & 0.0007 & $-10.7 \%$ & $0.9 \%$ \\
\hline 2 & EM-16 & $\mathrm{CaO}$ (wt\%) & 1.2827 & 1.3522 & 1.3200 & -0.0373 & 0.0322 & $-2.8 \%$ & $2.4 \%$ \\
\hline 2 & EM-16 & Ce2O3 (wt\%) & 0.2346 & 0.2346 & 0.2400 & -0.0054 & -0.0054 & $-2.3 \%$ & $-2.3 \%$ \\
\hline 2 & EM-16 & Cr2O3 (wt\%) & 0.0903 & 0.0922 & 0.1400 & -0.0497 & -0.0478 & $-35.5 \%$ & $-34.1 \%$ \\
\hline 2 & EM-16 & $\mathrm{CuO}(\mathrm{wt} \%)$ & 0.0457 & 0.0475 & 0.0400 & 0.0057 & 0.0075 & $14.2 \%$ & $18.9 \%$ \\
\hline 2 & EM-16 & Fe2O3 (wt\%) & 13.7144 & 14.3480 & 14.9500 & -1.2356 & -0.6020 & $-8.3 \%$ & $-4.0 \%$ \\
\hline 2 & EM-16 & La2O3 (wt\%) & 0.0821 & 0.0821 & 0.0800 & 0.0021 & 0.0021 & $2.6 \%$ & $2.6 \%$ \\
\hline 2 & EM-16 & Li2O (wt\%) & 3.9721 & 3.9510 & 4.0000 & -0.0279 & -0.0490 & $-0.7 \%$ & $-1.2 \%$ \\
\hline 2 & EM-16 & $\mathrm{MgO}$ (wt\%) & 0.7135 & 0.7715 & 0.7100 & 0.0035 & 0.0615 & $0.5 \%$ & $8.7 \%$ \\
\hline 2 & EM-16 & $\mathrm{MnO}$ (wt\%) & 2.8536 & 2.9573 & 2.9200 & -0.0664 & 0.0373 & $-2.3 \%$ & $1.3 \%$ \\
\hline 2 & EM-16 & Na2O (wt\%) & 15.0302 & 15.0950 & 15.4800 & -0.4498 & -0.3850 & $-2.9 \%$ & $-2.5 \%$ \\
\hline 2 & EM-16 & $\mathrm{NiO}(\mathrm{wt} \%)$ & 0.8822 & 0.9978 & 1.1400 & -0.2578 & -0.1422 & $-22.6 \%$ & $-12.5 \%$ \\
\hline 2 & EM-16 & $\mathrm{PbO}$ (wt\%) & 0.0899 & 0.0899 & 0.1000 & -0.0101 & -0.0101 & $-10.1 \%$ & $-10.1 \%$ \\
\hline 2 & EM-16 & $\mathrm{SiO} 2$ (wt\%) & 37.1703 & 39.9662 & 37.4200 & -0.2497 & 2.5462 & $-0.7 \%$ & $6.8 \%$ \\
\hline 2 & EM-16 & SO4 (wt\%) & 0.4269 & 0.4269 & 0.4500 & -0.0231 & -0.0231 & $-5.1 \%$ & $-5.1 \%$ \\
\hline 2 & EM-16 & TiO2 (wt\%) & 2.0225 & 2.1784 & 2.0000 & 0.0225 & 0.1784 & $1.1 \%$ & $8.9 \%$ \\
\hline 2 & EM-16 & $\mathrm{ZnO}$ (wt\%) & 0.0610 & 0.0610 & 0.0700 & -0.0090 & -0.0090 & $-12.9 \%$ & $-12.9 \%$ \\
\hline 2 & EM-16 & ZrO2 (wt\%) & 0.1800 & 0.1800 & 0.1700 & 0.0100 & 0.0100 & $5.9 \%$ & $5.9 \%$ \\
\hline 2 & EM-16 & ZZ sum & 97.2994 & 102.1072 & 99.9000 & -2.6006 & 2.2072 & $-2.6 \%$ & $2.2 \%$ \\
\hline 2 & EM-17 & Al2O3 (wt\%) & 13.5761 & 14.5096 & 14.0900 & -0.5139 & 0.4196 & $-3.6 \%$ & $3.0 \%$ \\
\hline 2 & EM-17 & B2O3 (wt\%) & 6.8584 & 6.8927 & 7.0000 & -0.1416 & -0.1073 & $-2.0 \%$ & $-1.5 \%$ \\
\hline 2 & EM-17 & $\mathrm{BaO}(\mathrm{wt} \%)$ & 0.0692 & 0.0781 & 0.0800 & -0.0108 & -0.0019 & $-13.5 \%$ & $-2.3 \%$ \\
\hline 2 & EM-17 & $\mathrm{CaO}$ (wt\%) & 1.2470 & 1.3145 & 1.3200 & -0.0730 & -0.0055 & $-5.5 \%$ & $-0.4 \%$ \\
\hline 2 & EM-17 & Ce2O3 (wt\%) & 0.2243 & 0.2243 & 0.2400 & -0.0157 & -0.0157 & $-6.5 \%$ & $-6.5 \%$ \\
\hline 2 & EM-17 & Cr2O3 (wt\%) & 0.0983 & 0.1004 & 0.1400 & -0.0417 & -0.0396 & $-29.8 \%$ & $-28.3 \%$ \\
\hline 2 & EM-17 & $\mathrm{CuO}(\mathrm{wt} \%)$ & 0.0507 & 0.0528 & 0.0400 & 0.0107 & 0.0128 & $26.7 \%$ & $31.9 \%$ \\
\hline 2 & EM-17 & Fe2O3 (wt\%) & 13.4713 & 14.0903 & 14.9500 & -1.4787 & -0.8597 & $-9.9 \%$ & $-5.8 \%$ \\
\hline 2 & EM-17 & La2O3 (wt\%) & 0.0783 & 0.0783 & 0.0800 & -0.0017 & -0.0017 & $-2.1 \%$ & $-2.1 \%$ \\
\hline 2 & EM-17 & Li2O (wt\%) & 3.9237 & 3.9027 & 4.0000 & -0.0763 & -0.0973 & $-1.9 \%$ & $-2.4 \%$ \\
\hline 2 & EM-17 & MgO (wt\%) & 0.7052 & 0.7624 & 0.7100 & -0.0048 & 0.0524 & $-0.7 \%$ & $7.4 \%$ \\
\hline 2 & EM-17 & $\mathrm{MnO}$ (wt\%) & 2.8245 & 2.9268 & 2.9200 & -0.0955 & 0.0068 & $-3.3 \%$ & $0.2 \%$ \\
\hline 2 & EM-17 & $\mathrm{Na} 2 \mathrm{O}$ (wt\%) & 13.0790 & 13.1359 & 13.6000 & -0.5210 & -0.4641 & $-3.8 \%$ & $-3.4 \%$ \\
\hline 2 & EM-17 & $\mathrm{NiO}(\mathrm{wt} \%)$ & 0.9296 & 1.0513 & 1.1400 & -0.2104 & -0.0887 & $-18.5 \%$ & $-7.8 \%$ \\
\hline 2 & EM-17 & $\mathrm{PbO}$ (wt\%) & 0.0883 & 0.0883 & 0.1000 & -0.0117 & -0.0117 & $-11.7 \%$ & $-11.7 \%$ \\
\hline 2 & EM-17 & SiO2 (wt\%) & 36.2611 & 38.9881 & 36.8100 & -0.5489 & 2.1781 & $-1.5 \%$ & $5.9 \%$ \\
\hline 2 & EM-17 & SO4 (wt\%) & 0.4239 & 0.4239 & 0.4500 & -0.0261 & -0.0261 & $-5.8 \%$ & $-5.8 \%$ \\
\hline 2 & EM-17 & $\mathrm{TiO} 2$ (wt\%) & 1.8431 & 1.9851 & 2.0000 & -0.1569 & -0.0149 & $-7.8 \%$ & $-0.7 \%$ \\
\hline 2 & EM-17 & $\mathrm{ZnO}$ (wt\%) & 0.0610 & 0.0610 & 0.0700 & -0.0090 & -0.0090 & $-12.9 \%$ & $-12.9 \%$ \\
\hline 2 & EM-17 & $\mathrm{ZrO} 2$ (wt\%) & 0.1726 & 0.1726 & 0.1700 & 0.0026 & 0.0026 & $1.5 \%$ & $1.5 \%$ \\
\hline 2 & EM-17 & ZZ sum & 95.9856 & 100.8392 & 99.9100 & -3.9244 & 0.9292 & $-3.9 \%$ & $0.9 \%$ \\
\hline 2 & EM-18 & $\mathrm{Al} 2 \mathrm{O} 3$ (wt\%) & 8.9704 & 9.3550 & 9.0900 & -0.1196 & 0.2650 & $-1.3 \%$ & $2.9 \%$ \\
\hline 2 & EM-18 & B2O3 (wt\%) & 6.8423 & 6.8767 & 7.0000 & -0.1577 & -0.1233 & $-2.3 \%$ & $-1.8 \%$ \\
\hline 2 & EM-18 & $\mathrm{BaO}$ (wt\%) & 0.0692 & 0.0779 & 0.0800 & -0.0108 & -0.0021 & $-13.5 \%$ & $-2.6 \%$ \\
\hline 2 & EM-18 & $\mathrm{CaO}(\mathrm{wt} \%)$ & 1.3425 & 1.3630 & 1.3200 & 0.0225 & 0.0430 & $1.7 \%$ & $3.3 \%$ \\
\hline 2 & EM-18 & Ce2O3 (wt\%) & 0.2269 & 0.2269 & 0.2400 & -0.0131 & -0.0131 & $-5.4 \%$ & $-5.4 \%$ \\
\hline 2 & EM-18 & Cr2O3 (wt\%) & 0.1049 & 0.1071 & 0.1400 & -0.0351 & -0.0329 & $-25.1 \%$ & $-23.5 \%$ \\
\hline 2 & EM-18 & $\mathrm{CuO}(\mathrm{wt} \%)$ & 0.0444 & 0.0465 & 0.0400 & 0.0044 & 0.0065 & $11.1 \%$ & $16.2 \%$ \\
\hline
\end{tabular}


SRNL-STI-2009-00778, Revision 0

Table D3. Average Measured and Bias-Corrected Chemical Compositions Versus Targeted Compositions by Oxide by Glass ID for the Matrix 2A Study

\begin{tabular}{|c|c|c|c|c|c|c|c|c|c|}
\hline & & & & Measured & & & & & \\
\hline & & & Measured & Bias-Corrected & Targeted & Diff of & Diff of & \% Diff of & $\%$ Diff of \\
\hline Set & Glass \# & Oxide & (wt\%) & (wt\%) & (wt\%) & Measured & Meas BC & Measured & Meas BC \\
\hline 2 & EM-18 & "Fe2O3 (wt\%) & $\begin{array}{c}13.7787 \\
\end{array}$ & 14.3913 & 14.9500 & -1.1713 & -0.5587 & $-7.8 \%$ & -3.7\% \\
\hline 2 & EM-18 & La2O3 (wt\%) & 0.0739 & 0.0739 & 0.0800 & -0.0061 & -0.0061 & $-7.6 \%$ & $-7.6 \%$ \\
\hline 2 & EM-18 & Li2O (wt\%) & 3.9183 & 3.8974 & 4.0000 & -0.0817 & -0.1026 & $-2.0 \%$ & $-2.6 \%$ \\
\hline 2 & EM-18 & $\mathrm{MgO}(\mathrm{wt} \%)$ & 0.6952 & 0.7486 & 0.7100 & -0.0148 & 0.0386 & $-2.1 \%$ & $5.4 \%$ \\
\hline 2 & EM-18 & $\mathrm{MnO}$ (wt\%) & 2.8729 & 2.9619 & 2.9200 & -0.0471 & 0.0419 & $-1.6 \%$ & $1.4 \%$ \\
\hline 2 & EM-18 & $\mathrm{Na2O}$ (wt\%) & 17.1533 & 16.6726 & 17.5100 & -0.3567 & -0.8374 & $-2.0 \%$ & $-4.8 \%$ \\
\hline 2 & EM-18 & $\mathrm{NiO}$ (wt\%) & 0.9566 & 1.0788 & 1.1400 & -0.1834 & -0.0612 & $-16.1 \%$ & $-5.4 \%$ \\
\hline 2 & EM-18 & $\mathrm{PbO}$ (wt\%) & 0.0856 & 0.0856 & 0.1000 & -0.0144 & -0.0144 & $-14.4 \%$ & $-14.4 \%$ \\
\hline 2 & EM-18 & $\mathrm{SiO} 2$ (wt\%) & 37.1169 & 39.9082 & 37.9000 & -0.7831 & 2.0082 & $-2.1 \%$ & $5.3 \%$ \\
\hline 2 & EM-18 & SO4 (wt\%) & 0.4172 & 0.4172 & 0.4500 & -0.0328 & -0.0328 & $-7.3 \%$ & $-7.3 \%$ \\
\hline 2 & EM-18 & $\mathrm{TiO} 2$ (wt\%) & 1.9766 & 2.0784 & 2.0000 & -0.0234 & 0.0784 & $-1.2 \%$ & $3.9 \%$ \\
\hline 2 & EM-18 & $\mathrm{ZnO}$ (wt\%) & 0.0557 & 0.0557 & 0.0700 & -0.0143 & -0.0143 & $-20.4 \%$ & $-20.4 \%$ \\
\hline 2 & EM-18 & $\mathrm{ZrO2}$ (wt\%) & 0.1732 & 0.1732 & 0.1700 & 0.0032 & 0.0032 & $1.9 \%$ & $1.9 \%$ \\
\hline 2 & EM-18 & ZZ sum & 96.8749 & 100.5959 & 99.9100 & -3.0351 & 0.6859 & $-3.0 \%$ & $0.7 \%$ \\
\hline 2 & EM-19 & Al2O3 (wt\%) & "6.5282 & 6.9771 & 6.5900 & -0.0618 & 0.3871 & $-0.9 \%$ & $5.9 \%$ \\
\hline 2 & EM-19 & B2O3 (wt\%) & 7.3816 & 7.4185 & 7.0000 & 0.3816 & 0.4185 & $5.5 \%$ & $6.0 \%$ \\
\hline 2 & EM-19 & $\mathrm{BaO}$ (wt\%) & 0.0675 & 0.0762 & 0.0800 & -0.0125 & -0.0038 & $-15.6 \%$ & $-4.7 \%$ \\
\hline 2 & EM-19 & $\mathrm{CaO}$ (wt\%) & 1.2985 & 1.3686 & 1.3200 & -0.0215 & 0.0486 & $-1.6 \%$ & $3.7 \%$ \\
\hline 2 & EM-19 & Ce2O3 (wt\%) & 0.2196 & 0.2196 & 0.2400 & -0.0204 & -0.0204 & $-8.5 \%$ & $-8.5 \%$ \\
\hline 2 & EM-19 & Cr2O3 (wt\%) & 0.1220 & 0.1247 & 0.1400 & -0.0180 & -0.0153 & $-12.8 \%$ & $-11.0 \%$ \\
\hline 2 & EM-19 & $w t \%)$ & 0.0404 & & 0.0400 & 0.0004 & 0.0020 & $0.9 \%$ & $5.0 \%$ \\
\hline 2 & EM-19 & $\mathrm{Fe} 2 \mathrm{O} 3(\mathrm{wt} \%)$ & 13.8538 & 14.4898 & 14.9500 & -1.0962 & -0.4602 & $-7.3 \%$ & $-3.1 \%$ \\
\hline 2 & EM-19 & $\mathrm{La} 2 \mathrm{O} 3$ (wt\%) & 0.0765 & 0.0765 & 0.0800 & -0.0035 & -0.0035 & $-4.3 \%$ & $-4.3 \%$ \\
\hline 2 & EM-19 & Li2O (wt\%) & 4.1174 & 4.0955 & 4.0000 & 0.1174 & 0.0955 & $2.9 \%$ & $2.4 \%$ \\
\hline 2 & EM-19 & $\mathrm{MgO}(\mathrm{wt} \%)$ & 0.6961 & 0.7526 & 0.7100 & -0.0139 & 0.0426 & $-2.0 \%$ & $6.0 \%$ \\
\hline 2 & EM-19 & $\mathrm{MnO}$ (wt\%) & 2.8729 & 2.9768 & 2.9200 & -0.0471 & 0.0568 & $-1.6 \%$ & $1.9 \%$ \\
\hline 2 & EM-19 & $\mathrm{Na} 2 \mathrm{O}$ (wt\%) & 17.1533 & 17.2280 & 17.9300 & -0.7767 & -0.7020 & $-4.3 \%$ & $-3.9 \%$ \\
\hline 2 & EM-19 & $\mathrm{NiO}$ (wt\%) & 0.9706 & 1.0977 & 1.1400 & -0.1694 & -0.0423 & $-14.9 \%$ & $-3.7 \%$ \\
\hline 2 & EM-19 & $\mathrm{PbO}$ (wt\%) & 0.0832 & 0.0832 & 0.1000 & -0.0168 & -0.0168 & $-16.8 \%$ & $-16.8 \%$ \\
\hline 2 & EM-19 & SiO2 (wt\%) & 39.2027 & 42.1523 & 39.9800 & -0.7773 & 2.1723 & $-1.9 \%$ & $5.4 \%$ \\
\hline 2 & EM-19 & SO4 (wt\%) & 0.4254 & 0.4254 & 0.4500 & -0.0246 & -0.0246 & $-5.5 \%$ & $-5.5 \%$ \\
\hline 2 & EM-19 & $\mathrm{TiO} 2$ (wt\%) & 2.0058 & 2.1602 & 2.0000 & 0.0058 & 0.1602 & $0.3 \%$ & $8.0 \%$ \\
\hline 2 & EM-19 & $\mathrm{ZnO}(\mathrm{wt} \%)$ & 0.0591 & & 0.0700 & -0.0109 & -0.0109 & $-15.5 \%$ & $-15.5 \%$ \\
\hline 2 & EM-19 & $\mathrm{ZrO} 2(\mathrm{wt} \%)$ & 0.1699 & 0.1699 & 0.1700 & -0.0001 & -0.0001 & $-0.1 \%$ & $-0.1 \%$ \\
\hline 2 & EM-19 & ZZ sum & 97.3446 & 101.9939 & 99.9100 & -2.5654 & 2.0839 & $-2.6 \%$ & $2.1 \%$ \\
\hline 2 & EM-20 & $\mathrm{Al} 2 \mathrm{O} 3$ (wt\%) & 6.5849 & 6.8672 & 6.5900 & -0.0051 & 0.2772 & $-0.1 \%$ & $4.2 \%$ \\
\hline 2 & EM-20 & $\mathrm{B} 2 \mathrm{O} 3$ (wt\%) & 6.8262 & 6.8640 & 7.0000 & -0.1738 & -0.1360 & $-2.5 \%$ & $-1.9 \%$ \\
\hline 2 & EM-20 & $\mathrm{BaO}$ (wt\%) & 0.0759 & 0.0854 & 0.0800 & -0.0041 & 0.0054 & $-5.1 \%$ & $6.8 \%$ \\
\hline 2 & EM-20 & $\mathrm{CaO}$ (wt\%) & 1.3194 & 1.3396 & 1.3200 & -0.0006 & 0.0196 & $0.0 \%$ & $1.5 \%$ \\
\hline 2 & EM-20 & Ce2O3 (wt\%) & 0.2266 & 0.2266 & 0.2400 & -0.0134 & -0.0134 & $-5.6 \%$ & $-5.6 \%$ \\
\hline 2 & EM-20 & Cr2O3 (wt\%) & 0.1125 & 0.1150 & 0.1400 & -0.0275 & -0.0250 & $-19.6 \%$ & $-17.9 \%$ \\
\hline 2 & EM-20 & $\mathrm{CuO}(\mathrm{wt} \%)$ & 0.0457 & 0.0478 & 0.0400 & 0.0057 & 0.0078 & $14.2 \%$ & $19.5 \%$ \\
\hline 2 & EM-20 & Fe2O3 (wt\%) & 16.0126 & 16.7242 & 17.4500 & -1.4374 & -0.7258 & $-8.2 \%$ & $-4.2 \%$ \\
\hline 2 & EM-20 & La2O3 (wt\%) & 0.0827 & & 0.0800 & 0.0027 & 0.0027 & $3.4 \%$ & $3.4 \%$ \\
\hline 2 & EM-20 & Li2O (wt\%) & 3.8106 & 3.8905 & 4.0000 & -0.1894 & -0.1095 & $-4.7 \%$ & $-2.7 \%$ \\
\hline 2 & EM-20 & $\mathrm{MgO}$ (wt\%) & 0.6874 & 0.7401 & 0.7100 & -0.0226 & 0.0301 & $-3.2 \%$ & $4.2 \%$ \\
\hline 2 & EM-20 & $\mathrm{MnO}$ (wt\%) & 2.8439 & 2.9319 & 2.9200 & -0.0761 & 0.0119 & $-2.6 \%$ & $0.4 \%$ \\
\hline 2 & EM-20 & $\mathrm{Na} 2 \mathrm{O}$ (wt\%) & 16.0075 & 15.5582 & 16.2700 & -0.2625 & -0.7118 & $-1.6 \%$ & $-4.4 \%$ \\
\hline 2 & EM-20 & $\mathrm{NiO}$ (wt\%) & 0.8853 & 0.9985 & 1.1400 & -0.2547 & -0.1415 & $-22.3 \%$ & $-12.4 \%$ \\
\hline 2 & EM-20 & $\mathrm{PbO}$ (wt\%) & 0.0856 & 0.0856 & 0.1000 & -0.0144 & -0.0144 & $-14.4 \%$ & $-14.4 \%$ \\
\hline 2 & EM-20 & $\mathrm{SiO} 2$ (wt\%) & 37.7586 & 40.9407 & 39.1400 & -1.3814 & 1.8007 & $-3.5 \%$ & $4.6 \%$ \\
\hline 2 & EM-20 & SO4 (wt\%) & 0.4187 & 0.4187 & 0.4500 & -0.0313 & -0.0313 & $-7.0 \%$ & $-7.0 \%$ \\
\hline 2 & EM-20 & $\mathrm{TiO} 2$ (wt\%) & 1.9766 & 2.0784 & 2.0000 & -0.0234 & 0.0784 & $-1.2 \%$ & $3.9 \%$ \\
\hline 2 & EM-20 & $\mathrm{ZnO}$ (wt\%) & 0.0569 & 0.0569 & 0.0700 & -0.0131 & -0.0131 & $-18.6 \%$ & $-18.6 \%$ \\
\hline 2 & EM-20 & $\mathrm{ZrO} 2(\mathrm{wt} \%)$ & 0.1729 & 0.1729 & 0.1700 & 0.0029 & 0.0029 & $1.7 \%$ & $1.7 \%$ \\
\hline 2 & EM-20 & ZZ sum & 95.9908 & 100.2250 & 99.9100 & -3.9192 & 0.3150 & $-3.9 \%$ & $0.3 \%$ \\
\hline 2 & EM-21 & Al2O3 (wt\%) & "6.9628 & 7.2613 & 6.5900 & 0.3728 & 0.6713 & $5.7 \%$ & $10.2 \%$ \\
\hline 2 & EM-21 & $\mathrm{B} 2 \mathrm{O} 3$ (wt\%) & 7.0274 & 7.0677 & 7.0000 & 0.0274 & 0.0677 & $0.4 \%$ & $1.0 \%$ \\
\hline
\end{tabular}


SRNL-STI-2009-00778, Revision 0

Table D3. Average Measured and Bias-Corrected Chemical Compositions Versus Targeted Compositions by Oxide by Glass ID for the Matrix 2A Study

\begin{tabular}{|c|c|c|c|c|c|c|c|c|c|}
\hline & & & & "Measured & & & & & \\
\hline & & & Measured & Bias-Corrected & Targeted & Diff of & Diff of & \% Diff of & \% Diff of \\
\hline Set & Glass \# & Oxide & (wt\%) & (wt\%) & (wt\%) & Measured & Meas BC & Measured & Meas BC \\
\hline 2 & EM-21 & BaO (wt\%) & 0.0703 & 0.0791 & 0.0800 & -0.0097 & -0.0009 & $-12.1 \%$ & $-1.1 \%$ \\
\hline 2 & EM-21 & $\mathrm{CaO}$ (wt\%) & 1.3926 & 1.4138 & 1.3200 & 0.0726 & 0.0938 & $5.5 \%$ & $7.1 \%$ \\
\hline 2 & EM-21 & Ce2O3 (wt\%) & 0.2284 & 0.2284 & 0.2400 & -0.0116 & -0.0116 & $-4.8 \%$ & $-4.8 \%$ \\
\hline 2 & EM-21 & Cr2O3 (wt\%) & 0.1114 & 0.1138 & 0.1400 & -0.0286 & -0.0262 & $-20.4 \%$ & $-18.7 \%$ \\
\hline 2 & EM-21 & $\mathrm{CuO}$ (wt\%) & 0.0457 & 0.0478 & 0.0400 & 0.0057 & 0.0078 & $14.2 \%$ & $19.5 \%$ \\
\hline 2 & EM-21 & Fe2O3 (wt\%) & 18.6218 & 19.4495 & 19.9500 & -1.3282 & -0.5005 & $-6.7 \%$ & $-2.5 \%$ \\
\hline 2 & EM-21 & La2O3 (wt\%) & 0.0710 & 0.0710 & 0.0800 & -0.0090 & -0.0090 & $-11.3 \%$ & $-11.3 \%$ \\
\hline 2 & EM-21 & Li2O (wt\%) & 3.8967 & 3.9784 & 4.0000 & -0.1033 & -0.0216 & $-2.6 \%$ & $-0.5 \%$ \\
\hline 2 & EM-21 & $\mathrm{MgO}$ (wt\%) & 0.7131 & 0.7678 & 0.7100 & 0.0031 & 0.0578 & $0.4 \%$ & $8.1 \%$ \\
\hline 2 & EM-21 & $\mathrm{MnO}$ (wt\%) & 2.9730 & 3.0651 & 2.9200 & 0.0530 & 0.1451 & $1.8 \%$ & $5.0 \%$ \\
\hline 2 & EM-21 & $\mathrm{Na} 2 \mathrm{O}(\mathrm{wt} \%)$ & 14.0529 & 13.6582 & 13.7700 & 0.2829 & -0.1118 & $2.1 \%$ & $-0.8 \%$ \\
\hline 2 & EM-21 & $\mathrm{NiO}$ (wt\%) & 0.9525 & 1.0742 & 1.1400 & -0.1875 & -0.0658 & $-16.5 \%$ & $-5.8 \%$ \\
\hline 2 & EM-21 & $\mathrm{PbO}(\mathrm{wt} \%)$ & 0.0908 & 0.0908 & 0.1000 & -0.0092 & -0.0092 & $-9.2 \%$ & $-9.2 \%$ \\
\hline 2 & EM-21 & $\mathrm{SiO} 2$ (wt $\%)$ & 38.6144 & 41.8695 & 39.1400 & -0.5256 & 2.7295 & $-1.3 \%$ & $7.0 \%$ \\
\hline 2 & EM-21 & SO4 (wt\%) & 0.4344 & 0.4344 & 0.4500 & -0.0156 & -0.0156 & $-3.5 \%$ & $-3.5 \%$ \\
\hline 2 & EM-21 & $\mathrm{TiO} 2$ (wt $\%)$ & 1.9015 & 1.9995 & 2.0000 & -0.0985 & -0.0005 & $-4.9 \%$ & $0.0 \%$ \\
\hline 2 & EM-21 & $\mathrm{ZnO}$ (wt\%) & 0.0619 & 0.0619 & 0.0700 & -0.0081 & -0.0081 & $-11.5 \%$ & $-11.5 \%$ \\
\hline 2 & EM-21 & $\mathrm{ZrO} 2$ (wt\%) & 0.1739 & 0.1739 & 0.1700 & 0.0039 & 0.0039 & $2.3 \%$ & $2.3 \%$ \\
\hline 2 & EM-21 & $\mathrm{ZZ} \mathrm{sum}$ & 98.3965 & 102.9062 & 99.9100 & -1.5135 & 2.9962 & $-1.5 \%$ & $3.0 \%$ \\
\hline 2 & EM-22 & $\mathrm{Al} 2 \mathrm{O} 3$ (wt\%) & 6.5329 & 6.8130 & 6.5900 & -0.0571 & 0.2230 & $-0.9 \%$ & $3.4 \%$ \\
\hline 2 & EM-22 & B2O3 (wt\%) & 7.0596 & 7.1004 & 7.0000 & 0.0596 & 0.1004 & $0.9 \%$ & $1.4 \%$ \\
\hline 2 & EM-22 & $\mathrm{BaO}$ (wt\%) & 0.0723 & 0.0814 & 0.0800 & -0.0077 & 0.0014 & $-9.6 \%$ & $1.7 \%$ \\
\hline 2 & EM-22 & $\mathrm{CaO}$ (wt\%) & 3.4315 & 3.4839 & 3.3200 & 0.1115 & 0.1639 & $3.4 \%$ & $4.9 \%$ \\
\hline 2 & EM-22 & Ce2O3 (wt\%) & 0.2290 & 0.2290 & 0.2400 & -0.0110 & -0.0110 & $-4.6 \%$ & $-4.6 \%$ \\
\hline 2 & EM-22 & $\mathrm{Cr} 2 \mathrm{O} 3$ (wt\%) & 0.1063 & 0.1086 & 0.1400 & -0.0337 & -0.0314 & $-24.0 \%$ & $-22.4 \%$ \\
\hline 2 & EM-22 & $\mathrm{CuO}(\mathrm{wt} \%)$ & 0.0476 & 0.0498 & 0.0400 & 0.0076 & 0.0098 & $18.9 \%$ & $24.4 \%$ \\
\hline 2 & EM-22 & $\mathrm{Fe} 2 \mathrm{O} 3(\mathrm{wt} \%)$ & 18.4074 & 19.2252 & 19.9500 & -1.5426 & -0.7248 & $-7.7 \%$ & $-3.6 \%$ \\
\hline 2 & EM-22 & La2O3 (wt\%) & 0.0827 & 0.0827 & 0.0800 & 0.0027 & 0.0027 & $3.4 \%$ & $3.4 \%$ \\
\hline 2 & EM-22 & Li2O (wt\%) & 3.8967 & 3.9784 & 4.0000 & -0.1033 & -0.0216 & $-2.6 \%$ & $-0.5 \%$ \\
\hline 2 & EM-22 & $\mathrm{MgO}$ (wt\%) & 0.6915 & 0.7446 & 0.7100 & -0.0185 & 0.0346 & $-2.6 \%$ & $4.9 \%$ \\
\hline 2 & EM-22 & $\mathrm{MnO}(\mathrm{wt} \%)$ & 2.8761 & 2.9651 & 2.9200 & -0.0439 & 0.0451 & $-1.5 \%$ & $1.5 \%$ \\
\hline 2 & EM-22 & $\mathrm{Na} 2 \mathrm{O}$ (wt\%) & 13.8170 & 13.4299 & 13.6000 & 0.2170 & -0.1701 & $1.6 \%$ & $-1.3 \%$ \\
\hline 2 & EM-22 & $\mathrm{NiO}$ (wt\%) & 0.8055 & 0.9084 & 1.1400 & -0.3345 & -0.2316 & $-29.3 \%$ & $-20.3 \%$ \\
\hline 2 & EM-22 & $\mathrm{PbO}(\mathrm{wt} \%)$ & 0.0843 & 0.0843 & 0.1000 & -0.0157 & -0.0157 & $-15.7 \%$ & $-15.7 \%$ \\
\hline 2 & EM-22 & $\mathrm{SiO} 2$ (wt\%) & 36.9029 & 40.0142 & 37.3100 & -0.4071 & 2.7042 & $-1.1 \%$ & $7.2 \%$ \\
\hline 2 & EM-22 & SO4 (wt\%) & 0.4299 & 0.4299 & 0.4500 & -0.0201 & -0.0201 & $-4.5 \%$ & $-4.5 \%$ \\
\hline 2 & EM-22 & TiO2 (wt\%) & 1.9682 & 2.0696 & 2.0000 & -0.0318 & 0.0696 & $-1.6 \%$ & $3.5 \%$ \\
\hline 2 & EM-22 & $\mathrm{ZnO}(\mathrm{wt} \%)$ & 0.0622 & 0.0622 & 0.0700 & -0.0078 & -0.0078 & $-11.1 \%$ & $-11.1 \%$ \\
\hline 2 & EM-22 & $\mathrm{ZrO2}$ (wt\%) & 0.1770 & 0.1770 & 0.1700 & 0.0070 & 0.0070 & $4.1 \%$ & $4.1 \%$ \\
\hline 2 & EM-22 & ZZ sum & 97.6808 & 102.0376 & 99.9100 & -2.2292 & 2.1276 & $-2.2 \%$ & $2.1 \%$ \\
\hline 2 & EM-23 & Al2O3 (wt\%) & 6.6416 & 7.0986 & 6.5900 & 0.0516 & 0.5086 & $0.8 \%$ & $7.7 \%$ \\
\hline 2 & EM-23 & B2O3 (wt\%) & 7.0596 & 7.0997 & 7.0000 & 0.0596 & 0.0997 & $0.9 \%$ & $1.4 \%$ \\
\hline 2 & EM-23 & $\mathrm{BaO}$ (wt\%) & 0.0673 & 0.0759 & 0.0800 & -0.0127 & -0.0041 & $-15.9 \%$ & $-5.1 \%$ \\
\hline 2 & EM-23 & $\mathrm{CaO}(\mathrm{wt} \%)$ & 4.2885 & 4.5216 & 4.3200 & -0.0315 & 0.2016 & $-0.7 \%$ & $4.7 \%$ \\
\hline 2 & EM-23 & Ce2O3 (wt\%) & 0.2243 & 0.2243 & 0.2400 & -0.0157 & -0.0157 & $-6.5 \%$ & $-6.5 \%$ \\
\hline 2 & EM-23 & Cr2O3 (wt\%) & 0.0998 & 0.1019 & 0.1400 & -0.0402 & -0.0381 & $-28.7 \%$ & $-27.2 \%$ \\
\hline 2 & EM-23 & $\mathrm{CuO}(\mathrm{wt} \%)$ & 0.0460 & 0.0479 & 0.0400 & 0.0060 & 0.0079 & $15.0 \%$ & $19.7 \%$ \\
\hline 2 & EM-23 & $\mathrm{Fe} 2 \mathrm{O} 3(\mathrm{wt} \%)$ & 18.1929 & 19.0315 & 19.9500 & -1.7571 & -0.9185 & $-8.8 \%$ & $-4.6 \%$ \\
\hline 2 & EM-23 & La2O3 (wt\%) & 0.0792 & 0.0792 & 0.0800 & -0.0008 & -0.0008 & $-1.0 \%$ & $-1.0 \%$ \\
\hline 2 & EM-23 & Li2O (wt\%) & 3.9452 & 4.0279 & 4.0000 & -0.0548 & 0.0279 & $-1.4 \%$ & $0.7 \%$ \\
\hline 2 & EM-23 & $\mathrm{MgO}(\mathrm{wt} \%)$ & 0.7044 & 0.7615 & 0.7100 & -0.0056 & 0.0515 & $-0.8 \%$ & $7.3 \%$ \\
\hline 2 & EM-23 & $\mathrm{MnO}(\mathrm{wt} \%)$ & 2.8536 & 2.9571 & 2.9200 & -0.0664 & 0.0371 & $-2.3 \%$ & $1.3 \%$ \\
\hline 2 & EM-23 & $\mathrm{Na} 2 \mathrm{O}$ (wt\%) & 13.3115 & 13.3687 & 13.6000 & -0.2885 & -0.2313 & $-2.1 \%$ & $-1.7 \%$ \\
\hline 2 & EM-23 & $\mathrm{NiO}(\mathrm{wt} \%)$ & 0.7759 & 0.8775 & 1.1400 & -0.3641 & -0.2625 & $-31.9 \%$ & $-23.0 \%$ \\
\hline 2 & EM-23 & $\mathrm{PbO}(\mathrm{wt} \%)$ & 0.0918 & 0.0918 & 0.1000 & -0.0082 & -0.0082 & $-8.2 \%$ & $-8.2 \%$ \\
\hline 2 & EM-23 & $\mathrm{SiO} 2$ (wt\%) & 37.5447 & 40.7106 & 36.3100 & 1.2347 & 4.4006 & $3.4 \%$ & $12.1 \%$ \\
\hline 2 & EM-23 & SO4 (wt\%) & 0.4389 & 0.4389 & 0.4500 & -0.0111 & -0.0111 & $-2.5 \%$ & $-2.5 \%$ \\
\hline 2 & EM-23 & $\mathrm{TiO} 2$ (wt $\%)$ & 1.9391 & 2.0886 & 2.0000 & -0.0610 & 0.0886 & $-3.0 \%$ & $4.4 \%$ \\
\hline
\end{tabular}


SRNL-STI-2009-00778, Revision 0

Table D3. Average Measured and Bias-Corrected Chemical Compositions Versus Targeted Compositions by Oxide by Glass ID for the Matrix 2A Study

\begin{tabular}{|c|c|c|c|c|c|c|c|c|c|}
\hline & & & & Measured & & & & & \\
\hline & & & Measured & Bias-Corrected & Targeted & Diff of & Diff of & \% Diff of & $\%$ Diff of \\
\hline Set & Glass \# & Oxide & (wt\%) & (wt\%) & (wt\%) & Measured & Meas BC & Measured & Meas BC \\
\hline 2 & EM-23 & "ZnO (wt\%) & 0.0585 & "0.0585 & 0.0700 & "-0.0115 & "-0.0115 & -16.4\% & "-16.4\% \\
\hline 2 & EM-23 & ZrO2 (wt\%) & 0.1739 & 0.1739 & 0.1700 & 0.0039 & 0.0039 & $2.3 \%$ & $2.3 \%$ \\
\hline 2 & EM-23 & ZZ sum & 98.5366 & 103.8356 & 99.9100 & -1.3734 & 3.9256 & $-1.4 \%$ & $3.9 \%$ \\
\hline 2 & EM-24 & Al2O3 (wt\%) & 11.7291 & 12.2319 & 11.5900 & 0.1391 & 0.6419 & $1.2 \%$ & $5.5 \%$ \\
\hline 2 & EM-24 & B2O3 (wt\%) & 7.0757 & 7.1108 & 7.0000 & 0.0757 & 0.1108 & $1.1 \%$ & $1.6 \%$ \\
\hline 2 & EM-24 & $\mathrm{BaO}(\mathrm{wt} \%)$ & 0.0698 & 0.0785 & 0.0800 & -0.0102 & -0.0015 & $-12.8 \%$ & $-1.9 \%$ \\
\hline 2 & EM-24 & $\mathrm{CaO}$ (wt\%) & 1.3387 & 1.3591 & 1.3200 & 0.0187 & 0.0391 & $1.4 \%$ & $3.0 \%$ \\
\hline 2 & EM-24 & Ce2O3 (wt\%) & 0.2261 & 0.2261 & 0.2400 & -0.0139 & -0.0139 & $-5.8 \%$ & $-5.8 \%$ \\
\hline 2 & EM-24 & Cr2O3 (wt\%) & 0.0921 & 0.0941 & 0.1400 & -0.0479 & -0.0459 & $-34.2 \%$ & $-32.8 \%$ \\
\hline 2 & EM-24 & $\mathrm{CuO}$ (wt\%) & 0.0448 & 0.0468 & 0.0400 & 0.0048 & 0.0068 & $11.9 \%$ & $17.1 \%$ \\
\hline 2 & EM-24 & Fe2O3 (wt\%) & 15.8697 & 16.5738 & 17.4500 & -1.5803 & -0.8762 & $-9.1 \%$ & $-5.0 \%$ \\
\hline 2 & EM-24 & $\mathrm{La} 2 \mathrm{O} 3$ (wt\%) & 0.0715 & 0.0715 & 0.0800 & -0.0085 & -0.0085 & $-10.6 \%$ & $-10.6 \%$ \\
\hline 2 & EM-24 & $\mathrm{Li} 2 \mathrm{O}$ (wt\%) & 4.0152 & 3.9937 & 4.0000 & 0.0152 & -0.0063 & $0.4 \%$ & $-0.2 \%$ \\
\hline 2 & EM-24 & $\mathrm{MgO}(\mathrm{wt} \%)$ & 0.7098 & 0.7643 & 0.7100 & -0.0002 & 0.0543 & $0.0 \%$ & $7.6 \%$ \\
\hline 2 & EM-24 & $\mathrm{MnO}(\mathrm{wt} \%)$ & 2.8536 & 2.9416 & 2.9200 & -0.0664 & 0.0216 & $-2.3 \%$ & $0.7 \%$ \\
\hline 2 & EM-24 & $\mathrm{Na} 2 \mathrm{O}(\mathrm{wt} \%)$ & 13.6418 & 13.2592 & 13.6000 & 0.0418 & -0.3408 & $0.3 \%$ & $-2.5 \%$ \\
\hline 2 & EM-24 & $\mathrm{NiO}$ (wt\%) & 0.8838 & 0.9967 & 1.1400 & -0.2562 & -0.1433 & $-22.5 \%$ & $-12.6 \%$ \\
\hline 2 & EM-24 & $\mathrm{PbO}(\mathrm{wt} \%)$ & 0.0891 & 0.0891 & 0.1000 & -0.0109 & -0.0109 & $-10.9 \%$ & $-10.9 \%$ \\
\hline 2 & EM-24 & $\mathrm{SiO2}$ (wt\%) & 37.2773 & 40.0803 & 36.8100 & 0.4673 & 3.2703 & $1.3 \%$ & $8.9 \%$ \\
\hline 2 & EM-24 & SO4 (wt\%) & 0.4269 & 0.4269 & 0.4500 & -0.0231 & -0.0231 & $-5.1 \%$ & $-5.1 \%$ \\
\hline 2 & EM-24 & TiO2 (wt\%) & 2.0183 & 2.1223 & 2.0000 & 0.0183 & 0.1223 & $0.9 \%$ & $6.1 \%$ \\
\hline 2 & EM-24 & & 0.0566 & 0.0566 & 0.0700 & -0.0134 & -0.0134 & $-19.1 \%$ & $-19.1 \%$ \\
\hline 2 & EM-24 & $\mathrm{ZrO} 2$ (wt\%) & 0.1901 & 0.1901 & 0.1700 & 0.0201 & 0.0201 & $11.8 \%$ & $11.8 \%$ \\
\hline 2 & EM-24 & $\mathrm{ZZ} \mathrm{sum}$ & 98.6797 & 102.7134 & 99.9100 & -1.2303 & 2.8034 & $-1.2 \%$ & $2.8 \%$ \\
\hline 2 & EM-25 & $\begin{array}{l}\mathrm{Al} 2 \mathrm{O} 3 \\
\end{array}$ & $\overline{c 11.4929}$ & $\begin{array}{l}11.9856 \\
\end{array}$ & $=11.5900$ & $\begin{array}{c}-0.0971 \\
\end{array}$ & 0.3956 & -0.8\% & 3.4\% \\
\hline 2 & EM-25 & B2O3 (wt\%) & 6.9630 & 7.0025 & 7.0000 & -0.0370 & 0.0025 & $-0.5 \%$ & $0.0 \%$ \\
\hline 2 & EM-25 & $\mathrm{BaO}$ (wt\%) & 0.0684 & 0.0769 & 0.0800 & -0.0116 & -0.0031 & $-14.5 \%$ & $-3.8 \%$ \\
\hline 2 & EM-25 & $\mathrm{CaO}(\mathrm{wt} \%)$ & 1.3541 & 1.3747 & 1.3200 & 0.0341 & 0.0547 & $2.6 \%$ & $4.1 \%$ \\
\hline 2 & EM-25 & Ce2O3 (wt\%) & 0.2249 & 0.2249 & 0.2400 & -0.0151 & -0.0151 & $-6.3 \%$ & $-6.3 \%$ \\
\hline 2 & EM-25 & Cr2O3 (wt\%) & 0.1067 & 0.1090 & 0.1400 & -0.0333 & -0.0310 & $-23.8 \%$ & $-22.2 \%$ \\
\hline 2 & EM-25 & $\mathrm{CuO}$ (wt\%) & 0.0448 & 0.0468 & 0.0400 & 0.0048 & 0.0068 & $11.9 \%$ & $17.1 \%$ \\
\hline 2 & EM-25 & Fe2O3 (wt\%) & 11.7128 & 12.2329 & 12.4500 & -0.7372 & -0.2171 & $-5.9 \%$ & $-1.7 \%$ \\
\hline 2 & EM-25 & $\mathrm{La} 2 \mathrm{O} 3$ (wt\%) & 0.0707 & 0.0707 & 0.0800 & -0.0093 & -0.0093 & $-11.7 \%$ & $-11.7 \%$ \\
\hline 2 & EM-25 & Li2O (wt\%) & 3.8483 & 3.9290 & 4.0000 & -0.1517 & -0.0710 & $-3.8 \%$ & $-1.8 \%$ \\
\hline 2 & EM-25 & $\mathrm{MgO}(\mathrm{wt} \%)$ & 0.6952 & 0.7486 & 0.7100 & -0.0148 & 0.0386 & $-2.1 \%$ & $5.4 \%$ \\
\hline 2 & EM-25 & $\mathrm{MnO}$ (wt\%) & 2.8955 & 2.9850 & 2.9200 & -0.0245 & 0.0650 & $-0.8 \%$ & $2.2 \%$ \\
\hline 2 & EM-25 & $\mathrm{Na} 2 \mathrm{O}$ (wt\%) & 17.1870 & 16.7057 & 17.5100 & -0.3230 & -0.8043 & $-1.8 \%$ & $-4.6 \%$ \\
\hline 2 & EM-25 & $\mathrm{NiO}$ (wt\%) & 0.9652 & 1.0885 & 1.1400 & -0.1748 & -0.0515 & $-15.3 \%$ & $-4.5 \%$ \\
\hline 2 & EM-25 & $\mathrm{PbO}(\mathrm{wt} \%)$ & 0.0816 & 0.0816 & 0.1000 & -0.0184 & -0.0184 & $-18.4 \%$ & $-18.4 \%$ \\
\hline 2 & EM-25 & SiO2 (wt\%) & 37.0634 & 40.1884 & 37.9000 & -0.8366 & 2.2884 & $-2.2 \%$ & $6.0 \%$ \\
\hline 2 & EM-25 & SO4 (wt\%) & 0.4202 & 0.4202 & 0.4500 & -0.0298 & -0.0298 & $-6.6 \%$ & $-6.6 \%$ \\
\hline 2 & EM-25 & $\mathrm{TiO} 2$ (wt\%) & 1.9933 & 2.0959 & 2.0000 & -0.0067 & 0.0959 & $-0.3 \%$ & $4.8 \%$ \\
\hline 2 & EM-25 & $\mathrm{ZnO}$ (wt\%) & 0.0610 & 0.0610 & 0.0700 & -0.0090 & -0.0090 & $-12.9 \%$ & $-12.9 \%$ \\
\hline 2 & EM-25 & $\mathrm{ZrO} 2$ (wt\%) & 0.1722 & 0.1722 & 0.1700 & 0.0022 & 0.0022 & $1.3 \%$ & $1.3 \%$ \\
\hline 2 & EM-25 & $\mathrm{ZZ} \mathrm{sum}$ & 97.4211 & 101.6002 & 99.9100 & -2.4889 & 1.6902 & $-2.5 \%$ & $1.7 \%$ \\
\hline 2 & EM-26 & בAl2O3 (wt\%) & 11.2472 & $\begin{array}{l}12.0208 \\
\end{array}$ & (11.5900 & $\begin{array}{c}-0.3428 \\
\end{array}$ & $\begin{array}{c}0.4308 \\
\end{array}$ & $-3.0 \%$ & 3.7\% \\
\hline 2 & EM-26 & B2O3 (wt\%) & 6.9067 & 6.9462 & 7.0000 & -0.0933 & -0.0538 & $-1.3 \%$ & $-0.8 \%$ \\
\hline 2 & EM-26 & $\mathrm{BaO}(\mathrm{wt} \%)$ & 0.0656 & 0.0740 & 0.0800 & -0.0144 & -0.0060 & $-18.0 \%$ & $-7.4 \%$ \\
\hline 2 & EM-26 & $\mathrm{CaO}$ (wt\%) & 1.2859 & 1.3554 & 1.3200 & -0.0341 & 0.0354 & $-2.6 \%$ & $2.7 \%$ \\
\hline 2 & EM-26 & Ce2O3 (wt\%) & 0.2275 & 0.2275 & 0.2400 & -0.0125 & -0.0125 & $-5.2 \%$ & $-5.2 \%$ \\
\hline 2 & EM-26 & Cr2O3 (wt\%) & 0.0884 & 0.0903 & 0.1400 & -0.0516 & -0.0497 & $-36.8 \%$ & $-35.5 \%$ \\
\hline 2 & EM-26 & $\mathrm{CuO}$ (wt\%) & 0.0435 & 0.0453 & 0.0400 & 0.0035 & 0.0053 & $8.8 \%$ & $13.2 \%$ \\
\hline 2 & EM-26 & Fe2O3 (wt\%) & 13.3891 & 14.0064 & 14.9500 & -1.5609 & -0.9436 & $-10.4 \%$ & $-6.3 \%$ \\
\hline 2 & EM-26 & La2O3 (wt\%) & 0.0780 & 0.0780 & 0.0800 & -0.0020 & -0.0020 & $-2.5 \%$ & $-2.5 \%$ \\
\hline 2 & EM-26 & Li2O (wt\%) & 3.8591 & 3.9399 & 4.0000 & -0.1409 & -0.0601 & $-3.5 \%$ & $-1.5 \%$ \\
\hline 2 & EM-26 & $\mathrm{MgO}(\mathrm{wt} \%)$ & 0.6944 & 0.7508 & 0.7100 & -0.0156 & 0.0408 & $-2.2 \%$ & $5.7 \%$ \\
\hline 2 & EM-26 & $\mathrm{MnO}$ (wt\%) & 3.7961 & 3.9340 & 3.9200 & -0.1239 & 0.0140 & $-3.2 \%$ & $0.4 \%$ \\
\hline 2 & EM-26 & $\mathrm{Na2O}$ (wt\%) & 13.2138 & 13.2712 & 13.6000 & -0.3862 & -0.3288 & $-2.8 \%$ & $-2.4 \%$ \\
\hline
\end{tabular}


SRNL-STI-2009-00778, Revision 0

Table D3. Average Measured and Bias-Corrected Chemical Compositions Versus Targeted Compositions by Oxide by Glass ID for the Matrix 2A Study

\begin{tabular}{|c|c|c|c|c|c|c|c|c|c|}
\hline & & & & Measured & & & & & \\
\hline & & & Measured & Bias-Corrected & Targeted & Diff of & Diff of & \% Diff of & \% Diff of \\
\hline Set & Glass \# & Oxide & (wt\%) & (wt\%) & (wt\%) & Measured & Meas BC & Measured & Meas BC \\
\hline 2 & EM-26 & "NiO (wt\%) & "0.7584 & "0.8577 & 1.1400 & -0.3816 & -0.2823 & -33.5\% & -24.8\% \\
\hline 2 & EM-26 & $\mathrm{PbO}$ (wt\%) & 0.0832 & 0.0832 & 0.1000 & -0.0168 & -0.0168 & $-16.8 \%$ & $-16.8 \%$ \\
\hline 2 & EM-26 & $\mathrm{SiO} 2$ (wt\%) & 37.5447 & 40.7101 & 38.3100 & -0.7653 & 2.4001 & $-2.0 \%$ & $6.3 \%$ \\
\hline 2 & EM-26 & SO4 (wt\%) & 0.4127 & 0.4127 & 0.4500 & -0.0373 & -0.0373 & $-8.3 \%$ & $-8.3 \%$ \\
\hline 2 & EM-26 & TiO2 (wt\%) & 1.9474 & 2.0975 & 2.0000 & -0.0526 & 0.0975 & $-2.6 \%$ & $4.9 \%$ \\
\hline 2 & EM-26 & $\mathrm{ZnO}$ (wt\%) & 0.0560 & 0.0560 & 0.0700 & -0.0140 & -0.0140 & $-20.0 \%$ & $-20.0 \%$ \\
\hline 2 & EM-26 & ZrO2 (wt\%) & 0.1719 & 0.1719 & 0.1700 & 0.0019 & 0.0019 & $1.1 \%$ & $1.1 \%$ \\
\hline 2 & EM-26 & ZZ sum & 95.8697 & 101.1291 & 99.9100 & -4.0403 & 1.2191 & $-4.0 \%$ & $1.2 \%$ \\
\hline 2 & EM-27 & Al2O3 (wt\%) & 11.1339 & 11.8997 & 11.5900 & -0.4561 & 0.3097 & $-3.9 \%$ & $2.7 \%$ \\
\hline 2 & EM-27 & B2O3 (wt\%) & 6.9308 & 6.9649 & 7.0000 & -0.0692 & -0.0351 & $-1.0 \%$ & $-0.5 \%$ \\
\hline 2 & EM-27 & $\mathrm{BaO}$ (wt\%) & 0.0673 & 0.0759 & 0.0800 & -0.0127 & -0.0041 & $-15.9 \%$ & $-5.1 \%$ \\
\hline 2 & EM-27 & $\mathrm{CaO}(\mathrm{wt} \%)$ & 1.2638 & 1.3322 & 1.3200 & -0.0562 & 0.0122 & $-4.3 \%$ & $0.9 \%$ \\
\hline 2 & EM-27 & Ce2O3 (wt\%) & 0.2234 & 0.2234 & 0.2400 & -0.0166 & -0.0166 & $-6.9 \%$ & $-6.9 \%$ \\
\hline 2 & EM-27 & Cr2O3 (wt\%) & 0.1082 & 0.1105 & 0.1400 & -0.0318 & -0.0295 & $-22.7 \%$ & $-21.1 \%$ \\
\hline 2 & EM-27 & $\mathrm{CuO}(\mathrm{wt} \%)$ & 0.0516 & 0.0537 & 0.0400 & 0.0116 & 0.0137 & $29.1 \%$ & $34.3 \%$ \\
\hline 2 & EM-27 & $\mathrm{Fe} 2 \mathrm{O} 3(\mathrm{wt} \%)$ & 13.8860 & 14.5272 & 14.9500 & -1.0640 & -0.4228 & $-7.1 \%$ & $-2.8 \%$ \\
\hline 2 & EM-27 & La2O3 (wt\%) & 0.0789 & 0.0789 & 0.0800 & -0.0011 & -0.0011 & $-1.4 \%$ & $-1.4 \%$ \\
\hline 2 & EM-27 & Li2O (wt\%) & 3.9721 & 3.9508 & 4.0000 & -0.0279 & -0.0492 & $-0.7 \%$ & $-1.2 \%$ \\
\hline 2 & EM-27 & & 0.6965 & 0.7530 & 0.7100 & -0.0135 & 0.0430 & $-1.9 \%$ & $6.1 \%$ \\
\hline 2 & EM-27 & $(w t \%)$ & 1.8561 & 1. & 1.9200 & -0.0639 & 0.0036 & $-3.3 \%$ & $0.2 \%$ \\
\hline 2 & EM-27 & $\mathrm{Na} 2 \mathrm{O}$ (wt\%) & 16.8837 & 16.9566 & 17.5100 & -0.6263 & -0.5534 & $-3.6 \%$ & $-3.2 \%$ \\
\hline 2 & EM-27 & $\mathrm{NiO}$ (wt\%) & 0.8968 & 1.0142 & 1.1400 & -0.2432 & -0.1258 & $-21.3 \%$ & $-11.0 \%$ \\
\hline 2 & EM-27 & $\mathrm{PbO}$ (wt\%) & 0.0873 & 0.0873 & 0.1000 & -0.0127 & -0.0127 & $-12.7 \%$ & $-12.7 \%$ \\
\hline 2 & EM-27 & $\mathrm{SiO} 2$ (wt\%) & 36.1007 & 38.8151 & 36.4000 & -0.2993 & 2.4151 & $-0.8 \%$ & $6.6 \%$ \\
\hline 2 & EM-27 & SO4 (wt\%) & 0.4322 & 0.4322 & 0.4500 & -0.0178 & -0.0178 & $-4.0 \%$ & $-4.0 \%$ \\
\hline 2 & EM-27 & TiO2 (wt\%) & 2.0225 & 2.1784 & 2.0000 & 0.0225 & 0.1784 & $1.1 \%$ & $8.9 \%$ \\
\hline 2 & EM-27 & $\mathrm{ZnO}$ (wt\%) & 0.0663 & 0.0663 & 0.0700 & -0.0037 & -0.0037 & $-5.3 \%$ & $-5.3 \%$ \\
\hline 2 & EM-27 & ZrO2 (wt\%) & 0.1729 & 0.1729 & 0.1700 & 0.0029 & 0.0029 & $1.7 \%$ & $1.7 \%$ \\
\hline 2 & EM-27 & $\mathrm{ZZ} \mathrm{sum}$ & 96.9308 & 101.6168 & 99.9100 & -2.9792 & 1.7068 & $-3.0 \%$ & $1.7 \%$ \\
\hline 2 & EM-28 & Al2O3 (wt\%) & (14.0154 & (14.6163 & (14.0900 & -0.0746 & 0.5263 & $-0.5 \%$ & $3.7 \%$ \\
\hline 2 & EM-28 & B2O3 (wt\%) & 6.9147 & 6.9530 & 7.0000 & -0.0853 & -0.0470 & $-1.2 \%$ & $-0.7 \%$ \\
\hline 2 & EM-28 & $\mathrm{BaO}$ (wt\%) & 0.0667 & 0.0751 & 0.0800 & -0.0133 & -0.0049 & $-16.6 \%$ & $-6.2 \%$ \\
\hline 2 & EM-28 & & 2.3996 & 2.4362 & 2.3200 & 0.0796 & 0.1162 & $3.4 \%$ & $5.0 \%$ \\
\hline 2 & EM-28 & & 0.2170 & & 0.2400 & -0.0230 & -0.0230 & $-9.6 \%$ & $-9.6 \%$ \\
\hline 2 & EM-28 & 3 (wt\%) & 0.0972 & & 0.1400 & -0.0428 & -0.0407 & $-30.6 \%$ & $-29.1 \%$ \\
\hline 2 & EM-28 & $\mathrm{CuO}$ (wt\%) & 0.0501 & 0.0524 & 0.0400 & 0.0101 & 0.0124 & $25.2 \%$ & $31.0 \%$ \\
\hline 2 & EM-28 & $\mathrm{Fe} 2 \mathrm{O} 3(\mathrm{wt} \%)$ & 11.5305 & 12.0426 & 12.9500 & -1.4195 & -0.9074 & $-11.0 \%$ & $-7.0 \%$ \\
\hline 2 & EM-28 & La2O3 (wt\%) & 0.0739 & 0.0739 & 0.0800 & -0.0061 & -0.0061 & $-7.6 \%$ & $-7.6 \%$ \\
\hline 2 & EM-28 & Li2O (wt\%) & 3.8322 & 3.9125 & 4.0000 & -0.1678 & -0.0875 & $-4.2 \%$ & $-2.2 \%$ \\
\hline 2 & EM-28 & $\mathrm{MgO}(\mathrm{wt} \%)$ & 0.6832 & 0.7357 & 0.7100 & -0.0268 & 0.0257 & $-3.8 \%$ & $3.6 \%$ \\
\hline 2 & EM-28 & $\mathrm{MnO}(\mathrm{wt} \%)$ & 2.8213 & 2.9085 & 2.9200 & -0.0987 & -0.0115 & $-3.4 \%$ & $-0.4 \%$ \\
\hline 2 & EM-28 & $\mathrm{Na} 2 \mathrm{O}$ (wt\%) & 14.6932 & 14.2810 & 14.5600 & 0.1332 & -0.2790 & $0.9 \%$ & $-1.9 \%$ \\
\hline 2 & EM-28 & $\mathrm{NiO}$ (wt\%) & 0.8294 & 0.9353 & 1.1400 & -0.3106 & -0.2047 & $-27.2 \%$ & $-18.0 \%$ \\
\hline 2 & EM-28 & & 0.0862 & 0.0862 & 0.1000 & -0.0138 & -0.0138 & $-13.8 \%$ & $-13.8 \%$ \\
\hline 2 & EM-28 & & 36.1542 & 39.2010 & 36.8500 & -0.6958 & 2.3510 & $-1.9 \%$ & $6.4 \%$ \\
\hline 2 & EM-28 & $4(\mathrm{wt} \%)$ & 0.4142 & & 0.4500 & -0.0358 & -0.0358 & $-8.0 \%$ & $-8.0 \%$ \\
\hline 2 & EM-28 & TiO2 (wt\%) & 2.0308 & 2.13 & 2.0000 & 0.0308 & 0.1354 & $1.5 \%$ & $6.8 \%$ \\
\hline 2 & EM-28 & $\mathrm{ZnO}$ (wt\%) & 0.0601 & 0.0601 & 0.0700 & -0.0099 & -0.0099 & $-14.2 \%$ & $-14.2 \%$ \\
\hline 2 & EM-28 & $\mathrm{ZrO2}$ (wt\%) & 0.1712 & 0.1712 & 0.1700 & 0.0012 & 0.0012 & $0.7 \%$ & $0.7 \%$ \\
\hline 2 & EM-28 & $\mathrm{ZZ} \mathrm{sum}$ & 97.1409 & 101.4066 & 99.9100 & -2.7691 & 1.4966 & $-2.8 \%$ & $1.5 \%$ \\
\hline 2 & EM-29 & Al2O3 (wt\%) & 13.7697 & 14.7167 & 14.0900 & -0.3203 & 0.6267 & $-2.3 \%$ & $4.4 \%$ \\
\hline 2 & EM-29 & B2O3 (wt\%) & 7.1240 & 7.1644 & 7.0000 & 0.1240 & 0.1644 & $1.8 \%$ & $2.3 \%$ \\
\hline 2 & EM-29 & $\mathrm{BaO}$ (wt\%) & 0.0695 & 0.0785 & 0.0800 & -0.0105 & -0.0015 & $-13.1 \%$ & $-1.9 \%$ \\
\hline 2 & EM-29 & $\mathrm{CaO}$ (wt\%) & 3.3301 & 3.5105 & 3.3200 & 0.0101 & 0.1905 & $0.3 \%$ & $5.7 \%$ \\
\hline 2 & EM-29 & Ce2O3 (wt\%) & 0.2255 & 0.2255 & 0.2400 & -0.0145 & -0.0145 & $-6.1 \%$ & $-6.1 \%$ \\
\hline 2 & EM-29 & Cr2O3 (wt\%) & 0.0928 & 0.0948 & 0.1400 & -0.0472 & -0.0452 & $-33.7 \%$ & $-32.3 \%$ \\
\hline 2 & EM-29 & $\mathrm{CuO}$ (wt\%) & 0.0438 & 0.0456 & 0.0400 & 0.0038 & 0.0056 & $9.5 \%$ & $14.0 \%$ \\
\hline 2 & EM-29 & $\mathrm{Fe} 2 \mathrm{O} 3(\mathrm{wt} \%)$ & 11.6699 & 12.2073 & 12.9500 & -1.2801 & -0.7427 & $-9.9 \%$ & $-5.7 \%$ \\
\hline
\end{tabular}


SRNL-STI-2009-00778, Revision 0

Table D3. Average Measured and Bias-Corrected Chemical Compositions Versus Targeted Compositions by Oxide by Glass ID for the Matrix 2A Study

\begin{tabular}{|c|c|c|c|c|c|c|c|c|c|}
\hline & & & & Measured & & & & & \\
\hline & & & Measured & Bias-Corrected & Targeted & Diff of & Diff of & \% Diff of & \% Diff of \\
\hline Set & Glass \# & Oxide & $(w t \%)$ & $(\mathrm{wt} \%)$ & (wt\%) & Measured & Meas BC & Measured & Meas BC \\
\hline 2 & EM-29 & La2O3 (wt\%) & 0.0759 & 0.0759 & 0.0800 & -0.0041 & -0.0041 & $-5.1 \%$ & $-5.1 \%$ \\
\hline 2 & EM-29 & Li2O (wt\%) & 3.9183 & 4.0004 & 4.0000 & -0.0817 & 0.0004 & $-2.0 \%$ & $0.0 \%$ \\
\hline 2 & EM-29 & MgO (wt\%) & 0.6990 & 0.7557 & 0.7100 & -0.0110 & 0.0457 & $-1.6 \%$ & $6.4 \%$ \\
\hline 2 & EM-29 & $\mathrm{MnO}(\mathrm{wt} \%)$ & 2.8245 & 2.9270 & 2.9200 & -0.0955 & 0.0070 & $-3.3 \%$ & $0.2 \%$ \\
\hline 2 & EM-29 & $\mathrm{Na2O}$ (wt\%) & 13.6822 & 13.7417 & 14.0600 & -0.3778 & -0.3183 & $-2.7 \%$ & $-2.3 \%$ \\
\hline 2 & EM-29 & $\mathrm{NiO}(\mathrm{wt} \%)$ & 0.8475 & 0.9585 & 1.1400 & -0.2925 & -0.1815 & $-25.7 \%$ & $-15.9 \%$ \\
\hline 2 & EM-29 & $\mathrm{PbO}(\mathrm{wt} \%)$ & 0.0862 & 0.0862 & 0.1000 & -0.0138 & -0.0138 & $-13.8 \%$ & $-13.8 \%$ \\
\hline 2 & EM-29 & SiO2 (wt\%) & 36.5285 & 39.6076 & 36.3500 & 0.1785 & 3.2576 & $0.5 \%$ & $9.0 \%$ \\
\hline 2 & EM-29 & SO4 (wt\%) & 0.4187 & 0.4187 & 0.4500 & -0.0313 & -0.0313 & $-7.0 \%$ & $-7.0 \%$ \\
\hline 2 & EM-29 & TiO2 (wt\%) & 1.9933 & 2.1469 & 2.0000 & -0.0067 & 0.1469 & $-0.3 \%$ & $7.3 \%$ \\
\hline 2 & EM-29 & $\mathrm{ZnO}$ (wt\%) & 0.0579 & 0.0579 & 0.0700 & -0.0121 & -0.0121 & $-17.3 \%$ & $-17.3 \%$ \\
\hline 2 & EM-29 & $\mathrm{ZrO} 2$ (wt\%) & 0.1743 & 0.1743 & 0.1700 & 0.0043 & 0.0043 & $2.5 \%$ & $2.5 \%$ \\
\hline 2 & EM-29 & ZZ sum & 97.6316 & 102.9939 & 99.9100 & -2.2784 & 3.0839 & $-2.3 \%$ & $3.1 \%$ \\
\hline 2 & EM-30 & $\mathrm{Al} 2 \mathrm{O} 3$ (wt\%) & 14.0862 & 14.6901 & 14.0900 & -0.0038 & 0.6001 & $0.0 \%$ & $4.3 \%$ \\
\hline 2 & EM-30 & B2O3 (wt\%) & 6.9711 & 7.0057 & 7.0000 & -0.0289 & 0.0057 & $-0.4 \%$ & $0.1 \%$ \\
\hline 2 & EM-30 & $\mathrm{BaO}(\mathrm{wt} \%)$ & 0.0662 & 0.0744 & 0.0800 & -0.0138 & -0.0056 & $-17.3 \%$ & $-7.0 \%$ \\
\hline 2 & EM-30 & $\mathrm{CaO}$ (wt\%) & 4.4739 & 4.5422 & 4.3200 & 0.1539 & 0.2222 & $3.6 \%$ & $5.1 \%$ \\
\hline 2 & EM-30 & Ce2O3 (wt\%) & 0.2161 & 0.2161 & 0.2400 & -0.0239 & -0.0239 & $-10.0 \%$ & $-10.0 \%$ \\
\hline 2 & EM-30 & Cr2O3 (wt\%) & 0.0899 & 0.0918 & 0.1400 & -0.0501 & -0.0482 & $-35.8 \%$ & $-34.4 \%$ \\
\hline 2 & EM-30 & $\mathrm{CuO}(\mathrm{wt} \%)$ & 0.0432 & 0.0452 & 0.0400 & 0.0032 & 0.0052 & $8.0 \%$ & $13.0 \%$ \\
\hline 2 & EM-30 & Fe2O3 (wt\%) & 11.7772 & 12.3002 & 12.9500 & -1.1728 & -0.6498 & $-9.1 \%$ & $-5.0 \%$ \\
\hline 2 & EM-30 & La2O3 (wt\%) & 0.0689 & 0.0689 & 0.0800 & -0.0111 & -0.0111 & $-13.9 \%$ & $-13.9 \%$ \\
\hline 2 & EM-30 & $\mathrm{Li} 2 \mathrm{O}(\mathrm{wt} \%)$ & 3.9667 & 3.9455 & 4.0000 & -0.0333 & -0.0545 & $-0.8 \%$ & $-1.4 \%$ \\
\hline 2 & EM-30 & $\mathrm{MgO}(\mathrm{wt} \%)$ & 0.6691 & 0.7205 & 0.7100 & -0.0409 & 0.0105 & $-5.8 \%$ & $1.5 \%$ \\
\hline 2 & EM-30 & $\mathrm{MnO}(\mathrm{wt} \%)$ & 2.8310 & 2.9185 & 2.9200 & -0.0890 & -0.0015 & $-3.0 \%$ & $-0.1 \%$ \\
\hline 2 & EM-30 & Na2O (wt\%) & 13.6148 & 13.2341 & 13.5600 & 0.0548 & -0.3259 & $0.4 \%$ & $-2.4 \%$ \\
\hline 2 & EM-30 & $\mathrm{NiO}$ (wt\%) & 0.8055 & 0.9084 & 1.1400 & -0.3345 & -0.2316 & $-29.3 \%$ & $-20.3 \%$ \\
\hline 2 & EM-30 & $\mathrm{PbO}(\mathrm{wt} \%)$ & 0.0768 & 0.0768 & 0.1000 & -0.0232 & -0.0232 & $-23.2 \%$ & $-23.2 \%$ \\
\hline 2 & EM-30 & SiO2 (wt\%) & 36.0472 & 38.7581 & 35.8500 & 0.1972 & 2.9081 & $0.6 \%$ & $8.1 \%$ \\
\hline 2 & EM-30 & SO4 (wt \%) & 0.4104 & 0.4104 & 0.4500 & -0.0396 & -0.0396 & $-8.8 \%$ & $-8.8 \%$ \\
\hline 2 & EM-30 & TiO2 (wt\%) & 2.0016 & 2.1047 & 2.0000 & 0.0016 & 0.1047 & $0.1 \%$ & $5.2 \%$ \\
\hline 2 & EM-30 & $\mathrm{ZnO}(\mathrm{wt} \%)$ & 0.0573 & 0.0573 & 0.0700 & -0.0127 & -0.0127 & $-18.2 \%$ & $-18.2 \%$ \\
\hline 2 & EM-30 & $\mathrm{ZrO} 2$ (wt\%) & 0.1668 & 0.1668 & 0.1700 & -0.0032 & -0.0032 & $-1.9 \%$ & $-1.9 \%$ \\
\hline 2 & EM-30 & ZZ sum & 98.4398 & 102.3357 & 99.9100 & -1.4702 & 2.4257 & $-1.5 \%$ & $2.4 \%$ \\
\hline
\end{tabular}




\section{Exhibit D1. Matrix 2A Sample Measurements in Analytical Sequence by Oxide}

Measured By Analytical Sequence Oxide $=\mathrm{Al} 2 \mathrm{O3}(\mathrm{wt} \%)$

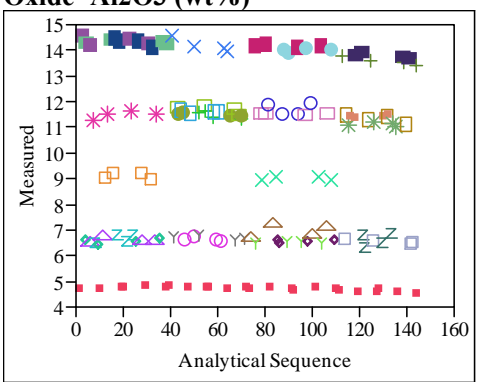

Measured By Analytical Sequence Oxide $=$ B2O3 (wt\%)

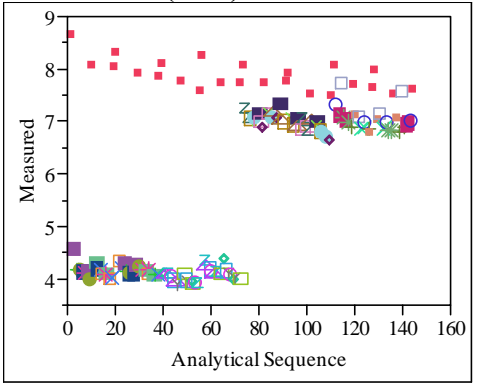

Measured By Analytical Sequence Oxide $=\mathrm{BaO}(\mathrm{wt} \%)$

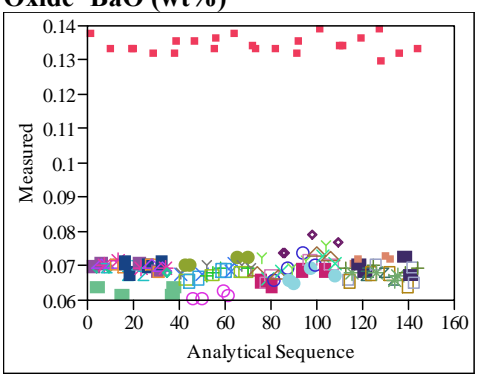

Measured By Analytical Sequence Oxide $=\mathrm{CaO}(\mathrm{wt} \%)$

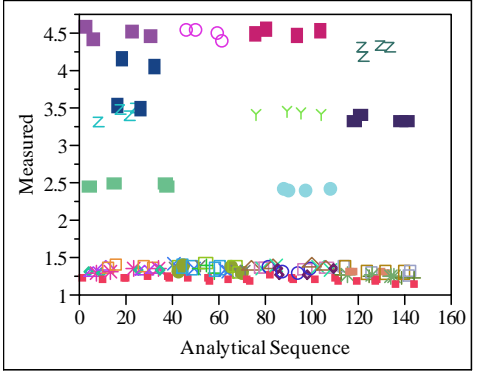

Measured By Analytical Sequence Oxide $=\operatorname{Ce} 203(w t \%)$

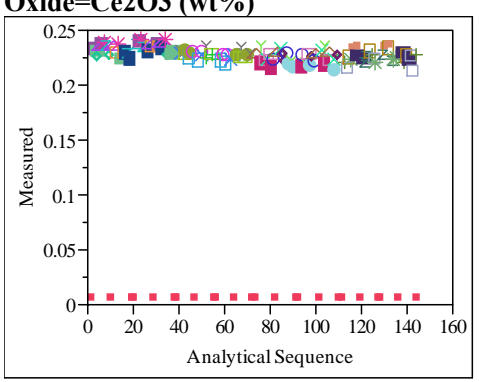

Measured By Analytical Sequence Oxide $=\mathrm{Cr} 2 \mathrm{O3}(\mathrm{wt} \%)$

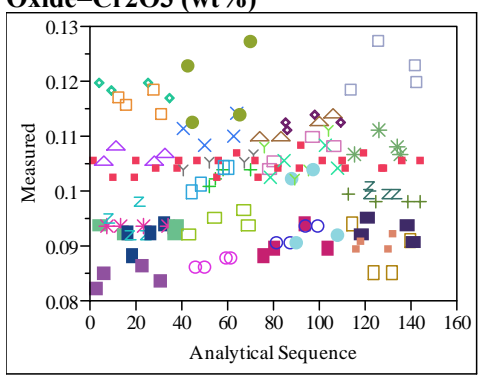

Measured By Analytical Sequence Oxide $=\mathrm{CuO}(\mathrm{wt} \%)$

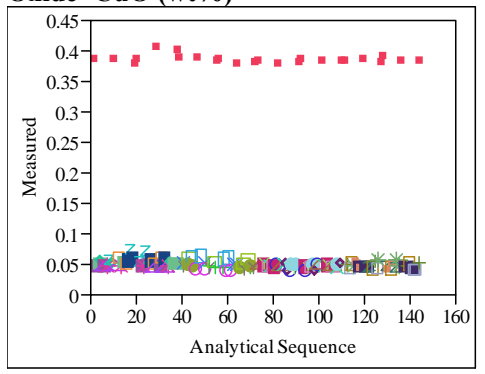

Measured By Analytical Sequence Oxide $=$ Fe2O3 (wt\%)

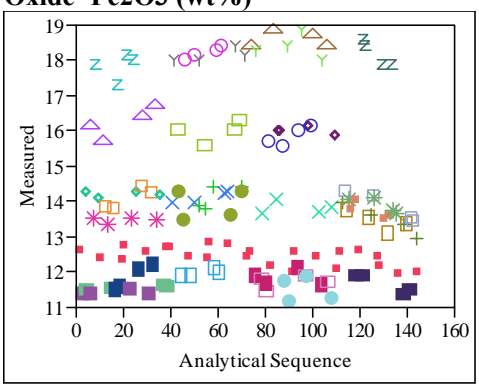

Measured By Analytical Sequence Oxide $=$ La2O3 (wt\%)

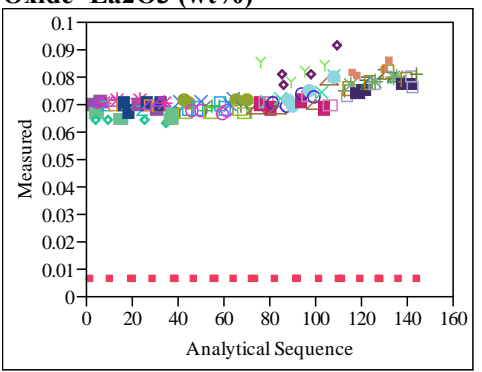

Measured By Analytical Sequence Oxide $=$ Li2O (wt\%)

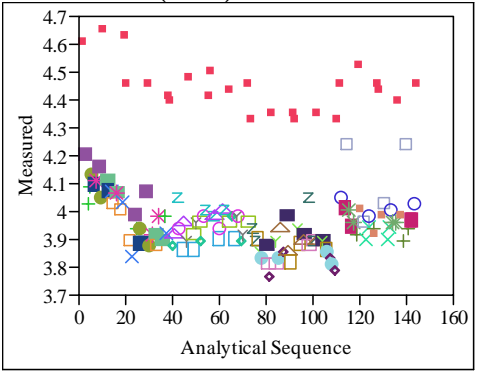

Measured By Analytical Sequence Oxide $=\operatorname{MgO}\left(w^{\circ} \%\right)$

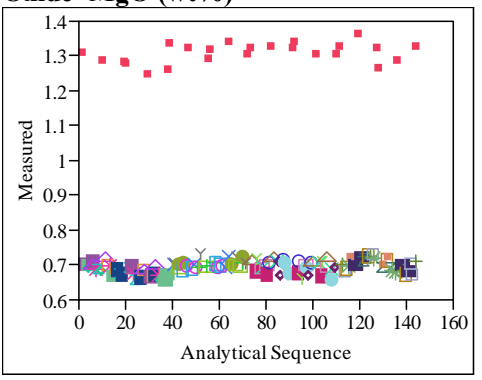

Measured By Analytical Sequence Oxide $=\mathrm{MnO}(\mathrm{wt} \%)$

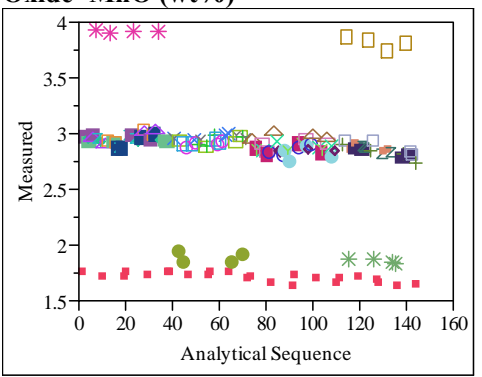




\section{Exhibit D1. Matrix 2A Sample Measurements in Analytical Sequence by Oxide}

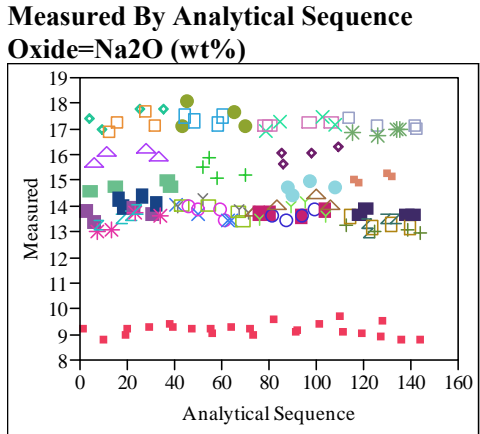

Measured By Analytical Sequence Oxide $=\mathrm{NiO}(\mathbf{w t} \%)$

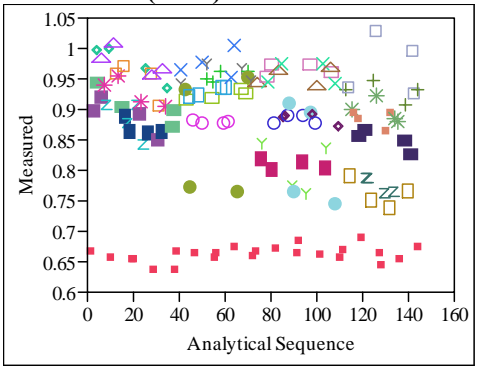

Measured By Analytical Sequence Oxide $=\operatorname{PbO}(w t \%)$

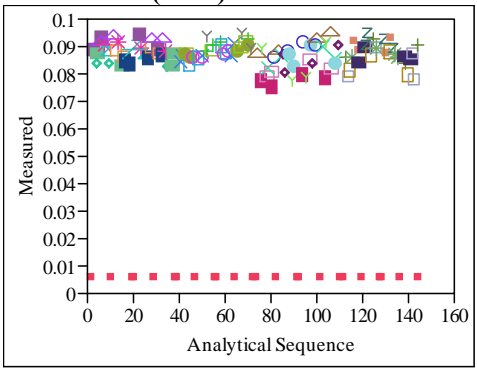

Measured By Analytical Sequence Oxide $=\mathrm{SiO} 2(\mathrm{wt} \%)$

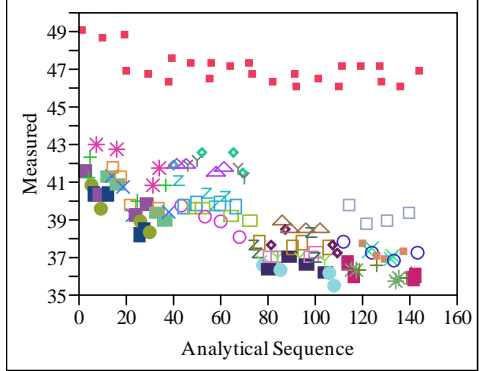

Measured By Analytical Sequence Oxide=SO4 (wt\%)

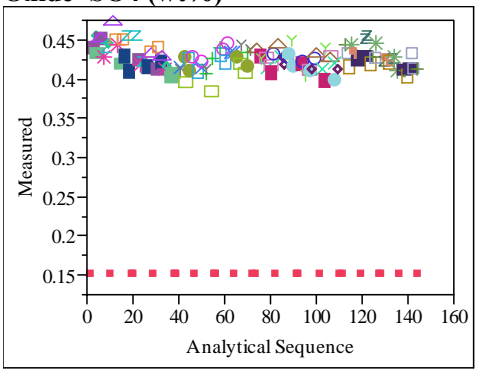

Measured By Analytical Sequence Oxide $=\mathrm{TiO2}(\mathrm{wt} \%)$

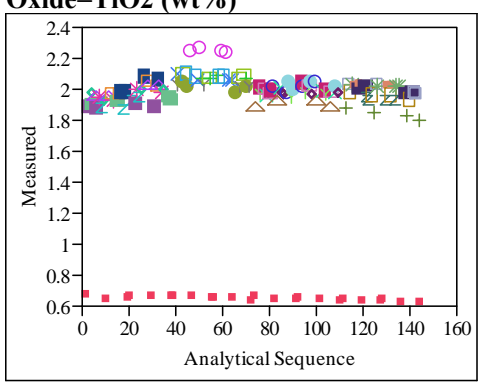

Measured By Analytical Sequence Oxide $=\mathrm{ZnO}(\mathbf{w t} \%)$

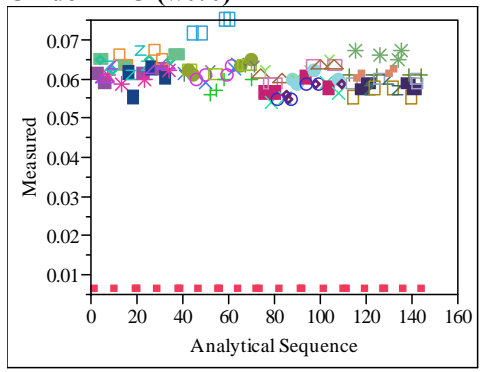

Measured By Analytical Sequence Oxide $=$ ZrO2 (wt\%)

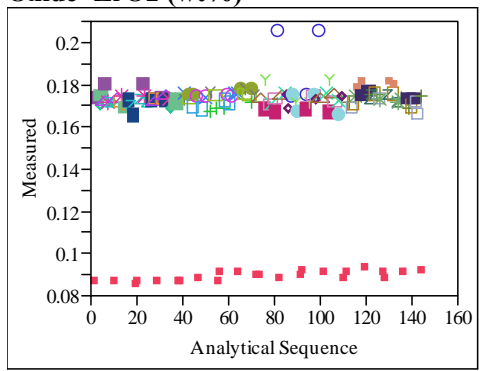




\section{Exhibit D2. Matrix 2A Sample Measurements by Lab ID within Glass ID by Oxide by Analytical Block}

Set $=1$, Oxide $=$ Al2O3 (wt $\%)$

Variability Chart for Measured

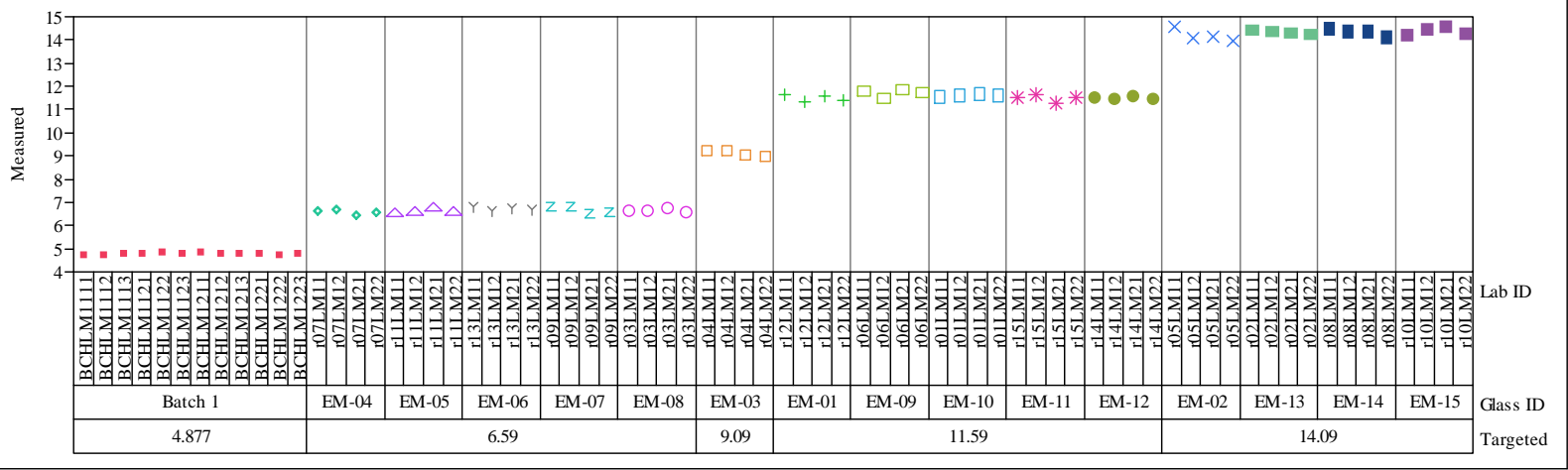

Set $=1$, Oxide $=$ B2O3 $\left(w_{t} \%\right)$

Variability Chart for Measured

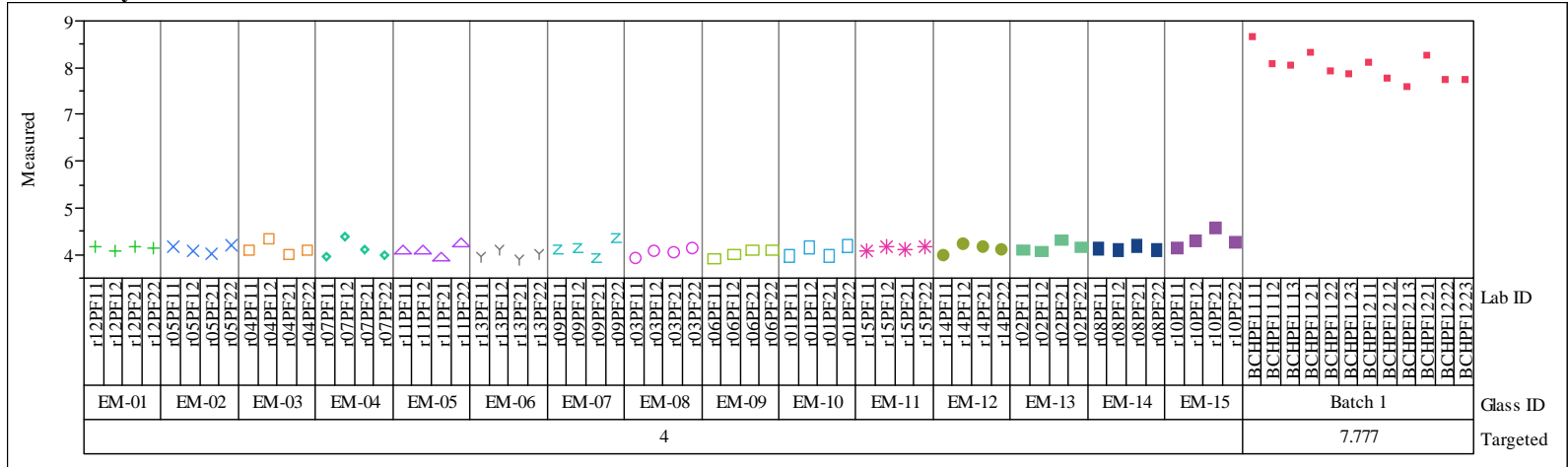

Set $=1$, Oxide $=$ BaO (wt $\%)$

Variability Chart for Measured

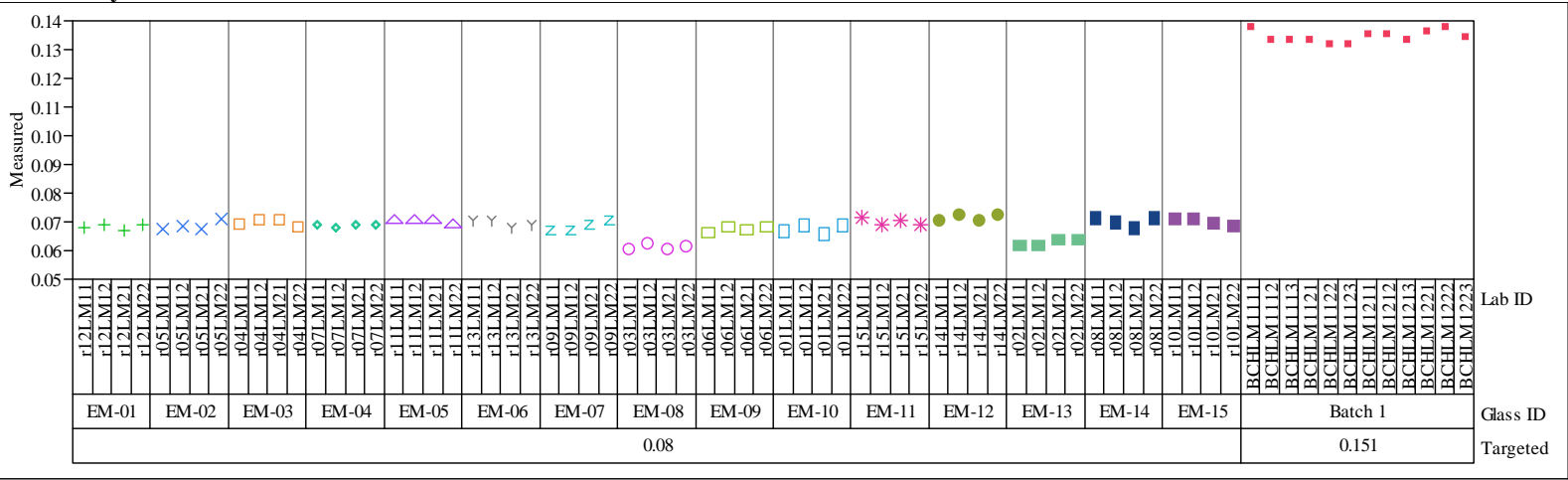




\section{Exhibit D2. Matrix 2A Sample Measurements by Lab ID within Glass ID by Oxide by Analytical Block}

Set $=1$, Oxide $=\mathrm{CaO}(\mathrm{wt} \%)$

Variability Chart for Measured

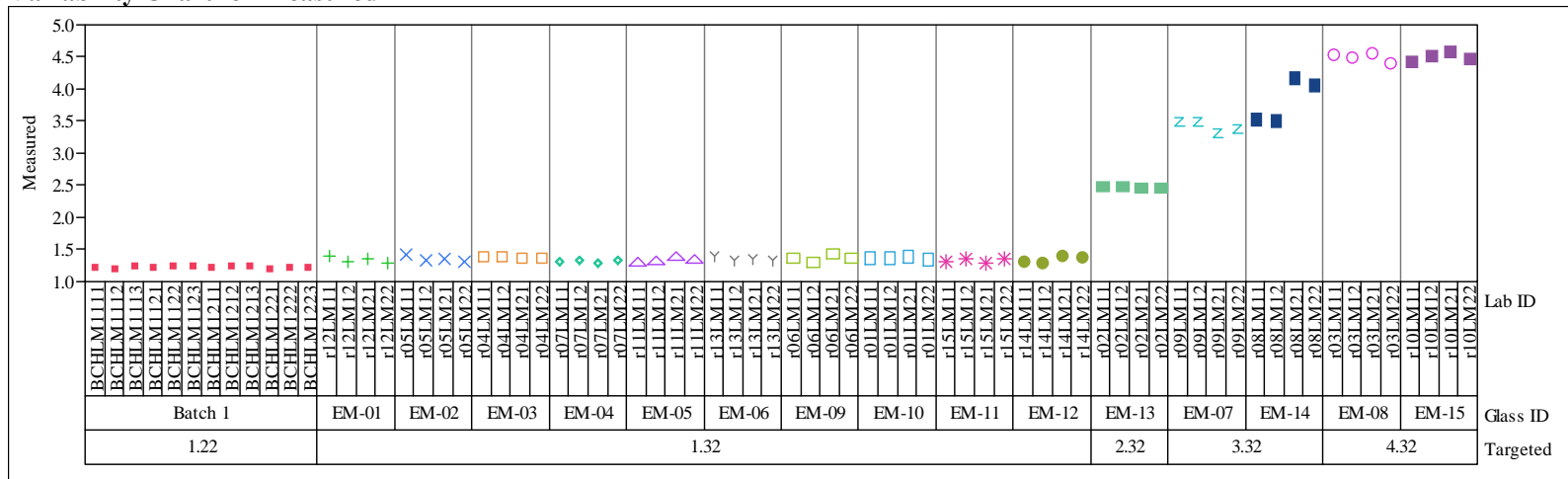

Set $=1$, Oxide $=\mathrm{Ce} 2 \mathrm{O3}(\mathrm{wt} \%)$

Variability Chart for Measured

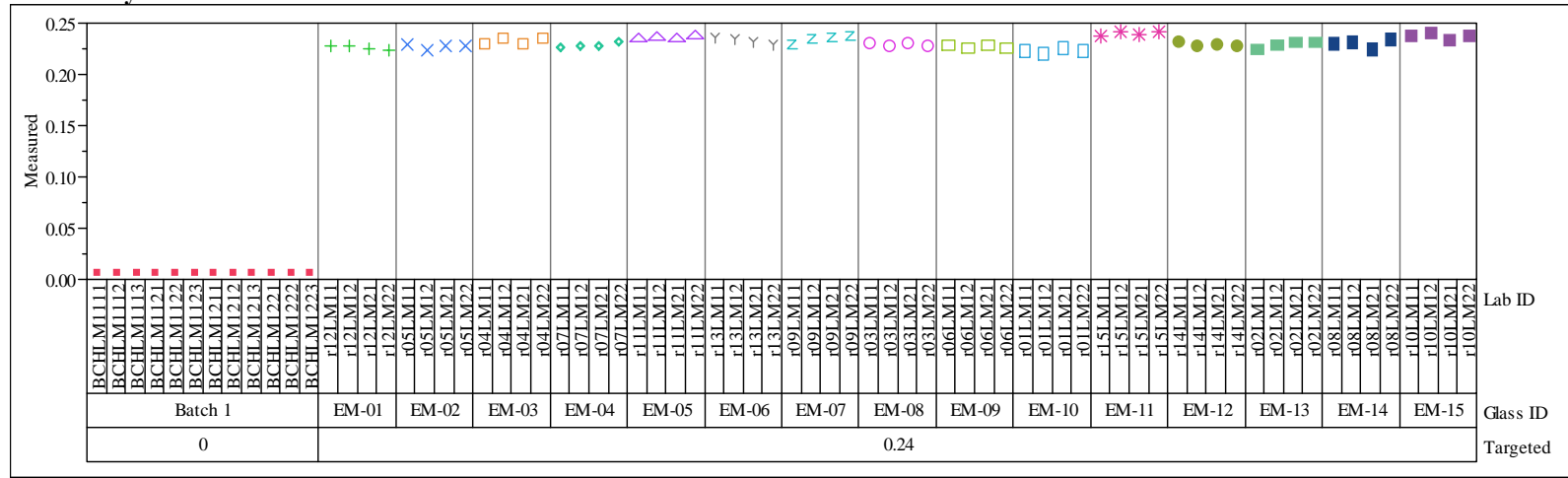

Set $=1$, Oxide $=\mathbf{C r} 203($ wt $\%)$

Variability Chart for Measured

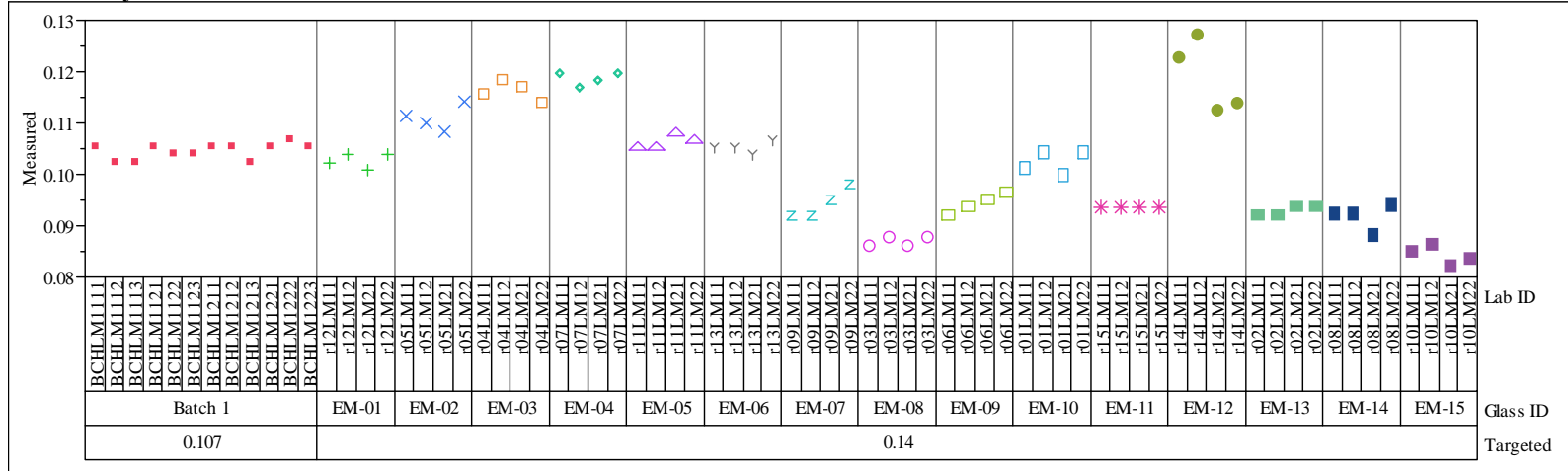




\section{Exhibit D2. Matrix 2A Sample Measurements by Lab ID within Glass ID by Oxide by Analytical Block}

Set $=1$, Oxide $=\mathrm{CuO}\left(\mathrm{wt}_{\mathrm{t}} \%\right)$

Variability Chart for Measured

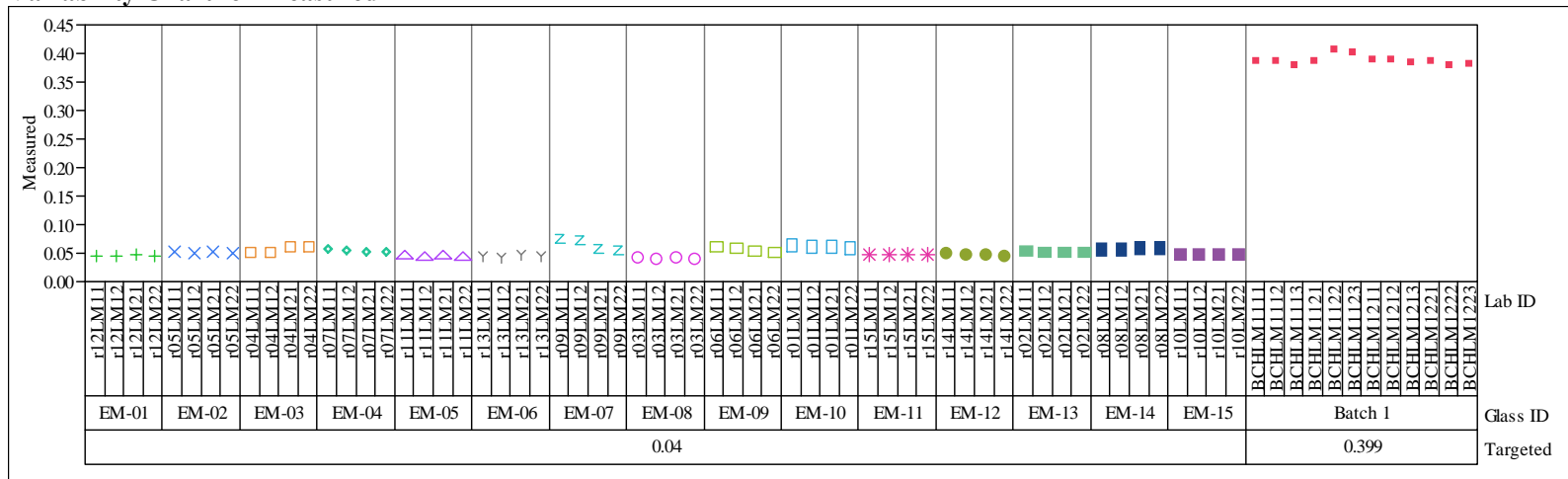

Set $=1$, Oxide $=\mathrm{Fe} 2 \mathrm{O} 3(\mathrm{wt} \%)$

Variability Chart for Measured

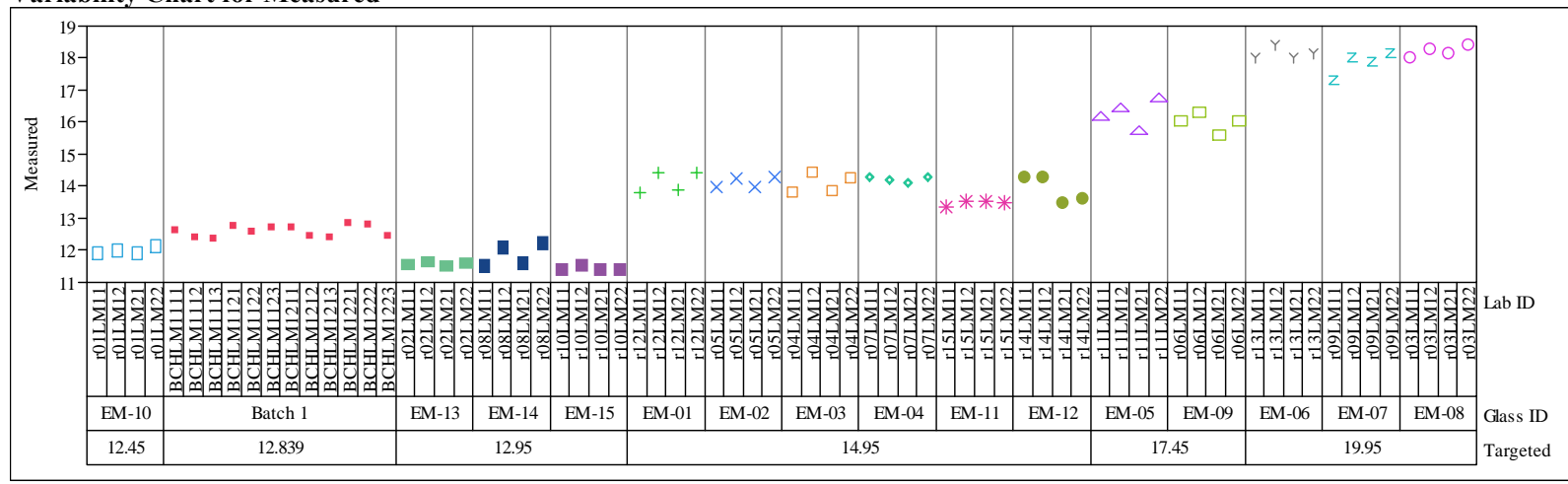

Set $=1$, Oxide $=$ La2O3 $($ wt $\%)$

Variability Chart for Measured

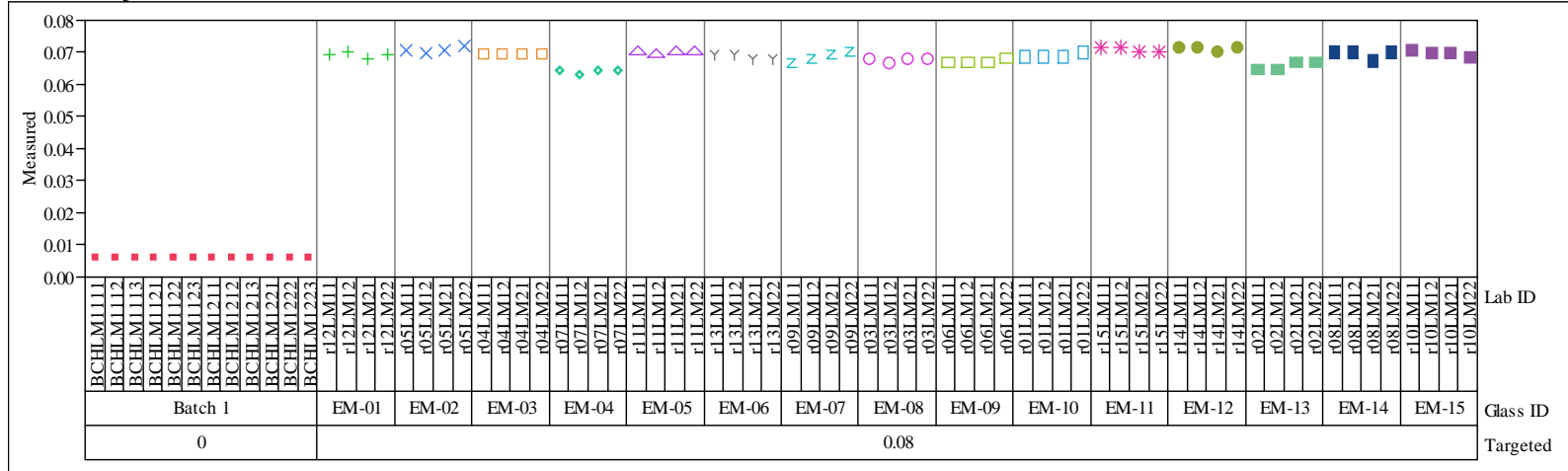




\section{Exhibit D2. Matrix 2A Sample Measurements by Lab ID within Glass ID by Oxide by Analytical Block}

Set $=1$, Oxide $=$ Li2O (wt $\%)$

Variability Chart for Measured

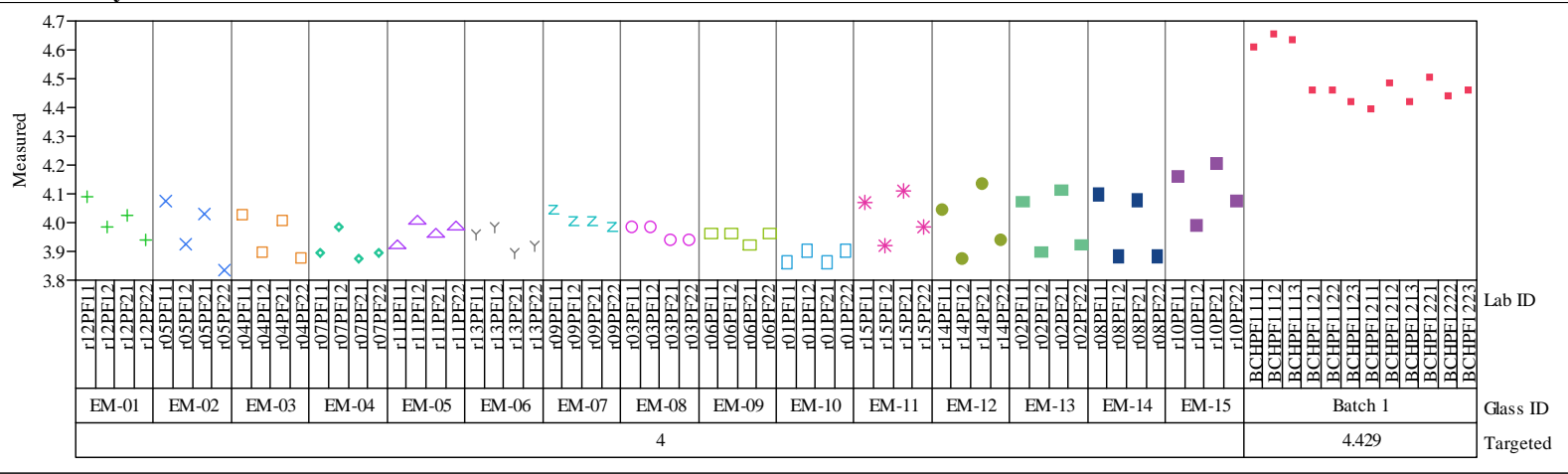

Set $=1$, Oxide $=\operatorname{MgO}(w t \%)$

Variability Chart for Measured

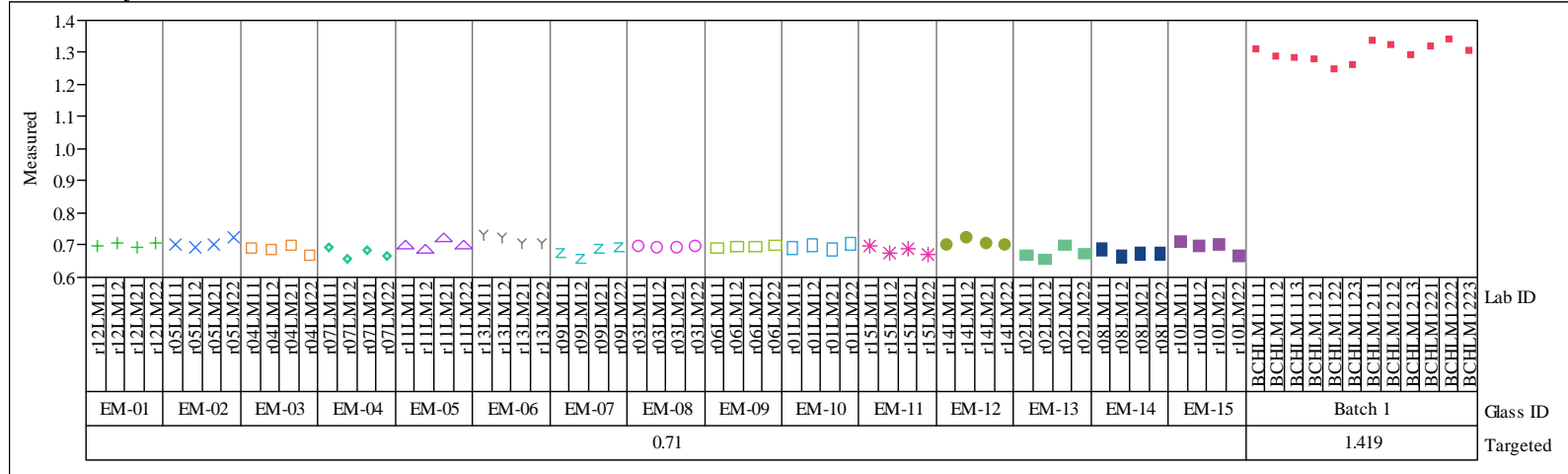

Set $=1$, Oxide $=\operatorname{MnO}($ wt $\%)$

Variability Chart for Measured

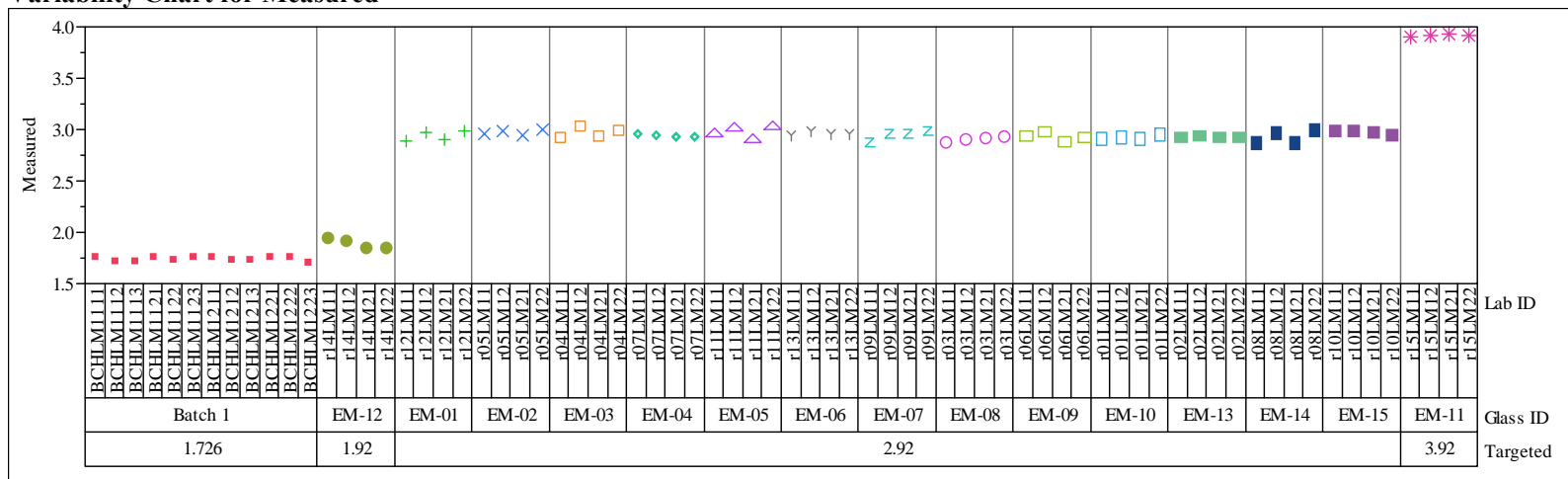




\section{Exhibit D2. Matrix 2A Sample Measurements by Lab ID within Glass ID} by Oxide by Analytical Block

Set $=1$, Oxide $=$ Na2O $\left(w_{t} \%\right)$

Variability Chart for Measured

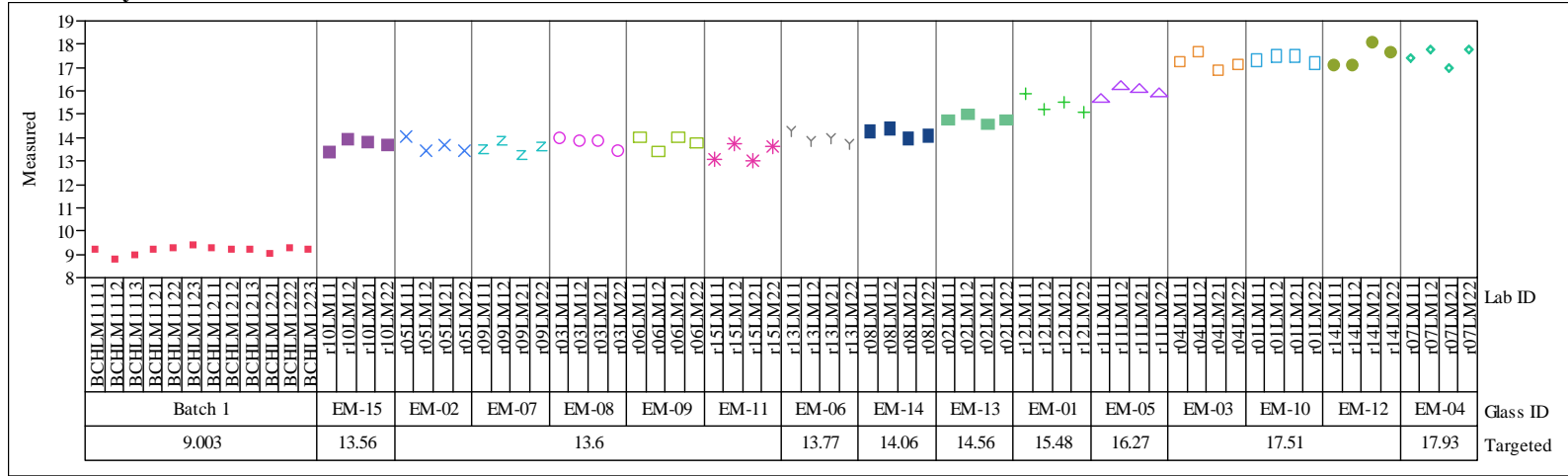

Set $=1$, Oxide $=$ NiO $(w t \%)$

Variability Chart for Measured

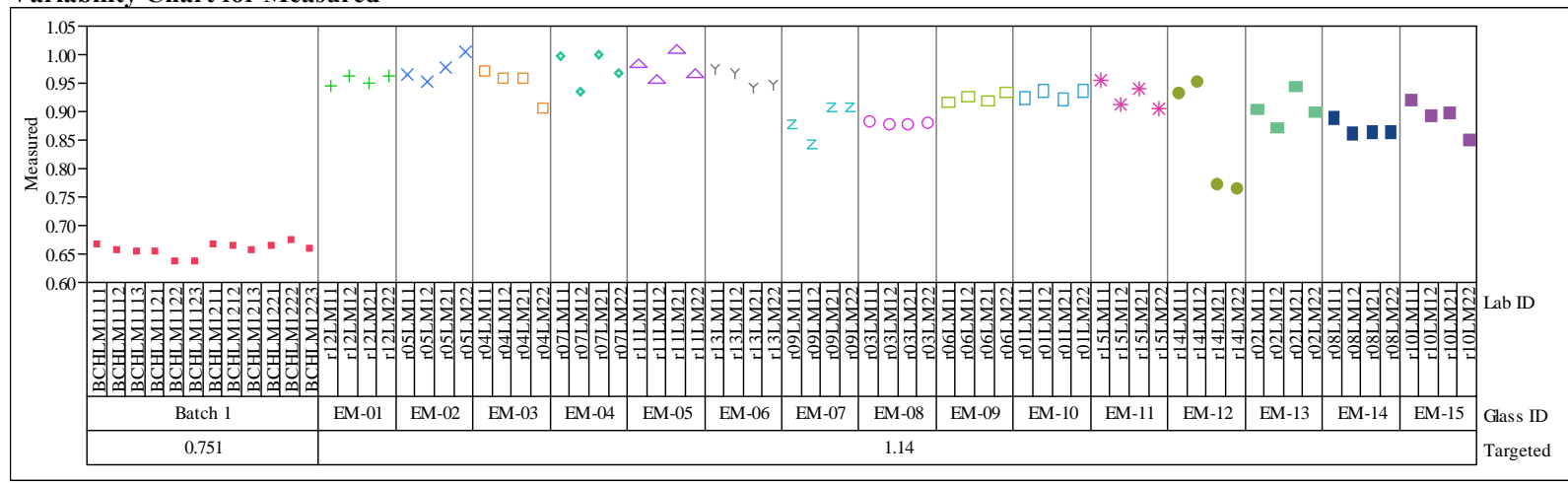

Set $=1$, Oxide $=\operatorname{PbO}\left(w_{t} \%\right)$

Variability Chart for Measured

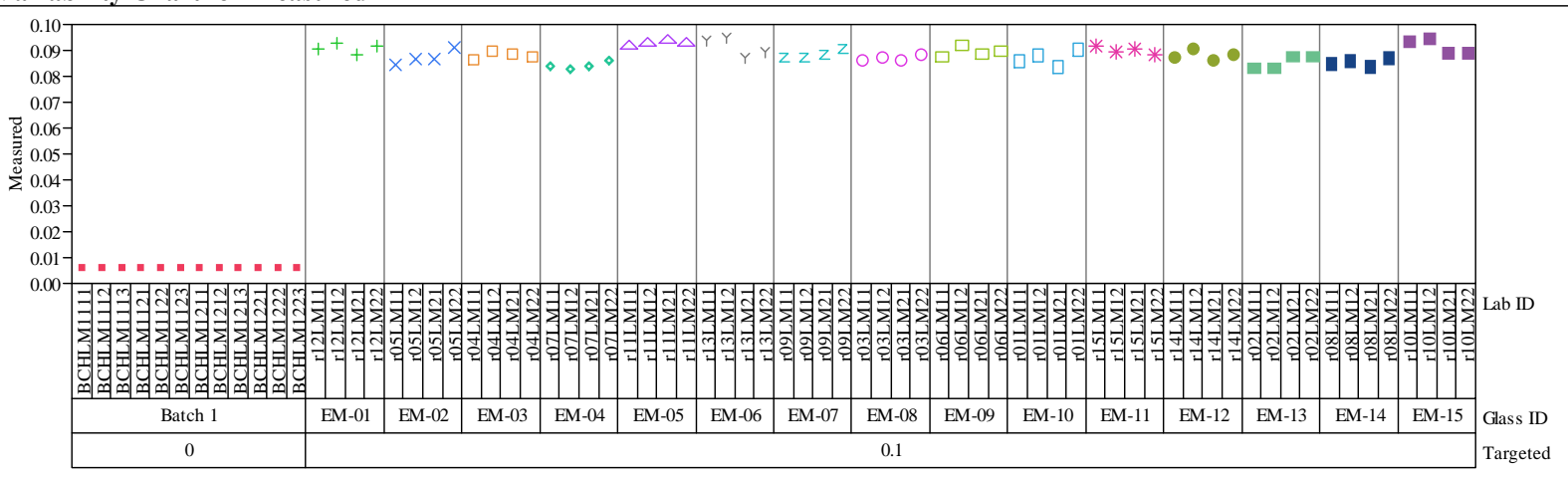




\section{Exhibit D2. Matrix 2A Sample Measurements by Lab ID within Glass ID by Oxide by Analytical Block}

Set $=1$, Oxide $=\mathrm{SiO} 2(\mathrm{wt} \%)$

Variability Chart for Measured

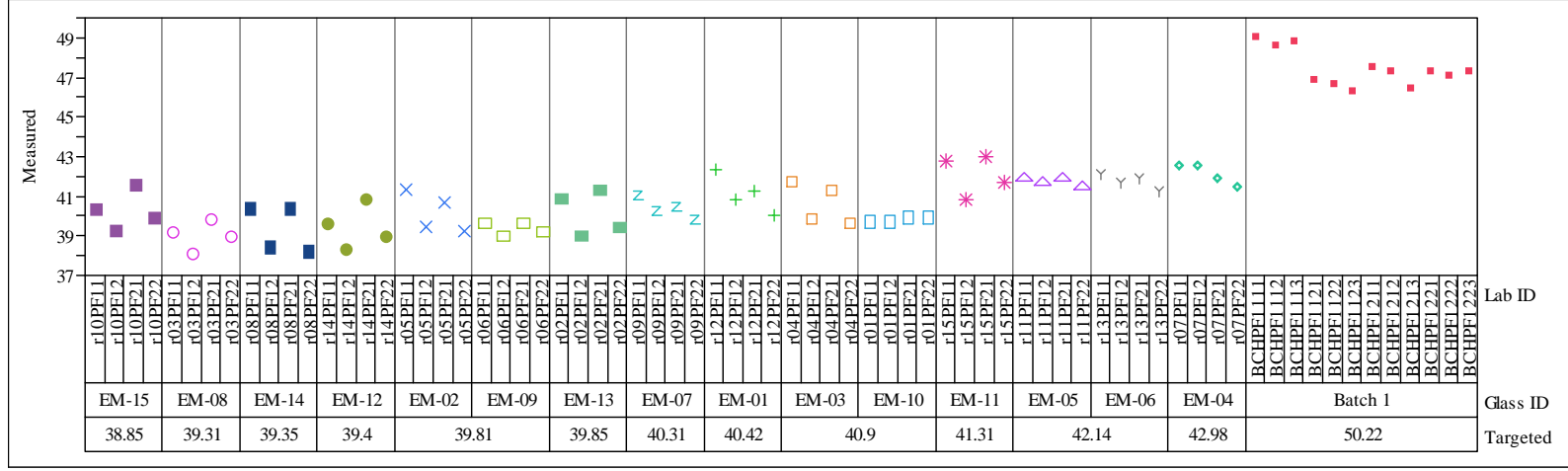

Set $=1$, Oxide $=$ SO4 $($ wt $\%)$

Variability Chart for Measured

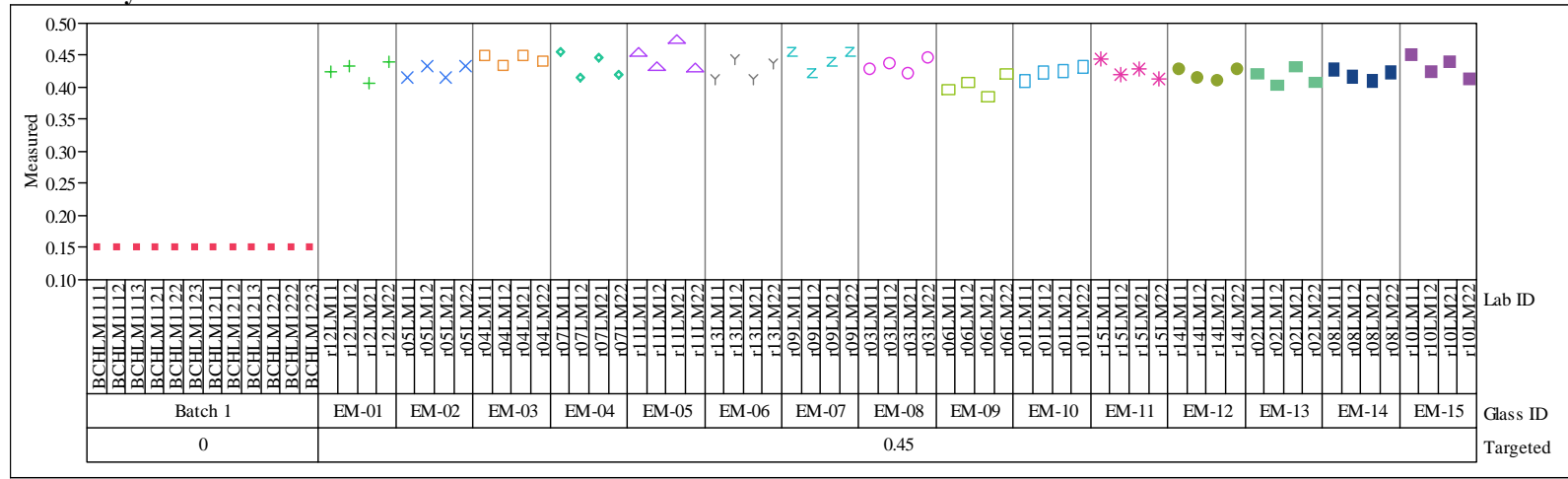

Set $=1$, Oxide $=$ TiO2 $(w t \%)$

Variability Chart for Measured

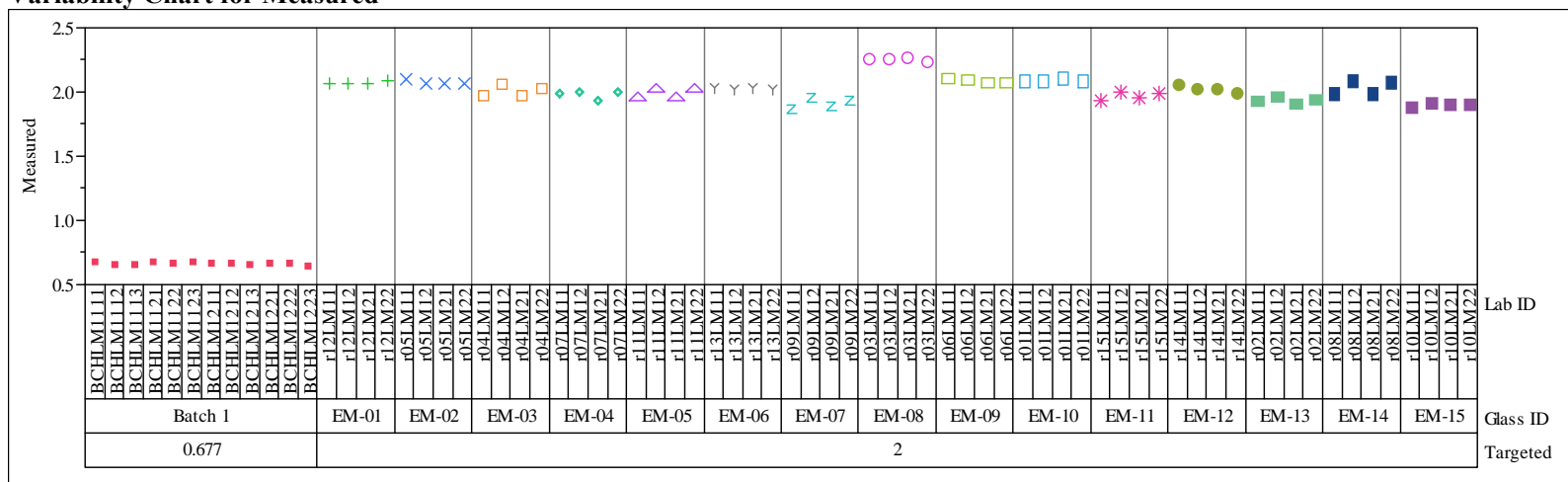




\section{Exhibit D2. Matrix 2A Sample Measurements by Lab ID within Glass ID} by Oxide by Analytical Block

Set $=1$, Oxide $=\mathrm{ZnO}($ wt $\%)$

Variability Chart for Measured

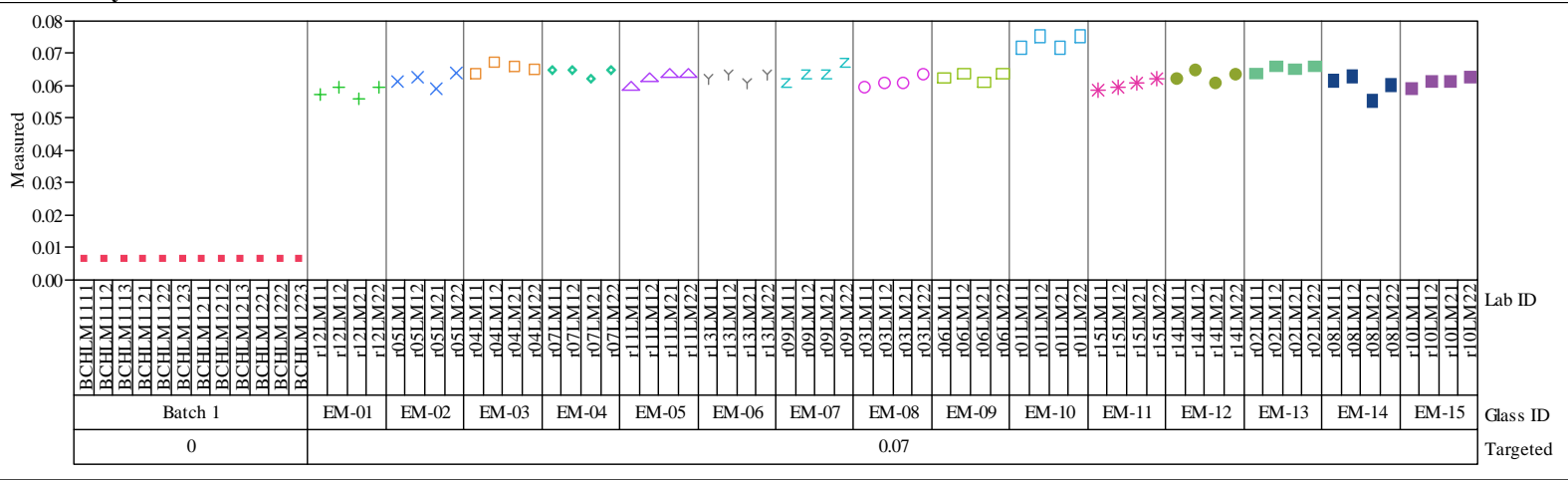

Set $=1$, Oxide $=\mathrm{ZrO} 2(\mathrm{wt} \%)$

Variability Chart for Measured

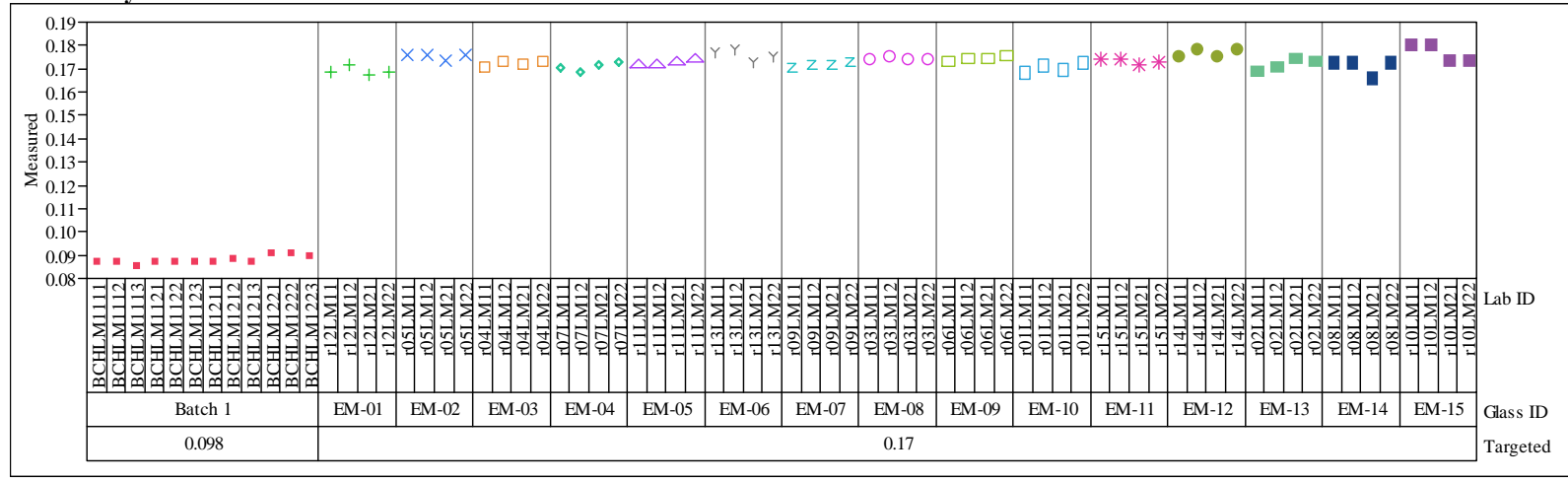

Set $=2$, Oxide $=\mathbf{A} 1203$ (wt $\%)$

Variability Chart for Measured

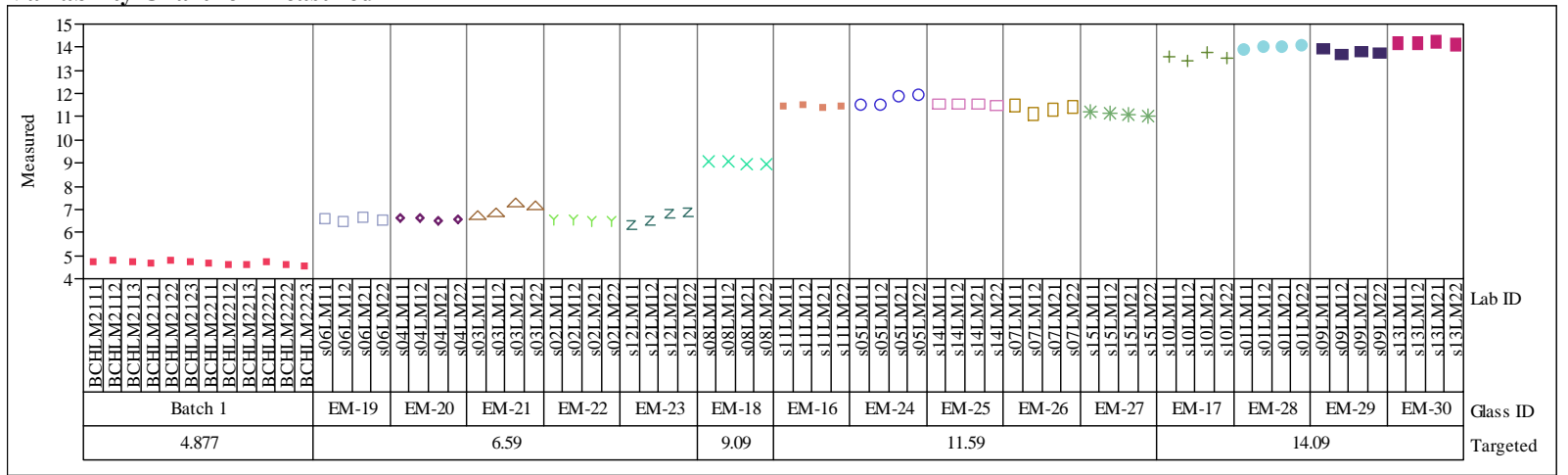




\section{Exhibit D2. Matrix 2A Sample Measurements by Lab ID within Glass ID by Oxide by Analytical Block}

Set $=2$, Oxide $=$ B2O3 $\left(w_{t} \%\right)$

Variability Chart for Measured

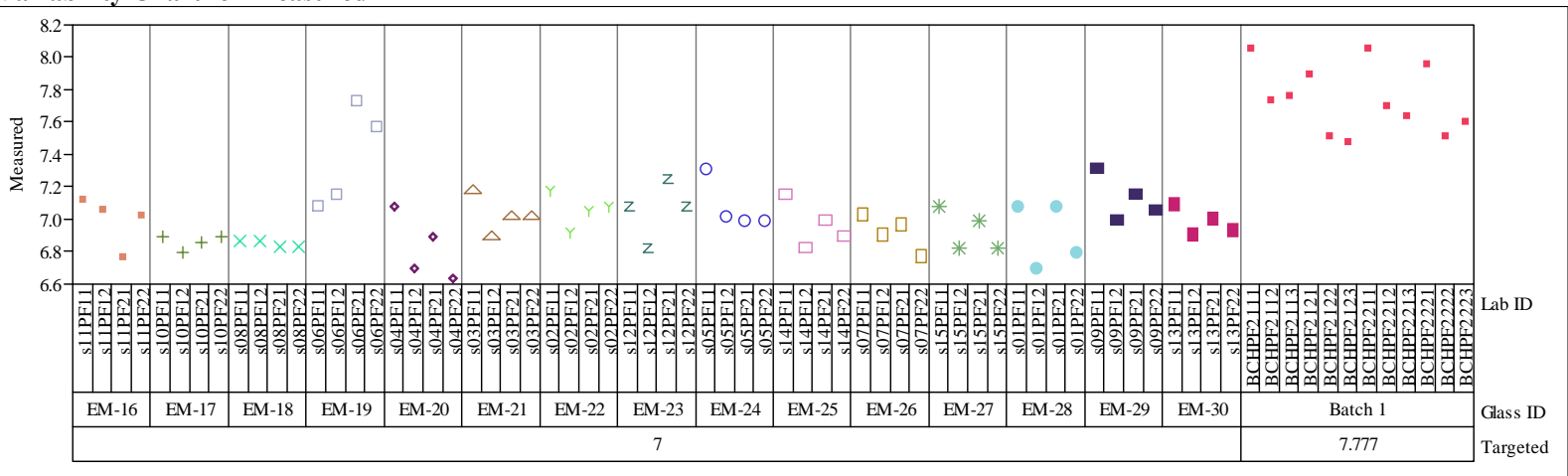

Set $=2$, Oxide $=\mathbf{B a O}(\mathbf{w t} \%)$

Variability Chart for Measured

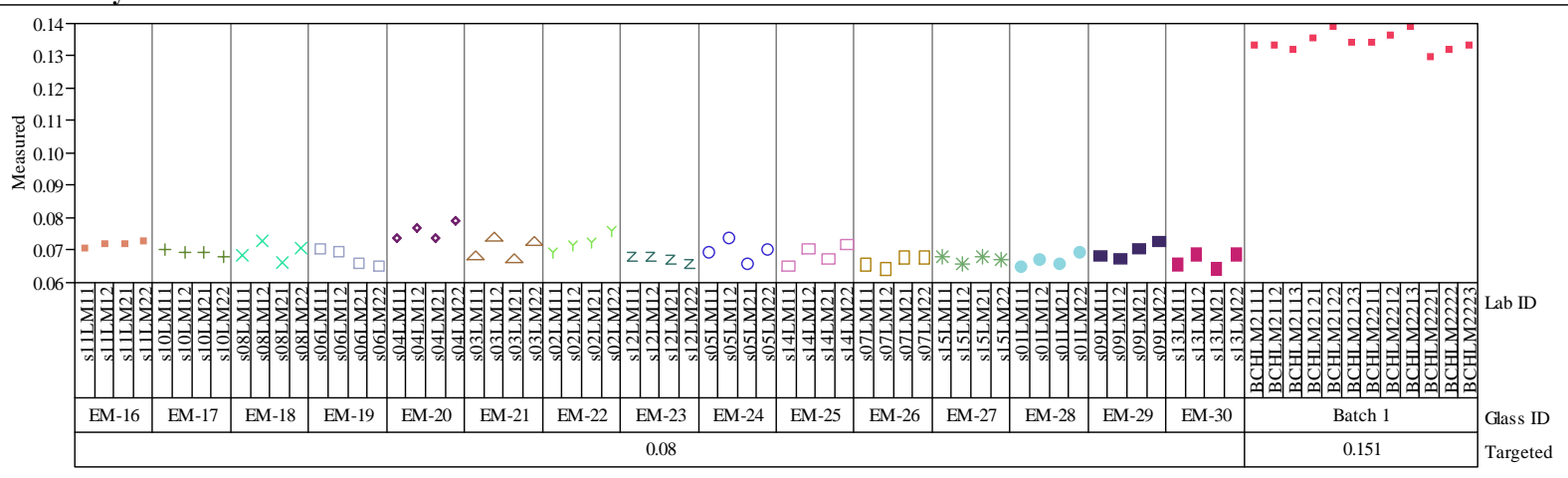

Set $=2$, Oxide $=\mathrm{CaO}(\mathrm{wt} \%)$

Variability Chart for Measured

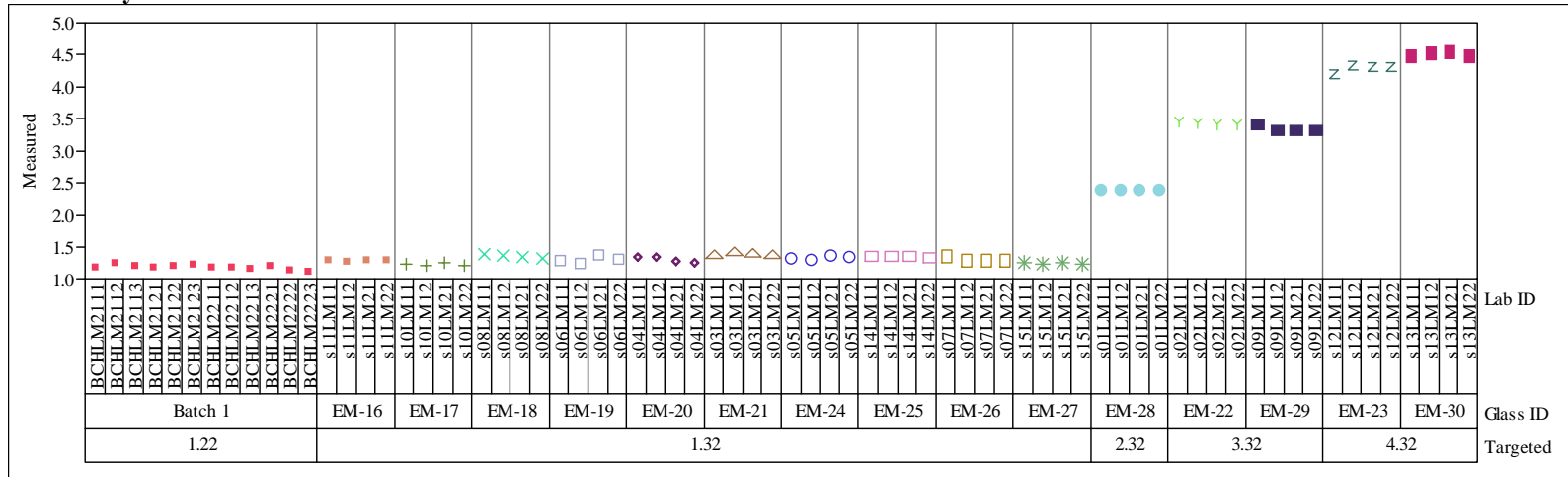




\section{Exhibit D2. Matrix 2A Sample Measurements by Lab ID within Glass ID by Oxide by Analytical Block}

Set $=2$, Oxide $=\mathrm{Ce} 2 \mathrm{O} 3(\mathrm{wt} \%)$

Variability Chart for Measured

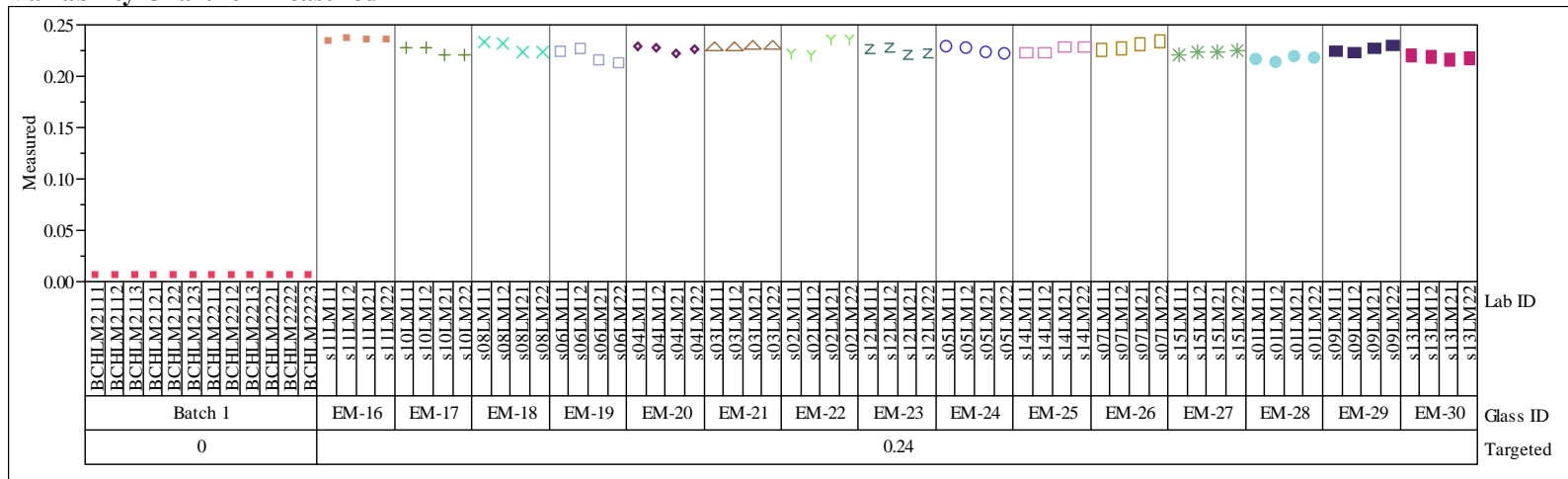

Set $=2$, Oxide $=$ Cr2O3 (wt $\%)$

Variability Chart for Measured

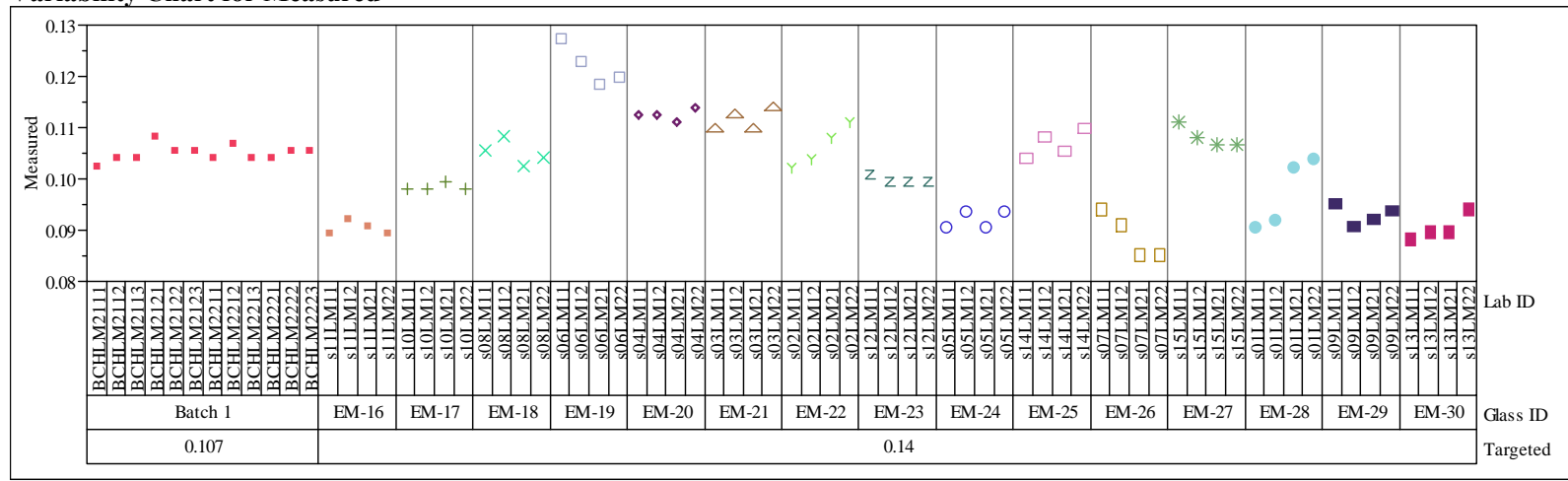

Set $=2$, Oxide $=\mathrm{CuO}(\mathrm{wt} \%)$

Variability Chart for Measured

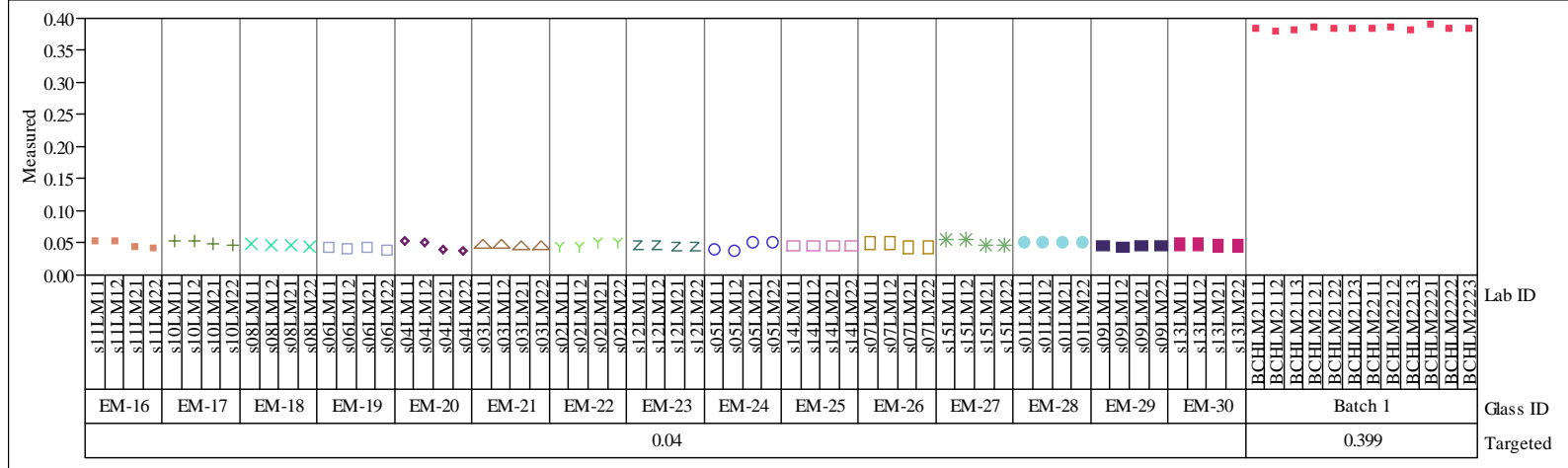




\section{Exhibit D2. Matrix 2A Sample Measurements by Lab ID within Glass ID by Oxide by Analytical Block}

Set $=2$, Oxide $=\mathrm{Fe} 2 \mathrm{O} 3(\mathrm{wt} \%)$

Variability Chart for Measured

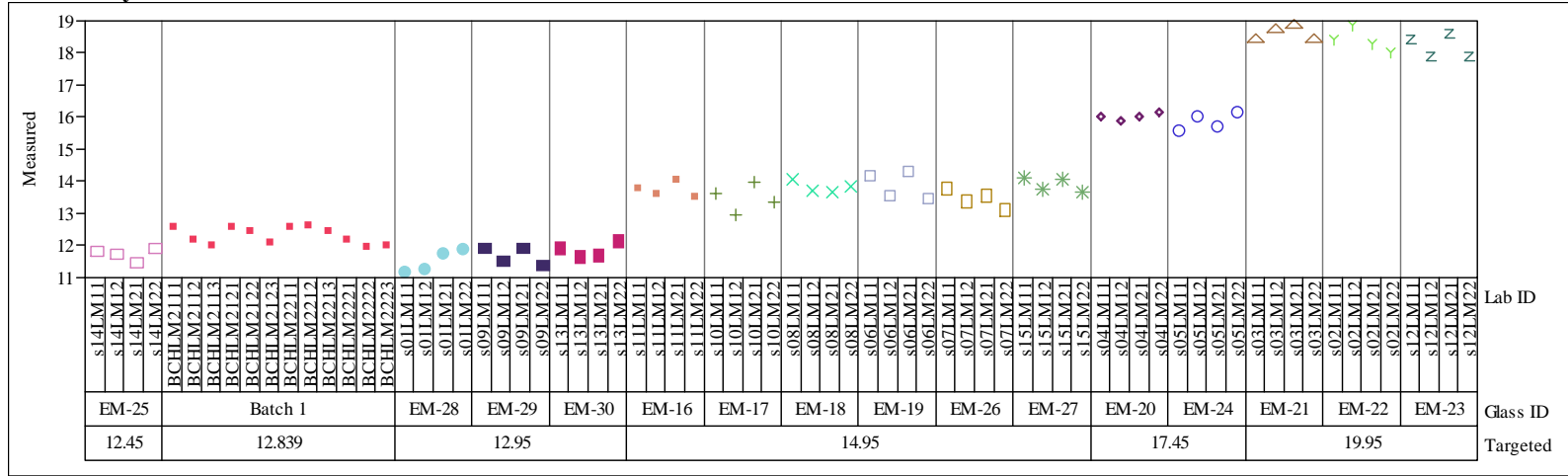

Set $=2$, Oxide $=$ La2O3 $($ wt $\%)$

Variability Chart for Measured

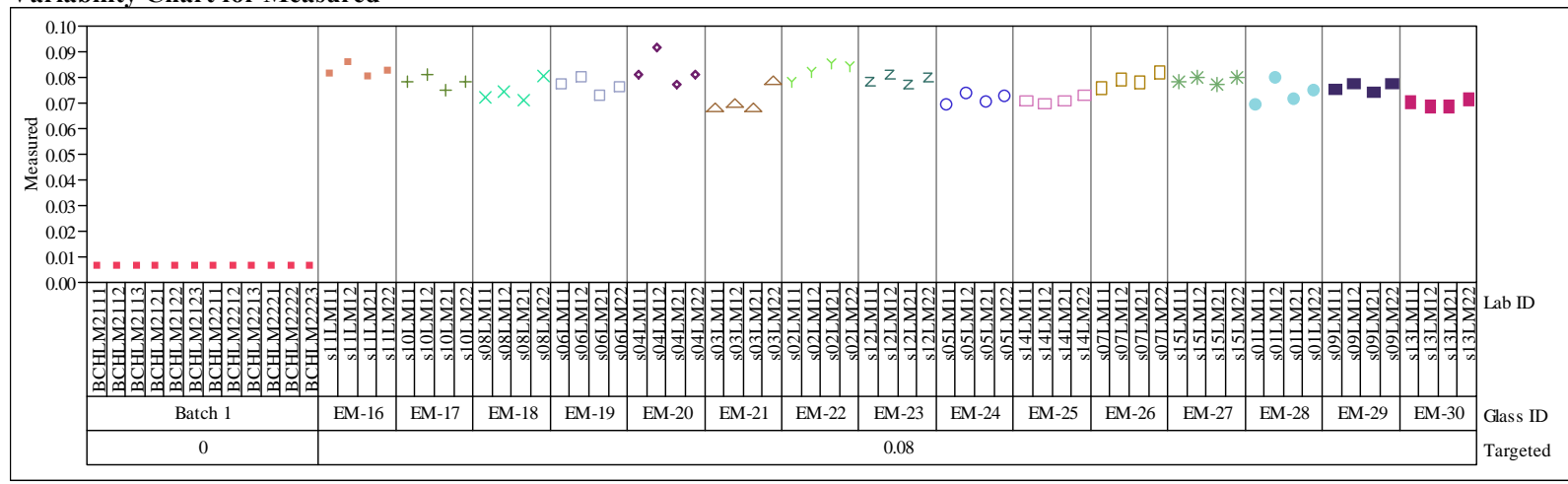

Set $=2$, Oxide $=$ Li2O $($ wt $\%)$

Variability Chart for Measured

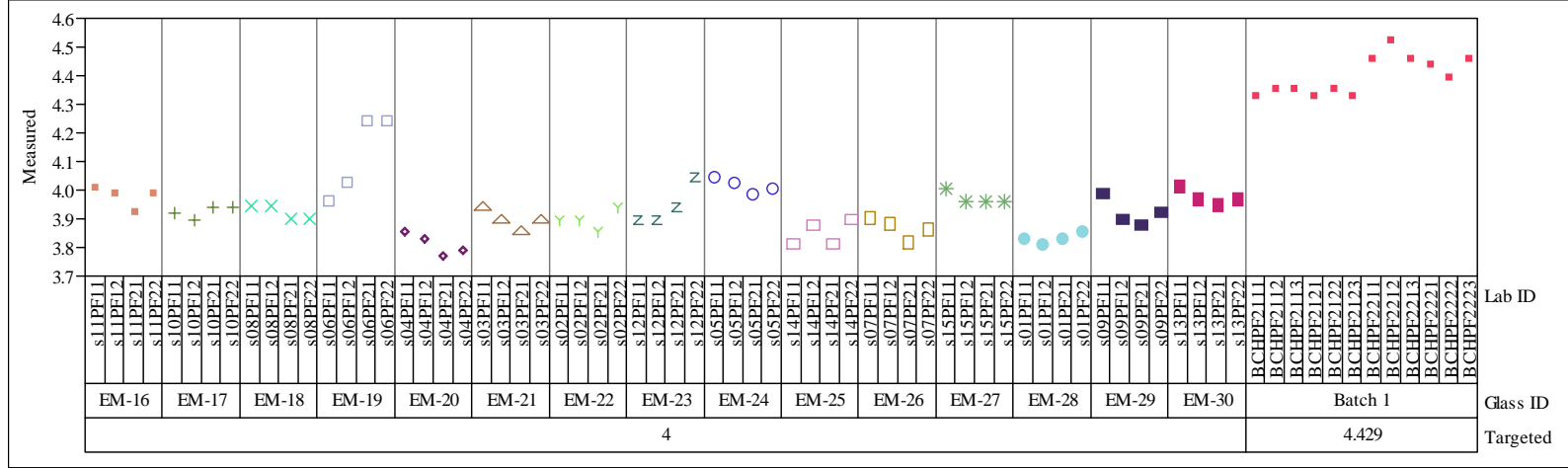




\section{Exhibit D2. Matrix 2A Sample Measurements by Lab ID within Glass ID by Oxide by Analytical Block}

Set $=2$, Oxide $=\operatorname{MgO}(w t \%)$

Variability Chart for Measured

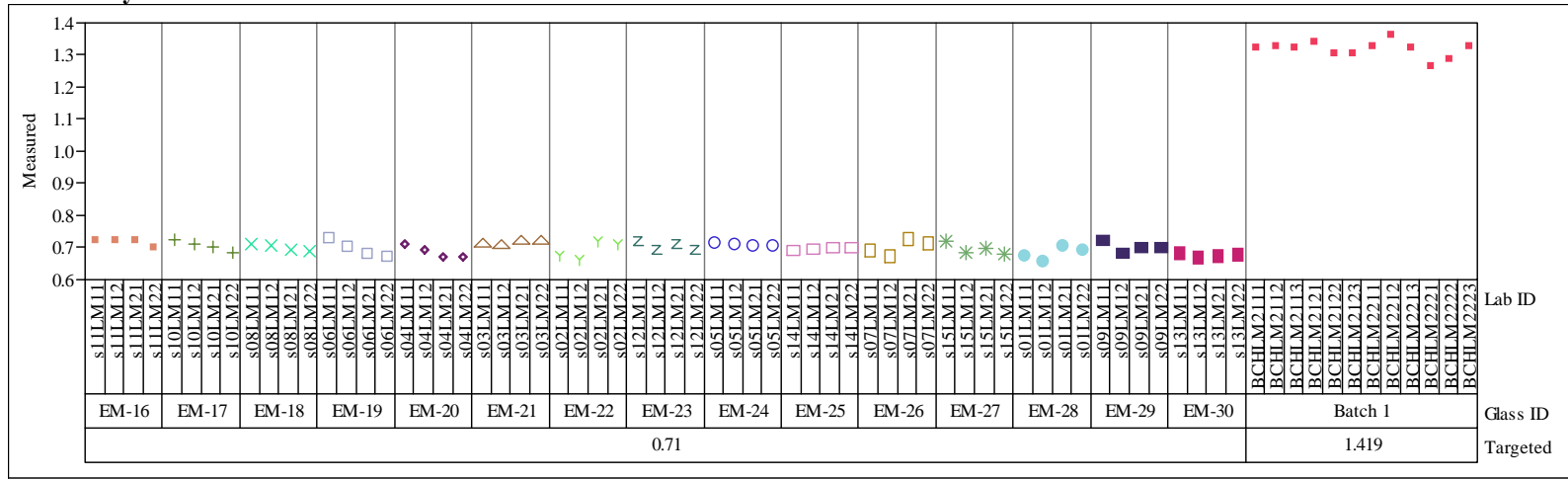

Set $=2$, Oxide $=\operatorname{MnO}(\mathbf{w t} \%)$

Variability Chart for Measured

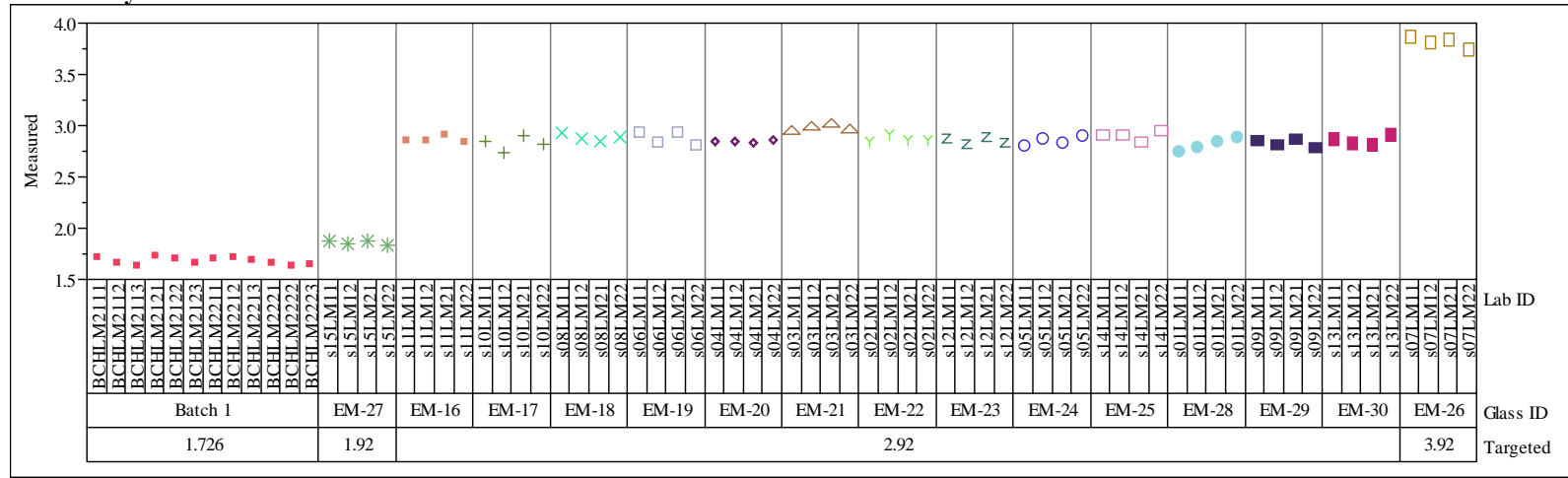

Set $=2$, Oxide $=\mathrm{Na} 2 \mathrm{O}(\mathrm{wt} \%)$

Variability Chart for Measured

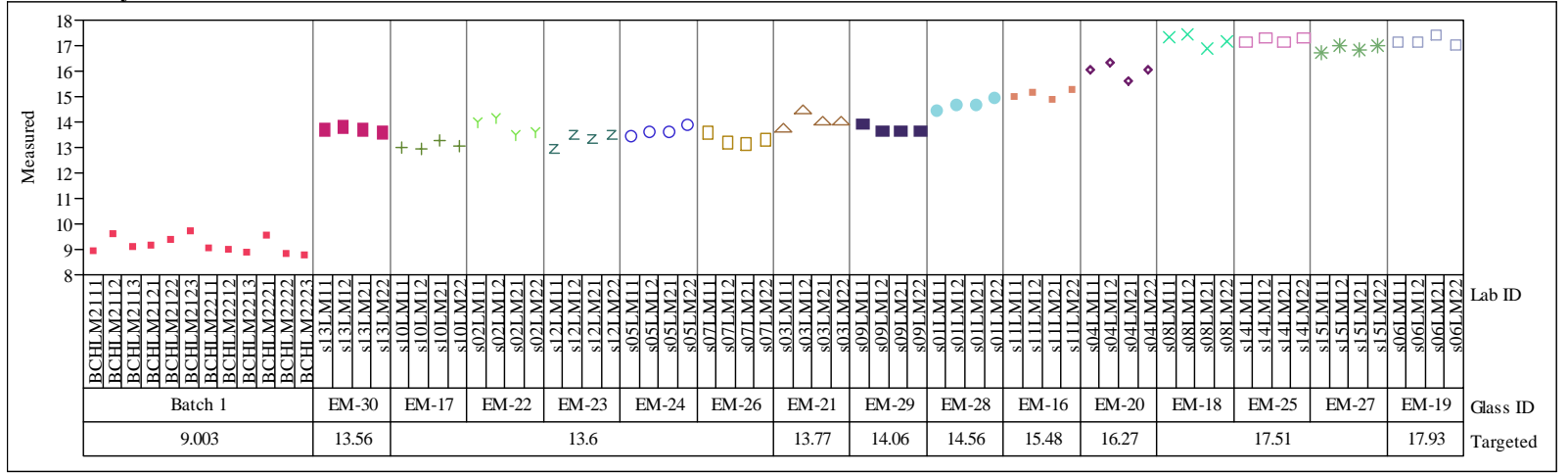




\section{Exhibit D2. Matrix 2A Sample Measurements by Lab ID within Glass ID by Oxide by Analytical Block}

Set $=2$, Oxide $=\mathrm{NiO}(\mathrm{wt} \%)$

Variability Chart for Measured

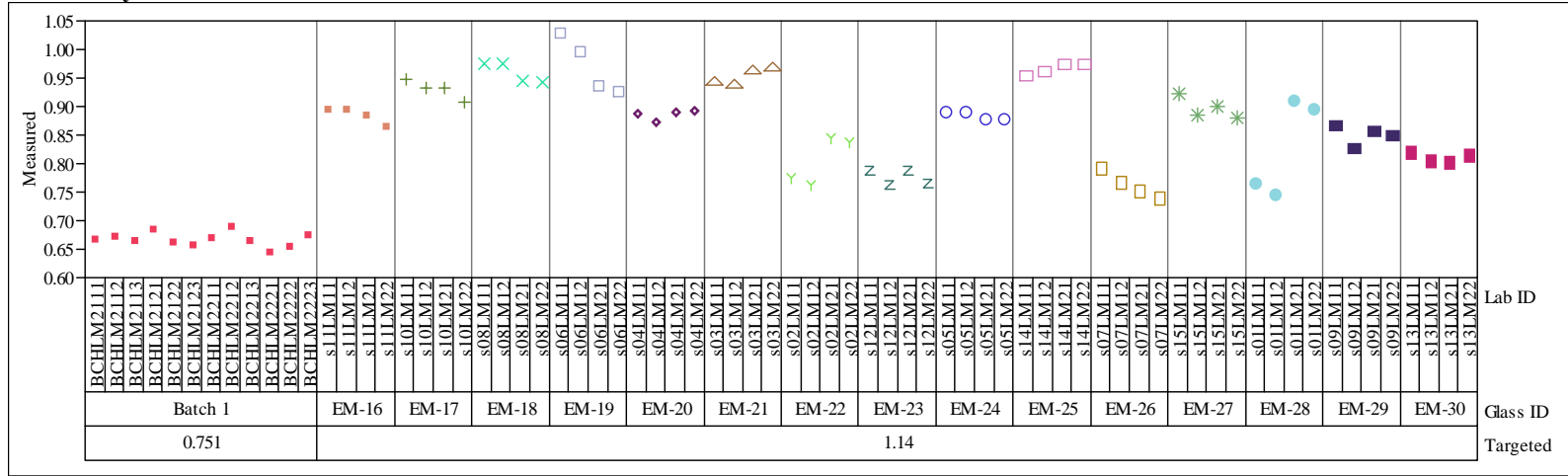

Set $=2$, Oxide $=\operatorname{PbO}(\mathbf{w t} \%)$

Variability Chart for Measured

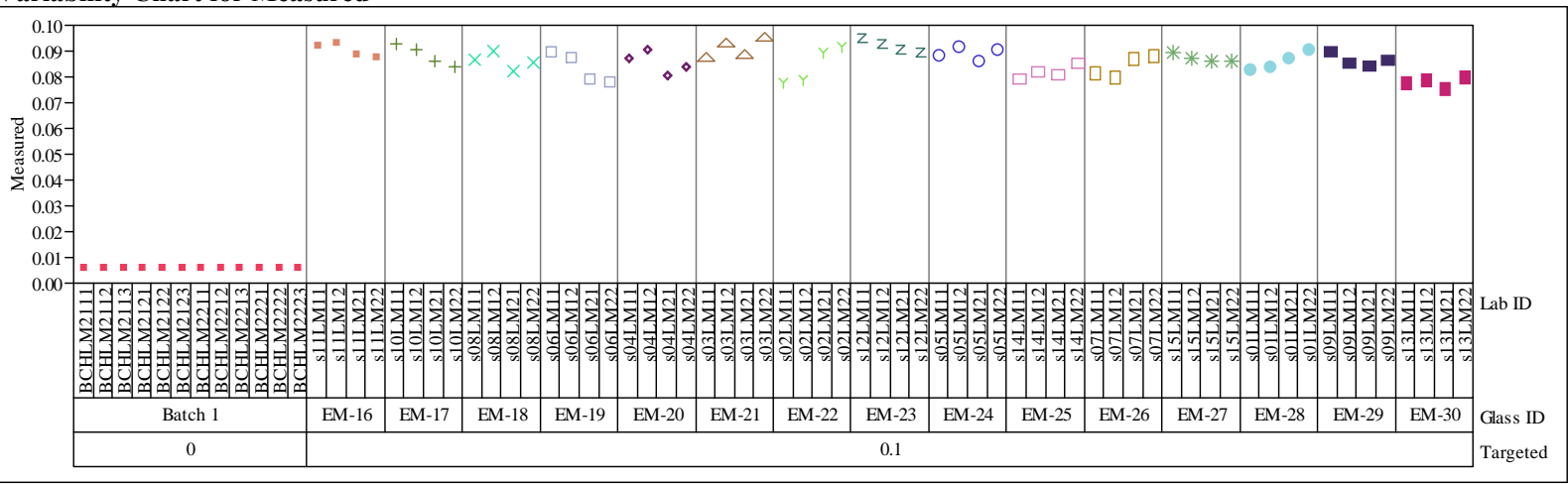

Set $=2$, Oxide $=\mathrm{SiO} 2(\mathrm{wt} \%)$

Variability Chart for Measured

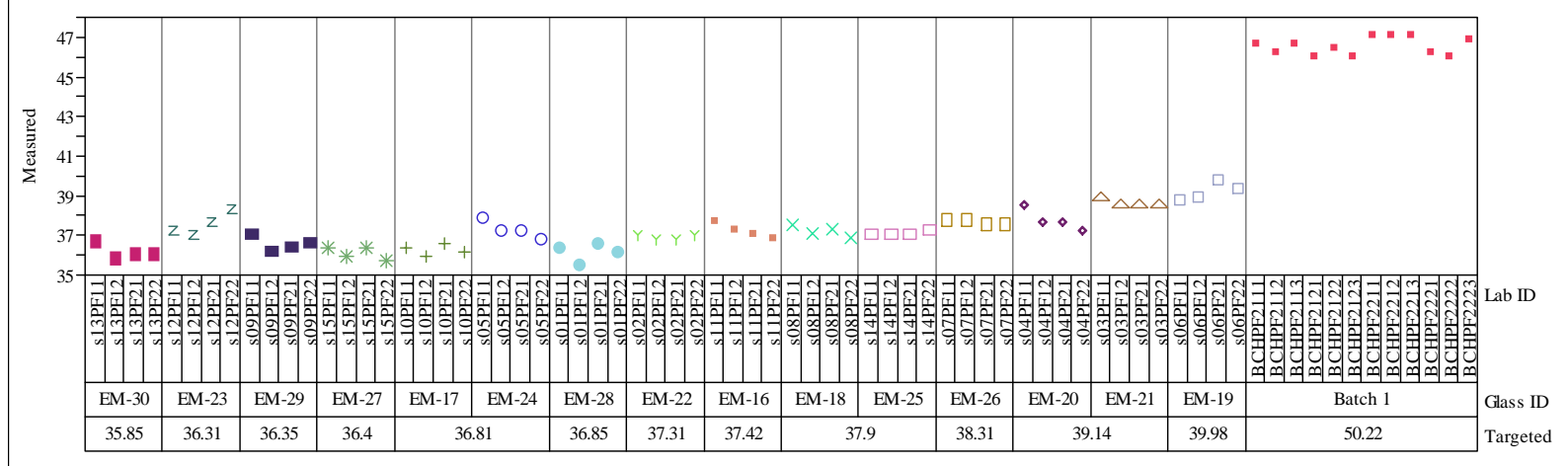




\section{Exhibit D2. Matrix 2A Sample Measurements by Lab ID within Glass ID} by Oxide by Analytical Block

Set $=2$, Oxide $=\mathrm{SO} 4(\mathrm{wt} \%)$

Variability Chart for Measured

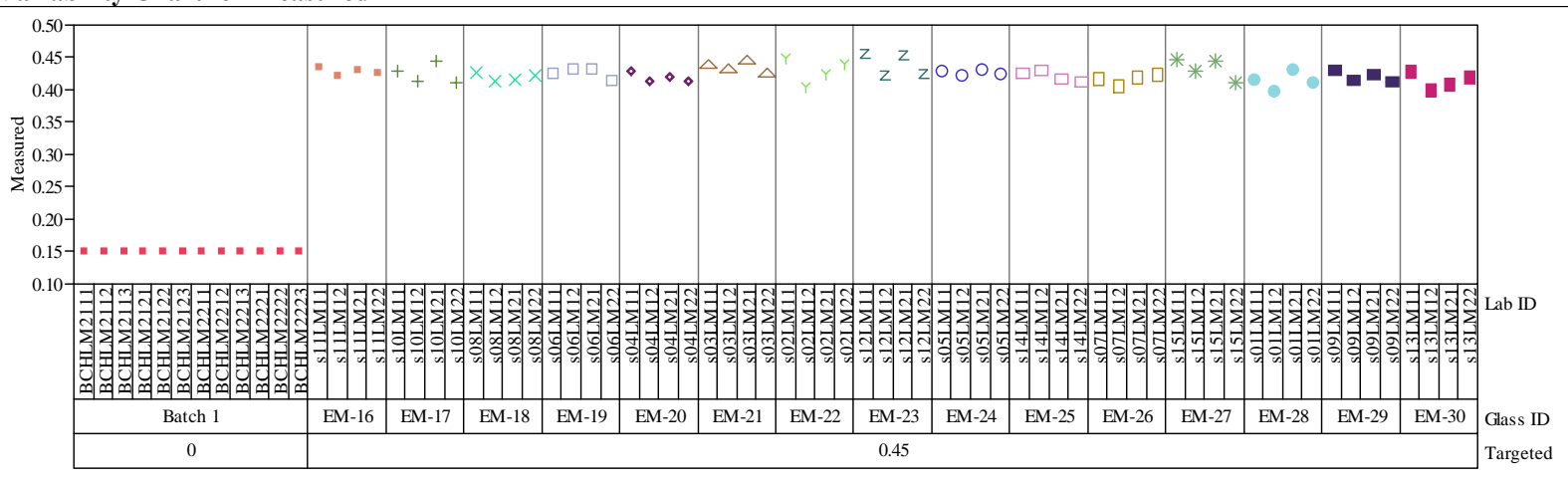

Set $=2$, Oxide $=$ TiO2 $(w t \%)$

Variability Chart for Measured

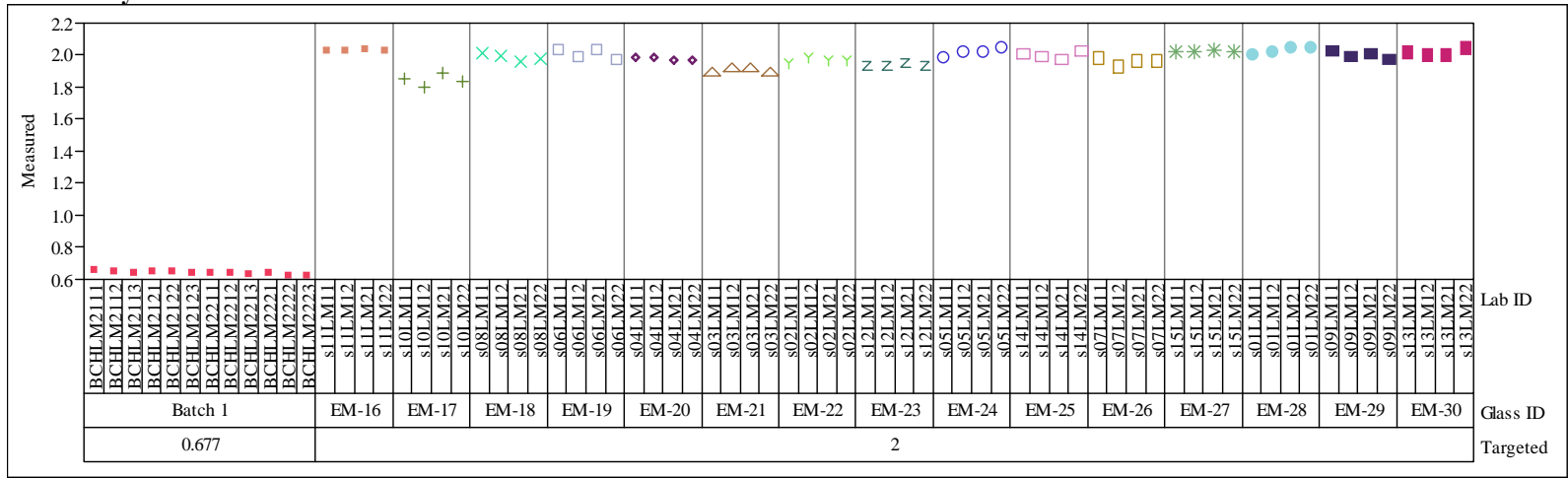

Set $=2$, Oxide $=\mathrm{ZnO}(\mathbf{w t} \%)$

Variability Chart for Measured

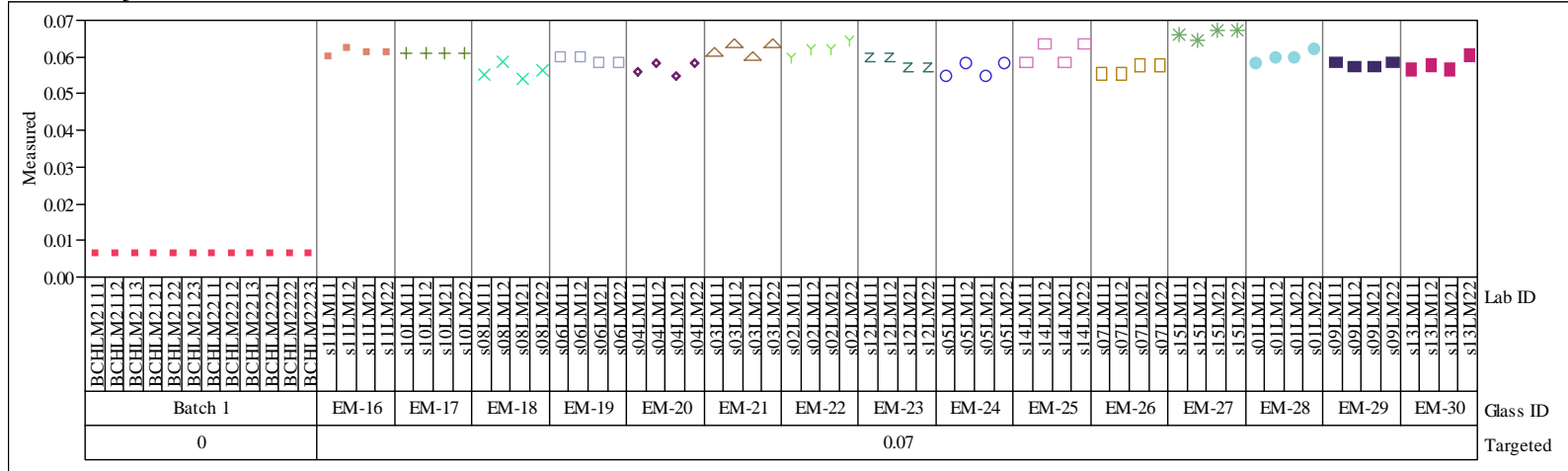


Exhibit D2. Matrix 2A Sample Measurements by Lab ID within Glass ID by Oxide by Analytical Block

Set $=2$, Oxide $=$ ZrO2 $\left(w^{\circ} \%\right)$

Variability Chart for Measured

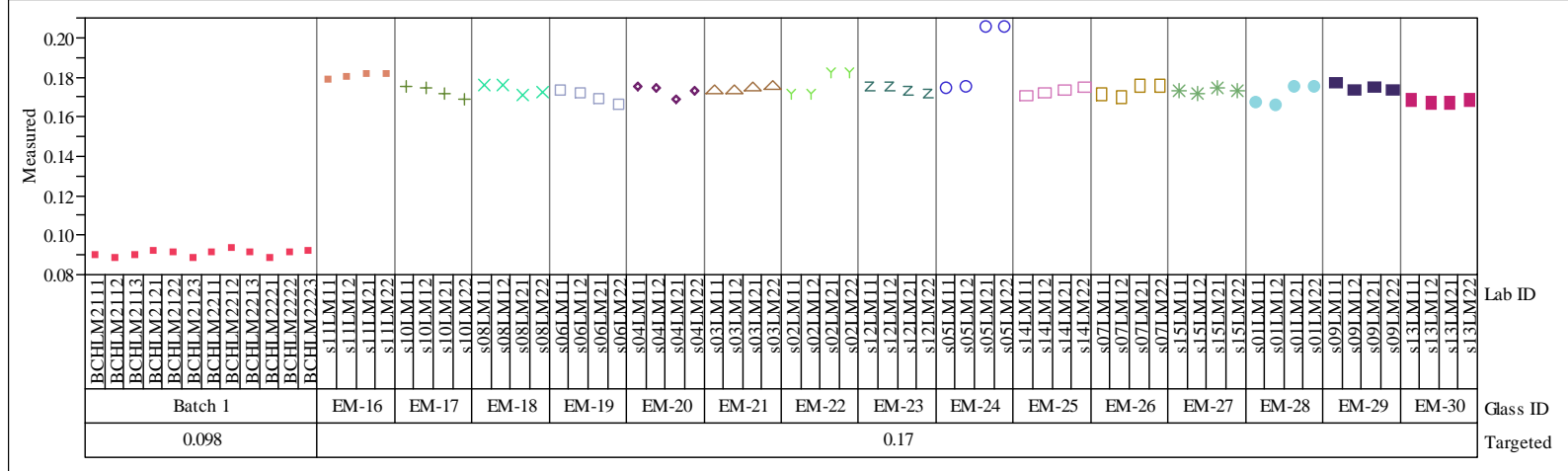


SRNL-STI-2009-00778, Revision 0

Exhibit D3. Batch 1 Sample Measurements by Block and Sub-Block by Oxide for Both Preparation Methods for the Matrix 2A Study

Oneway Analysis of Measured By Set/Block/Sub-Blk Oxide $=$ Al2O3 $\left(\mathrm{wt}^{\mathrm{O}} \%\right)$

Reference Value $=4.877 \mathrm{wt} \%$

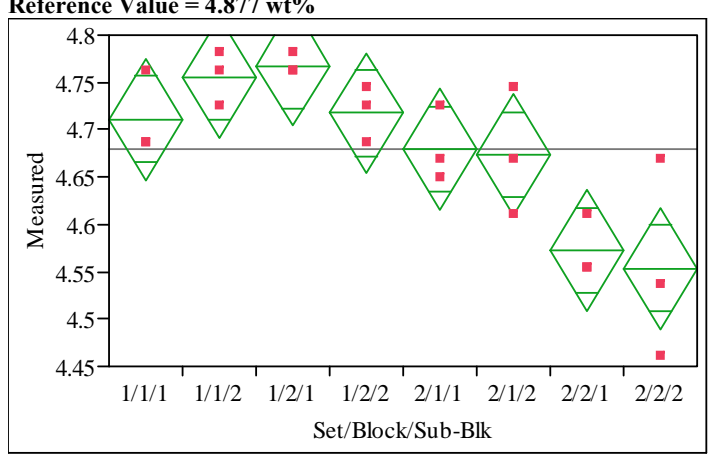

\section{Oneway Anova}

Summary of Fit

$\begin{array}{lr}\text { Rsquare } & 0.748777 \\ \text { Adj Rsquare } & 0.638867 \\ \text { Root Mean Square Error } & 0.052176 \\ \text { Mean of Response } & 4.678874 \\ \text { Observations (or Sum Wgts) } & 24\end{array}$

Analysis of Variance

$\begin{array}{llr}\text { Source } & \text { DF } & \begin{array}{r}\text { Sum of } \\ \text { Squares }\end{array}\end{array}$

Set/Block/Sub- $\quad 7 \quad 0.12982177$

Blk

Error

$\begin{array}{ll}16 & 0.04355657 \\ 23 & 0.17337834\end{array}$

$\begin{array}{ll}16 & 0.04355657 \\ 23 & 0.17337834\end{array}$

\section{Means for Oneway Anova}

$\begin{array}{lrrrrr}\text { Level Number } & \text { Mean } & \text { Std Error } & \text { Lower 95\% } & \text { Upper 95\% } \\ 1 / 1 / 1 & 3 & 4.71115 & 0.03012 & 4.6473 & 4.7750 \\ 1 / 1 / 2 & 3 & 4.75524 & 0.03012 & 4.6914 & 4.8191 \\ 1 / 2 / 1 & 3 & 4.76784 & 0.03012 & 4.7040 & 4.8317 \\ 1 / 2 / 2 & 3 & 4.71745 & 0.03012 & 4.6536 & 4.7813 \\ 2 / 1 / 1 & 3 & 4.67966 & 0.03012 & 4.6158 & 4.7435 \\ 2 / 1 / 2 & 3 & 4.67336 & 0.03012 & 4.6095 & 4.7372 \\ 2 / 2 / 1 & 3 & 4.57259 & 0.03012 & 4.5087 & 4.6364 \\ 2 / 2 / 2 & 3 & 4.55370 & 0.03012 & 4.4898 & 4.6176\end{array}$

Std Error uses a pooled estimate of error variance Oneway Analysis of Measured By Set/Block/Sub-Blk
Oxide=B2O3 (wt $\%$ )

Reference Value $=7.777 \mathrm{wt} \%$

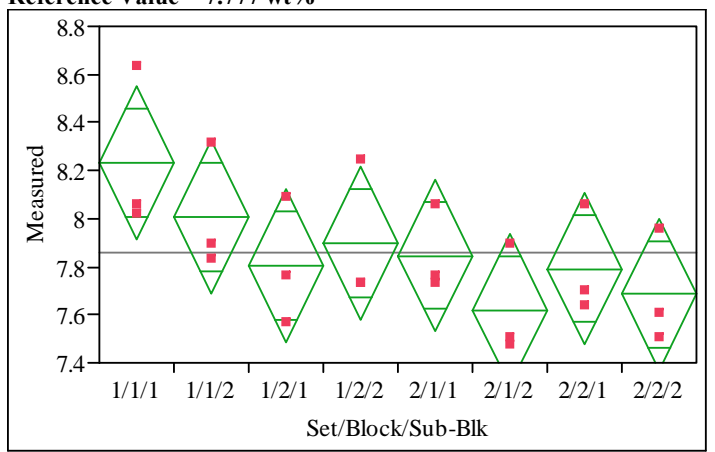

\section{Oneway Anova}

Summary of Fit

Adj Rsquare

Root Mean Square

Mean of Response

0.164713

0.25893

Observations (or Sum Wgts) $\quad 24$

Analysis of Variance

$\begin{array}{rrr}\text { Mean } & \text { F } & \text { Prob }> \\ \text { Square } & \text { Ratio } & \text { F } \\ 0.018546 & 6.8126 & 0.0008\end{array}$

Source

DF Sum of

$\begin{array}{llr}\text { Set/Block/Sub- } \quad 7 & 0.7733914\end{array}$

Blk

Error

$16 \quad 1.0727172$

Mean $F$ Prob >

Square Ratio

0.067045

Means for Oneway Anova

Level Number Mean Std Error Lower 95\% Upper 95\%

Level Number Mean Std Error Lower 95\% Upper 95\%

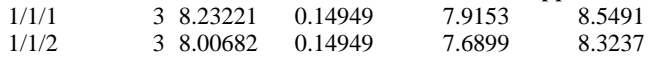

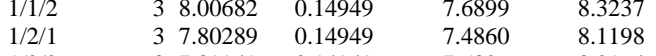

$\begin{array}{llllll}1 / 1 / 2 & 3 & 3.80289 & 0.14949 & 7.4860 & 8.1198 \\ 1 / 2 / 2 & 3 & 7.89949 & 0.14949 & 7.5826 & 8.2164\end{array}$

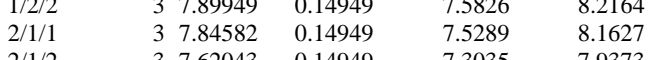

$\begin{array}{llllll}2 / 1 / 2 & 3 & 7.62043 & 0.14949 & 7.3035 & 7.9373 \\ 2 / 2 / 1 & 3 & 7.79216 & 0.14949 & 7.4752 & 8.1091\end{array}$

$\begin{array}{llllll}2 / 2 / 1 & 3 & 7.79216 & 0.14949 & 7.4752 & 8.1091 \\ 2 / 2 / 2 & 3 & 7.68483 & 0.14949 & 7.3679 & 8.0017\end{array}$

Std Error uses a pooled estimate of error variance
Oneway Analysis of Measured By Set/Block/Sub-Blk

Reference Value $=0.151 \mathrm{wt} \%$

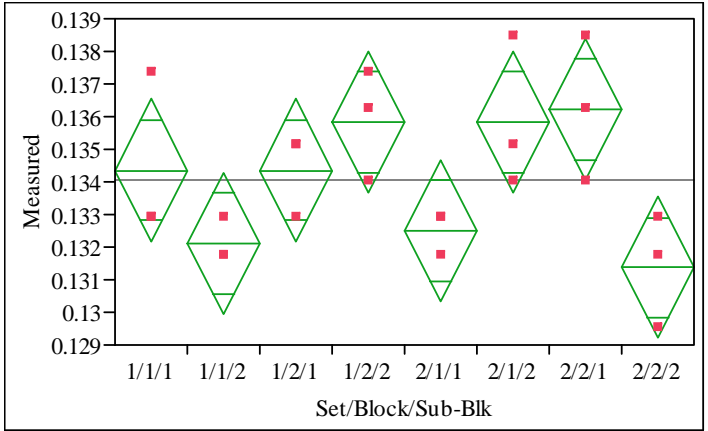

Oneway Anova

Summary of Fit

Rsquare

$\begin{array}{lr} & 0.414441 \\ \text { Root Mean Square Error } \quad 0.00178\end{array}$

$\begin{array}{lr}\text { Mean of Response } & 0.134073 \\ \text { Observations (or Sum Wgts) } & 24\end{array}$

Observations (or Sum Wgts)

Analysis of Variance

Source DF Sum of

$\begin{array}{rrr}\text { Squares } \\ \text { Set/Block/Sub- } \quad 7 & 0.00007376\end{array}$

Blk $\quad 16 \quad 0.00005069$

$\begin{array}{lll}\text { Error } & 16 & 0.00005069 \\ \text { C. Total } & 23 & 0.00012445\end{array}$

$\begin{array}{rrr}\text { Mean } & \text { F } & \text { Prob > } \\ \text { Square } & \text { Ratio } & \text { ( }\end{array}$ $0.000011 \quad 3.3255 \quad 0.0220$

3.168e-6

Means for Oneway Anova

Level Number Mean Std Error Lower 95\% Upper 95\%

$\begin{array}{llllll}1 / 1 / 1 & 3 & 0.134352 & 0.00103 & 0.13217 & 0.13653\end{array}$

$\begin{array}{llllll}1 / 1 / 2 & 3 & 0.132119 & 0.00103 & 0.12994 & 0.13430\end{array}$

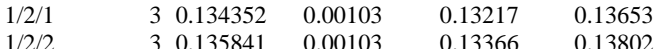

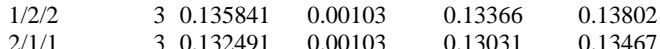

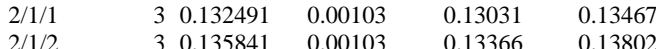

$\begin{array}{llllll}2 / 1 / 2 & 3 & 0.135841 & 0.00103 & 0.13366 & 0.13802 \\ 2 / 2 / 1 & 3 & 0.136213 & 0.00103 & 0.13403 & 0.13839\end{array}$

$\begin{array}{llllll}2 / 2 / 2 & 3 & 0.131375 & 0.00103 & 0.12920 & 0.13355\end{array}$

Std Error uses a pooled estimate of error variance 
SRNL-STI-2009-00778, Revision 0

Exhibit D3. Batch 1 Sample Measurements by Block and Sub-Block by Oxide for Both Preparation Methods for the Matrix 2A Study

Oneway Analysis of Measured By Set/Block/Sub-Blk Oxide $=\mathrm{CaO}\left(\mathrm{wt}_{\mathrm{T}} \%\right)$

Reference Value $=1.220 \mathrm{wt} \%$

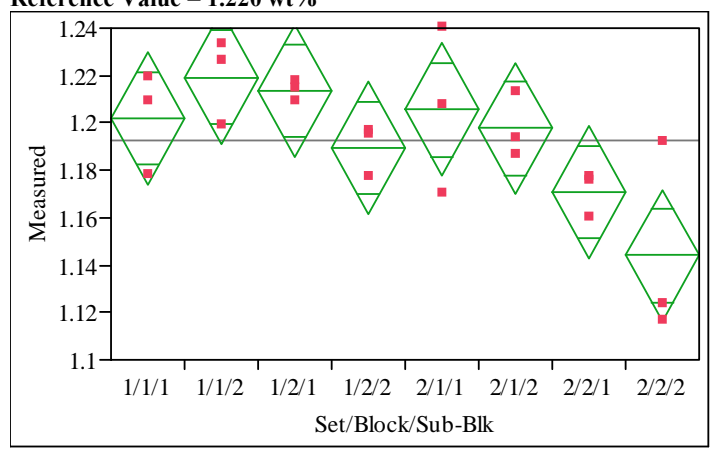

\section{Oneway Anova}

Summary of

Rsquare $\quad 0.607329$

Adj Rsquare $\quad 0.435535$

$\begin{array}{lr}\text { Root Mean Square Error } & 0.022765 \\ \text { Mean of Response } & 1.19276\end{array}$

Observations (or Sum Wgts)

24

Analysis of Variance

$\begin{array}{lll}\text { Analysis of } & \text { Variance } \\ \text { Source DF } & \text { Sum of }\end{array}$

$\begin{array}{lrr}\text { Source } & \text { DF } & \begin{array}{r}\text { Sum of } \\ \text { Squares }\end{array} \\ \text { Set/Block/Sub- } & 7 & 0.01282456\end{array}$

Blk

Error

$\begin{array}{ll}16 & 0.00829177\end{array}$

Means for Oneway Anova

Level Number Mean Std Error Lower 95\% Upper 95\%

$\begin{array}{llllll}\text { Level Num } & 3 & 1.20191 & 0.01314 & 1.1741 & 1.2298\end{array}$

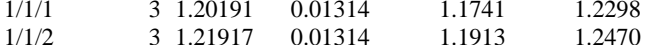

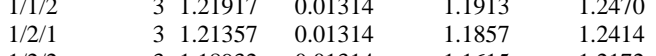

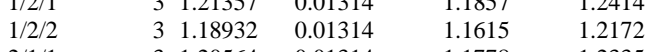

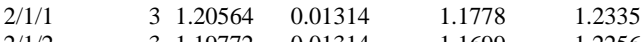

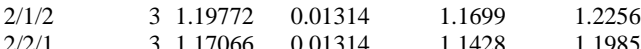

$\begin{array}{llllll}2 / 2 / 2 & 3 & 1.14408 & 0.01314 & 1.1162 & 1.1719\end{array}$

Std Error uses a pooled estimate of error variance
Oneway Analysis of Measured By Set/Block/Sub-Blk
Oxide $=$ Ce2O3 (wt $\%)$

Reference Value $=0.0 \mathrm{wt} \%$

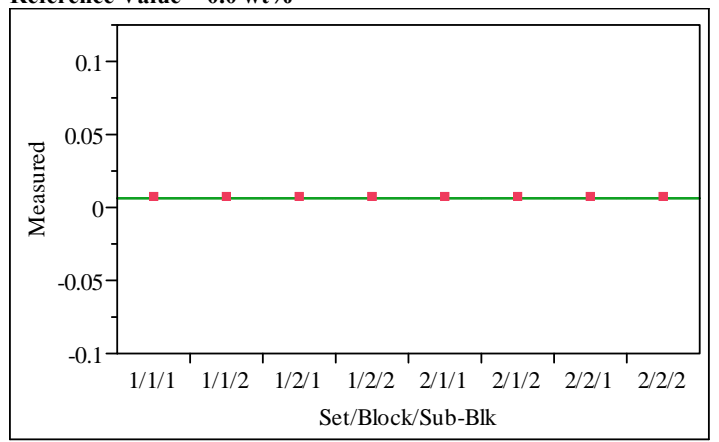

Oneway Anova

Summary of Fit

Rsquare

$\begin{array}{ll}\text { Root Mean Square Error } & \\ \text { Mean of Response } & 0.00585\end{array}$

Observations (or Sum Wgts) 24

Analysis of Variance
Source

Set/Block/Sub-

Error $\quad 16$

$$
\begin{array}{r}
\text { Sum of } \\
\text { Squares } \\
0
\end{array}
$$$$
23
$$

Means for Oneway Anova

Level Number Mean Std Error Lower 95\% Upper 95\%

$\begin{array}{llllll}1 / 1 / 1 & 3 & 0.005857 & 0 & 0.00586 & 0.00586\end{array}$

$\begin{array}{llllll}1 / 1 / 2 & 3 & 0.005857 & 0 & 0.00586 & 0.00586\end{array}$

$\begin{array}{llllll}1 / 2 / 1 & 3 & 0.005857 & 0 & 0.00586 & 0.00586\end{array}$

$\begin{array}{llllll}1 / 2 / 2 & 3 & 0.005857 & 0 & 0.00586 & 0.00586\end{array}$

$\begin{array}{llllll}2 / 1 / 1 & 3 & 0.005857 & 0 & 0.00586 & 0.00586\end{array}$

$\begin{array}{llllll}2 / 1 / 2 & 3 & 0.005857 & 0 & 0.00586 & 0.00586\end{array}$

$\begin{array}{llllll}2 / 2 / 1 & 3 & 0.005857 & 0 & 0.00586 & 0.00586 \\ 2 / 2 / 2 & 3 & 0.005857 & 0 & 0.00586 & 0.00586\end{array}$

Std Error uses a pooled estimate of error variance
Oneway Analysis of Measured By Set/Block/Sub-Blk

Oxide $=\mathbf{C r 2 O 3}(\mathbf{w t} \%)$

Reference Value $=0.107 \mathrm{wt} \%$

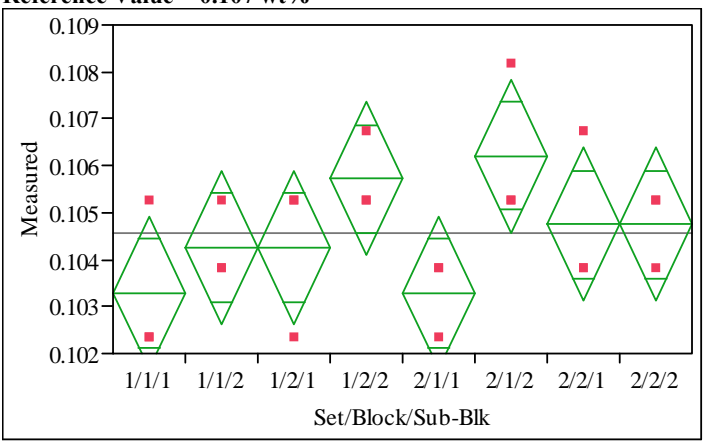

Oneway Anova

Summary of Fit

$\begin{array}{lr}\text { Rsquare } & 0.443478 \\ \text { Adj Rsquare } & 0.2\end{array}$

Root Mean Square Error $\quad 0.001334$ Mean of Response $\quad 0.104565$

Observations (or Sum Wgs)

Analysis of Variance

$\begin{array}{lrrrrr}\text { Source } & \text { DF } & \begin{array}{r}\text { Sum of } \\ \text { Squares }\end{array} & \begin{array}{r}\text { Mean } \\ \text { Square }\end{array} & \begin{array}{r}\text { F } \\ \text { Ratio }\end{array} & \begin{array}{r}\text { Prob }> \\ \text { F }\end{array} \\ \text { Set/Block/Sub- } & 7 & 0.00002270 & 3.2426 \mathrm{e}-6 & 1.8214 & 0.1517 \\ \text { Blk } & & & & & \\ \text { Error } & 16 & 0.00002848 & 1.7802 \mathrm{e}-6 & & \\ \text { C. Total } & 23 & 0.00005118 & & & \end{array}$

Means for Oneway Anova

Level Number Mean Std Error Lower 95\% Upper 95\% $\begin{array}{llllll}1 / 1 / 1 & 3 & 0.103286 & 0.00077 & 0.10165 & 0.10492\end{array}$

$\begin{array}{llllll}1 / 1 / 2 & 3 & 0.104261 & 0.00077 & 0.10263 & 0.10589\end{array}$

$\begin{array}{llllll}1 / 2 / 1 & 3 & 0.104261 & 0.00077 & 0.10263 & 0.10589\end{array}$

$\begin{array}{llllll}1 / 2 / 2 & 3 & 0.105722 & 0.00077 & 0.10409 & 0.10736\end{array}$

$\begin{array}{llllll}2 / 1 / 1 & 3 & 0.103286 & 0.00077 & 0.10165 & 0.10492\end{array}$

$\begin{array}{llllll}2 / 1 / 2 & 3 & 0.106210 & 0.00077 & 0.10458 & 0.10784\end{array}$

$\begin{array}{llllll}2 / 2 / 1 & 3 & 0.104748 & 0.00077 & 0.10311 & 0.10638\end{array}$

$\begin{array}{llllll}2 / 2 / 2 & 3 & 0.104748 & 0.00077 & 0.10311 & 0.10638\end{array}$

Std Error uses a pooled estimate of error variance 
SRNL-STI-2009-00778, Revision 0

Exhibit D3. Batch 1 Sample Measurements by Block and Sub-Block by Oxide for Both Preparation Methods for the Matrix 2A Study

Oneway Analysis of Measured By Set/Block/Sub-Blk Oxide $=\mathrm{CuO}(\mathrm{wt} \%)$

Reference Value $=0.399 \mathrm{wt} \%$

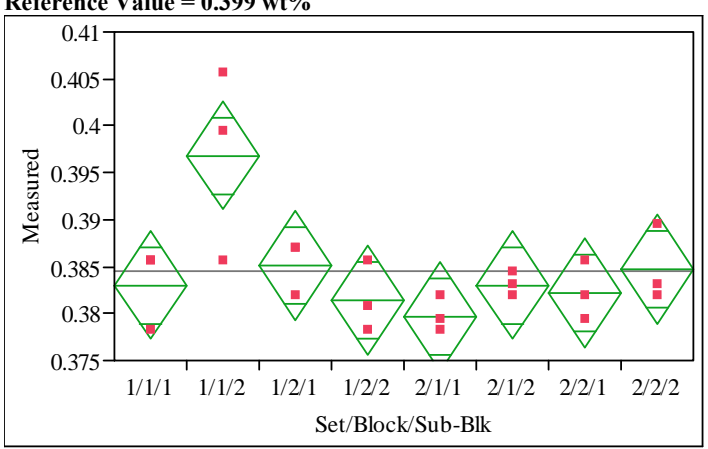

Oneway Anova
Summary of Fit

$\begin{array}{lrl}\text { Rsquare } & 0.62069 \\ \text { Adj } & 0.454741\end{array}$

0.454741
Root Mean Square Error $\quad 0.004719$

$\begin{array}{lr}\text { Mean of Response } & 0.384511 \\ \text { Observations (or Sum Wgts) } & 24\end{array}$

Analysis of Variance

$\begin{array}{lrr}\text { Analysis of Variance } & & \text { Sum of } \\ \text { Source } & \text { DF } & \text { Squares }\end{array}$

Squares
Set/Block/Sub- $\quad 7 \quad 0.00058293$

$\begin{array}{lll}\text { Blk } & & \\ \text { Error } & 16 & 0.00035623\end{array}$

C. Total

$\begin{array}{ll}16 & 0.00035623 \\ 23 & 0.00093916\end{array}$

Means for Oneway Anova

Level Number Mean Std Error Lower 95\% Upper 95\%

$\begin{array}{llrrrr}1 / 1 / 1 & 3 & 0.383051 & 0.00272 & 0.37728 & 0.38883\end{array}$

$\begin{array}{llllll}11 / 1 / 2 & 3 & 0.396821 & 0.00272 & 0.39105 & 0.40260\end{array}$

$\begin{array}{llllll}1 / 2 / 1 & 3 & 0.385137 & 0.00272 & 0.37936 & 0.39091\end{array}$

$\begin{array}{llllll}1 / 2 / 2 & 3 & 0.381382 & 0.00272 & 0.37561 & 0.38716\end{array}$

$\begin{array}{llllll}2 / 1 / 1 & 3 & 0.379713 & 0.00272 & 0.37394 & 0.38549\end{array}$

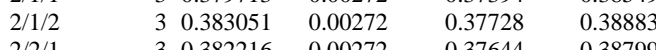

$\begin{array}{llllll}2 / 2 / 1 & 3 & 0.382216 & 0.00272 & 0.37644 & 0.38799 \\ 2 / 2 / 2 & 3 & 0.384720 & 0.00272 & 0.37894 & 0.39050\end{array}$

Std Error uses a pooled estimate of error variance
Oneway Analysis of Measured By Set/Block/Sub-Blk

Oxide $=\mathrm{Fe} 2 \mathrm{O} 3(\mathrm{wt} \%)$

Reference Value $=12.839 \mathrm{wt} \%$

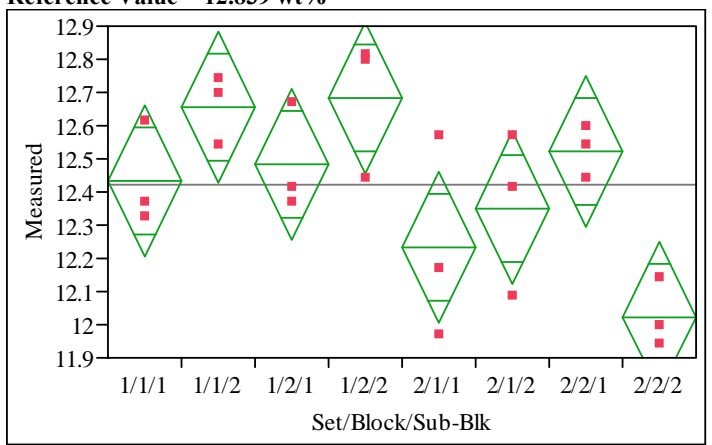

\section{Oneway Anova}

Summary of

Rsquare $\quad 0.644733$

Adj Rsquare $\quad 0.489304$

Root Mean Square Error $\quad 0.186273$
Mean of Response

\begin{tabular}{lr} 
Observations (or Sum Wgts) & 24 \\
\hline
\end{tabular}

Analysis of Variance

$\begin{array}{lll}\text { Analysis of Variance } & \\ \text { Source DF } & \text { DFm of }\end{array}$

$\begin{array}{lrr}\text { Source } & \text { DF } & \begin{array}{r}\text { Sum of } \\ \text { Squares }\end{array} \\ \text { Set/Block/Sub- } & 7 & 1.0074998\end{array}$

Set/Block/Sub- 7

Error

$\begin{array}{ll}16 & 0.5551618 \\ 23 & 1.5626617\end{array}$

C. Total

Means for Oneway Anova

Level Number Mean Std Error Lower 95\% Upper 95\%

$\begin{array}{llllll}1 / 1 / 1 & 3 & 12.4336 & 0.10754 & 12.206 & 12.662\end{array}$

$\begin{array}{llllll}1 / 1 / 2 & 3 & 12.6576 & 0.10754 & 12.430 & 12.886 \\ 1 / 2 / 1 & 3 & 12.4813 & 0.10754 & 12.253 & 12.709\end{array}$

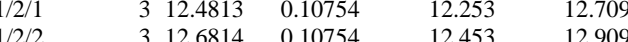

$1 / 2 / 2$

$2 / 1 / 1$

$2 / 2 / 1$

$\begin{array}{lll}3 & 12.6814 & 0.10754 \\ 3 & 12.2335 & 0.10754\end{array}$

$\begin{array}{lll}3 & 12.2335 & 0.10754 \\ 3 & 12.3526 & 0.10754\end{array}$

$\begin{array}{lll}3 & 12.3526 & 0.10754 \\ 3 & 12.5242 & 0.10754 \\ 3 & 12.0238 & 0.1075\end{array}$

12.453

12.005
12.125

12.296

$11.796-12.752$

Std Error uses a pooled estimate of error variance
Oneway Analysis of Measured By Set/Block/Sub-Blk

Oxide $=\mathrm{La} 2 \mathrm{O} 3(\mathrm{wt} \%)$

Reference Value $=0.0 \mathrm{wt} \%$

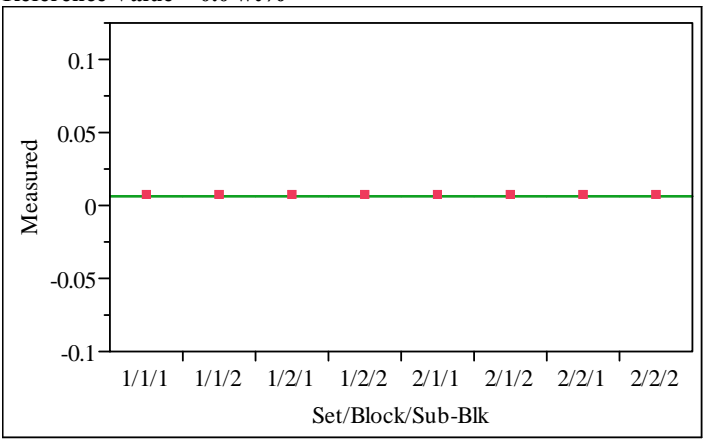

Oneway Anova

Summary of Fit

Rsquare

Adj Rsquare

$\begin{array}{lr}\text { Root Mean Square Error } & 0 \\ \text { Mean of Response } & 0.005864\end{array}$

Observations (or Sum Wgts)

Analysis of Variance

$\begin{array}{llrrrr}\text { Source } & \text { DF } & \begin{array}{r}\text { Sum of } \\ \text { Squares }\end{array} & \begin{array}{r}\text { Mean } \\ \text { Square }\end{array} & \begin{array}{r}F \\ \text { Ratio }\end{array} & \text { Prob }>\end{array}$

Square Ratio $r$ Prob

$\begin{array}{rrrl}\text { Square } & \text { Ratio } & \text { F } & \text { Se/Block/Sub- } \\ 0.143929 & 4.1481 & 0.0088 & \text { Set }\end{array}$

Blk

Error
C. Total

16
23

Means for Oneway Anova

Level Number Mean Std Error Lower 95\% Upper 95\%

$\begin{array}{llllll}1 / 1 / 1 & 3 & 0.005864 & 0 & 0.00586 & 0.00586 \\ 1 / 1 / 2 & 3 & 0.005864 & 0 & 0.00586 & 0.00586\end{array}$

$\begin{array}{llllll}1 / 1 / 2 & 3 & 0.005864 & 0 & 0.00586 & 0.00586\end{array}$

$\begin{array}{llllll}1 / 2 / 1 & 3 & 0.005864 & 0 & 0.00586 & 0.00586\end{array}$

$\begin{array}{llllll}1 / 2 / 2 & 3 & 0.005864 & 0 & 0.00586 & 0.00586\end{array}$

$\begin{array}{llllll}2 / 1 / 1 & 3 & 0.005864 & 0 & 0.00586 & 0.00586 \\ 21 / 2 & 3 & 0.005864 & 0 & 0.00586 & 0.00586\end{array}$

$\begin{array}{llllll}2 / 1 / 2 & 3 & 0.005864 & 0 & 0.00586 & 0.00586\end{array}$

$\begin{array}{llllll}2 / 2 / 1 & 3 & 0.005864 & 0 & 0.00586 & 0.00586 \\ 2 / 2 / 2 & 3 & 0.005864 & 0 & 0.00586 & 0.00586\end{array}$

Std Error uses a pooled estimate of error variance 
SRNL-STI-2009-00778, Revision 0

Exhibit D3. Batch 1 Sample Measurements by Block and Sub-Block by Oxide for Both Preparation Methods for the Matrix 2A Study

Oneway Analysis of Measured By Set/Block/Sub-Blk Oxide $=$ Li2O $($ wt $\%)$

Reference Value $=4.429 \mathrm{wt} \%$

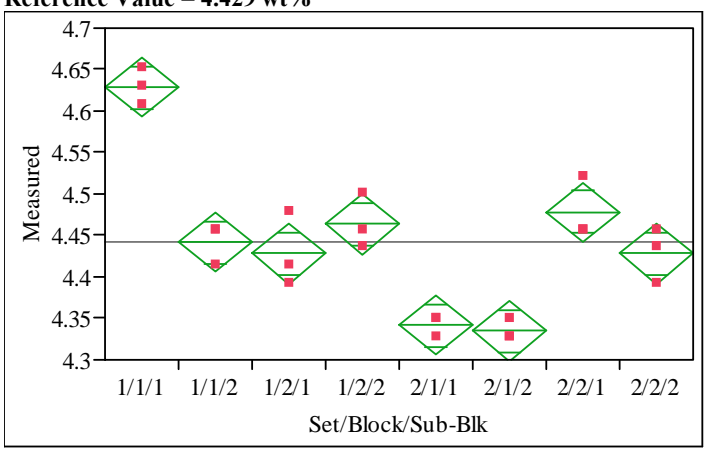

Oneway Anova

Summary o

Rsquare $\quad 0.926762$

Adj Rsquare $\quad 0.894721$

Root Mean Square Error $\quad 0.02948$

Observations (or Sum Wgts) $\quad 24$

Analysis of Variance

Source DF Sum of

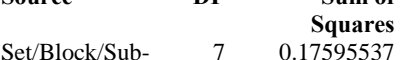

Blk

Error

$\begin{array}{ll}16 & 0.01390494 \\ 23 & 0.18986030\end{array}$

Square
0.02513628 .9238

0.000869

\section{Means for Oneway Anova}

Level Number Mean Std Error Lower 95\% Upper 95\%

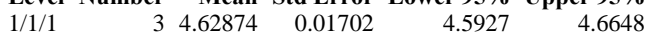

$\begin{array}{llllll}1 / 1 / 2 & 3 & 4.44215 & 0.01702 & 4.4061 & 4.4782\end{array}$

$\begin{array}{llllll}1 / 2 / 1 & 3 & 4.42780 & 0.01702 & 4.3917 & 4.4639\end{array}$

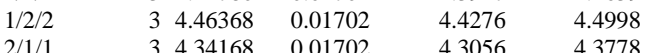

$\begin{array}{llllll}1 / 2 / 2 & 3 & 4.34168 & 0.01702 & 4.3056 & 4.3778 \\ 2 / 1 / 2 & 3 & 4.33451 & 0.01702 & 4.2984 & 4.3706\end{array}$

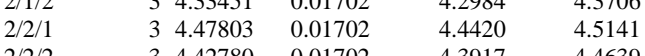

Std Error uses a pooled estimate of error variance
Oneway Analysis of Measured By Set/Block/Sub-Blk Oxide $=\operatorname{MaO}(\mathbf{w t} \%)$

Reference Value $=1.419 \mathrm{wt} \%$

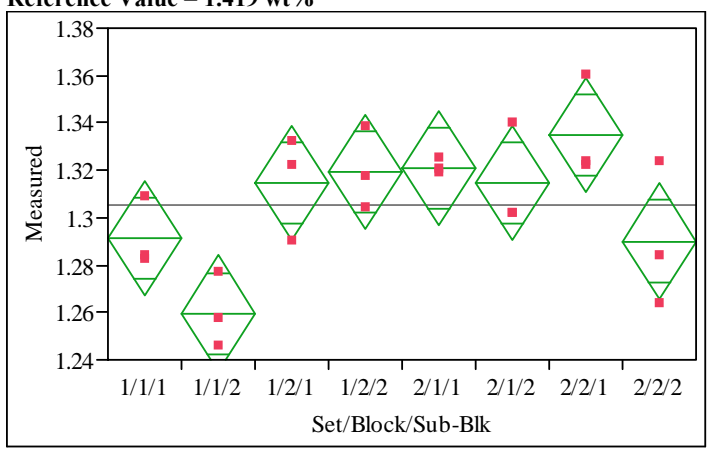

\section{Oneway Anova}

Summary of

Rsquare $\quad 0.656409$

$\begin{array}{ll}\text { Adj Rsquare } & 0.506088 \\ \text { Root Mean Square Error } & 0.019804\end{array}$

$\begin{array}{ll}\text { Root Mean Square Error } & 0.019804 \\ \text { Mean of Response } & 1.305704\end{array}$

$\begin{array}{lr}\text { Observations (or Sum Wgts) } & 24\end{array}$

Analysis of Variance

Source DF Sum of

Set/Block/Sub-

Blk

Error
C. Total

$\begin{array}{ll}16 & 0.00627541 \\ 23 & 0.01826420\end{array}$

Means for Oneway Anova

Level Number Mean Std Error Lower 95\% Upper 95\%

$\begin{array}{llllrr}1 / 1 / 1 & 3 & 1.29126 & 0.01143 & 1.2670 & 1.3155 \\ 1 / 1 / 2 & 3 & 1.25976 & 0.01143 & 1.2355 & 1.2840\end{array}$

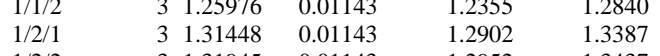

$1 / 2 / 2$

$1 / 2 / 2$
$2 / 1 / 1$
$2 / 1 / 2$
$2 / 2 / 1$

$2 / 2 / 1$

$\begin{array}{lll}3 & 1.31945 & 0.01143\end{array}$

$\begin{array}{lll}3 & 1.32111 & 0.01143 \\ 3 & 1.31448 & 0.01143\end{array}$

$\begin{array}{lll}3 & 1.31448 & 0.01143 \\ 3 & 1.33493 & 0.01143 \\ 3 & 1.29016 & 0.0143\end{array}$

$\begin{array}{lll}3 & 1.29016 & 0.01143\end{array}$

1.2952

$1.2969-1.3454$

$\begin{array}{ll}1.2902 & 1.3387 \\ 1.3107 & 1.3592\end{array}$

Std Error uses a pooled estimate of error variance

1.3144 Oneway Analysis of Measured By Set/Block/Sub-Blk

Reference Value $=1.726 \mathrm{wt} \%$

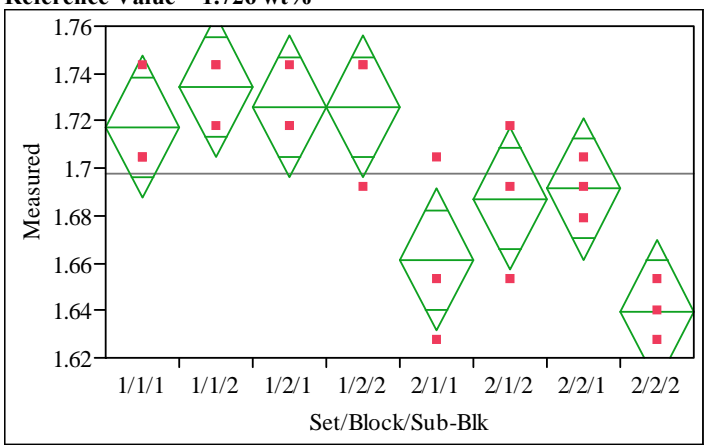

\section{Oneway Anova}

Summary of Fit

$\begin{array}{ll}\text { Rsquare } & 0.718954 \\ \text { Adj } & 0.595997\end{array}$

Adj Rsquare

Mean of Response $\quad 1.024792$

Observations (or Sum Wgts)

Analysis of Variance

Source

Source

DF

Sum of Squares
0.02445223

Set/Block/Sub-

Elk

Error

$\begin{array}{ll}16 & 0.00955860 \\ 23 & 0.03401083\end{array}$

Mean F Prob > $\begin{array}{rrr}\text { Square } & \text { Ratio } & \mathbf{F} \\ 0.003493 & 5.8472 & 0.0017\end{array}$

0.000597

Means for Oneway Anova

Level Number Mean Std Error Lower 95\% Upper 95\%

$\begin{array}{llllll}1 / 1 / 1 & 3 & 1.71730 & 0.01411 & 1.6874 & 1.7472\end{array}$

$\begin{array}{llllll}1 / 1 / 1 & 3 & 1.7730 & 0.0141 & 1.6874 & 1.7472 \\ 1 / 1 / 2 & 3 & 1.73451 & 0.01411 & 1.7046 & 1.7644\end{array}$

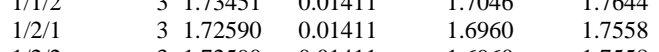

$\begin{array}{llllll}1 / 2 / 2 & 3 & 1.72590 & 0.01411 & 1.6960 & 1.7558\end{array}$

$2 / 1 / 1$

$2 / 1 / 2$

$2 / 2 / 1$

$\begin{array}{lll}3 & 1.68717 & 0.01411\end{array}$

$\begin{array}{llll}3 & 1.69147 & 0.01411\end{array}$

$\begin{array}{ll}1.6314 & 1.6913 \\ 1.6573 & 1.7171\end{array}$

Std Error uses a pooled estimate of error variance 
SRNL-STI-2009-00778, Revision 0

Exhibit D3. Batch 1 Sample Measurements by Block and Sub-Block by Oxide for Both Preparation Methods for the Matrix 2A Study

Oneway Analysis of Measured By Set/Block/Sub-Blk Oxide $=\mathrm{Na} 2 \mathrm{O}(\mathrm{wt} \%)$

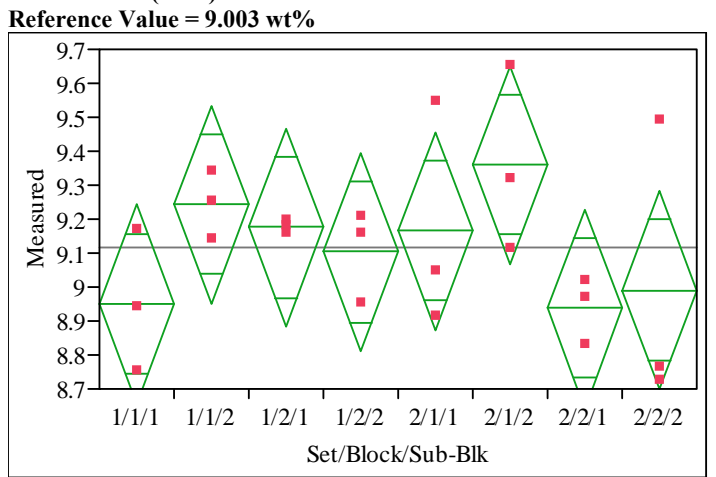

\section{Oneway Anova}

Summary of Fi

Rsquare

Adj Rsquare

Root Mean Square Error $\quad 0.051959$

Mean of Resporse Eiror $\quad 0.238406$

\begin{tabular}{lr} 
Observations (or Sum Wgts) & 9.11585 \\
\hline
\end{tabular}

Analysis of Variance

Source $\quad$ DF $\quad$ Sum of

$\begin{array}{llr}\text { Set/Block/Sub- } \quad 7 & 0.4695094\end{array}$

Blk

$\begin{array}{lll}\text { Error } & 16 & 0.9094000 \\ \text { C. Total } & 23 & 1.3789094\end{array}$

Mean F Prob $>$ Source DF

$\begin{array}{rrrr}\text { Square } & \text { Ratio } & \mathbf{F} & \\ 0.067073 & 1.1801 & 0.3671 & \text { Set/Block/Sub- } \quad 7\end{array}$

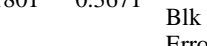

Means for Oneway Anova

Level Number Mean Std Error Lower 95\% Upper 95\%

$\begin{array}{llllll}1 / 1 / 1 & 3 & 8.95072 & 0.13764 & 8.6589 & 9.2425\end{array}$

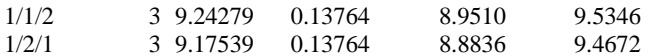

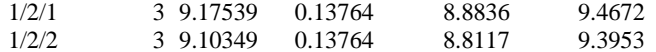

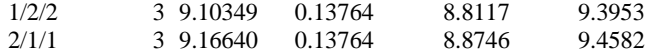

$\begin{array}{llllll}2 / 1 / 1 & 3 & 9.16640 & 0.13764 & 8.8746 & 9.4582 \\ 2 / 1 / 2 & 3 & 9.35961 & 0.13764 & 9.0678 & 9.6514\end{array}$

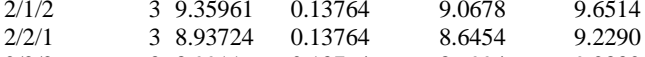

St/2 $38.99116 \quad 0.13764-8.6994$

Std Error uses a pooled estimate of error variance
Error

C. Total

$\begin{array}{ll}16 & 0.00171749 \\ 23 & 0.00360871\end{array}$

\section{Means for Oneway Anova}

Level Number Mean Std Error Lower 95\% Upper 95\%

Oneway Analysis of Measured By Set/Block/Sub-Blk

Reference Value $=0.751 \mathrm{wt} \%$

$\begin{array}{lllllr}1 / 1 / 1 & 3 & 0.656610 & 0.00598 & 0.64393 & 0.66929\end{array}$

$\begin{array}{llllll}1 / 1 / 2 & 3 & 0.660852 & 0.00598 & 0.64817 & 0.67353 \\ 1 / 2 / 2 & 3 & 0.663821 & 0.00598 & 0.65114 & 0.67650\end{array}$

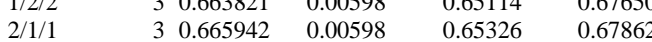

$\begin{array}{llllll}2 / 1 / 1 & 3 & 0.665942 & 0.00598 & 0.65326 & 0.67862 \\ 2 / 1 / 2 & 3 & 0.665942 & 0.00598 & 0.65326 & 0.67862\end{array}$

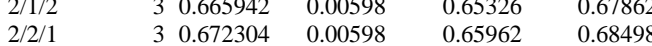

$2 / 2 / 2$

$\begin{array}{lll}3 & 0.672304 & 0.00598 \\ & 0.655762 & 0.00598\end{array}$

0.64308

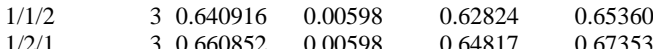

Oneway Analysis of Measured By Set/Block/Sub-Blk

Reference Value $=0.0 \mathrm{wt} \%$

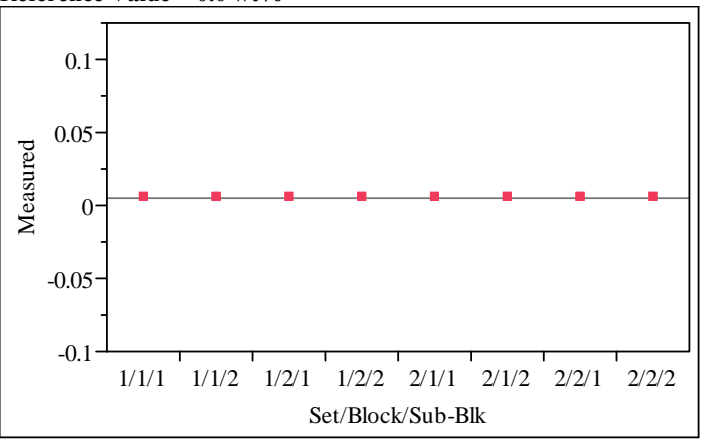

\section{Oneway Anova}

Summary of Fit

$\begin{array}{lr}\text { Rsquare } & 4 \\ \text { Adj Rsquare } & 5.3125 \\ \text { Root Mean Square Error } & \text {. } \\ \text { Mean of Response } & 0.005386 \\ \text { Observations (or Sum Wgts) } & 24\end{array}$

Analysis of Variance

Source DF Sum of

$\begin{array}{rrr}\text { Mean } & \mathbf{F} & \text { Prob }> \\ \text { Square } & \text { Ratio } & \mathbf{F}\end{array}$

$\begin{array}{llllll}\text { Set/Block/Sub- } & 7 & 7.2222 \mathrm{e}-35 & 1.032 \mathrm{e}-35 & -3.0476 & 0.0000\end{array}$

Error $\quad 16 \quad-5.417 \mathrm{e}-35 \quad-3.39 \mathrm{e}-36$

$\begin{array}{lll}\text { C. Total } & 23 & 1.8056 \mathrm{e}-35\end{array}$

Means for Oneway Anova

Level Number Mean Std Error Lower 95\% Upper 95\%

$\begin{array}{lll}1 / 1 / 1 & 3 & 0.005386 \\ 1 / 1 / 2 & 3 & 0.005386\end{array}$

$\begin{array}{lll}1 / 1 / 2 & 3 & 0.005386\end{array}$

$\begin{array}{lll}1 / 2 / 1 & 3 & 0.005386 \\ 1 / 2 / 2 & 3 & 0.005386\end{array}$

$\begin{array}{lll}1 / 2 / 2 & 3 & 0.005386 \\ 2 / 1 / 1 & 3 & 0.005386\end{array}$

$\begin{array}{lll}2 / 1 / 1 & 3 & 0.005386 \\ 2 / 1 / 2 & 3 & 0.005386\end{array}$

$\begin{array}{lll}2 / 1 / 2 & 3 & 0.005386 \\ 2 / 2 / 1 & 3 & 0.005386\end{array}$

$2 / 2 / 2 \quad 3 \quad 0.005386$

Std Error uses a pooled estimate of error variance 
SRNL-STI-2009-00778, Revision 0

Exhibit D3. Batch 1 Sample Measurements by Block and Sub-Block by Oxide for Both Preparation Methods for the Matrix 2A Study

Oneway Analysis of Measured By Set/Block/Sub-Blk Oxide $=\mathrm{SiO} 2(\mathrm{wt} \%)$

Reference Value $=\mathbf{5 0 . 2 2} \mathrm{wt} \%$

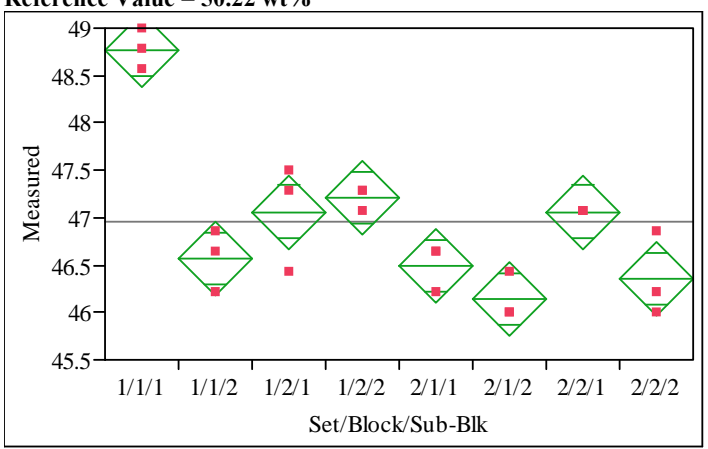

\section{Oneway Anova}

Summary of

Rsquare $\quad 0.899048$

Adj Rsquare $\quad 0.854881$

0.31791
Root Mean Square Error

\begin{tabular}{lr} 
Observations (or Sum Wgts) & 24 \\
\hline
\end{tabular}

Analysis of Variance

Source DF Sum of

Sum of
Squares

Blk

Error

$\begin{array}{rr}16 & 1.617067 \\ 23 & 16.018116\end{array}$

Mean

Square
2.0572920 .3558

0.10107

16.018116

\section{Means for Oneway Anova}

Level Number Mean Std Error Lower 95\% Upper 95\%

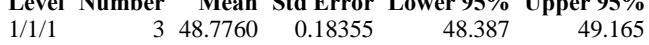

$\begin{array}{llllll}1 / 1 / 2 & 3 & 46.5654 & 0.18355 & 46.176 & 46.955\end{array}$

$\begin{array}{llllll}1 / 2 / 1 & 3 & 47.0646 & 0.18355 & 46.676 & 47.454\end{array}$

$\begin{array}{llllll}1 / 2 / 2 & 3 & 47.2072 & 0.18355 & 46.818 & 47.596\end{array}$

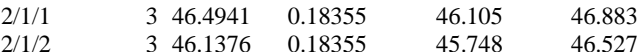

$\begin{array}{llllll}2 / 1 / 2 & 3 & 46.1376 & 0.18355 & 45.748 & 46.527 \\ 2 / 2 / 1 & 3 & 47.0646 & 0.18355 & 46.676 & 47.454\end{array}$

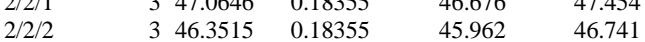

Std Error uses a pooled estimate of error variance
Oneway Analysis of Measured By Set/Block/Sub-Blk Oxide $=\mathrm{SO} 4(\mathrm{wt} \%)$

Reference Value $=0.0 \mathrm{wt} \%$

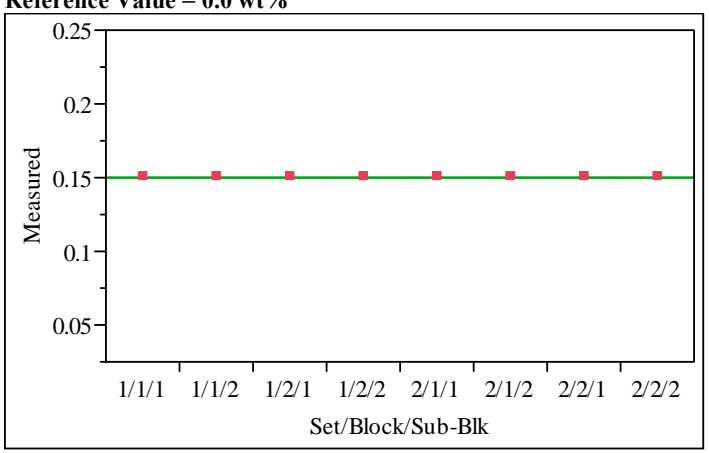

\section{Oneway Anova}

Summary of Fit

\section{Rsquare}

Adj Rsquare

Root Mean Square Error

$\begin{array}{lr}\text { Mean of Response } & 0.149795 \\ \text { Observations (or Sum Wgts) } & 24\end{array}$

Analysis of Variance

Source DF

Set/Block/Sub- 7

Blk
Erro

Error
C. Total

16
23

16
23

$$
\begin{array}{r}
\text { Sum of } \\
\text { Squares } \\
0
\end{array}
$$

Mean

Mean $\mathbf{F}$

Means for Oneway Anova

Level Number Mean Std Error Lower 95\% Upper 95\%

$\begin{array}{lrrrrr}1 / 1 / 1 & 3 & 0.149795 & 0 & 0.14980 & 0.14980\end{array}$

$\begin{array}{llllll}1 / 1 / 1 & 3 & 0.149795 & 0 & 0.14980 & 0.14980 \\ 1 / 1 / 2 & 3 & 0.149795 & 0 & 0.14980 & 0.14980\end{array}$

$\begin{array}{llll}1 / 2 / 1 & 3 & 0.149795 \\ 1 & 3 & 0.14995\end{array}$

$\begin{array}{lll}1 / 2 / 2 & 3 & 0.149795 \\ 1 / 1 / 2 & 3 & 0.1495\end{array}$

$\begin{array}{llll}1 / 1 / 1 & 3 & 0.149795 \\ & & 3 & 0.14995\end{array}$

$2 / 1 / 2-3 \quad 0.149795$

$\begin{array}{lll}2 / 2 / 1 & 3 & 0.149795 \\ 2 / 2 / 2 & 3 & 0.149795\end{array}$

0
0
0

$\begin{array}{lll}0 & 0.14980 & 0.14980 \\ 0 & 0.14980 & 0.14980\end{array}$

$\begin{array}{lll}0 & 0.14980 & 0.14980\end{array}$

$\begin{array}{lll}0 & 0.14980 & 0.14980 \\ 0 & 0.14980 & 0.14980\end{array}$

Std Error uses a pooled estimate of error variance
Oneway Analysis of Measured By Set/Block/Sub-Blk

Reference Value $=0.677 \mathrm{wt} \%$

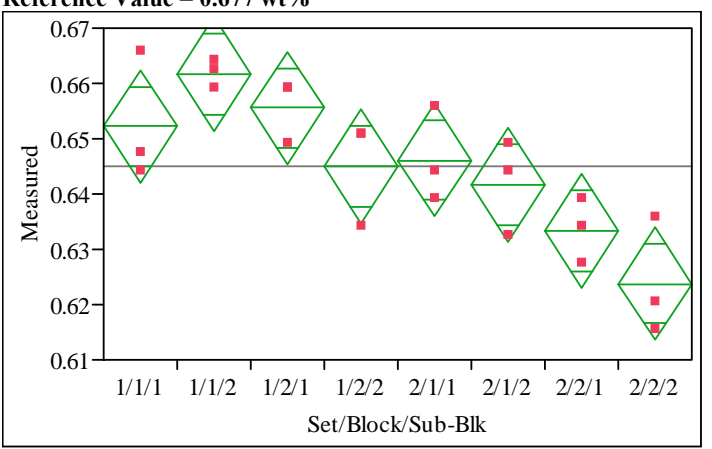

\section{Oneway Anova}

Summary of Fit

$\begin{array}{lr}\text { Rsquare } & 0.7359 \\ \text { Adj Rsquare } & 0.620356 \\ \text { Root Mean Square Error } & 0.008354 \\ \text { Mean of Response } & 0.644891 \\ \text { Observations (or Sum Wgts) } & 24\end{array}$

Analysis of Variance
Source DF

Sum of

$\begin{array}{llr}\text { Set/Block/Sub- } \quad 7 & 0.00311134\end{array}$

$\begin{array}{lll}\text { Blk } & 16 & 0.00111660 \\ \text { Error } & 23 & 0.00422794\end{array}$

$\begin{array}{lll}\text { C. Total } & 23 & 0.00422794\end{array}$

Mean F Prob >
Square $\begin{array}{rrr}\text { Square } & \text { Ratio } & \text { F } \\ 0.000444 & 6.3690 & 0.001\end{array}$

0.000070

Means for Oneway Anova

Level Number Mean Std Error Lower 95\% Upper 95\% $\begin{array}{llllll}1 / 1 / 1 & 3 & 0.652188 & 0.00482 & 0.64196 & 0.66241\end{array}$

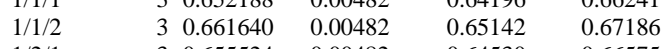
$\begin{array}{llllll}1 / 2 / 1 & 3 & 0.655524 & 0.00482 & 0.64530 & 0.66575\end{array}$ $\begin{array}{llllll}1 / 2 / 2 & 3 & 0.644960 & 0.00482 & 0.63474 & 0.65518\end{array}$ $\begin{array}{llllll}2 / 1 / 1 & 3 & 0.646072 & 0.00482 & 0.63585 & 0.65630\end{array}$

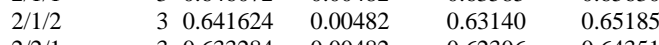

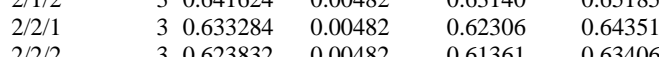

Std Error uses a pooled estimate of error variance 
Exhibit D3. Batch 1 Sample Measurements by Block and Sub-Block by Oxide for Both Preparation Methods for the Matrix 2A Study Oneway Analysis of Measured By Set/Block/Sub-Blk
Oxide $=\mathrm{ZnO}(\mathrm{wt} \%)$

Reference Value $=0.0 \mathrm{wt} \%$

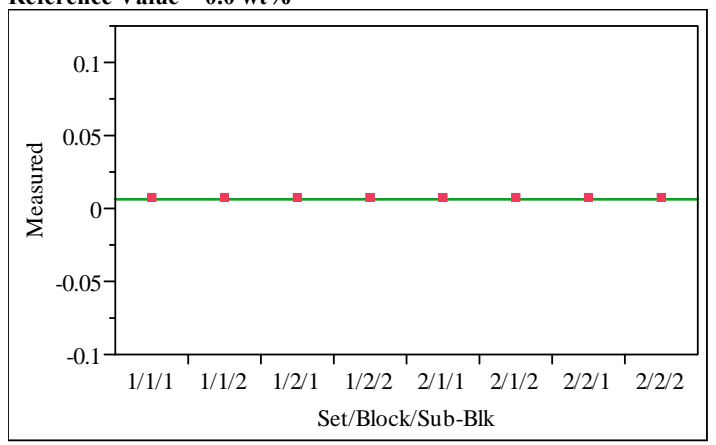

Oneway Anova

Summary of Fit

Rsquare

$\begin{array}{lr}\text { Root Mean Square Error } & 0 \\ \text { Mean of Response } & 0.006224\end{array}$

Analysis of Variance

Source DF

Set/Block/Sub-

Blk

C. Total

16
23

$$
\begin{array}{r}
\text { Sum of } \\
\text { Squares } \\
0
\end{array}
$$

23

0

Means for Oneway Anova

Level Number Mean Std Error Lower 95\% Upper 95\%

$\begin{array}{llllll}1 / 1 / 1 & 3 & 0.006224 & 0 & 0.00622 & 0.00622\end{array}$

$\begin{array}{llllll}1 / 1 / 2 & 3 & 0.006224 & 0 & 0.00622 & 0.00622\end{array}$

$\begin{array}{llllll}1 / 2 / 1 & 3 & 0.006224 & 0 & 0.00622 & 0.00622\end{array}$

$\begin{array}{llllll}1 / 2 / 2 & 3 & 0.006224 & 0 & 0.00622 & 0.00622\end{array}$

$\begin{array}{llllll}1 / 1 / 1 & 3 & 0.006224 & 0 & 0.00622 & 0.00622\end{array}$

$\begin{array}{llllll}2 / 1 / 2 & 3 & 0.006224 & 0 & 0.00622 & 0.00622\end{array}$

$\begin{array}{llllll}2 / 2 / 2 & 3 & 0.006224 & 0 & 0.00622 & 0.00622 \\ & & & 0 & 0.00622 & 0.00622\end{array}$

Std Error uses a pooled estimate of error variance
Oneway Analysis of Measured By Set/Block/Sub-Blk

Oxide $=\mathrm{ZrO} 2(\mathrm{wt} \%)$

Reference Value $=0.098 \mathrm{wt} \%$

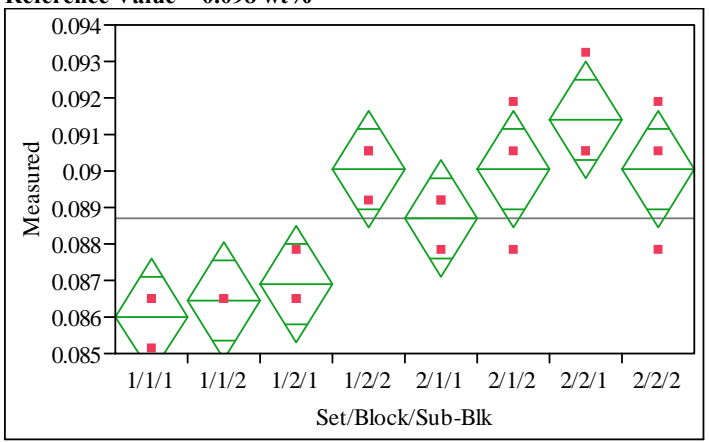

Oneway Anova

Summary of Fit

$\begin{array}{lr}\text { Rsquare } & 0.76087 \\ \text { Adj Rsquare } & 0.65625 \\ \text { Root Mean Square Error } & 0.001293 \\ \text { Mean of Response } & 0.088703 \\ \text { Observations (or Sum Wgts) } & 24\end{array}$

Observations (or Sum Wgts) 24

Analysis of Variance

Source DF Sum of

$\mathrm{F}$

Set/Block/Sub- $\quad$ Squares

70.00008515

$\begin{array}{lll}\text { Error } & 16 & 0.00002676\end{array}$

$\begin{array}{lll}\text { C. Total } & 23 & 0.00011191\end{array}$

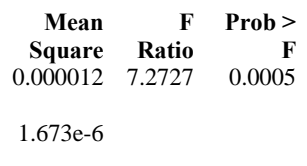

Means for Oneway Anova

Level Number Mean Std Error Lower 95\% Upper 95\%

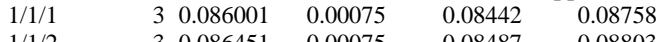

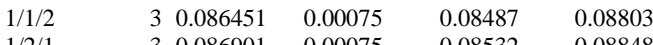

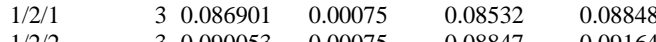

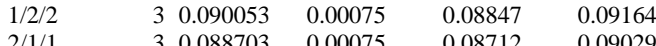

$\begin{array}{llllll}2 / 1 / 1 & 3 & 0.088703 & 0.00075 & 0.08712 & 0.09029\end{array}$

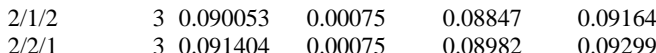

$\begin{array}{llllll}2 / 2 / 2 & 3 & 0.090053 & 0.00075 & 0.08847 & 0.09164\end{array}$

Std Error uses a pooled estimate of error variance 


\section{Exhibit D4. Average Measured and Bias-Corrected (bc) Versus Targeted Compositions by Glass ID by Oxide for the Matrix 2A Study}

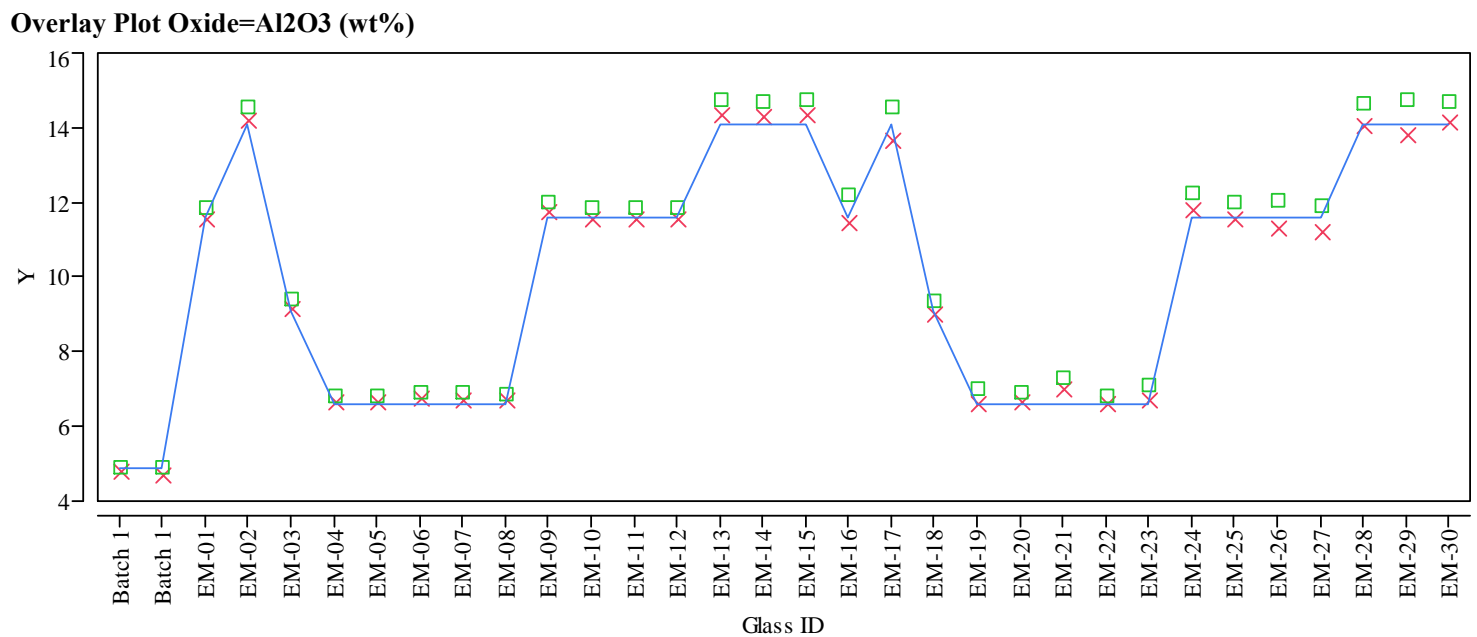

Overlay Plot Oxide=B2O3 (wt\%)

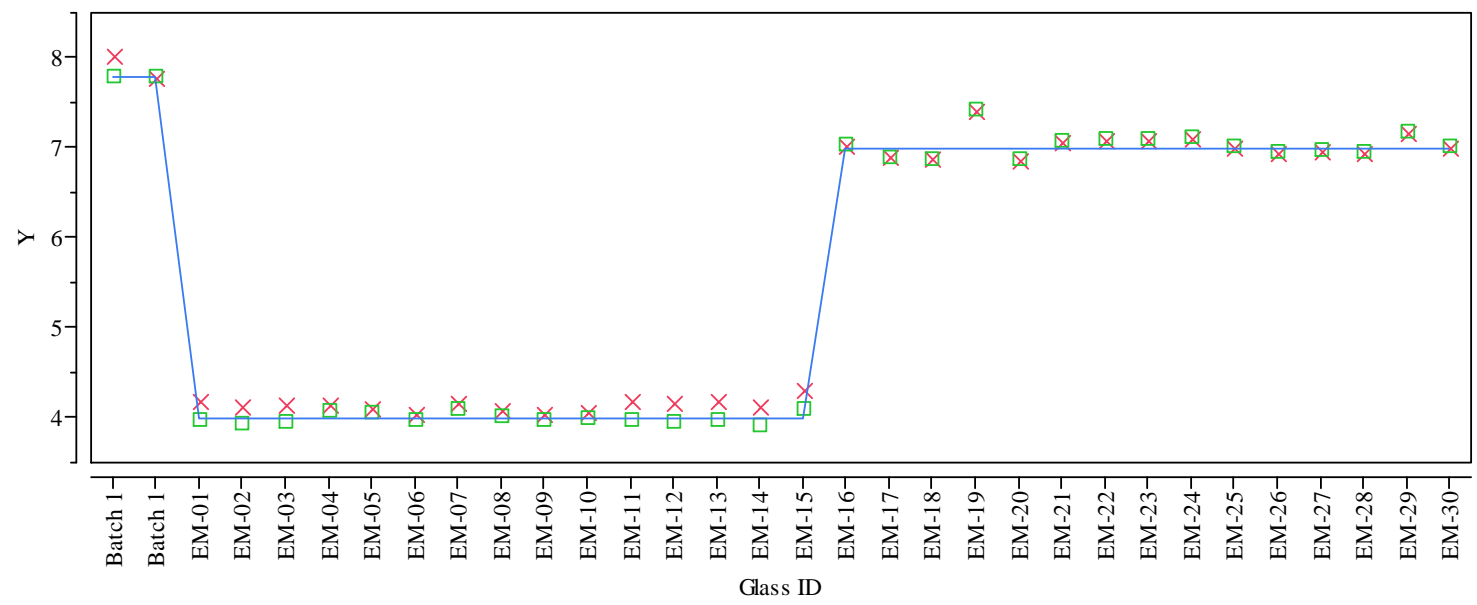

Overlay Plot Oxide $=\mathrm{BaO}($ wt $\%)$

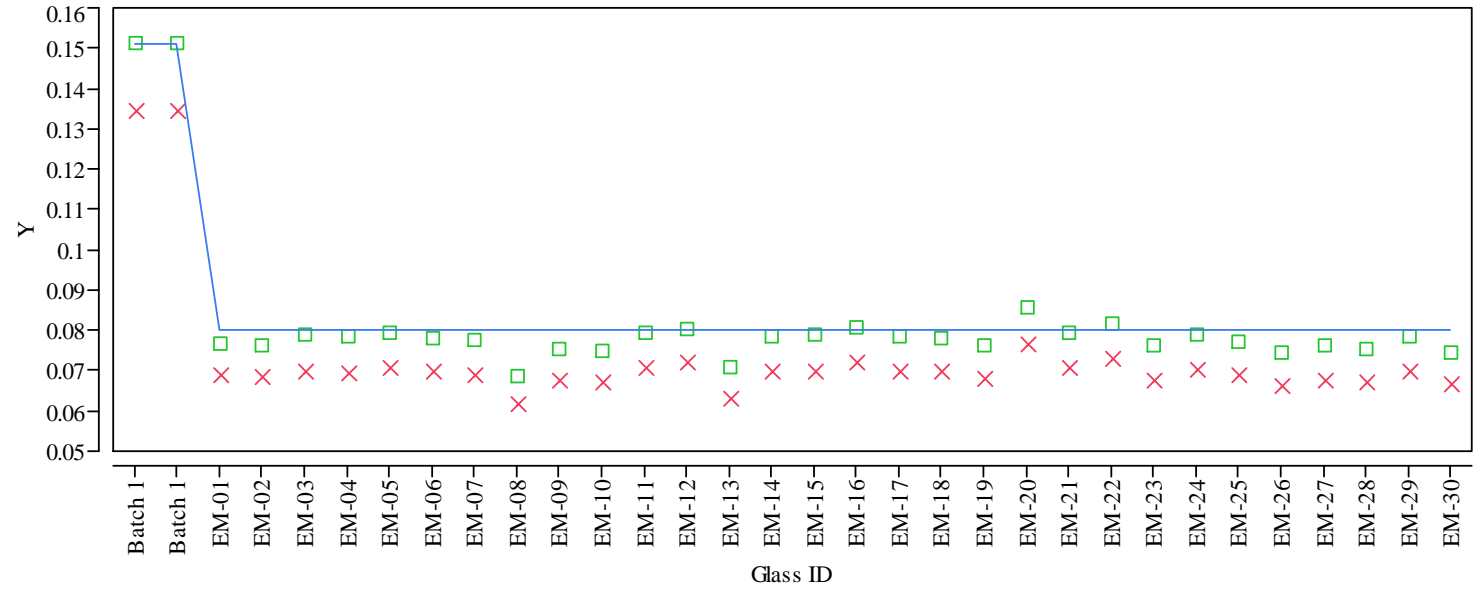

$\mathrm{Y} \times$ Measured $\square$ Measured bc —Targeted 
Exhibit D4. Average Measured and Bias-Corrected (bc) Versus Targeted Compositions by Glass ID by Oxide for the Matrix 2A Study
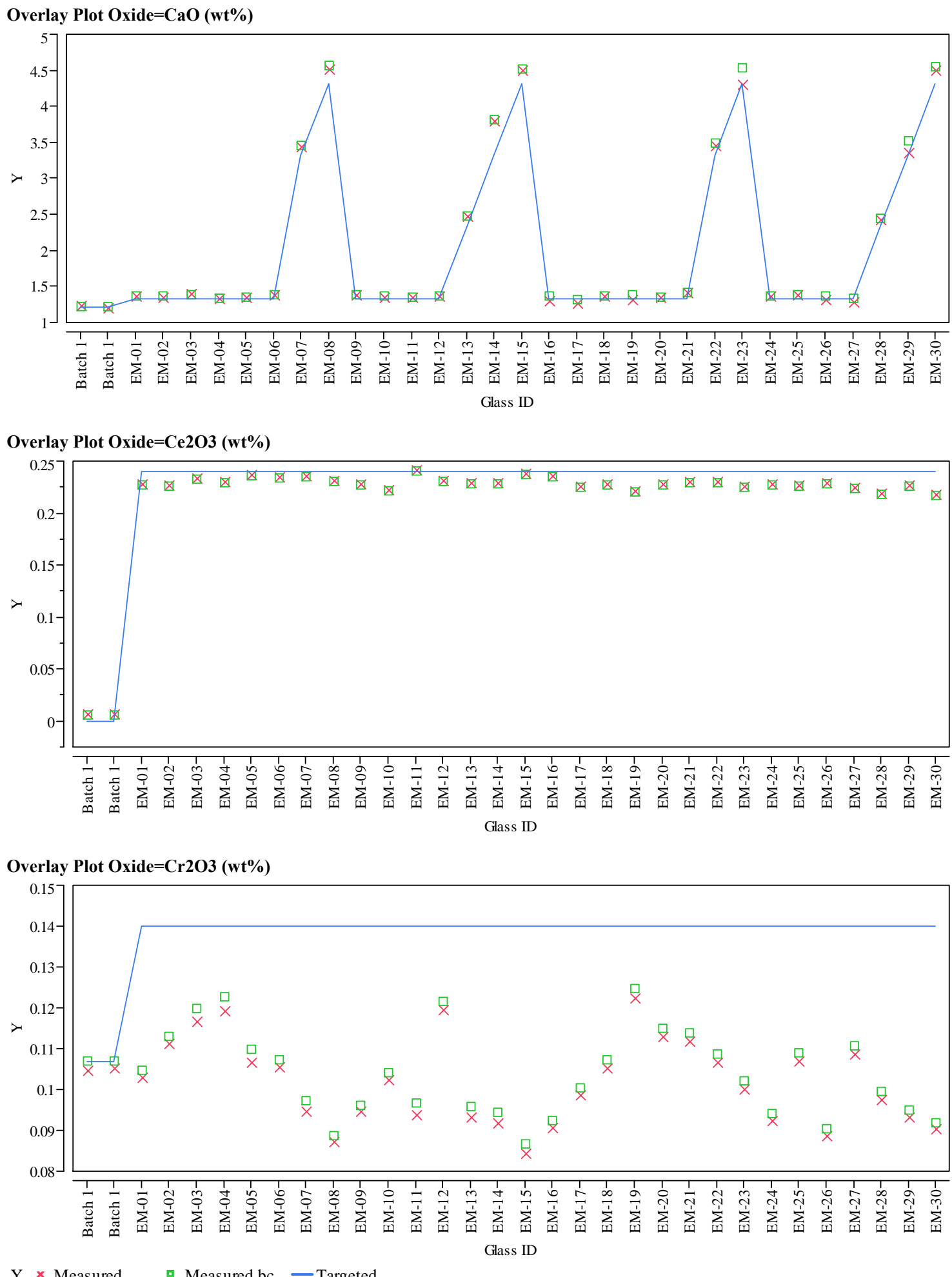
Exhibit D4. Average Measured and Bias-Corrected (bc) Versus Targeted Compositions by Glass ID by Oxide for the Matrix 2A Study

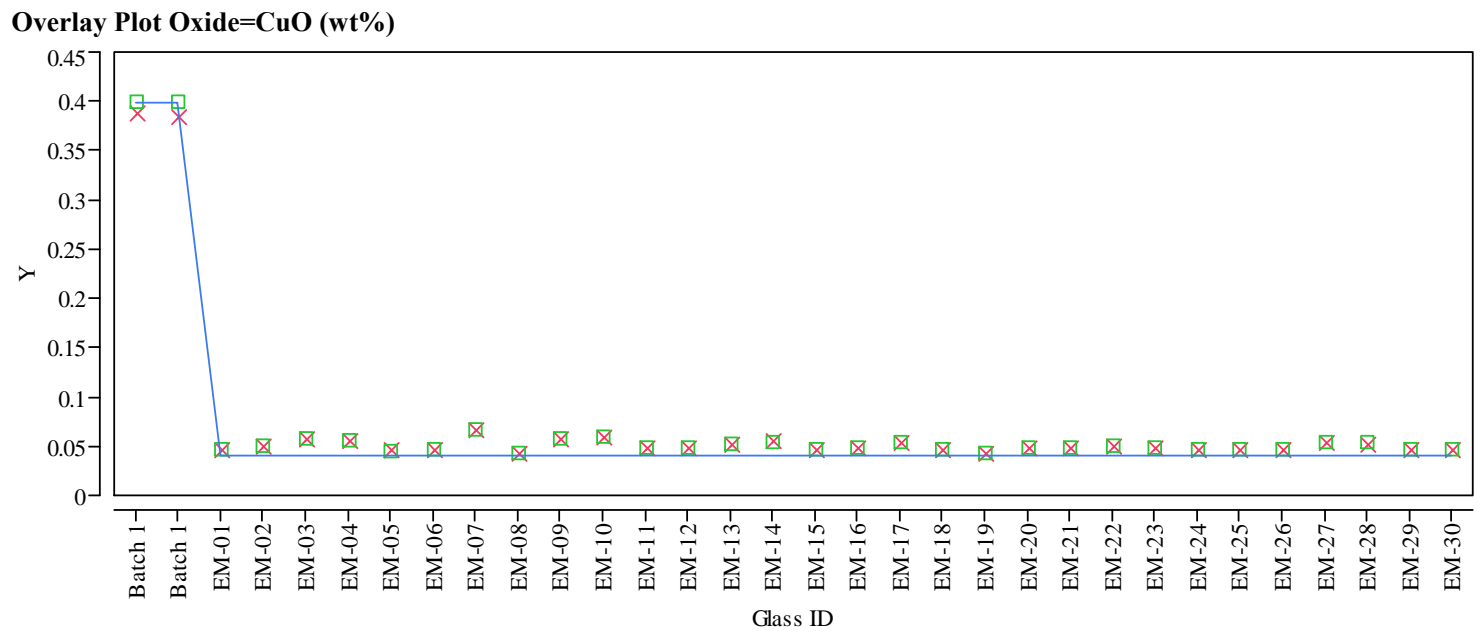

Overlay Plot Oxide $=\mathrm{Fe} 2 \mathrm{O} 3(\mathrm{wt} \%)$

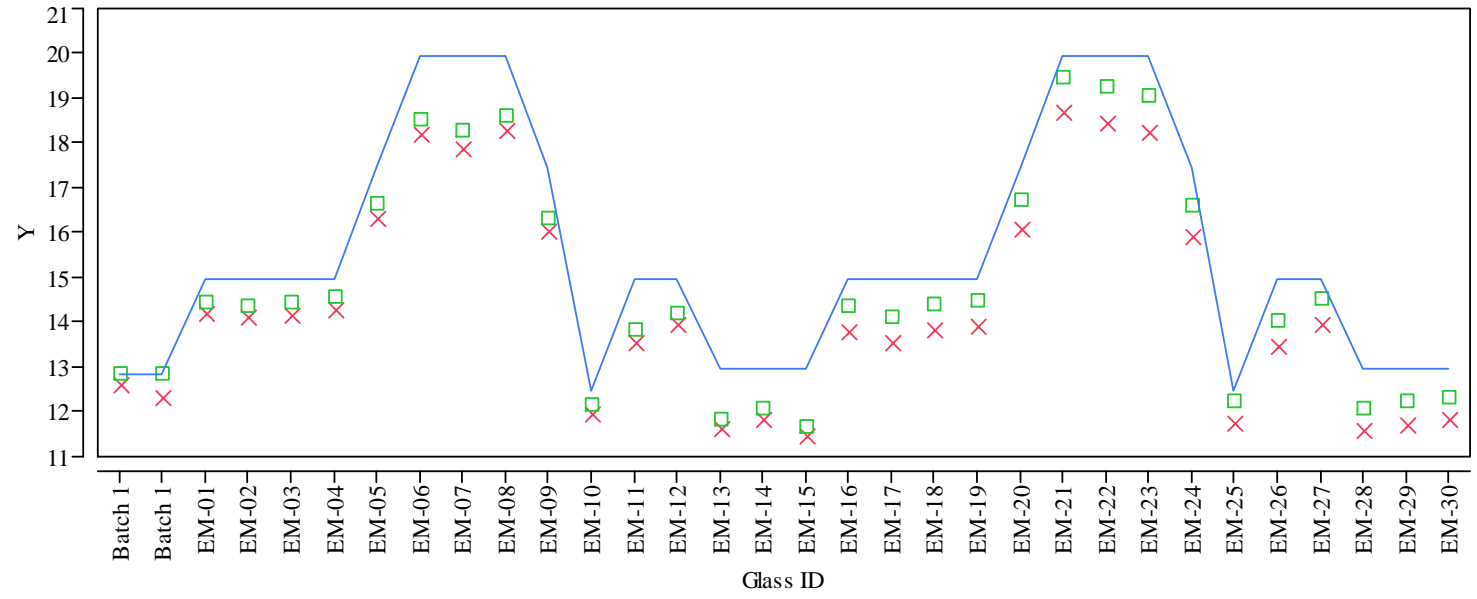

Overlay Plot Oxide $=\mathrm{La2O3}(\mathrm{wt} \%)$

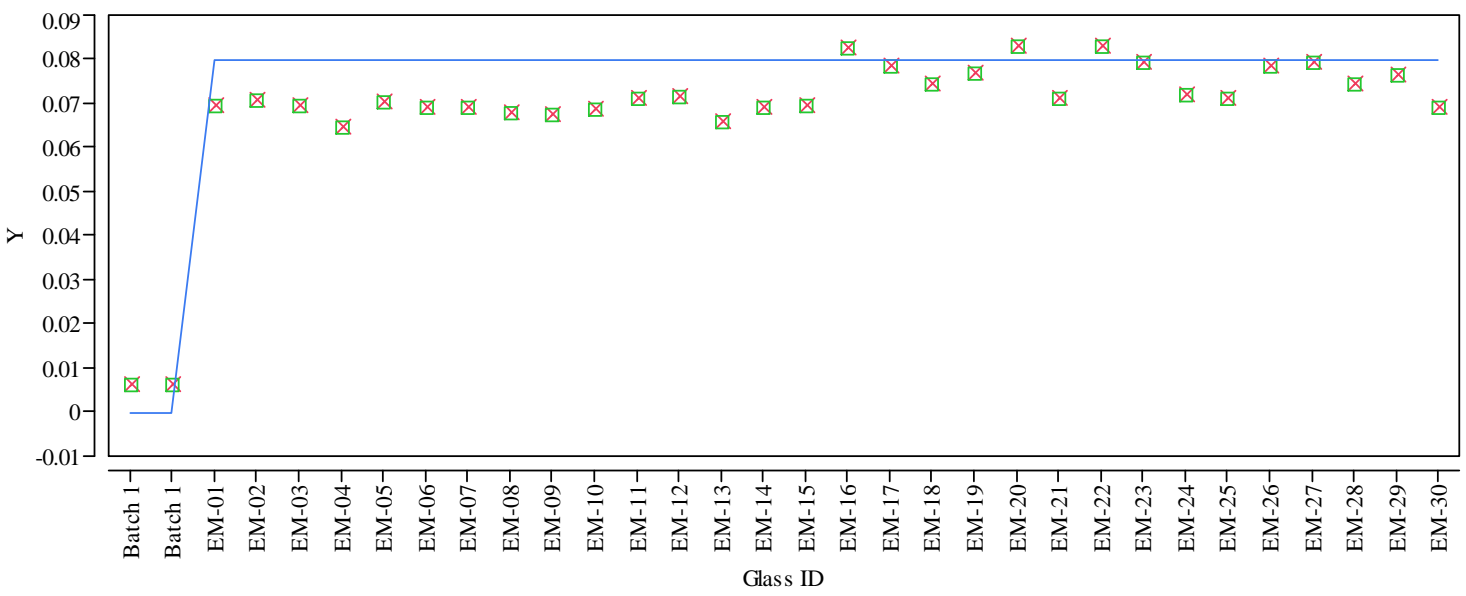

$\mathrm{Y} \times$ Measured $\square$ Measured bc — Targeted 
SRNL-STI-2009-00778, Revision 0

Exhibit D4. Average Measured and Bias-Corrected (bc) Versus Targeted Compositions by Glass ID by Oxide for the Matrix 2A Study

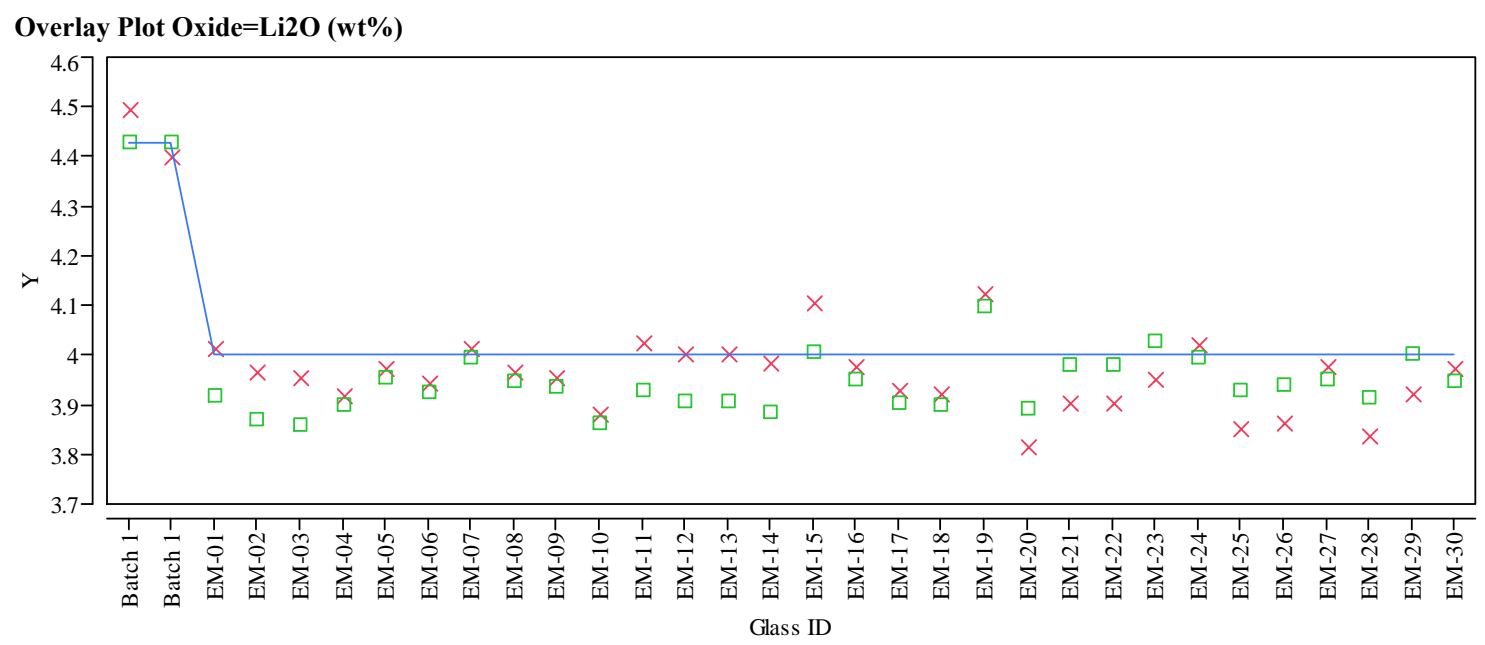

Overlay Plot Oxide $=\mathrm{MgO}(\mathrm{wt} \%)$

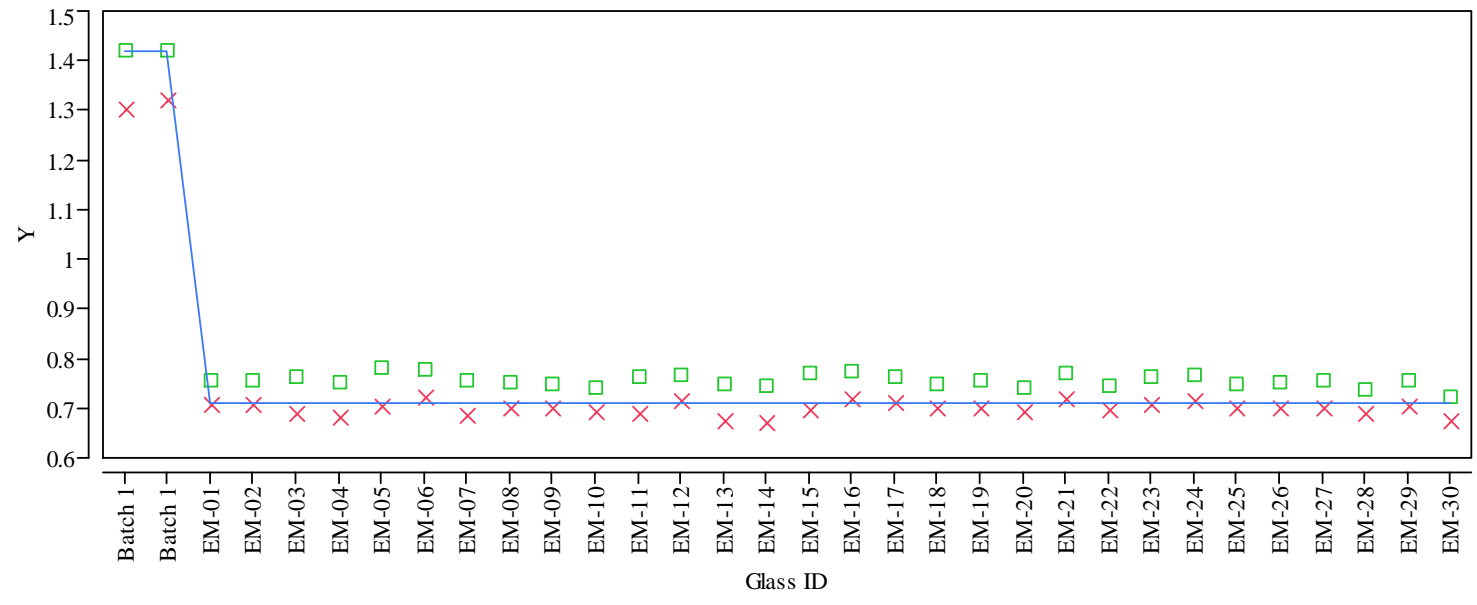

Overlay Plot Oxide $=\mathrm{MnO}(\mathrm{wt} \%)$

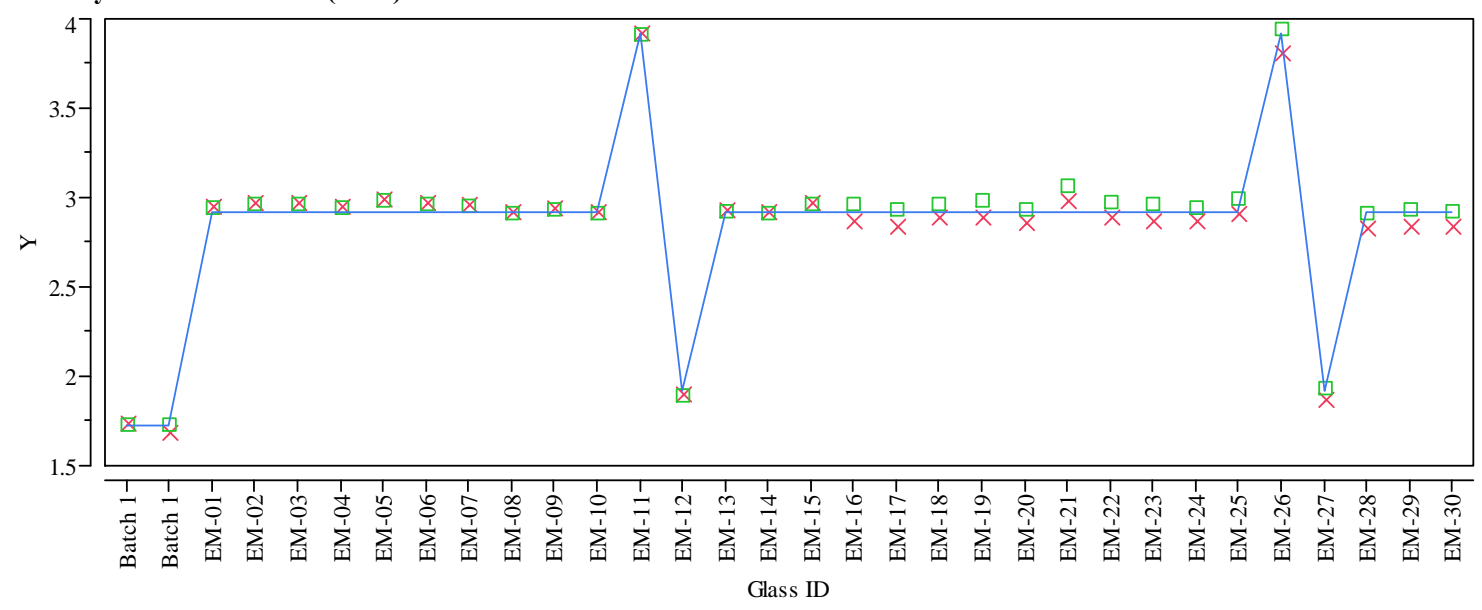

$\mathrm{Y} \times$ Measured $\square$ Measured bc — Targeted 


\section{Exhibit D4. Average Measured and Bias-Corrected (bc) Versus Targeted Compositions by Glass ID by Oxide for the Matrix 2A Study}
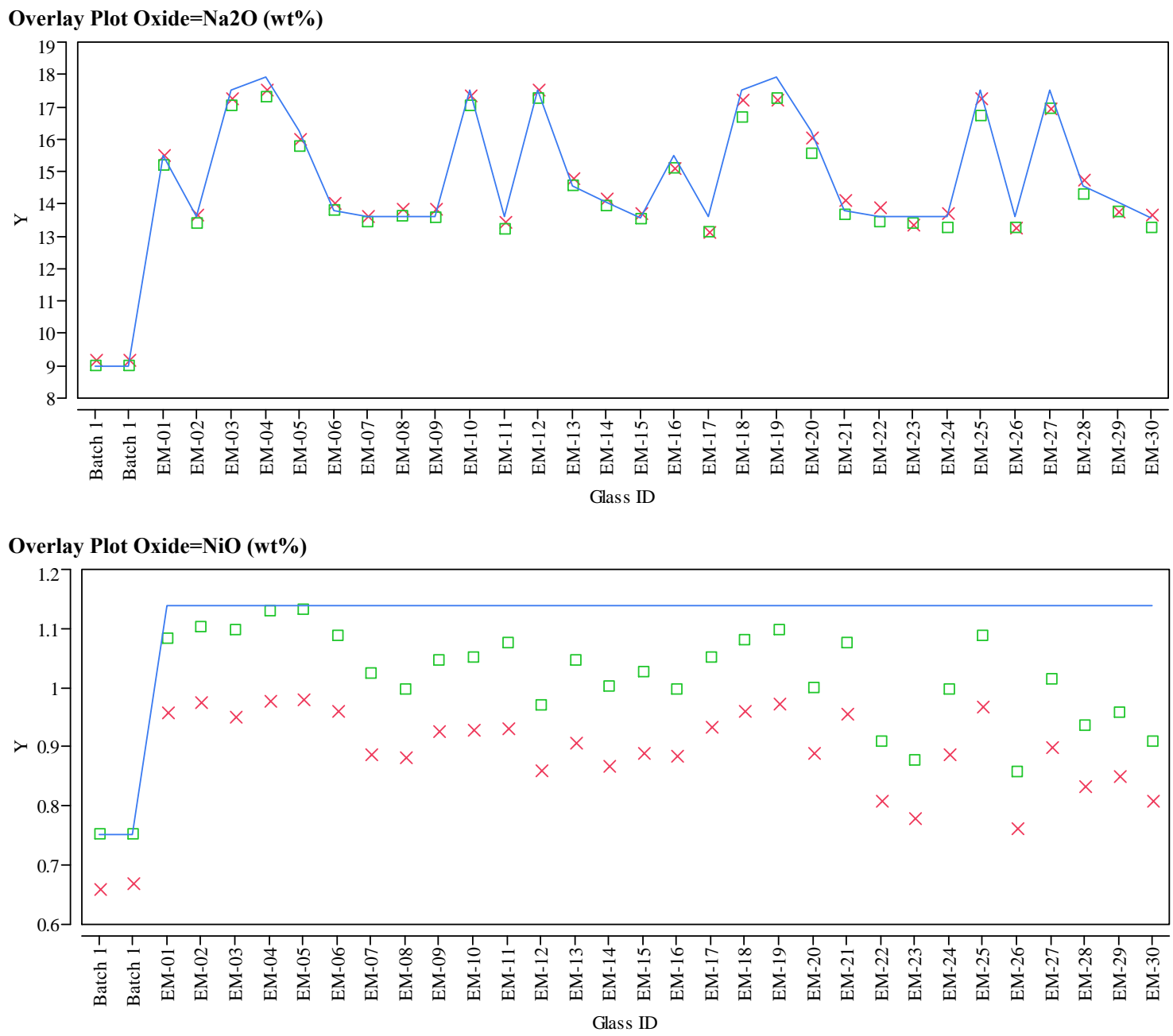

Overlay Plot Oxide $=\mathbf{P b O}(\mathbf{w t} \%)$

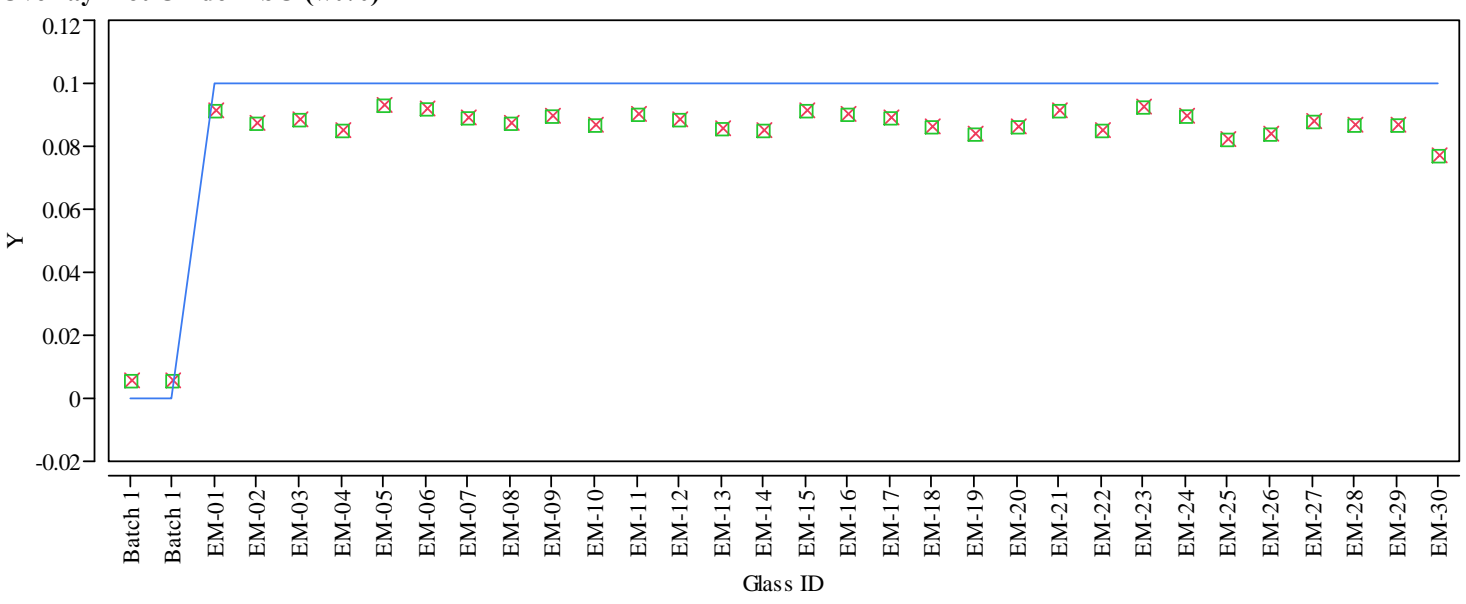

$\mathrm{Y} \times$ Measured Measured bc - Targeted 
Exhibit D4. Average Measured and Bias-Corrected (bc) Versus Targeted Compositions by Glass ID by Oxide for the Matrix 2A Study

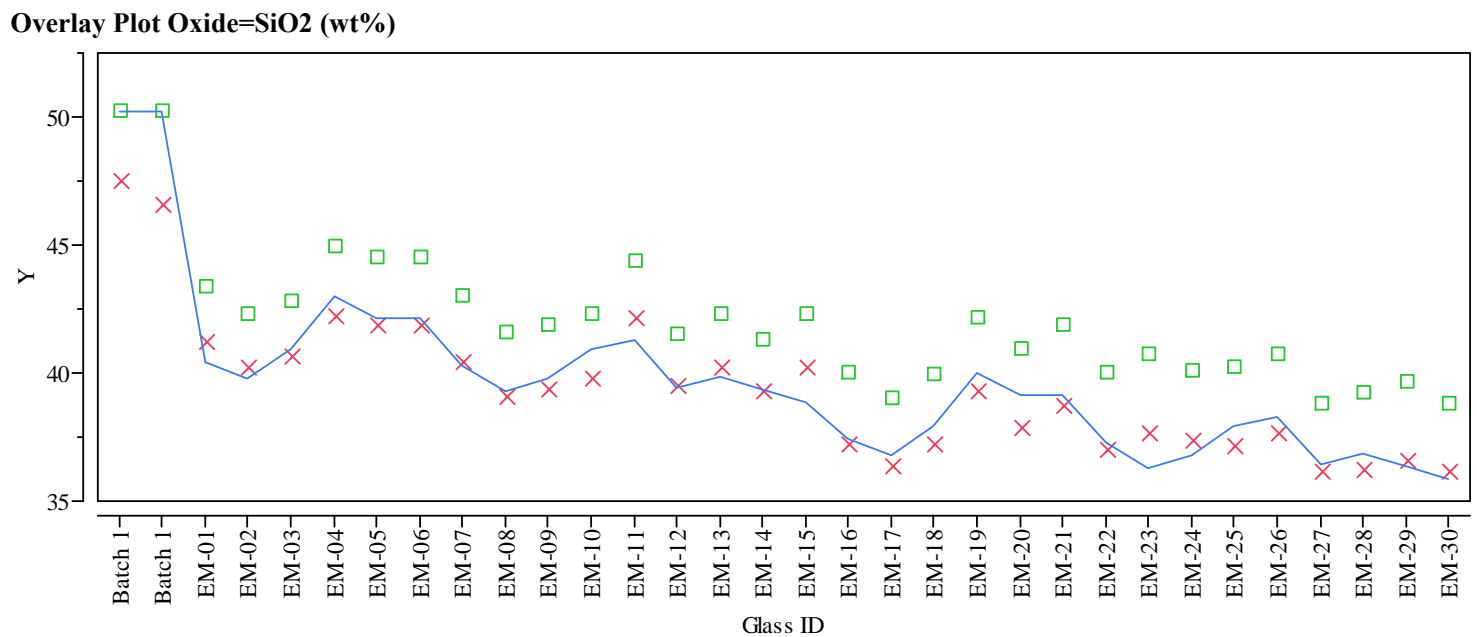

Overlay Plot Oxide=SO4 $(\mathbf{w t} \%)$

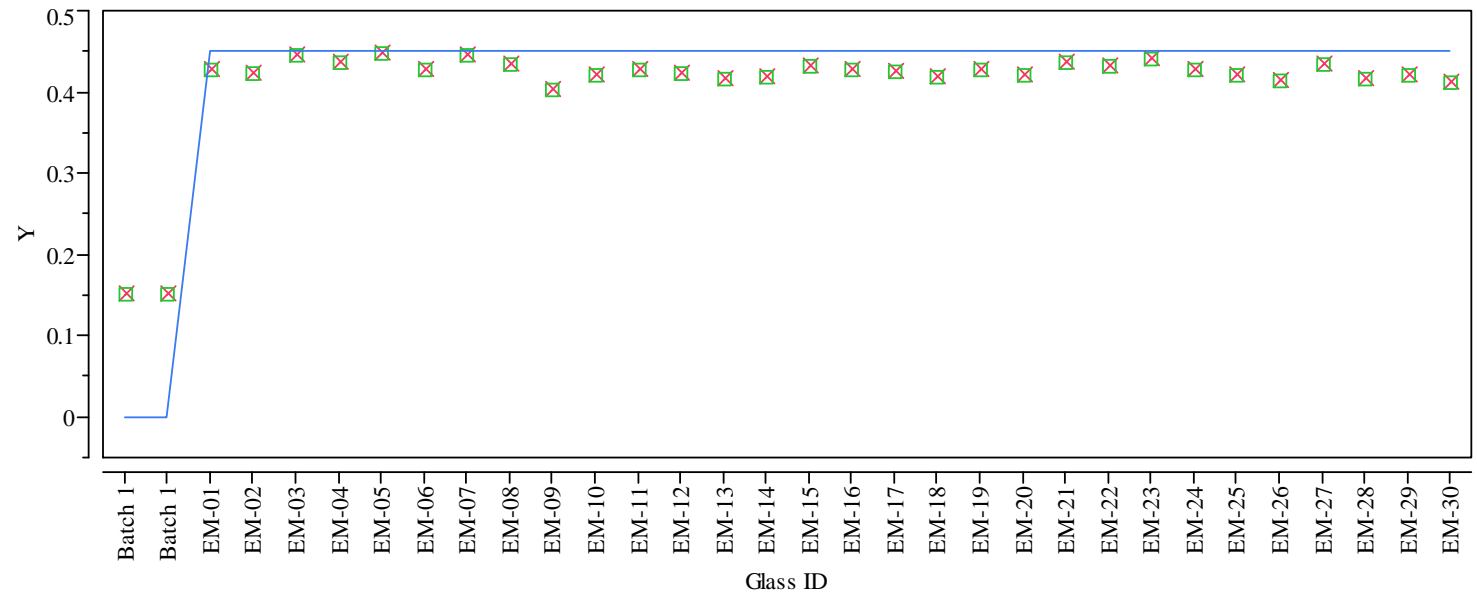

Overlay Plot Oxide=TiO2 $($ wt $\%)$

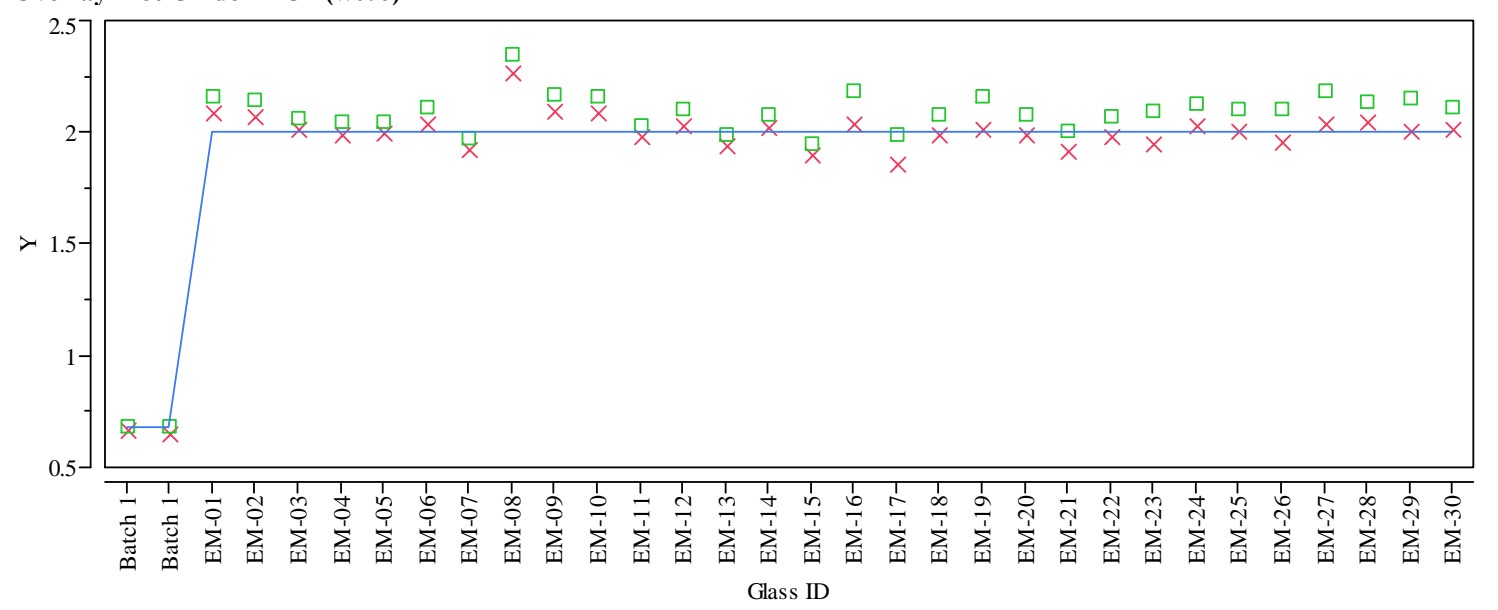

$\mathrm{Y} \times$ Measured $\square$ Measured bc — Targeted 
SRNL-STI-2009-00778, Revision 0

Exhibit D4. Average Measured and Bias-Corrected (bc) Versus Targeted Compositions by Glass ID by Oxide for the Matrix 2A Study

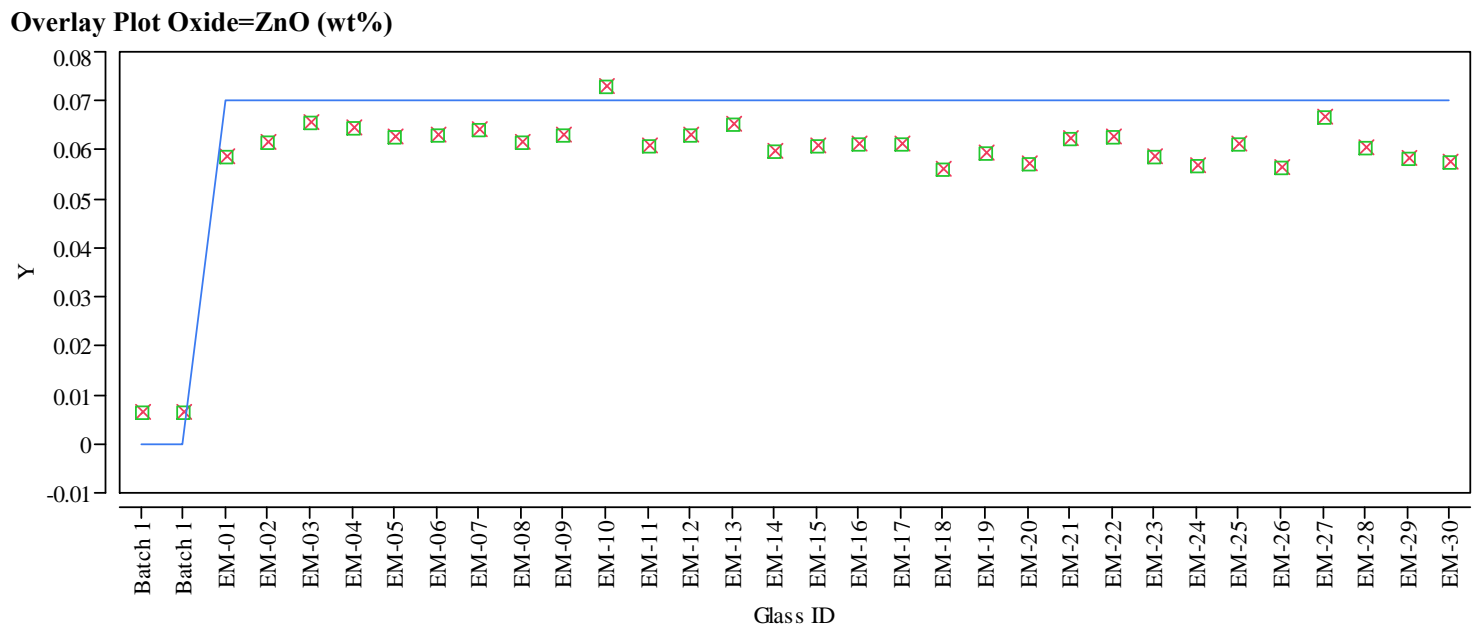

Overlay Plot Oxide $=\mathrm{ZrO} 2($ wt $\%)$

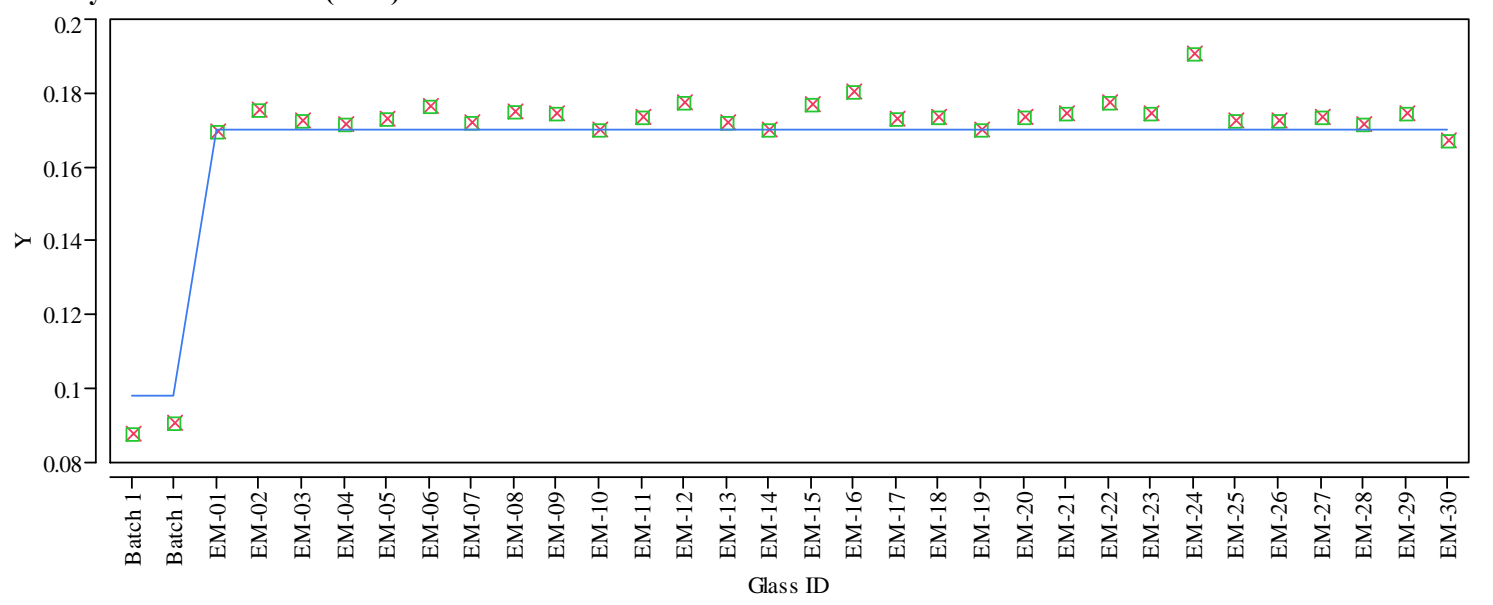

Overlay Plot Oxide $=$ Sum of Oxides

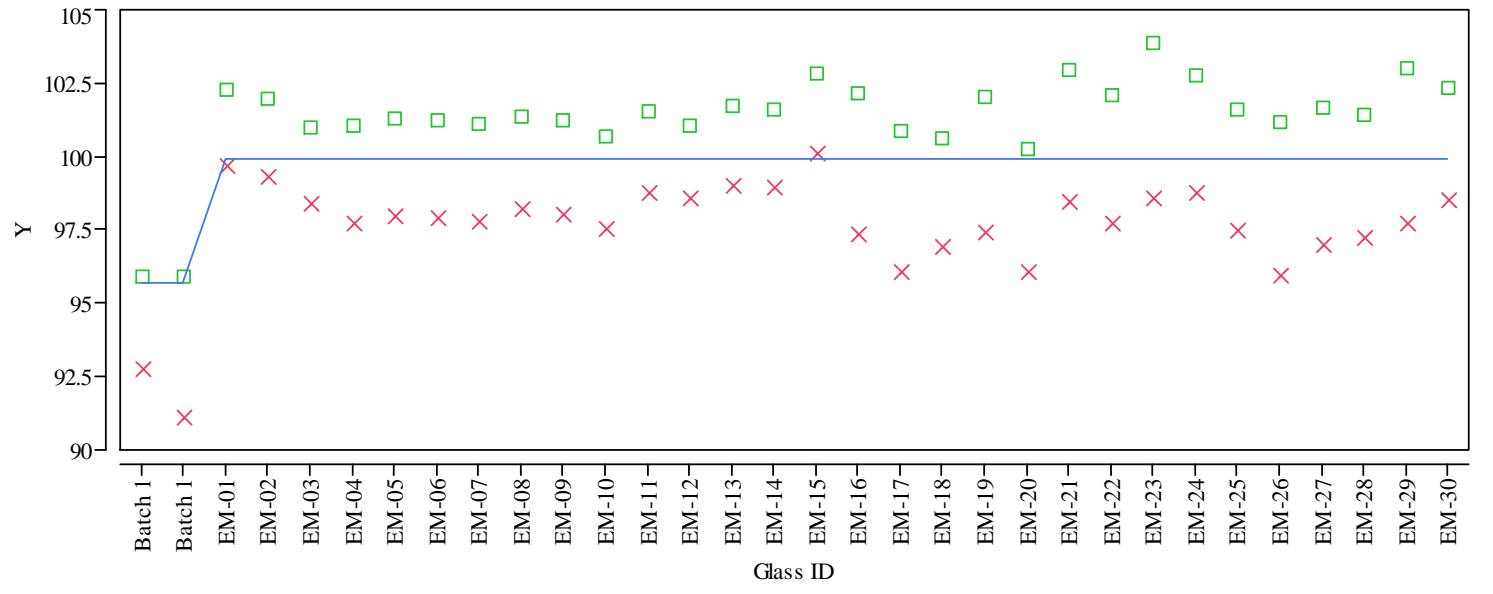

$\mathrm{Y} \times$ Measured $\square$ Measured bc - Targeted 


\section{Appendix E:}

\section{Tables and Exhibits Supporting the Analysis of the PCT Results for the Matrix 2A Study \\ Glasses}


SRNL-STI-2009-00778, Revision 0

This page intentionally left blank. 
SRNL-STI-2009-00778, Revision 0

Table E1. PSAL's Measurements of the Matrix 2A PCT Solutions As-Received (ar) and After Appropriate Adjustments (in ppm)

\begin{tabular}{|c|c|c|c|c|c|c|c|c|c|c|c|c|c|}
\hline Set & Glass ID & Heat Treatment & Block & Seq & Lab ID & B ar & Li ar & Na ar & Si ar & B (ppm) & Li (ppm) & Na (ppm) & Si (ppm) \\
\hline 1 & Soln Std & ref & 1 & 1 & std-11-1 & 21.0 & 9.95 & 80.6 & 53.3 & 21.00 & 9.95 & 80.60 & 53.30 \\
\hline 1 & EM-06 & CCC & 1 & 2 & n16 & 6.52 & 9.48 & 63.8 & 70.8 & 10.87 & 15.80 & 106.34 & 118.00 \\
\hline 1 & EM-07 & $\mathrm{CCC}$ & 1 & 3 & $\mathrm{n} 21$ & 6.93 & 11.0 & 68.9 & 65.1 & 11.55 & 18.33 & 114.84 & 108.50 \\
\hline 1 & EM-01 & CCC & 1 & 4 & $\mathrm{n} 17$ & 6.85 & 9.33 & 70.4 & 67.6 & 11.42 & 15.55 & 117.34 & 112.67 \\
\hline 1 & EM-10 & CCC & 1 & 5 & n34 & 86.8 & 98.2 & 452 & 309 & 144.67 & 163.67 & 753.35 & 515.01 \\
\hline 1 & EM-05 & quenched & 1 & 6 & n31 & 10.0 & 12.2 & 101 & 88.4 & 16.67 & 20.33 & 168.34 & 147.34 \\
\hline 1 & EM-06 & quenched & 1 & 7 & n13 & 8.54 & 11.2 & 6.89 & 75.6 & 14.23 & 18.67 & 11.48 & 126.00 \\
\hline 1 & blank & ref & 1 & 8 & n51 & $<0.100$ & $<0.100$ & $<0.100$ & $<0.100$ & 0.08 & 0.08 & 0.08 & 0.08 \\
\hline 1 & EM-03 & quenched & 1 & 9 & $\mathrm{n} 60$ & 8.48 & 10.6 & 107 & 80.3 & 14.13 & 17.67 & 178.34 & 133.84 \\
\hline 1 & EM-08 & CCC & 1 & 10 & n37 & 6.73 & 12.2 & 72.3 & 61.0 & 11.22 & 20.33 & 120.50 & 101.67 \\
\hline 1 & EM-08 & quenched & 1 & \begin{tabular}{|l|}
11 \\
\end{tabular} & n25 & 11.3 & 15.7 & 94.7 & 74.0 & 18.83 & 26.17 & 157.84 & 123.34 \\
\hline 1 & EM-03 & CCC & 1 & 12 & n63 & 7.65 & 11.9 & 114 & 89.3 & 12.75 & 19.83 & 190.00 & 148.84 \\
\hline 1 & ARM-1 & ref & 1 & 13 & n01 & 9.24 & 7.40 & 18.8 & 34.7 & 15.40 & 12.33 & 31.33 & 57.83 \\
\hline 1 & Soln Std & ref & 1 & 14 & std-11-2 & 19.7 & 9.50 & 79.0 & 50.7 & 19.70 & 9.50 & 79.00 & 50.70 \\
\hline 1 & EM-09 & quenched & 1 & 15 & n29 & 5.98 & 8.14 & 51.2 & 55.8 & 9.97 & 13.57 & 85.34 & 93.00 \\
\hline 1 & EM-10 & quenched & 1 & 16 & $\mathrm{n} 10$ & 6.75 & 8.64 & 91.1 & 68.0 & 11.25 & 14.40 & 151.84 & 113.34 \\
\hline 1 & EM-05 & CCC & 1 & \begin{tabular}{|l|}
17 \\
\end{tabular} & n65 & 8.87 & 12.7 & 102 & 97.8 & 14.78 & 21.17 & 170.00 & 163.00 \\
\hline 1 & EA & ref & 1 & 18 & n68 & 34.9 & 10.3 & 92.5 & 52.3 & 581.67 & 171.67 & 1541.67 & 871.67 \\
\hline 1 & EM-01 & quenched & 1 & \begin{tabular}{|l|}
19 \\
\end{tabular} & n07 & 7.12 & 8.73 & 69.6 & 63.0 & 11.87 & 14.55 & 116.00 & 105.00 \\
\hline 1 & EM-04 & CCC & 1 & 20 & n64 & 8.84 & 12.6 & 120 & 100 & 14.73 & 21.00 & 200.00 & 166.67 \\
\hline 1 & EM-02 & CCC & 1 & 21 & n12 & 17.6 & 24.2 & 77.0 & 85.4 & 29.33 & 40.33 & 128.34 & 142.34 \\
\hline 1 & EM-07 & quenched & 1 & 22 & n22 & 11.3 & 14.7 & 87.3 & 77.8 & 18.83 & 24.50 & 145.50 & 129.67 \\
\hline 1 & EM-04 & quenched & 1 & 23 & n41 & 10.4 & 12.7 & 126 & 104 & 17.33 & 21.17 & 210.00 & 173.34 \\
\hline 1 & EM-09 & CCC & 1 & 24 & n48 & 6.08 & 9.86 & 52.4 & 60.3 & 10.13 & 16.43 & 87.34 & 100.50 \\
\hline 1 & EM-02 & quenched & 1 & 25 & n14 & 4.71 & 7.12 & 43.5 & 50.1 & 7.85 & 11.87 & 72.50 & 83.50 \\
\hline 1 & Soln Std & ref & 1 & \begin{tabular}{|l|}
26 \\
\end{tabular} & std-11-3 & 19.4 & 9.41 & 77.1 & 50.0 & 19.40 & 9.41 & 77.10 & 50.00 \\
\hline 1 & Soln Std & ref & 2 & 1 & std-12-1 & 21.0 & 10.0 & 81.0 & 52.2 & 21.00 & 10.00 & 81.00 & 52.20 \\
\hline 1 & EM-08 & $\mathrm{CCC}$ & 2 & 2 & n53 & 7.33 & 12.5 & 74.2 & 61.2 & 12.22 & 20.83 & 123.67 & 102.00 \\
\hline 1 & EM-05 & quenched & 2 & 3 & n19 & 10.0 & 12.1 & 100 & 84.5 & 16.67 & 20.17 & 166.67 & 140.84 \\
\hline 1 & EM-02 & CCC & 2 & 4 & $\mathrm{n} 43$ & 17.9 & 24.7 & 80.3 & 86.1 & 29.83 & 41.17 & 133.84 & 143.50 \\
\hline 1 & EM-03 & quenched & 2 & 5 & n58 & 8.52 & 10.4 & 105 & 77.0 & 14.20 & 17.33 & 175.00 & 128.34 \\
\hline 1 & EM-09 & quenched & 2 & 6 & n09 & 5.75 & 8.28 & 50.7 & 55.9 & 9.58 & 13.80 & 84.50 & 93.17 \\
\hline 1 & EM-01 & quenched & 2 & 7 & n67 & 6.64 & 8.41 & 66.7 & 60.3 & 11.07 & 14.02 & 111.17 & 100.50 \\
\hline 1 & EM-10 & quenched & 2 & 8 & $\mathrm{n} 42$ & 6.60 & 8.56 & 88.6 & 66.3 & 11.00 & 14.27 & 147.67 & 110.50 \\
\hline 1 & EM-08 & quenched & 2 & 9 & n08 & 11.4 & 15.7 & 93.6 & 72.3 & 19.00 & 26.17 & 156.00 & 120.50 \\
\hline 1 & EM-07 & CCC & 2 & 10 & n61 & 6.90 & 11.0 & 68.4 & 64.1 & 11.50 & 18.33 & 114.00 & 106.84 \\
\hline 1 & EM-04 & CCC & 2 & \begin{tabular}{|l|}
11 \\
\end{tabular} & n56 & 8.63 & 12.5 & 120 & 95.0 & 14.38 & 20.83 & 200.00 & 158.34 \\
\hline 1 & ARM-1 & ref & 2 & \begin{tabular}{|l|}
12 \\
\end{tabular} & n27 & 9.47 & 7.50 & 19.3 & 34.7 & 15.78 & 12.50 & 32.17 & 57.83 \\
\hline 1 & EM-09 & CCC & 2 & 13 & n11 & 6.11 & 10.0 & 52.7 & 59.5 & 10.18 & 16.67 & 87.84 & 99.17 \\
\hline 1 & Soln Std & ref & 2 & 14 & std-12-2 & 19.6 & 9.52 & 76.8 & 50.1 & 19.60 & 9.52 & 76.80 & 50.10 \\
\hline 1 & EM-06 & CCC & 2 & 15 & n05 & 6.96 & 9.75 & 63.2 & 73.0 & 11.60 & 16.25 & 105.34 & 121.67 \\
\hline 1 & EM-02 & quenched & 2 & 16 & n57 & 4.95 & 7.18 & 43.3 & 49.2 & 8.25 & 11.97 & 72.17 & 82.00 \\
\hline 1 & EM-07 & quenched & 2 & \begin{tabular}{|l|}
17 \\
\end{tabular} & $\mathrm{n} 40$ & 11.5 & 14.7 & 88.2 & 76.2 & 19.17 & 24.50 & 147.00 & 127.00 \\
\hline 1 & EM-04 & quenched & 2 & 18 & n38 & 10.2 & 12.4 & 126 & 95.0 & 17.00 & 20.67 & 210.00 & 158.34 \\
\hline 1 & EM-05 & CCC & 2 & \begin{tabular}{|l|}
19 \\
\end{tabular} & n59 & 8.58 & 12.4 & 98.8 & 89.5 & 14.30 & 20.67 & 164.67 & 149.17 \\
\hline 1 & EM-03 & CCC & 2 & 20 & n62 & 7.73 & 11.9 & 114 & 86.6 & 12.88 & 19.83 & 190.00 & 144.34 \\
\hline 1 & EM-06 & quenched & 2 & 21 & n04 & 8.40 & 11.2 & 68.6 & 73.7 & 14.00 & 18.67 & 114.34 & 122.84 \\
\hline 1 & EA & ref & 2 & 22 & n36 & 33.0 & 10.3 & 91.0 & 50.8 & 550.00 & 171.67 & 1516.67 & 846.67 \\
\hline 1 & EM-10 & CCC & 2 & 23 & $\mathrm{n} 02$ & 85.5 & 95.0 & 443 & 289 & 142.50 & 158.34 & 738.35 & 481.68 \\
\hline 1 & EM-01 & CCC & 2 & 24 & n15 & 6.76 & 9.10 & 67.0 & 63.5 & 11.27 & 15.17 & 111.67 & 105.84 \\
\hline 1 & Soln Std & ref & 2 & 25 & std-12-3 & 19.5 & 9.54 & 77.5 & 50.0 & 19.50 & 9.54 & 77.50 & 50.00 \\
\hline 1 & Soln Std & ref & 3 & 1 & std-13-1 & 20.4 & 9.67 & 81.2 & 50.4 & 20.40 & 9.67 & 81.20 & 50.40 \\
\hline 1 & blank & ref & 3 & 2 & $\mathrm{n} 47$ & 0.432 & 0.256 & $<0.100$ & $<0.100$ & 0.72 & 0.43 & 0.08 & 0.08 \\
\hline 1 & EM-08 & quenched & 3 & 3 & n50 & 11.0 & 15.3 & 94.1 & 69.1 & 18.33 & 25.50 & 156.84 & 115.17 \\
\hline 1 & EM-02 & CCC & 3 & 4 & n28 & 17.6 & 24.4 & 81.5 & 84.9 & 29.33 & 40.67 & 135.84 & 141.50 \\
\hline 1 & EM-10 & CCC & 3 & 5 & $\mathrm{n} 20$ & 83.1 & 94.1 & 451 & 288.0 & 138.50 & 156.84 & 751.68 & 480.01 \\
\hline 1 & EM-06 & CCC & 3 & 6 & n33 & 6.27 & 9.49 & 64.9 & 72.0 & 10.45 & 15.82 & 108.17 & 120.00 \\
\hline 1 & EM-01 & $\mathrm{CCC}$ & 3 & 7 & $\mathrm{n} 49$ & 6.46 & 9.16 & 70.9 & 62.9 & 10.77 & 15.27 & 118.17 & 104.84 \\
\hline 1 & EM-04 & CCC & 3 & 8 & n55 & 8.46 & 12.4 & 123 & 98.0 & 14.10 & 20.67 & 205.00 & 163.34 \\
\hline 1 & EM-05 & CCC & 3 & 9 & $\mathrm{n} 18$ & 8.40 & 12.4 & 102 & 90.3 & 14.00 & 20.67 & 170.00 & 150.50 \\
\hline 1 & EM-03 & $\mathrm{CCC}$ & 3 & \begin{tabular}{|l|}
10 \\
\end{tabular} & n32 & 7.87 & 12.3 & 121 & 89.3 & 13.12 & 20.50 & 201.67 & 148.84 \\
\hline 1 & EM-04 & quenched & 3 & 11 & $\mathrm{n} 45$ & 9.86 & 12.2 & 126 & 97.3 & 16.43 & 20.33 & 210.00 & 162.17 \\
\hline 1 & EM-09 & quenched & 3 & \begin{tabular}{|l|}
12 \\
\end{tabular} & $\mathrm{n} 46$ & 5.53 & 8.28 & 52.3 & 55.8 & 9.22 & 13.80 & 87.17 & 93.00 \\
\hline 1 & EM-06 & quenched & 3 & 13 & n39 & 8.38 & 11.3 & 71.0 & 75.7 & 13.97 & 18.83 & 118.34 & 126.17 \\
\hline 1 & Soln Std & ref & 3 & \begin{tabular}{|l|}
14 \\
\end{tabular} & std-13-2 & 19.5 & 9.55 & 79.9 & 50.3 & 19.50 & 9.55 & 79.90 & 50.30 \\
\hline 1 & EM-08 & CCC & 3 & 15 & n66 & 7.66 & 12.3 & 76.4 & 60.1 & 12.77 & 20.50 & 127.34 & 100.17 \\
\hline 1 & EM-01 & quenched & 3 & \begin{tabular}{|l|}
16 \\
\end{tabular} & n52 & 6.83 & 8.26 & 68.5 & 60.6 & 11.38 & 13.77 & 114.17 & 101.00 \\
\hline 1 & EA & ref & 3 & \begin{tabular}{|l|}
17 \\
\end{tabular} & n35 & 33.6 & 10.6 & 92.1 & 52.6 & 560.00 & 176.67 & 1535.00 & 876.67 \\
\hline 1 & EM-10 & quenched & 3 & 18 & $\mathrm{n} 44$ & 6.89 & 8.57 & 91.0 & 66.9 & 11.48 & 14.28 & 151.67 & 111.50 \\
\hline 1 & EM-05 & quenched & 3 & \begin{tabular}{|l|}
19 \\
\end{tabular} & n23 & 9.75 & 12.0 & 102 & 89.8 & 16.25 & 20.00 & 170.00 & 149.67 \\
\hline 1 & EM-07 & quenched & 3 & 20 & n54 & 11.6 & 15.1 & 95.6 & 77.3 & 19.33 & 25.17 & 159.34 & 128.84 \\
\hline
\end{tabular}


SRNL-STI-2009-00778, Revision 0

Table E1. PSAL's Measurements of the Matrix 2A PCT Solutions As-Received (ar) and After Appropriate Adjustments (in ppm)

\begin{tabular}{|c|c|c|c|c|c|c|c|c|c|c|c|c|c|}
\hline Set & Glass ID & Heat Treatment & Block & Seq & Lab ID & B ar & Li ar & Na ar & Si ar & B (ppm) & Li (ppm) & $\mathrm{Na}$ (ppm) & Si (ppm) \\
\hline 1 & EM-09 & CCC & 3 & 21 & n06 & 6.02 & 9.80 & 54.1 & 58.3 & 10.03 & 16.33 & 90.17 & 97.17 \\
\hline 1 & EM-07 & CCC & 3 & 22 & n26 & 6.78 & 11.1 & 71.6 & 64.6 & 11.30 & 18.50 & 119.34 & 107.67 \\
\hline 1 & ARM-1 & ref & 3 & 23 & n24 & 9.44 & 7.64 & 20.3 & 35.1 & 15.73 & 12.73 & 33.83 & 58.50 \\
\hline 1 & EM-02 & quenched & 3 & 24 & n03 & 4.82 & 7.30 & 45.3 & 51.0 & 8.03 & 12.17 & 75.50 & 85.00 \\
\hline 1 & EM-03 & quenched & 3 & 25 & n30 & 8.45 & 10.7 & 110 & 79.5 & 14.08 & 17.83 & 183.34 & 132.50 \\
\hline 1 & Soln Std & ref & 3 & 26 & std-13-3 & 19.5 & 9.68 & 81.0 & 50.3 & 19.50 & 9.68 & 81.00 & 50.30 \\
\hline 2 & Soln Std & ref & 1 & 1 & STD-21-1 & 20.8 & 10.0 & 81.5 & 51.7 & 20.80 & 10.00 & 81.50 & 51.70 \\
\hline 2 & EM-16 & CCC & 1 & 2 & P14 & 23.4 & 17.3 & 97.6 & 74.7 & 39.00 & 28.83 & 162.67 & 124.50 \\
\hline 2 & EM-18 & quenched & 1 & 3 & P40 & 17.6 & 12.0 & 115 & 73.1 & 29.33 & 20.00 & 191.67 & 121.84 \\
\hline 2 & EM-20 & CCC & 1 & 4 & P65 & 17.6 & 13.6 & 101 & 81.5 & 29.33 & 22.67 & 168.34 & 135.84 \\
\hline 2 & EM-13 & CCC & 1 & 5 & P05 & 16.6 & 30.1 & 96.0 & 93.9 & 27.67 & 50.17 & 160.00 & 156.50 \\
\hline 2 & EM-15 & CCC & 1 & 6 & P29 & 4.61 & 15.3 & 46.5 & 39.0 & 7.68 & 25.50 & 77.50 & 65.00 \\
\hline 2 & EM-11 & CCC & 1 & 7 & P22 & 4.52 & 7.31 & 48.7 & 53.4 & 7.53 & 12.18 & 81.17 & 89.00 \\
\hline 2 & EM-12 & quenched & 1 & 8 & P64 & 7.47 & 9.32 & 107 & 72.1 & 12.45 & 15.53 & 178.34 & 120.17 \\
\hline 2 & EM-14 & quenched & 1 & 9 & P06 & 4.89 & 7.78 & 52.6 & 43.4 & 8.15 & 12.97 & 87.67 & 72.33 \\
\hline 2 & EM-12 & CCC & 1 & 10 & P04 & 73.6 & 83.3 & 420 & 270 & 122.67 & 138.84 & 700.01 & 450.01 \\
\hline 2 & EM-15 & quenched & 1 & \begin{tabular}{|l|}
11 \\
\end{tabular} & P15 & 5.27 & 8.26 & 52.4 & 39.4 & 8.78 & 13.77 & 87.34 & 65.67 \\
\hline 2 & EM-19 & quenched & 1 & 12 & P30 & 20.9 & 14.0 & 135 & 90.7 & 34.83 & 23.33 & 225.00 & 151.17 \\
\hline 2 & EM-18 & $\mathrm{CCC}$ & 1 & 13 & P02 & 42.4 & 28.9 & 196 & 113 & 70.67 & 48.17 & 326.67 & 188.34 \\
\hline 2 & Soln Std & ref & 1 & 14 & STD-21-2 & 20.3 & 9.91 & 81.0 & 50.9 & 20.30 & 9.91 & 81.00 & 50.90 \\
\hline 2 & EM-17 & quenched & 1 & \begin{tabular}{|l|}
15 \\
\end{tabular} & \begin{tabular}{|l|} 
P21 \\
\end{tabular} & 10.3 & 8.60 & 49.8 & 48.6 & 17.17 & 14.33 & 83.00 & 81.00 \\
\hline 2 & EA & ref & 1 & 16 & P17 & 31.1 & 10.2 & 85.2 & 50.5 & 518.33 & 170.00 & 1420.00 & 841.67 \\
\hline 2 & EM-11 & quenched & 1 & 17 & P37 & 5.87 & 8.17 & 48.2 & 54.6 & 9.78 & 13.62 & 80.33 & 91.00 \\
\hline 2 & EM-19 & CCC & 1 & 18 & P10 & 16.4 & 12.6 & 117 & 84.4 & 27.33 & 21.00 & 195.00 & 140.67 \\
\hline 2 & EM-13 & quenched & 1 & 19 & $\mathrm{P} 23$ & 5.15 & 7.42 & 55.5 & 47.8 & 8.58 & 12.37 & 92.50 & 79.67 \\
\hline 2 & blank & ref & 1 & \begin{tabular}{|l|}
20 \\
\end{tabular} & P35 & $<0.100$ & $<1.00$ & $<0.100$ & $<0.100$ & 0.08 & 0.83 & 0.08 & 0.08 \\
\hline 2 & ARM-1 & ref & 1 & 21 & P27 & 9.44 & 7.56 & 19.8 & 35.0 & 15.73 & 12.60 & 33.00 & 58.33 \\
\hline 2 & EM-20 & quenched & 1 & 22 & P31 & 22.0 & 15.4 & 121 & 86.6 & 36.67 & 25.67 & 201.67 & 144.34 \\
\hline 2 & EM-16 & quenched & 1 & 23 & P66 & 12.9 & 9.86 & 75.6 & 57.2 & 21.50 & 16.43 & 126.00 & 95.34 \\
\hline 2 & EM-17 & CCC & 1 & 24 & P28 & 39.7 & 29.2 & 98.2 & 83.5 & 66.17 & 48.67 & 163.67 & 139.17 \\
\hline 2 & EM-14 & CCC & 1 & 25 & P50 & 5.95 & 14.2 & 52.0 & 46.4 & 9.92 & 23.67 & 86.67 & 77.33 \\
\hline 2 & Soln Std & ref & 1 & 26 & \begin{tabular}{|l|} 
STD21-3 \\
\end{tabular} & 19.8 & 9.63 & 80.0 & 49.6 & 19.80 & 9.63 & 80.00 & 49.60 \\
\hline 2 & Soln Std & ref & 2 & 1 & \begin{tabular}{|l|} 
STD-22-1 \\
\end{tabular} & 21.0 & 9.73 & 82.4 & 50.8 & 21.00 & 9.73 & 82.40 & 50.80 \\
\hline 2 & ARM-1 & ref & 2 & 2 & P62 & 10.0 & 7.58 & 20.5 & 36.0 & 16.67 & 12.63 & 34.17 & 60.00 \\
\hline 2 & EM-16 & $\mathrm{CCC}$ & 2 & 3 & P67 & 24.0 & 17.1 & 99.1 & 73.9 & 40.00 & 28.50 & 165.17 & 123.17 \\
\hline 2 & EM-13 & quenched & 2 & 4 & $\mathrm{P} 43$ & 5.19 & 7.51 & 59.6 & 49.3 & 8.65 & 12.52 & 99.34 & 82.17 \\
\hline 2 & EM-15 & quenched & 2 & 5 & P36 & 4.40 & 7.93 & 51.7 & 38.6 & 7.33 & 13.22 & 86.17 & 64.33 \\
\hline 2 & EM-16 & quenched & 2 & 6 & P09 & 12.7 & 9.68 & 76.0 & 56.6 & 21.17 & 16.13 & 126.67 & 94.34 \\
\hline 2 & EA & ref & 2 & 7 & P38 & 34.0 & 10.7 & 95.2 & 52.6 & 566.67 & 178.33 & 1586.67 & 876.67 \\
\hline 2 & EM-15 & CCC & 2 & 8 & P54 & 4.19 & 15.3 & 46.6 & 38.1 & 6.98 & 25.50 & 77.67 & 63.50 \\
\hline 2 & EM-14 & CCC & 2 & 9 & P11 & 5.25 & 14.7 & 55.9 & 46.5 & 8.75 & 24.50 & 93.17 & 77.50 \\
\hline 2 & EM-17 & $\mathrm{CCC}$ & 2 & 10 & P32 & 39.9 & 28.7 & 100 & 81.0 & 66.50 & 47.83 & 166.67 & 135.00 \\
\hline 2 & EM-12 & quenched & 2 & \begin{tabular}{|l|}
11 \\
\end{tabular} & P53 & 7.14 & 8.76 & 104 & 67.9 & 11.90 & 14.60 & 173.34 & 113.17 \\
\hline 2 & EM-18 & CCC & 2 & 12 & P08 & 42.6 & 28.9 & 193 & 113 & 71.00 & 48.17 & 321.67 & 188.34 \\
\hline 2 & EM-11 & $\mathrm{CCC}$ & 2 & \begin{tabular}{|l|}
13 \\
\end{tabular} & P52 & 4.40 & 6.94 & 48.5 & 51.5 & 7.33 & 11.57 & 80.83 & 85.84 \\
\hline 2 & Soln Std & ref & 2 & \begin{tabular}{|l|}
14 \\
\end{tabular} & \begin{tabular}{|l|} 
STD-22-2 \\
\end{tabular} & 20.1 & 9.47 & 82.4 & 49.0 & 20.10 & 9.47 & 82.40 & 49.00 \\
\hline 2 & EM-20 & quenched & 2 & 15 & P13 & 20.8 & 14.0 & 113 & 78.2 & 34.67 & 23.33 & 188.34 & 130.34 \\
\hline 2 & EM-19 & CCC & 2 & 16 & $\mathrm{P} 03$ & 16.5 & 12.2 & 119 & 80.6 & 27.50 & 20.33 & 198.34 & 134.34 \\
\hline 2 & EM-20 & CCC & 2 & \begin{tabular}{|l|}
17 \\
\end{tabular} & P18 & 17.5 & 13.2 & 102 & 78.5 & 29.17 & 22.00 & 170.00 & 130.84 \\
\hline 2 & EM-19 & quenched & 2 & 18 & P20 & 19.1 & 12.6 & 128 & 81.5 & 31.83 & 21.00 & 213.34 & 135.84 \\
\hline 2 & EM-18 & quenched & 2 & 19 & $\mathrm{P} 25$ & 17.7 & 11.7 & 119 & 71.2 & 29.50 & 19.50 & 198.34 & 118.67 \\
\hline 2 & EM-13 & CCC & 2 & \begin{tabular}{|l|}
20 \\
\end{tabular} & P19 & 15.7 & 27.8 & 91.6 & 86.5 & 26.17 & 46.33 & 152.67 & 144.17 \\
\hline 2 & EM-12 & CCC & 2 & 21 & P60 & 72.2 & 81.0 & 423 & 257 & 120.34 & 135.00 & 705.01 & 428.34 \\
\hline 2 & EM-14 & quenched & 2 & 22 & P55 & 5.41 & 7.64 & 54.3 & 43.0 & 9.02 & 12.73 & 90.50 & 71.67 \\
\hline 2 & EM-17 & quenched & 2 & 23 & $\mathrm{P} 48$ & 9.45 & 7.85 & 48.1 & 44.5 & 15.75 & 13.08 & 80.17 & 74.17 \\
\hline 2 & EM-11 & quenched & 2 & \begin{tabular}{|l|}
24 \\
\end{tabular} & P51 & 5.22 & 7.67 & 48.6 & 51.4 & 8.70 & 12.78 & 81.00 & 85.67 \\
\hline 2 & Soln Std & ref & 2 & 25 & STD-22-3 & 19.7 & 9.62 & 82.0 & 49.4 & 19.70 & 9.62 & 82.00 & 49.40 \\
\hline 2 & Soln Std & ref & 3 & 1 & \begin{tabular}{|l|} 
STD-23-1 \\
\end{tabular} & 21.0 & 9.93 & 81.6 & 50.3 & 21.00 & 9.93 & 81.60 & 50.30 \\
\hline 2 & EM-15 & quenched & 3 & 2 & P26 & 5.30 & 8.24 & 51.5 & 38.8 & 8.83 & 13.73 & 85.84 & 64.67 \\
\hline 2 & EM-20 & quenched & 3 & 3 & P59 & 21.5 & 14.5 & 111 & 78.2 & 35.83 & 24.17 & 185.00 & 130.34 \\
\hline 2 & EM-13 & quenched & 3 & 4 & P63 & 5.71 & 7.58 & 55.5 & 48.5 & 9.52 & 12.63 & 92.50 & 80.83 \\
\hline 2 & EM-12 & quenched & 3 & 5 & P07 & 7.97 & 9.29 & 105 & 70.7 & 13.28 & 15.48 & 175.00 & 117.84 \\
\hline 2 & EM-17 & CCC & 3 & 6 & P46 & 40.4 & 28.9 & 97.7 & 81.2 & 67.33 & 48.17 & 162.84 & 135.34 \\
\hline 2 & EA & ref & 3 & 7 & P45 & 32.7 & 10.4 & 87.7 & 51.0 & 545.00 & 173.33 & 1461.67 & 850.00 \\
\hline 2 & EM-12 & CCC & 3 & 8 & P16 & 72.1 & 82.1 & 443 & 262 & 120.17 & 136.84 & 738.35 & 436.68 \\
\hline 2 & EM-19 & CCC & 3 & 9 & P47 & 17.7 & 12.8 & 117 & 83.6 & 29.50 & 21.33 & 195.00 & 139.34 \\
\hline 2 & blank & ref & 3 & \begin{tabular}{|l|}
10 \\
\end{tabular} & $\mathrm{P} 24$ & 0.703 & $<1.00$ & $<0.100$ & $<0.100$ & 1.17 & 0.83 & 0.08 & 0.08 \\
\hline 2 & EM-14 & CCC & 3 & \begin{tabular}{|l|}
11 \\
\end{tabular} & P42 & 6.22 & 15.3 & 57.2 & 47.9 & 10.37 & 25.50 & 95.34 & 79.83 \\
\hline 2 & EM-11 & quenched & 3 & 12 & P41 & 6.02 & 8.21 & 50.2 & 54.4 & 10.03 & 13.68 & 83.67 & 90.67 \\
\hline 2 & EM-15 & CCC & 3 & \begin{tabular}{|l|}
13 \\
\end{tabular} & P57 & 4.68 & 15.5 & 46.5 & 38.2 & 7.80 & 25.83 & 77.50 & 63.67 \\
\hline 2 & Soln Std & ref & 3 & 14 & \begin{tabular}{|l|} 
STD-23-2 \\
\end{tabular} & 20.8 & 10.2 & 83.8 & 51.3 & 20.80 & 10.20 & 83.80 & 51.30 \\
\hline
\end{tabular}


SRNL-STI-2009-00778, Revision 0

Table E1. PSAL's Measurements of the Matrix 2A PCT Solutions As-Received (ar) and After Appropriate Adjustments (in ppm)

\begin{tabular}{|c|c|c|c|c|c|c|c|c|c|c|c|c|c|}
\hline Set & Glass ID & Heat Treatment & Block & Seq & Lab ID & B ar & Li ar & Na ar & Si ar & B (ppm) & Li (ppm) & $\mathrm{Na}$ (ppm) & Si (ppm) \\
\hline 2 & EM-14 & quenched & 3 & 15 & P69 & 5.78 & 8.14 & 53.6 & 44.3 & 9.63 & 13.57 & 89.34 & 73.83 \\
\hline 2 & EM-18 & quenched & 3 & 16 & P49 & 17.7 & 11.8 & 111 & 70.3 & 29.50 & 19.67 & 185.00 & 117.17 \\
\hline 2 & EM-20 & CCC & 3 & 17 & P33 & 17.5 & 13.4 & 98.3 & 78.2 & 29.17 & 22.33 & 163.84 & 130.34 \\
\hline 2 & ARM-1 & ref & 3 & 18 & P34 & 10.1 & 7.68 & 20.2 & 35.1 & 16.83 & 12.80 & 33.67 & 58.50 \\
\hline 2 & EM-13 & CCC & 3 & 19 & P56 & 16.3 & 28.9 & 89.9 & 88.9 & 27.17 & 48.17 & 149.84 & 148.17 \\
\hline 2 & EM-19 & quenched & 3 & 20 & P12 & 20.5 & 13.6 & 128 & 85.7 & 34.17 & 22.67 & 213.34 & 142.84 \\
\hline 2 & EM-17 & quenched & 3 & 21 & P39 & 10.0 & 8.33 & 48.0 & 46.4 & 16.67 & 13.88 & 80.00 & 77.33 \\
\hline 2 & EM-11 & CCC & 3 & 22 & P58 & 4.89 & 7.44 & 49.7 & 53.1 & 8.15 & 12.40 & 82.83 & 88.50 \\
\hline 2 & EM-16 & quenched & 3 & 23 & P61 & 13.0 & 9.93 & 74.2 & 56.3 & 21.67 & 16.55 & 123.67 & 93.84 \\
\hline 2 & EM-18 & CCC & 3 & 24 & P01 & 43.6 & 29.8 & 195 & 116 & 72.67 & 49.67 & 325.01 & 193.34 \\
\hline 2 & EM-16 & CCC & 3 & 25 & P44 & 23.0 & 16.8 & 92.9 & 72.4 & 38.33 & 28.00 & 154.84 & 120.67 \\
\hline 2 & Soln Std & ref & 3 & 26 & STD-23-3 & 20.6 & 10.0 & 80.3 & 50.7 & 20.60 & 10.00 & 80.30 & 50.70 \\
\hline 3 & Soln Std & ref & 1 & 1 & STD-31-1 & 21.3 & 9.90 & 83.3 & 50.8 & 21.30 & 9.90 & 83.30 & 50.80 \\
\hline 3 & EM-25 & quenched & 1 & 2 & Q01 & 14.6 & 9.82 & 103 & 63.5 & 24.33 & 16.37 & 171.67 & 105.84 \\
\hline 3 & EM-30 & CCC & 1 & 3 & Q38 & 23.9 & 20.3 & 70.6 & 46.3 & 39.83 & 33.83 & 117.67 & 77.17 \\
\hline 3 & EM-27 & CCC & 1 & 4 & Q53 & 11.2 & 9.85 & 51.3 & 55.6 & 18.67 & 16.42 & 85.50 & 92.67 \\
\hline 3 & EM-24 & quenched & 1 & 5 & Q47 & 12.1 & 9.69 & 56.3 & 51.8 & 20.17 & 16.15 & 93.84 & 86.34 \\
\hline 3 & EM-30 & quenched & 1 & 6 & Q26 & 12.8 & 11.4 & 67.8 & 43.2 & 21.33 & 19.00 & 113.00 & 72.00 \\
\hline 3 & EM-26 & quenched & 1 & 7 & Q27 & 12.1 & 9.53 & 56.2 & 52.8 & 20.17 & 15.88 & 93.67 & 88.00 \\
\hline 3 & EM-27 & quenched & 1 & 8 & Q62 & 16.6 & 11.0 & 117 & 67.1 & 27.67 & 18.33 & 195.00 & 111.84 \\
\hline 3 & EM-21 & CCC & 1 & 9 & Q02 & 11.2 & 9.45 & 57.5 & 60.1 & 18.67 & 15.75 & 95.84 & 100.17 \\
\hline 3 & EM-22 & CCC & 1 & 10 & Q19 & 14.0 & 12.2 & 72.3 & 60.5 & 23.33 & 20.33 & 120.50 & 100.84 \\
\hline 3 & EM-28 & quenched & 1 & 11 & Q18 & 12.4 & 9.60 & 67.6 & 49.5 & 20.67 & 16.00 & 112.67 & 82.50 \\
\hline 3 & EA & ref & 1 & 12 & Q07 & 36.6 & 10.7 & 95.8 & 52.8 & 610.00 & 178.33 & 1596.67 & 880.00 \\
\hline 3 & EM-22 & quenched & 1 & 13 & Q10 & 36.1 & 24.6 & 135 & 86.9 & 60.17 & 41.00 & 225.00 & 144.84 \\
\hline 3 & Soln Std & ref & 1 & \begin{tabular}{|l|}
14 \\
\end{tabular} & \begin{tabular}{|l|} 
STD-31-2 \\
\end{tabular} & 20.8 & 9.88 & 82.6 & 51.0 & 20.80 & 9.88 & 82.60 & 51.00 \\
\hline 3 & EM-25 & CCC & 1 & 15 & Q44 & 151 & 102 & 469 & 240 & 251.67 & 170.00 & 781.68 & 400.01 \\
\hline 3 & EM-23 & quenched & 1 & 16 & Q43 & 52.6 & 35.9 & 195 & 99.2 & 87.67 & 59.83 & 325.01 & 165.34 \\
\hline 3 & EM-23 & CCC & 1 & \begin{tabular}{|l|}
17 \\
\end{tabular} & Q09 & 17.1 & 14.2 & 83.5 & 62.4 & 28.50 & 23.67 & 139.17 & 104.00 \\
\hline 3 & ARM-1 & ref & 1 & 18 & Q05 & 11.0 & 8.07 & 20.3 & 35.9 & 18.33 & 13.45 & 33.83 & 59.83 \\
\hline 3 & EM-29 & quenched & 1 & 19 & Q36 & 12.9 & 10.5 & 66.4 & 46.0 & 21.50 & 17.50 & 110.67 & 76.67 \\
\hline 3 & blank & ref & 1 & 20 & Q34 & 0.465 & $<1.00$ & $<0.100$ & $<0.100$ & 0.78 & 0.83 & 0.08 & 0.08 \\
\hline 3 & EM-26 & CCC & 1 & \begin{tabular}{|l|}
21 \\
\end{tabular} & Q64 & 271 & 163 & \begin{tabular}{|l|}
771 \\
\end{tabular} & 283 & 451.68 & 271.67 & 1285.03 & 471.68 \\
\hline 3 & EM-24 & CCC & 1 & 22 & Q55 & 18.2 & 12.6 & 60.7 & 60.6 & 30.33 & 21.00 & 101.17 & 101.00 \\
\hline 3 & EM-29 & $\mathrm{CCC}$ & 1 & 23 & Q08 & 48.5 & 34.7 & 115 & 73.0 & 80.83 & 57.83 & 191.67 & 121.67 \\
\hline 3 & EM-28 & CCC & 1 & 24 & Q21 & 99.9 & 69.2 & 220 & 115 & 166.50 & 115.34 & 366.67 & 191.67 \\
\hline 3 & EM-21 & quenched & 1 & 25 & Q59 & 22.5 & 14.8 & 85.5 & 74.0 & 37.50 & 24.67 & 142.50 & 123.34 \\
\hline 3 & Soln Std & ref & 1 & 26 & STD31-3 & 21.0 & 9.69 & 80.0 & 49.8 & 21.00 & 9.69 & 80.00 & 49.80 \\
\hline 3 & Soln Std & ref & 2 & 1 & \begin{tabular}{|l|} 
STD-32-1 \\
\end{tabular} & 20.9 & 10.1 & 81.5 & 50.5 & 20.90 & 10.10 & 81.50 & 50.50 \\
\hline 3 & EM-24 & quenched & 2 & 2 & Q31 & 12.2 & 10.0 & 57.7 & 52.0 & 20.33 & 16.67 & 96.17 & 86.67 \\
\hline 3 & EM-27 & quenched & 2 & 3 & Q49 & 15.9 & 11.0 & 114 & 66.6 & 26.50 & 18.33 & 190.00 & 111.00 \\
\hline 3 & EM-23 & CCC & 2 & 4 & Q54 & 15.3 & 14.1 & 82.8 & 61.2 & 25.50 & 23.50 & 138.00 & 102.00 \\
\hline 3 & EM-23 & quenched & 2 & 5 & Q28 & 49.8 & 35.4 & 238 & 97.9 & 83.00 & 59.00 & 396.67 & 163.17 \\
\hline 3 & EM-22 & quenched & 2 & 6 & Q37 & 36.7 & 25.6 & 144 & 88.1 & 61.17 & 42.67 & 240.00 & 146.84 \\
\hline 3 & EM-26 & CCC & 2 & 7 & Q46 & 292 & 176 & 858 & 306 & 486.68 & 293.34 & 1430.03 & 510.01 \\
\hline 3 & EM-25 & quenched & 2 & 8 & Q14 & 17.0 & 10.1 & 102 & 63.2 & 28.33 & 16.83 & 170.00 & 105.34 \\
\hline 3 & EM-24 & CCC & 2 & 9 & Q16 & 16.6 & 13.0 & 64.6 & 61.1 & 27.67 & 21.67 & 107.67 & 101.84 \\
\hline 3 & EM-26 & quenched & 2 & 10 & Q13 & 12.9 & 10.1 & 58.5 & 53.8 & 21.50 & 16.83 & 97.50 & 89.67 \\
\hline 3 & EM-21 & CCC & 2 & \begin{tabular}{|l|}
11 \\
\end{tabular} & Q12 & 11.4 & 9.80 & 57.9 & 60.8 & 19.00 & 16.33 & 96.50 & 101.34 \\
\hline 3 & EM-28 & CCC & 2 & 12 & Q56 & 112 & 71.7 & 246 & 128 & 186.67 & 119.50 & 410.01 & 213.34 \\
\hline 3 & EM-25 & CCC & 2 & 13 & Q63 & 167 & 108 & 543 & 269 & 278.34 & 180.00 & 905.02 & 448.34 \\
\hline 3 & Soln Std & ref & 2 & \begin{tabular}{|l|}
14 \\
\end{tabular} & STD-32-2 & 22.6 & 10.3 & 84.4 & 51.4 & 22.60 & 10.30 & 84.40 & 51.40 \\
\hline 3 & EM-30 & quenched & 2 & 15 & Q35 & 13.5 & 11.8 & 70.8 & 43.7 & 22.50 & 19.67 & 118.00 & 72.83 \\
\hline 3 & EM-21 & quenched & 2 & 16 & Q51 & 20.7 & 15.1 & 87.4 & 73.3 & 34.50 & 25.17 & 145.67 & 122.17 \\
\hline 3 & EA & ref & 2 & 17 & Q20 & 36.5 & 11.3 & 102 & 52.8 & 608.33 & 188.33 & 1700.00 & 880.00 \\
\hline 3 & EM-22 & CCC & 2 & \begin{tabular}{|l|}
18 \\
\end{tabular} & Q30 & 14.5 & 12.8 & 74.2 & 61.4 & 24.17 & 21.33 & 123.67 & 102.34 \\
\hline 3 & EM-28 & quenched & 2 & 19 & Q25 & 12.7 & 10.2 & 69.9 & 50.5 & 21.17 & 17.00 & 116.50 & 84.17 \\
\hline 3 & EM-27 & CCC & 2 & 20 & Q04 & 11.6 & 10.6 & 56.6 & 57.3 & 19.33 & 17.67 & 94.34 & 95.50 \\
\hline 3 & EM-29 & CCC & 2 & 21 & Q67 & 47.1 & 36.2 & \begin{tabular}{|l|}
120.7 \\
\end{tabular} & 73.4 & 78.50 & 60.33 & 201.17 & 122.34 \\
\hline 3 & EM-30 & CCC & 2 & 22 & Q29 & 23.9 & 20.7 & 73.4 & 46.5 & 39.83 & 34.50 & 122.34 & 77.50 \\
\hline 3 & EM-29 & quenched & 2 & \begin{tabular}{|l|}
23 \\
\end{tabular} & Q68 & 12.9 & 11.0 & 69.3 & 46.4 & 21.50 & 18.33 & 115.50 & 77.33 \\
\hline 3 & ARM-1 & ref & 2 & 24 & Q57 & 10.5 & 8.48 & 22.2 & 36.4 & 17.50 & 14.13 & 37.00 & 60.67 \\
\hline 3 & Soln Std & ref & 2 & 25 & STD-32-3 & 20.5 & 10.2 & 84.1 & 50.7 & 20.50 & 10.20 & 84.10 & 50.70 \\
\hline 3 & Soln Std & ref & 3 & 1 & STD-33-1 & 21.2 & 9.91 & 82.2 & 51.0 & 21.20 & 9.91 & 82.20 & 51.00 \\
\hline 3 & EM-30 & quenched & 3 & 2 & Q03 & 12.1 & 11.1 & 67.8 & 41.6 & 20.17 & 18.50 & 113.00 & 69.33 \\
\hline 3 & EM-28 & quenched & 3 & 3 & Q06 & 12.3 & 9.76 & 68.1 & 50.1 & 20.50 & 16.27 & 113.50 & 83.50 \\
\hline 3 & EM-27 & quenched & 3 & 4 & Q40 & 16.1 & 11.0 & 118.1 & 66.0 & 26.83 & 18.33 & 196.84 & 110.00 \\
\hline 3 & EM-26 & CCC & 3 & 5 & Q42 & 266 & 164 & 766 & 281 & 443.34 & 273.34 & 1276.69 & 468.34 \\
\hline 3 & EM-29 & quenched & 3 & 6 & Q50 & 15.2 & 10.4 & 67.0 & 45.6 & 25.33 & 17.33 & 111.67 & 76.00 \\
\hline 3 & EM-24 & CCC & 3 & 7 & Q66 & 16.6 & 12.5 & 63.6 & 60.8 & 27.67 & 20.83 & 106.00 & 101.34 \\
\hline 3 & EA & ref & 3 & 8 & Q58 & 37.4 & 10.8 & 97.5 & 52.7 & 623.33 & 180.00 & 1625.00 & 878.34 \\
\hline
\end{tabular}


SRNL-STI-2009-00778, Revision 0

Table E1. PSAL's Measurements of the Matrix 2A PCT Solutions As-Received (ar) and After Appropriate Adjustments (in ppm)

\begin{tabular}{|c|c|c|c|c|c|c|c|c|c|c|c|c|c|}
\hline Set & Glass ID & Heat Treatment & Block & Seq & Lab ID & B ar & Li ar & $\mathrm{Na}$ ar & Si ar & B (ppm) & Li (ppm) & $\mathrm{Na}$ (ppm) & Si (ppm) \\
\hline 3 & EM-23 & CCC & 3 & 9 & Q17 & 16.2 & 14.1 & 83.8 & 61.4 & 27.00 & 23.50 & 139.67 & 102.34 \\
\hline 3 & ARM-1 & ref & 3 & 10 & Q45 & 10.7 & 8.25 & 21.5 & 36.5 & 17.83 & 13.75 & 35.83 & 60.83 \\
\hline 3 & EM-24 & quenched & 3 & 11 & Q24 & 12.3 & 9.92 & 58.9 & 52.6 & 20.50 & 16.53 & 98.17 & 87.67 \\
\hline 3 & EM-28 & CCC & 3 & 12 & Q33 & 106 & 74.4 & 219 & 119 & 176.67 & 124.00 & 365.01 & 198.34 \\
\hline 3 & EM-25 & quenched & 3 & 13 & Q65 & 15.6 & 10.1 & 107 & 64.3 & 26.00 & 16.83 & 178.34 & 107.17 \\
\hline 3 & Soln Std & ref & 3 & 14 & STD-33-2 & 21.5 & 10.1 & 84.0 & 51.2 & 21.50 & 10.10 & 84.00 & 51.20 \\
\hline 3 & EM-26 & quenched & 3 & 15 & Q15 & 12.7 & 9.77 & 59.9 & 53.3 & 21.17 & 16.28 & 99.84 & 88.84 \\
\hline 3 & EM-27 & CCC & 3 & 16 & Q11 & 11.8 & 10.3 & 56.1 & 57.1 & 19.67 & 17.17 & 93.50 & 95.17 \\
\hline 3 & EM-22 & CCC & 3 & 17 & Q48 & 13.9 & 12.2 & 73.2 & 60.3 & 23.17 & 20.33 & 122.00 & 100.50 \\
\hline 3 & blank & ref & 3 & 18 & Q60 & $<0.100$ & $<1.00$ & $<0.100$ & $<0.100$ & 0.08 & 0.83 & 0.08 & 0.08 \\
\hline 3 & EM-21 & quenched & 3 & 19 & Q23 & 20.4 & 14.4 & 86.8 & 71.1 & 34.00 & 24.00 & 144.67 & 118.50 \\
\hline 3 & EM-25 & CCC & 3 & 20 & Q32 & 166 & 106 & 517 & 268 & 276.67 & 176.67 & 861.68 & 446.68 \\
\hline 3 & EM-29 & CCC & 3 & 21 & Q52 & 50.8 & 35.8 & 124 & 73.7 & 84.67 & 59.67 & 206.67 & 122.84 \\
\hline 3 & EM-21 & CCC & 3 & 22 & Q39 & 12.3 & 9.55 & 59.2 & 61.5 & 20.50 & 15.92 & 98.67 & 102.50 \\
\hline 3 & EM-23 & quenched & 3 & 23 & Q22 & 52.7 & 35.8 & 226 & 110 & 87.84 & 59.67 & 376.67 & 183.34 \\
\hline 3 & EM-30 & CCC & 3 & 24 & Q41 & 24.9 & 20.1 & 70.7 & 45.4 & 41.50 & 33.50 & 117.84 & 75.67 \\
\hline 3 & EM-22 & quenched & 3 & 25 & Q61 & 36.5 & 24.7 & 139 & 86.8 & 60.83 & 41.17 & 231.67 & 144.67 \\
\hline 3 & Soln Std & ref & 3 & 26 & STD-33-3 & 21.1 & 9.78 & 83.0 & 50.5 & 21.10 & 9.78 & 83.00 & 50.50 \\
\hline
\end{tabular}




\section{Exhibit E1. PCT Measurements in Analytical Sequence over All of the Analytical Plans for the Matrix 2A Study}
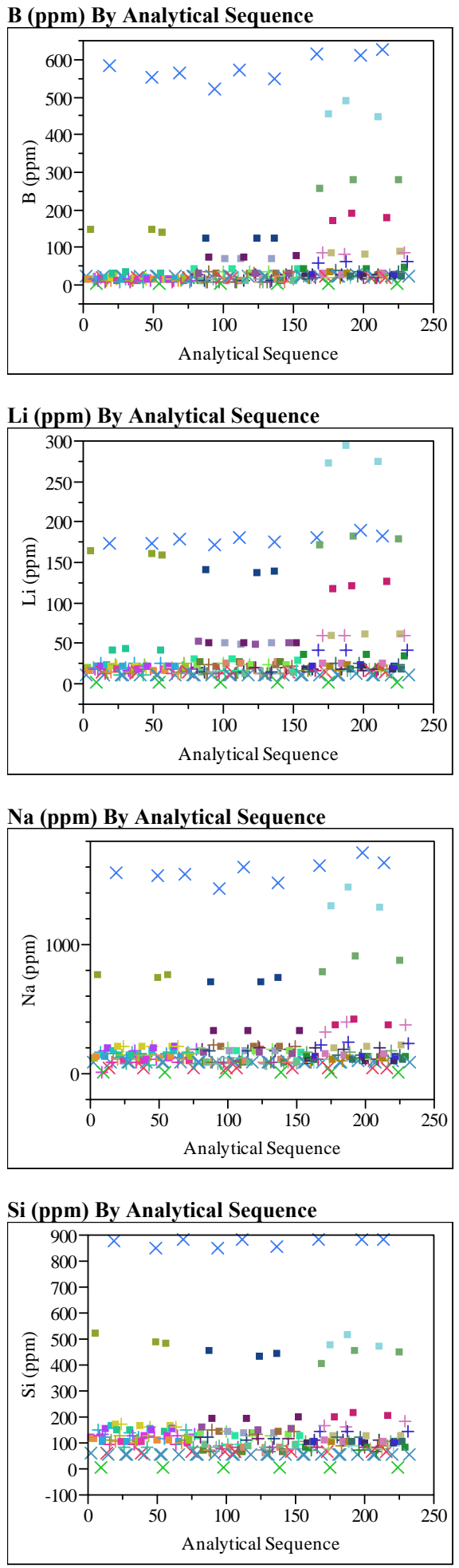

$\log [\mathrm{B}$ ppm] By Analytical Sequence

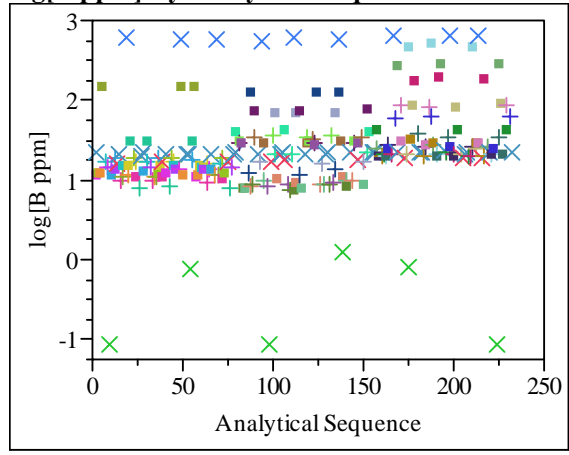

$\log [\mathrm{Li} \mathrm{ppm}]$ By Analytical Sequence

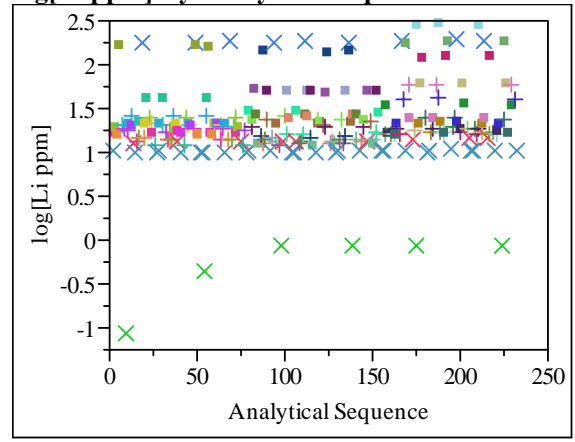

$\log [$ Na ppm] By Analytical Sequence
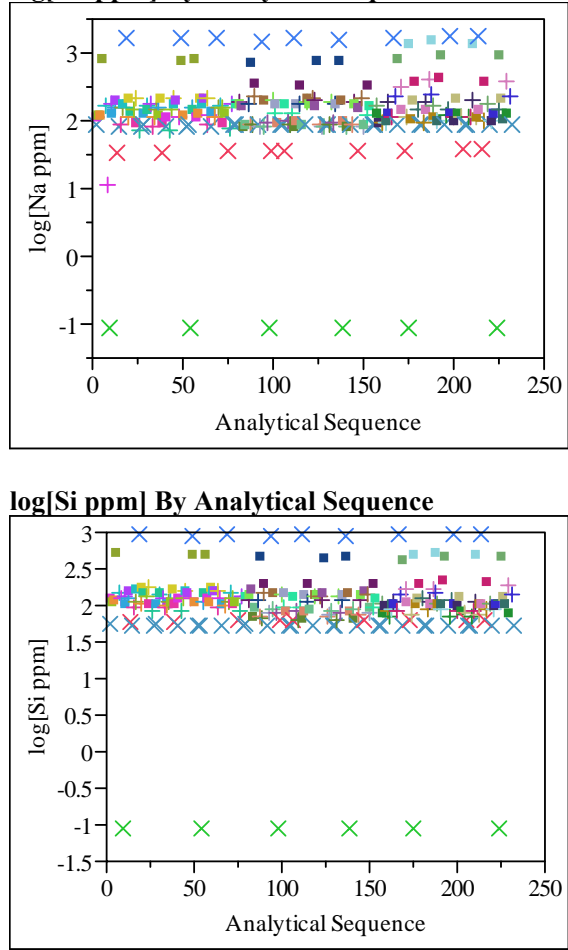
SRNL-STI-2009-00778, Revision 0

Exhibit E2. Measurements of the Multi-Element Solution Standard by ICP Block for the Matrix 2A Study

Oneway Analysis of B (ppm) By Set/Block

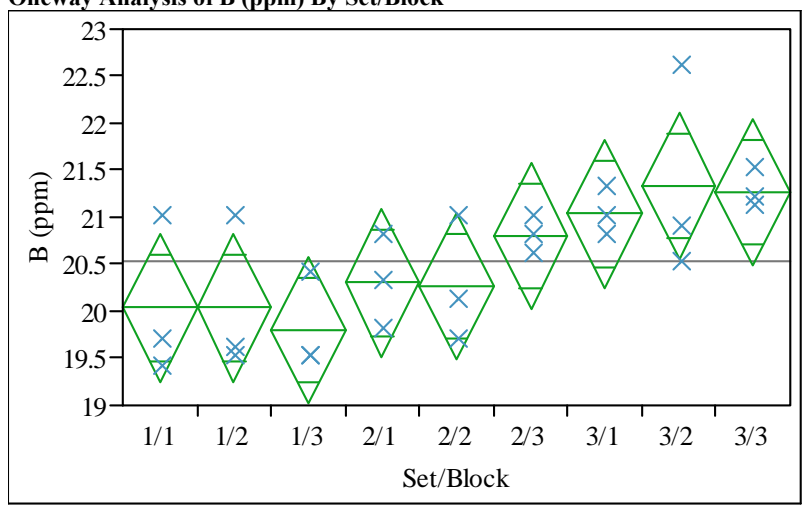

Oneway Anova

Summary of Fit

$\begin{array}{lr}\text { Rsquare } & 0.513676 \\ \text { Adj Rsquare } & 0.297532 \\ \text { Root Mean Square Error } & 0.648074 \\ \text { Mean of Response } & 20.54074 \\ \text { Observations (or Sum Wgts) } & 27\end{array}$

Analysis of Variance

Source DF Sum of Squares Mean Square F Ratio Prob $>$ F

$\begin{array}{llllll}\text { Set/Block } & 8 & 7.985185 & 0.998148 & 2.3765 & 0.0607\end{array}$

$\begin{array}{lrrr}\text { Error } & 18 & 7.560000 & 0.420000\end{array}$

Means for Oneway Anova

Level Number Mean Std Error Lower 95\% Upper 95\%

$\begin{array}{llllll}1 / 1 & 3 & 20.0333 & 0.37417 & 19.247 & 20.819\end{array}$

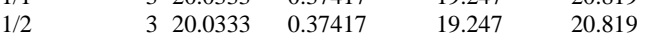

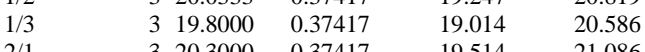

$\begin{array}{llllll}2 / 1 & 3 & 20.3000 & 0.37417 & 19.514 & 21.086\end{array}$

$\begin{array}{llllll}2 / 2 & 3 & 20.2667 & 0.37417 & 19.481 & 21.053 \\ 2 / 3 & 3 & 20.8000 & 0.37417 & 20.014 & 21.586\end{array}$

$\begin{array}{llllll}2 / 3 & 3 & 20.8000 & 0.37417 & 20.014 & 21.586 \\ 3 / 1 & 3 & 21.0333 & 0.37417 & 20.247 & 21.819\end{array}$

$\begin{array}{llllll}3 / 2 & 3 & 21.3333 & 0.37417 & 20.547 & 22.119 \\ 3 / 3 & 3 & 21.2667 & 0.37417 & 20.481 & 22.053\end{array}$

Std Error uses a pooled estimate of error variance

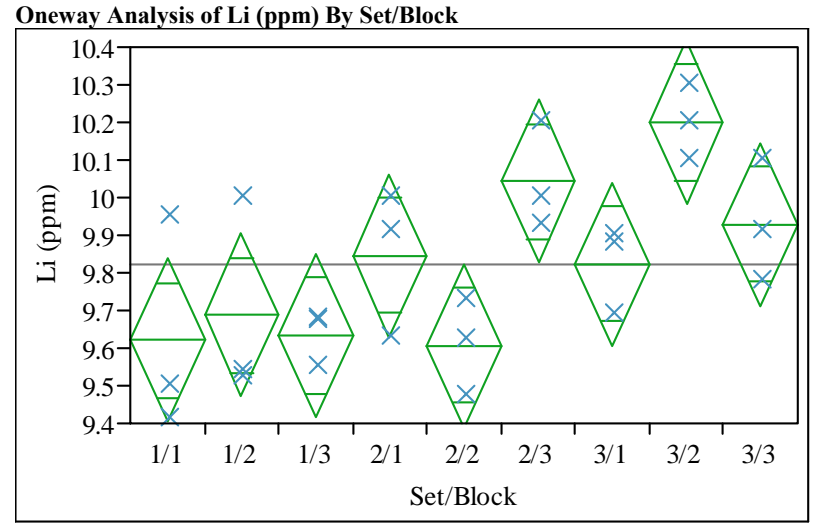

Oneway Anova

Summary of Fit

$\begin{array}{lr}\text { Rsquare } & 0.644285 \\ \text { Adj Rsquare } & 0.486189 \\ \text { Root Mean Square Error } & 0.178232 \\ \text { Mean of Response } & 9.821111 \\ \text { Observations (or Sum Wgts) } & 27\end{array}$

Observations (or Sum Wgts) 9.821111

Analysis of Variance

Source DF Sum of Squares Mean Square F Ratio Prob $>$ F

$\begin{array}{llllll}\text { Set/Block } & 8 & 1.0356667 & 0.129458 & 4.0753 & 0.0063\end{array}$

$\begin{array}{llll}\text { Error } & 18 & 0.5718000 & 0.031767 \\ \text { C. Total } & 26 & 16074667 & \end{array}$

Means for Oneway Anova

Level Number Mean Std Error Lower 95\% Upper 95\%

$\begin{array}{lllllr}1 / 1 & 3 & 9.6200 & 0.10290 & 9.4038 & 9.836\end{array}$

$\begin{array}{llllll}1 / 2 & 3 & 9.6867 & 0.10290 & 9.4705 & 9.903\end{array}$

$\begin{array}{llllll}1 / 3 & 3 & 9.6333 & 0.10290 & 9.4171 & 9.850\end{array}$

$\begin{array}{llllll}2 / 1 & 3 & 9.8467 & 0.10290 & 9.6305 & 10.063\end{array}$

$\begin{array}{llllll}2 / 2 & 3 & 9.6067 & 0.10290 & 9.3905 & 9.823\end{array}$

$\begin{array}{rrrrrr}2 / 3 & 3 & 10.0433 & 0.10290 & 9.8271 & 10.260 \\ 3 / 1 & 3 & 9.8233 & 0.10290 & 9.6071 & 10.040 \\ 3 / 2 & 3 & 10.2000 & 0.10290 & 9.9838 & 10.416\end{array}$

$\begin{array}{llllll}3 / 2 & 3 & 10.2000 & 0.10290 & 9.9838 & 10.040 \\ 3 / 3 & 3 & 9.9300 & 0.10290 & 9.713 & 10.416\end{array}$

$\begin{array}{llrlll}3 / 3 & 3 & 9.9300 & 0.10290 & 9.7138 & 10.146\end{array}$

Std Error uses a pooled estimate of error variance 
SRNL-STI-2009-00778, Revision 0

Exhibit E2. Measurements of the Multi-Element Solution Standard by ICP Block for the Matrix 2A Study

Oneway Analysis of Na (ppm) By Set/Block

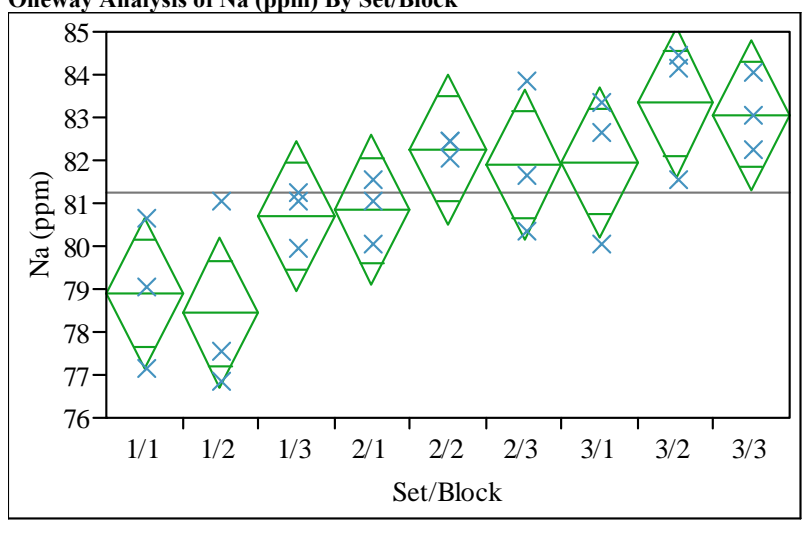

Oneway Anova

Summary of Fit

Rsquare

Adj Rsquare

Root Mean Square Error

0.652922

0.498665

1.444145

Mean of Response

27

Analysis of Variance

Source DF Sum of Squares Mean Square F Ratio Prob $>$ F

$\begin{array}{lrrrrr}\text { Set/Block } & 8 & 70.62000 & 8.82750 & 4.2327 & 0.0053\end{array}$

$\begin{array}{llr}\text { Error } & 18 & 37.54000\end{array}$

2.08556

Means for Oneway Anova

Level Number Mean Std Error Lower 95\% Upper 95\%

$\begin{array}{llrrrr}1 / 1 & 3 & 78.9000 & 0.83378 & 77.148 & 80.652\end{array}$

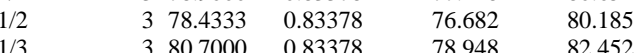

$\begin{array}{llllll}1 / 3 & 3 & 80.7000 & 0.83378 & 78.948 & 82.452 \\ 2 / 1 & 3 & 80.8333 & 0.8378 & 79.082 & 82.585\end{array}$

$\begin{array}{llllll}2 / 1 & 3 & 80.8333 & 0.83378 & 79.082 & 82.585 \\ 2 / 2 & 3 & 82.2667 & 0.83378 & 80.515 & 84.018\end{array}$

$\begin{array}{llllll}2 / 2 & 3 & 82.2667 & 0.83378 & 80.515 & 84.018 \\ 2 / 3 & 3 & 81.9000 & 0.83378 & 80.148 & 83.652\end{array}$

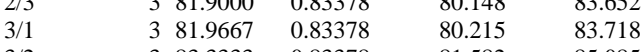

$\begin{array}{llllll}3 / 2 & 3 & 83.3333 & 0.83378 & 81.582 & 85.085 \\ 3 / 3 & 3 & 83.0667 & 0.83378 & 81.315 & 84.818\end{array}$

Std Error uses a pooled estimate of error variance

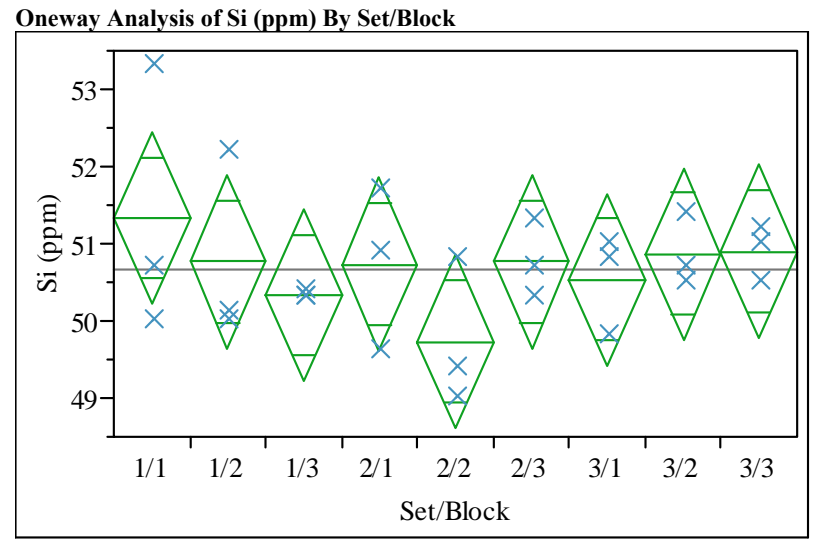

Oneway Anova

Summary of Fit

$\begin{array}{lr}\text { Rsquare } & 0.235625 \\ \text { Adj Rsquare } & -0.1041 \\ \text { Root Mean Square Error } & 0.91934 \\ \text { Mean of Response } & 50.66296 \\ \text { Observations (or Sum Wgts) } & 27\end{array}$

Analysis of Variance

Analysis of Variance
Source DF Sum of Squares Mean Square F Ratio Prob > F

$\begin{array}{lrrrrr}\text { Source } & \text { DF } & \text { Sum of Squares } & \text { Mean Square } & \text { F Ratio } & \text { Prob }>\text { F } \\ \text { Set/Block } & 8 & 4.689630 & 0.586204 & 0.6936 & 0.6928\end{array}$

$\begin{array}{lrrr}\text { Set/Block } & 8 & 4.689630 & 0.586204 \\ \text { Error } & 18 & 15.213333 & 0.845185\end{array}$

C. Total $\quad 26 \quad 19.902963$

Means for Oneway Anova

Level Number Mean Std Error Lower 95\% Upper 95\%

$\begin{array}{llllll}1 / 1 & 3 & 51.3333 & 0.53078 & 50.218 & 52.448 \\ 1 / 2 & 3 & 50.7667 & 0.53078 & 49.652 & 51.882\end{array}$

$\begin{array}{llllll}1 / 2 & 3 & 50.7667 & 0.53078 & 49.652 & 51.882 \\ 1 / 3 & 3 & 50.3333 & 0.53078 & 49.218 & 51.448\end{array}$

$\begin{array}{llllll}2 / 1 & 3 & 50.7333 & 0.53078 & 49.618 & 51.848 \\ 2 / 2 & 3 & 49.7333 & 0.53078 & 48.618 & 50.848\end{array}$

$\begin{array}{llllll}2 / 2 & 3 & 49.7333 & 0.53078 & 48.618 & 50.848 \\ 2 / 3 & 3 & 50.7667 & 0.53078 & 49.652 & 51.882\end{array}$

$\begin{array}{llllll}2 / 3 & 3 & 50.7667 & 0.53078 & 49.652 & 51.882 \\ 3 / 1 & 3 & 50.5333 & 0.53078 & 49.418 & 51.648\end{array}$

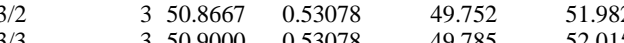

Std Error uses a pooled estimate of error variance 
Exhibit E3. PCT Measurements by Glass Identifier for the Matrix 2A Study Glasses and Standards

Set=1

Variability Chart for B (ppm)

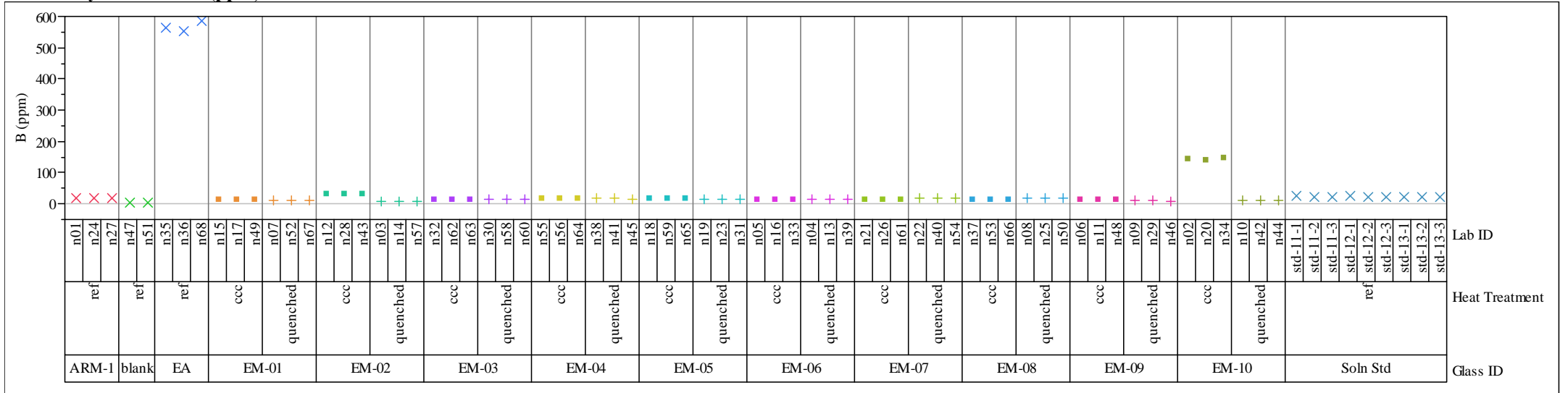

Set=1

Variability Chart for Li (ppm)

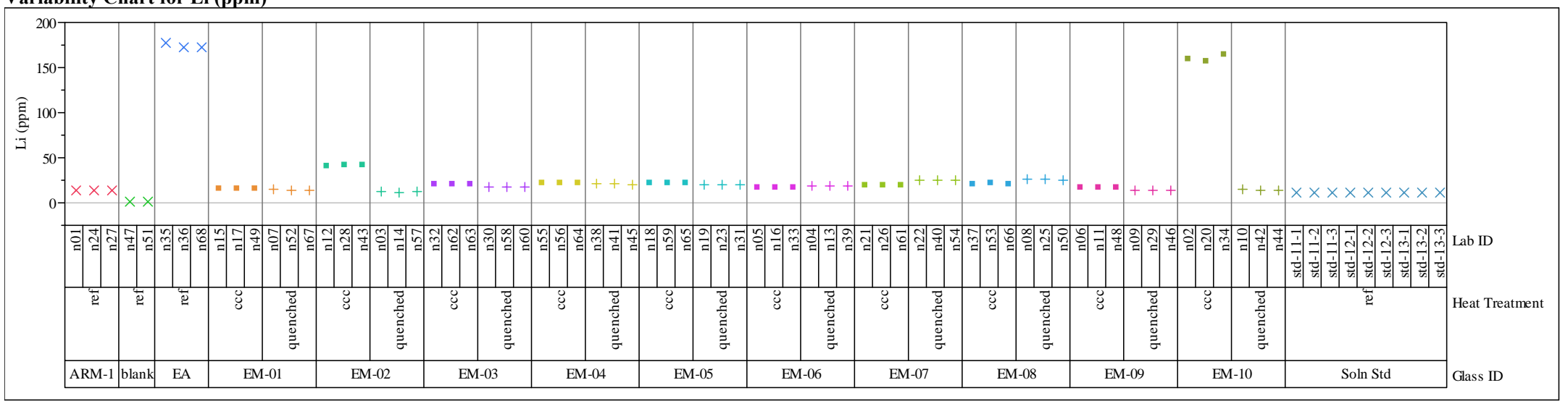


Exhibit E3. PCT Measurements by Glass Identifier for the Matrix 2A Study Glasses and Standards

Set=1

Variability Chart for Na (ppm)

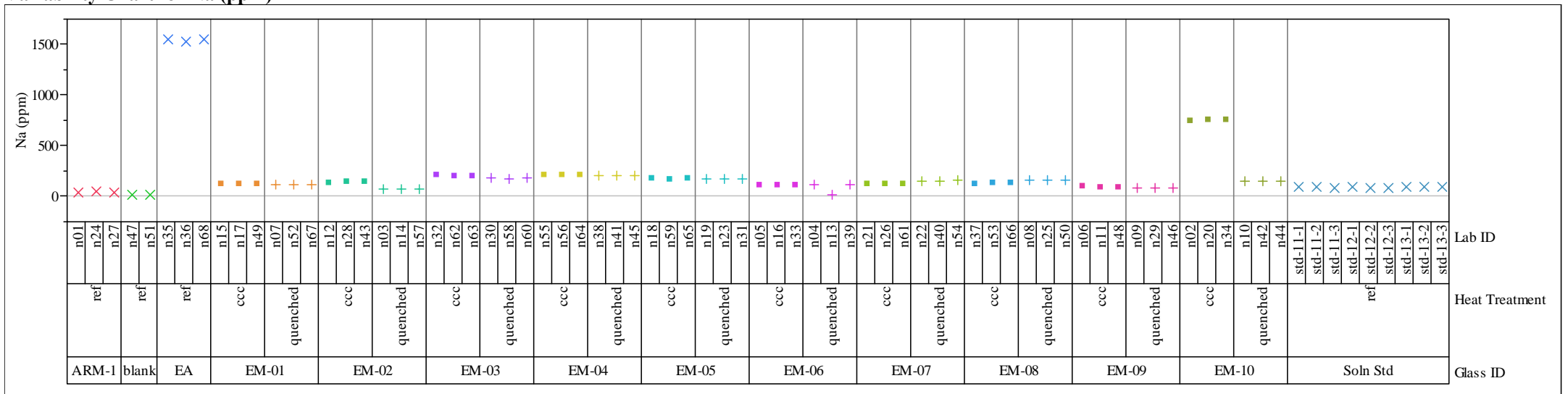

Set=1

Variability Chart for Si (ppm)

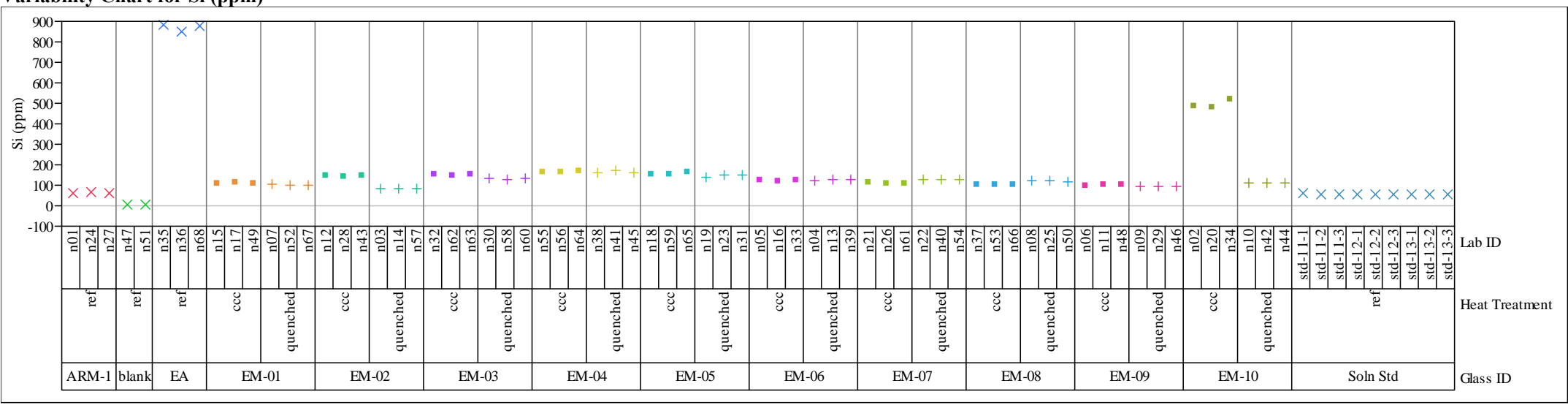


Exhibit E3. PCT Measurements by Glass Identifier for the Matrix 2A Study Glasses and Standards

Set=1

Variability Chart for $\log [\mathrm{B}$ ppm]

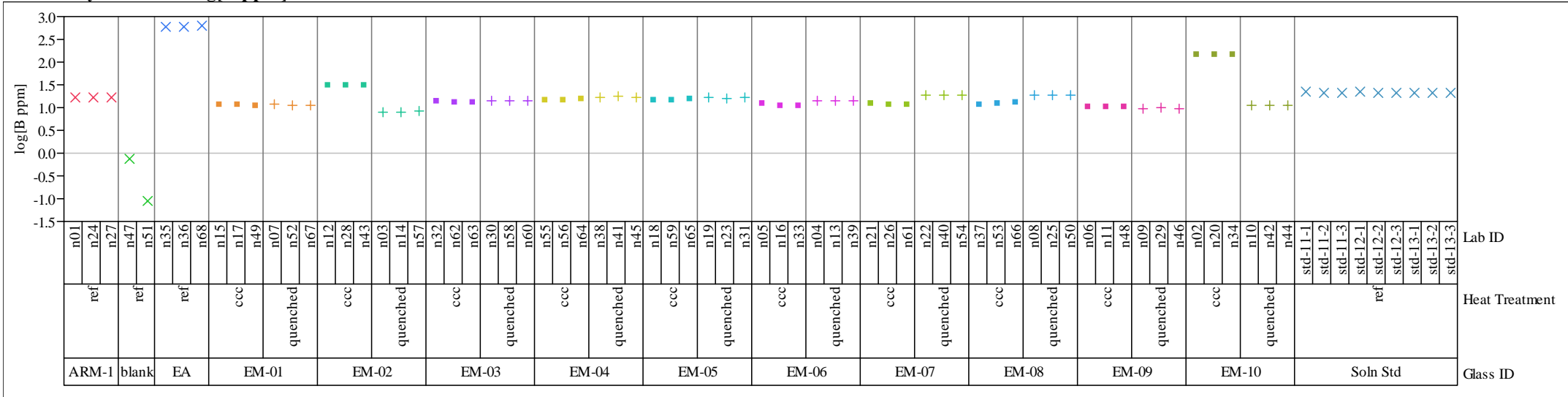

Set $=1$

Variability Chart for $\log [\mathrm{Li} \mathrm{ppm]}$

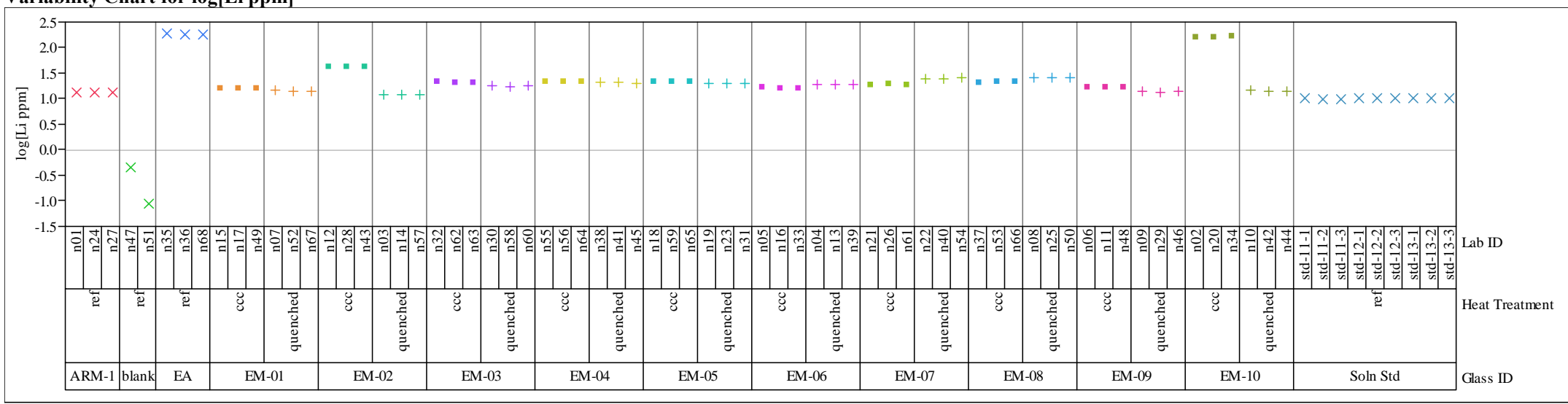


Exhibit E3. PCT Measurements by Glass Identifier for the Matrix 2A Study Glasses and Standards

Set=1

Variability Chart for log[Na ppm]

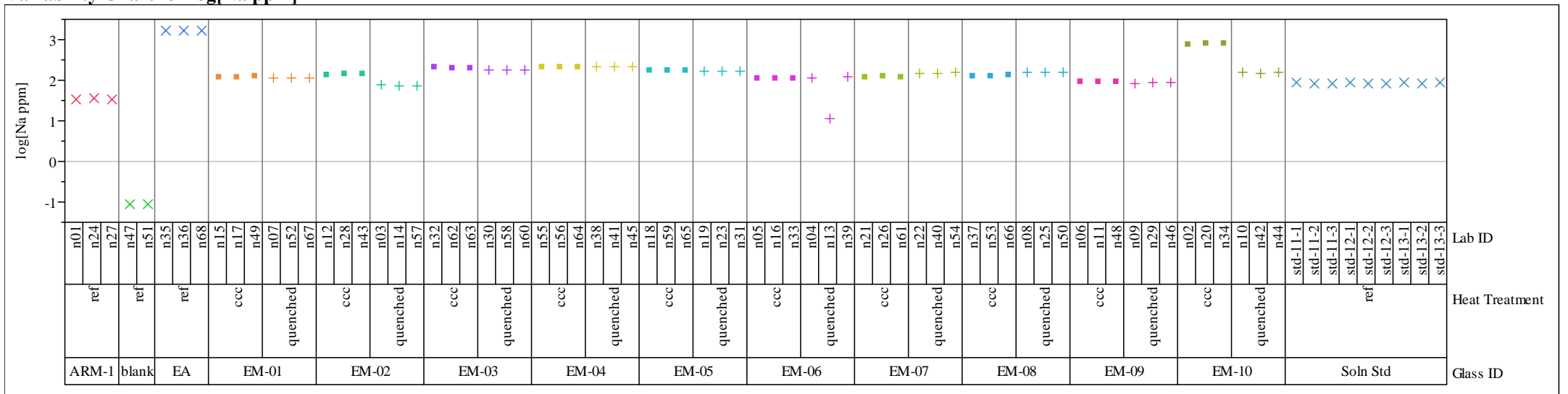

Set $=1$

Variability Chart for $\log [\mathrm{Si}$ ppm]

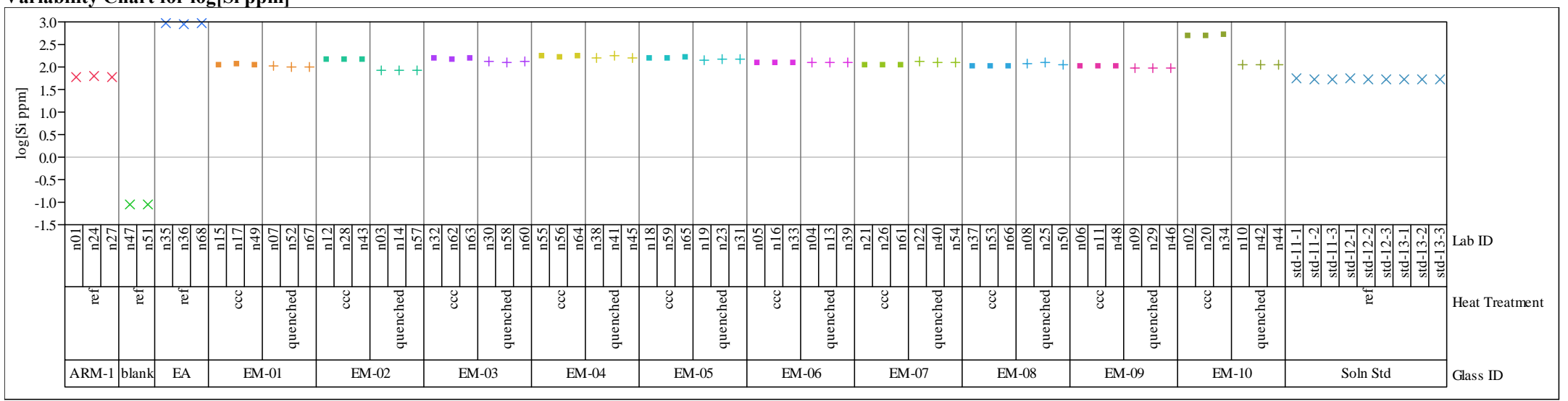


Exhibit E3. PCT Measurements by Glass Identifier for the Matrix 2A Study Glasses and Standards

Set $=2$

Variability Chart for B (ppm)

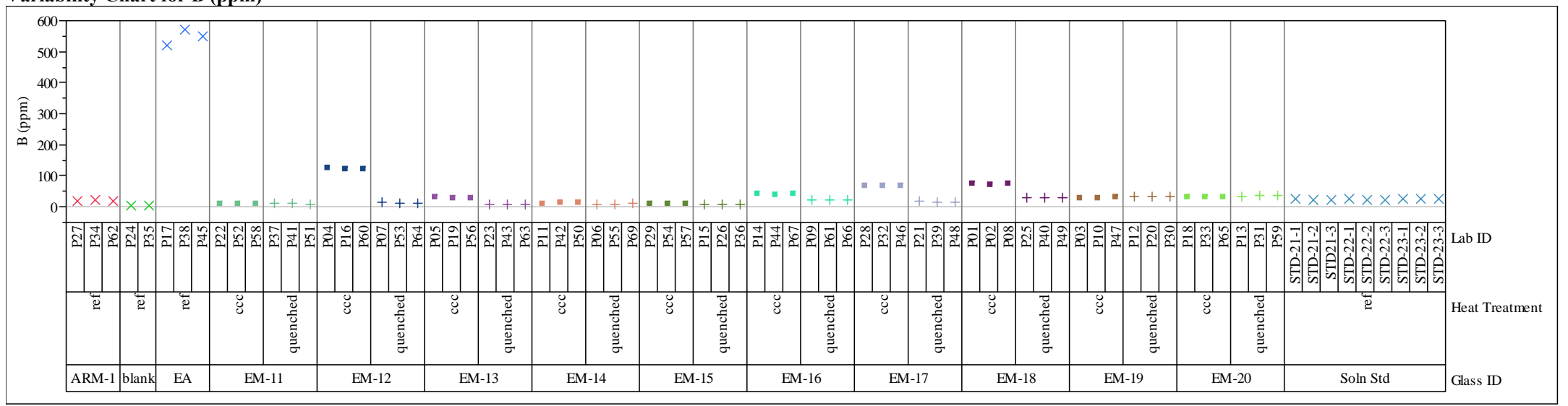

Set $=2$

Variability Chart for Li (ppm)

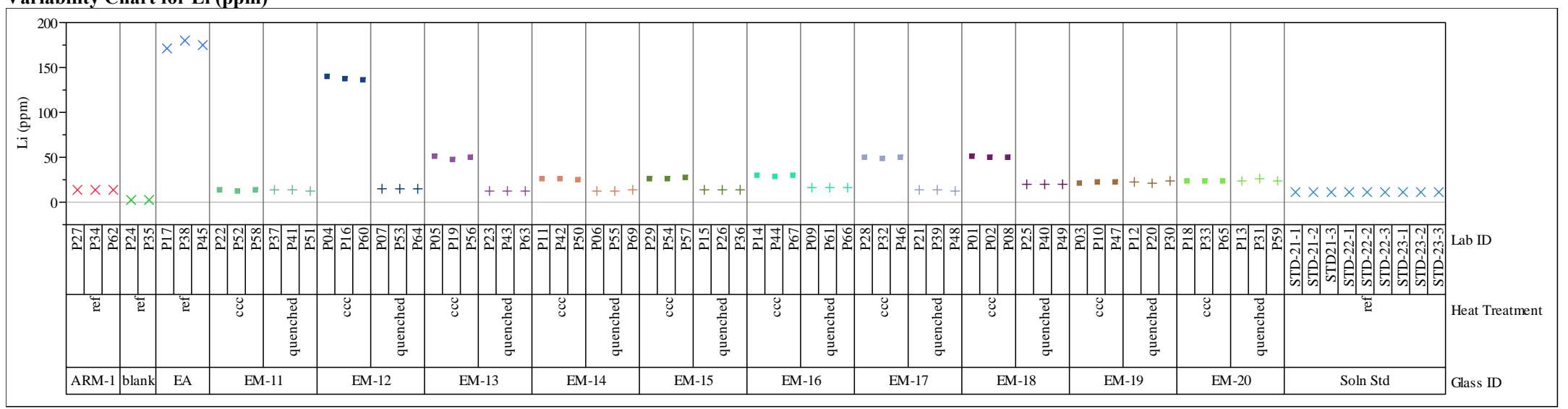


Exhibit E3. PCT Measurements by Glass Identifier for the Matrix 2A Study Glasses and Standards

Set $=2$

Variability Chart for Na (ppm)

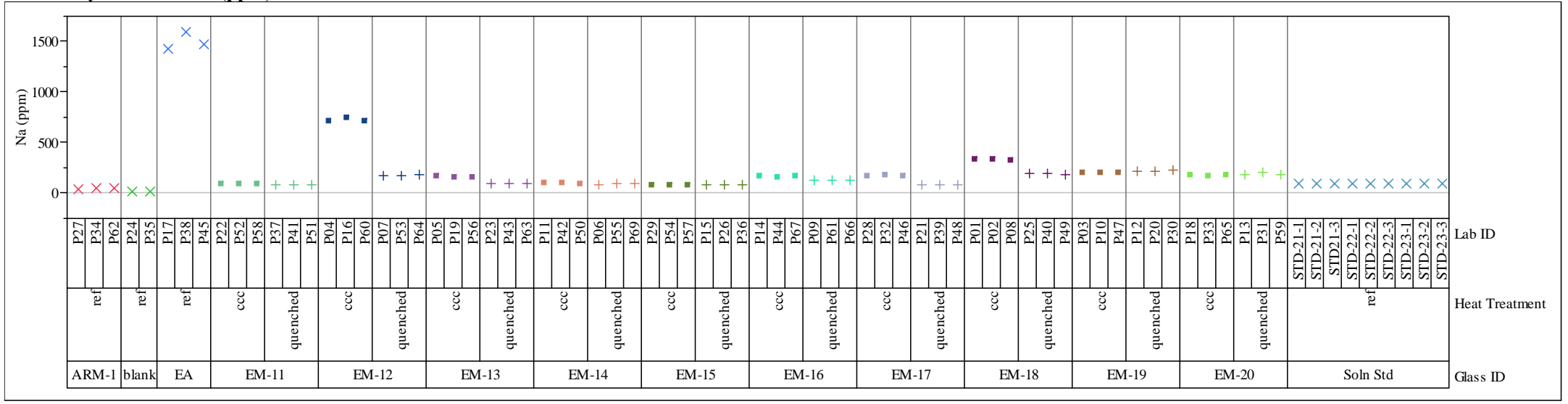

Set $=2$

Variability Chart for Si (ppm)

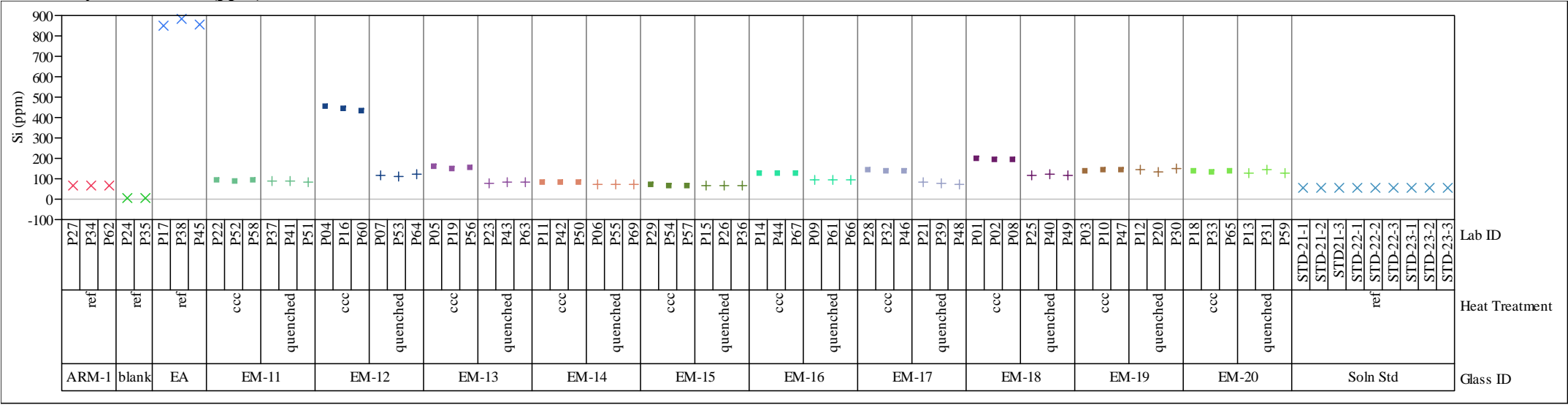




\section{Exhibit E3. PCT Measurements by Glass Identifier for the Matrix 2A Study Glasses and Standards}

Set $=2$

Variability Chart for $\log [\mathrm{B}$ ppm]

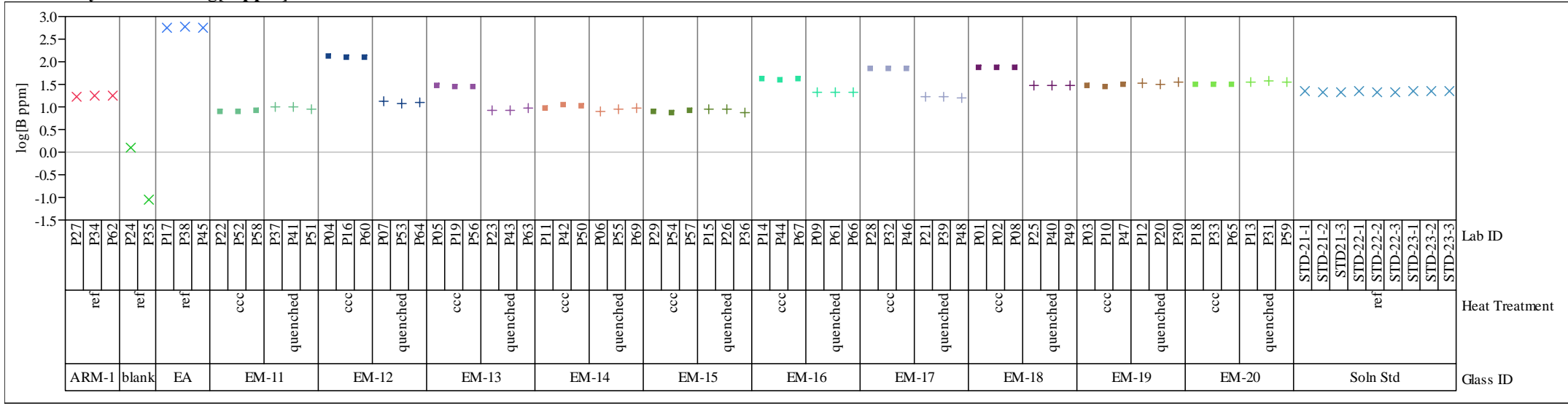

Set $=2$

Variability Chart for log[Li ppm]

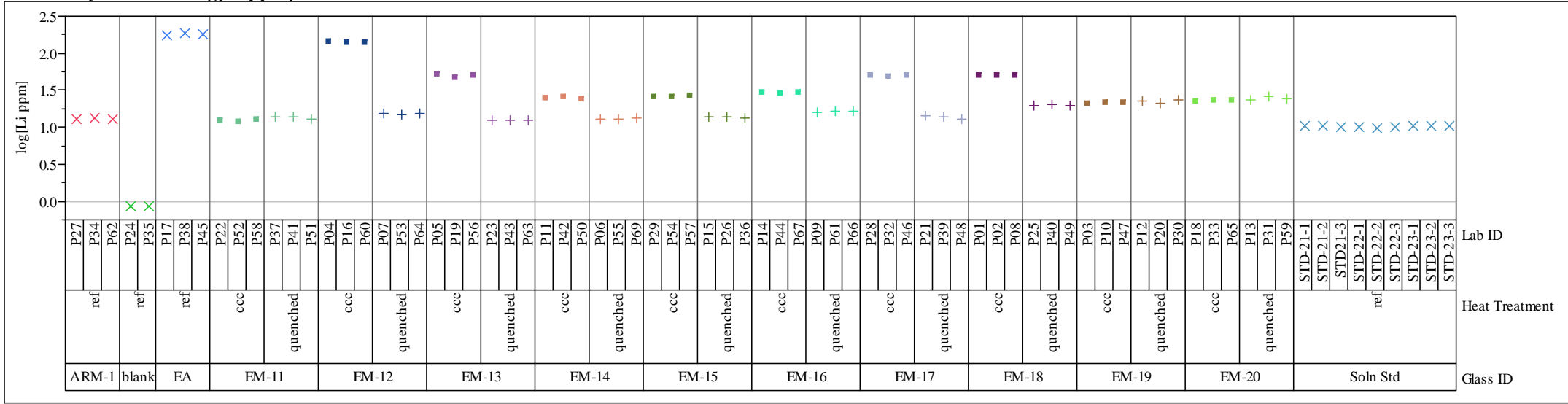


Exhibit E3. PCT Measurements by Glass Identifier for the Matrix 2A Study Glasses and Standards

Set $=2$

Variability Chart for log[Na ppm]

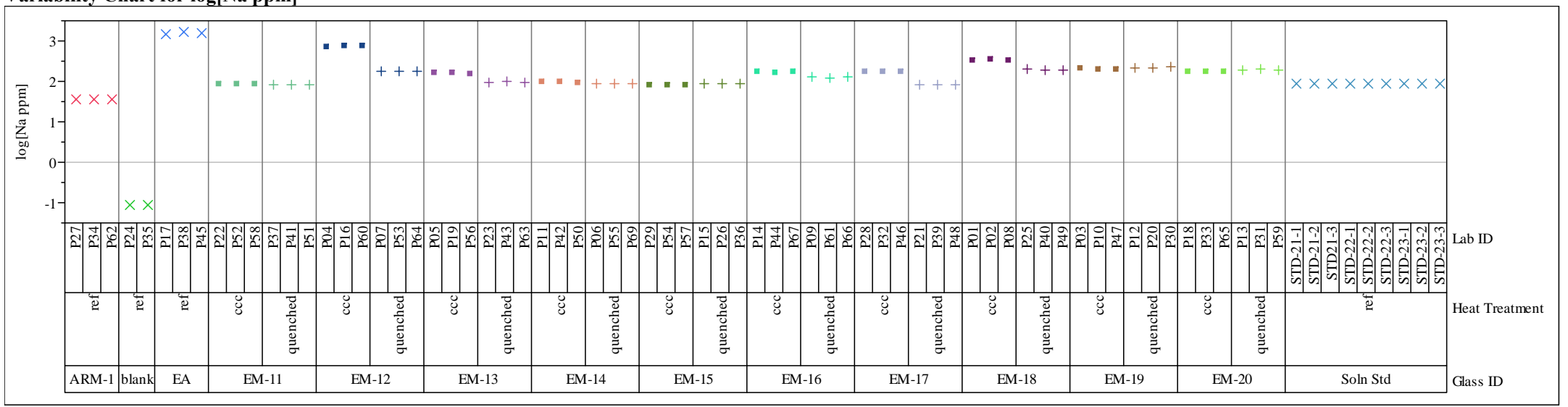

Set $=2$

Variability Chart for $\log [\mathrm{Si}$ ppm]

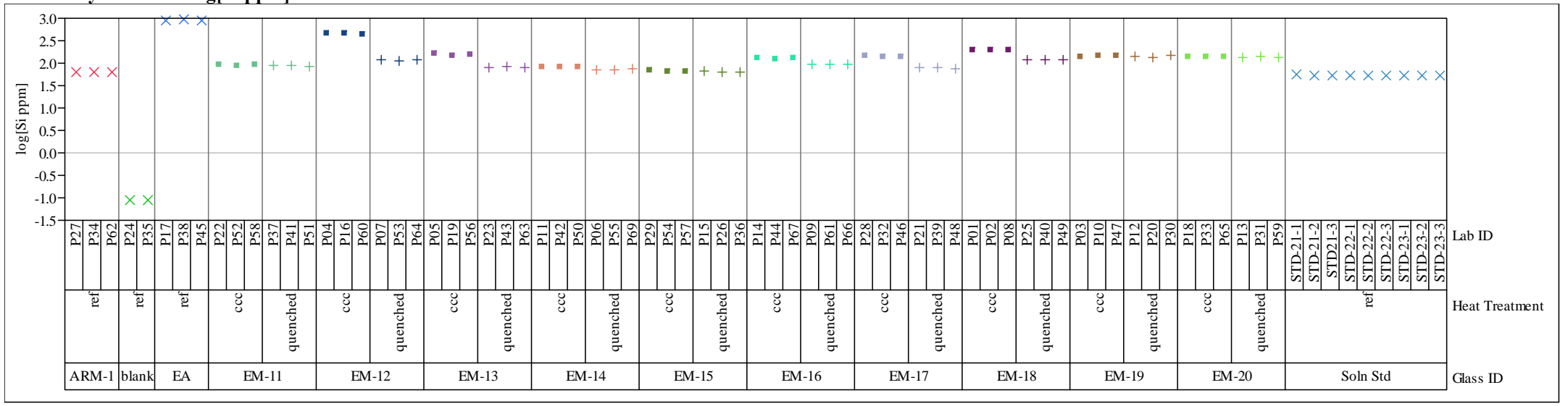


Exhibit E3. PCT Measurements by Glass Identifier for the Matrix 2A Study Glasses and Standards

Set $=3$

Variability Chart for B (ppm)

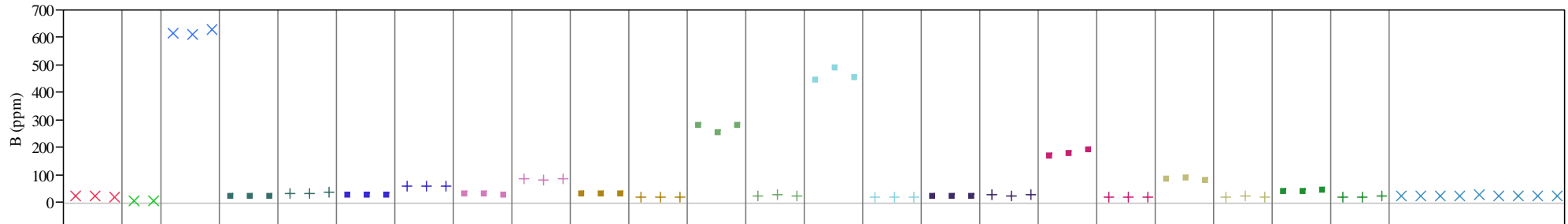

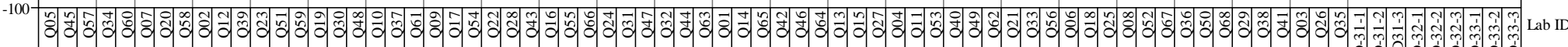
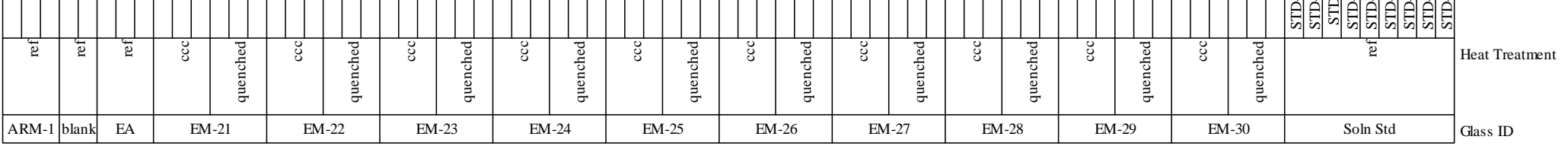

Set $=3$

Variability Chart for Li (ppm)

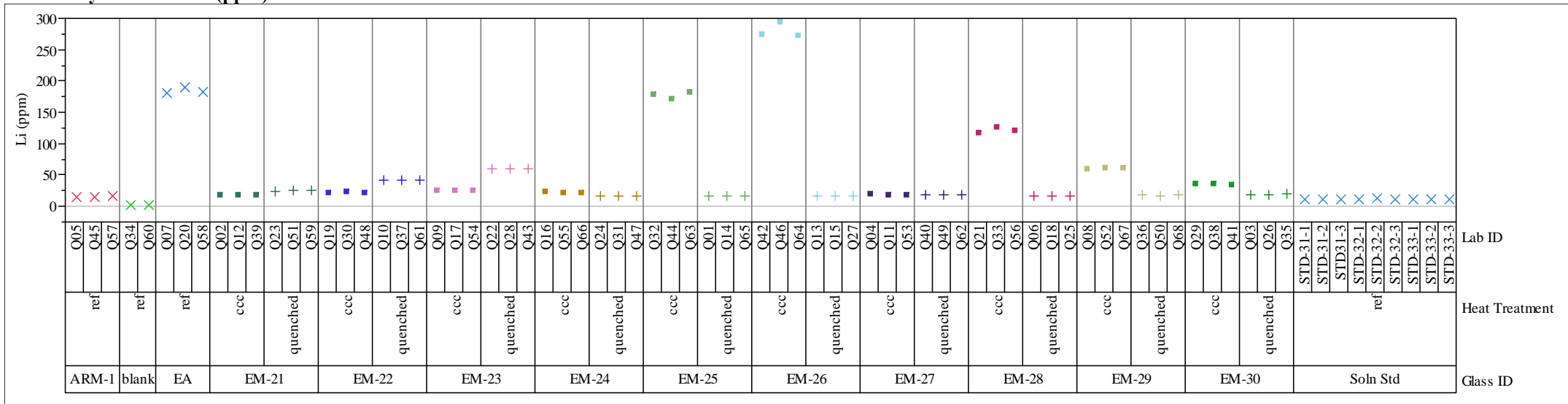


Exhibit E3. PCT Measurements by Glass Identifier for the Matrix 2A Study Glasses and Standards

Set $=3$

Variability Chart for Na (ppm)

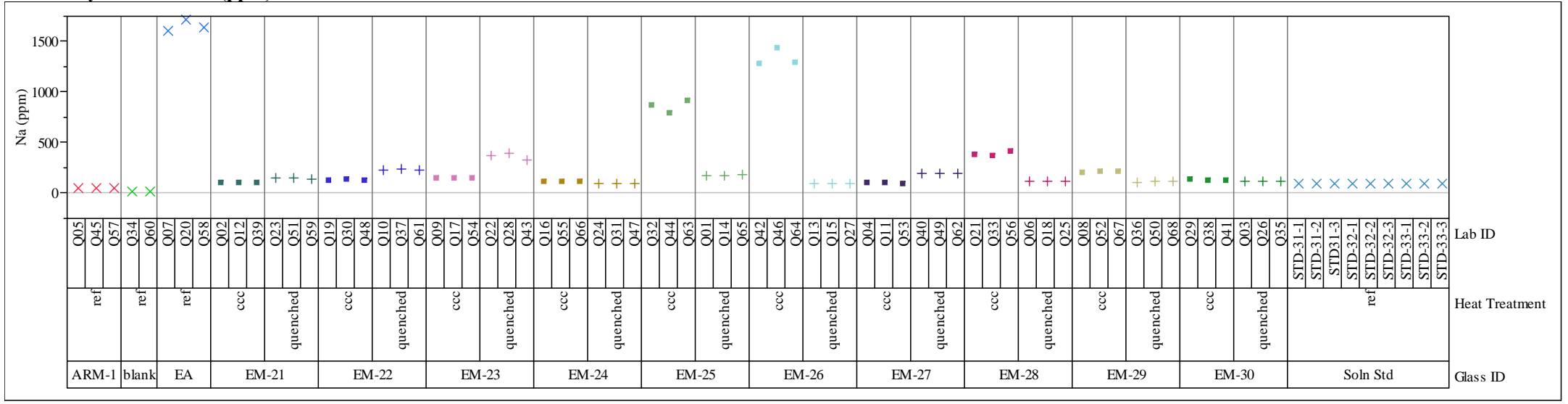

Set $=3$

Variability Chart for Si (ppm)

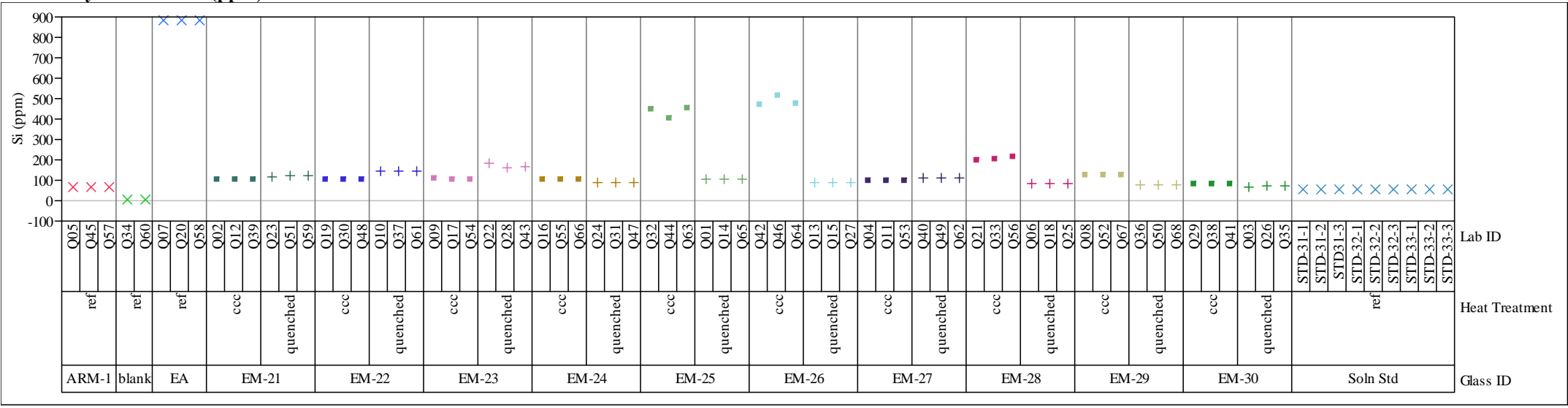


Exhibit E3. PCT Measurements by Glass Identifier for the Matrix 2A Study Glasses and Standards

Set $=3$

Variability Chart for $\log [\mathrm{B}$ ppm]

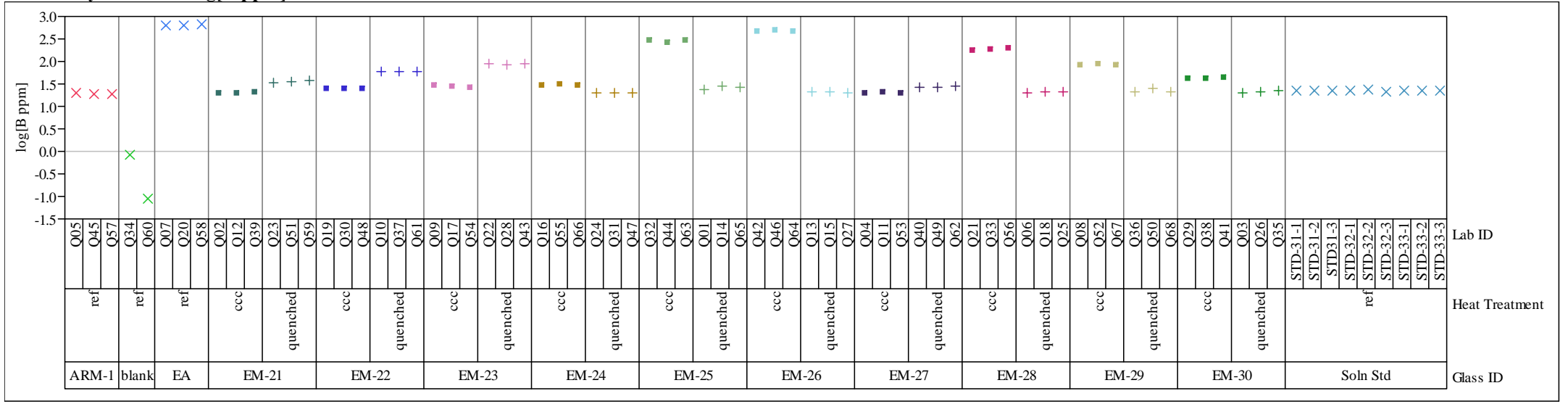

Set $=3$

Variability Chart for $\log [\mathrm{Li}$ ppm]

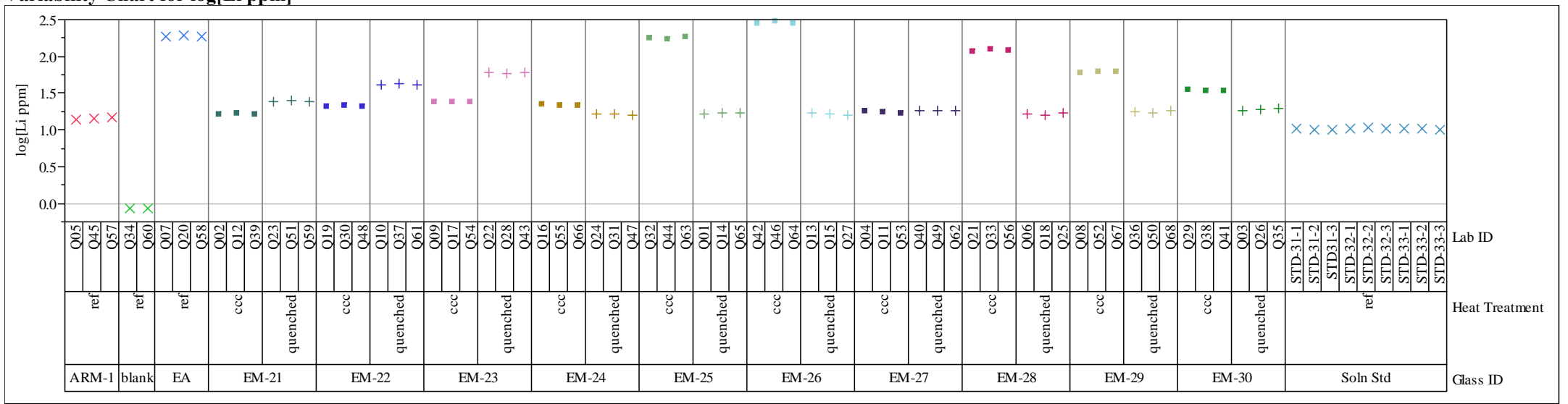


Exhibit E3. PCT Measurements by Glass Identifier for the Matrix 2A Study Glasses and Standards

Set $=3$

Variability Chart for log[Na ppm]

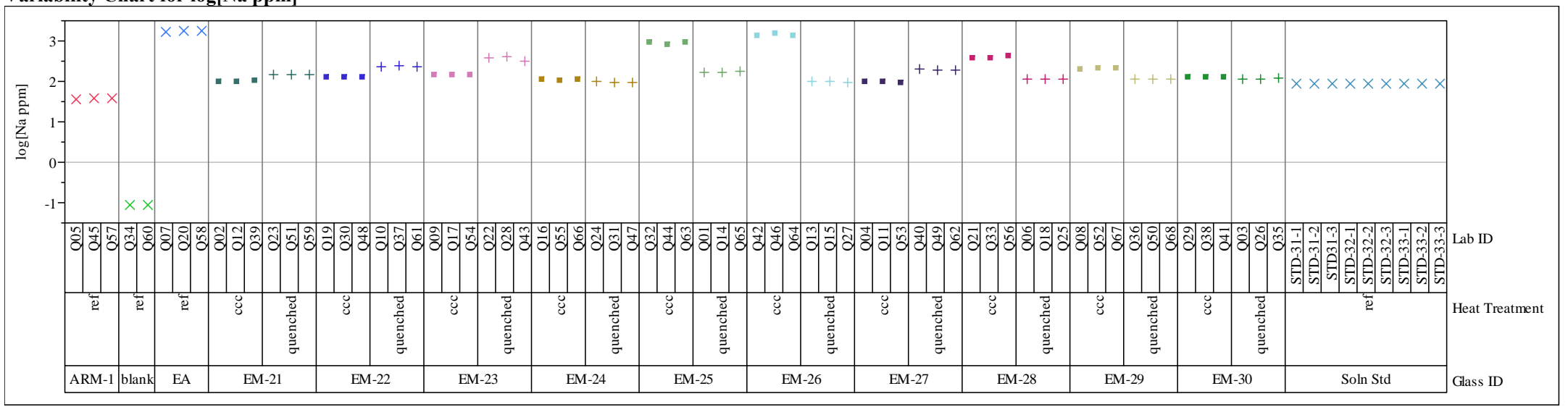

Set $=3$

Variability Chart for $\log [\mathrm{Si}$ ppm]

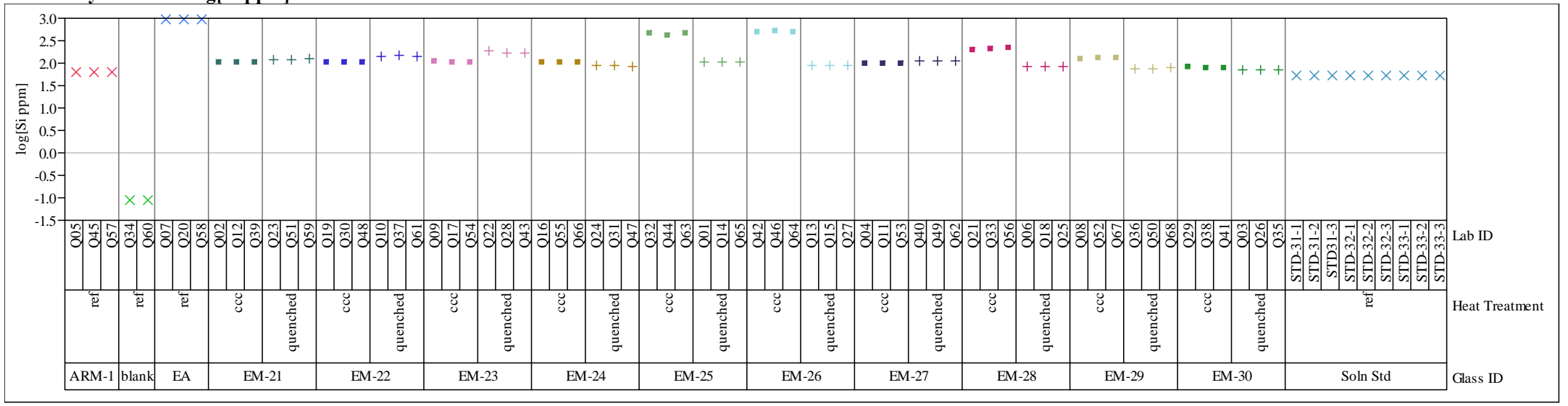


Exhibit E4. Correlations and Scatter Plots of Normalized PCTs Over All Compositional Views and Heat Treatments for the Matrix 2A Study

Multivariate

Correlations

$\log \mathrm{NL}[\mathrm{B}(\mathrm{g} / \mathrm{L})]$ $\log \mathrm{NL}[\mathrm{Li}(\mathrm{g} / \mathrm{L})]$ $\log \mathrm{NL}[\mathrm{Na}(\mathrm{g} / \mathrm{L})]$

$\begin{array}{rrrr}\log \mathbf{N L}[\mathbf{B}(\mathbf{g} / \mathbf{L})] & \log \mathbf{N L}[\mathbf{L i}(\mathbf{g} / \mathbf{L})] & \log \mathbf{N L}[\mathbf{N a}(\mathbf{g} / \mathbf{L})] & \log \mathbf{N L}[\mathbf{S i}(\mathbf{g} / \mathbf{L})] \\ 1.0000 & 0.9765 & 0.9476 & 0.9302 \\ 0.9765 & 1.0000 & 0.9153 & 0.8868 \\ 0.9476 & 0.9153 & 1.0000 & 0.9408 \\ 0.9302 & 0.8868 & 0.9408 & 1.0000\end{array}$

\section{Scatterplot Matrix}

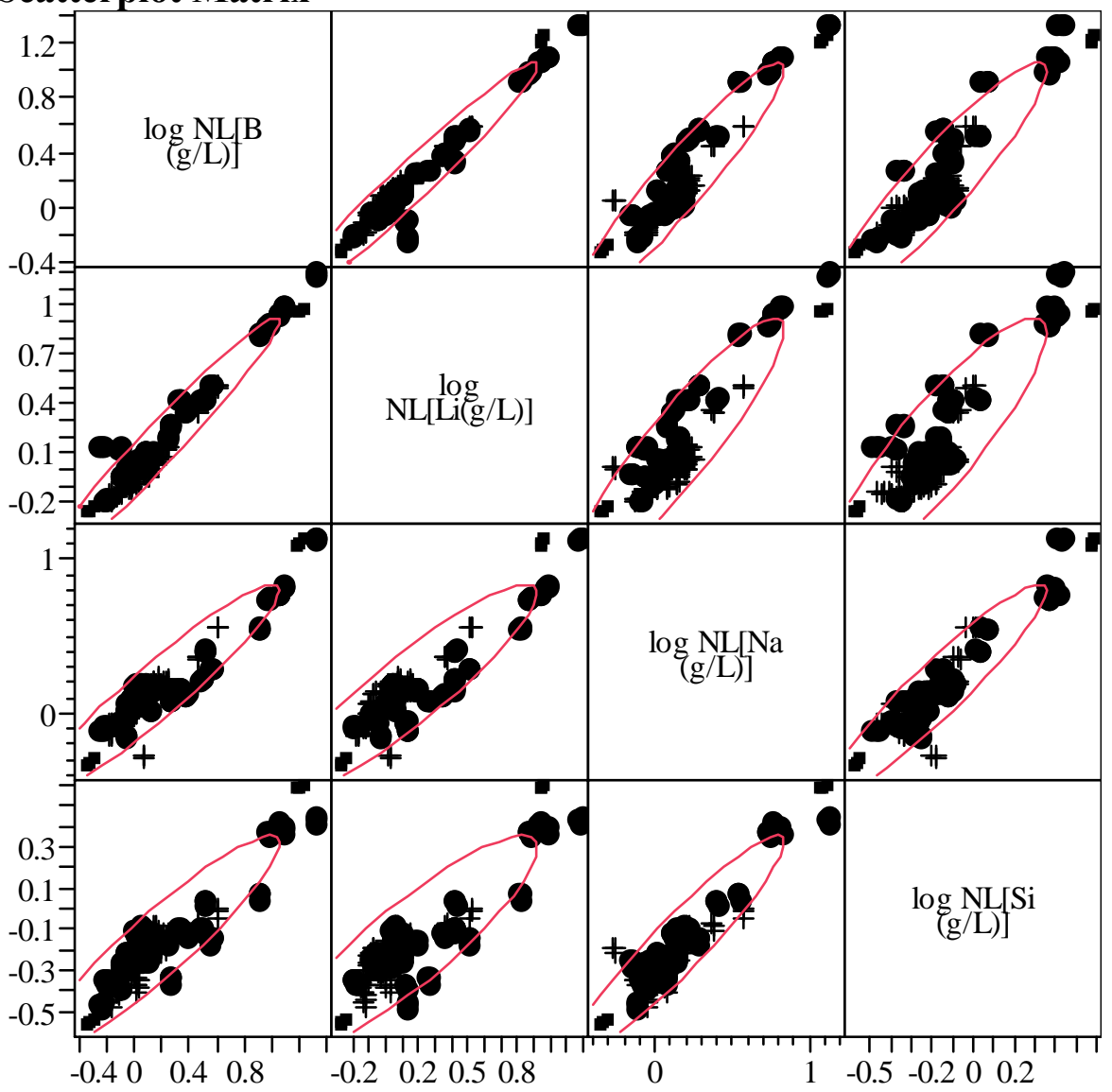


SRNL-STI-2009-00778, Revision 0

\section{Exhibit E5. Effects of Heat Treatment (HT) on PCT $\log (\mathrm{ppm})$ Response of the Matrix 2A Study Glasses}

Oneway Analysis of $\log [\mathrm{B}$ ppm] By Heat Treatment Glass ID=EM-01

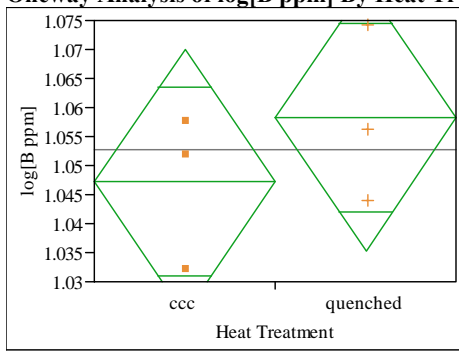

\section{Oneway Anova}

Summary of Fit

Rsquare

Adj Rsquare

1.05268

$\begin{array}{r}1.052681 \\ \hline\end{array}$

t Test

quenched-ccc

Assuming equal variances

Difference $\quad 0.01107$ t Ratio $\quad 0.945685$ Std Err Dif 0.01170 DF

Upper CL Dif 0.04356 Prob $>|t| \quad 0.3979$

$\begin{array}{lrr}\text { Lower CL Dif } & -0.02142 \text { Prob }>t & 0.1989 \\ \text { Confidence } & 0.95 \text { Prob }<t & 0.8011\end{array}$

Analysis of Variance

Source DF Sum of Squares Mean Square F Ratio Prob $>$ F $\begin{array}{llllll}\text { Heat Treatment } & 1 & 0.00018370 & 0.000184 & 0.8943 & 0.3979\end{array}$

Error 0.0008216 0.000205

Means for Oneway Anova

Level Number Mean Std Error Lower 95\% Upper 95\% $\begin{array}{llllll}\text { ccC } & 3 & 1.04715 & 0.00827 & 1.0242 & 1.070\end{array}$

Std Error uses a pooled estimate of error variance
Oneway Analysis of $\log [\mathrm{Li} \mathrm{ppm}]$ By Heat Treatment Glass ID=EM-01

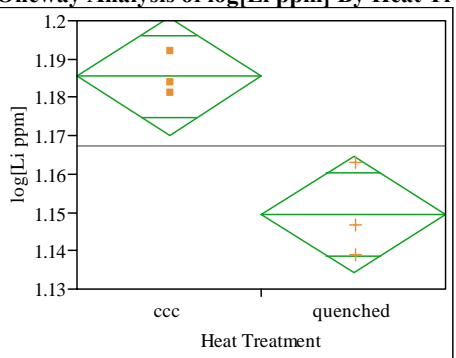

Oneway Anova

Summary of Fit

Root Mean Square Error $\quad 0.009536$

Mean of Response

1.167459

Observations (or Sum Wgts)

t Test

quenched-ccc

Assuming equal variances

Difference $\quad-0.03601$ t Ratio $\quad-4.62488$

Std Err Dif $\quad 0.00779 \mathrm{DF} \quad-4.62488$

Upper CL Dif -0.01439 Prob $>|t| 0.0098$

Lower CL Dif -0.05763 Prob $>t \quad 0.9951$

Confidence $\quad 0.95$ Prob $<\mathrm{t} \quad 0.0049$

Analysis of Variance

Source DF Sum of Squares Mean Square F Ratio Prob $>$ F $\begin{array}{llllll}\text { Heat Treatment } & 1 & 0.00194502 & 0.001945 & 21.3895 & 0.0098\end{array}$

Error

0.00036373
0.00230875

Means for Oneway Anova

Level Number Mean Std Error Lower 95\% Upper 95\%

$\begin{array}{llllll}\text { ccC } & 3 & 1.18546 & 0.00551 & 1.1702 & 1.2007\end{array}$

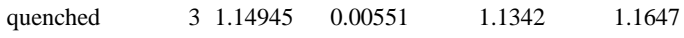

Std Error uses a pooled estimate of error variance

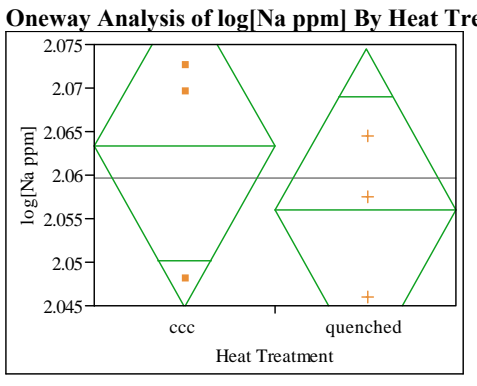

Oneway Anova

Summary of Fit

Rsquare

0.130111

0.08736
Root Mean Square Eror $\quad 0.011542$

Root Mean Square Error $\quad 0.011542$

$\begin{array}{lr}\text { Mean of Response } & 2.059644 \\ \text { Observations (or Sum Wgts) } & 6\end{array}$

t Test

quenched-ccc

Assuming equal variances

Difference $\quad-0.00729$ t Ratio $\quad-0.77349$ Std Err Dif $\quad 0.00942$ DF

Upper CL Dif 0.01888 Prob $>|t| \quad 0.4824$

$\begin{array}{lrr}\text { Lower CL Dif } & -0.03345 \text { Prob }>t & 0.7588 \\ \text { Confidence } & 0.95 \text { Prob }<\text { t } & 0.2412\end{array}$

Analysis of Variance

Source DF Sum of Squares Mean Square F Ratio Prob $>$ F $\begin{array}{llllll}\text { Heat Treatment } & 1 & 0.00007970 & 0.000080 & 0.5983 & 0.4824\end{array}$ $\begin{array}{lll}\text { Error } & 4 & 0.00053287\end{array}$

Means for Oneway Anova

Level Number Mean Std Error Lower 95\% Upper 95\%

$\begin{array}{lllrr}\text { ccc } & 32.06329 & 0.00666 & 2.0448 & 2.0818\end{array}$

$2.0375-2.0745$

Std Error uses a pooled estimate of error variance 
SRNL-STI-2009-00778, Revision 0

\section{Exhibit E5. Effects of Heat Treatment (HT) on PCT $\log (\mathrm{ppm})$ Response of the Matrix 2A Study Glasses}

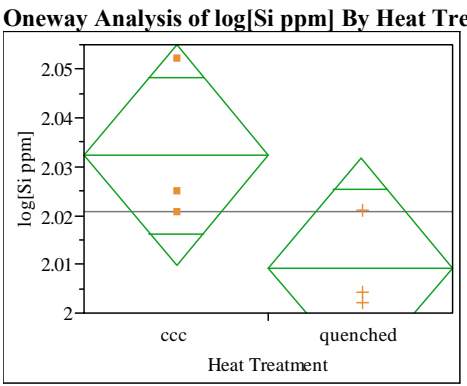

Oneway Anova

Summary of Fit

\begin{tabular}{|c|c|c|c|}
\hline \multirow{2}{*}{\multicolumn{2}{|c|}{$\begin{array}{l}\text { Rsquare } \\
\text { Adj Rsquare }\end{array}$}} & \multicolumn{2}{|c|}{0.501177} \\
\hline & & \multicolumn{2}{|c|}{0.376471} \\
\hline \multicolumn{2}{|c|}{ Root Mean Square Error } & \multicolumn{2}{|c|}{0.0141} \\
\hline \multicolumn{2}{|c|}{ Mean of Response } & \multicolumn{2}{|c|}{2.020774} \\
\hline \multicolumn{4}{|c|}{ Observations (or Sum Wgts) $\quad 6$} \\
\hline \multicolumn{4}{|l|}{$\begin{array}{l}\text { t Test } \\
\text { quenched-ccc }\end{array}$} \\
\hline \multicolumn{4}{|c|}{ Assuming equal variances } \\
\hline Difference & $-0.02308 \mathrm{t}$ & t Ratio & -2.00471 \\
\hline Std Err Dif & $0.01151 \mathrm{D}$ & DF & \\
\hline Upper CL Dif & $0.00888 \mathrm{P}$ & $\operatorname{Prob}>|t|$ & 0.11 \\
\hline Lower CL Dif & $-0.05505 \mathrm{P}$ & Prob $>t$ & 0.94 \\
\hline Confidence & $0.95 \mathrm{P}$ & Prob $<\mathrm{t}$ & 0.05 \\
\hline
\end{tabular}

Analysis of Variance

Source DF Sum of Squares Mean Square F Ratio Prob $>$ F

$\begin{array}{llllll}\text { Heat Treatment } & 1 & 0.00079904 & 0.000799 & 4.0189 & 0.1155 \\ \text { Error } & 4 & 0.00079529 & 0.000199 & & \end{array}$

0.000199

Means for Oneway Anova

Level Number Mean Std Error Lower 95\% Upper 95\% $\begin{array}{lllll}\text { ccc } & 32.03231 & 0.00814 & 2.0097 & 2.0549\end{array}$

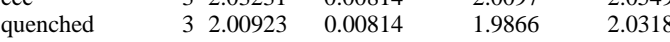

Std Error uses a pooled estimate of error variance
Oneway Analysis of $\log [\mathrm{B} \mathrm{ppm}]$ By Heat Treatment Glass ID=EM-02

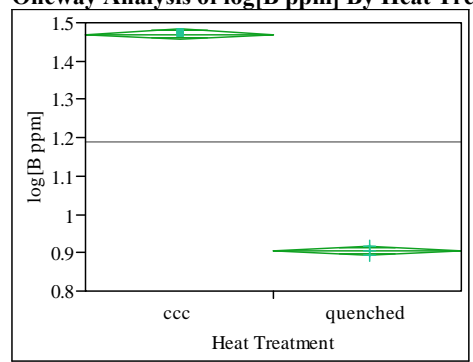

Oneway Anova

Summary of Fit

$\begin{array}{lr}\text { Rsquare } & 0.999437 \\ \text { Adj Rsquare } & 0.999296 \\ \text { Root Mean Square Error } & 0.008204 \\ \text { Mean of Response } & 1.187616 \\ \text { Observations (or Sum Wgts) } & 6\end{array}$

t Test

quenched-ccc

Assuming equal variances

Difference $\quad-0.56440$ t Ratio $\quad-84.2527$

$\begin{array}{lrl}\text { Std Err Dif } & 0.00670 \mathrm{DF} & 4 \\ \text { Upper CL Dif } & -0.54580 \text { Prob }>|t| & <.0001\end{array}$

$\begin{array}{lrr}\text { Upper CL Dif } & -0.54580 \text { Prob }>|t| & <.0001 \\ \text { Lower CL Dif }-0.58300 & \text { Prob }>t & 1.0000\end{array}$

$\begin{array}{lll}\text { Confidence } & 0.95 \text { Prob }<t \quad<.0001\end{array}$

Analysis of Variance

Source DF Sum of Squares Mean Square F Ratio Prob $>$ F $\begin{array}{llllll}\text { Heat Treatment } & 1 & 0.47782399 & 0.477824 & 7098.523 & <.0001 \\ \text { Error } & 4 & 0.00026925 & 0.000067 & & \end{array}$

Error 0.000067

Means for Oneway Anova

Level Number Mean Std Error Lower 95\% Upper 95\%

$\begin{array}{llrrrr}\text { ccC } & 3 & 1.46982 & 0.00474 & 1.4567 & 1.4830\end{array}$

$\begin{array}{llllll}\text { quenched } & 3 & 0.90542 & 0.00474 & 0.8923 & 0.9186\end{array}$

Std Error uses a pooled estimate of error variance

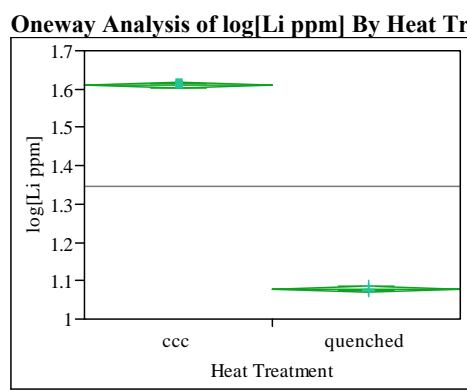

Oneway Anova

Summary of Fit

$\begin{array}{lr}\text { Rsquare } & 0.999761 \\ \text { Adj Rsquare } & 0.999702 \\ \text { Root Mean Square Error } & 0.005021 \\ \text { Mean of Response } & 1.344496 \\ \text { Observations (or Sum Wgts) } & 6\end{array}$

t Test

quenched-ccc

Assuming equal variances

Difference $\quad-0.53066$ t Ratio $\quad-129.448$

Std Err Dif 0.00410 DF $\quad-129.448$

Upper CL Dif -0.51928 Prob $>|t|<.0001$

Lower CL Dif -0.54204 Prob $>t \quad 1.0000$

$\begin{array}{lll}\text { Confidence } \quad 0.95 \text { Prob }<\mathrm{t} \quad<.0001 & \end{array}$

Analysis of Variance

Source DF Sum of Squares Mean Square F Ratio Prob $>$ F $\begin{array}{lrrrrr}\text { Heat Treatment } & 1 & 0.42239732 & 0.422397 & 16756.70 & <.0001\end{array}$ $\begin{array}{lll}\text { Error } & 4 & 0.00010083 \\ \text { C. Total } & 5 & 0.42249815\end{array}$

Means for Oneway Anova

Level Number Mean Std Error Lower 95\% Upper 95\%

$\begin{array}{llllll}\text { ccc } & 3 & 1.60982 & 0.00290 & 1.6018 & 1.6179 \\ \text { quenched } & 3 & 1.07917 & 0.00290 & 1.0711 & 1.0872\end{array}$

Std Error uses a pooled estimate of error variance 
SRNL-STI-2009-00778, Revision 0

Exhibit E5. Effects of Heat Treatment (HT) on PCT $\log (\mathrm{ppm})$ Response of the Matrix 2 A Study Glasses

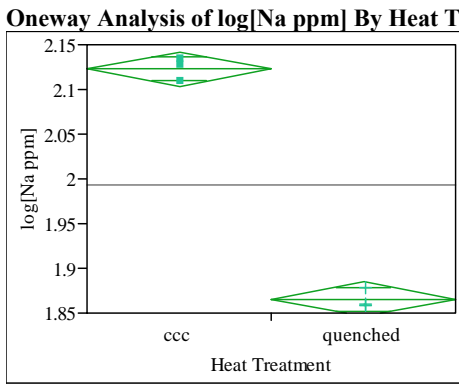

Oneway Anova

Summary of Fit

\begin{tabular}{|c|c|c|c|}
\hline \multicolumn{4}{|c|}{0.994381} \\
\hline \multicolumn{2}{|l|}{$\begin{array}{l}\text { Rsquare } \\
\text { Adj Rsquare }\end{array}$} & 0.992 & 976 \\
\hline Root Mean Square Error & uare Error & 0.011 & 835 \\
\hline \multicolumn{2}{|c|}{ Mean of Response } & 1.994 & 097 \\
\hline \multicolumn{2}{|c|}{ Observations (or Sum Wgts) } & & 6 \\
\hline \multicolumn{4}{|l|}{$\begin{array}{l}\text { t Test } \\
\text { quenched-ccc }\end{array}$} \\
\hline \multicolumn{4}{|c|}{ Assuming equal variances } \\
\hline Difference & $-0.25710 \mathrm{t}$ & Ratio & -26.6061 \\
\hline Std Err Dif & $0.00966 \mathrm{D}$ & DF & \\
\hline Upper CL Dif & $-0.23027 \mathrm{P}$ & $\operatorname{Prob}>|t|$ & $\frac{0.00}{0}$ \\
\hline Lower CL Dif & $-0.28393 \mathrm{P}$ & Prob $>t$ & 1.00 \\
\hline Confidence & $0.95 \mathrm{P}$ & Prob $<\mathrm{t}$ & $<.00$ \\
\hline
\end{tabular}

Analysis of Variance

$\begin{array}{lrrrrr}\text { Source } & \text { DF } & \text { Sum of Squares } & \text { Mean Square } & \text { F Ratio Prob }>\text { F } \\ \text { Heat Treatment } & 1 & 0.09914766 & 0.099148 & 707.8842 & <.000\end{array}$

0.00056025

0.09970791

0.000140

Means for Oneway Anova

Level Number Mean Std Error Lower 95\% Upper 95\%

$\begin{array}{llllll}\text { ccC } & 3 & 2.12265 & 0.00683 & 2.1037 & 2.1416\end{array}$

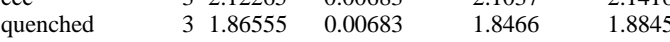

Std Error uses a pooled estimate of error variance
Oneway Analysis of $\log [\mathrm{Si}$ ppm] By Heat Treatment Glass ID=EM-02

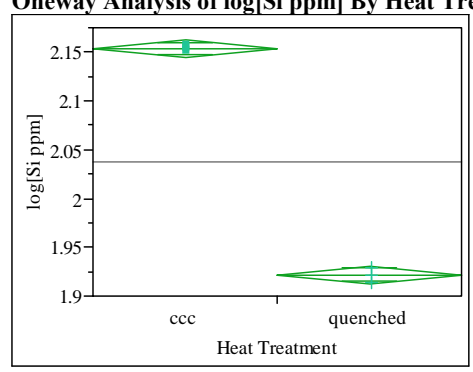

Oneway Anova

Summary of Fit

$\begin{array}{lr}\text { Rsquare } & 0.998263 \\ \text { Adj Rsquare } & 0.997828 \\ \text { Root Mean Square Error } & 0.005927 \\ \text { Mean of Response } & 2.037648 \\ \text { Observations (or Sum Wgts) } & 6\end{array}$

t Test

quenched-ccc

Assuming equal variances

Difference $\quad-0.23200$ t Ratio $\quad-47.9419$

Std Err Dif 0.00484 DF

Upper CL Dif -0.21856 Prob $>|t|<.0001$

Lower CL Dif -0.24543 Prob $>\mathrm{t} \quad 1.0000$

Confidence $\quad 0.95$ Prob $<\mathrm{t} \quad<.0001$

Analysis of Varia

Source DF Sum of Squares Mean Square F Ratio Prob $>$ F

$\begin{array}{llllll}\text { Heat Treatment } & 1 & 0.08073500 & 0.080735 & 2298.427 & <.0001 \\ \text { Error } & 4 & 0.00014050 & 0.000035 & & \end{array}$

0.000035

Means for Oneway Anova

Level Number Mean Std Error Lower 95\% Upper 95\%

$\begin{array}{llllll}\text { ccC } & 32.15365 & 0.00342 & 2.1441 & 2.1631\end{array}$

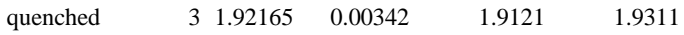

Std Error uses a pooled estimate of error variance

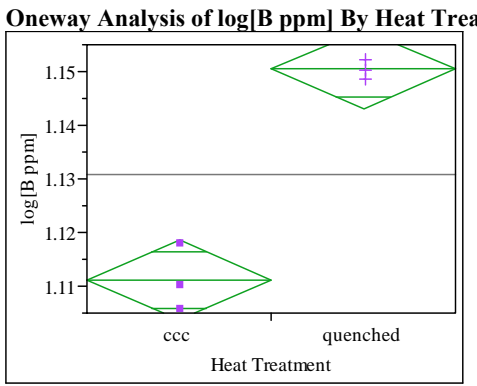

Oneway Anova

Summary of Fit

$\begin{array}{lr}\text { Rsquare } & 0.964973 \\ \text { Adj Rsquare } & 0.956217 \\ \text { Root Mean Square Error } & 0.004584 \\ \text { Mean of Response } & 1.130775 \\ \text { Observations (or Sum Wgts) } & 6\end{array}$

quenched-ccc

Assuming equal variances

Difference $\quad 0.039292$ t Ratio 10.4975

Std Err Dif 0.003743 DF

Upper CL Dif 0.049684 Prob $>|t| \quad 0.0005$

Lower CL Dif 0.028900 Prob $>t \quad 0.0002$

$\begin{array}{lll}\text { Confidence } & 0.95 \mathrm{Prob}<\mathrm{t} \quad 0.9998\end{array}$

Analysis of Variance

Source DF Sum of Squares Mean Square F Ratio Prob $>$ F $\begin{array}{llllll}\text { Heat Treatment } & 1 & 0.00231581 & 0.002316 & 110.1985 & 0.0005\end{array}$ $\begin{array}{llll}\text { Error } & 4 & 0.00008406 & 0.000021 \\ \text { C. Total } & 5 & 0.00239987 & \end{array}$

Means for Oneway Anova

Level Number Mean Std Error Lower 95\% Upper 95\%

$\begin{array}{llllll}\text { ccc } & 3 & 1.11113 & 0.00265 & 1.1038 & 1.1185 \\ \text { quenched } & 3 & 1.15042 & 0.00265 & 1.1431 & 1.1578\end{array}$

Std Error uses a pooled estimate of error variance 
SRNL-STI-2009-00778, Revision 0

\section{Exhibit E5. Effects of Heat Treatment (HT) on PCT $\log (\mathrm{ppm})$ Response of the Matrix 2A Study Glasses}

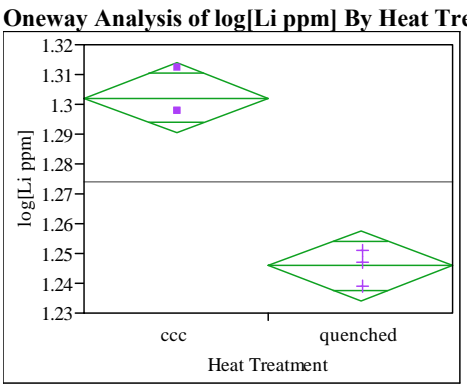

Oneway Anova

Summary of Fit

\begin{tabular}{|c|c|c|c|}
\hline \\
\hline \multicolumn{2}{|l|}{$\begin{array}{l}\text { Rsquare } \\
\text { Adj Rsquare }\end{array}$} & \multicolumn{2}{|c|}{$\begin{array}{l}0.956606 \\
0.945758\end{array}$} \\
\hline \multicolumn{4}{|c|}{ Root Mean Square Error } \\
\hline \multicolumn{4}{|c|}{ Mean of Response } \\
\hline \multicolumn{4}{|c|}{ Observations (or Sum Wgts) } \\
\hline \multicolumn{4}{|l|}{$\begin{array}{l}\text { t Test } \\
\text { quenched-ccc }\end{array}$} \\
\hline \multicolumn{4}{|c|}{ Assuming equal variances } \\
\hline Difference & $-0.05643 \mathrm{t}$ & Ratio & -9.39039 \\
\hline Std Err Dif & $0.00601 \mathrm{D}$ & DF & \\
\hline Upper CL Dif & $-0.03974 \mathrm{P}$ & Prob $>|t|$ & 0.00 \\
\hline Lower CL Dif & $-0.07311 \mathrm{P}$ & Prob $>t$ & 0.99 \\
\hline Confidence & $0.95 \mathrm{P}$ & Prob $<\mathrm{t}$ & 0.000 \\
\hline
\end{tabular}

Analysis of Variance

Source DF Sum of Squares Mean Square F Ratio Prob $>$ F

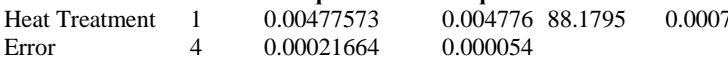

0.000054

Means for Oneway Anova

Level Number Mean Std Error Lower 95\% Upper 95\% $\begin{array}{llllll}\text { ccc } & 3 & 1.30219 & 0.00425 & 1.2904 & 1.3140 \\ \text { quenched } & 3 & 1.24577 & 0.00425 & 1.2340 & 1.2576\end{array}$

Std Error uses a pooled estimate of error variance

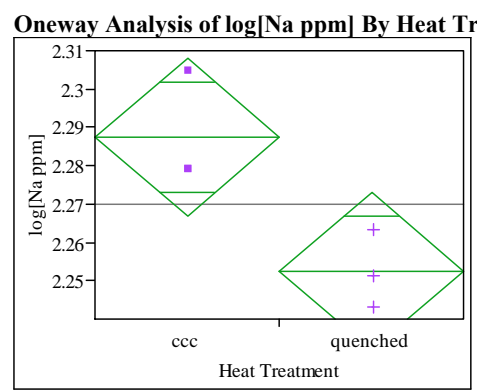

Oneway Anova

Summary of Fit

Rsquare

Adj Rsquare $\quad 0.670524$

Root Mean Square Error $\quad 0.012777$

Mean of Response 2.269951

t Test

quenched-ccc

Assuming equal variances

Difference $\quad-0.03488$ t Ratio $\quad-3.34299$

$\begin{array}{lrr}\text { Std Err Dif } & 0.01043 \text { DF } & 4 \\ \text { Upper CL Dif } & -0.00591 \text { Prob }>|t| & 0.0288\end{array}$

$\begin{array}{lrr}\text { Upper CL Dif }-0.00591 & \text { Prob }>|t| & 0.0288 \\ \text { Lower CL Dif }-0.06384 & \text { Prob }>t & 0.9856\end{array}$

$\begin{array}{lll}\text { Confidence } & 0.95 \text { Prob }<\mathrm{t} \quad 0.0144\end{array}$

Analysis of Variance

Source DF Sum of Squares Mean Square F Ratio Prob $>$ F $\begin{array}{llllll}\text { Heat Treatment } & 1 & 0.00182455 & 0.001825 & 11.1756 & 0.0288\end{array}$

Erro $4 \quad 0.00065305$ 0.000163

Means for Oneway Anova

Level Number Mean Std Error Lower 95\% Upper 95\%

$\begin{array}{llllrr}\text { ccc } & 3 & 2.28739 & 0.00738 & 2.2669 & 2.3079\end{array}$

$\begin{array}{llllll}\text { quenched } & 3 & 2.25251 & 0.00738 & 2.2320 & 2.2730\end{array}$

Std Error uses a pooled estimate of error variance

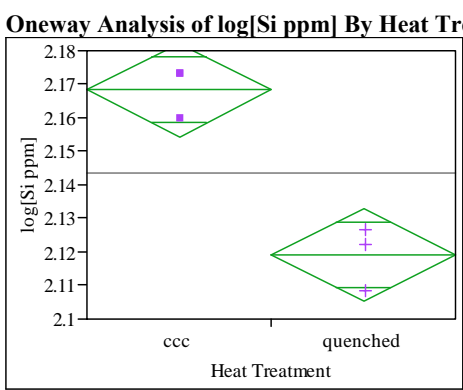

Oneway Anova

Summary of Fit

$\begin{array}{lr}\text { Rsquare } & 0.923793 \\ \text { Adj Rsquare } & 0.904741 \\ \text { Root Mean Square Error } & 0.008656 \\ \text { Mean of Response } & 2.143656 \\ \text { Observations (or Sum Wgts) } & 6\end{array}$

t Test

quenched-ccc

Assuming equal variances

Difference $\quad-0.04922$ t Ratio $\quad-6.96336$

Std Err Dif $\quad 0.00707$ DF $\quad-6.9636$

Upper CL Dif -0.02959 Prob $>|t| 0.0022$

Lower CL Dif -0.06884 Prob $>t \quad 0.9989$

$\begin{array}{lll}\text { Confidence } & 0.95 \text { Prob }<\mathrm{t} \quad 0.0011\end{array}$

Analysis of Variance

Source DF Sum of Squares Mean Square F Ratio Prob $>$ F

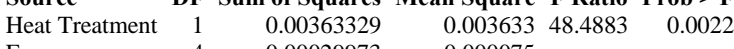

$\begin{array}{llll}\text { Error } & 4 & 0.00029973 & 0.000075 \\ \text { C. Total } & 5 & 0.00393302 & \end{array}$

Means for Oneway Anova

Level Number Mean Std Error Lower 95\% Upper 95\%

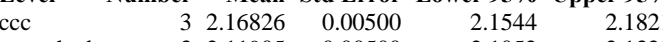

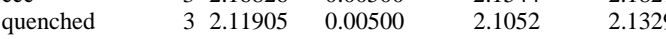

Std Error uses a pooled estimate of error variance 
SRNL-STI-2009-00778, Revision 0

Exhibit E5. Effects of Heat Treatment (HT) on PCT $\log (\mathrm{ppm})$ Response of the Matrix 2 A Study Glasses

Oneway Analysis of $\log [\mathrm{B}$ ppm $]$ By Heat Treatment Glass ID=EM-04

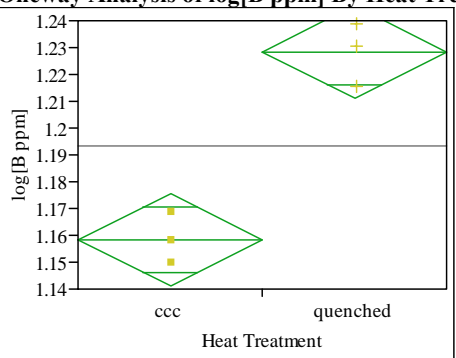

Oneway Anova

Summary of Fit

$\begin{array}{lr}\text { Rsquare } & 0.941256 \\ \text { Adj Rsquare } & 0.92657 \\ \text { Root Mean Square Error } & 0.010692 \\ \text { Mean of Response } & 1.193415 \\ \text { Observations (or Sum Wgts) } & 6 \\ & \end{array}$

t Test

quenched-ccc

Assuming equal variances

Difference $\quad 0.069892$ t Ratio 8.005759 Std Err Dif $\quad 0.008730$ DF

Upper CL Dif 0.094131 Prob $>|t| 0.0013$

Lower CL Dif 0.045653 Prob $>t \quad 0.0007$

Confidence $\quad 0.95 \mathrm{Prob}<\mathrm{t} \quad 0.9993$

Analysis of Variance

Source DF Sum of Squares Mean Square F Ratio Prob $>$ F $\begin{array}{llllll}\text { Heat Treatment } & 1 & 0.00732741 & 0.007327 & 64.0922 & 0.0013 \\ \text { Error } & 4 & 0.00045730 & 0.000114 & \end{array}$

Error 0.000114

Means for Oneway Anova

Level Number Mean Std Error Lower 95\% Upper 95\%

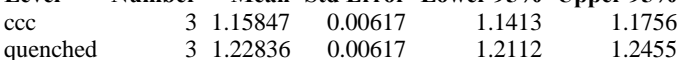

Std Error uses a pooled estimate of error variance
Oneway Analysis of $\log [\mathrm{Li} \mathrm{ppm}]$ By Heat Treatment Glass ID=EM-04

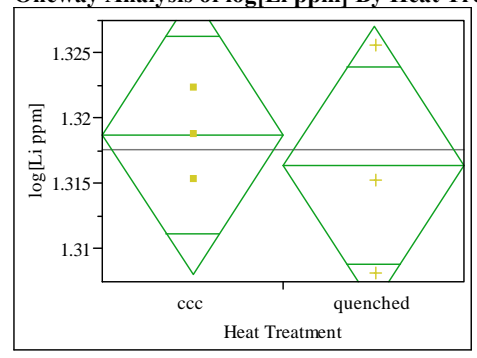

Oneway Anova

Summary of Fit

Rsquare

Adj Rsquare

$\begin{array}{ll}\text { Adj Rsquare } & -0.19344 \\ \text { Root Mean Square Error } & 0.006673\end{array}$

Mean of Respons

0.006673
1.317572

t Test

quenched-ccc

Assuming equal variances

Difference $\quad-0.00237$ t Ratio $\quad-0.4354$

Std Err Dif 0.00545 DF

Upper CL Dif 0.01276 Prob $>|t| 0.685$

Lower CL Dif $-0.01750 \mathrm{Prob}>\mathrm{t} \quad 0.657$

Confidence $\quad 0.95$ Prob $<\mathrm{t} \quad 0.3429$

Analysis of Variance

Source DF Sum of Squares Mean Square F Ratio Prob $>$ F $\begin{array}{llllll}\text { Heat Treatment } & 1 & 0.00000844 & 8.442 \mathrm{e}-6 & 0.1896 & 0.6857\end{array}$ Error 0.00017813 0.000045

Means for Oneway Anova

Level Number Mean Std Error Lower 95\% Upper 95\%

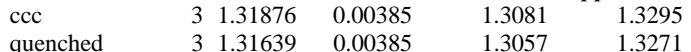

Std Error uses a pooled estimate of error variance

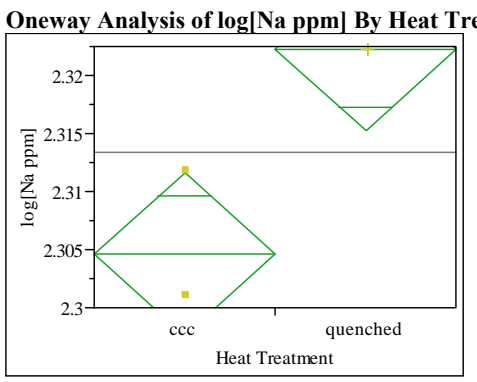

Oneway Anova

Summary of Fi

Rsquare

Adj Rsquare

Root Mean Square Error $\quad 0.004378$

Mean of Respons

t Test

quenched-ccc

Assuming equal variances

Difference $\quad 0.017615$ t Ratio $\quad 4.927704$

Std Err Dif 0.003575 DF Upper CL Dif 0.027539 Prob $>|t| \quad 0.0079$ $\begin{array}{lrr}\text { Lower CL Dif } & 0.007690 \text { Prob }>t & 0.0039 \\ \text { Confidence } & 0.95 \text { Prob }<\mathrm{t} & 0.9961\end{array}$

Analysis of Variance

Source DF Sum of Squares Mean Square F Ratio Prob $>$ F $\begin{array}{lrrrrr}\text { Heat Treatment } & 1 & 0.00046542 & 0.000465 & 24.2823 & 0.0079\end{array}$ $\begin{array}{lll}\text { Error } & 4 & 0.00007667\end{array}$

\section{Means for Oneway Anova}

Level Number Mean Std Error Lower 95\% Upper 95\%

$\begin{array}{lllllr}\text { ccc } & 3 & 2.30461 & 0.00253 & 2.2976 & 2.3116 \\ \text { qunched } & 3 & 2.32223 & 0.00253 & 2.3152 & 2.3292\end{array}$

Std Error uses a pooled estimate of error variance 
SRNL-STI-2009-00778, Revision 0

\section{Exhibit E5. Effects of Heat Treatment (HT) on PCT $\log (\mathrm{ppm})$ Response of the Matrix 2A Study Glasses}

Oneway Analysis of $\log [\mathrm{Si}$ ppm] By Heat Treatment Glass ID=EM-04

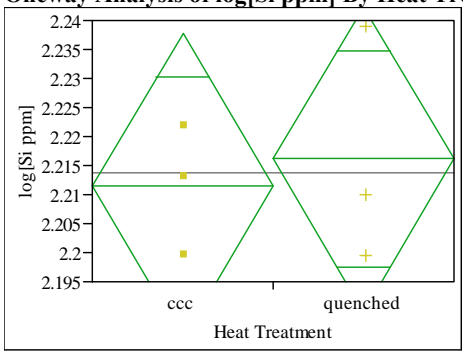

\section{Oneway Anova}

Summary of Fit

Rsquare $\quad 0.02899$

Adj Rsquare $\quad-0.21376$

Root Mean Square Error $\quad 0.016445$

Mean of Response 2.213827
6

t Test

quenched-ccc

Assuming equal variances

Difference $\quad 0.00464$ t Ratio 0.345575 Std Err Dif 0.01343 DF

Upper CL Dif 0.04192 Prob $>|t| \quad 0.747$

$\begin{array}{lrr}\text { Lower CL Dif } & -0.03264 \text { Prob }>t & 0.3735 \\ \text { Confidence } & 0.95 \text { Prob }<t & 0.6265\end{array}$

Analysis of Variance

Source DF Sum of Squares Mean Square F Ratio Prob $>$ F

$\begin{array}{llllll}\text { Heat Treatment } & 1 & 0.00003229 & 0.000032 & 0.1194 & 0.7471 \\ \text { Error } & 4 & 0.00108171 & 0.000270 & & \end{array}$

Means for Oneway Anova

Level Number Mean Std Error Lower 95\% Upper 95\% $\begin{array}{llllll}\text { ccC } & 3 & 2.21151 & 0.00949 & 2.1851 & 2.2379 \\ \text { quenched } & 3 & 2.21615 & 0.00949 & 2.1898 & 2.2425\end{array}$

Std Error uses a pooled estimate of error variance
Oneway Analysis of $\log [\mathrm{B}$ ppm] By Heat Treatment Glass ID=EM-05

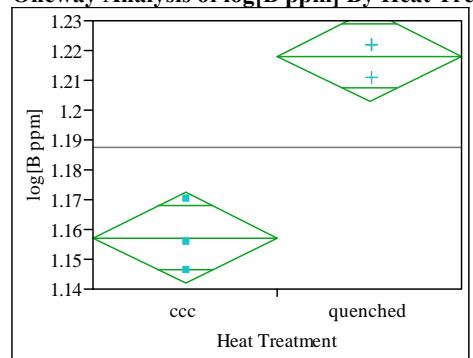

Oneway Anova

Summary of Fit

$\begin{array}{lr}\text { Rsquare } & 0.938867 \\ \text { Adj Rsquare } & 0.923584 \\ \text { Root Mean Square Error } & 0.009548 \\ \text { Mean of Response } & 1.18764 \\ \text { Observations (or Sum Wgts) } & 6\end{array}$

t Test

quenched-ccc

Assuming equal variances

Difference $\quad 0.061105$ t Ratio 7.837792

Std Err Dif 0.007796 DF

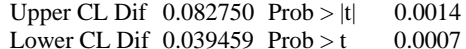

$\begin{array}{lrl}\text { Confidence } & 0.95 \text { Prob }<\mathrm{t} & 0.9993\end{array}$

Analysis of Variance

Source DF Sum of Squares Mean Square F Ratio Prob $>$ F $\begin{array}{llllll}\text { Heat Treatment } & 1 & 0.00560070 & 0.005601 & 61.4310 & 0.0014 \\ \text { Error } & 4 & 0.00036468 & 0.000091 & & \end{array}$

Error 0.000091

\section{Means for Oneway Anova}

Level Number Mean Std Error Lower 95\% Upper 95\%

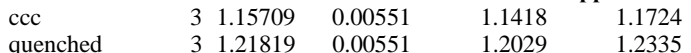

Std Error uses a pooled estimate of error variance
Oneway Analysis of $\log [\mathrm{Li}$ ppm] By Heat Treatment Glass ID=EM-05

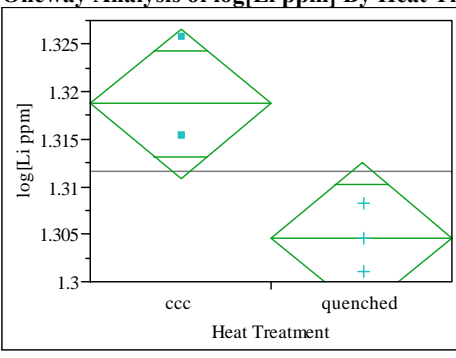

Oneway Anova

Summary of Fit

Rsquare

0.753556

Root Mean Square Eror -0.691945

Root Mean Square Error $\quad 0.00494$

Mean of Response

1.311686

t Test

quenched-ccc

Assuming equal variances

Difference $\quad-0.01411$ t Ratio $\quad-3.49726$ Std Err Dif 0.00403 DF

Upper CL Dif -0.00291 Prob $>|t| \quad 0.0250$

$\begin{array}{lrr}\text { Lower CL Dif } & -0.02531 \text { Prob }>t & 0.9875 \\ \text { Confidence } & 0.95 \text { Prob }<\text { t } & 0.0125\end{array}$

Analysis of Variance

Source DF Sum of Squares Mean Square F Ratio Prob $>$ F $\begin{array}{llllll}\text { Heat Treatment } & 1 & 0.00029851 & 0.000299 & 12.2309 & 0.0250\end{array}$ $\begin{array}{lll}\text { Error } & 4 & 0.00009762\end{array}$

Means for Oneway Anova

Level Number Mean Std Error Lower 95\% Upper 95\%

$\begin{array}{llllrr}\text { ccc } & 3 & 1.31874 & 0.00285 & 1.3108 & 1.3267\end{array}$

quench

Std Error uses a pooled estimate of error variance 
SRNL-STI-2009-00778, Revision 0

\section{Exhibit E5. Effects of Heat Treatment (HT) on PCT $\log (\mathrm{ppm})$ Response of the Matrix 2A Study Glasses}

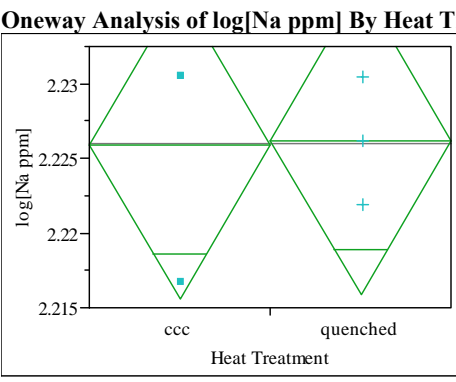

\section{Oneway Anova}

Summary of Fit

Rsquare $\quad 0.00094$

Adj Rsquare $\quad-0.24883$

Root Mean Square Error $\quad 0.006418$

Mean of Respons 2.226004
6

t Test

quenched-ccc

Assuming equal variances

Difference $\quad 0.00032$ t Ratio 0.061341 Std Err Dif 0.00524 DF

Upper CL Dif 0.01487 Prob $>|t| \quad 0.9540$

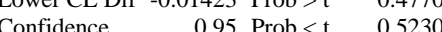

Analysis of Variance

Source DF Sum of Squares Mean Square F Ratio Prob $>$ F $\begin{array}{llllll}\text { Heat Treatment } & 1 & 0.00000015 & 1.55 \mathrm{e}-7 & 0.0038 & 0.9540\end{array}$ Error 0.00016474 0.0004

Means for Oneway Anova

Level Number Mean Std Error Lower 95\% Upper 95\% $\begin{array}{llllll}\text { ccc } & 3 & 2.22584 & 0.00371 & 2.2156 & 2.2361 \\ \text { quenched } & 3 & 2.22616 & 0.00371 & 2.2159 & 2.2365\end{array}$

Std Error uses a pooled estimate of error variance
Oneway Analysis of $\log [\mathrm{Si}$ ppm] By Heat Treatment Glass ID=EM-05

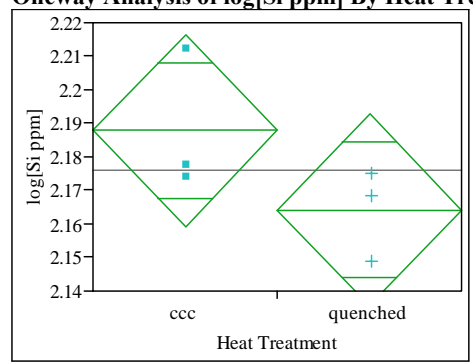

Oneway Anova

Summary of Fit

Adj Rsquare

Root Mean Square

Mean of Response

0.24853

Observations (or Sum Wgts)

t Test

quenched-ccc

Assuming equal variances

Difference $\quad-0.02375$ t Ratio -1.629

$\begin{array}{lrr}\text { Std Err Dif } & 0.01458 \text { DF } & 4 \\ \text { Upper } & & -1.629\end{array}$

$\begin{array}{lrl}\text { Upper CL Dif } & 0.01673 \text { Prob }>|t| & 0.1786 \\ \text { Lower CL Dif }-0.06424 & \text { Prob }>t \quad 0.9107\end{array}$

Confidence $\quad 0.95$ Prob $<\mathrm{t} \quad 0.0893$

Analysis of Variance

Source DF Sum of Squares Mean Square F Ratio Prob $>$ F $\begin{array}{llllll}\text { Heat Treatment } & 1 & 0.00084643 & 0.000846 & 2.6536 & 0.1786\end{array}$

Error $\begin{array}{ll}1 & 0.00084643 \\ 4 & 0.00127588\end{array}$ 0.000319

\section{Means for Oneway Anova}

Level Number Mean Std Error Lower 95\% Upper 95\%

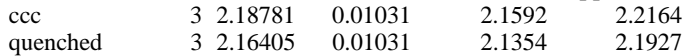

Std Error uses a pooled estimate of error variance

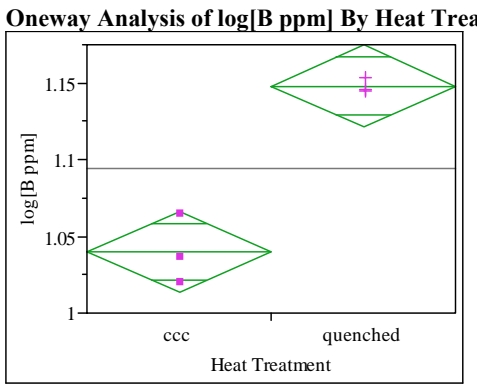

Oneway Anova

Summary of Fit

$\begin{array}{lr}\text { Rsquare } & 0.941667 \\ \text { Adj Rsquare } & 0.927084 \\ \text { Root Mean Square Error } & 0.016504 \\ \text { Mean of Response } & 1.094042 \\ \text { Observations (or Sum Wgts) } & 6\end{array}$

t Test

quenched-ccc

Assuming equal variances

Difference $\quad 0.108286$ t Ratio $\quad 8.035687$ Std Err Dif $\quad 0.013476$ DF

Upper CL Dif 0.145700 Prob $>|t| \quad 0.0013$ Lower CL Dif 0.070871 Prob $>t \quad 0.0007$ Confidence $\quad 0.95 \mathrm{Prob}<\mathrm{t} \quad 0.9993$

Analysis of Variance

Source DF Sum of Squares Mean Square F Ratio Prob $>$ F $\begin{array}{llllll}\text { Heat Treatment } & 1 & 0.01758866 & 0.017589 & 64.5723 & 0.0013\end{array}$ $\begin{array}{llll}\text { Error } & 4 & 0.00108955 & 0.000272 \\ \text { C. Total } & 5 & 0.01867821 & \end{array}$

Means for Oneway Anova

Level Number Mean Std Error Lower 95\% Upper 95\% $\begin{array}{lllllr}\text { ccC } & 3 & 1.03990 & 0.00953 & 1.0134 & 1.0664\end{array}$

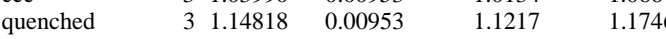

Std Error uses a pooled estimate of error variance 
SRNL-STI-2009-00778, Revision 0

Exhibit E5. Effects of Heat Treatment (HT) on PCT $\log (\mathrm{ppm})$ Response of the Matrix 2 A Study Glasses

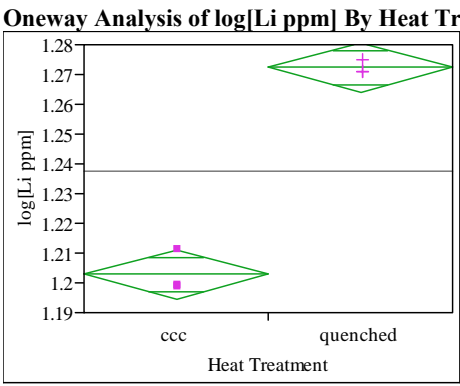

Oneway Anova

Summary of Fit

\begin{tabular}{|c|c|c|c|}
\hline \\
\hline \multicolumn{2}{|l|}{$\begin{array}{l}\text { Rsquare } \\
\text { Adj Rsquare }\end{array}$} & \multicolumn{2}{|c|}{$\begin{array}{l}0.985637 \\
0.982046\end{array}$} \\
\hline \multirow{2}{*}{\multicolumn{2}{|c|}{ Root Mean Square Error }} & 0.005 & \\
\hline \multirow{2}{*}{\multicolumn{2}{|c|}{$\begin{array}{l}\text { Mean of Response } \\
\text { Observations (or Sum Wgts }\end{array}$}} & 1.237 & \\
\hline & & & 6 \\
\hline \multicolumn{4}{|l|}{$\begin{array}{l}\text { t Test } \\
\text { quenched-ccc }\end{array}$} \\
\hline \multicolumn{4}{|c|}{ Assuming equal variances } \\
\hline Difference & $0.069478 \mathrm{t}$ & t Ratio & 16.567 \\
\hline Std Err Dif & $0.004194 \mathrm{I}$ & DF & \\
\hline Upper CL Dif & $0.081122 \mathrm{I}$ & $\operatorname{Prob}>|t|$ & $<.0001$ \\
\hline Lower CL Dif & $0.057835 \mathrm{I}$ & Prob $>t$ & \\
\hline Confidence & $0.95 \mathrm{I}$ & Prob $<$ t & 1.00 \\
\hline
\end{tabular}

Analysis of Variance

Source DF Sum of Squares Mean Square F Ratio Prob $>$

$\begin{array}{llllll}\text { Heat Treatment } & 1 & 0.00724088 & 0.007241 & 274.4884 & <.0001 \\ \text { Error } & 4 & 0.00010552 & 0.000026 & & \end{array}$

Error

0.000026

Means for Oneway Anova

Level Number Mean Std Error Lower 95\% Upper 95\%

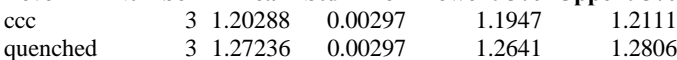

Std Error uses a pooled estimate of error variance

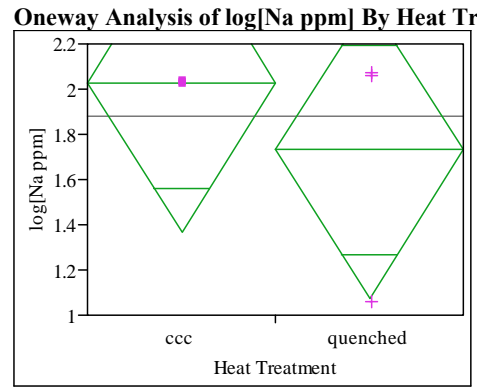

Oneway Anova

Summary of Fit

$\begin{array}{lr}\text { Rsquare } & 0.164338 \\ \text { Adj Rsquare } & -0.04458 \\ \text { Root Mean Square Error } & 0.410578 \\ \text { Mean of Response } & 1.879121 \\ \text { Observations (or Sum Wgts) } & 6\end{array}$

Observations (or Sum Wgts)

t Test

quenched-ccc

Assuming equal variances

$\begin{array}{lll}\text { Difference } & -0.2973 \text { t Ratio } \quad-0.88692\end{array}$

$\begin{array}{lrr}\text { Std Err Dif } & 0.3352 \text { DF } & \\ \text { Upper CL Dif } & 0.6334 \text { Prob }>|t| & 0.4252\end{array}$

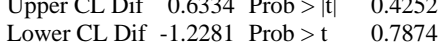

Confidence $\quad 0.95$ Prob $<\mathrm{t} \quad 0.2126$

Analysis of Variance

Source DF Sum of Squares Mean Square F Ratio Prob $>$ F $\begin{array}{llllll}\text { Heat Treatment } & 1 & 0.13260495 & 0.132605 & 0.7866 & 0.4252 \\ \text { Error } & 4 & 0.67429651 & 0.168574 & & \end{array}$ C. Total 0.80690146

Means for Oneway Anova

Level Number Mean Std Error Lower 95\% Upper 95\%

$\begin{array}{llllll}\text { ccc } & 3 & 2.02778 & 0.23705 & 1.3696 & 2.6859 \\ \text { quenched } & 3 & 1.73046 & 0.23705 & 1.0723 & 2.3886\end{array}$

Std Error uses a pooled estimate of error variance

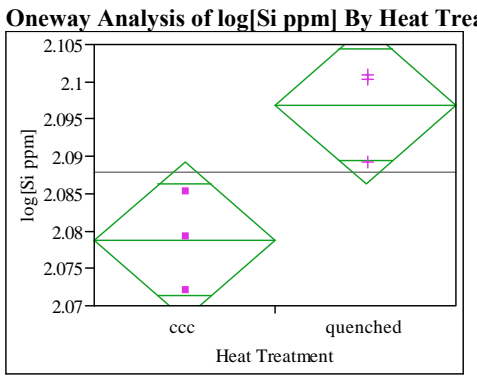

Oneway Anova

Summary of Fit

Rsquare

0.738632

Root Mean Square Error $\quad 0.67329$

Root Mean Square Error $\quad 0.006605$

$\begin{array}{lr}\text { Mean of Response } & 2.08782 \\ \text { Observations (or Sum Wgts) } & 6\end{array}$

t Test

quenched-ccc

Assuming equal variances

Difference $\quad 0.018132$ t Ratio 3.362159

Std Err Dif 0.005393 DF

Upper CL Dif 0.033106 Prob $>|t| \quad 0.0282$

$\begin{array}{lrr}\text { Lower CL Dif } & 0.003159 \text { Prob }>t & 0.0141 \\ \text { Confidence } & 0.95 \text { Prob }<t & 0.9859\end{array}$

Analysis of Variance

Source DF Sum of Squares Mean Square F Ratio Prob > F $\begin{array}{lllrlrl}\text { Heat Treatment } & 1 & 0.00049316 & 0.000493 & 11.3041 & 0.0282\end{array}$ $\begin{array}{lll}\text { Error } & 4 & 0.00017451 \\ \text { C. } & 5 & 0.00066767\end{array}$ 0.00044

Means for Oneway Anova

Level Number Mean Std Error Lower 95\% Upper 95\%

\begin{tabular}{lrrrrr} 
ccc & 3 & 2.07875 & 0.00381 & 2.0682 & 2.0893 \\
\hline
\end{tabular}

Std Error uses a pooled estimate of error variance 
SRNL-STI-2009-00778, Revision 0

Exhibit E5. Effects of Heat Treatment (HT) on PCT $\log (\mathrm{ppm})$ Response of the Matrix 2 A Study Glasses

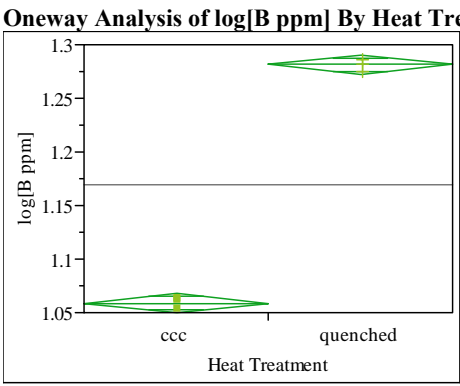

Oneway Anova

Summary of Fit

\begin{tabular}{|c|c|c|c|}
\hline \multicolumn{4}{|c|}{0.998415} \\
\hline \multicolumn{2}{|l|}{$\begin{array}{l}\text { square } \\
\text { di Rsquare }\end{array}$} & 0.9980 & \\
\hline \multicolumn{2}{|c|}{ Root Mean Square Error } & 0.005 & \\
\hline \multicolumn{2}{|c|}{ Mean of Response } & 1.170 & \\
\hline \multicolumn{2}{|c|}{ Observations (or Sum Wgts) } & & 6 \\
\hline \multicolumn{4}{|l|}{$\begin{array}{l}\text { t Test } \\
\text { quenched-ccc }\end{array}$} \\
\hline \multicolumn{4}{|c|}{ Assuming equal variances } \\
\hline Difference & $0.222474 \mathrm{t}$ & t Ratio & 50 \\
\hline Std Err Dif & $0.004432 \mathrm{I}$ & DF & \\
\hline Upper CL Dif & $0.234780 \mathrm{I}$ & $\operatorname{Prob}>|t|$ & \\
\hline Lower CL Dif & $0.210168 \mathrm{I}$ & Prob $>t$ & \\
\hline Confidence & $0.95 \mathrm{I}$ & Prob $<$ t & 1.00 \\
\hline
\end{tabular}

Analysis of Varianc

Source DF Sum of Squares Mean Square F Ratio Prob $>$

$\begin{array}{llllll}\text { Heat Treatment } & 1 & 0.07424208 & 0.074242 & 2519.453 & <.0001 \\ \text { Error } & 4 & 0.00011787 & 0.000029 & & \end{array}$

$$
0.0001178
$$

0.0743599

Means for Oneway Anova

Level Number Mean Std Error Lower 95\% Upper 95\%

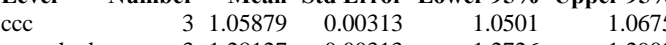

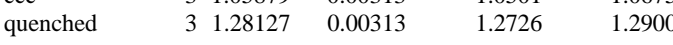

Std Error uses a pooled estimate of error variance
Oneway Analysis of $\log [\mathrm{Li}$ ppm] By Heat Treatment Glass ID=EM-07

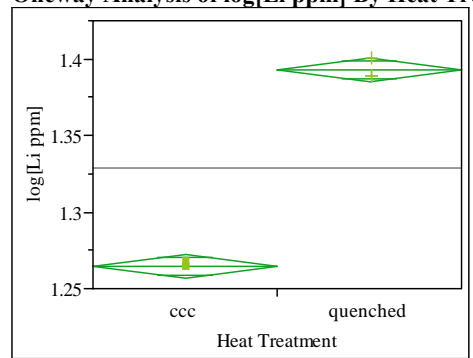

Oneway Anova

Summary of $\mathrm{F}$

Adj Rsquare

0.995942

Reot Mean Square Error $\quad 0.005023$

$\begin{array}{lr}\text { Mean of Response } & 1.328811 \\ \text { Observations (or Sum Wgts) } & 6\end{array}$

t Test

quenched-ccc

Assuming equal variances

Difference $\quad 0.128501$ t Ratio 31.33099

$\begin{array}{lrrr}\text { Std Err Dif } & 0.004101 \text { DF } & 4 \\ \text { Uper } & \text { DL } & <0001\end{array}$

$\begin{array}{lll}\text { Upper CL Dif } & 0.139888 \text { Prob }>|t| & <.0001 \\ \text { Lower CL Dif } 0.117114 \text { Prob }>t & <.0001\end{array}$

$\begin{array}{lr}\text { Confidence } & 0.95 \text { Prob }<\mathrm{t} \quad 1.0000\end{array}$

Analysis of Variance

Source DF Sum of Squares Mean Square F Ratio Prob $>$ F $\begin{array}{llllll}\text { Heat Treatment } & 1 & 0.02476879 & 0.024769 & 981.6307 & <.0001 \\ \text { Error } & 4 & 0.00010093 & 0.000025 & \end{array}$

Error 0.000025

\section{Means for Oneway Anova}

Level Number Mean Std Error Lower 95\% Upper 95\%

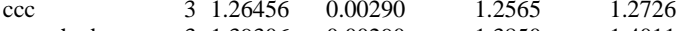

$\begin{array}{llllll}\text { quenched } & 3 & 1.39306 & 0.00290 & 1.3850 & 1.4011\end{array}$

Std Error uses a pooled estimate of error variance

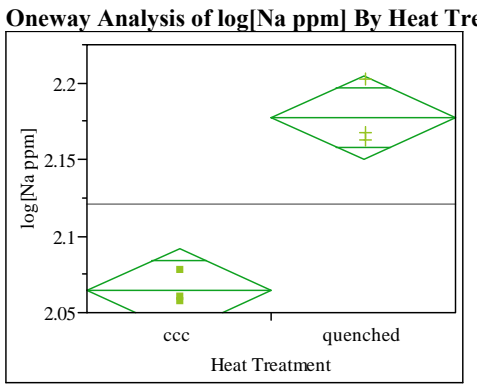

Oneway Anova

Summary of Fit

$\begin{array}{lr}\text { Rsquare } & 0.942772 \\ \text { Adj Rsquare } & 0.928465 \\ \text { Root Mean Square Error } & 0.017036 \\ \text { Mean of Response } & 2.121046 \\ \text { Observations (or Sum Wgts) } & 6\end{array}$

quenched-ccc

Assuming equal variances

Difference $\quad 0.112917$ t Ratio $\quad 8.117609$

Std Err Dif 0.013910 DF

Upper CL Dif 0.151538 Prob $>|t| \quad 0.0013$

Lower CL Dif 0.074297 Prob $>t \quad 0.0006$

Confidence $\quad 0.95$ Prob $<\mathrm{t} \quad 0.9994$

Analysis of Variance

Source DF Sum of Squares Mean Square F Ratio Prob $>$ F

$\begin{array}{llllll}\text { Heat Treatment } & 1 & 0.01912553 & 0.019126 & 65.8956 & 0.0013\end{array}$

$\begin{array}{llll}\text { Error } & 4 & 0.00116096 & 0.000290 \\ \text { C. Total } & 5 & 0.02028649 & \end{array}$

Means for Oneway Anova

Level Number Mean Std Error Lower 95\% Upper 95\%

$\begin{array}{llllll}\text { cCC } & 3 & 2.06459 & 0.00984 & 2.0373 & 2.091\end{array}$

$\begin{array}{llllll}\text { quenched } & 3 & 2.17750 & 0.00984 & 2.1502 & 2.2048\end{array}$

Std Error uses a pooled estimate of error variance 
SRNL-STI-2009-00778, Revision 0

Exhibit E5. Effects of Heat Treatment (HT) on PCT $\log (\mathrm{ppm})$ Response of the Matrix 2A Study Glasses

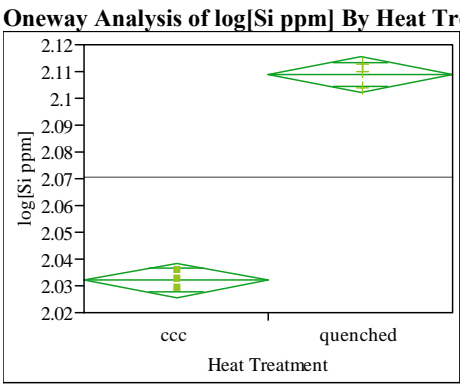

Oneway Anova

Summary of Fit

\begin{tabular}{|c|c|c|c|}
\hline \multicolumn{4}{|c|}{0.992679} \\
\hline \multicolumn{4}{|l|}{ Adj Rsquare } \\
\hline \multicolumn{2}{|c|}{ Root Mean Square Error } & 0.00 & 404 \\
\hline & 2.070 & \\
\hline \multicolumn{2}{|c|}{$\begin{array}{l}\text { Mean of Response } \\
\text { Observations (or Sum Wgts }\end{array}$} & & 6 \\
\hline \multicolumn{4}{|l|}{$\begin{array}{l}\text { t Test } \\
\text { quenched-ccc }\end{array}$} \\
\hline \multicolumn{4}{|c|}{ Assuming equal variances } \\
\hline Difference & $0.076814 \mathrm{t}$ & t Ratio & \\
\hline Std Err Dif & 0.003298 & DF & \\
\hline Upper CL Dif & $0.085972 \mathrm{I}$ & $\operatorname{Prob}>|t|$ & \\
\hline Lower CL Dif & 0.067656 & Prob $>t$ & \\
\hline Confidence & $0.95 \mathrm{I}$ & Prob $<$ t & \\
\hline
\end{tabular}

Analysis of Varianc

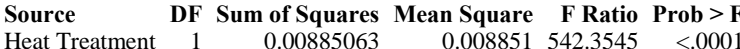

$\begin{array}{llllll}\text { Heat Treatment } & 1 & 0.00885063 & 0.008851 & 542.3545 & <.0001 \\ \text { Error } & 4 & 0.00006528 & 0.000016 & \end{array}$

0.000016

Means for Oneway Anova

Level Number Mean Std Error Lower 95\% Upper 95\%

$\begin{array}{llllll}\text { ccC } & 3 & 2.03208 & 0.00233 & 2.0256 & 2.0386\end{array}$

quenched $\quad \begin{array}{lllll}3 & 2.10890 & 0.00233 & 2.1024 & 2.1154\end{array}$

Std Error uses a pooled estimate of error variance
Oneway Analysis of $\log [\mathrm{B} \mathrm{ppm}]$ By Heat Treatment Glass ID=EM-08

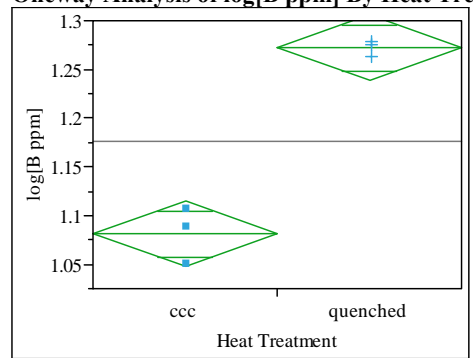

Oneway Anova

Summary of Fit

Rean Square Error $\quad 0.961091$

Mean of Response 1.17664

Observations (or Sum Wgts)

6

t Test

quenched-ccc

Assuming equal variances

Difference $\quad 0.191343$ t Ratio $\quad 11.15812$

$\begin{array}{lrr}\text { Std Err Dif } & 0.017148 \text { DF } & 4 \\ \text { Uper } & \text { D Dif } & 0.238954\end{array}$

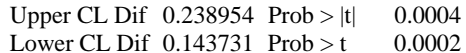

$\begin{array}{lll}\text { Confidence } & 0.95 \mathrm{Prob}<\mathrm{t} & 0.9998\end{array}$

Source DF Sum of Squares Mean Square F Ratio Prob $>$ F $\begin{array}{lllllll}\text { Heat Treatment } & 1 & 0.05491806 & 0.054918 & 124.5037 & 0.0004\end{array}$ Error 0.00176438 0.05668244 0.000441

\section{Means for Oneway Anova}

Level Number Mean Std Error Lower 95\% Upper 95\%

\begin{tabular}{llll} 
cCC & 31.08097 & 0.01213 \\
\hline
\end{tabular}

$\begin{array}{llllll}\text { quenched } & 3 & 1.27232 & 0.01213 & 1.2386 & 1.3060\end{array}$

Std Error uses a pooled estimate of error variance

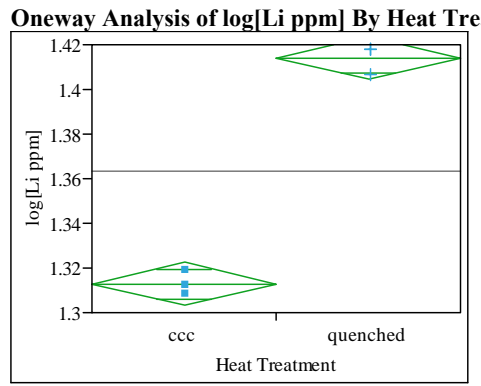

Oneway Anova

Summary of Fit

$\begin{array}{lr}\text { Rsquare } & 0.990863 \\ \text { Adj Rsquare } & 0.988578 \\ \text { Root Mean Square Error } & 0.005946 \\ \text { Mean of Response } & 1.363468 \\ \text { Observations (or Sum Wgts) } & 6\end{array}$

quenched-ccc

Assuming equal variances

Difference $\quad 0.101105$ t Ratio $\quad 20.82709$

Std Err Dif 0.004855 DF

Upper CL Dif 0.114584 Prob $>|t| \quad<.0001$

Lower CL Dif 0.087627 Prob $>t \quad<.0001$

Confidence $\quad 0.95 \mathrm{Prob}<\mathrm{t} \quad 1.0000$

Analysis of Variance

Source DF Sum of Squares Mean Square F Ratio Prob $>$ F $\begin{array}{llllll}\text { Heat Treatment } & 1 & 0.01533341 & 0.015333 & 433.7675 & <.0001\end{array}$ $\begin{array}{llll}\text { Error } & 4 & 0.00014140 & 0.000035\end{array}$ $\begin{array}{lll}\text { C. Total } & 5 & 0.01547481\end{array}$

Means for Oneway Anova

Level Number Mean Std Error Lower 95\% Upper 95\%

$\begin{array}{llllll}\text { cCC } & 3 & 1.31292 & 0.00343 & 1.3034 & 1.3224\end{array}$

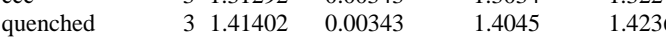

Std Error uses a pooled estimate of error variance 
SRNL-STI-2009-00778, Revision 0

Exhibit E5. Effects of Heat Treatment (HT) on PCT $\log (\mathrm{ppm})$ Response of the Matrix 2 A Study Glasses

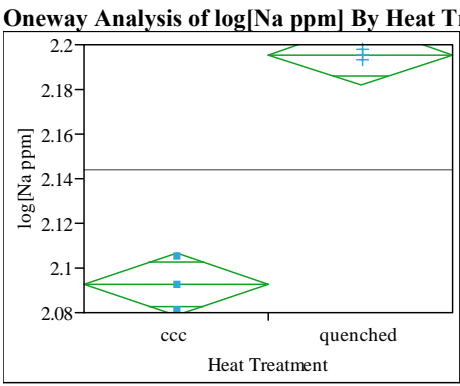

Oneway Anova

Summary of Fit

\begin{tabular}{|c|c|c|c|}
\hline \\
\hline \multicolumn{2}{|l|}{$\begin{array}{l}\text { Rsquare } \\
\text { Adj Rsquare }\end{array}$} & \multicolumn{2}{|c|}{$\begin{array}{l}0.981437 \\
0.976797\end{array}$} \\
\hline \multicolumn{2}{|c|}{ Root Mean Square Error } & 0.008 & \\
\hline \multirow{2}{*}{\multicolumn{2}{|c|}{$\begin{array}{l}\text { Mean of Response } \\
\text { Observations (or Sum Wgts) }\end{array}$}} & 2.144 & \\
\hline & & & 6 \\
\hline \multicolumn{4}{|l|}{$\begin{array}{l}\text { t Test } \\
\text { quenched-ccc }\end{array}$} \\
\hline \multicolumn{4}{|c|}{ Assuming equal variances } \\
\hline Difference & $0.102860 \mathrm{t}$ & t Ratio & 14.54 \\
\hline Std Err Dif & $0.007073 \mathrm{I}$ & DF & \\
\hline Upper CL Dif & $0.122498 \mathrm{I}$ & $\operatorname{Prob}>|t|$ & \\
\hline Lower CL Dif & $0.083222 \mathrm{~F}$ & Prob $>t$ & \\
\hline Confidence & $0.95 \mathrm{I}$ & Prob $<$ t & 0.99 \\
\hline
\end{tabular}

Analysis of Varia

Source DF Sum of Squares Mean Square F Ratio Prob $>$ $\begin{array}{llllll}\text { Heat Treatment } & 1 & 0.01587026 & 0.015870 & 211.4857 & 0.0001\end{array}$

$$
0.0003001
$$

0.01617043

0.000075

Means for Oneway Anova

Level Number Mean Std Error Lower 95\% Upper 95\%

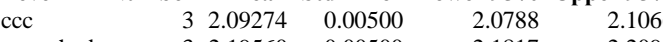

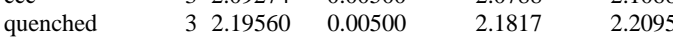

Std Error uses a pooled estimate of error variance
Oneway Analysis of $\log [\mathrm{Si}$ ppm] By Heat Treatment Glass ID=EM-08

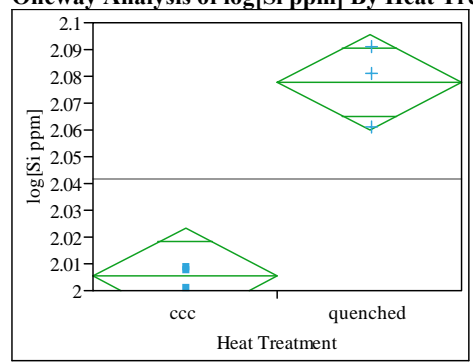

Oneway Anova

Summary of Fit

Adj Rsquare

\subsection{4}

Aj Rsquare Square Error $\quad 0.911103$

Mean of Response 2.041658

Observations (or Sum Wgts)

t Test

quenched-ccc

Assuming equal variances

Difference $\quad 0.072297$ t Ratio $\quad 7.974677$

$\begin{array}{lrr}\text { Std Err Dif } & 0.009066 \text { DF } & 4 \\ \text { Uper } & 0.0013\end{array}$

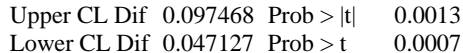

$\begin{array}{lll}\text { Confidence } & 0.95 \mathrm{Prob}<\mathrm{t} & 0.9993\end{array}$

Analysis of Variance

Source DF Sum of Squares Mean Square F Ratio Prob $>$ F

$\begin{array}{lllllll}\text { Heat Treatment } & 1 & 0.00784038 & 0.007840 & 63.5955 & 0.0013\end{array}$

Error

0.00049314

0.000123

Means for Oneway Anova

Level Number Mean Std Error Lower 95\% Upper 95\%

ccC 32.005510 .00641

$\begin{array}{llllll}\text { quenched } & 3 & 2.07781 & 0.00641 & 2.0600 & 2.0956\end{array}$

Std Error uses a pooled estimate of error variance

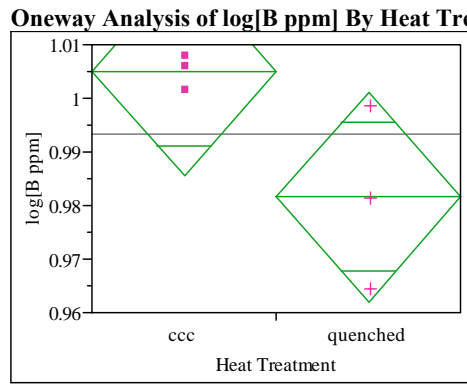

Oneway Anova

Summary of Fit

$\begin{array}{lr}\text { Rsquare } & 0.580089 \\ \text { Adj Rsquare } & 0.475111 \\ \text { Root Mean Square Error } & 0.012235 \\ \text { Mean of Response } & 0.993297 \\ \text { Observations (or Sum Wgts) } & 6\end{array}$

quenched-ccc

Assuming equal variances

Difference $\quad-0.02348$ t Ratio $\quad-2.3507$

Std Err Dif 0.00999 DF

Upper CL Dif 0.00425 Prob $>|t| 0.0785$

Lower CL Dif -0.05122 Prob $>$ t 0.9608

Confidence $\quad 0.95$ Prob $<\mathrm{t} \quad 0.0392$

Analysis of Variance

Source DF Sum of Squares Mean Square F Ratio Prob $>$ F $\begin{array}{llllll}\text { Heat Treatment } & 1 & 0.00082713 & 0.000827 & 5.5258 & 0.0785\end{array}$ $\begin{array}{lll}\text { Error } & 4 & 0.00059874 \\ \text { C. Total } & 5 & 0.00142587\end{array}$ 0.000150

Means for Oneway Anova

Level Number Mean Std Error Lower 95\% Upper 95\% $\begin{array}{llllll}\text { ccC } & 3 & 1.00504 & 0.00706 & 0.98543 & 1.0246\end{array}$ $\begin{array}{llllll}\text { quenched } & 3 & 0.98156 & 0.00706 & 0.96194 & 1.0012\end{array}$

Std Error uses a pooled estimate of error variance 
SRNL-STI-2009-00778, Revision 0

Exhibit E5. Effects of Heat Treatment (HT) on PCT $\log (\mathrm{ppm})$ Response of the Matrix 2A Study Glasses

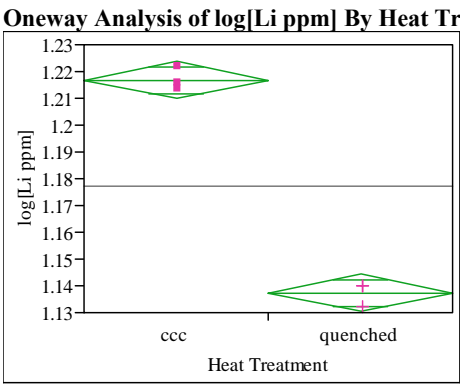

Oneway Anova

Summary of Fit

\begin{tabular}{|c|c|c|c|}
\hline \\
\hline \multicolumn{4}{|l|}{ Rsquare } \\
\hline \multicolumn{2}{|c|}{$\begin{array}{l}\text { Adj Rsquare } \\
\text { Root Mean Square Error }\end{array}$} & 0.004 & \\
\hline \multicolumn{2}{|c|}{ Mean of Response } & 1.177 & 7155 \\
\hline \multicolumn{4}{|c|}{ Observations (or Sum Wgts) 6} \\
\hline \multicolumn{4}{|l|}{$\begin{array}{l}\text { t Test } \\
\text { quenched-ccc }\end{array}$} \\
\hline \multicolumn{4}{|c|}{ Assuming equal variances } \\
\hline Difference & -0.07947 & t Ratio & -22.175 \\
\hline Std Err Dif & 0.00358 & DF & \\
\hline Upper CL Dif & -0.06952 & Prob $>|t|$ & $<.0001$ \\
\hline Lower CL Dif & -0.08942 & Prob $>t$ & 1.0000 \\
\hline Confidence & 0.95 & Prob $<\mathrm{t}$ & $<.0001$ \\
\hline
\end{tabular}

Analysis of Variance

Source DF Sum of Squares Mean Square F Ratio Prob $>$ F $\begin{array}{llllll}\text { Heat Treatment } & 1 & 0.00947385 & 0.009474 & 491.7297 & <.0001\end{array}$

$$
0.00007707
$$$$
0.00955092
$$$$
0.000019
$$

Means for Oneway Anova

Level Number Mean Std Error Lower 95\% Upper 95\%

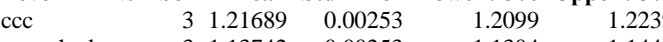

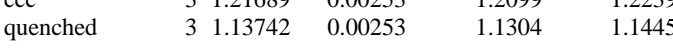

Std Error uses a pooled estimate of error variance

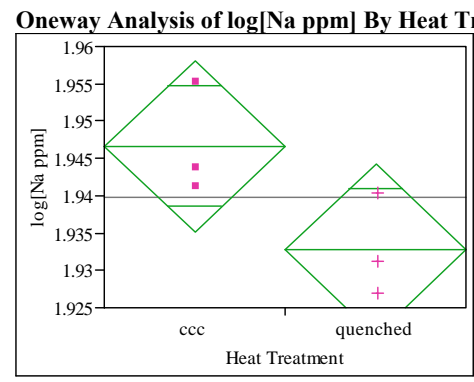

Oneway Anova

Summary of Fit

Rsquare

0.584643

Adj Rsquare $\quad 0.480804$

Root Mean Square Error $\quad 0.00715$

Mean of Response ${ }_{\text {Observations (or Sum Wgts) }}^{1.939711}$

t Test

quenched-ccc

Assuming equal variances

Difference $\quad-0.01385$ t Ratio $\quad-2.37282$

Std Err Dif $\quad 0.00584$ DF $\quad-2.37282$

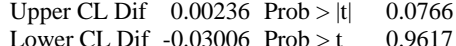

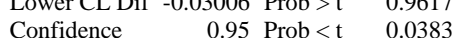

Analysis of Variance

Source DF Sum of Squares Mean Square F Ratio Prob $>$ F

$\begin{array}{lll}\text { Heat Treatment } & 1 & 0.00028787 \\ & 4 & 0.000204538\end{array}$

$\begin{array}{lll}\text { Error } & 4 & 0.00020451 \\ \text { C. Total } & 5 & 0.00049238\end{array}$

$5.6303 \quad 0.0766$

Means for Oneway Anova

Level Number Mean Std Error Lower 95\% Upper 95\%

$\begin{array}{llllll}\text { ccc } & 3 & 1.94664 & 0.00413 & 1.9352 & 1.9581\end{array}$

Std Error uses a pooled estimate of error variance

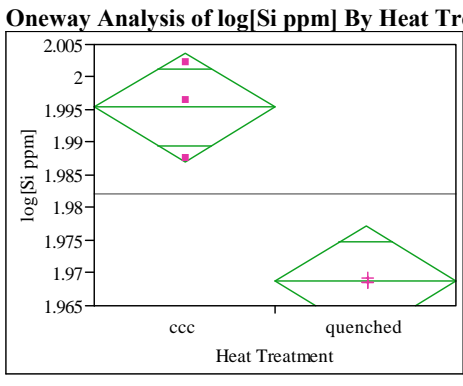

Oneway Anova

Summary of Fit

Rsquare

0.906723

Root Mean Square Eror $\quad 0.883404$

Root Mean Square Error $\quad 0.005226$

Mean of Response 1.982055

t Test

quenched-ccc

Assuming equal variances

Difference $\quad-0.02661$ t Ratio -6.23563 Std Err Dif $\quad 0.00427$ DF

Upper CL Dif -0.01476 Prob $>|t| \quad 0.0034$ $\begin{array}{lrr}\text { Lower CL Dif } & -0.03845 \text { Prob }>t & 0.9983 \\ \text { Confidence } & 0.95 \text { Prob }<t & 0.0017\end{array}$

Analysis of Variance

Source DF Sum of Squares Mean Square F Ratio Prob $>$ F $\begin{array}{llllll}\text { Heat Treatment } & 1 & 0.00106194 & 0.001062 & 38.8831 & 0.0034\end{array}$ \begin{tabular}{lll} 
Error & 4 & 0.00010924 \\
\hline
\end{tabular} 000027

Means for Oneway Anova

Level Number Mean Std Error Lower 95\% Upper 95\%

$\begin{array}{llllrr}\text { ccC } & 3 & 1.99536 & 0.00302 & 1.9870 & 2.0037\end{array}$

Std Error uses a pooled estimate of error variance 
SRNL-STI-2009-00778, Revision 0

Exhibit E5. Effects of Heat Treatment (HT) on PCT $\log (\mathrm{ppm})$ Response of the Matrix 2 A Study Glasses

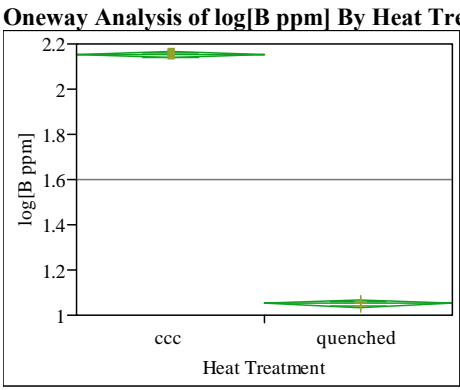

Oneway Anova

Summary of Fit

$\begin{array}{ll}\text { Rsquare } & 0.999803 \\ \text { Adj Rsquare } & 0.999753 \\ \text { Root Mean Square Error } & 0.009475 \\ \text { Mean of Response } & 1.601383\end{array}$

Mean of Response 1.601383

Observations (or Sum Wgts)

t Test

quenched-ccc

Assuming equal variances

Difference $\quad-1.1010$ t Ratio $\quad-142.319$

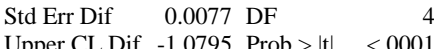

$\begin{array}{lll}\text { Upper CL Dif } & -1.0795 \text { Prob }>|t| & <.0001 \\ \text { Lower CL Dif }-1.1225 & \text { Prob }>t & 1.0000\end{array}$

Confidence $\quad 0.95$ Prob $<\mathrm{t} \quad<.0001$

Analysis of Variance

Source DF Sum of Squares Mean Square F Ratio Prob $>$ F $\begin{array}{llllll}\text { Heat Treatment } & 1 & 1.8183234 & 1.81832 & 20254.82 & <.0001 \\ \text { Error } & 4 & 0.0003591 & 8.977 \mathrm{e}-5 & & \end{array}$ Error $8.977 \mathrm{e}-5$

\section{Means for Oneway Anova}

Level Number Mean Std Error Lower 95\% Upper 95\%

$\begin{array}{llllll}\text { ccc } & 3 & 2.15189 & 0.00547 & 2.1367 & 2.1671 \\ \text { quenched } & 3 & 1.05088 & 0.00547 & 1.0357 & 1.0661\end{array}$

Std Error uses a pooled estimate of error variance
Oneway Analysis of $\log [\mathrm{Li}$ ppm] By Heat Treatment Glass ID=EM-10

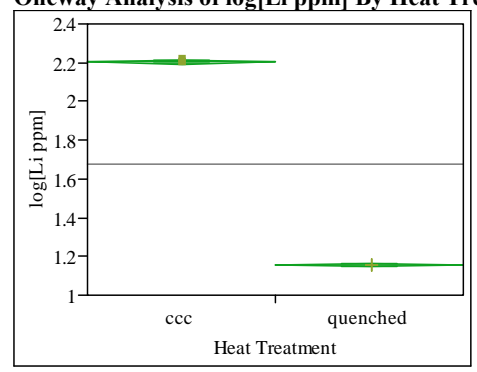

Oneway Anova

Summary of Fit

Rsquare $\quad 0.999879$

Adj Rsquare $\quad 0.999849$

Root Mean Square Error $\quad 0.007049$

Mean of Response 1.679423

Observations (or Sum Wgts)

t Test

quenched-ccc

Assuming equal variances

Difference $\quad-1.0472$ t Ratio $\quad-181.946$

$\begin{array}{lrrr}\text { Std Err Dif } & 0.0058 \text { DF } & 4 \\ \text { Upper CL Dif } & -1.0312 \text { Prob }>|t| & <0001\end{array}$

$\begin{array}{lll}\text { Upper CL Dif }-1.0312 \text { Prob }>|t| & <.0001 \\ \text { Lower CL Dif }-1.0631 & \text { Prob }>t & 1.0000\end{array}$

$\begin{array}{lll}\text { Confidence } & 0.95 \mathrm{Prob}<\mathrm{t} \quad<.0001\end{array}$

Analysis of Variance

Source DF Sum of Squares Mean Square F Ratio Prob $>$ F $\begin{array}{lllllll}\text { Heat Treatment } & 1 & 1.6447914 & 1.64479 & 33104.36 & <.0001\end{array}$ Error $\begin{array}{ll}1 & 1.6447914 \\ 4 & 0.0001987\end{array}$ 4.969e-5

\section{Means for Oneway Anova}

Level Number Mean Std Error Lower 95\% Upper 95\%

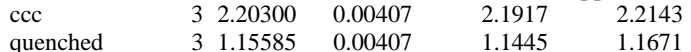

Std Error uses a pooled estimate of error variance

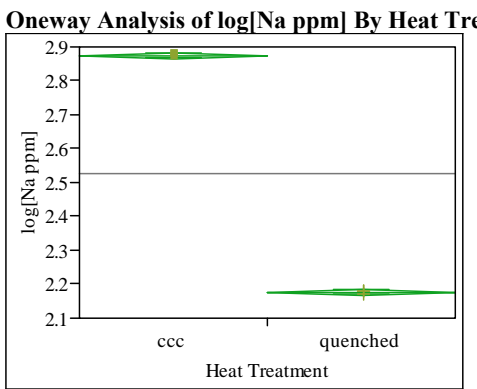

Oneway Anova

Summary of Fit

Rsquare

Adj Rsquare $\quad 0.999808$

Root Mean Square Error $\quad 0.99976$

Mean of Response 2.525476

Observations (or Sum Wgts)

t Test

quenched-ccc

Assuming equal variance

Difference $\quad-0.69658$ t Ratio $\quad-144.439$

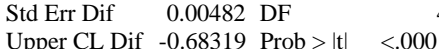

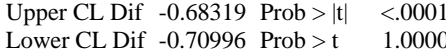

Confidence $\quad 0.95$ Prob $<\mathrm{t} \quad<.0001$

Analysis of Variance

Source DF Sum of Squares Mean Square F Ratio Prob $>$ F $\begin{array}{lllllll}\text { Heat Treatment } & 1 & 0.72782524 & 0.727825 & 20862.49 & <.0001\end{array}$ Error $\quad 4 \quad 0.00013955$ 0.000035

Means for Oneway Anova

Level Number Mean Std Error Lower 95\% Upper 95\% $\begin{array}{llllll}\text { ccc } & 3 & 2.87376 & 0.00341 & 2.8643 & 2.8832 \\ \text { quenched } & 3 & 2.17719 & 0.00341 & 2.1677 & 2.1867\end{array}$

Std Error uses a pooled estimate of error variance 
SRNL-STI-2009-00778, Revision 0

Exhibit E5. Effects of Heat Treatment (HT) on PCT $\log (\mathrm{ppm})$ Response of the Matrix 2A Study Glasses

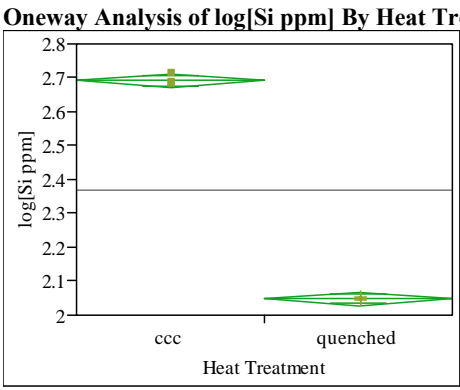

Summary of Fit

$\begin{array}{lr}\text { Rsquare } & 0.998946 \\ \text { Adj Rsquare } & 0.998682 \\ \text { Root Mean Square Error } & 0.012804 \\ \text { Mean of Response } & 2.37014 \\ \text { Observations (or Sum Wgts) } & 6\end{array}$

Test

quenched-ccc

Assuming equal variances

Difference $\quad-0.64360$ t Ratio $\quad-61.5604$

$\begin{array}{lrr}\text { Std Err Dif } & 0.01045 \text { DF } & 4 \\ \text { Upper CL Dif } & -0.61457 \text { Prob }>|t| & <0001\end{array}$

$\begin{array}{lll}\text { Upper CL Dif }-0.61457 \text { Prob }>|t| & <.0001 \\ \text { Lower CL Dif }-0.67263 & \text { Prob }>t & 1.0000\end{array}$

Confidence $\quad 0.95$ Prob $<\mathrm{t} \quad<.0001$

Analysis of Variance

Source DF Sum of Squares Mean Square F Ratio Prob $>$ F $\begin{array}{llllll}\text { Heat Treatment } & 1 & 0.62133160 & 0.621332 & 3789.687 & <.0001 \\ \text { Error } & 4 & 0.00065581 & 0.000164 & \end{array}$ Erro

C. Total 0.000164

Means for Oneway Anova

Level Number Mean Std Error Lower 95\% Upper 95\%

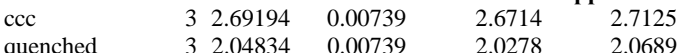

Std Error uses a pooled estimate of error variance
Oneway Analysis of $\log [\mathrm{B}$ ppm $]$ By Heat Treatment Glass ID=EM-11

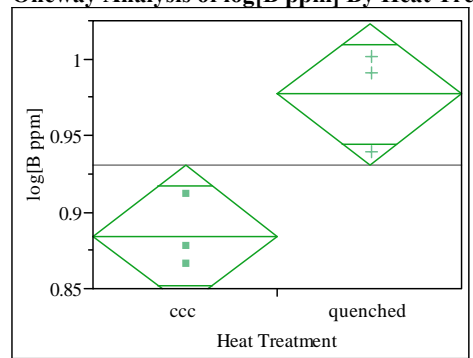

Oneway Anova

Summary of Fit

Adj Rsquare

\subsection{3}

0.795083
Root Mean Square Error $\quad 0.743854$
0.028809

Mean of Response 0.930825

Observations (or Sum Wgts)

t Test

quenched-ccc

Assuming equal variances

Difference $\quad 0.092668$ t Ratio 3.939557

$\begin{array}{lrr}\text { Std Err Dif } & 0.023523 \text { DF } & 4 \\ \text { Uper } & & 0.0170\end{array}$

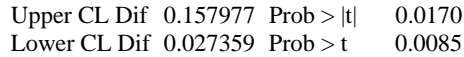

$\begin{array}{lll}\text { Confidence } & 0.95 \text { Prob }<\mathrm{t} \quad 0.9915\end{array}$

Analysis of Variance

Source DF Sum of Squares Mean Square F Ratio Prob $>$ F $\begin{array}{llllll}\text { Heat Treatment } & 1 & 0.01288114 & 0.012881 & 15.5201 & 0.0170 \\ \text { Error } & 4 & 0.00331986 & 0.000830 & & \end{array}$ 0.000830

\section{Means for Oneway Anova}

Level Number Mean Std Error Lower 95\% Upper 95\%

$\begin{array}{lllllr}\text { ccc } & 3 & 0.884491 & 0.01663 & 0.83831 & 0.9307\end{array}$

$\begin{array}{llllll}\text { quenched } & 3 & 0.977159 & 0.01663 & 0.93098 & 1.0233\end{array}$

Std Error uses a pooled estimate of error variance

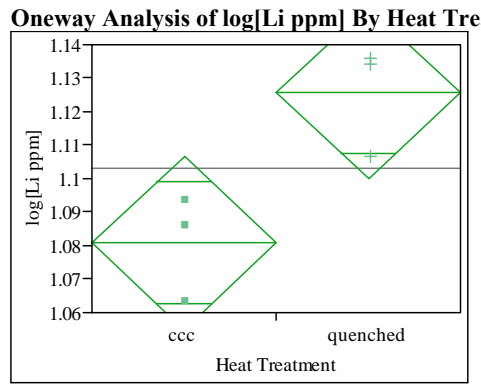

Oneway Anova

Summary of Fit

$\begin{array}{lr}\text { Rsquare } & 0.744164 \\ \text { Adj Rsquare } & 0.680205 \\ \text { Root Mean Square Error } & 0.016099 \\ \text { Mean of Response } & 1.103226 \\ \text { Observations (or Sum Wgts) } & 6\end{array}$

t Test

quenched-ccc

Assuming equal variances

Difference $\quad 0.044837$ t Ratio 3.411017 Std Err Dif $\quad 0.013145$ DF

Upper CL Dif 0.081333 Prob $>|t| \quad 0.0270$

Lower CL Dif 0.008341 Prob $>t \quad 0.0135$

$\begin{array}{lll}\text { Confidence } & 0.95 \mathrm{Prob}<\mathrm{t} \quad 0.9865\end{array}$

Analysis of Variance

Source DF Sum of Squares Mean Square F Ratio Prob $>$ F $\begin{array}{llllll}\text { Heat Treatment } & 1 & 0.00301553 & 0.003016 & 11.6350 & 0.0270\end{array}$ $\begin{array}{llll}\text { Error } & 4 & 0.00103670 & 0.000259 \\ \text { C. Total } & 5 & 0.00405223 & \end{array}$

Means for Oneway Anova

Level Number Mean Std Error Lower 95\% Upper 95\%

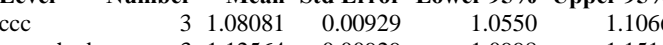

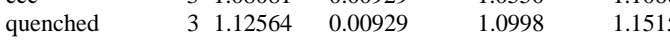

Std Error uses a pooled estimate of error variance 
SRNL-STI-2009-00778, Revision 0

\section{Exhibit E5. Effects of Heat Treatment (HT) on PCT $\log (\mathrm{ppm})$ Response of the Matrix 2A Study Glasses}

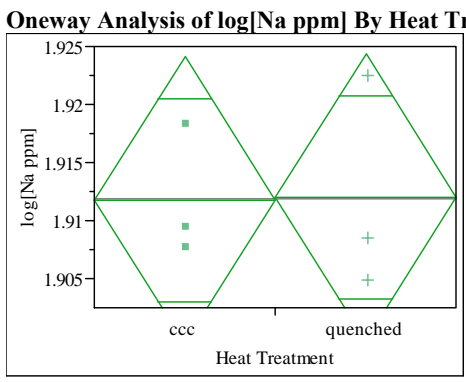

\section{Oneway Anova}

Summary of Fit

Rsquare $\quad 0.000403$

Adj Rsquare $\quad-0.2495$

Root Mean Square Error $\quad 0.007726$

$\begin{array}{lr}\text { Mean of Response } & 1.91186 \\ \text { Observations (or Sum Wgts) } & 6\end{array}$

t Test

quenched-ccc

Assuming equal variances

Difference $\quad 0.00025$ t Ratio $\quad 0.040155$ Std Err Dif 0.00631 DF

Upper CL Dif 0.0177 Prob $>|t| \quad 0.9699$

Lower CL Dif -0.01726 Prob $>t \quad 0.4849$

Analysis of Variance

Source DF Sum of Squares Mean Square F Ratio Prob $>$ F $\begin{array}{llllll}\text { Heat Treatment } & 1 & 9.62505 \mathrm{e}-8 & 9.625 \mathrm{e}-8 & 0.0016 & 0.9699\end{array}$

Error

0.00023877

Means for Oneway Anova

Level Number Mean Std Error Lower 95\% Upper 95\%

$\begin{array}{llllll}\text { ccc } & 3 & 1.91173 & 0.00446 & 1.8993 & 1.924\end{array}$

Std Error uses a pooled estimate of error variance
Oneway Analysis of $\log [\mathrm{Si}$ ppm] By Heat Treatment Glass ID=EM-11

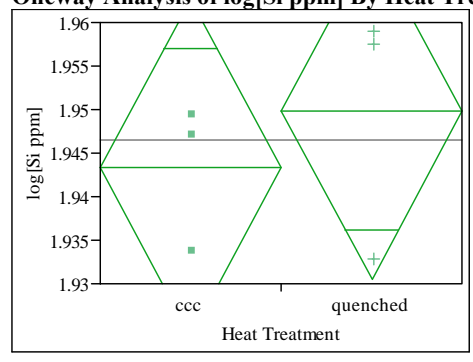

Oneway Anova

Summary of Fit

Rsquare $\quad 0.097423$

Adj Rsquare $\quad-0.1282$

Root Mean Square Eror -0.011998

Mean of Response 1.946557

t Test

quenched-ccc

Assuming equal variances

Difference $\quad 0.00644$ t Ratio $\quad 0.657082$

Std Err Dif 0.00980 D

Upper CL Dif 0.03364 Prob $>|t| \quad 0.5470$

Low CL Dif 0.02076 Prob $>t \quad 0.2735$

Analysis of Variance

Source DF Sum of Squares Mean Square F Ratio Prob $>$ F $\begin{array}{llllll}\text { Heat Treatment } & 1 & 0.00006216 & 0.000062 & 0.4318 & 0.5470\end{array}$ Error 0.00057585

Means for Oneway Anova

Level Number Mean Std Error Lower 95\% Upper 95\%

$\begin{array}{llllll}\text { ccc } & 3 & 1.94334 & 0.00693 & 1.9241 & 1.9626\end{array}$

Std Error uses a pooled estimate of error variance

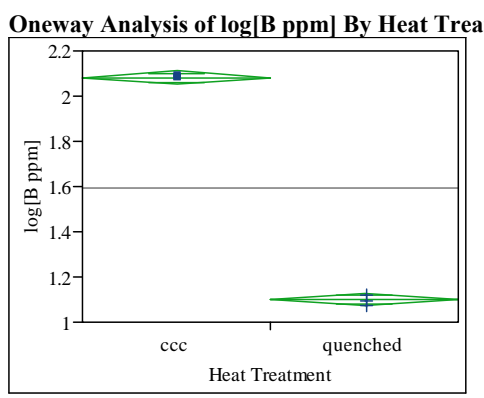

Oneway Anova

Summary of Fit

Rsquare

Root Mean Square Error $\quad 0.017339$

$\begin{array}{lr}\text { Mean of Response } & 1.590495 \\ \text { Observations (or Sum Wgts) } & 6\end{array}$

t Test

quenched-ccc

Assuming equal variance

Difference $\quad-0.9850$ t Ratio $\quad-69.5727$

Std Err Dif $\quad 0.0142$ DF 4

$\begin{array}{rrr}\text { Upper CL Dif } & -0.9457 \text { Prob }>|t| & <.0001 \\ \text { Lower CL Dif }-1.0243 \text { Prob }>t & 1.0000\end{array}$

$\begin{array}{lr}\text { Confidence } & 0.95 \text { Prob }<\mathrm{t} \quad<.0001\end{array}$

Analysis of Variance

Source DF Sum of Squares Mean Square F Ratio Prob $>$ F $\begin{array}{llllll}\text { Heat Treatment } & 1 & 1.4552125 & 1.45521 & 4840.362 & <.0001\end{array}$ $\begin{array}{lll}\text { Error } & 4 & 0.0012026\end{array}$ $\begin{array}{lll}\text { C. Total } & 5 & 1.4564151\end{array}$ 0.00030

Means for Oneway Anova

Level Number Mean Std Error Lower 95\% Upper 95\% $\begin{array}{llllll}\text { ccc } & 3 & 2.08297 & 0.01001 & 2.0552 & 2.1108 \\ \text { quenched } & 3 & 1.09802 & 0.01001 & 1.0702 & 1.1258\end{array}$

Std Error uses a pooled estimate of error variance 
SRNL-STI-2009-00778, Revision 0

Exhibit E5. Effects of Heat Treatment (HT) on PCT $\log (\mathrm{ppm})$ Response of the Matrix 2 A Study Glasses

Oneway Analysis of $\log [\mathrm{Li}$ ppm] By Heat Treatment Glass ID=EM-12

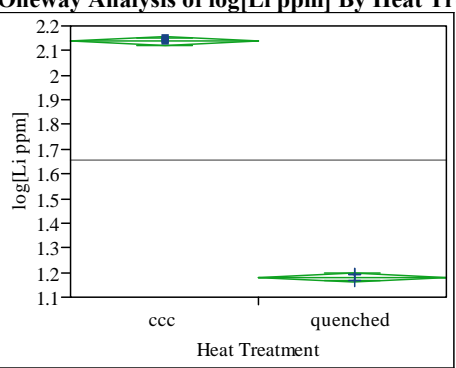

Oneway Anova

Summary of Fit

$\begin{array}{lr}\text { Rsquare } & 0.99961 \\ \text { Adj Rsquare } & 0.999513\end{array}$

Root Mean Square Error $\quad 0.011543$

Mean of Response 1.659092

Observations (or Sum Wgts)

t Test

quenched-ccc

Assuming equal variances

Difference $\quad-0.95451$ t Ratio $\quad-101.275$

$\begin{array}{lrr}\text { Std Err Dif } & 0.00942 \text { DF } & 4 \\ \text { Uper } & -10.0001\end{array}$

Lower CL Dif -0.98068 Prob $>t \quad 1.0000$

$\begin{array}{lll}\text { Confidence } \quad 0.95 \text { Prob }<\mathrm{t} \quad<.0001 & \end{array}$

Analysis of Variance

Source DF Sum of Squares Mean Square F Ratio Prob $>$ F $\begin{array}{llllll}\text { Heat Treatment } & 1 & 1.3666411 & 1.36664 & 10256.58 & <.0001\end{array}$ Error 0.0005330 0.00013 1.3671741

Means for Oneway Anova

Level Number Mean Std Error Lower 95\% Upper 95\%

$\begin{array}{llllll}\text { ccC } & 3 & 2.13635 & 0.00666 & 2.1178 & 2.1549\end{array}$

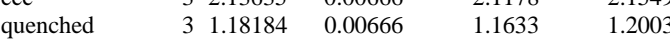

Std Error uses a pooled estimate of error variance
Oneway Analysis of $\log [\mathrm{Na}$ ppm] By Heat Treatment Glass ID=EM-12

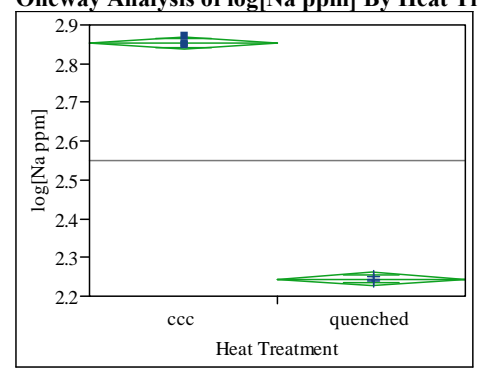

Oneway Anova

Summary of $\mathrm{Fit}$

Rsquare

Root Mean Square Error $\quad 0.009938$

Mean of Response 2.549124

Observations (or Sum Wgts)

6

quenched-ccc

Assuming equal variances

Difference $\quad-0.60946$ t Ratio $\quad-75.109$

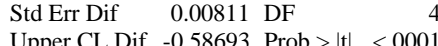
$\begin{array}{rrr}\text { Upper CL Dif }-0.58693 \text { Prob }>|t| & <.0001 \\ \text { Lower CL Dif }-0.63199 & \text { Prob }>t & 1.0000\end{array}$ $\begin{array}{lll}\text { Confidence } & 0.95 \text { Prob }<\mathrm{t} \quad<.0001\end{array}$

Analysis of Variance

Source DF Sum of Squares Mean Square F Ratio Prob $>$ F Heat Treatment $11 \quad 0.55716648$

C. Tot $4 \quad 0.00039506$ $0.5571665641 .355<0001$

Means for Oneway Anova

Level Number Mean Std Error Lower 95\% Upper 95\%

$\begin{array}{llllll}\text { ccc } & 3 & 2.85386 & 0.00574 & 2.8379 & 2.8698 \\ \text { quenched } & 3 & 2.24439 & 0.00574 & 2.2285 & 2.2603\end{array}$

Std Error uses a pooled estimate of error variance

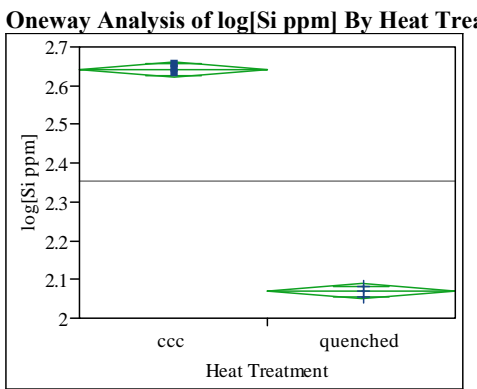

Oneway Anova

Summary of $F$

Rsquare

Adj Rsquare

0.998515

Root Mean Square Error $\quad 0.01211$

$\begin{array}{lr}\text { Mean of Response } & 2.354995 \\ \text { Observations (or Sum Wgts) } & 6\end{array}$

t Test

quenched-ccc

Assuming equal variance

Difference $\quad-0.57346$ t Ratio $\quad-57.9963$

$\begin{array}{lll}\text { Std Err Dif } & 0.00989 \text { DF } & \\ \text { Upper CL Dif } & -0.54600 \text { Prob }>|t| & <.000\end{array}$

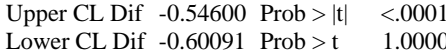

Confidence $\quad 0.95 \mathrm{Prob}<\mathrm{t} \quad<.0001$

Analysis of Variance

Source DF Sum of Squares Mean Square F Ratio Prob $>$ F $\begin{array}{lllllll}\text { Heat Treatment } & 1 & 0.49328096 & 0.493281 & 3363.573 & <.0001\end{array}$ $\begin{array}{lll}\text { Error } & 4 & 0.00058662 \\ \text { C. Total } & 5 & 0.49386757\end{array}$

Means for Oneway Anova

Level Number Mean Std Error Lower 95\% Upper 95\% $\begin{array}{llllll}\text { ccC } & 3 & 2.64172 & 0.00699 & 2.6223 & 2.6611 \\ \text { quenched } & 3 & 2.06827 & 0.00699 & 2.0489 & 2.0877\end{array}$

Std Error uses a pooled estimate of error variance 
SRNL-STI-2009-00778, Revision 0

\section{Exhibit E5. Effects of Heat Treatment (HT) on PCT $\log (\mathrm{ppm})$ Response of the Matrix 2A Study Glasses}

Oneway Analysis of $\log [\mathrm{B}$ ppm $]$ By Heat Treatment Glass ID=EM-13

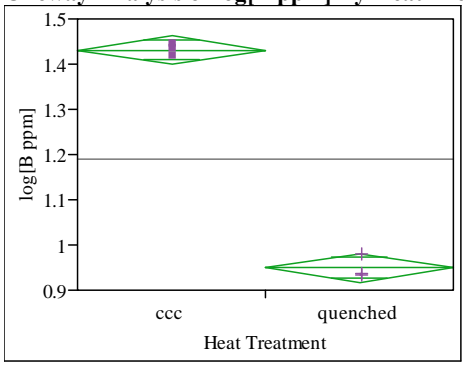

Oneway Anova

Summary of Fit

Rsquare $\quad 0.995559$

\begin{tabular}{ll}
0.994449 \\
\hline
\end{tabular}

Mean of Response 1.190492

Observations (or Sum Wgts)

Test

quenched-ccc

Assuming equal variances

Difference $\quad-0.48153$ t Ratio $\quad-29.9443$

$\begin{array}{lrr}\text { Std Err Dif } & 0.01608 \text { DF } & 4 \\ \text { Upper CL Dif } & -0.43688 \text { Prob }>|t| & <.0001\end{array}$

Lower CL Dif -0.52618 Prob $>\mathrm{t} \quad 1.0000$

$\begin{array}{lrl}\text { Confidence } & 0.95 \text { Prob }<\mathrm{t} \quad<.0001\end{array}$

Analysis of Variance

Source DF Sum of Squares Mean Square F Ratio Prob $>$ F $\begin{array}{llllll}\text { Heat Treatment } & 1 & 0.34780414 & 0.347804 & 896.6613 & <.0001\end{array}$ Error $0.00155155-0.000388$

Means for Oneway Anova

Level Number Mean Std Error Lower 95\% Upper 95\% $\begin{array}{llllll}\text { ccc } & 3 & 1.43126 & 0.01137 & 1.3997 & 1.4628\end{array}$

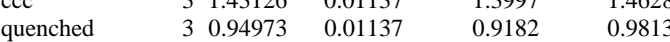

Std Error uses a pooled estimate of error variance
Oneway Analysis of $\log [\mathrm{Li} \mathrm{ppm}]$ By Heat Treatment Glass ID=EM-13

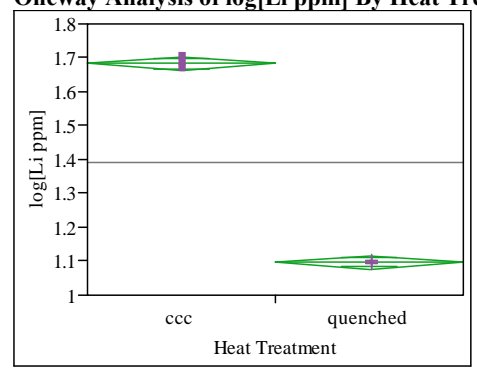

Oneway Anova

Summary of Fit

Rsquare 0.99876

Adj Rsquare $\quad 0.998451$

Root Mean Square Error $\quad 0.012641$

Mean of Response 1.39006

Observations (or Sum Wgts)

t Test

quenched-ccc

Assuming equal variances

Difference $\quad-0.58593$ t Ratio $\quad-56.7703$

$\begin{array}{llr}\text { Std Err Dif } & 0.01032 \text { DF } & 4 \\ \text { Upper } & -0.5728 \text { Prob }>|t| & <0001\end{array}$

$\begin{array}{llll}\text { Upper CL Dif } & -0.55728 & \text { Prob }>|t| & <.000 \\ \text { Lower CL Dif } & -0.61459 & \text { Prob }>t & 1.0000\end{array}$

$\begin{array}{lll}\text { Confidence } & 0.95 \mathrm{Prob}<\mathrm{t} \quad<.000\end{array}$

Analysis of Variance

Source DF Sum of Squares Mean Square F Ratio Prob $>$ F $\begin{array}{lllllll}\text { Heat Treatment } & 1 & 0.51497451 & 0.514975 & 3222.869 & <.0001\end{array}$ C. Tot $4 \quad 0.00063915$ 0.000160

Means for Oneway Anova

Level Number Mean Std Error Lower 95\% Upper 95\%

$\begin{array}{llllll}\text { ccc } & 3 & 1.68303 & 0.00730 & 1.6628 & 1.7033 \\ \text { quenched } & 3 & 1.09710 & 0.00730 & 1.0768 & 1.1174\end{array}$

Std Error uses a pooled estimate of error variance

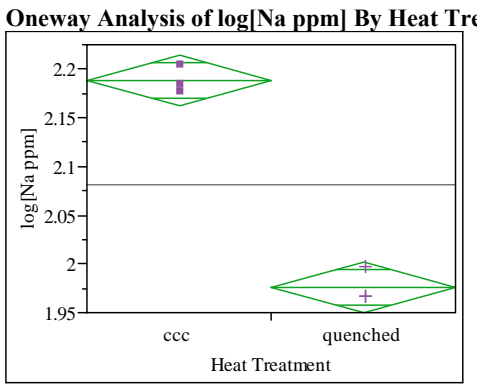

Oneway Anova

Summary of Fit

$\begin{array}{lr}\text { Rsquare } & 0.984281 \\ \text { Adj Rsquare } & 0.980352 \\ \text { Root Mean Square Error } & 0.016357 \\ \text { Mean of Response } & 2.082151 \\ \text { Observations (or Sum Wgts) } & 6\end{array}$

quenched-ccc

Assuming equal variances

Difference $\quad-0.21136$ t Ratio $\quad-15.8265$

Std Err Dif 0.01336 DF $>0.15 .82600$

Upper CL Dif -0.17428 Prob $>|t| \quad<.0001$

Lower CL Dif -0.24844 Prob $>t \quad 1.0000$

$\begin{array}{lll}\text { Confidence } & 0.95 \text { Prob }<\mathrm{t} \quad<.0001\end{array}$

Analysis of Variance

Source DF Sum of Squares Mean Square F Ratio Prob $>$ F $\begin{array}{lllllll}\text { Heat Treatment } & 1 & 0.06701257 & 0.067013 & 250.4767 & <.0001\end{array}$ $\begin{array}{lll}\text { Error } & 4 & 0.00107016\end{array}$ 0.000268

Means for Oneway Anova

Level Number Mean Std Error Lower 95\% Upper 95\%

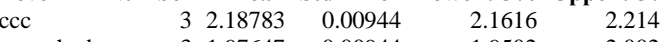

$\begin{array}{llllll}\text { quenched } & 3 & 1.97647 & 0.00944 & 1.9502 & 2.0027\end{array}$

Std Error uses a pooled estimate of error variance 
SRNL-STI-2009-00778, Revision 0

Exhibit E5. Effects of Heat Treatment (HT) on PCT $\log (\mathrm{ppm})$ Response of the Matrix 2A Study Glasses

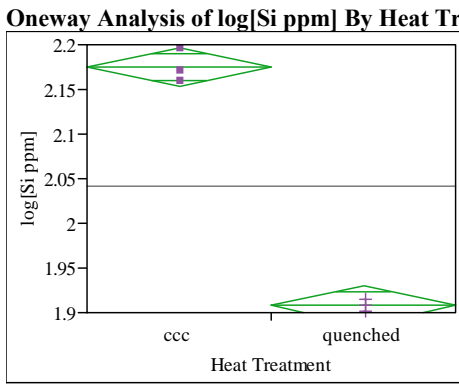

Oneway Anova

Summary of Fit

\begin{tabular}{|c|c|c|c|}
\hline \multicolumn{4}{|c|}{0.993036} \\
\hline \multicolumn{2}{|l|}{$\begin{array}{l}\text { quare } \\
\text { dj Rsquare }\end{array}$} & 0.991 & 295 \\
\hline Root Mean Square Error & uare Error & 0.013 & 685 \\
\hline \multicolumn{2}{|c|}{ Mean of Response } & 2.041 & 291 \\
\hline \multicolumn{2}{|c|}{ Observations (or Sum Wgts) } & & 6 \\
\hline \multicolumn{4}{|l|}{$\begin{array}{l}\text { t Test } \\
\text { quenched-ccc }\end{array}$} \\
\hline \multicolumn{4}{|c|}{ Assuming equal variances } \\
\hline Difference & $-0.26686 \mathrm{t}$ & t Ratio & -23.88 \\
\hline Std Err Dif & $0.01117 \mathrm{D}$ & DF & \\
\hline Upper CL Dif & $-0.23583 \mathrm{P}$ & Prob $>|t|$ & $<.000$ \\
\hline Lower CL Dif & $-0.29788 \mathrm{P}$ & Prob $>t$ & 1.00 \\
\hline Confidence & $0.95 \mathrm{P}$ & Prob $<$ t & $<.00$ \\
\hline
\end{tabular}

Analysis of Varia

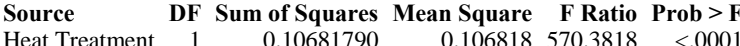

0.10681790

0.10756699

0.00018

C. Total

Std Error Lower 95\% Upper 95\% $\begin{array}{llllrr}\text { cCC } & 3 & 2.17472 & 0.00790 & 2.1528 & 2.1967\end{array}$ $\begin{array}{llllll}\text { quenched } & 3 & 1.90786 & 0.00790 & 1.8859 & 1.9298\end{array}$

Std Error uses a pooled estimate of error variance

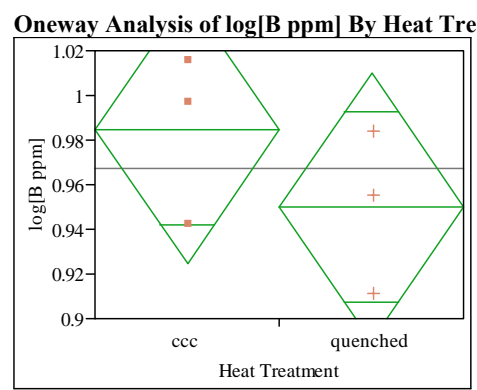

Oneway Anova Summary of Fit

Adj Rsquare

Root Mean Square Erro

Mean of Response

0.054911

t Test

quenched-ccc

Assuming equal variances

Difference $\quad-0.03468$ t Ratio $\quad-1.1360$

$\begin{array}{lrrr}\text { Std Err Dif } & 0.03053 \text { DF } & 4 \\ \text { Upper CL Dif } & 0.05008 \text { Prob }>|t| & 0.3194\end{array}$

$\begin{array}{lrr}\text { Upper CL Dif } & 0.05008 \text { Prob }>|t| & 0.3194 \\ \text { Lower CL Dif } & -0.11943 \text { Prob }>t & 0.8403\end{array}$

$\begin{array}{lrl}\text { Confidence } & 0.95 \text { Prob }<\mathrm{t} & 0.1597\end{array}$

Analysis of Variance

Source DF Sum of Squares Mean Square F Ratio Prob $>$

$\begin{array}{llllll}\text { Heat Treatment } & 1 & 0.00180380 & 0.001804 & 1.2905 & 0.3194 \\ \text { Error } & 4 & 0.00559097 & 0.001398 & & \end{array}$

Erro 0.001398

\section{Means for Oneway Anova}

Level Number Mean Std Error Lower 95\% Upper 95\%

$\begin{array}{llllll}\text { cCC } & 3 & 0.984680 & 0.02159 & 0.92475 & 1.0446\end{array}$

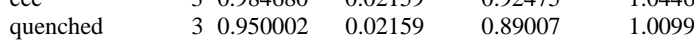

Std Error uses a pooled estimate of error variance

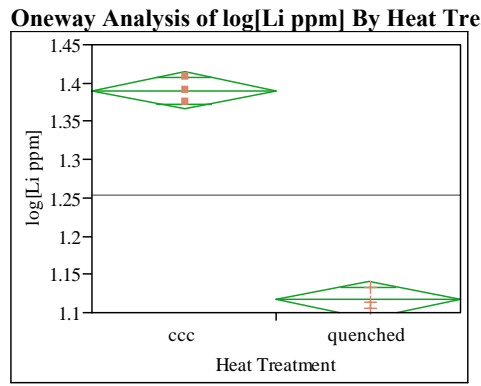

Oneway Anova

Summary of Fit

$\begin{array}{lr}\text { Rsquare } & 0.99178 \\ \text { Adj Rsquare } & 0.989725 \\ \text { Root Mean Square Error } & 0.015231 \\ \text { Mean of Response } & 1.253357 \\ \text { Observations (or Sum Wgts) } & 6\end{array}$

t Test

quenched-ccc

Assuming equal variances

Difference $\quad-0.27320$ t Ratio $\quad-21.9685$

Std Err Dif 0.01244 DF $\quad-21.9685$

Upper CL Dif -0.23867 Prob $>|t|<.0001$

Lower CL Dif -0.30773 Prob $>t \quad 1.0000$

$\begin{array}{lll}\text { Confidence } & 0.95 \text { Prob }<\mathrm{t} \quad<.0001\end{array}$

Analysis of Variance

Source DF Sum of Squares Mean Square $\quad$ F Ratio Prob $>$ F $\begin{array}{lllllll}\text { Heat Treatment } & 1 & 0.11195729 & 0.111957 & 482.6162 & <.0001\end{array}$ $\begin{array}{llll}\text { Error } & 4 & 0.00092792 & 0.000232 \\ \text { C. Total } & 5 & 0.11288521 & \end{array}$

Means for Oneway Anova

Level Number Mean Std Error Lower 95\% Upper 95\% $\begin{array}{llllll}\text { ccc } & 3 & 1.38996 & 0.00879 & 1.3655 & 1.4144 \\ \text { quenched } & 3 & 1.11676 & 0.00879 & 1.0923 & 1.1412\end{array}$

Std Error uses a pooled estimate of error variance 
SRNL-STI-2009-00778, Revision 0

\section{Exhibit E5. Effects of Heat Treatment (HT) on PCT $\log (\mathrm{ppm})$ Response of the Matrix 2A Study Glasses}

Oneway Analysis of $\log [\mathrm{Na}$ ppm] By Heat Treatment Glass ID=EM-14

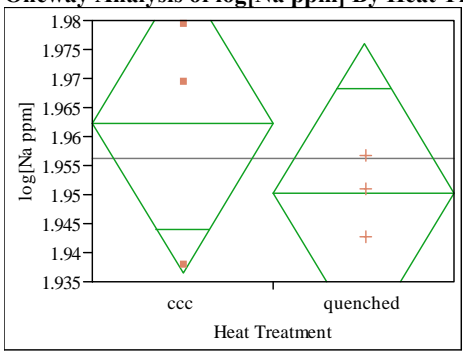

Oneway Anova

Summary of Fit

Rsquare $\quad 0.172293$

Adj Rsquare $\quad-0.03463$

Root Mean Square Error $\quad 0.016044$

Mean of Response 1.956151
6

t Test

quenched-ccc

Assuming equal variances

Difference $\quad-0.01195$ t Ratio $\quad-0.91248$

$\begin{array}{lrrr}\text { Std Err Dif } & 0.01310 \text { DF } & 4 \\ \text { Upper CL Dif } & 0.02442 \text { Prob }>|t| & 0.4131\end{array}$

$\begin{array}{lrrr}\text { Upper CL Dif } & 0.02442 & \text { Prob }>|t| & 0.4131 \\ \text { Lower CL Dif } & -0.04833 & \text { Prob }>t & 0.7934\end{array}$

$\begin{array}{rrr}\text { Lonfidence } & -0.95 \text { Prob }<t \quad 0.2066\end{array}$

Analysis of Variance

Source DF Sum of Squares Mean Square F Ratio Prob $>$ F $\begin{array}{llllll}\text { Heat Treatment } & 1 & 0.00021433 & 0.000214 & 0.8326 & 0.4131 \\ \text { Error } & 4 & 0.00102967 & 0.000257 & & \end{array}$

Error 0.00102967

Means for Oneway Anova

Level Number Mean Std Error Lower 95\% Upper 95\% $\begin{array}{llllll}\text { ccc } & 3 & 1.96213 & 0.00926 & 1.9364 & 1.9878 \\ \text { quenched } & 3 & 1.95017 & 0.00926 & 1.9245 & 1.9759\end{array}$

Std Error uses a pooled estimate of error variance
Oneway Analysis of $\log [\mathrm{Si}$ ppm] By Heat Treatment Glass ID=EM-14

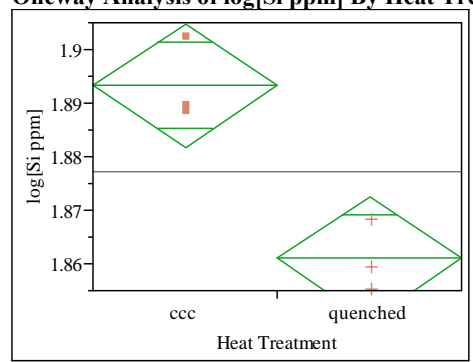

Oneway Anova

Summary of Fit

Rsquare

Adj Rsquare

Root Mean Square Error

0.854152

Mean of Response

0.007192

Observations (or Sum Wgts)

6

t Test

quenched-ccc

Assuming equal variances

Difference $\quad-0.03231$ t Ratio $\quad-5.50293$

Std Err Dif $\quad 0.00587$ DF $\quad 4$

Upper CL Dif -0.01601 Prob $>|t| 0.0053$

Lower CL Dif -0.04862 Prob $>t \quad 0.9973$

$\begin{array}{lll}\text { Confidence } & 0.95 \text { Prob }<\mathrm{t} \quad 0.0027\end{array}$

Analysis of Variance

Source DF Sum of Squares Mean Square F Ratio Prob $>$ F $\begin{array}{llllll}\text { Heat Treatment } & 1 & 0.00156637 & 0.001566 & 30.2822 & 0.0053 \\ \text { Error } & 4 & 0.00020690 & 0.000052 & & \end{array}$

Error 0.000052

\section{Means for Oneway Anova}

Level Number Mean Std Error Lower 95\% Upper 95\%

$\begin{array}{llllll}\text { ccC } & 3 & 1.89329 & 0.00415 & 1.8818 & 1.9048\end{array}$

$\begin{array}{llllll}\text { quenched } & 3 & 1.86098 & 0.00415 & 1.8494 & 1.8725\end{array}$

Std Error uses a pooled estimate of error variance

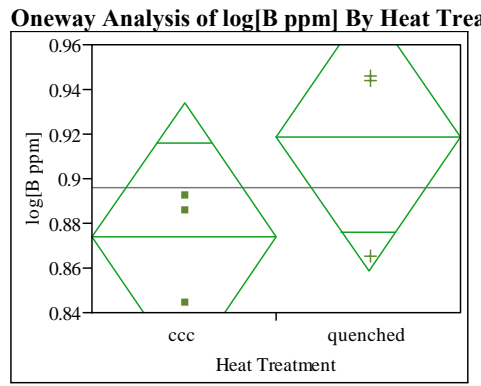

Oneway Anova

Summary of Fit

$\begin{array}{lr}\text { Rsquare } & 0.346855 \\ \text { Adj Rsquare } & 0.183569 \\ \text { Root Mean Square Error } & 0.03736 \\ \text { Mean of Response } & 0.896141 \\ \text { Observations (or Sum Wgts) } & 6\end{array}$

t Test

quenched-ccc

Assuming equal variances

Difference $\quad 0.04446$ t Ratio $\quad 1.45747$ Std Err Dif $\quad 0.03050$ D

Upper CL Dif 0.12915 Prob $>|t| \begin{array}{rr}4 & 0.2187\end{array}$ Lower CL Dif -0.04023 Prob $>t \quad 0.1094$ Confidence $\quad 0.95$ Prob $<\mathrm{t} \quad 0.8906$

Analysis of Variance

Source DF Sum of Squares Mean Square F Ratio Prob $>$ F $\begin{array}{llllll}\text { Heat Treatment } & 1 & 0.00296496 & 0.002965 & 2.1242 & 0.2187\end{array}$ $\begin{array}{llll}\text { Error } & 4 & 0.00558316 & 0.001396 \\ \text { C. Total } & 5 & 0.00854813 & \end{array}$

Means for Oneway Anova

Level Number Mean Std Error Lower 95\% Upper 95\%

$\begin{array}{lllllr}\text { ccc } & 3 & 0.873911 & 0.02157 & 0.81402 & 0.93380\end{array}$

$\begin{array}{llllll}\text { quenched } & 3 & 0.918370 & 0.02157 & 0.85848 & 0.97826\end{array}$

Std Error uses a pooled estimate of error variance 
SRNL-STI-2009-00778, Revision 0

Exhibit E5. Effects of Heat Treatment (HT) on PCT $\log (\mathrm{ppm})$ Response of the Matrix 2 A Study Glasses

Oneway Analysis of $\log [\mathrm{Li}$ ppm $]$ By Heat Treatment Glass ID=EM-15

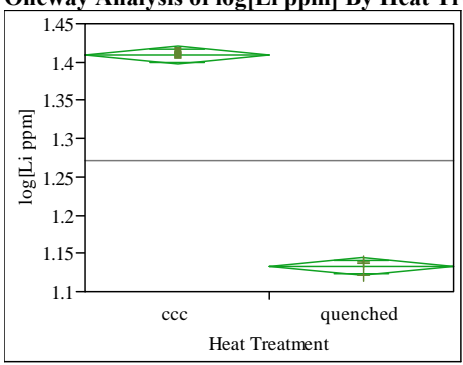

Oneway Anova

Summary of Fit

\begin{tabular}{|c|c|c|c|}
\hline \multicolumn{4}{|c|}{0.998089} \\
\hline Adj Rsquare & & 0.997 & 611 \\
\hline Root Mean Sq & uare Error & 0.007 & 392 \\
\hline Mean of Respo & onse & 1.270 & 1507 \\
\hline Observations ( & or Sum Wgt & & 6 \\
\hline $\begin{array}{l}\text { t Test } \\
\text { quenched-ccc }\end{array}$ & & & \\
\hline Assuming equa & al variances & & \\
\hline Difference & $-0.27584 \mathrm{t}$ & Ratio & -45.70 \\
\hline Std Err Dif & $0.00604 \mathrm{D}$ & DF & \\
\hline Upper CL Dif & $-0.25909 \mathrm{P}$ & $\operatorname{Prob}>|t|$ & $<.00$ \\
\hline Lower CL Dif & $-0.29260 \mathrm{P}$ & Prob $>t$ & 1.0000 \\
\hline Confidence & 0.95 & De & 00 \\
\hline
\end{tabular}

Analysis of Variance

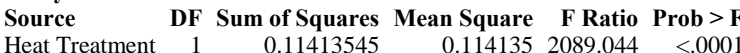

10.11413545

0.00021854

0.11435399

0.000055

Means for Oneway Anova

Level Number Mean Std Error Lower 95\% Upper 95\%

$\begin{array}{lllllr}\text { ccc } & 3 & 1.40843 & 0.00427 & 1.3966 & 1.4203\end{array}$

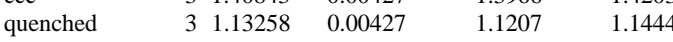

Std Error uses a pooled estimate of error variance

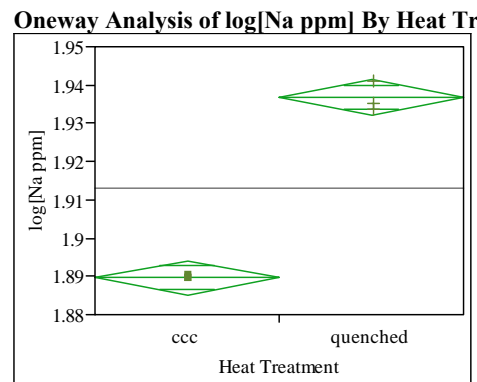

Oneway Anova

Summary of Fit

Adj Rsquare $\quad 0.98818$

Root Mean Square Error $\quad 0.002818$

Mean of Response 1.913178

t Test

quenched-ccc

Assuming equal variances

Difference $\quad 0.047112$ t Ratio $\quad 20.47504$

Std Err Dif 0.002301 DF $\quad 4$

$\begin{array}{lll}\text { Upper CL Dif } & 0.053501 \text { Prob }>|t| & <.0001 \\ \text { Lower CL Dif } & 0.040724 \text { Prob }>t & <.0001\end{array}$

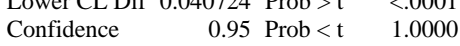

Analysis of Variance

Source DF Sum of Squares Mean Square F Ratio Prob $>$ F \begin{tabular}{llllll} 
Heat Treatment & 1 & 0.00332937 & 0.003329 & 419.2275 & $<.0001$ \\
Error & 4 & 0.00003177 & $7.942 \mathrm{e}-6$ & & \\
\hline C. & 5 & 0.00336114 & & &
\end{tabular}

C. Tot 0.00336114

Means for Oneway Anova

Level Number Mean Std Error Lower 95\% Upper 95\%

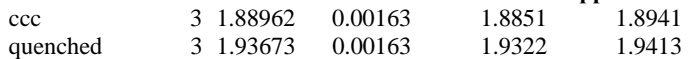

Std Error uses a pooled estimate of error variance

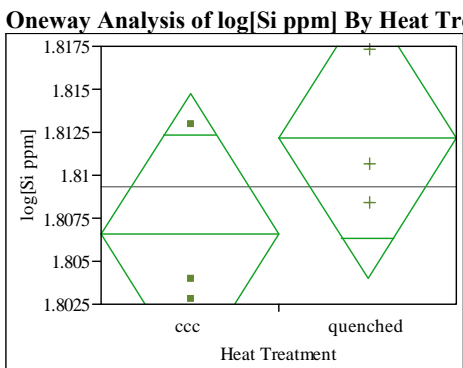

Oneway Anova

Summary of Fit

Rsquare

Adj Rsquare $\quad 0.311691$

Adj Rsquare $\quad 0.139614$

Reor of Square Eror -0.005115

Mean of Response 1.809352

t Test

quenched-ccc

Assuming equal variances

Difference $\quad 0.00562$ t Ratio 1.345861

Std Err Dif 0.00418 DF 4

Upper CL Dif 0.01722 Prob $>|t| 0.2496$

$\begin{array}{lrl}\text { Lower CL Dif }-0.00597 \text { Prob }>t & 0.1248 \\ \text { Confiden } & 0.95 \text { Prob }<\mathrm{t} & 0.8752\end{array}$

(n)

Source DF Sum of Squares Mean Square F Ratio Prob $>$ F $\begin{array}{lllrlr}\text { Heat Treatment } & 1 & 0.00004739 & 0.000047 & 1.8113 & 0.2496\end{array}$

$\begin{array}{llll}\text { Heat Treatment } & 1 & 0.00004739 & 0.000047 \\ \text { Error } & 4 & 0.00010465 & 0.000026\end{array}$

Means for Oneway Anova

Level Number Mean Std Error Lower 95\% Upper 95\%

$\begin{array}{llllll}\text { ccc } & 3 & 1.80654 & 0.00295 & 1.7983 & 1.814 \\ \text { quenched } & 3 & 1.81216 & 0.00295 & 1.8040 & 1.8204\end{array}$

Std Error uses a pooled estimate of error variance 
SRNL-STI-2009-00778, Revision 0

Exhibit E5. Effects of Heat Treatment (HT) on PCT $\log (\mathrm{ppm})$ Response of the Matrix 2A Study Glasses

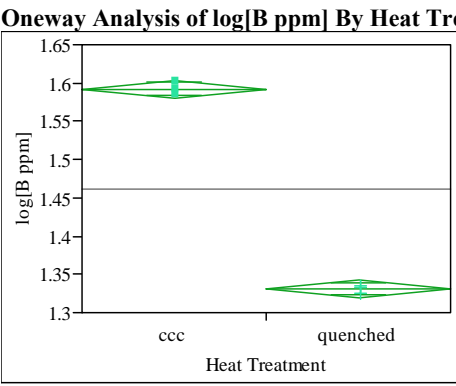

Oneway Anova

Summary of Fit

\begin{tabular}{|c|c|c|c|}
\hline \multicolumn{4}{|c|}{0.99779} \\
\hline \multirow{2}{*}{\multicolumn{2}{|c|}{$\begin{array}{l}\text { Adj Rsquare } \\
\text { Ront Mean Sauare Frror }\end{array}$}} & 0.997 & \\
\hline & & 0.007 & \\
\hline \multicolumn{2}{|c|}{ Mean of Response } & 1.461 & \\
\hline \multicolumn{4}{|c|}{ Observations (or Sum Wgts) } \\
\hline \multicolumn{4}{|l|}{$\begin{array}{l}\text { t Test } \\
\text { quenched-ccc }\end{array}$} \\
\hline \multicolumn{4}{|c|}{ Assuming equal variances } \\
\hline Difference & $-0.26094 \mathrm{t}$ & t Ratio & -42.4945 \\
\hline Std Err Dif & $0.00614 \mathrm{D}$ & DF & \\
\hline Upper CL Dif & $-0.24389 \mathrm{P}$ & Prob $>|t|$ & $<.00$ \\
\hline Lower CL Dif & $-0.27799 \mathrm{P}$ & Prob $>t$ & 1.0 \\
\hline Confidence & $0.95 \mathrm{P}$ & Prob $<$ t & $<.000$ \\
\hline
\end{tabular}

Analysis of Variance

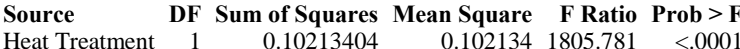
$\begin{array}{llllll}\text { Heat Treatment } & 1 & 0.10213404 & 0.102134 & 1805.781 & <.0001\end{array}$

0.00022624

0.00005

Means for Oneway Anova

Level Number Mean Std Error Lower 95\% Upper 95\%

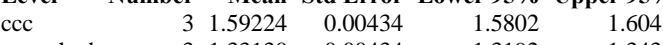

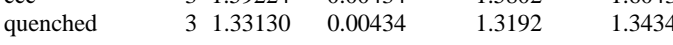

Std Error uses a pooled estimate of error variance

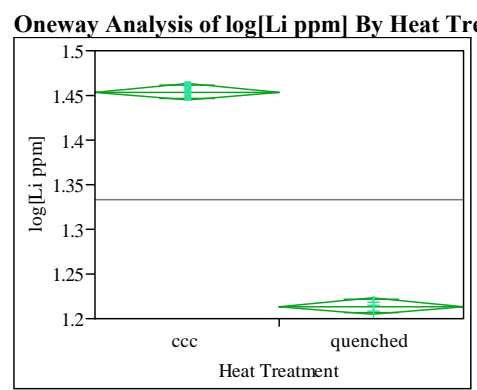

Oneway Anova

Summary of Fit

$\begin{array}{lr}\text { Rsquare } & 0.998292 \\ \text { Adj Rsquare } & 0.997866 \\ \text { Root Mean Square Error } & 0.006075 \\ \text { Mean of Response } & 1.334033 \\ \text { Observations (or Sum Wgts) } & 6\end{array}$

t Test

quenched-ccc

Assuming equal variances

Difference $\quad-0.23988$ t Ratio $\quad-48.359$

$\begin{array}{lrr}\text { Std Err Dif } & 0.00496 \text { DF } & 4 \\ \text { Upper CL Dif } & -0.22611 \text { Prob }>|t| & <0001\end{array}$

$\begin{array}{lll}\text { Upper CL Dif } & -0.22611 \text { Prob }>|t| & <.0001 \\ \text { Lower CL Dif } & -0.25366 \text { Prob }>t & 1.0000\end{array}$

$\begin{array}{lr}\text { Confidence } & 0.95 \text { Prob }<\mathrm{t} \quad<.0001\end{array}$

Analysis of Varianc

Source DF Sum of Squares Mean Square F Ratio Prob $>$ F $\begin{array}{llllll}\text { Heat Treatment } & 1 & 0.08631601 & 0.086316 & 2338.598 & <.0001 \\ \text { Error } & 4 & 0.00014764 & 0.000037 & & \end{array}$

C. Tot 0.08646365

Means for Oneway Anova

Level Number Mean Std Error Lower 95\% Upper 95\%

$\begin{array}{llllll}\text { ccC } & 3 & 1.45397 & 0.00351 & 1.4442 & 1.4637\end{array}$

$\begin{array}{llllll}\text { quenched } & 3 & 1.21409 & 0.00351 & 1.2044 & 1.2238\end{array}$

Std Error uses a pooled estimate of error variance

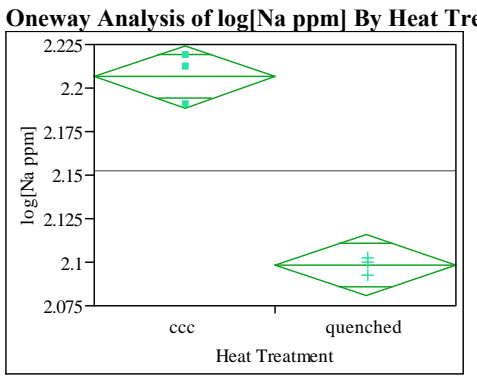

Oneway Anova

Summary of Fit

Rsquare

Adj Rsqutse 0.972723

Root Mean Square Error $\quad 0.965903$

Root Mean Square Error $\quad 0.011068$

$\begin{array}{lr}\text { Mean of Response } & 2.152404 \\ \text { Observations (or Sum Wgts) } & 6\end{array}$

t Test

quenched-ccc

Assuming equal variances

Difference $\quad-0.10793$ t Ratio $\quad-11.9433$ Std Err Dif $\quad 0.00904$ D

Upper CL Dif -0.08284 Prob $>|t| \quad 0.0003$

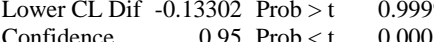

Analysis of Variance

Source DF Sum of Squares Mean Square F Ratio Prob $>$ F \begin{tabular}{lllllll} 
Heat Treatment & 1 & 0.01747440 & 0.017474 & 142.6425 & 0.0003 \\
\hline Crror & 4 & 0.0004902 & 0.000123 & &
\end{tabular} $\begin{array}{lll}\text { Error } & 4 & 0.00049002 \\ \text { C. } & 5 & 0.01796441\end{array}$

Means for Oneway Anova

Level Number Mean Std Error Lower 95\% Upper 95\%

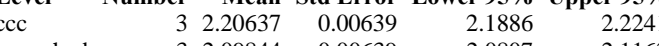

Std Error uses a pooled estimate of error variance 
SRNL-STI-2009-00778, Revision 0

Exhibit E5. Effects of Heat Treatment (HT) on PCT $\log (\mathrm{ppm})$ Response of the Matrix 2 A Study Glasses

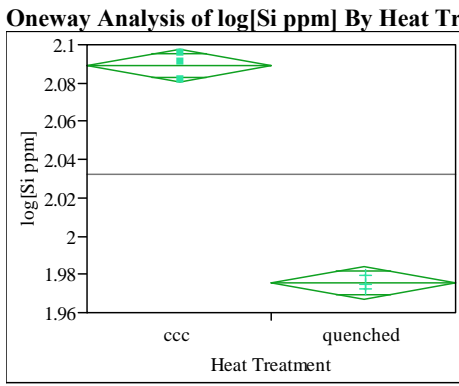

Oneway Anova

Summary of Fit

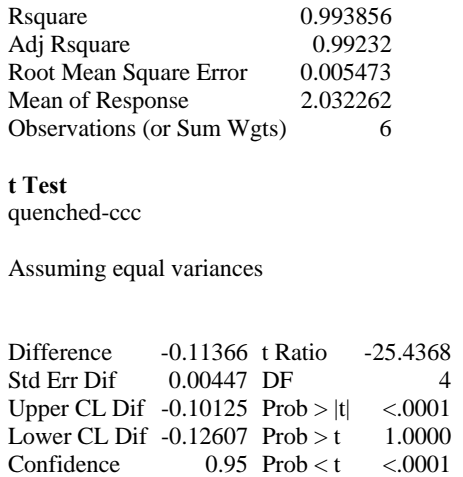

Analysis of Variance

Source DF Sum of Squares Mean Square F Ratio Prob >

$\begin{array}{llllll}\text { Heat Treatment } & 1 & 0.01937821 & 0.019378 & 647.0330 & <.0001 \\ \text { Error } & 4 & 0.00011980 & 0.000030 & & \end{array}$

0.01949800

0.000030

Means for Oneway Anova

Level Number Mean Std Error Lower 95\% Upper 95\%

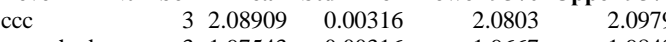

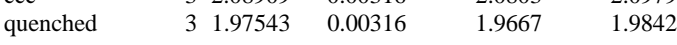

Std Error uses a pooled estimate of error variance

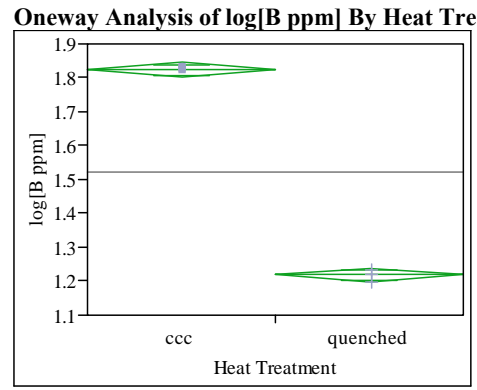

Oneway Anova

Summary of Fit

$\begin{array}{lr}\text { Rsquare } & 0.998635 \\ \text { Adj Rsquare } & 0.998293 \\ \text { Root Mean Square Error } & 0.013721 \\ \text { Mean of Response } & 1.520926 \\ \text { Observations (or Sum Wgts) } & 6\end{array}$

t Test

quenched-ccc

Assuming equal variances

Difference $\quad-0.60596$ t Ratio $\quad-54.0882$

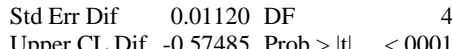

$\begin{array}{lrl}\text { Upper CL Dif } & -0.57485 \text { Prob }>|t| & <.000 \\ \text { Lower CL Dif }-0.63706 & \text { Prob }>t & 1.0000\end{array}$

$\begin{array}{lll}\text { Confidence } & 0.95 \mathrm{Prob}<\mathrm{t} \quad<.0001\end{array}$

Analysis of Variance

Source DF Sum of Squares Mean Square F Ratio Prob $>$ F $\begin{array}{lllllll}\text { Heat Treatment } & 1 & 0.55077870 & 0.550779 & 2925.536 & <.0001\end{array}$ Error \begin{tabular}{ll}
1 & 0.55077870 \\
\hline & 0.00075306
\end{tabular}

Means for Oneway Anova

Level Number Mean Std Error Lower 95\% Upper 95\%

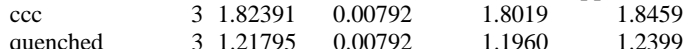

Std Error uses a pooled estimate of error variance

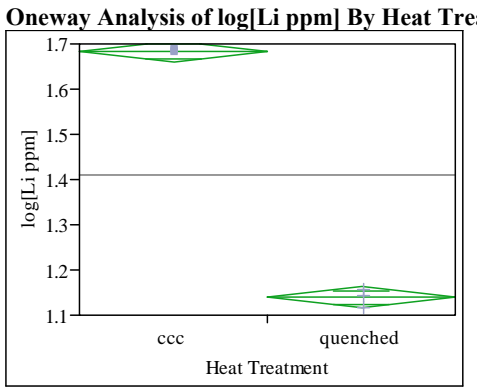

Oneway Anova

Summary of Fit

$\begin{array}{lr}\text { Rsquare } & 0.998122 \\ \text { Adj Rsquare } & 0.997653 \\ \text { Root Mean Square Error } & 0.014469 \\ \text { Mean of Response } & 1.410887 \\ \text { Observations (or Sum Wgts) } & 6\end{array}$

t Test

quenched-ccc

Assuming equal variance

Difference $\quad-0.54472$ t Ratio $\quad-46.1083$ Std Err Dif 0.01181 DF $\begin{array}{lll}\text { Upper CL Dif } & -0.51192 \text { Prob }>|t| & <.0001 \\ \text { Lower CL Dif }-0.57752 & \text { Prob }>t & 1.0000\end{array}$ $\begin{array}{lr}\text { Confidence } & 0.95 \mathrm{Prob}<\mathrm{t} \quad<.0001\end{array}$

Analysis of Variance

Source DF Sum of Squares Mean Square F Ratio Prob $>$ F $\begin{array}{lllllll}\text { Heat Treatment } & 1 & 0.44507409 & 0.445074 & 2125.978 & <.0001\end{array}$ $\begin{array}{lll}\text { Error } & 4 & 0.00083740 \\ \text { C. Total } & 5 & 0.44591149\end{array}$

Means for Oneway Anova

Level Number Mean Std Error Lower 95\% Upper 95\% $\begin{array}{llllll}\text { ccc } & 3 & 1.68324 & 0.00835 & 1.6601 & 1.7064 \\ & 3 & 1.13853 & 0.00835 & 1.1153 & 1.1617\end{array}$

Std Error uses a pooled estimate of error variance 
SRNL-STI-2009-00778, Revision 0

Exhibit E5. Effects of Heat Treatment (HT) on PCT $\log (\mathrm{ppm})$ Response of the Matrix 2 A Study Glasses

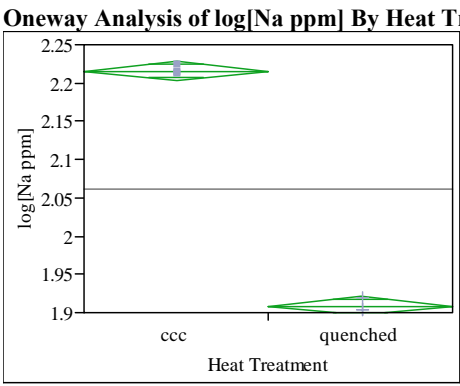

Oneway Anova

Summary of Fit

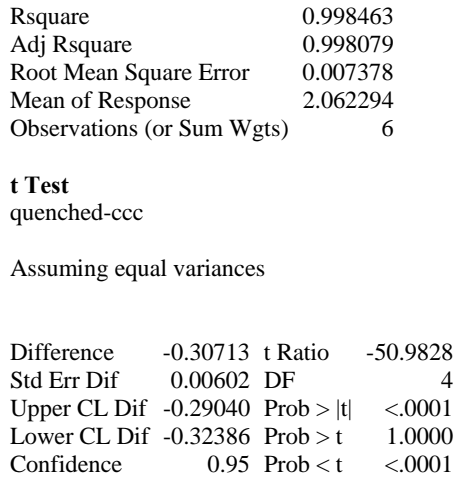

Analysis of Variance

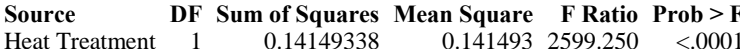

0.14149338

0.14171112

Means for Oneway Anova

Level Number Mean Std Error Lower 95\% Upper 95\%

$\begin{array}{llllll}\text { ccC } & 3 & 2.21586 & 0.00426 & 2.2040 & 2.227\end{array}$

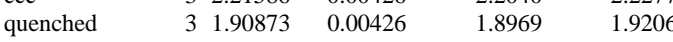

Std Error uses a pooled estimate of error variance

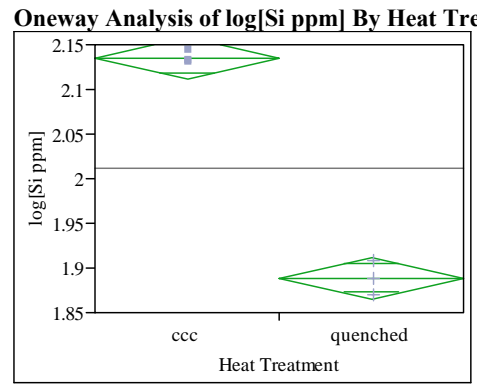

Oneway Anova

Summary of Fit

$\begin{array}{lr}\text { Rsquare } & 0.990829 \\ \text { Adj Rsquare } & 0.988536 \\ \text { Root Mean Square Error } & 0.014497 \\ \text { Mean of Response } & 2.012064 \\ \text { Observations (or Sum Wgts) } & 6\end{array}$

Observations (or Sum Wgts)

t Test

quenched-ccc

Assuming equal variances

Difference $\quad-0.24607$ t Ratio $\quad-20.788$

$\begin{array}{lrl}\text { Std Err Dif } & 0.01184 \text { DF } & -20.7881 \\ \text { Upper CL Dif }-0.21321 \text { Prob }>|t| & <.0001\end{array}$

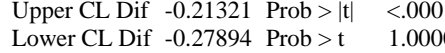

$\begin{array}{lll}\text { Confidence } & 0.95 \text { Prob }<\mathrm{t} \quad<.0001\end{array}$

Analysis of Variance

Source DF Sum of Squares Mean Square F Ratio Prob $>$ F $\begin{array}{llllll}\text { Heat Treatment } & 1 & 0.09082647 & 0.090826 & 432.1464 & <.0001 \\ \text { Error } & 4 & 0.00084070 & 0.000210 & \end{array}$

Error 0.09166717 0.000210

\section{Means for Oneway Anova}

Level Number Mean Std Error Lower 95\% Upper 95\%

$\begin{array}{llllll}\text { ccC } & 3 & 2.13510 & 0.00837 & 2.1119 & 2.1583\end{array}$

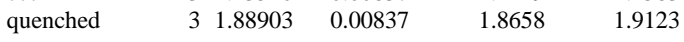

Std Error uses a pooled estimate of error variance

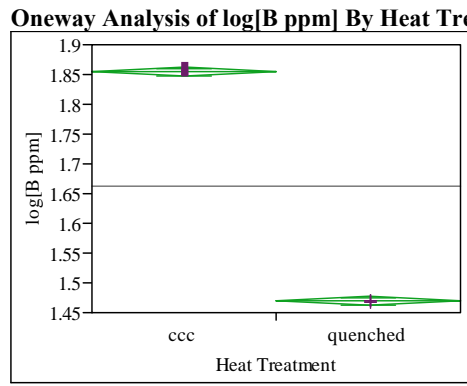

Oneway Anova

Summary of Fit

$\begin{array}{lr}\text { Rsquare } & 0.999603 \\ \text { Adj Rsquare } & 0.999504 \\ \text { Root Mean Square Error } & 0.004697 \\ \text { Mean of Response } & 1.661478 \\ \text { Observations (or Sum Wgts) } & 6\end{array}$

quenched-ccc

Assuming equal variances

Difference $\quad-0.38493$ t Ratio -100.372

Std Err Dif 0.00384 DF

Upper CL Dif -0.37429 Prob $>|t|<.0001$

Lower CL Dif -0.39558 Prob $>t \quad 1.0000$

$\begin{array}{lll}\text { Confidence } & 0.95 \text { Prob }<\mathrm{t} \quad<.0001\end{array}$

Analysis of Variance

Source DF Sum of Squares Mean Square F Ratio Prob $>$ F $\begin{array}{llllll}\text { Heat Treatment } & 1 & 0.22226156 & 0.222262 & 10074.58 & <.0001\end{array}$ $\begin{array}{llll}\text { Error } & 4 & 0.00008825 & 0.000022 \\ \text { C. Total } & 5 & 0.22234981 & \end{array}$

Means for Oneway Anova

Level Number Mean Std Error Lower 95\% Upper 95\%

$\begin{array}{llllll}\text { ccC } & 3 & 1.85394 & 0.00271 & 1.8464 & 1.8615 \\ \text { quenched } & 3 & 1.46901 & 0.00271 & 1.4615 & 1.4765\end{array}$

Std Error uses a pooled estimate of error variance 
SRNL-STI-2009-00778, Revision 0

Exhibit E5. Effects of Heat Treatment (HT) on PCT $\log (\mathrm{ppm})$ Response of the Matrix 2 A Study Glasses

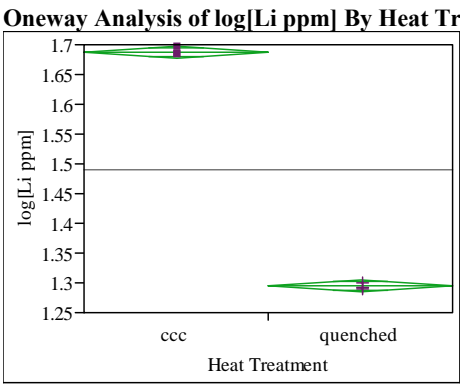

Oneway Anova

Summary of Fit

\begin{tabular}{|c|c|c|c|}
\hline \multicolumn{4}{|c|}{0.999217} \\
\hline \multirow{2}{*}{\multicolumn{4}{|c|}{$\begin{array}{l}\text { Adj Rsquare } \\
\text { Root Mean Square Error }\end{array}$}} \\
\hline & & & \\
\hline \multicolumn{4}{|c|}{ Mean of Response } \\
\hline \multicolumn{4}{|c|}{ Observations (or Sum Wgts) $\quad 6$} \\
\hline \multicolumn{4}{|l|}{$\begin{array}{l}\text { t Test } \\
\text { quenched-ccc }\end{array}$} \\
\hline \multicolumn{4}{|c|}{ Assuming equal variances } \\
\hline Difference & $-0.39225 \mathrm{t}$ & t Ratio & -71.4437 \\
\hline Std Err Dif & $0.00549 \mathrm{I}$ & DF & \\
\hline Upper CL Dif & $-0.37701 \mathrm{~F}$ & $\operatorname{Prob}>|t|$ & \\
\hline Lower CL Dif & $-0.40750 \mathrm{~F}$ & Prob $>t$ & 1.0 \\
\hline Confidence & $0.95 \mathrm{~F}$ & Prob $<\mathrm{t}$ & $<.000$ \\
\hline
\end{tabular}

Analysis of Varia

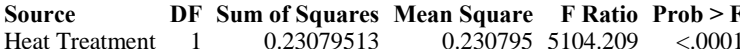
$\begin{array}{llllll}\text { Heat Treatment } & 1 & 0.23079513 & 0.230795 & 5104.209 & <.0001\end{array}$

$$
0.00018087
$$

0.23097599

0.000045

Means for Oneway Anova

Level Number Mean Std Error Lower 95\% Upper 95\%

$\begin{array}{llllll}\text { ccC } & 3 & 1.68719 & 0.00388 & 1.6764 & 1.698\end{array}$

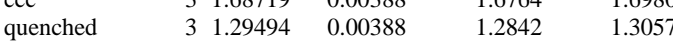

Std Error uses a pooled estimate of error variance
Oneway Analysis of $\log [\mathrm{Na}$ ppm] By Heat Treatment Glass ID=EM-18

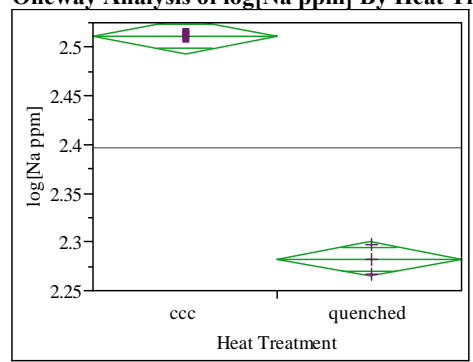

Oneway Anova

Summary of Fit

Adj Rsquare

0.992402

Mean of Response

Observations (or Sum Wgts)

6

t Test

quenched-ccc

Assuming equal variances

Difference $\quad-0.22876$ t Ratio $\quad-25.574$

Std Err Dif 0.00894 DF $>11<001$

Upper CL Dif -0.20392 Prob $>|t|<.0001$

Lower CL Dif -0.25360 Prob $>t \quad 1.0000$

Confidence $\quad 0.95$ Prob $<\mathrm{t} \quad<.0001$

nalysis of Variance

Source DF Sum of Squares Mean Square F Ratio Prob $>$ F $\begin{array}{llllll}\text { Heat Treatment } & 1 & 0.07849675 & 0.078497 & 654.0368 & <.0001 \\ \text { Error } & 4 & 0.00048008 & 0.000120 & \end{array}$ 0.07897683

0.000120

\section{Means for Oneway Anova}

Level Number Mean Std Error Lower 95\% Upper 95\%

$\begin{array}{lll}\text { CCC } & 32.51114 & 0.00633\end{array}$

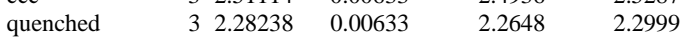

Std Error uses a pooled estimate of error variance

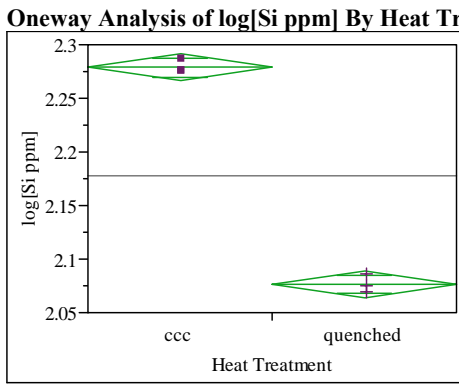

Oneway Anova

Summary of Fit

$\begin{array}{lr}\text { Rsquare } & 0.996175 \\ \text { Adj Rsquare } & 0.995218 \\ \text { Root Mean Square Error } & 0.007681 \\ \text { Mean of Response } & 2.177519 \\ \text { Observations (or Sum Wgts) } & 6\end{array}$

quenched-ccc

Assuming equal variances

Difference $\quad-0.20242$ t Ratio $\quad-32.2748$

Std Err Dif 0.00627 DF $>$ (

Upper CL Dif -0.18501 Prob $>|t|<.0001$

Lower CL Dif -0.21983 Prob $>t \quad 1.0000$

$\begin{array}{lrl}\text { Confidence } & 0.95 \text { Prob }<\mathrm{t} \quad<.0001\end{array}$

Analysis of Variance

Source DF Sum of Squares Mean Square $\quad$ F Ratio Prob $>$ F $\begin{array}{lllllll}\text { Heat Treatment } & 1 & 0.06146123 & 0.061461 & 1041.664 & <.0001\end{array}$ $\begin{array}{llll}\text { Error } & 4 & 0.00023601 & 0.000059 \\ \text { C. Total } & 5 & 0.06169724 & \end{array}$

Means for Oneway Anova

Level Number Mean Std Error Lower 95\% Upper 95\% $\begin{array}{llllll}\text { cCC } & 32.27873 & 0.00443 & 2.2664 & 2.2910\end{array}$

$\begin{array}{llllll}\text { quenched } & 3 & 2.07631 & 0.00443 & 2.0640 & 2.0886\end{array}$

Std Error uses a pooled estimate of error variance 
SRNL-STI-2009-00778, Revision 0

Exhibit E5. Effects of Heat Treatment (HT) on PCT $\log (\mathrm{ppm})$ Response of the Matrix 2 A Study Glasses

Oneway Analysis of $\log [B$ ppm] By Heat Treatment Glass ID=EM-19

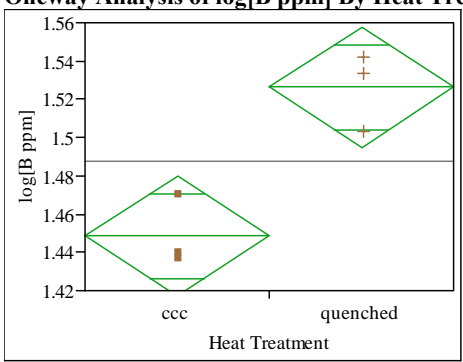

Oneway Anova

Summary of Fit

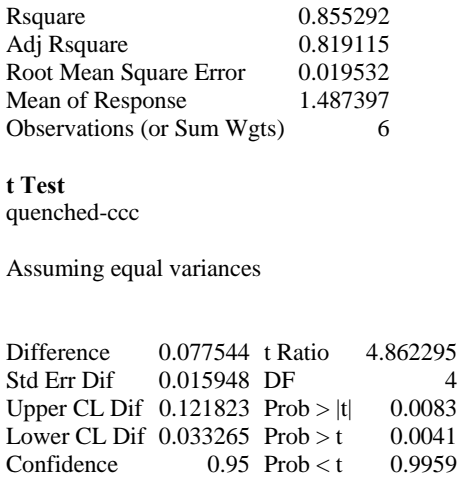

Analysis of Varia

Source DF Sum of Squares Mean Square F Ratio Prob $>$ F

$\begin{array}{llllll}\text { Heat Treatment } & 1 & 0.00901964 & 0.009020 & 23.6419 & 0.0083 \\ \text { Error } & 4 & 0.00152604 & 0.000382 & \end{array}$

0.000382

Means for Oneway Anova

Level Number Mean Std Error Lower 95\% Upper 95\%

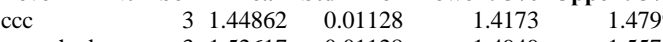

$\begin{array}{llllll}\text { quenched } & 3 & 1.52617 & 0.01128 & 1.4949 & 1.5575\end{array}$

Std Error uses a pooled estimate of error variance
Oneway Analysis of $\log [\mathrm{Li}$ ppm $]$ By Heat Treatment Glass ID=EM-19

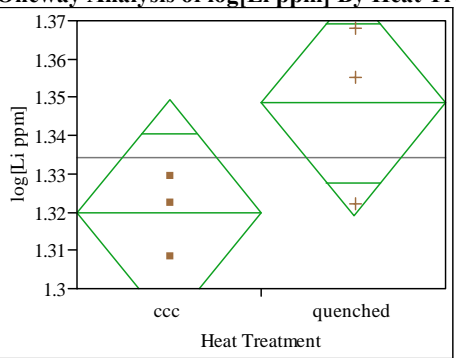

Oneway Anova

Summary of Fit

Adj Rsquare $\quad 0.34884$

Root Mean Square Error $\quad 0.018326$

Observations (or Sum Wgts)

t Test

quenched-ccc

Assuming equal variances

Difference $\quad 0.02870$ t Ratio 1.917969

Std Err Dif 0.01496 DF

Upper CL Dif 0.07024 Prob $>|t| \quad 0.1276$

Lower CL Dif -0.01285 Prob $>$ t 0.0638

Confidence $\quad 0.95$ Prob $<\mathrm{t} \quad 0.9362$

Analysis of Varianc

Source DF Sum of Squares Mean Square F Ratio Prob $>$ F $\begin{array}{llllll}\text { Heat Treatment } & 1 & 0.00123545 & 0.001235 & 3.6786 & 0.1276\end{array}$ $4 \quad 0.00134339$

Means for Oneway Anova

Level Number Mean Std Error Lower 95\% Upper 95\%

$\begin{array}{llll}\text { ccc } & 3 & 1.31984 & 0.01058\end{array}$

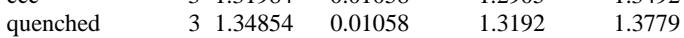

Std Error uses a pooled estimate of error variance

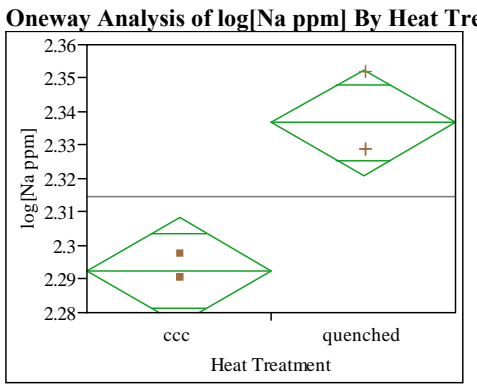

Oneway Anova

Summary of Fit

$\begin{array}{lr}\text { Rsquare } & 0.882225 \\ \text { Adj Rsquare } & 0.852781 \\ \text { Root Mean Square Error } & 0.009907 \\ \text { Mean of Response } & 2.314636 \\ \text { Observations (or Sum Wgts) } & 6\end{array}$

quenched-ccc

Assuming equal variances

Difference $\quad 0.044278$ t Ratio 5.47385 Std Err Dif 0.008089 D

Upper CL Dif 0.066737 Prob $>|t| \quad 0.0054$ Lower CL Dif 0.021820 Prob $>t \quad 0.0027$ Confidence $\quad 0.95$ Prob $<\mathrm{t} \quad 0.9973$

Analysis of Variance

Source DF Sum of Squares Mean Square F Ratio Prob $>$ F $\begin{array}{lllllll}\text { Heat Treatment } & 1 & 0.00294086 & 0.002941 & 29.9631 & 0.0054\end{array}$ Error $\quad 4 \quad 0.00039260$ 0.000098

Means for Oneway Anova

Level Number Mean Std Error Lower 95\% Upper 95\% $\begin{array}{llllll}\text { cCC } & 3 & 2.29250 & 0.00572 & 2.2766 & 2.3084\end{array}$ $\begin{array}{llllll}\text { quenched } & 3 & 2.33678 & 0.00572 & 2.3209 & 2.3527\end{array}$

Std Error uses a pooled estimate of error variance 
SRNL-STI-2009-00778, Revision 0

\section{Exhibit E5. Effects of Heat Treatment (HT) on PCT $\log (\mathrm{ppm})$ Response of the Matrix 2A Study Glasses}

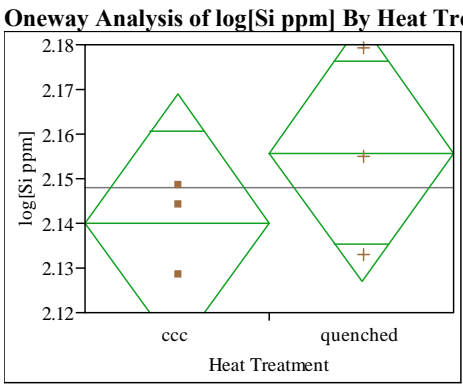

Oneway Anova

Summary of Fit

\begin{tabular}{|c|c|c|c|}
\hline \multicolumn{4}{|c|}{0.219271} \\
\hline Adj Rsquare & & 0.024 & 088 \\
\hline Root Mean Squ & uare Error & 0.01 & 805 \\
\hline Mean of Respo & onse & 2.147 & 962 \\
\hline Observations (o & or Sum Wgt & & 6 \\
\hline $\begin{array}{l}\text { t Test } \\
\text { quenched-ccc }\end{array}$ & & & \\
\hline Assuming equa & al variances & & \\
\hline Difference & $0.01562 \mathrm{t}$ & Ratio & 1.0595 \\
\hline Std Err Dif & $0.01474 \mathrm{I}$ & DF & \\
\hline Upper CL Dif & $0.05654 \mathrm{~F}$ & $\operatorname{Prob}>|t|$ & 0.34 \\
\hline Lower CL Dif & $-0.02530 \mathrm{~F}$ & Prob $>t$ & 0.17 \\
\hline Confidence & 0.95 & Prob $<t$ & \\
\hline
\end{tabular}

Analysis of Variance

Source DF Sum of Squares Mean Square F Ratio Prob $>$ F $\begin{array}{llllll}\text { Heat Treatment } & 1 & 0.00036601 & 0.000366 & 1.1234 & 0.3489\end{array}$

0.00130320

0.000326

Means for Oneway Anova

Level Number Mean Std Error Lower 95\% Upper 95\%

$\begin{array}{lllllr}\text { ccC } & 3 & 2.14015 & 0.01042 & 2.1112 & 2.1691 \\ \text { quenched } & 3 & 2.15577 & 0.01042 & 2.1268 & 2.1847\end{array}$

Std Error uses a pooled estimate of error variance
Oneway Analysis of $\log [\mathrm{B}$ ppm $]$ By Heat Treatment Glass ID=EM-20

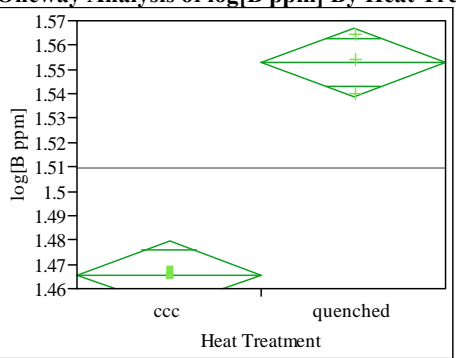

Oneway Anova

Summary of Fit

$\begin{array}{lr}\text { Rsquare } & 0.973989 \\ \text { Adj Rsquare } & 0.967486 \\ \text { Root Mean Square Error } & 0.008718 \\ \text { Mean of Response } & 1.509276 \\ \text { Observations (or Sum Wgts) } & 6\end{array}$

quenched-ccc

Assuming equal variances

Difference $\quad 0.087112$ t Ratio 12.23847

$\begin{array}{lrr}\text { Std Err Dif } & 0.007118 \text { DF } & 4 \\ \text { Uper } & & 0.0003\end{array}$

$\begin{array}{lrr}\text { Upper CL Dif } & 0.106874 \text { Prob }>|t| & 0.0003 \\ \text { Lower CL Dif } & 0.067350 \text { Prob }>t & 0.0001\end{array}$

$\begin{array}{lll}\text { Confidence } & 0.95 \mathrm{Prob}<\mathrm{t} & 0.9999\end{array}$

Analysis of Variance

Source DF Sum of Squares Mean Square F Ratio Prob $>$ F $\begin{array}{llllll}\text { Heat Treatment } & 1 & 0.01138273 & 0.011383 & 149.7802 & 0.0003 \\ \text { Error } & 4 & 0.00030398 & 0.000076 & & \end{array}$

Error 0.01168671

0.000076

Means for Oneway Anova

Level Number Mean Std Error Lower 95\% Upper 95\%

$\begin{array}{llllll}\text { ccC } & 3 & 1.46572 & 0.00503 & 1.4517 & 1.4797\end{array}$

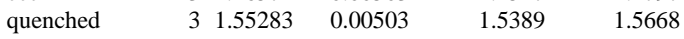

Std Error uses a pooled estimate of error variance

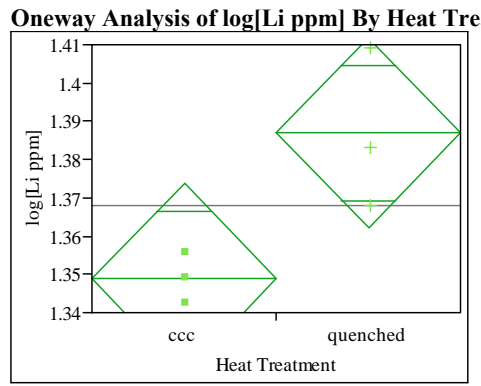

Oneway Anova

Summary of Fit

$\begin{array}{lr}\text { Rsquare } & 0.69202 \\ \text { Adj Rsquare } & 0.615025 \\ \text { Root Mean Square Error } & 0.015497 \\ \text { Mean of Response } & 1.367897 \\ \text { Observations (or Sum Wgts) } & 6\end{array}$

quenched-ccc

Assuming equal variances

Difference $\quad 0.037933$ t Ratio 2.997977 Std Err Dif 0.012653 DF $>$ Th Upper CL Dif 0.073063 Prob $>|t| \quad 0.0400$ Lower CL Dif 0.002803 Prob $>t \quad 0.0200$ Confidence $\quad 0.95$ Prob $<\mathrm{t} \quad 0.9800$

Analysis of Variance

Source DF Sum of Squares Mean Square F Ratio Prob $>$ F $\begin{array}{llllll}\text { Heat Treatment } & 1 & 0.00215837 & 0.002158 & 8.9879 & 0.0400\end{array}$ $\begin{array}{lll}\text { Error } & 4 & 0.00096057 \\ \text { C. Total } & 5 & 0.00311895\end{array}$

Means for Oneway Anova

Level Number Mean Std Error Lower 95\% Upper 95\%

$\begin{array}{llllll}\text { cCC } & 3 & 1.34893 & 0.00895 & 1.3241 & 1.3738\end{array}$

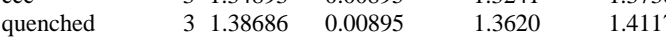

Std Error uses a pooled estimate of error variance 
SRNL-STI-2009-00778, Revision 0

Exhibit E5. Effects of Heat Treatment (HT) on PCT $\log (\mathrm{ppm})$ Response of the Matrix 2 A Study Glasses

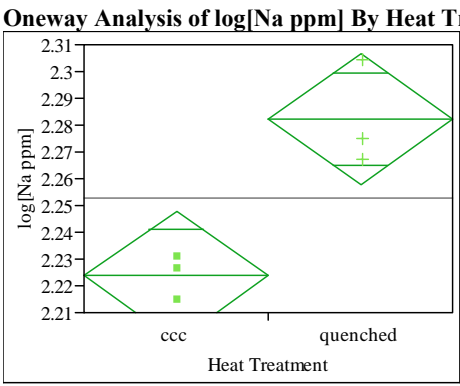

Oneway Anova

Summary of Fit

\begin{tabular}{|c|c|c|c|}
\hline \\
\hline \multicolumn{2}{|l|}{$\begin{array}{l}\text { Rsquare } \\
\text { Adj Rsquare }\end{array}$} & \multicolumn{2}{|c|}{$\begin{array}{r}0.848312 \\
0.81039\end{array}$} \\
\hline \multirow{2}{*}{\multicolumn{3}{|c|}{ Mean of Response }} & \\
\hline & & 2.2529 & \\
\hline \multicolumn{4}{|c|}{ Observations (or Sum Wgts) $\quad 6$} \\
\hline \multicolumn{4}{|l|}{$\begin{array}{l}\text { t Test } \\
\text { quenched-ccc }\end{array}$} \\
\hline \multicolumn{4}{|c|}{ Assuming equal variances } \\
\hline Difference & $0.058571 \mathrm{t}$ & t Ratio & \\
\hline Std Err Dif & $0.012384 \mathrm{I}$ & DF & \\
\hline Upper CL Dif & $0.092953 \mathrm{I}$ & $\operatorname{Prob}>|t|$ & \\
\hline Lower CL Dif & $0.024188 \mathrm{~F}$ & Prob $>t$ & \\
\hline Confidence & $0.95 \mathrm{I}$ & Prob $<\mathrm{t}$ & 0.99 \\
\hline
\end{tabular}

Analysis of Variance

Source DF Sum of Squares Mean Square F Ratio Prob $>$ F $\begin{array}{llllll}\text { Heat Treatment } & 1 & 0.00514577 & 0.005146 & 22.3699 & 0.0091\end{array}$

0.00092012

0.00606589

0.000230

Means for Oneway Anova

Level Number Mean Std Error Lower 95\% Upper 95\%

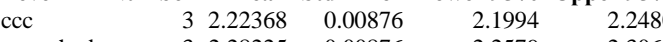

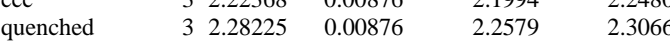

Std Error uses a pooled estimate of error variance

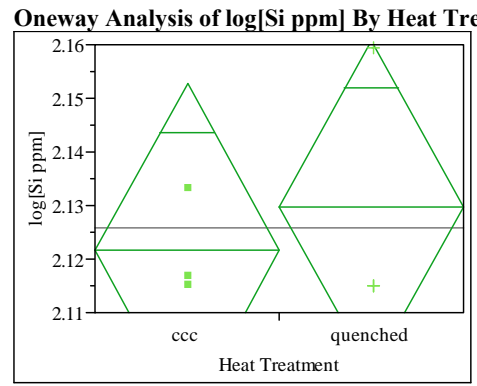

Oneway Anova

Summary of Fit

$\begin{array}{lr}\text { Rsquare } & 0.063245 \\ \text { Adj Rsquare } & -0.17094 \\ \text { Root Mean Square Error } & 0.019402 \\ \text { Mean of Response } & 2.125718 \\ \text { Observations (or Sum Wgts) } & 6\end{array}$

quenched-ccc

Assuming equal variances

$\begin{array}{lrlr}\text { Difference } & 0.00823 \text { t Ratio } & 0.519671 \\ \text { Std Err Dif } & 0.01584 \text { DF } & 4 \\ \text { Upper CL Dif } & 0.05222 \text { Prob }>|t| & 0.6307 \\ \text { Lower CL Dif } & -0.03575 \text { Prob }>\text { t } & 0.3154 \\ \text { Confidence } & 0.95 \text { Prob }<\mathrm{t} & 0.6846\end{array}$

Analysis of Variance

Source DF Sum of Squares Mean Square F Ratio Prob > $\begin{array}{llllll}\text { Heat Treatment } & 1 & 0.00010166 & 0.000102 & 0.2701 & 0.6307 \\ \text { Error } & 4 & 0.00150575 & 0.000376 & & \end{array}$ 0.000376

\section{Means for Oneway Anova}

Level Number Mean Std Error Lower 95\% Upper 95\%

$\begin{array}{llllll}\text { ccc } & 3 & 2.12160 & 0.01120 & 2.0905 & 2.1527\end{array}$

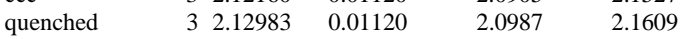

Std Error uses a pooled estimate of error variance

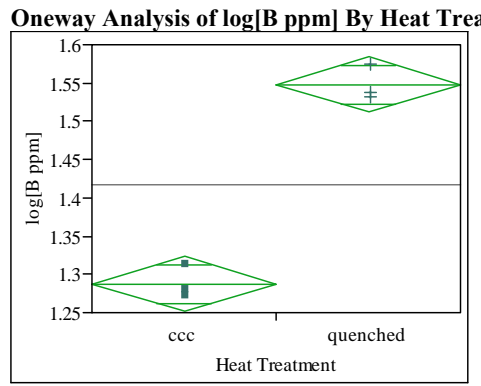

Oneway Anova

Summary of Fit

$\begin{array}{lr}\text { Rsquare } & 0.98085 \\ \text { Adj Rsquare } & 0.976063 \\ \text { Root Mean Square Error } & 0.022297 \\ \text { Mean of Response } & 1.417493 \\ \text { Observations (or Sum Wgts) } & 6\end{array}$

quenched-ccc

Assuming equal variances

Difference $\quad 0.260585$ t Ratio 14.31372

Std Err Dif 0.018205 DF

Upper CL Dif 0.311131 Prob $>|t| \quad 0.0001$

Lower CL Dif 0.210039 Prob $>t \quad<.0001$

Confidence $\quad 0.95 \mathrm{Prob}<\mathrm{t} \quad 0.9999$

Analysis of Variance

Source DF Sum of Squares Mean Square F Ratio Prob $>$ F $\begin{array}{llllll}\text { Heat Treatment } & 1 & 0.10185683 & 0.101857 & 204.8827 & 0.0001\end{array}$ $\begin{array}{llll}\text { Error } & 4 & 0.00198859 & 0.000497 \\ \text { C. Total } & 5 & 0.10384541\end{array}$

Means for Oneway Anova

Level Number Mean Std Error Lower 95\% Upper 95\% $\begin{array}{llllll}\text { cCC } & 3 & 1.28720 & 0.01287 & 1.2515 & 1.322\end{array}$

$\begin{array}{llllll}\text { quenched } & 3 & 1.54779 & 0.01287 & 1.5120 & 1.5835\end{array}$

Std Error uses a pooled estimate of error variance 
SRNL-STI-2009-00778, Revision 0

Exhibit E5. Effects of Heat Treatment (HT) on PCT $\log (\mathrm{ppm})$ Response of the Matrix 2A Study Glasses

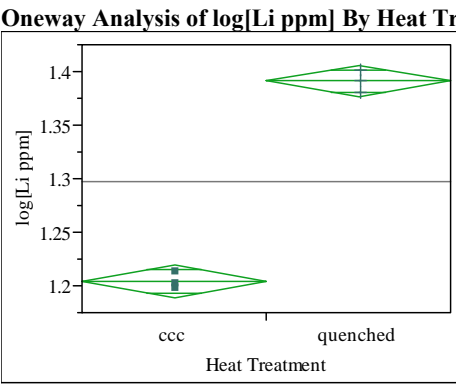

Oneway Anova

Summary of Fit

\begin{tabular}{|c|c|c|c|}
\hline \multicolumn{4}{|c|}{0.99344} \\
\hline \multicolumn{4}{|l|}{ Adj Rsquare } \\
\hline \multicolumn{2}{|c|}{ Root Mean Sauare Frror } & 0.009 & 304 \\
\hline \multirow{2}{*}{\multicolumn{2}{|c|}{$\begin{array}{l}\text { Mean of Response } \\
\text { Observations (or Sum Wgts }\end{array}$}} & 1.297 & 568 \\
\hline & & & 6 \\
\hline \multicolumn{4}{|l|}{$\begin{array}{l}\text { t Test } \\
\text { quenched-ccc }\end{array}$} \\
\hline \multicolumn{4}{|c|}{ Assuming equal variances } \\
\hline Difference & $0.186980 \mathrm{t}$ & t Ratio & 24.61 \\
\hline Std Err Dif & $0.007597 \mathrm{I}$ & DF & \\
\hline Upper CL Dif & $0.208072 \mathrm{I}$ & Prob $>|t|$ & \\
\hline Lower CL Dif & 0.165888 & Prob $>t$ & \\
\hline Confidence & $0.95 \mathrm{I}$ & $\operatorname{Prob}<\mathrm{t}$ & 1.00 \\
\hline
\end{tabular}

Analysis of Varianc

Source DF Sum of Squares Mean Square F Ratio Prob >

$\begin{array}{llllll}\text { Heat Treatment } & 1 & 0.05244226 & 0.052442 & 605.7964 & <.0001 \\ \text { Error } & 4 & 0.00034627 & 0.000087 & \end{array}$

$$
0.00034627
$$

0.0527885

Means for Oneway Anova

Level Number Mean Std Error Lower 95\% Upper 95\%

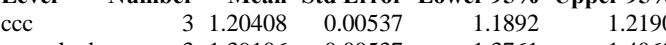

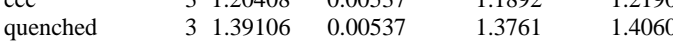

Std Error uses a pooled estimate of error variance
Oneway Analysis of $\log [\mathrm{Na}$ ppm] By Heat Treatment Glass ID=EM-21

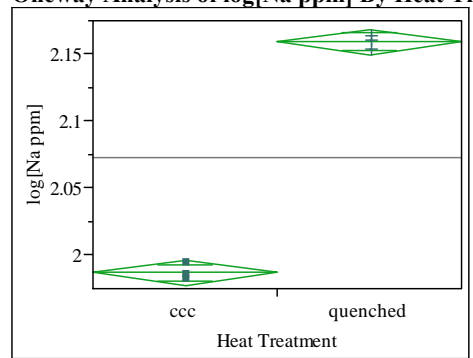

Oneway Anova

Summary of Fit

Adj Rsquare

0.996226

Root Mean Square Error $\quad 0.00581$

Observations (or Sum Wgts)

t Test

quenched-ccc

Assuming equal variances

Difference $\quad 0.172443$ t Ratio 36.34575

Std Err Di

Upper CL Dif 0.185616 Prob $>|t| \quad<.0001$

Lower CL Dif 0.159270 Prob $>t \quad<.0001$

Confidence $\quad 0.95$ Prob $<\mathrm{t} \quad 1.0000$

Analysis of Variance

Source DF Sum of Squares Mean Square F Ratio Prob $>$ F $\begin{array}{llllll}\text { Heat Treatment } & 1 & 0.04460491 & 0.044605 & 1321.013 & <.0001 \\ \text { Error } & 4 & 0.00013506 & 0.000034 & & \end{array}$

Error 0.000034

\section{Means for Oneway Anova}

Level Number Mean Std Error Lower 95\% Upper 95\%

$\begin{array}{llll}\text { ccC } & 3 & 1.98675 & 0.00335\end{array}$

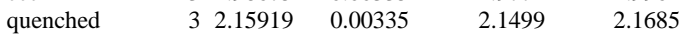

Std Error uses a pooled estimate of error variance

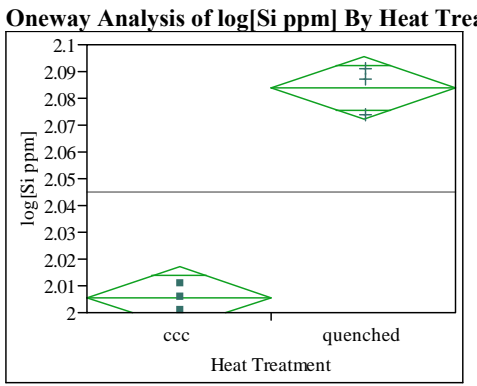

Oneway Anova

Summary of Fit

$\begin{array}{lr}\text { Rsquare } & 0.977136 \\ \text { Adj Rsquare } & 0.97142 \\ \text { Root Mean Square Error } & 0.007324 \\ \text { Mean of Response } & 2.044834 \\ \text { Observations (or Sum Wgts) } & 6\end{array}$

quenched-ccc

Assuming equal variances

Difference $\quad 0.078184$ t Ratio $\quad 13.07462$

Std Err Dif $\quad 0.005980$ D

$\begin{array}{ll}0.0002 \\ \text { Upper CL Dif } 0.094787 \text { Prob }>|t| & 0.000\end{array}$

Lower CL Dif 0.061581 Prob $>t \quad<.0001$

Confidence $\quad 0.95 \mathrm{Prob}<\mathrm{t} \quad 0.9999$

Analysis of Variance

Source DF Sum of Squares Mean Square F Ratio Prob $>$ F $\begin{array}{llllll}\text { Heat Treatment } & 1 & 0.00916912 & 0.009169 & 170.9457 & 0.0002\end{array}$ $\begin{array}{llll}\text { Error } & 4 & 0.00021455 & 0.000054 \\ \text { C. Total } & 5 & 0.00938367 & \end{array}$

Means for Oneway Anova

Level Number Mean Std Error Lower 95\% Upper 95\%

$\begin{array}{llllll}\text { cCC } & 3 & 2.00574 & 0.00423 & 1.9940 & 2.017\end{array}$

$\begin{array}{llllll}\text { quenched } & 3 & 2.08393 & 0.00423 & 2.0722 & 2.0957\end{array}$

Std Error uses a pooled estimate of error variance 
SRNL-STI-2009-00778, Revision 0

Exhibit E5. Effects of Heat Treatment (HT) on PCT $\log (\mathrm{ppm})$ Response of the Matrix 2A Study Glasses

Oneway Analysis of $\log [\mathrm{B}$ ppm $]$ By Heat Treatment Glass ID=EM-22

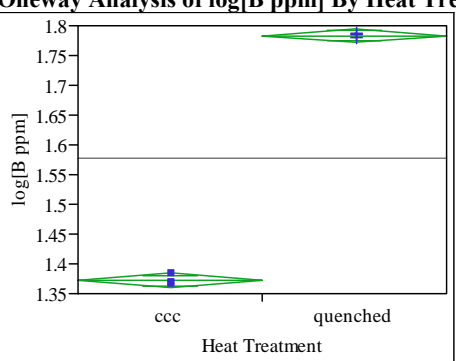

Oneway Anova

Summary of Fit

\begin{tabular}{|c|c|c|c|}
\hline \multicolumn{4}{|c|}{0.999136} \\
\hline \multicolumn{2}{|l|}{ Adj Rsquare } & 0.99 & \\
\hline Root Mean Square Error & uare Error & 0.007 & 408 \\
\hline \multicolumn{2}{|c|}{ Mean of Response } & 1.577 & \\
\hline \multicolumn{2}{|c|}{ Observations (or Sum Wgts) } & & 6 \\
\hline \multicolumn{4}{|l|}{$\begin{array}{l}\text { t Test } \\
\text { quenched-ccc }\end{array}$} \\
\hline \multicolumn{4}{|c|}{ Assuming equal variances } \\
\hline Difference & $0.411318 \mathrm{t}$ & t Ratio & \\
\hline Std Err Dif & $0.006049 \mathrm{I}$ & DF & \\
\hline Upper CL Dif & $0.428112 \mathrm{I}$ & $\operatorname{Prob}>|t|$ & \\
\hline Lower CL Dif & $0.394524 \mathrm{I}$ & Prob $>t$ & \\
\hline Confidence & $0.95 \mathrm{I}$ & Prob $<$ t & 1.00 \\
\hline
\end{tabular}

Analysis of Varianc

Source DF Sum of Squares Mean Square F Ratio Prob $>$

$\begin{array}{llllll}\text { Heat Treatment } & 1 & 0.25377428 & 0.253774 & 4624.077 & <.0001 \\ \text { Error } & 4 & 0.00021952 & 0.000055 & & \end{array}$$$
0.00021952
$$

0.25399380

Means for Oneway Anova

Level Number Mean Std Error Lower 95\% Upper 95\%

$\begin{array}{lllllr}\text { ccC } & 3 & 1.37203 & 0.00428 & 1.3602 & 1.3839\end{array}$

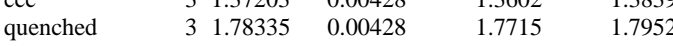

Std Error uses a pooled estimate of error variance
Oneway Analysis of $\log [\mathrm{Li} \mathrm{ppm}]$ By Heat Treatment Glass ID=EM-22

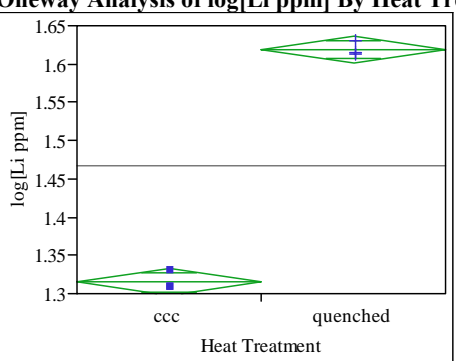

Oneway Anova

Summary of $\mathrm{F}$

Adj Rsquare

0.99576

Mean of Response $\quad 1.46715$

Observations (or Sum Wgts)

6

quenched-ccc

Assuming equal variances

Difference $\quad 0.303981$ t Ratio 34.30193

$\begin{array}{lrr}\text { Std Err Dif } & 0.008862 \text { DF } & 4 \\ \text { Uper } & & <.0001\end{array}$

$\begin{array}{lll}\text { Upper CL Dif } & 0.328585 \text { Prob }>|t| & <.0001 \\ \text { Lower CL Dif } & 0.279376 \text { Prob }>t & <.0001\end{array}$

$\begin{array}{lll}\text { Confidence } & 0.95 \mathrm{Prob}<\mathrm{t} \quad 1.0000\end{array}$

Source DF Sum of Squares Mean Square F Ratio Prob $>$ F $\begin{array}{llllll}\text { Heat Treatment } & 1 & 0.13860649 & 0.138606 & 1176.622 & <.0001 \\ \text { Error } & 4 & 0.00047120 & 0.000118 & & \end{array}$

Error 0.000118

\section{Means for Oneway Anova}

Level Number Mean Std Error Lower 95\% Upper 95\%

$\begin{array}{llllll}\text { ccC } & 3 & 1.31517 & 0.00627 & 1.2978 & 1.3326\end{array}$

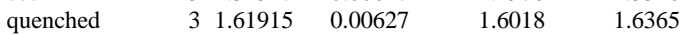

Std Error uses a pooled estimate of error variance

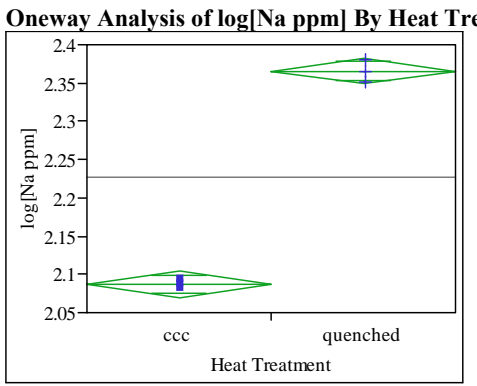

Oneway Anova

Summary of Fit

$\begin{array}{lr}\text { Rsquare } & 0.996103 \\ \text { Adj Rsquare } & 0.995129 \\ \text { Root Mean Square Error } & 0.010695 \\ \text { Mean of Response } & 2.226151 \\ \text { Observations (or Sum Wgts) } & 6\end{array}$

quenched-ccc

Assuming equal variances

Difference $\quad 0.279219$ t Ratio $\quad 31.97638$

0.008732 DF

Upper CL Dif 0.303463 Prob $>|t| \quad<.0001$

Lower CL Dif 0.254975 Prob $>t \quad<.0001$

Confidence $\quad 0.95 \mathrm{Prob}<\mathrm{t} \quad 1.0000$

Analysis of Variance

Source DF Sum of Squares Mean Square $F$ Ratio Prob $>$ F $\begin{array}{llllll}\text { Heat Treatment } & 1 & 0.11694509 & 0.116945 & 1022.489 & <.0001\end{array}$ $\begin{array}{llll}\text { Error } & 4 & 0.00045749 & 0.000114 \\ \text { C. Total } & 5 & 0.11740258 & \end{array}$

Means for Oneway Anova

Level Number Mean Std Error Lower 95\% Upper 95\%

$\begin{array}{llllll}\text { CCC } & 3 & 2.08654 & 0.00617 & 2.0694 & 2.1037\end{array}$

$\begin{array}{llllll}\text { quenched } & 3 & 2.36576 & 0.00617 & 2.3486 & 2.3829\end{array}$

Std Error uses a pooled estimate of error variance 
SRNL-STI-2009-00778, Revision 0

Exhibit E5. Effects of Heat Treatment (HT) on PCT $\log (\mathrm{ppm})$ Response of the Matrix 2 A Study Glasses

Oneway Analysis of $\log [\mathrm{Si}$ ppm] By Heat Treatment Glass ID=EM-22

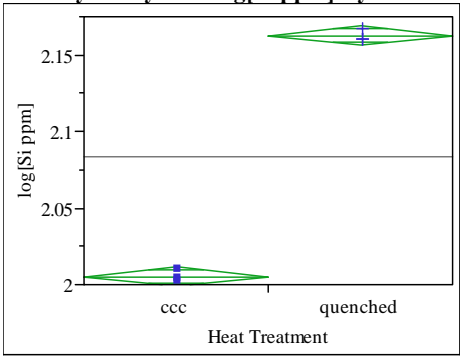

Oneway Anova

Summary of Fit

\begin{tabular}{|c|c|c|c|}
\hline \multicolumn{4}{|c|}{0.998369} \\
\hline \multicolumn{2}{|l|}{ Adj Rsquare } & 0.9979 & \\
\hline \multicolumn{2}{|c|}{ Root Mean Square Error } & 0.0038 & \\
\hline \multicolumn{2}{|c|}{ Mean of Response } & 2.0839 & \\
\hline \multicolumn{2}{|c|}{ Observations (or Sum Wgts) } & & 6 \\
\hline \multicolumn{4}{|l|}{$\begin{array}{l}\text { t Test } \\
\text { quenched-ccc }\end{array}$} \\
\hline \multicolumn{4}{|c|}{ Assuming equal variances } \\
\hline Difference & $0.157425 \mathrm{t}$ & t Ratio & 49. \\
\hline Std Err Dif & $0.003182 \mathrm{I}$ & DF & \\
\hline Upper CL Dif & $0.166259 \mathrm{I}$ & $\operatorname{Prob}>|t|$ & $<.00$ \\
\hline Lower CL Dif & $0.148590 \mathrm{I}$ & Prob $>t$ & $<.00$ \\
\hline Confidence & $0.95 \mathrm{I}$ & Prob $<\mathrm{t}$ & \\
\hline
\end{tabular}

Analysis of Variance

Source DF Sum of Squares Mean Square F Ratio Prob $>$ $\begin{array}{llllll}\text { Heat Treatment } & 1 & 0.03717384 & 0.037174 & 2447.774 & <.0001 \\ \text { Error } & 4 & 0.00006075 & 0.000015 & & \end{array}$ 0.000015

Means for Oneway Anova

Level Number Mean Std Error Lower 95\% Upper 95\%

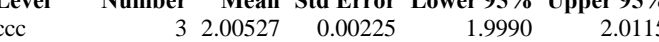

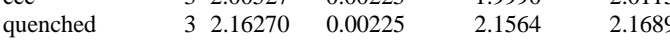

Std Error uses a pooled estimate of error variance
Oneway Analysis of $\log [\mathrm{B}$ ppm] By Heat Treatment Glass ID=EM-23

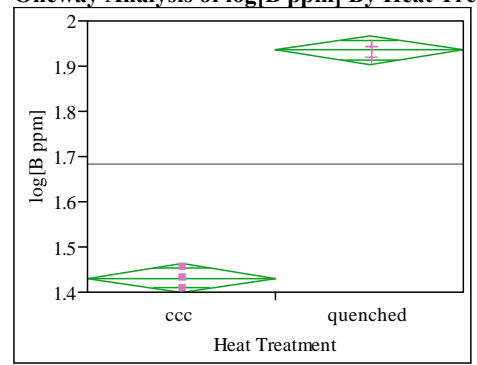

Oneway Anova

Summary of

$\begin{array}{ll}\text { Rsquare } & 0.995935 \\ \text { Adj Rsquare } & 0.994919\end{array}$

Root Mean Square Error $\quad 0.019728$

Mean of Response 1.683062

Observations (or Sum Wgts)

t Test

quenched-ccc

Assuming equal variances

Difference $\quad 0.504274$ t Ratio 31.30656

$\begin{array}{llr}\text { Std Err Dif } & 0.016108 \text { DF } & 4 \\ \text { U } & & \end{array}$

$\begin{array}{lrr}\text { Upper CL Dif } & 0.548996 \text { Prob }>|t| & <.0001 \\ \text { Lower CL Dif } & 0.459552 \text { Prob }>t & <.0001\end{array}$

Confidence $\quad 0.95$ Prob $<\mathrm{t} \quad 1.0000$

Analysis of Variance

Source DF Sum of Squares Mean Square F Ratio Prob $>$ F $\begin{array}{llllll}\text { Heat Treatment } & 1 & 0.38143898 & 0.381439 & 980.1010 & <.0001 \\ \text { Error } & 4 & 0.00155673 & 0.000389 & & \end{array}$ Error 0.000389

\section{Means for Oneway Anova}

Level Number Mean Std Error Lower 95\% Upper 95\%

$\begin{array}{llllll}\text { ccc } & 3 & 1.43092 & 0.01139 & 1.3993 & 1.4625 \\ \text { quenched } & 3 & 1.93520 & 0.01139 & 1.9036 & 1.9668\end{array}$

Std Error uses a pooled estimate of error variance

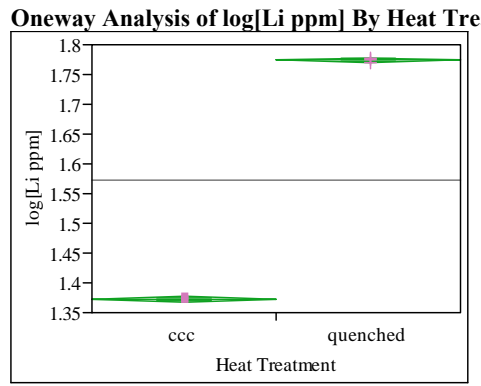

Oneway Anova

Summary of Fit

$\begin{array}{lr}\text { Rsquare } & 0.999889 \\ \text { Adj Rsquare } & 0.999861 \\ \text { Root Mean Square Error } & 0.002602 \\ \text { Mean of Response } & 1.573309 \\ \text { Observations (or Sum Wgts) } & 6\end{array}$

quenched-ccc

Assuming equal variances

Difference $\quad 0.402418$ t Ratio $\quad 189.4416$

Std Err Dif 0.002124 DF $>0.4001$

Upper CL Dif 0.408316 Prob $>|t| \quad<.0001$

Lower CL Dif 0.396520 Prob $>t \quad<.0001$

Confidence $\quad 0.95$ Prob $<\mathrm{t} \quad 1.0000$

Analysis of Variance

Source DF Sum of Squares Mean Square F Ratio Prob $>$ F $\begin{array}{lrrrrr}\text { Heat Treatment } & 1 & 0.24291044 & 0.242910 & 35888.12 & <.0001\end{array}$ $\begin{array}{lll}\text { Error } & 4 & 0.00002707\end{array}$ $6.769 \mathrm{e}-6$

Means for Oneway Anova

Level Number Mean Std Error Lower 95\% Upper 95\% $\begin{array}{llllll}\text { ccc } & 3 & 1.37210 & 0.00150 & 1.3679 & 1.3763 \\ \text { quenched } & 3 & 1.77452 & 0.00150 & 1.7703 & 1.7787\end{array}$

Std Error uses a pooled estimate of error variance 
SRNL-STI-2009-00778, Revision 0

\section{Exhibit E5. Effects of Heat Treatment (HT) on PCT $\log (\mathrm{ppm})$ Response of the Matrix 2A Study Glasses}

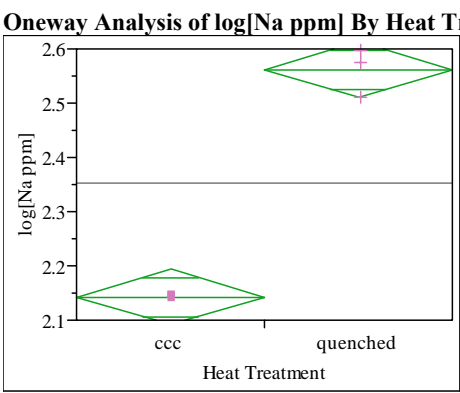

Summary of Fit

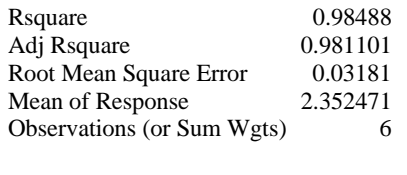

t Test

quenched-ccc

Assuming equal variances

Difference $\quad 0.419253$ t Ratio $\quad 16.14181$ $\begin{array}{lrr}\text { Std Err Dif } & 0.025973 \text { DF } & 4 \\ \text { Uper CL Dif } & 0.491366 \text { Prob }>|t| & <.0001\end{array}$ $\begin{array}{lll}\text { Upper CL Dif } & 0.491366 \text { Prob }>|t| & <.0001 \\ \text { Lower CL Dif } 0.347140 \text { Prob }>t & <.0001\end{array}$ Confidence $\quad 0.95 \mathrm{Prob}<\mathrm{t} \quad 1.0000$

Analysis of Variance

Source DF Sum of Squares Mean Square F Ratio Prob $>$ F $\begin{array}{lrrrrr}\text { Heat Treatment } & 1 & 0.26365969 & 0.263660 & 260.5581 & <.0001 \\ \text { Error } & 4 & 0.00404761 & 0.001012 & & \end{array}$ Error

Means for Oneway Anova

Level Number Mean Std Error Lower 95\% Upper 95\% $\begin{array}{llllll}\text { ccc } & 3 & 2.14284 & 0.01837 & 2.0919 & 2.1938\end{array}$ $\begin{array}{llllll}\text { quenched } & 3 & 2.56210 & 0.01837 & 2.5111 & 2.6131\end{array}$

Std Error uses a pooled estimate of error variance

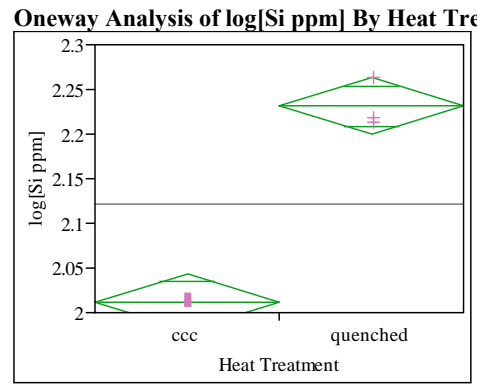

Oneway Anova

Summary of Fit

Adj Rsquare

$\begin{array}{ll}\text { Adj Rsquare } & 0.973314 \\ \text { Root Mean Square Error } & 0.019855\end{array}$

Mean of Response

Observations (or Sum Wgts)

6

t Test

quenched-ccc

Assuming equal variances

Difference $\quad 0.219528$ t Ratio 13.54115

$\begin{array}{lrr}\text { Std Err Dif } & 0.016212 \mathrm{DF} & 4 \\ \text { Uper } & & 0.0002\end{array}$

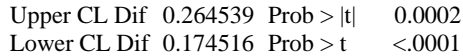

$\begin{array}{lr}\text { Confidence } & 0.95 \text { Prob }<\mathrm{t} \quad 0.9999\end{array}$

Analysis of Variance

$\begin{array}{lrrrrr}\text { Source } & \text { DF } & \text { Sum of Squares } & \text { Mean Square } & \text { F Ratio } & \text { Prob }>\text { F } \\ \text { Heat Treatment } & 1 & 0.07228852 & 0.072289 & 183.3628 & 0.0002\end{array}$ Heat

Error
C. Tota

1

0.07228852
0.00157695
0.07386547

0.07386547

Means for Oneway Anova

Level Number Mean Std Error Lower 95\% Upper 95\%

$\begin{array}{llllll}\text { ccc } & 3 & 2.01189 & 0.01146 & 1.9801 & 2.0437\end{array}$

$\begin{array}{llllll}\text { quenched } & 3 & 2.23142 & 0.01146 & 2.1996 & 2.2632\end{array}$

Std Error uses a pooled estimate of error variance

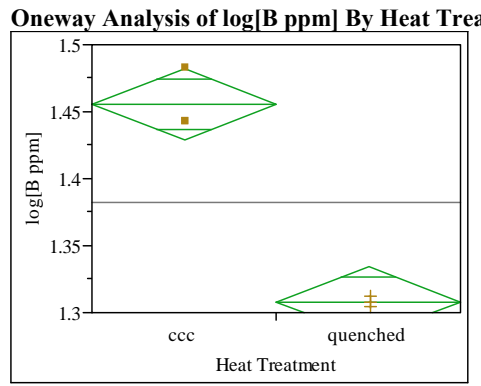

Oneway Anova

Summary of Fit

$\begin{array}{lr}\text { Rsquare } & 0.967498 \\ \text { Adj Rsquare } & 0.959373 \\ \text { Root Mean Square Error } & 0.016508 \\ \text { Mean of Response } & 1.381747 \\ \text { Observations (or Sum Wgts) } & 6\end{array}$

t Test

quenched-ccc

Assuming equal variances

Difference $\quad-0.14708$ t Ratio $\quad-10.9119$ Std Err Dif $\quad 0.01348$ DF

Upper CL Dif -0.10966 Prob $>|t| \quad 0.0004$ Lower CL Dif -0.18450 Prob $>t \quad 0.9998$ $\begin{array}{ll}\text { Confidence } & 0.95 \mathrm{Prob}<\mathrm{t} \quad 0.0002\end{array}$

Analysis of Variance

Source DF Sum of Squares Mean Square $\quad$ F Ratio Prob $>$ F $\begin{array}{lrrrrr}\text { Heat Treatment } & 1 & 0.03244839 & 0.032448 & 119.0706 & 0.0004\end{array}$ $\begin{array}{lll}\text { Error } & 4 & 0.00109006 \\ \text { C. Total } & 5 & 0.03353844\end{array}$

Means for Oneway Anova

Level Number Mean Std Error Lower 95\% Upper 95\% $\begin{array}{lllllr}\text { ccc } & 3 & 1.45529 & 0.00953 & 1.4288 & 1.4817 \\ \text { quenched } & 3 & 1.30821 & 0.00953 & 1.2817 & 1.3347\end{array}$

Std Error uses a pooled estimate of error variance 
SRNL-STI-2009-00778, Revision 0

Exhibit E5. Effects of Heat Treatment (HT) on PCT $\log (\mathrm{ppm})$ Response of the Matrix 2 A Study Glasses

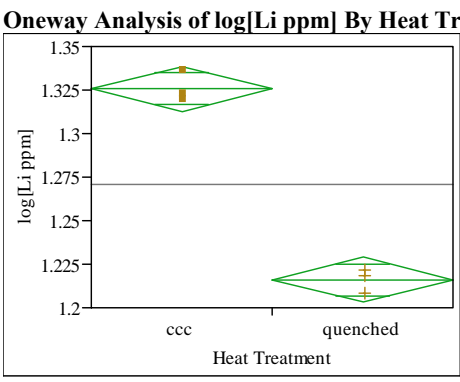

Oneway Anova

Summary of Fit

Rsquare $\quad 0.985572$

Adj Rsquare $\quad 0.981965$

Root Mean Square Error $\quad 0.00811$

Mean of Response

) 6

t Test

quenched-ccc

Assuming equal variances

Difference $\quad-0.10946$ t Ratio $\quad-16.53$

Std Err Dif 0.00662 DF

Upper CL Dif -0.09108 Prob $>|t|<.000$

Lower CL Dif -0.12785 Prob $>t \quad 1.0000$

Analysis of Variance

Source DF Sum of Squares Mean Square F Ratio Prob $>$ $\begin{array}{llllll}\text { Heat Treatment } & 1 & 0.01797316 & 0.017973 & 273.2412 & <.0001 \\ \text { Error } & 4 & 0.00026311 & 0.000066 & \end{array}$

Error 0.00026311 .000066

Means for Oneway Anova

Level Number Mean Std Error Lower 95\% Upper 95\% $\begin{array}{llllll}\text { CCC } & 3 & 1.32560 & 0.00468 & 1.3126 & 1.3386\end{array}$

Std Error uses a pooled estimate of error variance

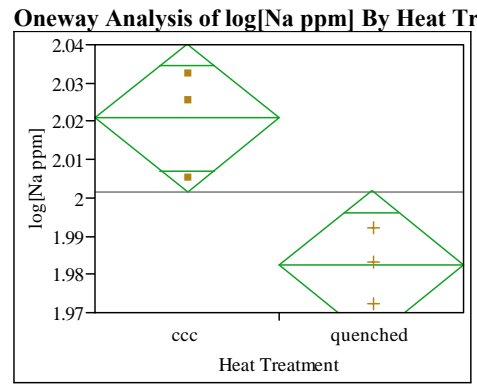

Oneway Anova

Summary of Fit

Adj Rsquare

Root Mean Square Error

\subsection{8}

Mean of Response

0.012132

Observations (or Sum Wgts)

6

t Test

quenched-ccc

Assuming equal variances

Difference $\quad-0.03836$ t Ratio $\quad-3.87247$

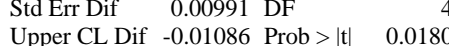

$\begin{array}{lll}\text { Upper CL Dif } & -0.01086 \text { Prob }>|t| & 0.0180 \\ \text { Lower CL Dif }-0.06586 \text { Prob }>t & 0.9910\end{array}$

Confidence $\quad 0.95$ Prob $<\mathrm{t} \quad 0.0090$

Analysis of Variance

Source DF Sum of Squares Mean Square F Ratio Prob $>$ F

$\begin{array}{lllllll}\text { Heat Treatment } & 1 & 0.00220719 & 0.002207 & 14.9960 & 0.0180\end{array}$

$4 \quad 0.00058874$

0.000147

\section{Means for Oneway Anova}

Level Number Mean Std Error Lower 95\% Upper 95\%

$\begin{array}{llllll}\text { ccC } & 3 & 2.02082 & 0.00700 & 2.0014 & 2.0403\end{array}$

$\begin{array}{llllll}\text { quenched } & 3 & 1.98246 & 0.00700 & 1.9630 & 2.0019\end{array}$

Std Error uses a pooled estimate of error variance

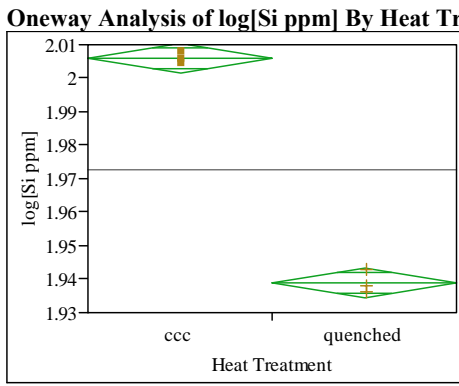

Oneway Anova

Summary of Fit

$\begin{array}{lr}\text { Rsquare } & 0.995506 \\ \text { Adj Rsquare } & 0.994383 \\ \text { Root Mean Square Error } & 0.002758 \\ \text { Mean of Response } & 1.97248 \\ \text { Observations (or Sum Wgts) } & 6\end{array}$

quenched-ccc

Assuming equal variances

Difference $\quad-0.06703$ t Ratio $\quad-29.7672$

Std Err Dif 0.00225 DF $>$ - $\quad<<001$

Upper CL Dif -0.06078 Prob $>|t| \quad<.0001$

Lower CL Dif -0.07329 Prob $>t \quad 1.0000$

$\begin{array}{lll}\text { Confidence } & 0.95 \text { Prob }<\mathrm{t} \quad<.0001\end{array}$

Analysis of Variance

Source DF Sum of Squares Mean Square F Ratio Prob $>$ F

$\begin{array}{lllllll}\text { Heat Treatment } & 1 & 0.00674011 & 0.006740 & 886.0862 & <.0001\end{array}$

$\begin{array}{llll}\text { Error } & 4 & 0.00003043 & 7.607 \mathrm{e}-6\end{array}$

Means for Oneway Anova

Level Number Mean Std Error Lower 95\% Upper 95\%

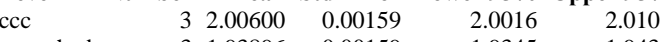

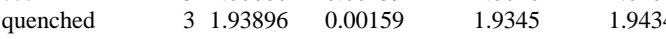

Std Error uses a pooled estimate of error variance 
SRNL-STI-2009-00778, Revision 0

Exhibit E5. Effects of Heat Treatment (HT) on PCT $\log (\mathrm{ppm})$ Response of the Matrix 2 A Study Glasses

Oneway Analysis of $\log [B$ ppm $]$ By Heat Treatment Glass ID=EM-25

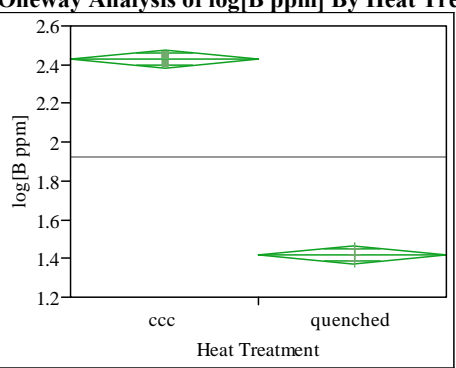

Oneway Anova

Summary of Fit

Rsquare $\quad 0.997788$

Adj Rsquare $\quad 0.997235$

Root Mean Square Error $\quad 0.029157$

Mean of Response 1.923479

Observations (or Sum Wgts)

t Test

quenched-ccc

Assuming equal variances

Difference $\quad-1.0113$ t Ratio $\quad-42.48$

Std Err Dif $\quad 0.0238$ DF $\quad 4$

Upper CL Dif -0.9452 Prob $>|t|<.0001$

Lower CL Dif -1.0774 Prob $>t \quad 1.0000$

Confidence $\quad 0.95$ Prob $<\mathrm{t}<.0001$

Analysis of Variance

Source DF Sum of Squares Mean Square F Ratio Prob $>$ F $\begin{array}{lrrrrr}\text { Heat Treatment } & 1 & 1.5340664 & 1.53407 & 1804.549 & <.0001 \\ \text { Error } & 4 & 0.0034004 & 0.00085 & & \end{array}$ Error 0.00085

C. Tota 1.5374668

Means for Oneway Anova

Level Number Mean Std Error Lower 95\% Upper 95\%

$\begin{array}{llllll}\text { ccC } & 32.42912 & 0.01683 & 2.3824 & 2.4759\end{array}$

quenched $\quad 31.41783 \quad 0.01683 \quad 1.3711 \quad 1.4646$

Std Error uses a pooled estimate of error variance
Oneway Analysis of $\log [\mathrm{Li}$ ppm] By Heat Treatment Glass ID=EM-25

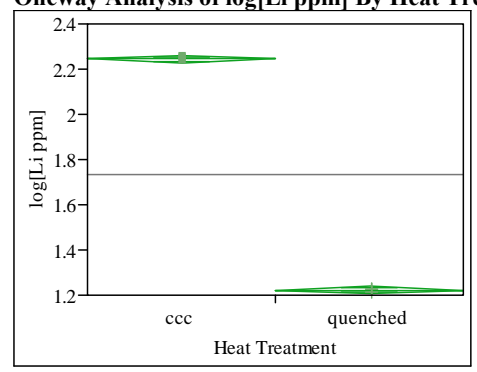

Oneway Anova

Summary of Fit

Rsquare $\quad 0.999732$

Adj Rsquare $\quad 0.999665$

Root Mean Square Error $\quad 0.010244$

Mean of Response 1.733205

Observations (or Sum Wgts)

t Test

quenched-ccc

Assuming equal variances

Difference $\quad-1.0222$ t Ratio $\quad-122.207$

$\begin{array}{lrr}\text { Std Err Dif } & 0.0084 \text { DF } & 4 \\ \text { Upper CL Dif } & -0.990 \text { Pro }>|t| & <0001\end{array}$

$\begin{array}{lll}\text { Upper CL Dif } & -0.9990 \text { Prob }>|t| & <.0001 \\ \text { Lower CL Dif }-1.0454 \text { Prob }>t & 1.0000\end{array}$

Confidence $\quad 0.95$ Prob $<\mathrm{t} \quad<.0001$

Analysis of Variance

Source DF Sum of Squares Mean Square F Ratio Prob $>$ F $\begin{array}{lllllll}\text { Heat Treatment } & 1 & 1.5673143 & 1.56731 & 14934.47 & <.0001\end{array}$ Error $\begin{array}{ll}1 & 1.5673143 \\ 4 & 0.0004198\end{array}$ 0.00010

\section{Means for Oneway Anova}

Level Number Mean Std Error Lower 95\% Upper 95\%

$\begin{array}{llllll}\text { ccc } & 3 & 2.24430 & 0.00591 & 2.2279 & 2.2607 \\ \text { quenched } & 3 & 1.22211 & 0.00591 & 1.2057 & 1.2385\end{array}$

Std Error uses a pooled estimate of error variance

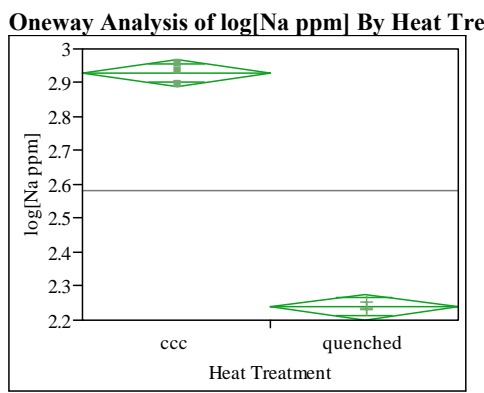

Oneway Anova

Summary of Fit

Rsquare

Root Mean Square Error $\quad 0.024182$

$\begin{array}{lr}\text { Mean of Response } & 2.583572 \\ \text { Observations (or Sum Wgts) } & 6\end{array}$

t Test

quenched-ccc

Assuming equal variance

Difference $\quad-0.68955$ t Ratio $\quad-34.9241$

$\begin{array}{lll}\text { Std Err Dif } & 0.01974 \text { DF } & \\ \text { Upper CL Dif } & -0.63473 \text { Prob }>|t| & <.000\end{array}$

$\begin{array}{lll}\text { Upper CL Dif } & -0.63473 \text { Prob }>|t| & <.0001 \\ \text { Lower CL Dif }-0.74437 \text { Prob }>t & 1.0000\end{array}$

Confidence $\quad 0.95$ Prob $<\mathrm{t} \quad<.0001$

Analysis of Variance

Source DF Sum of Squares Mean Square $\quad$ F Ratio Prob $>$ F $\begin{array}{lllllll}\text { Heat Treatment } & 1 & 0.71321332 & 0.713213 & 1219.695 & <.0001\end{array}$ $\begin{array}{lll}\text { Error } & 4 & 0.00233899 \\ \text { C. Total } & 5 & 0.71555230\end{array}$

Means for Oneway Anova

Level Number Mean Std Error Lower 95\% Upper 95\%

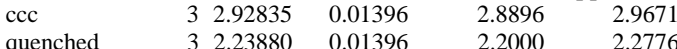

Std Error uses a pooled estimate of error variance 
SRNL-STI-2009-00778, Revision 0

Exhibit E5. Effects of Heat Treatment (HT) on PCT $\log (\mathrm{ppm})$ Response of the Matrix 2 A Study Glasses

Oneway Analysis of $\log [\mathrm{Si}$ ppm] By Heat Treatment Glass ID=EM-25

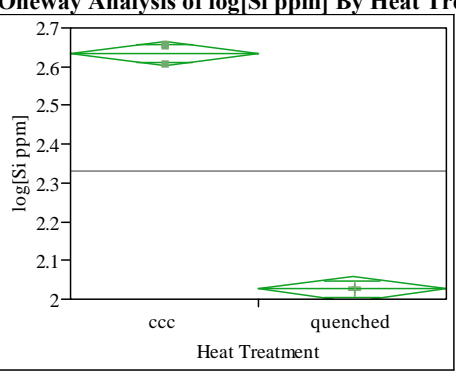

Oneway Anova

Summary of Fit

Rsquare $\quad 0.997104$

Adj Rsquan $\quad 0.99638$

Mean of Response $\quad 2.330157$

Observations (or Sum Wgts)

t Test

quenched-ccc

Assuming equal variances

Difference $\quad-0.60880$ t Ratio $\quad-37.1132$

$\begin{array}{lrr}\text { Std Err Dif } & 0.01640 \text { DF } & 4 \\ \text { Upper CL Dif } & -0.56325 \text { Prob }>|t| & <0001\end{array}$

$\begin{array}{lll}\text { Upper CL Dif } & -0.56325 \text { Prob }>|t| & <.0001 \\ \text { Lower CL Dif }-0.65434 & \text { Prob }>t & 1.0000\end{array}$

Confidence $\quad 0.95 \mathrm{Prob}<\mathrm{t} \quad<.0001$

Analysis of Variance

Source DF Sum of Squares Mean Square F Ratio Prob $>$ F $\begin{array}{lllllll}\text { Heat Treatment } & 1 & 0.55595405 & 0.555954 & 1377.388 & <.000\end{array}$ Erro$$
4 \quad 0.00161452
$$$$
0.000404
$$

C. Total 0.55756857

Means for Oneway Anova

Level Number Mean Std Error Lower 95\% Upper 95\%

$\begin{array}{llllll}\text { ccC } & 3 & 2.63456 & 0.01160 & 2.6024 & 2.6668 \\ \text { quenched } & 3 & 2.02576 & 0.01160 & 1.9936 & 2.0580\end{array}$

Std Error uses a pooled estimate of error variance
Oneway Analysis of $\log [\mathrm{B}$ ppm $]$ By Heat Treatment Glass ID=EM-26

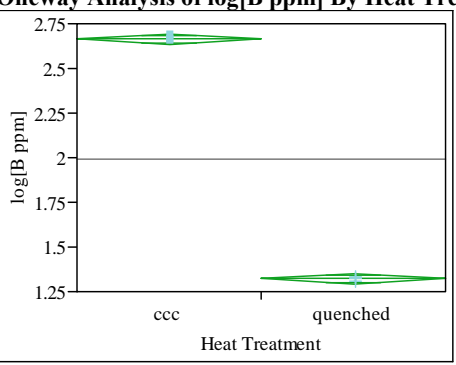

Oneway Anova

Summary of Fit

Adj Rsquare

0.999505

0.999381
0.018297

Mean of Response

1.99192

Observations (or Sum Wgts)

t Test

quenched-ccc

Assuming equal variances

Difference $\quad-1.3420$ t Ratio $\quad-89.8313$

$\begin{array}{lrl}\text { Std Err Dif } & 0.0149 \text { DF } & 4 \\ \text { Upper CL Dif } & -1.3005 \text { Prob }>|t|<.0001\end{array}$

$\begin{array}{lll}\text { Upper CL Dif }-1.3005 & \text { Prob }>|t| & <.0001 \\ \text { Lower CL Dif }-1.3835 \text { Prob }>t & 1.0000\end{array}$

$\begin{array}{lrl}\text { Confidence } & 0.95 \mathrm{Prob}<\mathrm{t} & <.0001\end{array}$

Analysis of Variance

$\begin{array}{lrrrrr}\text { Source } & \text { DF } & \text { Sum of Squares } & \text { Mean Square } & \text { F Ratio } & \text { Prob }>\text { F } \\ \text { Heat Treatment } & 1 & 2.7015198 & 2.70152 & 8069.659 & <.0001\end{array}$ Heat Treatment $1-2.7015198$

Error

4

0.0013391
2.7028589

Means for Oneway Anova

Level Number Mean Std Error Lower 95\% Upper 95\%

$\begin{array}{llllll}\text { ccC } & 3 & 2.66294 & 0.01056 & 2.6336 & 2.6923\end{array}$

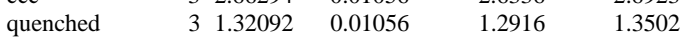

Std Error uses a pooled estimate of error variance

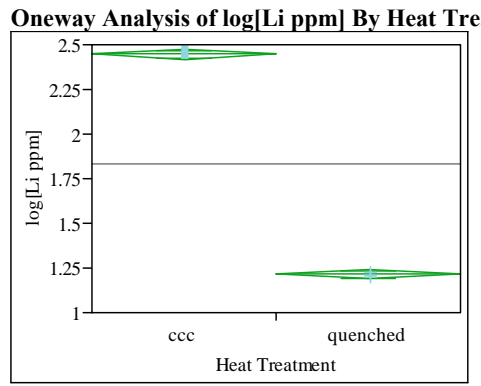

Oneway Anova

Summary of Fit

$\begin{array}{lr}\text { Rsquare } & 0.999559 \\ \text { Adj Rsquare } & 0.999449 \\ \text { Root Mean Square Error } & 0.015863 \\ \text { Mean of Response } & 1.8295 \\ \text { Observations (or Sum Wgts) } & 6\end{array}$

quenched-ccc

Assuming equal variances

Difference $\quad-1.2331$ t Ratio $\quad-95.2053$

Std Err Dif 0.0130 DF

Upper CL Dif -1.1971 Prob $>|t|<.0001$

Lower CL Dif -1.2690 Prob $>t \quad 1.0000$

$\begin{array}{lll}\text { Confidence } & 0.95 \text { Prob }<\mathrm{t} \quad<.0001\end{array}$

Analysis of Variance

Source DF Sum of Squares Mean Square F Ratio Prob $>$ F

$\begin{array}{llllll}\text { Heat Treatment } & 1 & 2.2807236 & 2.28072 & 9064.049 & <.0001\end{array}$

$\begin{array}{lll}\text { Error } & 4 & 0.0010065\end{array}$ 0.00025

Means for Oneway Anova

Level Number Mean Std Error Lower 95\% Upper 95\%

$\begin{array}{llllll}\text { CCC } & 3 & 2.44604 & 0.00916 & 2.4206 & 2.4715\end{array}$

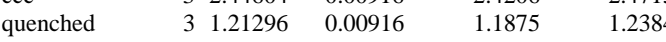

Std Error uses a pooled estimate of error variance 
SRNL-STI-2009-00778, Revision 0

Exhibit E5. Effects of Heat Treatment (HT) on PCT $\log (\mathrm{ppm})$ Response of the Matrix 2 A Study Glasses

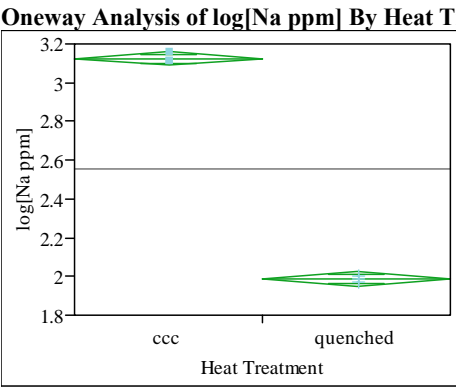

Oneway Anova

Summary of Fit

$\begin{array}{lr}\text { Rsquare } & 0.999009 \\ \text { Adj Rsquare } & 0.998762 \\ \text { Root Mean Square Error } & 0.02192 \\ \text { Mean of Response } & 2.555039\end{array}$

$\begin{array}{lr}\text { Mean of Response } & 2.555039 \\ \text { Observations (or Sum Wgts) } & 6\end{array}$

Test

quenched-ccc

Assuming equal variances

Difference $\quad-1.1368$ t Ratio $\quad-63.5164$

$\begin{array}{lrr}\text { Std Err Dif } & 0.0179 \text { DF } & 4 \\ \text { Upper CL Dif } & -1.0871 \text { Prob }>|t| & <0001\end{array}$

$\begin{array}{lll}\text { Upper CL Dif }-1.0871 \text { Prob }>|t| & <.0001 \\ \text { Lower CL Dif }-1.1865 \text { Prob }>t & 1.0000\end{array}$

Confidence $\quad 0.95$ Prob $<\mathrm{t} \quad<.0001$

Analysis of Variance

Source DF Sum of Squares Mean Square F Ratio Prob $>$ F $\begin{array}{lllllll}\text { Heat Treatment } & 1 & 1.9385298 & 1.93853 & 4034.333 & <.0001\end{array}$ Error 0.0019220 0.00048 1.9404519

Means for Oneway Anova

Level Number Mean Std Error Lower 95\% Upper 95\%

$\begin{array}{llllll}\text { ccc } & 3 & 3.12345 & 0.01266 & 3.0883 & 3.1586\end{array}$

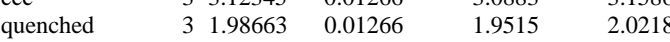

Std Error uses a pooled estimate of error variance
Oneway Analysis of $\log [\mathrm{Si}$ ppm] By Heat Treatment Glass ID=EM-26

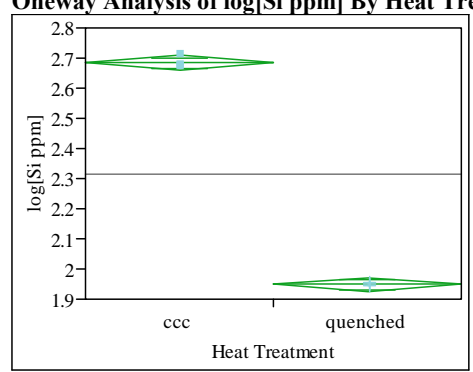

Oneway Anova

Summary of Fit

Rsquare $\quad 0.99892$

Adj Rsquare $\quad 0.99865$

Root Mean Square Error $\quad 0.014806$

Mean of Response 2.31625

Observations (or Sum Wgts)

t Test

quenched-ccc

Assuming equal variances

Difference $\quad-0.73536$ t Ratio $\quad-60.8264$

$\begin{array}{llr}\text { Std Err Dif } & 0.01209 \text { DF } & 4 \\ \text { Upper } & -0.70179 \text { Prob }>|t| & <0001\end{array}$

$\begin{array}{lrl}\text { Upper CL Dif } & -0.70179 \text { Prob }>|t| & <.0001 \\ \text { Lower CL Dif } & -0.76892 \text { Prob }>t & 1.0000\end{array}$

Confidence $\quad 0.95$ Prob $<\mathrm{t} \quad<.000$

Analysis of Variance

Source DF Sum of Squares Mean Square F Ratio Prob $>$ F $\begin{array}{lllllll}\text { Heat Treatment } & 1 & 0.81112471 & 0.811125 & 3699.848 & <.0001\end{array}$ Error $4 \quad 0.81112471$ 0.000219

\section{Means for Oneway Anova}

Level Number Mean Std Error Lower 95\% Upper 95\%

$\begin{array}{llllll}\text { ccc } & 3 & 2.68393 & 0.00855 & 2.6602 & 2.7077 \\ \text { quenched } & 3 & 1.94857 & 0.00855 & 1.9248 & 1.9723\end{array}$

Std Error uses a pooled estimate of error variance

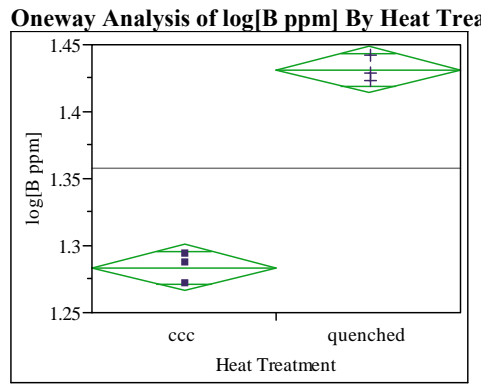

Oneway Anova

Summary of Fit

$\begin{array}{lr}\text { Rsquare } & 0.986345 \\ \text { Adj Rsquare } & 0.982932 \\ \text { Root Mean Square Error } & 0.010634 \\ \text { Mean of Response } & 1.357506 \\ \text { Observations (or Sum Wgts) } & 6\end{array}$

quenched-ccc

Assuming equal variances

Difference $\quad 0.147591$ t Ratio $\quad 16.99822$ Std Err Dif 0.008683 D

Upper CL Dif 0.171698 Prob $>|t|<.000$

Lower CL Dif 0.123484 Prob $>t \quad<.0001$

Confidence $\quad 0.95 \mathrm{Prob}<\mathrm{t} \quad 1.0000$

Analysis of Variance

Source DF Sum of Squares Mean Square F Ratio Prob $>$ F $\begin{array}{llllll}\text { Heat Treatment } & 1 & 0.03267467 & 0.032675 & 288.9395 & <.0001\end{array}$ $\begin{array}{lll}\text { Error } & 4 & 0.00045234 \\ \text { C. Total } & 5 & 0.03312700\end{array}$ 0.000113

Means for Oneway Anova

Level Number Mean Std Error Lower 95\% Upper 95\%

$\begin{array}{llllll}\text { ccC } & 3 & 1.28371 & 0.00614 & 1.2667 & 1.3008\end{array}$

$\begin{array}{llllll}\text { quenched } & 3 & 1.43130 & 0.00614 & 1.4143 & 1.4483\end{array}$

Std Error uses a pooled estimate of error variance 
SRNL-STI-2009-00778, Revision 0

\section{Exhibit E5. Effects of Heat Treatment (HT) on PCT $\log (\mathrm{ppm})$ Response of the Matrix 2 A Study Glasses}

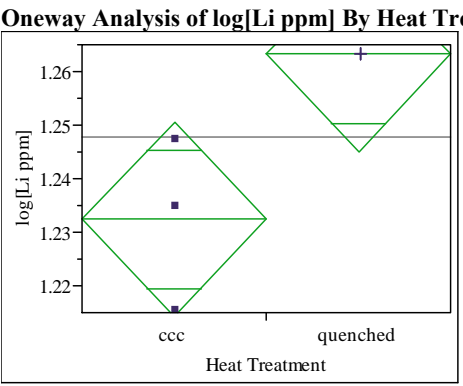

Oneway Anova

Summary of Fit

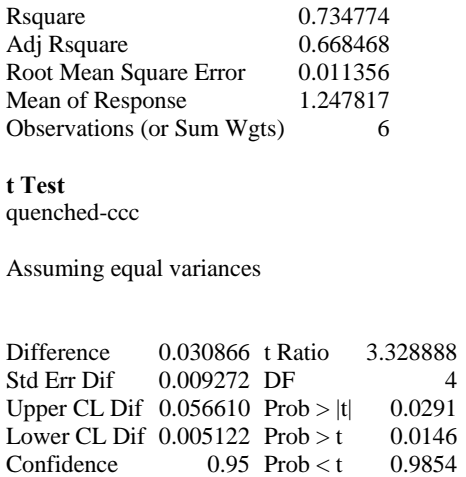

Analysis of Variance

Source DF Sum of Squares Mean Square F Ratio Prob $>$ F

$\begin{array}{llllll}\text { Heat Treatment } & 1 & 0.00142909 & 0.001429 & 11.0815 & 0.0291 \\ \text { Error } & 4 & 0.00051585 & 0.000129 & & \end{array}$

0.000129

Means for Oneway Anova

Level Number Mean Std Error Lower 95\% Upper 95\%

$\begin{array}{llllll}\text { cCC } & 3 & 1.23238 & 0.00656 & 1.2142 & 1.2506\end{array}$

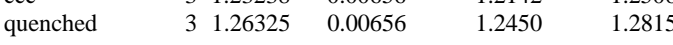

Std Error uses a pooled estimate of error variance

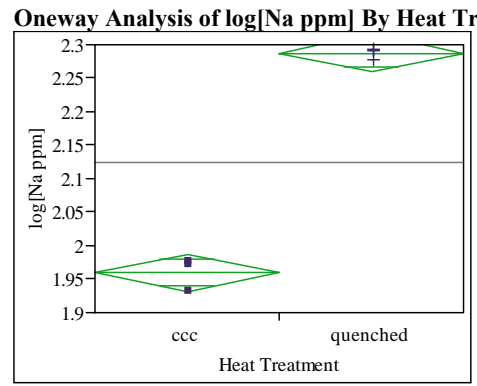

Oneway Anova

Summary of Fit

$\begin{array}{lr}\text { Rsquare } & 0.992384 \\ \text { Adj Rsquare } & 0.99048 \\ \text { Root Mean Square Error } & 0.017622 \\ \text { Mean of Response } & 2.123397 \\ \text { Observations (or Sum Wgts) } & 6\end{array}$

t Test

quenched-ccc

Assuming equal variances

Difference $\quad 0.328481$ t Ratio $\quad 22.83017$

$\begin{array}{lrrr}\text { Std Err Dif } & 0.014388 \text { DF } & 4 \\ \text { Uper } & <\text { Dif } & 0.368429 \text { Prob }>|t| & <.0001\end{array}$

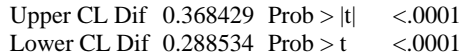

$\begin{array}{lll}\text { Confidence } & 0.95 \mathrm{Prob}<\mathrm{t} \quad 1.0000\end{array}$

Analysis of Variance

Source DF Sum of Squares Mean Square F Ratio Prob $>$ F $\begin{array}{llllll}\text { Heat Treatment } & 1 & 0.16184996 & 0.161850 & 521.2166 & <.0001 \\ \text { Error } & 4 & 0.00124209 & 0.000311 & & \end{array}$

Error 0.000311

\section{Means for Oneway Anova}

Level Number Mean Std Error Lower 95\% Upper 95\%

$\begin{array}{llllll}\text { ccc } & 3 & 1.95916 & 0.01017 & 1.9309 & 1.9874\end{array}$

$\begin{array}{llllll}\text { quenched } & 3 & 2.28764 & 0.01017 & 2.2594 & 2.3159\end{array}$

Std Error uses a pooled estimate of error variance

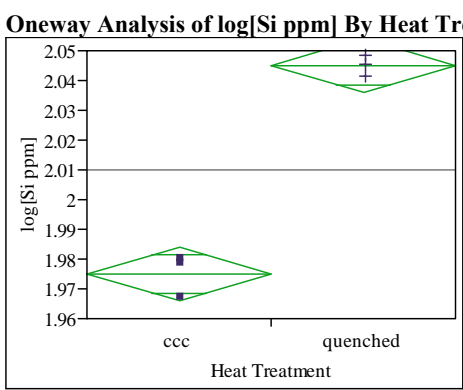

Oneway Anova Summary of Fit

$\begin{array}{lr}\text { Rsquare } & 0.982838 \\ \text { Adj Rsquare } & 0.978547 \\ \text { Root Mean Square Error } & 0.005661 \\ \text { Mean of Response } & 2.010125 \\ \text { Observations (or Sum Wgts) } & 6\end{array}$

quenched-ccc

Assuming equal variances

Difference $\quad 0.069958$ t Ratio 15.13496 Upper CL Dif 0.082792 Prob $>|t| \quad 0.000$ Lower CL Dif 0.057125 Prob $>t \quad<.0001$ Confidence $\quad 0.95 \mathrm{Prob}<\mathrm{t} \quad 0.9999$

Analysis of Variance

Source DF Sum of Squares Mean Square F Ratio Prob $>$ F $\begin{array}{lllllll}\text { Heat Treatment } & 1 & 0.00734126 & 0.007341 & 229.0670 & 0.0001\end{array}$ $\begin{array}{lll}\text { Error } & 4 & 0.00012819 \\ \text { C. Total } & 5 & 0.00746946\end{array}$

Means for Oneway Anova

Level Number Mean Std Error Lower 95\% Upper 95\% $\begin{array}{llllll}\text { cCC } & 3 & 1.97515 & 0.00327 & 1.9661 & 1.9842\end{array}$ $\begin{array}{llllll}\text { quenched } & 3 & 2.04510 & 0.00327 & 2.0360 & 2.0542\end{array}$

Std Error uses a pooled estimate of error variance 
SRNL-STI-2009-00778, Revision 0

Exhibit E5. Effects of Heat Treatment (HT) on PCT $\log (\mathrm{ppm})$ Response of the Matrix 2A Study Glasses

Oneway Analysis of $\log [\mathrm{B}$ ppm] By Heat Treatment Glass ID=EM-28

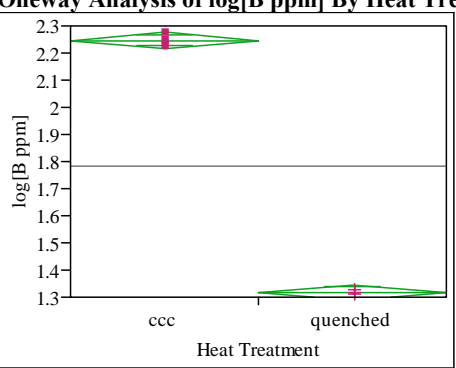

Oneway Anova

Summary of Fit

Rsquare $\quad 0.998968$

Adj Rsquare $\quad 0.99871$

Mean of Response 1.782061

Observations (or Sum Wgts)

t Test

quenched-ccc

Assuming equal variances

Difference $\quad-0.92899$ t Ratio $\quad-62.2168$ $\begin{array}{lrr}\text { Std Err Dif } & 0.01493 \text { DF } & 4 \\ \text { Upper CL Dif } & -0.88753 \text { Prob }>|t| & <.0001\end{array}$ $\begin{array}{lll}\text { Upper CL Dif }-0.88753 & \text { Prob }>|t| & <.0001 \\ \text { Lower CL Dif }-0.97044 & \text { Prob }>t & 1.0000\end{array}$ Confidence $\quad 0.95$ Prob $<\mathrm{t} \quad<.0001$

Analysis of Variance

Source DF Sum of Squares Mean Square F Ratio Prob $>$ F $\begin{array}{llllll}\text { Heat Treatment } & 1 & 1.2945233 & 1.29452 & 3870.934 & <.0001 \\ \text { Error } & 4 & 0.0013377 & 0.00033 & & \end{array}$ Error 0.00033 1.2958610

Means for Oneway Anova

Level Number Mean Std Error Lower 95\% Upper 95\%

$\begin{array}{llllll}\text { ccC } & 32.24655 & 0.01056 & 2.2172 & 2.2759\end{array}$

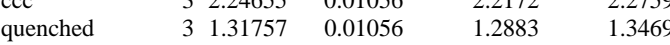

Std Error uses a pooled estimate of error variance
Oneway Analysis of $\log [\mathrm{Li} \mathrm{ppm}]$ By Heat Treatment Glass ID=EM-28

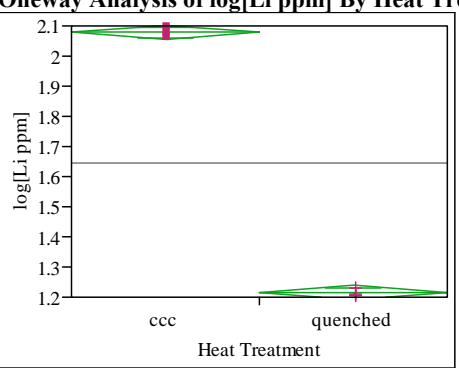

Oneway Anova

Summary of $\mathrm{Fit}$

Rsquare $\quad 0.999224$

Adj Rsquare $\quad 0.999031$

Root Mean Square Error $\quad 0.014711$

Mean of Response 1.646444

Observations (or Sum Wgts)

t Test

quenched-ccc

Assuming equal variances

Difference $\quad-0.86229$ t Ratio $\quad-71.7895$

$\begin{array}{llr}\text { Std Err Dif } & 0.01201 \text { DF } & 4 \\ \text { Upper CL Dif } & -0.82894 \text { Prob }>|t| & <.0001\end{array}$

$\begin{array}{lll}\text { Upper CL Dif } & -0.82894 \text { Prob }>|t| & <.0001 \\ \text { Lower CL Dif } & -0.89564 \text { Prob }>t & 1.0000\end{array}$

Confidence $\quad 0.95$ Prob $<\mathrm{t} \quad<.0001$

Analysis of Variance

Source DF Sum of Squares Mean Square F Ratio Prob $>$ F $\begin{array}{llllll}\text { Heat Treatment } & 1 & 1.1153221 & 1.11532 & 5153.727 & <.0001\end{array}$ Error 0.0008656

Means for Oneway Anova

Level Number Mean Std Error Lower 95\% Upper 95\%

$\begin{array}{llllll}\text { ccc } & 3 & 2.07759 & 0.00849 & 2.0540 & 2.1012 \\ \text { quenched } & 3 & 1.21530 & 0.00849 & 1.1917 & 1.2389\end{array}$

Std Error uses a pooled estimate of error variance

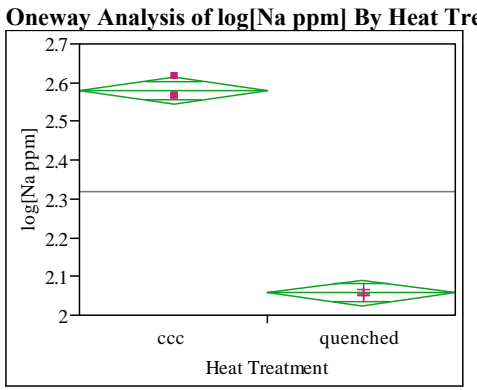

Oneway Anova

Summary of Fit

Rsquare

Adj Rsquare

0.020929

Root Mean Square Error $\quad 0.020929$

Mean of Response

6

t Tes

quenched-ccc

Assuming equal variance

Difference $\quad-0.52208$ t Ratio $\quad-30.5509$

Std Err Dif $\quad 0.01709 \mathrm{DF} \quad 4$

$\begin{array}{lll}\text { Upper CL Dif }-0.47463 & \text { Prob }>|t| & <.0001 \\ \text { Lower CL Dif }-0.56952 & \text { Prob }>t & 1.0000\end{array}$

$\begin{array}{lrl}\text { Confidence } & 0.95 \mathrm{Prob}<\mathrm{t} \quad<.0001\end{array}$

Analysis of Variance

Source DF Sum of Squares Mean Square F Ratio Prob $>$ F $\begin{array}{llll}\text { Heat Treatment } & 1 & 0.40884655 & 0.40884\end{array}$

$\begin{array}{llll}\text { Error } & 4 & 0.00175215 & 0.000438 \\ \text { C. Total } & 5 & 0.41059870 & \end{array}$

Means for Oneway Anova

Level Number Mean Std Error Lower 95\% Upper 95\% $\begin{array}{llllll}\text { ccC } & 3 & 2.57979 & 0.01208 & 2.5462 & 2.6133 \\ \text { quenched } & 3 & 2.05771 & 0.01208 & 2.0242 & 2.0913\end{array}$

Std Error uses a pooled estimate of error variance 
SRNL-STI-2009-00778, Revision 0

Exhibit E5. Effects of Heat Treatment (HT) on PCT $\log (\mathrm{ppm})$ Response of the Matrix 2 A Study Glasses

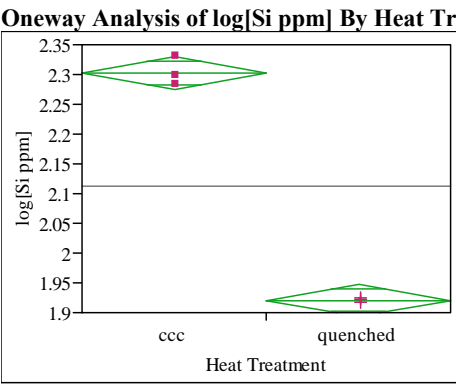

Oneway Anova

Summary of Fit

\begin{tabular}{|c|c|c|c|}
\hline Rsquare & & 0.994 & \\
\hline Adj Rsquare & & 0.993 & 367 \\
\hline Root Mean Sq & uare Error & 0.017 & 081 \\
\hline Mean of Respo & onse & 2.112 & 056 \\
\hline Observations ( & or Sum Wgt & & 6 \\
\hline $\begin{array}{l}\text { t Test } \\
\text { quenched-ccc }\end{array}$ & & & \\
\hline Assuming equa & al variances & & \\
\hline Difference & $-0.38191 \mathrm{t}$ & Ratio & -27.38 \\
\hline Std Err Dif & $0.01395 \mathrm{D}$ & DF & \\
\hline Upper CL Dif & $-0.34318 \mathrm{P}$ & $\operatorname{Prob}>|t|$ & $<.00$ \\
\hline Lower CL Dif & $-0.42063 \mathrm{P}$ & Prob $>t$ & 1.000 \\
\hline Confidence & 00 & De & \\
\hline
\end{tabular}

Analysis of Variance

Source DF Sum of Squares Mean Square F Ratio Prob $>$ $\begin{array}{llllll}\text { Heat Treatment } & 1 & 0.21877923 & 0.218779 & 749.8480 & <.0001\end{array}$

0.00116706

0.000292

Means for Oneway Anova

Level Number Mean Std Error Lower 95\% Upper 95\%

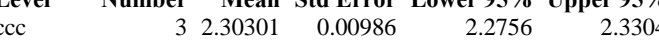

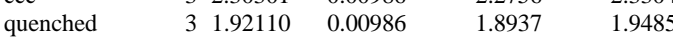

Std Error uses a pooled estimate of error variance
Oneway Analysis of $\log [\mathrm{B}$ ppm $]$ By Heat Treatment Glass ID=EM-29

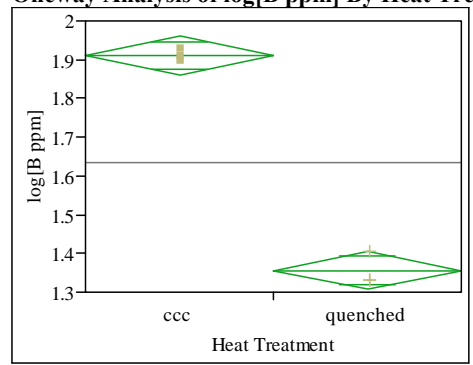

Oneway Anova

Summary of Fit

$\begin{array}{ll}\text { Rsquare } & 0.991525 \\ \text { Adj Rsquare } & 0.989406\end{array}$

Adj Rsquare

Mean of Response 1.633132

Observations (or Sum Wgts)

t Test

quenched-ccc

Assuming equal variances

Difference $\quad-0.55387$ t Ratio $\quad-21.6326$

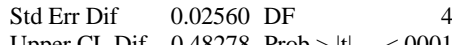

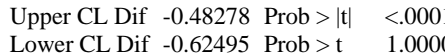

$\begin{array}{lll}\text { Confidence } & 0.95 \mathrm{Prob}<\mathrm{t} \quad<.0001\end{array}$

Analysis of Variance

Source DF Sum of Squares Mean Square F Ratio Prob $>$ F $\begin{array}{llllll}\text { Heat Treatment } & 1 & 0.46015428 & 0.460154 & 467.9696 & <.0001 \\ \text { Error } & 4 & 0.00393320 & 0.000983 & \end{array}$ Error

\section{Means for Oneway Anova}

Level Number Mean Std Error Lower 95\% Upper 95\%

$\begin{array}{llllll}\text { ccc } & 3 & 1.91007 & 0.01810 & 1.8598 & 1.9603 \\ \text { quenched } & 3 & 1.35620 & 0.01810 & 1.3059 & 1.4065\end{array}$

Std Error uses a pooled estimate of error variance

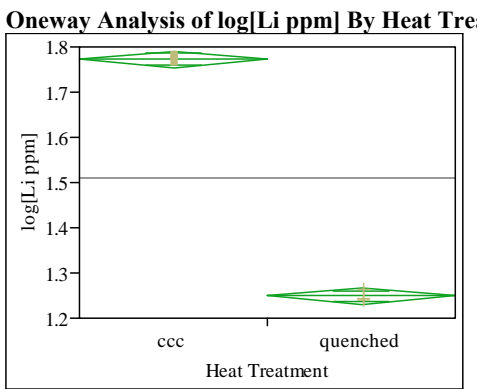

Oneway Anova

Summary of Fit

$\begin{array}{lr}\text { Rsquare } & 0.998738 \\ \text { Adj Rsquare } & 0.998423 \\ \text { Root Mean Square Error } & 0.011415 \\ \text { Mean of Response } & 1.510614 \\ \text { Observations (or Sum Wgts) } & 6\end{array}$

t Test

quenched-ccc

Assuming equal variance

Difference $\quad-0.52444$ t Ratio $\quad-56.2685$

Std Err Dif $\quad 0.00932$ DF $\quad 4$

$\begin{array}{lll}\text { Upper CL Dif } & -0.49856 \text { Prob }>|t| & <.0001 \\ \text { Lower CL Dif }-0.55031 & \text { Prob }>t & 1.0000\end{array}$

$\begin{array}{lr}\text { Confidence } & 0.95 \text { Prob }<\mathrm{t} \quad<.0001\end{array}$

Analysis of Variance

Source DF Sum of Squares Mean Square F Ratio Prob $>$ F $\begin{array}{lllllll}\text { Heat Treatment } & 1 & 0.41254850 & 0.412548 & 3166.139 & <.0001\end{array}$

$\begin{array}{llll}\text { Error } & 4 & 0.00052120 & 0.000130 \\ \text { C. Total } & 5 & 0.41306970 & \end{array}$

Means for Oneway Anova

Level Number Mean Std Error Lower 95\% Upper 95\%

$\begin{array}{llllll}\text { ccc } & 3 & 1.77283 & 0.00659 & 1.7545 & 1.7911 \\ \text { quenched } & 3 & 1.24840 & 0.00659 & 1.2301 & 1.2667\end{array}$

Std Error uses a pooled estimate of error variance 
SRNL-STI-2009-00778, Revision 0

Exhibit E5. Effects of Heat Treatment (HT) on PCT $\log (\mathrm{ppm})$ Response of the Matrix 2 A Study Glasses

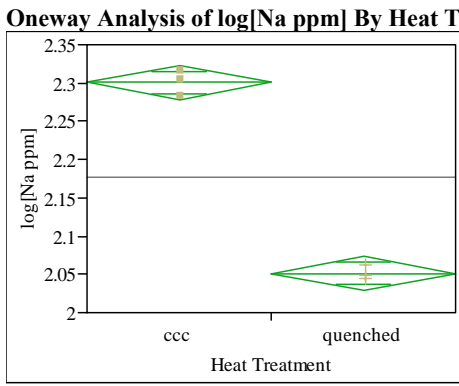

Oneway Anova

Summary of Fit

\begin{tabular}{|c|c|c|c|}
\hline \multicolumn{4}{|c|}{0.992088} \\
\hline Adj Rsquare & & 0.99 & 011 \\
\hline Root Mean Sq & uare Error & 0.013 & \\
\hline Mean of Respo & onse & 2.175 & 991 \\
\hline Observations ( & or Sum Wgt & & 6 \\
\hline $\begin{array}{l}\text { t Test } \\
\text { quenched-ccc }\end{array}$ & & & \\
\hline Assuming equa & al variances & & \\
\hline Difference & $-0.24895 \mathrm{t}$ & Ratio & -22.395 \\
\hline Std Err Dif & $0.01112 \mathrm{D}$ & DF & \\
\hline Upper CL Dif & $-0.21809 \mathrm{P}$ & $\operatorname{Prob}>|t|$ & $<.00$ \\
\hline Lower CL Dif & $-0.27981 \mathrm{P}$ & Prob $>t$ & 1.00 \\
\hline Confidence & 0.95 & $\mathrm{bb}<\mathrm{t}$ & \\
\hline
\end{tabular}

Analysis of Varia

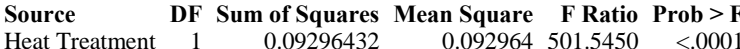

4

0.09296432

0.09370575

0.000185

Means for Oneway Anova

Level Number Mean Std Error Lower 95\% Upper 95\%

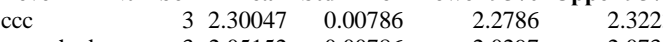

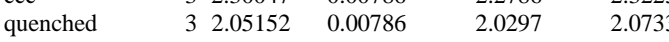

Std Error uses a pooled estimate of error variance

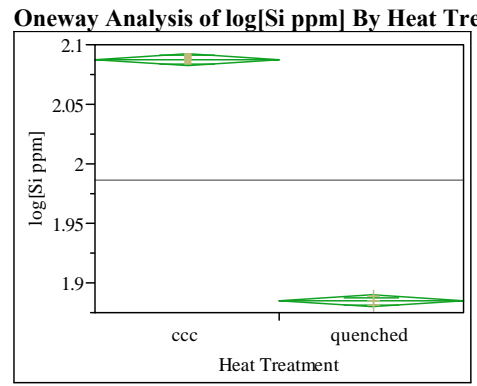

Oneway Anova

Summary of Fit

$\begin{array}{lr}\text { Rsquare } & 0.999397 \\ \text { Adj Rsquare } & 0.999247 \\ \text { Root Mean Square Error } & 0.003049 \\ \text { Mean of Response } & 1.985979 \\ \text { Observations (or Sum Wgts) } & 6\end{array}$

Observations (or Sum Wgts)

t Test

quenched-ccc

Assuming equal variances

Difference $\quad-0.20275$ t Ratio $\quad-81.4538$

$\begin{array}{lrl}\text { Std Err Dif } & 0.00249 \text { DF } & 4 \\ \text { Upper CL Dif } & -0.19584 \text { Prob }>|t| & <.0001\end{array}$

$\begin{array}{lll}\text { Upper CL Dif } & -0.19584 \text { Prob }>|t| & <.0001 \\ \text { Lower CL Dif } & -0.20966 \text { Prob }>t & 1.0000\end{array}$

$\begin{array}{lll}\text { Confidence } & 0.95 \text { Prob }<\mathrm{t} \quad<.0001\end{array}$

Analysis of Variance

Source DF Sum of Squares Mean Square F Ratio Prob $>$ F $\begin{array}{lllllll}\text { Heat Treatment } & 1 & 0.06166048 & 0.061660 & 6634.716 & <.0001\end{array}$

Error

Means for Oneway Anova

Level Number Mean Std Error Lower 95\% Upper 95\%

$\begin{array}{lll}\text { CCC } & 32.08735 & 0.00176\end{array}$

$\begin{array}{llllll}\text { quenched } & 3 & 1.88460 & 0.00176 & 1.8797 & 1.8895\end{array}$

Std Error uses a pooled estimate of error variance

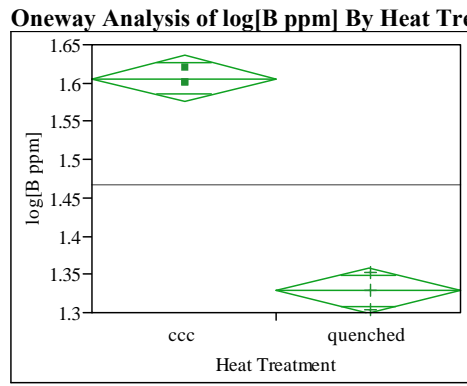

Oneway Anova

Summary of Fit

$\begin{array}{lr}\text { Rsquare } & 0.98852 \\ \text { Adj Rsquare } & 0.98565 \\ \text { Root Mean Square Error } & 0.018316 \\ \text { Mean of Response } & 1.467411 \\ \text { Observations (or Sum Wgts) } & 6\end{array}$

quenched-ccc

Assuming equal variances

Difference $\quad-0.27756$ t Ratio $\quad-18.559$

Std Err Dif 0.01496 DF

Upper CL Dif -0.23603 Prob $>|t|<.0001$

Lower CL Dif -0.31908 Prob $>t \quad 1.0000$

Confidence $\quad 0.95$ Prob $<\mathrm{t} \quad<.0001$

Analysis of Variance

Source DF Sum of Squares Mean Square $F$ Ratio Prob $>$ F $\begin{array}{llllll}\text { Heat Treatment } & 1 & 0.11555546 & 0.115555 & 344.4358 & <.0001\end{array}$ $\begin{array}{lll}\text { Error } & 4 & 0.00134197 \\ \text { C. Total } & 5 & 0.11689742\end{array}$ 0.000335

Means for Oneway Anova

Level Number Mean Std Error Lower 95\% Upper 95\%

$\begin{array}{llllll}\text { cCC } & 3 & 1.60619 & 0.01058 & 1.5768 & 1.6356\end{array}$

$\begin{array}{llllll}\text { quenched } & 3 & 1.32863 & 0.01058 & 1.2993 & 1.3580\end{array}$

Std Error uses a pooled estimate of error variance 
SRNL-STI-2009-00778, Revision 0

Exhibit E5. Effects of Heat Treatment (HT) on PCT $\log (\mathrm{ppm})$ Response of the Matrix 2 A Study Glasses

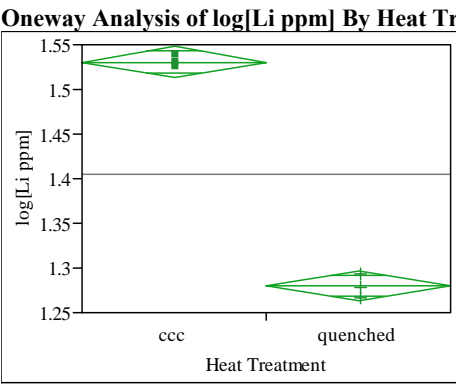

Oneway Anova

Summary of Fit

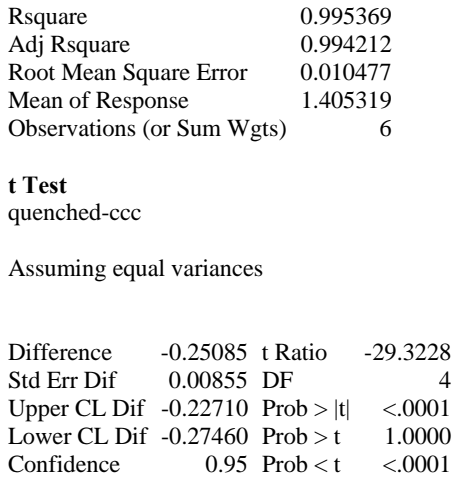

Analysis of Variance

Source DF Sum of Squares Mean Square F Ratio Prob $>$ F $\begin{array}{llllll}\text { Heat Treatment } & 1 & 0.09438924 & 0.094389 & 859.8276 & <.0001\end{array}$

$$
0.00043911
$$

0.09482834

0.000110

Means for Oneway Anova

Level Number Mean Std Error Lower 95\% Upper 95\%

$\begin{array}{llllll}\text { ccC } & 3 & 1.53074 & 0.00605 & 1.5139 & 1.5475\end{array}$

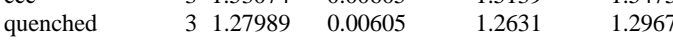

Std Error uses a pooled estimate of error variance

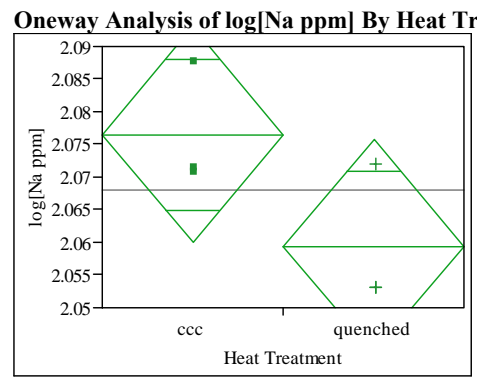

Oneway Anova

Summary of Fit

Rsquare $\quad 0.512523$

Adj Rsquare $\quad 0.39065$

Root Mean Square Error $\quad 0.010238$

Mean of Response
Observations (or Sum Wgts)

t Test

quenched-ccc

Assuming equal variances

Difference $\quad-0.01714$ t Ratio $\quad-2.05073$

Std Err Dif $\quad 0.00836$ DF

Upper CL Dif 0.00607 Prob $>|t| 0.1096$

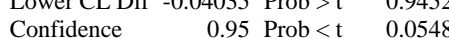

Analysis of Variance

Source DF Sum of Squares Mean Square F Ratio Prob $>$ F $\begin{array}{llllll}\text { Heat Treatment } & 1 & 0.00044080 & 0.000441 & 4.2055 & 0.1096\end{array}$ Error 0.00086006

Means for Oneway Anova

Level Number Mean Std Error Lower 95\% Upper 95\%

$\begin{array}{llllll}\text { ccC } & 3 & 2.07650 & 0.00591 & 2.0601 & 2.0929\end{array}$

Std Error uses a pooled estimate of error variance

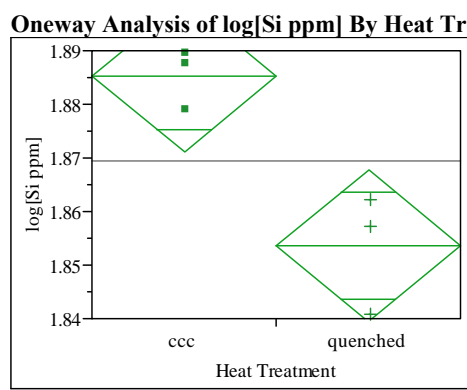

Oneway Anova

Summary of Fit

$\begin{array}{lr}\text { Rsquare } & 0.828401 \\ \text { Adj Rsquare } & 0.785501 \\ \text { Root Mean Square Error } & 0.008829 \\ \text { Mean of Response } & 1.869382 \\ \text { Observations (or Sum Wgts) } & 6\end{array}$

quenched-ccc

Assuming equal variances

Difference $\quad-0.03168$ t Ratio $\quad-4.39434$

Std Err Dif 0.00721 DF

Upper CL Dif -0.01166 Prob $>|t| \quad 0.0117$

Lower CL Dif -0.05169 Prob $>t \quad 0.9941$

Confidence $\quad 0.95$ Prob $<\mathrm{t} \quad 0.0059$

Analysis of Variance

Source DF Sum of Squares Mean Square F Ratio Prob $>$ F $\begin{array}{llllll}\text { Heat Treatment } & 1 & 0.00150516 & 0.001505 & 19.3102 & 0.0117\end{array}$ $\begin{array}{lllll}\text { Error } & 4 & 0.00031179 & 0.000078\end{array}$ $\begin{array}{lll}\text { C. Total } & 5 & 0.00181694\end{array}$

Means for Oneway Anova

Level Number Mean Std Error Lower 95\% Upper 95\%

$\begin{array}{llllll}\text { cCC } & 3 & 1.88522 & 0.00510 & 1.8711 & 1.8994\end{array}$

$\begin{array}{llllll}\text { quenched } & 3 & 1.85354 & 0.00510 & 1.8394 & 1.8677\end{array}$

Std Error uses a pooled estimate of error variance 
Exhibit E6. Effects of Heat Treatment for the Matrix 2A Study Glasses by Compositional View

Comp View=Measured

Variability Chart for $\log$ NL[B (g/L)]

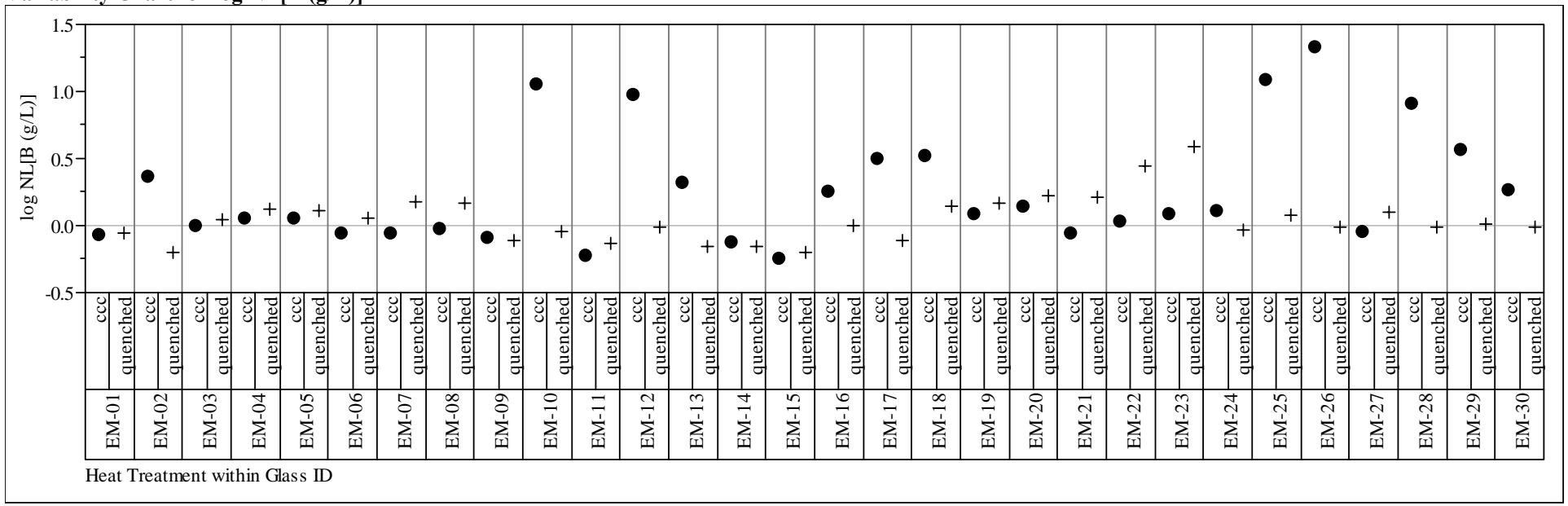

Comp View=Measured

Variability Chart for $\log \mathrm{NL}[\mathrm{Li}(\mathrm{g} / \mathrm{L})]$

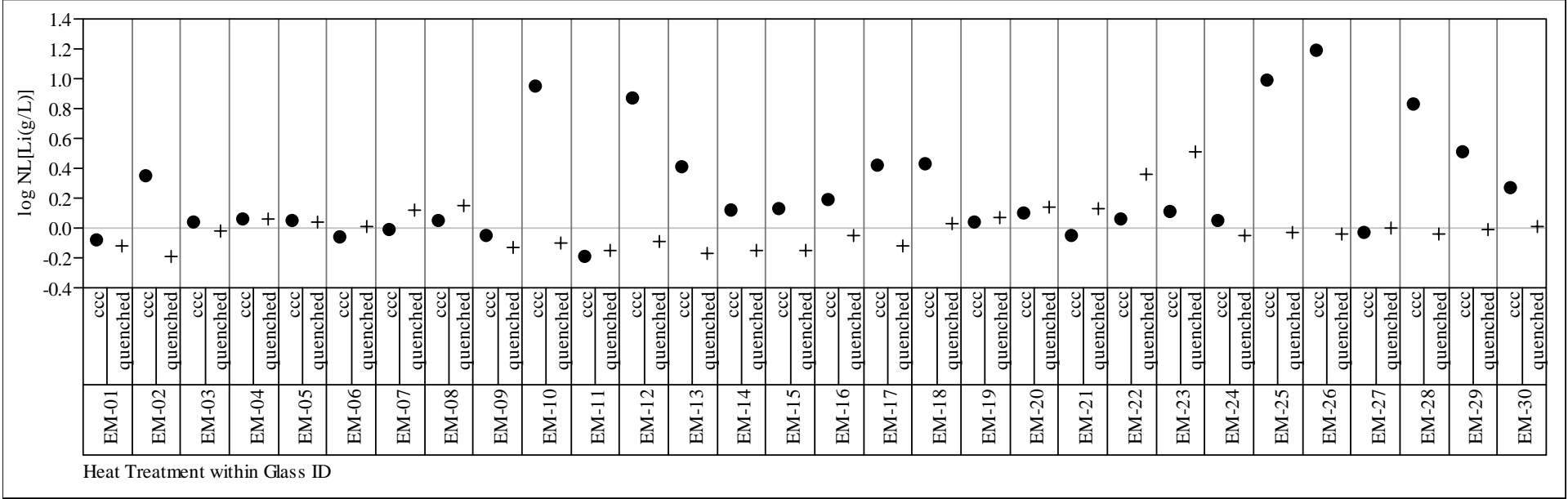


Exhibit E6. Effects of Heat Treatment for the Matrix 2A Study Glasses by Compositional View

Comp View=Measured

Variability Chart for log NL[Na (g/L)]

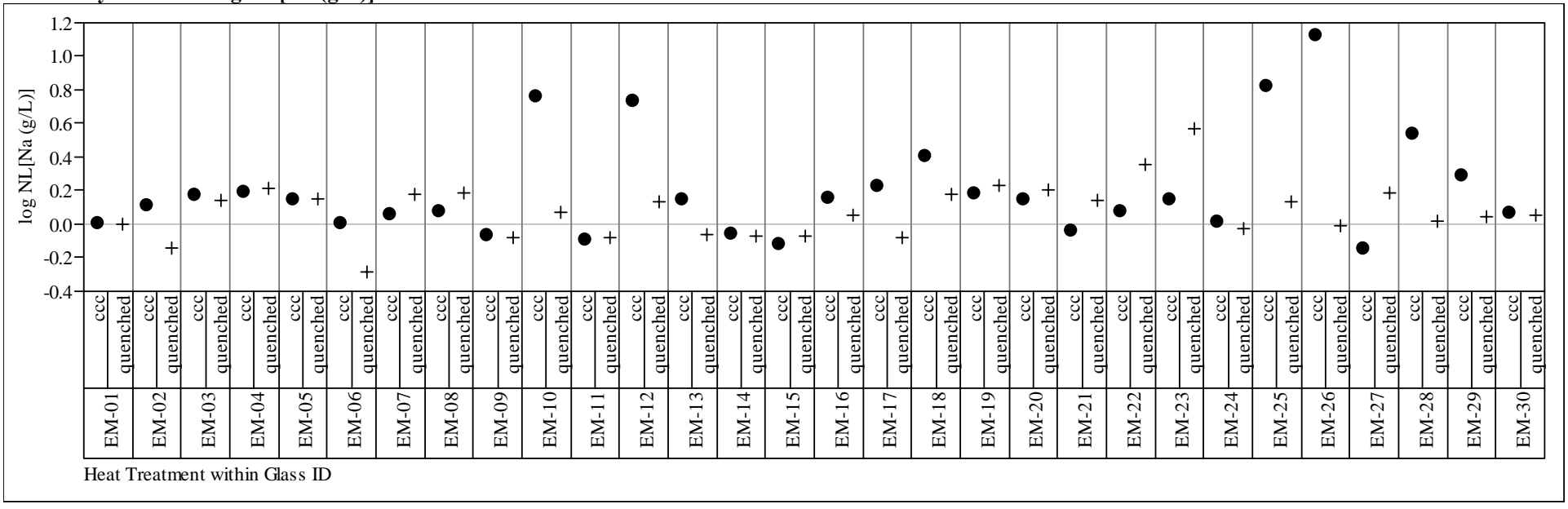

Comp View=Measured

Variability Chart for $\log \mathrm{NL}[\mathrm{Si}(\mathrm{g} / \mathrm{L})$

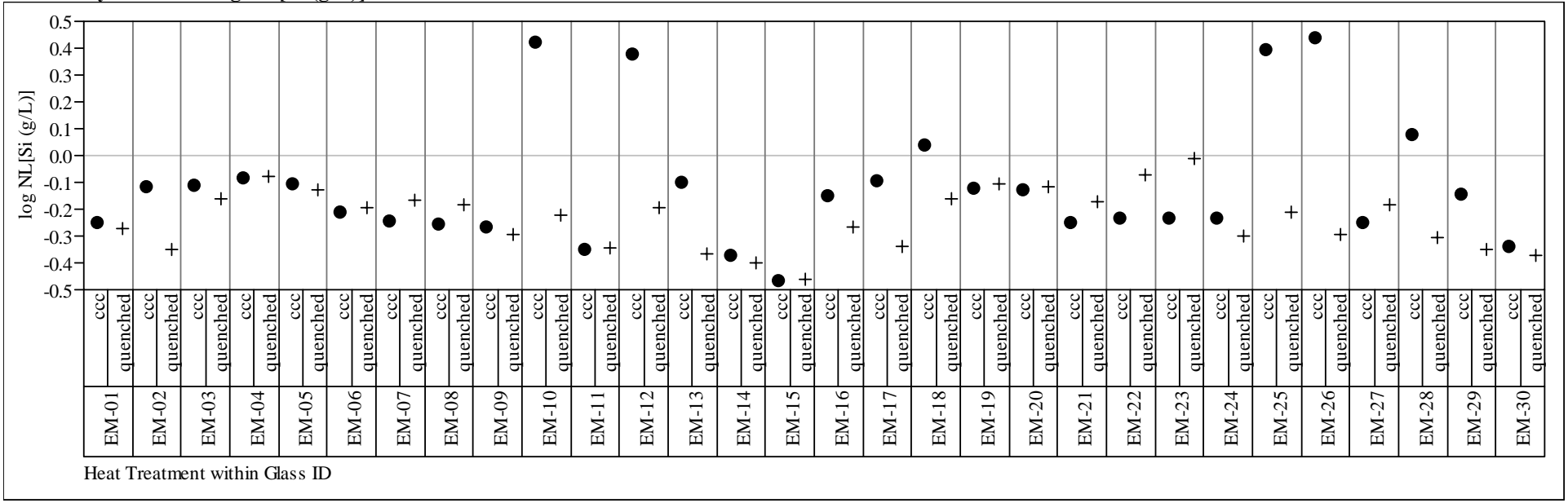


Exhibit E6. Effects of Heat Treatment for the Matrix 2A Study Glasses by Compositional View

Comp View=Measured bc

Variability Chart for $\log$ NL[B (g/L)]

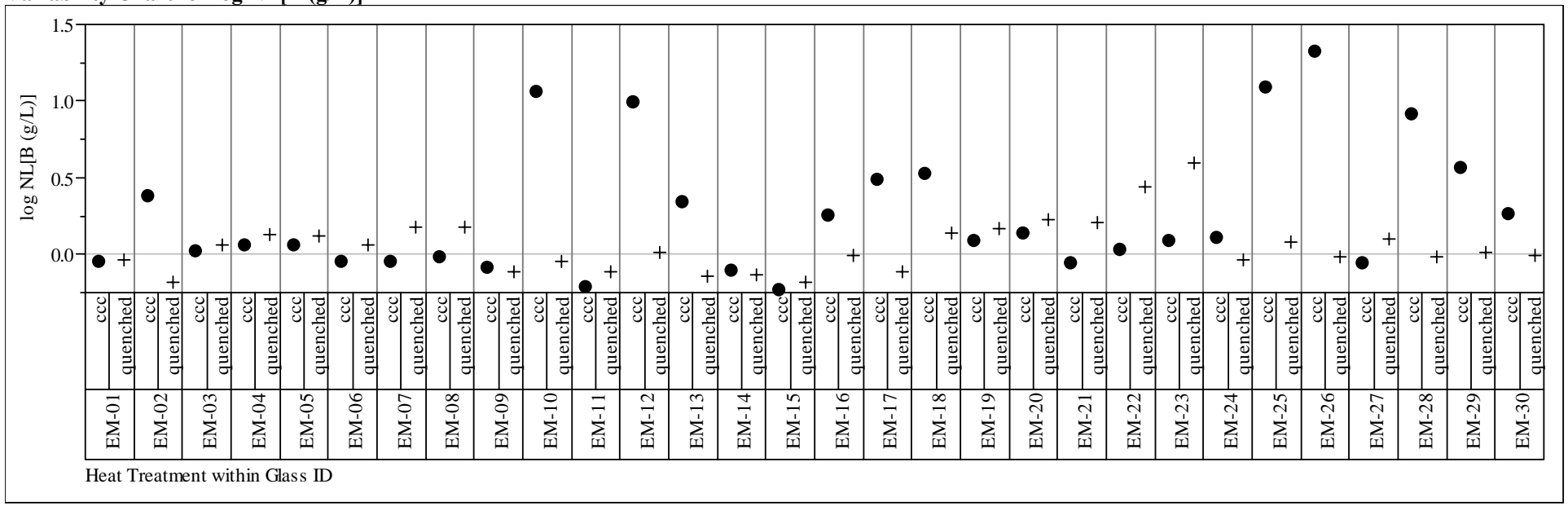

Comp View=Measured bc

Variability Chart for $\log \mathrm{NL}[\mathrm{Li}(\mathrm{g} / \mathrm{L})]$

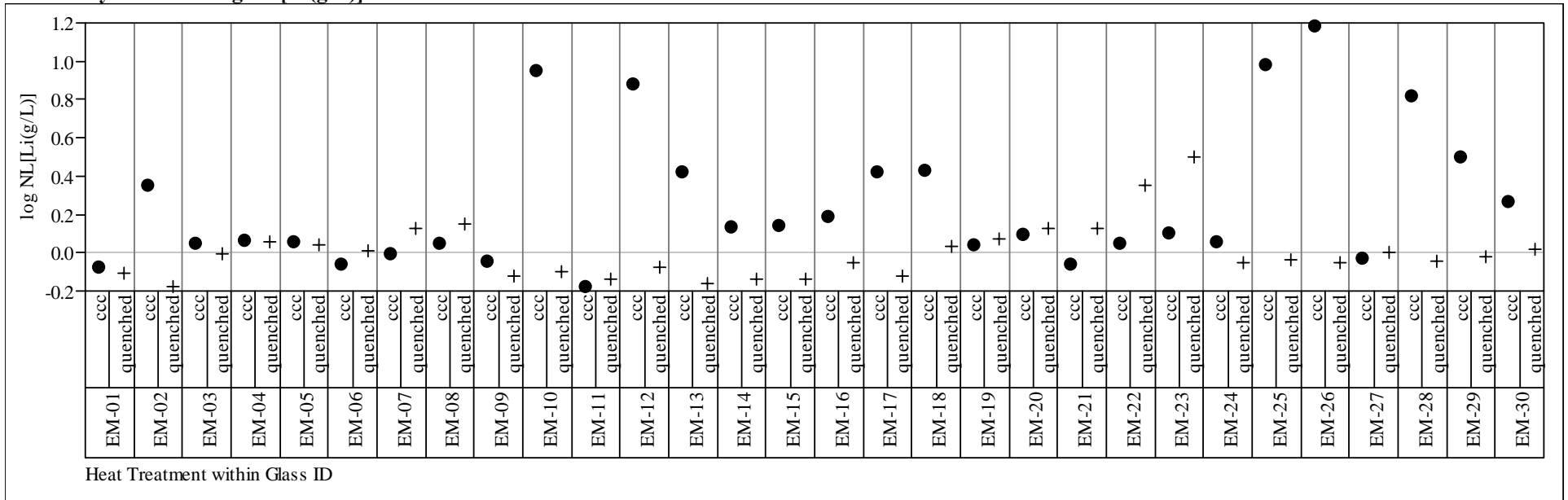


Exhibit E6. Effects of Heat Treatment for the Matrix 2A Study Glasses by Compositional View

Comp View=Measured bc

Variability Chart for log NL[Na (g/L)]

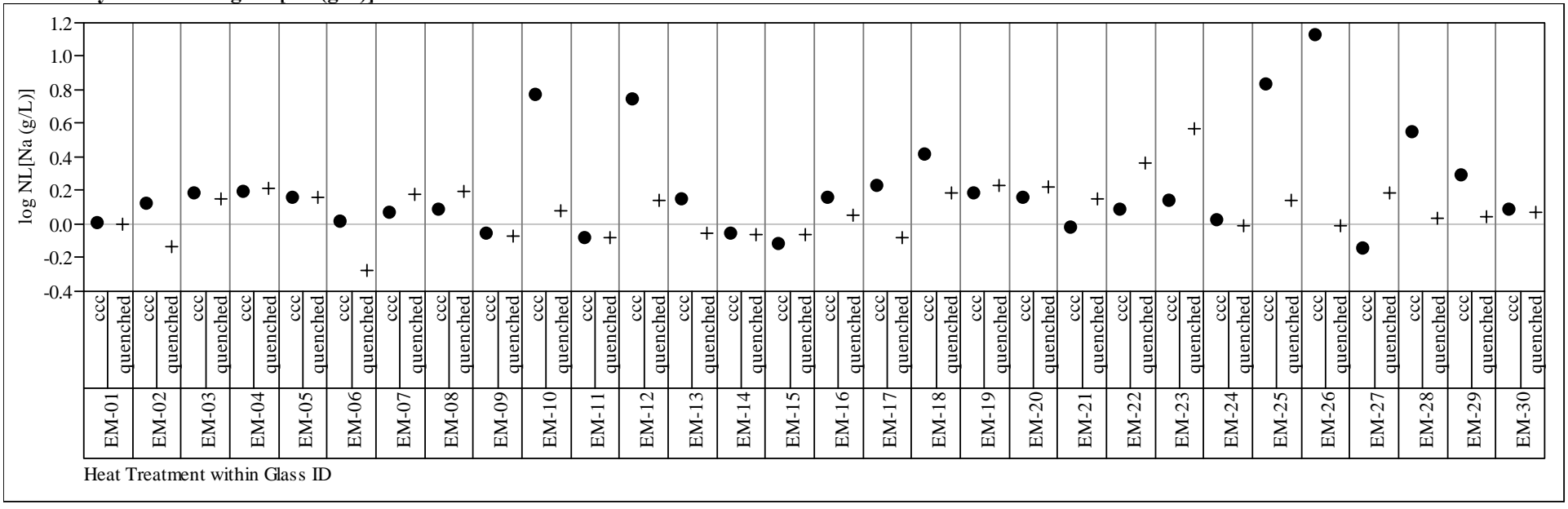

Comp View=Measured bc

Variability Chart for $\log \mathrm{NL}[\mathrm{Si}(\mathrm{g} / \mathrm{L})]$

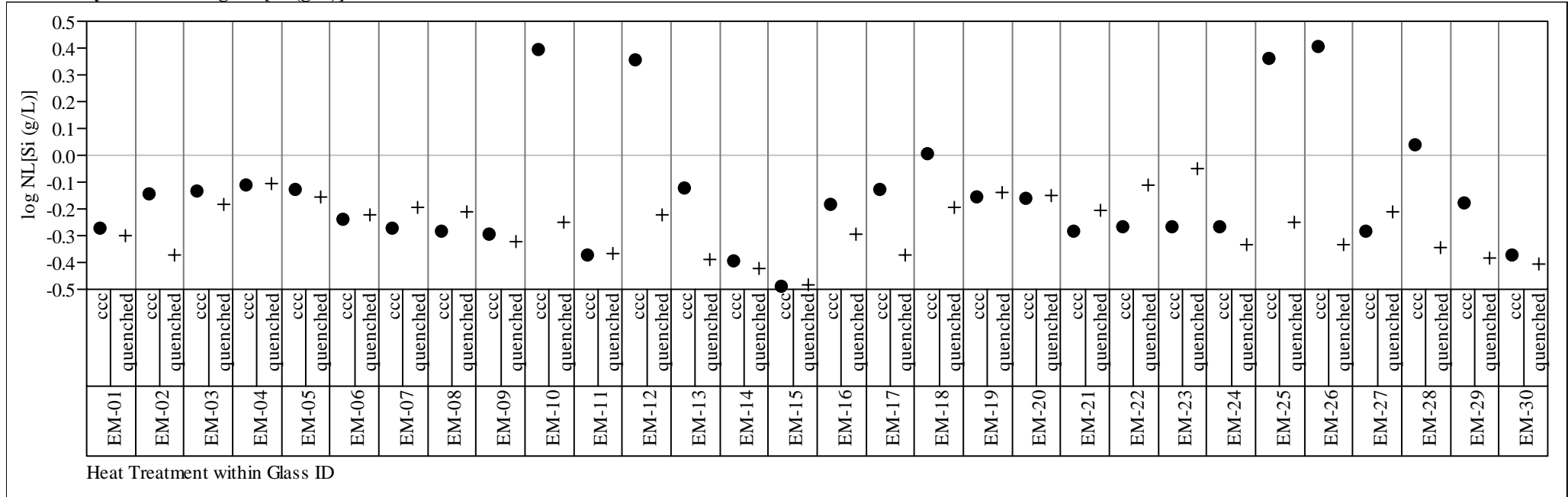


Exhibit E6. Effects of Heat Treatment for the Matrix 2A Study Glasses by Compositional View

Comp View=Targeted

Variability Chart for $\log \mathrm{NL}[\mathrm{B}(\mathrm{g} / \mathrm{L})]$

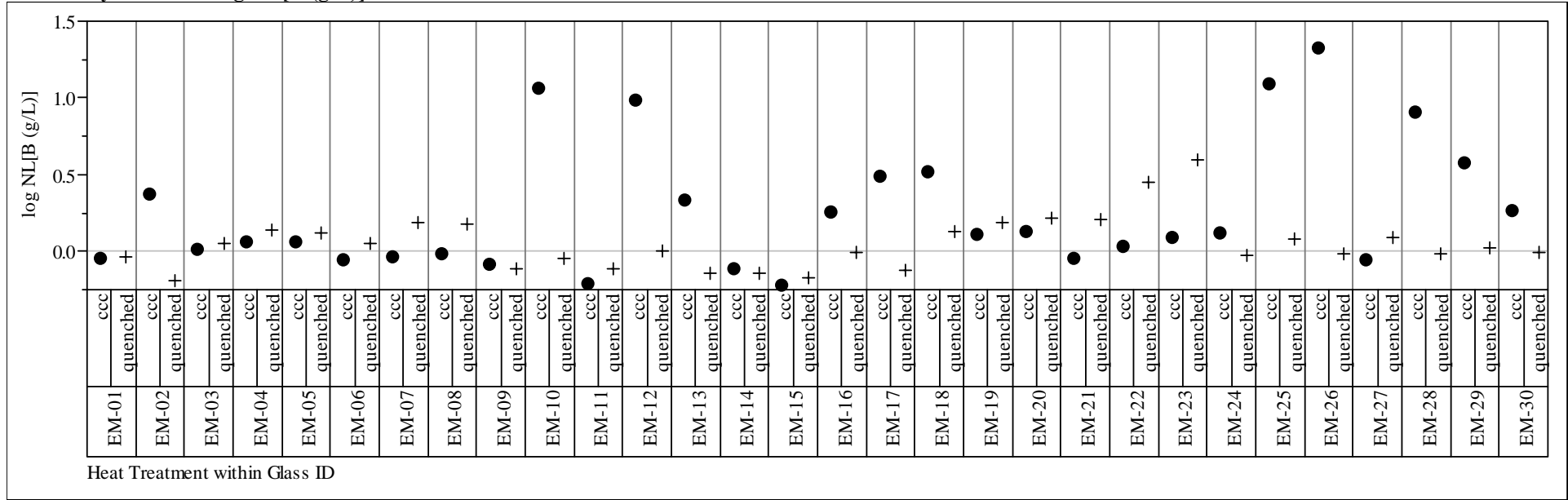

Comp View=Targeted

Variability Chart for $\log \mathrm{NL}[\mathrm{Li}(\mathrm{g} / \mathrm{L})]$

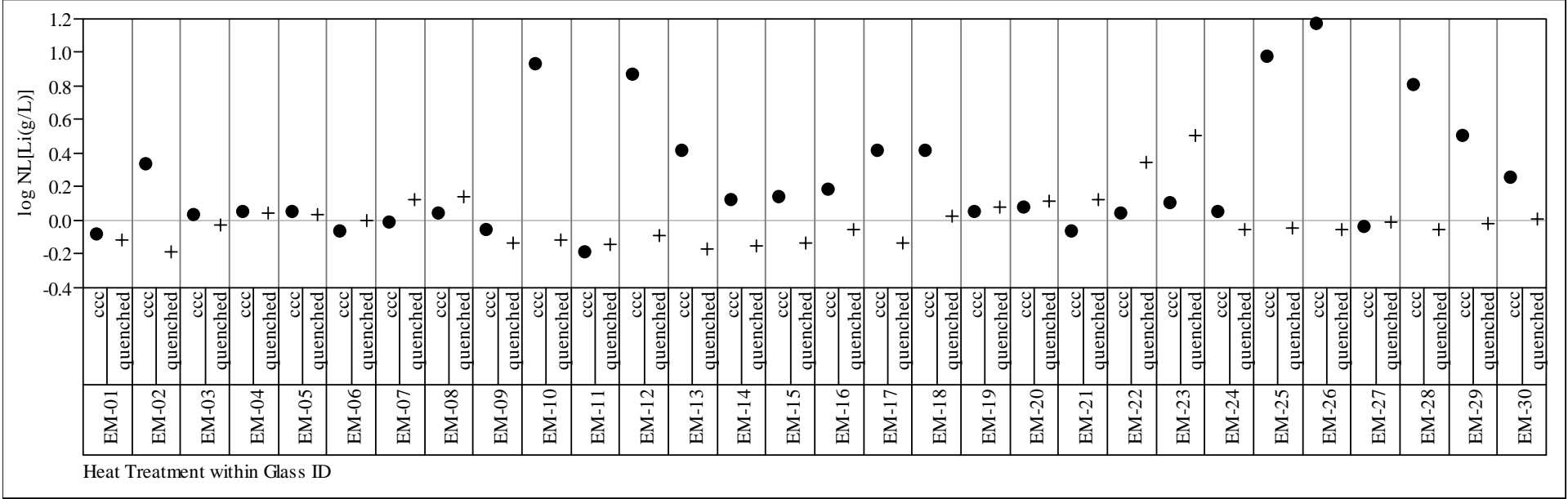


Exhibit E6. Effects of Heat Treatment for the Matrix 2A Study Glasses by Compositional View

Comp View=Targeted

Variability Chart for log NL[Na (g/L)]

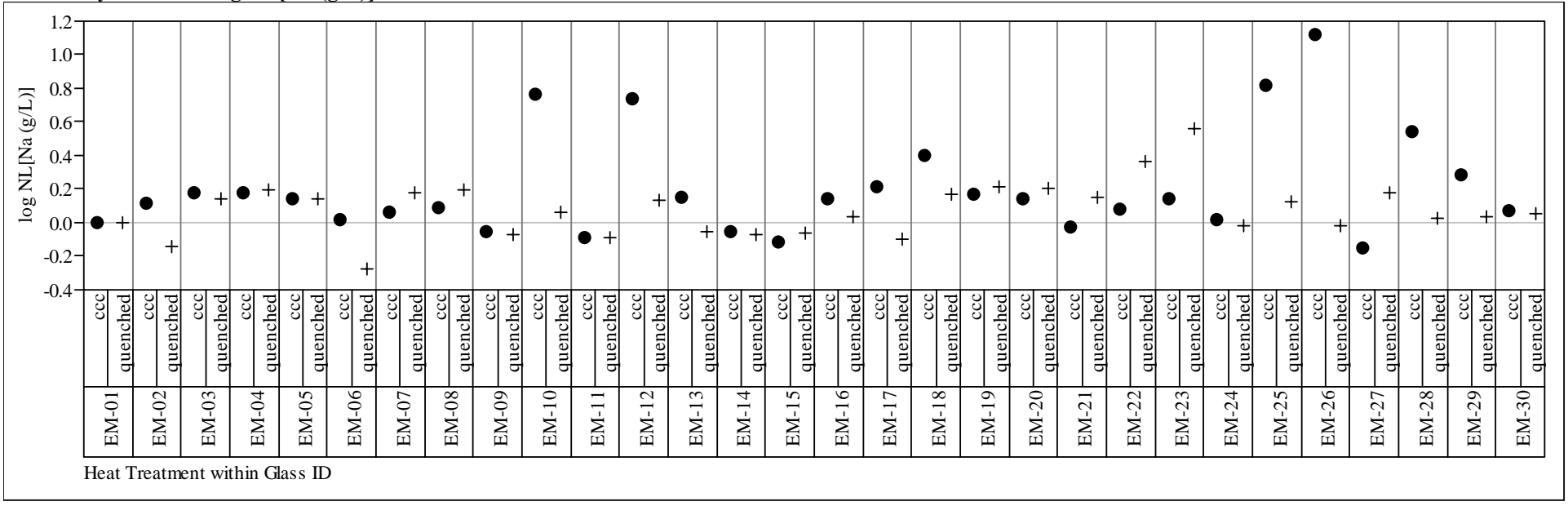

Comp View=Targeted

Variability Chart for $\log \mathrm{NL}[\mathrm{Si}(\mathrm{g} / \mathrm{L})]$

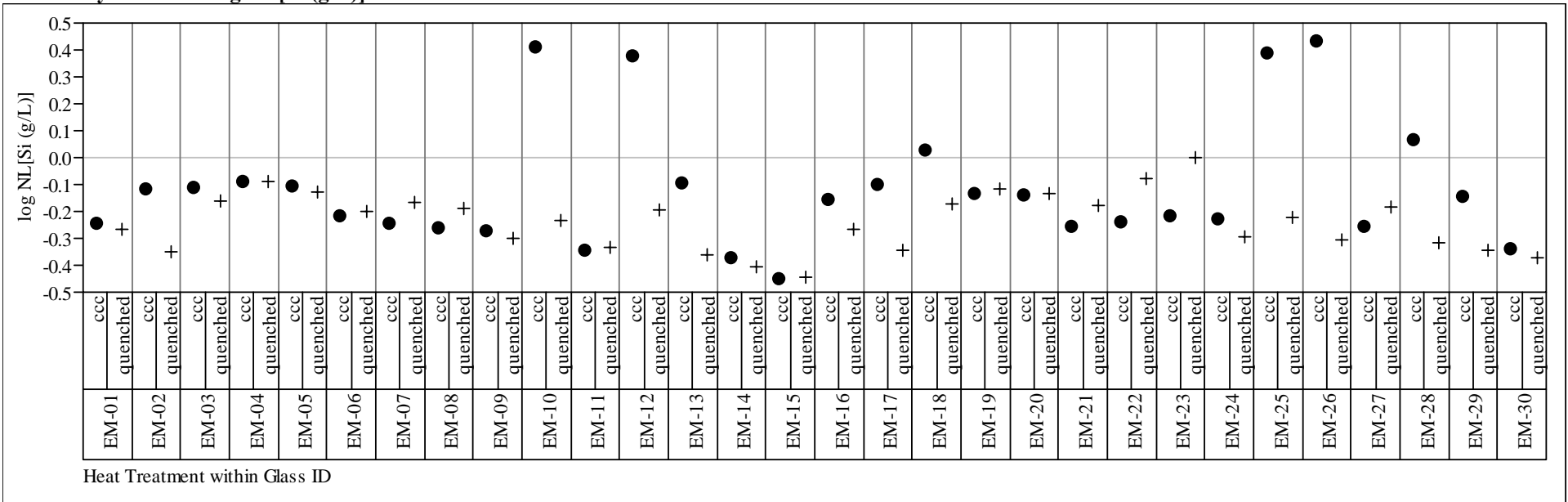


Exhibit E7. del $G p\left(\Delta G_{p}\right)$ Predictions versus Common Logarithm Normalized Leachate (log NL[.]) for B over All Compositional Views and Heat Treatments for the Matrix 2A Study Glasses

\begin{tabular}{|c|c|}
\multicolumn{1}{c}{ Legend } \\
\hline Symbol & $\begin{array}{c}\text { Standard/ } \\
\text { Comp View-Heat Treatment }\end{array}$ \\
\hline $\mathrm{z}$ & EA \\
\hline$\diamond$ & ARM \\
\hline$\square$ & Measured-ccc \\
\hline$\square$ & Measured bc -ccc \\
\hline$\square$ & Targeted-ccc \\
\hline$\bullet$ & Measured-quenched \\
\hline$\bullet$ & Measured bc - quenched \\
\hline$\bullet$ & Targeted- quenched \\
\hline
\end{tabular}

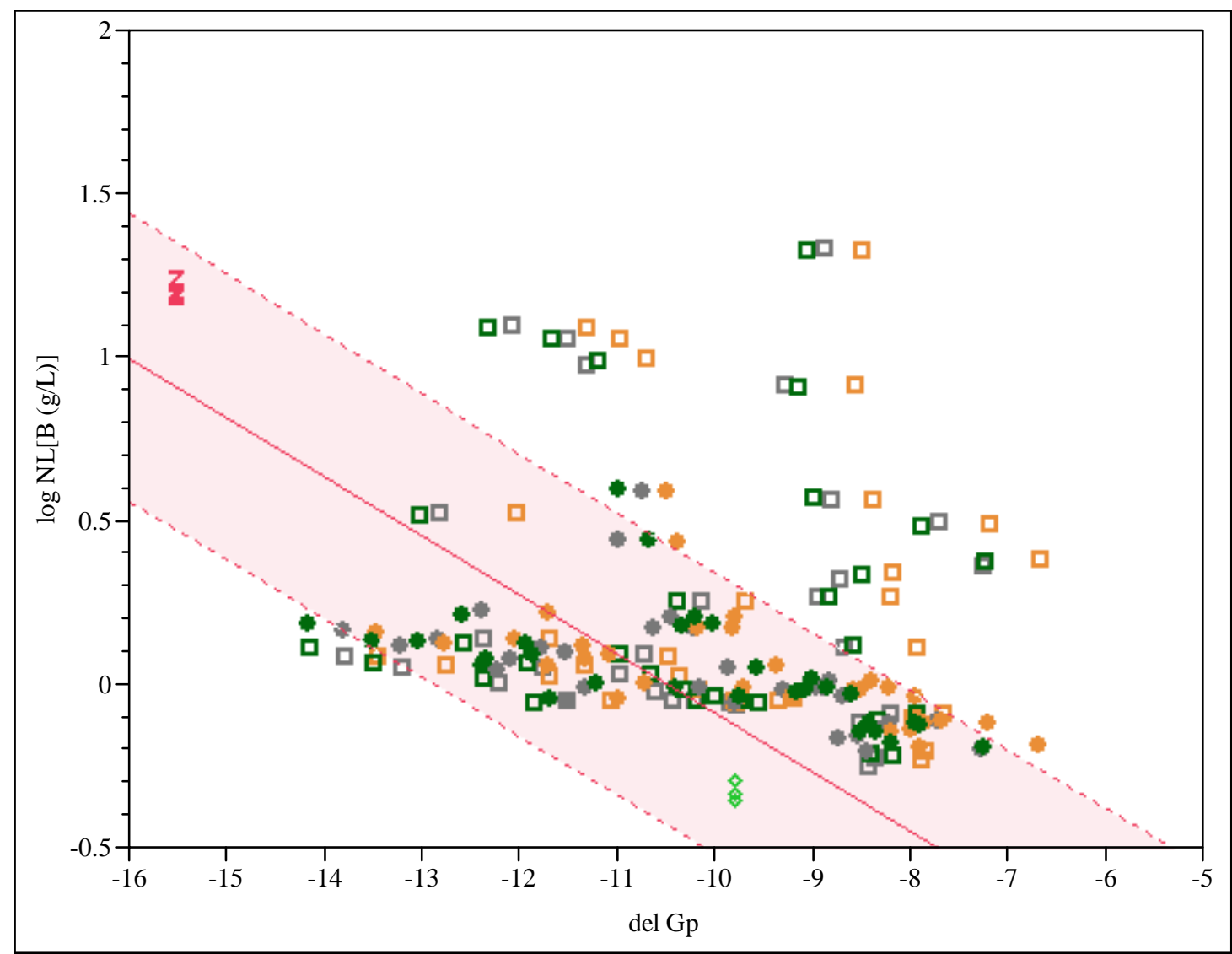


Exhibit E6. Effects of Heat Treatment for the Matrix 2A Study Glasses by Compositional View

Exhibit E8. del $G p\left(\Delta G_{p}\right)$ Predictions versus Common Logarithm Normalized Leachate $(\log \mathrm{NL}[]$.$) for Li over All Compositional Views and Heat Treatments for$ the Matrix 2A Study Glasses

\begin{tabular}{|c|c|}
\multicolumn{1}{c}{ Legend } \\
\hline Symbol & $\begin{array}{c}\text { Standard/ } \\
\text { Comp View-Heat Treatment }\end{array}$ \\
\hline $\mathrm{z}$ & EA \\
\hline$\diamond$ & ARM \\
\hline$\square$ & Measured-ccc \\
\hline$\square$ & Measured bc -ccc \\
\hline$\square$ & Targeted-ccc \\
\hline$\bullet$ & Measured-quenched \\
\hline$\bullet$ & Measured bc - quenched \\
\hline$\bullet$ & Targeted- quenched \\
\hline
\end{tabular}

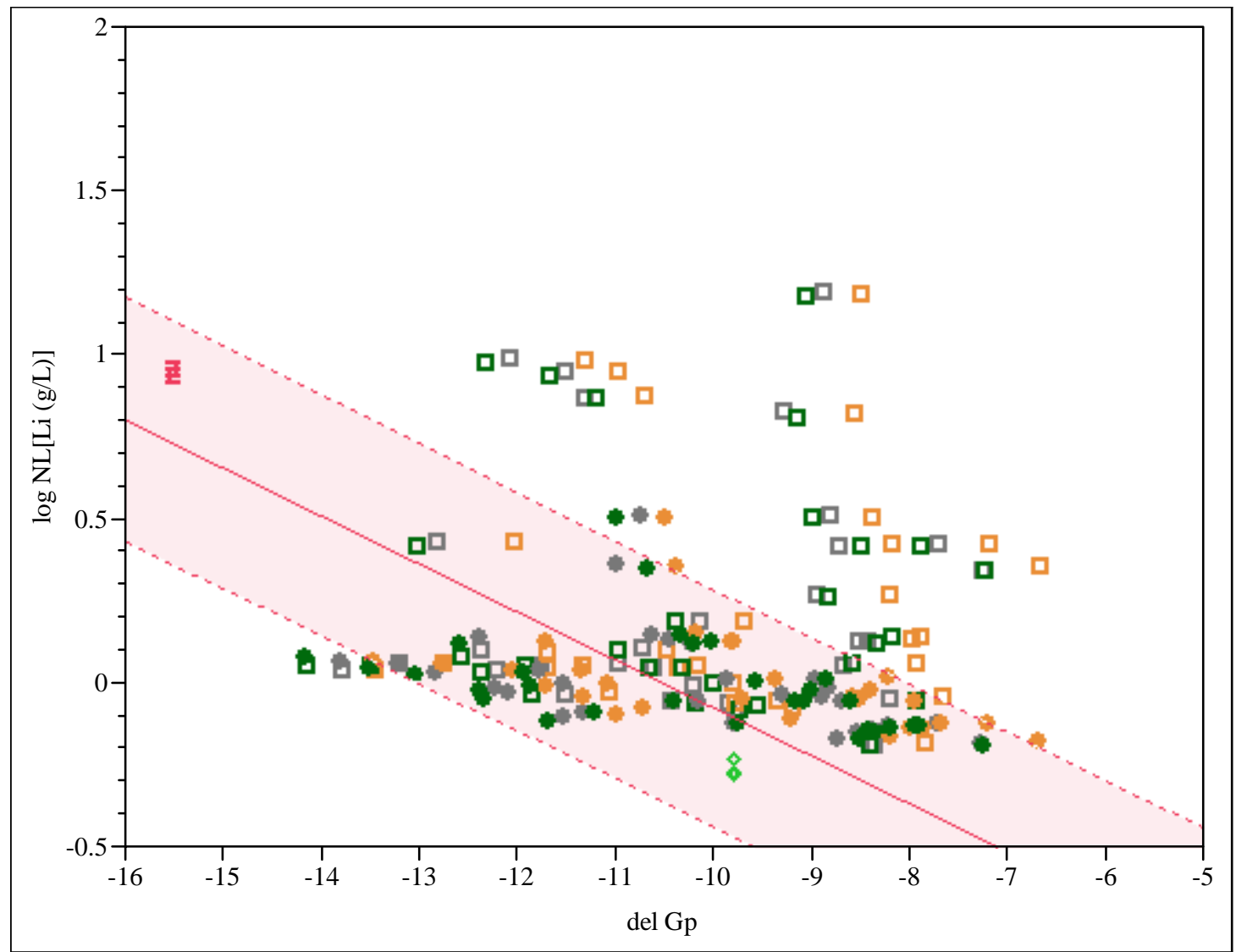


Exhibit E6. Effects of Heat Treatment for the Matrix 2A Study Glasses by Compositional View

Exhibit E9. del $\mathbf{G p}\left(\Delta \mathbf{G}_{\mathrm{p}}\right)$ Predictions versus Common Logarithm Normalized Leachate $(\log \mathrm{NL}[]$.$) for \mathrm{Na}$ over All Compositional Views and Heat Treatments for the Matrix 2A Study Glasses

\begin{tabular}{|c|c|}
\multicolumn{1}{c}{ Legend } \\
\hline Symbol & $\begin{array}{c}\text { Standard/ } \\
\text { Comp View-Heat Treatment }\end{array}$ \\
\hline $\mathrm{z}$ & EA \\
\hline$\diamond$ & ARM \\
\hline$\square$ & Measured-ccc \\
\hline$\square$ & Measured bc -ccc \\
\hline$\square$ & Targeted-ccc \\
\hline$\bullet$ & Measured-quenched \\
\hline$\bullet$ & Measured bc - quenched \\
\hline$\bullet$ & Targeted- quenched \\
\hline
\end{tabular}

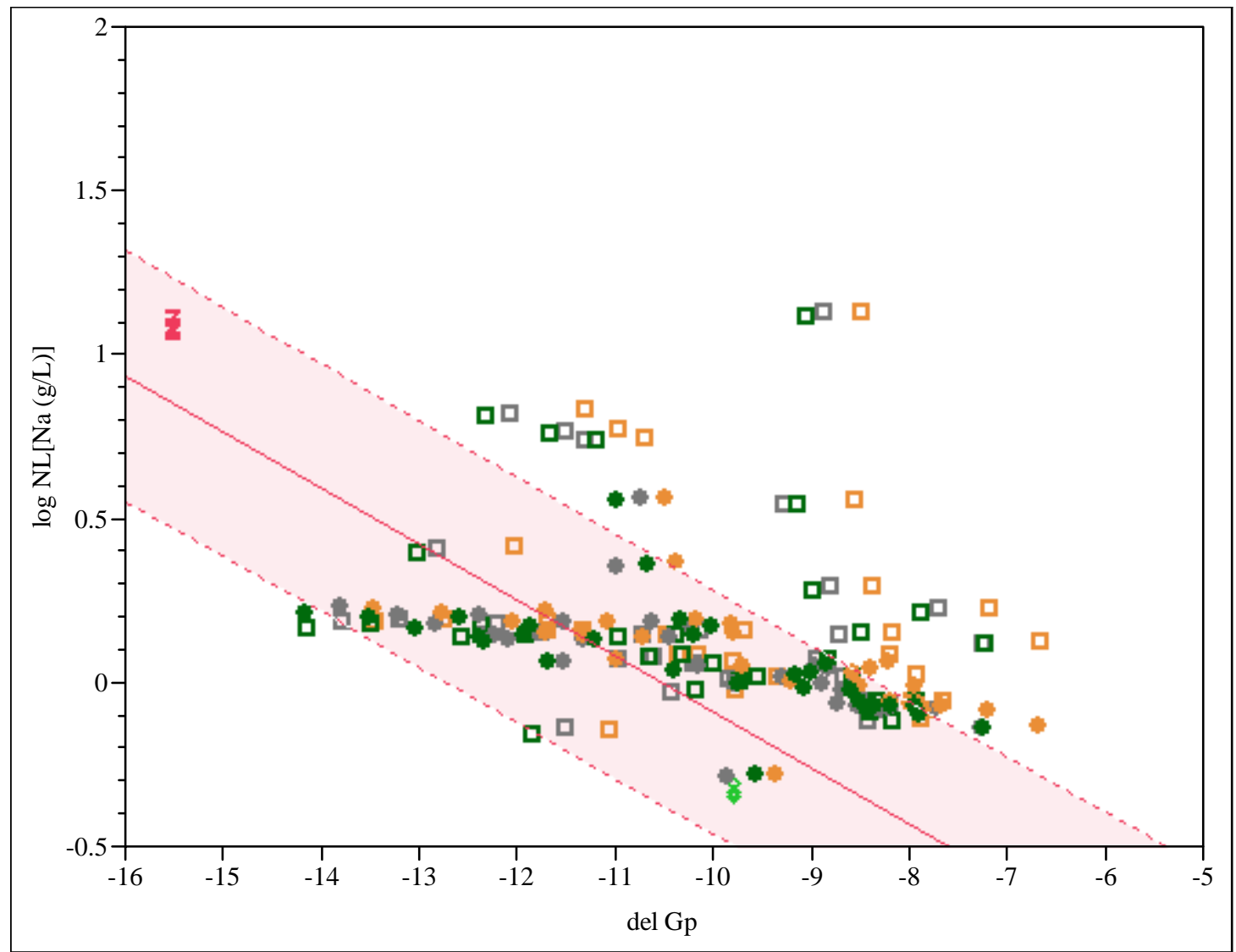


Exhibit E6. Effects of Heat Treatment for the Matrix 2A Study Glasses by Compositional View

Exhibit E10. del $G p\left(\Delta G_{p}\right)$ Predictions versus Common Logarithm Normalized Leachate $(\log \mathrm{NL}[]$.$) for Si over All Compositional Views and Heat Treatments for$ the Matrix 2A Study Glasses

\begin{tabular}{|c|c|}
\multicolumn{1}{c}{ Legend } \\
\hline Symbol & $\begin{array}{c}\text { Standard/ } \\
\text { Comp View-Heat Treatment }\end{array}$ \\
\hline $\mathrm{z}$ & EA \\
\hline$\diamond$ & ARM \\
\hline$\square$ & Measured-ccc \\
\hline$\square$ & Measured bc -ccc \\
\hline$\square$ & Targeted-ccc \\
\hline$\bullet$ & Measured-quenched \\
\hline$\bullet$ & Measured bc - quenched \\
\hline$\bullet$ & Targeted- quenched \\
\hline
\end{tabular}

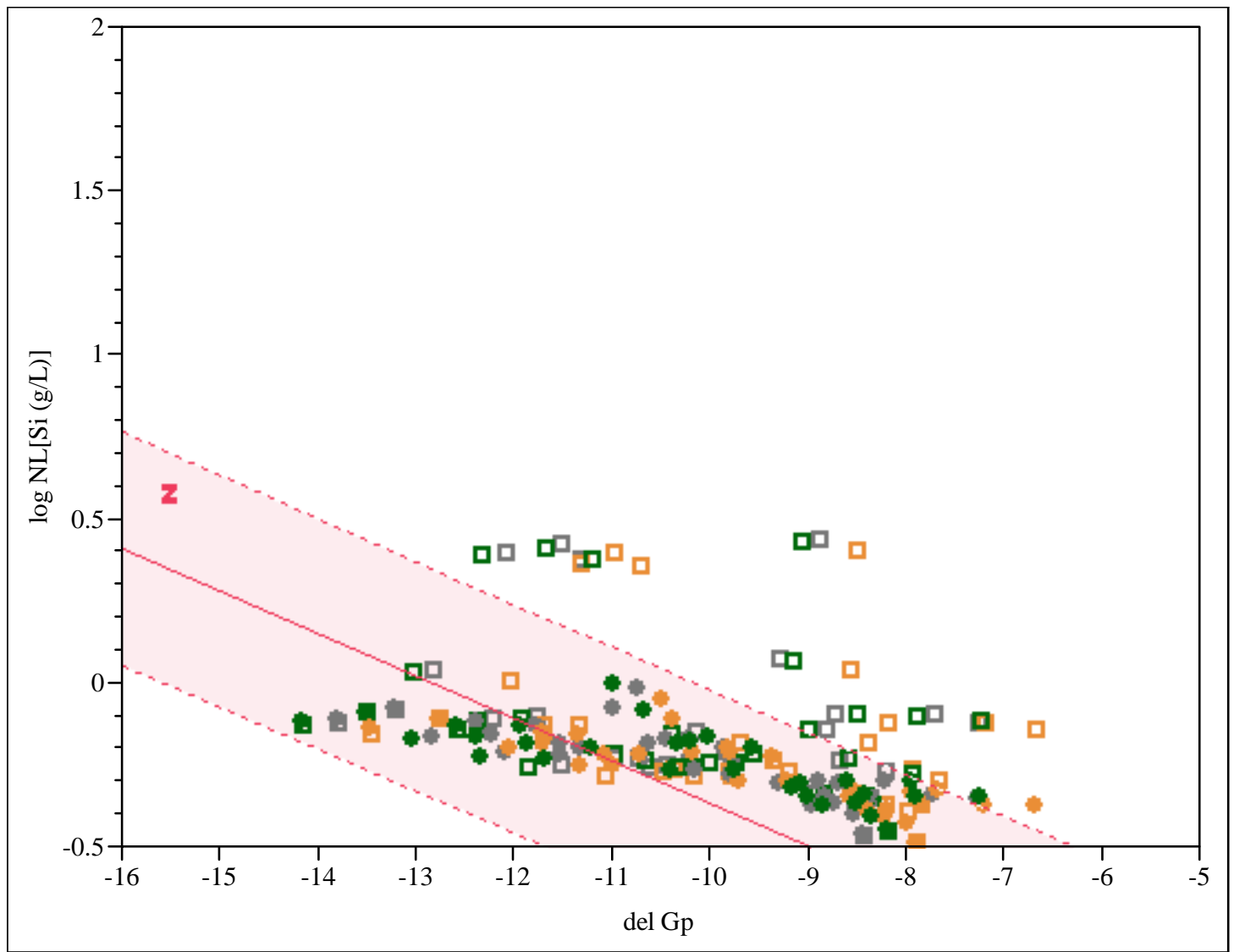




\section{Appendix F:}

\section{Exhibits Supporting the Analysis of the Viscosity Measurements of the FY07 Study Glasses}


Exhibit F1. VFT fit of HWL-01.

Nonlinear Fit Glass ID=HWL-01

Prediction Model

Response: $\ln (\mathrm{n}$; poise), Predictor: $\ln (\mathrm{n}$; VTF)

Control Panel

Converged in Gradient

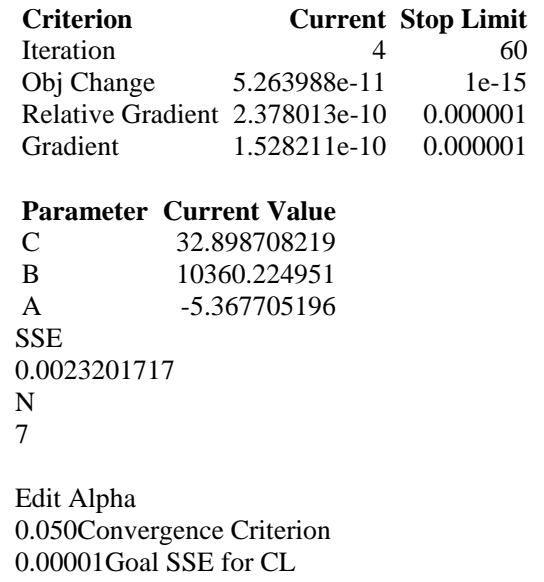

Plot

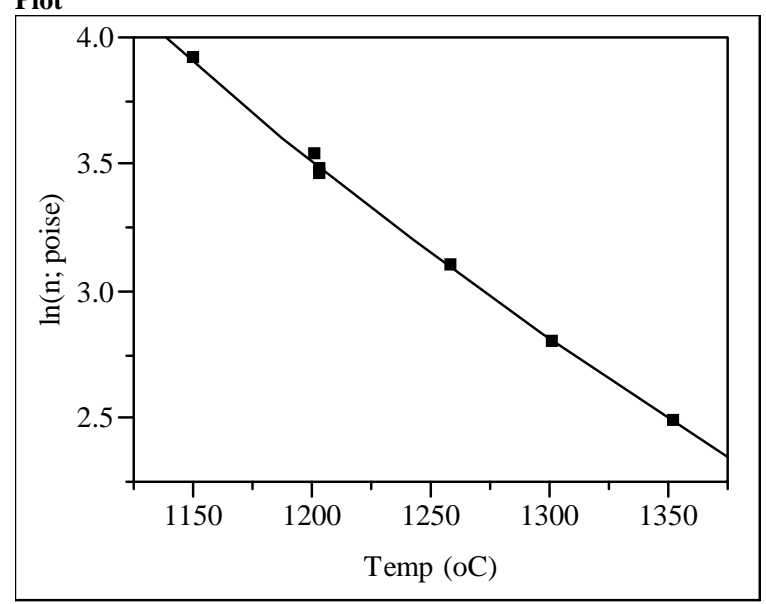

$$
\begin{array}{lrrr}
\text { Parameter } & \text { Estimate } & \text { Low } & \text { High } \\
\text { C } & 32.898708219 & 64.1049 & 192.315 \\
\text { B } & 10360.224951 & 3212.47 & 9637.41 \\
\text { A } & -5.367705196 & -5.8424 & -1.9475
\end{array}
$$

Solution

$$
\text { SSE DFE MSE RMSE }
$$$$
\begin{array}{lrrrr}
0.0023201717 & 4 & 0.00058 & 0.0240841
\end{array}
$$

$\begin{array}{lrr}\text { Parameter } & \text { Estimate } & \text { ApproxStdErr } \\ \text { C } & 32.898708219 & 487.792693 \\ \text { B } & 10360.224951 & 8337.50906 \\ \text { A } & -5.367705196 & 3.43123739\end{array}$

Solved By:

Analytic NR

\section{Exhibit F2. VFT fit of HWL-02.}

Nonlinear Fit Glass ID=HWL-02

Prediction Model

Response: $\ln (n$; poise), Predictor: $\ln (\mathrm{n}$; VTF)

Control Panel

Converged in Gradient

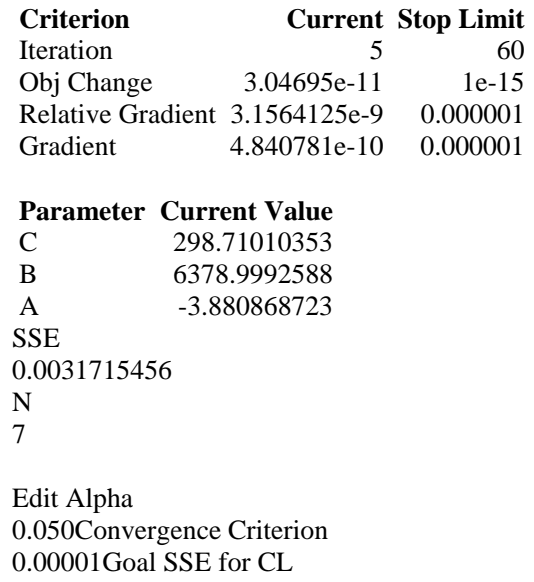

Plot

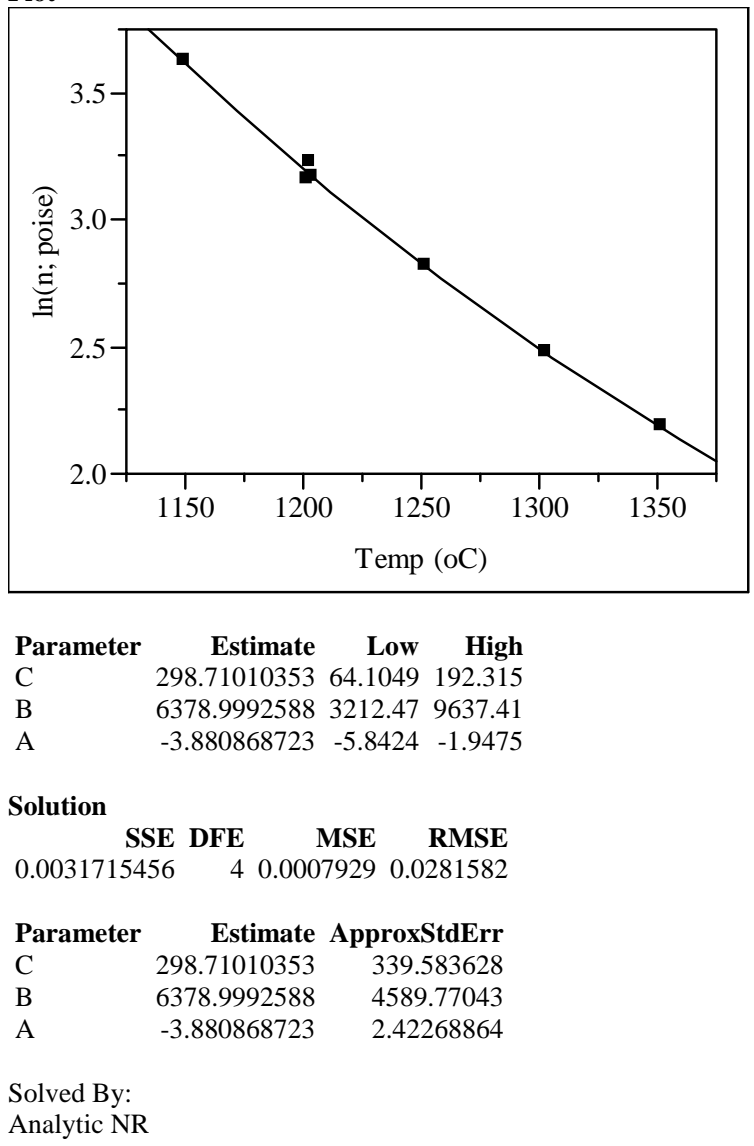




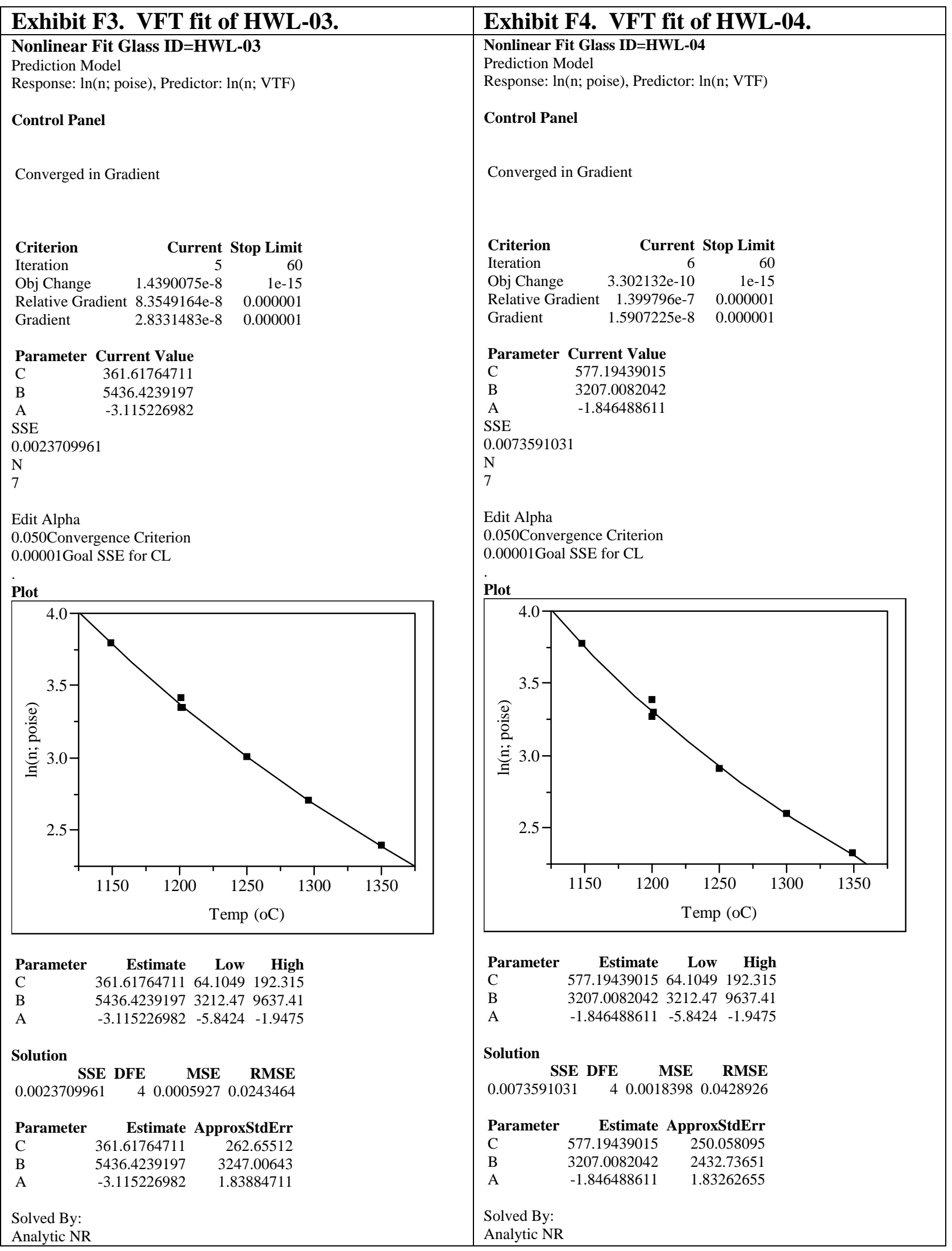




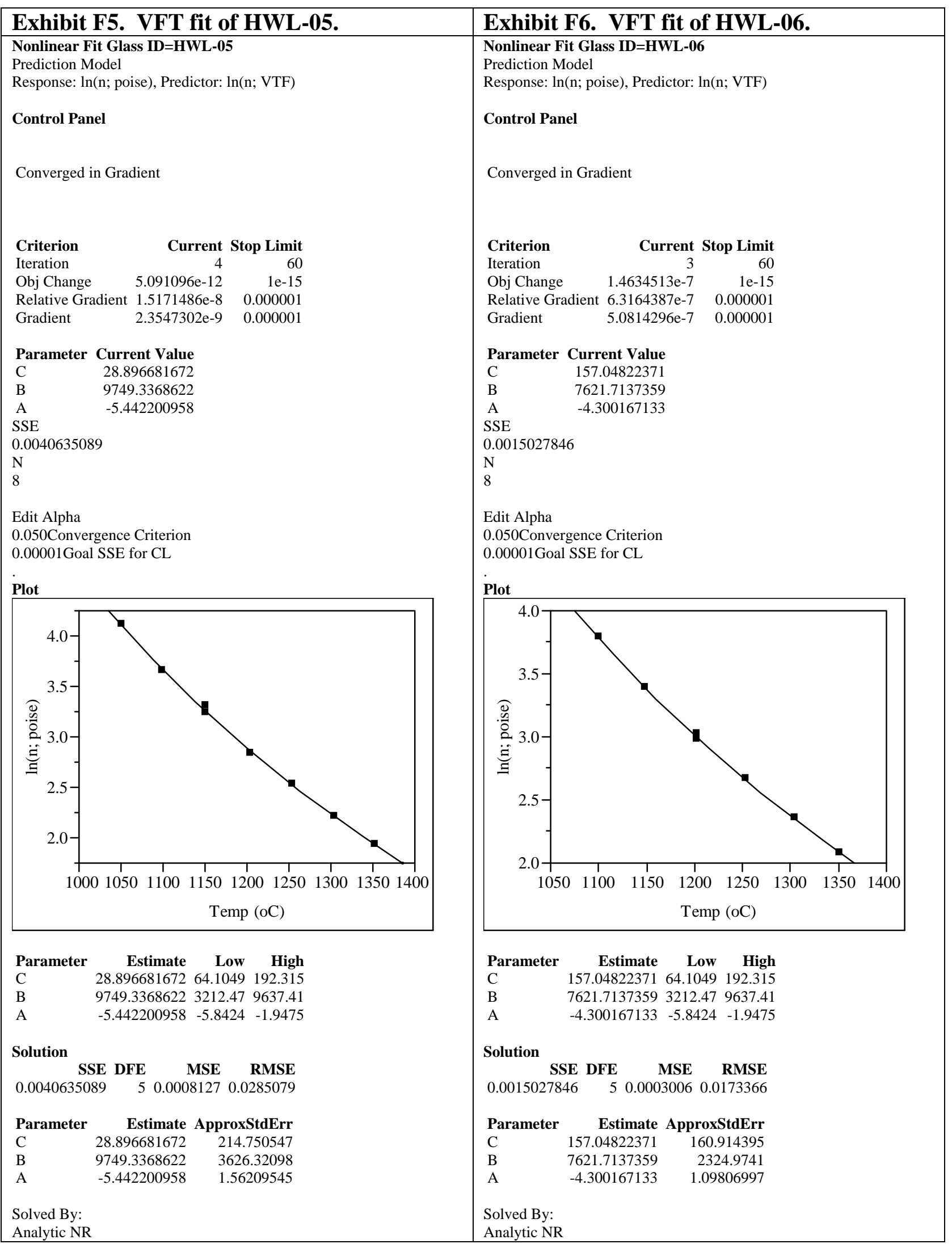




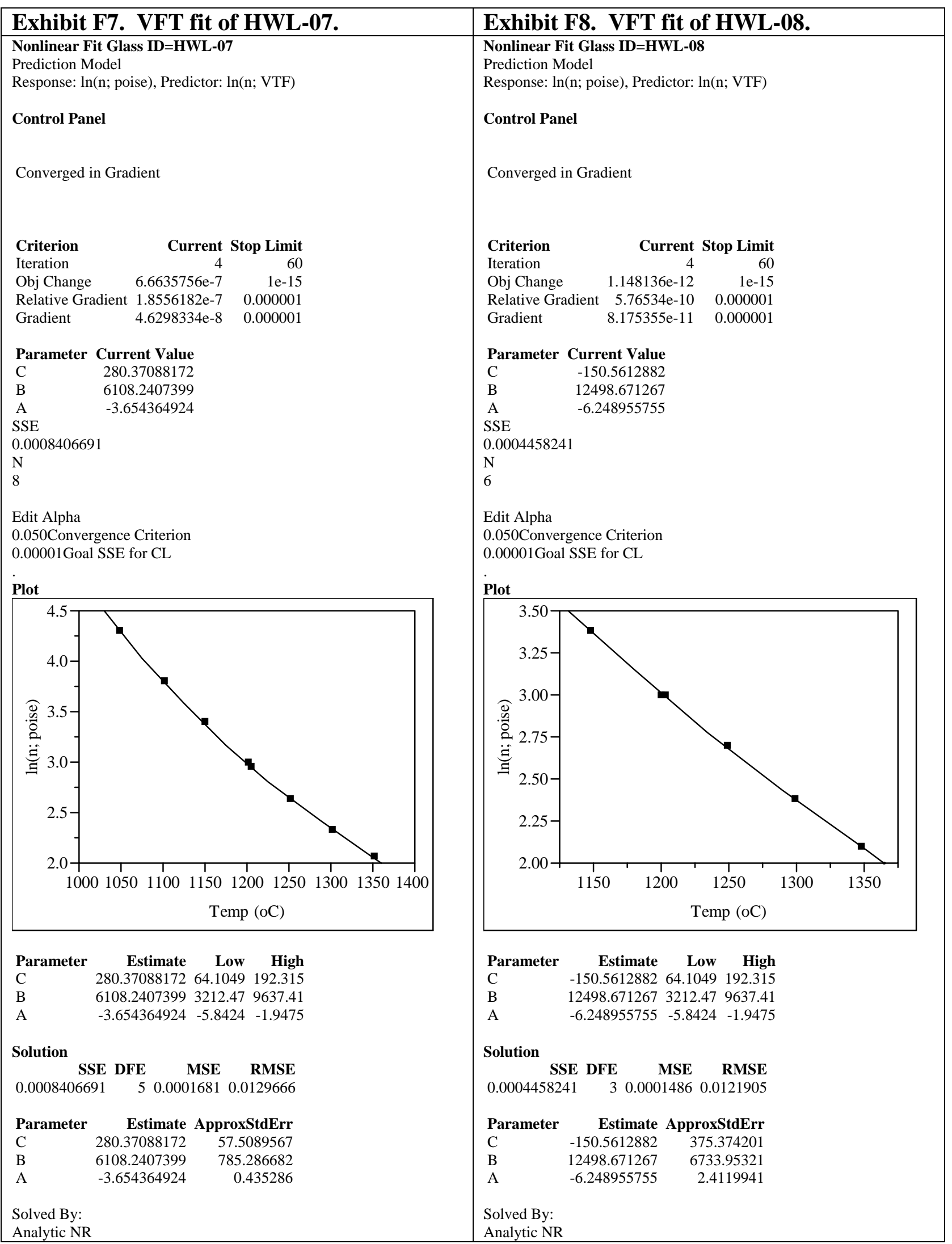




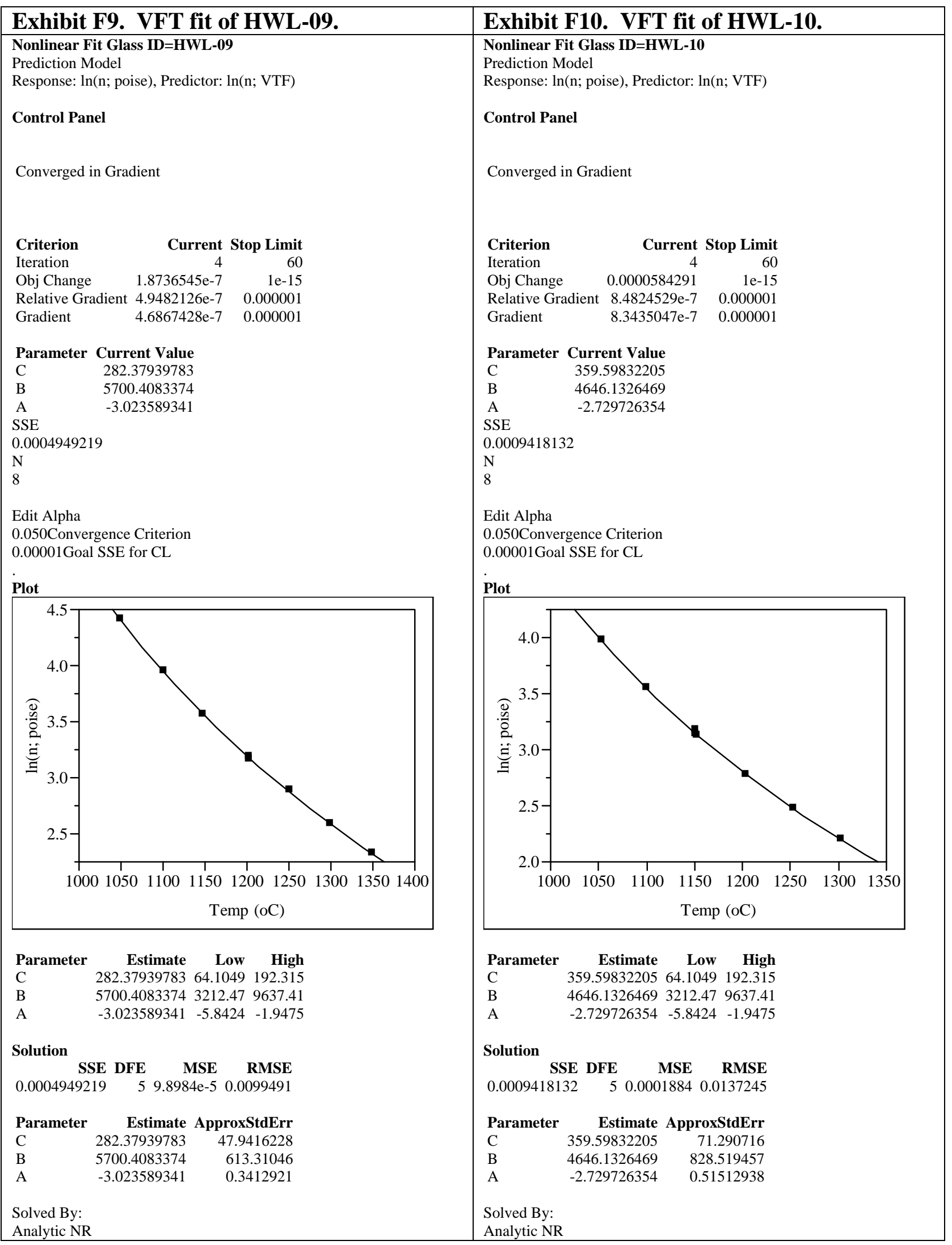




\begin{tabular}{|c|c|}
\hline Exhibit F11. VFT fit of HWL-11. & Exhibit F12. VFT fit of HWL-12. \\
\hline $\begin{array}{l}\text { Nonlinear Fit Glass ID=HWL-11 } \\
\text { Prediction Model } \\
\text { Response: } \ln (n ; \text { poise), Predictor: } \ln (n ; \text { VTF) }\end{array}$ & $\begin{array}{l}\text { Nonlinear Fit Glass ID=HWL-12 } \\
\text { Prediction Model } \\
\text { Response: } \ln (n \text {; poise), Predictor: } \ln (n ; \text { VTF) }\end{array}$ \\
\hline Control Panel & Control Panel \\
\hline Converged in Gradient & Converged in Gradient \\
\hline $\begin{array}{lrr}\text { Criterion } & \text { Current } & \text { Stop Limit } \\
\text { Iteration } & 3 & 60 \\
\text { Obj Change } & 3.4865106 \mathrm{e}-7 & 1 \mathrm{e}-15 \\
\text { Relative Gradient } & 4.1635725 \mathrm{e}-7 & 0.000001 \\
\text { Gradient } & 3.076968 \mathrm{e}-7 & 0.000001\end{array}$ & $\begin{array}{lrr}\text { Criterion } & \text { Current } & \text { Stop Limit } \\
\text { Iteration } & 4 & 60 \\
\text { Obj Change } & 1.204915 \mathrm{e}-11 & 1 \mathrm{e}-15 \\
\text { Relative Gradient } & 4.088051 \mathrm{e}-8 & 0.000001 \\
\text { Gradient } & 9.7855589 \mathrm{e}-9 & 0.000001\end{array}$ \\
\hline $\begin{array}{lr}\text { Parameter } & \text { Current Value } \\
\mathrm{C} & 77.810378009 \\
\mathrm{~B} & 8458.6788468 \\
\mathrm{~A} & -4.923236692 \\
\mathrm{SSE} & \\
0.0084790265 \\
\mathrm{~N} \\
8 \\
\\
\text { Edit Alpha } \\
0.050 \text { Convergence Criterion } \\
0.00001 \text { Goal SSE for CL } \\
\text { Plot }\end{array}$ & $\begin{array}{lr}\text { Parameter } & \text { Current Value } \\
\mathrm{C} & 194.73864068 \\
\mathrm{~B} & 6659.9801306 \\
\mathrm{~A} & -3.348813405 \\
\mathrm{SSE} & \\
0.0003630188 \\
\mathrm{~N} \\
7 \\
\\
\text { Edit Alpha } \\
0.050 \text { Convergence Criterion } \\
0.00001 \text { Goal SSE for CL } \\
\text { Plot }\end{array}$ \\
\hline 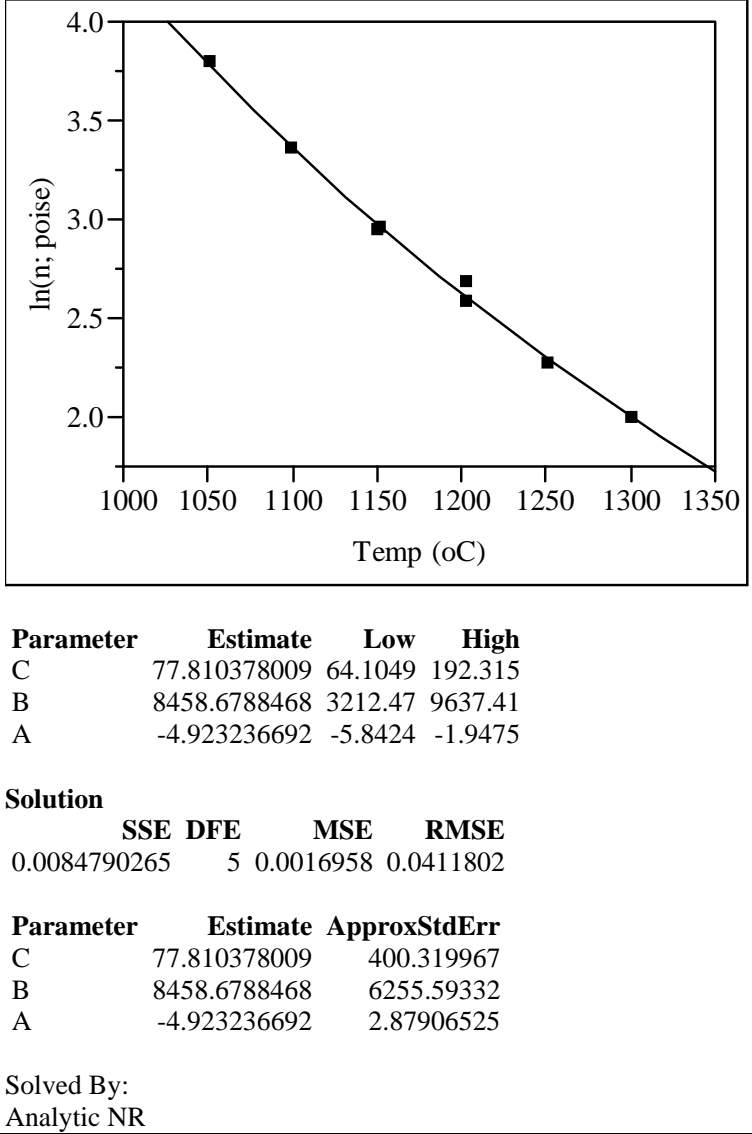 & 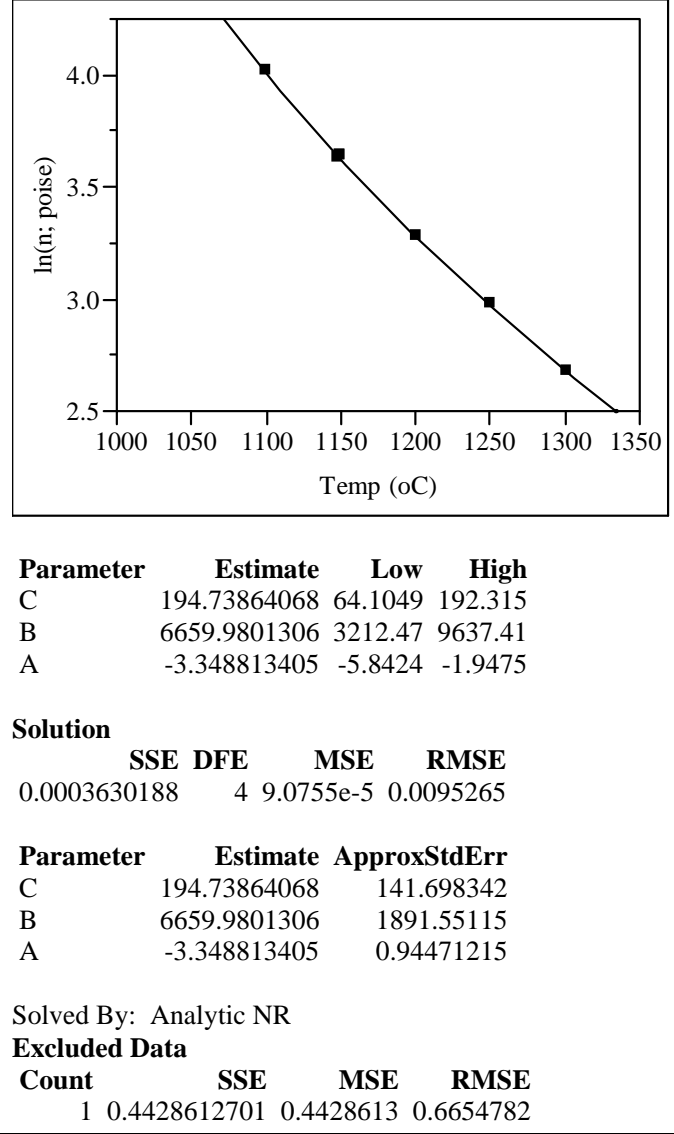 \\
\hline
\end{tabular}




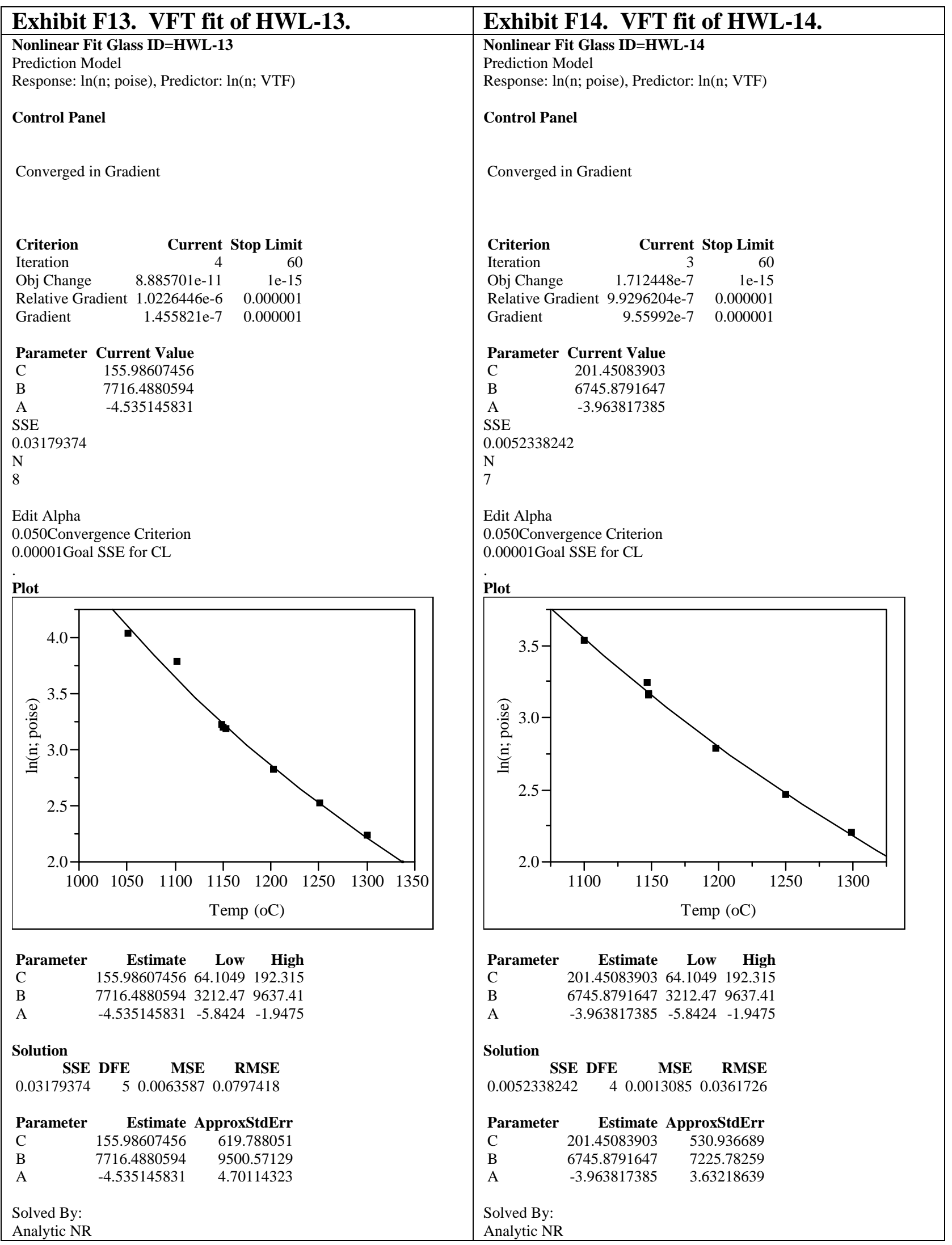




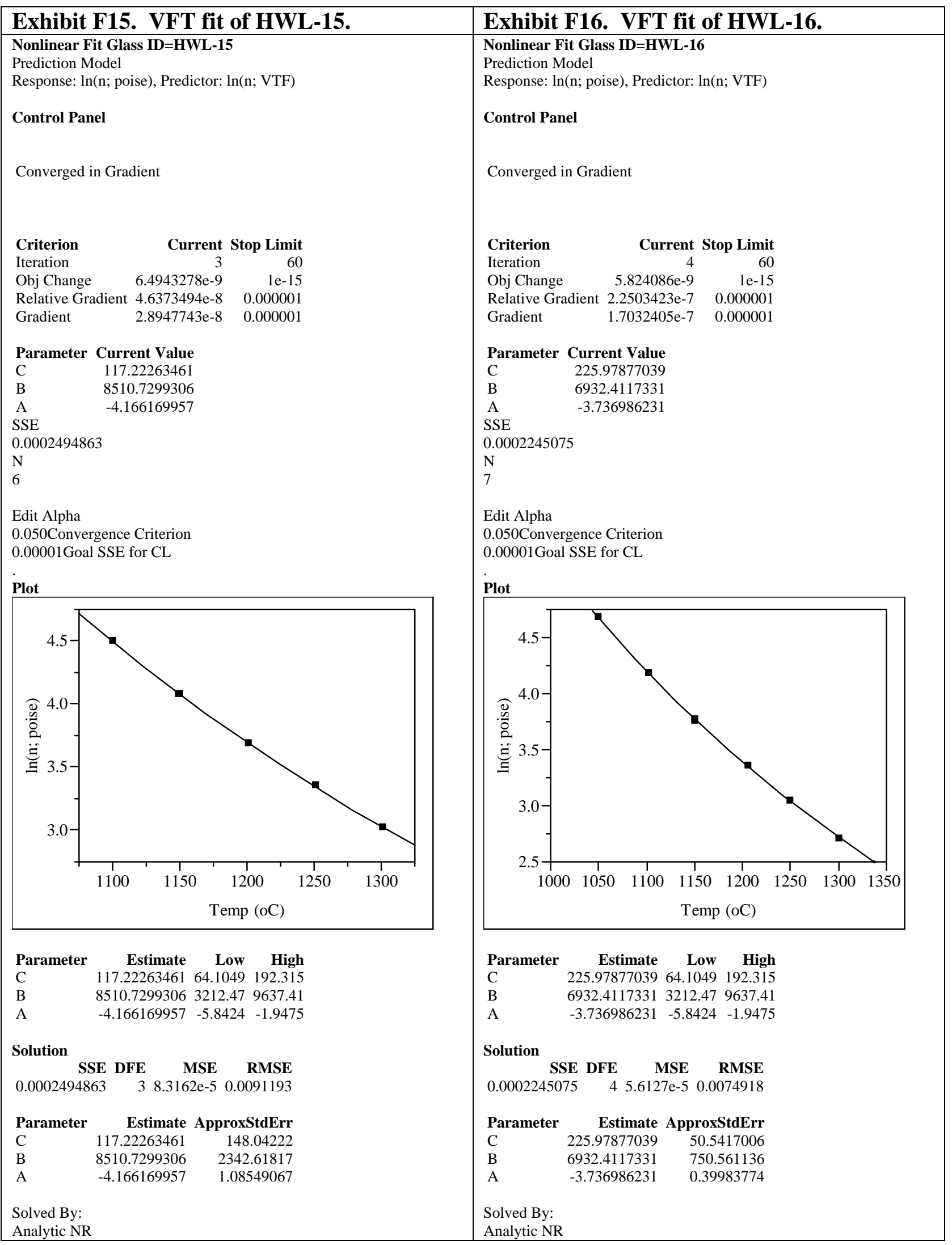




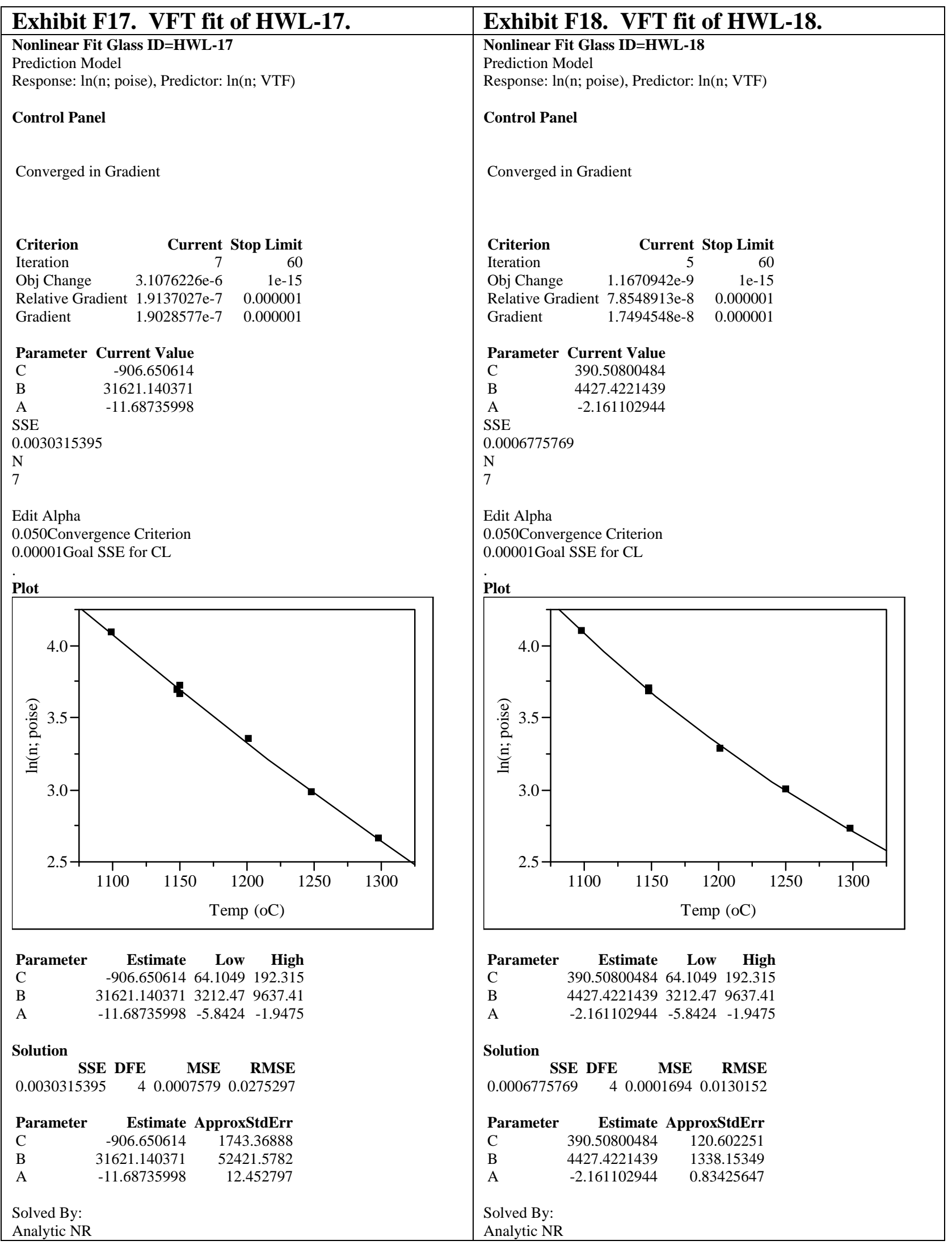




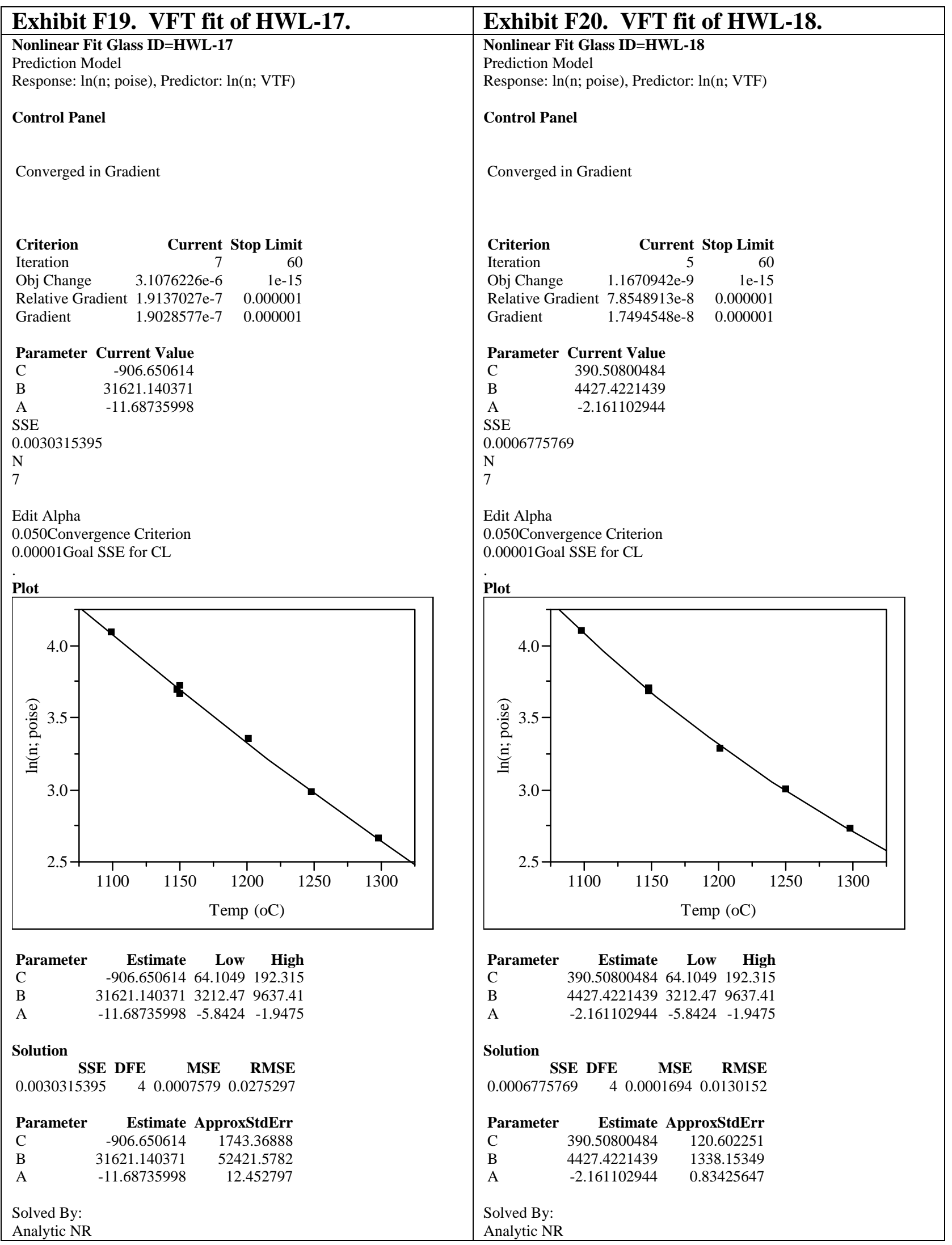




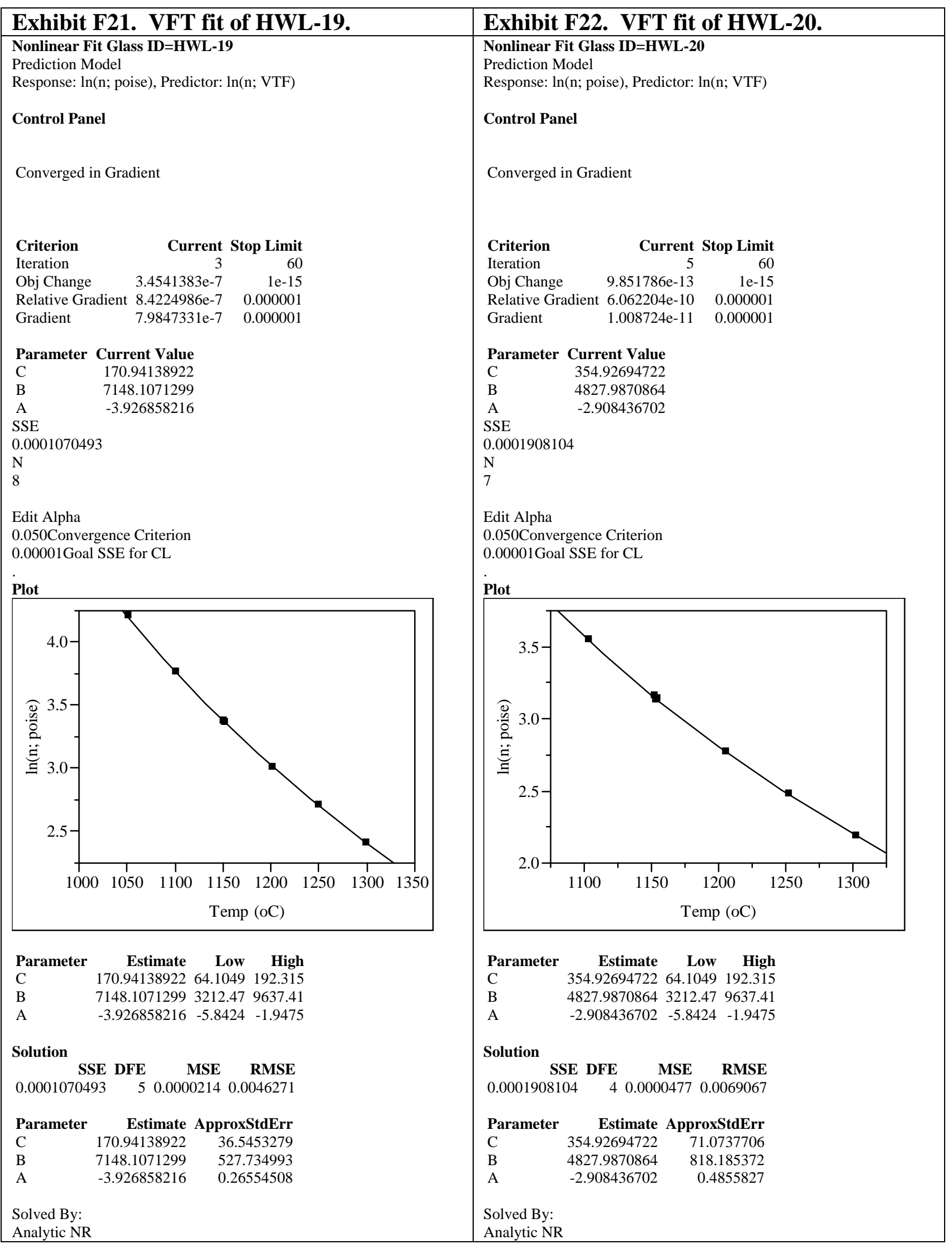




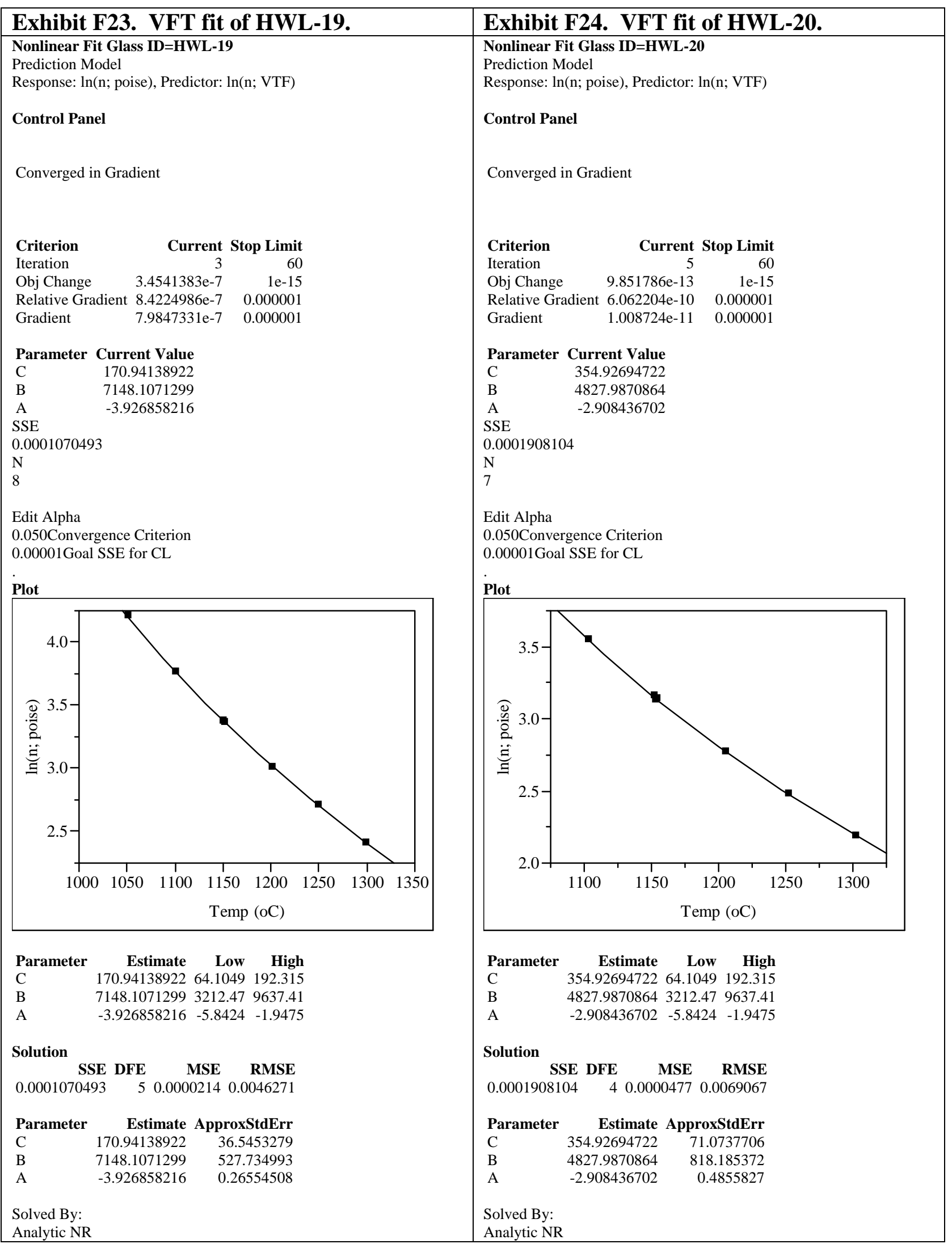




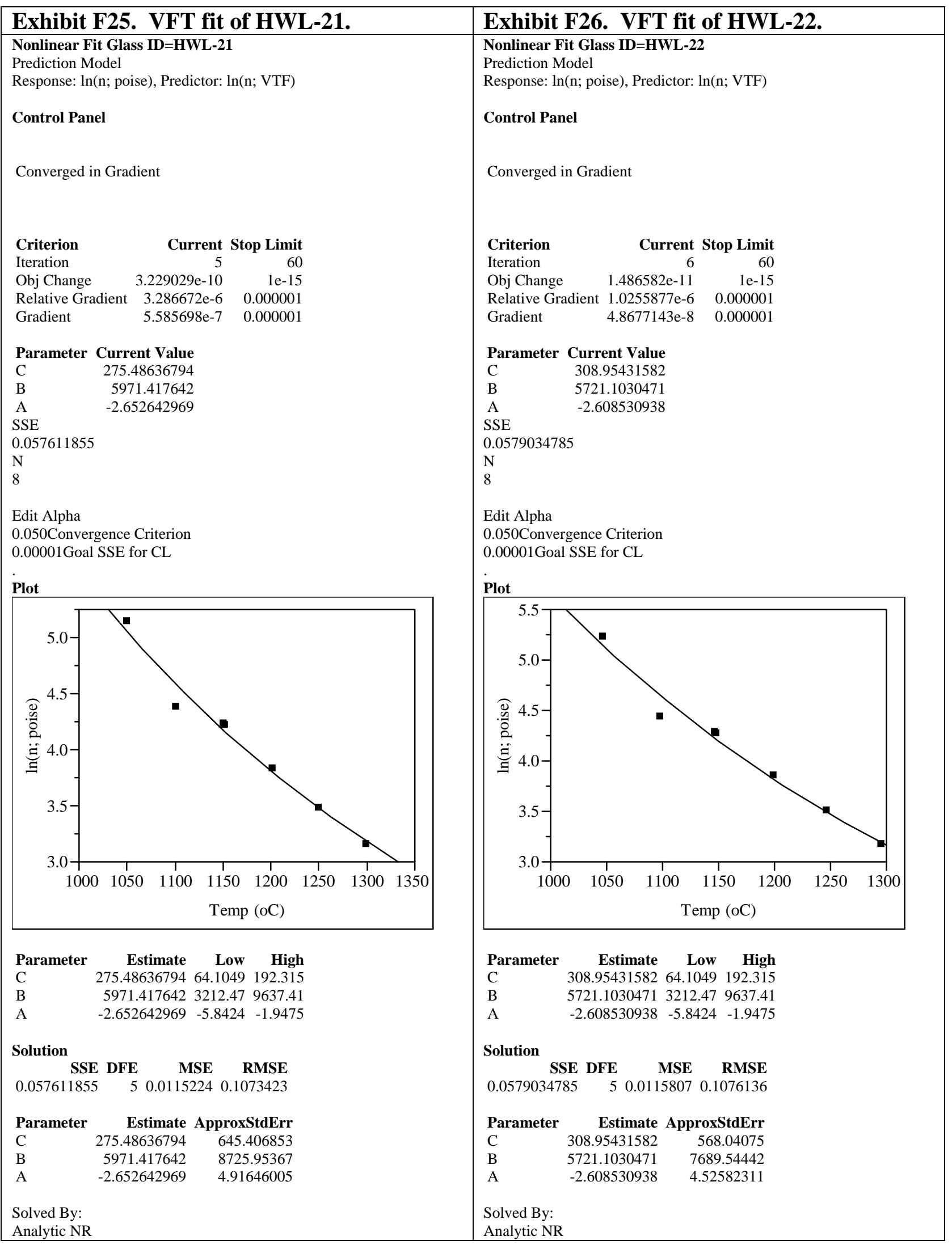


Table F1. Viscosity at $1150^{\circ} \mathrm{C}$ Determined from the Fitted Data

\begin{tabular}{|c|c|}
\hline Glass ID & Viscosity from Fit (P) \\
\hline HWL-01 & 49.72 \\
\hline HWL-02 & 37.06 \\
\hline HWL-03 & 43.84 \\
\hline HWL-04 & 42.61 \\
\hline HWL-05 & 25.89 \\
\hline HWL-06 & 29.24 \\
\hline HWL-07 & 29.07 \\
\hline HWL-08 & 28.83 \\
\hline HWL-09 & 34.69 \\
\hline HWL-10 & 23.30 \\
\hline HWL-11 & 19.41 \\
\hline HWL-12 & 37.45 \\
\hline HWL-13 & 25.22 \\
\hline HWL-14 & 23.29 \\
\hline HWL-15 & 58.82 \\
\hline HWL-16 & 43.18 \\
\hline HWL-17 & 39.95 \\
\hline HWL-18 & 39.19 \\
\hline HWL-19 & 29.20 \\
\hline HWL-20 & 23.66 \\
\hline HWL-21 & 65.08 \\
\hline HWL-22 & 66.28 \\
\hline
\end{tabular}

